\title{
Nitrogen-neighboured single cobalt sites enable heterogeneous oxidase-type catalysis
}

\section{Yong Cao ( $\nabla$ yongcao@fudan.edu.cn )}

Fudan University

Qi zhang

Mi Peng

Peking University

\section{Zirui Gao}

Peking University

\section{Wendi Guo}

Fudan University

\section{Zehui Sun}

Fudan University

\section{Yi Zhao}

Fudan University

\section{Wu Zhou}

University of Chinese Academy of Sciences https://orcid.org/0000-0002-6803-1095

\section{Meng Wang}

Peking University

\section{Bingbao Mei}

Shanghai Institute of Applied Physics

\section{Xianlong Du}

Shanghai Institute of Applied Physics

\section{Zheng Jiang}

Shanghai Advanced Research Institute https://orcid.org/0000-0003-4297-464X

\section{Wei Sun}

Lanzhou Institute of Chemical Physics

Chao Liu

Lanzhou Institute of Chemical Physics, Chinese Academy of Sciences https://orcid.org/0000-00022521-924X

\section{Yifeng Zhu}

Dalian Institute of Chemical Physics

\section{Yongmei Liu}

Fudan University

Heyong He 
Fudan University

Zhen Li

Fudan University

\section{Ding Ma}

Peking University https://orcid.org/0000-0002-3341-2998

\section{Physical Sciences - Article}

Keywords:

Posted Date: January 4th, 2022

DOI: https://doi.org/10.21203/rs.3.rs-1093028/v1

License: (c) (1) This work is licensed under a Creative Commons Attribution 4.0 International License. Read Full License 

catalysis

Authors: Qi Zhang, ${ }^{1,8}$ Mi Peng, ${ }^{2,8}$ Zirui Gao, ${ }^{2,8}$ Wendi Guo, ${ }^{1,8}$ Zehui Sun, ${ }^{1}$ Yi Zhao, ${ }^{1}$ Wu Zhou, ${ }^{3,4 *}$ Meng Wang, ${ }^{2}$ Bingbao Mei, ${ }^{5,6}$ Xianlong Du, ${ }^{5}$ Zheng Jiang, ${ }^{5,6}$ Wei Sun, ${ }^{7}$ Chao Liu, ${ }^{7}$ Yifeng Zhu, ${ }^{1}$ Yongmei Liu, ${ }^{1}$ Heyong He,${ }^{1}$ Zhen Hua Li, ${ }^{1} *$ Ding Ma, ${ }^{2 *}$ Yong $\mathrm{Cao}^{1 *}$

\section{Affiliations:}

${ }^{1}$ Shanghai Key Laboratory of Molecular Catalysis and Innovative Materials, Department of Chemistry, Fudan University, Shanghai, 200438, China.

${ }^{2}$ Beijing National Laboratory for Molecular Engineering, College of Chemistry and Molecular Engineering, Peking University, Beijing, 100871, China.

${ }^{3}$ School of Physical Sciences and CAS Key Laboratory of Vacuum Physics, University of Chinese Academy of Sciences, Beijing, 100049, China.

${ }^{4}$ CAS Center for Excellence in Topological Quantum Computation, University of Chinese Academy of Sciences, Beijing, 100049, China.

${ }^{5}$ Shanghai Institute of Applied Physics, Chinese Academy of Sciences, Shanghai, 201204, China.

${ }^{6}$ Shanghai Synchrotron Radiation Facility, Zhangjiang Lab, Shanghai Advanced Research Institute, Chinese Academy of Sciences, Shanghai, 201204, China.

${ }^{7}$ State Key Laboratory for Oxo Synthesis and Selective Oxidation, Suzhou Research Institute, Lanzhou Institute of Chemical Physics, Chinese Academy of Sciences, Lanzhou, 730000, China.

${ }^{8}$ These authors contributed equally to this work: Qi Zhang, Mi Peng, Zirui Gao, Wendi Guo.

*e-mail: yongcao@fudan.edu.cn (Y.C.); dma@pku.edu.cn (D.M.); lizhenhua@ fudan.edu.cn (Z.H.L.); wuzhou@ucas.ac.cn (W.Z.) 
Development of biomimetic catalytic systems that can imitate or even surpass natural enzymes remains an ongoing challenge ${ }^{1-3}$. This is particularly true in the context of accessing non-natural reactions by bioinspired approaches, which offer intriguing possibilities for benign and affordable chemical synthesis ${ }^{4}$. Exploiting the untapped potential of inorganic solids by translating complex knowledge in (bio)molecular-based systems may constitute a potentially useful strategy for such purpose ${ }^{\mathbf{5}}$, but efforts to capitalize on the minimum catalytic unit of a versatile solid matrix have been largely unsuccessful. Here, we show how an all-inorganic biomimetic system bearing robust nitrogen-neighboured single cobalt site/pyridinic-N site $\left(\mathrm{Co}-\mathrm{N}_{4} / \mathrm{Py}-\mathrm{N}\right)$ pairs can act cooperatively as an oxidase mimic, which renders an engaged coupling of oxygen $\left(\mathrm{O}_{2}\right)$ reduction with synthetically beneficial chemical transformations. By developing this broadly applicable platform, the scalable synthesis of greater than 100 industrially and pharmaceutically appealing $O$-silylated compounds via the unprecedented aerobic oxidation of hydrosilane under ambient conditions is demonstrated. Moreover, this heterogeneous oxidase mimic also offers potential for expanding the catalytic scope of enzymatic synthesis. We anticipate that the strategy demonstrated here will pave a new avenue for understanding the underlying nature of redox enzymes and open up a new class of material systems for artificial biomimetics.

Expansion of mild selective oxidation routes that use $\mathrm{O}_{2}$ is one of the ultimate goals of chemical research ${ }^{6}$. However, the exquisite control of $\mathrm{O}_{2}$-consuming reactions still remains a daunting task due to the kinetic non-reactivity of $\mathrm{O}_{2}$ without catalytic intervention ${ }^{7}$. In nature, evolution has afforded efficient and selective oxidases, which constitute a class of oxygenutilizing enzymes that can catalyse the oxidation of a substrate via the co-generation of $\mathrm{H}_{2} \mathrm{O}_{2}$ (or $\mathrm{H}_{2} \mathrm{O}$ ) using $\mathrm{O}_{2}$ as the terminal electron acceptor ${ }^{8}$. A particularly compelling scenario across the oxidase superfamilies hinges on the interplay between redox and base centres to drive a reaction cycle via the so-called proton-coupled hydride transfer (PCHT) pathway (Fig. 1A) ${ }^{9,10}$. 
Despite the fact that natural enzymes are synthetically beneficial in this context, they are expensive and highly sensitive to reaction conditions. Moreover, their inherent poor operational stability severely limits their practical utility. Therefore, it is imperative to develop new chemical approaches that mimic oxidase-like activity. In this regard, the molecular design of metal-ligand complexes as oxidase mimics has attracted attention ${ }^{11-14}$. However, attempts to mimic the remarkable ability of oxidases with organic-free materials have been mainly unsuccessful.

a
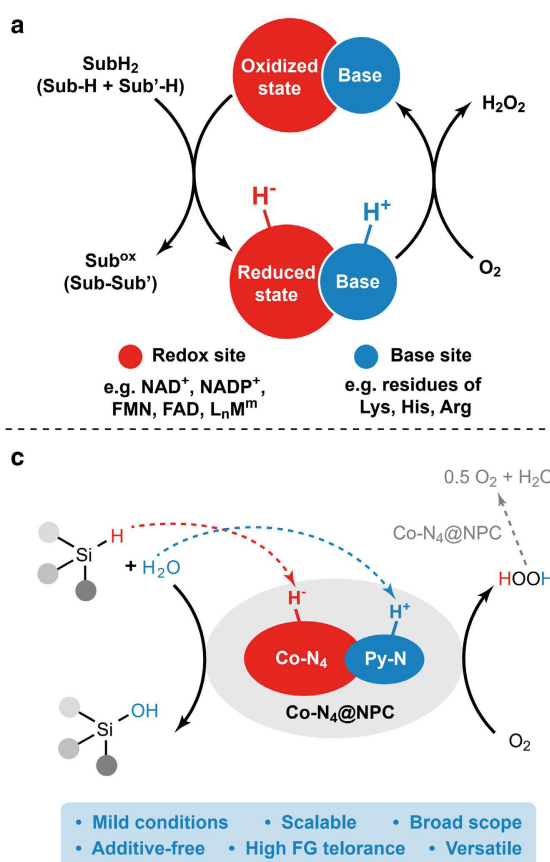

b

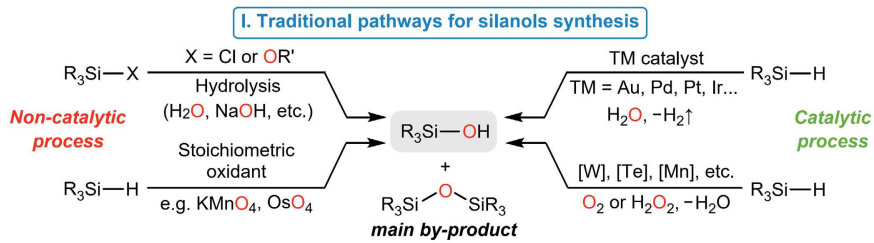

II. Silanols synthesis via enzymatic approach (oxygenase-type oxidation) - State of the Art $\mathrm{R}_{3} \mathrm{Si}-\mathrm{H}$ P450 variants, NADPH

$\mathrm{O}_{2},-\mathrm{H}_{2} \mathrm{O} \longrightarrow \mathrm{R}_{3} \mathrm{Si} \cdot \longrightarrow$ radical round Can silanols be synthesized via oxidase-type oxidation process?

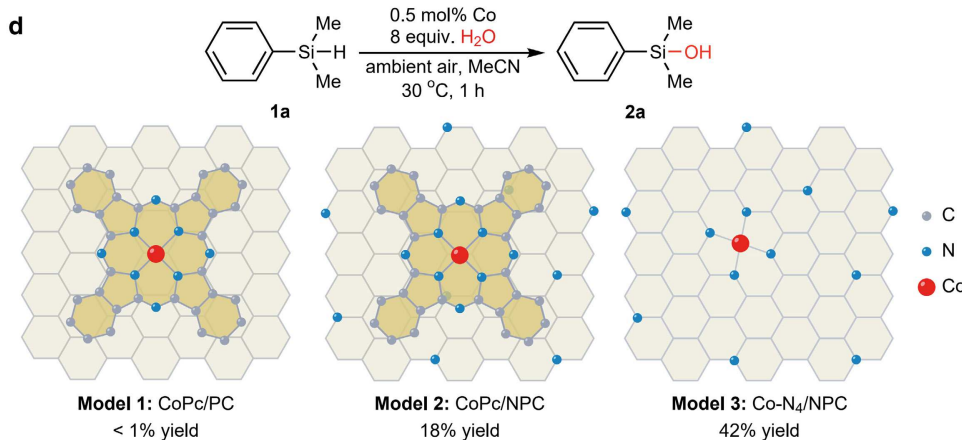

Fig. 1 | Oxidase-inspired aerobic hydrosilane-oxidizing. a, Simplified catalytic cycle of

oxidase-catalyzed aerobic oxidation via PCHT pathway. b, Classical silanol synthesis methods.

c, Proposed oxidase-type hydrosilane oxidation process catalyzed by all-inorganic biomimetics bearing independent redox-active Co- $\mathrm{N}_{4}$ sites and basic Py-N sites. d, CoPc-based or -derived oxidase-mimicking models for selective silane oxidation. Reactions were performed on $1 \mathrm{mmol}$ scale, see supplementary materials catalytic performance evaluation section for details. Yields were determined by GC using anisole as an internal standard. 
In this study, we present a new design of an all-inorganic biomimetic system which exhibits an oxidase-type catalytic activity far superior to that of natural enzymes. Silanols are key precursors for constructing Si-based structural motifs, which are found in a number of advanced functional materials ${ }^{15}$. In addition, silanols have been reported as pivotal intermediates for the synthesis of challenging bioactive agents in experimental pharmacology ${ }^{16,17}$ and as catalysts because of their anion recognition ability ${ }^{18}$. Currently, silanols are mainly synthesized by the hydrolysis of chlorosilanes or oxidation of hydrosilanes by using strong oxidants (e.g., permanganate and $\mathrm{OsO}_{4}$, etc.), which often produces toxic waste and involves tedious procedures (Fig. 1B, I, left paths) ${ }^{15,19}$. The transition-metal-catalysed hydrolytic conversion of hydrosilanes to silanols has been reported often using $\mathrm{H}_{2} \mathrm{O}_{2}$ as the oxidant (Fig. 1B, I, right paths) ${ }^{20}$. Recently, the selective oxygenase-type oxidation of silanes to silanols using $\mathrm{O}_{2}$ with engineered cytochrome $\mathrm{P} 450$ enzymes has been reported (Fig. $\left.1 \mathrm{~B}, \mathrm{II}\right)$ 21. Unfortunately, the reported catalytic activity is extremely dependent on the reaction conditions, and the associated high costs can be a significant limitation for large-scale synthesis.

To realize the selective oxidase-type oxidation of silanes, a reaction that involves the PCHT-initiated co-activation of silane and $\mathrm{H}_{2} \mathrm{O}$ and subsequent reaction of the resulting intermediate with $\mathrm{O}_{2}$ to yield silanols and $\mathrm{H}_{2} \mathrm{O}_{2}$ was envisioned. By accommodating isolated redox-active centers on a suitably basified surface, such a process was anticipated to be realized (Fig. 1C). Cobalt phthalocyanine $(\mathrm{CoPc})$ complexes are known to activate $\mathrm{O}_{2}$ under mild conditions ${ }^{22}$. It could be combined with an $\mathrm{N}$-doped carbon matrix bearing Lewis basic $\mathrm{N}$ sites to construct a dual-site biomimics, which is the starting point for the development of our catalyst. As a preliminary proof-of-concept, three designs were investigated in which the nature of cobalt sites as well as the availability of $\mathrm{N}$ species in the underlying carbonaceous supports derived from zeolitic imidazolate framework-8 (ZIF-8) [i.e. porous carbon (PC) and N-doped PC (NPC)] was changed (Supplementary Fig. 1): (i) CoPc deposited on PC (CoPc/PC), (ii) CoPc deposited on NPC (CoPc/NPC), and (iii) CoPc/NPC subjected to pyrolysis at $600{ }^{\circ} \mathrm{C}$ 
under flowing $\mathrm{Ar}\left(\mathrm{Co}-\mathrm{N}_{4} / \mathrm{NPC}\right)$. CoPc/PC and $\mathrm{CoPc} / \mathrm{NPC}$ were synthesized by the incipient wetness impregnation of the porous supports with $\mathrm{N}, \mathrm{N}$-dimethylformamide (DMF) solutions of CoPc and subsequent mild drying at $150{ }^{\circ} \mathrm{C}$. For the last catalyst, the precise location and coordination environment of Co sites were different from those of the CoPc/NPC sample (Supplementary Figs. 2 and 3).

For the aerobic oxidation of dimethylphenylsilane (1a, in a mixture of acetonitrile/ $\mathrm{H}_{2} \mathrm{O}$ under atmospheric air at $30{ }^{\circ} \mathrm{C}$ ), NPC-containing catalysts (i.e. $\mathrm{CoPc} / \mathrm{NPC}$ and $\mathrm{Co}-\mathrm{N}_{4} / \mathrm{NPC}$ ) afford the desired product (2a) in $18 \%$ and $42 \%$ yields, respectively (Fig. 1D), whereas the catalyst not doped with $\mathrm{N}$ in the corresponding support (CoPc/PC) was inactive. Furthermore, although CoPc/PC or NPC alone was barely active (Supplementary Table 1, entries 3 and 4), the reaction proceeded over a physical mixture of NPC and the CoPc/PC catalyst (Supplementary Table 1, entry 5), albeit with a considerably lower yield. In a set of control experiments conducted without $\mathrm{O}_{2}$ or $\mathrm{H}_{2} \mathrm{O}$ under otherwise similar reaction conditions, the transformation of the hydrosilane substrate was not observed (Supplementary Table 1, entries 6 and 7). In an additional control experiment using an electron-deficient analogue of CoPc/NPC (CoPc-16Cl/NPC), the yield of 2a noticeably increased (Supplementary Table 1, entry 8). For these experiments, benefits of coupling between $1 \mathrm{a} / \mathrm{H}_{2} \mathrm{O}$ and $\mathrm{O}_{2}$ were identified to be beneficial for this catalytic system. Notably, the high formation efficiency of $\mathbf{2 a}$ required extensive and substantive synergy, which was rendered by the close association of suitable single Co sites and reactive $\mathrm{N}$ species embedded in a carbon matrix.

To ensure an intimate proximity between the atomic Co and $\mathrm{N}$ sites and clarify the role of different $\mathrm{N}$ species, a series of ZIF-derived Co/N co-doped NPC catalysts $\left(\mathrm{Co}-\mathrm{N}_{4} @ \mathrm{NPC}-\mathrm{T}\right.$, where $\mathrm{T}$ denotes the pyrolysis temperature in ${ }^{\circ} \mathrm{C}$ ) were prepared by the confined pyrolysis of bimetallic $\mathrm{Co}_{1} \mathrm{Zn}_{50}$-ZIF crystals (see Methods and Supplementary Fig. 4 for catalyst preparation). Co-N $\mathrm{N}_{4} @$ NPC-900 exhibited the highest catalytic activity, with a turnover frequency (TOF) of $489 \mathrm{~h}^{-1}$ for the conversion of 1a (Supplementary Fig. 5, metal loading 
determined by inductively coupled plasma optical emission spectrometry in Supplementary Table 6). Under pressurized air, the reaction rate was further accelerated, and the TOF significantly increased up to $1853 \mathrm{~h}^{-1}$ (Fig. 2A, Supplementary Fig. 6). This performance was in contrast to that observed using the nanoparticulate Co catalyst embedded in the NPC matrix (Co-NPs@NPC), the performance of which was independent of $\mathrm{O}_{2}$ (Fig. 2A). Even under anaerobic conditions, Co-NPs@NPC afforded an equimolar amount of 2a and $\mathrm{H}_{2}$ (Supplementary Fig. 7), albeit with an extremely low efficiency. These results underscored the fact that the successful use of Co- $\mathrm{N}_{4} @ \mathrm{NPC}-900$ might implicate an $\mathrm{O}_{2}$-mediated PCHT pathway in lieu of a traditional hydrolytic oxidation process and that the efficiency of the Co site most likely depends on the access to a specific type of neighbouring $\mathrm{N}$ species.

The sole presence of isolated Co sites in Co- $\mathrm{N}_{4} @$ NPC-based catalysts was confirmed by aberration-corrected high-angle annular dark-field scanning transmission electron microscopy (HAADF-STEM) (Fig. 2B-D, Supplementary Fig. 8D-F) coupled with electron energy-loss spectroscopy (EELS) measurements (Fig. 2E, Supplementary Figs. 9 and 10). X-ray absorption spectroscopy (XAS) analysis revealed the absence of a Co-Co bond in the Co- $\mathrm{N}_{4} @ \mathrm{NPC}$ catalysts (Fig. 2F, Supplementary Fig. 11B). The single peak centred at $1.4 \AA$ verified the coordination of cobalt to nitrogen (and possibly carbon as well) in the host. Extended X-ray absorption fine structure (EXAFS) fitting revealed that the Co-N coordination number is $\sim 4$ (Supplementary Table 2), indicative of the presence of Co- $\mathrm{N}_{4}$ motifs. Notably, XAS measurements (Supplementary Fig. 12) and HAADF-STEM imaging revealed that $\mathrm{Zn}$ in the sample also was atomically dispersed with the absence of a $\mathrm{Zn}-\mathrm{Zn}$ bond. Co $2 p \mathrm{X}$-ray photoelectron spectroscopy (XPS) (Supplementary Fig. 13B) and Co K-edge X-ray absorption near-edge structure (XANES) (Supplementary Fig. 11A) analysis revealed identical results for all Co- $\mathrm{N}_{4} @$ NPC-based samples. The N $1 s$ XPS featured five species bearing different orders of Lewis basicity, among which Py-N with a binding energy of $398.3 \mathrm{eV}^{23}$ was the most relevant $\mathrm{N}$ species based on the correlation of the relative $\mathrm{N}$ content evolution with the 

Figs. 11 and 13). This characterization provided reasonable verification for our structural hypothesis as well as evidence substantiating the importance of the interplay between neighbouring Py-N and $\mathrm{Co}-\mathrm{N}_{4}$ sites to render oxidative catalysis.

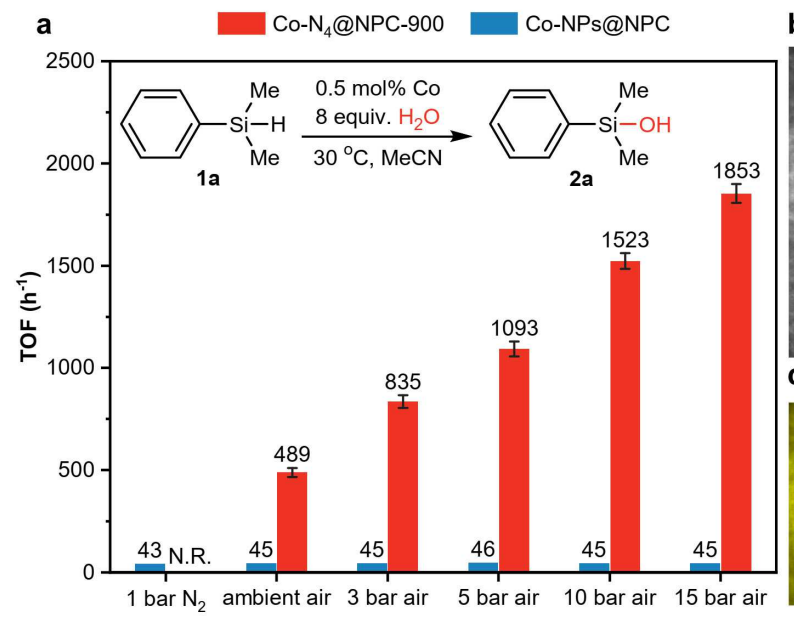

e
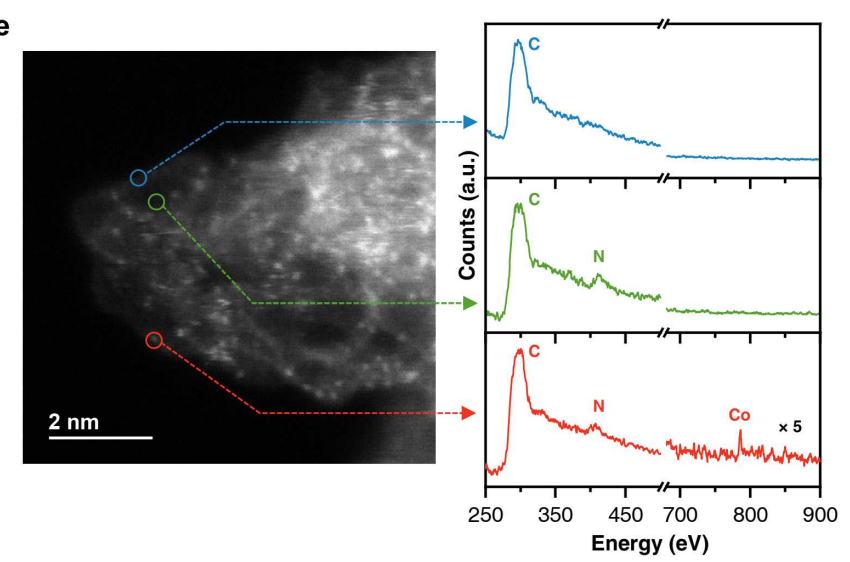

b d

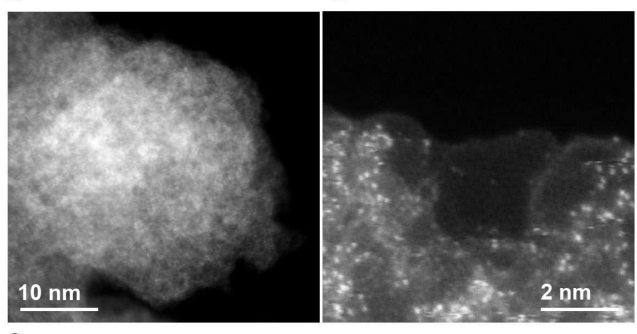

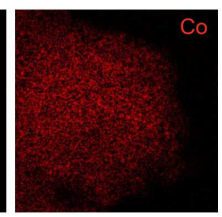

f

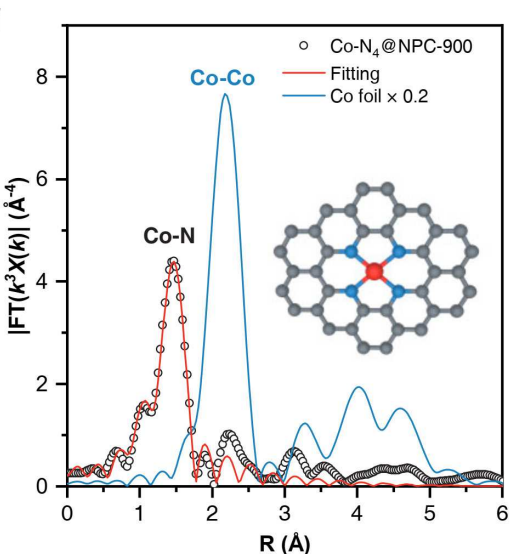

g

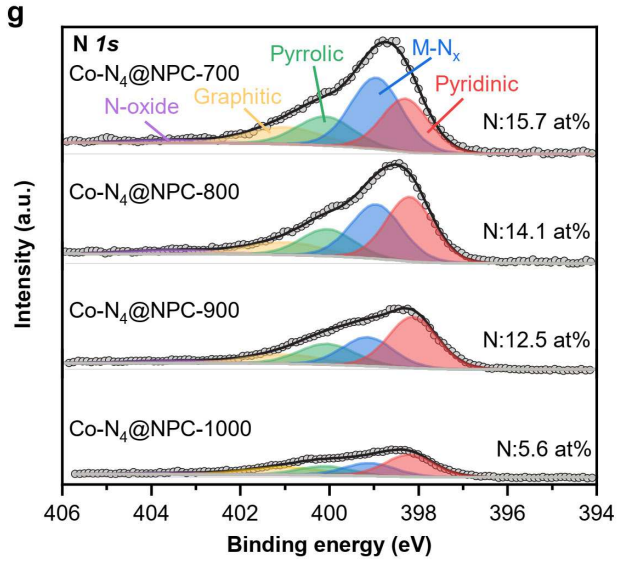

h

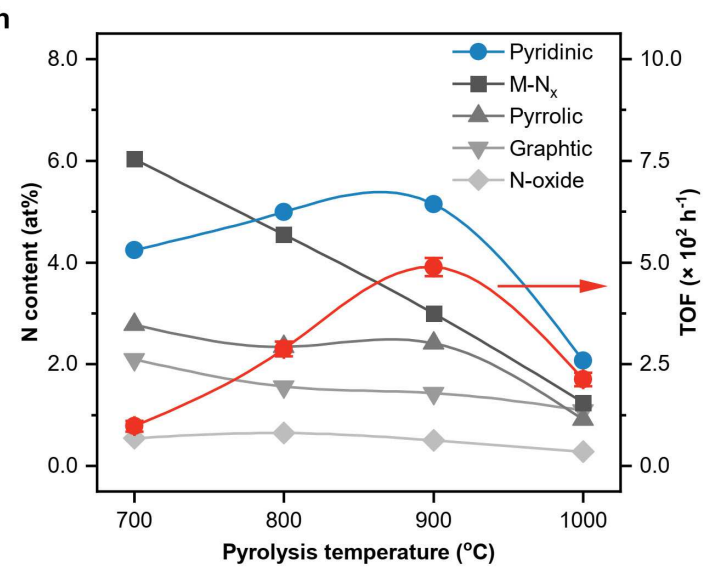

Fig. 2 | Performance and structural characterization of Co-N $\mathrm{N}_{4} @$ NPC-based catalysts. a,

The performance of Co- $\mathrm{N}_{4} @$ NPC-900 and Co-NPs@NPC in 1a oxidation under different 
1a conversion based on total Co atoms. Error bars represent standard deviation, calculated from at least three independent experiments. N.R. = no reaction. b,, $\mathbf{c}$, HADDF-STEM image $(\mathbf{b})$ and the corresponding elemental maps (c) of C (yellow), N (blue) and Co (red) of Co-N $\mathrm{N}_{4} @ \mathrm{NPC}-$ 900. d, Atomic-resolution HADDF-STEM image of Co-N ${ }_{4} @$ NPC-900. e, EELS point spectra of different regions on Co-N $\mathrm{N}_{4} @$ NPC-900.f, FT-EXAFS spectra of Co K-edge of Co-N ${ }_{4} @$ NPC-

On this basis, the overall reaction efficiency could be improved further by the increase in the surface density of vicinal Co- $\mathrm{N}_{4} / \mathrm{Py}-\mathrm{N}$ pairs via the re-introduction of $\mathrm{Co}-\mathrm{N}_{4}$ motifs into the Py-N-dominated Co-N $\mathrm{N}_{4} @$ NPC matrix (Supplementary Figs. 14-16). The resultant Co$\mathrm{N}_{4} @$ NPC-re catalyst exhibited an even higher TOF of $595 \mathrm{~h}^{-1}$ for $\mathbf{1 a}$ conversion under ambient air, and it effectively facilitated the reaction even under a catalyst loading of $0.01 \mathrm{~mol} \%$ (Supplementary Fig. 17). Notably, the Co-N $\mathrm{N}_{4} @$ NPC-re material readily afforded excellent yields of 2a at concentrations up to $4.3 \mathrm{M}$ starting with 1a (Supplementary Fig. 18), corresponding to conditions far better than those used for a natural or genetically engineered enzyme $^{21}$. The robustness and stability of Co- $\mathrm{N}_{4} @$ NPC-re were further investigated in a flowreaction mode for $300 \mathrm{~h}$ (supplementary information, continuous-flow reactions section including Supplementary Figs. 19-23). Notably, the catalyst was stable during the 300-h reaction without any noticeable structural change (Supplementary Figs. 24-27), affording a total turnover number (TON) of 130000 on the basis of the total amount of Co.

Real-time FTIR spectroscopy was employed to gain insights into the possible occurrence 
of proximity-driven oxidase-type catalysis (Fig. 3A, supplementary information, real-time FTIR experiments section including Supplementary Figs. 28-30). By monitoring the characteristic evolution of $\mathrm{Si}-\mathrm{OH}$ vs its respective $\mathrm{Si}-\mathrm{H}$ features during the aerobic oxidation of $1 \mathrm{a}$ in the presence of 2 equiv. $\mathrm{H}_{2}{ }^{18} \mathrm{O}$ over $0.1 \mathrm{~mol} \%$ of Co- $\mathrm{N}_{4} @ \mathrm{NPC}-\mathrm{re}$ at $30{ }^{\circ} \mathrm{C}$, FTIR results provided direct molecular-level evidence for the immediate and exclusive incorporation of the ${ }^{18} \mathrm{O}$-labelled hydroxyl species into the product. With the progress of the reaction, the Si${ }^{16} \mathrm{O}$ stretching band at $880 \mathrm{~cm}^{-1}$ emerged, and it lagged consistently behind that of $\mathrm{Si}^{-1}{ }^{18} \mathrm{O}$ at $865 \mathrm{~cm}^{-1}$ (Fig. 3B). This alteration in the oxygen source during the conversion of $1 \mathbf{a}$ to $2 \mathbf{a}$ was interpreted as a sequential $\mathrm{H}_{2} \mathrm{O}$-mediated oxygen-atom transfer. This also considered the $\mathrm{O}_{2}-$ coupled intermediate $\mathrm{H}_{2} \mathrm{O}_{2}$ formation (Supplementary Figs. 31, 32A and B) and the favourable impact of Co-N $\mathrm{N}_{4} @$ NPC-re for rapid $\mathrm{H}_{2} \mathrm{O}_{2}$ destruction (Supplementary Fig. 32C). Furthermore, based on results of the radical scavenger effect (Supplementary Fig. 33) and kinetic isotope effects associated with 1a conversion (Supplementary Table 3), this reaction did not involve reactive oxygen species (ROS) and silyl radicals as intermediates, and the rate-limiting step for biomimetic turnover involved the breaking of the $\mathrm{Si}-\mathrm{H}$ bond.

Density functional theory (DFT) calculations were employed to obtain additional information about the specific nature of oxidase-type PCHT. A $\mathrm{CoN}_{4} \mathrm{C}_{10}$ motif in combination with a Py-N moiety was used to represent the Co-N $\mathrm{N}_{4} @$ NPC-based catalyst (see computational details in Methods section including Supplementary Fig. 34 in supplementary information). Figure $3 \mathrm{C}$ shows the catalytic cycle for the aerobic-hydrolytic oxidation of $1 \mathbf{a}$ in $\mathrm{Co}-\mathrm{N}_{4} @ \mathrm{NPC}$, and Supplementary Fig. 35 shows the associated free-energy profile. The co-adsorption of 1a and $\mathrm{O}_{2}$ on the $\mathrm{CoN}_{4} \mathrm{C}_{10}$ motif was the first step in the catalytic reaction, where charge transfer from the axially ligated $\mathrm{O}_{2}$ to the Co atom enhanced the binding of $\mathbf{1 a}$ to the Co- $\mathrm{N}_{4}$ site. This aided in the activation of the $\mathrm{Si}-\mathrm{H}$ bond in a polarised manner. The proposed mechanism for $\mathrm{O}_{2}$-assisted $\mathrm{Si}-\mathrm{H}$ bond activation to form adduct $\mathbf{I}$ was completely supported by $\mathbf{1 a} / \mathrm{O}_{2}$ adsorption analysis based on in situ diffuse reflectance FTIR measurements (Supplementary 
Fig. 37). Then, activated 1a species served as the initial species in the catalytic cycle. The reaction was proposed to proceed in the following four steps. After the formation of adduct $\mathbf{I}$, the facile cleavage of the $\mathrm{H}_{2} \mathrm{O}$-assisted $\mathrm{Si}-\mathrm{H}$ bond to afford $\mathbf{2 a}$ occurred via a concerted linear $\mathrm{S}_{\mathrm{N}}$ 2-type dehydride mechanism $\left(\mathrm{CLS}_{\mathrm{N}} 2 \mathrm{D}\right.$, Step 2 in Fig. 3C), a pathway that required the participation of Py-N species to facilitate the deprotonation of $\mathrm{H}_{2} \mathrm{O}$ bonded to the $\mathrm{Si}$ atom in adduct $\mathbf{I}$.

To further investigate the validity of the proposed CLS $\mathrm{S}_{\mathrm{N}} 2 \mathrm{D}$ mechanism, an alternative pyrrolic-N or graphitic-N-assisted deprotonation pathway was calculated. The resulting barriers for such processes were elevated (with $\Delta \mathrm{G}=-22.2,14.5$ and $48.0 \mathrm{kcal} / \mathrm{mol}$ for initiating the crucial deprotonation step with $\mathrm{Py}-\mathrm{N}$, pyrrolic- $\mathrm{N}$ and graphitic-N, respectively, Supplementary Fig. 38), providing support for the $\mathrm{CLS}_{\mathrm{N}} 2 \mathrm{D}$ mechanism. Moreover, an experiment dealing with the oxidation of an optically active hydrosilane $\boldsymbol{R}-\mathbf{1 b}$ (95\% ee) with $\mathrm{O}_{2}$ revealed inversion of the configuration at silicon (Supplementary Fig. 39), thereby further supporting the proposed CLS $2 \mathrm{D}$ mechanism as the favoured pathway for silanol formation over a relatively straightforward process involving the hetero- or homolysis of the Si-H bond. Subsequently, the insertion of $\mathrm{O}_{2}$ into the resulting cobalt hydride III to form cobalt hydroperoxide IV occurred. The formation of IV enabled the rapid formation of $\mathrm{HOOH}^{*}$ without any barrier via the transfer of proton to the $\mathrm{O}$ atom bonded to the Co atom, ultimately affording $\mathrm{H}_{2} \mathrm{O}_{2}$ and regenerating the initial Co- $\mathrm{N}_{4}$ catalyst structure (Steps 3-5 in Fig. 3C). This reaction was exothermic by $17.3 \mathrm{kcal} / \mathrm{mol}$. The contribution of $\mathrm{H}_{2} \mathrm{O}_{2}$ to this oxidation reaction was examined. However, on evaluating the reaction efficacy by the experimental replacement of $\mathrm{O}_{2}$ with a stoichiometric amount of $\mathrm{H}_{2} \mathrm{O}_{2}$, the reaction barely proceeded (Supplementary Fig. 32D). This result suggested that $\mathrm{H}_{2} \mathrm{O}_{2}$ exerts a minimal effect on the aerobic oxidation of 1a, apparently as a result of the rapid disproportionation of $\mathrm{H}_{2} \mathrm{O}_{2}$ in the presence of Co-N $\mathrm{N}_{4} @$ NPC-re under (an)aerobic conditions (Supplementary Fig. 32C). 


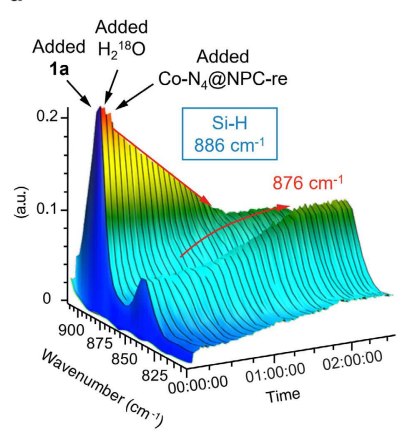

c

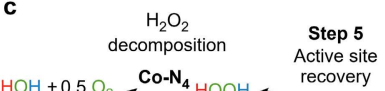

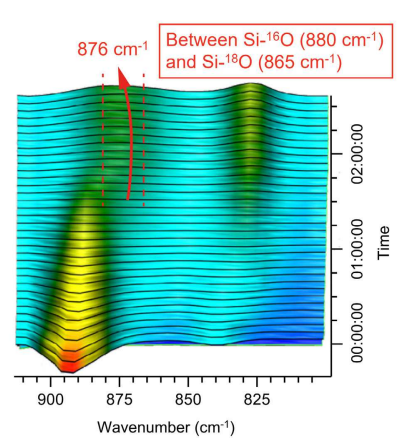
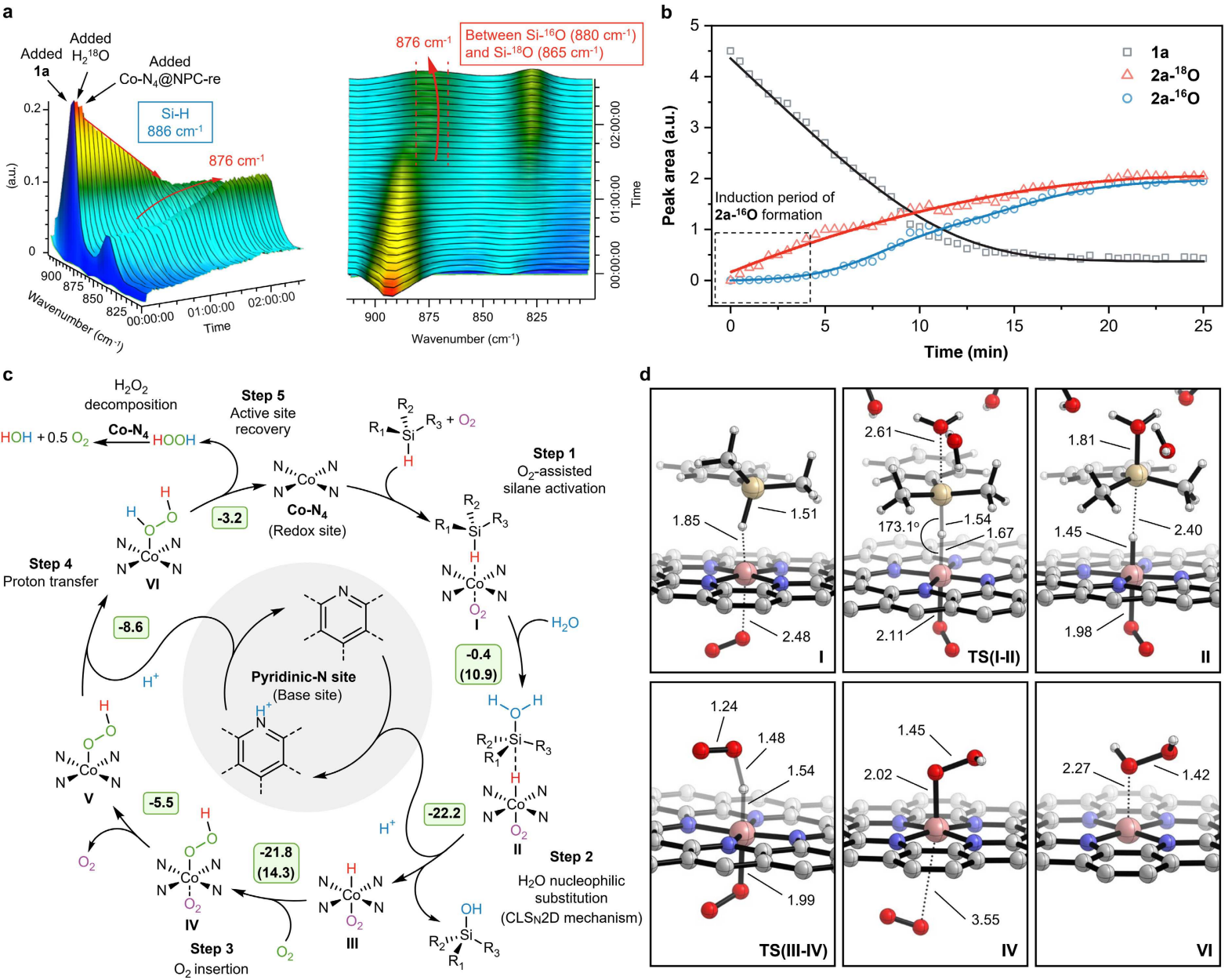

Fig. 3 | Reaction mechanism for Co-N $@$ @PC-catalyzed selective aerobic hydrosilane

oxidation. a, Real time FT-IR kinetic studies of the reaction of $\mathbf{1 a}$ in $\mathrm{MeCN} / \mathrm{H}_{2}{ }^{18} \mathrm{O}$ mixture under ambient air using Co- $\mathrm{N}_{4} @$ NPC-re as catalyst. Reaction conditions: 1a (5 mmol), $\mathrm{H}_{2}{ }^{18} \mathrm{O}$ (10 mmol), Co-N ${ }_{4} @$ NPC-re (Co $\left.0.1 \mathrm{~mol} \%\right)$, anhydrous MeCN $(15 \mathrm{~mL}), 30{ }^{\circ} \mathrm{C} . \mathbf{b}$, Temporal changes of the spectral intensity in $\mathrm{Si}^{-1}{ }^{16} \mathrm{O}$ stretching frequency of $\mathbf{2 a}^{-16} \mathbf{O}\left(880 \mathrm{~cm}^{-1}\right), \mathrm{Si}_{-}{ }^{18} \mathrm{O}$ stretching frequency of $\mathbf{2 a}^{\mathbf{1 8}} \mathbf{O}\left(865 \mathrm{~cm}^{-1}\right)$ and $\mathrm{Si}-\mathrm{H}$ bending frequency in $\mathbf{1 a}\left(886 \mathrm{~cm}^{-1}\right)$. The spectra were analyzed by deconvolution of the peaks between 840 to $920 \mathrm{~cm}^{-1}$. c, A proposed full catalytic cycle of Co-N $\mathrm{N}_{4} @$ NPC-catalyzed aerobic oxidation of hydrosilanes, as well as the free-energy changes and free-energy barriers (in parentheses) between the corresponding key steps. The energy values are in $\mathrm{kcal} / \mathrm{mol}$. d, DFT-optimized main intermediates and transition state structures of catalytic cycle. Co, pink; O, red; C, grey; N, blue; H, white. Key distances are given in $\AA$ and angles in degrees. For clarity, some hydrogen atoms were hidden. 
The general applicability of this catalyst was demonstrated by the selective oxidation of greater than 70 diverse organosilanes with different electronic and steric properties (Fig. 4A and Supplementary Fig. 40). The scope of this chemistry was further extended to the straightforward synthesis of borasiloxanes containing [B-O-Si] motifs by the direct oxidativehydrolytic conversion of silanes with boranes (Fig. 4B). These motifs are valuable precursors for borosilicate materials, heat- and chemical-resistant polymers, and polymer sensors ${ }^{24-26}$. Moreover, by the application of a single Co-mediated oxidase-type catalyst, selected silyl ethers were prepared starting from silanes and alcohols (Fig. 4C). Silyl ethers are of special interest due to their prevalence in a number of technologically important and functional materials ${ }^{27}$. This class of compounds is routinely prepared either by the dehydrogenative coupling of hydrosilanes with alcohols ${ }^{28}$ or by the reaction of halosilanes with alcohols in the presence of a base ${ }^{29}$. Compared with existing Si-alkoxylation processes, the developed single Co-catalysed synthesis of silyl ethers features advantages of more cost-effectiveness or more facile scalability.

Finally, the ability to accomplish oxidase-type oxidation chemistry with these vicinal Co$\mathrm{N}_{4} / \mathrm{Py}-\mathrm{N}$ pairs permitted for the oxidation of broader range of substrates without the incorporation of oxygen in the reaction products (Fig. 4D). For example, the oxidative dimerization of 2-naphthol (7) to 1,1'-binaphthyl-2,2'-diol (8) was successfully achieved. This product comprised binaphthyl backbones, a particularly important scaffold for asymmetric catalysis ${ }^{30}$. Similarly, the oxidative carbonylation of aniline (9) to N, $\mathrm{N}^{\prime}$-diphenyl urea (10) was accomplished. Remarkably, the aerobic oxidative cyclization of 2-aminophenol (11) also proceeded smoothly under green and mild conditions with an excellent product yield, permitting safe and facile access to biologically and pharmaceutically relevant 2aminophenoxazin-3-one (12). Therefore, inspiration from this developed strategy will exert a key impact on biomimicking chemistry by leading to the discovery of new redox-related pathways as well as enabling the generation of completely green, affordable biomimetic 


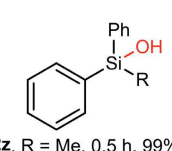

2z, $\mathrm{R}=\mathrm{Me}, 0.5 \mathrm{~h}, 99 \%$ 2aa, $R=E t, 2 h, 98 \%$ 2ab, $R={ }^{\mathrm{P}} \mathrm{Pr}, 6 \mathrm{~h}, 98 \%$ 2ac, $\mathrm{R}=\mathrm{Ph}, 2 \mathrm{~h}, 97 \%$

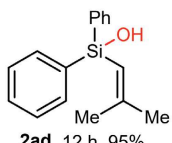

2ad, 12 h, $95 \%$

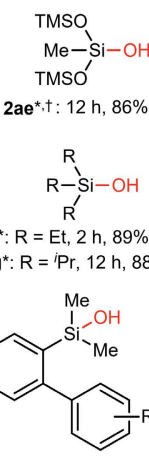

2an, $\mathrm{R}=\mathrm{H}, 8 \mathrm{~h}, 98 \%$ 2ao, $R=4-M e, 12 \mathrm{~h}, 97 \%$ 2ap, $R=3-M e, 12 \mathrm{~h}, 98 \%$ 2aq, $\mathrm{R}=4-\mathrm{CF}_{3}, 16 \mathrm{~h}, 97 \%$ 2ar, $R=4-O M e, 14 h, 92 \%$

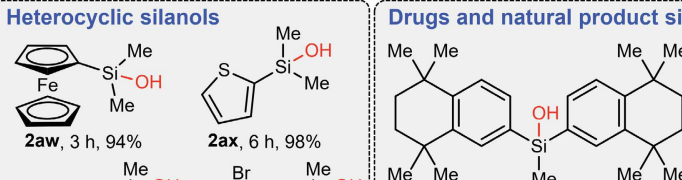

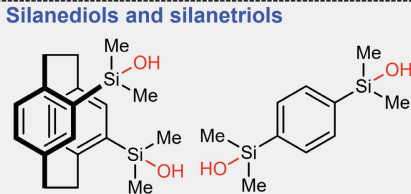<smiles>COc1ccccc1[Si](O)(O)c1ccccc1</smiles>

2ah, 12 h, 95\% 2ai, 4 h, 98\% 2aj, 0.25 h, 93\% $\quad \mathbf{2 a k}^{\star \star \star}, 0.5 \mathrm{~h}, 96 \%$

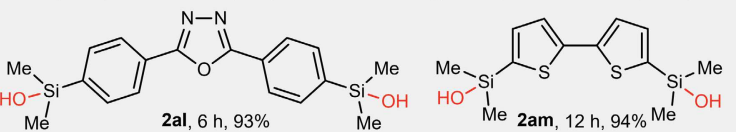<smiles>CC#Cc1ccc([Si](C)(C)N(C)C)cc1</smiles>
$6 \mathrm{a}, \mathrm{R}=\mathrm{Me}, 0.5 \mathrm{~h},(96 \%)$
$6 \mathrm{~b}, \mathrm{R}=\mathrm{Et}, 0.5 \mathrm{~h},(97 \%)$ Cd, $\mathrm{R}=\mathrm{Bu}, 0.5 \mathrm{~h}, 93 \%$

$6 e^{\dagger}, R={ }^{t} \mathrm{Bu}, 6 \mathrm{~h}, 90 \%$ 6f, $R=\operatorname{Pr}, 6 \mathrm{~h}, 92 \% \quad 6 \mathrm{C}=\mathrm{Me}, 1 \mathrm{~h}, 93 \%$ Condition $\mathrm{B}$ : alcohol $(1 \mathrm{mmol})$, silane $(2-5 \mathrm{mmol})$

\section{$n$-heptane $(3 \mathrm{~mL})$}

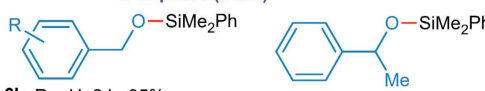

6k, $\mathrm{R}=\mathrm{H}, 2 \mathrm{~h}, 95 \%$ 6|\#, II, R $=4-\mathrm{OMe}, 16 \mathrm{~h}, 73 \% \quad 6 \mathrm{p}, 5 \mathrm{~h}, 64 \%$ $6 \mathrm{~m}, \mathrm{R}=4-\mathrm{Pr}, 2 \mathrm{~h}, 92 \% \quad \mathrm{PhMe}_{2} \mathrm{Si}-\mathrm{O} \quad \mathrm{O}-\mathrm{SiMe}_{2} \mathrm{Ph}$ $\mathbf{6 n}^{\#}, \mathrm{R}=4-\mathrm{F}, 2 \mathrm{~h}, 91 \% \mathrm{6q \pi}, 12 \mathrm{~h}, 55 \%$ 6o*\#,II, R = 4-Br, 16 h, 86\% $\mathrm{O}_{\mathrm{O}^{-}} \mathrm{SiMe}_{2} \mathrm{Ph} \mathrm{Me} \curvearrowright \mathrm{O}^{-} \mathrm{SiMe}_{2} \mathrm{Ph}$ $6 \mathrm{r}, 8 \mathrm{~h}, 80 \% \quad 6 \mathbf{s}, 20 \mathrm{~h}, 78 \%$ $\nabla_{\mathrm{O}^{-}}^{-\mathrm{SiMe}_{2} \mathrm{Ph}} \mathrm{O}_{\mathrm{O}^{-}-\mathrm{SiMe}_{2} \mathrm{Ph}}$ 6t, $3.5 \mathrm{~h}, 90 \% \quad 6 \mathrm{u}, 3 \mathrm{~h}, 66 \%$

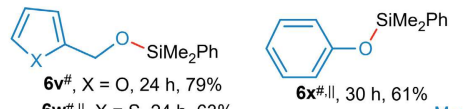
2ba, 6 h, $93 \% \quad$ 2bb, 5 h, $92 \%$ (b), $5 \mathrm{~h}, 02 \%$<smiles>COc1cnn(Cc2ccccc2)c(=O)c1[Si]([O-])(O)O</smiles>

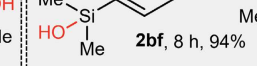
2bg, 6 h, $92 \%$
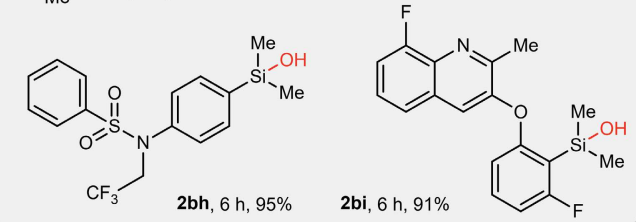

$6 w^{*}, \| x=S, 24 h, 0$

, $30 \mathrm{~h}, 61 \%$

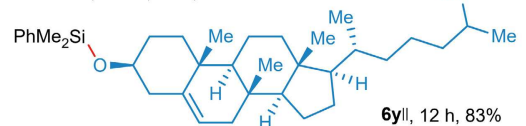
2bh, 6 h, $95 \% \quad$ 2bi, 6 h, $91 \%$ xidative cyclization
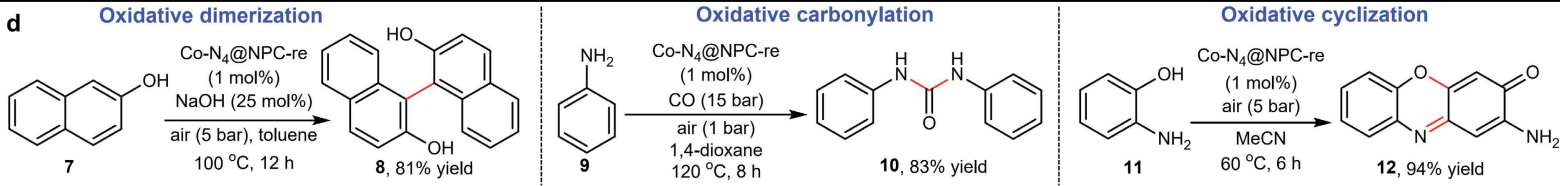
$120^{\circ} \mathrm{C}, 8 \mathrm{~h}$

$10,83 \%$ yield

Fig. 4 | Scope of Co-N $@$ @ NPC-catalyzed oxidase-type transformations. See supplementary under air atmosphere. Isolated yields are reported unless otherwise indicated. Yields in parentheses refer to GC yield. a, Substrate scope of silane substrates. b, Multicomponent 
synthesis of borosiloxanes. c, Oxidative cross-coupling of hydrosilanes and alcohols. d, Applications in a variety of other oxidase-like reactions. $* 10 \mathrm{mmol}$ scale. $†$ Under $\mathrm{O}_{2}(1 \mathrm{bar})$

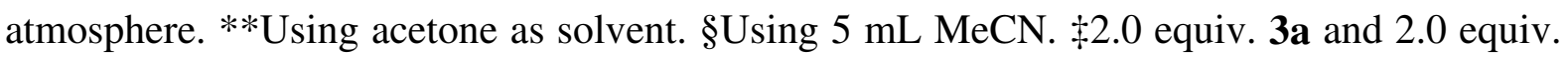
$\mathrm{H}_{2} \mathrm{O}$. \#Using toluene as solvent, 3.0 equiv. 1a. $\llbracket[U \operatorname{sing}$ toluene/MeCN $(\mathrm{v} / \mathrm{v}=2: 1)$ as solvent, 5.0 equiv. 1a. $\| 60{ }^{\circ} \mathrm{C}$. TMS, trimethylsilyl; Bn, benzyl; Ts, tosyl; Boc, tert-butyloxycarbonyl; HBPin, pinacolborane; HBCat, catecholborane.

Main References

1. Chen, J. et al. Carbonyl catalysis enables a biomimetic asymmetric Mannich reaction. Science 360, 1438-1442; 10.1126/science.aat4210 (2018).

2. Guan, J. et al. Water oxidation on a mononuclear manganese heterogeneous catalyst. Nat. Catal. 1, 870-877; 10.1038/s41929-018-0158-6 (2018).

3. Le Goff, A. et al. From hydrogenases to noble metal-free catalytic nanomaterials for $\mathrm{H}_{2}$ production and uptake. Science 326, 1384-1387; 10.1126/science.1179773 (2009).

4. Liu, W., Lavagnino, M. N., Gould, C. A., Alcázar, J. \& MacMillan, D. W. C. A biomimetic $\mathrm{S}_{\mathrm{H}} 2$ cross-coupling mechanism for quaternary $\mathrm{sp}^{3}$-carbon formation. Science, eabl4322; 10.1126/science.abl4322 (2021).

5. Ragg, R., Tahir, M. N. \& Tremel, W. Solids go bio: Inorganic nanoparticles as enzyme mimics. Eur. J. Inorg. Chem. 2016, 1906-1915; 10.1002/ejic.201501237 (2016).

6. Jiao, N. \& Stahl, S. S. (eds.). Green oxidation in organic synthesis (Wiley-VCH, 2019).

7. Borden, W. T., Hoffmann, R., Stuyver, T. \& Chen, B. Dioxygen: What makes this triplet diradical kinetically persistent? J. Am. Chem. Soc. 139, 9010-9018; 10.1021/jacs.7b04232 (2017).

8. Valentine, J. S., Foote, C. S., Greenberg, A. \& Liebman, J. F. Active oxygen in biochemistry (Springer US, Boston, MA, 1995). 
9. Romero, E., Gómez Castellanos, J. R., Gadda, G., Fraaije, M. W. \& Mattevi, A. Same substrate, many reactions: Oxygen activation in flavoenzymes. Chem. Rev. 118, 17421769; 10.1021/acs.chemrev.7b00650 (2018).

10. Ghisla, S. \& Massey, V. Mechanisms of flavoprotein-catalyzed reactions. Eur. J. Biochem. 181, 1-17; 10.1111/j.1432-1033.1989.tb14688.x (1989).

11. Roux, Y., Ricoux, R., Avenier, F. \& Mahy, J.-P. Bio-inspired electron-delivering system for reductive activation of dioxygen at metal centres towards artificial flavoenzymes. Nat. Commun. 6, 8509; 10.1038/ncomms9509 (2015).

12. Largeron, M. \& Fleury, M.-B. Bioinspired oxidation catalysts. Science 339, 43-44; 10.1126/science.1232220 (2013).

13. Wang, Y., DuBois, J. L., Hedman, B., Hodgson, K. O. \& Stack, T. D. Catalytic galactose oxidase models: Biomimetic Cu(II)-phenoxyl-radical reactivity. Science 279, 537-540; 10.1126/science.279.5350.537 (1998).

14. Chaudhuri, P., Hess, M., Flörke, U. \& Wieghardt, K. From structural models of galactose oxidase to homogeneous catalysis: Efficient aerobic oxidation of alcohols. Angew. Chem. Int. Ed. 37, 2217-2220; 10.1002/(SICI)1521-3773(19980904)37:16<2217::AIDANIE2217>3.0.CO;2-D (1998).

15. Jeon, M., Han, J. \& Park, J. Catalytic synthesis of silanols from hydrosilanes and applications. ACS Catal. 2, 1539-1549; 10.1021/cs300296x (2012).

16. Ramesh, R. \& Reddy, D. S. Quest for novel chemical entities through incorporation of silicon in drug scaffolds. J. Med. Chem. 61, 3779-3798; 10.1021/acs.jmedchem.7b00718 (2018).

17. Franz, A. K. \& Wilson, S. O. Organosilicon molecules with medicinal applications. J. Med. Chem. 56, 388-405; 10.1021/jm3010114 (2013).

18. Kondo, S.-I., Harada, T., Tanaka, R. \& Unno, M. Anion recognition by a silanediol-based receptor. Org. Lett. 8, 4621-4624; 10.1021/o1061822p (2006). 
19. Chandrasekhar, V., Boomishankar, R. \& Nagendran, S. Recent developments in the synthesis and structure of organosilanols. Chem. Rev. 104, 5847-5910; $10.1021 / \mathrm{cr} 0306135$ (2004).

20. Wang, K. et al. Selective manganese-catalyzed oxidation of hydrosilanes to silanols under neutral reaction conditions. Angew. Chem. Int. Ed. 58, 6380-6384; 10.1002/anie.201900342 (2019).

21. Bähr, S. et al. Selective enzymatic oxidation of silanes to silanols. Angew. Chem. Int. Ed. 59, 15507-15511; 10.1002/anie.202002861 (2020).

22. Jasinski, R. A new fuel cell cathode catalyst. Nature 201, 1212-1213; 10.1038/2011212a0 (1964).

23. He, L., Weniger, F., Neumann, H. \& Beller, M. Synthesis, characterization, and application of metal nanoparticles supported on nitrogen-doped carbon: Catalysis beyond electrochemistry. Angew. Chem. Int. Ed. 55, 12582-12594; 10.1002/anie.201603198 (2016).

24. Peña-Alonso, R., Mariotto, G., Gervais, C., Babonneau, F. \& Soraru, G. D. New insights on the high-temperature nanostructure evolution of SiOC and B-doped SiBOC polymerderived glasses. Chem. Mater. 19, 5694-5702; 10.1021/cm071203q (2007).

25. Puneet, P., Vedarajan, R. \& Matsumi, N. Alternating poly(borosiloxane) for solid state ultrasensitivity toward fluoride ions in aqueous media. ACS Sens. 1, 1198-1202; 10.1021/acssensors.6b00346 (2016).

26. Wu, T. \& Chen, B. Synthesis of multiwalled carbon nanotube-reinforced polyborosiloxane nanocomposites with mechanically adaptive and self-healing capabilities for flexible conductors. ACS Appl. Mater. Interfaces 8, 24071-24078; 10.1021/acsami.6b06137 (2016). 
27. Kamino, B. A. \& Bender, T. P. The use of siloxanes, silsesquioxanes, and silicones in organic semiconducting materials. Chem. Soc. Rev. 42, 5119-5130; 10.1039/c3cs35519e (2013).

28. Asensio, J. M., Bouzouita, D., van Leeuwen, P. W. N. M. \& Chaudret, B. $\sigma-H-H, \sigma-C-H$, and $\sigma-\mathrm{Si}-\mathrm{H}$ bond activation catalyzed by metal nanoparticles. Chem. Rev. 120, 10421084; 10.1021/acs.chemrev.9b00368 (2020).

\section{Methods}

\section{Chemicals}

All materials were used without further purification, unless otherwise stated. Cobalt nitrate hexahydrate $\left(\mathrm{Co}\left(\mathrm{NO}_{3}\right)_{2} \cdot 6 \mathrm{H}_{2} \mathrm{O}, \geq 98.5 \%\right)$, zinc nitrate hexahydrate $\left(\mathrm{Zn}\left(\mathrm{NO}_{3}\right)_{2} \cdot 6 \mathrm{H}_{2} \mathrm{O}, \geq 99.0 \%\right)$, cobalt acetate tetrahydrate $\left(\mathrm{Co}(\mathrm{OAc})_{2} \cdot 4 \mathrm{H}_{2} \mathrm{O}, \geq 99.5 \%\right)$, ammonium chloride $\left(\mathrm{NH}_{4} \mathrm{Cl}, \geq 99.5 \%\right)$, ammonium hydroxide $\left(\mathrm{NH}_{3} \cdot \mathrm{H}_{2} \mathrm{O}, 25.0-28.0 \%\right)$, methanol $(\mathrm{MeOH}, \geq 99.7 \%)$, ethanol $(\mathrm{EtOH}$, $\geq 99.7 \%$ ) and acetonitrile (MeCN, $\geq 99.5 \%$ ) were purchased from Sinopharm Chemical Reagent

Co., Ltd. 2-Methylimidazole (2-MeIm, 98\%), 1,10-phenanthroline (99\%) and dimethylphenylsilane (97\%) were purchased from Aladdin.

\section{Preparation of catalysts}

Synthesis of Co- $\mathrm{N}_{4} @ N P C-T$ catalysts. The Co- $\mathrm{N}_{4} @$ NPC-T catalysts, where T represents the carbonization temperature $\left(\mathrm{T}=700,800,900\right.$, and $\left.1000{ }^{\circ} \mathrm{C}\right)$, were synthesized by using low $\mathrm{Co} / \mathrm{Zn}$ ratio bimetallic $\mathrm{ZIF}\left(\mathrm{Co}_{1} \mathrm{Zn}_{50}-\mathrm{ZIF}\right)$ as precursor. The molar ratio of metal ions to 2- 
MeIm remained at 1:8 and the concentration of metal ions in final mixed solution was controlled at an optimal concentration of $25 \mathrm{mmol} / \mathrm{L}$. The typical synthesis process of Co$\mathrm{N}_{4} @$ NPC-900 is given as follows. A solution of 2-MeIm $(13.4 \mathrm{~g}, 163.2 \mathrm{mmol})$ in $408 \mathrm{~mL}$ $\mathrm{MeOH}$ was added to a solution of $\mathrm{Co}\left(\mathrm{NO}_{3}\right)_{2} \cdot 6 \mathrm{H}_{2} \mathrm{O}(0.116 \mathrm{~g}, 0.4 \mathrm{mmol})$ and $\mathrm{Zn}\left(\mathrm{NO}_{3}\right)_{2} \cdot 6 \mathrm{H}_{2} \mathrm{O}$ $(5.95 \mathrm{~g}, 20 \mathrm{mmol})$ in $408 \mathrm{~mL} \mathrm{MeOH}$. After stirred at room temperature (RT, $\left.\sim 25^{\circ} \mathrm{C}\right)$ for $24 \mathrm{~h}$, the obtained $\mathrm{Co}_{1} \mathrm{Zn}_{50}$-ZIF precipitates was were collected by centrifugation, wash with $\mathrm{MeOH}$ for three times and then dried under vacuum at RT overnight. Subsequently, $1.0 \mathrm{~g}$ as-prepared $\mathrm{Co}_{1} \mathrm{Zn}_{50}$-ZIF precursor was heated at $900{ }^{\circ} \mathrm{C}$ for $4 \mathrm{~h}$ under the flowing $\mathrm{Ar}$ atmosphere (80 $\mathrm{mL} / \mathrm{min}$ ) in a tube furnace, followed by naturally cooling down to RT. Co-N $\mathrm{N}_{4} @$ NPC-NL-900 was obtained as a black powder.

Next, ammonia treatment in $2.0 \mathrm{~mol} / \mathrm{L} \mathrm{NH}_{3} / \mathrm{NH}_{4} \mathrm{Cl}$ aqueous solution (sum concentration of initial ammonium ion and ammonia, the molar ratio of ammonium to ammonia was 1:1) at RT for $8 \mathrm{~h}$ was carried out to remove $\mathrm{Zn}$ species and to generate more favorable open porosity in Co-N4@NPC-NL-900 samples. Typically, $100 \mathrm{~mL}$ leaching solution was used for $100 \mathrm{mg}$ sample. An additional pyrolysis treatment at $900{ }^{\circ} \mathrm{C}$ under the flowing $\mathrm{Ar}$ atmosphere for $4 \mathrm{~h}$ is necessary to repair the carbon nanostructures. After naturally cooling to RT, Co-N 4 @NPC900 was obtained.

Co-N $4 @$ NPC-700, Co- $\mathrm{N}_{4} @$ NPC-800 and Co-N $4 @$ NPC-1000 were synthesized by using $\mathrm{Co}_{1} \mathrm{Zn}_{50}-\mathrm{ZIF}$ as precursor following similar procedure to that of $\mathrm{Co}-\mathrm{N}_{4} @ \mathrm{NPC}-900$ preparation except that the pyrolysis and additional pyrolysis temperature were 700,800 and $1000{ }^{\circ} \mathrm{C}$, respectively.

Synthesis of Co-N $N_{4} @ N P C$-re catalyst. For synthesis of Co- $\mathrm{N}_{4} @ N P C-r e, \mathrm{Co}(\mathrm{OAc})_{2} \cdot 4 \mathrm{H}_{2} \mathrm{O}(12.7$ $\mathrm{mg}, 0.05 \mathrm{mmol})$ and 1,10-phenanthroline $(27.6 \mathrm{mg}, 0.15 \mathrm{mmol})$ were dissolved in $25 \mathrm{~mL} \mathrm{EtOH}$ and stirred for $30 \mathrm{~min}$ at RT. Then $300 \mathrm{mg}$ ammonia-treated Co-N ${ }_{4} @$ NPC-NL-900 samples was added and stirred for $4 \mathrm{~h}$ at $60{ }^{\circ} \mathrm{C}$. The solvent was removed by rotary evaporation and thus obtained solid was dried under vacuum at RT overnight. A subsequent pyrolysis treatment 
at $900{ }^{\circ} \mathrm{C}$ under the flowing $\mathrm{Ar}$ atmosphere $(80 \mathrm{~mL} / \mathrm{min})$ for $4 \mathrm{~h}$ was conducted to obtain the final Co-N $\mathrm{N}_{4} @ \mathrm{NPC}-\mathrm{re}$ sample.

Synthesis of Co-NPs@NPC catalyst.Co-NPs@NPC catalysts were synthesized by using high $\mathrm{Co} / \mathrm{Zn}$ ratio bimetallic $\mathrm{ZIF}\left(\mathrm{Co}_{1} \mathrm{Zn}_{2}-\mathrm{ZIF}\right)$ as precursor. $\mathrm{Co}_{1} \mathrm{Zn}_{2}-\mathrm{ZIF}$ was synthesized using similar synthesis procedure of $\mathrm{Co}_{1} \mathrm{Zn}_{50}-\mathrm{ZIF}$ except that the $\mathrm{Zn} / \mathrm{Co}$ molar ratio in starting materials was 2:1. Then, as-prepared $\mathrm{Co}_{1} \mathrm{Zn}_{2}-\mathrm{ZIF}$ precursor was pyrolysized at $900{ }^{\circ} \mathrm{C}$ for $4 \mathrm{~h}$ under the flowing Ar atmosphere $(80 \mathrm{~mL} / \mathrm{min})$ in a tube furnace. After cooling to RT naturally, Co-NPs@NPC was obtained as a black powder without further treatment.

\section{Evaluation of catalytic performance}

Normal pressure reactions (ambient air or $N_{2}$ ). Dimethyl-phenylsilane (1a, $155 \mu \mathrm{L}, 1 \mathrm{mmol}$ ) and water $(145 \mu \mathrm{L}, 8 \mathrm{mmol})$ were mixed with solvent $(3 \mathrm{~mL})$ in a $15 \mathrm{~mL}$ oven-dried roundbottom tube with steady magnetic stirring $(800 \mathrm{rpm})$ at $30^{\circ} \mathrm{C}$ under an ambient atmosphere of air. The reaction was started by the addition of a certain amount of catalyst (metal: $0.5 \mathrm{~mol} \%$ ) and the mixture was allowed to stir for required reaction time. After completion of the reaction, the catalyst was filtered by $0.2 \mu \mathrm{m}$ syringe filter. For determining conversion and selectivity, anisole $(50 \mu \mathrm{L})$ used as an internal standard was added to $1 \mathrm{~mL}$ filtrate and the mixture was analyzed by GC (Agilent $7820 \mathrm{~A}$ gas chromatograph with FID detector and $30 \mathrm{~m} \times 0.32$ $\mathrm{mm} \times 0.25 \mu \mathrm{m}$ HP-INNOWax capillary column). The initial TOF was measured at $\mathbf{1 a}$ conversion below $20 \%$ based on total Co atoms.

For evaluating the catalytic performances under $\mathrm{N}_{2}$ atmosphere, reactions were carried out in a $\mathrm{N}_{2}$-filled glovebox (MIKROUNA Super; typically $<1 \mathrm{ppm}$ oxygen and water). The catalysts used in such reaction conditions were first evacuated under dynamic vacuum $(\sim 0.1$ torr) at $150^{\circ} \mathrm{C}$ for $4 \mathrm{~h}$ to remove gas molecules in nanopores and stored under inert atmosphere. Solvent was degassed by three freeze-pump-thaw procedure cycles before used. The reaction procedure and analytical method was the same as that used for catalytic performance evaluation under ambient air atmosphere. 
Pressurized reactions. In a $\mathrm{N}_{2}$-filled glovebox, a mixture of $1 \mathbf{a}(155 \mu \mathrm{L}, 1 \mathrm{mmol})$, water (145 $\mu \mathrm{L}, 8 \mathrm{mmol}$ ) and degassed solvent $(3 \mathrm{~mL})$ was charged into a $25 \mathrm{~mL}$ Hastelloy-C high pressure reactor (Beijing Century Senlang Experimental Apparatus Co., Ltd.; contain a stainless steel sampling tube with filter). Then, catalyst (metal: $0.5 \mathrm{~mol} \%$ ) was added and the reactor was sealed. After removing from the glovebox, the reactor was immersed into a pre-heated water bath $\left(30{ }^{\circ} \mathrm{C}\right)$ and connected with a gas cylinder of air. The reaction was started by pressurizing the reactor to given pressure (3-15 bar) and the gas source was kept connected during the reaction. After stirring for a certain period of time, the reaction liquid was taken from the reactor through sampling tube. The conversion and selectivity were determined by GC using anisole as an internal standard.

\section{Catalyst characterization}

Element analysis. The contents of metal elements in the samples were measured by inductively coupled plasma optical emission spectroscopy (ICP-OES) using PerkinElmer Optima 2100DV spectrometer. The samples were first digested by hot perchloric acid and then dissolved in hot aqua regia.

XRD analysis. The XRD patterns were collected on a Bruker D8 Advance X-ray diffractometer using a monochromatic $\mathrm{Cu} \mathrm{K \alpha}(\lambda=1.5418 \AA)$ source at $40 \mathrm{kV}$ and $40 \mathrm{~mA}$.

Raman spectroscopy. Raman spectra were collected on a HORIBA LabRAM HR Evolution high resolution Raman spectrometer. A $514.5 \mathrm{~nm} \mathrm{Ar}^{+}$laser was used as the excitation source. Nitrogen-adsorption and desorption isotherms. The test was performed at $77 \mathrm{~K}$ using Micromeritics ASAP 2460 Surface Area and Porosity Analyzer. The specific surface area of sample was estimated by Brunauer-Emmett-Teller (BET) method. The samples were first evacuated under dynamic vacuum $\left(<10^{-3}\right.$ torr $)$ at $150{ }^{\circ} \mathrm{C}$ for $12 \mathrm{~h}$ to remove the guest molecules and adsorbed moisture before re-measuring the accurate sample weight.

FT-IR spectroscopy. FT-IR transmittance spectra were collected on a Nicolet iS10 TF-IR spectrometer with a DTGS detector. The samples were mixed with $\mathrm{KBr}$ and made into a small 
tablet for test. The spectra were obtained by averaging 64 scans with a resolution of $0.5 \mathrm{~cm}^{-1}$ over wavenumbers ranging from 400 to $4000 \mathrm{~cm}^{-1}$.

Electron microscopy. The SEM images were acquired on a Zeiss Merlin Compact microscope operated at $10 \mathrm{kV}$. The samples were prepared by drop-drying the samples from their diluted EtOH suspensions onto silicon slices. The TEM and HRTEM images were acquired on a FEI Tecnai G2 F20 S-Twin operated at $200 \mathrm{kV}$. Atomic resolution HAADF-STEM images and EELS spectra of samples were captured on a Nion UltraSTEM U100 operated at $60 \mathrm{kV}$. The HAADF-STEM images were collected with an annular dark field detector in the range of 86200 mrad with a convergence angle of $30 \mathrm{mrad}$. EELS was carried out with the same experimental set-up. Samples for TEM were prepared by drop-drying the samples from their diluted EtOH suspensions onto TEM grids.

XPS analysis. The XPS measurements were conducted on a Kratos Axis Ultra Imaging Photoelectron Spectrometer equipped with a monochromatic Al Ka $(1486.6 \mathrm{eV})$ source. The samples were fixed on conductive tapes and introduced into the UHV chamber. The pressure in the analysis chamber was maintained below $5 \times 10^{-9}$ Torr for data acquisition. The highresolution spectra were performed at a pass energy of $20 \mathrm{eV}$ and steps of $0.1 \mathrm{eV}$. The photoelectrons were detected at a takeoff angle of $0^{\circ}$ with respect to the surface normal. The binding energy scale was calibrated by assigning the main $\mathrm{C} 1 \mathrm{~s}$ peak at $284.6 \mathrm{eV}$. XAFS analysis. XAFS measurements at Co K-edge and Zn K-edge under fluorescence mode were performed at the BL14W1 in Shanghai Synchrotron Radiation Facility (SSRF) using a Lytle detector. The electron beam energy was $3.5 \mathrm{GeV}$ and the stored current was $230 \mathrm{~mA}$ (top-up). A 38-pole wiggler with the maximum magnetic field of $1.2 \mathrm{~T}$ inserted in the straight section of the storage ring was used. The station was operated with a fixed-exit double-crystal Si (111) monochromator. The XAFS samples were prepared by mixing with paraffin wax (Sigma-Aldrich, $\mathrm{mp} 58-62^{\circ} \mathrm{C}$ ), then making into a small tablet $(8.0 \mathrm{~mm}$ diameter) and sealing in a chamber with Kapton tape. Co foil and $\mathrm{Zn}$ foil were used as standard reference materials 
and the X-ray absorption data were recorded in transmission mode using ion chambers. The raw data analysis was performed using IFEFFIT software package ${ }^{31}$ according to the standard data analysis procedures. The spectra were calibrated, averaged, pre-edge background subtracted, and post-edge normalized using Athena program in IFEFFIT software package. The Fourier transformation of the $k^{3}$-weighted EXAFS oscillations, $k^{3} \cdot \chi(k)$, from $\mathrm{k}$ space to $\mathrm{R}$ space was performed to obtain a radial distribution function. And data fitting was done by Artemis program in IFEFFIT. The wavelet transform (WT) analysis was using FORTRAN program HAMA developed by H. Funke and M. Chukalina ${ }^{32}$. The $k$-space file of each sample which contains the data of exact $k$ range of Fourier transformation was processed after running the code, following which the $k$ space, $R$ space spectra and the continuous wavelet transform figure were generated automatically.

DRIFT spectroscopy. The DRIFT spectra of chemisorbed 1a were recorded in an in-situ diffuse reflectance cell (Harrick) equipped with a $\mathrm{CaF}_{2}$ window on a ThermoFisher Nicolet 6700 FTIR spectrometer, using an MCT detector. Samples (ca. $20 \mathrm{mg}$ ) were pretreated at $250{ }^{\circ} \mathrm{C}$ for 1 h with flowing $\mathrm{He}(20 \mathrm{~mL} / \mathrm{min})$, then treated by $1 \mathrm{a}$ at $15^{\circ} \mathrm{C}$ with air (presence of $\mathrm{O}_{2}$ ) or $\mathrm{He}$ (absence of $\mathrm{O}_{2}$ ) gas flow $(20 \mathrm{~mL} / \mathrm{min})$. The collected spectra were subtracted from the sample background spectra in He to obtain the spectra for chemisorbed 1a.

\section{Computational details}

Quantum chemical calculations were all performed at the density functional theory (DFT) level using the Gaussian 16 software package ${ }^{33}$. The DFT functional used is the MN15 functional ${ }^{34}$. The models of $\mathrm{CoPc}, \mathrm{CoPc}-16 \mathrm{Cl}, \mathrm{CoN}_{4} \mathrm{C}_{10}$ motif (based on XANES spectra analysis, see Supplementary Figs. 41 and 42 and Supplementary Discussion 1) and N-doped graphene sheets are reported in Supplementary Fig. 4. In this work, the working catalyst, Co- $\mathrm{N}_{4} @$ NPC-based catalyst was modeled by a $\mathrm{CoN}_{4} \mathrm{C}_{10}$ cluster model. Cluster and slab models are two commonly used models to approximate the activity centers of heterogeneous catalysts. Using cluster model, we can carefully choose a well-validated DFT functional, MN15, which is very accurate 
for reaction energetics. This cannot be done if a slab model was used because there are just a few functionals, such as the PBE and PW91 functionals, implemented in the commonly used periodic software packages and these functionals are not very accurate in computing energy barriers. In addition, solvation effect is difficult to deal with if slab model was used but it plays a critical role in our catalytic system. Here we carefully designed the cluster model so that it can well represent the catalytic center. Besides, our experiments showed that there are some similarities between the model $\mathrm{CoPc}$ and $\mathrm{CoPc}-16 \mathrm{Cl}$ catalysts and the working catalyst. Because $\mathrm{CoPc}$ and $\mathrm{CoPc}-16 \mathrm{Cl}$ are molecular catalysts, the similarity between the CoPc/CoPc$16 \mathrm{Cl}$ catalysts and the Co embedded $\mathrm{N}$-doped graphene catalyst indicates that it is reasonable to use the $\mathrm{CoN}_{4} \mathrm{C}_{10}$ cluster to model the catalyst.

In the simulation, a moderate basis set (MBS), which was a combined basis set in which the aug-cc-pVDZ basis set ${ }^{35}$ was employed to Co atom, the $6-31+G(d, p)$ basis set ${ }^{36,37}$ was employed for the atoms of the reaction center (four $\mathrm{N}$ atoms, $\mathrm{Si}$ atom, three $\mathrm{O}$ atoms on $\mathrm{O}_{2}$ and $\mathrm{H}_{2} \mathrm{O}$, three hydrogen atoms on $\mathrm{Si}-\mathrm{H}$ and $\mathrm{H}_{2} \mathrm{O}$, respectively) and the 6-31G(d) basis set ${ }^{36,37}$ was employed for the other atoms, was used for geometry optimization. Single-point energies were calculated under a larger basis set (LBS) on the geometries that optimized with the modest basis set to provide more accurate energetic results. For single-point energies calculation, the aug-cc-pVTZ basis set was employed to Co atom, the $6-311++\mathrm{G}(3 \mathrm{df}, 2 \mathrm{p})$ basis set ${ }^{38,39}$ was employed for the atoms of the reaction center and the $6-311+G(d, p)$ basis set ${ }^{38,39}$ was employed for the other atoms. Except $\mathrm{O}_{2}$ molecule, DFT calculations for other species were performed in the MeCN solution which was modelled by the polarizable continuum solvation model (IEFPCM) ${ }^{40}$ with radii and non-electrostatic terms for Truhlar and coworkers' SMD solvation model ${ }^{41}$. This solvation model is by far the most reliable one in predicting solvation free energies. The convergence criteria used for the geometry optimization were $4.50 \times 10^{-4}$ au. for gradients, and $1.80 \times 10^{-3}$ au. for displacements. Harmonic vibrational analyses were carried out to confirm if the optimized structure is a local minimum structure or a first order transition state 
and to provide zero-point vibrational energy corrections and thermal corrections to various thermodynamic properties. Transition states were further confirmed by IRC calculations. Optimized DFT structures were illustrated using CYLView ${ }^{42}$.

The free energy of each species at $298.15 \mathrm{~K}$ under standard state $\left(G_{298}\right)$ was calculated by:

$$
G_{298}=G_{\text {solv }}+Z P E+\Delta G_{0 \sim 298}+\Delta G_{C}
$$

where $G_{\text {solv }}$ is the single-point energy of species that calculated under LBS after considering the solvation effect (for $\mathrm{O}_{2}$, it is the electron energy in the gas phase), ZPE is the zero-point vibrational energy that approximately calculated by harmonic vibration approximation under MBS, $\Delta G_{0 \sim 298}$ is the Gibbs free-energy change from 0 to $298.15 \mathrm{~K}$ calculated under harmonic (for $\mathrm{O}_{2}$ it is 0 , for species in solvent it is $1.90 \mathrm{kcal} / \mathrm{mol}$ ).

\section{Data availability}

The data supporting the findings of this study are available within the article and its Supplementary Information files. All other relevant source data are available from the corresponding authors upon reasonable request.

\section{Methods References}

31. Ravel, B. \& Newville, M. ATHENA, ARTEMIS, HEPHAESTUS: Data analysis for Xray absorption spectroscopy using IFEFFIT. J. Synchrotron. Rad. 12, 537-541; $10.1107 /$ S0909049505012719 (2005).

32. Funke, H., Scheinost, A. C. \& Chukalina, M. Wavelet analysis of extended x-ray absorption fine structure data. Phys. Rev. B 71; 10.1103/PhysRevB.71.094110 (2005).

33. Frisch, M. J. et al. Gaussian 16. Gaussian, Inc. (Wallingford CT, 2016). 
34. Yu, H. S., He, X., Li, S. L. \& Truhlar, D. G. MN15: A Kohn-Sham global-hybrid exchange-correlation density functional with broad accuracy for multi-reference and single-reference systems and noncovalent interactions. Chem. Sci. 7, 5032-5051; 10.1039/c6sc00705h (2016).

35. Woon, D. E. \& Dunning, T. H. Gaussian basis sets for use in correlated molecular calculations. III. The atoms aluminum through argon. J. Chem. Phys. 98, 1358-1371; 10.1063/1.464303 (1993).

36. Francl, M. M. et al. Self-consistent molecular orbital methods. XXIII. A polarization-type basis set for second-row elements. J. Chem. Phys. 77, 3654-3665; 10.1063/1.444267 (1982).

37. Ditchfield, R., Hehre, W. J. \& Pople, J. A. Self-consistent molecular-orbital methods. IX. An extended Gaussian-type basis for molecular-orbital studies of organic molecules. $J$. Chem. Phys. 54, 724-728; 10.1063/1.1674902 (1971).

38. Krishnan, R., Binkley, J. S., Seeger, R. \& Pople, J. A. Self-consistent molecular orbital methods. XX. A basis set for correlated wave functions. J. Chem. Phys. 72, 650-654; 10.1063/1.438955 (1980).

39. McLean, A. D. \& Chandler, G. S. Contracted Gaussian basis sets for molecular calculations. I. Second row atoms, Z =11-18. J. Chem. Phys. 72, 5639-5648; 10.1063/1.438980 (1980).

40. Tomasi, J., Mennucci, B. \& Cammi, R. Quantum mechanical continuum solvation models. Chem. Rev. 105, 2999-3093; 10.1021/cr9904009 (2005).

41. Marenich, A. V., Cramer, C. J. \& Truhlar, D. G. Universal solvation model based on solute electron density and on a continuum model of the solvent defined by the bulk dielectric constant and atomic surface tensions. J. Phys. Chem. B 113, 6378-6396; 10.1021/jp810292n (2009).

42. Legault, C. Y. CYLview (Université de Sherbrooke, 2009). 
Acknowledgments This work was supported by the National Natural Science Foundation of China (21773033, 21725301, 21972024, 91645201, 21821004, 91645115, 91845201, 22088101, 21961160722), Science \& Technology Commission of Shanghai Municipality (19DZ2270100), Beijing Outstanding Young Scientist Program (BJJWZYJH01201914430039) and SINOPEC (X514005). X-ray absorption spectroscopy (XAS) experiments were performed in Shanghai Synchrotron Radiation Facility (SSRF) BL14W. D. M. acknowledges support from the Tencent Foundation through the XPLORER PRIZE.

Author contributions Y.C. and D.M. designed the study. Q.Z. and W.G. performed most of catalyst preparation, characterization, and catalytic tests. Z.S. and Y.Z. assisted with the

\section{Additional information}

Supplementary Information is available for this paper.

Correspondence and requests for materials should be addressed to Yong Cao, Ding Ma, Zhen Hua Li or Wu Zhou.

Reprints and permissions information is available at www.nature.com/reprints. 


\section{Supplementary Information for}

\section{Nitrogen-neighbored single cobalt sites enable \\ heterogeneous oxidase-type catalysis}

Qi Zhang, ${ }_{1,8}^{1,8}$ Mi Peng, ${ }^{2,8}$ Zirui Gao, ${ }^{2,8}$ Wendi Guo, ${ }^{1,8}$ Zehui Sun, ${ }^{1}$ Yi Zhao, ${ }^{1}$ Wu Zhou, ${ }^{3,4 *}$ Meng Wang, ${ }^{2}$ Bingbao Mei, ${ }^{5,6}$ Xianlong Du, ${ }^{5}$ Zheng Jiang, ${ }^{5,6}$ Wei Sun, ${ }^{7}$ Chao Liu, ${ }^{7}$ Yifeng Zhu, ${ }^{1}$ Yongmei Liu, ${ }^{1}$ Heyong He, ${ }^{1}$ Zhen Hua Li,${ }^{1 *}$ Ding Ma, ${ }^{2 *}$ Yong $\mathrm{Cao}^{1 *}$

${ }^{1}$ Shanghai Key Laboratory of Molecular Catalysis and Innovative Materials, Department of Chemistry, Fudan University, Shanghai, 200438, China.

${ }^{2}$ Beijing National Laboratory for Molecular Engineering, College of Chemistry and Molecular Engineering, Peking University, Beijing, 100871, China.

${ }^{3}$ School of Physical Sciences and CAS Key Laboratory of Vacuum Physics, University of Chinese Academy of Sciences, Beijing, 100049, China.

${ }^{4}$ CAS Center for Excellence in Topological Quantum Computation, University of Chinese Academy of Sciences, Beijing, 100049, China.

${ }^{5}$ Shanghai Institute of Applied Physics, Chinese Academy of Sciences, Shanghai, 201204, China.

${ }^{6}$ Shanghai Synchrotron Radiation Facility, Zhangjiang Lab, Shanghai Advanced Research Institute, Chinese Academy of Sciences, Shanghai, 201204, China.

${ }^{7}$ State Key Laboratory for Oxo Synthesis and Selective Oxidation, Suzhou Research Institute, Lanzhou Institute of Chemical Physics, Chinese Academy of Sciences, Lanzhou, 730000, China.

${ }^{8}$ These authors contributed equally to this work: Qi Zhang, Mi Peng, Zirui Gao, Wendi Guo.

*Email: yongcao@fudan.edu.cn (Y.C.); dma@pku.edu.cn (D.M.); zhenhuali@fudan.edu.cn (Z.H.L.); wuzhou@ucas.ac.cn (W.Z.)

\section{This PDF file includes:}

Supplementary Methods

Supplementary Figures 1 to 53

Supplementary Tables 1 to 13

Supplementary Discussions 1 to 3

NMR Spectra

Supplementary References 


\section{Table of Contents}

Supplementary Methods............................................................................................................ 3

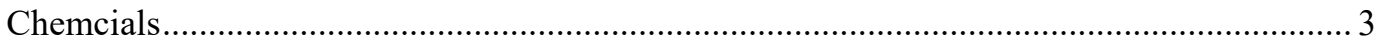

Synthesis of CoPc-based or -derives catalysts ..................................................................... 3

Supplementary Figures, Tables and Discussions .................................................................................5

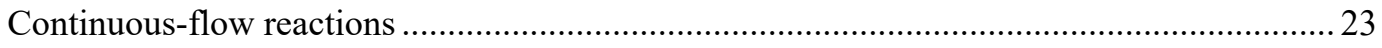

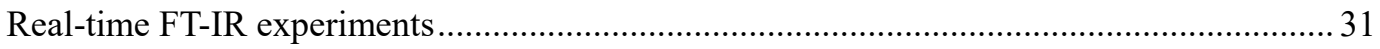

Scopes and Limitations Studies ......................................................................................................6 65

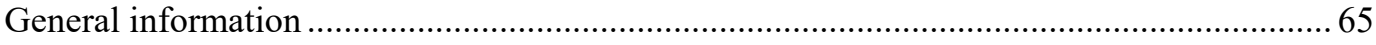

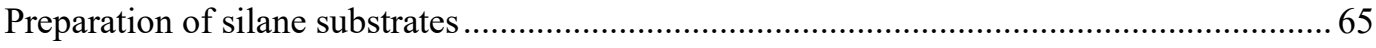

General procedure for Co- $\mathrm{N}_{4} @ \mathrm{NPC}$-catalyzed selective oxidation of hydrosilanes under optimized reaction conditions in batch reactors (GP A) ...................................................... 91

General procedure for $\mathrm{Co}_{-} \mathrm{N}_{4} @ \mathrm{NPC}$-catalyzed multicomponent synthesis of borosiloxanes (GP

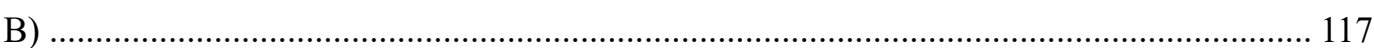

General procedure for Co- $\mathrm{N}_{4} @$ NPC-catalyzed oxidative cross-coupling of hydrosilanes and

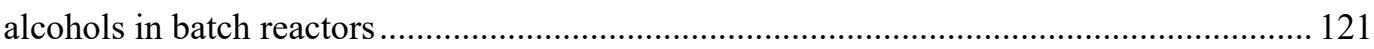

Oxidative cross-coupling of hydrosilanes with simple alkyl alcohols (GP C) .............. 121

Oxidative cross-coupling of hydrosilanes with complex alcohols (GP D).................... 125

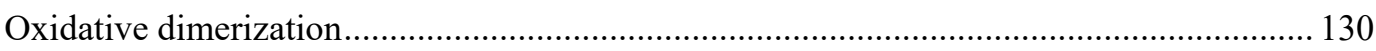

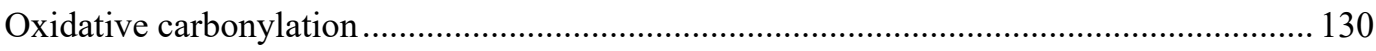

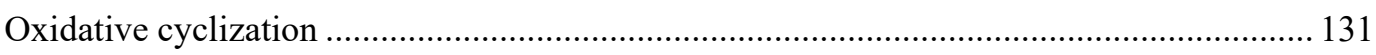

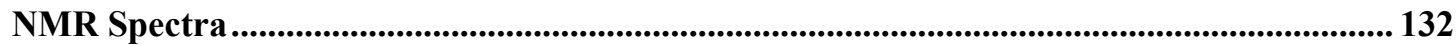

Geometries and Cartesian coordinates for optimized structures ...........................................493

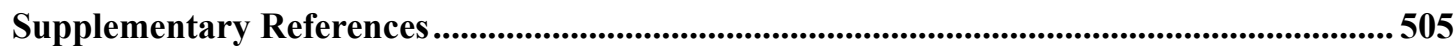




\section{Supplementary Methods}

\section{Chemcials}

All chemicals were used without further purification, unless otherwise stated. cobalt chloride hexahydrate $\left(\mathrm{CoCl}_{2} \cdot 6 \mathrm{H}_{2} \mathrm{O}, \geq 99.0 \%\right)$, zinc nitrate hexahydrate $\left(\mathrm{Zn}\left(\mathrm{NO}_{3}\right)_{2} \cdot 6 \mathrm{H}_{2} \mathrm{O}, \geq 99.0 \%\right)$, urea $\left(\mathrm{CO}\left(\mathrm{NH}_{2}\right)_{2}, \geq 99.0 \%\right)$, methanol ( $\mathrm{MeOH}, \geq 99.7 \%$ ), ethanol (EtOH, $\geq 99.7 \%$ ), N,N-dimethylformamide (DMF, HPLC grade), chloroform $\left(\mathrm{CHCl}_{3}, \geq 99.0 \%\right)$, acetone $\left(\mathrm{CO}\left(\mathrm{CH}_{3}\right)_{2}, \geq 99.5 \%\right)$, hydrochloric acid $(\mathrm{HCl}, 36.0-38.0 \%)$ and sodium hydroxide $(\mathrm{NaOH}, \geq 96.0 \%)$ were purchased from Sinopharm Chemical Reagent Co., Ltd. Tetrachlorophthalic anhydride (98\%) was purchased from Aladdin. Ammonium molybdate tetrahydrate $\left(\left(\mathrm{NH}_{4}\right)_{6} \mathrm{Mo}_{7} \mathrm{O}_{24} \cdot 4 \mathrm{H}_{2} \mathrm{O}, \geq 99.0 \%\right)$ were purchased from Sigma-Aldrich. Cobalt (II) phthalocyanine $(\mathrm{CoPc},>93.0 \%)$ was purchased from TCI. $\mathrm{TiO}_{2}$ (AEROXIDE P25, specific surface area: $50 \mathrm{~m}^{2} / \mathrm{g}$ ) was purchased from Evonik.

\section{Synthesis of CoPc-based or -derives catalysts}

A) Synthesis of cobalt (II) hexadecachlorophthalocyanine (CoPc-16Cl)

The following procedure is adapted from the literature ${ }^{1}$.
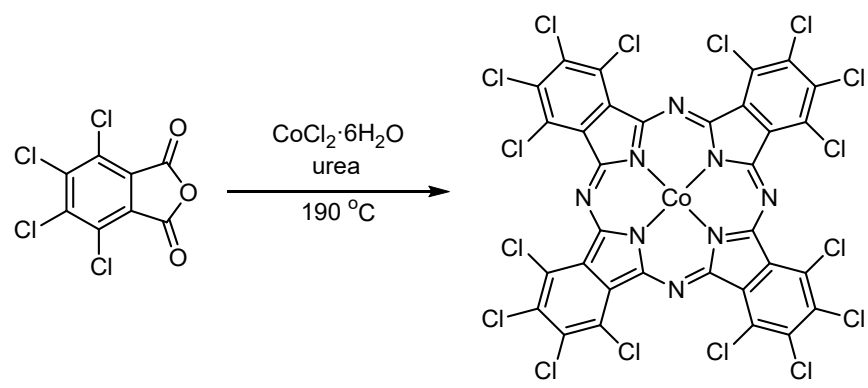

A finely ground mixture of $\mathrm{CoCl}_{2} \cdot 6 \mathrm{H}_{2} \mathrm{O}(2.38 \mathrm{~g} 10 \mathrm{mmol})$, tetrachlorophthalic anhydride $(11.4 \mathrm{~g}, 40 \mathrm{mmol})$, urea $(24.0 \mathrm{~g}, 400 \mathrm{mmol})$ and $\left(\mathrm{NH}_{4}\right)_{6} \mathrm{Mo}_{7} \mathrm{O}_{24} \cdot 4 \mathrm{H}_{2} \mathrm{O}(0.125 \mathrm{~g}, 0.1 \mathrm{mmol})$ was reacted in solid state at $190{ }^{\circ} \mathrm{C}$ for $5 \mathrm{~h}$. The resulting solid was ground and further stirred in $500 \mathrm{~mL} \mathrm{HCl}$ aqueous solution $(1 \mathrm{~mol} / \mathrm{L})$ and $500 \mathrm{~mL} \mathrm{NaOH}$ aqueous solution $(1 \mathrm{~mol} / \mathrm{L})$ at $100{ }^{\circ} \mathrm{C}$ for $1 \mathrm{~h}$, respectively. The residue was then filtered, washed with water, and dried under vacuum to obtain crude $\mathrm{CoPc}-16 \mathrm{Cl}$. The crude product was transferred to a Soxhlet thimble and extracted using $\mathrm{CHCl}_{3}$, EtOH and acetone for $48 \mathrm{~h}$, successively. After drying at 60 ${ }^{\circ} \mathrm{C}$ for $3 \mathrm{~h}, \mathrm{CoPc}-16 \mathrm{Cl}$ was obtained as dark green solid.

UV-Vis (DMF, $\left.\lambda_{\max }=\mathbf{n m}\right): 660(\mathrm{~s}), 498(\mathrm{sh}), 306(\mathrm{sh})$.

FT-IR (KBr, $\left.\mathbf{~ c m}^{-1}\right):$ 1600, 1459, 1393, 1383, 1340, 1315, 1275, 1215, 1201, 1163, 1130, 1103, 1037, 955, $778,764,752$.

\section{B) Synthesis of PC and NPC supports}

PC and NPC supports were synthesized by using ZIF-8 as precursor following one-step thermal activation under different conditions. For synthesis of ZIF-8 precursor, a solution of 2-MeIm (13.1 g, $160 \mathrm{mmol})$ in $400 \mathrm{~mL} \mathrm{MeOH}$ was added to a solution of $\mathrm{Zn}\left(\mathrm{NO}_{3}\right)_{2} \cdot 6 \mathrm{H}_{2} \mathrm{O}(5.95 \mathrm{~g}, 20 \mathrm{mmol})$ in $400 \mathrm{~mL}$ methanol. After stirred at room temperature $\left(25^{\circ} \mathrm{C}\right)$ for $24 \mathrm{~h}$, the obtained ZIF-8 precipitates were collected by centrifugation, wash with $\mathrm{MeOH}$ for three times and then dried under vacuum at room temperature overnight.

For synthesis of NPC support, $1.0 \mathrm{~g}$ as-prepared ZIF-8 precursor was heated at $1000{ }^{\circ} \mathrm{C}$ for $4 \mathrm{~h}$ with a heating rate of $2{ }^{\circ} \mathrm{C} / \mathrm{min}$ under the flowing Ar atmosphere $(80 \mathrm{~mL} / \mathrm{min})$ in a tube furnace, followed by 
naturally cooling down to room temperature. NPC was obtained as a black powder. The product was used directly without further treatment.

PC support was synthesized using similar procedure but pyrolyzed at $1400{ }^{\circ} \mathrm{C}$ for $8 \mathrm{~h}$.

\section{C) Synthesis of $\mathrm{CoPc} / \mathrm{PC}, \mathrm{CoPc} / \mathrm{NPC}, \mathrm{CoPc}-16 \mathrm{Cl} / \mathrm{NPC}, \mathrm{CoPc} / \mathrm{TiO}_{2}$ and $\mathrm{CoPc}-16 \mathrm{Cl} / \mathrm{TiO} \mathrm{O}_{2}$}

A series of supported phthalocyanine cobalt catalysts with the Co loading of $0.25 \mathrm{wt} \%$ were prepared by incipient wetness impregnation. The synthesis of $\mathrm{CoPc} / \mathrm{NPC}$ is given as follows. A certain amount of $\mathrm{CoPc}$ was dissolved in $15 \mathrm{~mL}$ DMF and $0.5 \mathrm{~g} \mathrm{NPC}$ was dispersed in the solution. After stirring at room temperature for $12 \mathrm{~h}$, the solvent was removed by rotary evaporation. The sample was dried under dynamic vacuum ( 0.1 torr) at $150{ }^{\circ} \mathrm{C}$ for $3 \mathrm{~h}$ and $\mathrm{CoPc} / \mathrm{NPC}$ was obtained. Other catalysts were prepared according to the same procedure by using different substituted cobalt phthalocyanines or supports.

\section{D) Synthesis of $\mathrm{Co}-\mathrm{N}_{4} / \mathrm{NPC}$}

$0.5 \mathrm{~g}$ as-prepared $\mathrm{CoPc} / \mathrm{NPC}$ was heated at $600{ }^{\circ} \mathrm{C}$ for $2 \mathrm{~h}$ with a heating rate of $2{ }^{\circ} \mathrm{C} /$ min under the flowing Ar atmosphere $(80 \mathrm{~mL} / \mathrm{min})$ in a tube furnace. After cooling down to room temperature, Co- $\mathrm{N}_{4} / \mathrm{NPC}$ was obtained. 


\section{Supplementary Figures, Tables and Discussions}

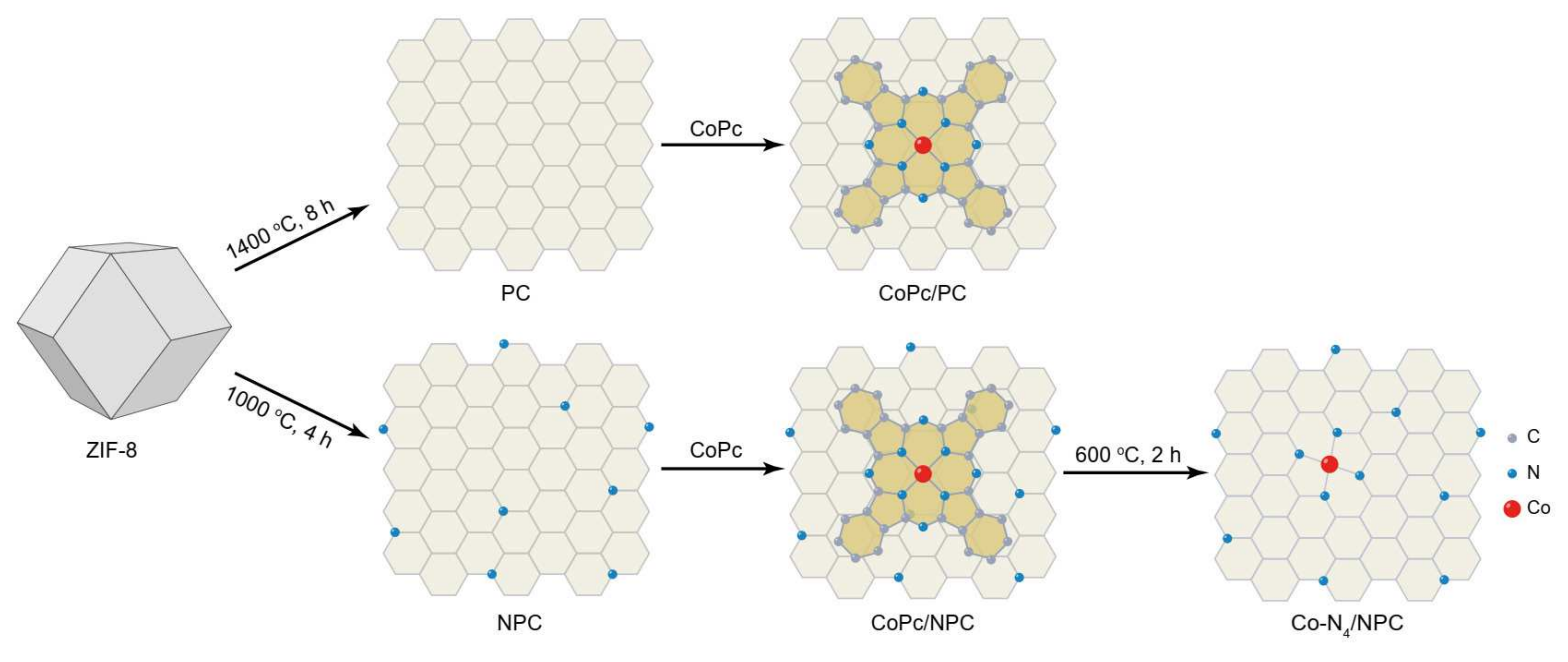

Supplementary Fig. 1 | Procedures for the synthesis CoPc-based or -derived biomimetic catalyst models. 


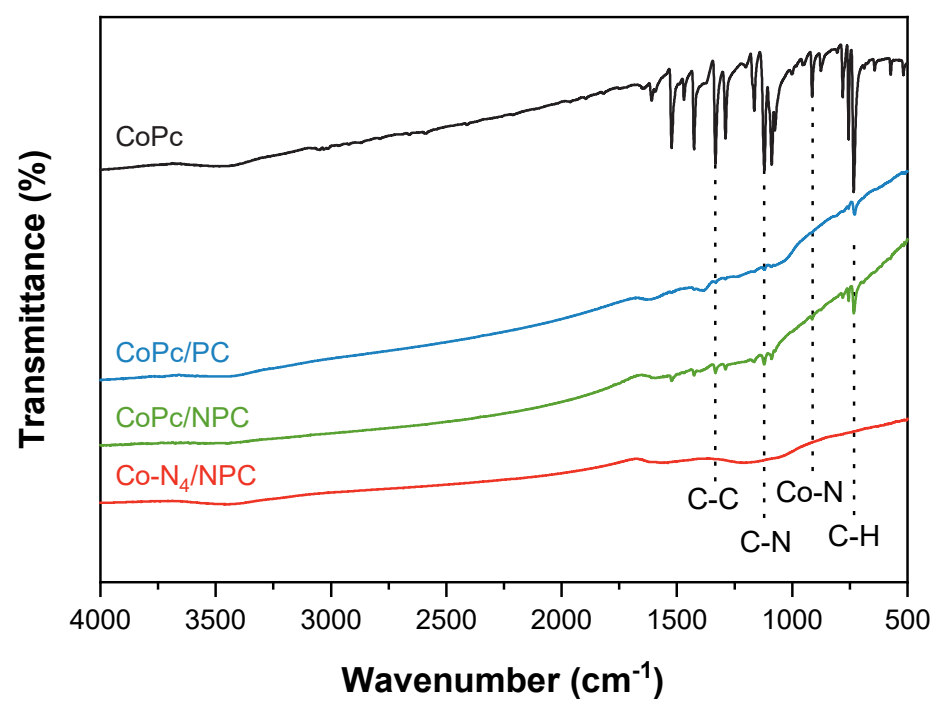

Supplementary Fig. 2 | FT-IR spectra of CoPc, $\mathrm{CoPc} / \mathrm{PC}, \mathrm{CoPc} / \mathrm{NPC}$ and $\mathrm{Co}-\mathrm{N}_{4} / \mathrm{NPC}$. The characteristic peaks of CoPc could obtain in $\mathrm{CoPc} / \mathrm{PC}$ and $\mathrm{CoPc} / \mathrm{NPC}$ samples, confirming the existence of $\mathrm{CoPc}$ on the carbon-based supports. While the Co- $\mathrm{N}_{4} / \mathrm{NPC}$ sample has no characteristic peak of $\mathrm{CoPc}$, indicating the CoPc molecules were decomposed after pyrolysis step. 

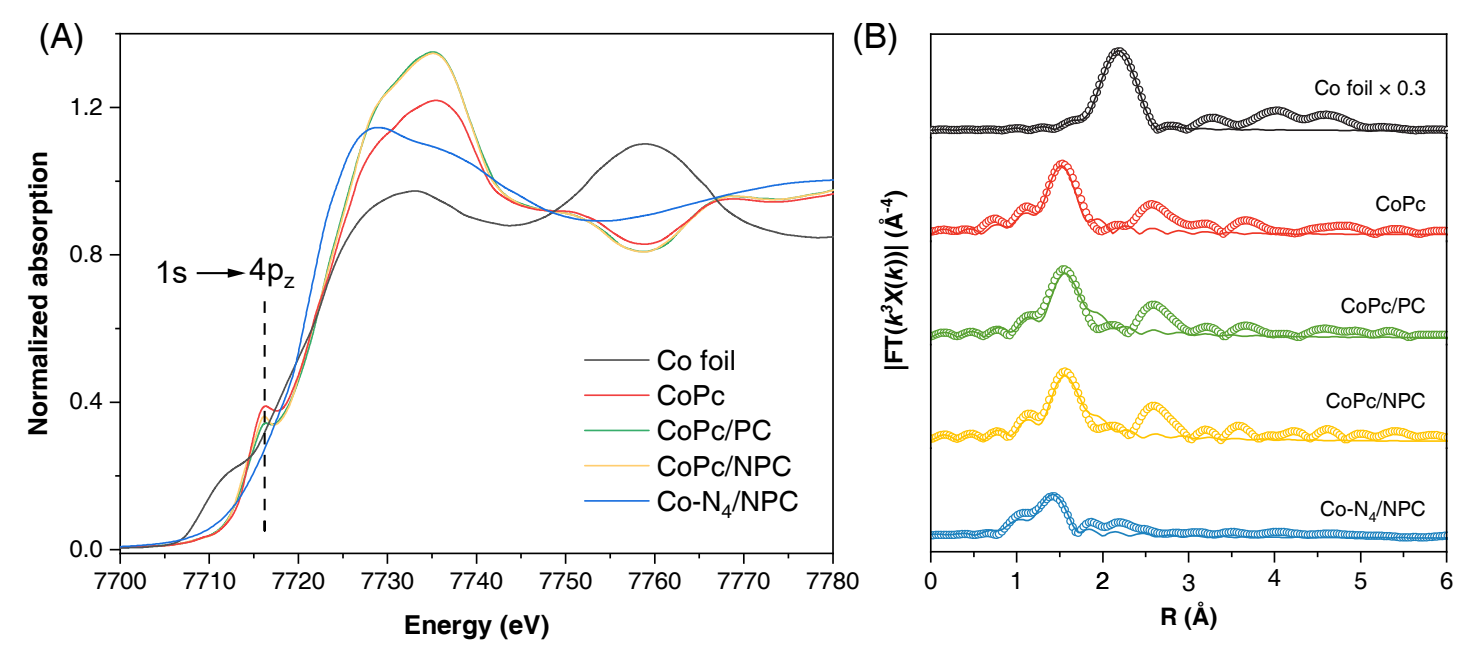

Supplementary Fig. 3 | (A) Co K-edge XANES spectra and (B) R-space Co K-edge EXAFS spectra for CoPc-based or -derived catalysts, $\mathrm{CoPc}$ and $\mathrm{Co}$ foil (open circles stand for as obtained data and solid lines denote for fitting curves). The fitting results are listed in Supplementary Table 2. In Supplementary Fig. $\mathbf{3 A}$, the shoulder at $7715.7 \mathrm{eV}$ arising from $1 \mathrm{~s} \rightarrow 4 \mathrm{p}_{\mathrm{z}}$ shakedown transition is a straightforward fingerprint for a square-planar Co- $\mathrm{N}_{4}$ configuration with $\mathrm{D}_{4 \mathrm{~h}}$ symmetry ${ }^{2}$. However, as this shoulder is relatively weak for $\mathrm{Co}-\mathrm{N}_{4} / \mathrm{NPC}$ as compared to that for $\mathrm{CoPc}, \mathrm{CoPc} / \mathrm{PC}$ and $\mathrm{CoPc} / \mathrm{NPC}$, indicating that the $\mathrm{D}_{4 \mathrm{~h}}$ symmetry is broken. This result further confirmed that the macrocyclic structure of $\mathrm{CoPc}$ was decomposed after pyrolysis step. 

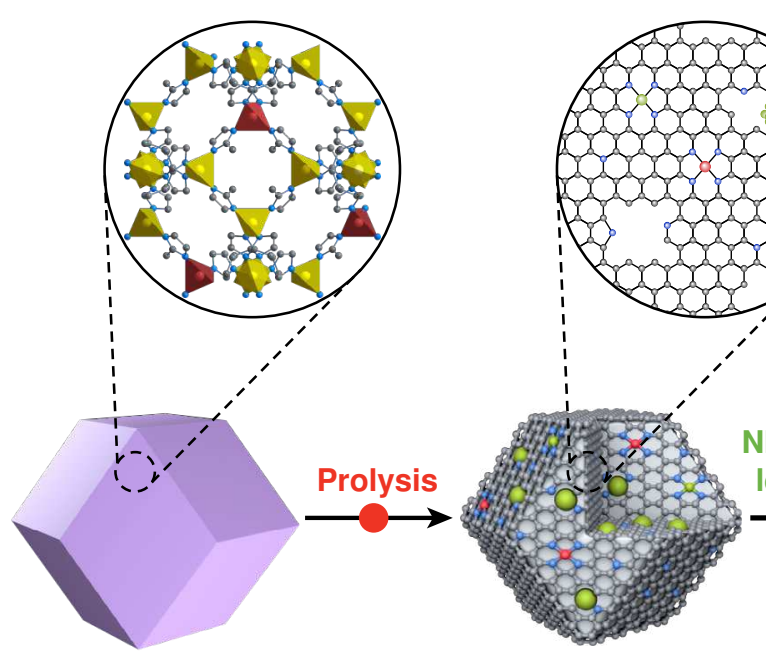

leaching activation

$\mathrm{Co}_{1} \mathrm{Zn}_{50}$-ZIF

Co-N $4 @ N P C-N L$
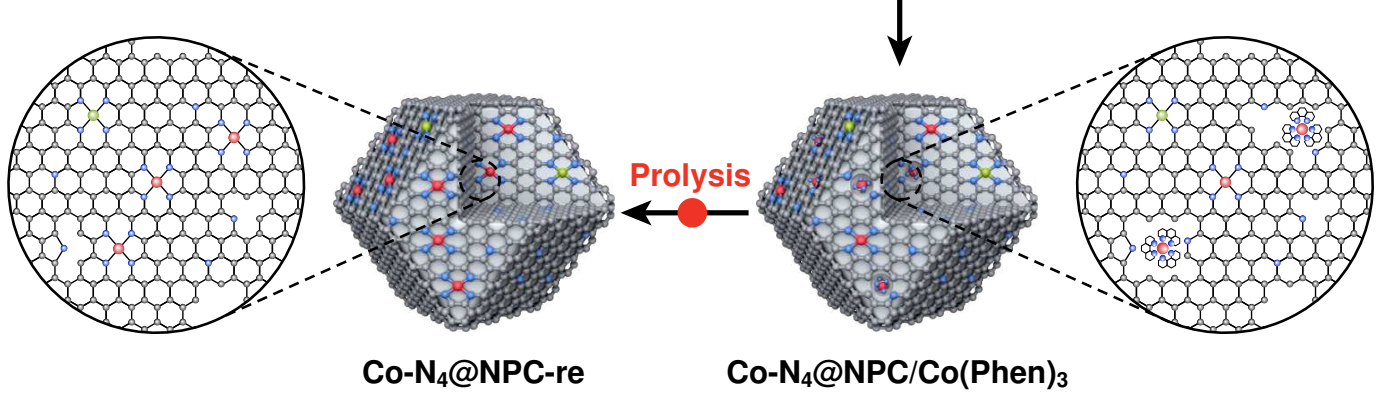

co $\mathrm{N} O \mathrm{c}$

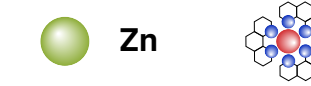

Co phenanthroline complex

Supplementary Fig. 4 | Procedures for the synthesis of Co-N4@NPC-based materials. 


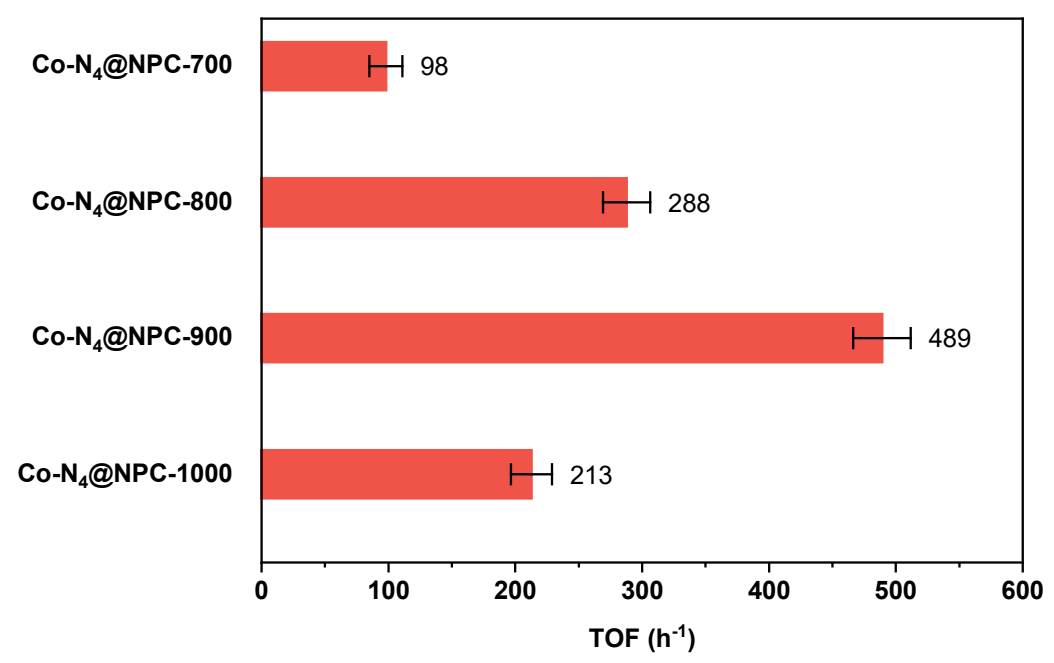

Supplementary Fig. 5 | Catalytic performance for oxidation of 1a over Co- $\mathrm{N}_{4} @$ NPC-based catalyst prepared with different pyrolysis temperatures. Reaction conditions: $\mathbf{1 a}(1 \mathrm{mmol}), \mathrm{H}_{2} \mathrm{O}(8 \mathrm{mmol}), \mathrm{MeCN}(3$ $\mathrm{mL}$ ), catalyst (Co $0.5 \mathrm{~mol} \%$ ), air atmosphere, $30{ }^{\circ} \mathrm{C}$. TOF was measured at $1 \mathrm{a}$ conversion below $20 \%$ based on total Co atoms. Error bars represent the standard deviation from at least three independent experiments. For all reactions, $>99 \%$ selectivity of $\mathbf{2 a}$ were obtained. 


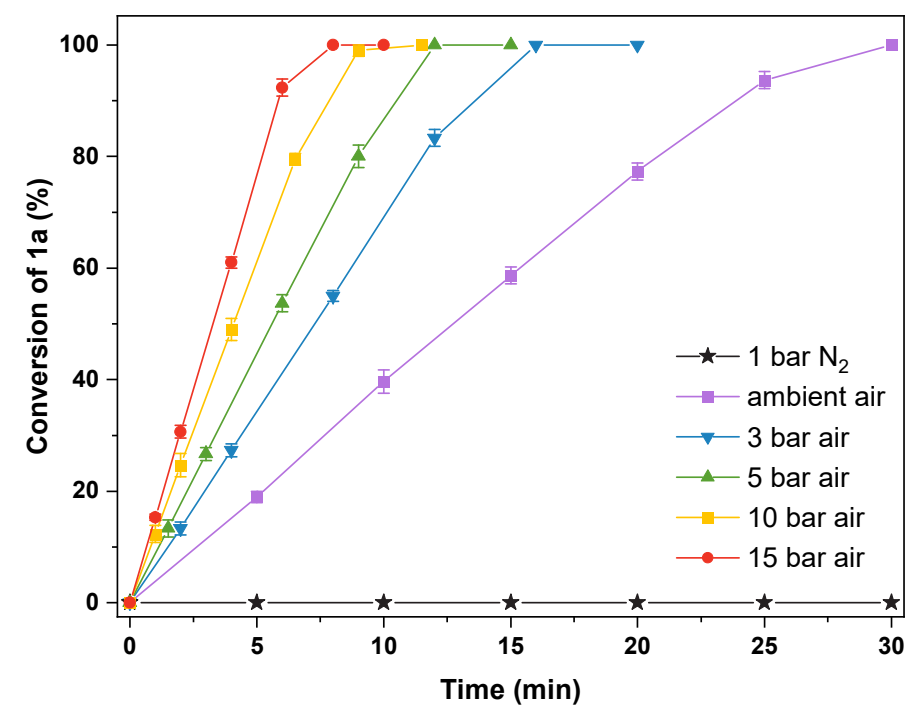

Supplementary Fig. 6 | Reaction profile for oxidation of 1a under different atmosphere over Co- $\mathrm{N}_{4} @$ NPC900 catalyst. Reaction conditions: 1a $(1 \mathrm{mmol}), \mathrm{H}_{2} \mathrm{O}(8 \mathrm{mmol}), \mathrm{MeCN}(3 \mathrm{~mL}), \mathrm{Co}-\mathrm{N}_{4} @ \mathrm{NPC}-900$ (Co 0.5 mol\%), $30{ }^{\circ} \mathrm{C}$. The conversion was determined by GC using anisole as an internal standard. 


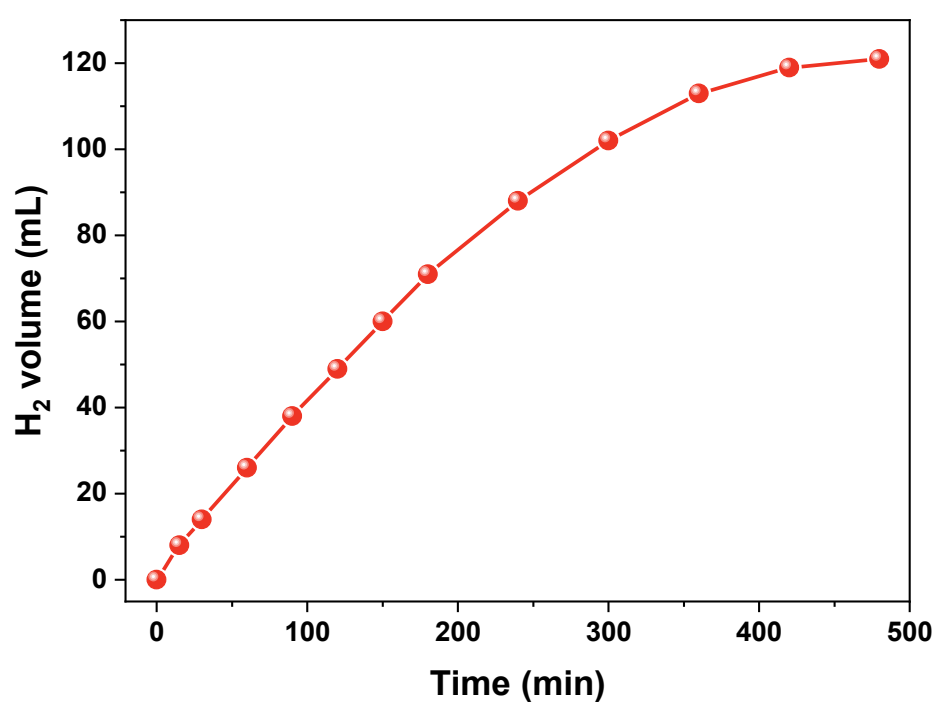

Supplementary Fig. 7 | $\mathrm{H}_{2}$ evolution by hydrolytic dehydrogenation of 1a catalyzed by Co-NPs@NPC. Reaction conditions: 1a (5 mmol), $\mathrm{H}_{2} \mathrm{O}(40 \mathrm{mmol}), \mathrm{MeCN}(15 \mathrm{~mL}), \mathrm{Co}-\mathrm{NPs} @ \mathrm{NPC}(0.5 \mathrm{~mol} \%), 30^{\circ} \mathrm{C}, \mathrm{N}_{2}$ atmosphere. The volume change of $\mathrm{H}_{2}$ was detected by a gas barrette. After $8 \mathrm{~h}$ reaction, $121 \mathrm{~mL} \mathrm{H}_{2}$ was produced. 

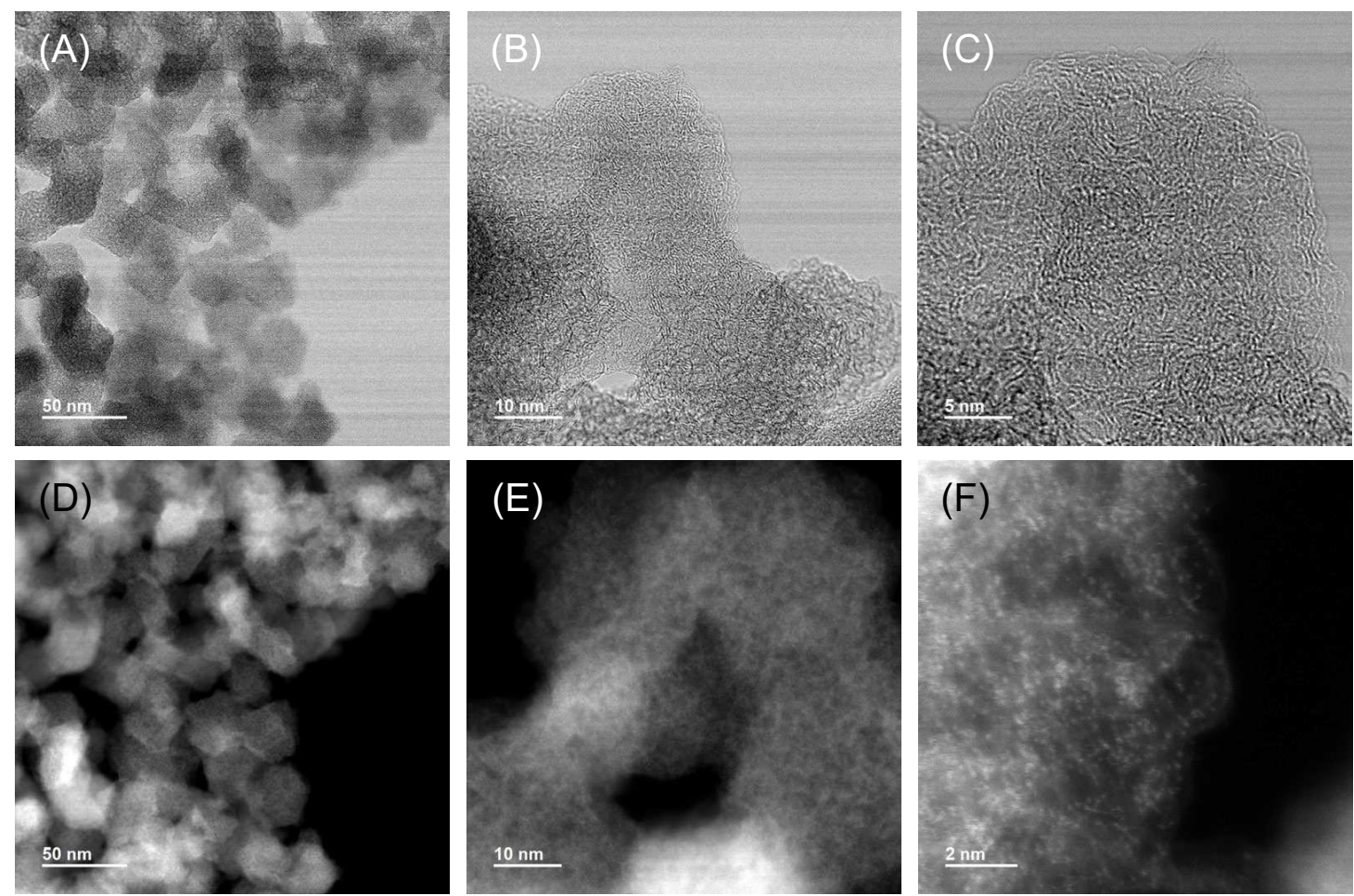

Supplementary Fig. 8 | Additional electron microscopy characterization results of Co-N4@NPC-900.

Low magnifi-cation BF-STEM (bright-field) (A) and HAADF-STEM images (D) showed that Co$\mathrm{N}_{4} @$ NPC-900 sample has a highly porous appearance, formed by the fusion and aggregation of $\mathrm{Co}_{1} \mathrm{Zn}_{50^{-}}$ ZIF precursor $(\sim 35 \mathrm{~nm})$ under high-temperature pyrolysis conditions. BF-STEM images (B and C) revealed that Co-N $\mathrm{N}_{4} @$ NPC-900 sample has highly disordered carbon structures with randomly oriented graphitic domains. The pore-like structure in carbon species was confirmed by HAADF-STEM image (E). Furthermore, atomic-resolution HAADF-STEM image of different region in Co-N 4 @NPC-900 sample (F) also confirmed the uniformly atomic dispersed Co and $\mathrm{Zn}$ sites (bright spots). 

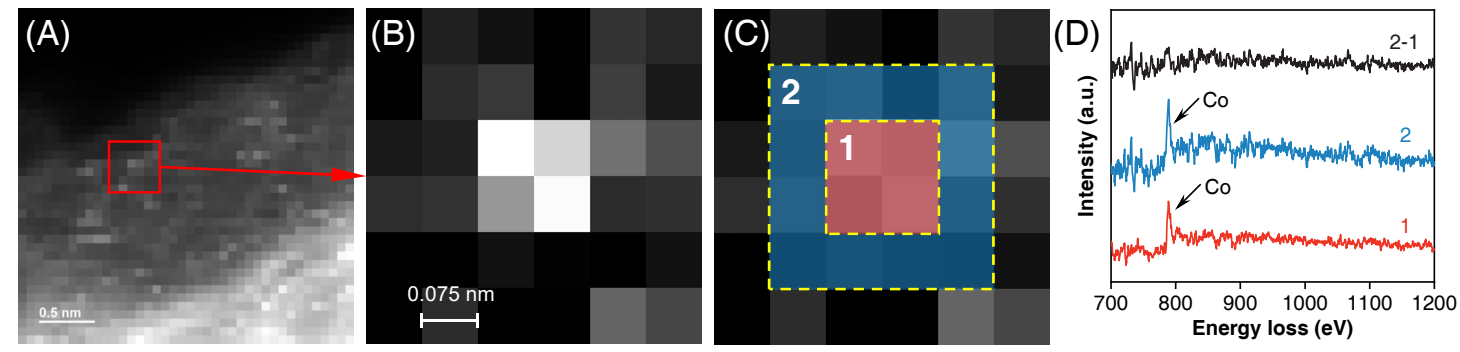

(E)
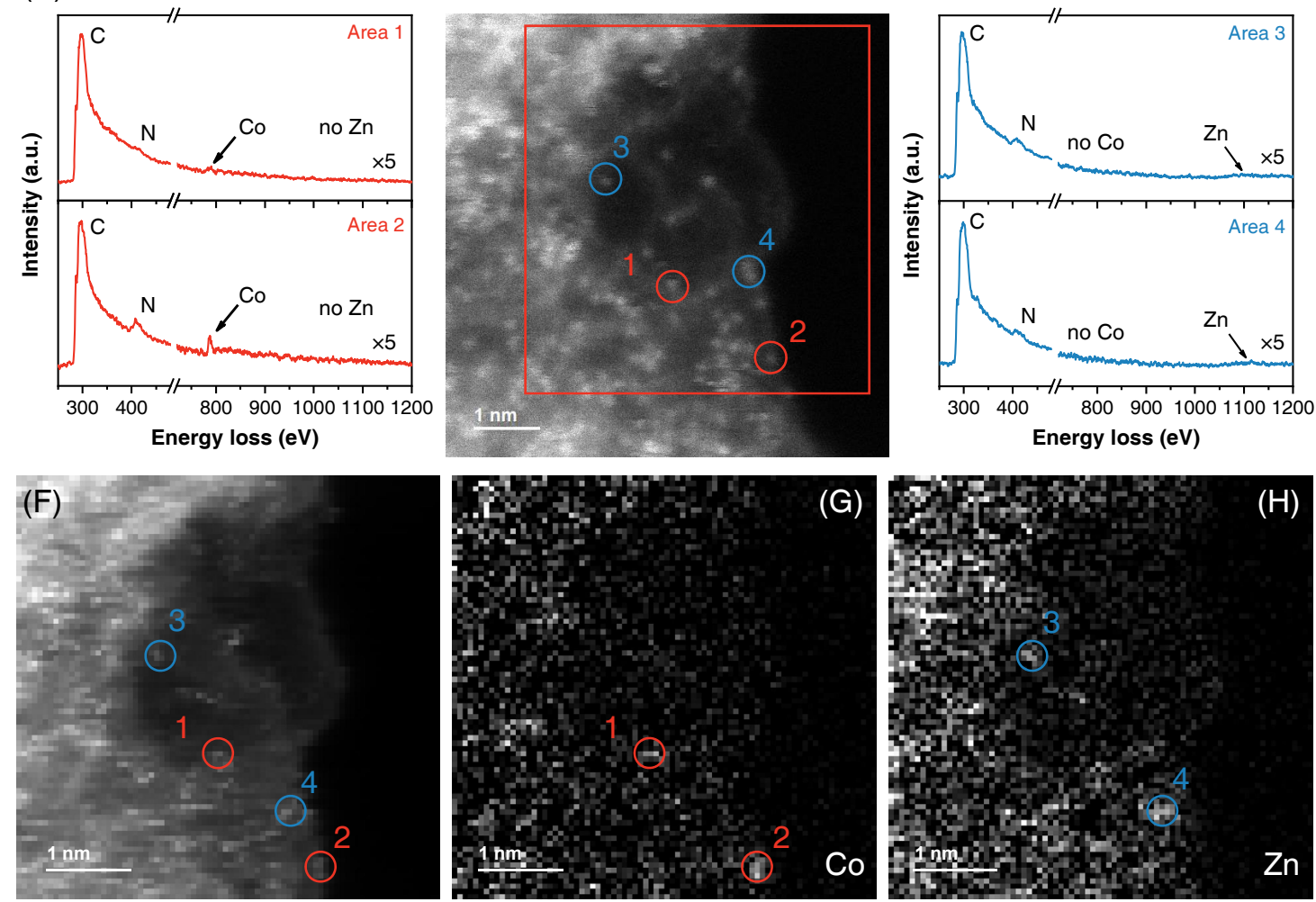

Supplementary Fig. 9 | Identify the M-N $\mathbf{x}_{\mathbf{x}}$ sites in Co-N 4 @NPC-900 by EELS analysis. (A) Regions of HAADF spectrum-image for EELS analysis. (B) Local enlarged STEM-EELS spectrum image of the red box in Supplementary Fig. 9A with $6 \times 6$ pixels. (C) The actual analysis regions with different size (region$1,0.15 \times 0.15 \mathrm{~nm}$ or region-2, $0.30 \times 0.30 \mathrm{~nm}$ ). (D) The EELS spectra acquired from region-1 (red line), region-2 (blue line) and corresponding difference spectrum (black line) of two areas. The EELS spectra acquired from the same bright spot position with different size (region-1 or region-2) in Co-N 4 @NPC-900 showing the similar intensity of Co signal. The difference spectrum of region-1 and region-2 further proved that the Co signal only exists in region-1. (E) HADDF-STEM image and EELS spectra of four different regions in the image. EELS spectra acquired from area 1 and 2 (red region) showed the coexistence of Co and $\mathrm{N}$ but not $\mathrm{Zn}$; while the EELS spectra acquired from area 3 and 4 (blue region) only showed the coexistence of Zn and N. The STEM-EELS spectrum image of the red box in Supplementary Fig. 9 (F) and corresponding elemental EELS map of $\mathrm{Co}(\mathrm{G})$ and $\mathrm{Zn}(\mathrm{H})$ further confirmed the regional distribution of Co and $\mathrm{Zn}$ (the Co signal only increases in area 1 and 2; while the $\mathrm{Zn}$ signal only increases in area 3 and 4). These results suggested the presence of independent Co- $\mathrm{N}_{\mathrm{x}}$ and Zn- $\mathrm{N}_{\mathrm{x}}$ sites in Co- $\mathrm{N}_{4} @ \mathrm{NPC}-900$. 

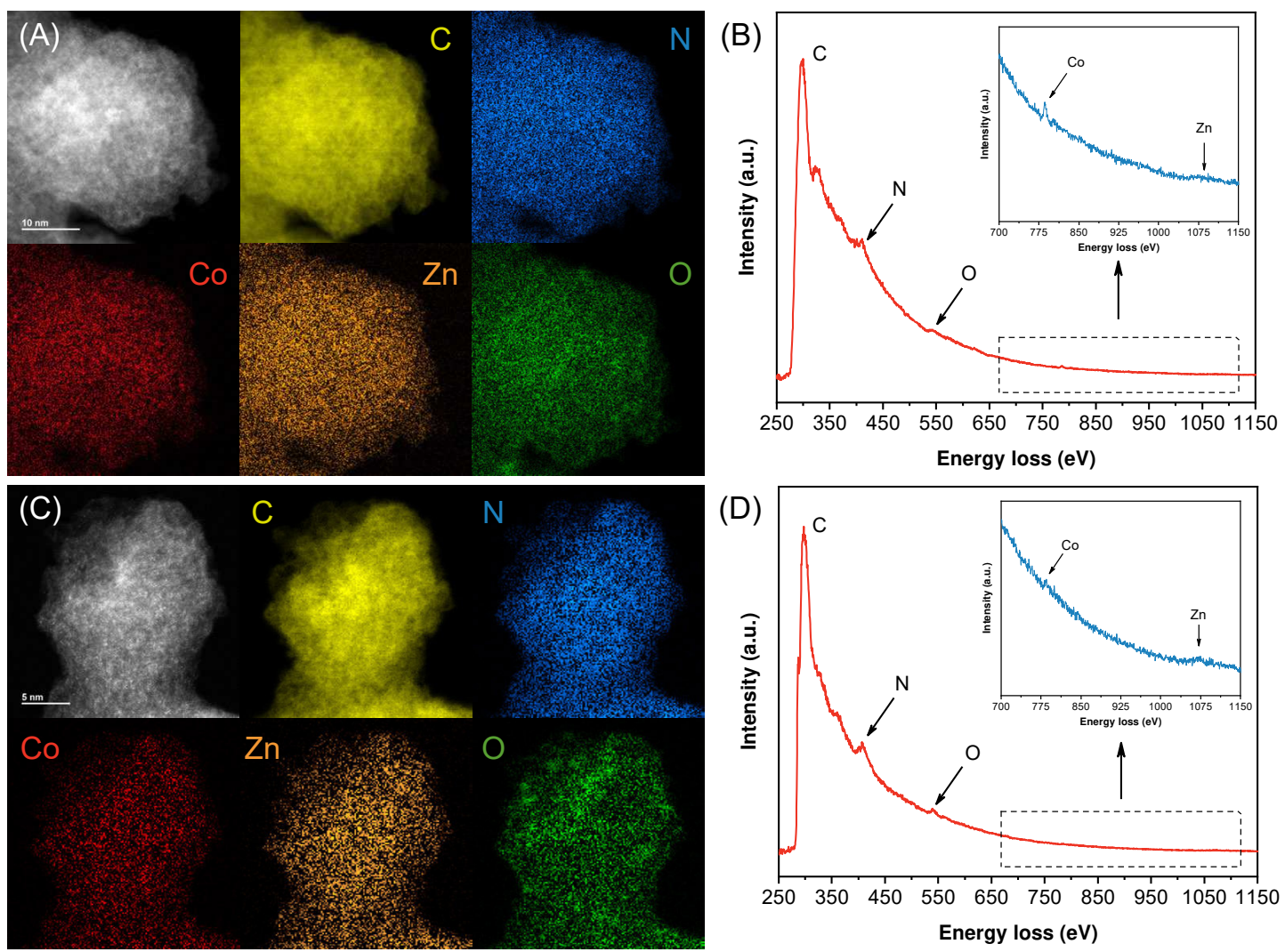

Supplementary Fig. 10 | (A, C) HAADF-STEM images and corresponding EELS elemental maps of C (yellow), N (blue), O (green), Co (red) and Zn (orange) from two different regions in Co-N $\mathrm{N}_{4} @ \mathrm{NPC}-900$. The Supplementary Fig. 10A also shows in Fig. 2B in the main text. (B, D) EELS spectra of the entire region in Supplementary Fig. 10, A and C, respectively. The inset shows the local enlarged spectrum of 700-1150 eV. Distinguishable signals for C, N, O, Co and Zn were observed in the EELS elemental maps and EELS spectra across the carbon species, indicating the uniform distribution of each element. 

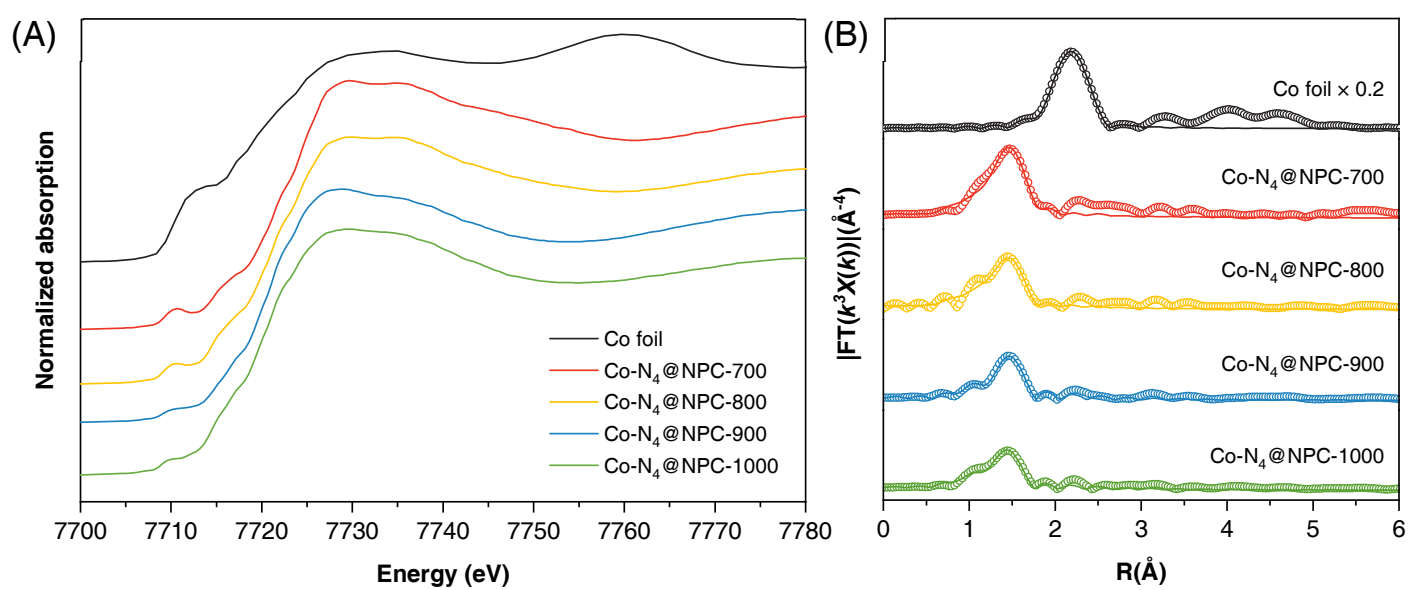

Supplementary Fig. 11 | (A) Co K-edge XANES spectra and (B) R-space Co K-edge EXAFS spectra for Co-N ${ }_{4} @$ NPC-T catalysts prepared with different pyrolysis temperatures and Co foil (open circles stand for as obtained data and solid lines denote for fitting curves). The fitting results are listed in Supplementary Table 2. 

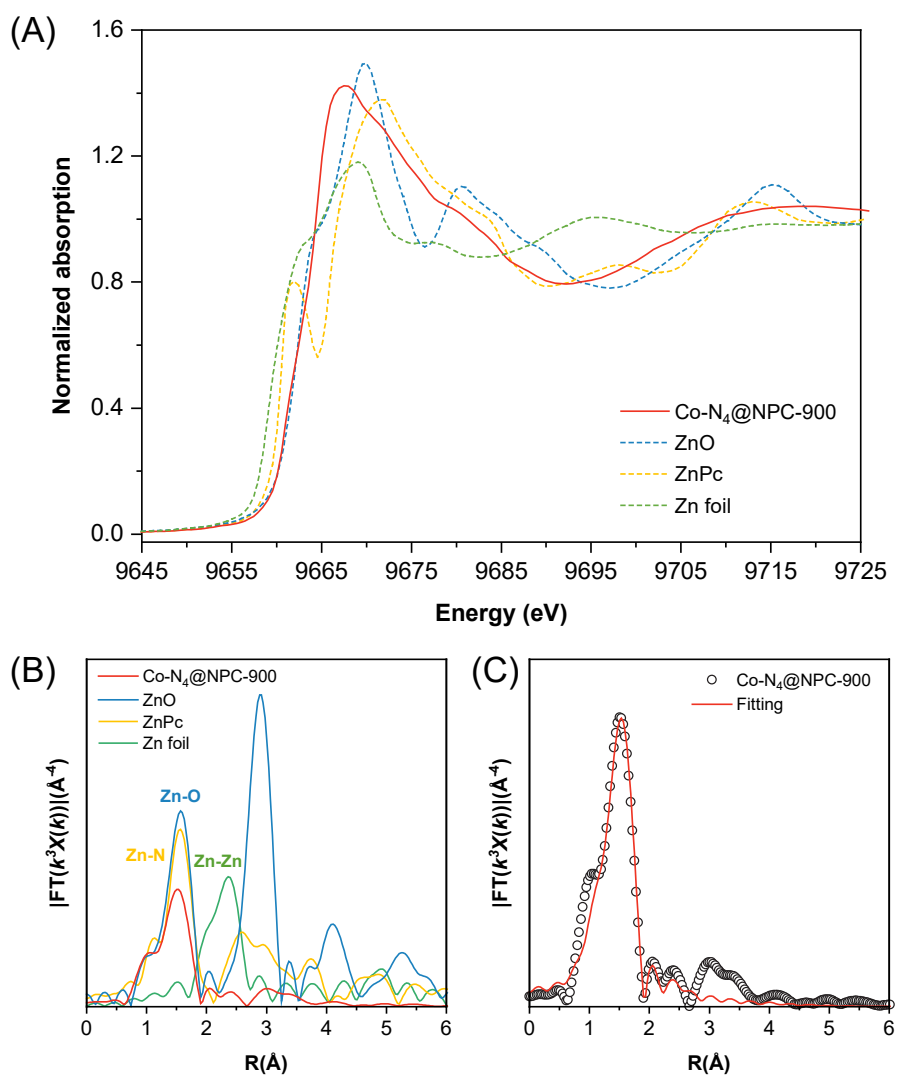

Supplementary Fig. 12 | (A) Zn K-edge XANES spectra and (B) R-space Zn K-edge EXAFS spectra for Co-N 4 @NPC-900 catalyst and reference samples. (C) First shell fitting of Zn K-edge EXAFS spectrum for Co-N ${ }_{4} @$ NPC-900. The fitting results are listed in Supplementary Table 2. 
(A)
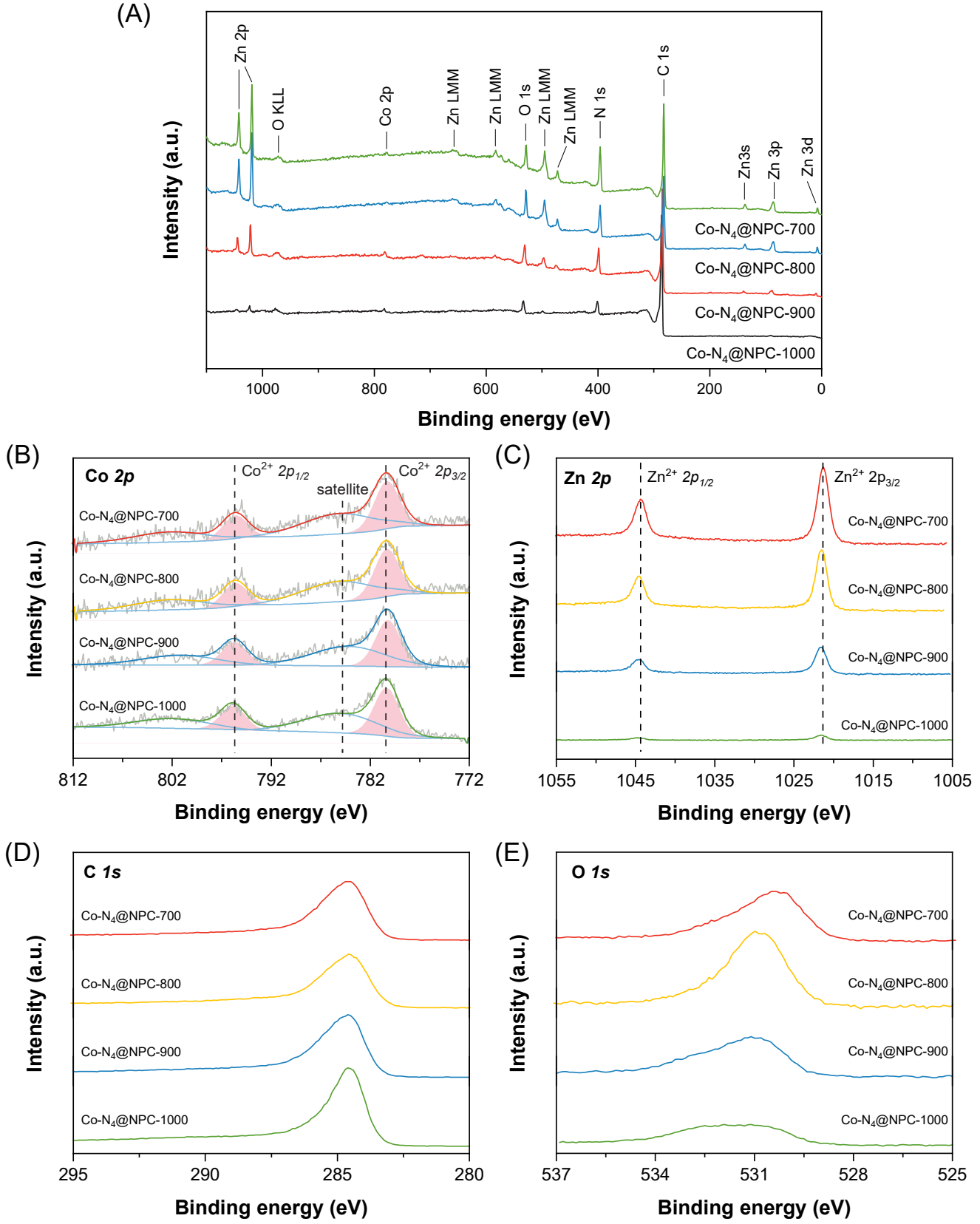

Supplementary Fig. 13 | Survey (A), Co $2 p$ (B), Zn $2 p$ (C), C $1 s$ (D) and O $1 s$ (E) XPS spectra for Co$\mathrm{N}_{4} @$ NPC-T samples prepared with different pyrolysis temperatures. It should be noted that only $\mathrm{Co}^{2+}$ species $(780.3 \mathrm{eV})$ was found in all the samples, revealing that the majority of Co is in atomically-dispersed Co- $\mathrm{N}_{\mathrm{x}}$ sites as evidenced by XAFS results (Fig. 2F, Supplementary Fig. 11). 


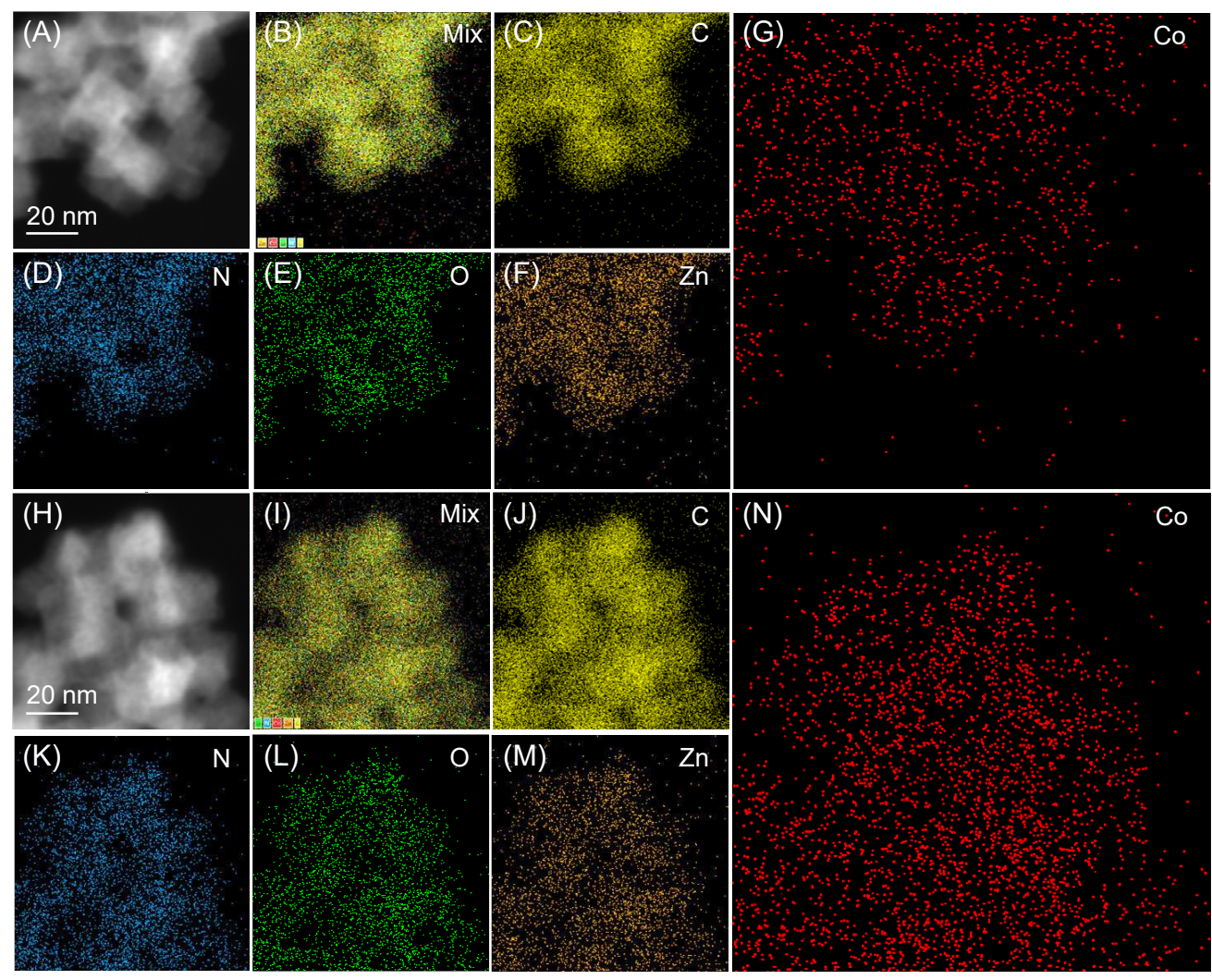

(O)

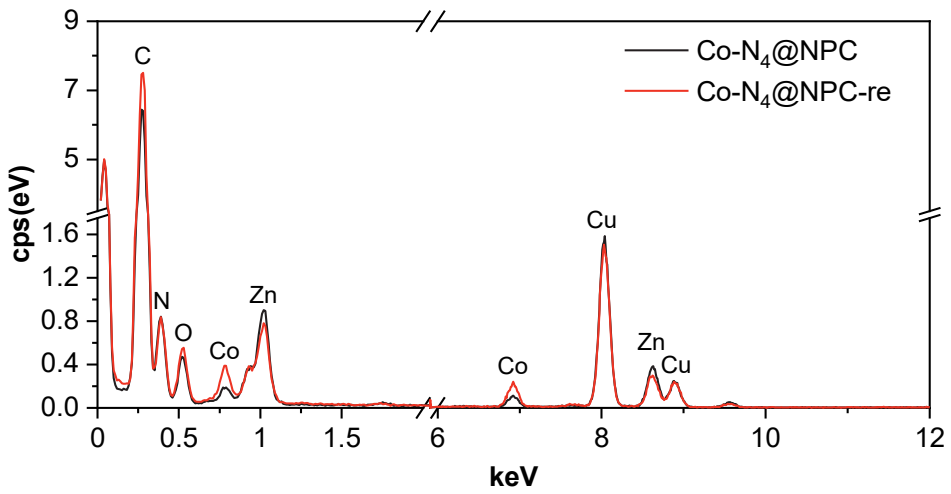

Supplementary Fig. 14 | STEM images and corresponding EDS elemental maps of Co-N $\mathrm{N}_{4} @$ NPC-900 (AG) and Co- $\mathrm{N}_{4} @$ NPC-re (H-N). (O) The corresponding EDS spectra of the region in $(\mathrm{A})$ and $(\mathrm{H})$. 


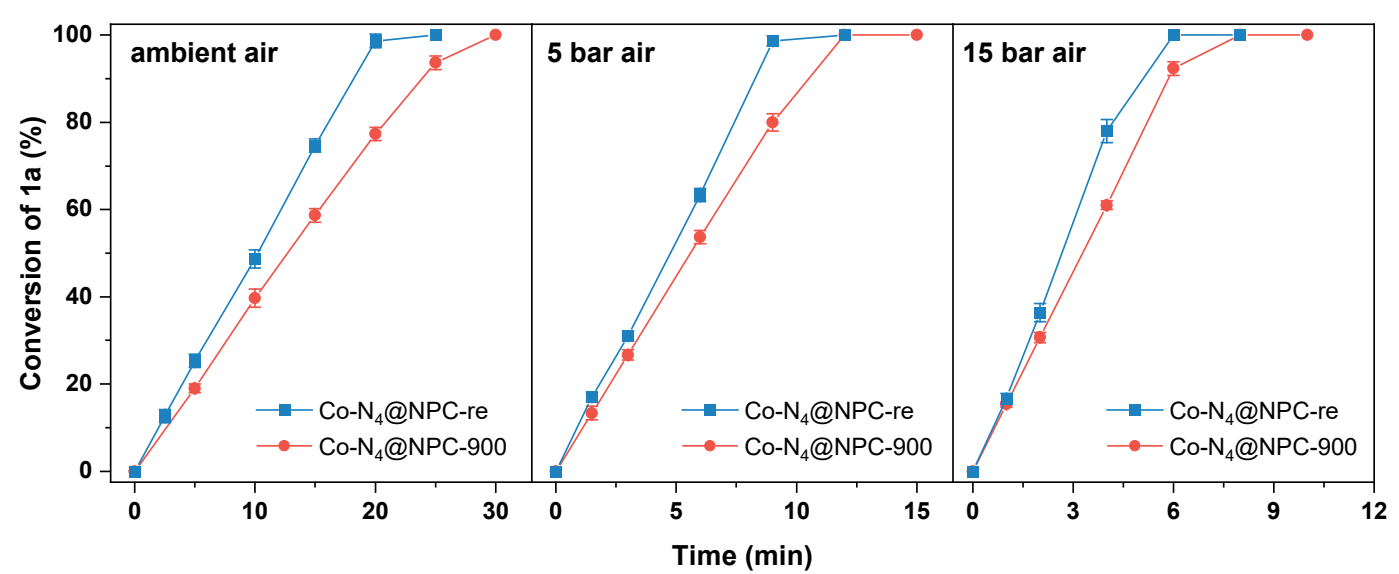

Supplementary Fig. 15 | Reaction profile for oxidation of 1a under different atmosphere over Co-N 4 @NPC-

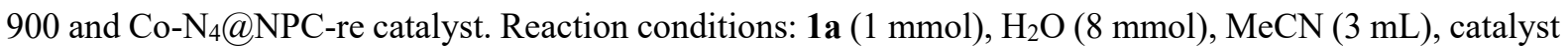
(Co $0.5 \mathrm{~mol} \%$ ), $30^{\circ} \mathrm{C}$. The conversion was determined by $\mathrm{GC}$ using anisole as an internal standard. 


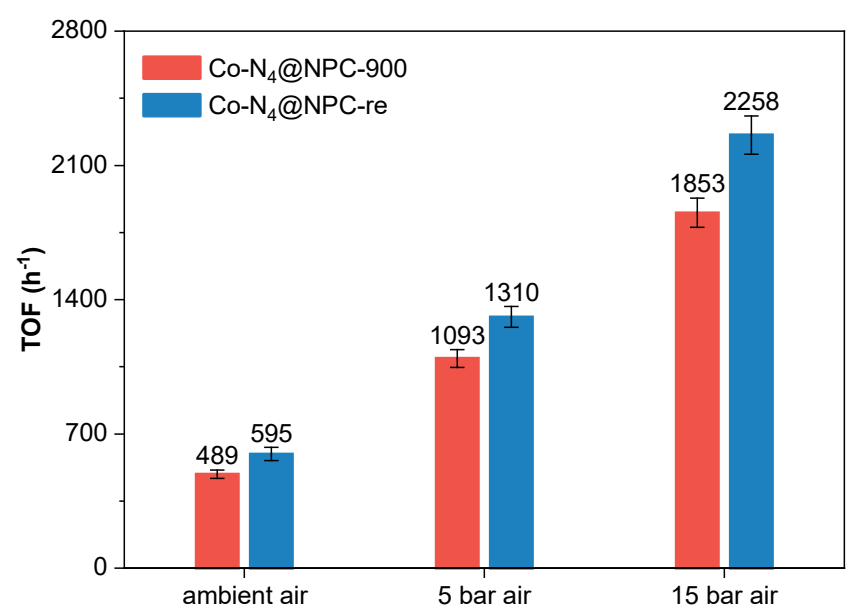

Supplementary Fig. 16 | Catalytic performance for oxidation of 1a over Co- $\mathrm{N}_{4} @$ NPC-900 and Co$\mathrm{N}_{4} @$ NPC-re catalysts under different atmosphere. Reaction conditions: Reaction conditions: 1a $(1 \mathrm{mmol})$, $\mathrm{H}_{2} \mathrm{O}(8 \mathrm{mmol}), \mathrm{MeCN}(3 \mathrm{~mL})$, catalyst $(\mathrm{Co} 0.5 \mathrm{~mol} \%), 30{ }^{\circ} \mathrm{C}$. TOF was measured at 1a conversion below $20 \%$ based on total Co atoms. Error bars represent the standard deviation from at least three independent experiments. For all reactions, $>99 \%$ selectivity of $\mathbf{2 a}$ were obtained. 


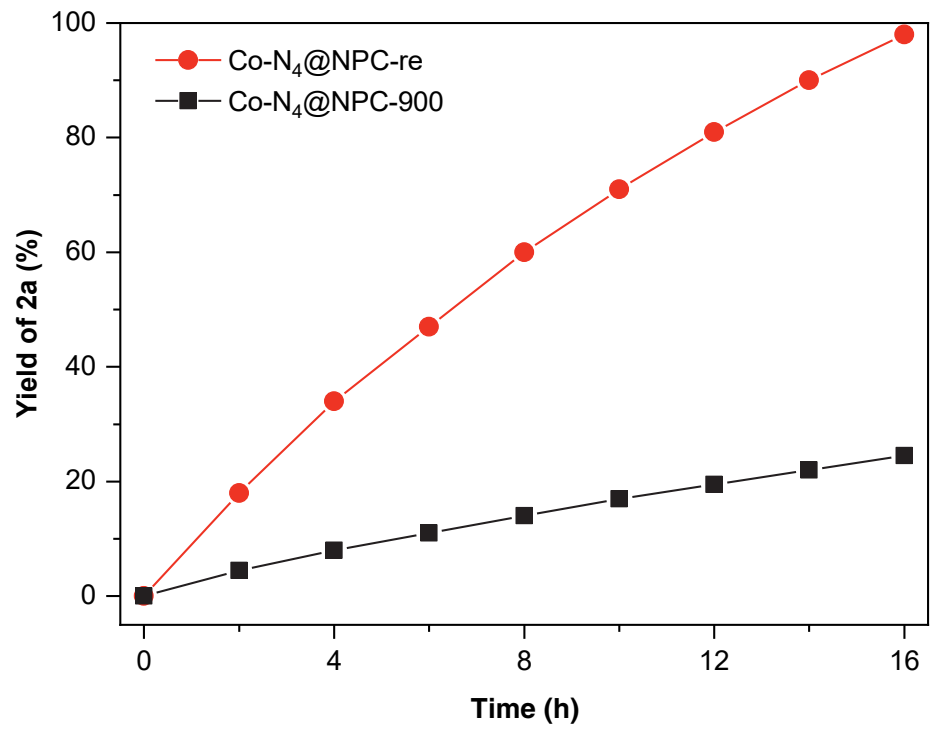

Supplementary Fig. $17 \mid$ Comparison of catalytic performance between Co- $\mathrm{N}_{4} @ N P C-r e$ and Co- $\mathrm{N}_{4} @ \mathrm{NPC}-$ 900 in 1a oxidation under $0.01 \mathrm{~mol} \%$ Co level. Reaction conditions: 1a (50 mmol), $\mathrm{H}_{2} \mathrm{O}$ (400 mmol), MeCN $(60 \mathrm{~mL})$, catalyst $(\mathrm{Co} 0.01 \mathrm{~mol} \%), \mathrm{O}_{2}(15 \mathrm{bar}), 30^{\circ} \mathrm{C}$. The yield of $\mathbf{2 a}$ was determined by GC using anisole as an internal standard. 
(A)
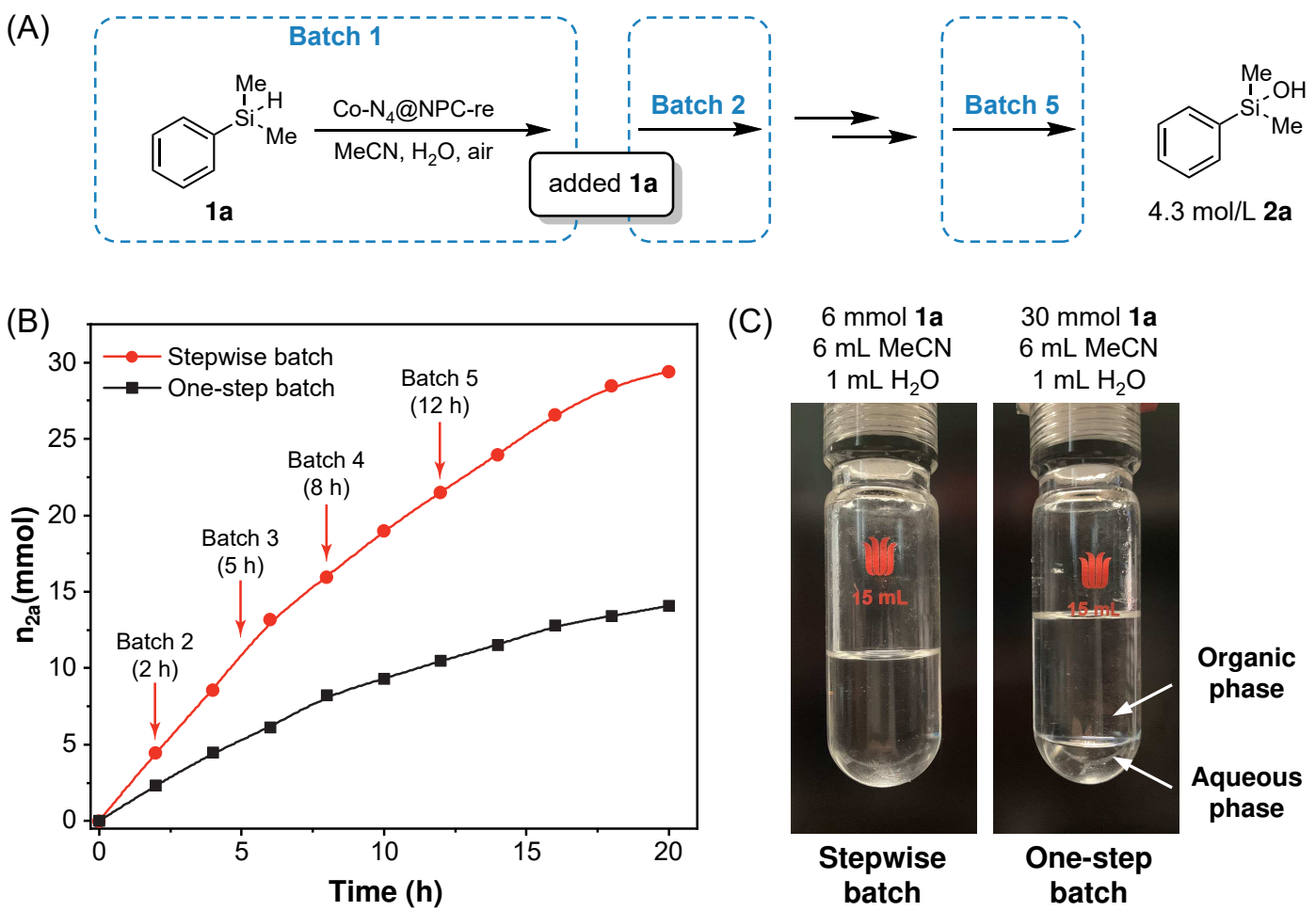

Organic

phase

Aqueous

phase

Supplementary Fig. $18 \mid$ Development of high-concentration silanol synthesis. (A) Schematic illustration of stepwise approach for high-concentration silanol synthesis. (B) The detail comparison between stepwise and one-step approach. (C) Initial state of reaction solution of stepwise batch (left) and one-step batch (right) approach. Reaction conditions: 1a (30 mmol), $\mathrm{H}_{2} \mathrm{O}(1 \mathrm{~mL}), \mathrm{Co}-\mathrm{N}_{4} @$ NPC-re (Co $\left.0.05 \mathrm{~mol} \%\right), \mathrm{MeCN}(6 \mathrm{~mL})$, $30{ }^{\circ} \mathrm{C}$, air atmosphere. (For the one-step batch, $30 \mathrm{mmol} \mathbf{1 a}$ was added at the beginning of the reaction; for the stepwise batch, $30 \mathrm{mmol} 1 \mathrm{a}$ was added repetitively for 5 times during the reaction.)

Although silane (such as 1a) was usually miscible with $\mathrm{MeCN}$, a phase separation occurred when a large amount of silane added to $\mathrm{MeCN}-\mathrm{H}_{2} \mathrm{O}$ mixture (Supplementary Fig. 18, right). Considering phase separation may reduce mass transfer efficiency of reaction, a semi-continuous procedure was developed, allowing the process-intensified conversion of high-concentration 1a in $\mathrm{MeCN}-\mathrm{H}_{2} \mathrm{O}$ mixture. It was thus possible to obtain a solution of $4.3 \mathrm{~mol} / \mathrm{L} 2 \mathrm{a}$ in $\mathrm{MeCN} / \mathrm{H}_{2} \mathrm{O}(6: 1)$ mixture by subjecting Co- $\mathrm{N}_{4} @ \mathrm{NPC}$-re catalyst to a five-stage-repetitive injection of $6 \mathrm{mmol} 1 \mathrm{a}$ during the reaction. The oxidation of 1a readily proceeded to give $>98 \%$ 2a yield in $20 \mathrm{~h}$ and only $<1 \%$ by-product disiloxane was obtained. In contrast, the conventional one-stage procedure gave only $47 \%$ yield after reaction for $20 \mathrm{~h}$. The stepwise reaction mode reduces the working concentration of $\mathbf{1 a}$ in solution, which not only avoids phase separation of reaction system, but also helps to keep optimal silane $/ \mathrm{H}_{2} \mathrm{O}$ molar ratio for high reaction efficiency. 


\section{Continuous-flow reactions}

The reactions were performed on a ThalesNano ${ }^{\circledR}$ Phoenix Flow Reactor (Supplementary Fig. 19). An HPLC pump was used for substrate solution introduction. Air was introduced by gas module. The liquid and gas were intensively mixed by liquid-gas mixer and flowed by up-flow through the reaction column. The system pressure was regulated by a back pressure valve. While the product solution was mainly collected in product container, the change valve can bypass the product container to sample the reaction mixture.

The stainless steel (SS) reaction column used in research contained a SS tube $(110 \mathrm{~mm} \times 2.1 \mathrm{~mm}$ I.D. $\times$ 1/4 in. O.D.), two nut/ferrule sets, two frits (pore size $50 \mu \mathrm{m}$ ) and two endcaps (Supplementary Fig. 20).

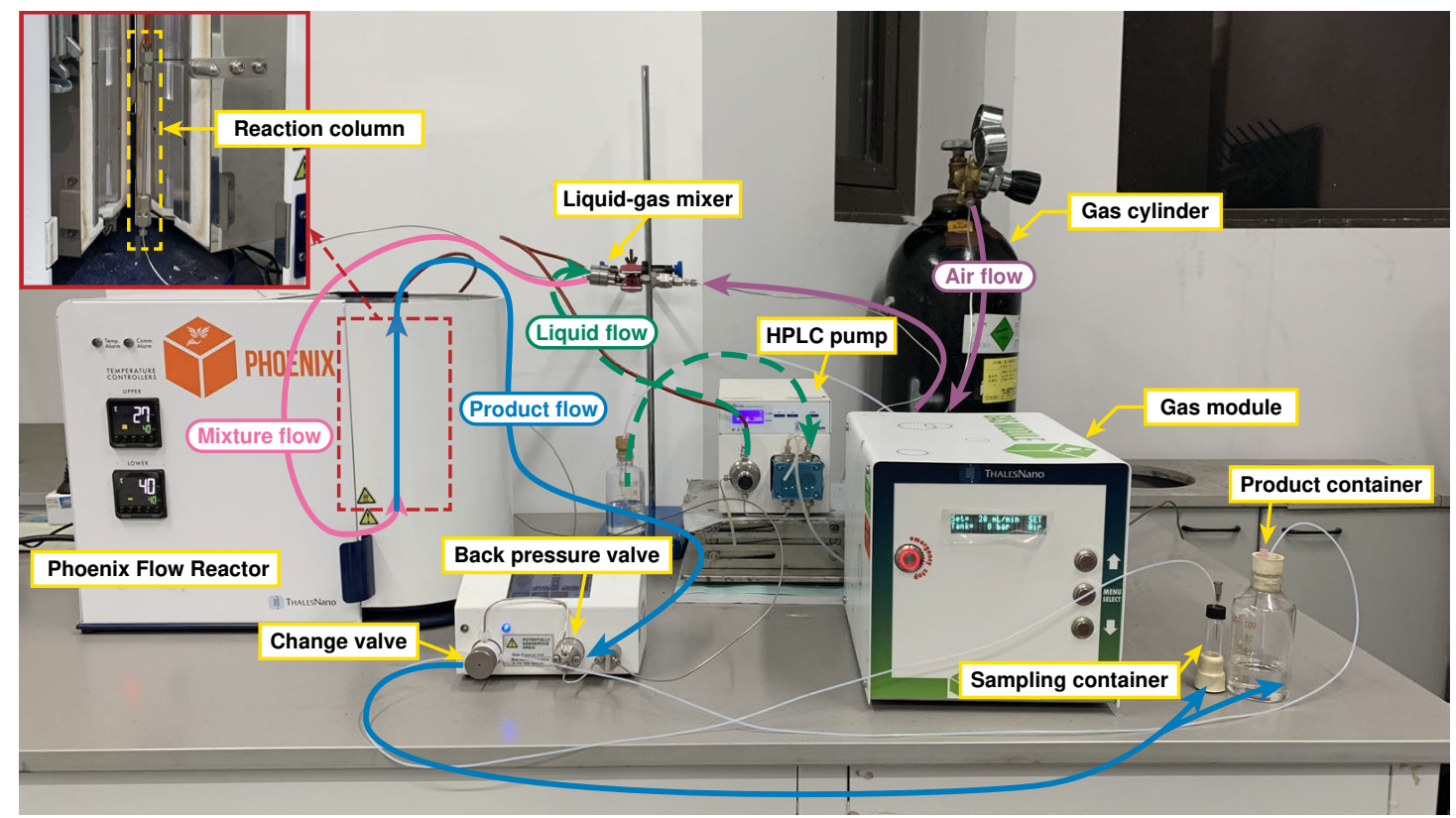

Supplementary Fig. 19 | Photographic image of ThalesNano ${ }^{\circledR}$ Phoenix Flow Reactor.

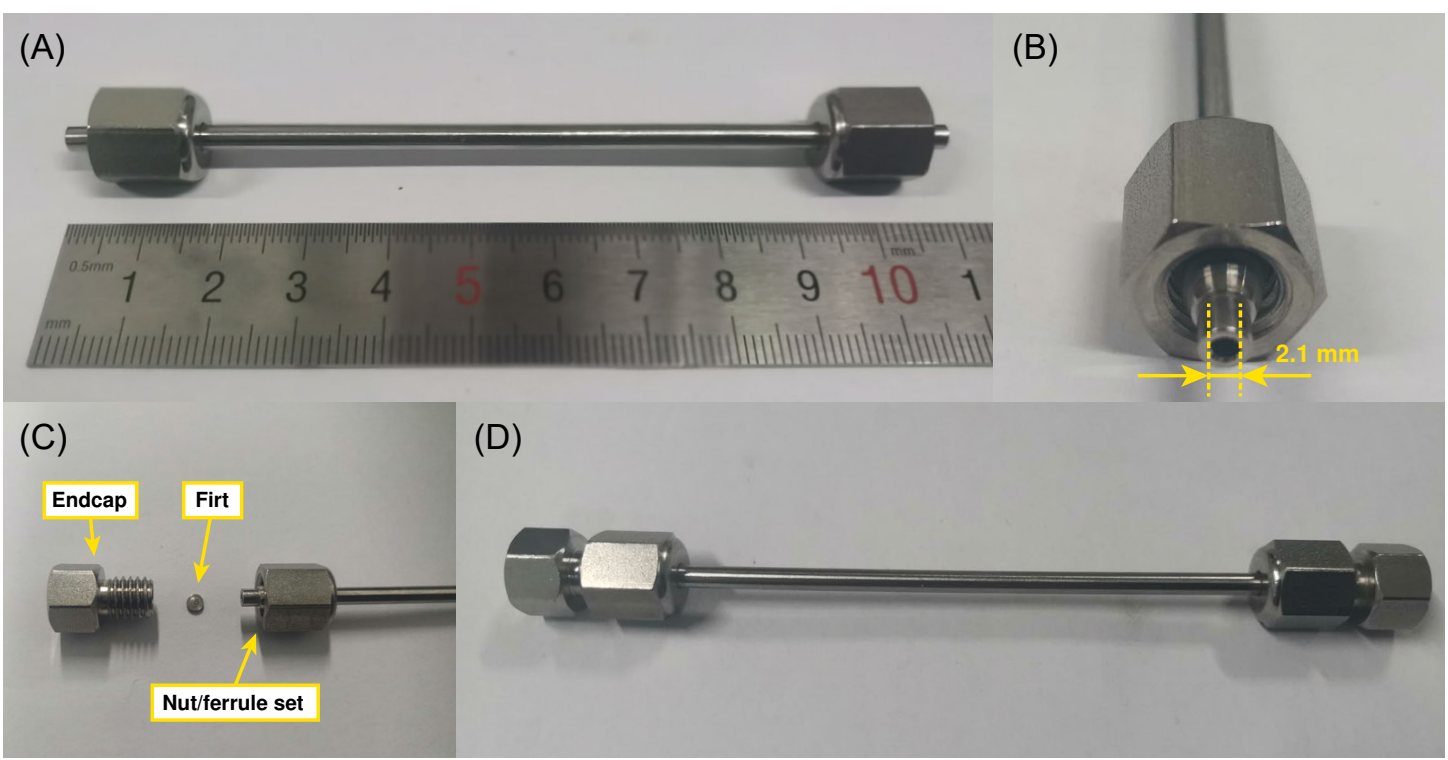

Supplementary Fig. 20 | (A) Stainless steel tube. (B) Close up. (C) End sealing structure. (D) Completely assembled reaction column. 
Under a typical continuous-flow procedure (as shown in Supplementary Fig. 21), 15 mg Co-N $4 @$ NPCre was diluted with $135 \mathrm{mg} \mathrm{SiO}$ (AEROSIL 380), and then pressed, crushed and sieved to 80-100 mesh. The mixture was packed into reaction column and held in place between quartz sand (80-100 mesh) at either end of the column. The solution $\left(0.3 \mathrm{~mol} / \mathrm{L} \mathrm{1a}\right.$ and $2.4 \mathrm{~mol} / \mathrm{L} \mathrm{H}_{2} \mathrm{O}$ in $\left.\mathrm{MeCN}, 0.20 \mathrm{~mL} / \mathrm{min}\right)$ and air $(20$ $\mathrm{mL} / \mathrm{min}$ ) was introduced simultaneously by up flow. The reaction was carried out under the condition of 30 bar air and $50{ }^{\circ} \mathrm{C}$. After stabilization, the fraction was collected to sampling container every $2 \mathrm{~h}$ during the continuous flow and analyzed by GC using anisole as an internal standard. For stability evaluation, the 2a yield was measured for more than $300 \mathrm{~h}$. The main product solution collected in product container was concentrated on a rotary evaporator and further purified by vacuum distillation $\left(62-64{ }^{\circ} \mathrm{C}, 0.5\right.$ torr) to obtain pure 2a.
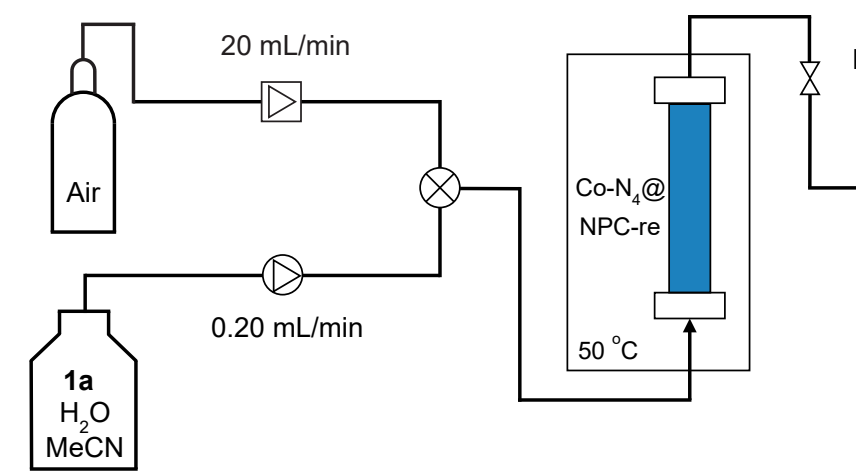

Back pressure Vacuum distillation $\nabla$ Gas module $\otimes$ HPLC pump $\otimes$ Gas-liquid mixer $\quad \nabla$ Back pressure valve

Supplementary Fig. 21 | Diagram of continuous-flow reaction. 


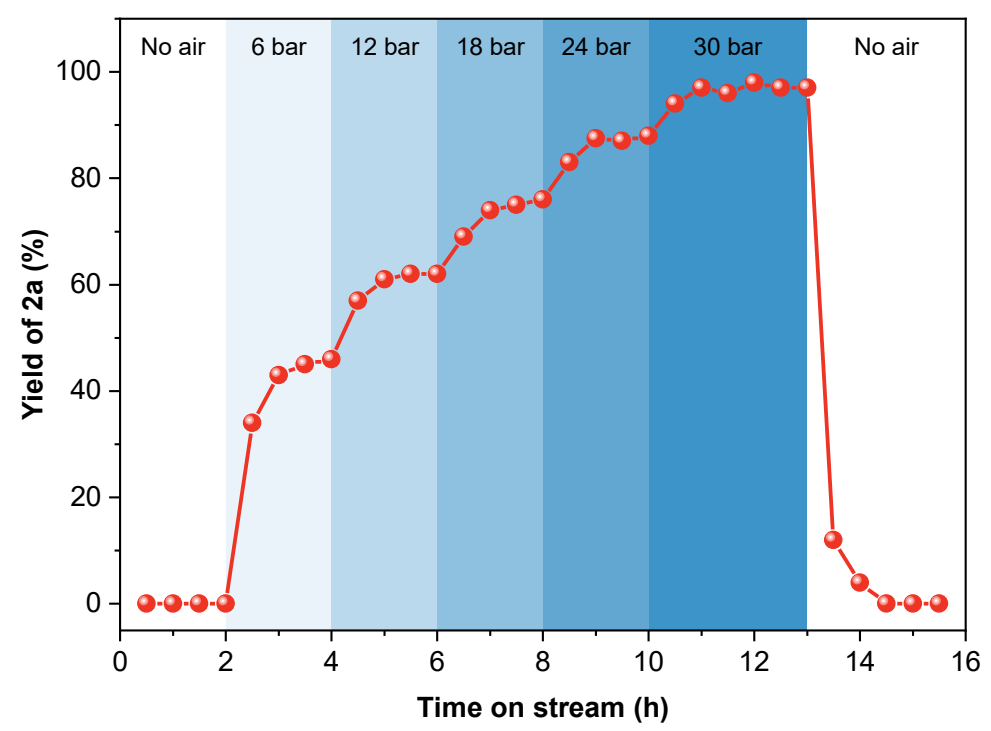

Supplementary Fig. 22 | Catalytic performance of a Co- $\mathrm{N}_{4} @ N P C-r e$ catalyst for 1a oxidation under different air pressure in continuous-flow system. Reaction conditions: catalyst $\left(\mathrm{Co}-\mathrm{N}_{4} @ \mathrm{NPC}-\mathrm{re} / \mathrm{SiO}_{2}=1 / 9\right.$, $150 \mathrm{mg}), 1 \mathrm{a}(0.3 \mathrm{~mol} / \mathrm{L}), \mathrm{H}_{2} \mathrm{O}(2.4 \mathrm{~mol} / \mathrm{L}), \mathrm{MeCN}$ as solvent, liquid flow rate $0.20 \mathrm{~mL} / \mathrm{min}$, gas flow rate $20 \mathrm{~mL} / \mathrm{min}$ (if needed), $50{ }^{\circ} \mathrm{C}$.

The influence of air pressure in continuous-flow system was investigated. As shown in Supplementary Fig. 22, when the flow reaction was conducted only using $\mathbf{1 a}$ and $\mathrm{H}_{2} \mathrm{O}$ as reactant $(0-2 \mathrm{~h})$, no reaction occurred. In contrast, 2a could be obtained when air was introduced into the flow reaction system simultaneously. Under a certain gas $(20 \mathrm{~mL} / \mathrm{min})$ and liquid $(0.20 \mathrm{~mL} / \mathrm{min})$ flow rate, further increasing the air pressure led to a higher yield of $\mathbf{2 a}$ and $\mathbf{2 a}$ reached near quantitative yield under 30 bar air at $50{ }^{\circ} \mathrm{C}$. The catalytic performance in continuous-flow system further confirmed that Co- $\mathrm{N}_{4} @ \mathrm{NPC}-\mathrm{re}$ system for 1a oxidation followed a $\mathrm{O}_{2}$-coupled hydrolytic pathway, $\mathrm{O}_{2}$ was served as another essential reactant. 


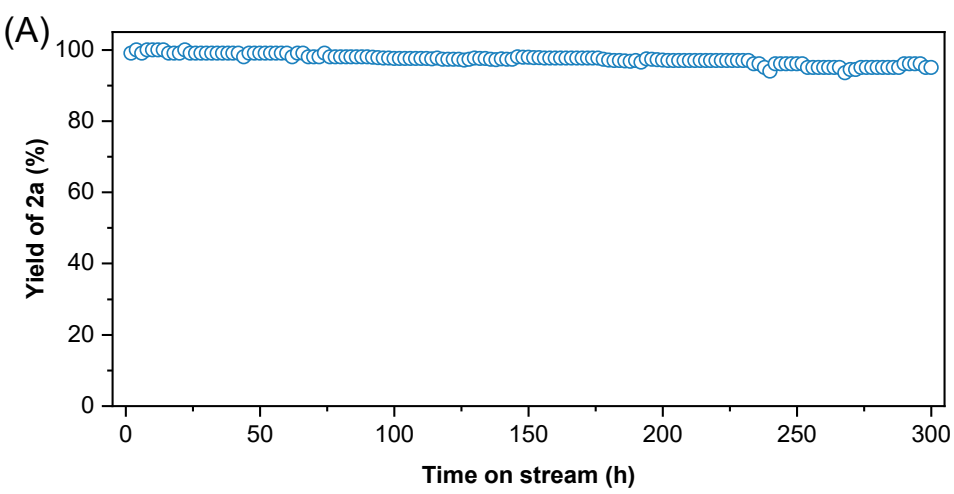

(B)

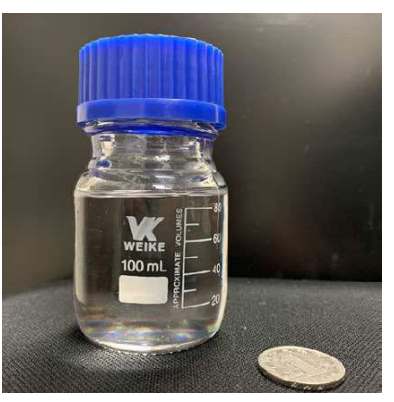

Supplementary Fig. 23 | (A) The stability evaluation of a Co- $\mathrm{N}_{4} @ N$ NP-re catalyst for 1a oxidation under continuous-flow conditions. Reaction conditions: catalyst (Co-N $\left.\mathrm{N}_{4} @ \mathrm{NPC}-\mathrm{re} / \mathrm{SiO}_{2}=1 / 9,150 \mathrm{mg}\right), 1 \mathrm{a}(0.3$ $\mathrm{mol} / \mathrm{L}), \mathrm{H}_{2} \mathrm{O}(2.4 \mathrm{~mol} / \mathrm{L}), \mathrm{MeCN}$ as solvent, air (30 bar), liquid flow rate $0.20 \mathrm{~mL} / \mathrm{min}$, gas flow rate 20 $\mathrm{mL} / \mathrm{min}, 50^{\circ} \mathrm{C}$. (B) Image of $2 \mathrm{a}$ after purification.

The Co- $\mathrm{N}_{4} @$ NPC-re catalyst exhibited stable performance over 300 hours. The 2a yield remained $>95 \%$ during a 300-hour stability test. The space-time yield of $2 \mathrm{a}$ was $\sim 7.5 \mathrm{~mol}_{2 \mathrm{a}} / \mathrm{g}_{\mathrm{Co}} / \mathrm{h}$ and total turnover number reached up to 130000 . 

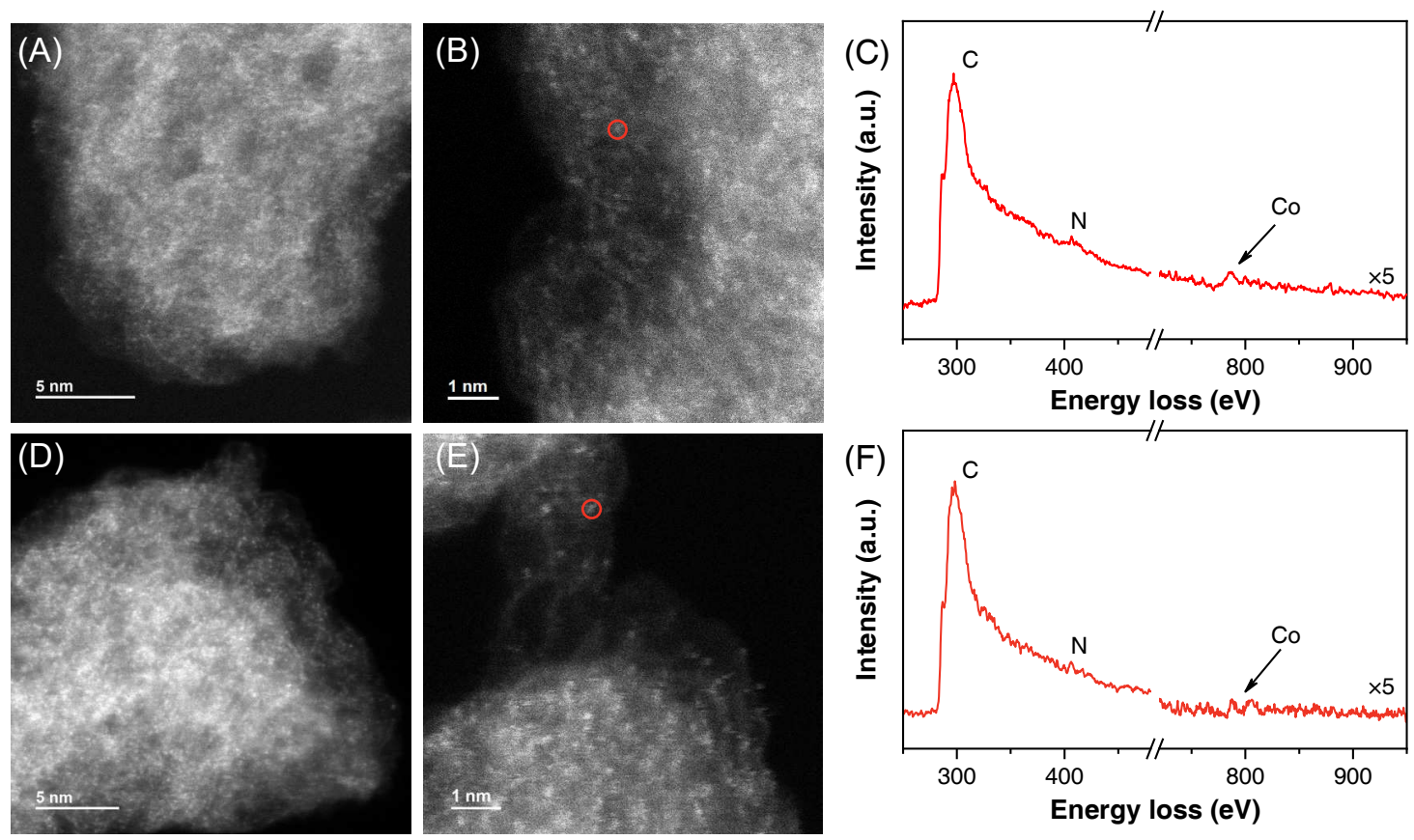

Supplementary Fig. 24 | HAADF-STEM images with accompanying EELS point spectra for Co-N $\mathrm{N}_{4} @$ NPCre catalyst before (A-C) and after (D-F) 300-hour continuous-flow reaction stability test. The microstructure in the catalyst maintained nearly the same and Co- $\mathrm{N}_{\mathrm{x}}$ sites could clearly obtained in spent catalyst by EELS point spectra, indicated high stability of Co- $\mathrm{N}_{4} @$ NPC-re catalyst. 

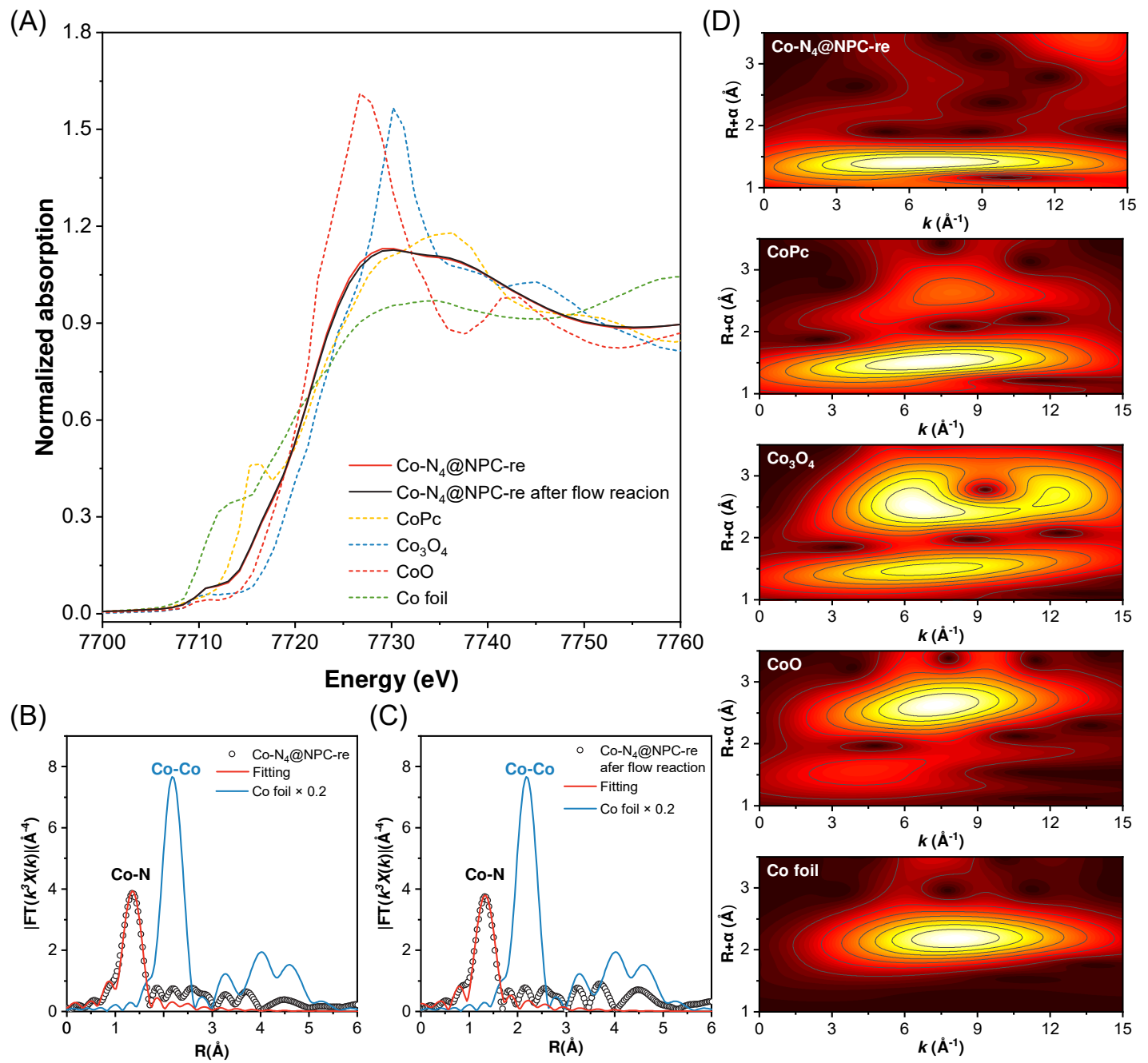

(C)
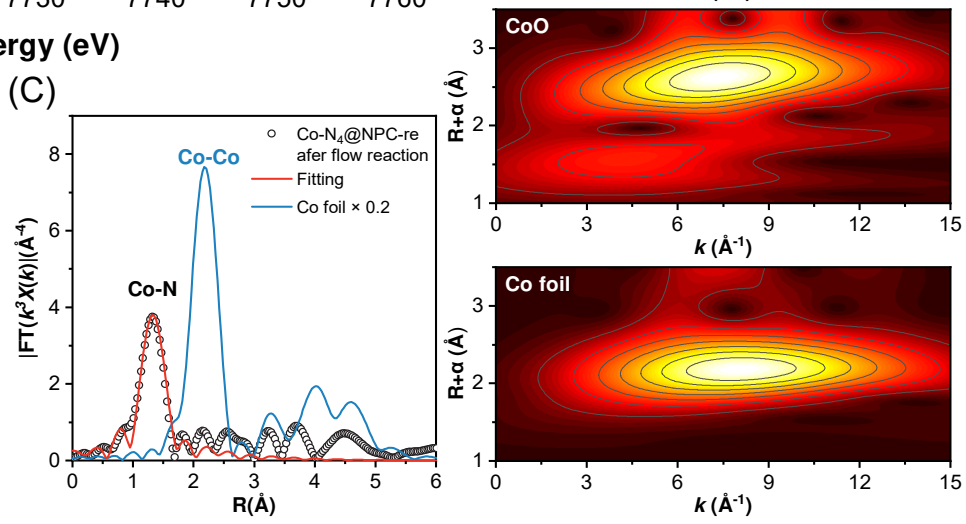

Supplementary Fig. 25 | (A) Co K-edge XANES spectra of Co-N ${ }_{4} @ N P C-r e$ catalyst before (red) and after (black) 300-hour continuous-flow reaction stability test and reference samples. (B and C) R-space Co Kedge EXAFS spectra and fittings of Co- $\mathrm{N}_{4} @ N P C-r e$ catalyst before and after stability test, respectively. The fitting results are listed in Supplementary Table 2. (D) WT of Co K-edge for Co- $\mathrm{N}_{4} @ \mathrm{NPC}-\mathrm{re}, \mathrm{CoPc}, \mathrm{Co}_{3} \mathrm{O}_{4}$, $\mathrm{CoO}$ and $\mathrm{Co}$ foil. 

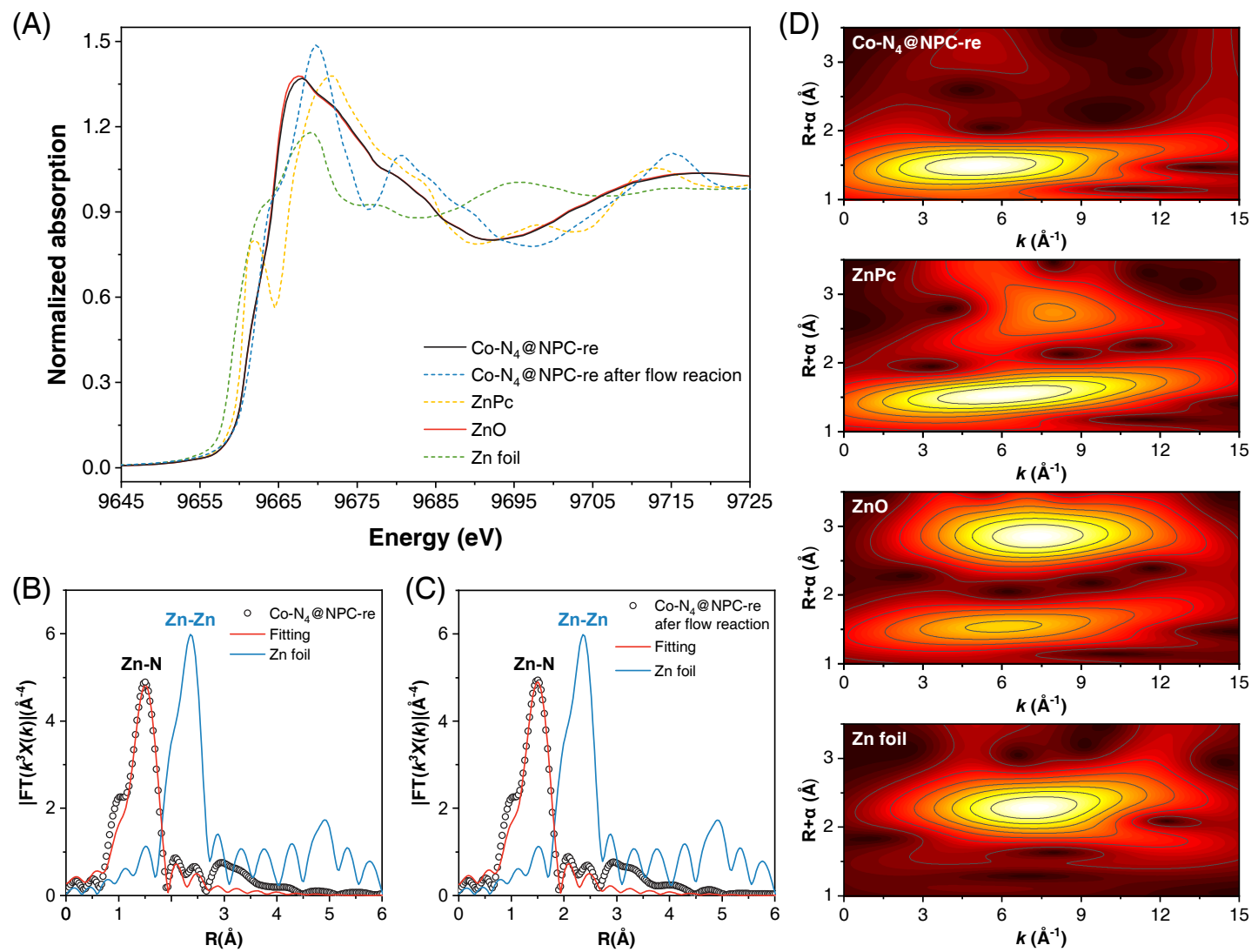

Supplementary Fig. 26 | (A) Zn K-edge XANES spectra of Co-N ${ }_{4} @ N P C-r e$ catalyst before (red) and after (black) 300-hour continuous-flow reaction stability test and reference samples. (B and C) R-space Zn Kedge EXAFS spectra and fittings of Co-N $\mathrm{N}_{4} @$ NPC-re catalyst before and after stability test, respectively. The fitting results are listed in Supplementary Table 2. (D) WT of Zn K-edge for Co- $\mathrm{N}_{4} @ \mathrm{NPC}-\mathrm{re}, \mathrm{ZnPc}, \mathrm{ZnO}$ and $\mathrm{Zn}$ foil. 

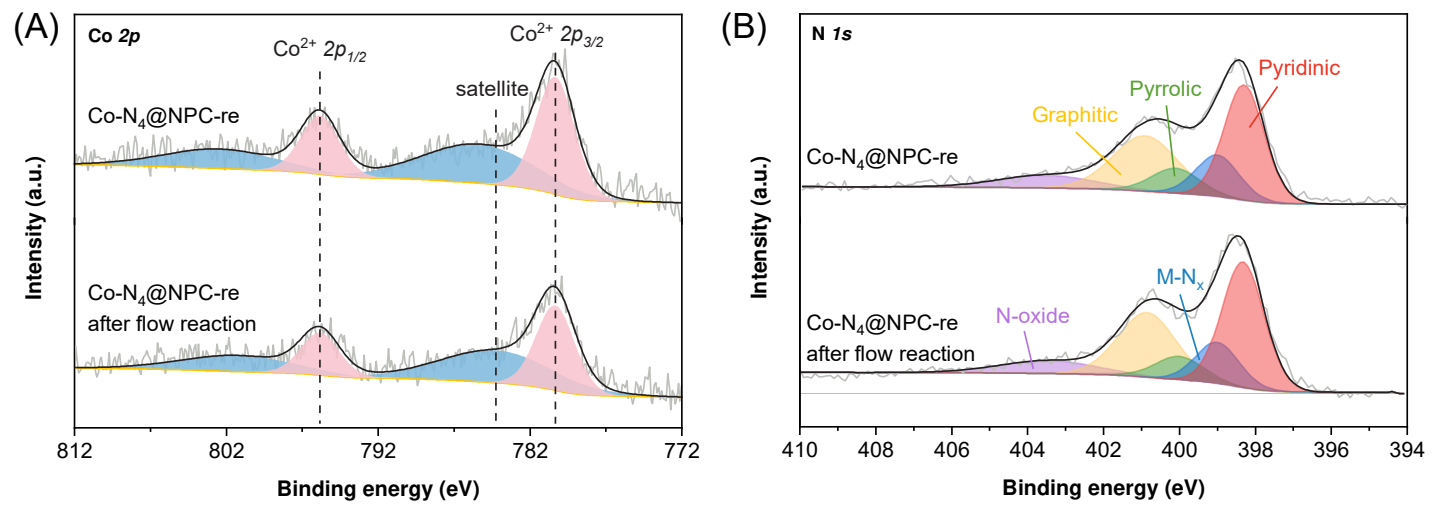

Supplementary Fig. 27 | Co $2 p$ (A) and N $1 s$ (B) XPS spectra for Co-N $\mathrm{N}_{4} @ N P C-r e$ catalyst before and after 300 -hour continuous-flow reaction stability test. Detailed fitting results of N $1 s$ XPS spectra are listed in Supplementary table 5. 


\section{Real-time FT-IR experiments}

The reaction spectra were recorded using an IC 15 from Mettler-Toledo AutoChem fitted with a diamondtipped probe and an MCT detector. Data manipulation was carried out using the iC IR software, version 7.0.
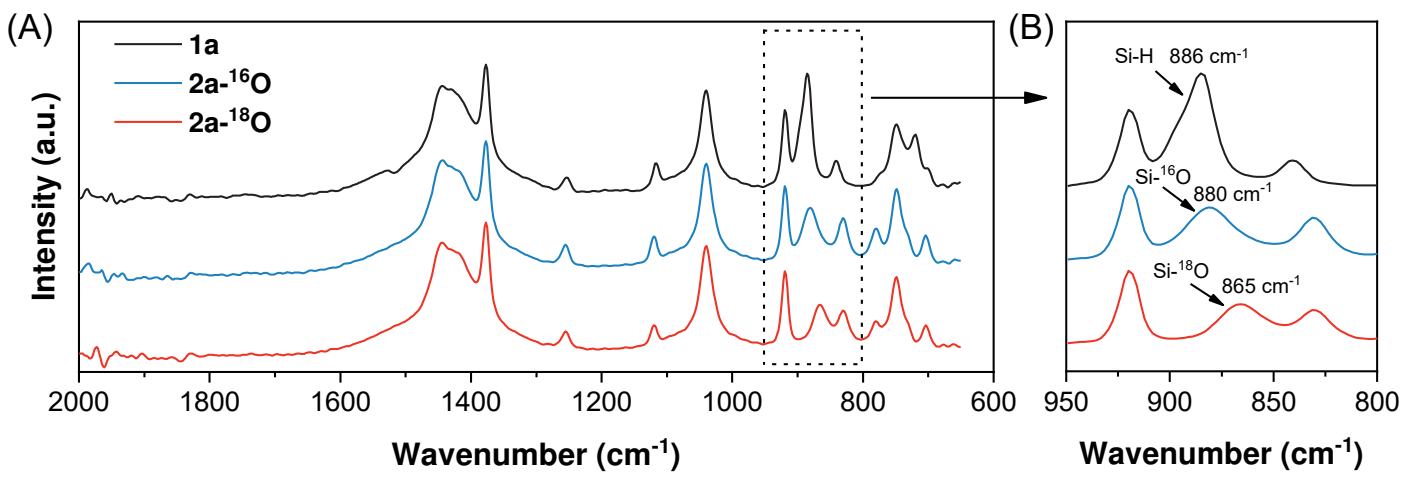

Supplementary Fig. $28 \mid$ (A) Characteristic IR band of 1a, $2 \mathbf{2 a}^{16} \mathrm{O}$ and $\mathbf{2 a}-{ }^{18} \mathrm{O}$ in MeCN. (B) Local enlarged spectra of the region from 950 to $800 \mathrm{~cm}^{-1}$.

As shown in Supplementary Fig. 28, the IR band of 1a at $886 \mathrm{~cm}^{-1}$ assigned to bending vibration of Si$\mathrm{H}$ bond. For $2 \mathbf{2 a}^{-16} \mathrm{O}$, the stretching vibration of $\mathrm{Si}^{-16} \mathrm{O}$ bond was located at $880 \mathrm{~cm}^{-1}$. While for $2 \mathbf{2}^{-18} \mathbf{O}$, due to the ${ }^{16} \mathrm{O}$ atom was substituted with the higher mass ${ }^{18} \mathrm{O}$ isotope, a downshifted Si-O vibrational frequency occurred and the characteristic stretching vibration of $\mathrm{Si}^{-18} \mathrm{O}$ bond was obtained at $865 \mathrm{~cm}^{-1}$.

The catalytic reaction was carried out as follows: a three-necked reaction vessel was fitted with a magnetic stirring bar. The IR probe was inserted through an adapter into the middle neck; the other two necks were capped with septa for injections and an air balloon. The reaction vessel was kept at $30^{\circ} \mathrm{C}$. Then, the threenecked vessel was charged with $15 \mathrm{~mL}$ anhydrous $\mathrm{MeCN}$ and the data collection was started. After $5 \mathrm{~min}$, 1a $(681.5 \mathrm{mg}, 5 \mathrm{mmol})$ was added. Then $5 \mathrm{~min}$ later, $\mathrm{H}_{2}{ }^{18} \mathrm{O}(200.2 \mathrm{mg}, 10 \mathrm{mmol})$ was added. After another $5 \mathrm{~min}$, the reaction was initiated by the addition of Co-N $4 @$ NPC-re $(9.5 \mathrm{mg}, 0.1 \mathrm{~mol} \% \mathrm{Co})$. The IR spectra were recorded over the course of the reaction.
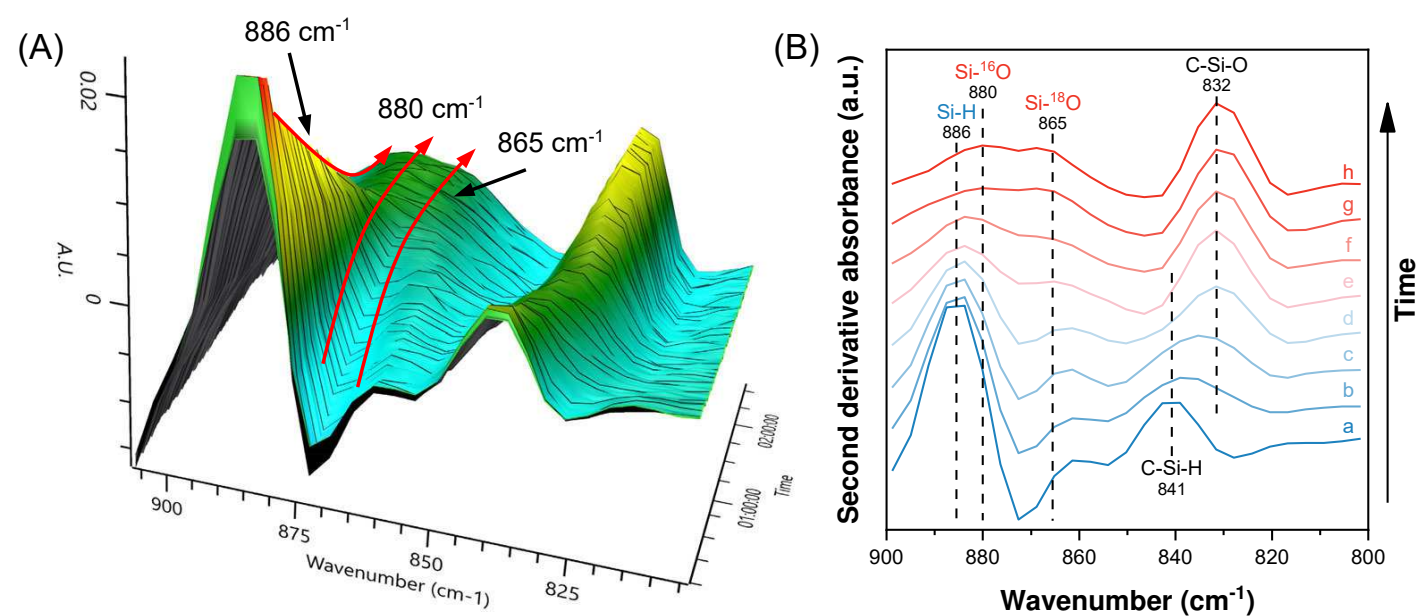

Supplementary Fig. 29 | (A) Three-dimensional (3D) stack plot of the second derivative IR spectra of the oxidation of 1a in $\mathrm{MeCN} / \mathrm{H}_{2}{ }^{18} \mathrm{O}$ mixture under air atmosphere using Co- $\mathrm{N}_{4} @ \mathrm{NPC}$-re as catalyst. The original 3D stack plot of IR spectra was shown in Fig. 3A. (B) Selected second derivative spectra of different times. From bottom to top, a-h: 15, 35, 55, 75, 95, 115, 135, and 155 min.

In the real reaction system, due to the partially overlapped of the characteristic band of $1 \mathbf{a}\left(886 \mathrm{~cm}^{-1}\right)$ and 2a $\left(880 \mathrm{~cm}^{-1}\right.$ for $\mathbf{2 a}-{ }^{16} \mathbf{O}$ and $865 \mathrm{~cm}^{-1}$ for $\left.\mathbf{2 a}^{-18} \mathbf{O}\right)$, second derivative spectroscopy was applied to identify 
the potential peaks in IR spectra. The second derivative spectra clearly showed the peak at $886 \mathrm{~cm}^{-1}$ decreased and the peaks at 880 and $865 \mathrm{~cm}^{-1}$ increased during the reaction (Supplementary Fig. 29), indicated the consumption of $1 \mathbf{a}$ and the formation $2 \mathbf{a}^{-16} \mathrm{O}$ and $\mathbf{2 a}-{ }^{18} \mathrm{O}$. Considering only oxygen molecules contained ${ }^{16} \mathrm{O}$ atoms in the reaction system, these results provided directed evidence that $\mathrm{O}_{2}$ participates in the reaction as a reactant.

Based on the results of second derivative spectral analysis, a curve-fitting was further used to quantitatively calculate the peak area of each component in the mixture at different reaction times. A part of the fitting results were shown in Supplementary Fig. 30 and the respective corresponding changes were shown in Fig. 3B in the main text. The fitting results indicated that the initially formed product was $\mathbf{2} \mathbf{2}-{ }^{18} \mathbf{O}$ accompanied by the delayed appearance of $\mathbf{2} \mathbf{a}^{-1}{ }^{16} \mathbf{O}$. Combining the result that in such Co- $\mathrm{N}_{4} @$ NPC-based catalytic system, $\mathrm{O}_{2}$ could not directly oxidize $1 \mathrm{a}$ in the absence of $\mathrm{H}_{2} \mathrm{O}$ (Supplementary Table 8, entry 6), the ${ }^{16} \mathrm{O}$ atom in product $2 \mathbf{2 a}^{-16} \mathrm{O}$ should come from the reduction product of $\mathrm{O}_{2}$ (such as $\mathrm{H}_{2} \mathrm{O}_{2}$ and $\mathrm{H}_{2} \mathrm{O}$ ) which was gradually formed during the reaction.
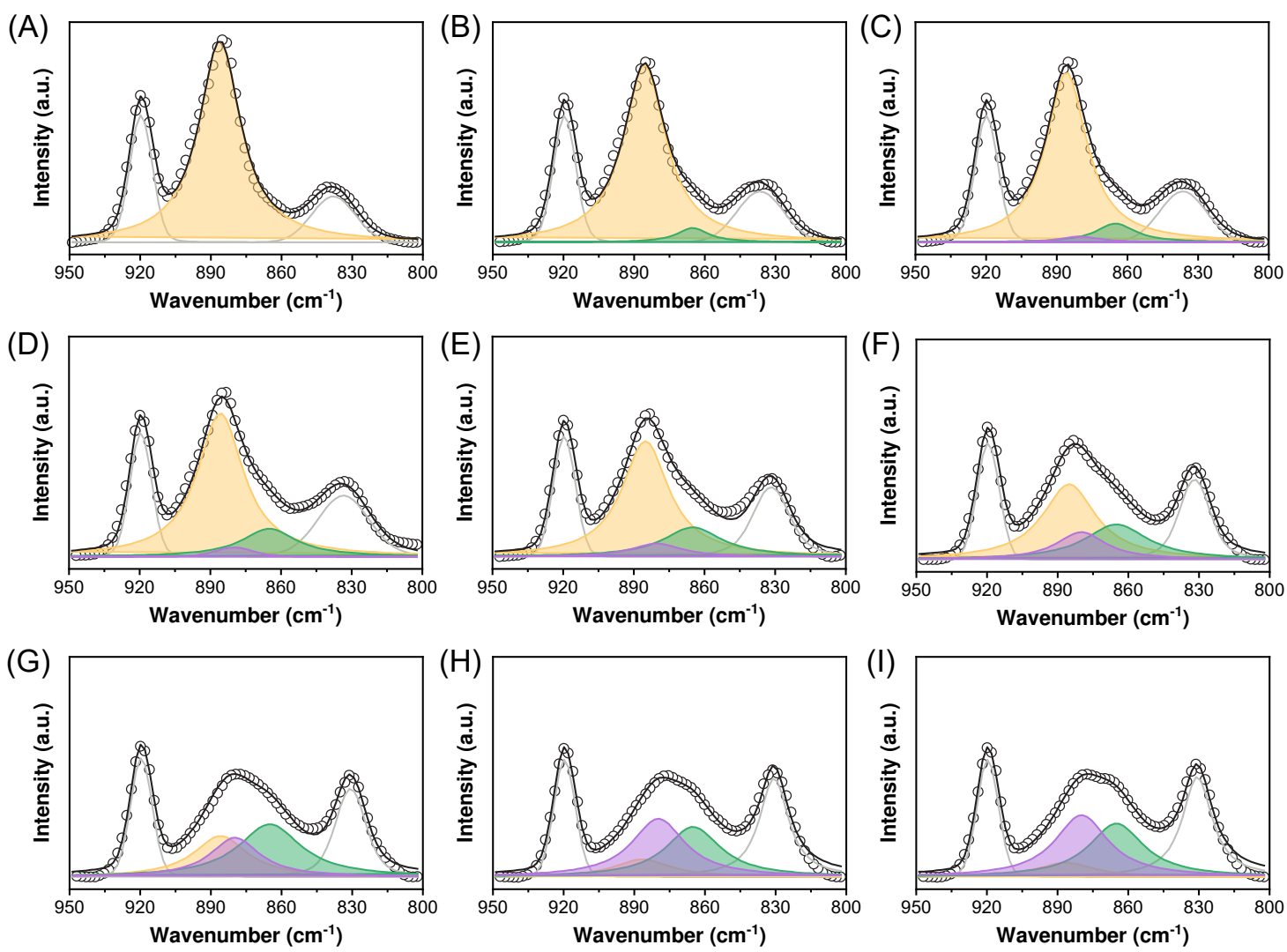

Supplementary Fig. 30 | Raw data and fitting results of selected IR spectra at (A) $15 \mathrm{~min}$, (B) $30 \mathrm{~min}$, (C) 45 min, (D) 60 min, (E) 75 min, (F) 90 min, (G) 105 min, (H) 130 min and (I) $155 \mathrm{~min}$. 


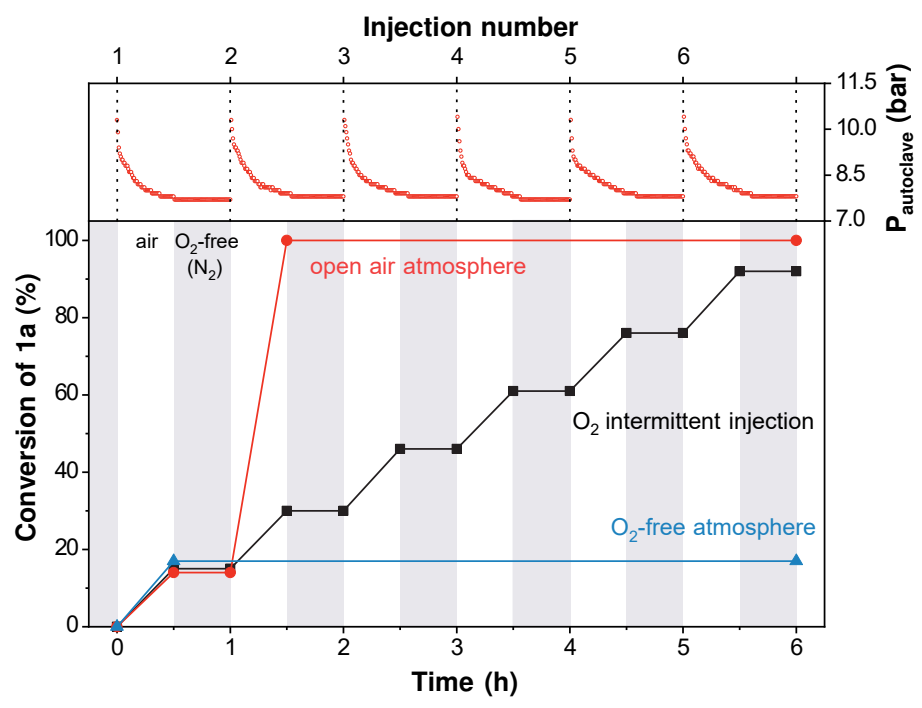

\begin{tabular}{cccccccc}
\hline Step & $\mathbf{P}_{\mathbf{i}}(\mathbf{b a r})$ & $\begin{array}{c}\mathbf{P}_{\mathbf{f}} \\
(\mathbf{b a r})\end{array}$ & $\boldsymbol{\Delta \mathbf { P } ( \mathbf { b a r } )}$ & $\mathbf{n}_{\mathbf{0 x y g e n}}(\mathbf{m o l})^{\mathbf{a}}$ & $\Delta$ Conv. of 1a (\%) & $\mathbf{n}_{\mathbf{1 a}}(\mathbf{m o l})$ & $\mathbf{n}_{\mathbf{o x y g e n}}: \mathbf{n}_{\mathbf{1 a}}$ \\
\hline 1 & 10.3 & 7.7 & 2.6 & 2.06 & 15.2 & 3.80 & $1: 1.84$ \\
2 & 10.3 & 7.8 & 2.5 & 1.98 & 14.9 & 3.72 & $1: 1.88$ \\
3 & 10.3 & 7.8 & 2.5 & 1.98 & 15.7 & 3.92 & $1: 1.98$ \\
4 & 10.4 & 7.7 & 2.7 & 2.14 & 15.5 & 3.88 & $1: 1.83$ \\
5 & 10.3 & 7.8 & 2.5 & 1.98 & 15.3 & 3.82 & $1: 1.93$ \\
6 & 10.4 & 7.8 & 2.6 & 2.06 & 16.2 & 4.05 & $1: 1.97$ \\
\hline Sum & - & - & - & 12.2 & 92.8 & 23.2 & $1: 1.90$ \\
\hline
\end{tabular}

${ }^{\text {a }}$ Calculated by ideal gas equation, $n_{\text {oxygen }}=\frac{\Delta P V_{h}}{R T}$.

Supplementary Fig. 31 | Intermittent oxygen injection experiments. Reaction conditions: 1a (25 mmol), $\mathrm{H}_{2} \mathrm{O}(200 \mathrm{mmol})$, degassing MeCN (75 mL), Co- $\mathrm{N}_{4} @$ NPC-re (0.1 mol\%), 10 bar air (each injection), $30^{\circ} \mathrm{C}$. The table shows the detail pressure and $\mathbf{1 a}$ conversion profile. $\mathrm{P}_{\mathrm{i}}$, initial pressure; $\mathrm{P}_{\mathrm{f}}$, final pressure; Conv., conversion. Conv. determined by GC using anisole as the internal standard.

The capacity of used $120 \mathrm{~mL}$ autoclave was determined via water displacement to calculate the injected mole number of oxygen $\left(\mathrm{n}_{\text {oxygen }}\right)$. When equipped with quartz liner, reactants, solvent and stir bar, the surplus volume of headspace $\left(\mathrm{V}_{\mathrm{h}}\right)$ within the autoclave was $20 \mathrm{~mL}$. During each reaction step, the reactor pressure dropped rapidly at the beginning and finally kept constant. The decreased pressure $\Delta \mathrm{P}$ roughly equal the partial pressure of $\mathrm{O}_{2}$ in pressurized air, further GC-TCD analysis showed the $\mathrm{O}_{2}$ was almost completely consumed after reaction. In addition, by stopping inject oxygen after the first injection, we observed no additional product during the next $5 \mathrm{~h}$ (blue line). Contrarily, exposing the solution to open air atmosphere resulting a full conversion of $1 \mathrm{a}$ within $0.5 \mathrm{~h}$ (red line). These results further suggested $\mathrm{O}_{2}$ participates reaction as another reactant, which was in close agreement with the results from real-time FT-IR experiments. Further quantitative analysis showed that the stoichiometry of the overall reaction of $\mathrm{O}_{2}$ with 1a was approximately 1:2. Combining the fact of identifying $\mathrm{H}_{2} \mathrm{O}_{2}$ as an intermediate, the possible pathway for Co$\mathrm{N}_{4} @$ NPC-re-catalyzed silane oxidation can be described as follows:

$$
\begin{aligned}
& \left\{\begin{array}{l}
\text { Reaction 1: } \quad \mathrm{RSi}-\mathrm{H}+\mathrm{O}_{2}+\mathrm{H}_{2} \mathrm{O} \stackrel{\text { Co- } \mathrm{N}_{4} @ N P C-r e}{\longrightarrow} \mathrm{RSi}-\mathrm{OH}+\mathrm{H}_{2} \mathrm{O}_{2} \\
\text { Reaction 2: } \\
\text { Overall reaction: } \quad 2 \mathrm{RSi}-\mathrm{H}+\mathrm{O}_{2} \longrightarrow 2 \mathrm{Co- \textrm {N } _ { 4 } @ N P C - r e}
\end{array} \mathrm{H}_{2} \mathrm{O}+0.5 \mathrm{O}_{2}\right.
\end{aligned}
$$


(A)

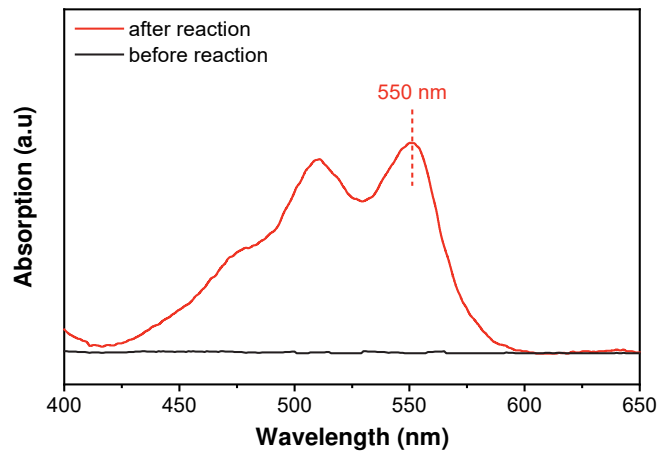

(C)

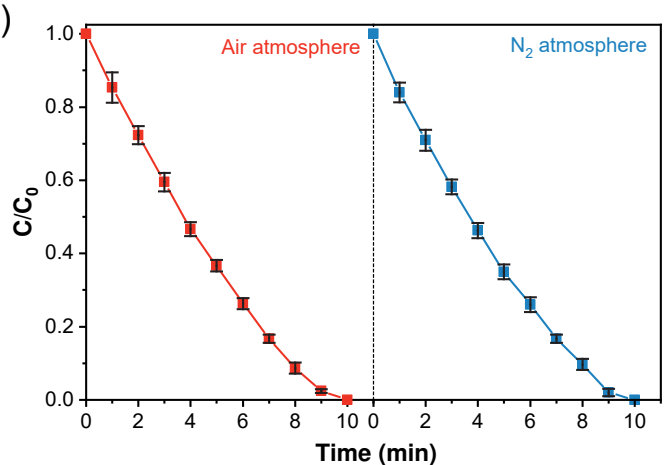

(B)

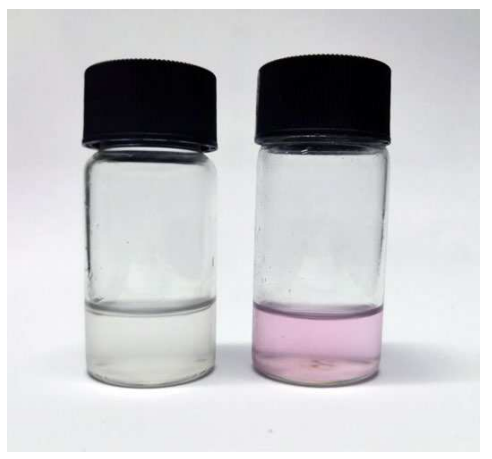

(D)

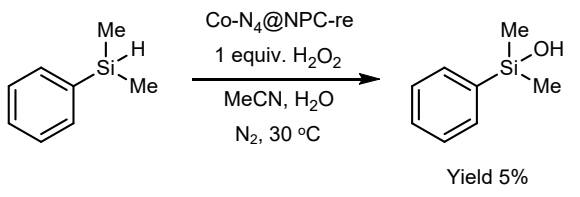

Supplementary Fig. 32 (A) Colorimetric detection of $\mathrm{H}_{2} \mathrm{O}_{2}$ in reaction solution. (B) Images of $\mathrm{H}_{2} \mathrm{O}_{2}$ determination for reaction solution before (left) and after (right) reaction. (C) The evolution of $\mathrm{H}_{2} \mathrm{O}_{2}$ in Co$\mathrm{N}_{4} @$ NPC-re-catalyzed $\mathrm{H}_{2} \mathrm{O}_{2}$ disproportionation under different atmosphere. Reaction conditions: $0.1 \mathrm{~mol} / \mathrm{L}$ $\mathrm{H}_{2} \mathrm{O}_{2}$ aqueous solution $(100 \mathrm{~mL}), \mathrm{Co}-\mathrm{N}_{4} @ \mathrm{NPC}-\mathrm{re}(\mathrm{Co} 0.02 \mathrm{~mol} \%), 30^{\circ} \mathrm{C}$. Error bars represent the standard deviation from at least three independent experiments. (D) Control experiment with $\mathrm{H}_{2} \mathrm{O}_{2}$ as oxidant.

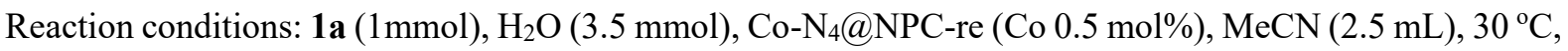
$\mathrm{N}_{2}$ atmosphere (in a $\mathrm{N}_{2}$-filled glovebox), $30 \mathrm{~min}, 100 \mu \mathrm{L} 30 \% \mathrm{H}_{2} \mathrm{O}_{2}$ aqueous solution (contain $1 \mathrm{mmol}_{2} \mathrm{O}_{2}$ and $4.5 \mathrm{mmol} \mathrm{H}_{2} \mathrm{O}$ ) was dissolved in $0.5 \mathrm{~mL} \mathrm{MeCN}$ and injected over 5 min via syringe pump.

For determination of $\mathrm{H}_{2} \mathrm{O}_{2}$, a modified chromogenic method using $\mathrm{Fe}_{3} \mathrm{O}_{4}$ magnetic nanoparticles as a peroxidase mimetic and $N, N$-diethyl- $p$-phenylenediamine sulfate (DPD) as an indicator was employed ${ }^{3}$. The oxidation product (DPD+) of DPD has a strong absorption maximum at $550 \mathrm{~nm}$. In this way, we were able to clearly identify the formation of $\mathrm{H}_{2} \mathrm{O}_{2}$ during the reaction (Supplementary Fig. 32, A and B).

The essential role of $\mathrm{H}_{2} \mathrm{O}_{2}$ was further explored via some control experiments. $\mathrm{H}_{2} \mathrm{O}_{2}$ was found to undergo disproportionation in the presence of Co- $\mathrm{N}_{4} @$ NPC-re under (an)aerobic conditions in the absence of 1a (Supplementary Fig. 32C). The TOF reaches up to more than $44000 \mathrm{~h}^{-1}$ in both air and $\mathrm{N}_{2}$ atmosphere, which is over 70-fold higher than the TOF of catalytic 1a oxidation. In addition, separate experiment (Supplementary Fig. 32D) showed that using $\mathrm{H}_{2} \mathrm{O}_{2}$ as oxidant only gives very low 2a yield under actual reaction system (in absence of $\mathrm{O}_{2}$ ), while large amounts of $\mathrm{O}_{2}$ evolution were observed. Together, these results suggest that $\mathrm{H}_{2} \mathrm{O}_{2}$, formed during the reaction, will undergo rapid disproportionation in preference to oxidizing 1a. 
(A)<smiles>C[Si](C)([Mg])c1ccccc1</smiles>

$1 a$

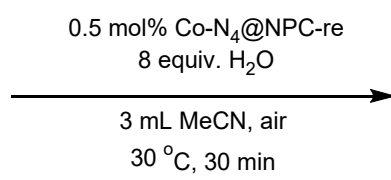

without additive

with Galvinoxyl (1 equiv.)

with TEMPO (1 equiv.)<smiles>CS(=O)(=O)c1ccccc1</smiles>

$2 a$

$>99 \%$ yield

$93 \%$ yield

$95 \%$ yield

(B)

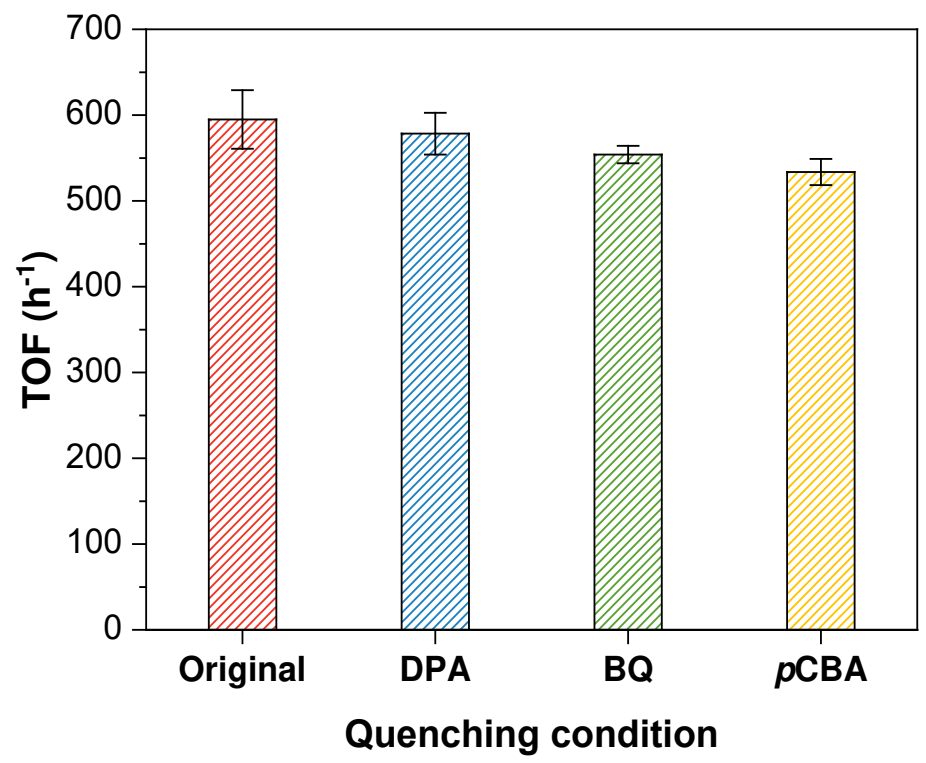

Supplementary Fig. 33 | (A) Control reactions performed in the presence of various radical scavengers. (B) Effect of the addition of various ROS scavengers on 1a oxidation over Co- $\mathrm{N}_{4} @ \mathrm{NPC}$-re catalyst. Reaction

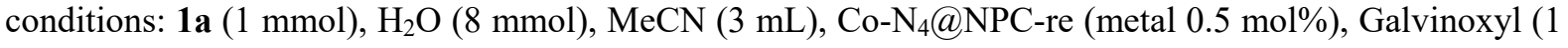
$\mathrm{mmol}$, if needed), TEMPO ( $1 \mathrm{mmol}$, if needed), DPA ( $0.5 \mathrm{mmol}$, if needed), BQ $(0.5 \mathrm{mmol}$, if needed), $p$ CBA $\left(0.5 \mathrm{mmol}\right.$, if needed), air atmosphere, $30{ }^{\circ} \mathrm{C}$. The yield was determined by $\mathrm{GC}$ using anisole as an internal standard. TOF was measured at 1 a conversion below $20 \%$ based on total Co atoms. Error bars represent the standard deviation from at least three independent experiments. DPA, 9,10diphenylanthracene; $\mathrm{BQ}, p$-benzoquinone; $p \mathrm{CBA}$, 4-chlorobenzoic acid. 


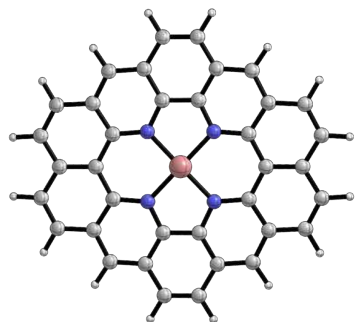

$\mathrm{CoC}_{4} \mathrm{~N}_{10}$

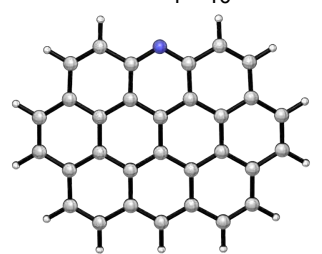

Pyridinic N

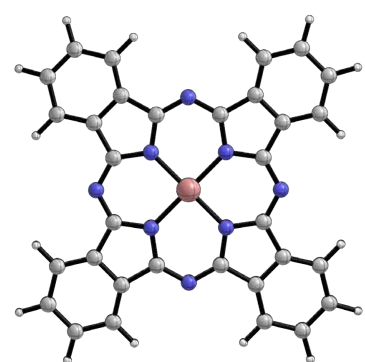

$\mathrm{CoPc}$

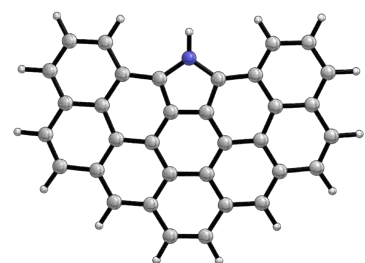

Pyrrolic N
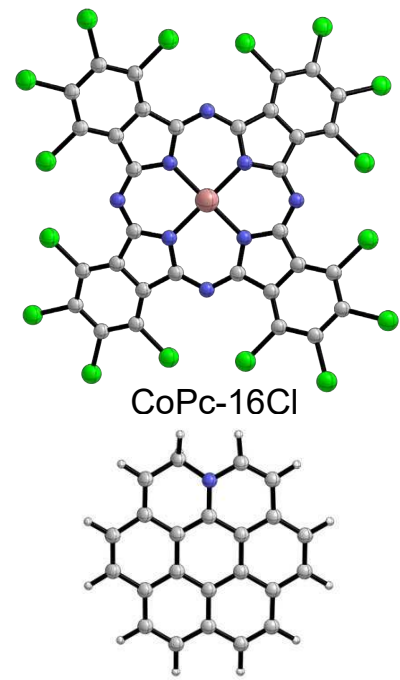

Graphitic N

Supplementary Fig. 34 | DFT-optimized cluster models of $\mathrm{CoN}_{4} \mathrm{C}_{10}$, CoPc, CoPc-16Cl and N-doped graphene sheets. Co, pink; $\mathrm{C}$, grey; $\mathrm{N}$, blue; $\mathrm{H}$, white; $\mathrm{Cl}$, green. 


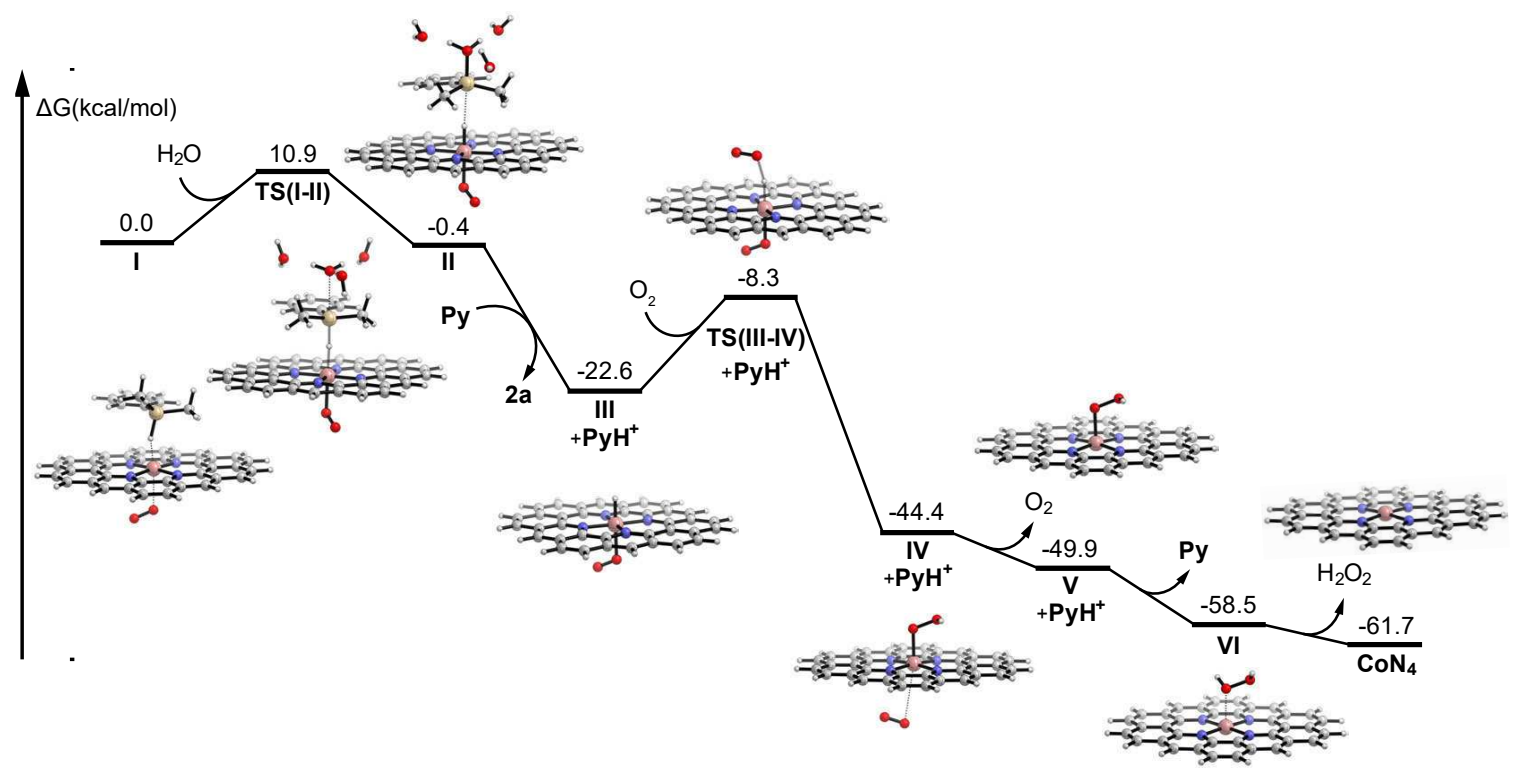

Supplementary Fig. 35 | Free energy profiles for Co-N $4 /$ pyridinic-N catalyzed silane oxidation at $298.15 \mathrm{~K}$. 


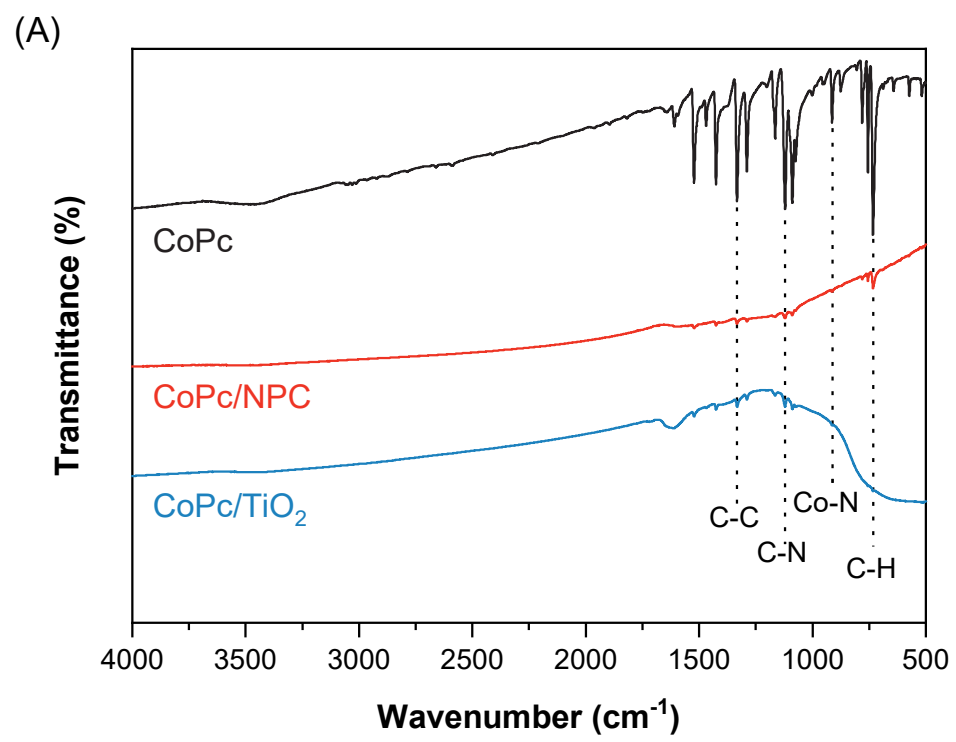

(B)

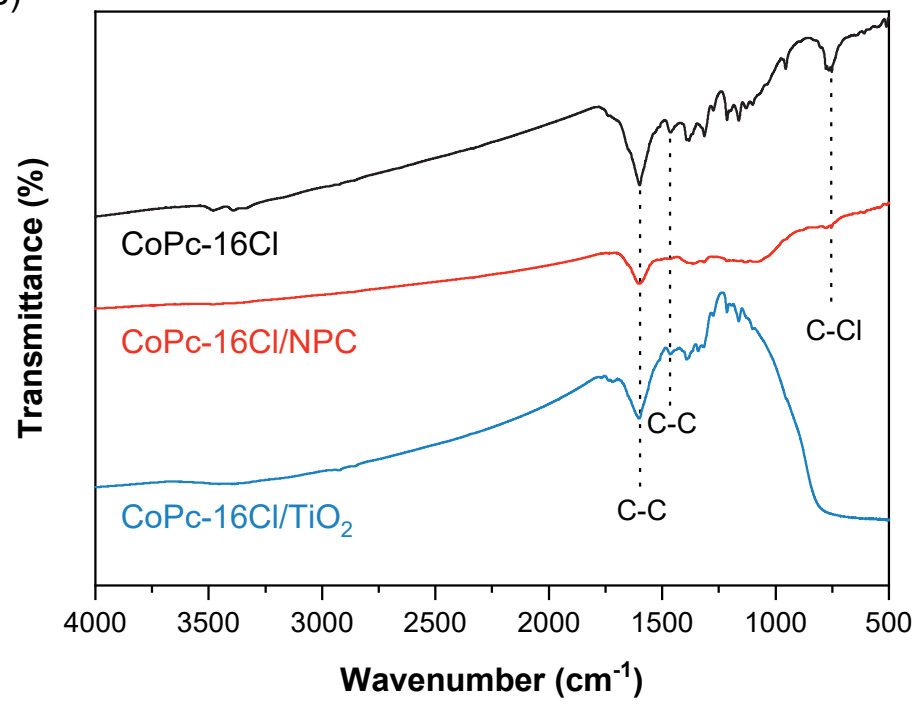

Supplementary Fig. 36 | (A) FT-IR spectra of $\mathrm{CoPc}$, $\mathrm{CoPc} / \mathrm{NPC}$ and $\mathrm{CoPc} / \mathrm{TiO}_{2}$. (B) FT-IR spectra of CoPc$16 \mathrm{Cl}, \mathrm{CoPc}-16 \mathrm{Cl} / \mathrm{NPC}$ and $\mathrm{CoPc}-16 \mathrm{Cl} / \mathrm{TiO}_{2}$. 


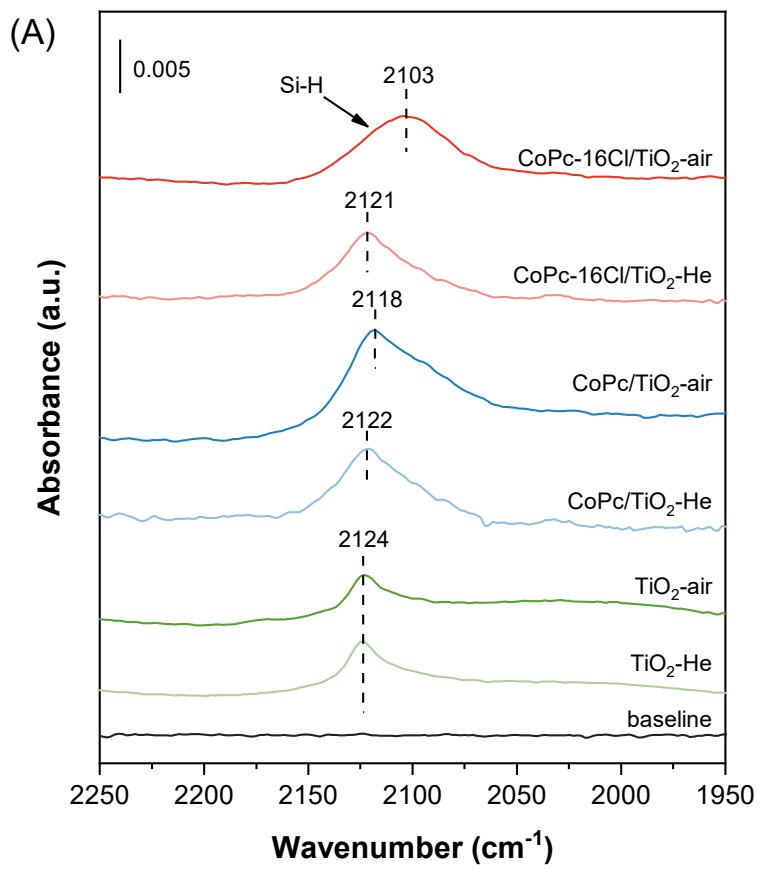

(B)

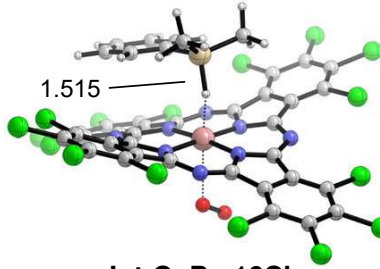

Int-CoPc-16Cl

$v(\mathrm{Si}-\mathrm{H})_{\text {theory }}=2087.4 \mathrm{~cm}^{-1}$

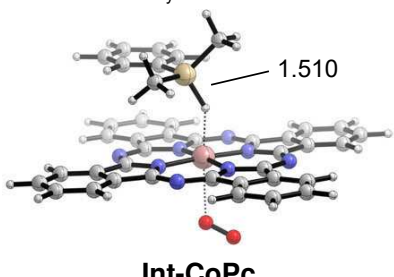

Int-CoPc

$v(\mathrm{Si}-\mathrm{H})_{\text {theory }}=2109.0 \mathrm{~cm}^{-1}$

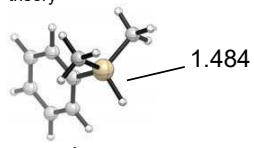

1a

$v(\mathrm{Si}-\mathrm{H})_{\text {theory }}=2224.3 \mathrm{~cm}^{-1}$

Supplementary Fig. $37 \mid$ (A) DRIFT spectra of $1 \mathrm{a}$ adsorbed on $\mathrm{TiO}_{2}, \mathrm{CoPc} / \mathrm{TiO}_{2}$ and $\mathrm{CoPc}-16 \mathrm{Cl} / \mathrm{TiO}_{2}$ under different atmosphere at $15{ }^{\circ} \mathrm{C}$. (B) DFT-optimized geometries and corresponding theoretical stretching vibrations of Si-H bond of 1a-CoPc-16Cl-O $\mathbf{O}_{2}, \mathbf{1 a - C o P c - O} \mathbf{O}_{2}$ and 1a structures. Si-H bond lengths are given in $\AA$.

$\mathrm{TiO}_{2}$-supported CoPc-based samples were chosen as the model of actual catalyst instead of Co- $\mathrm{N}_{4}-$ or CoPc-based carbon catalysts, because the reflectance of such $\mathrm{Co}-\mathrm{N}_{4}-$ or CoPc-based carbon catalysts are insufficient for performing IR measurements. As catalytic performance evaluation of CoPc-based or -derived catalyst models (Supplementary Table 1) clearly showed that $\mathrm{N}$-coordinated Co atomic site was the reaction site of silane and the reaction behavior of CoPc-based carbon catalysts is similar to that of Co- $\mathrm{N}_{4} @ \mathrm{NPC}$ based catalysts, the IR studies of $\mathrm{TiO}_{2}$-supported CoPc-based samples will reveal the interaction between silane and Co site in some extent.

As shown in Supplementary Fig. 37A, the atmosphere during the treatment of $\mathrm{TiO}_{2}$ with $\mathbf{1 a}$ has no influence for 1a adsorption. An absorption band assigned to a $\mathrm{Si}-\mathrm{H}$ bond stretching vibration appeared at $v$ $=2124 \mathrm{~cm}^{-1}$ in the absence or presence of $\mathrm{O}_{2}$. As a comparison, when $\mathrm{CoPc} / \mathrm{TiO}_{2}$ treated with $\mathbf{1 a}$ in the presence of $\mathrm{O}_{2}$, slight redshift of stretching band of $\mathrm{Si}-\mathrm{H}$ bond could be clearly verified. And more importantly, the extent of such redshift was more pronounced on $\mathrm{CoPc}-16 \mathrm{Cl} / \mathrm{TiO}_{2}$ in the presence of $\mathrm{O}_{2}$. These phenomena reveal that $\mathrm{O}_{2}$ promotes the activation of $\mathrm{Si}-\mathrm{H}$ bond in hydrosilane that adsorbed on $\mathrm{N}$ coordinated $\mathrm{Co}$ atomic site. Moreover, electron-deficient $\mathrm{Co}$ site can active $\mathrm{Si}-\mathrm{H}$ bond more efficiently in the presence of $\mathrm{O}_{2}$, which is in agreement with the higher catalytic activity of CoPc-16Cl/NC-900.

It was reported in electrocatalytic oxygen reduction reaction that $\mathrm{O}_{2}$ can easily interact with such $\mathrm{Co}-\mathrm{N}_{\mathrm{x}}$ center through end-on adsorption and cause the electron transfer from $\mathrm{Co}$ atom to $\mathrm{O}$ atom, which decreases the electron density of Co site ${ }^{4}$. These results make us to propose that in our system, $\mathrm{O}_{2}$ can act as a ligand to optimize the electronic structure of $\mathrm{Co}$ and co-adsorb with silane on Co atom to boost the activation capacity of Co site for the $\mathrm{Si}-\mathrm{H}$ bond. We employed DFT calculations to study the interaction of $\mathrm{O}_{2}, \mathbf{1 a}$ and $\mathrm{CoPc}$ or $\mathrm{CoPc}-16 \mathrm{Cl}$ (Supplementary Fig. 37B). The optimal geometries of both adducts 1a-CoPc-16Cl-O and $\mathbf{1 a}-\mathrm{CoPc}-\mathrm{O}_{2}$ show a greater $\mathrm{Si}-\mathrm{H}$ bond length than that of individual 1a, suggesting the $\mathrm{O}_{2}$-coordinated Co- $\mathrm{N}_{4}$ site can active $\mathrm{Si}-\mathrm{H}$ bond effectively, which is consistent with the observation of DRIFT spectra. 
(A)

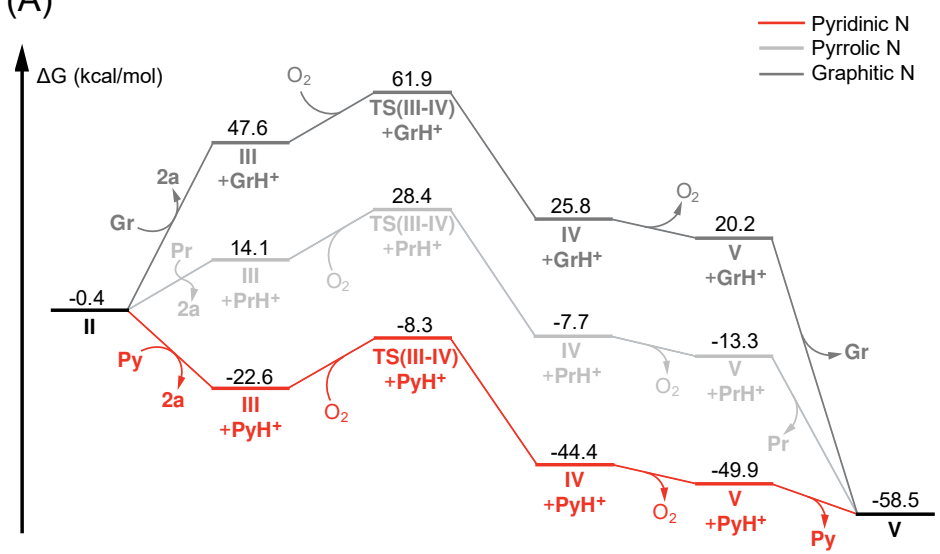

(B)

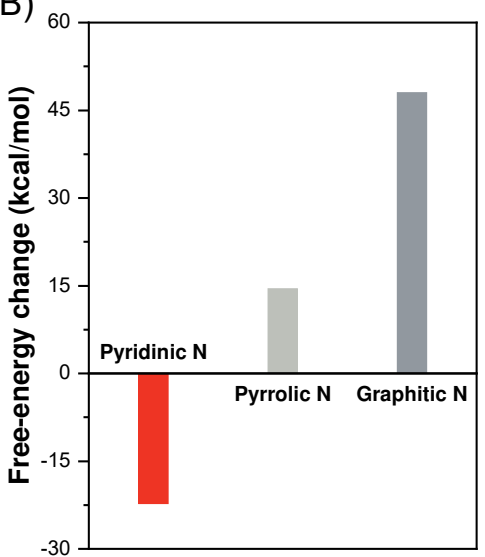

Supplementary Fig. 38 | (A) Free energy profiles for with different N-doped graphene structures at 298.15K. (B) The free-energy changes of the deprotonation step "II $\rightarrow$ III" with different N-doped graphene structures. Py, Pyridinic N; Pr, Pyrrolic N; Gr, Graphitic N. 


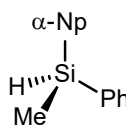

$95 \%$ ee

$R-1 b$

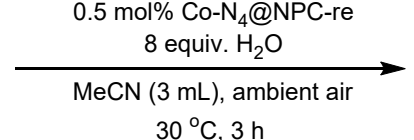

$30{ }^{\circ} \mathrm{C}, 3 \mathrm{~h}$

(1)

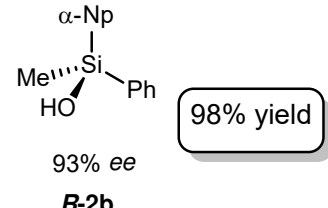

$R-2 b$

Supplementary Fig. 39 | Oxidation of optically active hydrosilane $\boldsymbol{R}-\mathbf{1 b}$ over Co-N $@$ @NPC-re catalyst. As seen from the below Chiral HPLC data, the inversion of the configuration at silicon was obtained.

\section{Chromatogram of $\mathbf{1 b}$}

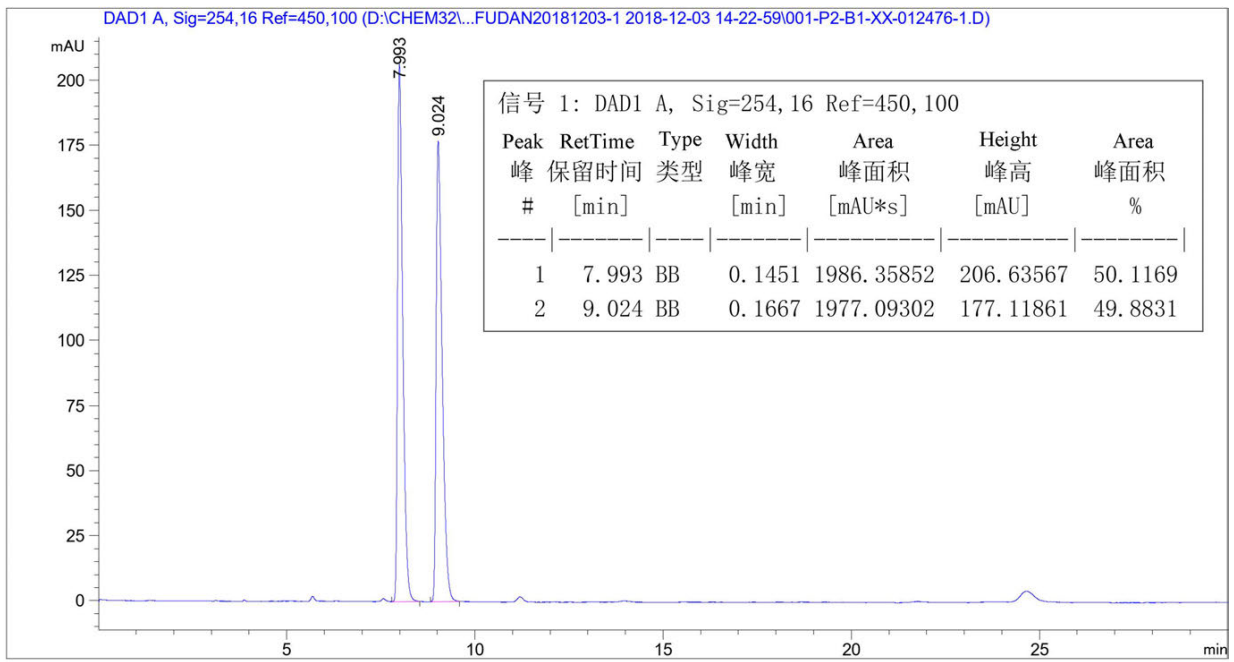

\section{Chromatogram of $\boldsymbol{R}-\mathbf{1 b}$}

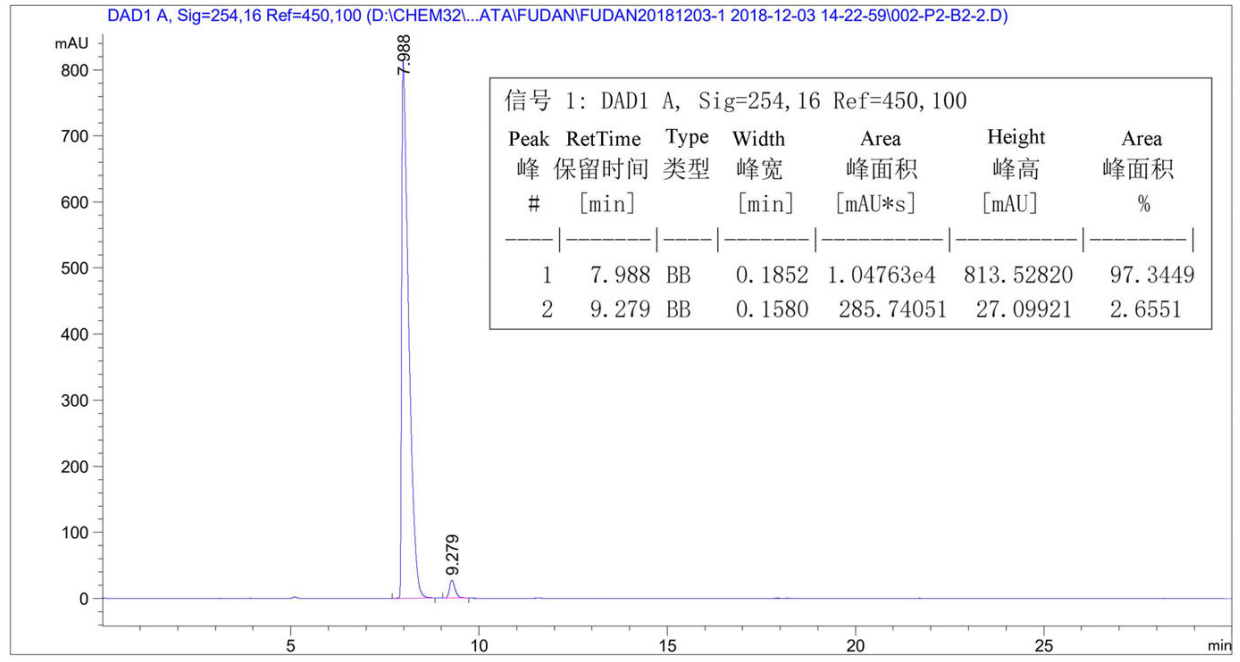




\section{Chromatogram of $\mathbf{2 b}$}

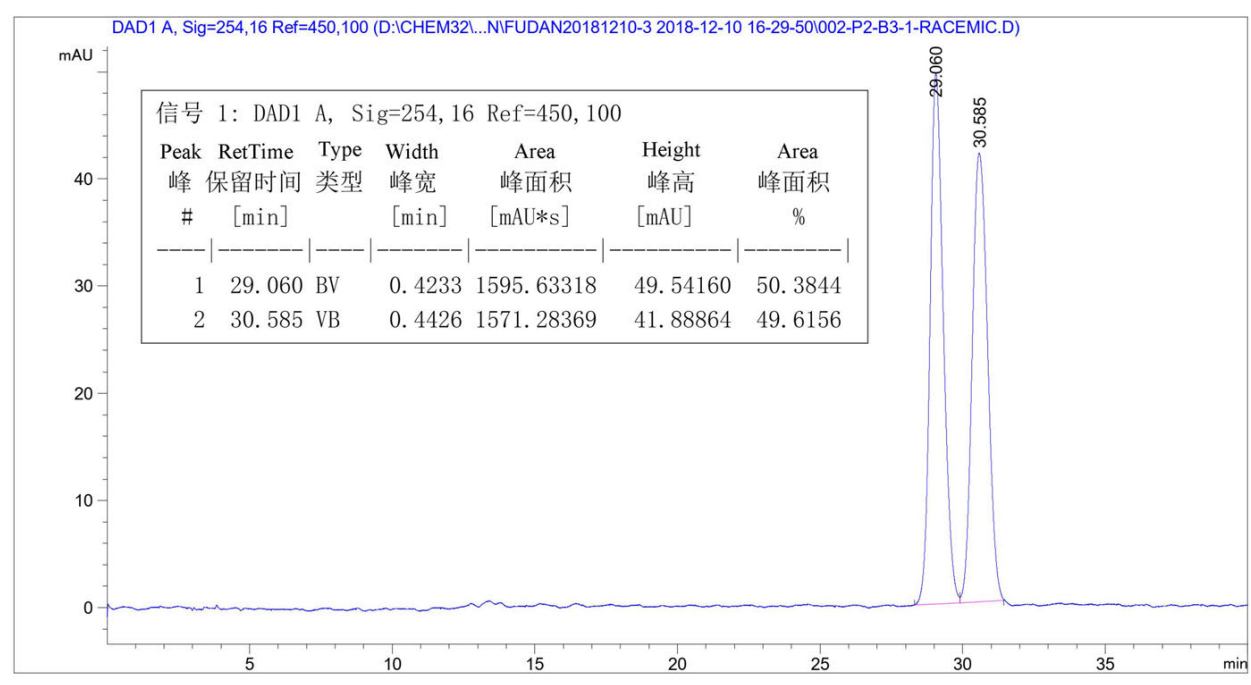

\section{Chromatogram of $\boldsymbol{R}-\mathbf{2 b}$}

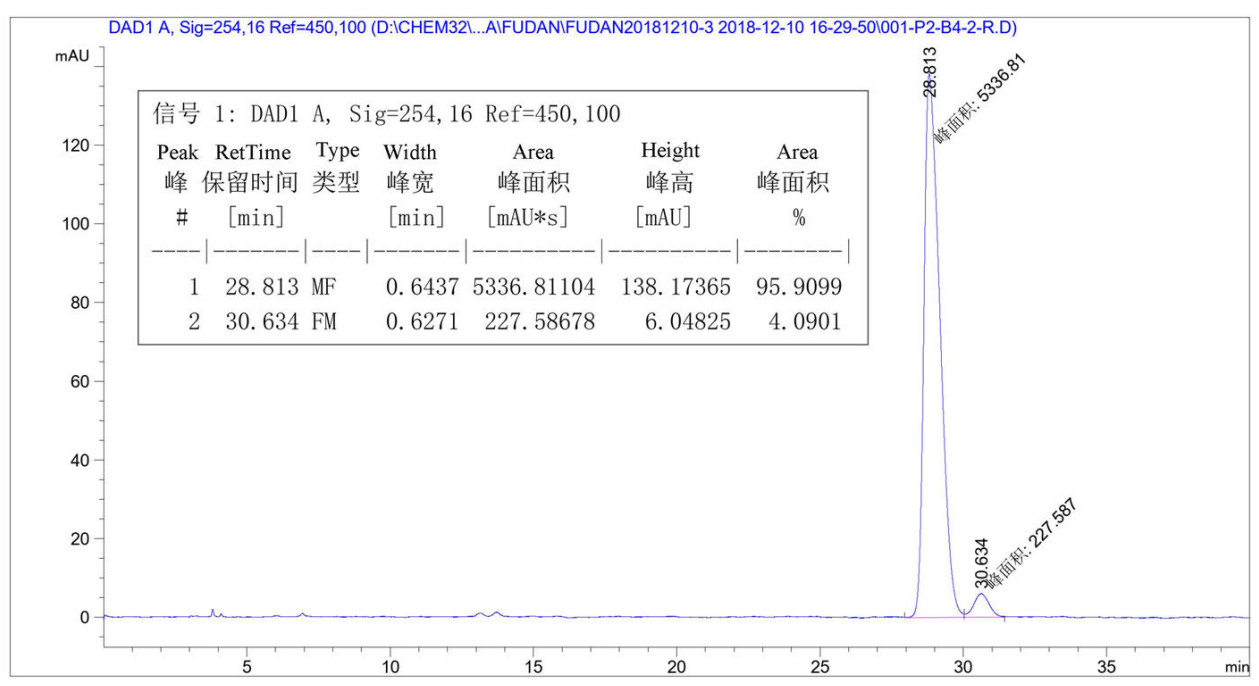




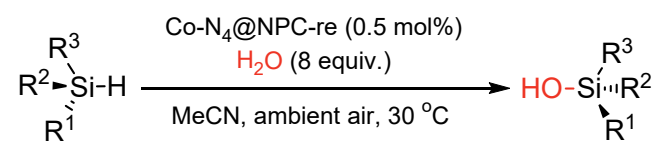

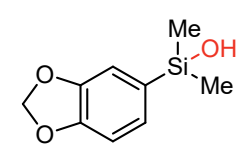

2bj, 3 h, 97\%

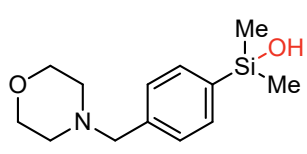

2bn, 6 h, 95\%

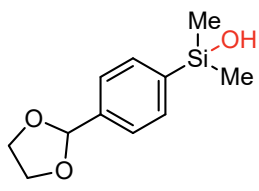

2bq, 4 h, $94 \%$<smiles>C[Si](O)(O)c1ccncc1</smiles>

2bk, 4 h, $93 \%$

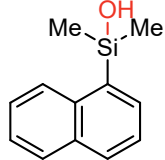

2bl, 3 h, 98\%

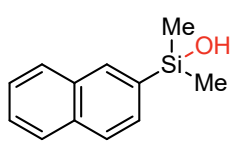

2bm, 1 h, 97\%<smiles>C=C[Si](C)(O)c1ccccc1</smiles>

2br, 4 h, $97 \%$

2bo, 5 h, $96 \%$<smiles>C[Si](O)(O)c1ccc(C(=O)N2CCCCC2)cc1</smiles><smiles>C[Si](C)(O)Cc1ccccc1</smiles>

2bs, 4 h, $98 \%$ 2bp, 3 h, $97 \%$<smiles>C[Si](O)(O)c1ccc(OC2CCCCO2)cc1</smiles>

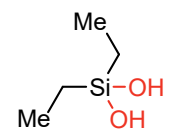

2bt, 2 h, $96 \%$

Supplementary Fig. 40 | Extended substrate scope of silane oxidation continued from Fig. 4A. Reaction conditions: silane (1 mmol), $\mathrm{H}_{2} \mathrm{O}(8 \mathrm{mmol}), \mathrm{MeCN}(3 \mathrm{~mL}), \mathrm{Co}_{4} \mathrm{~N}_{4} @ \mathrm{NPC}-\mathrm{re}(\mathrm{Co} 0.5 \mathrm{~mol} \%), 30{ }^{\circ} \mathrm{C}$, air atmosphere. Isolated yields are reported unless otherwise indicated. 
(A)

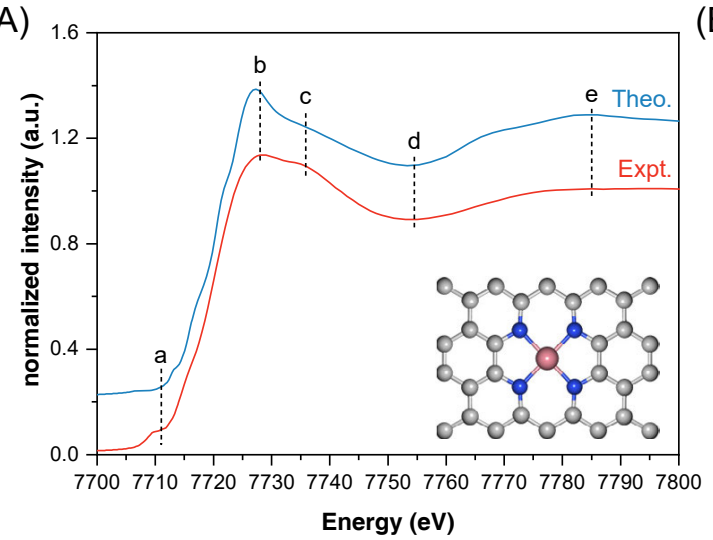

(B)

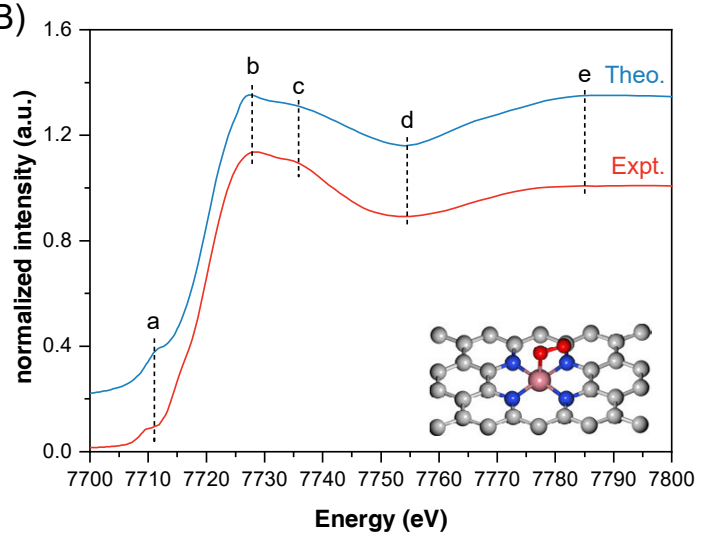

Supplementary Fig. 41 | Comparison between the experimental Co K-edge XANES spectrum of Co$\mathrm{N}_{4} @ \mathrm{NPC}$-re and the theoretical spectra of $\mathrm{CoN}_{4} \mathrm{C}_{12}$ and $\mathrm{CoN}_{4} \mathrm{C}_{12}-\mathrm{O}_{2}$ structure.

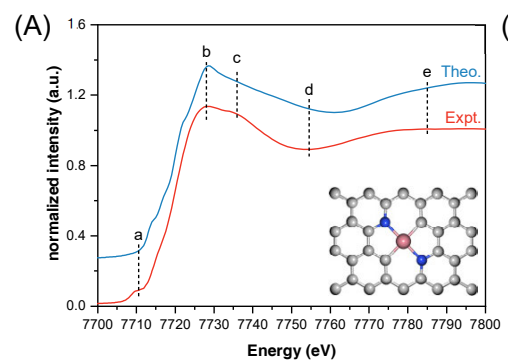

(D)

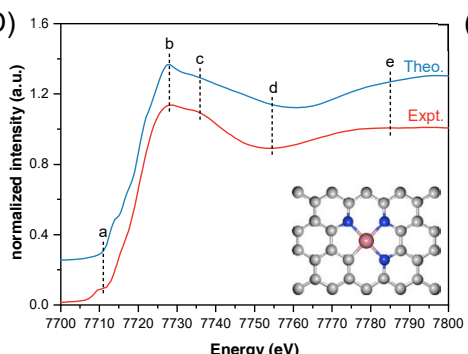

(G)

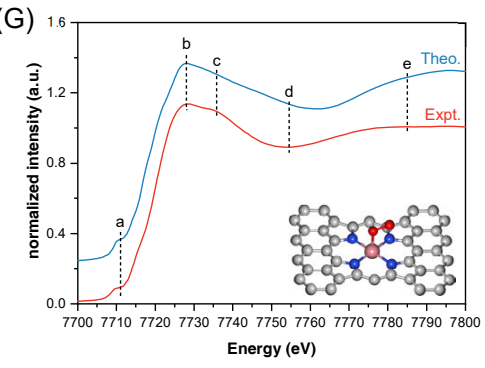

(B)

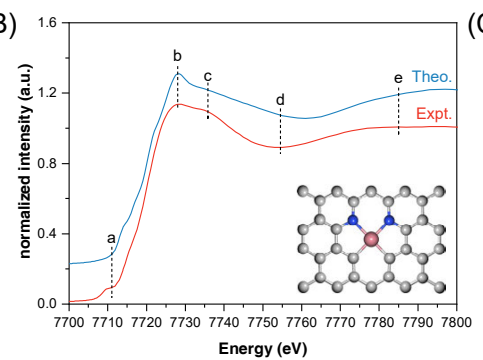

(E)

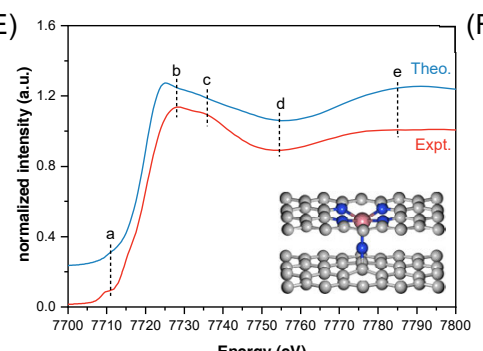

(H)

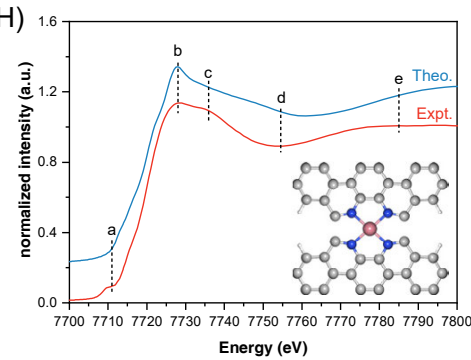

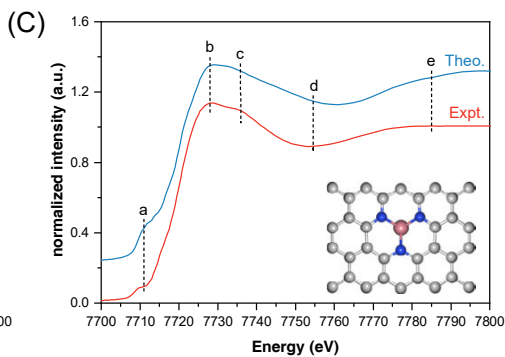

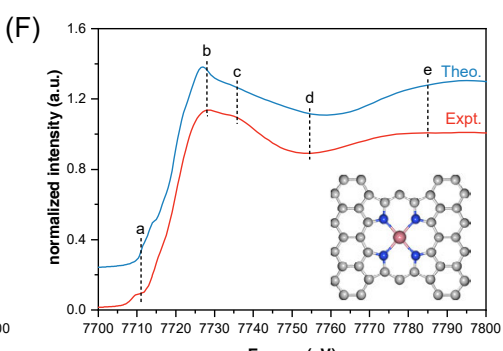

(I)

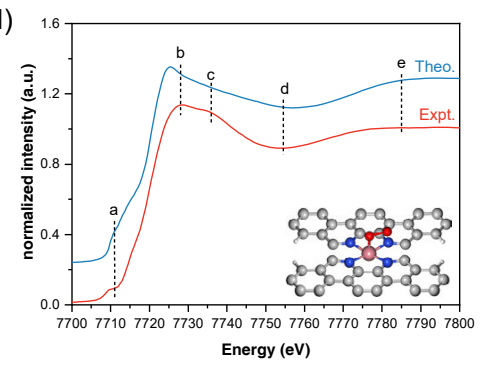

Supplementary Fig. 42 | Comparison between the experimental Co K-edge XANES spectrum of Co$\mathrm{N}_{4} @$ NPC-re and the theoretical spectra of other potential Co-N-C structures.

The Co K-edge XANES simulation was conducted with the FDMNES code in multiple scattering mode (Green's functions) using the muffin-tin potential. The calculated radius was $6.5 \AA$ with a self-consistent calculated radius of $6.0 \AA$. Parameter optimization was performed by comparing the theoretical and experimental spectra to acquire the most appropriate convolution parameters. The calculated models were built based on DFT calculations to avoid manual bias. 


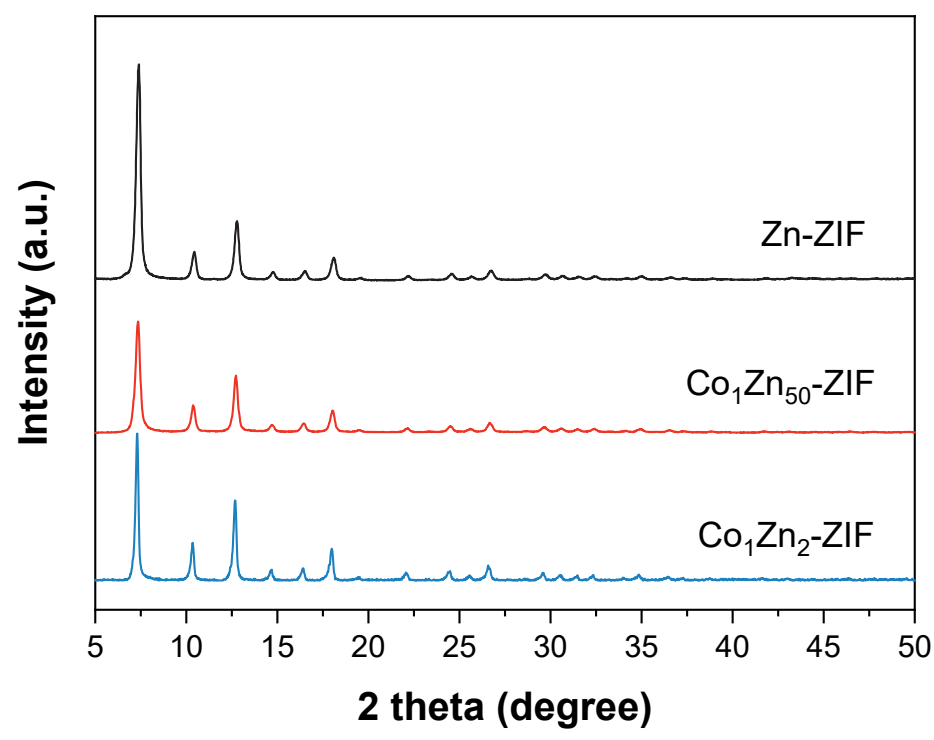

Supplementary Fig. $43 \mid$ XRD patterns of ZIF precursors.

(A)

(B)

(C)

Supplementary Fig. 44 | Photographs of (A) Zn-ZIF, (B) $\mathrm{Co}_{1} \mathrm{Zn}_{50}-\mathrm{ZIF}$ and (C) $\mathrm{Co}_{1} \mathrm{Zn}_{2}-\mathrm{ZIF}$. 

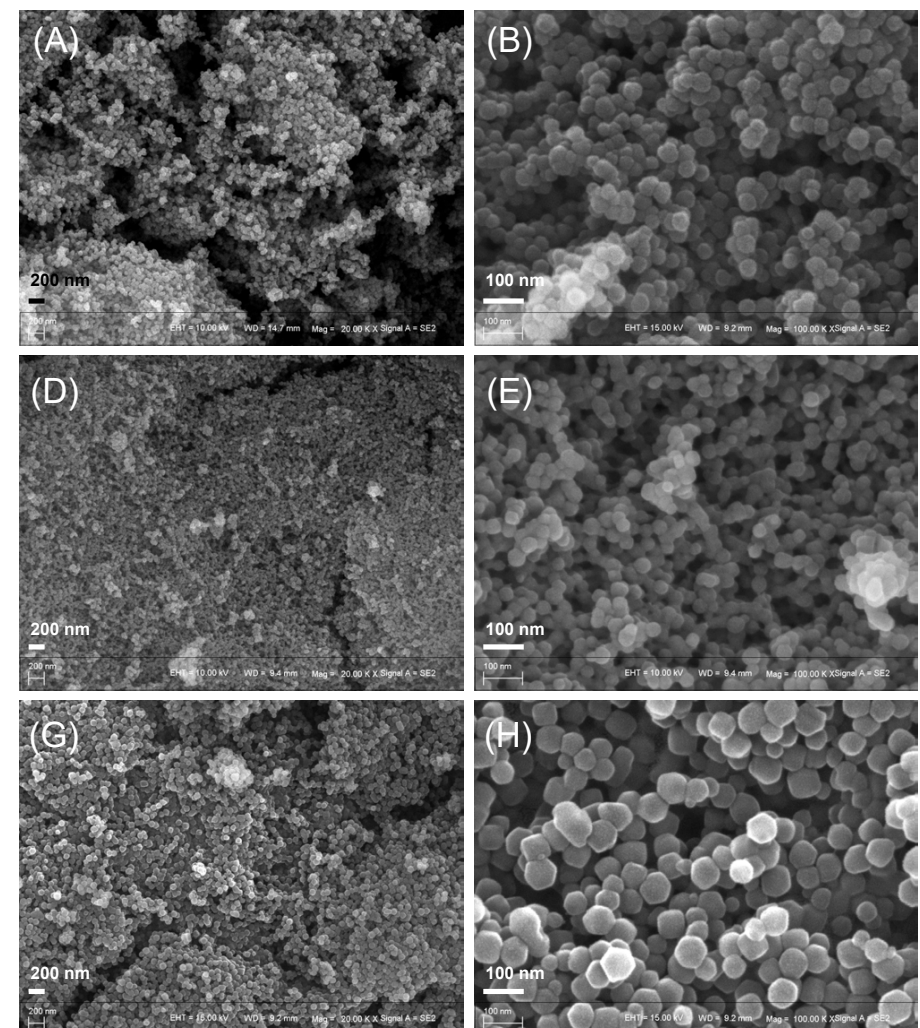

Supplementary HIg. $\mathbf{4 5}$ (A-C) SElv mages (A, B) and particle size distribution diagram (C) for Zn-ZIF.

(D-F) SEM images (D, E) and particle size distribution diagram (F) for $\mathrm{Co}_{1} \mathrm{Zn}_{50}$-ZIF. (G-I) SEM images (G, $\mathrm{H})$ and particle size distribution diagram (I) for $\mathrm{Co}_{1} \mathrm{Zn}_{2}$-ZIF. 


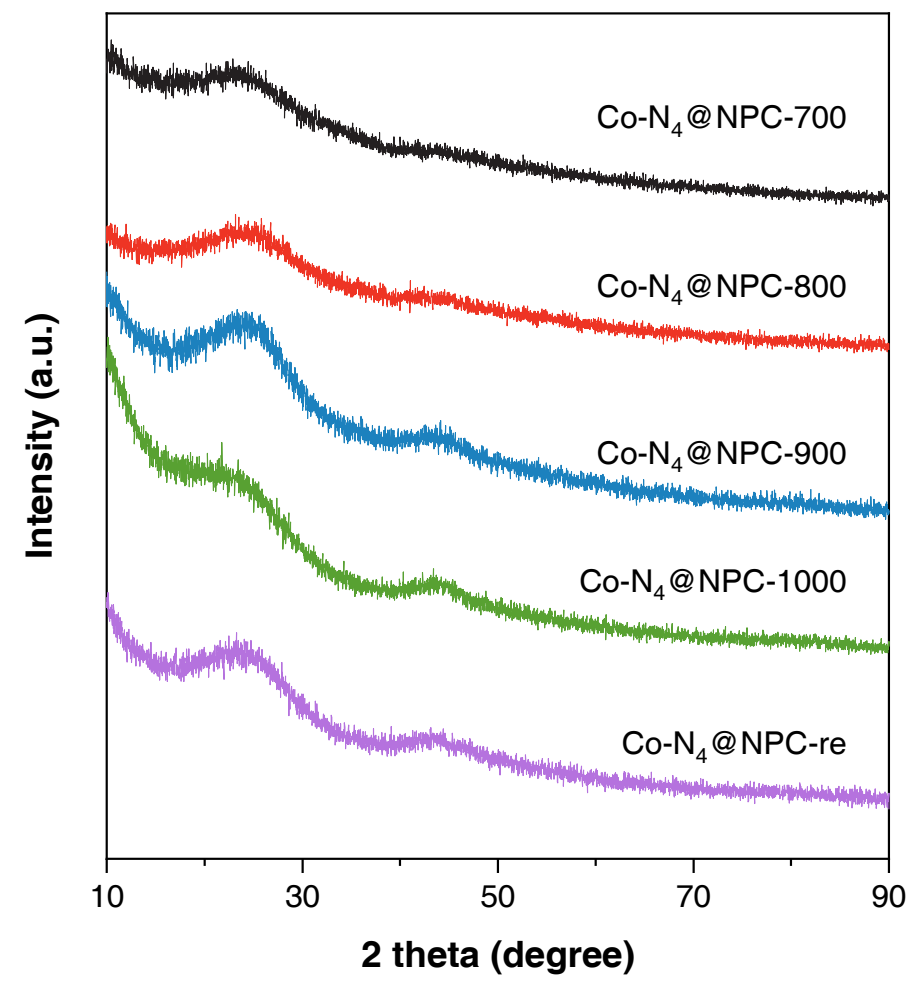

Supplementary Fig. 46 | XRD patterns for $\mathrm{Co}_{1} \mathrm{Zn}_{50}$-ZIF-derived Co- $\mathrm{N}_{4} @ \mathrm{NPC}$-based samples with different synthesis conditions. 

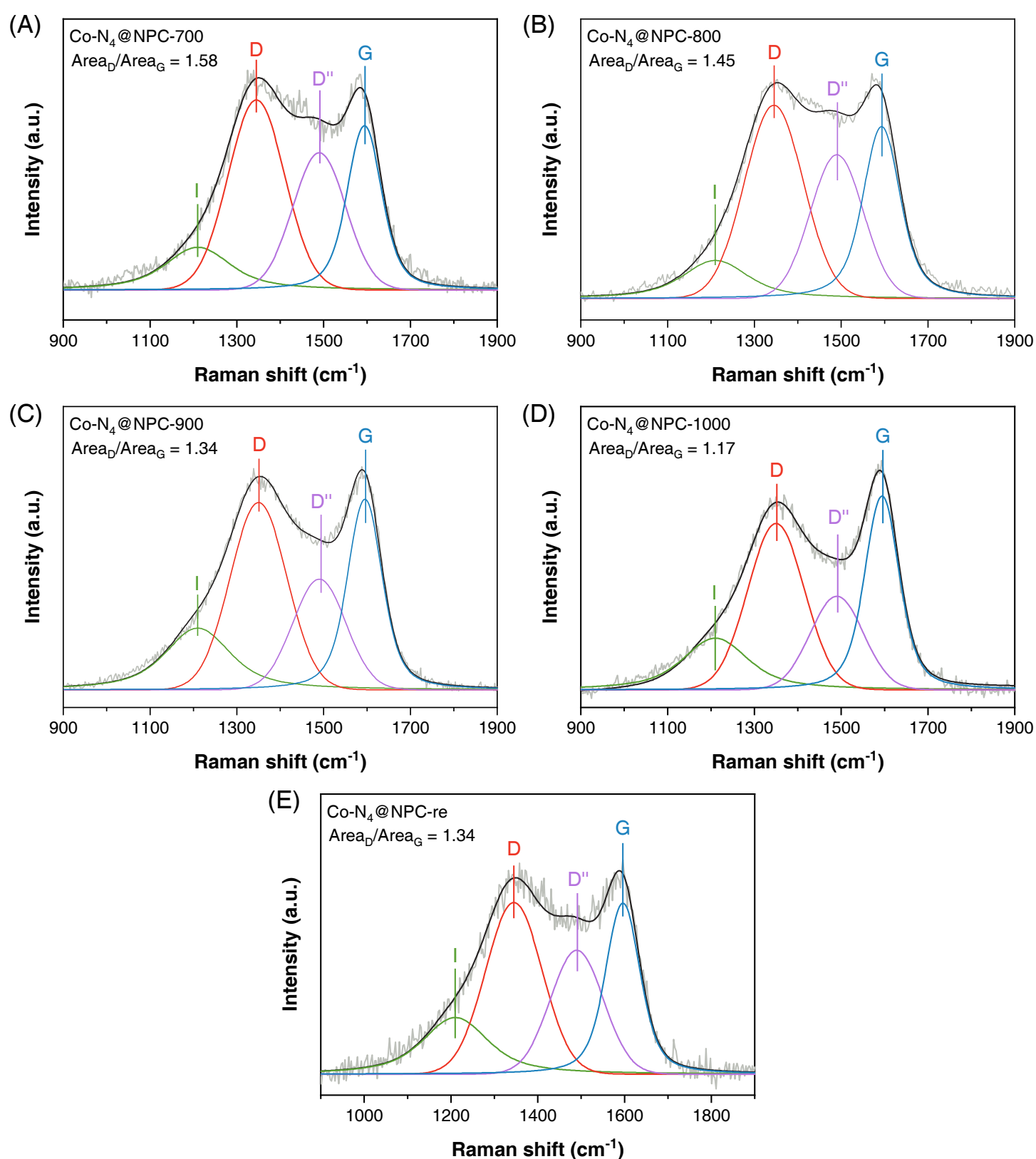

Supplementary Fig. 47 | Raman spectra for $\mathrm{Co}_{1} \mathrm{Zn}_{50}-\mathrm{ZIF}$-derived Co- $\mathrm{N}_{4} @ \mathrm{NPC}$-based samples with different synthesis conditions. With the increase of pyrolysis temperature, the ratio of Area $/ \mathrm{Area}_{\mathrm{G}}$ decreased gradually, indicating the significant promotion of graphitization degree. In addition, peak around $1490 \mathrm{~cm}^{-1}$ becomes obviously smaller with an increase in heating temperatures, suggesting a reduction of nitrogen doping and other irregular carbon structures, i.e. five-side ring ${ }^{5}$. The ratio of $\mathrm{Area}_{\mathrm{D}} / \mathrm{Area}_{\mathrm{G}}$ in $\mathrm{Co}_{\mathrm{N}} \mathrm{N}_{4} @ \mathrm{NPC}-$ re is closed to Co-N $\mathrm{N}_{4} @$ NPC-900, revealing the similar partially graphitized carbon structures in such samples. 
(A)

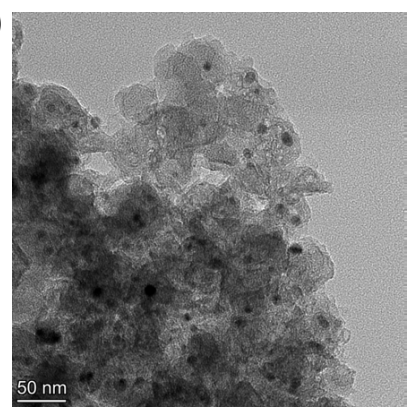

(B)

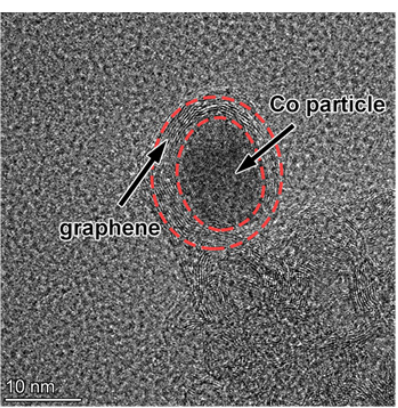

(C)

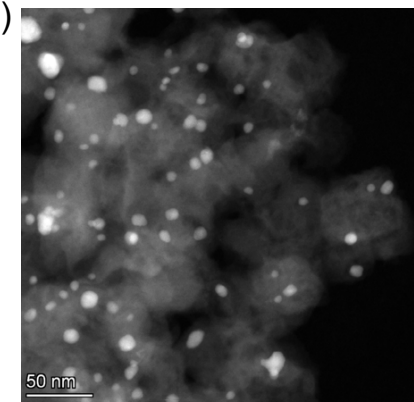

(D)

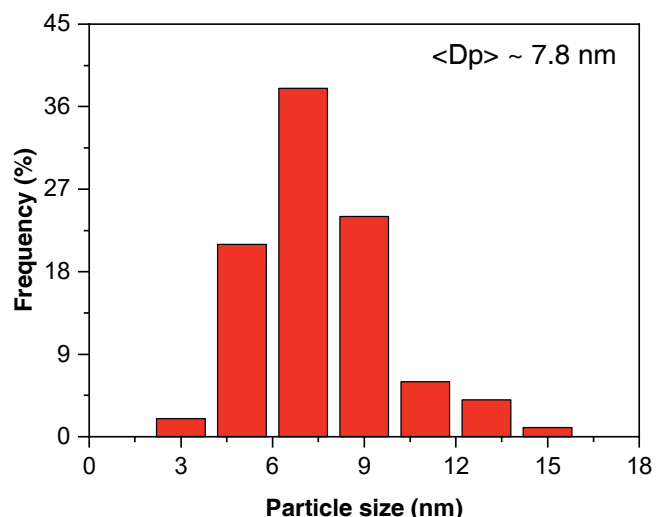

(E)

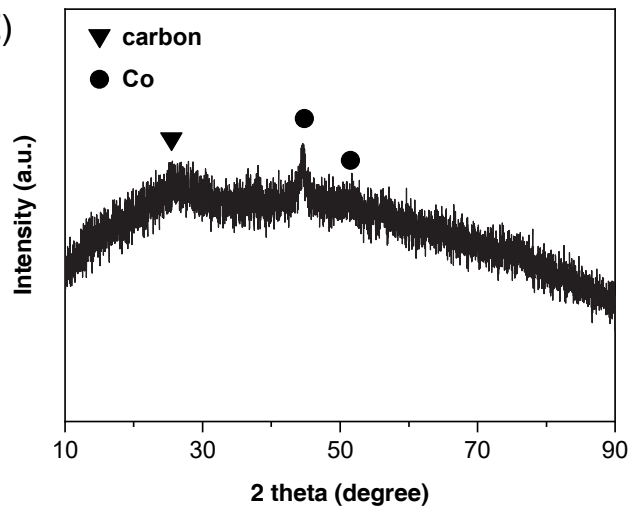

$(\mathrm{F})$

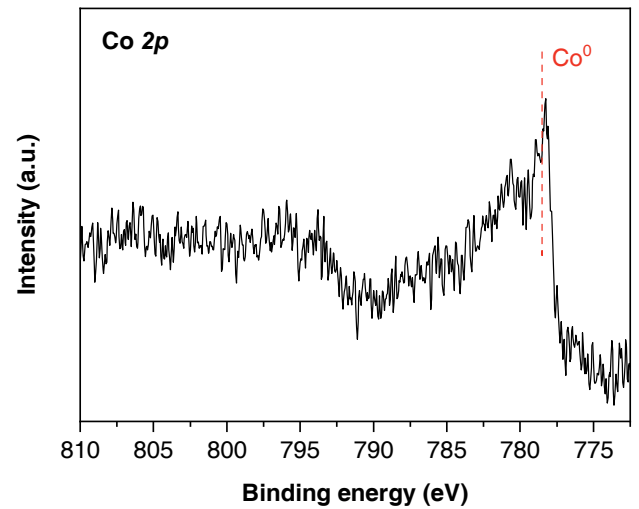

(G)

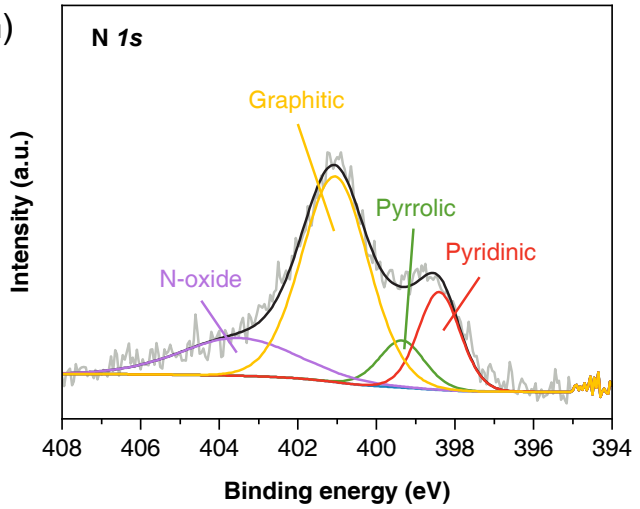

(H)

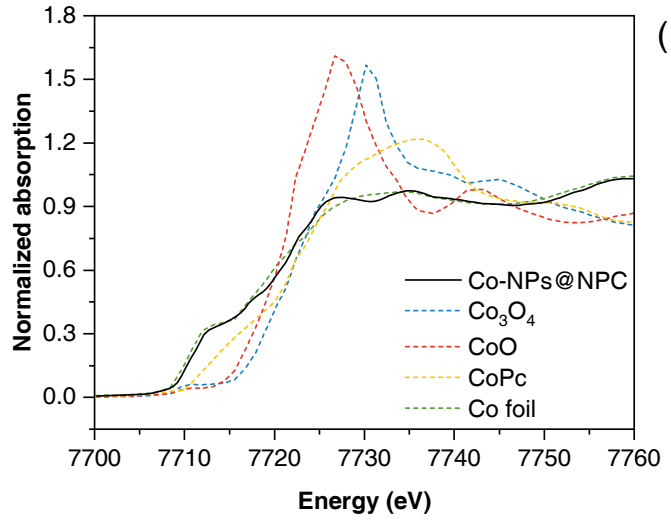

Supplementary Fig. 48 | Characterizations of Co-NPs@NPC. (A) TEM image. (B) High resolution TEM image. The graphene-encapsulated Co nanoparticle was obtained. (C) STEM image. (D) Particle size distribution diagram based on STEM images. The average size of Co particles is $7.8 \mathrm{~nm}$. (E) XRD pattern. (F) Co $2 p$ XPS spectrum. (G) N $1 s$ XPS spectrum. (H) Co K-edge XANES spectra of Co-NPs@NPC, $\mathrm{Co}_{3} \mathrm{O}_{4}$, $\mathrm{CoO}, \mathrm{CoPc}$ and Co foil. $(\mathrm{H}) \mathrm{R}$-space Co K-edge EXAFS spectra and fittings of Co-NPs@NPC. The fitting results are listed in Supplementary Table 2. 

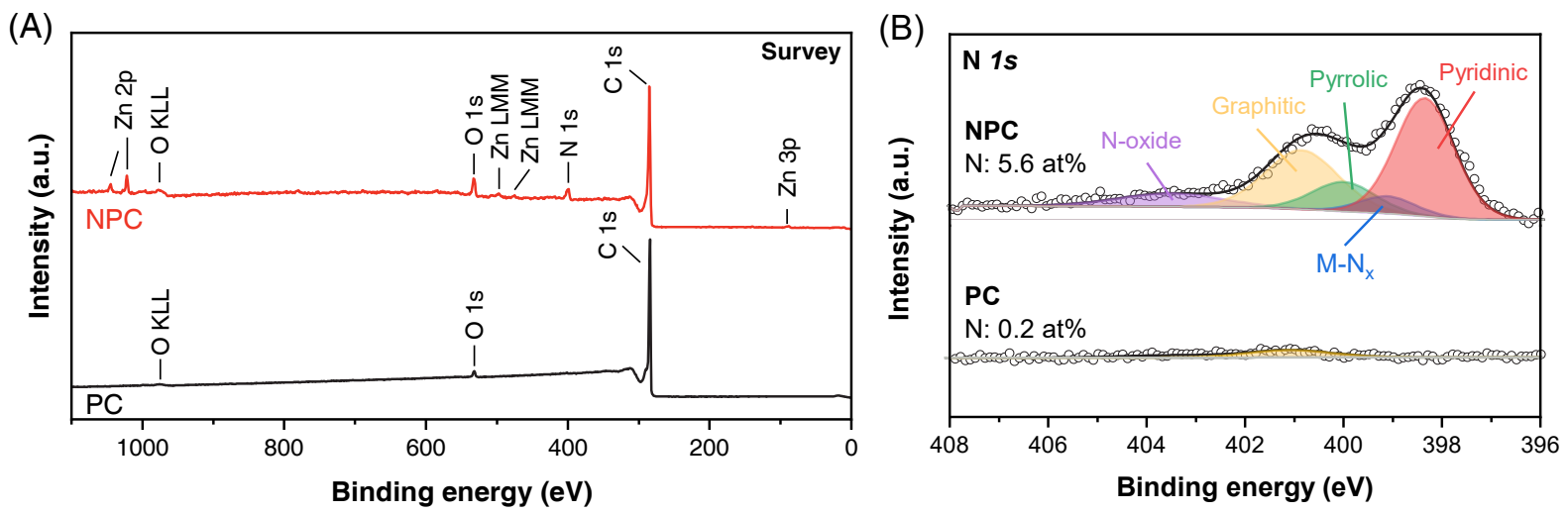

Supplementary Fig. 49 | (A) Survey and (B) N $1 s$ XPS spectra of PC and NPC. Compared to the N concentration of NPC sample (5.6 at $\%$ ), that of PC is negligible ( 0.2 at $\%)$. Moreover, analysis for $\mathrm{N} 1 \mathrm{~s}$ spectrum of PC showed the residue $\mathrm{N}$ species is mainly graphitic $\mathrm{N}(401.1 \mathrm{eV})^{6}$. Thus, the PC sample can be used as a quasi $\mathrm{N}$-free porous carbon.

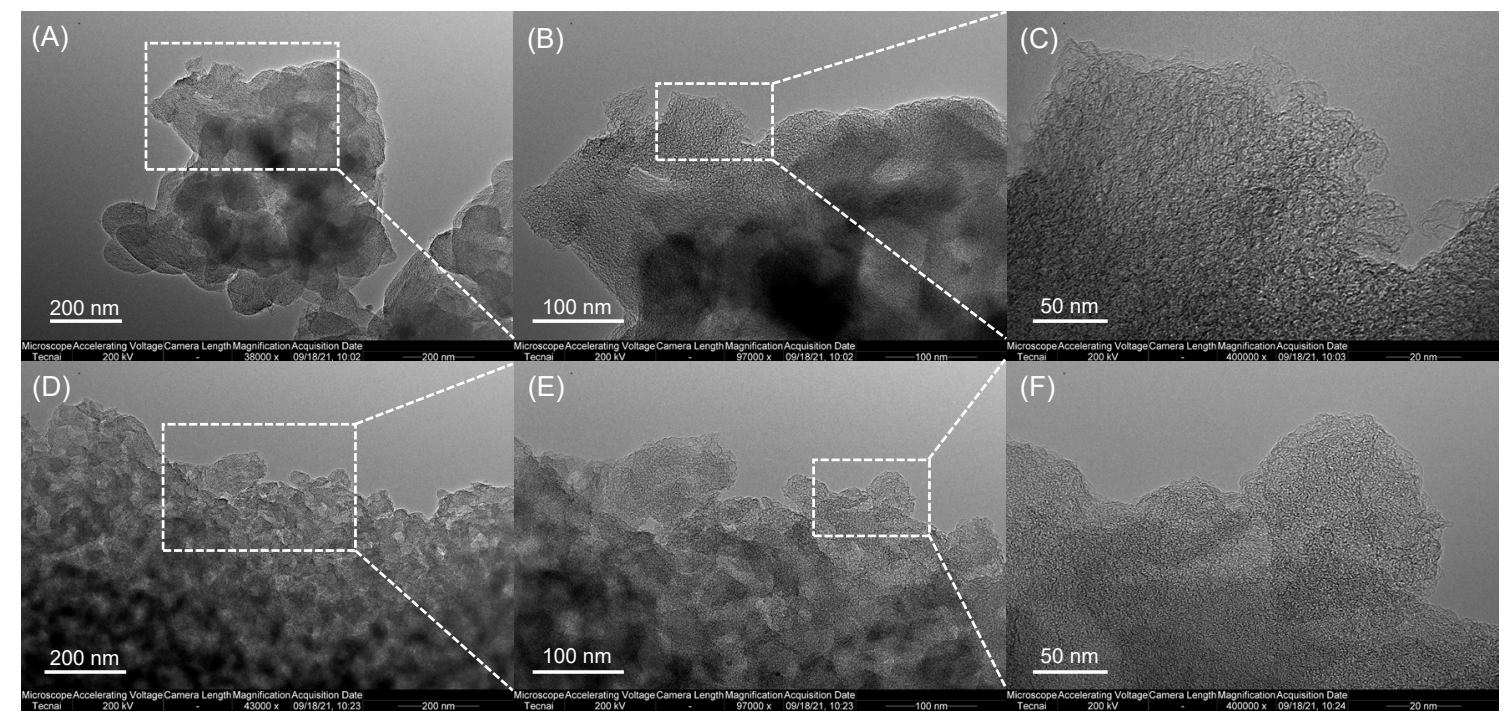

Supplementary Fig. 50 | TEM images of PC (A-C) and NPC (D-F). 


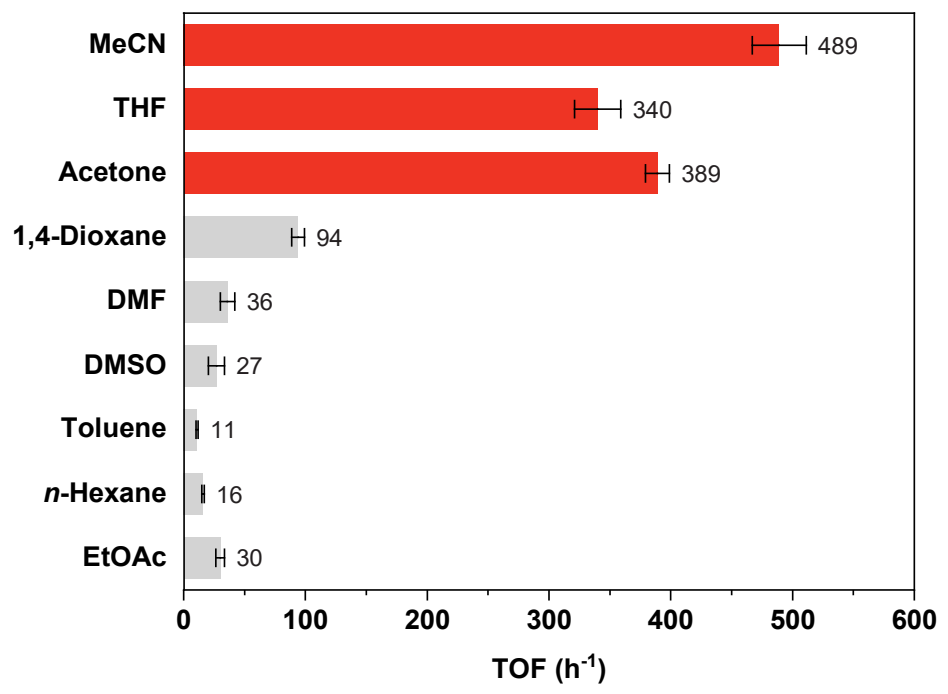

Supplementary Fig. 51 | Catalytic performance for oxidation of 1a over Co- $\mathrm{N}_{4} @ N$ NC-900 catalyst in different solvent media. Reaction conditions: $1 a(1 \mathrm{mmol}), \mathrm{H}_{2} \mathrm{O}(8 \mathrm{mmol})$, solvent $(3 \mathrm{~mL}), \mathrm{Co}-\mathrm{N}_{4} @ \mathrm{NPC}-$ 900 (Co $0.5 \mathrm{~mol} \%$ ), $30{ }^{\circ} \mathrm{C}$, air atmosphere. TOF was measured at $1 \mathrm{a}$ conversion below $20 \%$ based on total Co atoms. For all reactions, $>99 \%$ selectivity of $\mathbf{2 a}$ were obtained. 
(A)

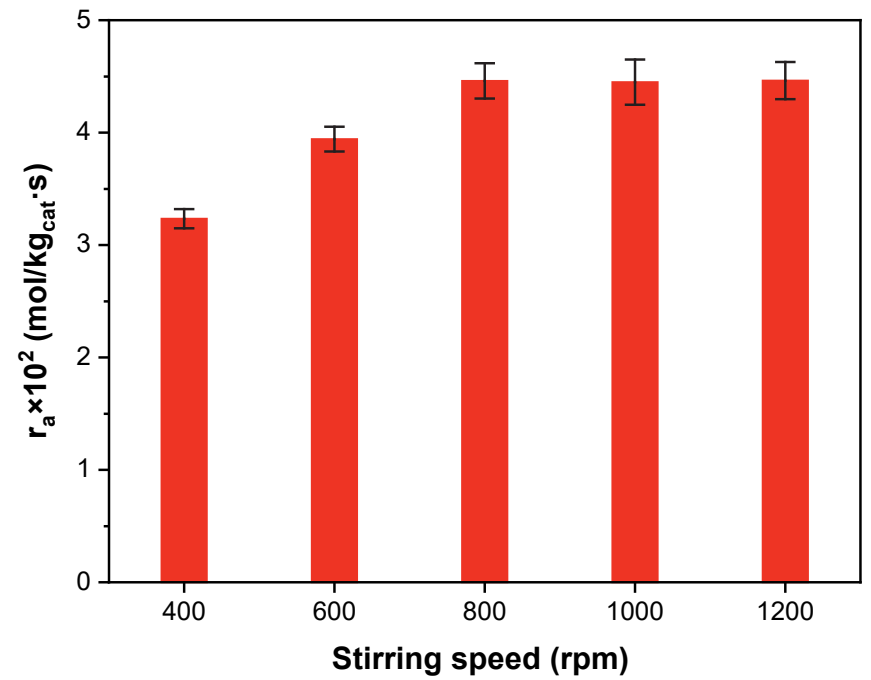

(B)

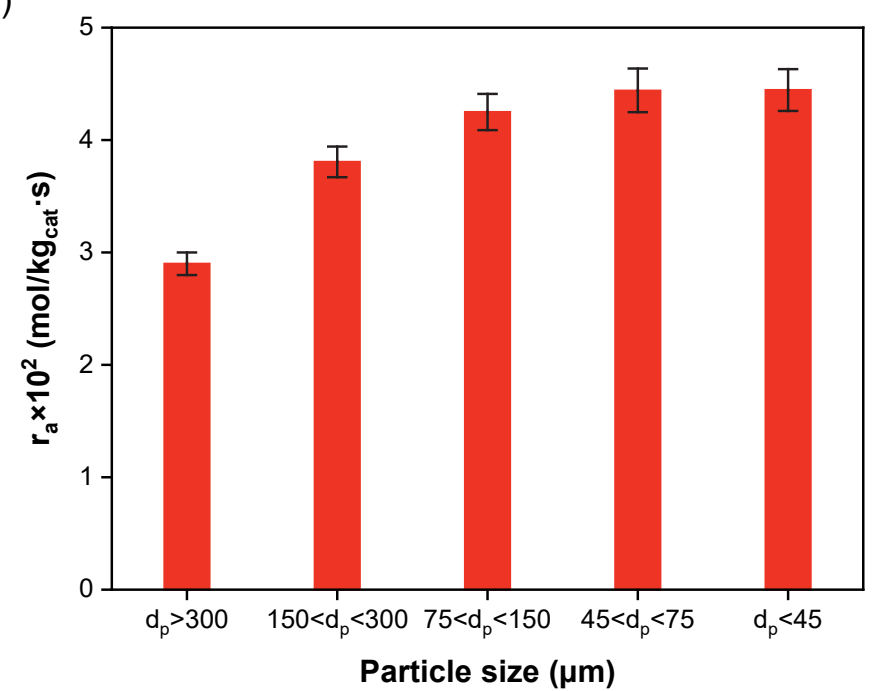

Supplementary Fig. 52 Dependence of the initial reaction rates $\left(r_{a}\right)$ of Co- $\mathrm{N}_{4} @ N P C-900$-catalyzed 1a oxidation on (A) stirring speed and (B) particle size. Reaction conditions: 1a (1 mmol), $\mathrm{H}_{2} \mathrm{O}(8 \mathrm{mmol})$, solvent (3 mL), Co-N 4 @NPC-900 (Co 0.5 mol\%), $30{ }^{\circ} \mathrm{C}$, air atmosphere. Error bars represent the standard deviation from at least three independent experiments. $r_{a}$ was calculated based on the corresponding TOF values. 
(A)

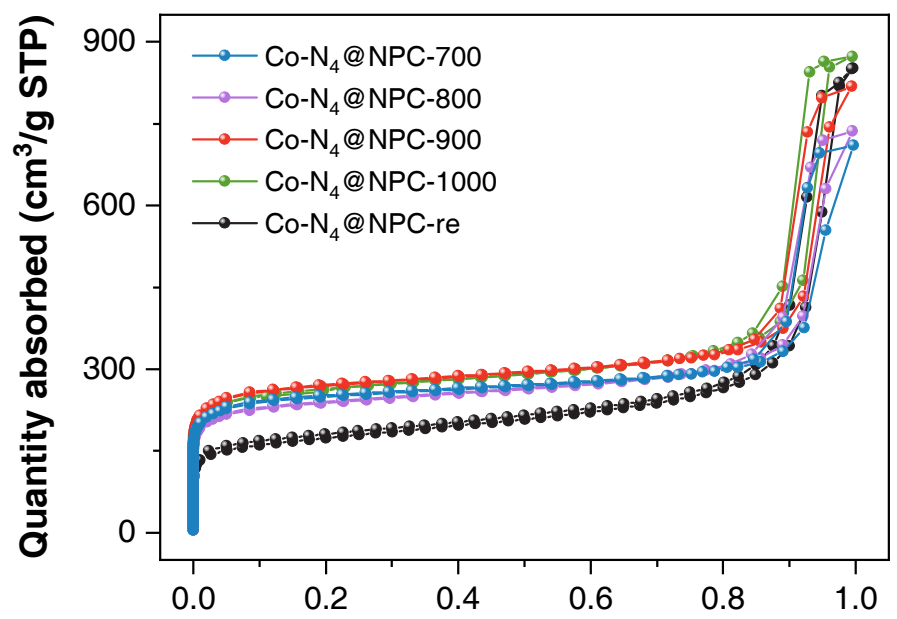

(B)

Relative pressure $\left(P / P_{0}\right)$

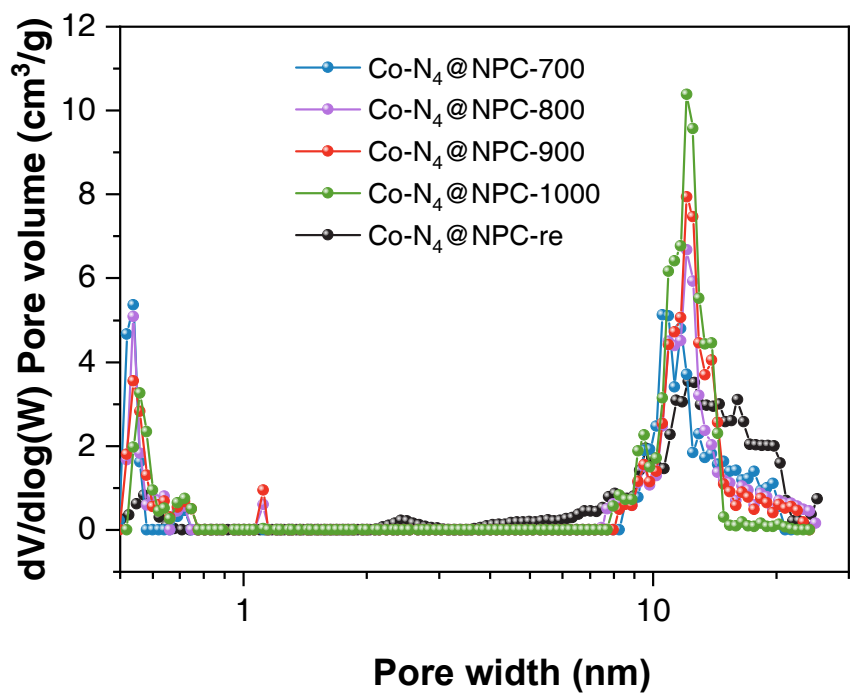

Supplementary Fig. 53 $\mid \mathrm{N}_{2}$ adsorption/desorption isotherms (A) and pore size distribution (B) of different Co-N ${ }_{4} @$ NPC samples. The pore size distribution was determined from non-local density functional theory (NLDFT) model of the $\mathrm{N}_{2}$ adsorption data. 
Supplementary Table 1 | Catalytic results of oxidase-type selective 1a oxidation over CoPc-based or derived biomimetic catalyst models.

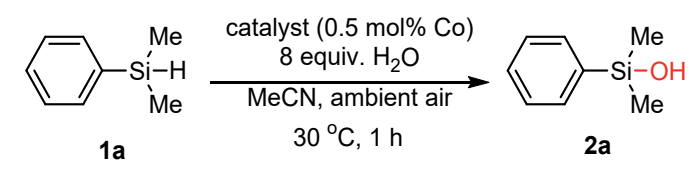

\begin{tabular}{ccc}
\hline Entry $^{\mathbf{a}}$ & Catalyst & Yield of 2a (\%) $^{\mathbf{b}}$ \\
\hline 1 & $\mathrm{CoPc} / \mathrm{NPC}$ & 18 \\
2 & $\mathrm{Co}-\mathrm{N}_{4} / \mathrm{NPC}$ & 42 \\
3 & $\mathrm{CoPc} / \mathrm{PC}$ & $<1$ \\
$4^{\mathrm{c}}$ & $\mathrm{NPC}$ & $\mathrm{N} . \mathrm{R}$. \\
$5^{\mathrm{c}}$ & $\mathrm{CoPc} / \mathrm{PC}+\mathrm{NPC}$ & 10 \\
$6^{\mathrm{d}}$ & $\mathrm{Co}-\mathrm{N}_{4} / \mathrm{NPC}$ & $\mathrm{N} . \mathrm{R}$. \\
$7^{\mathrm{e}}$ & $\mathrm{Co}-\mathrm{N}_{4} / \mathrm{NPC}$ & $\mathrm{N} . \mathrm{R}$. \\
8 & $\mathrm{CoPc}-16 \mathrm{Cl} / \mathrm{NPC}$ & 39 \\
\hline
\end{tabular}

${ }^{\text {a }}$ Reaction conditions: 1a $(1 \mathrm{mmol}), \mathrm{H}_{2} \mathrm{O}(8 \mathrm{mmol}), \mathrm{MeCN}(3 \mathrm{~mL})$, catalyst (Co $\left.0.5 \mathrm{~mol} \%\right)$, air atmosphere, $30{ }^{\circ} \mathrm{C}, 1 \mathrm{~h} .{ }^{\mathrm{b}}$ The yield was determined by GC using anisole as an internal standard, N.R. = no reaction. ${ }^{\mathrm{c}}$ Added $120 \mathrm{mg} \mathrm{NPC} .{ }^{\mathrm{d}}$ Under $\mathrm{N}_{2}$ atmosphere. ${ }^{\mathrm{e}}$ Without adding $\mathrm{H}_{2} \mathrm{O}$ and using anhydrous $\mathrm{MeCN}(3 \mathrm{~mL})$ as solvent. 
Supplementary Table 2 | Structure parameters extracted from the EXAFS fitting ${ }^{\mathrm{a}}$.

\begin{tabular}{|c|c|c|c|c|c|c|c|}
\hline Sample & Edge & Shell & $\mathbf{C N}$ & $\mathbf{R}(\AA ̊)$ & $\begin{array}{l}\Delta \mathbf{E}_{0} \\
(\mathrm{eV})\end{array}$ & $\begin{array}{c}\sigma^{2 * 10^{3}} \\
\left(\AA^{2}\right)\end{array}$ & R-factor \\
\hline Co foil & Co K-edge & $\mathrm{Co}-\mathrm{Co}$ & 12.0 & 2.49 & 8.3 & 6.1 & 0.002 \\
\hline $\mathrm{CoPc}$ & Co K-edge & $\mathrm{Co}-\mathrm{N}$ & 4.0 & 1.91 & 1.8 & 1.3 & 0.05 \\
\hline $\mathrm{Zn}$ foil & Zn K-edge & $\mathrm{Zn}-\mathrm{Zn}$ & 6.0 & 2.64 & -2.5 & 13.7 & 0.03 \\
\hline $\mathrm{ZnPc}$ & Zn K-edge & $\mathrm{Zn}-\mathrm{N}$ & 4.0 & 1.97 & 4.2 & 3.5 & 0.02 \\
\hline Co-N ${ }_{4} @ N P C-700$ & Co K-edge & Co-N & 3.2 & 1.89 & -5.2 & 0.6 & 0.004 \\
\hline Co-N ${ }_{4} @ N P C-800$ & Co K-edge & $\mathrm{Co}-\mathrm{N}$ & 3.4 & 1.89 & -5.2 & 3.4 & 0.005 \\
\hline \multirow{2}{*}{ Co-N $4 @$ NPC-900 } & Co K-edge & $\mathrm{Co}-\mathrm{N}$ & 3.6 & 1.98 & -3.5 & 10.0 & 0.03 \\
\hline & Zn K-edge & $\mathrm{Zn}-\mathrm{N}$ & 4.2 & 2.01 & -1.0 & 10.2 & 0.003 \\
\hline Co-N4@NPC-1000 & Co K-edge & Co-N & 3.8 & 1.94 & -6.0 & 10.3 & 0.007 \\
\hline \multirow{2}{*}{ Co-N $4 @$ NPC-re (fresh) } & Co K-edge & Co-N & 4.1 & 1.97 & -4.3 & 4.3 & 0.02 \\
\hline & Zn K-edge & $\mathrm{Zn}-\mathrm{N}$ & 4.0 & 2.01 & -0.6 & 10.4 & 0.004 \\
\hline \multirow{2}{*}{ Co-N4@NPC-re (used) } & Co K-edge & Co-N & 4.0 & 2.01 & -2.0 & 3.7 & 0.02 \\
\hline & Zn K-edge & $\mathrm{Zn}-\mathrm{N}$ & 4.0 & 2.01 & -0.9 & 8.2 & 0.004 \\
\hline Co-NPs@NPC & Co K-edge & $\mathrm{Co}-\mathrm{Co}$ & 9.8 & 2.50 & -3.6 & 6.3 & 0.003 \\
\hline $\mathrm{CoPc} / \mathrm{PC}$ & Co K-edge & Co-N & 4.2 & 1.96 & -0.1 & 1.1 & 0.05 \\
\hline $\mathrm{CoPc} / \mathrm{NPC}$ & Co K-edge & $\mathrm{Co}-\mathrm{N}$ & 4.3 & 1.96 & -0.1 & 1.1 & 0.04 \\
\hline $\mathrm{Co}-\mathrm{N}_{4} / \mathrm{NPC}$ & Co K-edge & Co-N & 4.0 & 2.03 & -1.5 & 9.7 & 0.01 \\
\hline NPC & Zn K-edge & $\mathrm{Zn}-\mathrm{N}$ & 3.5 & 2.01 & 0.2 & 8.2 & 0.004 \\
\hline
\end{tabular}

${ }^{a} \mathrm{R}$ : absorber-backscatterer distance; $\mathrm{CN}$ : coordination number; $\Delta \mathrm{E}_{0}$ : inner potential correction, $\sigma^{2}$ : DebyeWaller factor. Error bounds (accuracies) that characterize the structural parameters obtained by EXAFS spectroscopy were estimated as $\mathrm{CN} \pm 20 \% ; \mathrm{R} \pm 1 \% ; \Delta \mathrm{E}_{0} \pm 20 \% ; \sigma^{2} \pm 20 \%$. 
Supplementary Table 3 | Kinetic isotope effect study for oxidation of 1a over Co-N $\mathrm{N}_{4} @$ NPC-re catalyst.

\begin{tabular}{ccccc}
\hline Entry $^{\mathbf{a}}$ & Silane & Water & TOF $\left(\mathbf{h}^{-1}\right)^{\mathbf{b}}$ & KIE $^{\mathbf{c}}$ \\
\hline 1 & $\mathrm{PhMe}_{2} \mathrm{SiH}$ & $\mathrm{H}_{2} \mathrm{O}$ & $595 \pm 34$ & - \\
2 & $\mathrm{PhMe}_{2} \mathrm{SiD}$ & $\mathrm{H}_{2} \mathrm{O}$ & $256 \pm 12$ & $2.32 \pm 0.17$ \\
3 & $\mathrm{PhMe}_{2} \mathrm{SiH}$ & $\mathrm{D}_{2} \mathrm{O}$ & $572 \pm 30$ & $1.04 \pm 0.08$ \\
\hline
\end{tabular}

${ }^{\text {a }}$ Reaction conditions: silane (1 mmol), water (8 mmol), Co- $\mathrm{N}_{4} @$ NPC-re (Co $\left.0.5 \mathrm{~mol} \%\right), \mathrm{MeCN}(3 \mathrm{~mL}), 30$ ${ }^{\circ} \mathrm{C}$, air atmosphere. ${ }^{\mathrm{b}}$ TOF was measured at $1 \mathrm{a}$ conversion below $20 \%$ based on total Co atoms. ${ }^{\mathrm{c}} \mathrm{KIE}=\mathrm{TOF}$ (entry 1)/TOF (entry 2 ) or TOF (entry 1 )/TOF (entry 3 ).

Supplementary Table 4 | Elemental quantification (at \%) determined by XPS for different Co catalysts.

\begin{tabular}{|c|c|c|c|c|c|}
\hline Sample & $\mathrm{C}(\mathrm{at} \%)$ & N (at\%) & $\mathrm{O}(\mathbf{a t} \%)$ & Co $(\operatorname{at} \%)$ & $\mathrm{Zn}(\mathbf{a t} \%)$ \\
\hline Co-N $4 @ N P C-700$ & 76.3 & 15.7 & 6.4 & 0.1 & 1.5 \\
\hline Co-N ${ }_{4} @ N P C-800$ & 78.6 & 14.1 & 5.9 & 0.1 & 1.3 \\
\hline Co-N4@NPC-900 & 80.5 & 12.5 & 6.0 & 0.2 & 0.8 \\
\hline Co-N $4 @$ NPC-1000 & 90.3 & 5.6 & 3.7 & 0.2 & 0.2 \\
\hline Co-N $4 @$ NPC-re (fresh) & 83.7 & 9.8 & 5.7 & 0.3 & 0.5 \\
\hline Co-N4@@NPC-re (used) & 84.1 & 9.7 & 5.4 & 0.3 & 0.5 \\
\hline
\end{tabular}


Supplementary Table $5 \mid$ The total nitrogen content and percentage of different nitrogen species in different Co catalysts derived from XPS analysis.

\begin{tabular}{ccccccc}
\hline Sample & $\begin{array}{c}\text { N content } \\
\mathbf{a t \% )}\end{array}$ & $\begin{array}{c}\text { Pyridinic } \\
\mathbf{( \% )}\end{array}$ & $\begin{array}{c}\text { M-N } \\
\mathbf{( \% )}\end{array}$ & $\begin{array}{c}\text { Pyrrolic } \\
\mathbf{( \% )}\end{array}$ & $\begin{array}{c}\text { Graphitic } \\
\mathbf{( \% )}\end{array}$ & $\begin{array}{c}\text { N-oxide } \\
\mathbf{( \% )}\end{array}$ \\
\hline Co-N $@$ @NPC-700 & 15.7 & 27.0 & 38.4 & 17.7 & 13.4 & 3.5 \\
Co-N4@NPC-800 & 14.1 & 35.4 & 32.3 & 16.6 & 11.1 & 4.6 \\
Co-N4@NPC-900 & 12.5 & 41.2 & 24.0 & 19.3 & 11.5 & 4.0 \\
Co-N4@NPC-1000 & 5.6 & 37.0 & 22.2 & 16.3 & 19.4 & 5.1 \\
Co-N $@$ NPC-re (fresh) & 9.8 & 37.2 & 14.6 & 9.8 & 28.6 & 9.8 \\
Co-N $@$ NPC-re (used) & 9.7 & 39.8 & 13.6 & 8.7 & 28.4 & 9.5 \\
\hline
\end{tabular}

Supplementary Table 6 | ICP-OES results of metal loading amount of different Co- $\mathrm{N}_{4} @$ NPC-based catalysts.

\begin{tabular}{|c|c|c|}
\hline Sample & Co $(w t \%)$ & $\operatorname{Zn}(w t \%)$ \\
\hline Co-N ${ }_{4} @ N P C-700$ & 1.31 & 10.55 \\
\hline Co-N ${ }_{4} @ N P C-800$ & 1.57 & 7.88 \\
\hline Co-N ${ }_{4} @ N P C-900$ & 1.93 & 4.18 \\
\hline Co-N $4 @$ NPC-1000 & 2.11 & 1.07 \\
\hline Co-NPs@NPC & 15.23 & 0.08 \\
\hline Co-N $4 @$ NPC-re (fresh) & 2.75 & 4.10 \\
\hline Co-N ${ }_{4} @$ NPC-re (used) & 2.74 & 4.02 \\
\hline $\mathrm{Co}-\mathrm{N}_{4} / \mathrm{NPC}$ & 0.32 & 1.28 \\
\hline
\end{tabular}


Supplementary Table 7 | TEM-EDS element analysis of Co- $\mathrm{N}_{4} @ N P C$ and Co-N 4 @NPC-re catalysts.

\begin{tabular}{ccccc}
\hline \multirow{2}{*}{ Element } & \multicolumn{2}{c}{$\mathbf{C o N}_{\mathbf{4}} @ \mathbf{N P C - 9 0 0}$} & \multicolumn{2}{c}{$\mathbf{C o N}_{\mathbf{4}} @ \mathbf{N P C}-\mathbf{r e}$} \\
\cline { 2 - 5 } & Atomic\% & $\mathbf{w t \%}$ & Atomic\% & $\mathbf{w t \%}$ \\
\hline $\mathrm{C}$ & 89.06 & 83.15 & 90.25 & 84.97 \\
$\mathrm{~N}$ & 6.29 & 6.85 & 5.97 & 6.50 \\
$\mathrm{O}$ & 3.51 & 4.36 & 2.82 & 3.51 \\
$\mathrm{Co}$ & 0.33 & 1.50 & 0.49 & 2.26 \\
$\mathrm{Zn}$ & 0.81 & 4.14 & 0.54 & 2.76 \\
\hline
\end{tabular}

Supplementary Table 8 | Reaction condition optimization for oxidation of 1a over Co- $\mathrm{N}_{4} @ \mathrm{NPC}-900$ catalyst.

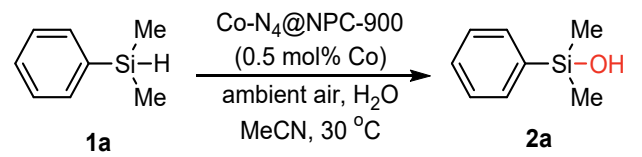

\begin{tabular}{cccccc}
\hline Entry $^{\mathbf{a}}$ & $\mathbf{V}_{\mathbf{M e C N}}(\mathbf{m L})$ & $\mathbf{V}_{\text {water }}(\boldsymbol{\mu L})$ & $\mathbf{n}_{\mathbf{w a t e r}} / \mathbf{n}_{\mathbf{1 a}}$ & $\mathbf{c}_{\mathbf{1 a}}(\mathbf{m o l} / \mathbf{L})$ & Yield of 2a (\%) \\
\hline 1 & 1 & 145 & 8 & 1.0 & 54 \\
2 & 2 & 145 & 8 & 0.5 & 62 \\
3 & 3 & 145 & 8 & 0.33 & 71 \\
4 & 4 & 145 & 8 & 0.25 & 45 \\
5 & 5 & 145 & 8 & 0.20 & 38 \\
6 & 3 & 0 & 0 & 0.33 & N.R. \\
7 & 3 & 55 & 3 & 0.33 & 40 \\
8 & 3 & 90 & 5 & 0.33 & 62 \\
9 & 3 & 200 & 11 & 0.33 & 46 \\
10 & 3 & 270 & 15 & 0.33 & 41 \\
\hline
\end{tabular}

${ }^{a}$ Reaction conditions: $1 \mathrm{a}(1 \mathrm{mmol}), \mathrm{Co}-\mathrm{N}_{4} @ \mathrm{NPC}-900$ (Co $\left.0.5 \mathrm{~mol} \%\right), 30^{\circ} \mathrm{C}$, air atmosphere, $20 \mathrm{~min}, \mathrm{~N} . \mathrm{R} .=$ no reaction. ${ }^{\mathrm{b}}$ The yield was determined by GC using anisole as an internal standard. 
Supplementary Table 9| $\mathrm{N}_{2}$ physisorption characteristics of different Co-N4@NPC-based catalysts.

\begin{tabular}{ccccccc}
\hline Sample & $\begin{array}{c}\mathbf{S}_{\text {BET }} \\
\left(\mathbf{m}^{2} / \mathbf{g}\right)\end{array}$ & $\begin{array}{c}\mathbf{S}_{\text {Micro }} \\
\left(\mathbf{m}^{2} / \mathbf{g}\right)\end{array}$ & $\begin{array}{c}\mathbf{S}_{\text {Exter }} \\
\left(\mathbf{m}^{2} / \mathbf{g}\right)\end{array}$ & $\begin{array}{c}\mathbf{V}_{\text {Total }} \\
\left(\mathbf{c m}^{3} / \mathbf{g}\right)\end{array}$ & $\begin{array}{c}\text { t-plot } \\
\left(\mathbf{c m}_{\text {Micro }} / \mathbf{g}\right)\end{array}$ & $\begin{array}{c}\mathbf{V}_{\text {Meso }} \\
\left(\mathbf{c m}^{3} / \mathbf{g}\right)\end{array}$ \\
\hline $\mathrm{CoN}_{4} @ \mathrm{NPC}-700$ & 756 & 546 & 210 & 1.10 & 0.29 & 0.81 \\
$\mathrm{CoN} 4$ @NPC-800 & 729 & 493 & 236 & 1.14 & 0.26 & 0.88 \\
$\mathrm{CoN}_{4} @ \mathrm{NPC}-900$ & 842 & 600 & 242 & 1.27 & 0.31 & 0.96 \\
$\mathrm{CoN}_{4} @ \mathrm{NPC}-1000$ & 804 & 545 & 259 & 1.35 & 0.29 & 1.06 \\
$\mathrm{CoN}_{4} @ \mathrm{NPC}-\mathrm{re}$ & 636 & 411 & 225 & 1.24 & 0.18 & 1.06 \\
\hline
\end{tabular}

Supplementary Table 10 | Raman fitting results of Co-N 4 @NPC catalysts with different pyrolysis temperature and synthesis steps.

\begin{tabular}{cccccc}
\hline Sample & I peak\% & D peak\% & D” peak\% & G peak\% & Area $_{\mathbf{D}} /$ Area $_{\mathbf{G}}$ \\
\hline CoN 4 @NPC-700 & 9.4 & 39.9 & 25.4 & 25.3 & 1.58 \\
CoN 4 @NPC-800 & 10.9 & 37.8 & 25.3 & 26.0 & 1.45 \\
CoN 4 @NPC-900 & 8.6 & 35.7 & 19.8 & 26.6 & 1.34 \\
$\mathrm{CoN}_{4} @ \mathrm{NPC}-1000$ & 16.6 & 35.0 & 18.5 & 29.9 & 1.17 \\
$\mathrm{CoN}_{4} @ \mathrm{NPC}-\mathrm{re}$ & 19.9 & 34.8 & 21.1 & 24.1 & 1.44 \\
\hline
\end{tabular}


Supplementary Table 11 | Reaction condition optimization for Co- $\mathrm{N}_{4} @$ NPC-re catalyzed oxidation of $1 \mathbf{a}$ and 3g.

\begin{tabular}{|c|c|c|c|c|}
\hline Entry $^{a}$ & 1a (equiv.) & solvent & Time (h) & Yield of $4 \mathrm{k}(\%)^{\mathrm{b}}$ \\
\hline 1 & 1 & $n$-heptane & 1 & 74 \\
\hline 2 & 1 & $n$-heptane & 1.5 & 74 \\
\hline 3 & 1.5 & $n$-heptane & 1.5 & 87 \\
\hline 4 & 1.5 & $n$-heptane & 2 & 91 \\
\hline 5 & 2 & $n$-heptane & 1 & 93 \\
\hline 6 & 2 & $n$-heptane & 1.5 & 97 \\
\hline 7 & 2 & $n$-heptane & 2 & 98 \\
\hline 8 & 3 & $n$-heptane & 3 & 99 \\
\hline $9^{c}$ & 2 & $n$-heptane & 3 & N.R. \\
\hline 10 & 2 & toluene & 1.5 & 68 \\
\hline 11 & 2 & toluene & 2 & 68 \\
\hline 12 & 3 & toluene & 1.5 & 78 \\
\hline 13 & 3 & toluene & 2 & 85 \\
\hline 14 & 2 & acetone & 2 & 16 \\
\hline 15 & 2 & THF & 2 & 16 \\
\hline 16 & 2 & EtOAc & 2 & 31 \\
\hline
\end{tabular}

${ }^{a}$ Reaction conditions: 3g (1 mmol), Co- $\mathrm{N}_{4} @ N P C-r e(C o 0.5 \mathrm{~mol} \%), 30{ }^{\circ} \mathrm{C}$, air atmosphere, N.R.= no reaction. ${ }^{\mathrm{b}}$ The yield was calculated based on $\mathbf{3 g}$ and determined by $\mathrm{GC}$ using anisole as an internal standard. ${ }^{\mathrm{c}}$ Under $\mathrm{N}_{2}$ atmosphere. 


\section{Supplementary Discussion 1 | Structure identification of Co-N $\mathbf{N}_{4} @$ NPC-re}

XAFS measurements were carried out to verify the atomic dispersion and precise coordination structure of Co and Zn species in Co-N ${ }_{4} @$ NPC-re. As shown in Supplementary Fig. 25, only one intensity maximum peak located at approximately $1.4 \AA$ was found in FT-EXAFS curve of Co K-edge in $\mathrm{Co}_{\mathrm{N}} \mathrm{N}_{4} @$ NPC-re, which originates from the interactions between Co and light atoms. This signal was attributed to the contribution of Co-N coordination. In comparison with the Co foil reference, which displayed a main peak at approximately $2.2 \AA$ assigned to the Co-Co bond, it was clear that this metallic interaction was not present in Co-N $\mathrm{N}_{4} @ \mathrm{NPC}-\mathrm{re}$ sample, indicating that the Co species in Co-N 4 @NPC-re sample are atomically dispersed. The same observation can be made in FT-EXAFS curve of Zn K-edge in Co- $\mathrm{N}_{4} @ \mathrm{NPC}$-re with the intensity maximum peak located at $1.5 \AA$ (Supplementary Fig. 26), which indicated the existence of $\mathrm{Zn}-\mathrm{N}$ coordination. Furthermore, the WT plots of Co and Zn K-edge EXAFS spectra both detect only one intensity maximum at approximately $5.0 \AA^{-1}$, which are similar to those in the WT plots of corresponding metal phthalocyanines (Supplementary Figs. 25 and 26), providing unequivocal support for the existence of Co$\mathrm{N}$ and $\mathrm{Zn}-\mathrm{N}$ scattering paths. The fitting results of EXAFS data further suggested that first shell coordination number of Co-N and $\mathrm{Zn}-\mathrm{N}$ are 4.1 and 4.0, respectively (Supplementary Table 2), revealing the metal species in $\mathrm{Co}_{-} \mathrm{N}_{4} @ \mathrm{NPC}-\mathrm{re}$ are $\mathrm{Co}-\mathrm{N}_{4}$ and $\mathrm{Zn}-\mathrm{N}_{4}$ sites.

To further identify the geometric configuration of Co- $\mathrm{N}_{4}$ sites in Co- $\mathrm{N}_{4} @ \mathrm{NPC}$-re, we calculated XANES spectra of various Co-N-C structures with different coordination geometries (Supplementary Figs. 41 and 42). It turned out that the Co- $\mathrm{N}_{4}$ sites in the pyridinic coordination environment (labeled as $\mathrm{CoN}_{4} \mathrm{C}_{10}$ ) could well reproduce the main features of the experimental spectrum. Furthermore, adding an oxygen molecule axial adsorbed on Co center (labeled as $\mathrm{CoN}_{4} \mathrm{C}_{10}-\mathrm{O}_{2}$ ) caused $\mathrm{I}_{\mathrm{b}} / \mathrm{I}_{\mathrm{c}}$ ratios in the theoretical and experimental spectra to become more similar and displaying better agreement over the entire XANES energy range. Such structure was also reported in the literatures for Co-N-C catalyst which synthesized using similar ZIF precursors ${ }^{7}$. It was reported that due to the weak interaction of $\mathrm{O}_{2}$ and $\mathrm{Co}-\mathrm{N}_{4}$ site, the adsorbed $\mathrm{O}_{2}$ molecule is not very stable ${ }^{8}$. Such $\mathrm{CoN}_{4} \mathrm{C}_{10}$ and $\mathrm{CoN}_{4} \mathrm{C}_{10}-\mathrm{O}_{2}$ structures are interconvertible when the sample is placed under different atmosphere $\left(\mathrm{N}_{2}\right.$ or $\left.\mathrm{O}_{2}\right)$. Thus, it is highly likely that in the real sample these two kinds of structure are co-exist and in dynamic equilibrium. 


\section{Supplementary Discussion 2 | Re-doping approach for increasing Co- $\mathbf{N}_{4}$ sites.}

As shown in XPS and EDS element analysis (Supplementary Tables 4 and 7), in Co- $\mathrm{N}_{4} @$ NPC, the content of pyridinic-N sites is much higher than that of Co- $\mathrm{N}_{4}$ sites. Thus, we tried to introduce more Co- $\mathrm{N}_{4}$ sites into Co- $\mathrm{N}_{4} @ \mathrm{NPC}$ to balance the Co- $\mathrm{N}_{4} /$ pyridinic-N ratio in catalyst for further improving the catalytic activity. Inspired by the recent advances of ORR catalyst in PEMFCs ${ }^{9}$, we developed a tandem ammonialeaching and adsorption approach for re-doping Co- $\mathrm{N}_{4}$ sites in Co- $\mathrm{N}_{4} @ \mathrm{NPC}$ matrix (Supplementary Fig. 4). In brief, $\mathrm{Co}-\mathrm{N}_{4} @ \mathrm{NPC}-\mathrm{NL}$ was first treated $\mathrm{NH}_{3} / \mathrm{NH}_{4} \mathrm{Cl}$ aqueous solution. Then the obtained sample was used as a host to adsorb Co phenanthroline complex $\left(\mathrm{Co}(\mathrm{Phen})_{3}\right)$ to form $\mathrm{Co}-\mathrm{N}_{4} @ \mathrm{NPC} / \mathrm{Co}(\mathrm{Phen})_{3}$. During the following pyrolysis step, $\mathrm{Co}(\mathrm{Phen})_{3}$ transformed to $\mathrm{Co}-\mathrm{N}_{4}$ active sites and doped into porous carbon matrix to generate Co-N $\mathrm{N}_{4} @ \mathrm{NPC}-\mathrm{re}$. The density of Co atoms is approximately $0.44 \mathrm{Co}$ atom $/ \mathrm{nm}^{2}$ (calculated based on BET surface area and loading of Co) in Co- $\mathrm{N}_{4} @$ NPC-re, which is much higher than that of Co$\mathrm{N}_{4} @ \mathrm{NPC}\left(0.24 \mathrm{Co}\right.$ atom $\left./ \mathrm{nm}^{2}\right)$. Catalytic performance evaluation showed that re-doping approach leads to significantly enhanced activity for Co- $\mathrm{N}_{4} @ \mathrm{NPC}$ sample (Supplementary Figs. 15 and 16). Considering the comparable N dopant and carbon structure (Supplementary Tables 7 and 10) of Co-N 4 @NPC-re and Co$\mathrm{N}_{4} @ \mathrm{NPC}$, the enhanced performance may originate from the optimization of $\mathrm{Co}-\mathrm{N}_{4} /$ pyridinic-N ratio caused by the increase of Co- $\mathrm{N}_{4}$ sites concentration. It should be pointed out that although the re-doping approach has been proved to be an effective means to optimize the Co- $\mathrm{N}_{4} /$ pyridinic-N ratio in sample, the content of pyridinic-N sites in Co- $\mathrm{N}_{4} @$ NPC-re is still excess, suggesting there is still room to further optimize catalyst structure. The possible ways include change solvent during adsorption step, use different Co complexes as Co precursor, and introduce more nanopores in carbon host, etc. These are under investigation now. 


\section{Supplementary Discussion 3 | The elimination of mass transfer limitation}

To establish the influence of external and internal mass transfer limitations, the effect of agitation speed and particle size on initial reaction rates $r_{a}$ was examined. Supplementary Fig. 52A shows that $r_{a}$ is independent of stirring speed in the range of 800-1200 rpm, which indicates external diffusion limitation is absent when the stirring speed falls within this range. Supplementary Fig. 52B shows that internal diffusion limitation is negligible for particles smaller than $75 \mu \mathrm{m}$. Therefore, the catalytic activity testing experiments in this work were carried out at a stirring speed of $1000 \mathrm{rpm}$ with catalyst particles smaller than $45 \mu \mathrm{m}$ (the catalyst was passed through 325 mesh sifting screen before used) to avoid both external and internal mass transfer limitations.

The importance of internal (intraparticle) diffusion was further evaluated by Weisz-Prater criterion ${ }^{10}$ :

$$
\Phi=\frac{r_{a} \rho_{p} L^{2}}{C_{s, i} D_{e, i}}=\eta \varphi_{i}^{2}
$$

where $r_{a}=$ observed initial reaction rate per unit mass of catalyst, $\mathrm{mol} / \mathrm{kg} \cdot \mathrm{s}$

$\rho_{p}=$ density of catalyst, $\mathrm{kg} / \mathrm{m}^{3}$

$L=$ characteristic length of catalyst particle, $\mathrm{m}$

$C_{s, i}=$ surface reactant concentration of specie $i, \mathrm{~mol} / \mathrm{m}^{3}$

$D_{e, i}=$ effective liquid phase diffusivity of specie $i, \mathrm{~m}^{2} / \mathrm{s}$

If $\Phi \leq 0.3$, the internal diffusion effect can be neglected.

In the current work, the shape of catalyst particle was assumed as sphere and particle size $d_{p}$ was less than $45 \mu \mathrm{m}$, thus $L=d_{p} / 6=7.5 \mu \mathrm{m}$. For Co-N $4 @$ NPC-900 catalyst, $\rho_{p}=250 \mathrm{~kg} / \mathrm{m}^{3}$ and $r_{a}=$ $4.45 \times 10^{-2} \mathrm{~mol} / \mathrm{kg}_{\text {cat }}$ s. A series of correlations ${ }^{11}$ were used to estimate the diffusion coefficients $D$ of $\mathbf{1 a}$, $\mathrm{H}_{2} \mathrm{O}$ and $\mathrm{O}_{2}$. $D_{e, i}$ was further calculated by the expression derived by Ternan ${ }^{12}$ :

$$
D_{e, i}=D_{i} \frac{(1-\lambda)^{2}}{1+P \lambda}
$$

where $D_{i}=$ diffusion coefficients of specie $i, \mathrm{~m}^{2} / \mathrm{s}$

$\lambda=$ the ratio of diffusing molecule radius to average pore radius of the catalyst, $r_{\text {molecule }} / r_{\text {pore }}$

$P=$ fitting parameter

$P$ is an empirical constant which is determined individually for catalysts. Based on the report of Massoth ${ }^{13}$, the value of $P$ was calculated to be 11.0 , which was employed in this work to estimate $D_{e, i}$ in the Co$\mathrm{N}_{4} @$ NPC-900 catalyst. The average pore radius of Co- $\mathrm{N}_{4} @ \mathrm{NPC}-900$ sample analyzed by BJH method from $\mathrm{N}_{2}$ adsorption-desorption isotherm was $8.9 \mathrm{~nm}$.

When $1 \mathbf{a}$ was considered as the diffusing reactant, the diffusivity of $\mathbf{1 a}$ in $\mathrm{H}_{2} \mathrm{O}$ and $\mathrm{MeCN}$ mixture can be estimated using eq 1-4.

$$
\begin{gathered}
D_{1 \boldsymbol{a} / \text { mix }}=\frac{\left(D_{\left.1 a / H_{2} O^{0} \eta_{H_{2} O}^{0.5}\right)^{x_{H_{2} O} O}\left(D_{1 / M e C N}^{0} \eta_{M e C N}^{0.5}\right.}\right)^{x_{M e C N}}}{\eta_{\text {mix }}^{0.5}} \\
D_{1 a / M e C N}^{0}=4.4 \times 10^{-15} \frac{T}{\eta_{M e C N}}\left(\frac{V_{M e C N}}{V_{1 a}}\right)^{1 / 6}\left(\frac{L_{M e C N}^{v a p}}{L_{1 a}^{v a d}}\right)^{1 / 2} \\
D_{1 a / H_{2} O}^{0}=\frac{8.621 \times 10^{-14}}{\eta_{H_{2} O}^{1.14} V_{1 a}^{0.589}} \\
\eta_{\text {mix }}^{0}=\eta_{M e C N}^{x_{M e C N}} \eta_{H_{2} O}^{x_{H_{2} O} O}
\end{gathered}
$$

Due to the low mole fraction of $1 \mathrm{a}$ in reaction solution $(1.5 \mathrm{~mol} \%)$, the influence of $1 \mathrm{a}$ to $D_{\mathrm{H}_{2} \mathrm{O}}$ and $D_{\mathrm{O}_{2}}$ can be neglected. The diffusivity of $\mathrm{H}_{2} \mathrm{O}$ in $\mathrm{MeCN}$ was estimated using eq 4-7. 


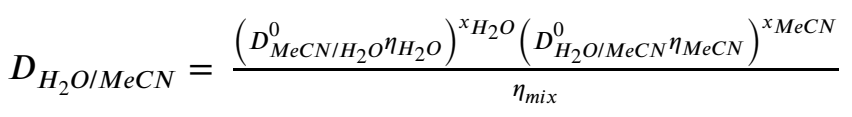

$$
\begin{aligned}
& D_{\mathrm{H}_{2} \mathrm{O} / \mathrm{MeCN}}^{0}=4.4 \times 10^{-15} \frac{T}{\eta_{M e C N}}\left(\frac{V_{M e C N}}{V_{\mathrm{H}_{2} \mathrm{O}}}\right)^{1 / 6}\left(\frac{L_{M e C N}^{v a p}}{L_{\mathrm{H}_{2} \mathrm{O}}^{\text {vap }}}\right)^{1 / 2} \\
& D_{\mathrm{MeCN} / \mathrm{H}_{2} \mathrm{O}}^{0}=\frac{8.621 \times 10^{-14}}{\eta_{\mathrm{H}_{2} \mathrm{O}}^{1.14} V_{\mathrm{MeCN}}^{.0589}}
\end{aligned}
$$

The diffusivity of $\mathrm{O}_{2}$ in $\mathrm{H}_{2} \mathrm{O}$ and $\mathrm{MeCN}$ mixture was estimated using eq 4, 8-10.

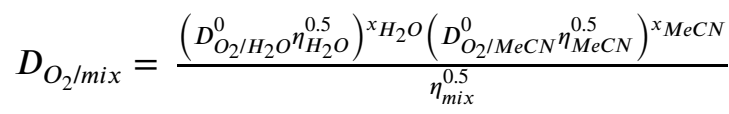

$$
\begin{aligned}
& D_{O_{2} / M e C N}^{0}=1.1728 \times 10^{-16} \frac{T \sqrt{\chi_{M e C N} M_{M e C N}}}{\eta_{M e C N} V_{O_{2}}^{0.6}} \\
& D_{\mathrm{O}_{2} / \mathrm{H}_{2} \mathrm{O}}^{0}=1.1728 \times 10^{-16} \frac{T \sqrt{\chi_{\mathrm{H}_{2} \mathrm{O}} \mathrm{M}_{\mathrm{H}_{2} \mathrm{O}}}}{\eta_{\mathrm{H}_{2} \mathrm{O}} V_{O_{2}}^{0.6}}
\end{aligned}
$$

The definitions of the quantities involved in eq 1-10 are listed as following:

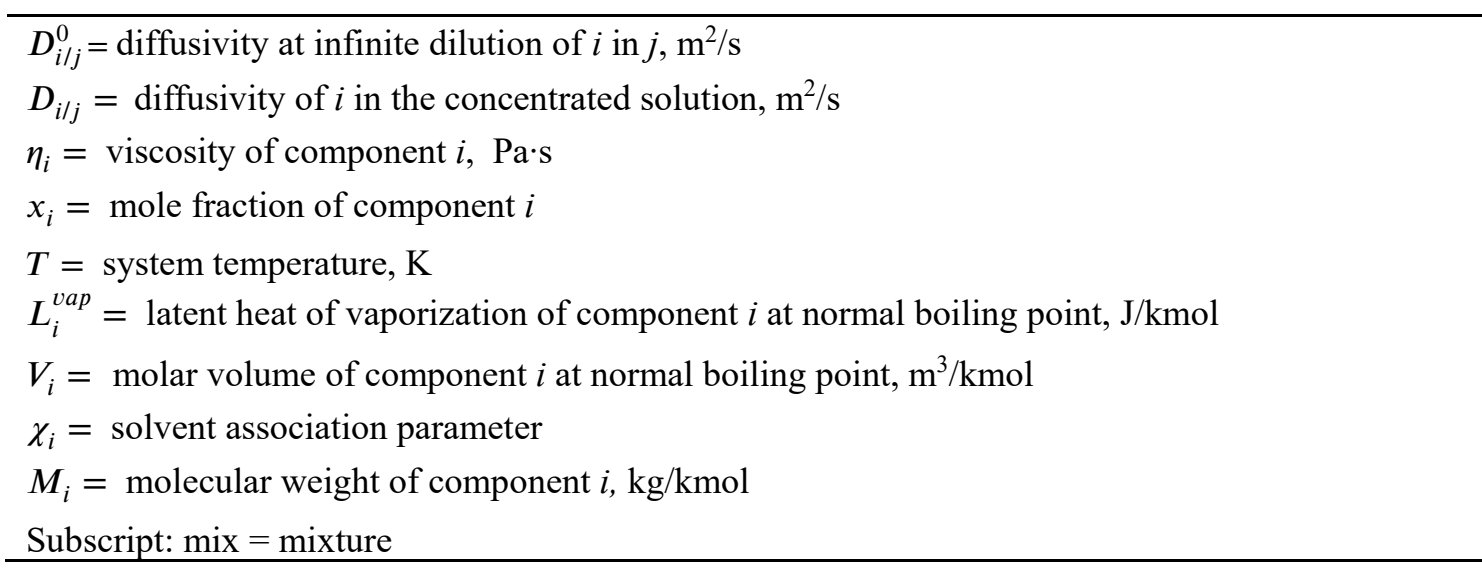

The properties of the solute and solvents used in calculations are given in Supplementary table 12 ${ }^{14-16}$.

Supplementary Table $12 \mid$ Physical properties of 1a, $\mathrm{H}_{2} \mathrm{O}, \mathrm{MeCN}$ and $\mathrm{O}_{2}$

\begin{tabular}{cccccccc}
\hline Component & $\begin{array}{c}r \\
(\mathrm{~nm})\end{array}$ & $\begin{array}{c}M_{i} \\
(\mathrm{~kg} / \mathrm{mol})\end{array}$ & $\begin{array}{c}V_{i} \\
\left(\mathrm{~m}^{3} / \mathrm{kmol}\right)\end{array}$ & $\chi_{i}$ & $\begin{array}{c}\eta_{i} @ 303 \mathrm{~K} \\
\left(\times 10^{-4} \mathrm{~Pa} \cdot \mathrm{s}\right)\end{array}$ & $x_{i}$ & $\begin{array}{c}L_{i}^{\text {vap }} \\
\left(\times 10^{7} \mathrm{~J} / \mathrm{kmol}\right)\end{array}$ \\
\hline $\mathbf{1 a}$ & 0.38 & 136.3 & 0.153 & - & - & - & 3.72 \\
$\mathrm{H}_{2} \mathrm{O}$ & 0.13 & 18.02 & 0.0187 & 2.6 & 8.01 & 0.122 & 4.07 \\
$\mathrm{MeCN}$ & - & 41.06 & 0.0574 & 1 & 3.25 & 0.878 & 3.03 \\
$\mathrm{O}_{2}$ & 0.17 & 32.00 & 0.0280 & - & - & - & - \\
\hline
\end{tabular}

Supplementary Table 13 | $\Phi$ of $1 \mathrm{a}, \mathrm{H}_{2} \mathrm{O}$ and $\mathrm{O}_{2}$ under reaction conditions

\begin{tabular}{cccccc}
\hline Component & $\lambda$ & $\begin{array}{c}C_{s, i} \\
\left(\mathrm{~mol} / \mathrm{m}^{3}\right)\end{array}$ & $\begin{array}{c}D_{i / j} \\
\left(\times 10^{-9} \mathrm{~m}^{2} / \mathrm{s}\right)\end{array}$ & $\begin{array}{c}D_{e, i} \\
\left(\times 10^{-9} \mathrm{~m}^{2} / \mathrm{s}\right)\end{array}$ & $\Phi$ \\
\hline 1a & 0.0427 & 303 & 2.69 & 1.68 & $2.3 \times 10^{-3}$ \\
$\mathrm{O}_{2}$ & 0.0191 & 2.42 & 5.41 & 4.30 & $5.9 \times 10^{-2}$ \\
$\mathrm{H}_{2} \mathrm{O}$ & 0.0146 & 2424 & 3.78 & 3.16 & $1.5 \times 10^{-4}$ \\
\hline
\end{tabular}

As shown in Supplementary table 13, the value of $\Phi$ for each reactant is less than 0.3 , which also proves the absence of mass transfer limitation in Co- $\mathrm{N}_{4} @$ NPC-catalyzed silane oxidation reaction. 


\section{Scopes and Limitations Studies}

\section{General information}

All reactions were carried out under an ambient atmosphere of air using oven-dried glasswares, unless otherwise specified. Anhydrous 2-methyltetrahydrofuran (2-MeTHF), toluene and $n$-heptane were purchased from Sinopharm Chemical Reagent Co., Ltd. and distilled from sodium-benzophenone under $\mathrm{N}_{2}$ atmosphere. Anhydrous MeCN was purchased from Sinopharm Chemical Reagent Co., Ltd. and distilled from $\mathrm{CaH}_{2}$ under $\mathrm{N}_{2}$ atmosphere. Anhydrous alcohols (MeOH, EtOH, $i-\mathrm{PrOH}, n-\mathrm{BuOH}, i-\mathrm{BuOH}$ and $\left.t-\mathrm{BuOH}\right)$ were purchased from J\&K Scientific (SuperDry) and used as received. Other solvents and reagents were purchased at the highest commercial quality and used directly without further purification. Reactions were monitored by GC (Agilent 7820A gas chromatograph with FID detector and $30 \mathrm{~m} \times 0.32 \mathrm{~mm} \times 0.25 \mu \mathrm{m}$ HP-INNOWax capillary column) and/or thin layer chromatography (TLC). TLC was performed using silica gel GF254 precoated TLC plates, using short-wave UV light $\left(254 \mathrm{~nm}\right.$ ) as the visualizing agent, and $\mathrm{I}_{2}$, or $\mathrm{KMnO}_{4}$ and heating as developing agents. All work-up and purification procedures were carried out with reagent grade solvents. Flash column chromatography was preformed using 300-400 mesh silica gel (Huanghai, Shandong) or 200-300 mesh neutral alumina (Aladdin, Shanghai) as stationary phase. NMR spectra were recorded on Bruker AVANCE III HD 400 and AVANCE NEO 400 instruments and were calibrated using residual undeuterated solvent (chloroform at $7.26 \mathrm{ppm}{ }^{1} \mathrm{H}$ NMR, $77.00 \mathrm{ppm}{ }^{13} \mathrm{C} \mathrm{NMR}$; acetone at $2.05 \mathrm{ppm}{ }^{1} \mathrm{H} \mathrm{NMR}$, $29.84 \mathrm{ppm}{ }^{13} \mathrm{C}$ NMR; DMSO at $2.50 \mathrm{ppm}{ }^{1} \mathrm{H}$ NMR, $39.52 \mathrm{ppm}{ }^{13} \mathrm{C}$ NMR). The following abbreviations (or combinations thereof) were used to explain multiplicities: $\mathrm{s}=$ singlet, $\mathrm{d}=$ doublet, $\mathrm{t}=$ triplet, $\mathrm{q}=$ quartet, hept $=$ heptet, $\mathrm{m}=$ multiplet, $\mathrm{br}=$ broad. Coupling constants, $J$, were reported in Hertz unit $(\mathrm{Hz})$. Highresolution mass spectra (HRMS) were recorded on a Bruker MicrOTOF II mass spectrometer by ESI-TOF (electrospray ionization-time of flight) or APCI-TOF (atmospheric pressure chemical ionization-time of flight) experiments. The enantiomeric excess (ee) of chiral compounds was determined on an Agilent 1260 Infinity II LC system using Daicel CHIRALPAK IB analytic column. Optical rotations were recorded on a Rudolph Autopol IV automatic polarimeter.

\section{Preparation of silane substrates}

\section{-List of silane substrates}<smiles>[14CH3][Si]([14CH3])([Mg])c1ccccc1</smiles>

1a

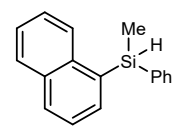

1b<smiles></smiles>

$R-1 \mathrm{~b}$

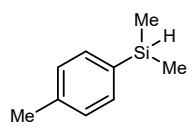

$1 c$

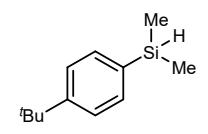

$1 d$<smiles>C[N+](C)(C)c1ccc([N+]([N-])=O)cc1</smiles>

$1 \mathrm{e}$<smiles>C[N+](C)(C)c1ccc(F)cc1</smiles>

$1 f$<smiles>[14CH3][Si]([14CH3])([Mg])c1ccc(Cl)cc1</smiles>

$1 \mathrm{~g}$<smiles>C[Si](C)([Na])c1ccc(Br)cc1</smiles>

$1 \mathrm{~h}$<smiles>CC(C)(C)c1ccc(Br)cc1</smiles>

$1 i$<smiles>COc1ccc([N+](C)(C)C)cc1</smiles>

1j

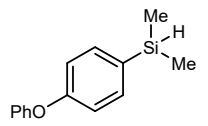

$1 k$

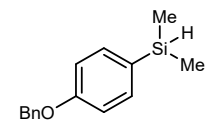<smiles>C[N+](C)(C)c1ccc(Oc2ccccc2)cc1</smiles>

$1 \mathrm{~m}$<smiles>[13CH3][Si]([14CH3])([14CH3])c1ccc(C#N)cc1</smiles>

1n<smiles>CN(C)c1ccc(N(C)C)cc1</smiles>

10<smiles>Cc1ccc(N(C)C)cc1</smiles>

$1 p$<smiles>CN(C)c1ccc(N(C)C)cc1</smiles>

19<smiles>CN(C)c1ccc(N(C)C)cc1</smiles>

$1 \mathrm{r}$

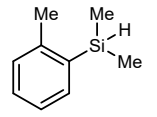

1s

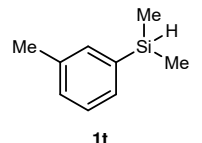



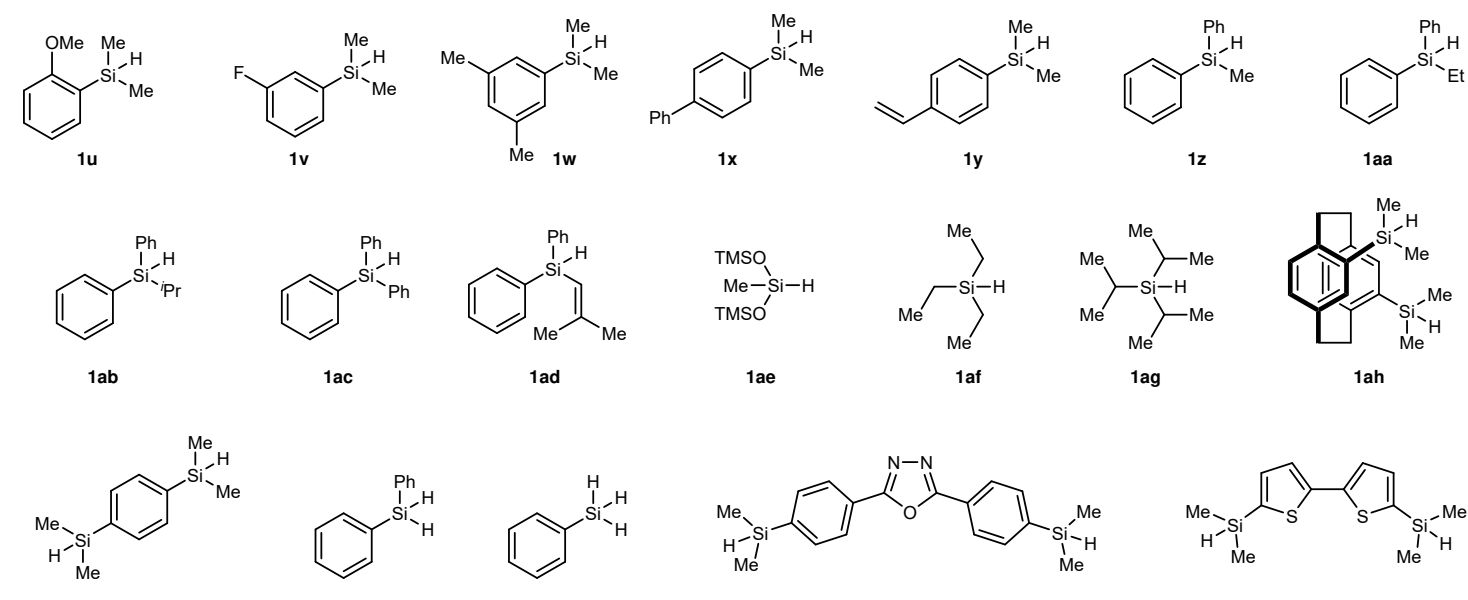

1aj<smiles>CN(C)c1ccccc1-c1ccccc1C(C)(C)C</smiles>

1al

1 am<smiles>C[Si](C)([Na])c1ccccc1-c1ccccc1</smiles>

Ian<smiles>[Y9][SiH]([Y19])c1ccc(C#Cc2ccccc2)cc1</smiles>

1at<smiles>Cc1ccc(-c2ccccc2[Si]([NH3+])([O-])[O-])cc1</smiles>

1ao<smiles>C[N+](=O)c1ccccc1C1Cc2cc(Br)ccc21</smiles>

1aq<smiles>CC(C)(C)c1ccccc1-c1ccc(O)cc1</smiles>

1ar

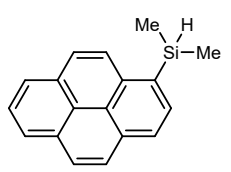

1as

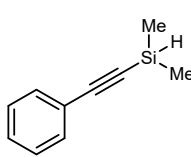

1au<smiles>C[Si](C)(C)c1ccc(/N=N/c2ccccc2)cc1</smiles>

1 av

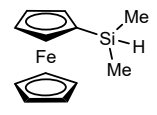

1aw

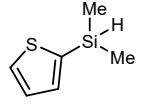

$1 a x$<smiles>C[Si](C)(C)[Si]1Cc2ccccc2O1</smiles>

1 ay

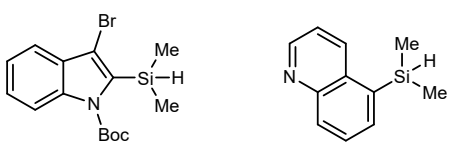

laz<smiles>C[13CH](N)c1cnc2ccccc2c1</smiles>

$1 \mathrm{bb}$<smiles>COc1cnn(Cc2ccccc2)c(=O)c1[N+](C)(C)C</smiles>

$1 \mathrm{bc}$

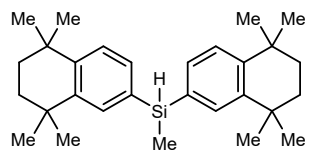

1bd

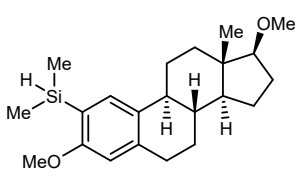

1 be<smiles>CC(C)[C@H]1CC[C@@H](C)C[C@H]1COCc1ccc(N(C)C)cc1</smiles>

$1 \mathrm{bf}$

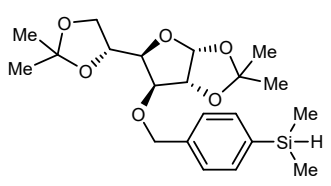

1 bg<smiles>[Y][SiH]([R])c1ccc(N(CC(F)(F)F)S(=O)(=O)c2ccccc2)cc1</smiles>

1bh<smiles>[Y6]c1nc2c(F)cccc2cc1Oc1cccc(F)c1[Si]([14CH3])([14CH3])[14CH3]</smiles><smiles>[Y9][Si]([14CH3])([14CH3])c1ccc2c(c1)OCO2</smiles>

1bj<smiles>[Y9][Si]([Na])(c1ccncc1)[N+]([O-])([O-])c1ccncc1</smiles>

$1 \mathrm{bk}$<smiles>C[Si](C)(C)c1cccc2ccccc12</smiles>

1bl
$1 b q$<smiles>C[N+](C)(C)c1ccc(C2OCCO2)cc1</smiles>

1 bp<smiles>C[Si](C)(N)c1ccc(OC2CCCCO2)cc1</smiles><smiles>C=C[As](C)(C)c1ccccc1</smiles>

$1 \mathrm{br}$

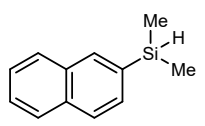

$1 \mathrm{bm}$

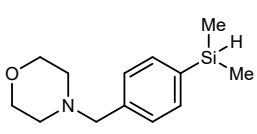

$1 \mathrm{bn}$

A) 1a, 1z, 1ac, 1ae, 1af, 1ag, 1ai, 1aj, 1ak, $1 \mathrm{bs}$ and $1 \mathrm{bt}$ were purchased from commercial suppliers and used without addition purification.

B) General procedure for the preparation of $1 \mathrm{c}, 1 \mathrm{~d}, 1 \mathrm{e}, 1 \mathrm{f}, 1 \mathrm{~g}, 1 \mathrm{i}, 1 \mathrm{j}, 1 \mathrm{k}, 1 \mathrm{p}, 1 \mathrm{~s}, 1 \mathrm{t}, 1 \mathrm{u}, 1 \mathrm{v}, 1 \mathrm{w}, 1 \mathrm{x}, 1 \mathrm{an}, 1 \mathrm{ax}$, $\underline{1 b f}, \mathbf{1 b g}, 1 \mathbf{b j}, 1 \mathbf{b l}, \mathbf{1 b m}, \mathbf{1 b p}$ and $\mathbf{1 b q}$ according to a modified literature procedure by $\mathrm{Mg}$ insertion ${ }^{17}$.<smiles>[R]c1cc(Br)cc2cc([Si](C)(C)[R17]([H])([H])[H])ccc12</smiles> 
Under $\mathrm{N}_{2}$ atmosphere, magnesium shavings $(0.583 \mathrm{~g}, 24 \mathrm{mmol}, 1.2$ equiv. $)$ and $30 \mathrm{~mL}$ anhydrous 2-MeTHF was added to a $100 \mathrm{~mL}$ two-neck round bottom flask. To this suspension was added 2 drops of 1,2dibromoethane. A solution of aryl bromide ( $20 \mathrm{mmol}, 1.0$ equiv.) in $10 \mathrm{~mL}$ anhydrous 2-MeTHF was then added slowly to the suspension of $\mathrm{Mg}$ at room temperature. After addition, the resulting mixture was stirred at $50{ }^{\circ} \mathrm{C}$ for $3 \mathrm{~h}$ and then cooled to room temperature. Chlorodimethylsilane ( $2.84 \mathrm{~g}, 30 \mathrm{mmol}, 1.5$ equiv.) was added and the mixture was stirred overnight. After quenched with saturated aqueous $\mathrm{NH}_{4} \mathrm{Cl}$ solution, the mixture was extracted with diethyl ether and the combined organic phases are dried over anhydrous $\mathrm{Na}_{2} \mathrm{SO}_{4}$. After evaporation of the solvents under reduced pressure, the crude product was purified by flash column chromatography on silica gel using suitable eluents to give the corresponding silane.

\section{Compound 1c}<smiles>Cc1ccc([As](C)(=O)([O-])[O-])cc1</smiles>

\section{(4-Methylphenyl)dimethylsilane}

Physical state: colorless oil.

${ }^{1}$ H NMR (400 MHz, Chloroform- $d$ ): $\delta=7.44(\mathrm{~d}, J=7.6 \mathrm{~Hz}, 2 \mathrm{H}), 7.18(\mathrm{~d}, J=7.5 \mathrm{~Hz}, 2 \mathrm{H}), 4.41$ (hept, $J=$ $3.8 \mathrm{~Hz}, 1 \mathrm{H}), 2.34(\mathrm{~s}, 3 \mathrm{H}), 0.32(\mathrm{~d}, J=3.7 \mathrm{~Hz}, 6 \mathrm{H})$.

${ }^{13}$ C NMR (101 MHz, Chloroform- $\boldsymbol{d}$ ): $\delta=139.06,134.03,128.69,126.80,21.46,-3.68$.

The characterization data are consistent with that reported in the literature ${ }^{18}$.

\section{Compound 1d}<smiles>C[As](=O)([Mg])c1ccc(Br)cc1</smiles>

\section{(4-tert-Butylphenyl)dimethylsilane}

Physical state: colorless oil.

${ }^{1}$ H NMR (400 MHz, Chloroform- $\left.d\right): \delta=7.55(\mathrm{~d}, J=6.3 \mathrm{~Hz}, 2 \mathrm{H}), 7.46(\mathrm{~d}, J=6.3 \mathrm{~Hz}, 2 \mathrm{H}), 4.51-4.46(\mathrm{~m}$, $1 \mathrm{H}), 1.39$ (s, 9H), 0.40 (d, $J=1.6 \mathrm{~Hz}, 6 \mathrm{H})$.

${ }^{13}$ C NMR (101 MHz, Chloroform-d): $\delta=152.19,133.93,124.85,31.23,-3.72$.

The characterization data are consistent with that reported in the literature ${ }^{19}$.

\section{Compound 1e}<smiles>Cc1ccc(S(=O)(=O)N(C)C)cc1</smiles>

\section{(4-Trimethylphenyl)dimethylsilylsilane}

Physical state: colorless oil.

${ }^{1}$ H NMR (400 MHz, Chloroform- $d$ ): $\delta=7.58$ (d, $J=5.6 \mathrm{~Hz}, 4 \mathrm{H}$ ), 4.47 (tt, $J=7.4,3.6 \mathrm{~Hz}, 1 \mathrm{H}$ ), 0.39 (dd, $J=5.3,3.8 \mathrm{~Hz}, 6 \mathrm{H}), 0.32(\mathrm{~d}, J=5.6 \mathrm{~Hz}, 9 \mathrm{H})$.

${ }^{13}$ C NMR (101 MHz, Chloroform-d): $\delta=141.56,137.95,133.29,132.72,-1.22,-3.85$.

The characterization data are consistent with that reported in the literature ${ }^{20}$. 


\section{Compound 1f}<smiles></smiles>

\section{(4-Fluorophenyl)dimethylsilane}

Physical state: colorless oil.

${ }^{1}$ H NMR (400 MHz, Chloroform- $d$ ): $\delta=7.50(\mathrm{dd}, J=8.3,6.3 \mathrm{~Hz}, 2 \mathrm{H}), 7.05(\mathrm{t}, J=8.9 \mathrm{~Hz}, 2 \mathrm{H}), 4.41$ (dq, $J=7.3,3.7 \mathrm{~Hz}, 1 \mathrm{H}), 0.33(\mathrm{~d}, J=3.7 \mathrm{~Hz}, 6 \mathrm{H})$.

${ }^{13}$ C NMR (101 MHz, Chloroform- $d$ ): $\delta=165.04,162.58,135.91,135.84,115.11,114.92,-3.67$.

${ }^{19}$ F NMR (376 MHz, Chloroform- $d$ ): $\delta=-111.82$.

The characterization data are consistent with that reported in the literature ${ }^{18}$.

\section{Compound 1g}<smiles>C[As](C)(=O)c1ccc(Cl)cc1</smiles>

(4-Chlorophenyl)dimethylsilane (1f)

Physical state: colorless oil.

${ }^{1}$ H NMR (400 MHz, Chloroform- $\boldsymbol{d}$ ): $\delta=7.50$ - 7.44 (m, 2H), 7.38 - 7.31 (m, 2H), 4.43 (hept, $J=3.8 \mathrm{~Hz}, 1 \mathrm{H}$ ), $0.35(\mathrm{~d}, J=3.8 \mathrm{~Hz}, 6 \mathrm{H})$.

${ }^{13}$ C NMR (101 MHz, Chloroform-d): $\delta=135.67,135.49,135.31,128.06,-3.86$.

The characterization data are consistent with that reported in the literature ${ }^{21}$.

\section{Compound 1i}

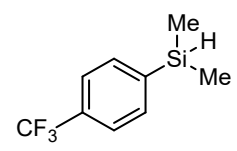

(4-Trifluoromethylphenyl)dimethylsilane

Physical state: colorless oil.

${ }^{1}$ H NMR (400 MHz, Chloroform- $\left.d\right): \delta=7.65(\mathrm{~d}, J=7.7 \mathrm{~Hz}, 2 \mathrm{H}), 7.59(\mathrm{~d}, J=7.7 \mathrm{~Hz}, 2 \mathrm{H}), 4.58-4.33(\mathrm{~m}$, $1 \mathrm{H}), 0.37(\mathrm{~d}, J=3.5 \mathrm{~Hz}, 6 \mathrm{H})$.

${ }^{13}$ C NMR (101 MHz, Chloroform-d): $\delta=142.45,134.29,131.04,125.57,124.41,124.37,124.33,124.30$, $122.86,-4.04$.

${ }^{19}$ F NMR (376 MHz, Chloroform- $d$ ): $\delta=-62.96$.

The characterization data are consistent with that reported in the literature ${ }^{18}$.

\section{Compound 1j}<smiles>COc1ccc([As](C)(=O)=O)cc1</smiles>

\section{(4-Methoxyphenyl)dimethylsilane}

Physical state: colorless oil.

${ }^{1}$ H NMR (400 MHz, Chloroform- $d$ ): $\delta=7.52(\mathrm{~d}, J=8.4 \mathrm{~Hz}, 2 \mathrm{H}), 6.97(\mathrm{~d}, J=8.4 \mathrm{~Hz}, 2 \mathrm{H}), 4.53-4.40$ (m, $1 \mathrm{H}), 3.86(\mathrm{~s}, 3 \mathrm{H}), 0.37(\mathrm{~d}, J=3.7 \mathrm{~Hz}, 6 \mathrm{H})$. 
${ }^{13}$ C NMR (101 MHz, Chloroform-d): $\delta=160.55,135.41,128.17,113.64,55.00,-3.55$.

The characterization data are consistent with that reported in the literature ${ }^{18}$.

\section{Compound 1k}<smiles>C[Si](C)(C)c1ccc(Oc2ccccc2)cc1</smiles>

\section{(4-Phenoxyphenyl)dimethylsilane}

Physical state: colorless oil.

${ }^{1}$ H NMR (400 MHz, Chloroform-d): $\delta=7.53(\mathrm{dd}, J=10.2,3.4 \mathrm{~Hz}, 2 \mathrm{H}), 7.38(\mathrm{t}, J=5.8 \mathrm{~Hz}, 2 \mathrm{H}), 7.15(\mathrm{t}$, $J=6.4 \mathrm{~Hz}, 1 \mathrm{H}), 7.04(\mathrm{dt}, J=14.1,7.0 \mathrm{~Hz}, 4 \mathrm{H}), 4.47(\mathrm{dd}, J=6.3,2.6 \mathrm{~Hz}, 1 \mathrm{H}), 0.38(\mathrm{~d}, J=3.6 \mathrm{~Hz}, 6 \mathrm{H})$.

${ }^{13}$ C NMR (101 MHz, Chloroform-d): $\delta=158.51,156.78,135.62,131.31,129.77,123.49,119.28,118.11$, 3.59 .

The characterization data are consistent with that reported in the literature ${ }^{22}$.

\section{Compound 1p}<smiles>C[Si](C)(C)c1ccc(S(C)(=O)=O)cc1</smiles>

(4-Methylthiophenyl)dimethylsilane

Physical state: colorless oil.

${ }^{1}$ H NMR (400 MHz, Chloroform-d): $\delta=7.48$ (d, $\left.J=8.0 \mathrm{~Hz}, 2 \mathrm{H}\right), 7.29$ (t, $\left.J=6.6 \mathrm{~Hz}, 2 \mathrm{H}\right), 4.55$ - 4.36 (m, $1 \mathrm{H}), 2.51(\mathrm{~s}, 3 \mathrm{H}), 0.37(\mathrm{~d}, J=3.7 \mathrm{~Hz}, 6 \mathrm{H})$.

${ }^{13}$ C NMR (101 MHz, Chloroform-d): $\delta=139.96,134.34,128.76,125.62,15.28,-3.77$.

The characterization data are consistent with that reported in the literature ${ }^{19}$.

\section{Compound 1s}<smiles>Cc1ccccc1[Si](C)(C)O</smiles>

\section{(2-Methylphenyl)dimethylsilane}

Physical state: colorless oil.

${ }^{1}$ H NMR (400 MHz, Chloroform-d): $\delta=7.48(\mathrm{~d}, J=6.6 \mathrm{~Hz}, 1 \mathrm{H}), 7.35-7.23(\mathrm{~m}, 1 \mathrm{H}), 7.17(\mathrm{~d}, J=6.7 \mathrm{~Hz}$, $2 \mathrm{H}), 4.55(\mathrm{dd}, J=6.6,3.1 \mathrm{~Hz}, 1 \mathrm{H}), 2.46(\mathrm{~d}, J=5.5 \mathrm{~Hz}, 3 \mathrm{H}), 0.37(\mathrm{~d}, J=3.5 \mathrm{~Hz}, 6 \mathrm{H})$.

${ }^{13}$ C NMR (101 MHz, Chloroform-d): $\delta=143.68,136.12,134.59,129.54,129.46,125.07,22.34,-3.55$.

The characterization data are consistent with that reported in the literature ${ }^{23}$.

\section{Compound 1t}<smiles>Cc1cccc([Si](C)([Na])[Na])c1</smiles>

(3-Methylphenyl)dimethylsilane

Physical state: colorless oil.

${ }^{1}$ H NMR (400 MHz, Chloroform-d): $\delta=7.38$ (d, $\left.J=8.1 \mathrm{~Hz}, 2 \mathrm{H}\right), 7.33-7.17$ (m, 2H), 4.47 - 4.42 (m, 1H), 
$2.39(\mathrm{~s}, 3 \mathrm{H}), 0.37(\mathrm{~d}, J=3.4 \mathrm{~Hz}, 6 \mathrm{H})$.

${ }^{13}$ C NMR (101 MHz, Chloroform-d): $\delta=137.28,137.20,134.67,130.98,129.97,127.80,21.45,-3.76$.

The characterization data are consistent with that reported in the literature ${ }^{24}$.

\section{Compound 1u}<smiles></smiles>

\section{(2-Methoxyphenyl)dimethylsilane}

Physical state: colorless oil.

${ }^{1} \mathrm{H}$ NMR (400 MHz, Chloroform- $\boldsymbol{d}$ ): ${ }^{1} \mathrm{H}$ NMR $(400 \mathrm{MHz}$, Chloroform-d) $\delta=7.48$ (d, $J=7.2 \mathrm{~Hz}, 1 \mathrm{H}), 7.41$ $(\mathrm{d}, J=7.6 \mathrm{~Hz}, 1 \mathrm{H}), 7.00(\mathrm{~d}, J=7.5 \mathrm{~Hz}, 1 \mathrm{H}), 6.88(\mathrm{~d}, J=8.2 \mathrm{~Hz}, 1 \mathrm{H}), 4.46(\mathrm{dq}, J=7.8,3.9,3.5 \mathrm{~Hz}, 1 \mathrm{H}), 3.86$ (s, $3 \mathrm{H}), 0.38(\mathrm{~s}, 6 \mathrm{H})$.

${ }^{13}$ C NMR (101 MHz, Chloroform-d): $\delta=164.28,135.73,131.14,125.42,120.52,109.45,55.14,-3.83$.

The characterization data are consistent with that reported in the literature ${ }^{21}$.

\section{Compound 1v}

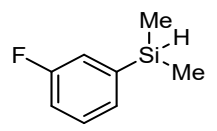

\section{(3-Fluorophenyl)dimethylsilane}

Physical state: colorless oil.

${ }^{1}$ H NMR (400 MHz, Chloroform- $d$ ): $\delta=7.43-7.31(\mathrm{~m}, 1 \mathrm{H}), 7.25(\mathrm{dd}, J=31.7,8.0 \mathrm{~Hz}, 2 \mathrm{H}), 7.04(\mathrm{t}, J=$ $8.3 \mathrm{~Hz}, 1 \mathrm{H}), 4.54-4.31(\mathrm{~m}, 1 \mathrm{H}), 0.34(\mathrm{~d}, J=2.6 \mathrm{~Hz}, 6 \mathrm{H})$.

${ }^{13}$ C NMR (101 MHz, Chloroform-d): $\delta=163.80,161.33,140.52,140.48,129.67,129.61,129.51,129.48$, $120.37,120.19,116.19,115.98,-3.93$.

${ }^{19}$ F NMR (376 MHz, Chloroform- $d$ ): $\delta=-113.69$.

The characterization data are consistent with that reported in the literature ${ }^{25}$.

\section{Compound 1w}<smiles>COc1cc([N+](=O)[O-])cc([N+](=O)[O-])c1</smiles>

\section{(3,5-Dimethylphenyl)dimethylsilane}

Physical state: colorless oil.

${ }^{1}$ H NMR (400 MHz, Chloroform- $\boldsymbol{d}$ ): $\delta=7.22(\mathrm{~s}, 2 \mathrm{H}), 7.07$ (s, 1H), 4.48 - 4.44 (m, 1H), $2.38(\mathrm{~s}, 6 \mathrm{H}), 0.39$ $(\mathrm{d}, J=4.0 \mathrm{~Hz}, 6 \mathrm{H})$.

${ }^{13}$ C NMR (101 MHz, Chloroform-d $): \delta=137.19,131.70,130.94,21.29,-3.71$.

The characterization data are consistent with that reported in the literature ${ }^{21}$. 


\section{Compound 1x}<smiles>Cc1ccc([As](C)(=O)([O-])c2ccc(C)cc2)cc1</smiles>

(1,1'-Biphenyl)-4-yldimethylsilane

Physical state: colorless oil.

${ }^{1}$ H NMR (400 MHz, Chloroform- $d$ ): $\delta=7.70(\mathrm{dd}, J=11.3,7.1 \mathrm{~Hz}, 6 \mathrm{H}), 7.53(\mathrm{t}, J=6.9 \mathrm{~Hz}, 2 \mathrm{H}), 7.44(\mathrm{~d}$, $J=6.7 \mathrm{~Hz}, 1 \mathrm{H}), 4.91-4.56(\mathrm{~m}, 1 \mathrm{H}), 0.47(\mathrm{~d}, J=3.6 \mathrm{~Hz}, 6 \mathrm{H})$.

${ }^{13}$ C NMR (101 MHz, Chloroform-d): $\delta=141.99,141.03,136.13,134.50,128.76,127.39,127.14,126.60$, -3.73 .

The characterization data are consistent with that reported in the literature ${ }^{26}$.

\section{Compound 1an}<smiles>C[N+]([O-])([O-])c1ccccc1-c1ccccc1</smiles>

(1,1'-Biphenyl)-2-yldimethylsilane

Physical state: colorless oil.

${ }^{1}$ H NMR (400 MHz, Chloroform- $d$ ): $\delta=7.70-7.62(\mathrm{~m}, 1 \mathrm{H}), 7.50-7.29(\mathrm{~m}, 8 \mathrm{H}), 4.37$ (hept, $J=3.7 \mathrm{~Hz}$, $1 \mathrm{H}), 0.09(\mathrm{~d}, J=3.8 \mathrm{~Hz}, 6 \mathrm{H})$.

${ }^{13}$ C NMR (101 MHz, Chloroform-d): $\delta=149.27,143.68,135.10,129.21,129.18,129.09,127.86,127.11$, $126.38,-3.02$.

The characterization data are consistent with that reported in the literature ${ }^{27}$.

\section{Compound 1ax}

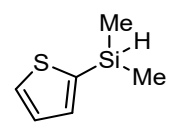

\section{Dimethyl(thiophen-2-yl)silane}

Physical state: colorless oil.

${ }^{1}$ H NMR (400 MHz, Chloroform- $\boldsymbol{d}$ ): $\delta=7.62(\mathrm{~d}, J=4.6 \mathrm{~Hz}, 1 \mathrm{H}), 7.33$ (bd, $\left.J=3.3 \mathrm{~Hz}, 1 \mathrm{H}\right), 7.21$ (dd, $J=$ 4.6, 3.3 Hz, 1H), 4.57 (hept, $J=3.7 \mathrm{~Hz}, 1 \mathrm{H}), 0.40(\mathrm{~d}, J=3.7 \mathrm{~Hz}, 6 \mathrm{H})$.

${ }^{13}$ C NMR (101 MHz, Chloroform-d): $\delta=135.07,131.05,128.23,-2.77$.

The characterization data are consistent with that reported in the literature ${ }^{28}$.

\section{Compound 1bf}<smiles>CC(C)C1CC[C@@H](C)CC1OCc1ccc([As])cc1</smiles>

(4-((((1R,2S,5R)-2-isopropyl-5-methylcyclohexyl)oxy)methyl)phenyl)dimethylsilane

Physical state: colorless oil. 
${ }^{1}$ H NMR (400 MHz, Chloroform- $d$ ): $\delta=7.58-7.48(\mathrm{~m}, 2 \mathrm{H}), 7.36(\mathrm{~d}, J=7.3 \mathrm{~Hz}, 2 \mathrm{H}), 4.67(\mathrm{~d}, J=11.5 \mathrm{~Hz}$, $1 \mathrm{H}), 4.48-4.37$ (m, 2H), 3.19 (td, $J=10.6,4.0 \mathrm{~Hz}, 1 \mathrm{H}), 2.32(\mathrm{p}, J=7.3 \mathrm{~Hz}, 1 \mathrm{H}), 2.21(\mathrm{~d}, J=11.8 \mathrm{~Hz}, 1 \mathrm{H}), 1.66$ (t, $J=11.7 \mathrm{~Hz}, 2 \mathrm{H}), 1.38-1.24(\mathrm{~m}, 2 \mathrm{H}), 0.93(\mathrm{dd}, J=16.5,7.7 \mathrm{~Hz}, 9 \mathrm{H}), 0.73(\mathrm{~d}, J=8.6 \mathrm{~Hz}, 3 \mathrm{H}), 0.34(\mathrm{~d}, J=3.8$ $\mathrm{Hz}, 6 \mathrm{H})$.

${ }^{13}$ C NMR (101 MHz, Chloroform- $\boldsymbol{d}$ ): $\delta=140.20,136.33,134.01,127.22,78.86,70.31,48.32,40.30,34.57$, $31.57,25.51,23.25,22.37,21.02,16.06,-3.74$.

HRMS (ESI-TOF): calcd for $\mathrm{C}_{19} \mathrm{H}_{32} \mathrm{OSiNa}^{+}[\mathrm{M}+\mathrm{Na}]^{+}: 327.2115$, found: 327.2130 .

\section{Compound 1 bg}

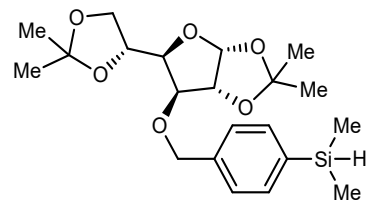

(4-((((3aR,5R,6S,6aR)-5-((R)-2,2-dimethyl-1,3-dioxolan-4-yl)-2,2-dimethyltetrahydrofuro-[2,3d][1,3]dioxol-6-yl)oxy)methyl)phenyl)dimethylsilane

Physical state: white solid.

${ }^{1}$ H NMR (400 MHz, Chloroform- $d$ ): $\delta=7.55(\mathrm{~d}, J=7.5 \mathrm{~Hz}, 2 \mathrm{H}), 7.37$ (d, $\left.J=5.6 \mathrm{~Hz}, 2 \mathrm{H}\right), 5.92(\mathrm{~d}, J=3.3$ $\mathrm{Hz}, 1 \mathrm{H}), 4.69$ (d, $J=7.6 \mathrm{~Hz}, 2 \mathrm{H}), 4.61(\mathrm{~d}, J=3.5 \mathrm{~Hz}, 1 \mathrm{H}), 4.49-4.43(\mathrm{~m}, 1 \mathrm{H}), 4.42-4.37(\mathrm{~m}, 1 \mathrm{H}), 4.15$ (dd, $J=16.2,7.8 \mathrm{~Hz}, 2 \mathrm{H}), 4.04(\mathrm{~d}, J=6.8 \mathrm{~Hz}, 2 \mathrm{H}), 1.52$ (s, 3H), 1.45 (s, 3H), 1.40 (s, 3H), $1.34(\mathrm{~s}, 3 \mathrm{H})$, $0.37(\mathrm{~d}, J=3.6 \mathrm{~Hz}, 6 \mathrm{H})$.

${ }^{13}$ C NMR (101 MHz, Chloroform-d): $\delta=138.63,137.01,134.10,127.06,111.77,108.95,105.29,82.66$, $81.72,81.32,72.50,72.27,67.39,26.83,26.76,26.23,25.42,-3.80$.

HRMS (ESI-TOF): calcd for $\mathrm{C}_{21} \mathrm{H}_{32} \mathrm{O}_{6} \mathrm{SiNa}^{+}[\mathrm{M}+\mathrm{Na}]^{+}: 431.1860$, found: 431.1878 .

\section{Compound 1bj}<smiles>CS(=O)(=O)c1ccc2c(c1)OCO2</smiles>

\section{1,3-Benzodioxol-5-yl(dimethyl)silane}

Physical state: colorless oil.

${ }^{1}$ H NMR (400 MHz, Chloroform-d): $\delta=7.08$ - $6.94(\mathrm{~m}, 2 \mathrm{H}), 6.85-6.81(\mathrm{~m}, 1 \mathrm{H}), 5.92(\mathrm{~s}, 2 \mathrm{H}), 4.51$ - 4.27 $(\mathrm{m}, 1 \mathrm{H}), 0.31(\mathrm{~d}, J=3.5 \mathrm{~Hz}, 6 \mathrm{H})$.

${ }^{13}$ C NMR (101 MHz, Chloroform-d): $\delta=148.70,147.55,130.41,128.11,121.75,113.39,108.79,100.67$, -3.37 .

HRMS (ESI-TOF): calcd for $\mathrm{C}_{9} \mathrm{H}_{12} \mathrm{O}_{2} \mathrm{SiNa}^{+}[\mathrm{M}+\mathrm{Na}]^{+}: 203.0499$, found: 203.0493 .

\section{Compound 1bl}<smiles>C[SiH](N)c1cccc2ccccc12</smiles>

Dimethyl(naphthalen-1-yl)silane

Physical state: colorless oil.

${ }^{1}$ H NMR (400 MHz, Chloroform- $d$ ): $\delta=8.19$ (d, $\left.J=7.9 \mathrm{~Hz}, 1 \mathrm{H}\right), 7.97$ - $7.68(\mathrm{~m}, 3 \mathrm{H}), 7.64$ - 7.39 (m, 3H), 
$5.01-4.92(\mathrm{~m}, 1 \mathrm{H}), 0.56(\mathrm{~d}, J=4.0 \mathrm{~Hz}, 6 \mathrm{H})$.

${ }^{13}$ C NMR (101 MHz, Chloroform-d): $\delta=136.92,135.61,133.61,133.17,129.98,128.94,127.59,125.91$, $125.52,125.16,-3.26$.

The characterization data are consistent with that reported in the literature ${ }^{21}$.

\section{Compound 1bm}<smiles>C[As](C)(N)c1ccc2ccccc2c1</smiles>

\section{Dimethyl(naphthalen-2-yl)silane}

Physical state: colorless oil.

${ }^{1}$ H NMR (400 MHz, Chloroform-d): ${ }^{1} \mathrm{H}$ NMR (400 MHz, Chloroform-d) $\delta=8.11$ (s, 1H), $7.91-7.87$ (m, $3 \mathrm{H}), 7.67(\mathrm{~d}, J=8.0 \mathrm{~Hz}, 1 \mathrm{H}), 7.56-7.52(\mathrm{~m}, 2 \mathrm{H}), 4.66-4.60(\mathrm{~m}, 1 \mathrm{H}), 0.50(\mathrm{~d}, J=4.0 \mathrm{~Hz}, 6 \mathrm{H})$.

${ }^{13}$ C NMR (101 MHz, Chloroform-d): $\delta=134.85,134.69,133.82,132.96,130.17,127.99,127.74,127.09$, $126.38,125.95,-3.69$.

The characterization data are consistent with that reported in the literature ${ }^{21}$.

\section{Compound 1bp}<smiles>C[As](C)(=O)c1ccc(OC2CCCCO2)cc1</smiles>

\section{Dimethyl(4-((tetrahydro-2H-pyran-2-yl)oxy)phenyl)silane}

Physical state: colorless oil.

${ }^{1}$ H NMR (400 MHz, Chloroform- $d$ ): $\delta=7.49(\mathrm{~d}, J=7.8 \mathrm{~Hz}, 2 \mathrm{H}), 7.10(\mathrm{~d}, J=7.8 \mathrm{~Hz}, 2 \mathrm{H}), 5.49(\mathrm{~s}, 1 \mathrm{H})$, $4.44(\mathrm{dt}, J=7.1,3.5 \mathrm{~Hz}, 1 \mathrm{H}), 4.00-3.87(\mathrm{~m}, 1 \mathrm{H}), 3.71-3.57(\mathrm{~m}, 1 \mathrm{H}), 2.06(\mathrm{tt}, J=15.4,7.7 \mathrm{~Hz}, 1 \mathrm{H}), 1.93$ - $1.86(\mathrm{~m}, 2 \mathrm{H}), 1.68(\mathrm{ddd}, J=19.3,14.6,7.9 \mathrm{~Hz}, 3 \mathrm{H}), 0.35(\mathrm{~d}, J=3.6 \mathrm{~Hz}, 6 \mathrm{H})$.

${ }^{13}$ C NMR (101 MHz, Chloroform- $d$ ): $\delta=158.06,135.33,129.26,116.04,96.03,61.96,30.31,25.20,18.71$, -3.58 .

HRMS (ESI-TOF): calcd for $\mathrm{C}_{13} \mathrm{H}_{20} \mathrm{O}_{2} \mathrm{SiNa}^{+}[\mathrm{M}+\mathrm{Na}]^{+}: 259.1125$, found: 259.1128 .

\section{Compound 1 bq}<smiles>C[N+](C)([Na])c1ccc(C2OCCO2)cc1</smiles>

\section{2-(4-(Dimethylsilyl)phenyl)-1,3-dioxolane}

Physical state: colorless oil.

${ }^{1}$ H NMR (400 MHz, Chloroform- $\left.d\right): \delta=7.61(\mathrm{~d}, J=8.0 \mathrm{~Hz}, 2 \mathrm{H}), 7.51(\mathrm{~d}, J=8.0 \mathrm{~Hz}, 2 \mathrm{H}), 5.87(\mathrm{~s}, 1 \mathrm{H})$, 4.52 - 4.47 (m, 1H), 4.16 (s, 2H), 4.07 (s, 2H), 0.38 (d, $J=1.9 \mathrm{~Hz}, 6 \mathrm{H})$.

${ }^{13}$ C NMR (101 MHz, Chloroform- $d$ ) $\delta=138.86,138.64,134.02,125.73,103.62,65.27,-3.85$.

The characterization data are consistent with that reported in the literature ${ }^{29}$. 

procedure by Grignard reagents exchange 18 .

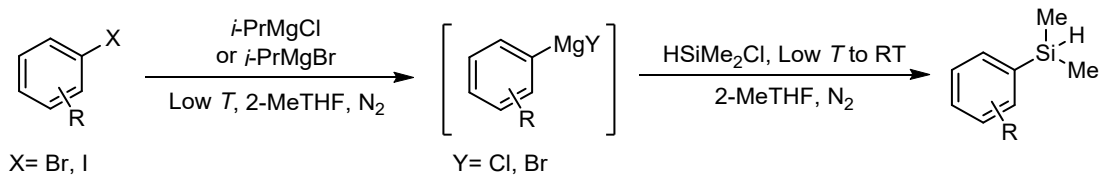

Under $\mathrm{N}_{2}$ atmosphere, a solution of aryl halogen (10 mmol, 1.0 equiv.) in $15 \mathrm{~mL}$ anhydrous 2-MeTHF was cooled to target temperature $\boldsymbol{T}$. Then Grignard reagents $(11 \mathrm{mmol}, 1.1$ equiv., commercially-available THF solution from J\&K Scientific) was slowly added and stirred at $\boldsymbol{T}$ for appropriate time. Chlorodimethylsilane (1.42 g, $15 \mathrm{mmol}, 1.5$ equiv.) was added and the mixture was allowed to warm to room temperature and stirred overnight. After quenched with saturated aqueous $\mathrm{NH}_{4} \mathrm{Cl}$ solution, the mixture was extracted with diethyl ether, and the combined organic phases are dried anhydrous $\mathrm{Na}_{2} \mathrm{SO}_{4}$. After evaporation of the solvents under reduced pressure, the crude product was purified by flash column chromatography on silica gel using suitable eluents to give the corresponding silane.

\section{Compound 1m}<smiles>C[Si](C)([14CH3])c1ccc(O[13CH3])cc1</smiles>

\section{4-(Dimethylsilyl)phenyl 4-methylbenzenesulfonate}

Prepared from 4-iodophenyl 4-methylbenzenesulfonate (Org. Lett. 2017, 19, 2486.) by magnesium-iodine exchange with $i-\mathrm{PrMgCl}$ ( 1.1 equiv.) at $-20^{\circ} \mathrm{C}$ for $2 \mathrm{~h}$ and then reacted with chlorodimethylsilane (1.5 equiv.).

Physical state: colorless oil.

${ }^{1}$ H NMR (400 MHz, Chloroform-d): $\delta=7.72(\mathrm{~d}, J=7.7 \mathrm{~Hz}, 2 \mathrm{H}), 7.46(\mathrm{~d}, J=7.5 \mathrm{~Hz}, 2 \mathrm{H}), 7.32(\mathrm{~d}, J=7.7$ $\mathrm{Hz}, 2 \mathrm{H}), 6.98$ (d, $J=7.6 \mathrm{~Hz}, 2 \mathrm{H}), 4.40$ (hept, $J=3.5 \mathrm{~Hz}, 1 \mathrm{H}), 2.45$ (s, 3H), 0.32 (d, $J=1.6 \mathrm{~Hz}, 6 \mathrm{H})$.

${ }^{13}$ C NMR (101 MHz, Chloroform- $\left.d\right): \delta=150.48,145.30,136.56,135.33,132.47,129.71,128.40,121.68$, $21.65,-3.90$.

HRMS (ESI-TOF): calcd for $\mathrm{C}_{15} \mathrm{H}_{19} \mathrm{SO}_{3} \mathrm{Si}^{+}[\mathrm{M}+\mathrm{H}]^{+}: 307.0819$, found: 307.0815 .

\section{Compound 1n}<smiles>C[Si](C)(C)c1ccc(C#N)cc1</smiles>

\section{4-(Dimethylsilyl)benzonitrile}

Prepared from 4-iodobenzonitrile by magnesium-iodine exchange with $i$ - $\mathrm{PrMgCl}$ (1.1 equiv.) at $-20{ }^{\circ} \mathrm{C}$ for $1 \mathrm{~h}$ and then reacted with chlorodimethylsilane (1.5 equiv.).

Physical state: yellow oil.

${ }^{1}$ H NMR (400 MHz, Chloroform-d): $\delta=7.63$ (s, 4H), 4.45 (d, $\left.J=3.4 \mathrm{~Hz}, 1 \mathrm{H}\right), 0.37$ (s, 6H).

${ }^{13}$ C NMR (101 MHz, Chloroform-d): $\delta=144.25,134.43,130.99,118.85,112.75,-4.22$.

The characterization data are consistent with that reported in the literature ${ }^{18}$. 


\section{Compound 10}<smiles></smiles>

\section{Methyl-4-(dimethylsilyl)benzoate}

Prepared from methyl 4-iodobenzoate by magnesium-iodine exchange with $i-\mathrm{PrMgCl}\left(1.1\right.$ equiv.) at $-20{ }^{\circ} \mathrm{C}$ for $1 \mathrm{~h}$ and then reacted with chlorodimethylsilane (1.5 equiv.).

Physical state: colorless oil.

${ }^{1}$ H NMR (400 MHz, Chloroform- $d$ ): $\delta=8.03$ (d, $\left.J=7.4 \mathrm{~Hz}, 2 \mathrm{H}\right), 7.64(\mathrm{~d}, J=7.5 \mathrm{~Hz}, 2 \mathrm{H}), 4.52-4.44$ (m, $1 \mathrm{H}), 3.94(\mathrm{~s}, 3 \mathrm{H}), 0.39$ (d, $J=2.9 \mathrm{~Hz}, 6 \mathrm{H})$.

${ }^{13}$ C NMR (101 MHz, Chloroform-d): $\delta=167.13,143.72,137.68,133.95,128.56,52.03,-4.05$.

The characterization data are consistent with that reported in the literature ${ }^{30}$.

\section{Compound 1bc}<smiles>COc1cnn(Cc2ccccc2)c(=O)c1[Si](C)(C)C</smiles>

\section{2-Benzyl-4-(dimethylsilyl)-5-methoxypyridazin-3(2H)-one}

Prepared from 2-benzyl-4-bromo-5-methoxypyridazin-3(2H)-one (J. Org. Chem. 2009, 74, 9440.) by magnesium-bromide exchange with $i-\mathrm{PrMgCl}\left(1.1\right.$ equiv.) at $-20{ }^{\circ} \mathrm{C}$ for $10 \mathrm{~min}$ in $40 \mathrm{~mL}$ anhydrous 2MeTHF and then reacted with chlorodimethylsilane (1.5 equiv.).

Physical state: white solid.

${ }^{1}$ H NMR (400 MHz, Chloroform- $d$ ): $\delta=7.73(\mathrm{~s}, 1 \mathrm{H}), 7.44$ - $7.38(\mathrm{~m}, 2 \mathrm{H}), 7.34$ - $7.24(\mathrm{~m}, 3 \mathrm{H}), 5.27$ (s, 2H), 4.47 (hept, $J=3.8 \mathrm{~Hz}, 1 \mathrm{H}), 3.90$ (s, 3H), 0.34 (d, $J=3.8 \mathrm{~Hz}, 6 \mathrm{H})$.

${ }^{13}$ C NMR (101 MHz, Chloroform-d): $\delta=164.05,136.62,128.68,128.49,127.70,126.35,117.29,56.59$, $54.58,-4.14$.

HRMS (ESI-TOF): calcd for $\mathrm{C}_{14} \mathrm{H}_{17} \mathrm{~N}_{2} \mathrm{O}_{2} \mathrm{Si}^{+}[\mathrm{M}-\mathrm{H}]^{+}:$: 273.1054, found: 273.1069 .

\section{Compound 1bh}<smiles>C[N+](C)([O-])c1ccc(N(CC(F)(F)F)S(=O)(=O)c2ccccc2)cc1</smiles>

\section{N-(4-(Dimethylsilyl)phenyl)-N-(2,2,2-trifluoroethyl)benzenesulfonamide}

Prepared from N-(4-iodophenyl)-N-(2,2,2-trifluoroethyl)benzenesulfonamide (Angew. Chem. Int. Ed. 2018, 57, 6858., using 4-iodoaniline instead of 4-bromoaniline as starting material) by magnesium-iodine exchange with $i-\operatorname{Pr} \operatorname{MgBr}\left(1.1\right.$ equiv.) at $0{ }^{\circ} \mathrm{C}$ for $2 \mathrm{~h}$ and then reacted with chlorodimethylsilane (1.5 equiv.). Physical state: white solid.

${ }^{1}$ H NMR (400 MHz, Chloroform- $\boldsymbol{d}$ ): $\delta=7.61(\mathrm{t}, J=7.7 \mathrm{~Hz}, 3 \mathrm{H}), 7.48(\mathrm{t}, J=7.8 \mathrm{~Hz}, 4 \mathrm{H}), 7.04$ (d, $J=7.6$ $\mathrm{Hz}, 2 \mathrm{H}), 4.53-4.32(\mathrm{~m}, 1 \mathrm{H}), 4.23(\mathrm{q}, J=8.2 \mathrm{~Hz}, 2 \mathrm{H}), 0.35$ (d, $J=3.2 \mathrm{~Hz}, 6 \mathrm{H})$.

${ }^{13}$ C NMR (101 MHz, Chloroform-d): $\delta=139.75,138.79,138.25,135.04,134.02,133.26,128.98,128.39$, $127.70,125.07,122.29,52.60,52.25,51.91,51.56,-3.97$.

${ }^{19}$ F NMR (376 MHz, Chloroform- $d$ ): $\delta=-70.48(\mathrm{t})$. 
HRMS (ESI-TOF): calcd for $\mathrm{C}_{16} \mathrm{H}_{19} \mathrm{NO}_{2} \mathrm{SF}_{3} \mathrm{SiNa}^{+}[\mathrm{M}+\mathrm{Na}]^{+}:$: 374.0852, found: 374.0843 .

\section{Compound 1 bo}<smiles>C[As](C)(=O)c1ccc(C(=O)N2CCCCC2)cc1</smiles>

\section{(4-(dimethylsilyl)phenyl)(piperidin-1-yl)methanone}

Prepared from (4-iodophenyl)(piperidin-1-yl)methanone (Angew. Chem. Int. Ed. 2018, 57, 12347.) by magnesium-iodine exchange with $i$ - $\operatorname{PrMgBr}\left(1.1\right.$ equiv.) at $-20{ }^{\circ} \mathrm{C}$ for $1 \mathrm{~h}$ and then reacted with chlorodimethylsilane (1.5 equiv.).

Physical state: brown oil.

${ }^{1}$ H NMR (400 MHz, Chloroform- $\boldsymbol{d}$ ): $\delta=7.56(\mathrm{~d}, J=7.4 \mathrm{~Hz}, 2 \mathrm{H}), 7.36(\mathrm{~d}, J=7.4 \mathrm{~Hz}, 2 \mathrm{H}), 4.43$ (hept, $J=$ $3.5 \mathrm{~Hz}, 1 \mathrm{H}$ ), 3.52 (two brs, 4H), 1.63 (two brs, $6 \mathrm{H}$ ), 0.34 (d, $J=3.5 \mathrm{~Hz}, 6 \mathrm{H}$ ).

${ }^{13}$ C NMR (101 MHz, Chloroform-d): $\delta=170.16,139.06,137.07,133.96,125.96,48.50,43.00,26.00$, $24.52,-3.95$.

HRMS (ESI-TOF): calcd for $\mathrm{C}_{14} \mathrm{H}_{22} \mathrm{NOSi}^{+}[\mathrm{M}+\mathrm{H}]^{+}: 248.1465$, found: 248.1468 .

D) General procedure for the preparation of $1 \mathrm{~h}, 1 \mathrm{l}, 1 \mathrm{q}, 1 \mathrm{v}, 1 \mathrm{ah}, 1 \mathrm{al}, 1 \mathrm{am}, 1 \mathrm{as}, 1 \mathrm{at}, 1 \mathrm{av}, 1 \mathrm{aw}, 1 \mathrm{av}, 1 \mathrm{ba}, 1 \mathrm{bb}$,

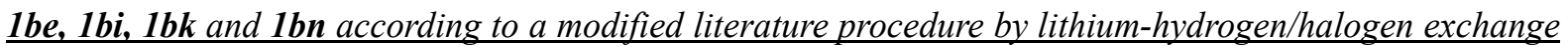
18

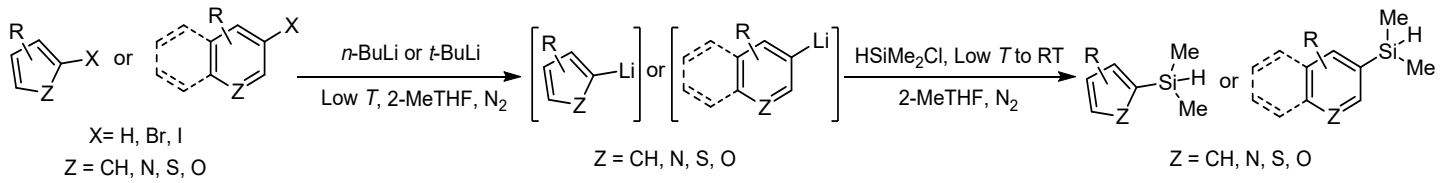

Under $\mathrm{N}_{2}$ atmosphere, a solution of aryl compound (10 mmol, 1.0 equiv.) in $15 \mathrm{~mL}$ anhydrous 2-MeTHF was cooled to target temperature $\boldsymbol{T}$. Then $n-\mathrm{BuLi}(6.9 \mathrm{~mL}$ of a $1.6 \mathrm{M}$ solution in hexane, $11 \mathrm{mmol}, 1.1$ equiv., commercially-available solution from J\&K Scientific) or $t$-BuLi $(16.9 \mathrm{~mL}$ of a $1.3 \mathrm{M}$ solution in pentane, 22 mmol, 2.2 equiv., commercially-available solution from Aladdin) was slowly added and stirred at $\boldsymbol{T}$ for appropriate time. Caution: $\boldsymbol{t}$-BuLi is strongly pyrophoric and should be handled extremely carefully! Chlorodimethylsilane (1.42 g, $15 \mathrm{mmol}, 1.5$ equiv.) was then added and the mixture was allowed to warm to room temperature and stirred overnight. After quenched with saturated aqueous $\mathrm{NH}_{4} \mathrm{Cl}$ solution, the mixture was extracted with diethyl ether, and the combined organic phases are dried over anhydrous $\mathrm{Na}_{2} \mathrm{SO}_{4}$. After evaporation of the solvents under reduced pressure, the crude product was purified by flash column chromatography on silica gel using suitable eluents to give the corresponding silane.

\section{Compound 1h}<smiles>C[As](C)(=O)c1ccc(Br)cc1</smiles>

\section{(4-Bromophenyl)dimethylsilane}

Prepared from 1,4-dibromobenzene by lithium-bromide exchange with $n$-BuLi (1.1 equiv.) at $-78{ }^{\circ} \mathrm{C}$ for $3 \mathrm{~h}$ and then reacted with chlorodimethylsilane (1.5 equiv.).

Physical state: colorless oil. 
${ }^{1}$ H NMR (400 MHz, Chloroform- $d$ ): $\delta=7.53(\mathrm{~d}, J=8.2 \mathrm{~Hz}, 2 \mathrm{H}), 7.43(\mathrm{~d}, J=8.2 \mathrm{~Hz}, 2 \mathrm{H}), 4.45(\mathrm{dq}, J=7.5$, $3.7 \mathrm{~Hz}, 1 \mathrm{H}), 0.37$ (d, $J=3.8 \mathrm{~Hz}, 6 \mathrm{H})$.

${ }^{13}$ C NMR (101 MHz, Chloroform- $d$ ): $\delta=136.16,135.55,130.99,123.99,-3.87$.

The characterization data are consistent with that reported in the literature ${ }^{18}$.

\section{Compound 11}

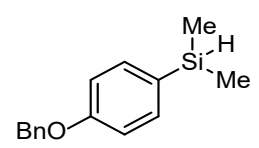

\section{(4-(Benzyloxy)phenyl)dimethylsilane}

Prepared from 1-(benzyloxy)-4-bromobenzene (J. Am. Chem. Soc. 2020, 142, 13246.) by lithium-bromide exchange with $n$-BuLi (1.1 equiv.) at $-78^{\circ} \mathrm{C}$ for $2.5 \mathrm{~h}$ and then reacted with chlorodimethylsilane (1.5 equiv.). Physical state: colorless oil.

${ }^{1}$ H NMR (400 MHz, Chloroform- $d$ ): $\delta=7.64$ - $7.34(\mathrm{~m}, 7 \mathrm{H}), 7.11(\mathrm{~d}, J=7.5 \mathrm{~Hz}, 2 \mathrm{H}), 5.17(\mathrm{~s}, 2 \mathrm{H}), 4.58$ $4.54(\mathrm{~m}, 1 \mathrm{H}), 0.44(\mathrm{~d}, J=1.3 \mathrm{~Hz}, 6 \mathrm{H})$.

${ }^{13}$ C NMR (101 MHz, Chloroform-d): $\delta=159.76,136.91,135.42,132.23,128.53,127.90,127.40,116.63$, $114.51,69.66,-3.57$.

The characterization data are consistent with that reported in the literature ${ }^{30}$.

\section{Compound 1q}<smiles>CN(C)c1ccc([N+](=O)[O-])cc1</smiles>

\section{4-(Dimethylsilyl)-N,N-dimethylaniline}

Prepared from 4-bromo-N,N-dimethylaniline by lithium-bromide exchange with $n$-BuLi (1.1 equiv.) at -78 ${ }^{\circ} \mathrm{C}$ for $2 \mathrm{~h}$ and then reacted with chlorodimethylsilane (1.5 equiv.).

Physical state: pale yellow liquid.

${ }^{1}$ H NMR (400 MHz, Chloroform- $\boldsymbol{d}$ ): $\delta=7.57$ (d, $\left.J=7.5 \mathrm{~Hz}, 2 \mathrm{H}\right), 6.89$ (d, $\left.J=7.4 \mathrm{~Hz}, 2 \mathrm{H}\right), 4.61-4.57$ (m, $1 \mathrm{H}), 3.09$ (s, 6H), 0.47 (d, $J=3.7 \mathrm{~Hz}, 6 \mathrm{H})$.

${ }^{13}$ C NMR (101 MHz, Chloroform-d): $\delta=151.10,134.98,122.18,111.96,40.10,-3.45$.

The characterization data are consistent with that reported in the literature ${ }^{31}$.

\section{Compound 1y}<smiles>C=Cc1ccc([Si](C)(C)[N+])cc1</smiles>

\section{Dimethyl(4-vinylphenyl)silane}

Prepared from 1-bromo-4-vinylbenzene by lithium-bromide exchange with $n$-BuLi (1.1 equiv.) at $-78{ }^{\circ} \mathrm{C}$ for $2.5 \mathrm{~h}$ and then reacted with chlorodimethylsilane (1.5 equiv.).

Physical state: colorless oil.

${ }^{1}$ H NMR (400 MHz, Chloroform- $d$ ): $\delta=7.56(\mathrm{t}, J=5.6 \mathrm{~Hz}, 2 \mathrm{H}), 7.45(\mathrm{~d}, J=6.2 \mathrm{~Hz}, 2 \mathrm{H}), 6.83-6.67$ (m, $1 \mathrm{H}), 5.83(\mathrm{dd}, J=17.6,3.7 \mathrm{~Hz}, 1 \mathrm{H}), 5.31(\mathrm{dd}, J=10.8,3.5 \mathrm{~Hz}, 1 \mathrm{H}), 4.50-4.44(\mathrm{~m}, 1 \mathrm{H}), 0.39$ (d, $J=2.0$ $\mathrm{Hz}, 6 \mathrm{H})$. 
${ }^{13}$ C NMR (101 MHz, Chloroform-d): $\delta=138.34,137.03,136.79,134.24,125.63,114.28,-3.78$.

The characterization data are consistent with that reported in the literature ${ }^{21}$.

\section{Compound 1ah}

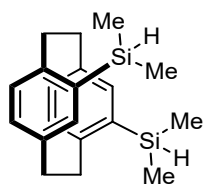

\section{rac-4,12-Bis(dimethylsilyl)[2.2]paracyclophane}

Prepared from pseudo-ortho-4,12-Dibromo[2.2]paracyclophane (Eur. J. Org. Chem. 2017, 1760.) by lithium-bromide exchange with $t$-BuLi (4.2 equiv.) at $-78{ }^{\circ} \mathrm{C}$ for $1 \mathrm{~h}$ and then reacted with chlorodimethylsilane (3 equiv.).

Physical state: white solid.

${ }^{1}$ H NMR (400 MHz, Chloroform-d): $\delta=6.80(\mathrm{~s}, 2 \mathrm{H}), 6.63(\mathrm{~d}, \mathrm{~J}=7.8 \mathrm{~Hz}, 2 \mathrm{H}), 6.57(\mathrm{~d}, \mathrm{~J}=7.7 \mathrm{~Hz}, 2 \mathrm{H})$, $4.66(\mathrm{~m}, 2 \mathrm{H}), 3.45$ (m, 2H), 3.18 (m, 6H), 0.42 (d, $J=1.2 \mathrm{~Hz}, 6 \mathrm{H}), 0.34(\mathrm{~d}, J=1.2 \mathrm{~Hz}, 6 \mathrm{H})$.

${ }^{13}$ C NMR (101 MHz, Chloroform-d): $\delta=146.12,138.03,137.01,134.23,133.31,35.63,34.53,-2.64$, 4.44 .

The characterization data are consistent with that reported in the literature ${ }^{32}$.

\section{Compound 1al}<smiles>C[Si](C)(C)c1ccc(-c2nnc(-c3ccc([As](C)(C)C)cc3)o2)cc1</smiles>

\section{2,5-Bis(4-(dimethylsilyl)phenyl)-1,3,4-oxadiazole}

Prepared from 2,5-bis(4-bromophenyl)-1,3,4-oxadiazole ( $R S C A d v .2017,7,25444$.) by lithium-bromide exchange with $n$-BuLi ( 3 equiv.) at $-78{ }^{\circ} \mathrm{C}$ for $1.5 \mathrm{~h}$ and then reacted with chlorodimethylsilane (3 equiv.).

Physical state: white solid.

${ }^{1}$ H NMR (400 MHz, Chloroform-d): $\delta=8.14(\mathrm{~d}, J=7.5 \mathrm{~Hz}, 4 \mathrm{H}), 7.73(\mathrm{~d}, J=7.7 \mathrm{~Hz}, 4 \mathrm{H}), 4.51$ (hept, $J=$ $3.6 \mathrm{~Hz}, 2 \mathrm{H}), 0.44(\mathrm{~d}, J=4.0 \mathrm{~Hz}, 12 \mathrm{H})$.

${ }^{13}$ C NMR (101 MHz, Chloroform-d): $\delta=164.67,142.48,134.63,126.02,124.41,-3.98$.

The characterization data are consistent with that reported in the literature ${ }^{33}$.

\section{Compound 1am}<smiles>C[Si](C)(C)c1ccc(-c2ccc([Si](=O)(=O)O)s2)s1</smiles>

\section{5,5'-Bis(dimethylsilyl)-2,2'-bithiophene}

Prepared from 5,5'-dibromo-2,2'-bithiophene by lithium-bromide exchange with $n$-BuLi (3 equiv.) at $-20{ }^{\circ} \mathrm{C}$ for $1 \mathrm{~h}$ and then reacted with chlorodimethylsilane (3 equiv.).

Physical state: colorless oil.

${ }^{1}$ H NMR (400 MHz, Chloroform- $d$ ): $\delta=7.27(\mathrm{~d}, J=3.2 \mathrm{~Hz}, 2 \mathrm{H}), 7.21$ (d, $\left.J=3.2 \mathrm{~Hz}, 2 \mathrm{H}\right), 4.59$ (hept, $J=$ $3.6 \mathrm{~Hz}, 2 \mathrm{H}), 0.43(\mathrm{~d}, J=1.4 \mathrm{~Hz}, 12 \mathrm{H})$.

${ }^{13}$ C NMR (101 MHz, Chloroform- $\boldsymbol{d}$ ): $\delta=142.88,136.01,135.88,125.29,-2.88$.

The characterization data are consistent with that reported in the literature ${ }^{34}$. 


\section{Compound 1as}

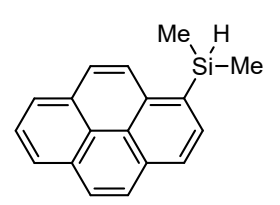

\section{Dimethyl(pyren-1-yl)silane}

Prepared from 1-bromopyrene by lithium-bromide exchange with $n$-BuLi (1.1 equiv.) at $-78{ }^{\circ} \mathrm{C}$ for $1 \mathrm{~h}$ and then reacted with chlorodimethylsilane (1.5 equiv.).

Physical state: white solid.

${ }^{1}$ H NMR (400 MHz, Chloroform- $\boldsymbol{d}$ ): $\delta=8.43(\mathrm{t}, J=7.9 \mathrm{~Hz}, 1 \mathrm{H}), 8.28-8.14(\mathrm{~m}, 5 \mathrm{H}), 8.14-7.99(\mathrm{~m}, 3 \mathrm{H})$, 5.13 (hept, $J=2.6 \mathrm{~Hz}, 1 \mathrm{H}), 0.65(\mathrm{~d}, J=4.2 \mathrm{~Hz}, 6 \mathrm{H})$.

${ }^{13}$ C NMR (101 MHz, Chloroform-d): $\delta=135.67,132.90,132.25,131.26,130.71,127.96,127.48,127.39$, $127.09,125.81,125.23,125.13,124.43,124.15,-2.92$.

The characterization data are consistent with that reported in the literature ${ }^{35}$.

\section{Compound 1at}<smiles>Cc1ccc([Si](=O)(=O)N(C)C)cc1</smiles>

\section{Dimethyl(4-(phenylethynyl)phenyl)silane}

Prepared from 1-bromo-4-(phenylethynyl)benzene by lithium-bromide exchange with $n$-BuLi (1.1 equiv.) at $-78^{\circ} \mathrm{C}$ for $1 \mathrm{~h}$ and then reacted with chlorodimethylsilane (1.5 equiv.).

Physical state: yellow solid.

${ }^{1}$ H NMR (400 MHz, Chloroform-d): $\delta=7.60-7.52(\mathrm{~m}, 6 \mathrm{H}), 7.38(\mathrm{~d}, J=4.9 \mathrm{~Hz}, 3 \mathrm{H}), 4.50-4.46(\mathrm{~m}, 1 \mathrm{H})$, $0.39(\mathrm{~d}, J=0.6 \mathrm{~Hz}, 6 \mathrm{H})$.

${ }^{13}$ C NMR (101 MHz, Chloroform-d): $\delta=137.90,133.89,131.62,130.76,128.32,128.27,123.97,123.21$, $89.98,89.42,-3.90$.

HRMS (ESI-TOF): calcd for $\mathrm{C}_{16} \mathrm{H}_{15} \mathrm{Si}^{+}[\mathrm{M}-\mathrm{H}]^{+}:$:235.0938, found: 235.0936 .

\section{Compound 1av}<smiles>C[N+]([N+])([O-])c1ccc(N=Nc2ccccc2)cc1</smiles>

\section{(E)-1-(4-(dimethylsilyl)phenyl)-2-phenyldiazene}

Prepared from (E)-1-(4-iodophenyl)-2-phenyldiazene (Org. Lett. 2019, 21, 7380.) by lithium-iodine exchange with $n$-BuLi (1.1 equiv.) at $-105^{\circ} \mathrm{C}$ for $1 \mathrm{~h}$ and then reacted with chlorodimethylsilane (1.5 equiv.). Physical state: red oil.

${ }^{1}$ H NMR (400 MHz, Chloroform- $d$ ): $\delta=7.93(\mathrm{dd}, J=13.7,7.6 \mathrm{~Hz}, 4 \mathrm{H}), 7.72(\mathrm{~d}, J=7.6 \mathrm{~Hz}, 2 \mathrm{H}), 7.58$ 7.46 (m, 3H), 4.51 (hept, $J=3.2 \mathrm{~Hz}, 1 \mathrm{H}), 0.41$ (d, $J=3.2 \mathrm{~Hz}, 6 \mathrm{H})$.

${ }^{13}$ C NMR (101 MHz, Chloroform- $\boldsymbol{d}$ ): $\delta=153.15,152.69,141.36,134.80,131.05,129.08,122.88,122.00$, -3.82 .

HRMS (ESI-TOF): calcd for $\mathrm{C}_{14} \mathrm{H}_{17} \mathrm{~N}_{2} \mathrm{Si}^{+}[\mathrm{M}+\mathrm{H}]^{+}:$: 241.1156, found: 241.1165. 


\section{Compound 1aw}

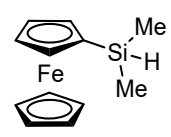

\section{(Dimethylsilyl)ferrocene}

Prepared from ferrocene by lithium-hydrogen exchange with potassium $t$-butoxide ( 0.15 equiv.) and $t$-BuLi (2.1 equiv.) at $-78{ }^{\circ} \mathrm{C}$ for $1.5 \mathrm{~h}$ and then reacted with chlorodimethylsilane (1.5 equiv.).

Physical state: red oil.

${ }^{1}$ H NMR (400 MHz, Chloroform- $d$ ): $\delta=4.47$ - 4.41 (m, 1H), 4.37 (d, $\left.J=16.1 \mathrm{~Hz}, 2 \mathrm{H}\right), 4.22(\mathrm{~s}, 2 \mathrm{H}), 4.18$ (s, $5 \mathrm{H}), 0.34(\mathrm{~d}, J=1.5 \mathrm{~Hz}, 6 \mathrm{H})$.

${ }^{13}$ C NMR (101 MHz, Chloroform- $d$ ): $\delta=73.40,71.08,68.38,68.10,-3.14$.

The characterization data are consistent with that reported in the literature ${ }^{35}$.

\section{Compound 1ay}<smiles>C[Si](C)([Mg])c1cc2ccccc2o1</smiles>

\section{Benzofuran-2-yldimethylsilane}

Prepared from benzofuran by lithium-hydrogen exchange with $n$-BuLi (1.1 equiv.) at $-78{ }^{\circ} \mathrm{C}$ for $1 \mathrm{~h}$ and then reacted with chlorodimethylsilane (1.5 equiv.).

Physical state: colorless oil.

${ }^{1}$ H NMR (400 MHz, Chloroform- $d$ ): $\delta=7.65(\mathrm{~d}, J=7.5 \mathrm{~Hz}, 1 \mathrm{H}), 7.59(\mathrm{~d}, J=8.0 \mathrm{~Hz}, 1 \mathrm{H}), 7.36$ (t, $J=7.4$ $\mathrm{Hz}, 1 \mathrm{H}), 7.28$ (t, $J=7.0 \mathrm{~Hz}, 1 \mathrm{H}), 7.11(\mathrm{~s}, 1 \mathrm{H}), 4.61$ (hept, $J=3.2 \mathrm{~Hz}, 1 \mathrm{H}), 0.50$ (d, $J=3.5 \mathrm{~Hz}, 6 \mathrm{H})$.

${ }^{13}$ C NMR (101 MHz, Chloroform-d): $\delta=160.36,158.24,127.85,124.56,122.41,121.04,117.44,111.29$, -4.77 .

The characterization data are consistent with that reported in the literature ${ }^{30}$.

\section{Compound 1ba}<smiles>C[As](C)(C)c1cccc2ncccc12</smiles>

\section{5-(Dimethylsilyl)quinoline}

Prepared from 5-bromoquinoline by lithium-bromide exchange with $n$-BuLi (1.1 equiv.) at $-78{ }^{\circ} \mathrm{C}$ for $1 \mathrm{~h}$ and then reacted with chlorodimethylsilane (1.5 equiv.).

Physical state: brown oil.

${ }^{1}$ H NMR (400 MHz, Chloroform- $\boldsymbol{d}$ ): $\delta=8.91(\mathrm{~d}, J=3.8 \mathrm{~Hz}, 1 \mathrm{H}), 8.41$ (d, $\left.J=8.4 \mathrm{~Hz}, 1 \mathrm{H}\right), 8.13$ (d, $J=8.3$ $\mathrm{Hz}, 1 \mathrm{H}), 7.78-7.62(\mathrm{~m}, 2 \mathrm{H}), 7.39(\mathrm{dd}, J=8.3,4.0 \mathrm{~Hz}, 1 \mathrm{H}), 4.89-4.82(\mathrm{~m}, 1 \mathrm{H}), 0.46(\mathrm{~d}, J=3.2 \mathrm{~Hz}, 6 \mathrm{H})$. ${ }^{13}$ C NMR (101 MHz, Chloroform-d): $\delta=149.82,148.14,136.46,135.41,133.87,131.71,131.20,128.68$, $120.72,-3.46$.

The characterization data are consistent with that reported in the literature ${ }^{36}$. 


\section{Compound $1 \mathrm{bb}$}<smiles>C[Si](C)(C)c1cncc2ccccc12</smiles>

\section{4-(Dimethylsilyl)isoquinoline}

Prepared from 4-bromoisoquinoline by lithium-bromide exchange with $n$-BuLi (1.1 equiv.) at $-78^{\circ} \mathrm{C}$ for $1 \mathrm{~h}$ and then reacted with chlorodimethylsilane (1.5 equiv.).

Physical state: brown oil.

${ }^{1}$ H NMR (400 MHz, Chloroform-d): $\delta=9.26(\mathrm{~s}, 1 \mathrm{H}), 8.64(\mathrm{~s}, 1 \mathrm{H}), 8.04$ (d, $\left.J=8.4 \mathrm{~Hz}, 1 \mathrm{H}\right), 7.97$ (d, $J=$ $8.1 \mathrm{~Hz}, 1 \mathrm{H}), 7.73$ (t, $J=7.6 \mathrm{~Hz}, 1 \mathrm{H}), 7.61$ (t, $J=7.5 \mathrm{~Hz}, 1 \mathrm{H}), 4.85$ (hept, $J=3.6 \mathrm{~Hz}, 1 \mathrm{H}), 0.51$ (d, $J=3.6$ $\mathrm{Hz}, 6 \mathrm{H})$.

${ }^{13}$ C NMR (101 MHz, Chloroform-d): $\delta=154.25,148.70,139.27,130.36,128.62,127.88,127.21,126.89$, $126.37,-3.67$.

The characterization data are consistent with that reported in the literature ${ }^{36}$.

\section{Compound 1be}

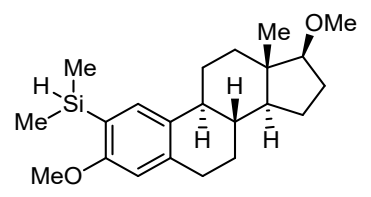

\section{2-Dimethylsilyl-3,17-dimethoxy- $\beta$-estra-1,3,5(10)-triene (1bo)}

Prepared from 2-bromo-3,17-dimethoxy- $\beta$-estra-1,3,5(10)-triene (Org. Lett. 2010, 12, 3352.) by lithiumbromide exchange with $n$-BuLi (1.1 equiv.) at $-78{ }^{\circ} \mathrm{C}$ for $1 \mathrm{~h}$ and then reacted with chlorodimethylsilane (1.5 equiv.).

Physical state: white solid.

${ }^{1}$ H NMR (400 MHz, Chloroform- $d$ ): $\delta=7.37$ (s, 1H), 6.57 (s, 1H), 4.38 (h, $\left.J=3.4 \mathrm{~Hz}, 1 \mathrm{H}\right), 3.79$ (s, 3H), 3.39 (s, 3H), $3.33(\mathrm{t}, J=8.3 \mathrm{~Hz}, 1 \mathrm{H}), 2.88(\mathrm{~m}, 2 \mathrm{H}), 2.44-1.99(\mathrm{~m}, 4 \mathrm{H}), 1.96-1.80(\mathrm{~m}, 1 \mathrm{H}), 1.79-1.63(\mathrm{~m}$, $1 \mathrm{H}), 1.51-1.22(\mathrm{~m}, 7 \mathrm{H}), 0.80(\mathrm{~s}, 3 \mathrm{H}), 0.33(\mathrm{~d}, J=2.9 \mathrm{~Hz}, 6 \mathrm{H})$.

${ }^{13}$ C NMR (101 MHz, Chloroform- $\boldsymbol{d}$ ): $\delta=162.27,140.00,132.93,126.32,113.75,109.91,90.78,57.88$, $55.21,50.24,43.98,43.22,38.72,38.06,30.20,27.74,27.18,26.44,23.04,11.53,-3.63$.

HRMS (ESI-TOF): calcd for $\mathrm{C}_{22} \mathrm{H}_{32} \mathrm{O}_{3} \mathrm{Si}^{+}[\mathrm{M}-\mathrm{H}]^{+}: 357.2244$, found: 375.2229 .

\section{Compound 1bi}<smiles>Cc1nc2c(F)cccc2cc1Oc1ccccc1[N+](C)(C)C</smiles>

\section{3-(2-(Dimethylsilyl)-3-fluorophenoxy)-8-fluoro-2-methylquinoline}

Prepared from 3-(2-bromo-3-fluorophenoxy)-8-fluoro-2-methylquinoline (Patent WO2017/178408) by lithium-bromide exchange with $n$-BuLi (1.1 equiv.) at $-78{ }^{\circ} \mathrm{C}$ for $2 \mathrm{~h}$ and then reacted with chlorodimethylsilane (1.5 equiv.).

Physical state: pale solid. 
${ }^{1}$ H NMR (400 MHz, Chloroform-d): $\delta=7.38$ (s, 3H), 7.29 (s, 2H), $6.93(\mathrm{t}, J=8.3 \mathrm{~Hz}, 1 \mathrm{H}), 6.71$ (d, $J=$ $8.0 \mathrm{~Hz}, 1 \mathrm{H}), 4.52(\mathrm{dp}, J=7.7,4.1 \mathrm{~Hz}, 1 \mathrm{H}), 2.82(\mathrm{~s}, 3 \mathrm{H}), 0.39$ (d, $J=3.7 \mathrm{~Hz}, 6 \mathrm{H})$.

Note: Due to an issue with rotational isomers, the ${ }^{13} \mathrm{C}$ NMR spectrum is complex.

${ }^{13}$ C NMR (101 MHz, Chloroform-d): $\delta=169.21,166.79,160.76,160.62,158.90,156.36,153.86,151.24$, $132.51,132.40,129.88,126.34,126.26,122.22$, 122.18, 118.22, 114.44, 112.08, 111.88, 111.67, 111.41, $20.77,-3.29,-3.32$.

${ }^{19}$ F NMR (376 MHz, Chloroform- $d$ ): $\delta=-97.13,-125.64$ (d).

HRMS (ESI-TOF): calcd for $\mathrm{C}_{18} \mathrm{H}_{18} \mathrm{NOF}_{2} \mathrm{Si}^{+}[\mathrm{M}+\mathrm{H}]^{+}: 330.1120$, found: 330.1130 .

\section{Compound 1bk}<smiles>C[As](C)(=O)c1ccncc1</smiles>

\section{4-(Dimethylsilyl)pyridine}

Prepared from 4-bromopyridine by lithium-bromide exchange with $n$-BuLi (1.1 equiv.) at $-78{ }^{\circ} \mathrm{C}$ for $2 \mathrm{~h}$ and then reacted with chlorodimethylsilane (1.5 equiv.).

Physical state: colorless oil.

${ }^{1}$ H NMR (400 MHz, Chloroform- $d$ ): $\delta=8.57(\mathrm{~d}, J=5.3 \mathrm{~Hz}, 2 \mathrm{H}), 7.41$ (d, $\left.J=5.5 \mathrm{~Hz}, 2 \mathrm{H}\right), 4.69-4.10$ (m, $1 \mathrm{H}), 0.37(\mathrm{~d}, J=3.8 \mathrm{~Hz}, 6 \mathrm{H})$.

${ }^{13}$ C NMR (101 MHz, Chloroform-d): $\delta=148.64,147.28,128.68,-4.61$.

The characterization data are consistent with that reported in the literature ${ }^{37}$.

\section{Compound 1bn}<smiles>N#[Sb](N)(=O)c1ccc(CN2CCOCC2)cc1</smiles>

\section{4-(4-(Dimethylsilyl)benzyl)morpholine}

Prepared from 4-(4-bromobenzyl)morpholine ( $\mathrm{Org}$. Lett. 2013, 15, 2210.) by lithium-bromide exchange with $n$-BuLi (1.1 equiv.) at $-78^{\circ} \mathrm{C}$ for $1 \mathrm{~h}$ and then reacted with chlorodimethylsilane (1.5 equiv.).

Physical state: yellow oil.

${ }^{1}$ H NMR (400 MHz, Chloroform- $d$ ): $\delta=7.53$ (d, $\left.J=7.3 \mathrm{~Hz}, 2 \mathrm{H}\right), 7.36$ (d, $\left.J=7.3 \mathrm{~Hz}, 2 \mathrm{H}\right), 4.46$ (hept, $J=$ $1.6 \mathrm{~Hz} 1 \mathrm{H}), 3.74$ (t, $J=2.0 \mathrm{~Hz} 4 \mathrm{H}), 3.53(\mathrm{~s}, 2 \mathrm{H}), 2.48(\mathrm{t}, J=2.0 \mathrm{~Hz}, 4 \mathrm{H}), 0.37$ (d, $J=1.9 \mathrm{~Hz}, 6 \mathrm{H})$.

${ }^{13}$ C NMR (101 MHz, Chloroform- $d$ ): $\delta=138.77,135.96,133.91,128.62,66.90,63.32,53.56,-3.80$.

HRMS (ESI-TOF): calcd for $\mathrm{C}_{13} \mathrm{H}_{22} \mathrm{NOSi}^{+}[\mathrm{M}+\mathrm{H}]^{+}:$: 236.1465, found: 236.1465 .

\section{E) General procedure for the preparation of 1,1'-biphenyl-2-ylsilanes 1ao, 1ap, 1aq and 1ar ${ }^{27}$.}

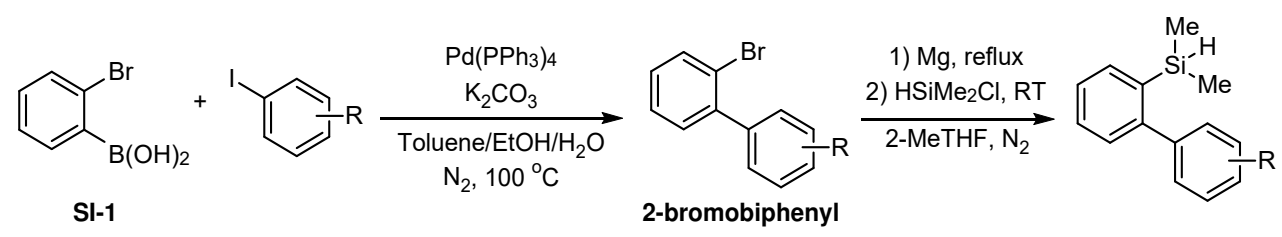

To a mixture of aryl iodide (10 mmol, 1 equiv.), 2-bromophenylboronic acid SI-1 (2.21 g, $11 \mathrm{mmol}, 1.1$ equiv.) and $\mathrm{K}_{2} \mathrm{CO}_{3}(1.73 \mathrm{~g}, 12.5 \mathrm{mmol}, 1.25$ equiv. $)$ in a mixed solvents of toluene/EtOH/water $(84 \mathrm{~mL}$, v:v:v $=15: 7: 2)$ was added $\mathrm{Pd}\left(\mathrm{PPh}_{3}\right)_{4}(231 \mathrm{mg}, 0.2 \mathrm{mmol}, 2 \mathrm{~mol} \% \mathrm{Pd})$ under $\mathrm{N}_{2}$ atmosphere. The resulting reaction 
mixture was stirred at $100{ }^{\circ} \mathrm{C}$ for $5 \mathrm{~h}$. After cooling to room temperature, the mixture was extracted with EA, and the combined organic phases are dried over anhydrous $\mathrm{Na}_{2} \mathrm{SO}_{4}$. After evaporation of the solvents under reduced pressure, the crude product was purified by flash column chromatography on silica gel using cyclohexane as eluents to give the corresponding 2-bromobiphenyl.

Under $\mathrm{N}_{2}$ atmosphere, magnesium shavings ( $0.144 \mathrm{~g}, 6 \mathrm{mmol}, 1.2$ equiv.) and $10 \mathrm{~mL}$ anhydrous 2-MeTHF was added to a $50 \mathrm{~mL}$ two-neck round bottom flask. To this suspension was added 2 drops of 1,2dibromoethane. A solution of 2-bromobiphenyl (5 mmol, 1.0 equiv.) in $10 \mathrm{~mL}$ anhydrous 2-MeTHF was then added slowly to the suspension of $\mathrm{Mg}$ at room temperature. After addition, the resulting mixture was stirred under reflux for $3 \mathrm{~h}$ and then cooled to room temperature. Chlorodimethylsilane $(0.709 \mathrm{~g}, 7.5 \mathrm{mmol}, 1.5$ equiv.) was added and the mixture was stirred overnight. After quenched with saturated aqueous $\mathrm{NH}_{4} \mathrm{Cl}$ solution, the mixture was extracted with diethyl ether, and the combined organic phases are dried over anhydrous $\mathrm{Na}_{2} \mathrm{SO}_{4}$. After evaporation of the solvents under reduced pressure, the crude product was purified by flash column chromatography on silica gel using hexanes as eluent to give the corresponding silane.

\section{Compound 1ao}<smiles></smiles>

(4'-Methyl-[1,1'-biphenyl]-2-yl)dimethylsilane

Physical state: colorless oil.

${ }^{1}$ H NMR (400 MHz, Chloroform- $\boldsymbol{d}$ ): $\delta=7.72(\mathrm{~d}, J=5.8 \mathrm{~Hz}, 1 \mathrm{H}), 7.54-7.27(\mathrm{~m}, 7 \mathrm{H}), 4.47-4.42(\mathrm{~m}, 1 \mathrm{H})$, $2.50(\mathrm{~s}, 3 \mathrm{H}), 0.18(\mathrm{~d}, J=3.6 \mathrm{~Hz}, 6 \mathrm{H})$.

${ }^{13}$ C NMR (101 MHz, Chloroform-d): $\delta=149.29,140.83,136.73,135.97,135.07,129.30,129.07,128.55$, $126.21,26.91,21.19,-2.95$.

The characterization data are consistent with that reported in the literature ${ }^{27}$.

\section{Compound 1ap}<smiles>C[N+]([O-])([O-])c1cccc(-c2ccccc2)c1</smiles>

(3'-Methyl-[1,1'-biphenyl]-2-yl)dimethylsilane

Physical state: colorless oil.

${ }^{1}$ H NMR (400 MHz, Chloroform- $d$ ): $\delta=7.66(\mathrm{dd}, J=7.3,0.8 \mathrm{~Hz}, 1 \mathrm{H}), 7.45(\mathrm{td}, J=7.4,1.3 \mathrm{~Hz}, 1 \mathrm{H}), 7.38$ (dd, $J=7.3,6.4 \mathrm{~Hz}, 1 \mathrm{H}), 7.35-7.29(\mathrm{~m}, 2 \mathrm{H}), 7.19$ (t, $J=8.4 \mathrm{~Hz}, 3 \mathrm{H}), 4.36$ (dp, $J=7.4,3.6 \mathrm{~Hz}, 1 \mathrm{H}), 2.43$ (s, 3H), 0.10 (d, $J=3.7 \mathrm{~Hz}, 6 \mathrm{H})$.

${ }^{13}$ C NMR (101 MHz, Chloroform-d): $\delta=149.39,143.60,137.39,135.94,135.11,130.01,129.10,129.05$, 127.76, 126.28, 126.21, 21.41, -2.94.

The characterization data are consistent with that reported in the literature ${ }^{27}$. 


\section{Compound 1aq}<smiles>[N+]#[Sb]c1ccccc1-c1ccc(C(F)(F)F)cc1</smiles>

(4'-Trifluoromethyl-[1,1'-biphenyl]-2-yl)dimethylsilane

Physical state: colorless oil.

${ }^{1}$ H NMR (400 MHz, Chloroform- $\boldsymbol{d}$ ): $\delta=7.74-7.69$ (m, 3H), 7.49 (dd, $\left.J=24.3,8.7 \mathrm{~Hz}, 4 \mathrm{H}\right), 7.32$ (d, $J=$ $7.3 \mathrm{~Hz}, 1 \mathrm{H}), 4.36$ (h, $J=3.5 \mathrm{~Hz}, 1 \mathrm{H}), 0.14(\mathrm{~d}, J=3.2 \mathrm{~Hz}, 6 \mathrm{H})$.

${ }^{13}$ C NMR (101 MHz, Chloroform-d): $\delta=147.71,147.27,135.22,129.55,129.26,129.11,128.98,128.18$, $127.42,127.28,127.06,125.68,124.92,124.88,124.84,-3.06$.

${ }^{19}$ F NMR (376 MHz, Chloroform- $d$ ): $\delta=-62.25$.

The characterization data are consistent with that reported in the literature ${ }^{27}$.

\section{Compound 1ar}<smiles>COc1ccc(-c2ccccc2[N+](=O)[O-])cc1</smiles>

(4'-Methoxy-[1,1'-biphenyl]-2-yl)dimethylsilane

Physical state: colorless oil.

${ }^{1}$ H NMR (400 MHz, Chloroform- $d$ ): $\delta=7.68(\mathrm{~d}, J=7.2 \mathrm{~Hz}, 1 \mathrm{H}), 7.51-7.29(\mathrm{~m}, 5 \mathrm{H}), 7.03-6.95(\mathrm{~m}, 2 \mathrm{H})$, $4.43(\mathrm{dt}, J=7.5,3.7 \mathrm{~Hz}, 1 \mathrm{H}), 3.91(\mathrm{~s}, 3 \mathrm{H}), 0.15(\mathrm{~d}, J=3.8 \mathrm{~Hz}, 6 \mathrm{H})$.

${ }^{13}$ C NMR (101 MHz, Chloroform-d): $\delta=158.86,148.94,136.26,136.09,135.08,130.24,129.38,129.10$, $126.14,113.23,55.25,-3.00$.

The characterization data are consistent with that reported in the literature ${ }^{27}$.

F) General procedure for the preparation of $1 \mathbf{a} a, 1 \mathbf{a b}$ and $1 \mathbf{a d}$.

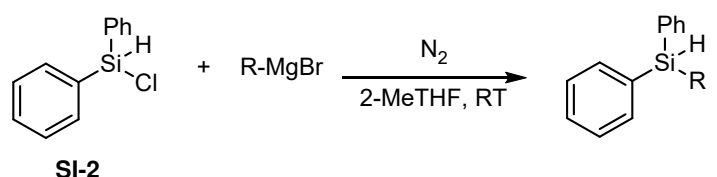

Under $\mathrm{N}_{2}$ atmosphere, aliphatic magnesium bromide (10 mmol, 1 equiv., commercially-available THF solution from $\mathrm{J} \& \mathrm{~K}$ Scientific) was added to $15 \mathrm{~mL}$ anhydrous 2-MeTHF at room temperature. Chlorodiphenylsilane SI-2 (2.73 g, 12.5 mmol, 1.25 equiv.) was then added dropweise, and the mixture was stirred for $6 \mathrm{~h}$. After quenched with saturated aqueous $\mathrm{NH}_{4} \mathrm{Cl}$ solution, the mixture was extracted with diethyl ether, and the combined organic phases are dried over anhydrous $\mathrm{Na}_{2} \mathrm{SO}_{4}$. After evaporation of the solvents under reduced pressure, the crude product was purified by flash column chromatography on silica gel using hexanes as eluent to give the corresponding silane.

\section{Compound 1aa}<smiles>CC[Si](c1ccccc1)(c1ccccc1)c1ccccc1</smiles> 


\section{Ethyldiphenylsilane}

Physical state: colorless oil.

${ }^{1}$ H NMR (400 MHz, Chloroform-d): $\delta=7.59$ - $7.49(\mathrm{~m}, 4 \mathrm{H}), 7.41-7.27(\mathrm{~m}, 6 \mathrm{H}), 4.82(\mathrm{t}, J=3.3 \mathrm{~Hz}, 1 \mathrm{H})$, $1.20-0.97(\mathrm{~m}, 5 \mathrm{H})$.

${ }^{13}$ C NMR (101 MHz, Chloroform-d): $\delta=135.14,134.40,129.48,127.94,8.13,4.13$.

The characterization data are consistent with that reported in the literature ${ }^{38}$.

\section{Compound 1ab}<smiles>[PH3-][Si](c1ccccc1)(c1ccccc1)c1ccccc1</smiles>

\section{Isopropyldiphenylsilane}

Physical state: colorless oil.

${ }^{1}$ H NMR (400 MHz, Chloroform- $d$ ): $\delta=7.65$ - $7.56(\mathrm{~m}, 4 \mathrm{H}), 7.56-7.29(\mathrm{~m}, 6 \mathrm{H}), 4.73(\mathrm{~d}, J=3.2 \mathrm{~Hz}, 1 \mathrm{H})$, 1.50 (ddd, $J=15.0,7.6,3.3 \mathrm{~Hz}, 1 \mathrm{H}), 1.13$ (d, $J=7.4 \mathrm{~Hz}, 6 \mathrm{H}$ ).

${ }^{13}$ C NMR (101 MHz, Chloroform- $\boldsymbol{d}$ ): $\delta=135.48,133.86,129.45,127.88,18.38,11.66$.

The characterization data are consistent with that reported in the literature ${ }^{39}$.

\section{Compound 1ad}<smiles></smiles>

\section{(2-Methylprop-1-en-1-yl)diphenylsilane}

Physical state: colorless oil.

${ }^{1}$ H NMR (400 MHz, Chloroform- $\left.\boldsymbol{d}\right)$ : $\delta=7.60$ - $7.55(\mathrm{~m}, 4 \mathrm{H}), 7.38$ - $7.32(\mathrm{~m}, 6 \mathrm{H}), 5.58$ - $5.50(\mathrm{~m}, 1 \mathrm{H}), 5.23$ $(\mathrm{d}, J=5.1 \mathrm{~Hz}, 1 \mathrm{H}), 1.96(\mathrm{~d}, J=1.1 \mathrm{~Hz}, 3 \mathrm{H}), 1.85(\mathrm{~d}, J=0.5 \mathrm{~Hz}, 3 \mathrm{H})$.

${ }^{13}$ C NMR (101 MHz, Chloroform-d): $\delta$ = 156.93, 135.22, 134.98, 129.35, 127.92, 116.66, 29.46, 23.75 .

The characterization data are consistent with that reported in the literature ${ }^{28}$.

\section{G) Preparation of $\mathbf{1 r}$.}

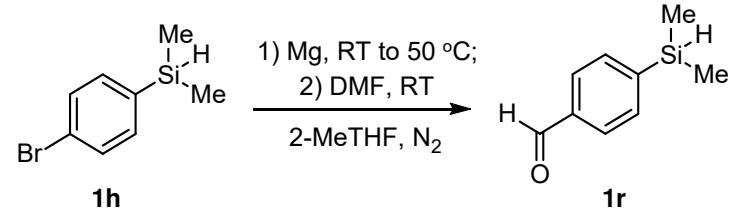

Under $\mathrm{N}_{2}$ atmosphere, magnesium shavings $(0.146 \mathrm{~g}, 6 \mathrm{mmol}, 1.2$ equiv.) and $10 \mathrm{~mL}$ anhydrous 2-MeTHF was added to a $50 \mathrm{~mL}$ two-neck round bottom flask. To this suspension was added 2 drops of 1,2dibromoethane. A solution of $\mathbf{1 h}(1.08 \mathrm{~g}, 5 \mathrm{mmol}, 1.0$ equiv.) in $5 \mathrm{~mL}$ anhydrous 2-MeTHF was then added slowly to the suspension of $\mathrm{Mg}$ at room temperature. After addition, the resulting mixture was stirred at 50 ${ }^{\circ} \mathrm{C}$ for $3 \mathrm{~h}$ and then cooled to room temperature. Anhydrous N,N-dimethylformamide $(0.439 \mathrm{~g}, 6 \mathrm{mmol}, 1.2$ equiv.) was added and the mixture was stirred for $6 \mathrm{~h}$. After quenched with saturated aqueous $\mathrm{NH}_{4} \mathrm{Cl}$ solution, the mixture was extracted with EA, and the combined organic phases are dried over anhydrous $\mathrm{Na}_{2} \mathrm{SO}_{4}$. After evaporation of the solvents under reduced pressure, the crude product was purified by flash column chromatography on silica gel using $\mathrm{EA} / \mathrm{PE}=1: 10$ as eluent to give compound $\mathbf{1 r}$. 


\section{Compound 1r}<smiles>N#[As]c1ccc(C=O)cc1</smiles>

\section{4-(Dimethylsilyl)benzaldehyde}

Physical state: colorless oil.

${ }^{1}$ H NMR (400 MHz, Chloroform- $\boldsymbol{d}$ ): $\delta=10.03(\mathrm{~s}, 1 \mathrm{H}), 7.85(\mathrm{~d}, J=7.3 \mathrm{~Hz}, 2 \mathrm{H}), 7.72(\mathrm{~d}, J=7.4 \mathrm{~Hz}, 2 \mathrm{H})$, $4.60-4.29(\mathrm{~m}, 1 \mathrm{H}), 0.39(\mathrm{~d}, J=2.8 \mathrm{~Hz}, 6 \mathrm{H})$.

${ }^{13}$ C NMR (101 MHz, Chloroform- $d$ ): $\delta=192.47,146.03,136.71,134.50,128.65,-4.11$.

The characterization data are consistent with that reported in the literature ${ }^{40}$.

\section{H) Preparation of $1 a u^{28}$}

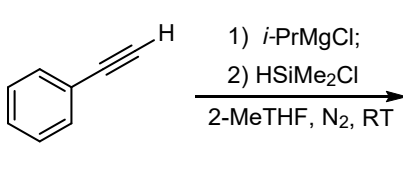

SI-3

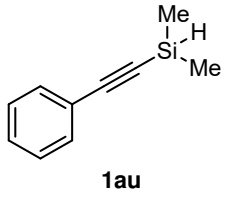

1au

Under $\mathrm{N}_{2}$ atmosphere, $i$ - $\mathrm{PrMgCl}$ ( 24 mmol, 1.2 equiv., commercially-available THF solution from $\mathrm{J} \& \mathrm{~K}$ Scientific) was slowly added to a solution of phenylacetylene SI-3 (2.04 g, $20 \mathrm{mmol}, 1$ equiv.) in $30 \mathrm{~mL}$ anhydrous 2-MeTHF at room temperature and stirred for $2 \mathrm{~h}$. Chlorodimethylsilane ( $2.84 \mathrm{~g}, 30 \mathrm{mmol}, 1.5$ equiv.) was added and the mixture and stirred for another $6 \mathrm{~h}$. After quenched with saturated aqueous $\mathrm{NH}_{4} \mathrm{Cl}$ solution, the mixture was extracted with diethyl ether, and the combined organic phases are dried over anhydrous $\mathrm{Na}_{2} \mathrm{SO}_{4}$. After evaporation of the solvents under reduced pressure, the crude product was purified by flash column chromatography using hexanes as eluent on silica gel to give compound 1au.

\section{Compound 1au}<smiles>C[As](C)(C)C#Cc1ccccc1</smiles>

\section{Dimethyl(phenylethynyl)silane}

Physical state: colorless oil.

${ }^{1}$ H NMR (400 MHz, Chloroform- $d$ ): $\delta=7.52(\mathrm{dd}, J=7.1,1.6 \mathrm{~Hz}, 2 \mathrm{H}), 7.35(\mathrm{t}, J=5.8 \mathrm{~Hz}, 3 \mathrm{H}), 4.31$ (dd, $J=7.2,3.6 \mathrm{~Hz}, 1 \mathrm{H}), 0.36(\mathrm{~d}, J=3.7 \mathrm{~Hz}, 6 \mathrm{H})$.

${ }^{13}$ C NMR (101 MHz, Chloroform- $d$ ): $\delta=131.96,128.71,128.22,122.79,106.39,91.06,-2.97$.

The characterization data are consistent with that reported in the literature ${ }^{21}$.

\section{I) Preparation of $1 a z$}

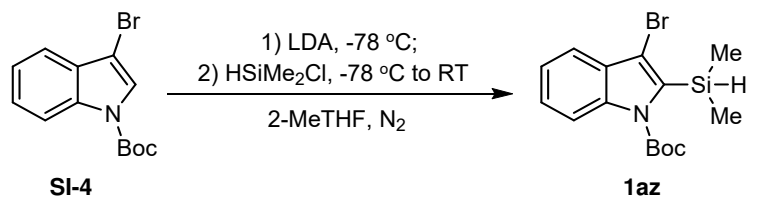

Under $\mathrm{N}_{2}$ atmosphere, a solution of 1-Boc-3-bromoindolen SI-4 (1.78 g, $6 \mathrm{mmol}, 1.0$ equiv.) in $15 \mathrm{~mL}$ anhydrous 2-MeTHF was cooled to $-78^{\circ} \mathrm{C}$. Then lithium diisopropylamide (LDA, $3.3 \mathrm{~mL}$ of a $2.0 \mathrm{M}$ solution 
in heptane/THF/ethylbenzene, $5.5 \mathrm{mmol}, 1.1$ equiv., commercially-available solution from J\&K Scientific) was slowly added and stirred at $-78^{\circ} \mathrm{C}$ for $3 \mathrm{~h}$. Chlorodimethylsilane $(0.852 \mathrm{~g}, 9 \mathrm{mmol}, 1.5$ equiv. $)$ was added and mixture was allowed to warm to room temperature and stirred overnight. After quenched with saturated aqueous $\mathrm{NH}_{4} \mathrm{Cl}$ solution, the mixture was extracted with EA, and the combined organic phases are dried over anhydrous $\mathrm{Na}_{2} \mathrm{SO}_{4}$. After evaporation of the solvents under reduced pressure, the crude product was purified by flash column chromatography on silica gel using EA/PE $=1: 15$ as eluent to give compound $\mathbf{1 a z}$.

\section{Compound 1az}<smiles></smiles>

tert-Butyl 3-bromo-2-(dimethylsilyl)-1H-indole-1-carboxylate

Physical state: white solid.

${ }^{1}$ H NMR (400 MHz, Chloroform-d): $\delta=7.97(\mathrm{~d}, J=8.4 \mathrm{~Hz}, 1 \mathrm{H}), 7.59(\mathrm{~d}, J=7.8 \mathrm{~Hz}, 1 \mathrm{H}), 7.36$ (t, $J=7.7$ $\mathrm{Hz}, 1 \mathrm{H}), 7.30$ (t, $J=7.5 \mathrm{~Hz}, 1 \mathrm{H}), 4.99-4.91(\mathrm{~m}, 1 \mathrm{H}), 1.73(\mathrm{~s}, 9 \mathrm{H}), 0.47$ (d, $J=3.0 \mathrm{~Hz}, 6 \mathrm{H})$.

${ }^{13}$ C NMR (101 MHz, Chloroform-d): $\delta=150.84,136.20,135.98,130.94,125.76,123.04,120.01,115.16$, $111.88,84.79,28.12,-2.96$.

HRMS (ESI-TOF): calcd for $\mathrm{C}_{11} \mathrm{H}_{11} \mathrm{NO}_{2} \mathrm{Si}^{79} \mathrm{Br}^{+}\left[\mathrm{M}-\mathrm{C}\left(\mathrm{CH}_{3}\right)_{3}\right]^{+}$: 295.9737, found: 295.9745; calcd for $\mathrm{C}_{11} \mathrm{H}_{11} \mathrm{NO}_{2} \mathrm{Si}^{81} \mathrm{Br}\left[\mathrm{M}-\mathrm{C}\left(\mathrm{CH}_{3}\right)_{3}\right]^{+}: 297.9717$, found: 297.9726.

\section{J) Preparation of $\mathbf{1} \mathbf{b d}^{41}$.}

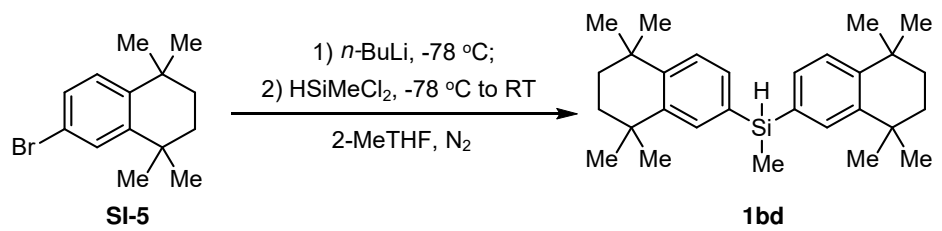

Under $\mathrm{N}_{2}$ atmosphere, a solution of 6-bromo-1,1,4,4-tetramethyl-1,2,3,4-tetrahydronaphthalene SI-5 (2.67 $\mathrm{g}, 10 \mathrm{mmol}, 1$ equiv.) in $30 \mathrm{~mL}$ anhydrous 2-MeTHF was cooled to $-78^{\circ} \mathrm{C}$. Then $n$-BuLi (6.9 mL of a $1.6 \mathrm{M}$ solution in THF, $11 \mathrm{mmol}, 1.1$ equiv.) was slowly added and the mixture was stirred at $-78{ }^{\circ} \mathrm{C}$ for $2.5 \mathrm{~h}$. Dichloromethylsilane $(0.57 \mathrm{~g}, 5 \mathrm{mmol}, 0.5$ equiv.) was added and the mixture was allowed to warm to room temperature and stirred overnight. After quenched with saturated aqueous $\mathrm{NH}_{4} \mathrm{Cl}$ solution, the mixture was extracted with EA, and the combined organic phases are dried over anhydrous $\mathrm{Na}_{2} \mathrm{SO}_{4}$. After evaporation of the solvents under reduced pressure, the crude product was purified by flash column chromatography on silica gel using hexanes as eluent to give compound $\mathbf{1 b d}$.

\section{Compound 1bd}<smiles>CC1(C)CCC(C)(C)C(C)(C)c2ccc([As](C)(C)C)cc21</smiles>

Methylbis(5,5,8,8-tetramethyl-5,6,7,8-tetrahydronaphthalen-2-yl)silane

Physical state: white solid.

${ }^{1}$ H NMR (400 MHz, Chloroform-d): $\delta=7.54$ (s, 2H), 7.33 (s, 4H), 4.90 (q, $\left.J=2.1 \mathrm{~Hz}, 1 \mathrm{H}\right), 1.70$ (s, 8H), 
$1.29(\mathrm{~s}, 24 \mathrm{H}), 0.61(\mathrm{~d}, J=1.8 \mathrm{~Hz}, 3 \mathrm{H})$.

${ }^{13}$ C NMR (101 MHz, Chloroform-d): $\delta=146.16,144.14,133.29,131.94,131.81,126.03,35.17,35.03$, $34.27,34.17,31.88,31.77,-4.78$.

The characterization data are consistent with that reported in the literature ${ }^{41}$.

K) Preparation of $\mathbf{1 b r}$.

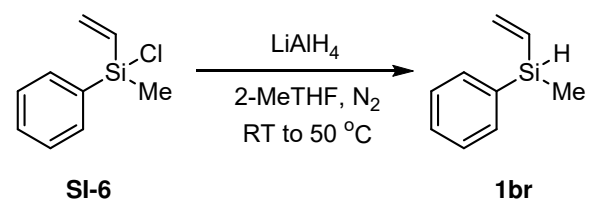

Under $\mathrm{N}_{2}$ atmosphere, chloromethylphenylvinylsilane SI-6 (1.83 g, $10 \mathrm{mmol}, 2.0$ equiv.) was added dropwise to a stirred solution of $\mathrm{LiAlH}_{4}(0.190 \mathrm{~g}, 5 \mathrm{mmol}, 1.0$ equiv. $)$ in $10 \mathrm{~mL}$ anhydrous 2-MeTHF at room temperature. After addition, the resulting mixture was stirred at $50{ }^{\circ} \mathrm{C}$ for $10 \mathrm{~h}$ and then cooled to room temperature. After quenched with aqueous solution of $\mathrm{NaOH}(10 \mathrm{ml}, 10 \mathrm{wt} \%)$, the mixture was extracted with diethyl ether, and the combined organic phases are dried over anhydrous $\mathrm{Na}_{2} \mathrm{SO}_{4}$. After evaporation of the solvents under reduced pressure, the crude product was purified by flash column chromatography on silica gel using hexanes as eluent to give compound $\mathbf{1 b r}$.

\section{Compound 1br}<smiles>C=C[Si](C)(C)c1ccccc1</smiles>

\section{Methyl(phenyl)(vinyl)silane}

Physical state: colorless oil.

${ }^{1}$ H NMR (400 MHz, Chloroform- $\boldsymbol{d}$ ): $\delta=7.60-7.49$ (m, 2H), 7.35 (dd, $\left.J=9.8,4.7 \mathrm{~Hz}, 3 \mathrm{H}\right), 6.28$ (ddd, $J$ $=20.0,14.5,2.7 \mathrm{~Hz}, 1 \mathrm{H}), 6.11(\mathrm{dd}, J=14.5,3.8 \mathrm{~Hz}, 1 \mathrm{H}), 5.85(\mathrm{dd}, J=20.0,3.8 \mathrm{~Hz}, 1 \mathrm{H}), 4.68-4.47(\mathrm{~m}$, $1 \mathrm{H}), 0.42(\mathrm{~d}, J=3.8 \mathrm{~Hz}, 3 \mathrm{H})$.

${ }^{13}$ C NMR (101 MHz, Chloroform- $d$ ): $\delta=135.31,134.78,134.52,129.40,127.91,-5.68$.

The characterization data are consistent with that reported in the literature ${ }^{42}$.

\section{L) Preparation of $\mathbf{1 b}$ and $\boldsymbol{R}-\mathbf{1} \boldsymbol{b}^{43}$.}

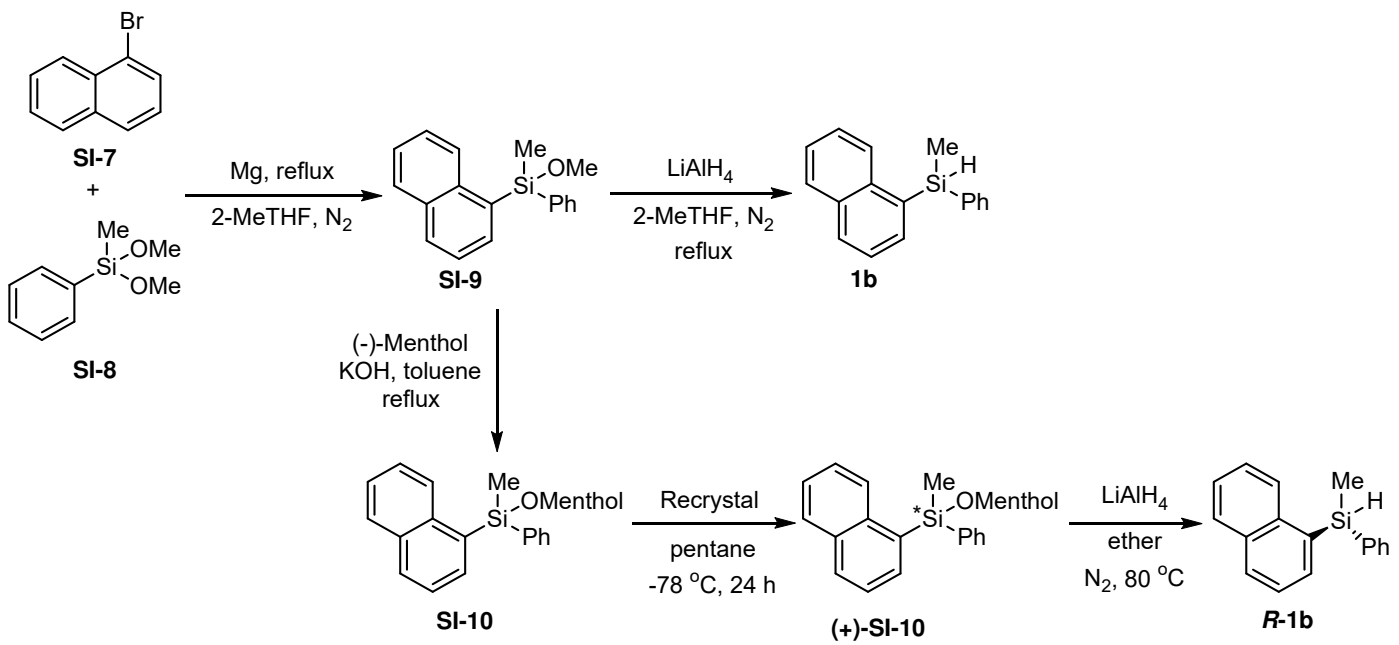


Under $\mathrm{N}_{2}$ atmosphere, magnesium shavings $(2.19 \mathrm{~g}, 90 \mathrm{mmol}, 1.2$ equiv.) and $30 \mathrm{~mL}$ anhydrous 2-MeTHF was added to a $100 \mathrm{~mL}$ two-neck round bottom flask. To this suspension was added 2 drops of 1,2dibromoethane. A solution of 1-bromonaphthalene SI-7 (15.53 g, $75 \mathrm{mmol}, 1.0$ equiv.) in $10 \mathrm{~mL}$ anhydrous 2-MeTHF was then added slowly to the suspension of $\mathrm{Mg}$ at room temperature. After addition, the resulting mixture was stirred under reflux for $12 \mathrm{~h}$ and then cooled to room temperature. A solution of dimethoxymethylphenylsilane SI-8 (13.67 g, 75 mmol, 1.0 equiv.) was added and the mixture was stirred overnight. After quenched with saturated aqueous $\mathrm{NH}_{4} \mathrm{Cl}$ solution, the mixture was extracted with diethyl ether, and the combined organic phases are dried over anhydrous $\mathrm{Na}_{2} \mathrm{SO}_{4}$. After evaporation of the solvents under reduced pressure, the crude product was purified by fractional distillation followed by crystallization to give $18.21 \mathrm{~g}$ (87 \% yield) purified methoxy-methyl(1-naphthyl)phenylsilane SI-9 as colorless crystals.

For synthesis of $\mathbf{1 b}$, a solution of SI-9 $(2.78 \mathrm{~g}, 10 \mathrm{mmol}, 1$ equiv.) in $10 \mathrm{~mL}$ anhydrous 2-MeTHF was added dropwise to a stirred solution of $\mathrm{LiAlH}_{4}(0.380 \mathrm{~g}, 10 \mathrm{mmol}, 1.0$ equiv. $)$ in $10 \mathrm{~mL}$ anhydrous 2-MeTHF under $\mathrm{N}_{2}$ atmosphere at room temperature. After addition, the resulting mixture was stirred at $50{ }^{\circ} \mathrm{C}$ for $12 \mathrm{~h}$ and then cooled to room temperature. After quenched with aqueous solution of $\mathrm{NaOH}(15 \mathrm{ml}, 10 \mathrm{wt} \%)$, the mixture was extracted with diethyl ether, and the combined organic phases are dried over anhydrous $\mathrm{Na}_{2} \mathrm{SO}_{4}$. After evaporation of the solvents under reduced pressure, the crude product was purified by flash column chromatography on silica gel using hexanes as eluent to give compound $\mathbf{1 b}$.

For synthesis of $\boldsymbol{R}-\mathbf{1 b},(-)-$ menthol $(6.25 \mathrm{~g}, 40 \mathrm{mmol}, 1$ equiv.) and solid $\mathrm{NaOH}$ (0.160 g, $4.0 \mathrm{mmol}, 0.1$ equiv.) were added into a solution of methoxymethyl(1-naphthyl)phenylsilane SI-9 (11.14 g, $40 \mathrm{mmol}$ ) in $20 \mathrm{~mL}$ toluene. The reaction mixture was refluxed overnight with a Vigreux column was used to distilled the $\mathrm{MeOH}$-toluene azeotrope. After $12 \mathrm{~h}$, the reaction mixture was cooled down to room temperature and $\mathrm{NaOH}$ was removed by passing the reaction mixture through a short column of silica gel with diethyl ether as an eluent. The filtrate was concentrated to afford a pale yellow oil containing a mixture of the diastereomers. The oil was diluted with twice its volume of pentane and chilled under $-78^{\circ} \mathrm{C}$ for over $24 \mathrm{~h}$. The white solid were formed, followed by recrystallization from pentane to give $1.54 \mathrm{~g}$ (10\% yield) menthoxymethyl(1naphthyl)phenylsilane (+)-SI-10 as white solid.

Under $\mathrm{N}_{2}$ atmosphere, a solution of (+)-SI-10 (1.21 g, 3 mmol, 1 equiv.) in $5 \mathrm{~mL} n$-butyl ether was added dropwise to a stirred solution of $\mathrm{LiAlH}_{4}(0.480 \mathrm{~g}, 12 \mathrm{mmol}, 4.0$ equiv. $)$ in $5 \mathrm{~mL}$ anhydrous 2-MeTHF at room temperature. After addition, the resulting mixture was stirred at $80{ }^{\circ} \mathrm{C}$ for $12 \mathrm{~h}$ and then cooled to room temperature. Water was added to quench the excess hydride species and then concentrated hydrochloric acid was also added, the mixture was extracted with diethyl ether, and the combined organic phases are dried over anhydrous $\mathrm{Na}_{2} \mathrm{SO}_{4}$. After evaporation of the solvents under reduced pressure, the crude product was purified by flash column chromatography on silica gel using hexanes as eluent to give $0.63 \mathrm{~g}$ ( $85 \%$ yield, $95 \%$ ee) compound $\boldsymbol{R}-\mathbf{1 b}$.

\section{Compound 1b}

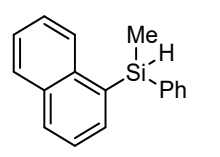

rac-Methyl(naphthalen-1-yl)phenylsilane

Physical state: white solid.

${ }^{1}$ H NMR (400 MHz, Chloroform- $\boldsymbol{d}$ ): $\delta=8.09(\mathrm{~d}, J=8.0 \mathrm{~Hz}, 1 \mathrm{H}), 7.95-7.86(\mathrm{~m}, 2 \mathrm{H}), 7.77(\mathrm{~d}, J=6.7 \mathrm{~Hz}$, 1H), $7.67-7.56(\mathrm{~m}, 2 \mathrm{H}), 7.55-7.33(\mathrm{~m}, 6 \mathrm{H}), 5.39(\mathrm{q}, J=3.8 \mathrm{~Hz}, 1 \mathrm{H}), 0.79(\mathrm{~d}, J=3.8 \mathrm{~Hz}, 3 \mathrm{H})$.

${ }^{13}$ C NMR (101 MHz, Chloroform-d): $\delta=137.07,135.36,135.21,134.88,133.36,133.24,130.46,129.49$, 
$128.87,128.00,127.96,126.05,125.62,125.20,-4.53$.

The characterization data are consistent with that reported in the literature ${ }^{43}$.

\section{Compound $R-1 b$}<smiles>[H][Y5]([H])([H])c1cccc2ccccc12</smiles>

\section{(R)-Methyl(naphthalen-1-yl)phenylsilane}

Physical state: white solid.

${ }^{1}$ H NMR (400 MHz, Chloroform- $\left.\boldsymbol{d}\right): \delta=8.09(\mathrm{~d}, J=8.0 \mathrm{~Hz}, 1 \mathrm{H}), 7.99-7.86(\mathrm{~m}, 2 \mathrm{H}), 7.78(\mathrm{~d}, J=6.7 \mathrm{~Hz}$, $1 \mathrm{H}), 7.65-7.57(\mathrm{~m}, 2 \mathrm{H}), 7.57-7.33(\mathrm{~m}, 6 \mathrm{H}), 5.39(\mathrm{q}, J=3.8 \mathrm{~Hz}, 1 \mathrm{H}), 0.80(\mathrm{~d}, J=3.8 \mathrm{~Hz}, 3 \mathrm{H})$.

${ }^{13}$ C NMR (101 MHz, Chloroform-d): $\delta=137.10,135.39,135.21,134.88,133.37,133.24,130.47,129.49$, $128.87,128.01,127.97,126.04,125.62,125.21,-4.52$.

$[\alpha]_{\mathbf{D}}{ }^{24}=+32.1(\mathrm{c}=1.0$, cyclohexane $)$.

The characterization data are consistent with that reported in the literature ${ }^{43}$.

Chiral HPLC: CHIRALPAK IB $(250 \mathrm{~mm} \times 4.6 \mathrm{~mm} \times 5 \mu \mathrm{m})$; hexanes: $i$-PrOH (100:0), $1 \mathrm{~mL} / \mathrm{min}, 254 \mathrm{~nm}$; $\mathrm{t}_{\mathrm{R}}($ major $)=8.0 \mathrm{~min}, \mathrm{t}_{\mathrm{R}}($ minor $)=9.0 \mathrm{~min}, 95 \%$ ee.

\section{M) Preparation of $\mathbf{1 a - d _ { 1 } { } ^ { 4 4 }}$.}

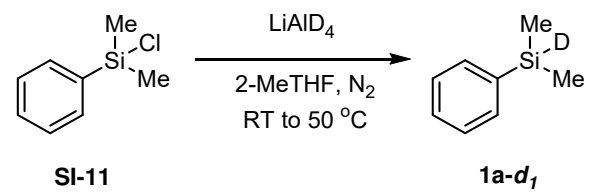

Under $\mathrm{N}_{2}$ atmosphere, chlorodimethylphenylsilane SI-11 (1.71 g, $10 \mathrm{mmol}, 2.0$ equiv.) was added dropwise to a stirred solution of $\mathrm{LiAlD}_{4}(0.210 \mathrm{~g}, 5 \mathrm{mmol}, 1.0$ equiv. $)$ in $10 \mathrm{~mL}$ anhydrous 2-MeTHF at room temperature. After addition, the resulting mixture was stirred at $50{ }^{\circ} \mathrm{C}$ for $10 \mathrm{~h}$ and then cooled to room temperature. After quenched with aqueous solution of $\mathrm{NaOH}(10 \mathrm{ml}, 10 \mathrm{wt} \%)$, the mixture was extracted with diethyl ether, and the combined organic phases are dried over anhydrous $\mathrm{Na}_{2} \mathrm{SO}_{4}$. After evaporation of the solvents under reduced pressure, the crude product was purified by flash column chromatography using hexanes as eluent on silica gel to give compound $\mathbf{1 a}-\boldsymbol{d}_{\boldsymbol{l}}$.

\section{Compound 1a- $d_{1}$}<smiles>C[Si](C)(O)c1ccccc1</smiles>

\section{Dimethylphenylsilane- $d_{1}$}

Physical state: colorless oil.

${ }^{1}$ H NMR (400 MHz, Chloroform- $d$ ): $\delta=7.65-7.54(\mathrm{~m}, 2 \mathrm{H}), 7.40$ (d, $\left.J=4.7 \mathrm{~Hz}, 3 \mathrm{H}\right), 0.39$ (s, 6H).

${ }^{13}$ C NMR (101 MHz, Chloroform-d): $\delta=137.43,133.99,129.17,127.85,-3.88$.

The characterization data are consistent with that reported in the literature ${ }^{44}$. 
General procedure for Co- $\mathrm{N}_{4} @$ NPC-catalyzed selective oxidation of hydrosilanes under optimized reaction conditions in batch reactors (GP A)

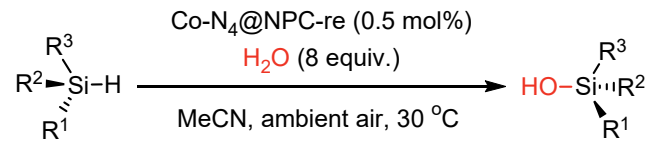

Silane ( $1 \mathrm{mmol}, 1$ equiv.) and water ( $145 \mu \mathrm{L}, 8 \mathrm{mmol}, 8$ equiv.) were mixed with $\mathrm{MeCN}(3 \mathrm{~mL})$ in a $15 \mathrm{~mL}$ oven-dried round-bottom tube with steady magnetic stirring $(800 \mathrm{rpm})$ at $30^{\circ} \mathrm{C}$ under an ambient atmosphere of air. The reaction was started by the addition of Co- $\mathrm{N}_{4} @ \mathrm{NPC}-\mathrm{re}(0.5 \mathrm{~mol} \% \mathrm{Co})$ catalyst and the mixture was allowed to stir for given reaction time. After completion of the reaction (monitored by GC and/or TLC), the catalyst was removed by filtration and washed with EA $(3 \times 5 \mathrm{~mL})$. The combined organics were washed with brine, dried over anhydrous $\mathrm{Na}_{2} \mathrm{SO}_{4}$ and solvent was removed under reduced pressure. In most cases, correspond silanol was obtained without further purification. If necessary, the product was purified via flash column chromatography or vacuum distillation.

\section{Compound 2a}<smiles>C[Si](O)(O)c1ccccc1</smiles>

\section{Dimethyl(phenyl)silanol}

The reaction was conducted for $0.5 \mathrm{~h}$ following GP A on $1 \mathrm{mmol}$ scale with $1 \mathrm{a}$. After dried over anhydrous $\mathrm{Na}_{2} \mathrm{SO}_{4}$ and evaporated the solvent, the title compound $2 \mathrm{a}$ was obtained in $97 \%$ yield (147 mg) without further purification.

Physical state: colorless oil.

${ }^{1}$ H NMR (400 MHz, Chloroform- $\boldsymbol{d}$ ): $\delta=7.65-7.52(\mathrm{~m}, 2 \mathrm{H}), 7.43-7.31(\mathrm{~m}, 3 \mathrm{H}), 2.10(\mathrm{br} \mathrm{s}, 1 \mathrm{H}), 0.39$ (s, $6 \mathrm{H})$.

${ }^{13}$ C NMR (101 MHz, Chloroform- $\boldsymbol{d}$ ): $\delta=139.09,133.03,129.53,127.81,-0.10$.

The characterization data are consistent with that reported in the literature ${ }^{21}$.

\section{Compound 2b}<smiles>O=S(=O)(O)c1cccc2ccccc12</smiles>

\section{rac-Methyl(naphthalen-1-yl)phenylsilanol}

The reaction was conducted for $3 \mathrm{~h}$ following GP A on $1 \mathrm{mmol}$ scale with $\mathbf{1 b}$. After dried over anhydrous $\mathrm{Na}_{2} \mathrm{SO}_{4}$ and evaporated the solvent, the title compound $\mathbf{2} \mathbf{b}$ was obtained in $97 \%$ yield $(256 \mathrm{mg}$ ) without further purification.

Physical state: white solid.

${ }^{1}$ H NMR (400 MHz, Chloroform- $\left.d\right): \delta=8.01(\mathrm{~d}, J=8.2 \mathrm{~Hz}, 1 \mathrm{H}), 7.78(\mathrm{~d}, J=8.2 \mathrm{~Hz}, 1 \mathrm{H}), 7.73(\mathrm{~d}, J=8.1$ $\mathrm{Hz}, 1 \mathrm{H}), 7.67(\mathrm{~d}, J=6.7 \mathrm{~Hz}, 1 \mathrm{H}), 7.48(\mathrm{~d}, J=7.7 \mathrm{~Hz}, 2 \mathrm{H}), 7.26(\mathrm{~m}, 6 \mathrm{H}), 2.99(\mathrm{br} \mathrm{s}, 1 \mathrm{H}), 0.65(\mathrm{~s}, 3 \mathrm{H})$.

${ }^{13}$ C NMR (101 MHz, Chloroform-d): $\delta=137.60,136.70,134.74,134.65,133.91,133.30,130.66,129.77$, $128.84,128.41,127.91,125.95,125.47,124.99,-0.10$.

The characterization data are consistent with that reported in the literature ${ }^{28}$. 


\section{Compound $\boldsymbol{R}-\mathbf{2 b}$}

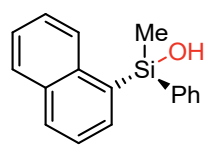

\section{$R$-Methyl(naphthalen-1-yl)phenylsilanol}

The reaction was conducted for $3 \mathrm{~h}$ following GPA on $1 \mathrm{mmol}$ scale with $\boldsymbol{R}-\mathbf{1 b}$. After dried over anhydrous $\mathrm{Na}_{2} \mathrm{SO}_{4}$ and evaporated the solvent, the title compound $\boldsymbol{R}-\mathbf{2} \mathbf{b}$ was obtained in $98 \%$ yield $(258 \mathrm{mg}$ ) without further purification.

Physical state: white solid.

${ }^{1}$ H NMR (400 MHz, Chloroform- $d$ ): $\delta=8.10(\mathrm{~d}, J=8.2 \mathrm{~Hz}, 1 \mathrm{H}), 7.90(\mathrm{~d}, J=8.2 \mathrm{~Hz}, 1 \mathrm{H}), 7.85$ (d, $J=9.4$ $\mathrm{Hz}, 1 \mathrm{H}), 7.78$ (d, $J=6.8 \mathrm{~Hz}, 1 \mathrm{H}), 7.60$ (d, $J=6.1 \mathrm{~Hz}, 2 \mathrm{H}), 7.49-7.29$ (m, 6H), $2.44(\mathrm{br} \mathrm{s}), 0.79(\mathrm{~s}, 3 \mathrm{H})$.

${ }^{13}$ C NMR (101 MHz, Chloroform-d): $\delta=137.65,136.75,134.76,134.67,133.94,133.38,130.75,129.87$, $128.90,128.42,127.98,126.02,125.53,125.02,-0.00$.

$[\alpha]_{\mathbf{D}}{ }^{24}=-15.6(\mathrm{c}=1.0$, diethyl ether $)$.

The characterization data are consistent with that reported in the literature ${ }^{28}$.

Chiral HPLC: CHIRALPAK IB $(250 \mathrm{~mm} \times 4.6 \mathrm{~mm} \times 5 \mu \mathrm{m})$; hexanes: $i$-PrOH $(99: 1), 1 \mathrm{~mL} / \mathrm{min}, 254 \mathrm{~nm}$; $t_{R}($ major $)=28.8 \mathrm{~min}, t_{R}($ minor $)=30.6 \mathrm{~min}, 92 \%$ ee .

\section{Compound 2c}<smiles>Cc1ccc([As](C)(=O)(O)O[Na])cc1</smiles>

\section{(4-Methylphenyl)dimethylsilanol}

The reaction was conducted for $0.5 \mathrm{~h}$ following GP A on $1 \mathrm{mmol}$ scale with $1 \mathbf{c}$. After dried over anhydrous $\mathrm{Na}_{2} \mathrm{SO}_{4}$ and evaporated the solvent, the title compound $2 \mathrm{c}$ was obtained in $97 \%$ yield $(161 \mathrm{mg})$ without further purification.

Physical state: colorless oil.

${ }^{1}$ H NMR (400 MHz, Chloroform-d): $\delta=7.51(\mathrm{~d}, J=7.8 \mathrm{~Hz}, 2 \mathrm{H}), 7.23(\mathrm{~d}, J=7.7 \mathrm{~Hz}, 2 \mathrm{H}), 2.39$ (s, 3H), 2.12 (br s, $1 \mathrm{H}), 0.41(\mathrm{~s}, 6 \mathrm{H})$.

${ }^{13}$ C NMR (101 MHz, Chloroform- $\boldsymbol{d}$ ): $\delta=139.55,135.51,133.09,128.68,21.48,-0.01$.

The characterization data are consistent with that reported in the literature ${ }^{45}$.

\section{Compound 2d}<smiles>O=S(=O)(O)c1ccc(Br)cc1</smiles>

\section{(4-tert-Butylphenyl)dimethylsilanol}

The reaction was conducted for $1.5 \mathrm{~h}$ following GPA on $1 \mathrm{mmol}$ scale with $\mathbf{1 d}$. After dried over anhydrous $\mathrm{Na}_{2} \mathrm{SO}_{4}$ and evaporated the solvent, the title compound $\mathbf{2 d}$ was obtained in $97 \%$ yield (203 mg) without further purification.

Physical state: white solid.

${ }^{1}$ H NMR (400 MHz, Chloroform- $d$ ): $\delta=7.56(\mathrm{dd}, J=8.0,1.7 \mathrm{~Hz}, 2 \mathrm{H}), 7.44(\mathrm{dd}, J=8.0,2.0 \mathrm{~Hz}, 2 \mathrm{H}), 2.07$ (br s, $1 \mathrm{H}), 1.35$ (d, $J=4.0 \mathrm{~Hz}, 9 \mathrm{H}), 0.41(\mathrm{~s}, 6 \mathrm{H})$.

${ }^{13}$ C NMR (101 MHz, Chloroform-d): $\delta=152.70,135.63,133.01,124.86,31.20,-0.02$. 
HRMS (ESI-TOF): calcd for $\mathrm{C}_{12} \mathrm{H}_{20} \mathrm{OSiNa}^{+}[\mathrm{M}+\mathrm{Na}]^{+}$: 231.1176, found: 231.1175 .

\section{Compound 2e}<smiles>Cc1ccc([As](C)(=O)(O)O[Na])cc1</smiles>

\section{(4-Trimethylphenyl)dimethylsilylsilanol}

The reaction was conducted for $0.5 \mathrm{~h}$ following GPA on $1 \mathrm{mmol}$ scale with 1e. After dried over anhydrous $\mathrm{Na}_{2} \mathrm{SO}_{4}$ and evaporated the solvent, the title compound $2 \mathbf{e}$ was obtained in $98 \%$ yield $(220 \mathrm{mg})$ without further purification.

Physical state: white solid.

${ }^{1}$ H NMR (400 MHz, Chloroform-d): $\delta=7.65-7.56$ (m, 4H), 2.13 (br s, 1H), 0.42 (s, 6H), 0.30 (s, 9H).

${ }^{13}$ C NMR (101 MHz, Chloroform-d): $\delta=142.16,139.50,132.75,132.28,-0.08,-1.25$.

The characterization data are consistent with that reported in the literature ${ }^{46}$.

\section{Compound 2f}<smiles>O=S(=O)(O)c1ccc(F)cc1</smiles>

\section{(4-Fluorophenyl)dimethylsilanol}

The reaction was conducted for $0.5 \mathrm{~h}$ following GP A on $1 \mathrm{mmol}$ scale with 1 f. After dried over anhydrous $\mathrm{Na}_{2} \mathrm{SO}_{4}$ and evaporated the solvent, the title compound $2 \mathrm{f}$ was obtained in $99 \%$ yield (168 $\mathrm{mg}$ ) without further purification.

Physical state: colorless oil.

${ }^{1} \mathrm{H}$ NMR (400 MHz, Chloroform- $\left.d\right): \delta=7.51(\mathrm{t}, J=7.1 \mathrm{~Hz}, 2 \mathrm{H}), 7.02(\mathrm{t}, J=8.8 \mathrm{~Hz}, 2 \mathrm{H}), 2.56(\mathrm{br} \mathrm{s}, 1 \mathrm{H})$, $0.34(\mathrm{~s}, 6 \mathrm{H})$.

${ }^{13}$ C NMR (101 MHz, Chloroform-d): $\delta=165.20,162.73,135.11,135.03,115.07,114.88,0.03$.

${ }^{19}$ F NMR (377 MHz, Chloroform- $d$ ): $\delta=-111.02$.

The characterization data are consistent with that reported in the literature ${ }^{47}$.

\section{Compound 2g}<smiles>O=[As](O)(O)c1ccc(Cl)cc1</smiles>

\section{(4-Chlorophenyl)dimethylsilanol}

The reaction was conducted for $1 \mathrm{~h}$ following GP A on $1 \mathrm{mmol}$ scale with $\mathbf{1 g}$. After dried over anhydrous $\mathrm{Na}_{2} \mathrm{SO}_{4}$ and evaporated the solvent, the title compound $2 \mathrm{~g}$ was obtained in $97 \%$ yield $(181 \mathrm{mg})$ without further purification.

Physical state: colorless oil.

${ }^{1}$ H NMR (400 MHz, Chloroform- $d$ ): $\delta=7.51(\mathrm{~d}, J=7.6 \mathrm{~Hz}, 2 \mathrm{H}), 7.35(\mathrm{~d}, J=7.6 \mathrm{~Hz}, 2 \mathrm{H}), 1.92(\mathrm{br} \mathrm{s}, 1 \mathrm{H})$, 0.39 (s, 3H).

${ }^{13}$ C NMR (101 MHz, Chloroform- $\boldsymbol{d}$ ): $\delta=137.26,135.86,134.42,128.06,-0.07$.

The characterization data are consistent with that reported in the literature ${ }^{21}$. 


\section{Compound 2h}<smiles>O=S(=O)(O)c1ccc(Br)cc1</smiles>

\section{(4-Bromophenyl)dimethylsilanol}

The reaction was conducted for $1 \mathrm{~h}$ following GP A on $1 \mathrm{mmol}$ scale with $\mathbf{1 h}$. After dried over anhydrous $\mathrm{Na}_{2} \mathrm{SO}_{4}$ and evaporated the solvent, the title compound $\mathbf{2 h}$ was obtained in $98 \%$ yield $(226 \mathrm{mg})$ without further purification.

Physical state: colorless oil.

${ }^{1} \mathrm{H}$ NMR (400 MHz, Chloroform- $d$ ): $\delta=7.51(\mathrm{~d}, J=7.6 \mathrm{~Hz}, 2 \mathrm{H}), 7.43(\mathrm{~d}, J=7.5 \mathrm{~Hz}, 2 \mathrm{H}), 2.13(\mathrm{br} \mathrm{s}, 1 \mathrm{H})$, $0.38(\mathrm{~s}, 6 \mathrm{H})$.

${ }^{13}$ C NMR (101 MHz, Chloroform- $d$ ): $\delta=137.82,134.64,131.04,124.46,-0.03$.

The characterization data are consistent with that reported in the literature ${ }^{48}$.

\section{Compound 2i}<smiles>O=S(=O)(O)c1ccc(C(F)(F)F)cc1</smiles>

\section{(4-Trifluoromethylphenyl)dimethylsilanol}

The reaction was conducted for $4 \mathrm{~h}$ following GP A on $1 \mathrm{mmol}$ scale with 1i. After dried over anhydrous $\mathrm{Na}_{2} \mathrm{SO}_{4}$ and evaporated the solvent, the title compound $2 \mathbf{i}$ was obtained in $98 \%$ yield $(216 \mathrm{mg}$ ) without further purification.

Physical state: colorless oil.

${ }^{1} \mathrm{H}$ NMR (400 MHz, Chloroform- $d$ ): $\delta=7.70(\mathrm{~d}, J=7.6 \mathrm{~Hz}, 2 \mathrm{H}), 7.62(\mathrm{~d}, J=7.7 \mathrm{~Hz}, 2 \mathrm{H}), 2.58(\mathrm{br} \mathrm{s}, 1 \mathrm{H})$, $0.42(\mathrm{~s}, 6 \mathrm{H})$.

${ }^{13}$ C NMR (101 MHz, Chloroform-d): $\delta=143.79,133.34,133.20,131.98,131.66,131.34,131.02,125.49$, 124.46, 124.42, 124.38, 124.34, 122.79, 0.05 .

${ }^{19}$ F NMR (376 MHz, Chloroform- $d$ ): $\delta=-62.99$.

The characterization data are consistent with that reported in the literature ${ }^{21}$.

\section{Compound 2j}<smiles>COc1ccc([As](C)(=O)(O)O[Na])cc1</smiles>

\section{(4-Methoxyphenyl)dimethylsilanol}

The reaction was conducted for $2 \mathrm{~h}$ following GP A on $1 \mathrm{mmol}$ scale with $\mathbf{1 j}$. After dried over anhydrous $\mathrm{Na}_{2} \mathrm{SO}_{4}$ and evaporated the solvent, the title compound $\mathbf{2} \mathbf{j}$ was obtained in $96 \%$ yield $(175 \mathrm{mg}$ ) without further purification.

Physical state: colorless oil.

${ }^{1} \mathrm{H}$ NMR (400 MHz, Chloroform- $\boldsymbol{d}$ ): $\delta=7.49(\mathrm{~d}, J=8.6 \mathrm{~Hz}, 2 \mathrm{H}), 6.90(\mathrm{~d}, J=8.6 \mathrm{~Hz}, 2 \mathrm{H}), 3.79(\mathrm{~s}, 3 \mathrm{H})$, 2.44 (br s, 1H), 0.34 (s, 6H).

${ }^{13}$ C NMR (101 MHz, Chloroform- $d$ ): $\delta=160.71,134.59,130.19,113.53,54.98,0.03$.

The characterization data are consistent with that reported in the literature ${ }^{21}$. 


\section{Compound 2k}<smiles>COc1ccc(S(=O)(=O)O)cc1</smiles>

\section{(4-Phenoxyphenyl)dimethylsilanol}

The reaction was conducted for $4 \mathrm{~h}$ following GP A on $1 \mathrm{mmol}$ scale with $\mathbf{1 k}$. After dried over anhydrous $\mathrm{Na}_{2} \mathrm{SO}_{4}$ and evaporated the solvent, the title compound $\mathbf{2} \mathbf{k}$ was obtained in $97 \%$ yield $(237 \mathrm{mg})$ without further purification.

Physical state: colorless oil.

${ }^{1}$ H NMR (400 MHz, Chloroform-d): $\delta=7.56(\mathrm{~d}, J=7.6 \mathrm{~Hz}, 2 \mathrm{H}), 7.36(\mathrm{t}, J=7.6 \mathrm{~Hz}, 2 \mathrm{H}), 7.15(\mathrm{t}, J=7.3$ $\mathrm{Hz}, 1 \mathrm{H}), 7.04$ (t, $J=9.3 \mathrm{~Hz}, 4 \mathrm{H}), 2.77$ (br s, 1H), 0.40 (s, 6H).

${ }^{13}$ C NMR (101 MHz, Chloroform-d): $\delta=158.73,156.60,134.77,133.11,129.74,123.52,119.28,117.94$, 0.04 .

HRMS (ESI-TOF): calcd for $\mathrm{C}_{14} \mathrm{H}_{15} \mathrm{O}_{2} \mathrm{Si}^{-}[\mathrm{M}-\mathrm{H}]^{-}: 243.0836$, found: 243.0842 .

\section{Compound 21}

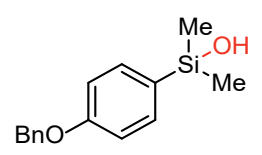

\section{(4-(Benzyloxy)phenyl)dimethylsilanol}

The reaction was conducted for $4 \mathrm{~h}$ following GPA on $1 \mathrm{mmol}$ scale 1l. After dried over anhydrous $\mathrm{Na}_{2} \mathrm{SO}_{4}$ and evaporated the solvent, the title compound $2 \mathbf{I}$ was obtained in $98 \%$ yield $(253 \mathrm{mg}$ ) without further purification.

Physical state: white solid.

${ }^{1} \mathrm{H}$ NMR (400 MHz, Chloroform- $d$ ): $\delta=7.55(\mathrm{~d}, J=8.5 \mathrm{~Hz}, 2 \mathrm{H}), 7.49-7.34(\mathrm{~m}, 5 \mathrm{H}), 7.03(\mathrm{~d}, J=8.5 \mathrm{~Hz}$, $2 \mathrm{H}), 5.11$ (s, 2H), 2.33 (br s, 1H), 0.41 (s, 6H).

${ }^{13}$ C NMR (101 MHz, Chloroform-d): $\delta=159.98,136.84,134.62,130.48,128.55,127.93,127.40,114.44$, $69.68,0.03$.

HRMS (ESI-TOF): calcd for $\mathrm{C}_{15} \mathrm{H}_{17} \mathrm{O}_{2} \mathrm{Si}^{-}[\mathrm{M}-\mathrm{H}]^{-}:$: 257.0992, found: 257.0987 .

\section{Compound 2m}<smiles>COc1ccc(S(=O)(=O)O[Na])cc1</smiles>

\section{4-(Hydroxydimethylsilyl)phenyl 4-methylbenzenesulfonate}

The reaction was conducted for $5 \mathrm{~h}$ following GP A on $1 \mathrm{mmol}$ scale with $\mathbf{1 m}$. After dried over anhydrous $\mathrm{Na}_{2} \mathrm{SO}_{4}$ and evaporated the solvent, the title compound $\mathbf{2 m}$ was obtained in $98 \%$ yield $(317 \mathrm{mg}$ ) without further purification.

Physical state: colorless viscous oil..

${ }^{1}$ H NMR (400 MHz, Chloroform- $d$ ): $\delta=7.72(\mathrm{~d}, J=8.3 \mathrm{~Hz}, 2 \mathrm{H}), 7.51(\mathrm{~d}, J=8.5 \mathrm{~Hz}, 2 \mathrm{H}), 7.33(\mathrm{~d}, J=8.0$ $\mathrm{Hz}, 2 \mathrm{H}), 6.99$ (d, $J=8.4 \mathrm{~Hz}, 2 \mathrm{H}), 2.46$ (s, 3H), 2.32 (br s, 1H), 0.37 (s, 6H).

${ }^{13}$ C NMR (101 MHz, Chloroform-d): $\delta=150.69,145.36,138.31,134.51,132.43,129.74,128.40,121.67$, $21.65,-0.03$.

HRMS (ESI-TOF): calcd for $\mathrm{C}_{15} \mathrm{H}_{17} \mathrm{SO}_{4} \mathrm{Si}^{-}[\mathrm{M}-\mathrm{H}]^{-}$: 321.0611 , found: 321.0629 . 


\section{Compound 2n}<smiles>N#[W]c1ccc(S(=O)(=O)O)cc1</smiles>

\section{4-(Hydroxydimethylsilyl)benzonitrile}

The reaction was conducted for $6 \mathrm{~h}$ following GP A on $1 \mathrm{mmol}$ scale with $\mathbf{1 n}$. After purification by flash column chromatography (silica gel, EA/PE $=1: 10)$, the title compound $\mathbf{2 n}$ was obtained in $93 \%$ yield $(165$ $\mathrm{mg})$.

Physical state: colorless oil.

${ }^{1} \mathrm{H}$ NMR (400 MHz, Chloroform- $d$ ): $\delta=7.69(\mathrm{~d}, J=8.2 \mathrm{~Hz}, 2 \mathrm{H}), 7.59(\mathrm{~d}, J=8.2 \mathrm{~Hz}, 2 \mathrm{H}), 3.53(\mathrm{br} \mathrm{s}, 1 \mathrm{H})$, $0.40(\mathrm{~s}, 6 \mathrm{H})$.

${ }^{13}$ C NMR (101 MHz, Chloroform- $d$ ): $\delta=145.99,133.47,130.93,118.73,112.51,-0.26$.

The characterization data are consistent with that reported in the literature ${ }^{45}$.

\section{Compound 20}<smiles></smiles>

\section{Methyl-4-(hydroxydimethylsilyl)benzoate}

The reaction was conducted for $4 \mathrm{~h}$ following GP A on $1 \mathrm{mmol}$ scale with 1o. After purification by flash column chromatography (silica gel, EA/PE $=1: 10$ ), the title compound 20 was obtained in $94 \%$ yield (198 $\mathrm{mg})$.

Physical state: colorless oil.

${ }^{1}$ H NMR (400 MHz, Chloroform- $\left.\boldsymbol{d}\right): \delta=7.95(\mathrm{~d}, J=8.2 \mathrm{~Hz}, 2 \mathrm{H}), 7.62(\mathrm{~d}, J=8.2 \mathrm{~Hz}, 2 \mathrm{H}), 3.89(\mathrm{~s}, 3 \mathrm{H})$, 3.42 (br s, $1 \mathrm{H}), 0.38$ (s, 6H).

${ }^{13}$ C NMR (101 MHz, Chloroform-d): $\delta=167.33,145.38,132.98,130.59,128.52,52.11,-0.16$.

The characterization data are consistent with that reported in the literature ${ }^{49}$.

\section{Compound 2p}<smiles>Cc1ccc([As](C)(=O)(O)O[Na])cc1</smiles>

\section{(4-Methylthiophenyl)dimethylsilanol}

The reaction was conducted for $3 \mathrm{~h}$ following GP A on $1 \mathrm{mmol}$ scale with $\mathbf{1 p}$. After dried over anhydrous $\mathrm{Na}_{2} \mathrm{SO}_{4}$ and evaporated the solvent, the title compound $\mathbf{2 p}$ was obtained in $98 \%$ yield (194 $\mathrm{mg}$ ) without further purification.

Physical state: colorless oil.

${ }^{1}$ H NMR (400 MHz, Chloroform- $d$ ): $\delta=7.51(\mathrm{dd}, J=7.9,1.1 \mathrm{~Hz}, 2 \mathrm{H}), 7.28(\mathrm{~d}, J=7.5 \mathrm{~Hz}, 2 \mathrm{H}), 2.51$ (s, $3 \mathrm{H}), 2.40$ (br s, $1 \mathrm{H}), 0.40$ (s, 6H).

${ }^{13}$ C NMR (101 MHz, Chloroform-d): $\delta=140.46,135.02,133.43,125.56,15.22,-0.04$.

HRMS (ESI-TOF): calcd for $\mathrm{C}_{9} \mathrm{H}_{13} \mathrm{SOSi}^{-}[\mathrm{M}-\mathrm{H}]^{-}: 197.0451$, found: 197.0465 . 


\section{Compound 2q}<smiles>O=[N+]([O-])c1ccc(S(=O)(=O)O)cc1</smiles>

\section{(4-(Dimethylamino)phenyl)dimethylsilanol}

The reaction was conducted for $10 \mathrm{~h}$ following GPA on $1 \mathrm{mmol}$ scale with 1q. After purification by flash column chromatography (silica gel, EA/PE/NEt ${ }_{3}=1: 3: 0.01$ ), the title compound $\mathbf{2 q}$ was obtained in $95 \%$ yield (185 mg).

Physical state: white solid.

${ }^{1}$ H NMR (400 MHz, Chloroform- $d$ ): $\delta=7.53(\mathrm{~d}, J=7.9 \mathrm{~Hz}, 2 \mathrm{H}), 6.81(\mathrm{~d}, J=8.1 \mathrm{~Hz}, 2 \mathrm{H}), 3.02(\mathrm{~s}, 6 \mathrm{H})$, 2.63 (br s, $1 \mathrm{H}), 0.42(\mathrm{~s}, 6 \mathrm{H})$.

${ }^{13}$ C NMR (101 MHz, Chloroform- $d$ ): $\delta=151.36,134.22,128.99,124.63,111.91,40.16,0.00$.

The characterization data are consistent with that reported in the literature ${ }^{50}$.

\section{Compound 2r}<smiles>O=Cc1ccc(S(=O)(=O)O)cc1</smiles>

\section{4-(Hydroxydimethylsilyl)benzaldehyde}

The reaction was conducted for $4 \mathrm{~h}$ following GP A on $1 \mathrm{mmol}$ scale with $1 \mathbf{r}$. After purification by flash column chromatography (silica gel, $\mathrm{EA} / \mathrm{PE}=1: 9)$, the title compound $\mathbf{2 r}$ was obtained in $96 \%$ yield $(173$ $\mathrm{mg})$.

Physical state: colorless oil.

${ }^{1}$ H NMR (400 MHz, Chloroform- $d$ ): $\delta=9.99(\mathrm{~s}, 1 \mathrm{H}), 7.84(\mathrm{~d}, J=6.4 \mathrm{~Hz}, 2 \mathrm{H}), 7.75(\mathrm{~d}, J=7.4 \mathrm{~Hz}, 2 \mathrm{H})$, 2.85 (br s, $1 \mathrm{H}), 0.43(\mathrm{~s}, 6 \mathrm{H})$.

${ }^{13}$ C NMR (101 MHz, Chloroform- $\boldsymbol{d}$ ): $\delta=192.80,134.64,133.57,132.49,128.77,-0.08$.

HRMS (ESI-TOF): calcd for $\mathrm{C}_{9} \mathrm{H}_{11} \mathrm{O}_{2} \mathrm{Si}^{-}[\mathrm{M}-\mathrm{H}]^{-}:$: 179.0523, found: 179.0523 .

\section{Compound 2s}<smiles>Cc1ccccc1S(=O)(=O)O</smiles>

\section{(2-Methylphenyl)dimethylsilanol}

The reaction was conducted for $1 \mathrm{~h}$ following GP A on $1 \mathrm{mmol}$ scale with $1 \mathrm{~s}$. After dried over anhydrous $\mathrm{Na}_{2} \mathrm{SO}_{4}$ and evaporated the solvent, the title compound $2 \mathrm{~s}$ was obtained in $98 \%$ yield (163 $\mathrm{mg}$ ) without further purification.

Physical state: colorless oil.

${ }^{1}$ H NMR (400 MHz, Chloroform- $d$ ): $\delta=7.56(\mathrm{~d}, J=7.2 \mathrm{~Hz}, 1 \mathrm{H}), 7.32(\mathrm{t}, J=7.4 \mathrm{~Hz}, 1 \mathrm{H}), 7.20$ (t, $J=7.8$ $\mathrm{Hz}, 2 \mathrm{H}), 2.52$ (s, 3H), 2.36 (br s, 1H), 0.46 (s, 6H).

${ }^{13}$ C NMR (101 MHz, Chloroform-d): $\delta=143.23,137.33,134.08,129.86,129.79,124.91,22.72,0.99$.

The characterization data are consistent with that reported in the literature ${ }^{51}$. 


\section{Compound 2t}<smiles>Cc1cccc(S(=O)(=O)O[Na])c1</smiles>

\section{(3-Methylphenyl)dimethylsilanol}

The reaction was conducted for $0.5 \mathrm{~h}$ following GP A on $1 \mathrm{mmol}$ scale with $\mathbf{1 t}$. After dried over anhydrous $\mathrm{Na}_{2} \mathrm{SO}_{4}$ and evaporated the solvent, the title compound $\mathbf{2 t}$ was obtained in $97 \%$ yield $(161 \mathrm{mg}$ ) without further purification.

Physical state: colorless oil.

${ }^{1}$ H NMR (400 MHz, Chloroform- $d$ ): $\delta=7.39(\mathrm{~d}, J=10.7 \mathrm{~Hz}, 2 \mathrm{H}), 7.28(\mathrm{t}, J=7.3 \mathrm{~Hz}, 1 \mathrm{H}), 7.21$ (d, $J=$ $7.5 \mathrm{~Hz}, 1 \mathrm{H}), 2.36$ (s, 3H), 2.09 (br s, 1H), 0.39 (s, 6H).

${ }^{13}$ C NMR (101 MHz, Chloroform-d): $\delta$ = 138.95, 137.22, 133.66, 130.38, 130.02, 127.81, 21.44, -0.05 .

The characterization data are consistent with that reported in the literature ${ }^{46}$.

\section{Compound 2u}<smiles>COc1ccccc1S(=O)(=O)O</smiles>

\section{(2-Methoxyphenyl)dimethylsilanol}

The reaction was conducted for $12 \mathrm{~h}$ following GPA on $1 \mathrm{mmol}$ scale with $1 \mathbf{u}$. After dried over anhydrous $\mathrm{Na}_{2} \mathrm{SO}_{4}$ and evaporated the solvent, the title compound $\mathbf{2 u}$ was obtained in $97 \%$ yield $(177 \mathrm{mg})$ without further purification.

Physical state: colorless oil.

${ }^{1}$ H NMR (400 MHz, Chloroform- $d$ ): $\delta=7.43(\mathrm{~d}, J=7.0 \mathrm{~Hz}, 1 \mathrm{H}), 7.39$ (t, $\left.J=7.9 \mathrm{~Hz}, 1 \mathrm{H}\right), 7.00$ (t, $J=7.2$ $\mathrm{Hz}, 1 \mathrm{H}), 6.87(\mathrm{~d}, J=8.2 \mathrm{~Hz}, 1 \mathrm{H}), 3.85(\mathrm{~s}, 3 \mathrm{H}), 2.47$ (br s, $1 \mathrm{H}), 0.40$ (s, 6H).

${ }^{13}$ C NMR (101 MHz, Chloroform-d): $\delta$ = 163.80, 134.51, 131.26, 126.83, 120.77, 109.58, 55.16, 0.27.

The characterization data are consistent with that reported in the literature ${ }^{21}$.

\section{Compound 2v}<smiles>C[As](C)(O)(O)c1cccc(F)c1</smiles>

\section{(3-Fluorophenyl)dimethylsilanol}

The reaction was conducted for $3 \mathrm{~h}$ following GP A on $1 \mathrm{mmol}$ scale with $\mathbf{1 v}$. After dried over anhydrous $\mathrm{Na}_{2} \mathrm{SO}_{4}$ and evaporated the solvent, the title compound $2 \mathrm{v}$ was obtained in $97 \%$ yield (166 mg) without further purification.

Physical state: colorless oil.

${ }^{1}$ H NMR (400 MHz, Chloroform- $\boldsymbol{d}$ ): $\delta=7.46-7.33(\mathrm{~m}, 2 \mathrm{H}), 7.28(\mathrm{~d}, J=8.5 \mathrm{~Hz}, 1 \mathrm{H}), 7.10(\mathrm{t}, J=7.0 \mathrm{~Hz}$, $1 \mathrm{H}), 2.51$ (br s, 1H), $0.42(\mathrm{~s}, 6 \mathrm{H})$.

${ }^{13}$ C NMR (101 MHz, Chloroform-d): $\delta=163.84,161.37,142.12,134.43,129.76,129.69,128.54,128.51$, $128.10,119.48,119.30,116.59,116.38,-0.12$.

${ }^{19}$ F NMR (376 MHz, Chloroform-d): $\delta=-113.48$.

HRMS (ESI-TOF): calcd for $\mathrm{C}_{8} \mathrm{H}_{10} \mathrm{FOSi}^{-}[\mathrm{M}-\mathrm{H}]^{-}: 169.0479$, found: 169.0472 . 


\section{Compound 2w}<smiles>Cc1cc([N+](=O)[O-])cc(S(=O)(=O)O)c1</smiles>

\section{(3,5-Dimethylphenyl)dimethylsilanol}

The reaction was conducted for $1 \mathrm{~h}$ following GP A on $1 \mathrm{mmol}$ scale with $\mathbf{1 w}$. After dried over anhydrous $\mathrm{Na}_{2} \mathrm{SO}_{4}$ and evaporated the solvent, the title compound $\mathbf{2 w}$ was obtained in $98 \%$ yield $(177 \mathrm{mg}$ ) without further purification.

Physical state: colorless oil.

${ }^{1}$ H NMR (400 MHz, Chloroform- $d$ ): $\delta=7.23$ (s, 2H), 7.07 (s, 1H), 2.36 (s, 6H), 2.12 (br s, 1H), 0.41 (s, $6 \mathrm{H})$.

${ }^{13}$ C NMR (101 MHz, Chloroform-d): $\delta=138.88,137.22,131.33,130.71,21.29,-0.01$.

The characterization data are consistent with that reported in the literature ${ }^{21}$.

\section{Compound 2x}<smiles>O=S(=O)(O)c1ccc(-c2ccccc2)cc1</smiles>

\section{(1,1'-Biphenyl)-4-yldimethylsilanol}

The reaction was conducted for $3 \mathrm{~h}$ following GP A on $1 \mathrm{mmol}$ scale with $\mathbf{1 x}$. After dried over anhydrous $\mathrm{Na}_{2} \mathrm{SO}_{4}$ and evaporated the solvent, the title compound $\mathbf{2 x}$ was obtained in $97 \%$ yield (223 $\mathrm{mg}$ ) without further purification.

Physical state: white solid.

${ }^{1}$ H NMR (400 MHz, Chloroform- $\left.d\right)$ : $\delta=7.66(\mathrm{~d}, J=7.4 \mathrm{~Hz}, 2 \mathrm{H}), 7.63-7.56(\mathrm{~m}, 4 \mathrm{H}), 7.44(\mathrm{t}, J=7.2 \mathrm{~Hz}$, $2 \mathrm{H}), 7.36$ (d, $J=7.3 \mathrm{~Hz}, 1 \mathrm{H}), 2.30$ (br s, $1 \mathrm{H}), 0.43$ (s, 6H).

${ }^{13}$ C NMR (101 MHz, Chloroform-d): $\delta=142.38,140.96,137.76,133.56,128.77,127.45,127.15,126.62$, 0.03 .

HRMS (ESI-TOF): calcd for $\mathrm{C}_{14} \mathrm{H}_{15} \mathrm{OSi}^{-}\left[\mathrm{M}^{-\mathrm{H}}\right]^{-}:$: 227.0887, found: 227.0885 .

\section{Compound 2y}<smiles>C=Cc1ccc([As](C)(=O)(O)O[Na])cc1</smiles>

\section{Dimethyl(4-vinylphenyl)silanol}

The reaction was conducted for $2 \mathrm{~h}$ following GP A on $1 \mathrm{mmol}$ scale with $\mathbf{1 y}$. After purification by flash column chromatography (silica gel, EA/PE $=1: 10$ ), the title compound $\mathbf{2 y}$ was obtained in $94 \%$ yield (167 $\mathrm{mg})$.

Physical state: colorless oil.

${ }^{1}$ H NMR (400 MHz, Chloroform-d): $=7.53(\mathrm{~d}, J=7.9 \mathrm{~Hz}, 2 \mathrm{H}), 7.40(\mathrm{~d}, J=7.9 \mathrm{~Hz}, 2 \mathrm{H}), 6.71(\mathrm{dd}, J=$ 17.6, $10.9 \mathrm{~Hz}, 1 \mathrm{H}), 5.78$ (d, $J=17.6 \mathrm{~Hz}, 1 \mathrm{H}), 5.27$ (d, $J=10.9 \mathrm{~Hz}, 1 \mathrm{H}), 2.31$ (br s, 1H), 0.37 (s, 6H).

${ }^{13}$ C NMR (101 MHz, Chloroform- $d$ ): $\delta=138.67,136.73,133.30,125.63,114.48,-0.04$.

HRMS (ESI-TOF): calcd for $\mathrm{C}_{9} \mathrm{H}_{11} \mathrm{O}_{2} \mathrm{Si}^{-}[\mathrm{M}-\mathrm{H}]^{-}:$: 177.0741, found: 177.0746 . 


\section{Compound 2z}<smiles>C[Si](c1ccccc1)(c1ccccc1)c1ccccc1</smiles>

\section{Methyldiphenylsilanol}

The reaction was conducted for $0.5 \mathrm{~h}$ following GP A on $1 \mathrm{mmol}$ scale with 1z. After dried over anhydrous $\mathrm{Na}_{2} \mathrm{SO}_{4}$ and evaporated the solvent, the title compound $\mathbf{2 z}$ was obtained in $99 \%$ yield (212 mg) without further purification.

Physical state: colorless oil.

${ }^{1}$ H NMR (400 MHz, Chloroform-d): $\delta=7.58$ (d, $\left.J=7.6 \mathrm{~Hz}, 4 \mathrm{H}\right), 7.37$ (ddd, $\left.J=14.3,7.6,2.4 \mathrm{~Hz}, 6 \mathrm{H}\right)$, 2.56 (br s, 1H), 0.63 (s, 3H).

${ }^{13}$ C NMR (101 MHz, Chloroform-d): $\delta=137.16,134.09,130.01,128.04,-1.15$.

The characterization data are consistent with that reported in the literature ${ }^{21}$.

\section{Compound 2aa}<smiles>CC[Si](O)(c1ccccc1)c1ccccc1</smiles>

\section{Ethyldiphenylsilanol}

The reaction was conducted for $2 \mathrm{~h}$ following GP A on $1 \mathrm{mmol}$ scale with 1 aa. After dried over anhydrous $\mathrm{Na}_{2} \mathrm{SO}_{4}$ and evaporated the solvent, the title compound 2 aa was obtained in $98 \%$ yield (224 mg) without further purification.

Physical state: colorless oil.

${ }^{1}$ H NMR (400 MHz, Chloroform-d): $\delta=7.73$ - 7.54 (m, 4H), 7.52 - $7.30(\mathrm{~m}, 6 \mathrm{H}), 2.46$ (br s, $\left.1 \mathrm{H}\right), 1.22$ $1.02(\mathrm{~m}, 5 \mathrm{H})$.

${ }^{13}$ C NMR (101 MHz, Chloroform-d): $\delta=136.03,134.20,129.80,127.85,6.78,6.62$.

The characterization data are consistent with that reported in the literature ${ }^{52}$.

\section{Compound 2ab}<smiles>CCC[Si](Pc1ccccc1)(c1ccccc1)c1ccccc1</smiles>

\section{Isopropyldiphenylsilanol}

The reaction was conducted for $6 \mathrm{~h}$ following GP A on $1 \mathrm{mmol}$ scale with $\mathbf{1 a b}$. After dried over anhydrous $\mathrm{Na}_{2} \mathrm{SO}_{4}$ and evaporated the solvent, the title compound $2 \mathbf{a b}$ was obtained in $98 \%$ yield (237 mg) without further purification.

Physical state: white solid.

${ }^{1}$ H NMR (400 MHz, Chloroform-d): $\delta=7.66$ (d, $\left.J=7.0 \mathrm{~Hz}, 4 \mathrm{H}\right), 7.54-7.35$ (m, 6H), 2.57 (br s, $\left.1 \mathrm{H}\right), 1.61$ - $1.39(\mathrm{~m}, 1 \mathrm{H}), 1.13(\mathrm{~d}, J=7.4 \mathrm{~Hz}, 6 \mathrm{H})$.

${ }^{13}$ C NMR (101 MHz, Chloroform-d): $\delta=135.23,134.51,129.73,127.78,17.00,13.36$.

The characterization data are consistent with that reported in the literature ${ }^{53}$. 


\section{Compound 2ac}<smiles>c1ccc([Si](c2ccccc2)(c2ccccc2)c2ccccc2)cc1</smiles>

\section{Triphenylsilanol}

The reaction was conducted for $2 \mathrm{~h}$ following GP A on $1 \mathrm{mmol}$ scale with 1ac. After dried over anhydrous $\mathrm{Na}_{2} \mathrm{SO}_{4}$ and evaporated the solvent, the title compound $2 \mathrm{ac}$ was obtained in $97 \%$ yield $(268 \mathrm{mg}$ ) without further purification.

Physical state: white solid.

${ }^{1}$ H NMR (400 MHz, Chloroform-d): $\delta=7.70$ - $7.53(\mathrm{~m}, 6 \mathrm{H}), 7.45-7.38(\mathrm{~m}, 3 \mathrm{H}), 7.38-7.32(\mathrm{~m}, 6 \mathrm{H}), 2.66$ (br s, 1H).

${ }^{13}$ C NMR (101 MHz, Chloroform-d): $\delta=135.07,134.96,130.08,127.89$.

The characterization data are consistent with that reported in the literature ${ }^{21}$.

\section{Compound 2ad}

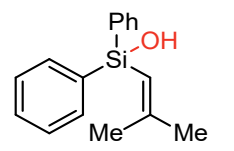

\section{(2-Methylprop-1-en-1-yl)diphenylsilanol}

The reaction was conducted for $12 \mathrm{~h}$ following GP A on $1 \mathrm{mmol}$ scale with 1ad. After purification by flash column chromatography (silica gel, EA/PE $=1: 10$ ), the title compound 2 ad was obtained in $95 \%$ yield (242 $\mathrm{mg})$.

Physical state: white solid.

${ }^{1}$ H NMR (400 MHz, Chloroform-d): $\delta=7.70(\mathrm{dd}, J=17.3,7.0 \mathrm{~Hz}, 4 \mathrm{H}), 7.47$ - 7.34 (m, 6H), $5.65(\mathrm{~s}, 1 \mathrm{H})$, 2.33 (br s, 1H), 2.00 (s, 3H), 1.85 (s, 3H).

${ }^{13}$ C NMR (101 MHz, Chloroform- $d$ ): $\delta=157.90,137.10,134.35,129.69,127.85,119.29,29.55,24.19$.

The characterization data are consistent with that reported in the literature ${ }^{28}$.

\section{Compound 2ae \\ TMSO \\ $\mathrm{Me}-\mathrm{Si}-\mathrm{OH}$}

TMSO'

\section{1,1,1,3,5,5,5-heptamethyltrisiloxan-3-ol}

The reaction was conducted for $12 \mathrm{~h}$ following GP A on $10 \mathrm{mmol}$ scale with 1ae under $\mathrm{O}_{2}$ atmosphere instead of ambient air. GC analysis showed the yield of title compound $\mathbf{2 a e}$ was $99 \%$. After vacuum distillation (17 Torr, $\left.75-78{ }^{\circ} \mathrm{C}\right), 2.05 \mathrm{~g}$ (86\% yield) 2 ac was obtained.

Physical state: colorless oil.

${ }^{1}$ H NMR (400 MHz, Chloroform- $\boldsymbol{d}$ ): $\delta=2.08$ (br s, 1H), 0.12 (s, 18H), 0.09 (s, 3H).

${ }^{13}$ C NMR (101 MHz, Chloroform- $d$ ): $\delta=1.60,1.43$.

The characterization data are consistent with that reported in the literature ${ }^{54}$.

\section{Compound 2af}

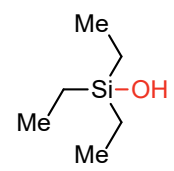




\section{Triethylsilanol}

The reaction was conducted for $2 \mathrm{~h}$ following GP A on $10 \mathrm{mmol}$ scale with $\mathbf{1 a f}$. GC analysis showed the yield of title compound $\mathbf{2 a f}$ was $99 \%$. After vacuum distillation ( $\left.10 \mathrm{Torr}, 54-57^{\circ} \mathrm{C}\right), 1.18 \mathrm{~g}(89 \%$ yield) $\mathbf{2 a d}$ was obtained.

Physical state: colorless oil.

${ }^{1}$ H NMR (400 MHz, Chloroform- $d$ ): $\delta=1.85(\mathrm{br} \mathrm{s}, 1 \mathrm{H}), 1.02-0.92(\mathrm{~m}, 9 \mathrm{H}), 0.65-0.55(\mathrm{~m}, 6 \mathrm{H})$.

${ }^{13}$ C NMR (101 MHz, Chloroform- $d$ ): $\delta=6.55,5.74$.

The characterization data are consistent with that reported in the literature ${ }^{21}$.

\section{Compound 2ag}<smiles>CC(C)[Si](C(C)C)(C(C)C)C(C)C</smiles>

\section{Triisopropylsilanol}

The reaction was conducted for $12 \mathrm{~h}$ following GP A on $10 \mathrm{mmol}$ scale with $1 \mathrm{ag}$ at $60{ }^{\circ} \mathrm{C}$ instead of $30{ }^{\circ} \mathrm{C}$. GC analysis showed the yield of title compound 2 ag was $97 \%$. After vacuum distillation ( 0.5 Torr, $42-45$ $\left.{ }^{\circ} \mathrm{C}\right), 1.53 \mathrm{~g}$ ( $88 \%$ yield) 2 ae was obtained.

Physical state: colorless oil.

${ }^{1}$ H NMR (400 MHz, Chloroform-d): $\delta=1.57(\mathrm{br} \mathrm{s}, 1 \mathrm{H}), 1.08-1.00(\mathrm{~m}, 21 \mathrm{H})$.

${ }^{13}$ C NMR (101 MHz, Chloroform- $d$ ): $\delta=17.67,12.26$.

The characterization data are consistent with that reported in the literature ${ }^{21}$.

\section{Compound 2ah}

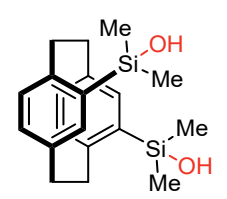

\section{rac-4,12-Bis(hydroxydimethylsilyl)[2.2] paracyclophane}

The reaction was conducted for $12 \mathrm{~h}$ following GP A on $1 \mathrm{mmol}$ scale with 1ah. After purification by flash column chromatography (silica gel, $\mathrm{Et}_{2} \mathrm{O} / \mathrm{PE}=1: 2$ ), the title compound $\mathbf{2 a h}$ was obtained in $95 \%$ yield (338 $\mathrm{mg})$.

Physical state: white solid.

${ }^{1}$ H NMR (400 MHz, Chloroform-d): $\delta=6.95(\mathrm{~s}, 2 \mathrm{H}), 6.62(\mathrm{~d}, J=7.8 \mathrm{~Hz}, 2 \mathrm{H}), 6.55(\mathrm{~d}, J=7.7 \mathrm{~Hz}, 2 \mathrm{H})$, 3.97 (br s, 2H), $3.42-3.35$ (m, 2H), $3.21-3.12(\mathrm{~m}, 6 \mathrm{H}), 0.60(\mathrm{~s}, 6 \mathrm{H}), 0.26(\mathrm{~s}, 6 \mathrm{H})$.

${ }^{13}$ C NMR (101 MHz, Chloroform-d): $\delta=145.36,138.01,137.89,137.75,134.57,133.42,35.95,35.52$, 2.42, 1.74 .

The characterization data are consistent with that reported in the literature ${ }^{32}$.

\section{Compound 2ai}<smiles>C[N+]([O-])([O-])c1ccc([As](C)(=O)O)cc1</smiles> 


\section{1,4-Phenylenebis(dimethylsilanol)}

The reaction was conducted for $4 \mathrm{~h}$ following GP A on $1 \mathrm{mmol}$ scale with 1ai. After dried over anhydrous $\mathrm{Na}_{2} \mathrm{SO}_{4}$ and evaporated the solvent, the title compound 2ai was obtained in $98 \%$ yield (221 $\mathrm{mg}$ ) without further purification.

Physical state: white solid.

${ }^{1}$ H NMR (400 MHz, DMSO-d $\boldsymbol{d}_{\boldsymbol{\sigma}}$ ): $\delta=7.52$ (s, 4H), 5.87 (br s, 2H), 0.23 (s, 12H).

${ }^{13}$ C NMR (101 MHz, DMSO-d $\left.\boldsymbol{d}_{6}\right): \delta=141.28,132.07,0.57$.

The characterization data are consistent with that reported in the literature ${ }^{21}$.

\section{Compound 2aj}<smiles>O[SnH](O)c1ccccc1</smiles>

\section{Diphenylsilanediol}

The reaction was conducted for $0.25 \mathrm{~h}$ following GP A on $1 \mathrm{mmol}$ scale with 1aj. After purification by flash column chromatography (silica gel, EA/PE $=1: 2$ ), the title compound 2aj was obtained in $93 \%$ yield (201 $\mathrm{mg})$.

Physical state: white solid.

${ }^{1}$ H NMR (400 MHz, DMSO-d d : $\delta=7.59$ (dd, $\left.J=10.0,4.9 \mathrm{~Hz}, 4 \mathrm{H}\right), 7.40-7.29$ (m, 6H), 6.94 (br s, 2H).

${ }^{13}$ C NMR (101 MHz, DMSO- $\left.\boldsymbol{d}_{\mathbf{6}}\right): \delta=137.80,134.07,129.33,127.46$.

The characterization data are consistent with that reported in the literature ${ }^{21}$.

\section{Compound 2ak}<smiles>O[Sb](O)(O)c1ccccc1</smiles>

\section{Phenylsilanetriol}

The reaction was conducted for $0.5 \mathrm{~h}$ following GP A on $1 \mathrm{mmol}$ scale with $\mathbf{1 a k}$ using acetone as solvent instead of $\mathrm{MeCN}$. After completion of the reaction, the catalyst was removed by filtration and washed with acetone $(3 \times 5 \mathrm{~mL})$. The combined organics were dried over anhydrous $\mathrm{Na}_{2} \mathrm{SO}_{4}$ and solvent was removed by rotary evaporation below $15^{\circ} \mathrm{C}$. The title compound 2ak was obtained in $96 \%$ yield $(150 \mathrm{mg})$ without further purification.

Physical state: white solid.

${ }^{1}$ H NMR (400 MHz, Acetone- $\boldsymbol{d}_{\boldsymbol{6}}$ ): $\delta=7.75-7.68$ (m, 2H), $7.39-7.28$ (m, 3H), 5.81 (br s, 3H).

${ }^{13}$ C NMR (101 MHz, Acetone- $\left.d_{6}\right)$ : $\delta=137.01,135.02,130.03,128.05$.

The characterization data are consistent with that reported in the literature ${ }^{55}$.

\section{Compound 2al}

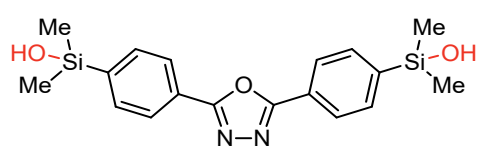

\section{((1,3,4-oxadiazole-2,5-diyl)bis(4,1-phenylene))bis(dimethylsilanol)}

The reaction was conducted for $6 \mathrm{~h}$ following GP A on $1 \mathrm{mmol}$ scale with 1al. After purification by flash column chromatography (silica gel, $\mathrm{CH}_{2} \mathrm{Cl}_{2}$ ), the title compound $\mathbf{2 a l}$ was obtained in $93 \%$ yield $(345 \mathrm{mg}$ ). 
Physical state: white solid.

${ }^{1}$ H NMR (400 MHz, Chloroform- $d$ ): $\delta=8.10(\mathrm{~d}, J=7.3 \mathrm{~Hz}, 4 \mathrm{H}), 7.75$ (d, $\left.J=7.3 \mathrm{~Hz}, 4 \mathrm{H}\right), 2.39$ (br s, 2H), $0.46(\mathrm{~s}, 12 \mathrm{H})$.

${ }^{13}$ C NMR (101 MHz, Chloroform-d): $\delta=164.63,144.05,133.71,126.05,124.51,-0.01$.

HRMS (ESI-TOF): calcd for $\mathrm{C}_{18} \mathrm{H}_{21} \mathrm{~N}_{2} \mathrm{O}_{3} \mathrm{Si}_{2}{ }^{-}[\mathrm{M}-\mathrm{H}]^{-}:$: 369.1085, found: 369.1097 .

\section{Compound 2am}<smiles>C[As](O)(O)c1ccc(-c2ccc(S(=O)(=O)O)s2)s1</smiles>

\section{[2,2'-Bithiophene]-5,5'-diylbis(dimethylsilanol)}

The reaction was conducted for $12 \mathrm{~h}$ following GP A on $1 \mathrm{mmol}$ scale with $1 \mathrm{am}$. After purification by flash column chromatography (silica gel, $\mathrm{CH}_{2} \mathrm{Cl}_{2}$ ), the title compound 2 am was obtained in $94 \%$ yield $(295 \mathrm{mg}$ ). Physical state: white solid.

${ }^{1}$ H NMR (400 MHz, Chloroform-d): $\delta=7.28(\mathrm{~d}, J=3.3 \mathrm{~Hz}, 2 \mathrm{H}), 7.25$ (d, $\left.J=3.4 \mathrm{~Hz}, 2 \mathrm{H}\right), 2.35$ (br s, 2H), $0.49(\mathrm{~s}, 12 \mathrm{H})$.

${ }^{13}$ C NMR (101 MHz, Chloroform- $\boldsymbol{d}$ ): $\delta=142.81,138.41,135.45,125.39,0.83$.

HRMS (ESI-TOF): calcd for $\mathrm{C}_{12} \mathrm{H}_{17} \mathrm{~S}_{2} \mathrm{O}_{2} \mathrm{Si}_{2}{ }^{-}[\mathrm{M}-\mathrm{H}]^{-}:$: 313.0203, found: 313.0216 .

\section{Compound 2an}<smiles>C[N+]([O-])(O)c1ccccc1-c1ccccc1</smiles>

\section{(1,1'-Biphenyl)-2-yldimethylsilanol}

The reaction was conducted for $8 \mathrm{~h}$ following GP A on $1 \mathrm{mmol}$ scale with 1an. After dried over anhydrous $\mathrm{Na}_{2} \mathrm{SO}_{4}$ and evaporated the solvent, the title compound $2 \mathrm{an}$ was obtained in $98 \%$ yield (224 $\mathrm{mg}$ ) without further purification.

Physical state: white solid.

${ }^{1}$ H NMR (400 MHz, Chloroform- $\boldsymbol{d}$ ): $\delta=7.80(\mathrm{~d}, J=6.8 \mathrm{~Hz}, 1 \mathrm{H}), 7.54-7.39(\mathrm{~m}, 7 \mathrm{H}), 7.34(\mathrm{~d}, J=7.6 \mathrm{~Hz}$, $1 \mathrm{H}), 2.55$ (br s, 1H), 0.19 (s, 6H).

${ }^{13}$ C NMR (101 MHz, Chloroform-d): $\delta=148.35,144.07,137.72,134.20,129.34,129.11,127.95,127.30$, $126.41,1.45$.

The characterization data are consistent with that reported in the literature ${ }^{27}$.

\section{Compound 2ao}<smiles>Cc1ccc(-c2ccccc2[N+](C)([O-])O)cc1</smiles>

\section{(4'-Methyl-[1,1'-biphenyl]-2-yl)dimethylsilanol}

The reaction was conducted for $12 \mathrm{~h}$ following GP A on $1 \mathrm{mmol}$ scale with 1ao. After dried over anhydrous $\mathrm{Na}_{2} \mathrm{SO}_{4}$ and evaporated the solvent, the title compound $2 \mathrm{ao}$ was obtained in $97 \%$ yield $(236 \mathrm{mg}$ ) without further purification. 
Physical state: white solid.

${ }^{1}$ H NMR (400 MHz, Chloroform- $d$ ): $\delta=7.83$ (dd, $J=7.3,1.2 \mathrm{~Hz}, 1 \mathrm{H}$ ), 7.49 (ddd, $J=16.1,7.3,1.4 \mathrm{~Hz}$, $2 \mathrm{H}), 7.36(\mathrm{dt}, J=17.6,5.0 \mathrm{~Hz}, 5 \mathrm{H}), 2.52(\mathrm{~s}, 3 \mathrm{H}), 2.31$ (br s, $1 \mathrm{H}), 0.25$ (s, 6H).

${ }^{13}$ C NMR (101 MHz, Chloroform-d): $\delta=148.36,141.19,137.79,137.01,134.11,129.47,129.08,128.97$, $128.68,126.28,21.13,1.52$.

HRMS (ESI-TOF): calcd for $\mathrm{C}_{15} \mathrm{H}_{18} \mathrm{OSiNa}^{+}[\mathrm{M}+\mathrm{Na}]^{+}: 265.1019$, found: 265.1022 .

\section{Compound 2ap}<smiles>C[N+]([O-])([O-])c1cccc(-c2ccccc2[As]([O-])(O)O)c1</smiles>

\section{(3'-Methyl-[1,1'-biphenyl]-2-yl)dimethylsilanol}

The reaction was conducted for $12 \mathrm{~h}$ following GP A on $1 \mathrm{mmol}$ scale with $\mathbf{1 a p}$. After dried over anhydrous $\mathrm{Na}_{2} \mathrm{SO}_{4}$ and evaporated the solvent, the title compound 2ap was obtained in $98 \%$ yield (237 $\mathrm{mg}$ ) without further purification.

Physical state: white solid.

${ }^{1}$ H NMR (400 MHz, Chloroform-d): $\delta=7.76(\mathrm{~d}, J=7.2 \mathrm{~Hz}, 1 \mathrm{H}), 7.51-7.40$ (m, 2H), 7.35 (dd, $J=14.3$, $7.1 \mathrm{~Hz}, 2 \mathrm{H}), 7.23$ (dd, $J=13.5,8.7 \mathrm{~Hz}, 3 \mathrm{H}), 2.46$ (s, 3H), 1.95 (br s, $1 \mathrm{H}), 0.20$ (s, 6H).

${ }^{13}$ C NMR (101 MHz, Chloroform-d): $\delta=148.43,144.99,137.71,137.57,134.11,129.93,129.22,129.04$, $128.01,127.91,126.34,126.08,21.32,1.53$.

HRMS (ESI-TOF): calcd for $\mathrm{C}_{15} \mathrm{H}_{18} \mathrm{OSiNa}^{+}[\mathrm{M}+\mathrm{Na}]^{+}: 265.1019$, found: 265.1018 .

\section{Compound 2aq}<smiles>O=S(=O)(O)c1ccccc1-c1ccc(C(F)(F)F)cc1</smiles>

\section{(4'-Trifluoromethyl-[1,1'-biphenyl]-2-yl)dimethylsilanol}

The reaction was conducted for $16 \mathrm{~h}$ following GP A on $1 \mathrm{mmol}$ scale with $\mathbf{1 a q}$. After dried over anhydrous $\mathrm{Na}_{2} \mathrm{SO}_{4}$ and evaporated the solvent, the title compound $2 \mathbf{a q}$ was obtained in $97 \%$ yield (287 $\mathrm{mg}$ ) without further purification.

Physical state: white solid.

${ }^{1}$ H NMR (400 MHz, Chloroform- $d$ ): $\delta=7.80(\mathrm{~d}, J=7.0 \mathrm{~Hz}, 1 \mathrm{H}), 7.70(\mathrm{~d}, J=8.1 \mathrm{~Hz}, 2 \mathrm{H}), 7.53-7.44$ (m, $4 \mathrm{H}), 7.28(\mathrm{~d}, J=8.0 \mathrm{~Hz}, 1 \mathrm{H}), 2.35$ (br s, 1H), $0.11(\mathrm{~s}, 6 \mathrm{H})$.

${ }^{13}$ C NMR (101 MHz, Chloroform-d): $\delta=147.73,146.98,137.49,134.54,129.63,129.38,129.33,127.03$, $124.90,124.86,124.82,124.78,1.42$.

${ }^{19}$ F NMR (376 MHz, Chloroform- $d$ ): $\delta=-62.28$.

HRMS (ESI-TOF): calcd for $\mathrm{C}_{15} \mathrm{H}_{15} \mathrm{OF}_{3} \mathrm{SiNa}^{+}[\mathrm{M}+\mathrm{Na}]^{+}:$319.0736, found: 319.0755 . 


\section{Compound 2ar}<smiles></smiles>

(4'-Methoxy-[1,1'-biphenyl]-2-yl)dimethylsilanol

The reaction was conducted for $14 \mathrm{~h}$ following GPA on $1 \mathrm{mmol}$ scale with $1 \mathrm{ar}$. After purification by flash column chromatography (silica gel, EA/PE $=1: 10$ ), the title compound 2ar was obtained in $92 \%$ yield (238 $\mathrm{mg})$.

Physical state: white solid.

${ }^{1}$ H NMR (400 MHz, Chloroform- $d$ ): $\delta=7.75(\mathrm{~d}, J=7.2 \mathrm{~Hz}, 1 \mathrm{H}), 7.42(\mathrm{dt}, J=23.4,7.2 \mathrm{~Hz}, 2 \mathrm{H}), 7.31$ (t, $J=8.8 \mathrm{~Hz}, 3 \mathrm{H}), 6.99$ (d, $J=8.4 \mathrm{~Hz}, 2 \mathrm{H}), 3.88$ (s, 3H), 2.20 (br s, $1 \mathrm{H}), 0.18$ (s, 6H).

${ }^{13}$ C NMR (101 MHz, Chloroform-d): $\delta=158.87,148.04,137.83,136.49,134.19,130.17,129.60,129.09$, $126.17,113.31,55.18,1.47$.

HRMS (ESI-TOF): calcd for $\mathrm{C}_{15} \mathrm{H}_{18} \mathrm{O}_{2} \mathrm{SiNa}^{+}[\mathrm{M}+\mathrm{Na}]^{+}:$281.0968, found: 281.0974 .

\section{Compound 2as}

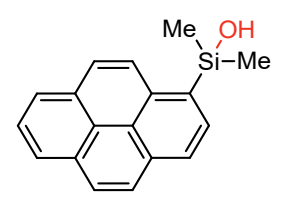

\section{Dimethyl(pyren-1-yl)silanol}

The reaction was conducted for $4 \mathrm{~h}$ following GP A on $1 \mathrm{mmol}$ scale with 1 as. After dried over anhydrous $\mathrm{Na}_{2} \mathrm{SO}_{4}$ and evaporated the solvent, the title compound 2 as was obtained in $98 \%$ yield $(271 \mathrm{mg})$ without further purification.

Physical state: white solid.

${ }^{1}$ H NMR (400 MHz, Chloroform- $\boldsymbol{d}$ ): $\delta=8.57(\mathrm{~d}, J=9.2 \mathrm{~Hz}, 1 \mathrm{H}), 8.27-8.19(\mathrm{~m}, 3 \mathrm{H}), 8.15-8.00(\mathrm{~m}, 5 \mathrm{H})$, 2.69 (br s, 1H), $0.72(\mathrm{~s}, 6 \mathrm{H})$.

${ }^{13}$ C NMR (101 MHz, Chloroform-d): $\delta=135.50,134.18,132.42,131.68,131.18,130.57,128.08,127.53$, $127.39,125.77,125.21,125.13,124.88,124.76,124.51,124.00,1.64$.

HRMS (ESI-TOF): calcd for $\mathrm{C}_{18} \mathrm{H}_{15} \mathrm{OSi}^{-}[\mathrm{M}-\mathrm{H}]^{-}:$: 275.0887, found: 275.0899 .

\section{Compound 2at}<smiles>C[As](C)(=O)(O)c1ccc(C#Cc2ccccc2)cc1</smiles>

\section{Dimethyl(4-(phenylethynyl)phenyl)silane}

The reaction was conducted for $6 \mathrm{~h}$ following GP A on $1 \mathrm{mmol}$ scale with 1at. After purification by flash column chromatography (silica gel, EA/PE $=1: 10$ ), the title compound 2at was obtained in $94 \%$ yield (237 $\mathrm{mg})$.

Physical state: white solid.

${ }^{1}$ H NMR (400 MHz, Chloroform- $\boldsymbol{d}$ ): $\delta=7.54(\mathrm{t}, J=6.1 \mathrm{~Hz}, 6 \mathrm{H}), 7.33(\mathrm{~s}, 3 \mathrm{H}), 2.28$ (br s, 1H), 0.39 (s, 6H).

${ }^{13}$ C NMR (101 MHz, Chloroform-d): $\delta=139.38,132.95,131.63,130.82,128.32,124.40,123.17,90.15$, 
$89.37,-0.08$.

HRMS (ESI-TOF): calcd for $\mathrm{C}_{16} \mathrm{H}_{15} \mathrm{OSi}^{-}[\mathrm{M}-\mathrm{H}]^{-}:$: 251.0887, found: 251.0896 .

\section{Compound 2au}<smiles>C[As](C)(O)(O)C#Cc1ccccc1</smiles>

\section{Dimethyl(phenylethynyl)silanol}

The reaction was conducted for $0.25 \mathrm{~h}$ following GPA on $1 \mathrm{mmol}$ scale with 1 au. After purification by flash column chromatography (silica gel, EA/PE $=1: 10)$, the title compound 2au was obtained in $92 \%$ yield (162 $\mathrm{mg})$.

Physical state: colorless oil.

${ }^{1}$ H NMR (400 MHz, Chloroform- $d$ ): $\delta=7.47$ (d, $\left.J=6.8 \mathrm{~Hz}, 2 \mathrm{H}\right), 7.36-7.23(\mathrm{~m}, 3 \mathrm{H}), 2.35$ (br s, $\left.1 \mathrm{H}\right), 0.38$ (s, 6H).

${ }^{13}$ C NMR (101 MHz, Chloroform-d): $\delta=132.03,128.88,128.23,122.42,104.74,92.62,1.50$.

The characterization data are consistent with that reported in the literature ${ }^{21}$.

\section{Compound 2av}<smiles>C[As](=O)(O)c1ccc(N=Nc2ccccc2)cc1</smiles>

\section{(E)-Dimethyl(4-(phenyldiazenyl)phenyl)silanol}

The reaction was conducted for $4 \mathrm{~h}$ following GP A on $1 \mathrm{mmol}$ scale with 1av. After purification by flash column chromatography (silica gel, $\mathrm{EA} / \mathrm{PE}=1: 10$ ), the title compound $2 \mathrm{av}$ was obtained in $95 \%$ yield (243 $\mathrm{mg})$.

Physical state: red oil.

${ }^{1}$ H NMR (400 MHz, Chloroform- $\boldsymbol{d}$ ): $\delta=7.93$ (dd, $\left.J=14.4,7.5 \mathrm{~Hz}, 4 \mathrm{H}\right), 7.74(\mathrm{~d}, J=7.6 \mathrm{~Hz}, 2 \mathrm{H}), 7.61$ 7.29 (m, 3H), 2.68 (br s, 1H), 0.45 (s, 6H).

${ }^{13}$ C NMR (101 MHz, Chloroform-d): $\delta=153.26,152.61,142.73,133.87,131.10,129.06,122.89,122.02$, 0.02 .

HRMS (ESI-TOF): calcd for $\mathrm{C}_{14} \mathrm{H}_{15} \mathrm{NOSi}^{-}[\mathrm{M}-\mathrm{H}]^{-}:$: 255.0948, found: 255.0957 .

\section{Compound 2aw}

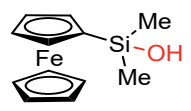

\section{(Hydroxydimethylsilyl)ferrocene}

The reaction was conducted for $3 \mathrm{~h}$ following GP A on $1 \mathrm{mmol}$ scale with 1aw. After purification by flash column chromatography (silica gel, EA/PE $=1: 5$ ), the title compound $2 \mathbf{a w}$ was obtained in $94 \%$ yield (244 $\mathrm{mg})$.

Physical state: red soild.

${ }^{1}$ H NMR (400 MHz, Chloroform-d): $\delta=4.42$ (s, 2H), 4.22 (s, 2H), 4.19 (s, 5H), 1.84 (br s, 1H), 0.39 (s, $6 \mathrm{H})$. 
${ }^{13}$ C NMR (101 MHz, Chloroform- $\boldsymbol{d}$ ): $\delta=73.10,71.45,70.50,68.54,0.41$.

HRMS (ESI-TOF): calcd for $\mathrm{C}_{12} \mathrm{H}_{15} \mathrm{FeOSi}^{-}[\mathrm{M}-\mathrm{H}]^{-}:$: 259.0236, found: 259.0242 .

\section{Compound 2ax}<smiles>O=S(=O)(O)c1cccs1</smiles>

\section{Dimethyl(thiophen-2-yl)silanol}

The reaction was conducted for $6 \mathrm{~h}$ following GPA on $1 \mathrm{mmol}$ scale with $1 \mathrm{ax}$. After dried over anhydrous $\mathrm{Na}_{2} \mathrm{SO}_{4}$ and evaporated the solvent, the title compound $2 \mathrm{ax}$ was obtained in $98 \%$ yield (155 $\mathrm{mg}$ ) without further purification.

Physical state: colorless oil.

${ }^{1}$ H NMR (400 MHz, Chloroform- $d$ ): $\delta=7.62(\mathrm{dd}, J=4.6,0.8 \mathrm{~Hz}, 1 \mathrm{H}), 7.35(\mathrm{dd}, J=3.3,0.9 \mathrm{~Hz}, 1 \mathrm{H}), 7.20$ (dd, $J=4.6,3.3 \mathrm{~Hz}, 1 \mathrm{H}), 2.65$ (br s, $1 \mathrm{H}), 0.44$ (s, 6H).

${ }^{13}$ C NMR (101 MHz, Chloroform- $d$ ): $\delta=138.53,134.64,131.02,128.15,0.85$.

The characterization data are consistent with that reported in the literature ${ }^{49}$.

\section{Compound 2ay}<smiles>C[Si](O)([Si])c1cc2ccccc2o1</smiles>

\section{benzofuran-2-yldimethylsilanol}

The reaction was conducted for $2 \mathrm{~h}$ following GP A on $1 \mathrm{mmol}$ scale with 1 ay. After dried over anhydrous $\mathrm{Na}_{2} \mathrm{SO}_{4}$ and evaporated the solvent, the title compound 2ay was obtained in $97 \%$ yield (186 mg) without further purification.

Physical state: colorless oil.

${ }^{1}$ H NMR (400 MHz, Chloroform- $d$ ): $\delta=7.62(\mathrm{~d}, J=7.6 \mathrm{~Hz}, 1 \mathrm{H}), 7.55(\mathrm{~d}, J=8.2 \mathrm{~Hz}, 1 \mathrm{H}), 7.34(\mathrm{t}, J=7.6$ $\mathrm{Hz}, 1 \mathrm{H}), 7.26$ (t, $J=7.4 \mathrm{~Hz}, 1 \mathrm{H}), 7.09$ (s, 1H), 2.79 (br s, 1H), 0.52 (s, 6H).

${ }^{13}$ C NMR (101 MHz, Chloroform-d): $\delta=161.33,157.84,127.56,124.82,122.47,121.33,116.78,111.41$, -0.51 .

The characterization data are consistent with that reported in the literature ${ }^{50}$.

\section{Compound 2az}<smiles>C[As](C)(O)c1c(Br)c2ccccc2n1O</smiles>

\section{tert-Butyl 3-bromo-2-(hydroxydimethylsilyl)-1H-indole-1-carboxylate}

The reaction was conducted for $12 \mathrm{~h}$ following GP A on $1 \mathrm{mmol}$ scale with 1az. After dried over anhydrous $\mathrm{Na}_{2} \mathrm{SO}_{4}$ and evaporated the solvent, the title compound $2 \mathrm{az}$ was obtained in $98 \%$ yield (362 mg) without further purification.

Physical state: white solid.

${ }^{1}$ H NMR (400 MHz, Chloroform- $d$ ): $\delta=7.92(\mathrm{~d}, J=8.4 \mathrm{~Hz}, 1 \mathrm{H}), 7.56$ (dd, $\left.J=7.8,0.5 \mathrm{~Hz}, 1 \mathrm{H}\right), 7.36$ (ddd, $J=8.5,7.2,1.4 \mathrm{~Hz}, 1 \mathrm{H}), 7.31-7.25(\mathrm{~m}, 1 \mathrm{H}), 3.19$ (br s, $1 \mathrm{H}), 1.71(\mathrm{~s}, 9 \mathrm{H}), 0.57$ (s, 6H).

${ }^{13}$ C NMR (101 MHz, Chloroform-d): $\delta=151.77,136.65,136.06,130.87,126.10,123.27,120.17,115.27$, 
$110.61,85.46,28.10,2.28$.

HRMS (ESI-TOF): calcd for $\left.\mathrm{C}_{10} \mathrm{H}_{11} \mathrm{NOSi}^{79} \mathrm{Br}^{-}{ }^{[M-B o c}\right]^{-}:$: 267.9798, found: 267.9792 ; calcd for $\mathrm{C}_{10} \mathrm{H}_{11} \mathrm{~N}-$ $\mathrm{OSi}^{81} \mathrm{Br}^{-}$[M-Boc] $]^{-}: 269.9778$, found: 269.9769 .

\section{Compound 2ba}<smiles>C[Si](O)(O)c1cccc2ncccc12</smiles>

\section{Dimethyl(quinolin-5-yl)silanol}

The reaction was conducted for $6 \mathrm{~h}$ following GP A on $1 \mathrm{mmol}$ scale with 1ba. After purification by flash column chromatography (silica gel, silica gel, $\mathrm{EA} / \mathrm{PE}=1: 3$ ), the title compound $\mathbf{2 b a}$ was obtained in $93 \%$ yield (189 mg).

Physical state: pale yellow solid.

${ }^{1}$ H NMR (400 MHz, Chloroform- $d$ ): $\delta=8.73(\mathrm{~d}, J=8.4 \mathrm{~Hz}, 1 \mathrm{H}), 8.55(\mathrm{~s}, 1 \mathrm{H}), 7.94(\mathrm{~d}, J=8.4 \mathrm{~Hz}, 1 \mathrm{H})$, $7.75(\mathrm{~d}, J=6.6 \mathrm{~Hz}, 1 \mathrm{H}), 7.50$ (t, $J=7.5 \mathrm{~Hz}, 1 \mathrm{H}), 7.28-7.13(\mathrm{~m}, 1 \mathrm{H}), 6.86(\mathrm{br} \mathrm{s}, 1 \mathrm{H}), 0.51(\mathrm{~s}, 6 \mathrm{H})$.

${ }^{13}$ C NMR (101 MHz, Chloroform-d): $\delta=148.74,147.31$, 139.19, 137.17, 133.52, 131.54, 130.11, 128.67, $120.46,1.36$.

HRMS (ESI-TOF): calcd for $\mathrm{C}_{11} \mathrm{H}_{12} \mathrm{NOSi}^{-}[\mathrm{M}-\mathrm{H}]^{-}:$: 202.0683, found: 202.0695 .

\section{Compound 2bb}<smiles>C[Si](O)(O)c1cncc2ccccc12</smiles>

\section{Isoquinolin-4-yldimethylsilanol}

The reaction was conducted for $5 \mathrm{~h}$ following GP A on $1 \mathrm{mmol}$ scale with $\mathbf{1 b b}$. After purification by flash column chromatography (silica gel, EA/PE $=1: 1$ ), the title compound $\mathbf{2 b b}$ was obtained in $92 \%$ yield (186 $\mathrm{mg})$.

Physical state: pale yellow viscous oil.

${ }^{1}$ H NMR (400 MHz, Chloroform- $d$ ): $\delta=9.02(\mathrm{~s}, 1 \mathrm{H}), 8.49(\mathrm{~s}, 1 \mathrm{H}), 8.30(\mathrm{~d}, J=8.4 \mathrm{~Hz}, 1 \mathrm{H}), 7.85$ (d, $J=$ $8.0 \mathrm{~Hz}, 1 \mathrm{H}), 7.77-7.65(\mathrm{~m}, 1 \mathrm{H}), 7.57$ (t, $J=7.2 \mathrm{~Hz}, 1 \mathrm{H}), 5.63$ (br s, 1H), 0.54 (s, 6H).

${ }^{13}$ C NMR (101 MHz, Chloroform-d): $\delta=153.54,147.24,139.20,130.62,129.96,128.64,127.92,127.32$, 126.96, 1.19 .

HRMS (ESI-TOF): calcd for $\mathrm{C}_{11} \mathrm{H}_{12} \mathrm{NOSi}^{-}[\mathrm{M}-\mathrm{H}]^{-}:$: 202.0683, found: 202.0689 .

\section{Compound 2bc}<smiles>COc1cnn(Cc2ccccc2)c(=O)c1[Si](=O)(=O)O</smiles>

\section{2-Benzyl-4-(hydroxydimethylsilyl)-5-methoxypyridazin-3(2H)-one}

The reaction was conducted for $6 \mathrm{~h}$ following GP A on $1 \mathrm{mmol}$ scale with $1 \mathrm{bc}$. After purification by flash column chromatography (silica gel, $\mathrm{EA} / \mathrm{PE}=1: 2$ to $2: 1$ ), the title compound $\mathbf{2 b c}$ was obtained in $91 \%$ yield (263 mg). 
Physical state: white solid.

2bn was found by NMR spectroscopy to exist as a pair of isomers (A and B) in a 55:45 ratio due to the tautomerism of amide bond (the major tautomer is represented by $A$ ).

${ }^{1}$ H NMR (400 MHz, Chloroform- $\left.\boldsymbol{d}\right): \delta=7.82\left(\mathrm{~s}, 1 \mathrm{H}_{\mathrm{A}}\right), 7.48\left(\mathrm{~s}, 1 \mathrm{H}_{\mathrm{B}}\right), 7.43-7.39\left(\mathrm{~m}, 2 \mathrm{H}_{\mathrm{A} / \mathrm{B}}\right), 7.35-7.28$ $\left(\mathrm{m}, 3 \mathrm{H}_{\mathrm{A} / \mathrm{B}}\right), 5.30\left(\mathrm{~s}, 2 \mathrm{H}_{\mathrm{A}}\right), 5.23\left(\mathrm{~s}, 2 \mathrm{H}_{\mathrm{B}}\right), 5.05\left(\mathrm{br} \mathrm{s}, 1 \mathrm{H}_{\mathrm{A} / \mathrm{B}}\right), 3.93\left(\mathrm{~s}, 3 \mathrm{H}_{\mathrm{A}}\right), 3.70\left(\mathrm{~s}, 1 \mathrm{H}_{\mathrm{B}}\right), 0.36\left(\mathrm{~s}, 6 \mathrm{H}_{\mathrm{A}}\right), 0.36$ $\left(\mathrm{s}, 6 \mathrm{H}_{\mathrm{B}}\right)$.

${ }^{13}$ C NMR (101 MHz, Chloroform- $\boldsymbol{d}$ ): $\delta=165.64(\mathrm{~B}), 163.92,136.67(\mathrm{~B}), 136.17(\mathrm{~A}), 128.81(\mathrm{~B}), 128.60$, 128.45(A), 127.91(B), 127.73(A), 126.85(A), 126.40(B), 118.01(A), 117.27(B), 56.58(B), 56.20(A), 54.96(A), 54.63(B), 2.19(A), 0.97(B).

HRMS (ESI-TOF): calcd for $\mathrm{C}_{14} \mathrm{H}_{19} \mathrm{~N}_{2} \mathrm{O}_{3} \mathrm{Si}^{+}[\mathrm{M}+\mathrm{H}]^{+}: 291.1159$, found: 291.1141 .

\section{Compound 2bd}<smiles>CC1(C)CCC(C)(C)c2cc([Si](C)(C)c3ccc4c(c3)C(C)(C)CCC4(C)C)ccc21</smiles>

\section{Methylbis(5,5,8,8-tetramethyl-5,6,7,8-tetrahydronaphthalen-2-yl)silanol}

The reaction was conducted for $18 \mathrm{~h}$ following GPA on $1 \mathrm{mmol}$ scale with $\mathbf{1 b d}$. After purification by flash column chromatography (silica gel, EA/PE $=1: 5$ ), the title compound $\mathbf{2 b d}$ was obtained in $90 \%$ yield (390 $\mathrm{mg})$.

Physical state: white solid.

${ }^{1} \mathrm{H}$ NMR (400 MHz, Chloroform- $d$ ): $\delta 7.60$ (s, 2H), 7.39 (d, $\left.J=7.7 \mathrm{~Hz}, 2 \mathrm{H}\right), 7.34(\mathrm{~d}, J=7.5 \mathrm{~Hz}, 2 \mathrm{H}), 2.04$ (br s, 1H), $1.71(\mathrm{~s}, 8 \mathrm{H}), 1.30(\mathrm{~d}, J=5.0 \mathrm{~Hz}, 24 \mathrm{H}), 0.67(\mathrm{~s}, 3 \mathrm{H})$.

${ }^{13}$ C NMR (101 MHz, Chloroform-d): $\delta=146.59,144.09,133.71,132.39,131.03,125.97,35.14,34.97$, $34.30,34.17,31.87,31.74,-1.09$.

The characterization data are consistent with that reported in the literature ${ }^{41}$.

\section{Compound 2be}

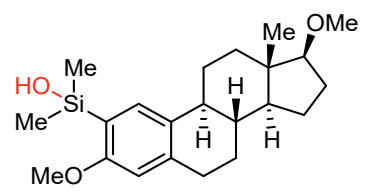

((8R,9S,13S,14S,17S)-3,17-dimethoxy-13-methyl-7,8,9,11,12,13,14,15,16,17-decahydro-6Hcyclopenta[a]phenanthren-2-yl)dimethylsilanol

The reaction was conducted for $12 \mathrm{~h}$ following GP A on $1 \mathrm{mmol}$ scale with $\mathbf{1 b e}$ in $5 \mathrm{~mL} \mathrm{MeCN}$. After purification by flash column chromatography (silica gel, $\mathrm{CH}_{2} \mathrm{Cl}_{2} / \mathrm{PE}=1: 2$ ), the title compound 2 be was obtained in $91 \%$ yield (340 mg).

Physical state: white solid.

${ }^{1}$ H NMR (400 MHz, Chloroform-d): $\delta=7.36(\mathrm{~s}, 1 \mathrm{H}), 6.59$ (s, 1H), $3.82(\mathrm{~s}, 3 \mathrm{H}), 3.39$ (s, 3H), 3.33 (t, $J=$ $8.4 \mathrm{~Hz}, 1 \mathrm{H}), 2.94-2.82(\mathrm{~m}, 2 \mathrm{H}), 2.38-2.17(\mathrm{~m}, 3 \mathrm{H}), 2.12-2.01(\mathrm{~m}, 2 \mathrm{H}), 1.94-1.84(\mathrm{~m}, 1 \mathrm{H}), 1.76-1.64$ $(\mathrm{m}, 1 \mathrm{H}), 1.54-1.24(\mathrm{~m}, 7 \mathrm{H}), 0.80(\mathrm{~s}, 3 \mathrm{H}), 0.39(\mathrm{~s}, 6 \mathrm{H})$.

${ }^{13}$ C NMR (101 MHz, Chloroform- $d$ ): $\delta=161.78,140.16,131.59,126.29,113.75,109.99,90.76,57.85$, $55.19,50.23,43.94,43.21,38.69,38.02,30.19,27.72,27.12,26.44,23.02,11.52,0.49,0.44$.

HRMS (ESI-TOF): calcd for $\mathrm{C}_{22} \mathrm{H}_{34} \mathrm{O}_{3} \mathrm{Si}^{+}[\mathrm{M}+\mathrm{H}]^{+}:$: 375.2350, found: 375.2333 . 
<smiles>CC(C)C1CC[C@@H](C)CC1OCc1ccc(S(=O)(=O)O)cc1</smiles>

\section{(4-((((1R,2S,5R)-2-Isopropyl-5-methylcyclohexyl)oxy)methyl)phenyl)dimethylsilanol}

The reaction was conducted for $8 \mathrm{~h}$ following GP A on $1 \mathrm{mmol}$ scale with $\mathbf{1 b f}$. After purification by flash column chromatography (silica gel, EA/PE $=1: 5$ ), the title compound $\mathbf{2 b f}$ was obtained in $94 \%$ yield (302 $\mathrm{mg})$.

Physical state: viscous oil.

${ }^{1}$ H NMR (400 MHz, Chloroform- $\left.\boldsymbol{d}\right): \delta=7.59(\mathrm{~d}, J=7.9 \mathrm{~Hz}, 2 \mathrm{H}), 7.39(\mathrm{~d}, J=7.8 \mathrm{~Hz}, 2 \mathrm{H}), 4.69$ (d, $J=$ $11.6 \mathrm{~Hz}, 1 \mathrm{H}), 4.43(\mathrm{~d}, J=11.6 \mathrm{~Hz}, 1 \mathrm{H}), 3.20(\mathrm{td}, J=10.5,4.1 \mathrm{~Hz}, 1 \mathrm{H}), 2.33(\mathrm{dtd}, J=14.0,6.9,2.5 \mathrm{~Hz}, 1 \mathrm{H})$, 2.22 (d, $J=11.0 \mathrm{~Hz}, 1 \mathrm{H}), 2.07$ (br s, 1H), $1.74-1.62(\mathrm{~m}, 2 \mathrm{H}), 1.43-1.28(\mathrm{~m}, 2 \mathrm{H}), 0.95$ (dd, $J=15.7,6.8$ $\mathrm{Hz}, 9 \mathrm{H}), 0.75(\mathrm{~d}, J=6.9 \mathrm{~Hz}, 3 \mathrm{H}), 0.41(\mathrm{~s}, 6 \mathrm{H})$.

${ }^{13}$ C NMR (101 MHz, Chloroform-d): $\delta=140.61,138.06,133.07,127.22,78.90,70.27,48.29,40.27,34.55$, $31.55,25.50,23.23,22.35,21.00,16.05,0.01$.

HRMS (ESI-TOF): calcd for $\mathrm{C}_{19} \mathrm{H}_{31} \mathrm{O}_{2} \mathrm{Si}^{-}[\mathrm{M}-\mathrm{H}]^{-}: 319.2088$, found: 319.2096.

\section{Compound 2bg}

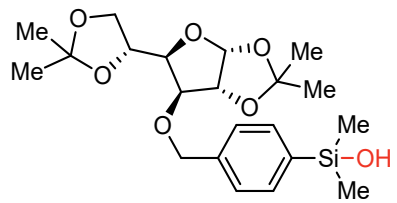

(4-((((3aR,5R,6S,6aR)-5-((R)-2,2-dimethyl-1,3-dioxolan-4-yl)-2,2-dimethyltetrahydrofuro[2,3d][1,3]dioxol-6-yl)oxy)methyl)phenyl)dimethylsilanol

The reaction was conducted for $6 \mathrm{~h}$ following GP A on $1 \mathrm{mmol}$ scale with $\mathbf{1 b g}$. After purification by flash column chromatography (silica gel, EA/PE $=1: 3$ ), the title compound $\mathbf{2 b g}$ was obtained in $92 \%$ yield (390 $\mathrm{mg})$..

Physical state: white solid.

${ }^{1}$ H NMR (400 MHz, Chloroform- $d$ ): $\delta=7.58(\mathrm{~d}, J=7.9 \mathrm{~Hz}, 2 \mathrm{H}), 7.36(\mathrm{~d}, J=7.8 \mathrm{~Hz}, 2 \mathrm{H}), 5.89$ (d, $J=3.6$ Hz, 1H), 4.66 (q, $J=12.0 \mathrm{~Hz}, 2 \mathrm{H}), 4.59$ (d, $J=3.7 \mathrm{~Hz}, 1 \mathrm{H}), 4.36$ (dd, $J=13.6,6.1 \mathrm{~Hz}, 1 \mathrm{H}), 4.12$ (ddd, $J=$ $14.8,8.1,4.6 \mathrm{~Hz}, 2 \mathrm{H}), 4.04-3.93(\mathrm{~m}, 2 \mathrm{H}), 2.21$ (br s, 1H), 1.49 (s, 3H), 1.42 (s, 3H), 1.37 (s, 3H), 1.31 (s, $3 \mathrm{H}), 0.39(\mathrm{~s}, 6 \mathrm{H})$.

${ }^{13}$ C NMR (101 MHz, Chloroform-d): $\delta=139.01,138.76,133.16,127.02,111.78,108.97,105.25,82.60$, $81.72,81.28,72.48,72.21,67.35,26.80,26.74,26.21,25.41,0.02$.

HRMS (ESI-TOF): calcd for $\mathrm{C}_{21} \mathrm{H}_{31} \mathrm{O}_{7} \mathrm{Si}^{-}[\mathrm{M}-\mathrm{H}]^{-}:$: 423.1834, found: 423.1824 .

\section{Compound 2bh}<smiles>CN(c1ccc([Si](=O)(=O)O)cc1)S(=O)(=O)c1ccccc1</smiles>

N-(4-(Hydroxydimethylsilyl)phenyl)-N-(2,2,2-trifluoroethyl)benzenesulfonamide 
The reaction was conducted for $6 \mathrm{~h}$ following GP A on $1 \mathrm{mmol}$ scale with $\mathbf{1 b h}$. After purification by flash column chromatography (silica gel, EA/PE $=1: 2$ ), the title compound $\mathbf{2 b h}$ was obtained in $95 \%$ yield (368 $\mathrm{mg})$.

Physical state: white solid.

${ }^{1}$ H NMR (400 MHz, Chloroform- $d$ ): $\delta=7.62(\mathrm{~d}, J=7.3 \mathrm{~Hz}, 3 \mathrm{H}), 7.54(\mathrm{~d}, J=7.7 \mathrm{~Hz}, 2 \mathrm{H}), 7.48(\mathrm{t}, J=7.4$ $\mathrm{Hz}, 2 \mathrm{H}), 7.06$ (d, $J=7.7 \mathrm{~Hz}, 2 \mathrm{H}), 4.23$ (q, $J=8.2 \mathrm{~Hz}, 2 \mathrm{H}), 2.59$ (br s, $1 \mathrm{H}), 0.38$ (s, 6H).

${ }^{13}$ C NMR (101 MHz, Chloroform-d): $\delta=140.44,140.00,138.18,134.15,133.28,128.99,128.36,127.66$, $125.05,122.26,52.56,52.22,51.87,51.52,-0.07$.

${ }^{19}$ F NMR (376 MHz, Chloroform- $d$ ): $\delta=-70.47(\mathrm{t})$.

HRMS (ESI-TOF): calcd for $\mathrm{C}_{16} \mathrm{H}_{17} \mathrm{~F}_{3} \mathrm{NSO}_{3} \mathrm{Si}^{-}[\mathrm{M}-\mathrm{H}]^{-}: 388.0645$, found: 388.0658 .

\section{Compound 2bi}

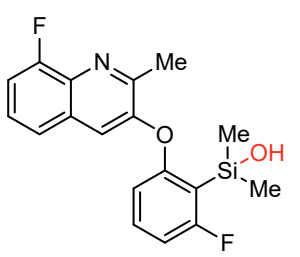

\section{(2-Fluoro-6-((8-fluoro-2-methylquinolin-3-yl)oxy)phenyl)dimethylsilanol}

The reaction was conducted for $6 \mathrm{~h}$ following GP A on $1 \mathrm{mmol}$ scale with $\mathbf{1 b i}$. After purification by flash column chromatography (silica gel, EA/PE $=1: 2$ ), the title compound 2 bi was obtained in $91 \%$ yield (315 $\mathrm{mg})$.

Physical state: white solid.

${ }^{1}$ H NMR (400 MHz, Chloroform- $\boldsymbol{d}$ ): $\delta=7.47$ (s, 1H), 7.44 - 7.37 (m, 2H), 7.37 - $7.28(\mathrm{~m}, 2 \mathrm{H}), 6.88(\mathrm{t}, J$ $=8.4 \mathrm{~Hz}, 1 \mathrm{H}), 6.55(\mathrm{~d}, J=8.1 \mathrm{~Hz}, 1 \mathrm{H}), 2.77(\mathrm{~s}, 3 \mathrm{H}), 2.71(\mathrm{br} \mathrm{s}, 1 \mathrm{H}), 0.49(\mathrm{~d}, J=1.3 \mathrm{~Hz}, 6 \mathrm{H})$.

Note: Due to an issue with rotational isomers, the ${ }^{13} \mathrm{C} N M R$ spectrum is complex.

${ }^{13}$ C NMR (101 MHz, Chloroform-d): $\delta=168.67,166.26,161.26,161.11,158.86,156.31,154.16,150.21$, $132.61,132.51,129.82,126.57,126.49,122.40,122.36,120.78,113.12,112.60,112.41,111.38,111.11$, $20.75,1.90$.

${ }^{19}$ F NMR (376 MHz, Chloroform- $d$ ): $\delta=-99.10,-125.27$ (d).

HRMS (APCI-TOF): calcd for $\mathrm{C}_{18} \mathrm{H}_{18} \mathrm{~F}_{2} \mathrm{NO}_{2} \mathrm{Si}^{+}[\mathrm{M}+\mathrm{H}]^{+}: 346.1069$, found: 346.1074 .

\section{Compound 2bj}<smiles>O=S(=O)(O)c1ccc2c(c1)OCO2</smiles>

\section{1,3-Benzodioxol-5-yl(dimethyl)silanol}

The reaction was conducted for $3 \mathrm{~h}$ following GP A on $1 \mathrm{mmol}$ scale with $\mathbf{1 b j}$. After dried over anhydrous $\mathrm{Na}_{2} \mathrm{SO}_{4}$ and evaporated the solvent, the title compound $\mathbf{2 b j}$ was obtained in $97 \%$ yield (190 $\mathrm{mg}$ ) without further purification.

Physical state: colorless oil.

${ }^{1}$ H NMR (400 MHz, Chloroform- $\boldsymbol{d}$ ): $\delta=7.19-6.96$ (m, 2H), 6.87 (d, $\left.J=7.5 \mathrm{~Hz}, 1 \mathrm{H}\right), 5.95$ (s, 2H), 3.05 (br s, 1H), 0.37 (s, 6H).

${ }^{13}$ C NMR (101 MHz, Chloroform-d): $\delta$ = 148.70, 147.31, 132.29, 127.21, 112.45, 108.54, 100.48, 0.01 . The characterization data are consistent with that reported in the literature ${ }^{46}$. 


\section{Compound 2bk}<smiles>CS(=O)(=O)c1ccncc1</smiles>

\section{Dimethyl(pyridin-4-yl)silanol}

The reaction was conducted for $4 \mathrm{~h}$ following GP A on $1 \mathrm{mmol}$ scale with $\mathbf{1 b k}$. After dried over anhydrous $\mathrm{Na}_{2} \mathrm{SO}_{4}$ and evaporated the solvent, the title compound $\mathbf{2 b k}$ was obtained in $93 \%$ yield (142 $\mathrm{mg}$ ) without further purification.

Note: $2 \boldsymbol{b i}$ is unstable and dimerize slowly during storage.

Physical state: turbid oil.

${ }^{1}$ H NMR (400 MHz, Chloroform- $d$ ): $\delta=8.57$ (d, $\left.J=5.5 \mathrm{~Hz}, 2 \mathrm{H}\right), 7.38$ (dd, $\left.J=4.3,1.3 \mathrm{~Hz}, 2 \mathrm{H}\right), 2.35$ (br $\mathrm{s}, 1 \mathrm{H}), 0.37$ (s, 6H).

${ }^{13}$ C NMR (101 MHz, Chloroform- $d$ ): $\delta=148.84,127.67,0.43$.

The characterization data are consistent with that reported in the literature ${ }^{56}$.

\section{Compound 2bl}<smiles>C[Si](C)(O)c1cccc2ccccc12</smiles>

\section{Dimethyl(naphthalen-1-yl)silanol}

The reaction was conducted for $3 \mathrm{~h}$ following GP A on $1 \mathrm{mmol}$ scale with $\mathbf{1 b l}$. After dried over anhydrous $\mathrm{Na}_{2} \mathrm{SO}_{4}$ and evaporated the solvent, the title compound $\mathbf{2 b l}$ was obtained in $98 \%$ yield (198 $\mathrm{mg}$ ) without further purification.

Physical state: white solid.

${ }^{1}$ H NMR (400 MHz, Chloroform- $d$ ): $\delta=8.25(\mathrm{~d}, J=7.8 \mathrm{~Hz}, 1 \mathrm{H}), 7.86(\mathrm{t}, J=7.7 \mathrm{~Hz}, 2 \mathrm{H}), 7.74(\mathrm{~d}, J=6.7$ $\mathrm{Hz}, 1 \mathrm{H}), 7.53-7.40$ (m, 3H), 2.25 (br s, 1H), 0.55 (s, 6H).

${ }^{13}$ C NMR (101 MHz, Chloroform- $d$ ): $\delta=136.90,136.56,133.36,133.22,130.38,128.99,128.09,126.00$, $125.50,125.00,1.31$.

The characterization data are consistent with that reported in the literature ${ }^{21}$.

\section{Compound 2bm}<smiles>C[Sn]([O-])(O)c1ccc2ccccc2c1</smiles>

\section{Dimethyl(naphthalen-2-yl)silanol}

The reaction was conducted for $1 \mathrm{~h}$ following GPA on $1 \mathrm{mmol}$ scale with $\mathbf{1 b m}$. After dried over anhydrous $\mathrm{Na}_{2} \mathrm{SO}_{4}$ and evaporated the solvent, the title compound $\mathbf{2} \mathbf{b m}$ was obtained in $97 \%$ yield (196 $\mathrm{mg}$ ) without further purification.

Physical state: white solid.

${ }^{1}$ H NMR (400 MHz, Chloroform- $\left.\boldsymbol{d}\right): \delta=8.03(\mathrm{~s}, 1 \mathrm{H}), 7.77(\mathrm{dd}, J=9.5,5.5 \mathrm{~Hz}, 3 \mathrm{H}), 7.59(\mathrm{~d}, J=8.1 \mathrm{~Hz}$, $1 \mathrm{H}), 7.51-7.39(\mathrm{~m}, 2 \mathrm{H}), 3.00$ (br s, 1H), $0.41(\mathrm{~s}, 6 \mathrm{H})$.

${ }^{13}$ C NMR (101 MHz, Chloroform-d): $\delta=136.46,133.92,133.83,132.77,129.15,128.17,127.66,127.11$, $126.48,125.92,0.02$. 
The characterization data are consistent with that reported in the literature ${ }^{21}$.

\section{Compound 2bn}<smiles>C[As](N)(=O)c1ccc(CN2CCOCC2)cc1</smiles>

\section{Dimethyl(4-(morpholinomethyl)phenyl)silanol}

The reaction was conducted for $6 \mathrm{~h}$ following GP A on $1 \mathrm{mmol}$ scale with $\mathbf{1 b n}$. After purification by flash column chromatography ( silica gel, EA/PE $=1: 3)$, the title compound $\mathbf{2 b n}$ was obtained in $95 \%$ yield $(238$ $\mathrm{mg})$.

Physical state: white solid.

${ }^{1}$ H NMR (400 MHz, Chloroform- $d$ ): $\delta=7.56(\mathrm{~d}, J=7.5 \mathrm{~Hz}, 2 \mathrm{H}), 7.35(\mathrm{~d}, J=7.6 \mathrm{~Hz}, 2 \mathrm{H}), 3.70$ (t, $J=4.6$ $\mathrm{Hz}, 4 \mathrm{H}), 3.51$ (br s, 2H), 2.45 (t, $J=4.7 \mathrm{~Hz}, 4 \mathrm{H}), 0.41$ (s, 6H).

${ }^{13}$ C NMR (101 MHz, Chloroform-d): $\delta=138.92,138.09,133.09,128.77,66.84,63.33,53.51,0.04$.

HRMS (ESI-TOF): calcd for $\mathrm{C}_{13} \mathrm{H}_{20} \mathrm{NO}_{2} \mathrm{Si}^{-}[\mathrm{M}-\mathrm{H}]^{-}:$: 250.1258, found: 250.1279 .

\section{Compound 2bo}<smiles>C[As](O)(O)c1ccc(C(=O)N2CCCCC2)cc1</smiles>

\section{(4-(Hydroxydimethylsilyl)phenyl)(piperidin-1-yl)methanone}

The reaction was conducted for $5 \mathrm{~h}$ following GP A on $1 \mathrm{mmol}$ scale with $\mathbf{1 b o}$. After purification by flash column chromatography (silica gel, EA/PE $=1: 1$ ), the title compound $\mathbf{2 b o}$ was obtained in $96 \%$ yield (225 $\mathrm{mg})$.

Physical state: yellow solid.

$2 \boldsymbol{b n}$ was found by NMR spectroscopy to exist as a pair of isomers (A and B) in a 70:30 ratio due to the tautomerism of amide bond (the major tautomer is represented by $A$ ).

${ }^{1} \mathrm{H}$ NMR (400 MHz, Chloroform- $\left.\boldsymbol{d}\right): \delta=7.57\left(\mathrm{~d}, J=4.4 \mathrm{~Hz}, 2 \mathrm{H}_{\mathrm{B}}\right), 7.56\left(\mathrm{~d}, J=4.6 \mathrm{~Hz}, 2 \mathrm{H}_{\mathrm{A}}\right), 7.36(\mathrm{~d}, J=$ $\left.8.0 \mathrm{~Hz}, 2 \mathrm{H}_{\mathrm{A}}\right), 7.33\left(\mathrm{~d}, J=7.9 \mathrm{~Hz}, 2 \mathrm{H}_{\mathrm{B}}\right), 3.71\left(\mathrm{br} \mathrm{s}, 4 \mathrm{H}_{\mathrm{A} / \mathrm{B}}\right), 3.34$ (br s, 4H $\left.\mathrm{H}_{\mathrm{A} / \mathrm{B}}\right), 2.67\left(\mathrm{~s}, 1 \mathrm{H}_{\mathrm{A}}\right), 1.68$ (br s, $4 \mathrm{H}), 1.52\left(\right.$ br s, $\left.2 \mathrm{H}_{\mathrm{A} / \mathrm{B}}\right), 1.26$ (br s, $\left.1 \mathrm{H}_{\mathrm{B}}\right), 0.35\left(\mathrm{~s}, 6 \mathrm{H}_{\mathrm{B}}\right), 0.35\left(\mathrm{~s}, 6 \mathrm{H}_{\mathrm{A}}\right)$.

${ }^{13}$ C NMR (101 MHz, Chloroform- $\boldsymbol{d}$ ): $\delta=170.34(\mathrm{~B}), 170.25(\mathrm{~A}), 141.34(\mathrm{~B}), 141.18(\mathrm{~A}), 137.17(\mathrm{~A})$, 137.04(B), 133.05(B), 132.95(A), 125.91(A), 125.85(B), 48.68, 43.05, 26.48, 25.56, 24.52, 0.77(A), $0.03(\mathrm{~B})$.

HRMS (ESI-TOF): calcd for $\mathrm{C}_{14} \mathrm{H}_{20} \mathrm{NO}_{2} \mathrm{Si}^{-}[\mathrm{M}-\mathrm{H}]^{-}:$: 262.1258, found: 262.1268 .

\section{Compound 2bp}<smiles>O=[As](O)(O)c1ccc(OC2CCCCO2)cc1</smiles>

\section{Dimethyl(4-((tetrahydro-2H-pyran-2-yl)oxy)phenyl)silanol}

The reaction was conducted for $3 \mathrm{~h}$ following GPA on $1 \mathrm{mmol}$ scale with $\mathbf{1 b p}$. After dried over anhydrous $\mathrm{Na}_{2} \mathrm{SO}_{4}$ and evaporated the solvent, the title compound $2 \mathbf{b p}$ was obtained in $97 \%$ yield (244 $\mathrm{mg}$ ) without further purification. 
Physical state: colorless oil.

${ }^{1}$ H NMR (400 MHz, Chloroform- $d$ ): $\delta=7.53(\mathrm{~d}, J=8.5 \mathrm{~Hz}, 2 \mathrm{H}), 7.09$ (d, $\left.J=8.5 \mathrm{~Hz}, 2 \mathrm{H}\right), 5.49$ (t, $J=2.8$ $\mathrm{Hz}, 1 \mathrm{H}), 3.96-3.87$ (m, 1H), $3.71-3.54(\mathrm{~m}, 1 \mathrm{H}), 2.29$ (br s, 1H), 2.04 (dt, $J=16.4,7.9 \mathrm{~Hz}, 1 \mathrm{H}), 1.89$ (dt, $J=7.5,3.7 \mathrm{~Hz}, 2 \mathrm{H}), 1.76-1.61(\mathrm{~m}, 3 \mathrm{H}), 0.40(\mathrm{~s}, 6 \mathrm{H})$.

${ }^{13}$ C NMR (101 MHz, Chloroform- $\boldsymbol{d}$ ): $\delta=158.28,134.52$, 115.95, 95.93, 61.96, 30.25, 25.15, 18.65, 0.04 . HRMS (ESI-TOF): calcd for $\mathrm{C}_{13} \mathrm{H}_{19} \mathrm{O}_{3} \mathrm{Si}^{-}[\mathrm{M}-\mathrm{H}]^{-}: 251.1098$, found: 251.1093 .

\section{Compound 2bq}<smiles>C[N+](C)(O)c1ccc(C2OCCO2)cc1</smiles>

\section{(4-(1,3-Dioxolan-2-yl)phenyl)dimethylsilanol}

The reaction was conducted for $4 \mathrm{~h}$ following GP A on $1 \mathrm{mmol}$ scale with $\mathbf{1 b q}$. After purification by flash column chromatography (silica gel, EA:PE $=1: 3)$, the title compound $\mathbf{2 b q}$ was obtained in $94 \%$ yield $(210$ $\mathrm{mg})$.

Physical state: colorless oil.

${ }^{1}$ H NMR (400 MHz, Chloroform-d): $\delta=7.55(\mathrm{~d}, J=8.0 \mathrm{~Hz}, 2 \mathrm{H}), 7.43(\mathrm{~d}, J=7.9 \mathrm{~Hz}, 2 \mathrm{H}), 5.78(\mathrm{~s}, 1 \mathrm{H})$, 4.11 - $3.96(\mathrm{~m}, 4 \mathrm{H}), 3.05$ (br s, 1H), 0.32 (s, 6H).

${ }^{13}$ C NMR (101 MHz, Chloroform- $d$ ): $\delta=140.49,138.81,133.03,125.69,103.53,65.16,-0.12$.

The characterization data are consistent with that reported in the literature ${ }^{50}$.

\section{Compound 2br}<smiles>C=C[As](C)(O)c1ccccc1</smiles>

\section{Methyl(phenyl)(vinyl)silanol}

The reaction was conducted for $4 \mathrm{~h}$ following GP A on $1 \mathrm{mmol}$ scale with $\mathbf{1 b r}$. After dried over anhydrous $\mathrm{Na}_{2} \mathrm{SO}_{4}$ and evaporated the solvent, the title compound $\mathbf{2} \mathbf{b r}$ was obtained in $97 \%$ yield $(160 \mathrm{mg}$ ) without further purification.

Physical state: colorless oil.

${ }^{1}$ H NMR (400 MHz, Chloroform- $\boldsymbol{d}$ ): $\delta=7.64$ (dd, $\left.J=7.4,1.7 \mathrm{~Hz}, 2 \mathrm{H}\right), 7.49$ - 7.36 (m, 3H), 6.34 (dd, $J=$ 20.2, $14.9 \mathrm{~Hz}, 1 \mathrm{H}), 6.17$ (dd, $J=14.9,3.8 \mathrm{~Hz}, 1 \mathrm{H}), 5.92$ (dd, $J=20.2,3.9 \mathrm{~Hz}, 1 \mathrm{H}), 2.35$ (br s, 1H), 0.51 (s, $3 \mathrm{H})$.

${ }^{13}$ C NMR (101 MHz, Chloroform-d): $\delta=137.20,136.50,134.54,133.59,129.80,127.88,-1.75$.

The characterization data are consistent with that reported in the literature ${ }^{21}$.

\section{Compound 2bs}

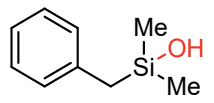

\section{Benzyldimethylsilanol}

The reaction was conducted for $4 \mathrm{~h}$ following GP A on $1 \mathrm{mmol}$ scale with $\mathbf{1 b s}$. After dried over anhydrous $\mathrm{Na}_{2} \mathrm{SO}_{4}$ and evaporated the solvent, the title compound $2 \mathbf{b s}$ was obtained in $98 \%$ yield $(163 \mathrm{mg}$ ) without further purification. 
Physical state: colorless oil.

${ }^{1}$ H NMR (400 MHz, Chloroform- $d$ ): $\delta=7.25$ (t, $\left.J=7.5 \mathrm{~Hz}, 2 \mathrm{H}\right), 7.10$ (dd, $J=17.8,7.5 \mathrm{~Hz}, 3 \mathrm{H}$ ), 2.19 (s, 2H), 1.91 (br s, 1H), 0.15 (s, 6H).

${ }^{13}$ C NMR (101 MHz, Chloroform-d): $\delta=138.98,128.38,128.14,124.25,28.00,-0.76$.

The characterization data are consistent with that reported in the literature ${ }^{21}$.

\section{Compound 2bt}

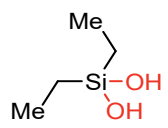

\section{Diethylsilanediol}

The reaction was conducted for $2 \mathrm{~h}$ following GP A on $1 \mathrm{mmol}$ scale with $\mathbf{1 b t}$. After dried over anhydrous $\mathrm{Na}_{2} \mathrm{SO}_{4}$ and evaporated the solvent, the title compound $\mathbf{2 b t}$ was obtained in $96 \%$ yield $(115 \mathrm{mg})$ without further purification.

Physical state: white solid.

${ }^{1}$ H NMR (400 MHz, Chloroform-d): $\delta=3.61$ (br s, 2H), $1.05-0.88$ (m, 6H), $0.71-0.44$ (m, 4H).

${ }^{13}$ C NMR (101 MHz, Chloroform- $d$ ): $\delta=6.61,6.38$.

The characterization data are consistent with that reported in the literature ${ }^{21}$. 


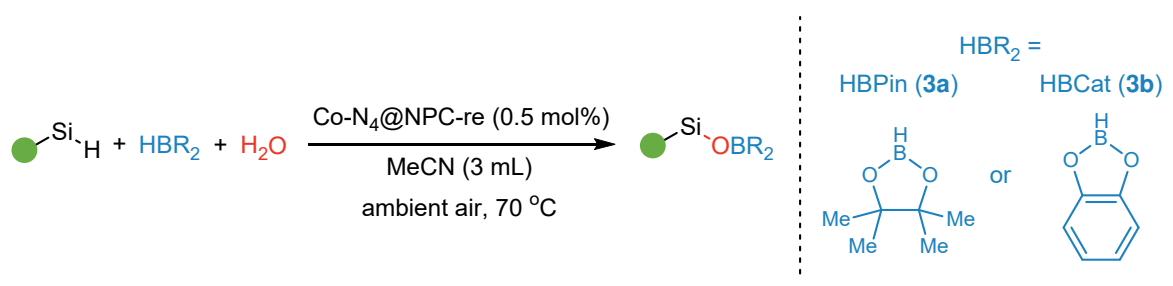

Silane (1mmol), borane (1 mmol) and water $(1 \mathrm{mmol})$ were mixed with $\mathrm{MeCN}(3 \mathrm{~mL})$ in a $10 \mathrm{~mL}$ oven-dried round-bottom flask with reflux condenser and steady magnetic stirring $(800 \mathrm{rpm})$ at $30^{\circ} \mathrm{C}$ under an ambient atmosphere of air. Then catalyst Co-N $4 @$ NPC-re $(0.5 \mathrm{~mol} \% \mathrm{Co})$ was added and the mixture was heat at 70 ${ }^{\circ} \mathrm{C}$ for given reaction time. After completion of the reaction (monitored by GC), the catalyst was removed by filtration and washed with EA $(3 \times 5 \mathrm{~mL})$. The combined organics were washed with brine, dried over anhydrous $\mathrm{Na}_{2} \mathrm{SO}_{4}$ and solvent was removed under reduced pressure. Correspond borosiloxane was obtained without further purification.

\section{Compound 4a}

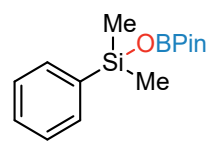

\section{Dimethyl(phenyl)((4,4,5,5-tetramethyl-1,3,2-dioxaborolan-2-yl)oxy)silane}

The reaction was conducted for $6 \mathrm{~h}$ following GP B on $1 \mathrm{mmol}$ scale with 1a and 3a. After dried over anhydrous $\mathrm{Na}_{2} \mathrm{SO}_{4}$ and evaporated the solvent, the title compound $\mathbf{4 a}$ was obtained in $97 \%$ yield $(269 \mathrm{mg})$ without further purification.

Physical state: colorless oil.

${ }^{1}$ H NMR (400 MHz, Chloroform- $\boldsymbol{d}$ ): $\delta=7.67-7.59$ (m, 2H), 7.38 (tdd, $\left.J=3.8,1.4,0.7 \mathrm{~Hz}, 3 \mathrm{H}\right), 1.24$ (s, $12 \mathrm{H}), 0.45(\mathrm{~s}, 6 \mathrm{H})$.

${ }^{13}$ C NMR (101 MHz, Chloroform-d): $\delta=138.15,133.11,129.47,127.64,82.21,24.52,-0.27$.

The characterization data are consistent with that reported in the literature ${ }^{57}$.

\section{Compound 4b}

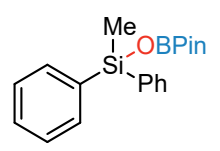

\section{Methyldiphenyl((4,4,5,5-tetramethyl-1,3,2-dioxaborolan-2-yl)oxy)silane}

The reaction was conducted for $6 \mathrm{~h}$ following GP B on $1 \mathrm{mmol}$ scale with $\mathbf{1 x}$ and 3a. After dried over anhydrous $\mathrm{Na}_{2} \mathrm{SO}_{4}$ and evaporated the solvent, the title compound $\mathbf{4 b}$ was obtained in $98 \%$ yield (334 $\mathrm{mg}$ ) without further purification.

Physical state: colorless oil.

${ }^{1}$ H NMR (400 MHz, Chloroform- $\left.\boldsymbol{d}\right): \delta=7.68-7.61(\mathrm{~m}, 4 \mathrm{H}), 7.43-7.32(\mathrm{~m}, 6 \mathrm{H}), 1.23(\mathrm{~s}, 12 \mathrm{H}), 0.74(\mathrm{~s}, 3 \mathrm{H})$.

${ }^{13}$ C NMR (101 MHz, Chloroform-d): $\delta=136.29,133.97,129.67,127.61,82.34,24.45,-1.59$.

The characterization data are consistent with that reported in the literature ${ }^{57}$. 


\section{Compound 4c}<smiles>C=C[Ge](C=C)(O[Na])c1ccccc1</smiles>

\section{Methyl(phenyl)((4,4,5,5-tetramethyl-1,3,2-dioxaborolan-2-yl)oxy)(vinyl)silane}

The reaction was conducted for $8 \mathrm{~h}$ following GP B on $1 \mathrm{mmol}$ scale with $\mathbf{1 b q}$ and 3a. After dried over anhydrous $\mathrm{Na}_{2} \mathrm{SO}_{4}$ and evaporated the solvent, the title compound $\mathbf{4 c}$ was obtained in $98 \%$ yield $(284 \mathrm{mg})$ without further purification.

Physical state: colorless oil.

${ }^{1}$ H NMR (400 MHz, Chloroform- $d$ ): $\delta=7.64$ (dd, $\left.J=7.3,1.5 \mathrm{~Hz}, 2 \mathrm{H}\right), 7.44-7.34$ (m, 3H), 6.32 (dd, $J=$ 20.3, $14.9 \mathrm{~Hz}, 1 \mathrm{H}), 6.13$ (dd, $J=14.9,3.8 \mathrm{~Hz}, 1 \mathrm{H}), 5.90$ (dd, $J=20.3,3.8 \mathrm{~Hz}, 1 \mathrm{H}), 1.25$ (s, 12H), 0.53 (s, $3 \mathrm{H})$.

${ }^{13}$ C NMR (101 MHz, Chloroform-d): $\delta=136.40,135.82,134.49,133.70,129.68,127.67,82.36,24.54$, 2.02 .

The characterization data are consistent with that reported in the literature ${ }^{57}$.

\section{Compound 4d}<smiles>CC[Sb](CC)(CC)O[Na]</smiles>

\section{Triethyl((4,4,5,5-tetramethyl-1,3,2-dioxaborolan-2-yl)oxy)silane}

The reaction was conducted for $12 \mathrm{~h}$ following GP B on $1 \mathrm{mmol}$ scale with $\mathbf{1 a d}$ and 3a. After dried over anhydrous $\mathrm{Na}_{2} \mathrm{SO}_{4}$ and evaporated the solvent, the title compound $\mathbf{4 d}$ was obtained in $94 \%$ yield $(243 \mathrm{mg})$ without further purification.

Physical state: colorless oil.

${ }^{1}$ H NMR (400 MHz, Chloroform- $d$ ): $\delta=1.23$ (s, 12H), $0.95(\mathrm{t}, J=7.9 \mathrm{~Hz}, 9 \mathrm{H}), 0.63(\mathrm{q}, J=7.9 \mathrm{~Hz}, 6 \mathrm{H})$.

${ }^{13}$ C NMR (101 MHz, Chloroform- $d$ ): $\delta=81.94,24.53,6.44,5.38$.

The characterization data are consistent with that reported in the literature ${ }^{57}$.

\section{Compound 4e}<smiles>[InH][SbH3](c1ccccc1)c1ccccc1</smiles>

\section{Triphenyl((4,4,5,5-tetramethyl-1,3,2-dioxaborolan-2-yl)oxy)silane}

The reaction was conducted for $6 \mathrm{~h}$ following GP B on $1 \mathrm{mmol}$ scale with 1aa and 3a. After dried over anhydrous $\mathrm{Na}_{2} \mathrm{SO}_{4}$ and evaporated the solvent, the title compound $4 \mathbf{e}$ was obtained in $98 \%$ yield $(394 \mathrm{mg}$ ) without further purification.

Physical state: white solid.

${ }^{1} \mathrm{H}$ NMR (400 MHz, Chloroform-d): $\delta=7.67$ (d, $\left.J=6.8 \mathrm{~Hz}, 6 \mathrm{H}\right), 7.41$ (dt, $\left.J=24.6,6.9 \mathrm{~Hz}, 9 \mathrm{H}\right), 1.21$ (s, 12H).

${ }^{13}$ C NMR (101 MHz, Chloroform- $\boldsymbol{d}$ ): $\delta=135.22,134.51,130.00,127.68,82.56,24.51$.

The characterization data are consistent with that reported in the literature ${ }^{57}$. 


\section{Compound 4f}

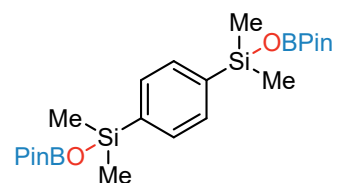

\section{1,4-Bis(dimethyl((4,4,5,5-tetramethyl-1,3,2-dioxaborolan-2-yl)oxy)silyl)benzene}

The reaction was conducted for $8 \mathrm{~h}$ following GP B on $1 \mathrm{mmol}$ scale with 1ag and $2 \mathrm{mmol}$ 3a instead of 1 mmol. After dried over anhydrous $\mathrm{Na}_{2} \mathrm{SO}_{4}$ and evaporated the solvent, the title compound $\mathbf{4 f}$ was obtained in $96 \%$ yield $(457 \mathrm{mg})$ without further purification.

Physical state: colorless oil.

${ }^{1}$ H NMR (400 MHz, Chloroform- $\boldsymbol{d}$ ): $\delta=7.64$ - 7.59 (m, 4H), 1.23 (s, 24H), 0.43 (s, 12H).

${ }^{13}$ C NMR (101 MHz, Chloroform-d): $\delta=139.47,132.27,82.20,24.52,-0.28$.

HRMS (ESI-TOF): calcd for $\mathrm{C}_{22} \mathrm{H}_{40} \mathrm{~B}_{2} \mathrm{O}_{6} \mathrm{Si}_{2}{ }^{+}[\mathrm{M}]^{+}:$: 48.2544, found: 478.2538 .

\section{Compound 4g}<smiles>CCOB(O[GaH])Oc1ccccc1O</smiles>

\section{(Benzo[d][1,3,2]dioxaborol-2-yloxy)triethylsilane}

The reaction was conducted for $12 \mathrm{~h}$ following GP B on $1 \mathrm{mmol}$ scale with $\mathbf{1 a d}$ and $\mathbf{3 b}$. After dried over anhydrous $\mathrm{Na}_{2} \mathrm{SO}_{4}$ and evaporated the solvent, the title compound $4 \mathrm{~g}$ was obtained in $96 \%$ yield $(240 \mathrm{mg})$ without further purification.

Physical state: colorless oil.

${ }^{1}$ H NMR (400 MHz, Chloroform- $d$ ): $\delta=7.12$ - $7.06(\mathrm{~m}, 2 \mathrm{H}), 7.04-6.97(\mathrm{~m}, 2 \mathrm{H}), 1.04(\mathrm{t}, J=7.9 \mathrm{~Hz}, 9 \mathrm{H})$, $0.77(\mathrm{q}, J=7.9 \mathrm{~Hz}, 6 \mathrm{H})$.

${ }^{13}$ C NMR (101 MHz, Chloroform-d): $\delta=148.29,122.00,111.80,6.37,5.34$.

The characterization data are consistent with that reported in the literature ${ }^{58}$.

\section{Compound 4h}

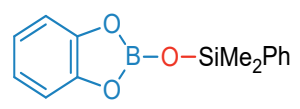

\section{(Benzo[d][1,3,2]dioxaborol-2-yloxy)dimethyl(phenyl)silane}

The reaction was conducted for $12 \mathrm{~h}$ following GP B on $1 \mathrm{mmol}$ scale with $\mathbf{1 a}$ and $\mathbf{3 b}$. After dried over anhydrous $\mathrm{Na}_{2} \mathrm{SO}_{4}$ and evaporated the solvent, the title compound $\mathbf{4 h}$ was obtained in $98 \%$ yield $(264 \mathrm{mg}$ ) without further purification.

Physical state: colorless oil.

${ }^{1}$ H NMR (400 MHz, Chloroform- $d$ ): $\delta=7.74-7.61(\mathrm{~m}, 2 \mathrm{H}), 7.42(\mathrm{~d}, J=1.4 \mathrm{~Hz}, 3 \mathrm{H}), 7.12-7.05$ (m, 2H), $7.03-6.95(\mathrm{~m}, 2 \mathrm{H}), 0.58(\mathrm{~d}, J=3.1 \mathrm{~Hz}, 6 \mathrm{H})$.

${ }^{13}$ C NMR (101 MHz, Chloroform-d): $\delta=148.00,136.88,133.11,130.04,127.97,122.11,111.90,-0.37$. HRMS (ESI-TOF): calcd for $\mathrm{C}_{14} \mathrm{H}_{15} \mathrm{BO}_{3} \mathrm{Si}^{+}[\mathrm{M}]^{+}: 270.0878$, found: 270.0869 .

\section{Compound 4i}<smiles>[CH2+]C[Si](CC)O[PbH2]</smiles>

Diethylbis((4,4,5,5-tetramethyl-1,3,2-dioxaborolan-2-yl)oxy)silane 
The reaction was conducted for $12 \mathrm{~h}$ following GP B on $1 \mathrm{mmol}$ scale with $1 \mathbf{b s}$ and $2 \mathrm{mmol}$ 3a instead of 1 mmol. After dried over anhydrous $\mathrm{Na}_{2} \mathrm{SO}_{4}$ and evaporated the solvent, the title compound $4 \mathbf{i}$ was obtained in $95 \%$ yield $(353 \mathrm{mg})$ without further purification.

Physical state: colorless oil.

${ }^{1}$ H NMR (400 MHz, Chloroform- $d$ ): $\delta=1.23$ (s, 24H), 0.97 (t, $\left.J=7.7 \mathrm{~Hz}, 6 \mathrm{H}\right), 0.68$ (dd, $J=15.9,8.0 \mathrm{~Hz}$, $4 \mathrm{H})$.

${ }^{13}$ C NMR (101 MHz, Chloroform-d): $\delta=82.20,24.56,5.95,5.87$.

The characterization data are consistent with that reported in the literature ${ }^{57}$.

\section{Compound $4 j$}

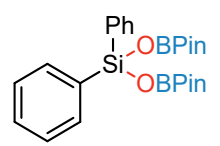

\section{Diphenylbis((4,4,5,5-tetramethyl-1,3,2-dioxaborolan-2-yl)oxy)silane}

The reaction was conducted for $8 \mathrm{~h}$ following GP B on $1 \mathrm{mmol}$ scale with 1 ah and $2 \mathrm{mmol}$ 3a instead of 1 mmol. After dried over anhydrous $\mathrm{Na}_{2} \mathrm{SO}_{4}$ and evaporated the solvent, the title compound $\mathbf{4} \mathbf{j}$ was obtained in $98 \%$ yield $(458 \mathrm{mg})$ without further purification.

Physical state: colorless oil.

${ }^{1}$ H NMR (400 MHz, Chloroform-d): $\delta=7.76-7.70(\mathrm{~m}, 4 \mathrm{H}), 7.42-7.30(\mathrm{~m}, 6 \mathrm{H}), 1.23(\mathrm{~s}, 24 \mathrm{H})$.

${ }^{13}$ C NMR (101 MHz, Chloroform- $\boldsymbol{d}$ ): $\delta=134.47,133.17,130.23,127.51,82.54,24.49$.

The characterization data are consistent with that reported in the literature ${ }^{58}$. 
General procedure for $\mathrm{Co}-\mathrm{N}_{4} @$ NPC-catalyzed oxidative cross-coupling of hydrosilanes and alcohols in batch reactors

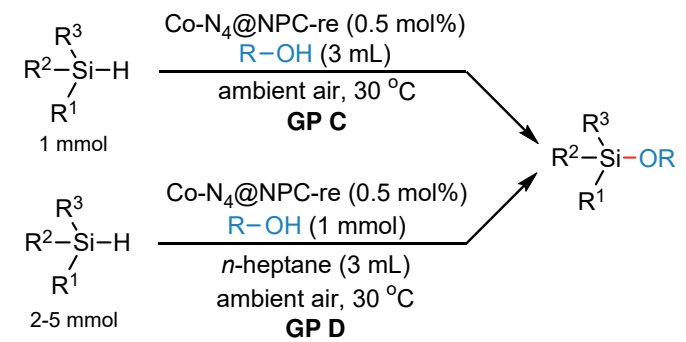

\section{-List of alcohol substrates}

$$
\text { GP D }
$$

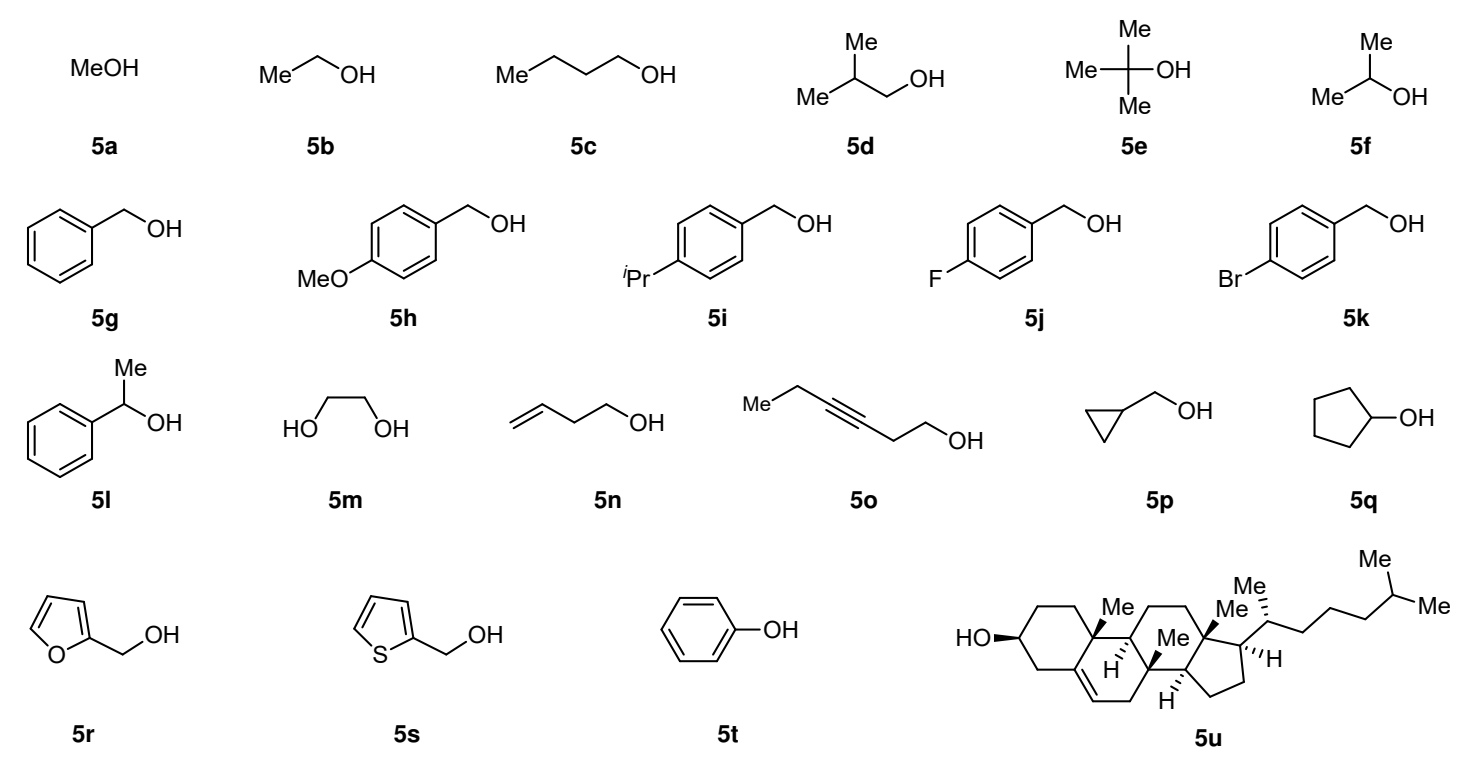

\section{Oxidative cross-coupling of hydrosilanes with simple alkyl alcohols (GP C)}

Silane $(1 \mathrm{mmol})$ and anhydrous alcohol $(3 \mathrm{~mL})$ were mixed in a $15 \mathrm{~mL}$ oven-dried round-bottom tube with steady magnetic stirring $(800 \mathrm{rpm})$ at $30^{\circ} \mathrm{C}$ under an ambient atmosphere of air. The reaction was started by the addition of Co- $\mathrm{N}_{4} @ \mathrm{NPC}-\mathrm{re}(0.5 \mathrm{~mol} \% \mathrm{Co})$ catalyst and the mixture was allowed to stir for given reaction time. After completion of the reaction (monitored by GC and/or TLC), the catalyst was removed by filtration and washed with EA $(3 \times 5 \mathrm{~mL})$. The combined organics were washed with brine, dried over anhydrous $\mathrm{Na}_{2} \mathrm{SO}_{4}$ and solvent was removed under reduced pressure. The crude product was purified via flash column chromatography or vacuum distillation to obtain corresponding silyl ether.

Note: Drying treatment of alcohols is essential to maximize the yield of silyl ether.

\section{Compound 6a}<smiles>C[Si](C)(C)c1ccccc1</smiles>

\section{Methoxydimethyl(phenyl)silane}

The reaction was conducted for $0.5 \mathrm{~h}$ following GP C on $1 \mathrm{mmol}$ scale with $1 \mathrm{a}$ and $\mathrm{MeOH}$ 5a. GC analysis showed the yield of title compound $\mathbf{6 a}$ was $96 \%$. For a gram-scale synthesis, the reaction was followed GP C with $1.36 \mathrm{~g} 1 \mathrm{a}(10 \mathrm{mmol})$ and $20 \mathrm{~mL}$ 5a at $30{ }^{\circ} \mathrm{C}$ for $1 \mathrm{~h}$. Purification by vacuum distillation (5 Torr, 51$53{ }^{\circ} \mathrm{C}$ ) afforded $6 \mathrm{a}$ in $83 \%$ yield $(1.38 \mathrm{~g})$.

Physical state: colorless oil. 
${ }^{1}$ H NMR (400 MHz, Chloroform- $\boldsymbol{d}$ ): $\delta=7.60$ - $7.55(\mathrm{~m}, 2 \mathrm{H}), 7.40-7.35(\mathrm{~m}, 3 \mathrm{H}), 3.44(\mathrm{~s}, 3 \mathrm{H}), 0.38(\mathrm{~s}, 6 \mathrm{H})$.

${ }^{13}$ C NMR (101 MHz, Chloroform- $\boldsymbol{d}$ ): $\delta=137.36,133.43,129.63,127.85,50.64,-2.37$.

The characterization data are consistent with that reported in the literature ${ }^{59}$.

\section{Compound 6b}<smiles>CCO[As](C)(=O)c1ccccc1</smiles>

\section{Ethoxydimethyl(phenyl)silane}

The reaction was conducted for $0.5 \mathrm{~h}$ following GP C on $1 \mathrm{mmol}$ scale with $\mathbf{1 a}$ and EtOH $\mathbf{5 b}$. GC analysis showed the yield of title compound $\mathbf{6 b}$ was $97 \%$. For a gram-scale synthesis, the reaction was followed $\mathbf{G P}$ C with $1.36 \mathrm{~g} \mathbf{1 a}(10 \mathrm{mmol})$ and $30 \mathrm{~mL} \mathbf{5 b}$ at $30^{\circ} \mathrm{C}$ for $1 \mathrm{~h}$. Purification by vacuum distillation (15 Torr, 82$84{ }^{\circ} \mathrm{C}$ ) afforded $\mathbf{6 b}$ in $85 \%$ yield $(1.53 \mathrm{~g})$.

Physical state: colorless oil.

${ }^{1}$ H NMR (400 MHz, Chloroform- $\boldsymbol{d}$ ): $\delta=7.59-7.57$ (m, 2H), 7.39-7.36 (m, 3H), 3.67 (q, $\left.J=7.0 \mathrm{~Hz}, 2 \mathrm{H}\right)$, $1.18(\mathrm{t}, J=7.0 \mathrm{~Hz}, 2 \mathrm{H}), 0.38(\mathrm{~s}, 6 \mathrm{H})$.

${ }^{13}$ C NMR (101 MHz, Chloroform-d): $\delta=137.98,133.44,129.52,127.80,58.66,18.40,-1.76$.

The characterization data are consistent with that reported in the literature ${ }^{59}$.

\section{Compound 6c}<smiles>C[As](C)(=O)c1ccccc1</smiles>

\section{Butoxydimethyl(phenyl)silane}

The reaction was conducted for $0.5 \mathrm{~h}$ following GP C on $1 \mathrm{mmol}$ scale with $1 \mathrm{a}$ and $n$-BuOH 5c. After purification by flash column chromatography (silica gel, EA:PE $=1: 10$ ), the title compound $\mathbf{6} \mathbf{c}$ was obtained in $93 \%$ yield (194 mg).

Physical state: colorless oil.

${ }^{1}$ H NMR (400 MHz, Chloroform- $\left.\boldsymbol{d}\right): \delta=7.68-7.58(\mathrm{~m}, 2 \mathrm{H}), 7.44-7.37(\mathrm{~m}, 3 \mathrm{H}), 3.64(\mathrm{t}, J=6.6 \mathrm{~Hz}, 2 \mathrm{H})$, $1.61-1.49$ (m, 2H), 1.38 (q, $J=7.4 \mathrm{~Hz}, 2 \mathrm{H}), 0.93$ (t, $J=7.3 \mathrm{~Hz}, 3 \mathrm{H}), 0.42$ (s, 6H).

${ }^{13}$ C NMR (101 MHz, Chloroform- $\boldsymbol{d}$ ): $\delta=138.04,133.43,129.47,127.77,62.82,34.71,18.93,13.83,-1.80$. The characterization data are consistent with that reported in the literature ${ }^{59}$.

\section{Compound 6d}<smiles>[O-][As]([O-])([Mg])c1ccccc1</smiles>

\section{Isobutoxydimethyl(phenyl)silane}

The reaction was conducted for $2 \mathrm{~h}$ following GP C on $1 \mathrm{mmol}$ scale with $1 \mathrm{a}$ and $i$-BuOH $5 \mathbf{d}$. After purification by flash column chromatography (silica gel, EA:PE $=1: 10$ ), the title compound $\mathbf{6 d}$ was obtained in $95 \%$ yield (198 mg).

Physical state: colorless oil.

${ }^{1}$ H NMR (400 MHz, Chloroform- $\left.\boldsymbol{d}\right): \delta=7.62-7.55(\mathrm{~m}, 2 \mathrm{H}), 7.39-7.33(\mathrm{~m}, 3 \mathrm{H}), 3.39(\mathrm{~d}, J=6.5 \mathrm{~Hz}, 2 \mathrm{H})$, $1.93-1.67$ (m, 1H), 0.90 (d, $J=6.6 \mathrm{~Hz}, 6 \mathrm{H}), 0.41$ (s, 6H). 
${ }^{13}$ C NMR (101 MHz, Chloroform-d): $\delta=138.15,133.46,129.45,127.76,69.74,30.66,18.99,-1.79$.

The characterization data are consistent with that reported in the literature ${ }^{60}$.

\section{Compound 6e}<smiles>[O-][As]([O-])([Mg])c1ccccc1</smiles>

\section{tert-Butoxydimethyl(phenyl)silane}

The reaction was conducted for $6 \mathrm{~h}$ following GP $\mathbf{C}$ on $1 \mathrm{mmol}$ scale with 1a and $t$-BuOH 5e. After purification by flash column chromatography (silica gel, EA:PE $=1: 10$ ), the title compound $6 \mathbf{e}$ was obtained in $90 \%$ yield $(187 \mathrm{mg})$.

Physical state: colorless oil.

${ }^{1}$ H NMR (400 MHz, Chloroform- $d$ ): $\delta=7.61-7.59(\mathrm{~m}, 2 \mathrm{H}), 7.36-7.34(\mathrm{~m}, 3 \mathrm{H}), 1.25(\mathrm{~s}, 9 \mathrm{H}), 0.38(\mathrm{~s}$, $6 \mathrm{H})$.

${ }^{13}$ C NMR (101 MHz, Chloroform- $d$ ): $\delta=140.75,133.34,129.02,127.60,72.75,32.06,1.38$

The characterization data are consistent with that reported in the literature ${ }^{61}$.

\section{Compound 6f}<smiles>C[As](C)(=O)(O)c1ccccc1</smiles>

\section{Isopropoxydimethyl(phenyl)silane}

The reaction was conducted for $6 \mathrm{~h}$ following GP C on $1 \mathrm{mmol}$ scale with $1 \mathrm{a}$ and $i$-PrOH $\mathbf{5 f}$. After purification by flash column chromatography (silica gel, EA:PE $=1: 10$ ), the title compound $\mathbf{6} \mathbf{f}$ was obtained in $92 \%$ yield (197 mg).

Physical state: colorless oil.

${ }^{1}$ H NMR (400 MHz, Chloroform-d): $\delta=7.66-7.62(\mathrm{~m}, 2 \mathrm{H}), 7.44-7.42(\mathrm{~m}, 3 \mathrm{H}), 4.04$ (hept, $J=6.1 \mathrm{~Hz}$, $1 \mathrm{H}), 1.18(\mathrm{~d}, J=6.1 \mathrm{~Hz}, 6 \mathrm{H}), 0.43(\mathrm{~s}, 6 \mathrm{H})$.

${ }^{13}$ C NMR (101 MHz, Chloroform-d): $\delta=138.50,133.47,129.42,127.73,65.27,25.64,-1.17$.

The characterization data are consistent with that reported in the literature ${ }^{61}$.

\section{Compound 6g}

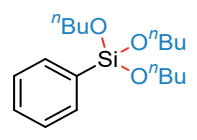

\section{Tributoxy(phenyl)silane}

The reaction was conducted for $0.5 \mathrm{~h}$ following GP C on $1 \mathrm{mmol}$ scale with 1ai and $n$-BuOH $\mathbf{5 c}$. After purification by flash column chromatography (silica gel, EA:PE $=1: 10$ ), the title compound $\mathbf{6 g}$ was obtained in $94 \%$ yield (305 mg).

Physical state: colorless oil.

${ }^{1}$ H NMR (400 MHz, Chloroform- $\left.\boldsymbol{d}\right)$ : $\delta=7.72-7.68(\mathrm{~m}, 2 \mathrm{H}), 7.47-7.38(\mathrm{~m}, 3 \mathrm{H}), 3.84(\mathrm{t}, J=6.6 \mathrm{~Hz}, 6 \mathrm{H})$, $1.66-1.55(\mathrm{~m}, 6 \mathrm{H}), 1.47-1.36(\mathrm{~m}, 6 \mathrm{H}), 0.94(\mathrm{t}, J=7.4 \mathrm{~Hz}, 9 \mathrm{H})$.

${ }^{13}$ C NMR (101 MHz, Chloroform-d): $\delta=134.79,131.03,130.20,127.74,62.72,34.51,18.86,13.77$.

The characterization data are consistent with that reported in the literature ${ }^{59}$. 


\section{Compound 6h}<smiles></smiles>

\section{Dibutoxydiphenylsilane}

The reaction was conducted for $1 \mathrm{~h}$ following GP C on $1 \mathrm{mmol}$ scale with 1 ah and $n$-BuOH 5c. After purification by flash column chromatography (silica gel, EA:PE =1:10), the title compound $\mathbf{6 h}$ was obtained in $96 \%$ yield $(315 \mathrm{mg})$.

Physical state: colorless oil.

${ }^{1}$ H NMR (400 MHz, Chloroform-d): $\delta=7.82$ - $7.58(\mathrm{~m}, 4 \mathrm{H}), 7.54-7.28(\mathrm{~m}, 6 \mathrm{H}), 3.79(\mathrm{t}, J=6.5 \mathrm{~Hz}, 4 \mathrm{H})$, $1.66-1.50(\mathrm{~m}, 4 \mathrm{H}), 1.48-1.33(\mathrm{~m}, 4 \mathrm{H}), 0.90(\mathrm{t}, J=7.4 \mathrm{~Hz}, 6 \mathrm{H})$.

${ }^{13}$ C NMR (101 MHz, Chloroform-d): $\delta=134.88,133.27,130.08,127.73,62.86,34.59,18.94,13.82$.

The characterization data are consistent with that reported in the literature ${ }^{59}$.

\section{Compound 6i}

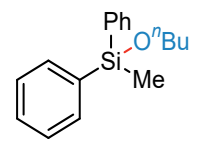

\section{Butoxy(methyl)diphenylsilane}

The reaction was conducted for $1 \mathrm{~h}$ following GP $\mathbf{C}$ on $1 \mathrm{mmol}$ scale with $\mathbf{1 x}$ and $n$-BuOH 5c. After purification by flash column chromatography ( in $93 \%$ yield $(251 \mathrm{mg})$.

Physical state: colorless oil.

${ }^{1}$ H NMR (400 MHz, Chloroform-d): $\delta=7.64$ - 7.59 (m, 4H), $7.43-7.36(\mathrm{~m}, 6 \mathrm{H}), 3.71(\mathrm{t}, J=6.5 \mathrm{~Hz}, 2 \mathrm{H})$, $1.62-1.53(\mathrm{~m}, 2 \mathrm{H}), 1.44-1.33(\mathrm{~m}, 2 \mathrm{H}), 0.89(\mathrm{~s}, 3 \mathrm{H}), 0.65(\mathrm{~s}, 3 \mathrm{H})$.

${ }^{13}$ C NMR (101 MHz, Chloroform-d): $\delta=136.29,134.32,129.70,127.79,63.26,34.70,18.96,13.84,-3.05$. The characterization data are consistent with that reported in the literature ${ }^{61}$.

\section{Compound 6j}<smiles>[OH2+][R17](c1ccccc1)[Si](c1ccccc1)(c1ccccc1)c1ccccc1</smiles>

\section{Butoxytriphenylsilane}

The reaction was conducted for $2 \mathrm{~h}$ following $\mathbf{G P} \mathbf{C}$ on $1 \mathrm{mmol}$ scale with 1 aa and $n$-BuOH 5c. After purification by flash column chromatography ( silica gel, EA:PE = 1:10), the title compound $\mathbf{6 j}$ was obtained in $94 \%$ yield (312 $\mathrm{mg})$.

Physical state: colorless oil.

${ }^{1}$ H NMR (400 MHz, Chloroform-d): $\delta=7.70$ - $7.66(\mathrm{~m}, 6 \mathrm{H}), 7.50-7.39(\mathrm{~m}, 9 \mathrm{H}), 3.85(\mathrm{t}, J=6.5 \mathrm{~Hz}, 2 \mathrm{H})$, $1.69-1.57(\mathrm{~m}, 2 \mathrm{H}), 1.50-1.39(\mathrm{~m}, 2 \mathrm{H}), 0.91(\mathrm{t}, J=7.5 \mathrm{~Hz}, 3 \mathrm{H})$.

${ }^{13}$ C NMR (101 MHz, Chloroform-d): $\delta=35.36,134.45,129.90,127.79,63.64,34.65,18.95,13.83$.

The characterization data are consistent with that reported in the literature ${ }^{60}$. 


\section{Oxidative cross-coupling of hydrosilanes with complex alcohols (GP D)}

Silane ( 2 mmol, 2 equiv.) and alcohol ( $1 \mathrm{mmol}, 1$ equiv.) were mixed with anhydrous $n$-heptane ( $3 \mathrm{~mL})$ in a $15 \mathrm{~mL}$ oven-dried round-bottom tube with steady magnetic stirring $(800 \mathrm{rpm})$ at $30{ }^{\circ} \mathrm{C}$ under an ambient atmosphere of air. The reaction was started by the addition of Co- $\mathrm{N}_{4} @ \mathrm{NPC}-\mathrm{re}$ (Co $\left.0.5 \mathrm{~mol} \%\right)$ catalyst and the mixture was allowed to stir for given reaction time. After completion of the reaction (monitored by GC and/or TLC), the catalyst was removed by filtration and washed with EA $(3 \times 5 \mathrm{~mL})$. The combined organics were washed with brine, dried over anhydrous $\mathrm{Na}_{2} \mathrm{SO}_{4}$ and solvent was removed under reduced pressure. The crude product was purified via flash column chromatography to obtain corresponding silyl ether.

Note: Drying treatment of $n$-heptane is essential to maximize the yield of silyl ether.

\section{Compound 6k}<smiles>c1ccc(CO[AsH2]c2ccccc2)cc1</smiles>

\section{(Benzyloxy)dimethyl(phenyl)silane}

The reaction was conducted for $2 \mathrm{~h}$ following GP D on $1 \mathrm{mmol}$ scale with benzyl alcohol $\mathbf{5 g}$ and $\mathbf{1 a}$. After purification by flash column chromatography (silica gel, EA:PE $=1: 20$ ), the title compound $\mathbf{6 k}$ was obtained in $95 \%$ yield $(230 \mathrm{mg})$.

Physical state: colorless oil.

${ }^{1}$ H NMR (400 MHz, Chloroform- $d$ ): $\delta=7.64$ - 7.57 (m, 2H), 7.42 - $7.36(\mathrm{~m}, 3 \mathrm{H}), 7.33$ - $7.29(\mathrm{~m}, 4 \mathrm{H}), 7.26$ - $7.22(\mathrm{~m}, 1 \mathrm{H}), 4.70(\mathrm{~s}, 2 \mathrm{H}), 0.41(\mathrm{~s}, 6 \mathrm{H})$.

${ }^{13}$ C NMR (101 MHz, Chloroform-d): $\delta=140.70,137.58,133.53,129.67,128.25,127.88,127.10,126.53$, 64.97, -1.71 .

The characterization data are consistent with that reported in the literature ${ }^{62}$.

\section{Compound 61}<smiles>COc1ccc(CO[AsH2]c2ccccc2)cc1</smiles>

\section{((4-Methoxybenzyl)oxy)dimethyl(phenyl)silane}

The reaction was conducted at $60{ }^{\circ} \mathrm{C}$ for $16 \mathrm{~h}$ following GP D on $1 \mathrm{mmol}$ scale with 4-methoxyphenylmethanol $\mathbf{5 h}$ and $3 \mathrm{mmol} \mathbf{1 a}$ using toluene as solvent. After purification by flash column chromatography (silica gel, EA:PE = 1:10), the title compound 61 was obtained in 73\% yield (199 mg).

Physical state: colorless oil.

${ }^{1}$ H NMR (400 MHz, Chloroform- $d$ ): $\delta=7.61$ - 7.57 (m, 2H), $7.41-7.36(\mathrm{~m}, 3 \mathrm{H}), 7.20(\mathrm{~d}, J=8.6 \mathrm{~Hz}, 2 \mathrm{H})$, $6.84(\mathrm{~d}, J=8.6 \mathrm{~Hz}, 2 \mathrm{H}), 4.62(\mathrm{~s}, 2 \mathrm{H}), 3.78(\mathrm{~s}, 3 \mathrm{H}), 0.39(\mathrm{~s}, 6 \mathrm{H})$.

${ }^{13}$ C NMR (101 MHz, Chloroform- $d$ ): $\delta=158.79,137.64,133.52,132.82,129.62,128.16,127.84,113.65$, $64.72,55.22,-1.67$.

The characterization data are consistent with that reported in the literature ${ }^{62}$.

\section{Compound 6m}<smiles>Pc1ccc(CO[AsH2]c2ccccc2)cc1</smiles>

((4-Isopropylbenzyl)oxy)dimethyl(phenyl)silane 
The reaction was conducted for $2 \mathrm{~h}$ following GP D on $1 \mathrm{mmol}$ scale with 4-isopropylphenylmethanol $\mathbf{5 i}$ and 1a. After purification by flash column chromatography (silica gel, EA:PE $=1: 20$ ), the title compound 6m was obtained in $92 \%$ yield $(262 \mathrm{mg})$.

Physical state: colorless oil.

${ }^{1}$ H NMR (400 MHz, Chloroform- $\boldsymbol{d}$ ): $\delta=7.64$ - 7.57 (m, 2H), 7.40 - $7.33(\mathrm{~m}, 3 \mathrm{H}), 7.25$ - $7.14(\mathrm{~m}, 4 \mathrm{H}), 4.65$ (s, 2H), 2.88 (p, $J=6.9 \mathrm{~Hz}, 1 \mathrm{H}), 1.23$ (d, $J=6.9 \mathrm{~Hz}, 6 \mathrm{H}), 0.40$ (s, 6H).

${ }^{13}$ C NMR (101 MHz, Chloroform-d): $\delta=147.81,138.04,137.68,133.54,129.62,127.85,126.74,126.31$, $64.89,33.82,24.03,-1.70$.

The characterization data are consistent with that reported in the literature ${ }^{63}$ ).

\section{Compound 6n}<smiles>Fc1ccc(CO[14CH2]c2ccccc2)cc1</smiles>

\section{((4-Fluorobenzyl)oxy)dimethyl(phenyl)silane}

The reaction was conducted for $2 \mathrm{~h}$ following GP D on 1 mmol scale with 4-fluorophenylmethanol $5 \mathbf{j}$ and 3 mmol 1a using toluene as solvent. After purification by flash column chromatography (silica gel, EA:PE = $1: 20)$, the title compound $\mathbf{6 n}$ was obtained in $91 \%$ yield $(238 \mathrm{mg}$ ).

Physical state: colorless oil.

${ }^{1}$ H NMR (400 MHz, Chloroform-d): $\delta=7.65-7.55(\mathrm{~m}, 2 \mathrm{H}), 7.46-7.33(\mathrm{~m}, 3 \mathrm{H}), 7.24(\mathrm{dd}, J=8.0,5.8$ $\mathrm{Hz}, 2 \mathrm{H}), 6.99(\mathrm{t}, J=8.7 \mathrm{~Hz}, 2 \mathrm{H}), 4.64(\mathrm{~s}, 2 \mathrm{H}), 0.41(\mathrm{~s}, 6 \mathrm{H})$.

${ }^{13}$ C NMR (101 MHz, Chloroform-d): $\delta=163.25,160.81,137.41,136.43,133.51,129.74,128.27,128.19$, 128.92, 115.15, 114.94, 64.36, -1.75.

${ }^{19}$ F NMR (376 MHz, Chloroform- $d$ ): $\delta=-115.81$.

The characterization data are consistent with that reported in the literature ${ }^{62}$.

\section{Compound 6o}<smiles>Brc1ccc(CO[AsH2]c2ccccc2)cc1</smiles>

\section{((4-Bromobenzyl)oxy)dimethyl(phenyl)silane}

The reaction was conducted at $60{ }^{\circ} \mathrm{C}$ for $16 \mathrm{~h}$ following GP D on $1 \mathrm{mmol}$ scale with 4-bromophenylmethanol 5k and $3 \mathrm{mmol} \mathbf{1 a}$ using toluene as solvent. After purification by flash column chromatography (silica gel, $\mathrm{EA}: \mathrm{PE}=1: 20)$, the title compound 60 was obtained in $86 \%$ yield $(276 \mathrm{mg})$.

Physical state: colorless oil.

${ }^{1}$ H NMR (400 MHz, Chloroform- $\left.\boldsymbol{d}\right): \delta=7.68-7.59(\mathrm{~m}, 2 \mathrm{H}), 7.52-7.25(\mathrm{~m}, 5 \mathrm{H}), 7.22(\mathrm{~d}, J=8.4 \mathrm{~Hz}, 2 \mathrm{H})$, $4.68(\mathrm{~s}, 2 \mathrm{H}), 0.46(\mathrm{~s}, 6 \mathrm{H})$.

${ }^{13}$ C NMR (101 MHz, Chloroform-d $): \delta=139.77,137.28,133.48,131.31,129.78,128.16,127.93,120.83$, $64.28,-1.76$.

The characterization data are consistent with that reported in the literature ${ }^{62}$.

\section{Compound 6p}<smiles>[C]C(O[Na])c1ccccc1</smiles>

\section{Dimethyl(phenyl)(1-phenylethoxy)silane}


The reaction was conducted for $5 \mathrm{~h}$ following GP D on $1 \mathrm{mmol}$ scale with 1-phenylethan-1-ol $\mathbf{5 l}$ and $\mathbf{1 a}$. After purification by flash column chromatography (silica gel, EA:PE $=1: 20$ ), the title compound $\mathbf{6} \mathbf{p}$ was obtained in $64 \%$ yield $(164 \mathrm{mg})$.

Physical state: colorless oil.

${ }^{1}$ H NMR (400 MHz, Chloroform-d): $\delta=7.58$ - $7.53(\mathrm{~m}, 2 \mathrm{H}), 7.44-7.35(\mathrm{~m}, 4 \mathrm{H}), 7.35$ - $7.27(\mathrm{~m}, 4 \mathrm{H}), 4.88$ (q, $J=6.4 \mathrm{~Hz}, 1 \mathrm{H}), 1.44(\mathrm{~d}, J=6.4 \mathrm{~Hz}, 3 \mathrm{H}), 0.36(\mathrm{~s}, 3 \mathrm{H}), 0.31(\mathrm{~s}, 3 \mathrm{H})$.

${ }^{13}$ C NMR (101 MHz, Chloroform-d): $\delta=146.21,138.10,133.51,129.50,128.11,127.75,126.86,125.38$, $71.06,26.81,-0.87,-1.42$.

The characterization data are consistent with that reported in the literature ${ }^{64}$.

\section{Compound 6q}

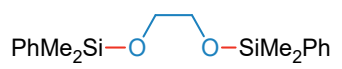

\section{2,7-Dimethyl-2,7-diphenyl-3,6-dioxa-2,7-disilaoctane}

The reaction was conducted for $12 \mathrm{~h}$ following GP D on $1 \mathrm{mmol}$ scale with ethylene glycol $\mathbf{5 m}$ and $5 \mathrm{mmol}$ 1a using toluene/ $\mathrm{MeCN}(\mathrm{v} / \mathrm{v}=2: 1)$ as the solvent. After purification by flash column chromatography (silica gel, $\mathrm{EA}: \mathrm{PE}=1: 20)$, the title compound $\mathbf{6 q}$ was obtained in $55 \%$ yield $(182 \mathrm{mg})$.

Physical state: colorless oil.

${ }^{1}$ H NMR (400 MHz, Chloroform- $d$ ): $\delta=7.59-7.53(\mathrm{~m}, 4 \mathrm{H}), 7.41-7.36(\mathrm{~m}, 6 \mathrm{H}), 3.66(\mathrm{~s}, 4 \mathrm{H}), 0.37$ (s, $12 \mathrm{H})$.

${ }^{13}$ C NMR (101 MHz, Chloroform- $\boldsymbol{d}$ ): $\delta=137.76,133.50,129.55,127.79,64.18,-1.78$.

The characterization data are consistent with that reported in the literature ${ }^{65}$.

\section{Compound 6r}<smiles>C=CCCO[Hg+](c1ccccc1)c1ccccc1</smiles>

\section{(But-3-en-1-yloxy)dimethyl(phenyl)silane}

The reaction was conducted for $8 \mathrm{~h}$ following GP D on $1 \mathrm{mmol}$ scale with but-3-en-1-ol $\mathbf{5 n}$ and 1a. After purification by flash column chromatography (silica gel, EA:PE $=1: 20$ ), the title compound $\mathbf{6} \mathbf{r}$ was obtained in $80 \%$ yield $(165 \mathrm{mg})$.

Physical state: colorless oil.

${ }^{1}$ H NMR (400 MHz, Chloroform- $\boldsymbol{d}$ ): $\delta=7.60$ - 7.56 (m, 2H), 7.40 - 7.37 (m, 3H), 5.79 (ddt, $J=17.1,10.3$, $6.8 \mathrm{~Hz}, 1 \mathrm{H}), 5.10-4.98(\mathrm{~m}, 2 \mathrm{H}), 3.65(\mathrm{t}, J=6.9 \mathrm{~Hz}, 2 \mathrm{H}), 2.29$ (qt, $J=6.9,1.4 \mathrm{~Hz}, 2 \mathrm{H}), 0.38(\mathrm{~s}, 6 \mathrm{H})$.

${ }^{13}$ C NMR (101 MHz, Chloroform-d): $\delta=137.89,135.17,133.47,129.56,127.81,116.45,62.61,37.13$, 1.77 .

The characterization data are consistent with that reported in the literature ${ }^{66}$.

\section{Compound 6s}

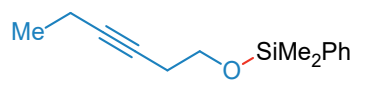

\section{(Hex-3-yn-1-yloxy)dimethyl(phenyl)silane}

The reaction was conducted for $20 \mathrm{~h}$ following GP D on $1 \mathrm{mmol}$ scale with hex-3-yn-1-ol 5o and 1a. After purification by flash column chromatography (silica gel, EA:PE $=1: 20$ ), the title compound $\mathbf{6 s}$ was obtained in $78 \%$ yield $(182 \mathrm{mg})$. 
Physical state: colorless oil.

${ }^{1}$ H NMR (400 MHz, Chloroform- $d$ ): $\delta=7.59$ (dq, $\left.J=4.7,2.3 \mathrm{~Hz}, 2 \mathrm{H}\right), 7.41-7.37(\mathrm{~m}, 3 \mathrm{H}), 3.68$ (t, $J=$ $7.3 \mathrm{~Hz}, 2 \mathrm{H}), 2.38$ (tt, $J=7.4,2.4 \mathrm{~Hz}, 2 \mathrm{H}), 2.14$ (qt, $J=7.6,2.4 \mathrm{~Hz}, 2 \mathrm{H}), 1.10$ (t, $J=7.5 \mathrm{~Hz}, 3 \mathrm{H}), 0.39$ (s, $6 \mathrm{H})$.

${ }^{13}$ C NMR (101 MHz, Chloroform-d): $\delta$ = 137.66, 133.47, 129.62, 127.83, 82.96, 76.01, 62.14, 22.89, 14.15, $12.38,-1.78$.

HRMS (ESI-TOF): calcd for $\mathrm{C}_{14} \mathrm{H}_{19} \mathrm{~N}_{2} \mathrm{O}_{3} \mathrm{Si}^{+}[\mathrm{M}+\mathrm{H}]^{+}: 233.1356$, found: 233.1368 .

\section{Compound 6t}<smiles></smiles>

\section{(Cyclopropylmethoxy)dimethyl(phenyl)silane}

The reaction was conducted for $3.5 \mathrm{~h}$ following GP D on $1 \mathrm{mmol}$ scale with cyclopropylmethanol $\mathbf{5 p}$ and 1a. After purification by flash column chromatography (neutral alumina, $\mathrm{Et}_{2} \mathrm{O}$ :hexanes $=1: 10$ ), the title compound $6 \mathbf{t}$ was obtained in $90 \%$ yield $(186 \mathrm{mg})$.

Note: $4 \mathrm{t}$ is volatile under high vacuum.

Physical state: colorless oil.

${ }^{1}$ H NMR (400 MHz, Chloroform- $d$ ): $\delta=7.62-7.56(\mathrm{~m}, 2 \mathrm{H}), 7.42-7.35(\mathrm{~m}, 3 \mathrm{H}), 3.44(\mathrm{~d}, J=6.7 \mathrm{~Hz}, 2 \mathrm{H})$, 1.02 (ttt, $J=8.1,6.5,4.9 \mathrm{~Hz}, 1 \mathrm{H}), 0.51-0.43(\mathrm{~m}, 2 \mathrm{H}), 0.40(\mathrm{~s}, 6 \mathrm{H}), 0.16-0.10(\mathrm{~m}, 2 \mathrm{H})$.

${ }^{13}$ C NMR (101 MHz, Chloroform- $d$ ): $\delta=138.01,133.51,129.51,127.79,67.81,13.17,2.98,-1.65$.

The characterization data are consistent with that reported in the literature ${ }^{62}$.

\section{Compound 6u}

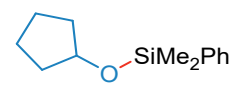

\section{(Cyclopentyloxy)dimethyl(phenyl)silane}

The reaction was conducted for $3 \mathrm{~h}$ following GP D on $1 \mathrm{mmol}$ scale with cyclopentanol $\mathbf{5 q}$ and 1a. After purification by flash column chromatography (silica gel, EA:PE $=1: 20$ ), the title compound $6 \mathbf{u}$ was obtained in $66 \%$ yield $(145 \mathrm{mg})$.

Physical state: colorless oil.

${ }^{1}$ H NMR (400 MHz, Chloroform- $\boldsymbol{d}$ ): $\delta=7.65-7.56(\mathrm{~m}, 2 \mathrm{H}), 7.41-7.36(\mathrm{~m}, 3 \mathrm{H}), 4.33-4.15(\mathrm{~m}, 1 \mathrm{H}), 1.80$ - $1.65(\mathrm{~m}, 4 \mathrm{H}), 1.61-1.45(\mathrm{~m}, 4 \mathrm{H}), 0.38(\mathrm{~s}, 6 \mathrm{H})$.

${ }^{13}$ C NMR (101 MHz, Chloroform-d): $\delta=138.67,133.47,129.36,127.72,74.70,35.49,23.13,-1.10$.

The characterization data are consistent with that reported in the literature ${ }^{67}$.

\section{Compound 6v}

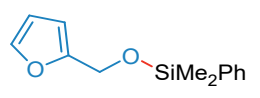

\section{(Furan-2-ylmethoxy)dimethyl(phenyl)silane}

The reaction was conducted for $24 \mathrm{~h}$ following GP D on $1 \mathrm{mmol}$ scale with furfuryl alcohol $\mathbf{5 r}$ and $3 \mathrm{mmol}$ 1a using toluene as solvent. After purification by flash column chromatography (silica gel, EA:PE = 1:10), the title compound $6 \mathbf{v}$ was obtained in $79 \%$ yield $(183 \mathrm{mg})$.

Physical state: colorless oil. 
${ }^{1}$ H NMR (400 MHz, Chloroform- $d$ ): $\delta=7.58(\mathrm{dd}, J=5.0,2.4 \mathrm{~Hz}, 2 \mathrm{H}), 7.38(\mathrm{dd}, J=12.2,5.9 \mathrm{~Hz}, 4 \mathrm{H})$, $6.29(\mathrm{~d}, J=1.8 \mathrm{~Hz}, 1 \mathrm{H}), 6.18(\mathrm{~d}, J=3.1 \mathrm{~Hz}, 1 \mathrm{H}), 4.59$ (s, 2H), 0.39 (s, 6H).

${ }^{13}$ C NMR (101 MHz, Chloroform-d): $\delta=153.58,142.30,137.28,133.55,129.67,127.85,110.19,107.83$, $57.71,-1.79$.

The characterization data are consistent with that reported in the literature ${ }^{62}$.

\section{Compound 6w}<smiles></smiles>

\section{Dimethyl(phenyl)(thiophen-2-ylmethoxy)silane}

The reaction was conducted at $60{ }^{\circ} \mathrm{C}$ for $24 \mathrm{~h}$ following GP D on $1 \mathrm{mmol}$ scale with thiophen-2-ylmethanol 5s and 3 mmol 1 a using toluene as solvent. After purification by flash column chromatography (silica gel, $\mathrm{EA}: \mathrm{PE}=1: 10)$, the title compound $\mathbf{6} \mathbf{w}$ was obtained in $63 \%$ yield $(171 \mathrm{mg})$.

Physical state: colorless oil.

${ }^{1}$ H NMR (400 MHz, Chloroform-d): $\delta=7.67-7.60(\mathrm{~m}, 2 \mathrm{H}), 7.42(\mathrm{~d}, J=7.1 \mathrm{~Hz}, 3 \mathrm{H}), 7.26(\mathrm{dd}, J=5.1$, $1.3 \mathrm{~Hz}, 1 \mathrm{H}), 6.99-6.88(\mathrm{~m}, 2 \mathrm{H}), 4.86(\mathrm{~s}, 2 \mathrm{H}), 0.44(\mathrm{~s}, 6 \mathrm{H})$.

${ }^{13}$ C NMR (101 MHz, Chloroform- $d$ ): $\delta=133.57,129.75,127.90,126.53,124.99,124.68,60.32,-1.70$.

The characterization data are consistent with that reported in the literature ${ }^{62}$.

\section{Compound 6x}

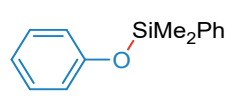

\section{Dimethyl(phenoxy)(phenyl)silane}

The reaction was conducted at $60{ }^{\circ} \mathrm{C}$ for $30 \mathrm{~h}$ following GP D on $1 \mathrm{mmol}$ scale with phenol $5 \mathrm{t}$ and $3 \mathrm{mmol}$ 1a using toluene as solvent. After purification by flash column chromatography (silica gel, EA:PE = 1:20), the title compound $\mathbf{6} \mathbf{x}$ was obtained in $61 \%$ yield $(139 \mathrm{mg})$.

Physical state: colorless oil.

${ }^{1}$ H NMR (400 MHz, Chloroform-d): $\delta=7.70$ - $7.64(\mathrm{~m}, 2 \mathrm{H}), 7.46-7.34(\mathrm{~m}, 3 \mathrm{H}), 7.22(\mathrm{t}, J=7.4 \mathrm{~Hz}, 2 \mathrm{H})$, $6.97(\mathrm{t}, J=7.2 \mathrm{~Hz}, 1 \mathrm{H}), 6.85(\mathrm{~d}, J=7.7 \mathrm{~Hz}, 2 \mathrm{H}), 0.55(\mathrm{~s}, 6 \mathrm{H})$.

${ }^{13}$ C NMR (101 MHz, Chloroform-d): $\delta=155.04,137.19,132.98,129.88,129.38,127.94,121.48,120.02$, -1.14 .

The characterization data are consistent with that reported in the literature ${ }^{66}$.

\section{Compound 6y}

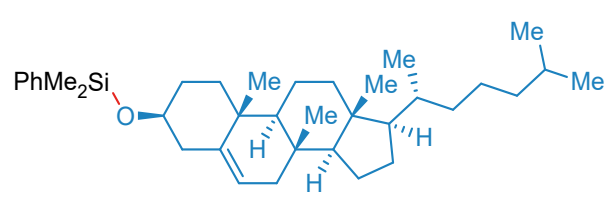

Dimethyl(phenyl)(((3S,8S,9S,10R,13R,14R,17R)-8,10,13-trimethyl-17-((R)-6-methylheptan-2-yl)2,3,4,7,8,9,10,11,12,13,14,15,16,17-tetradecahydro-1H-cyclopenta[a]phenanthren-3-yl)oxy)silane The reaction was conducted at $60{ }^{\circ} \mathrm{C}$ for $12 \mathrm{~h}$ following GP D on $1 \mathrm{mmol}$ scale with cholesterol $5 \mathbf{u}$ and $1 \mathbf{a}$. After purification by flash column chromatography (silica gel, EA:PE $=1: 10$ ), the title compound $\mathbf{6 y}$ was obtained in $83 \%$ yield $(443 \mathrm{mg})$.

Physical state: colorless oil. 
${ }^{1}$ H NMR (400 MHz, Chloroform- $\boldsymbol{d}$ ): $\delta=7.63-7.54(\mathrm{~m}, 2 \mathrm{H}), 7.38-7.32(\mathrm{~m}, 3 \mathrm{H}), 5.24(\mathrm{~d}, J=5.0 \mathrm{~Hz}, 1 \mathrm{H})$, $3.56-3.45(\mathrm{~m}, 1 \mathrm{H}), 2.37-2.23(\mathrm{~m}, 1 \mathrm{H}), 2.15$ (ddd, $J=13.4,4.6,1.8 \mathrm{~Hz}, 1 \mathrm{H}), 2.03-1.90(\mathrm{~m}, 2 \mathrm{H}), 1.86-$ $1.74(\mathrm{~m}, 2 \mathrm{H}), 1.69$ (d, $J=13.0 \mathrm{~Hz}, 1 \mathrm{H}), 1.60-1.18(\mathrm{~m}, 12 \mathrm{H}), 1.14-0.85(\mathrm{~m}, 21 \mathrm{H}), 0.66(\mathrm{~s}, 3 \mathrm{H}), 0.38(\mathrm{~d}, J$ $=2.0 \mathrm{~Hz}, 6 \mathrm{H})$.

${ }^{13}$ C NMR (101 MHz, Chloroform-d): $\delta=141.18,138.51,133.41,129.41,127.72,121.32,72.73,56.76$, $56.15,50.14,42.56,42.29,39.78,39.52,37.30,36.49,36.19,35.77,31.86,28.22,27.98,24.27,23.83,22.81$, $22.56,21.03,19.34,18.72,11.83,-0.97,-1.06$.

The characterization data are consistent with that reported in the literature ${ }^{68}$.

\section{Oxidative dimerization}

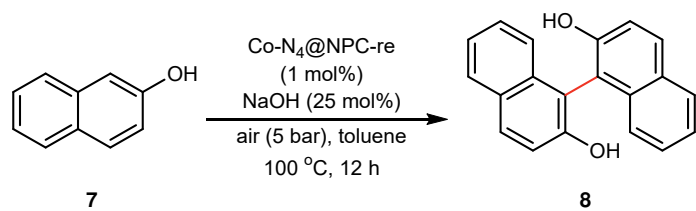

A mixture of 7 (144 mg, $1 \mathrm{mmol}$ ), NaOH (10 mg, $0.25 \mathrm{mmol}$ ), Co-N $\mathrm{N}_{4} @ \mathrm{NPC}-\mathrm{re}$ (Co $\left.1 \mathrm{~mol} \%\right)$ and toluene (3 $\mathrm{mL}$ ) was charged into a $25 \mathrm{~mL}$ Hastelloy-C high pressure Parr reactor. After the reactor was sealed, the reactor was pressured to 5 bar air and then heated to $100{ }^{\circ} \mathrm{C}$. The mixture of substrates and catalyst was stirred at a rate of $800 \mathrm{rpm}$ for $16 \mathrm{~h}$. After completion of the reaction, $5 \mathrm{~mL} 0.1 \mathrm{~mol} / \mathrm{L} \mathrm{HCl}$ aqueous solution was added. Then the mixture was filtered over a short pad of silica and washed with EA $(3 \times 5 \mathrm{~mL})$. The combined organics were washed with brine, dried over anhydrous $\mathrm{Na}_{2} \mathrm{SO}_{4}$ and solvent was removed under reduced pressure. The crude product was purified via flash column chromatography (silica gel, EA:PE = $1: 10)$ to afford compound $\mathbf{8}$ in $81 \%$ yield (114 $\mathrm{mg}$ ).

\section{Compound 8}<smiles>Oc1ccc2ccccc2c1-c1c(O)ccc2ccccc12</smiles>

\section{1,1'-Binaphthalene-2,2'-diol}

Physical state: white solid.

${ }^{1}$ H NMR (400 MHz, DMSO- $\left.\boldsymbol{d}_{6}\right): \delta=9.20(\mathrm{~s}, 2 \mathrm{H}), 7.85(\mathrm{dd}, J=8.6,3.8 \mathrm{~Hz}, 4 \mathrm{H}), 7.33(\mathrm{~d}, J=8.9 \mathrm{~Hz}, 2 \mathrm{H})$, 7.23 (t, $J=7.4 \mathrm{~Hz}, 2 \mathrm{H}), 7.17$ (t, $J=7.6 \mathrm{~Hz}, 2 \mathrm{H}), 6.94$ (d, $J=8.4 \mathrm{~Hz}, 2 \mathrm{H})$.

${ }^{13}$ C NMR (101 MHz, DMSO- $\left.\boldsymbol{d}_{\mathbf{6}}\right): \delta=152.99,134.11,128.62,128.11,127.84,125.81,124.39,122.25$, $118.53,115.39$.

The characterization data are consistent with that reported in the literature ${ }^{69}$.

\section{Oxidative carbonylation}

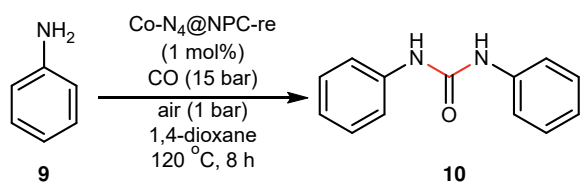

A mixture of 9 (93 mg, 1 mmol), Co-N $4 @$ NPC-re (Co 1 mol\%) and 1,4-dioxane (3 mL) was charged into a $25 \mathrm{~mL}$ Hastelloy-C high pressure Parr reactor. After the reactor was sealed, the reactor was charged with 15 
bar CO and 1 bar air. The mixture of substrates and catalyst was heated to $120^{\circ} \mathrm{C}$ and stirred at a rate of 800 rpm for $8 \mathrm{~h}$. After completion of the reaction, the mixture was filtered over a short pad of silica and washed with EA $(3 \times 5 \mathrm{~mL})$. The combined organics were washed with brine, dried over anhydrous $\mathrm{Na}_{2} \mathrm{SO}_{4}$ and solvent was removed under reduced pressure. The crude product was purified via flash column chromatography (silica gel, EA:PE $=1: 4$ ) to afford compound $\mathbf{1 0}$ in $83 \%$ yield $(88 \mathrm{mg}$ ).

\section{Compound 10}<smiles>O=C(Nc1ccccc1)Nc1ccccc1</smiles>

\section{1,3-Diphenylurea}

Physical state: white solid.

${ }^{1}$ H NMR (400 MHz, DMSO-d $\left.\mathbf{d}_{6}\right): \delta=8.66$ (s, 2H), 7.51 - 7.43 (m, 4H), 7.33 - 7.23 (m, 4H), 7.01 - 6.92 (m, $2 \mathrm{H})$.

${ }^{13}$ C NMR (101 MHz, DMSO- $\left.\boldsymbol{d}_{\mathbf{6}}\right): \delta=152.55,139.72,128.78,121.80,118.19$.

The characterization data are consistent with that reported in the literature ${ }^{70}$.

\section{Oxidative cyclization}

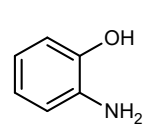

11

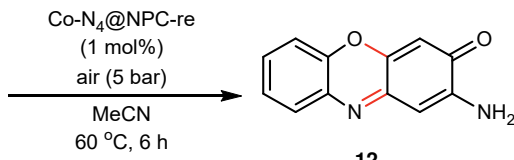

12

A mixture of 11 (109 mg, 1 mmol), Co-N $\mathrm{N}_{4} @ \mathrm{NPC}-\mathrm{re}(\mathrm{Co} 1 \mathrm{~mol} \%)$ and MeCN (3 mL) was charged into a 25 $\mathrm{mL}$ Hastelloy-C high pressure Parr reactor. After the reactor was sealed, the reactor was pressured to 5 bar air and then heated to $60{ }^{\circ} \mathrm{C}$. The mixture of substrates and catalyst was stirred at a rate of $800 \mathrm{rpm}$ for $6 \mathrm{~h}$. After completion of the reaction, the mixture was filtered over a short pad of silica and washed with THF (3 $\times 5 \mathrm{~mL}$ ). The combined organics were washed with brine, dried over anhydrous $\mathrm{Na}_{2} \mathrm{SO}_{4}$ and solvent was removed under reduced pressure. The crude product was purified via flash column chromatography (silica gel, $\mathrm{EA}: \mathrm{PE}=3: 7)$ to afford compound 12 in $94 \%$ yield $(100 \mathrm{mg})$.

\section{Compound 12}<smiles></smiles>

\section{2-Amino-3H-phenoxazin-3-one}

Physical state: red solid.

${ }^{1}$ H NMR (400 MHz, DMSO- $\left.\boldsymbol{d}_{6}\right): \delta=7.71(\mathrm{dd}, J=7.9,1.6 \mathrm{~Hz}, 1 \mathrm{H}), 7.54-7.43(\mathrm{~m}, 2 \mathrm{H}), 7.39(\mathrm{td}, J=7.5$, $1.8 \mathrm{~Hz}, 1 \mathrm{H}), 6.81$ (br s, 2H), 6.36 (s, 2H).

${ }^{13}$ C NMR (101 MHz, DMSO- $\left.\boldsymbol{d}_{\mathbf{6}}\right): \delta=180.22,148.88,148.25,147.38,141.93,133.74,128.80,127.98$, 125.28, 115.94, 103.43, 98.35 .

The characterization data are consistent with that reported in the literature ${ }^{71}$. 


\section{NMR Spectra}

\section{Compound 1c ${ }^{1} \mathrm{H}$ NMR}

(me

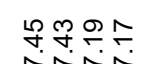

iñin

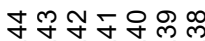

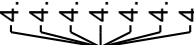

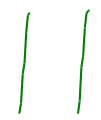

$\int$

$$
\mid
$$
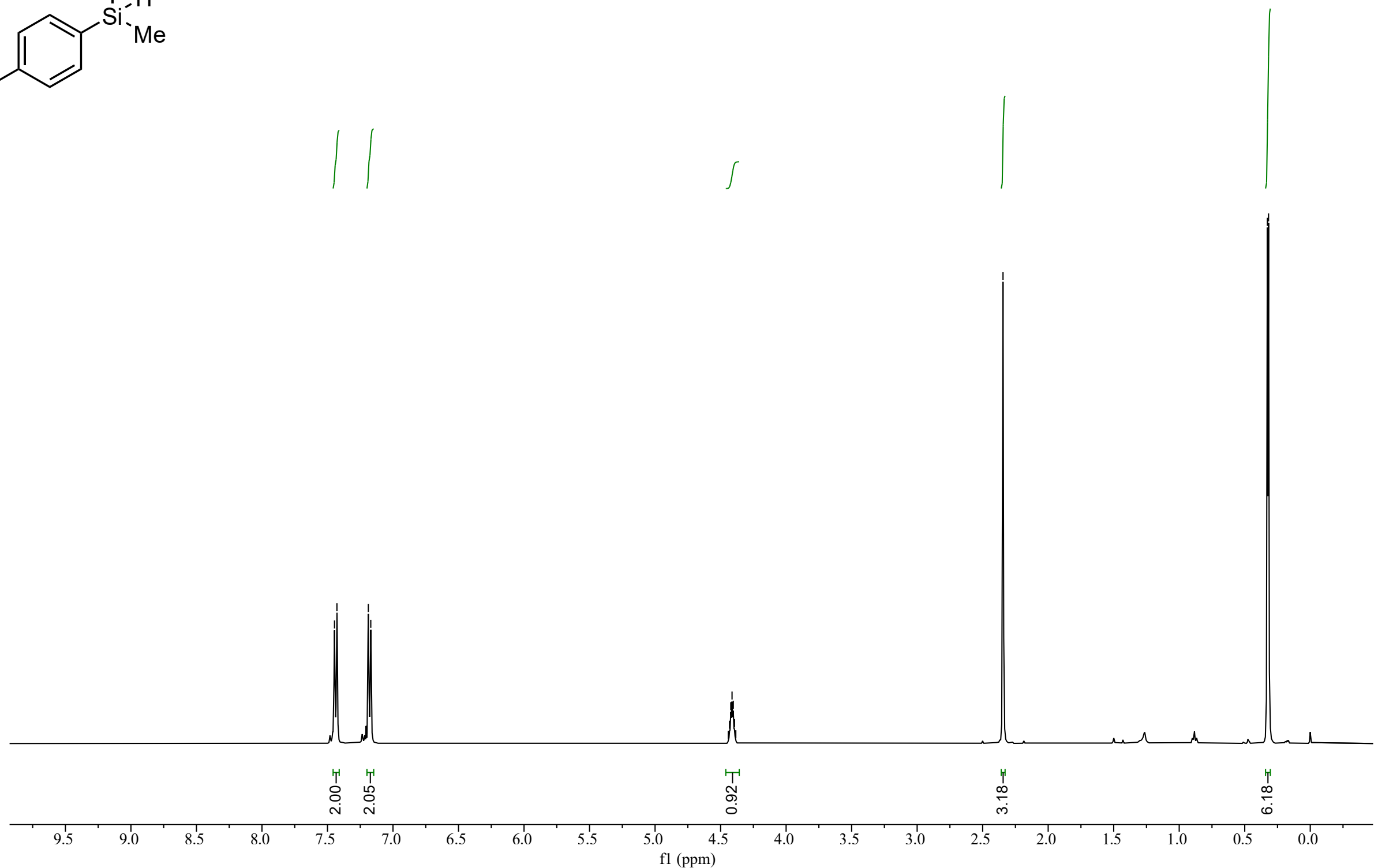
Compound 1c ${ }^{13} \mathrm{C}$ NMR

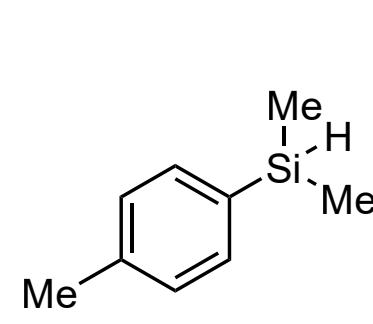

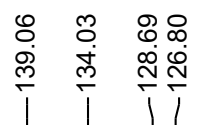

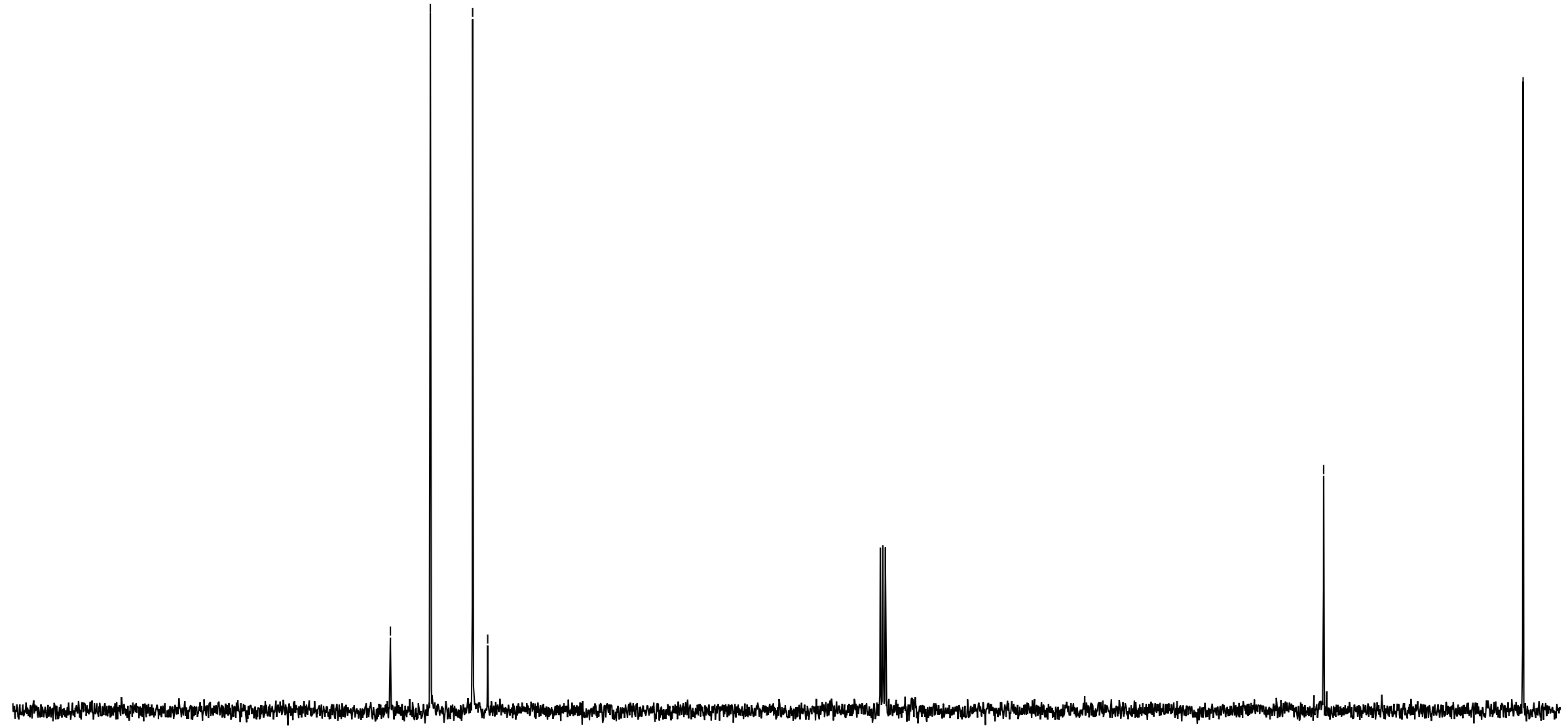




\section{Compound 1d ${ }^{1} \mathrm{H}$ NMR}

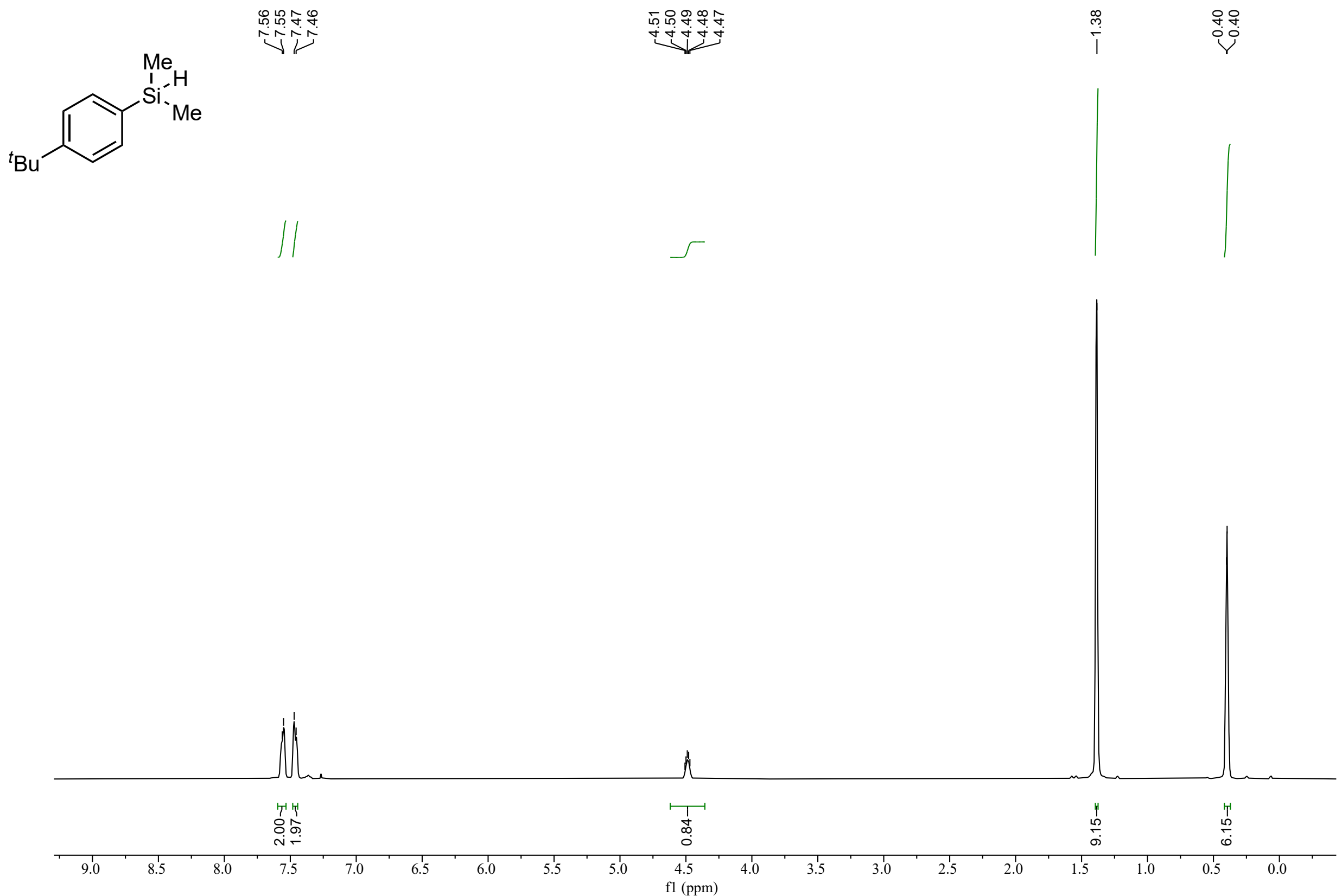


Compound 1d ${ }^{13} \mathrm{C}$ NMR
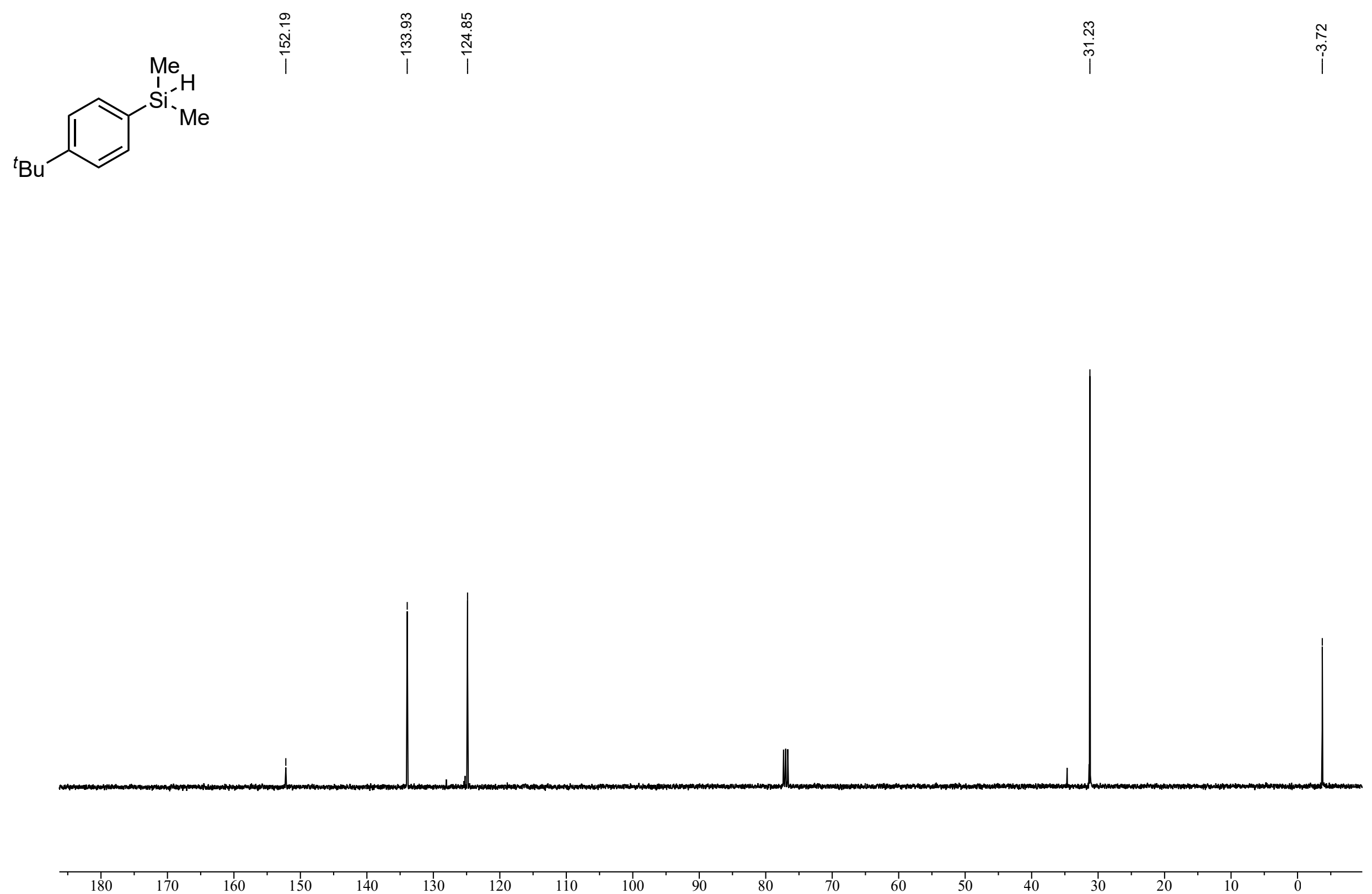


\section{Compound 1e ${ }^{1} \mathrm{H}$ NMR}

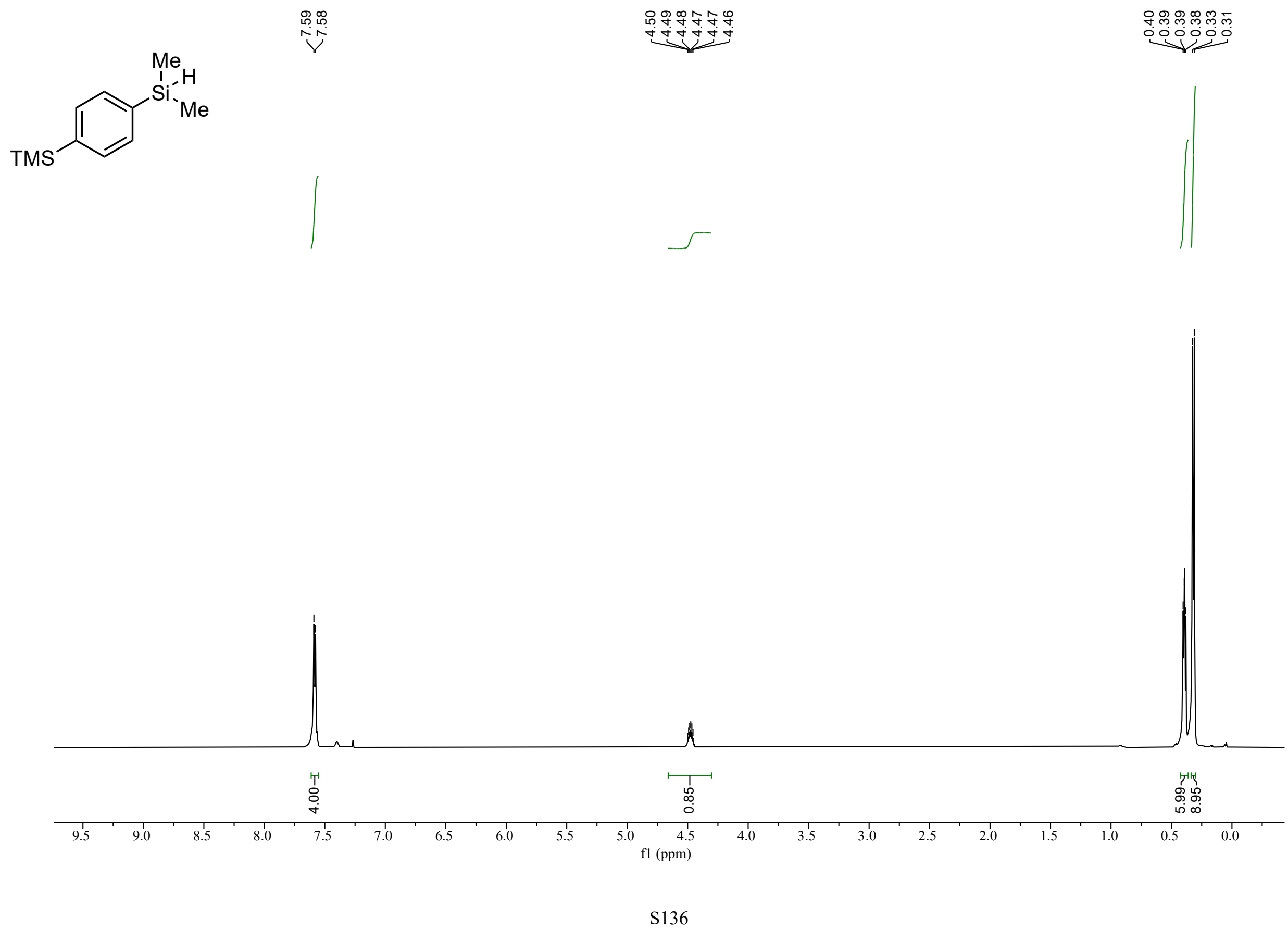


Compound 1e ${ }^{13} \mathrm{C}$ NMR

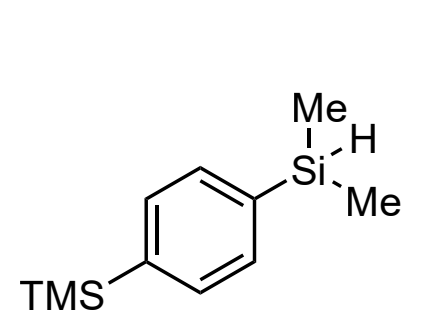

웅ํำำำ

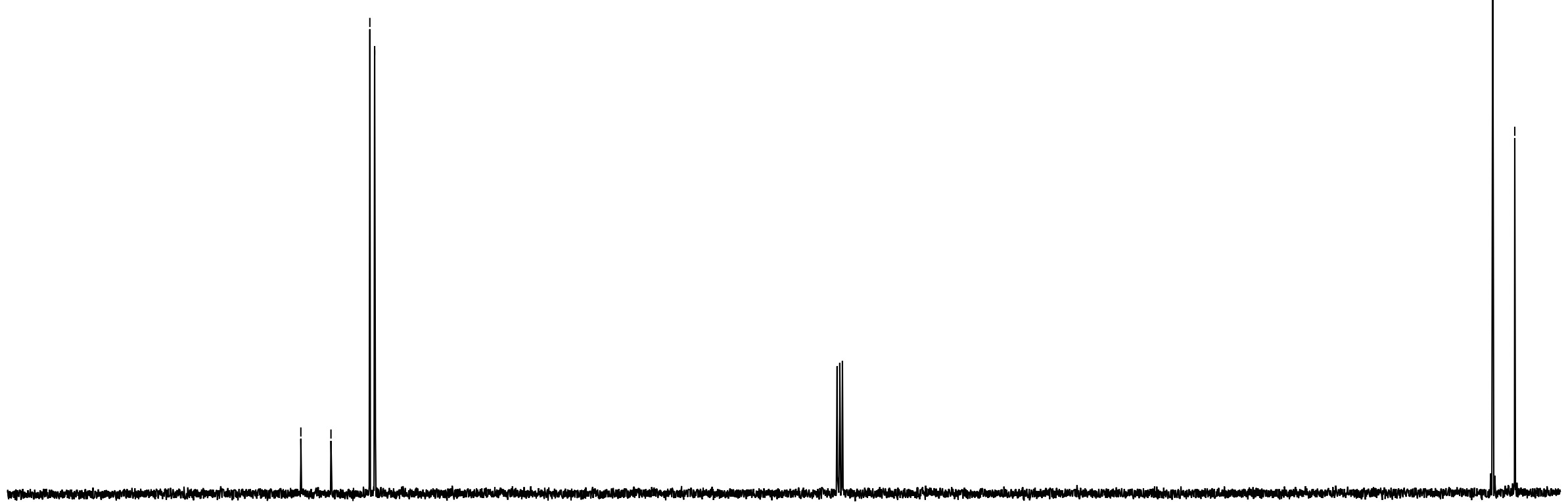


Compound 1f ${ }^{1} \mathrm{H}$ NMR
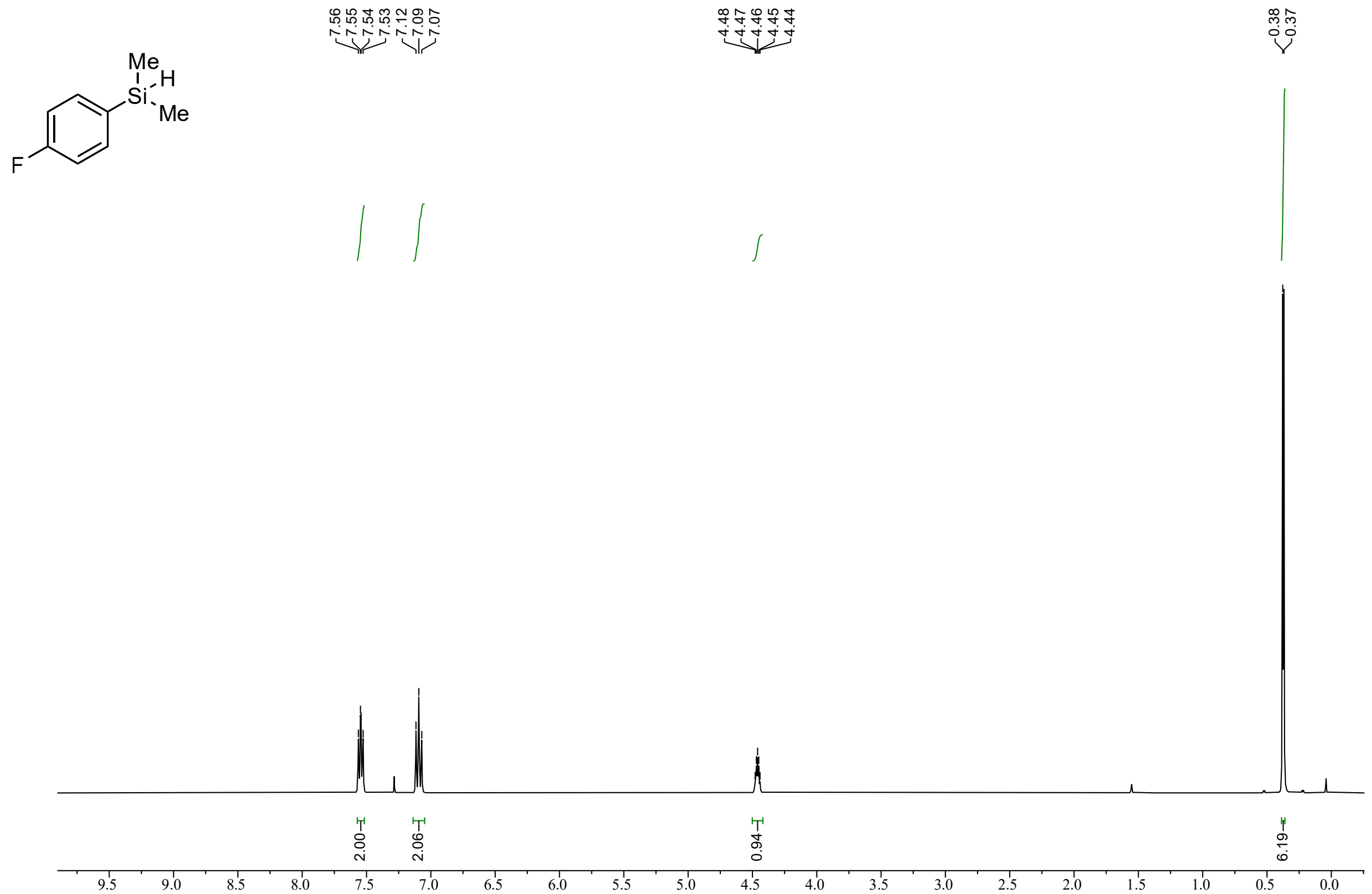
Compound $1 \mathrm{If}^{13} \mathrm{C}$ NMR
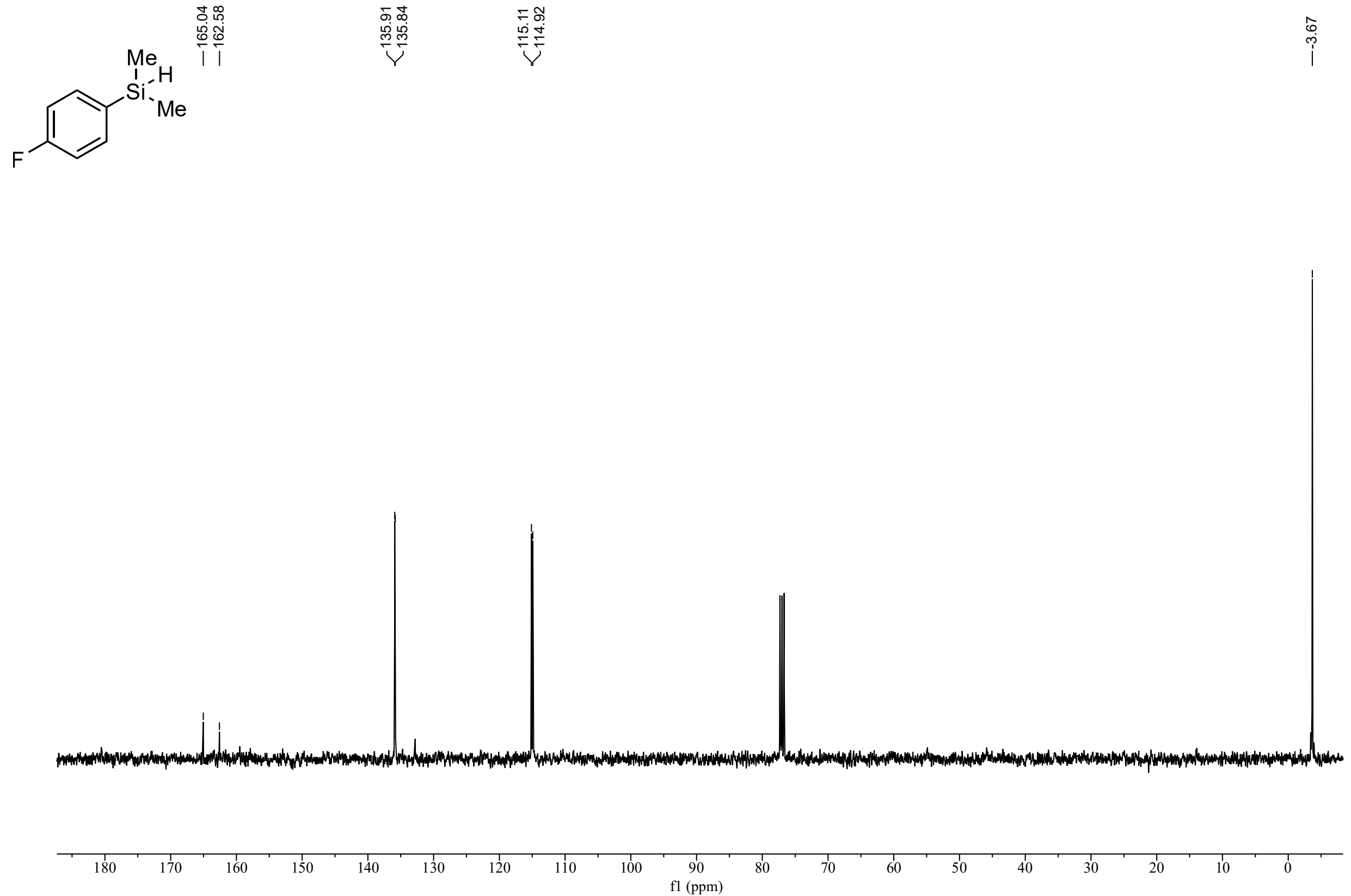
Compound 1f ${ }^{19} \mathrm{~F}$ NMR
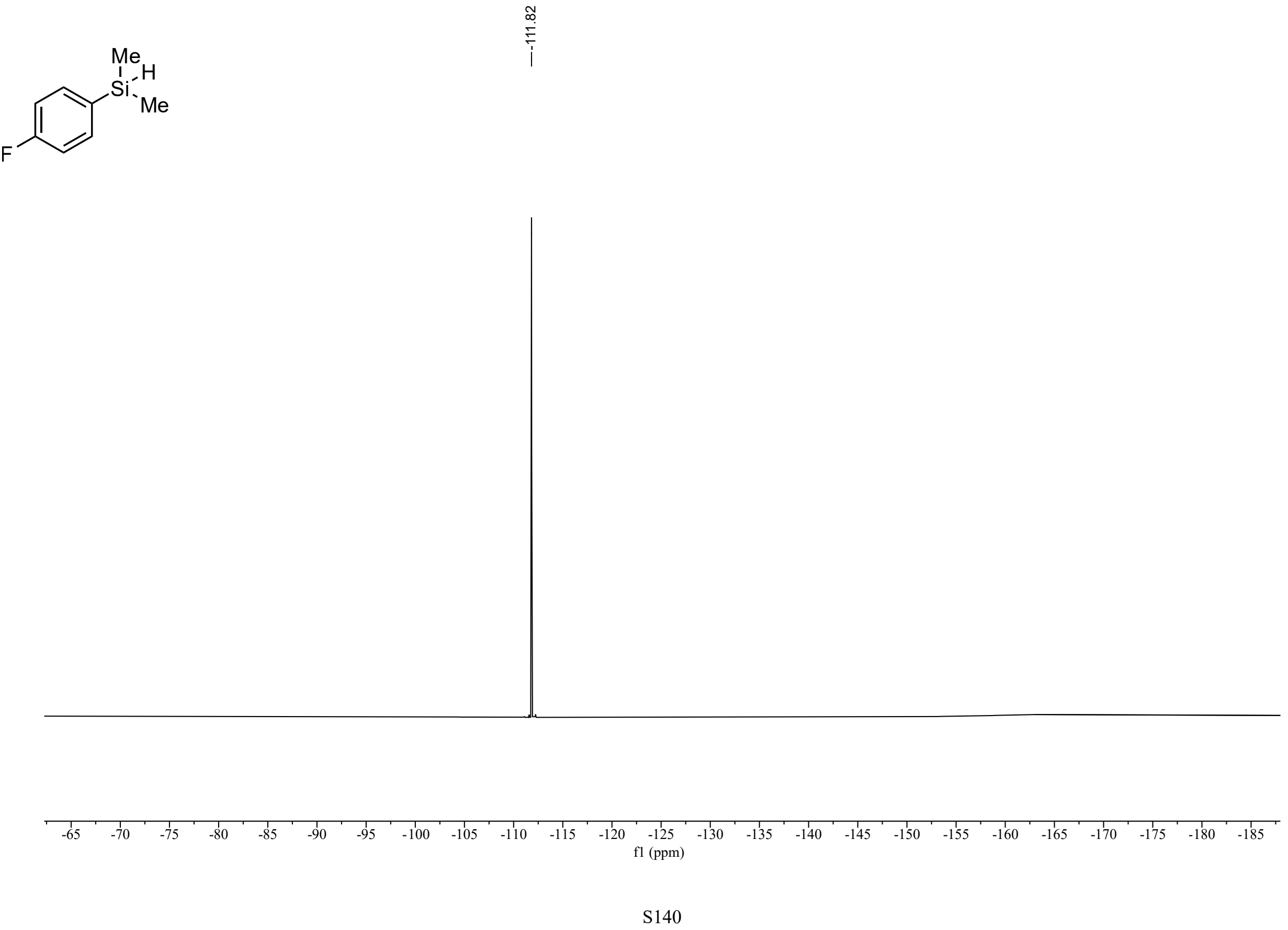
Compound 1g ${ }^{1} \mathrm{H}$ NMR

(1)

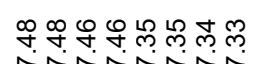

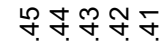

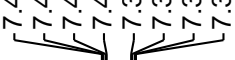

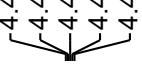

iv
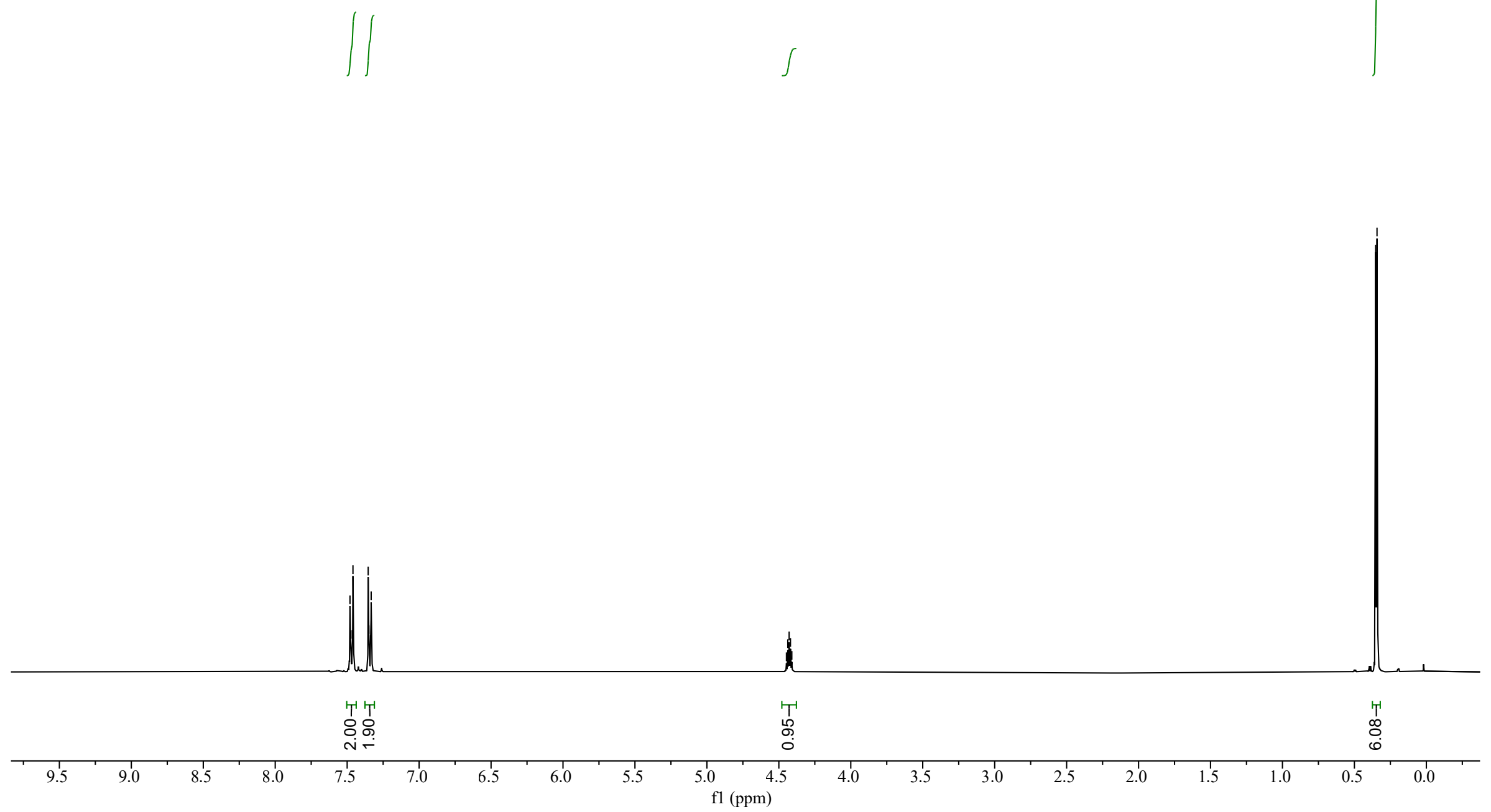
Compound 1g ${ }^{13} \mathrm{C}$ NMR

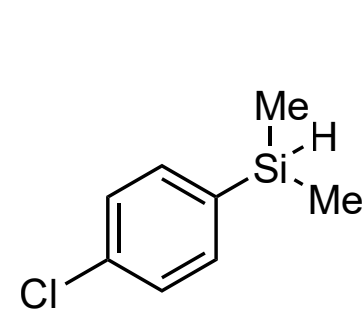

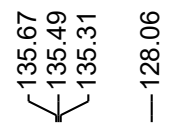

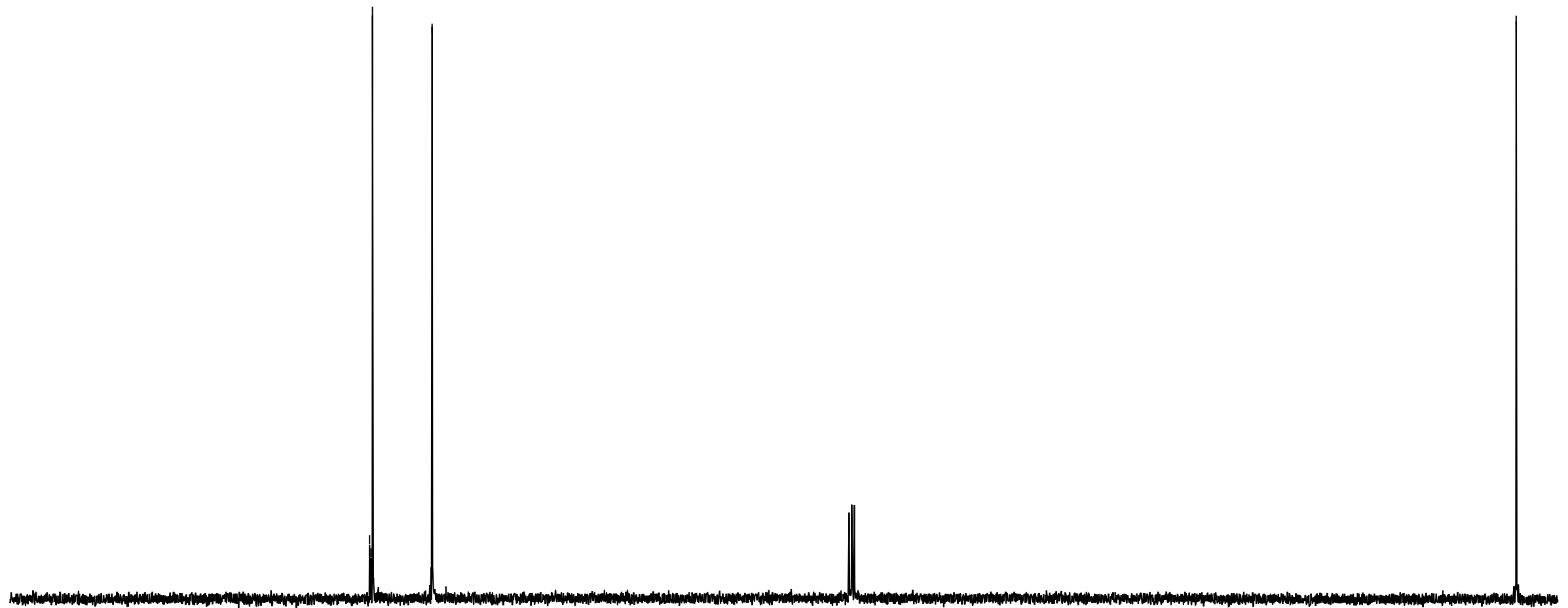


Compound $\mathbf{1 h}{ }^{1} \mathrm{H}$ NMR
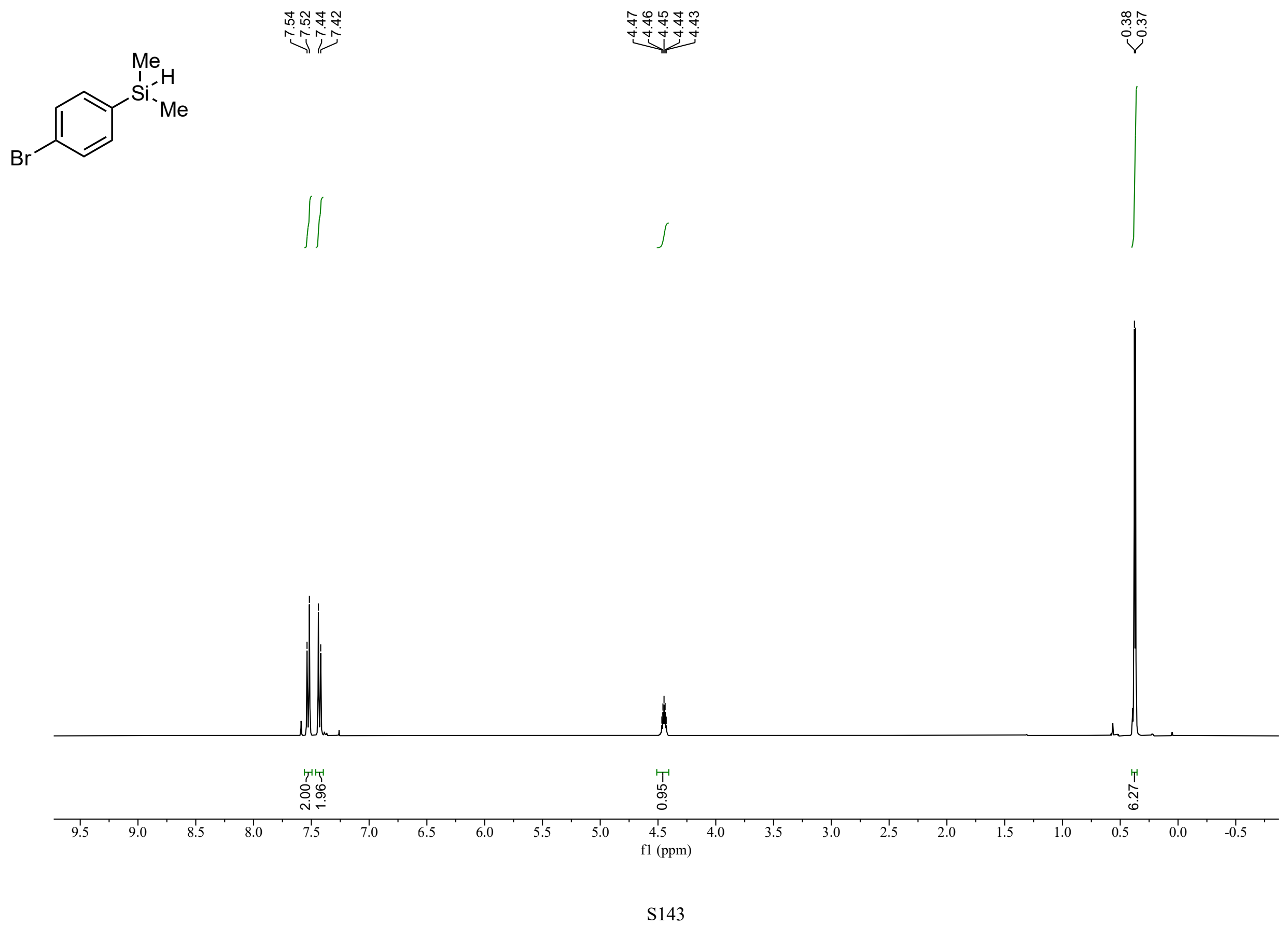
Compound $\mathbf{1 h}{ }^{13} \mathrm{C}$ NMR
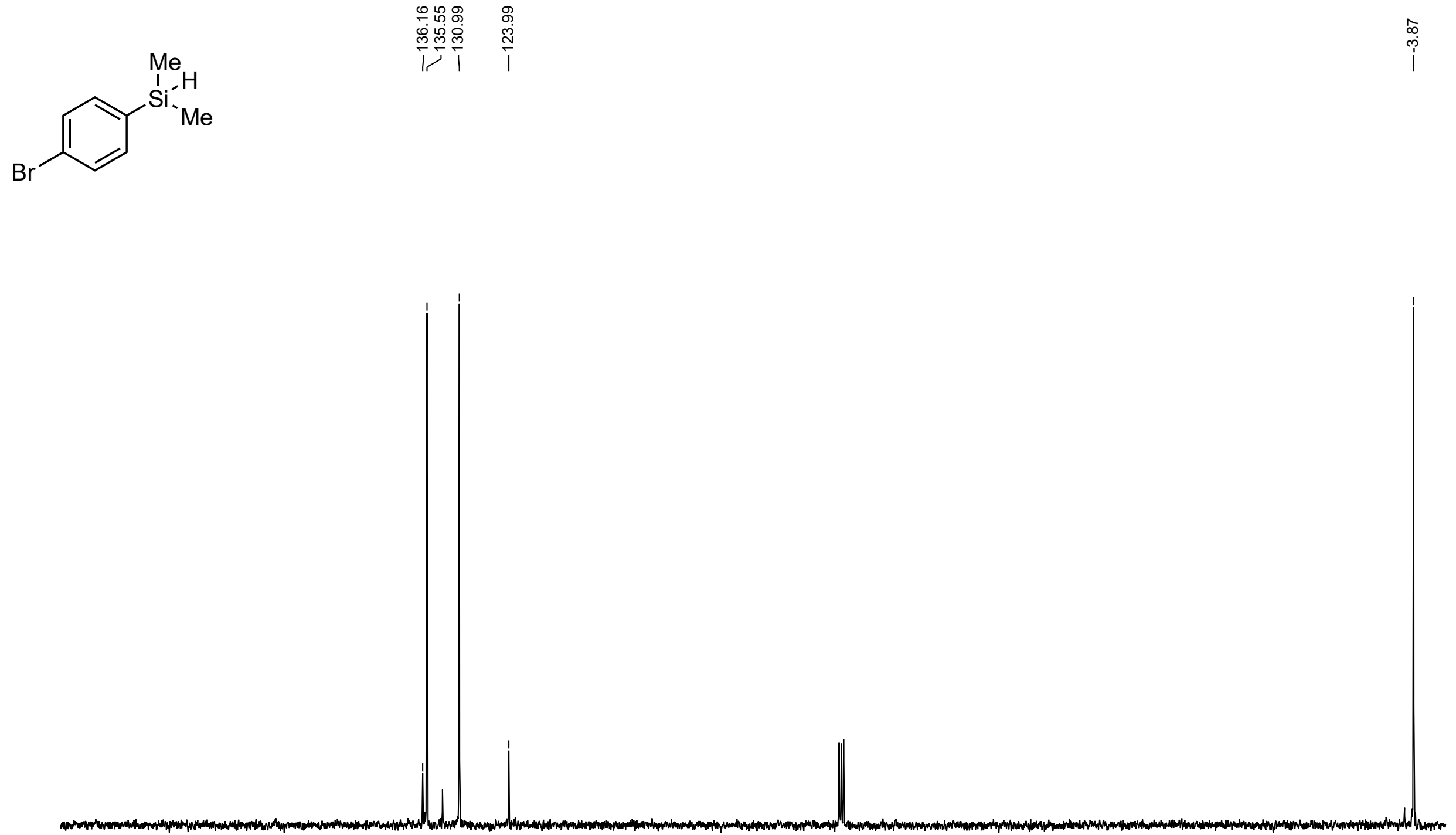


\section{Compound $1 \mathbf{i}{ }^{1} \mathrm{H}$ NMR}
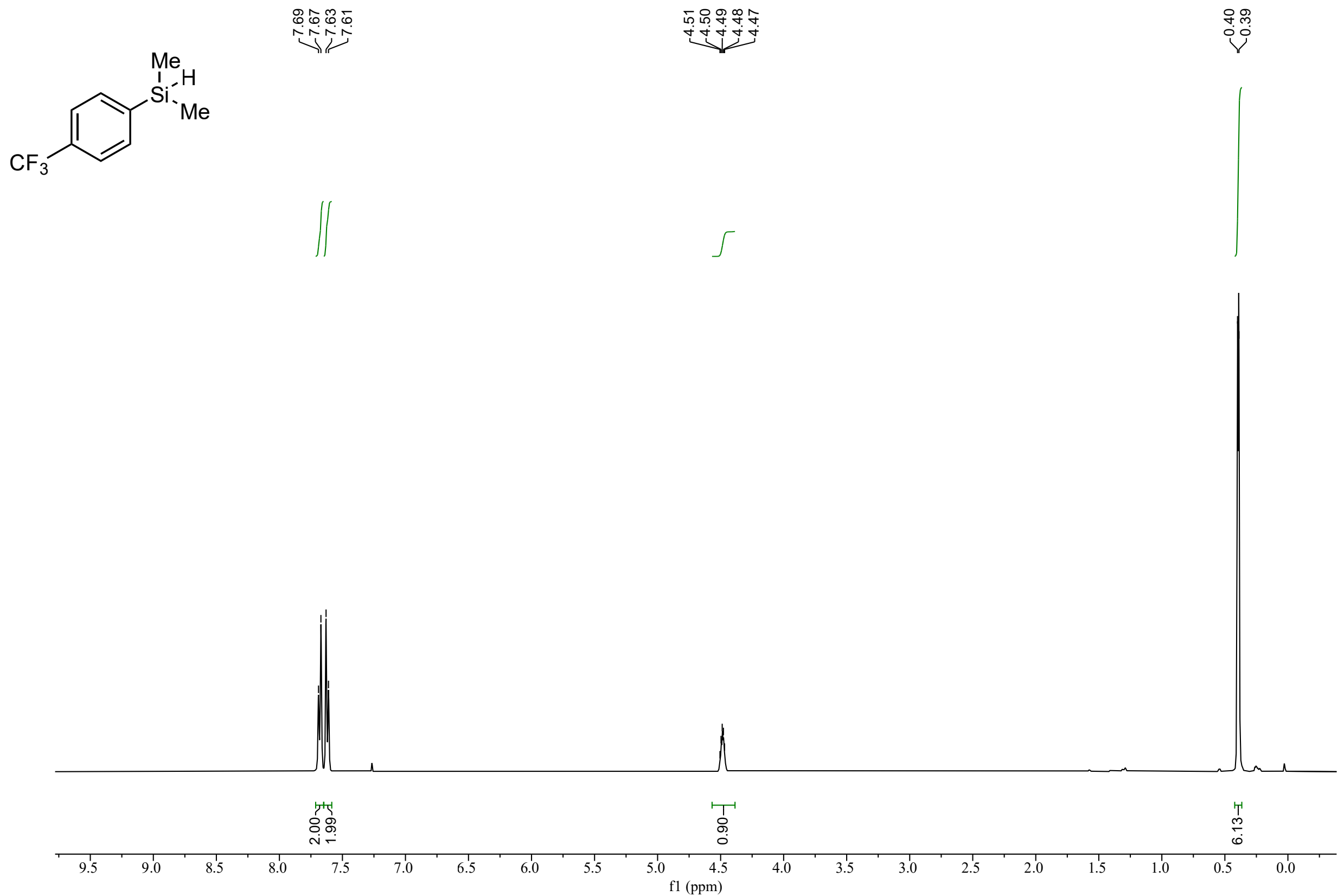
Compound $1 \mathbf{i}^{13} \mathrm{C}$ NMR

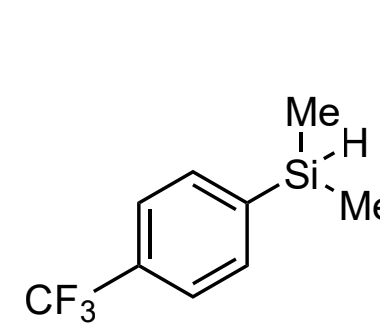

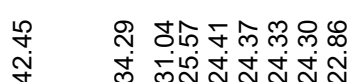

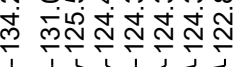
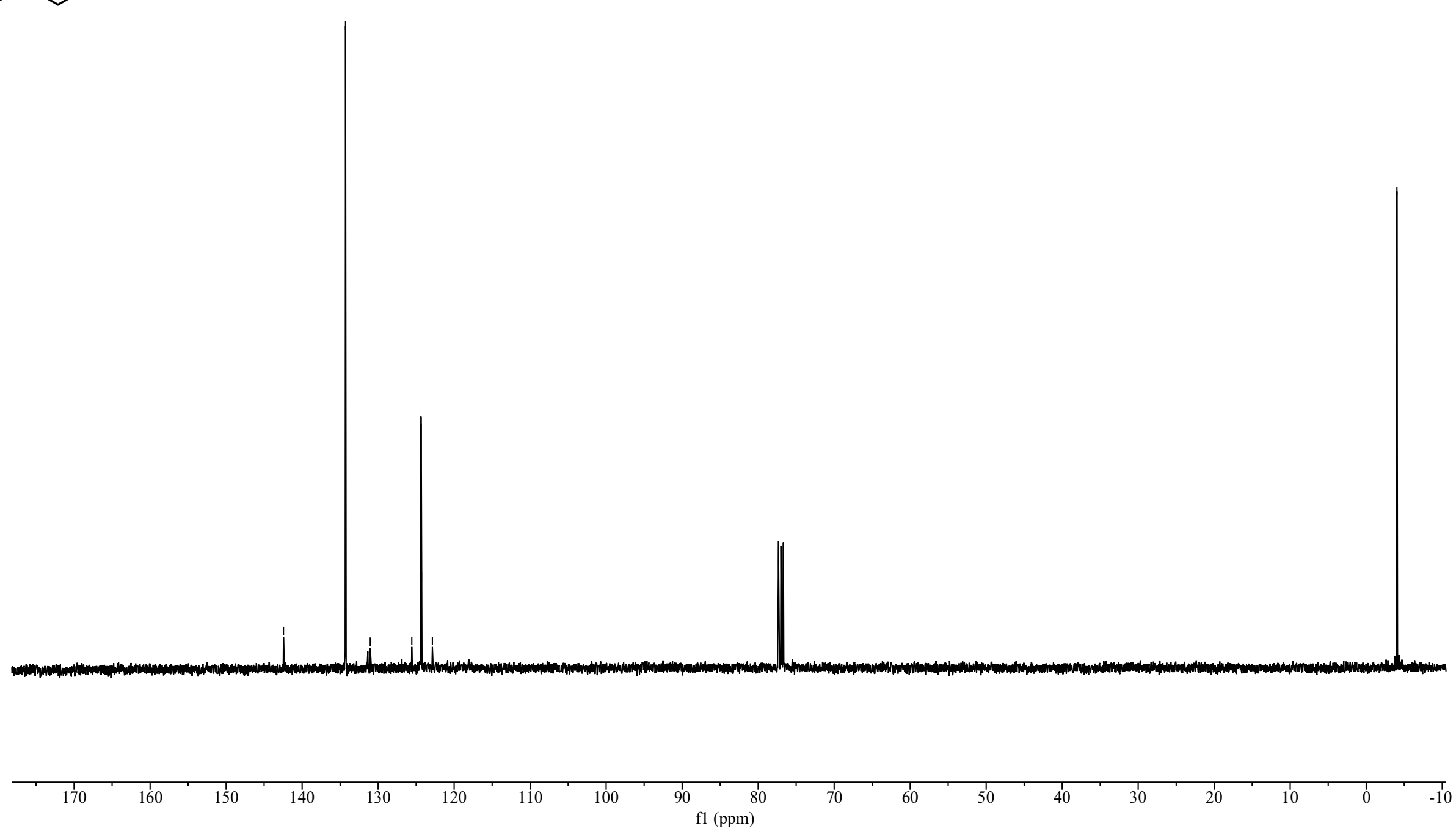
Compound 1i ${ }^{19} \mathrm{~F}$ NMR<smiles>C[Si](C)(C)c1ccc(C(F)(F)F)cc1</smiles> 


\section{Compound $\mathbf{1 j}{ }^{1} \mathrm{H}$ NMR}
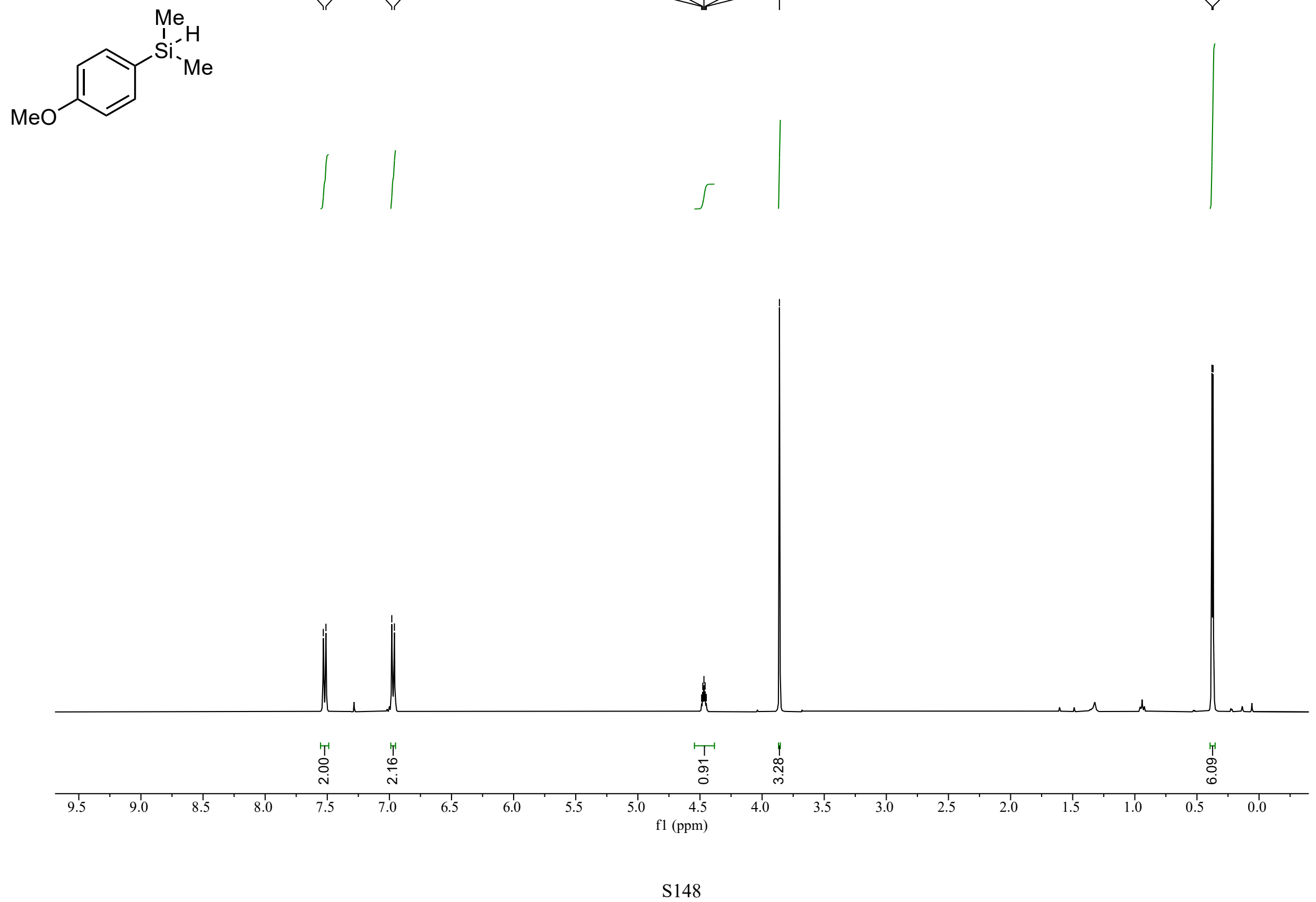
Compound $\mathbf{1 j}{ }^{13} \mathrm{C}$ NMR
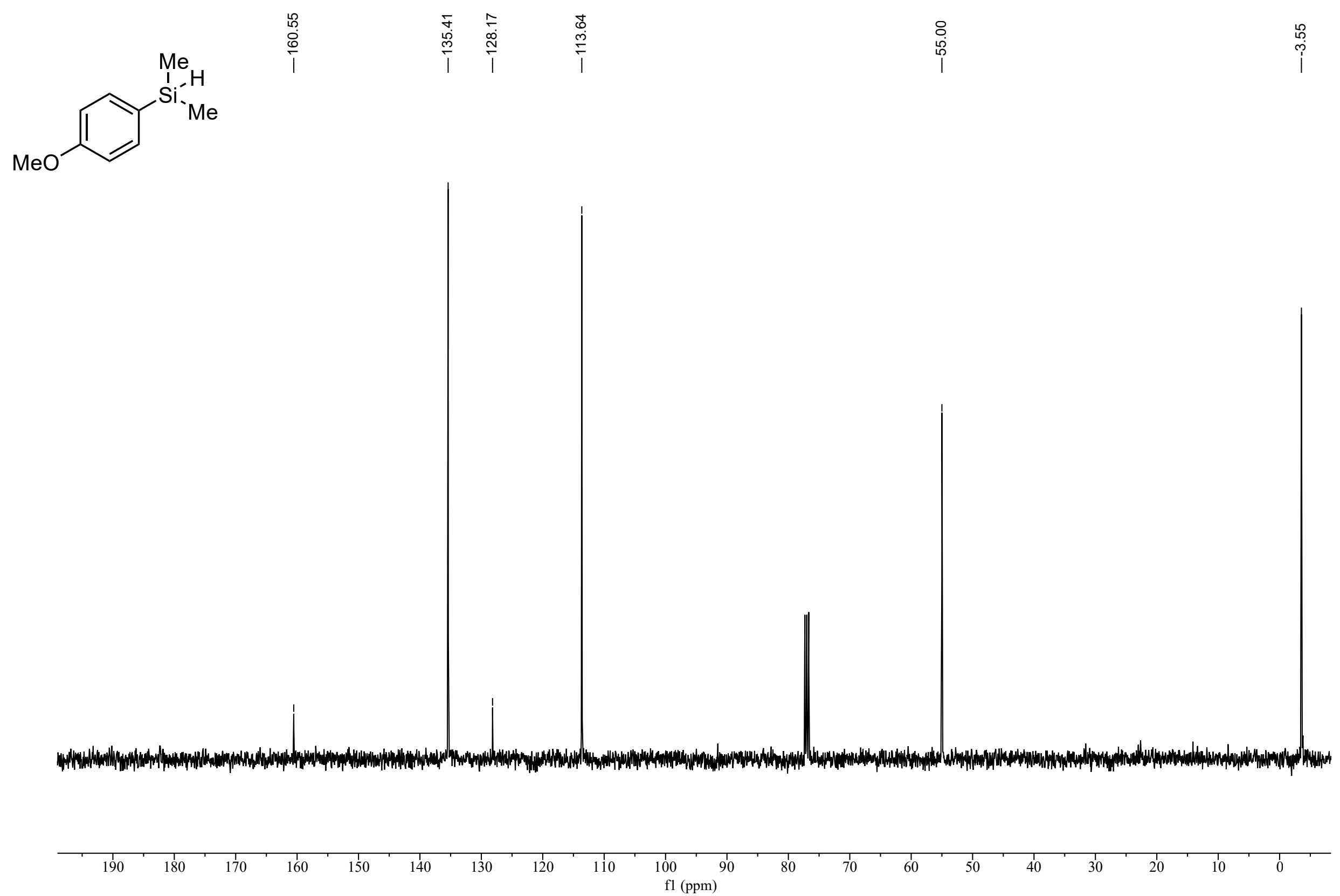

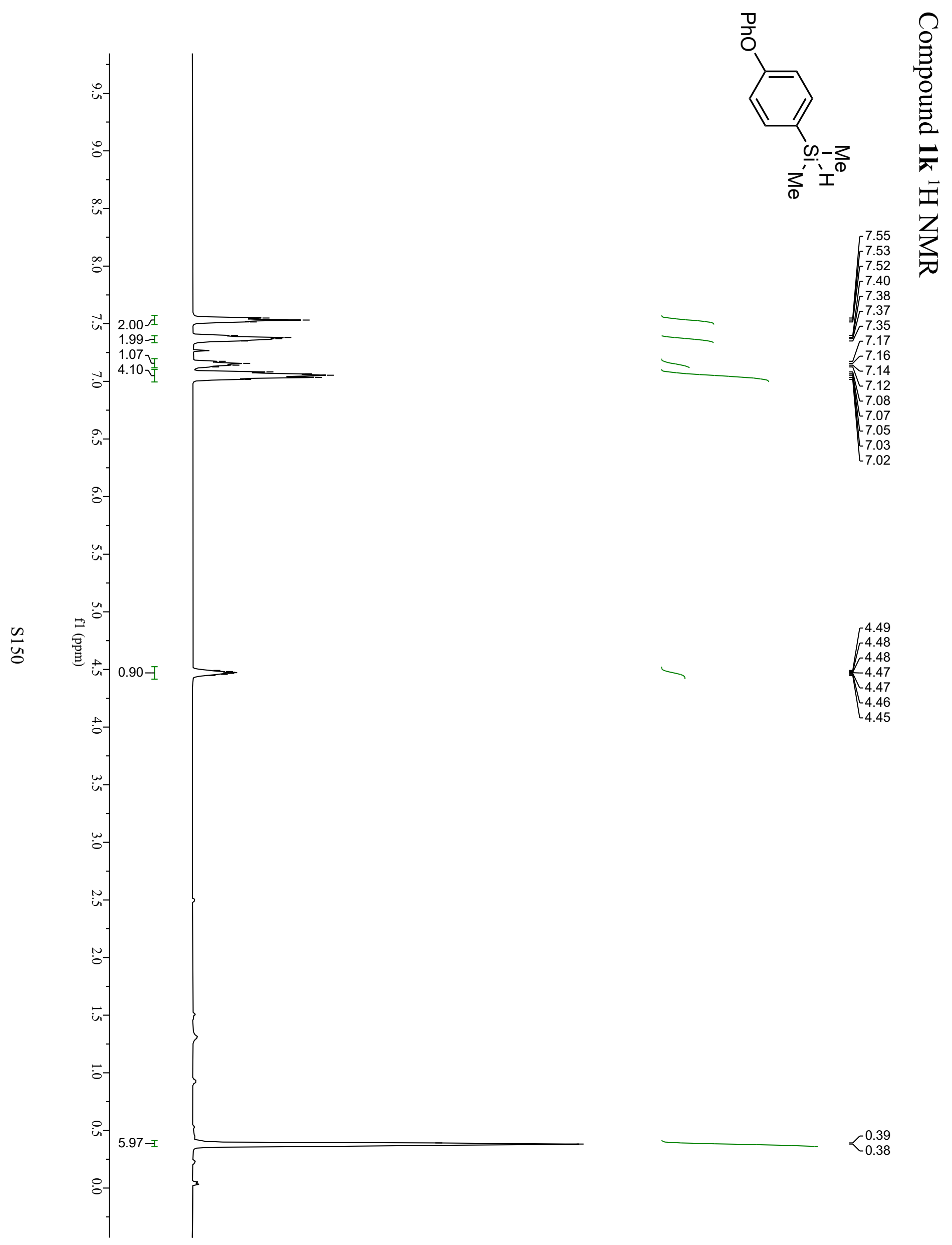
Compound $1 \mathbf{k}{ }^{13} \mathrm{C}$ NMR
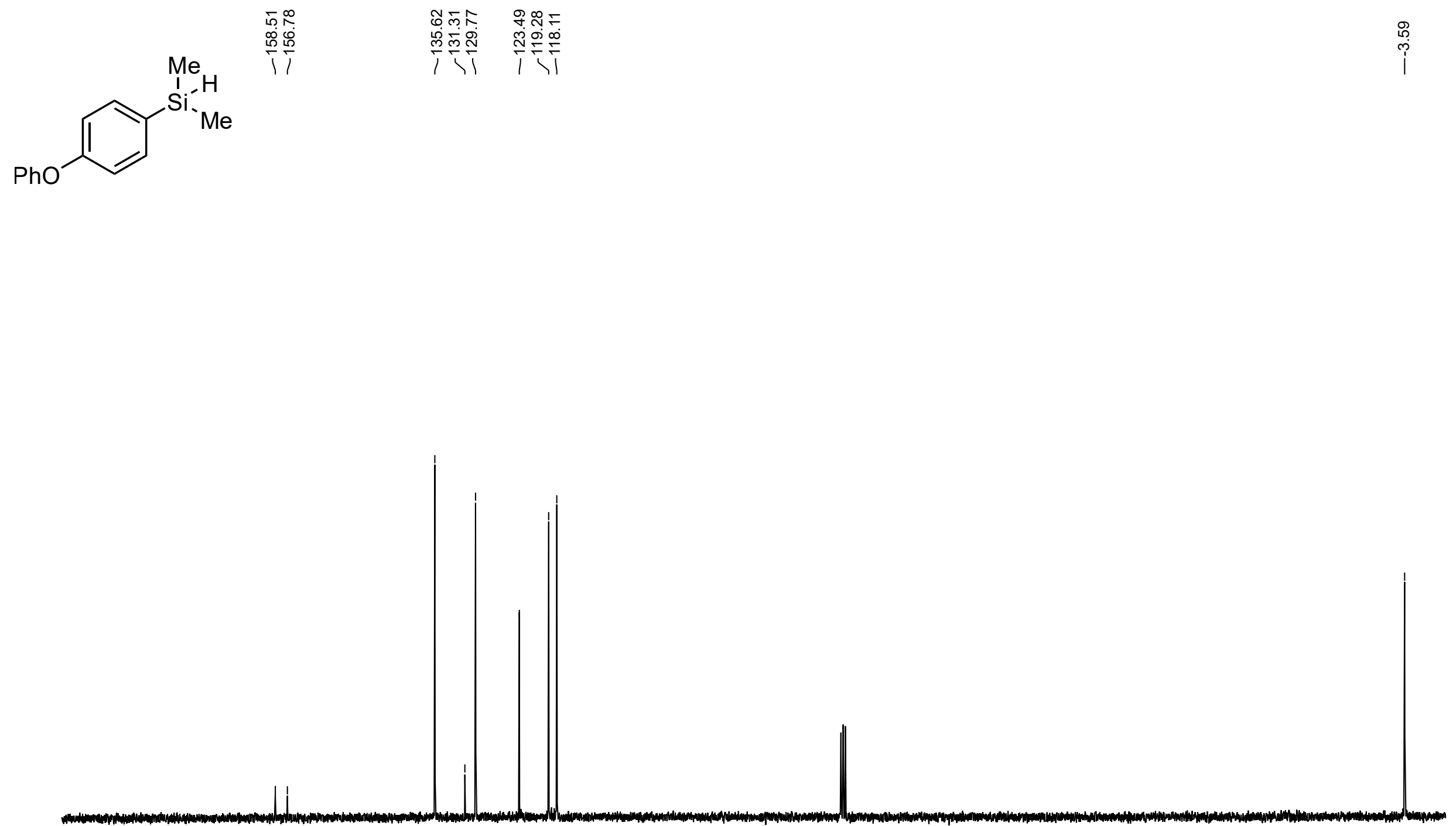

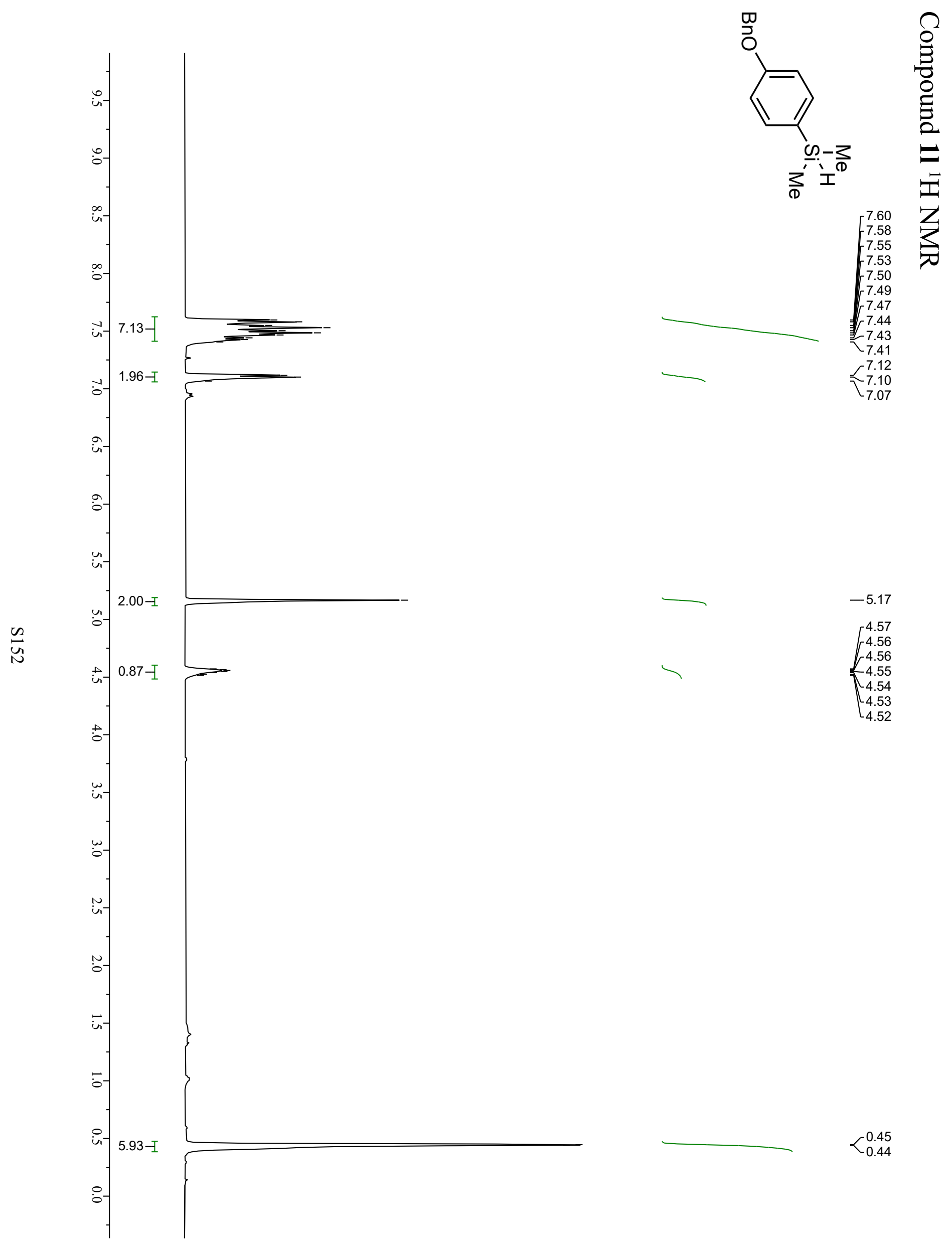
Compound $11^{13} \mathrm{C}$ NMR
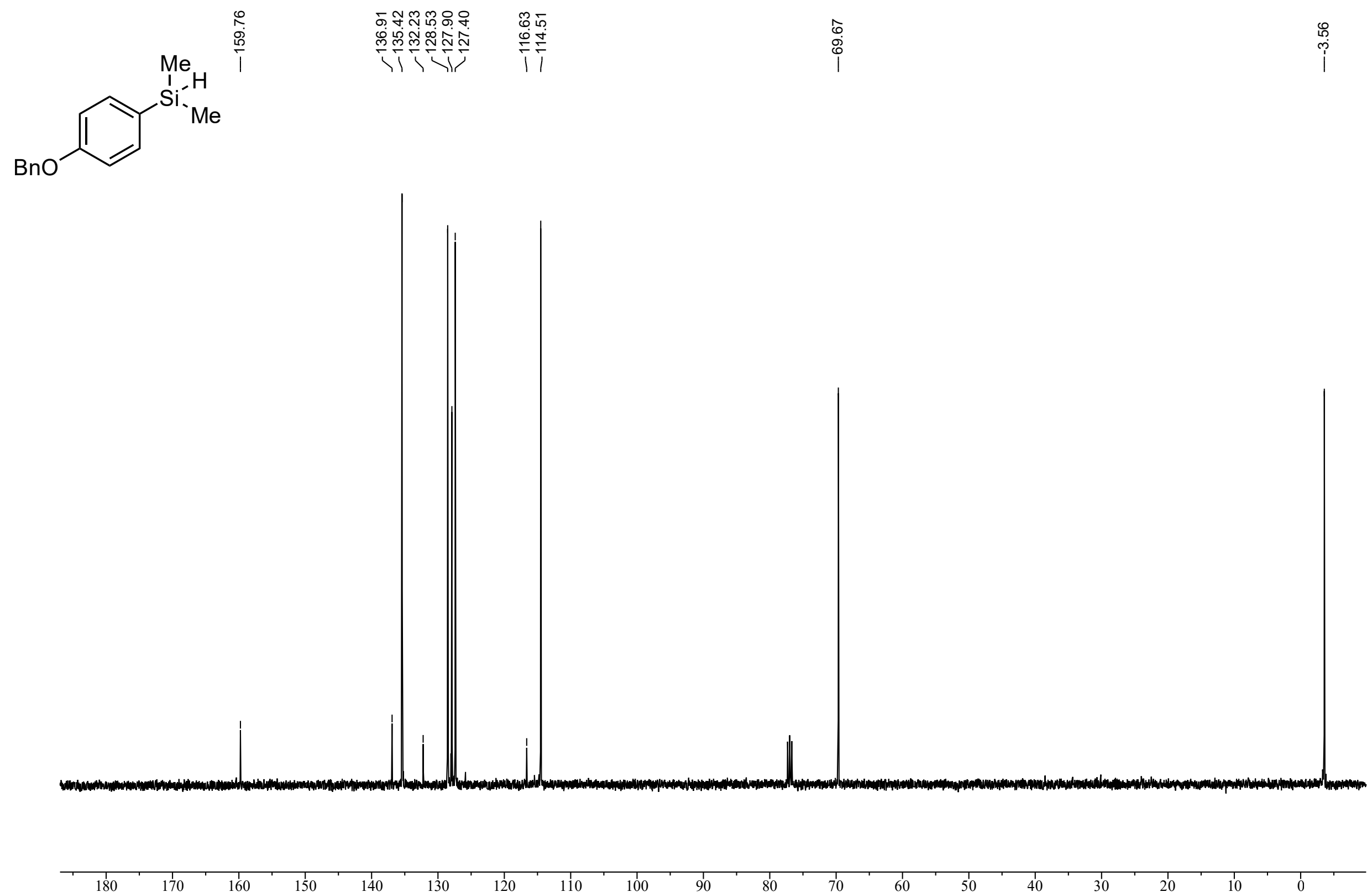

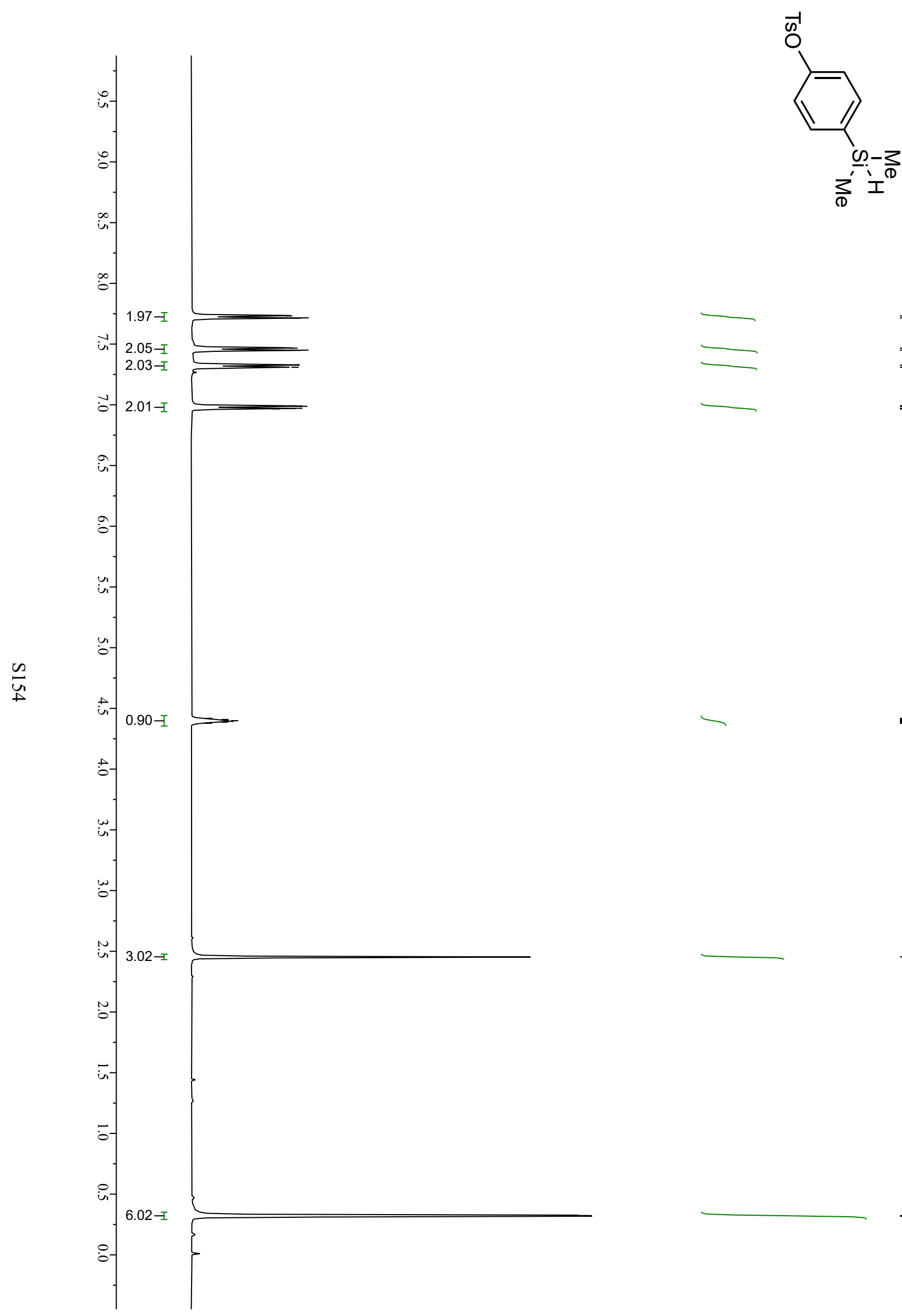

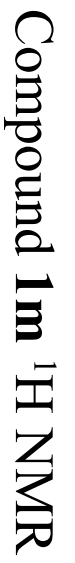

7.73
$\Upsilon_{7} .72$

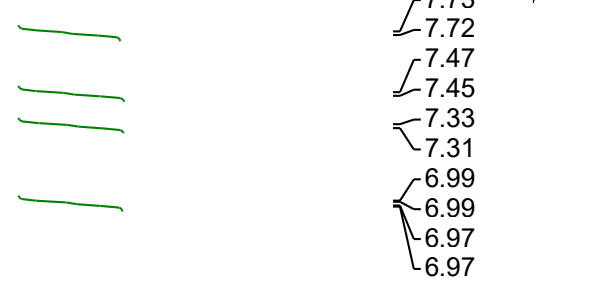

4.42
4.41
4.40

4.40

4.39

$-4.39$

$-2.45$

$<_{0.32}^{0.32}$ 
Compound $1 \mathbf{m}{ }^{13} \mathrm{C}$ NMR

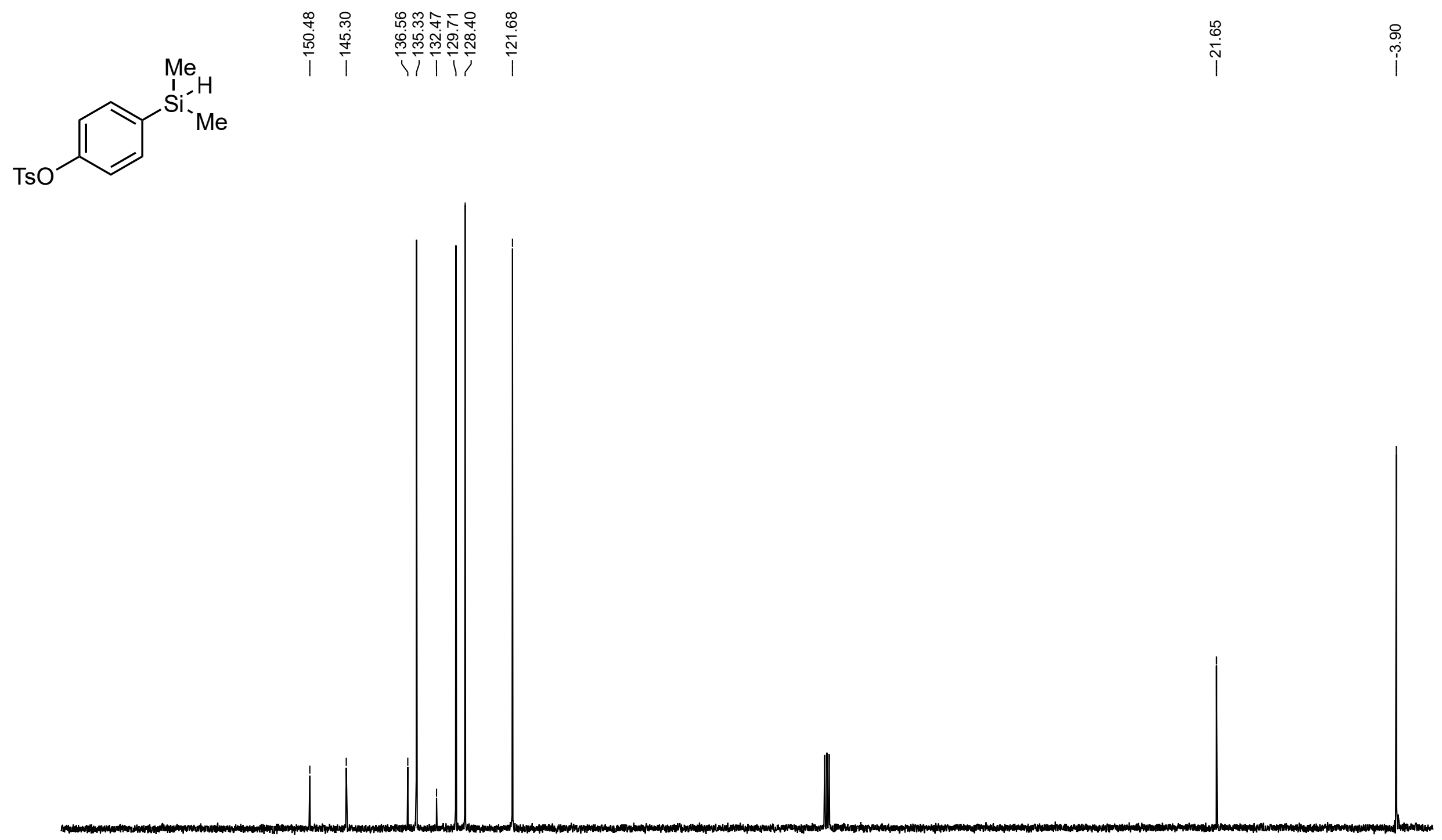


Compound 1n ${ }^{1} \mathrm{H}$ NMR
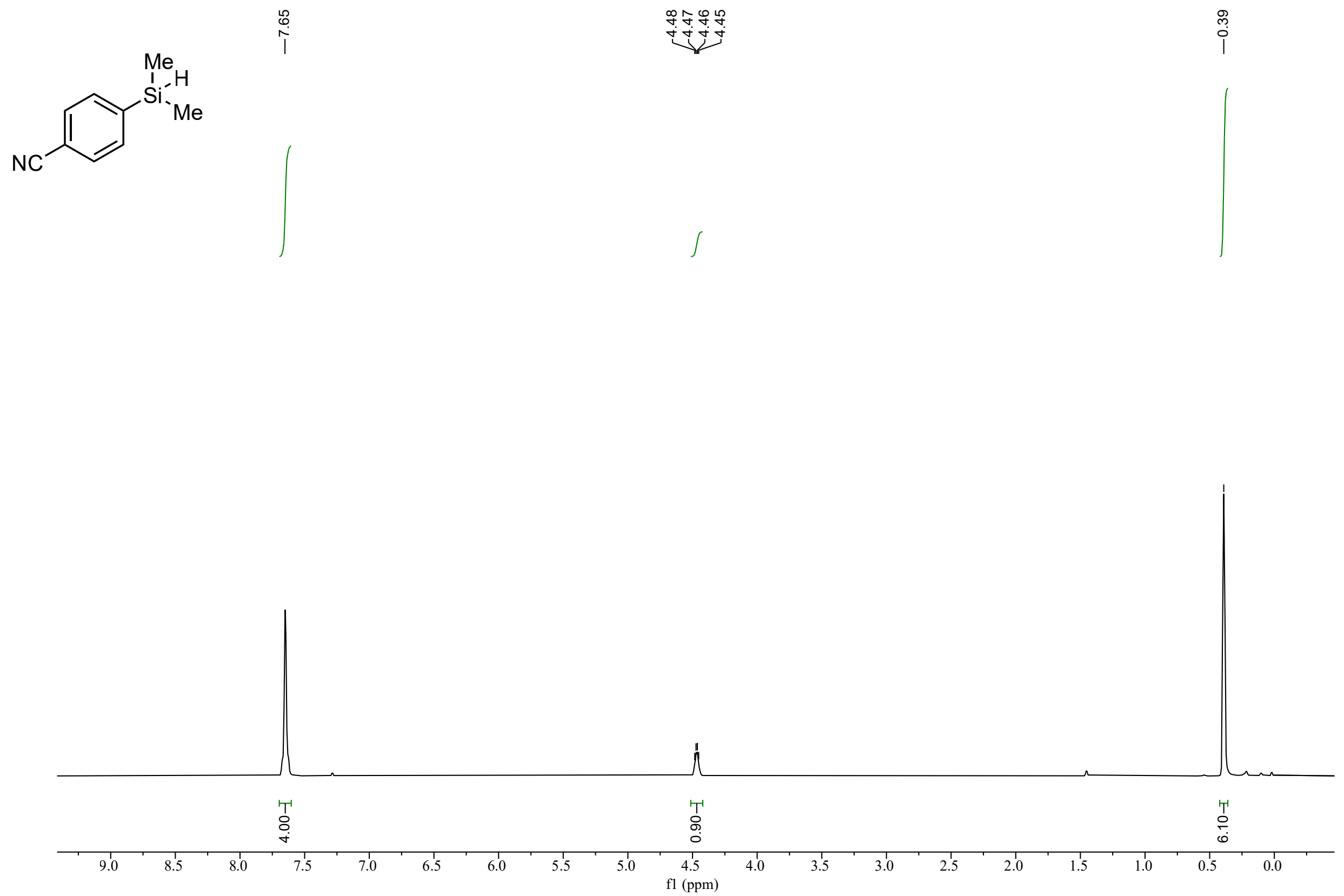
Compound 1n ${ }^{13} \mathrm{C}$ NMR

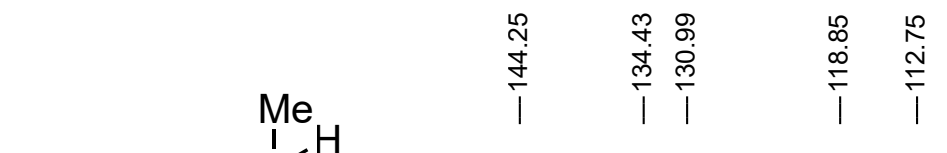

$\mathrm{NC}$

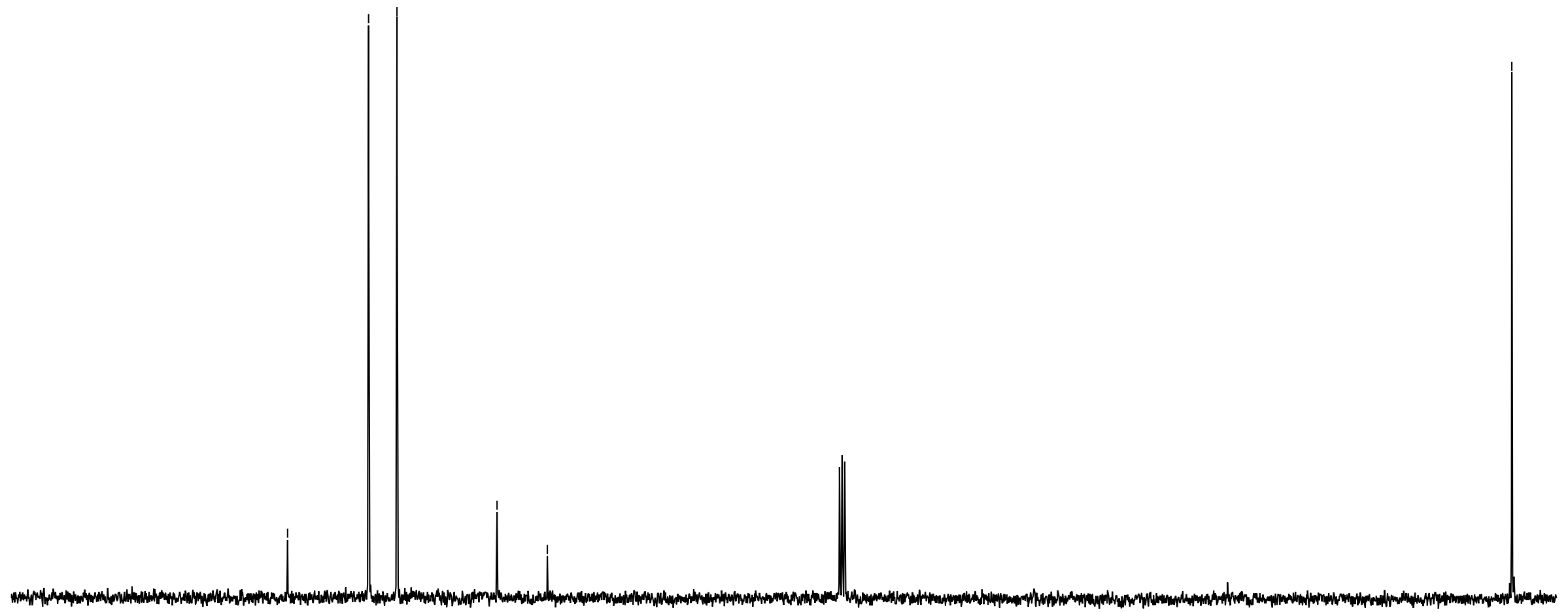

$170 \quad 160$ 
Compound $10{ }^{1} \mathrm{H}$ NMR

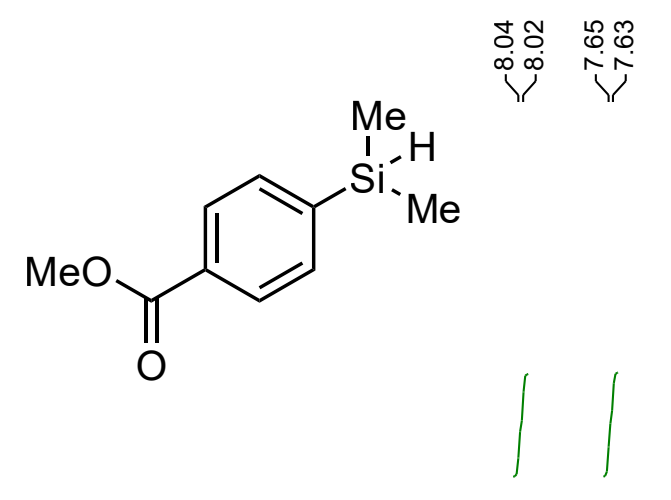

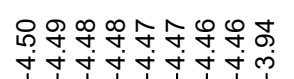

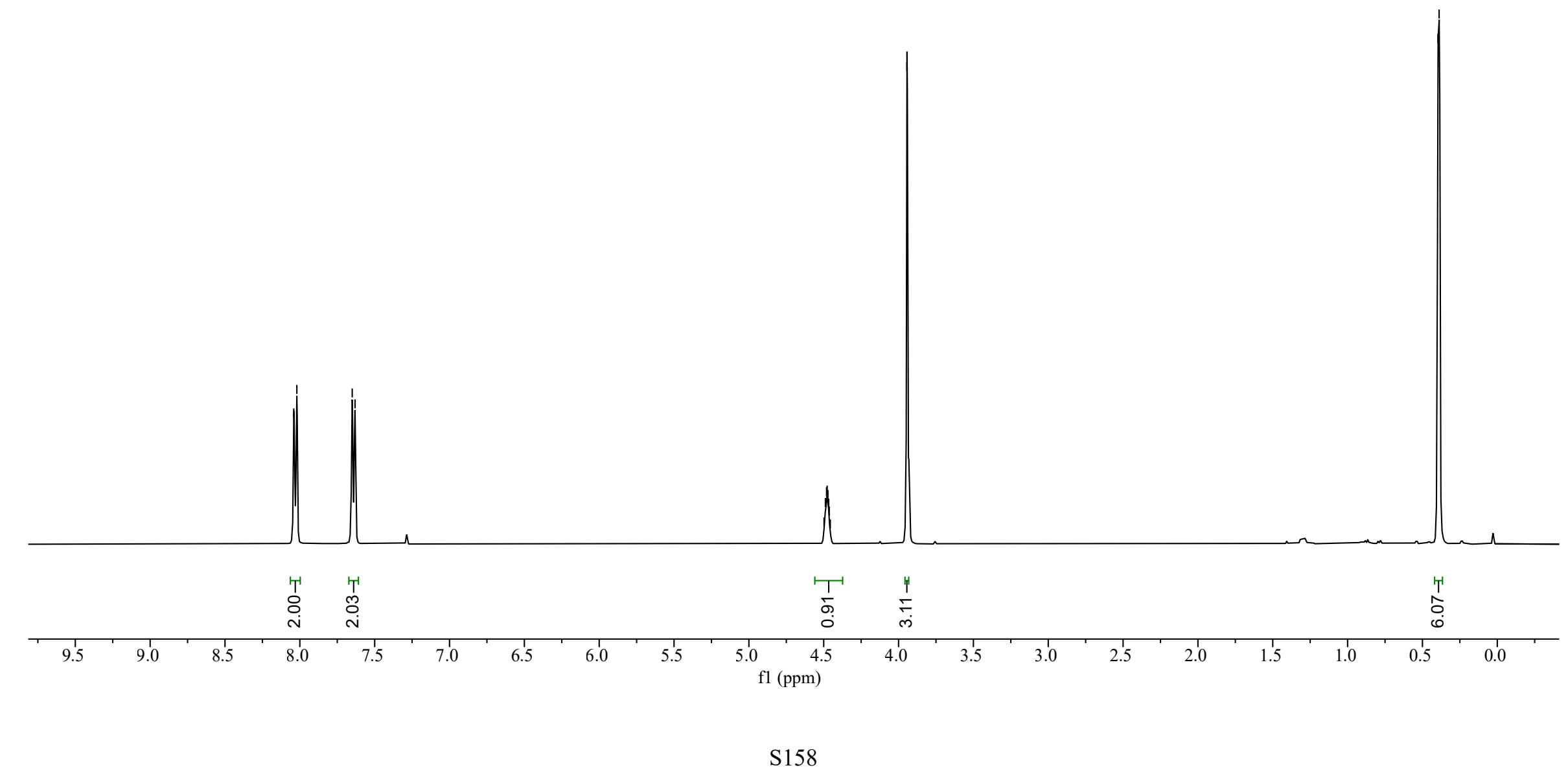


Compound 1o ${ }^{13} \mathrm{C}$ NMR
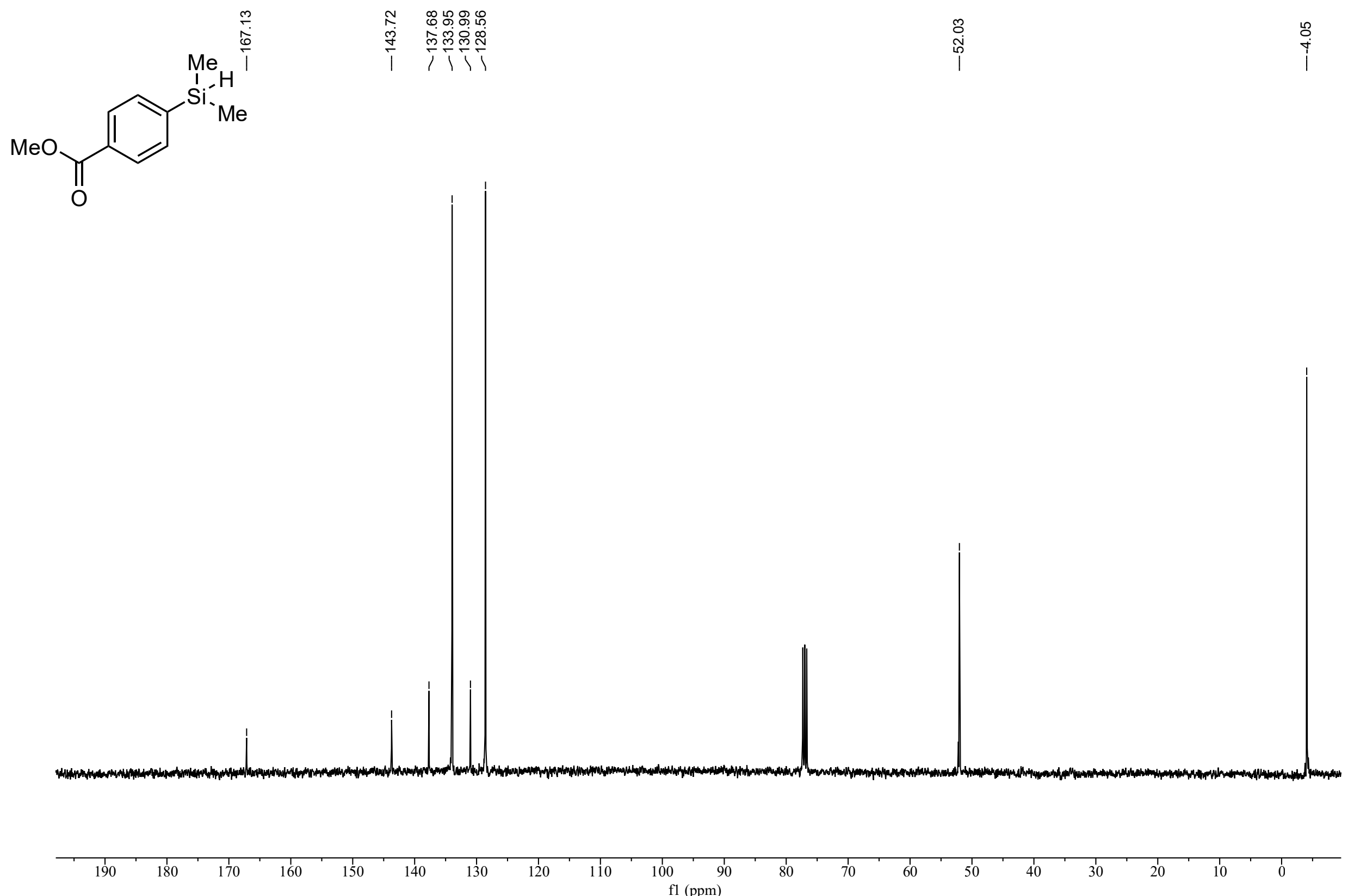
Compound 1p ${ }^{1} \mathrm{H}$ NMR

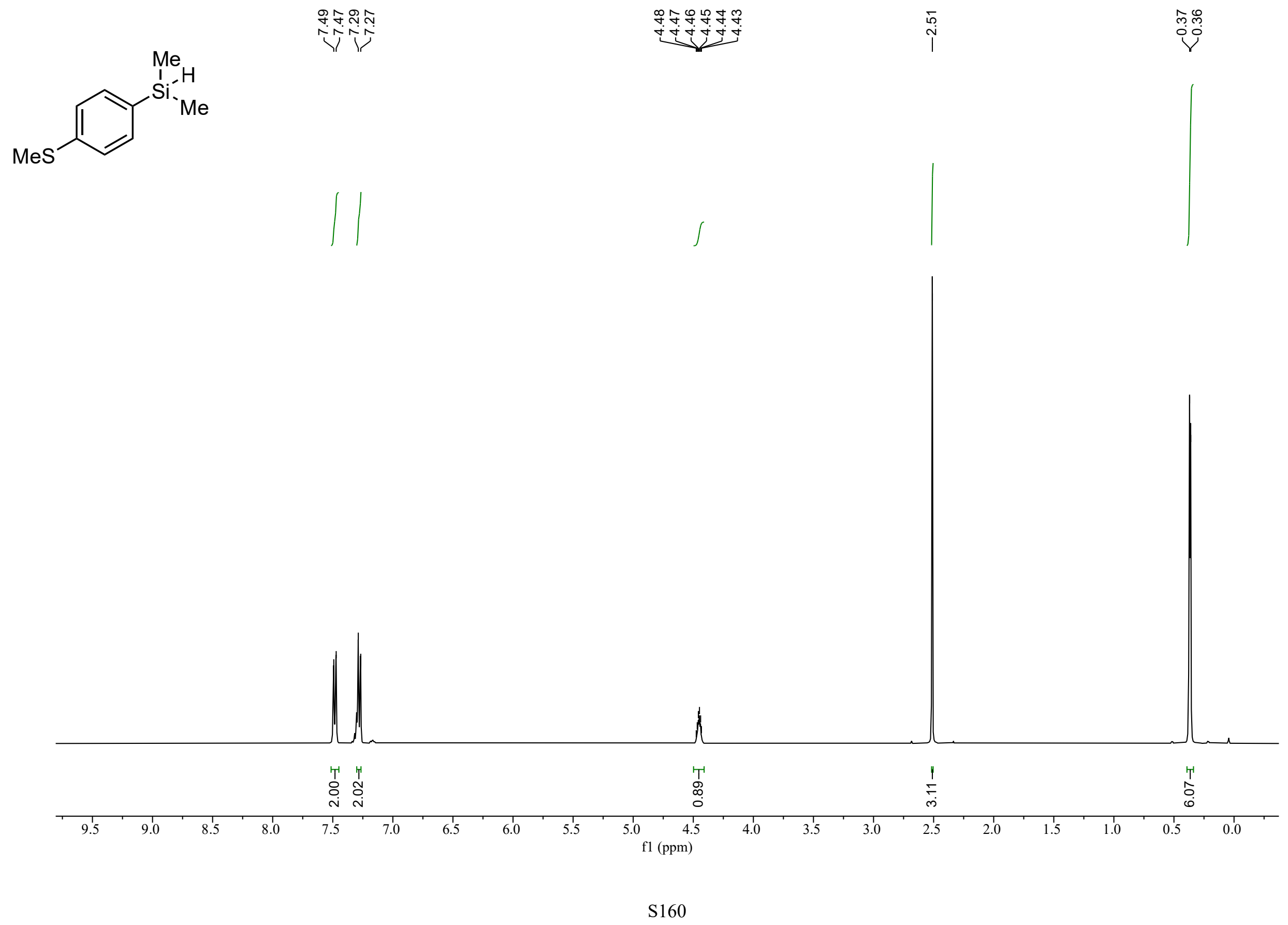


Compound $1 p{ }^{13} \mathrm{C}$ NMR
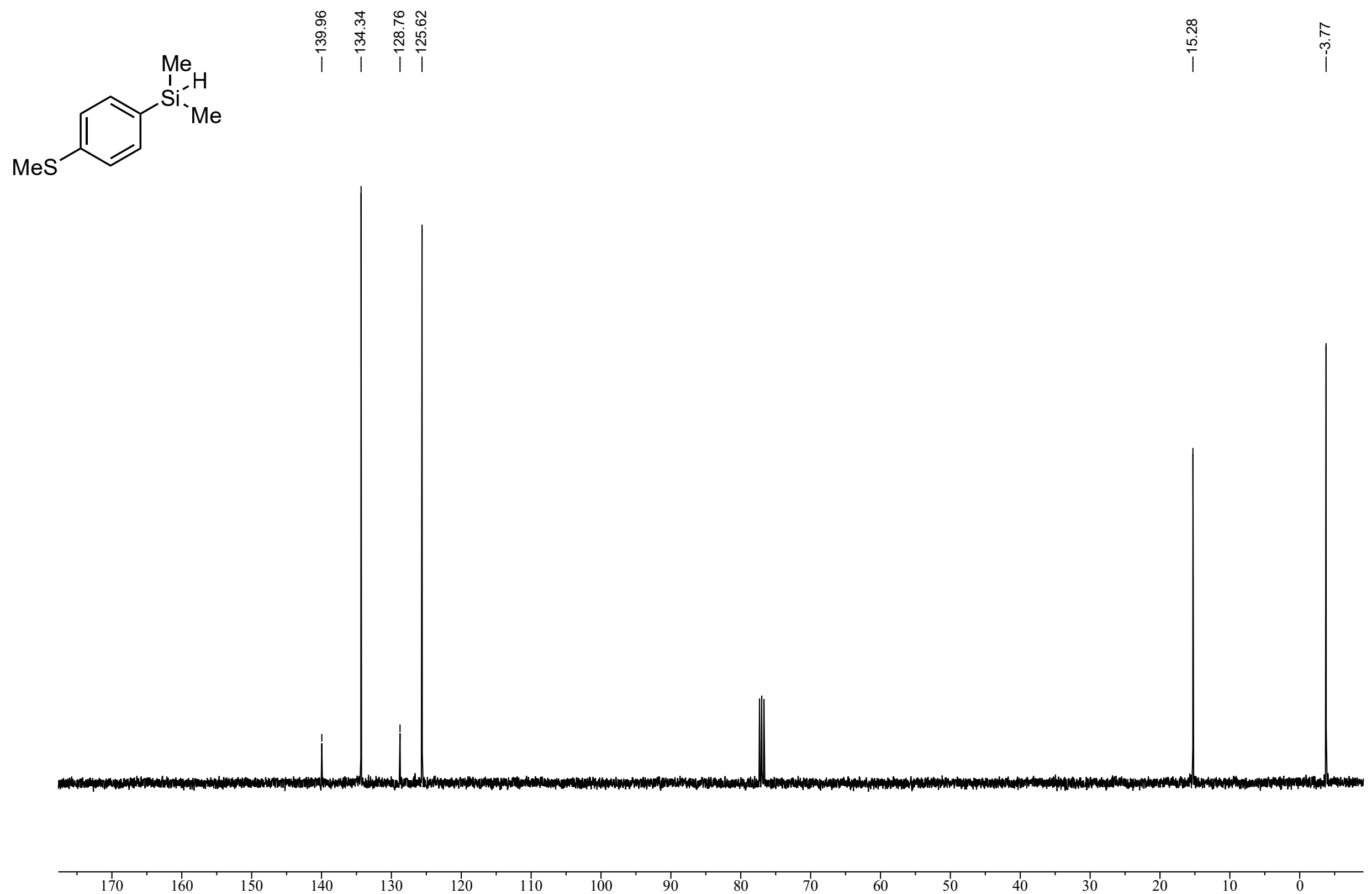
Compound 1q ${ }^{1} \mathrm{H}$ NMR

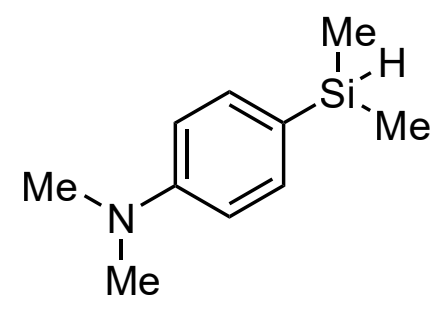

点

1.

$\mathrm{Me}$
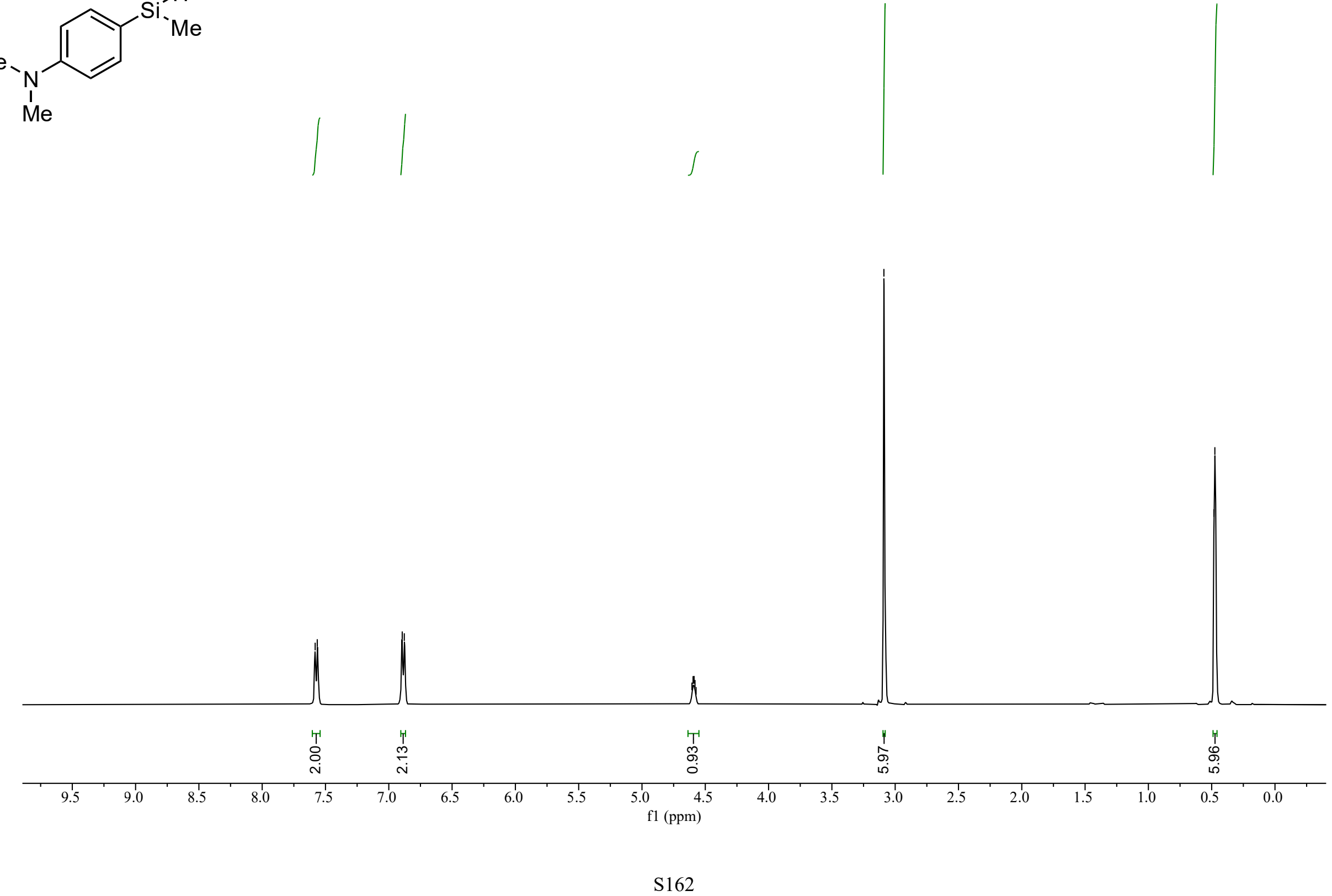
Compound 1q ${ }^{13} \mathrm{C}$ NMR
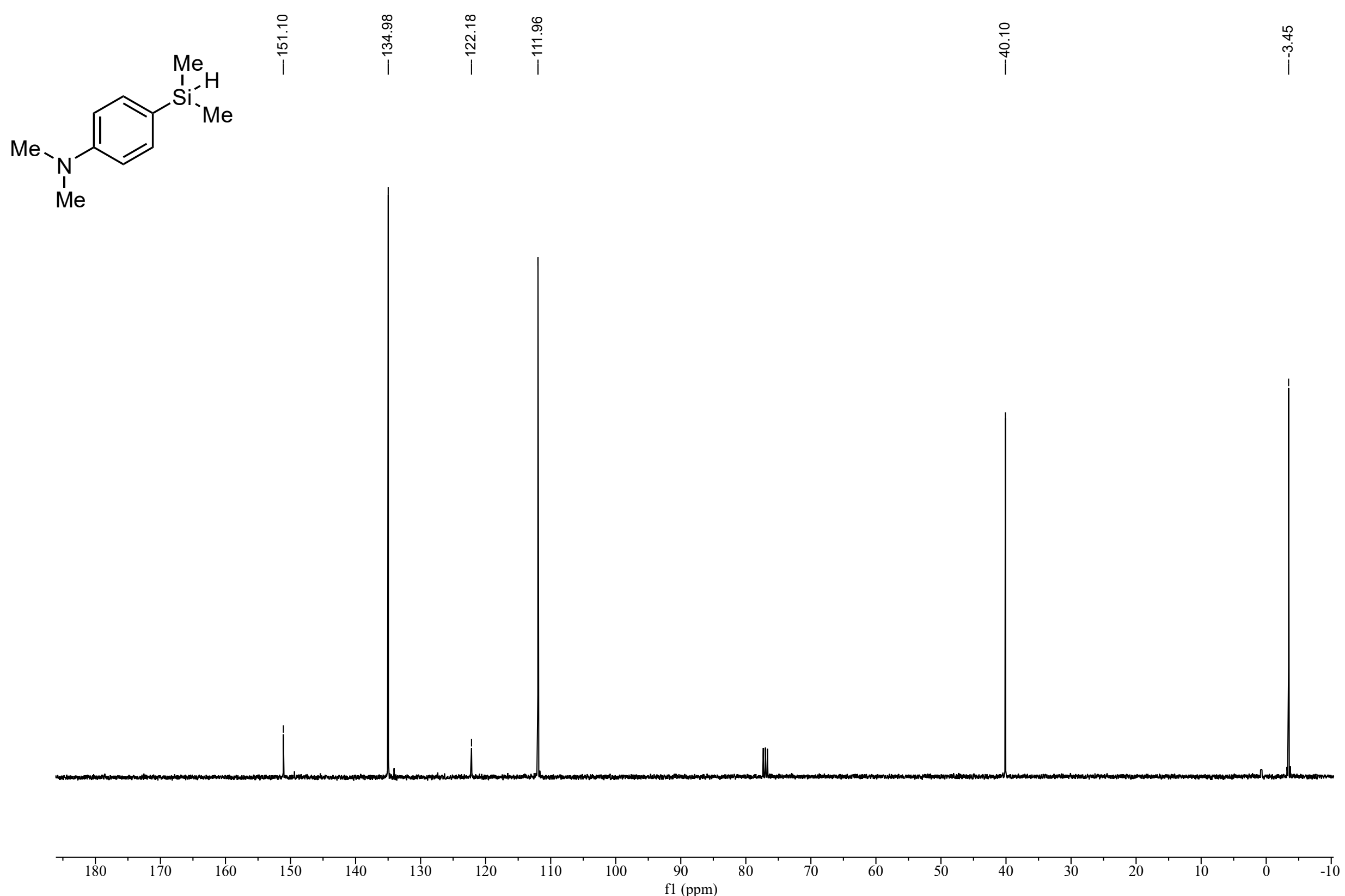


\section{Compound 1r ${ }^{1} \mathrm{H}$ NMR}

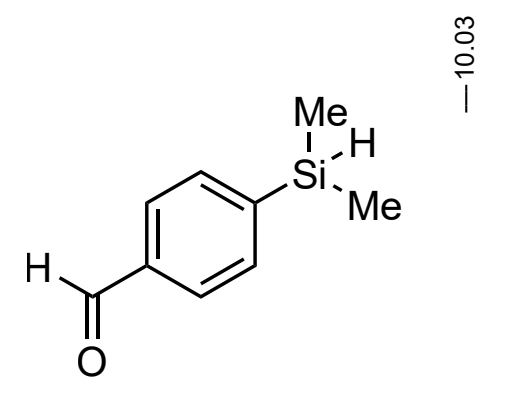

我

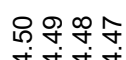

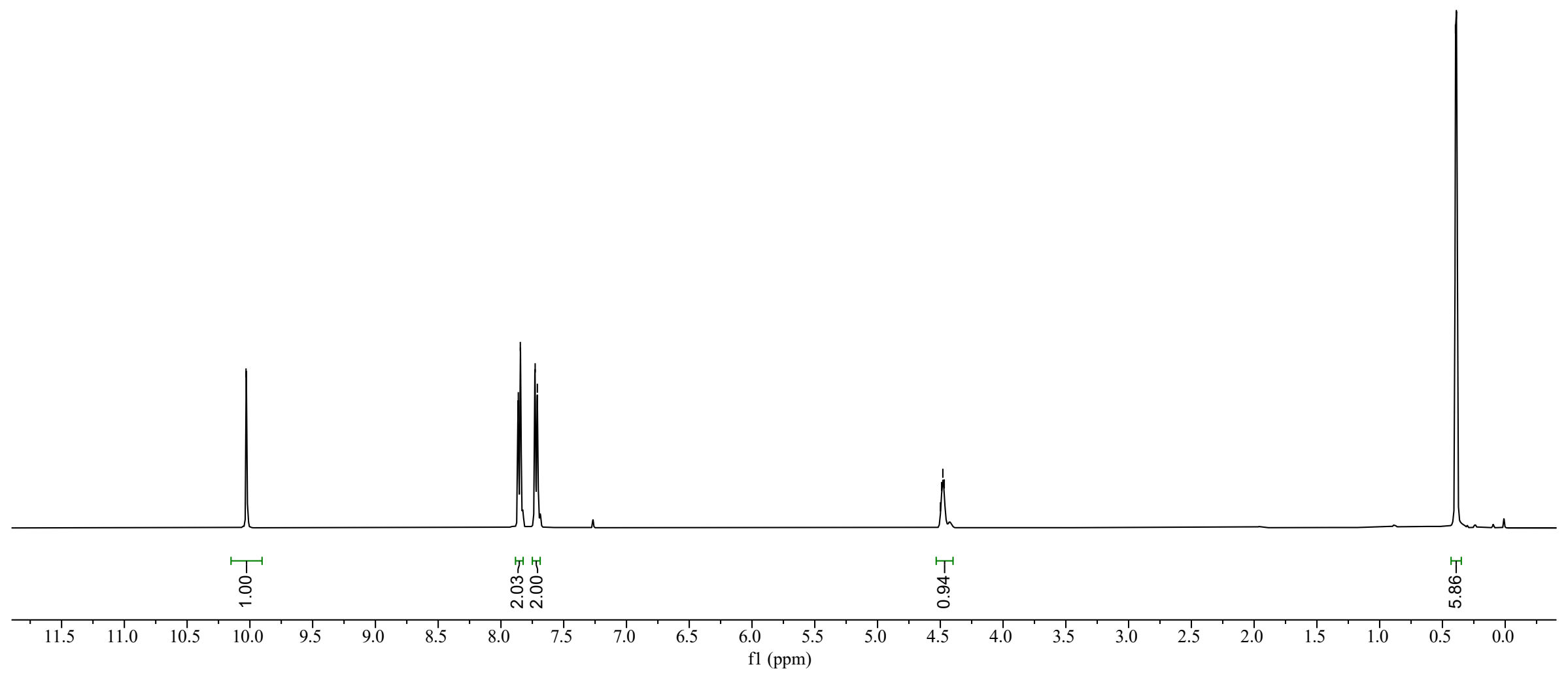


Compound $1 \mathbf{r}{ }^{13} \mathrm{C}$ NMR
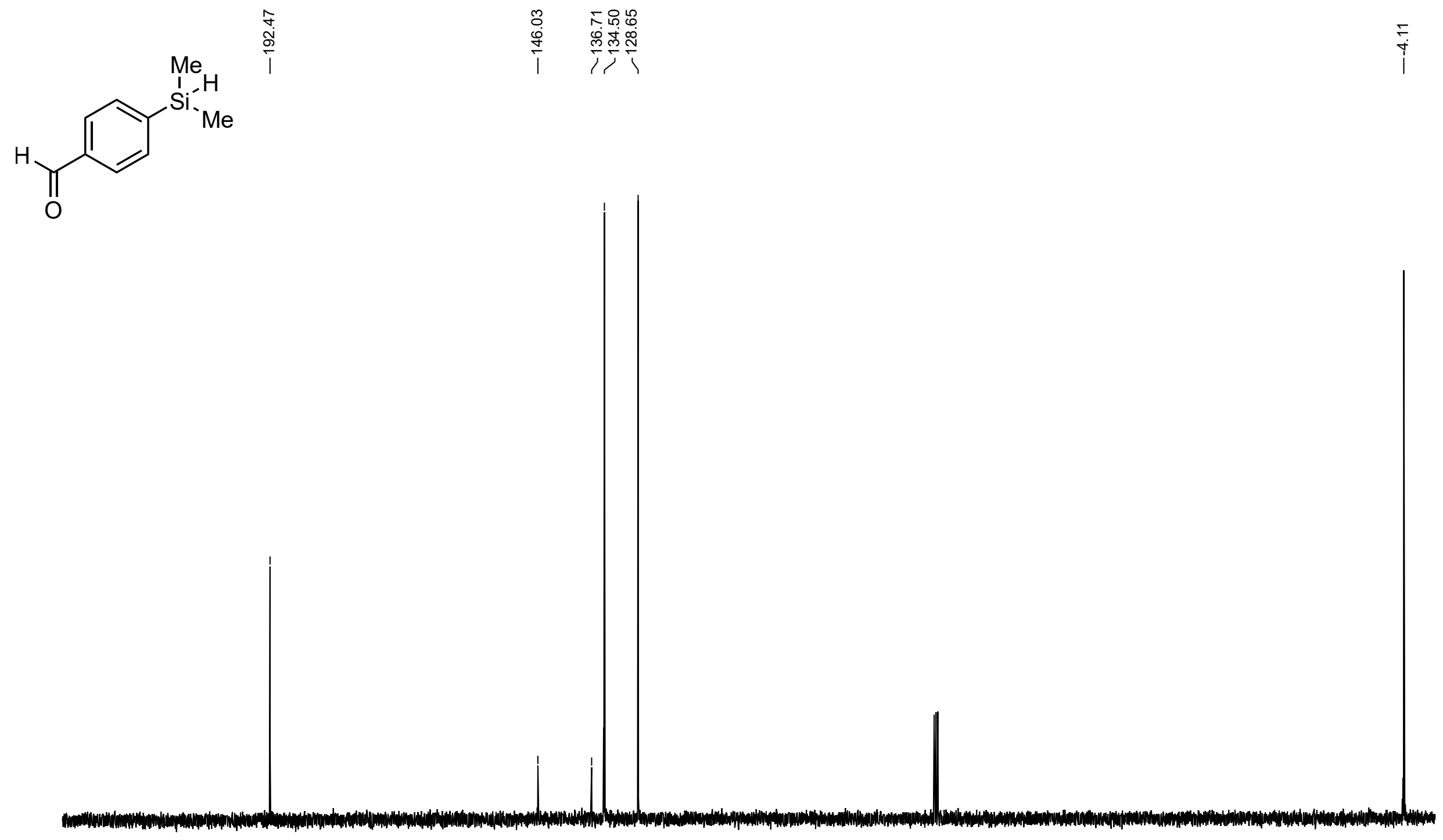

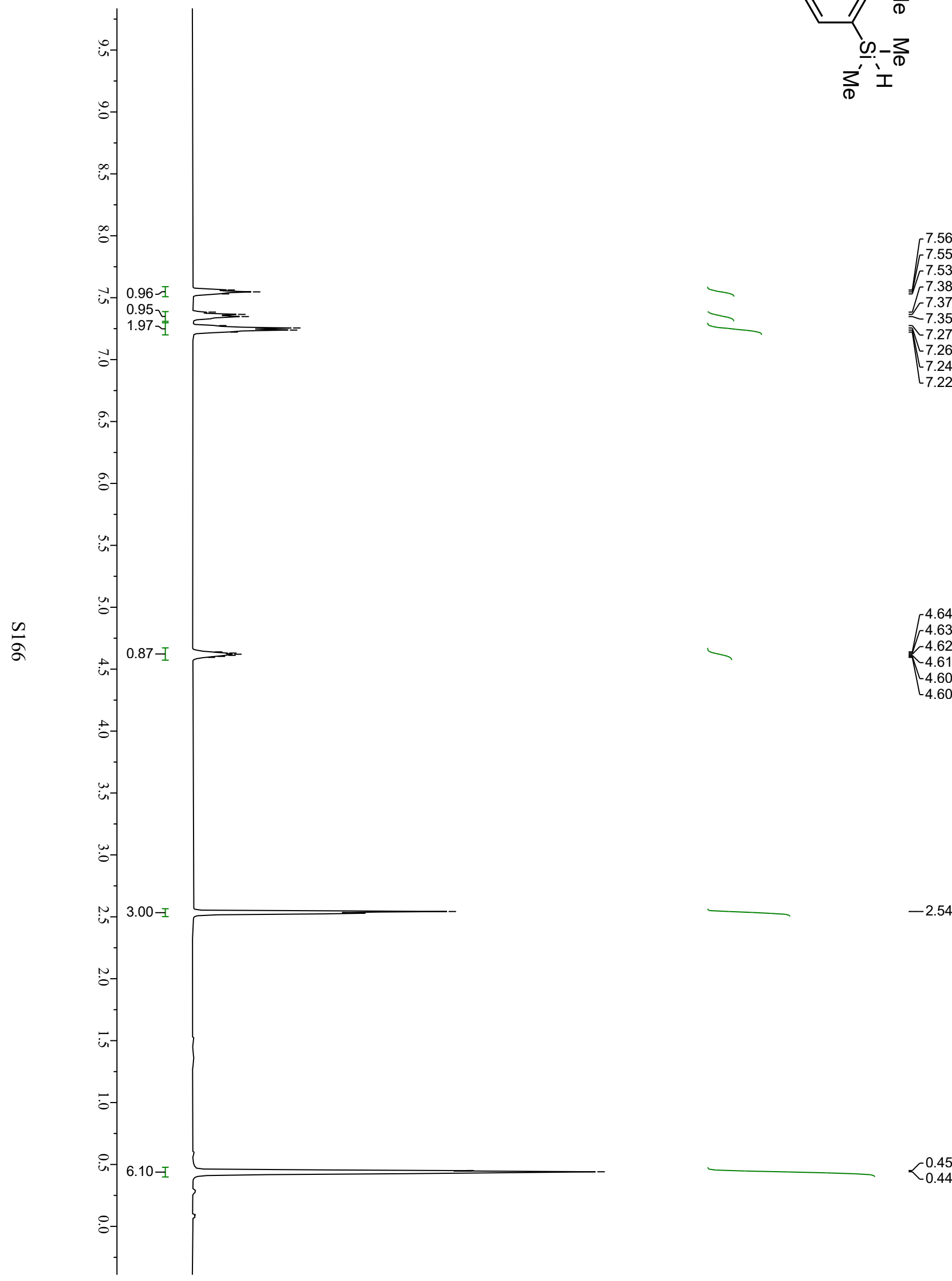
Compound $1 \mathrm{~s}{ }^{13} \mathrm{C}$ NMR

$\underbrace{M e}_{M e}$

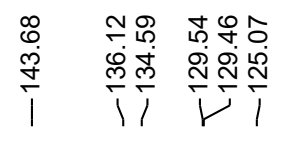
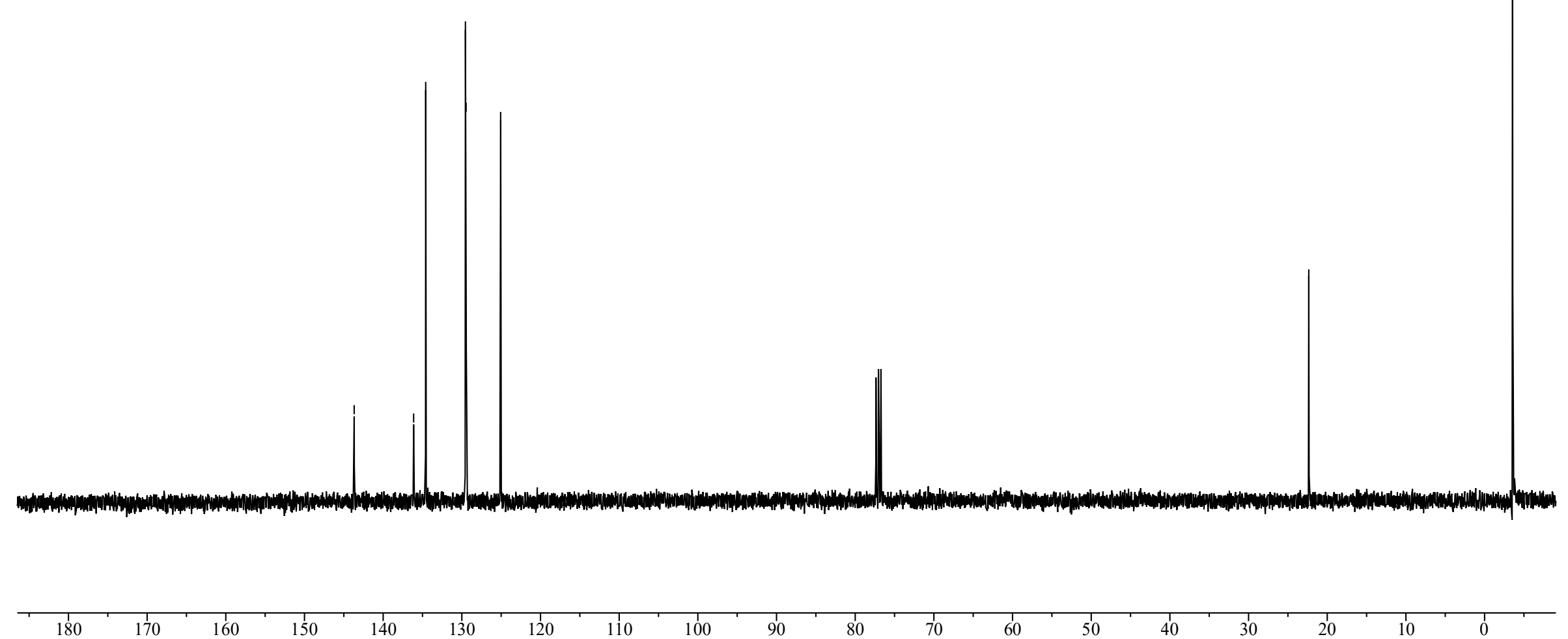


\section{Compound $1 \mathrm{t}^{1} \mathrm{H}$ NMR}

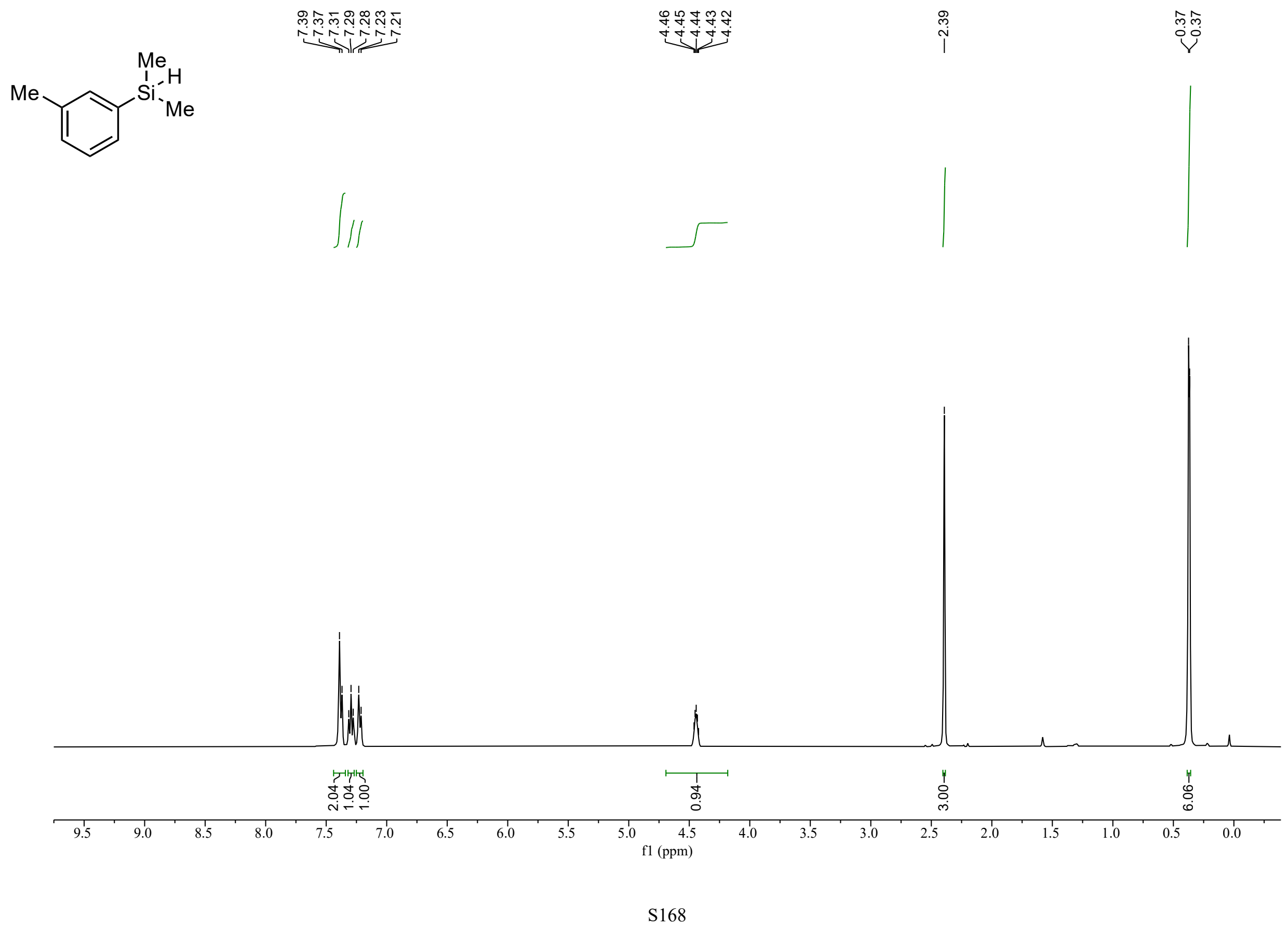


Compound $1 t^{13} \mathrm{C}$ NMR

Me

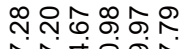

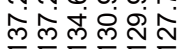

प)

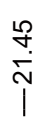

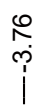

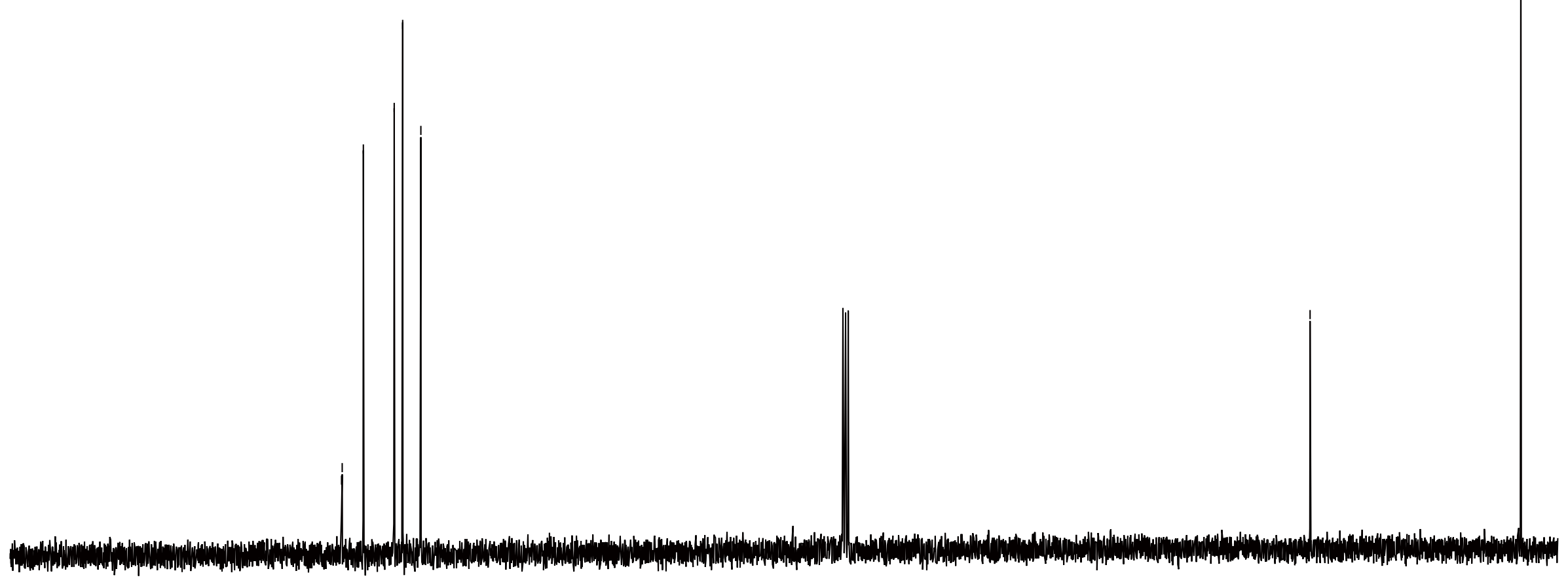




\section{Compound $1 \mathbf{u}{ }^{1} \mathrm{H}$ NMR}

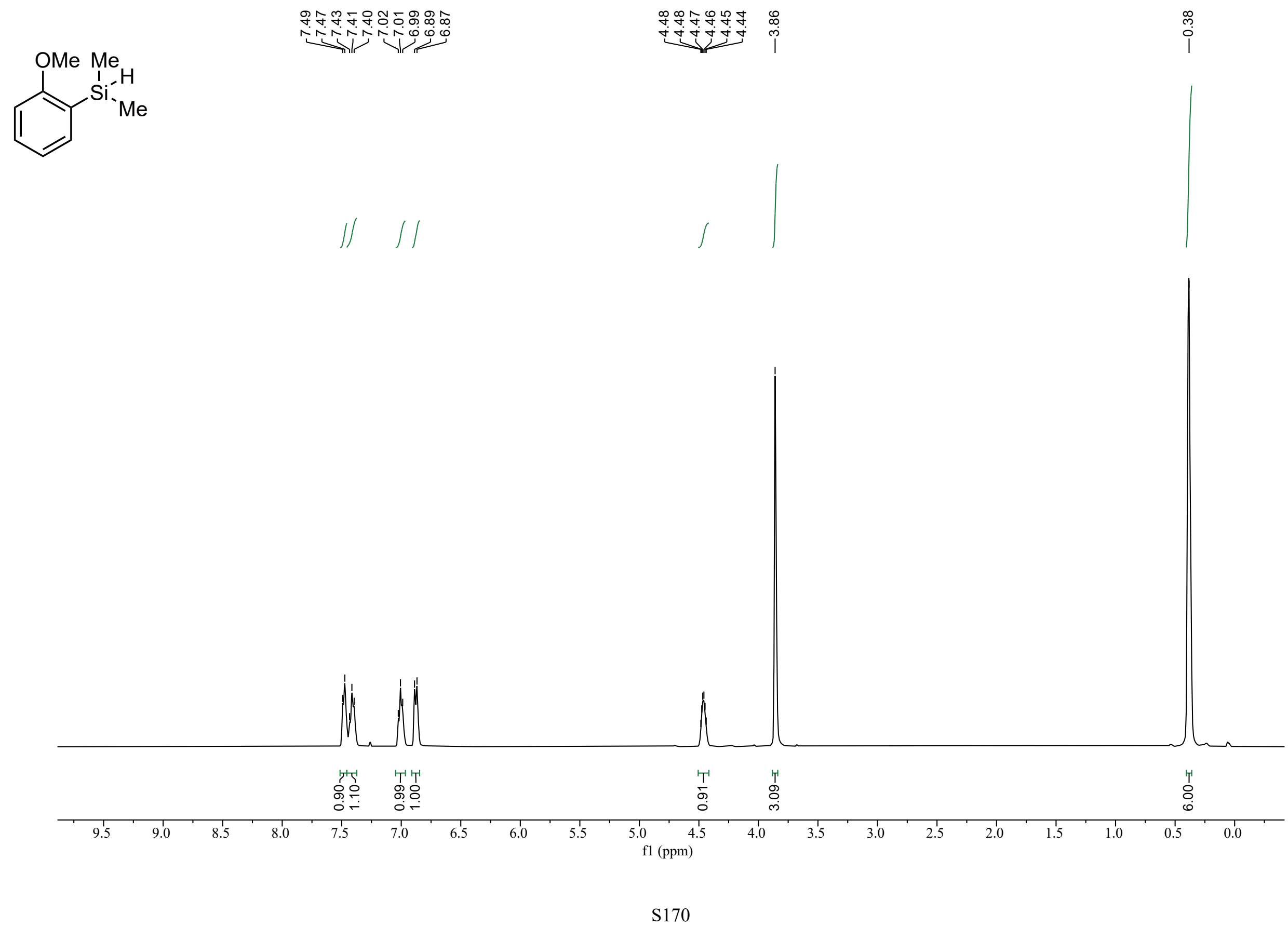


Compound $1 \mathbf{u}{ }^{13} \mathrm{C}$ NMR

OMe Me

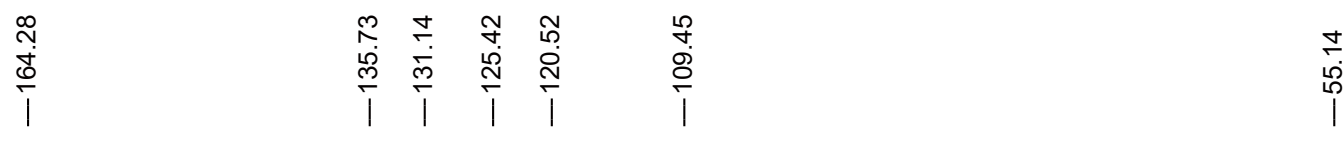

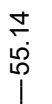
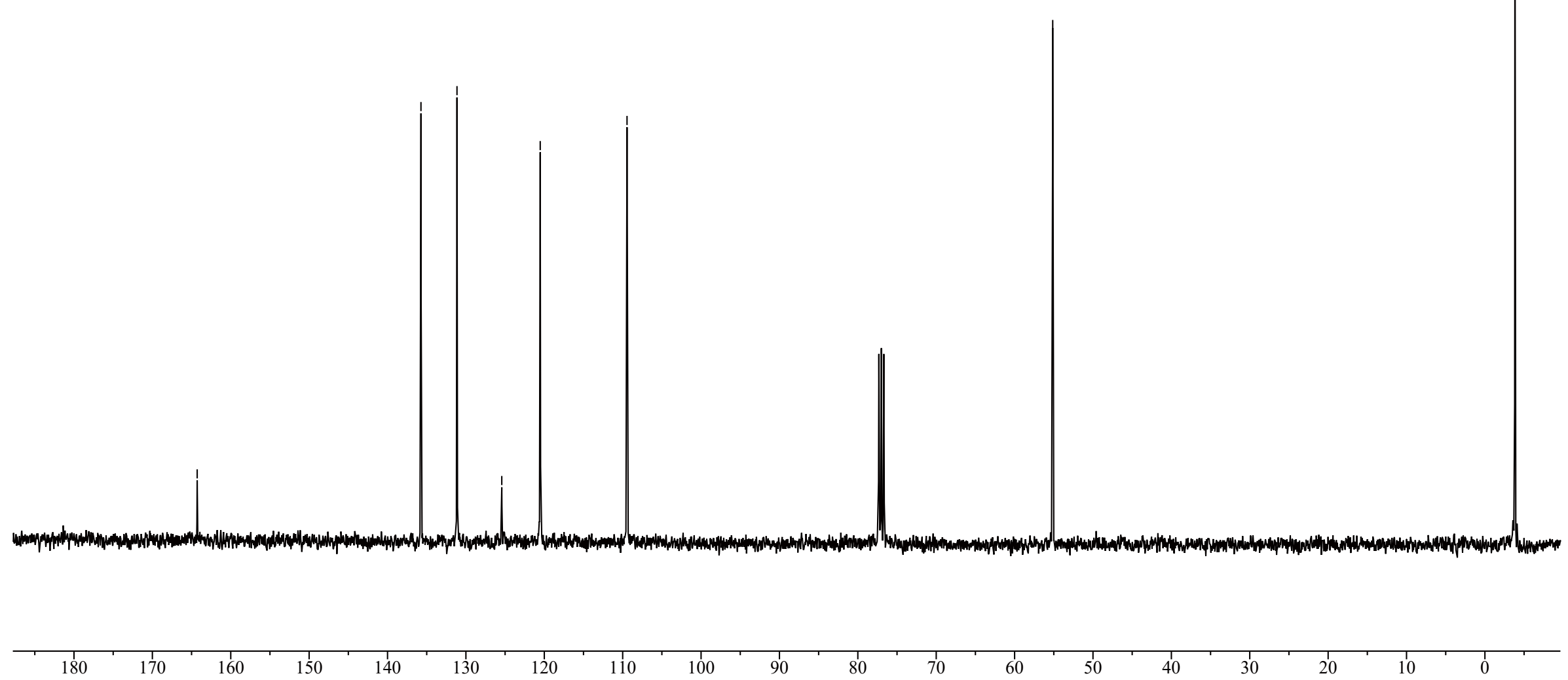


\section{Compound 1v ${ }^{1} \mathrm{H}$ NMR}
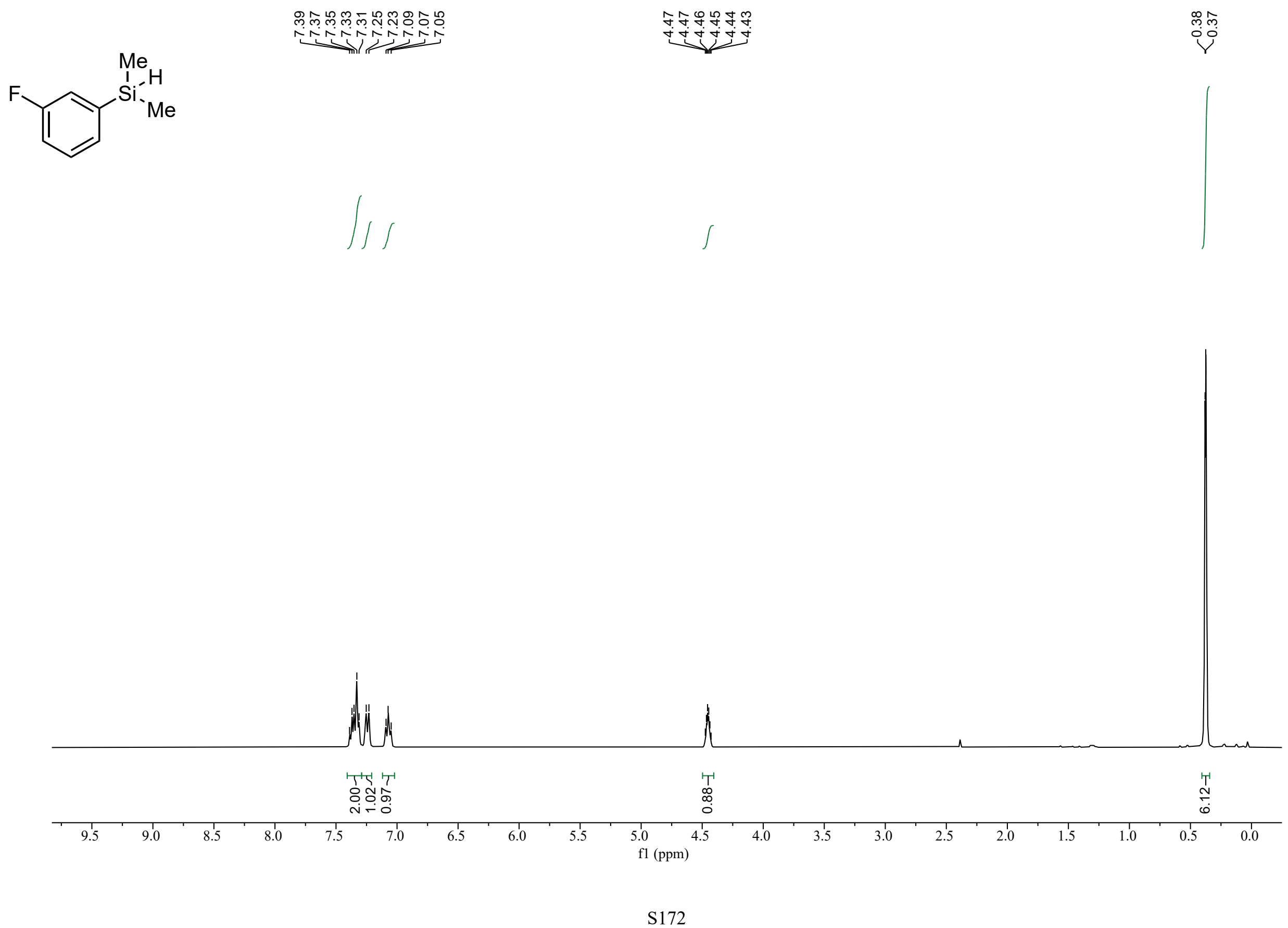
Compound $1 \mathbf{v}{ }^{13} \mathrm{C}$ NMR
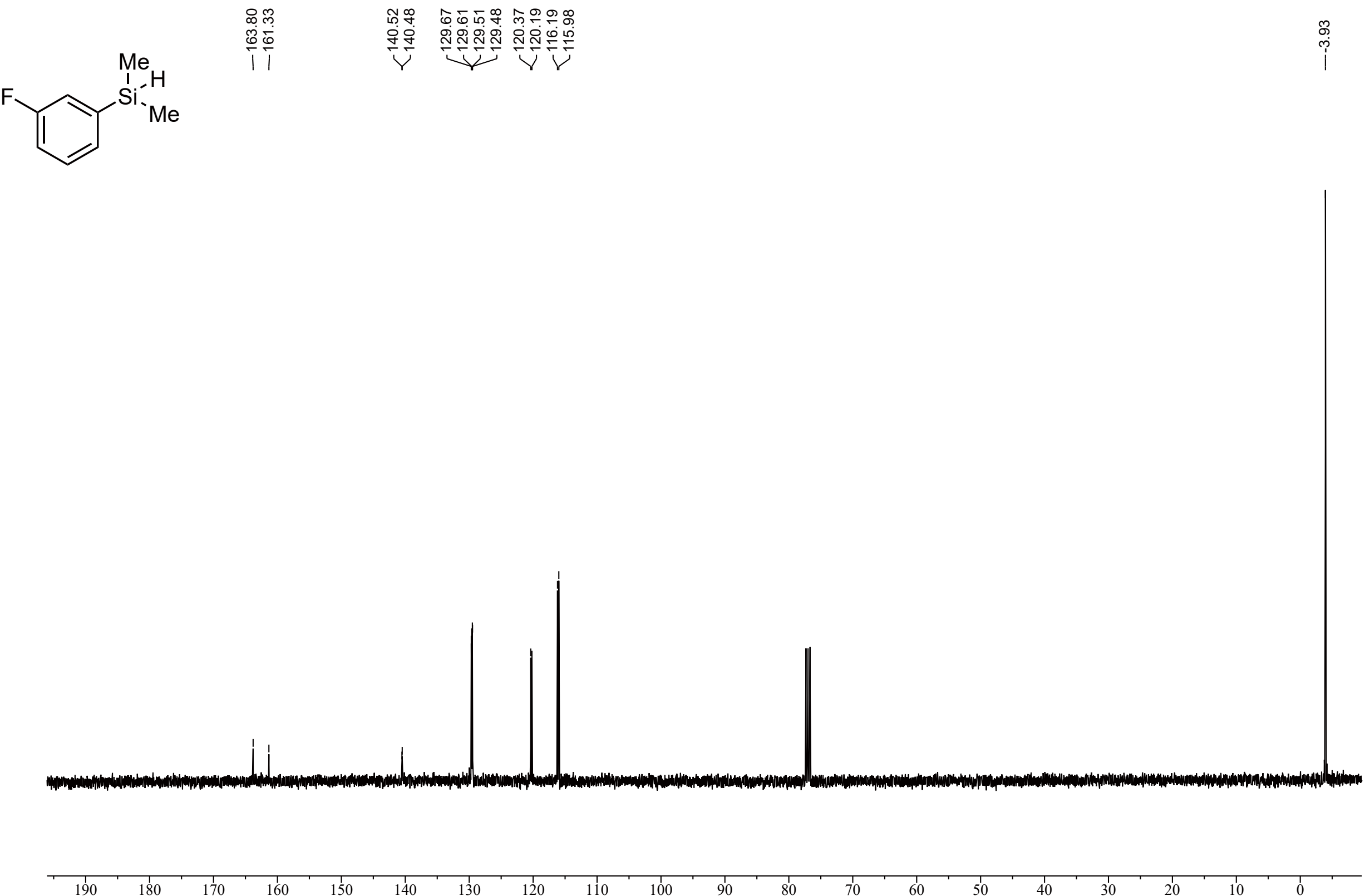
Compound 1v ${ }^{19}$ F NMR
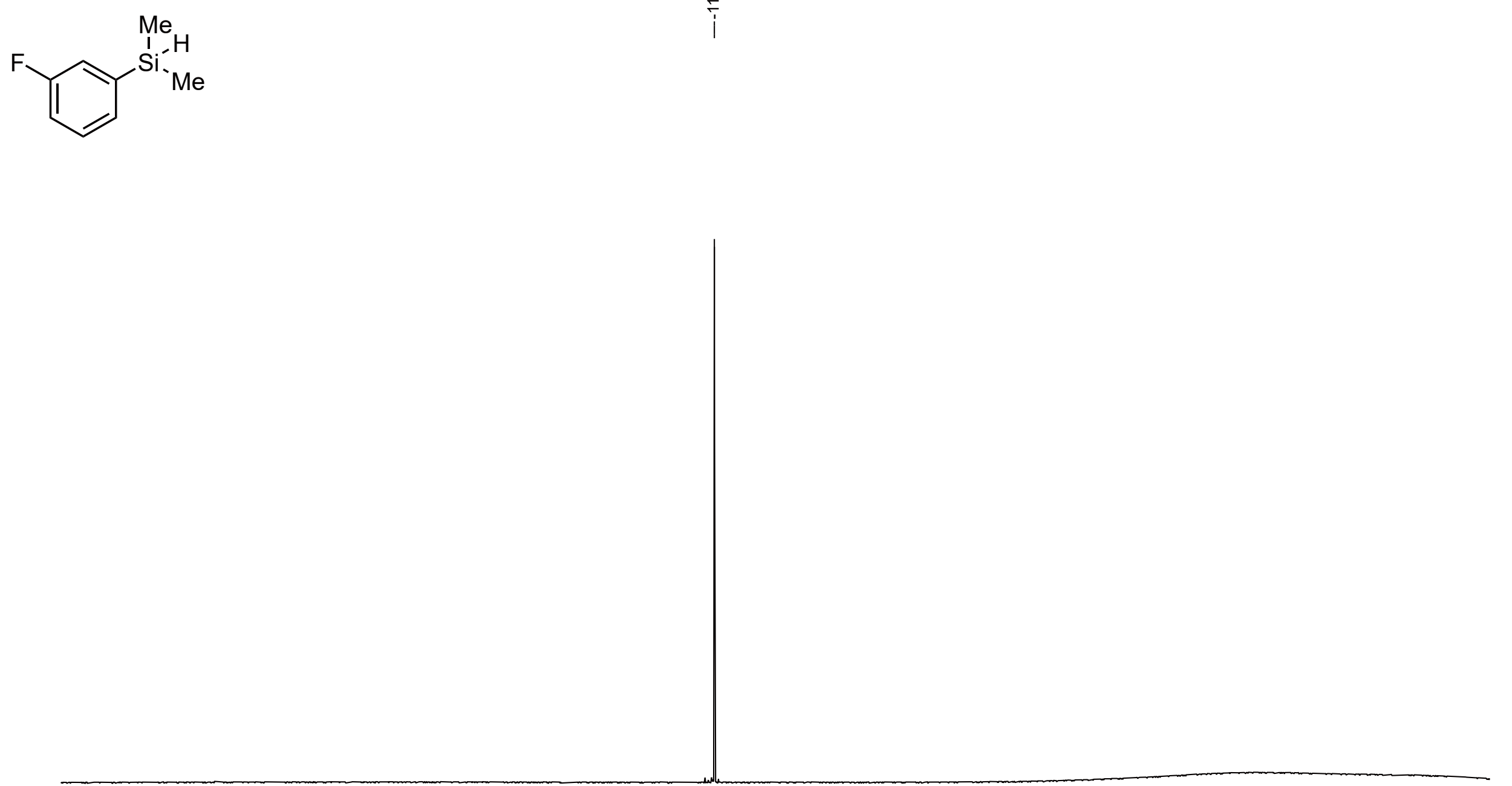

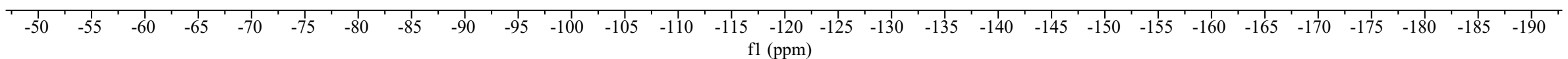


Compound 1w ${ }^{1} \mathrm{H}$ NMR

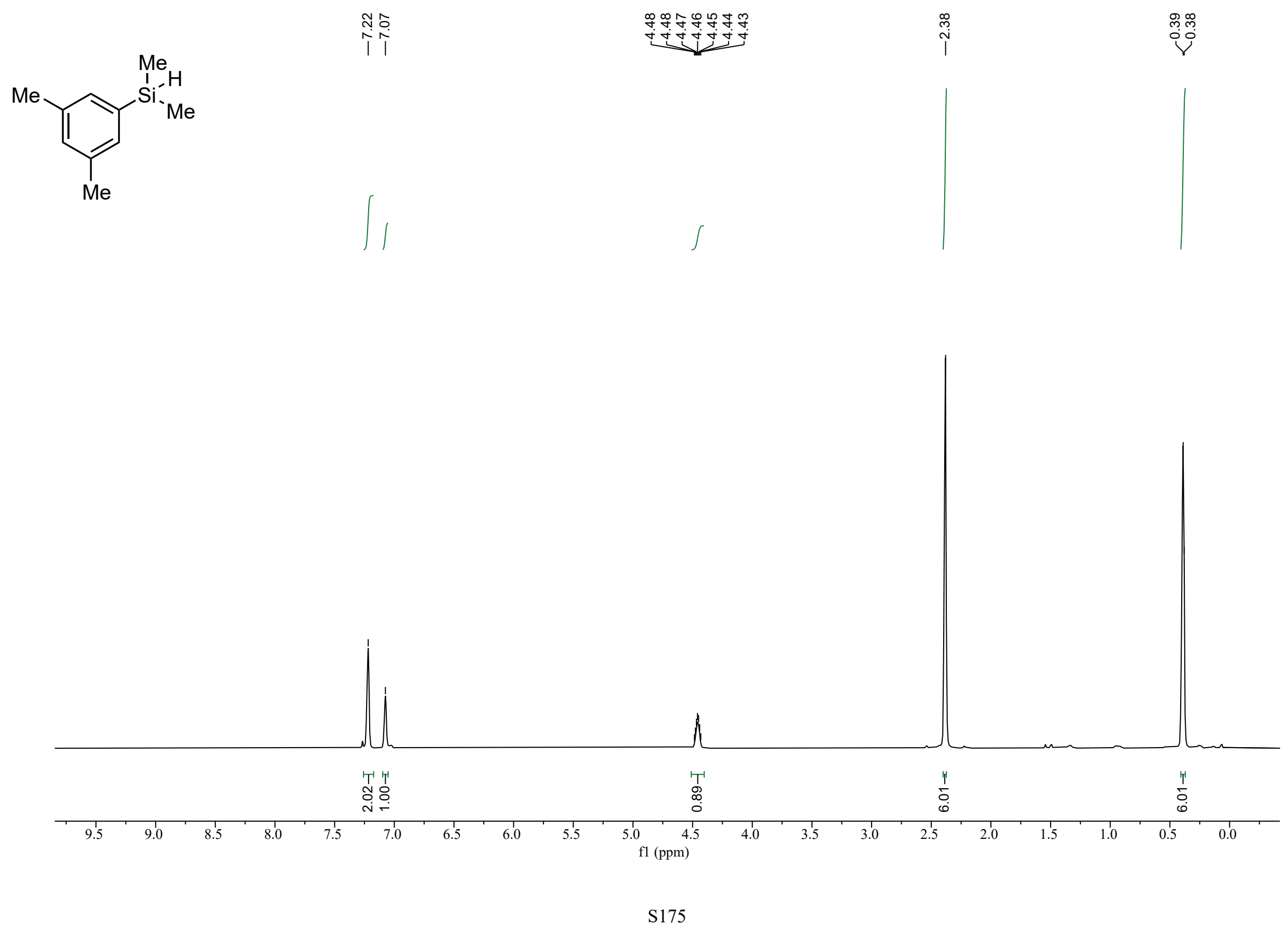


Compound $\mathbf{1 w}{ }^{13} \mathrm{C}$ NMR
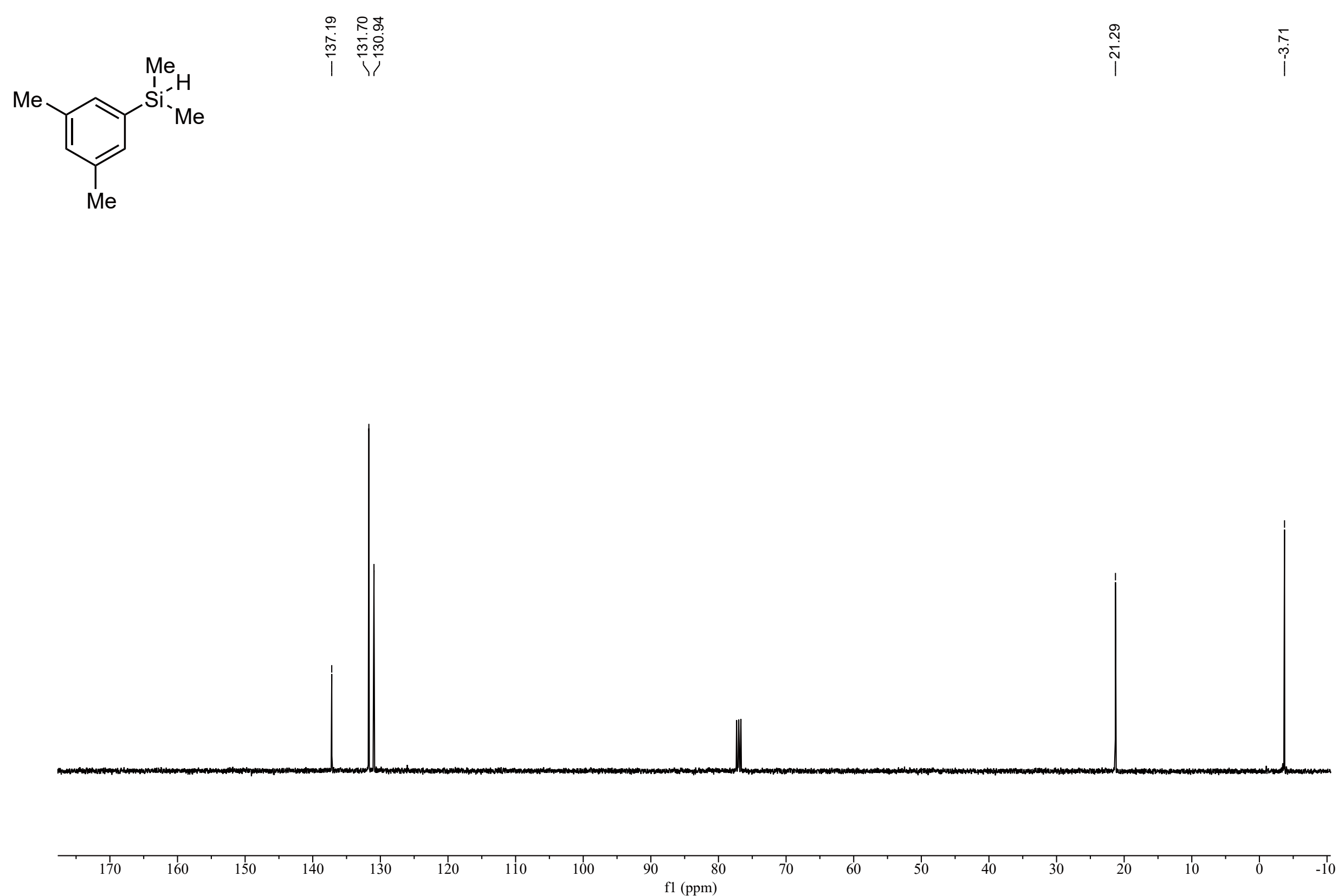


\section{Compound 1x ${ }^{1} \mathrm{H}$ NMR}

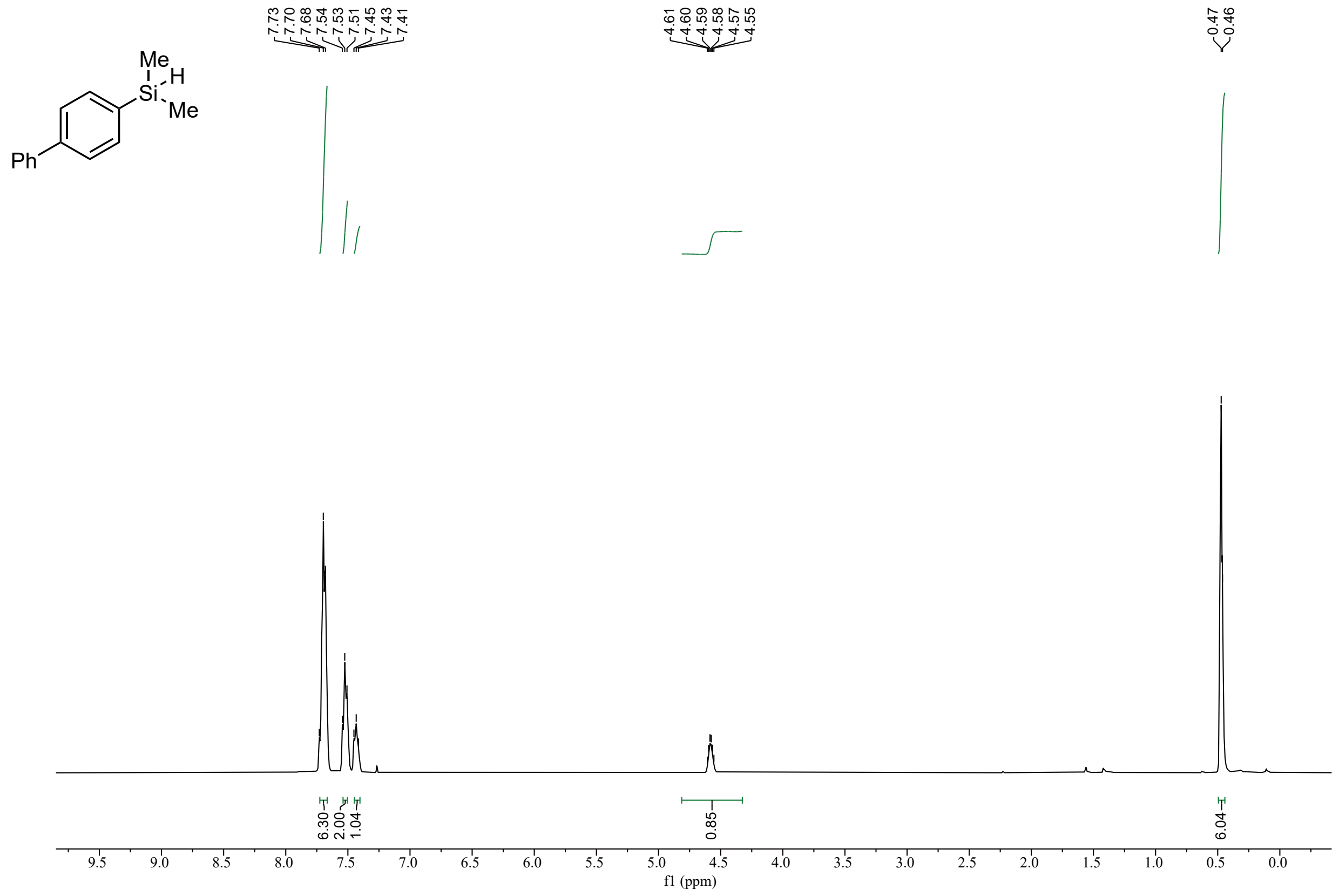


Compound $1 \mathbf{x}{ }^{13} \mathrm{C}$ NMR<smiles>C[Si](C)(C)c1ccc(-c2ccccc2)cc1</smiles>

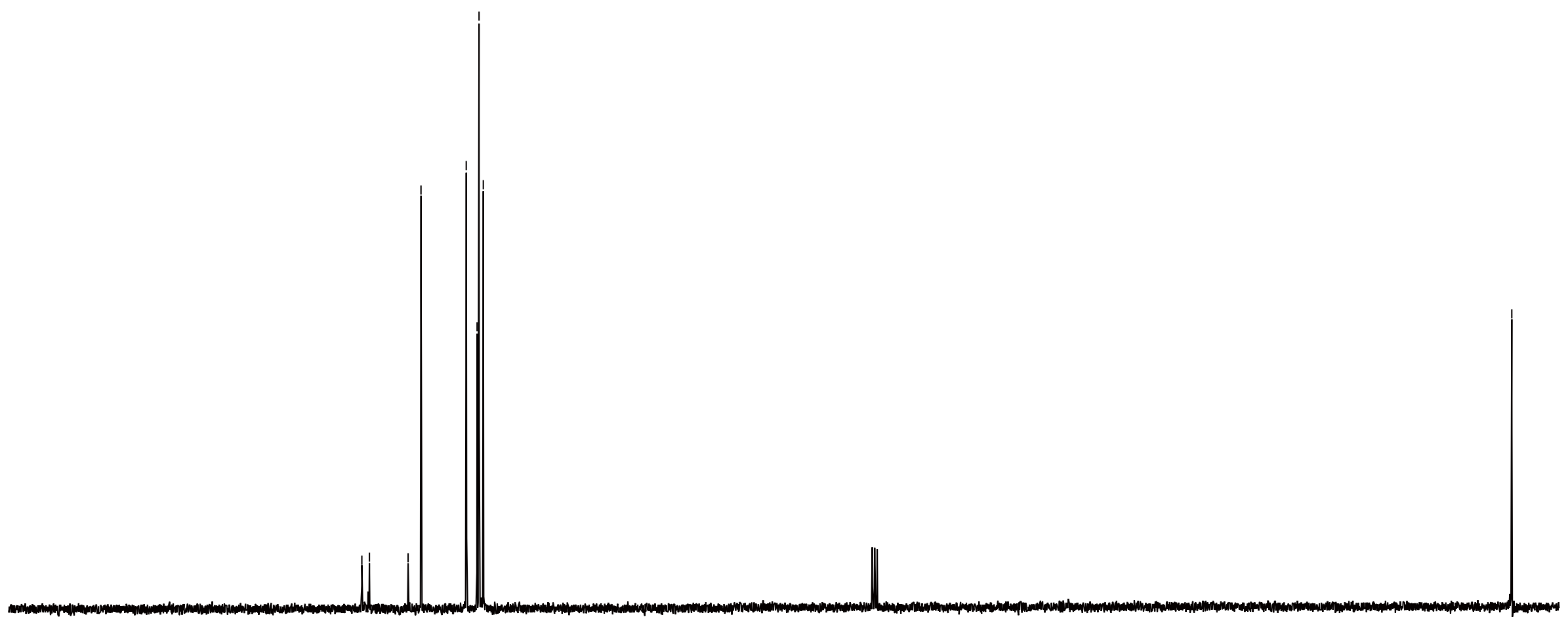



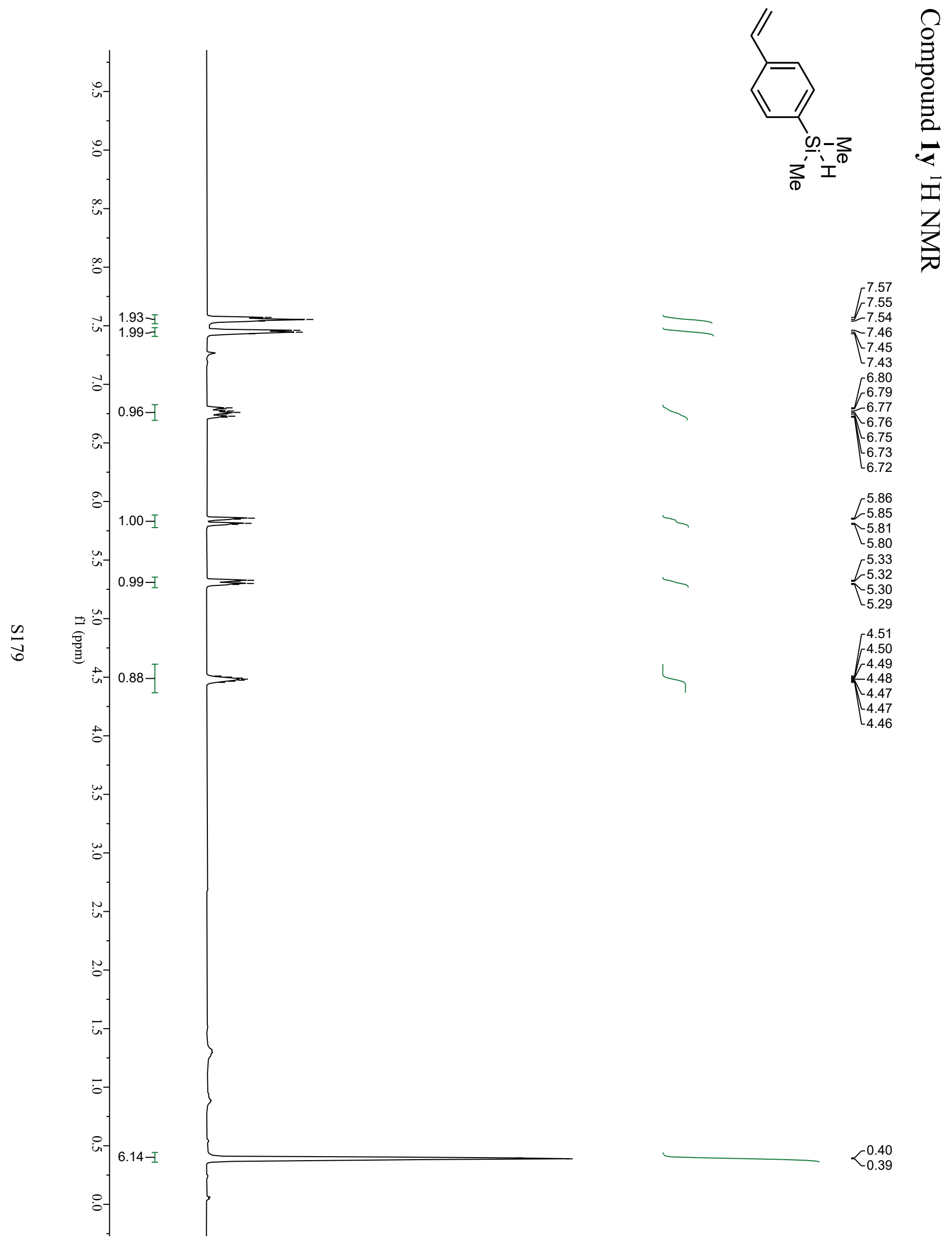
Compound $1 \mathrm{y}{ }^{13} \mathrm{C}$ NMR
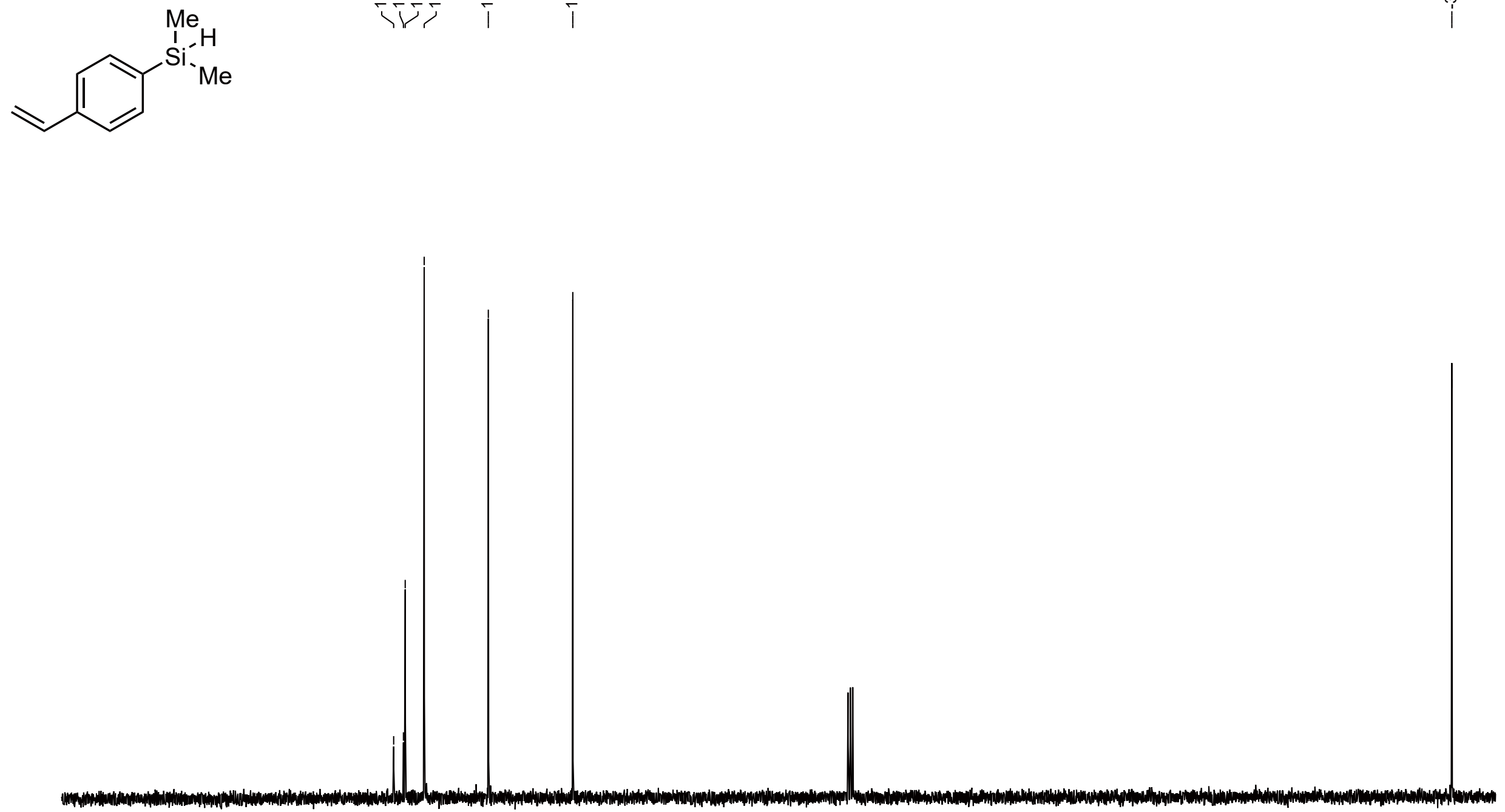

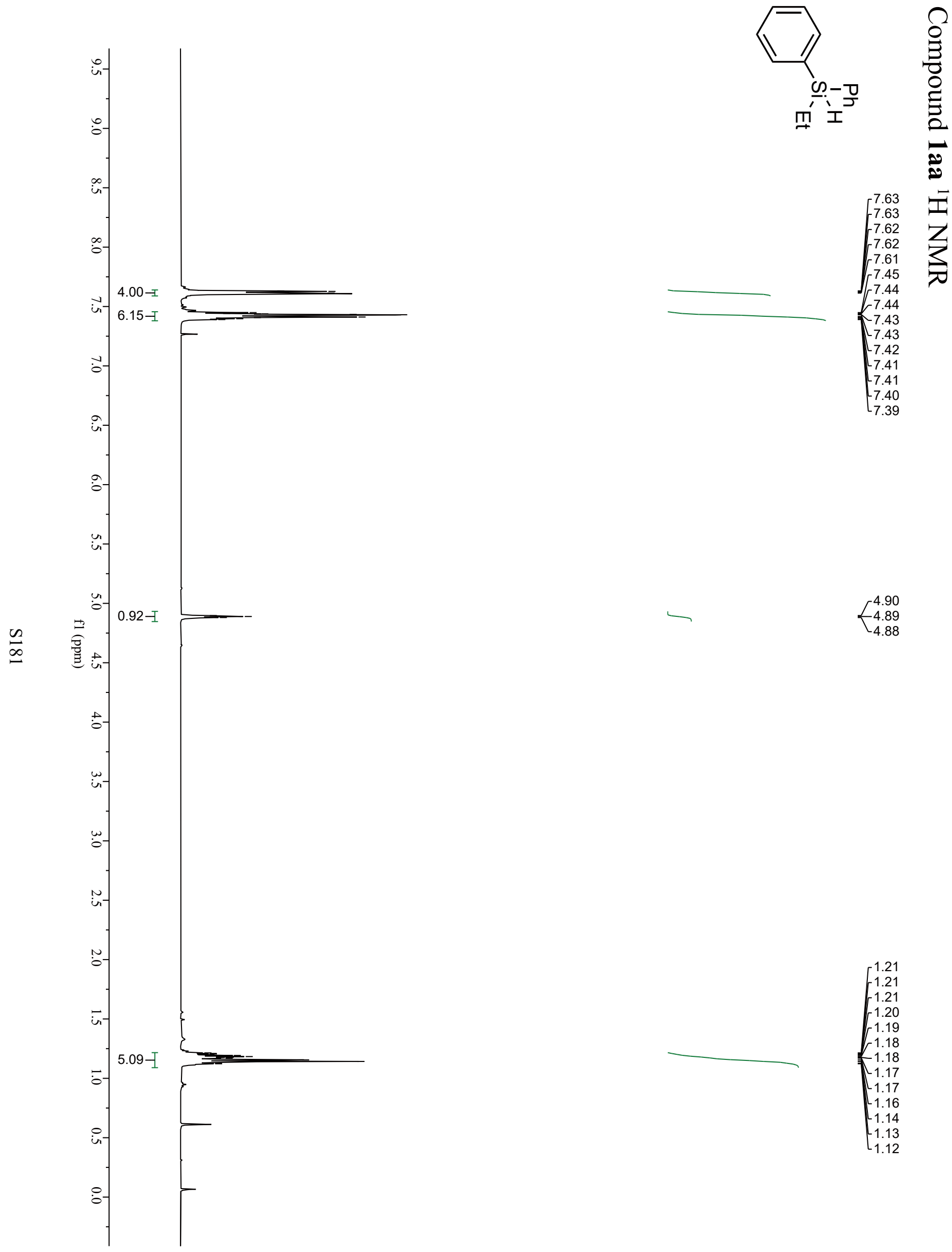
Compound 1aa ${ }^{13} \mathrm{C}$ NMR
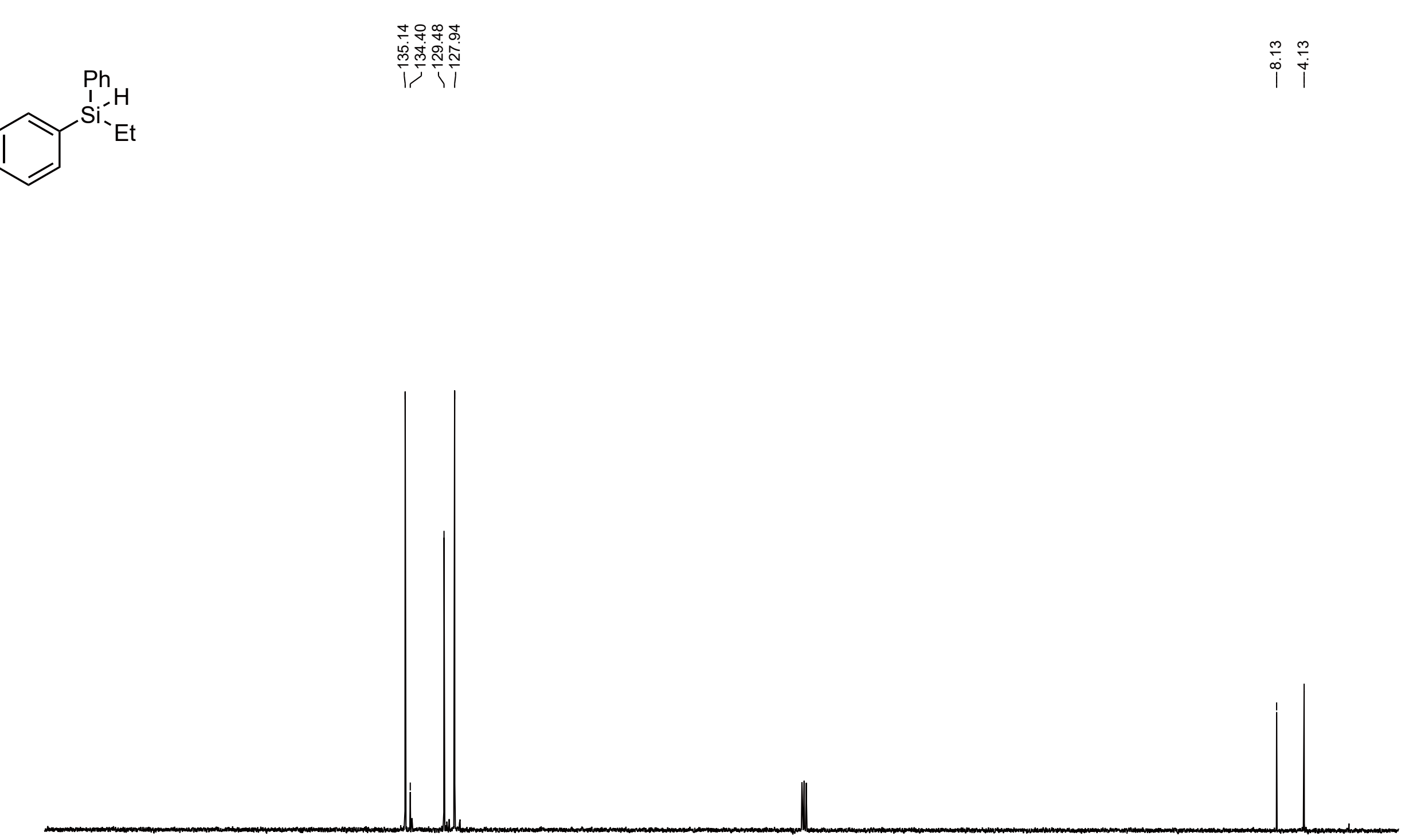


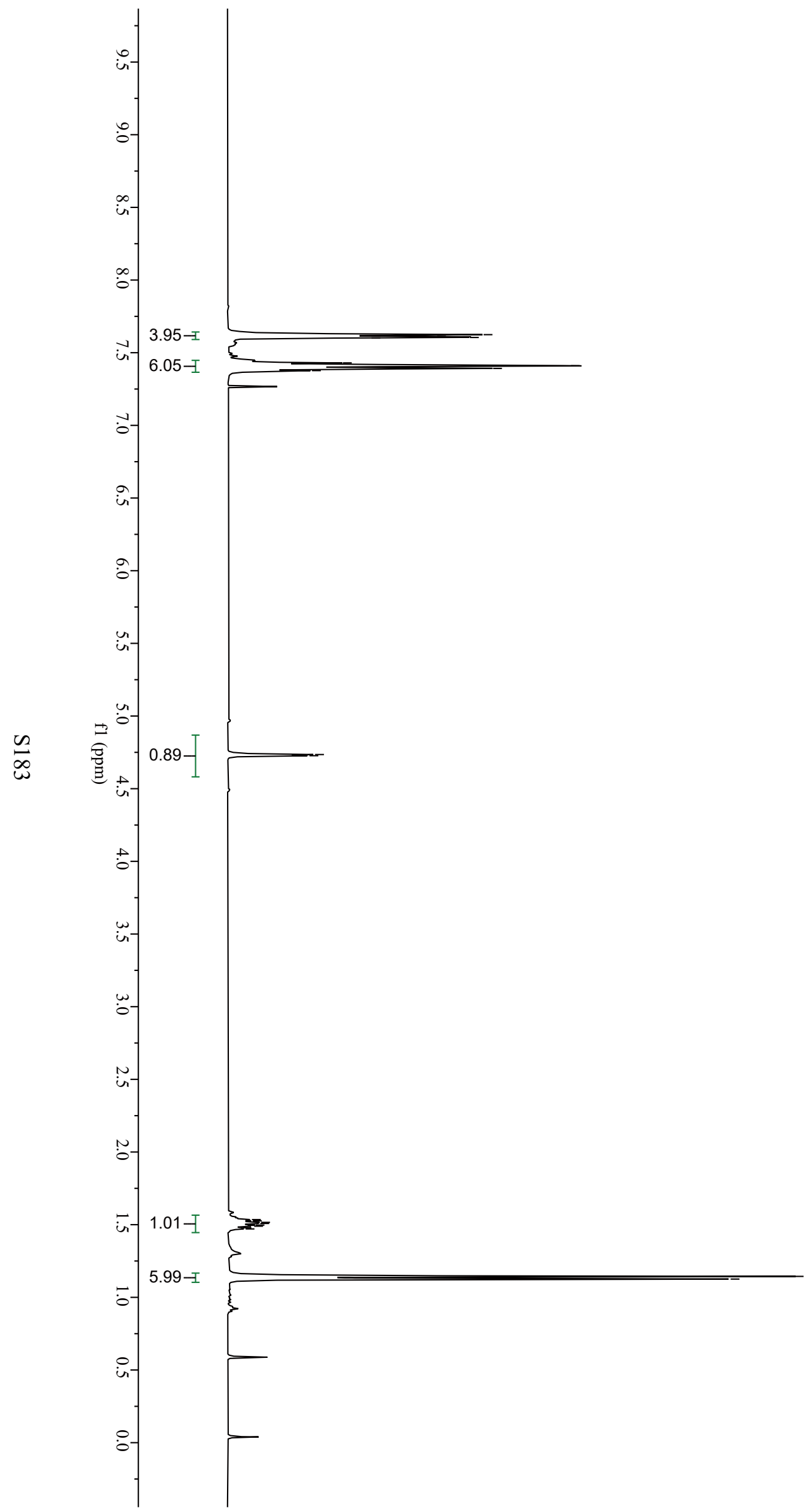

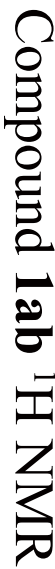
7.61

$+7.60$ 7.43

7.41

$\left[\begin{array}{r}7.39 \\ 7.38\end{array}\right.$

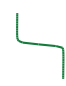

$<_{4.73}^{4.74}$

1.54 1.53 $-1.52$

1.51

1.50

$-1.49$

$-1.48$

1.47 $乙_{1.13}^{1.14}$ 
Compound $\mathbf{1 a b}{ }^{13} \mathrm{C}$ NMR

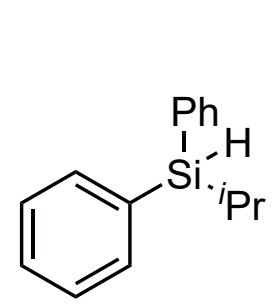

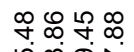

ผ․

$\stackrel{\infty}{\infty} \underset{\infty}{\infty} \stackrel{\wp}{\leftarrow}$

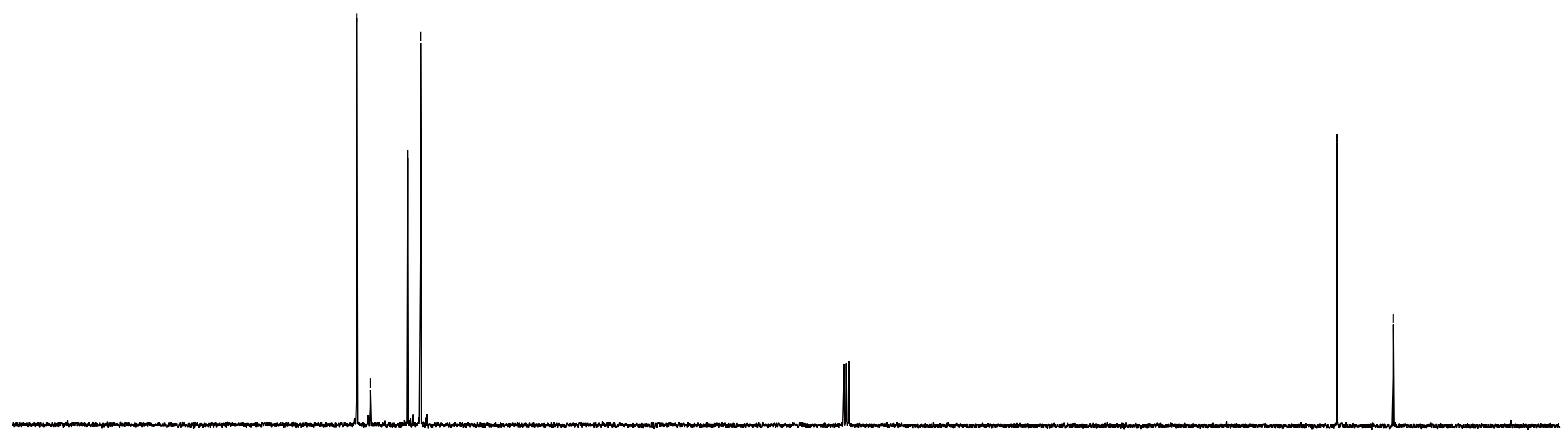




$$
=1
$$


Compound 1ad ${ }^{13} \mathrm{C}$ NMR
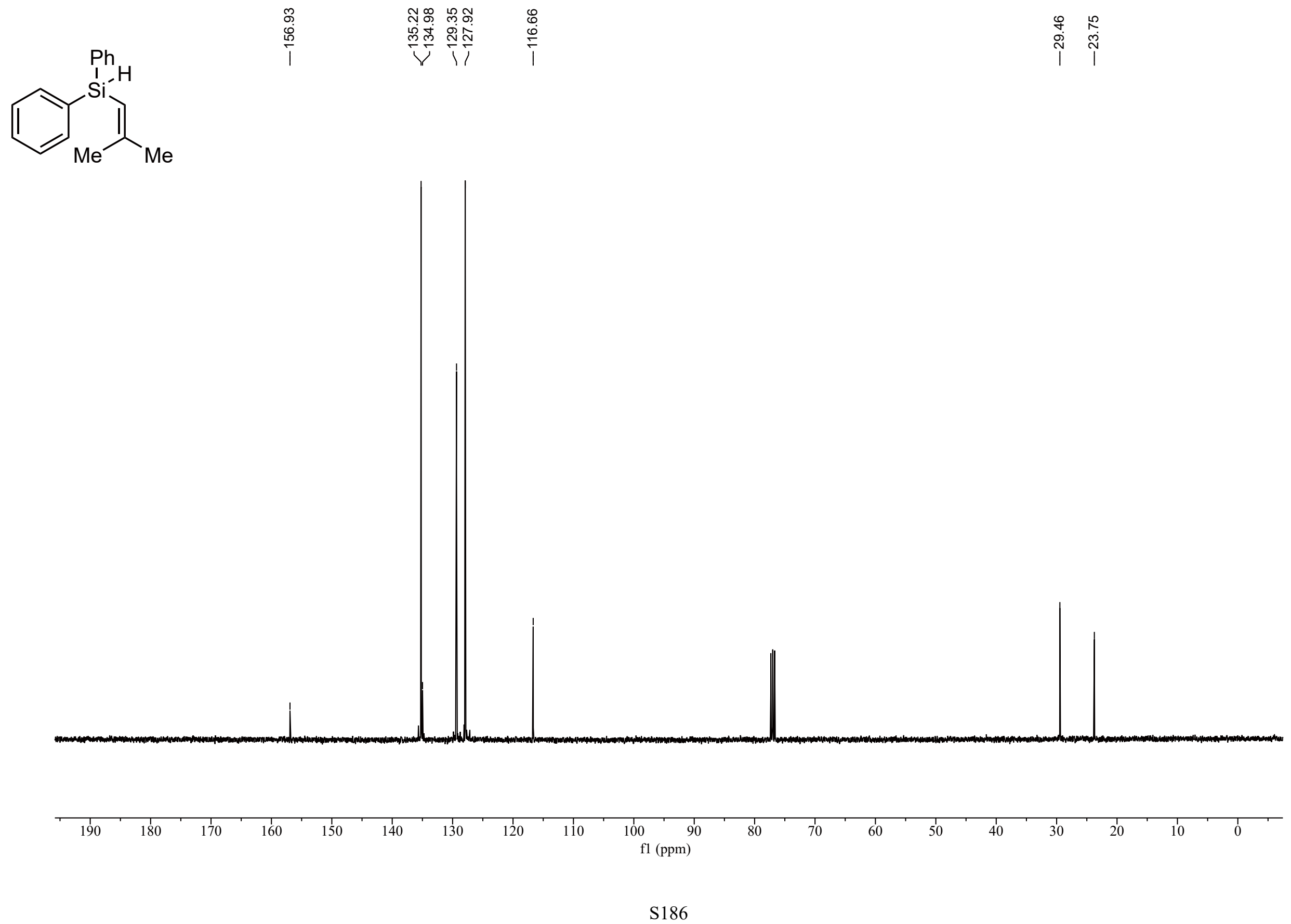

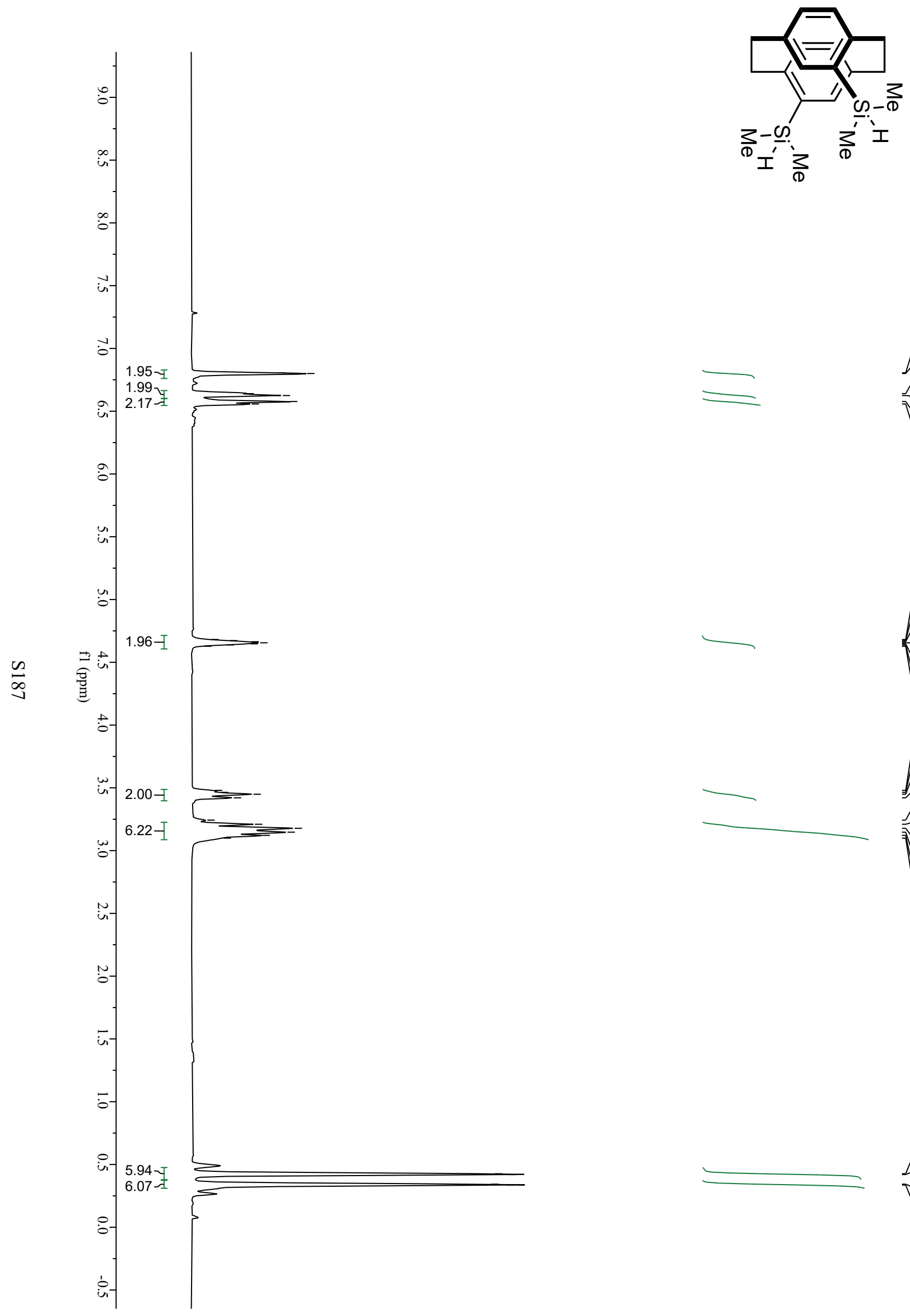

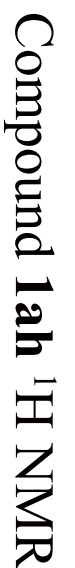
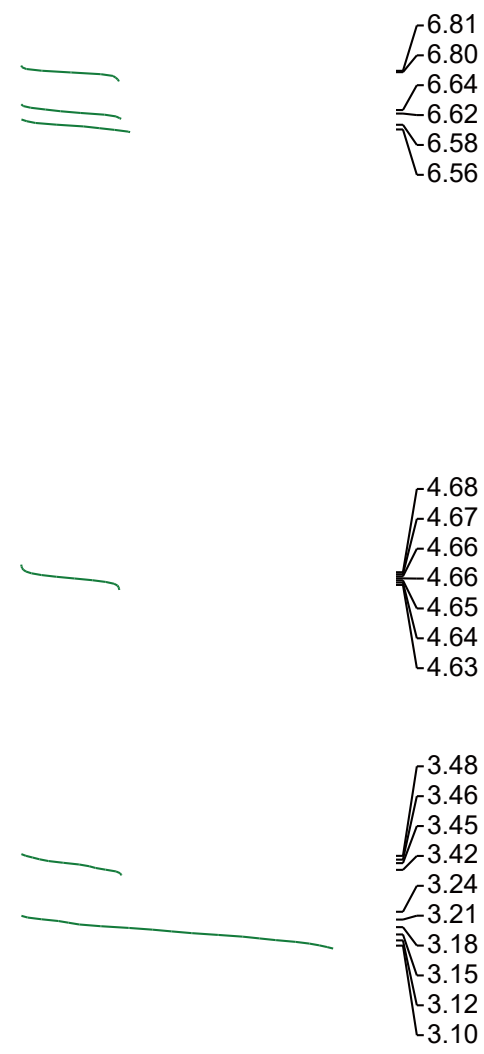

$\Sigma_{0.42}^{0.43}$

$-0.34$

$\tau_{0.34}$ 
Compound 1ah ${ }^{13} \mathrm{C}$ NMR
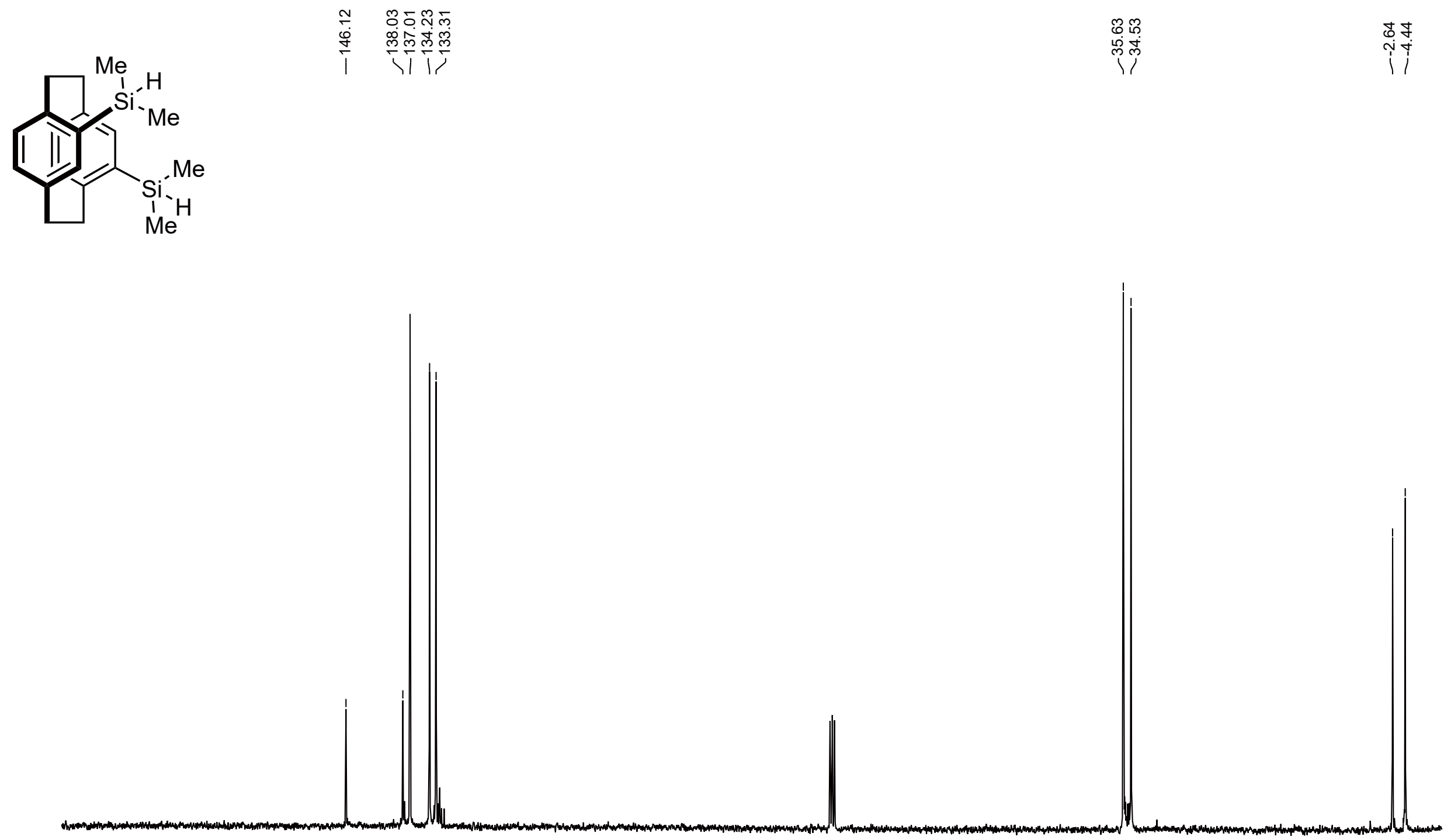


\section{Compound 1al ${ }^{1} \mathrm{H}$ NMR}

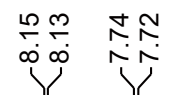
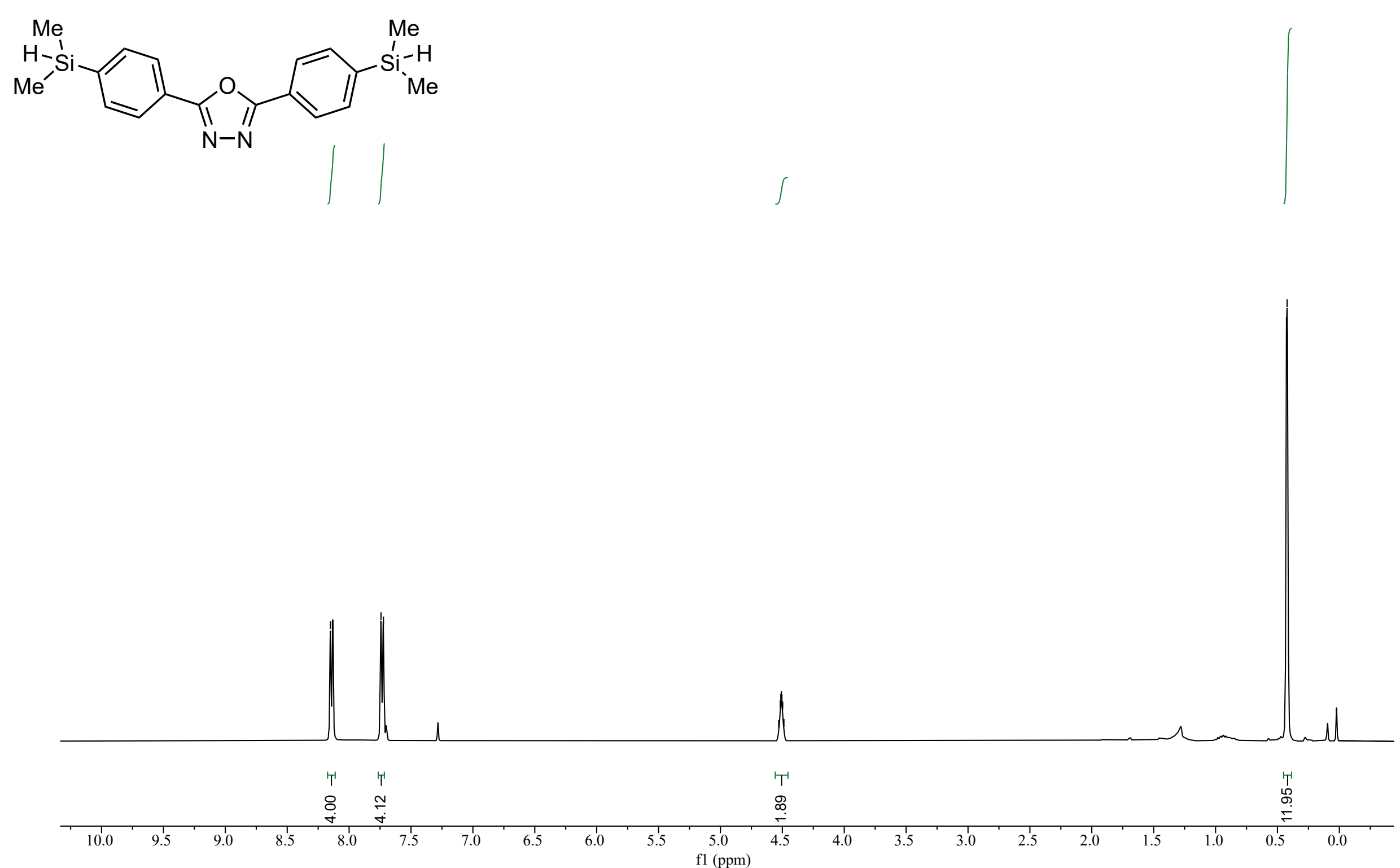
Compound 1al ${ }^{13} \mathrm{C}$ NMR

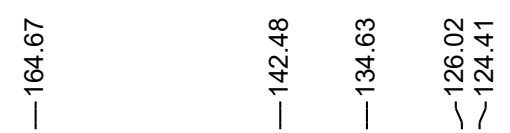
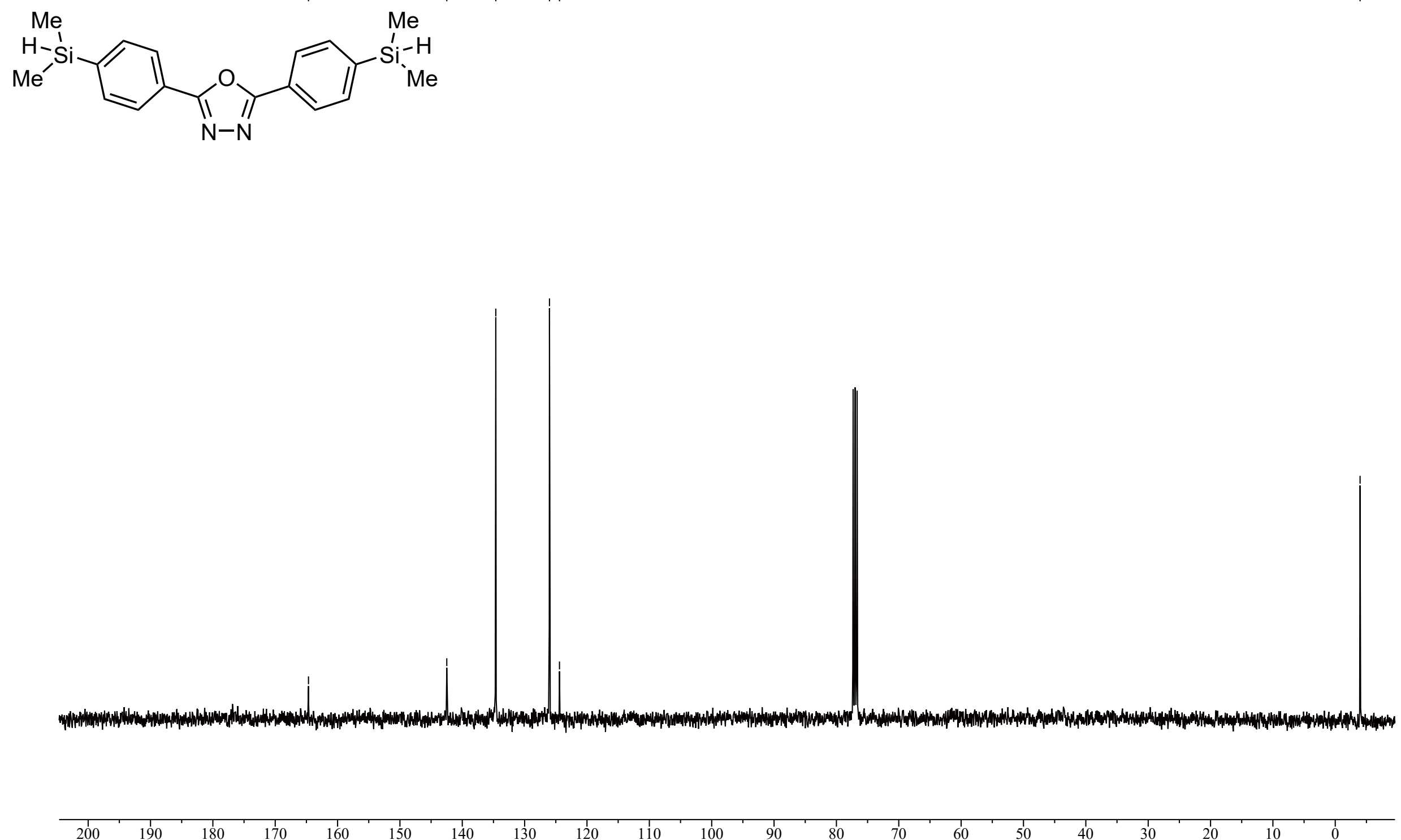


\section{Compound 1am ${ }^{1} \mathrm{H}$ NMR}<smiles>C[Si](C)(C)c1ccc(-c2ccc([Si](C)(C)C)s2)s1</smiles>

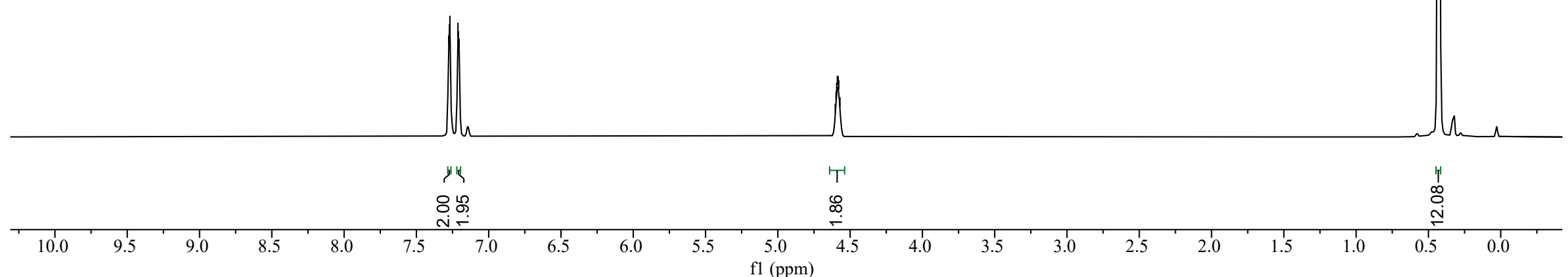


Compound 1am ${ }^{13} \mathrm{C}$ NMR
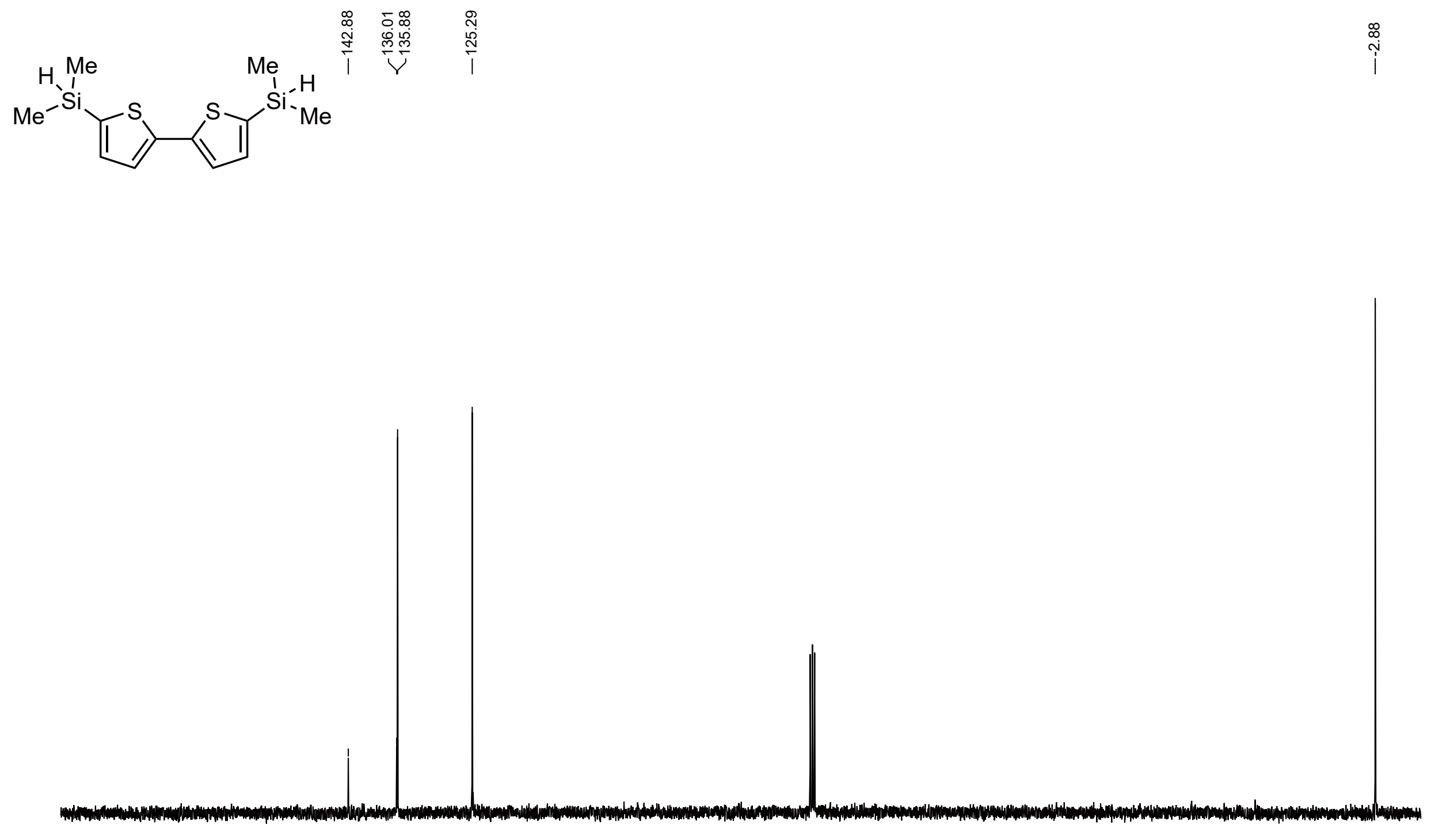

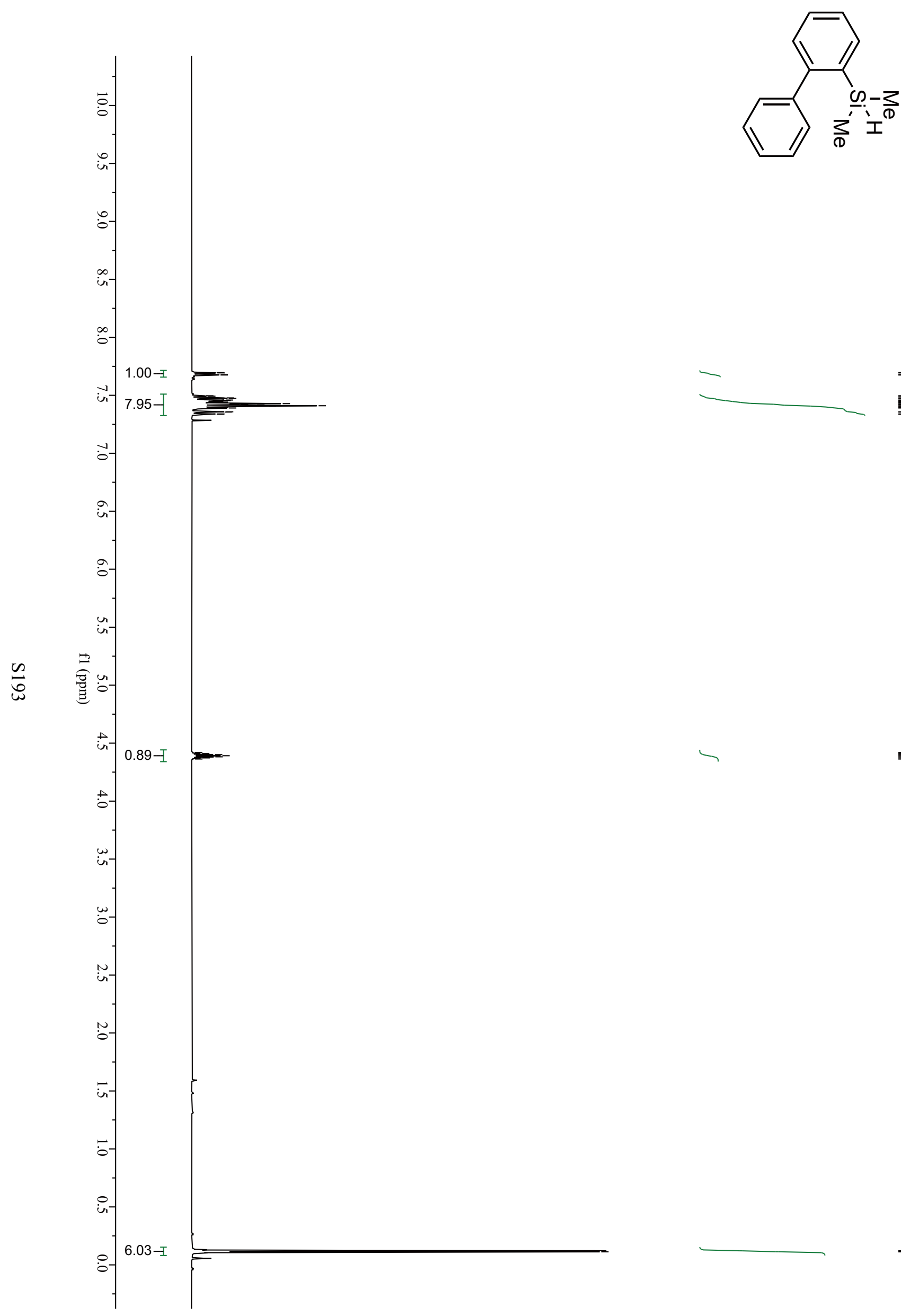

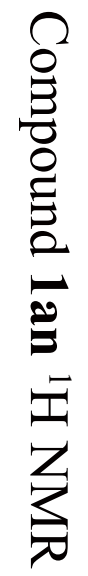

$\left\{\begin{array}{r}4.42 \\ 4.41 \\ 4.40 \\ 4.39 \\ 4.38 \\ 4.37 \\ 4.36\end{array}\right.$

$<_{0.11}^{0.12}$ 
Compound 1an ${ }^{13} \mathrm{C}$ NMR

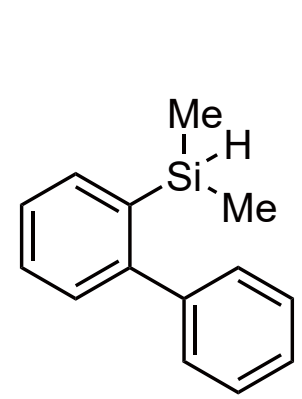

i

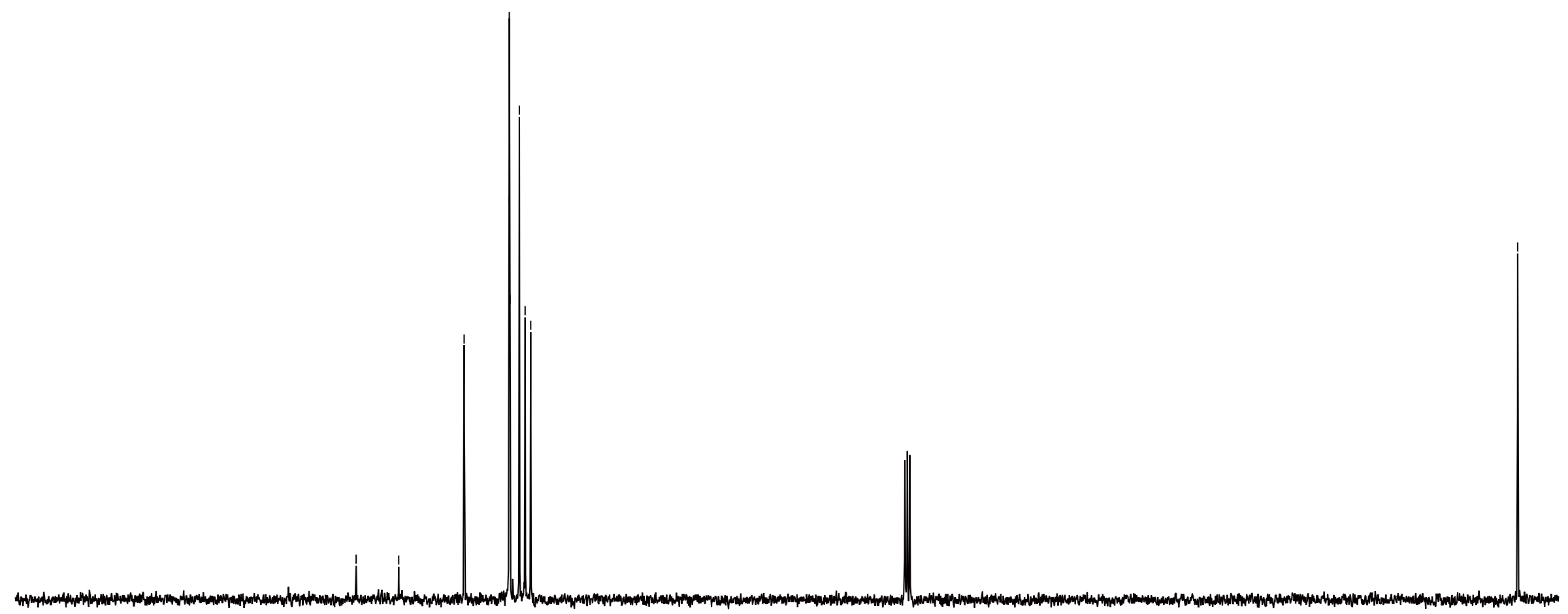



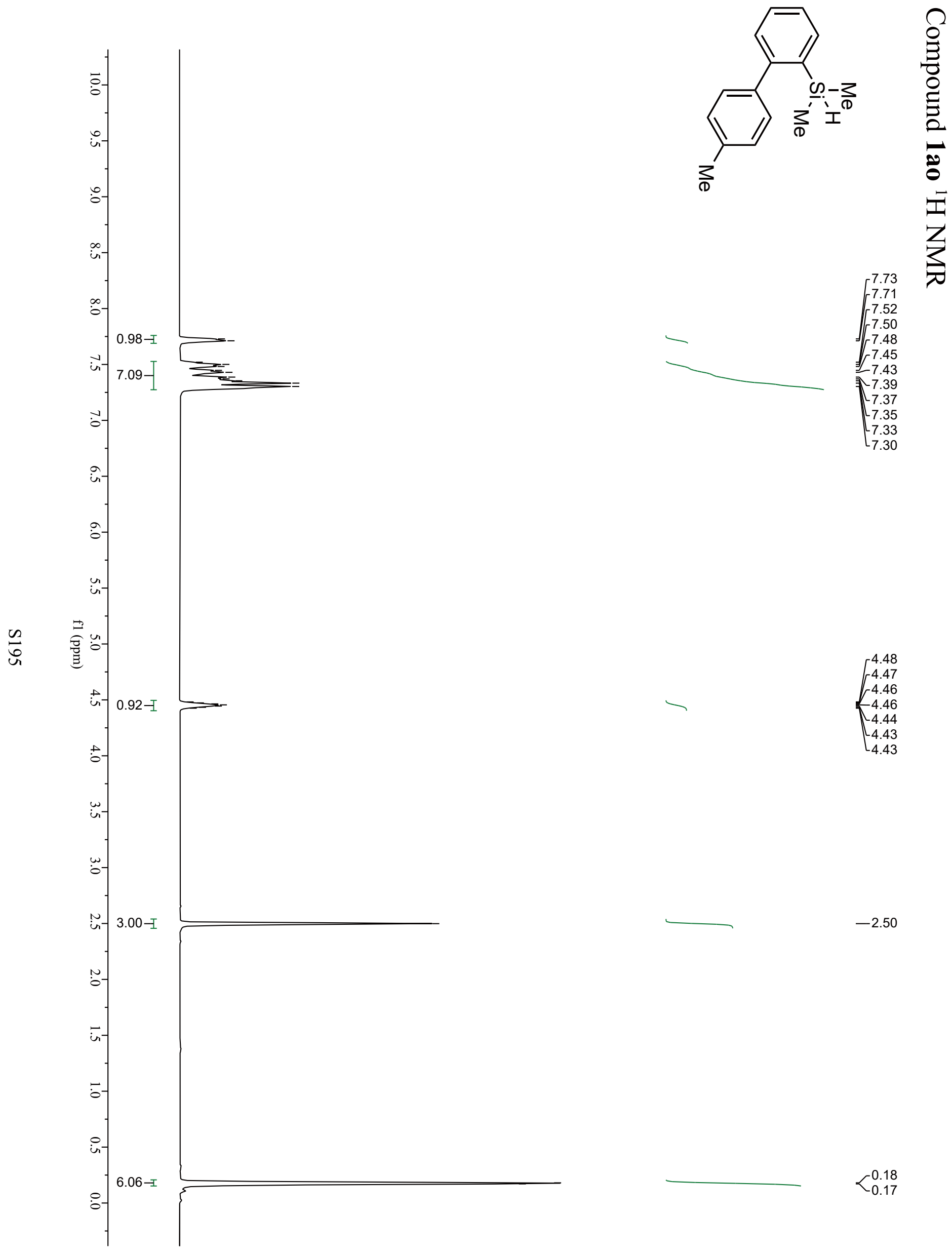
Compound 1ao ${ }^{13} \mathrm{C}$ NMR
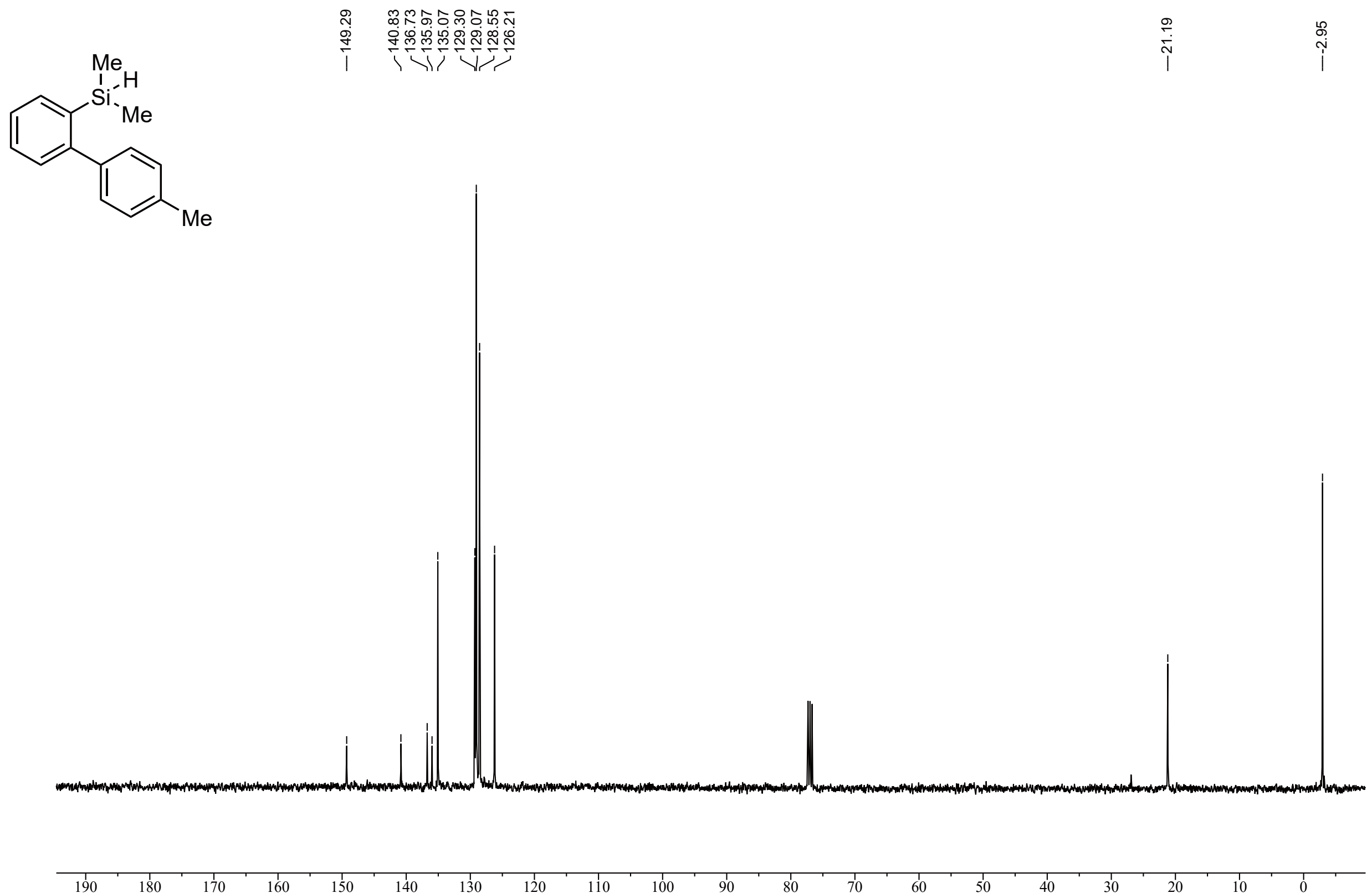

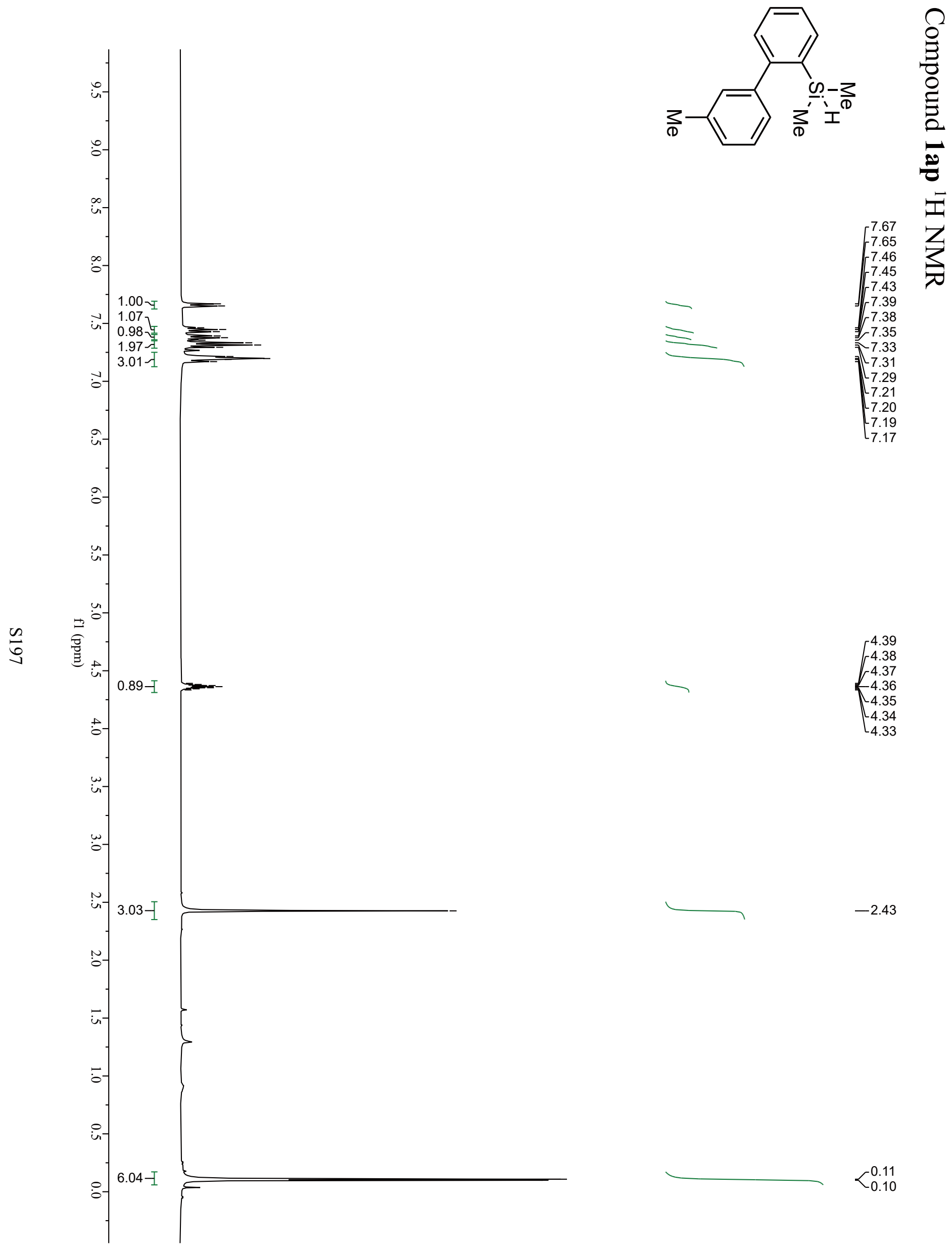
Compound 1ap ${ }^{13} \mathrm{C}$ NMR
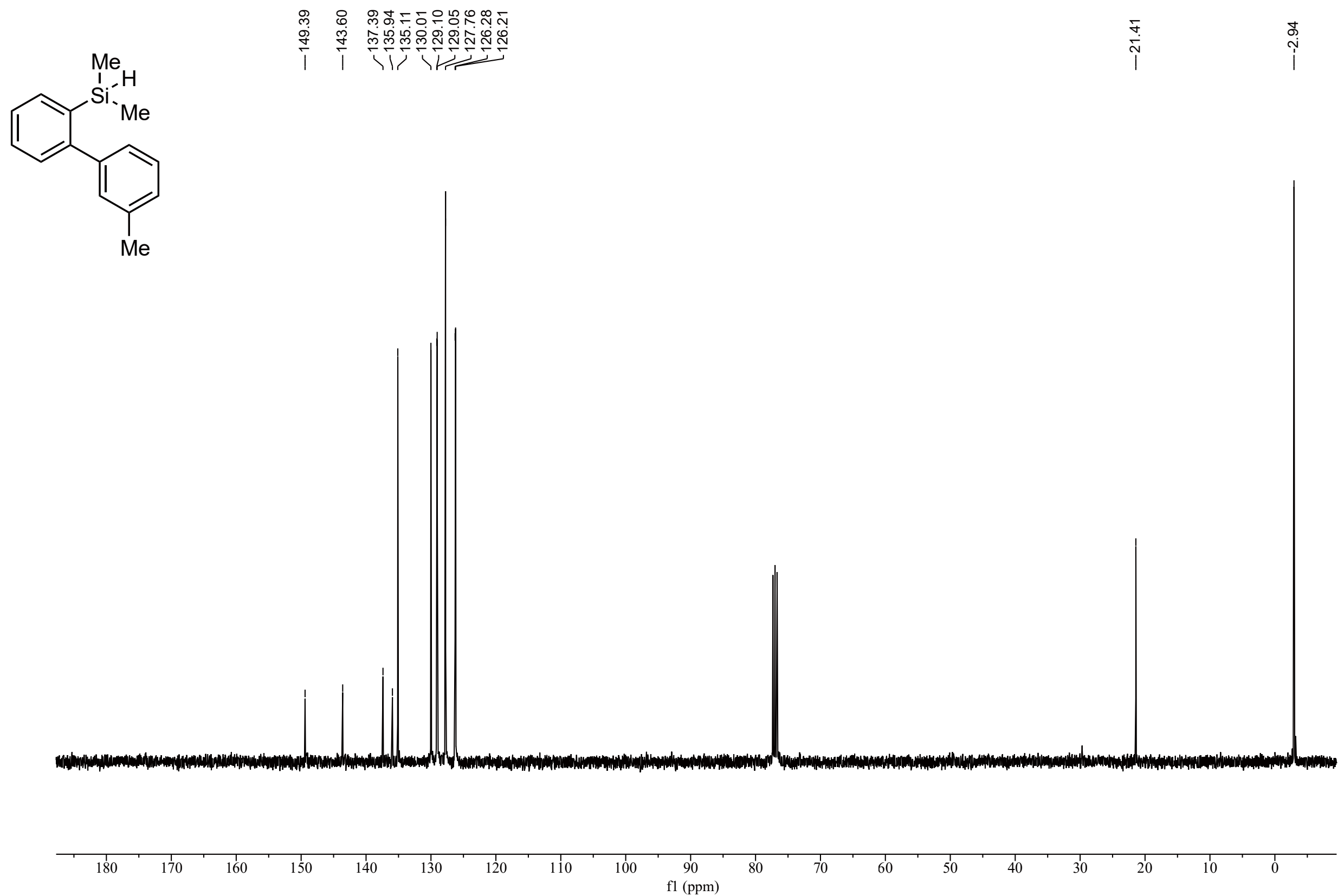
Compound 1 aq ${ }^{1} \mathrm{H}$ NMR

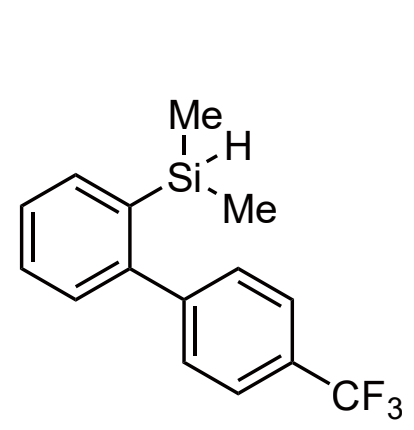

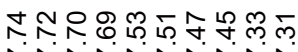

NiñNiñN

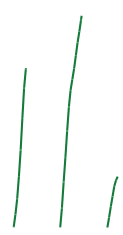

$\sqrt{4}$

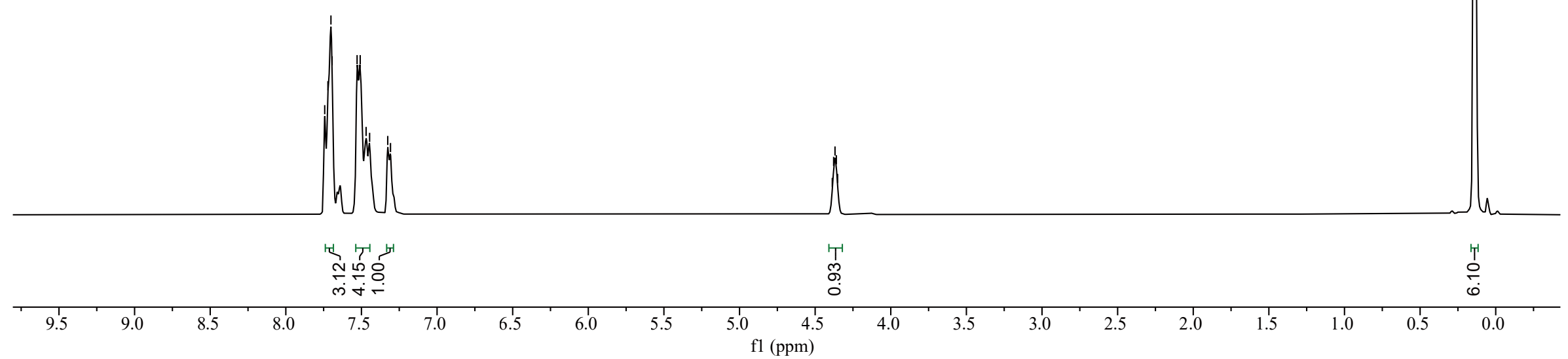



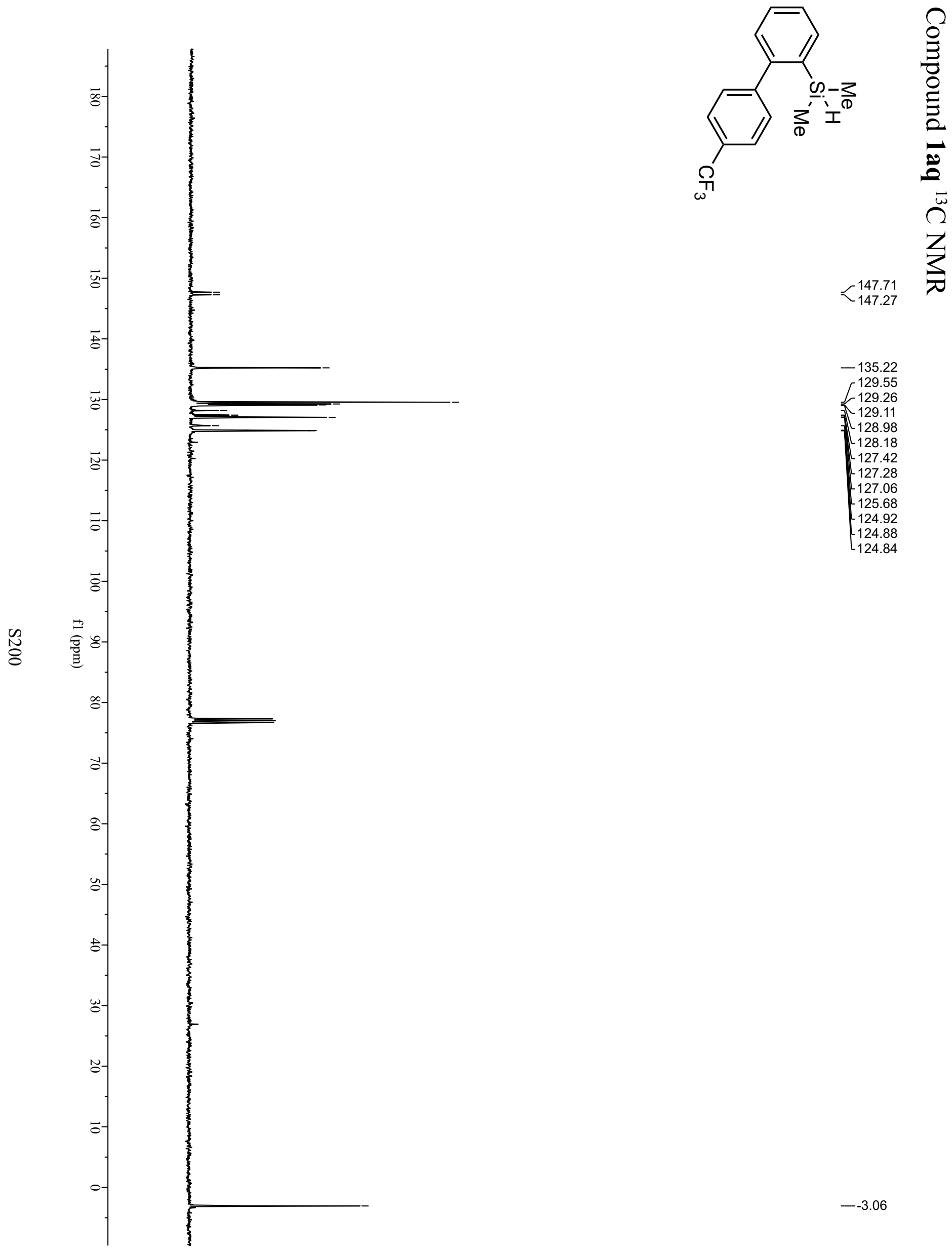

$--3.06$ 
Compound 1aq ${ }^{19} \mathrm{~F}$ NMR
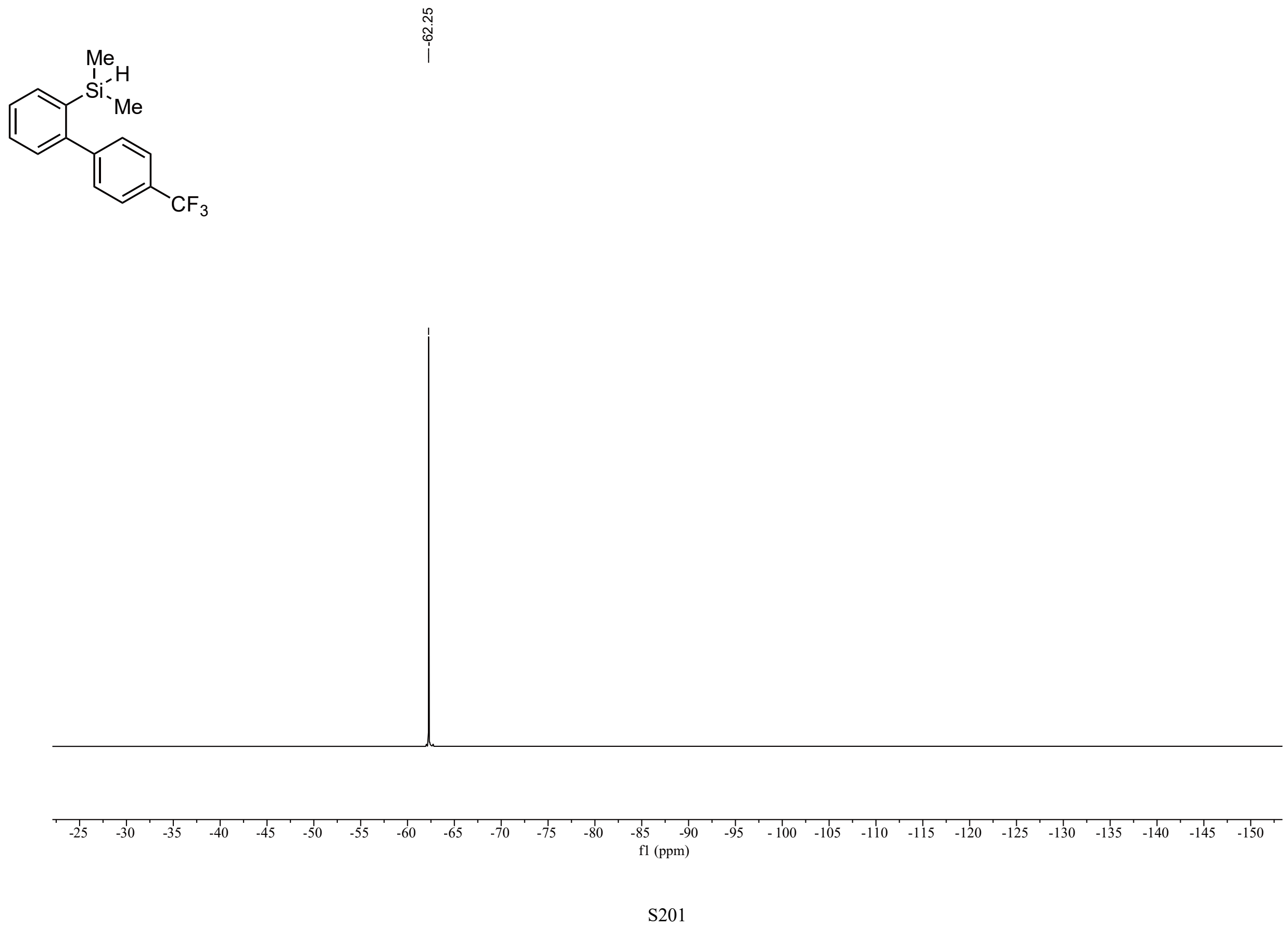

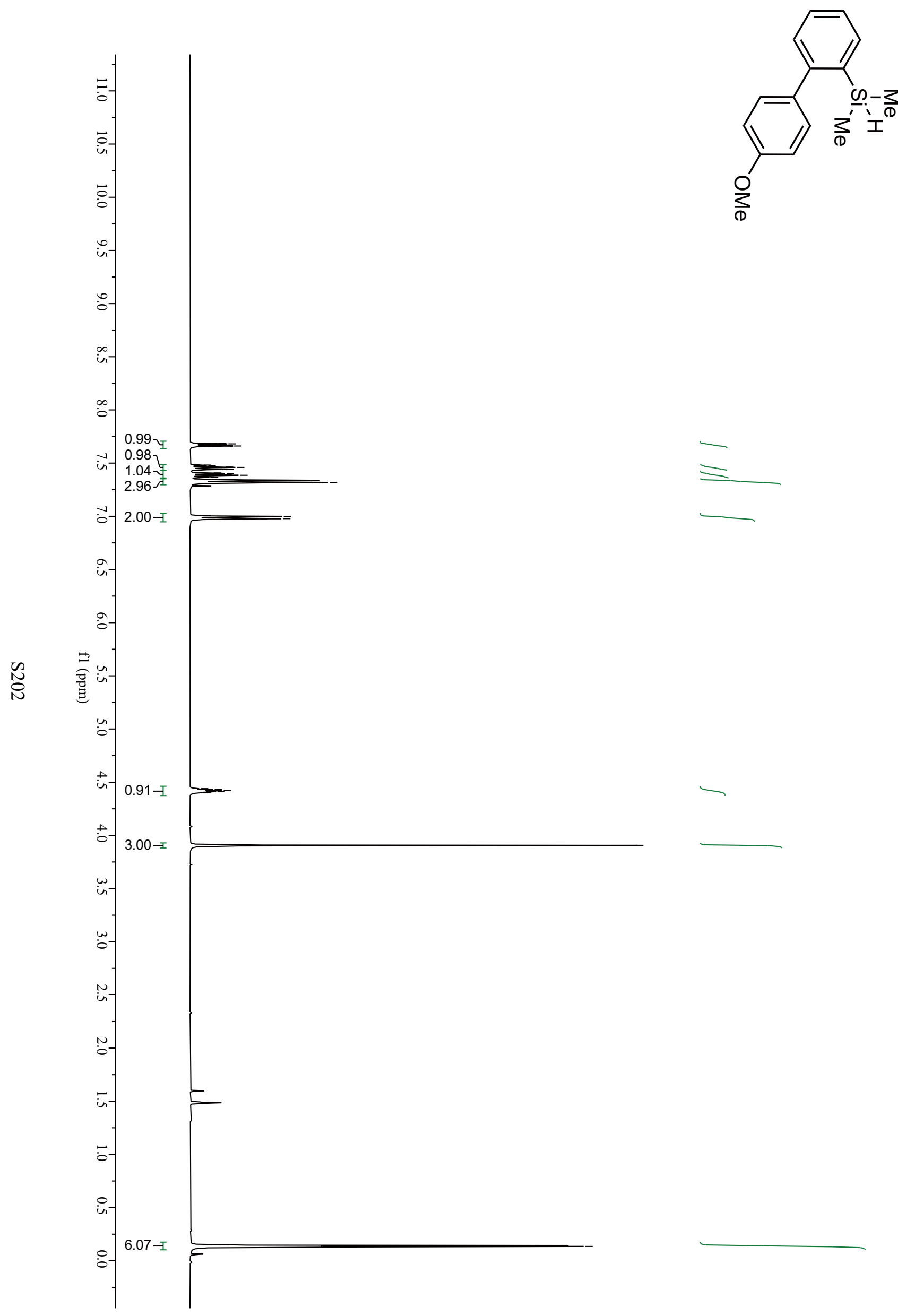

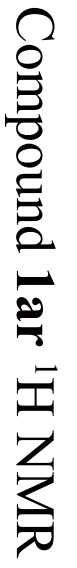

4.44

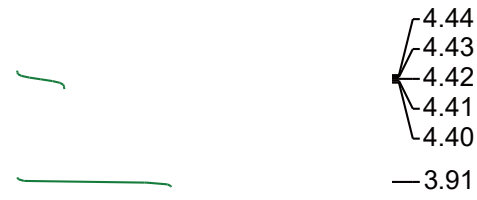

$<_{0.13}^{0.14}$ 
Compound 1 ar ${ }^{13} \mathrm{C}$ NMR

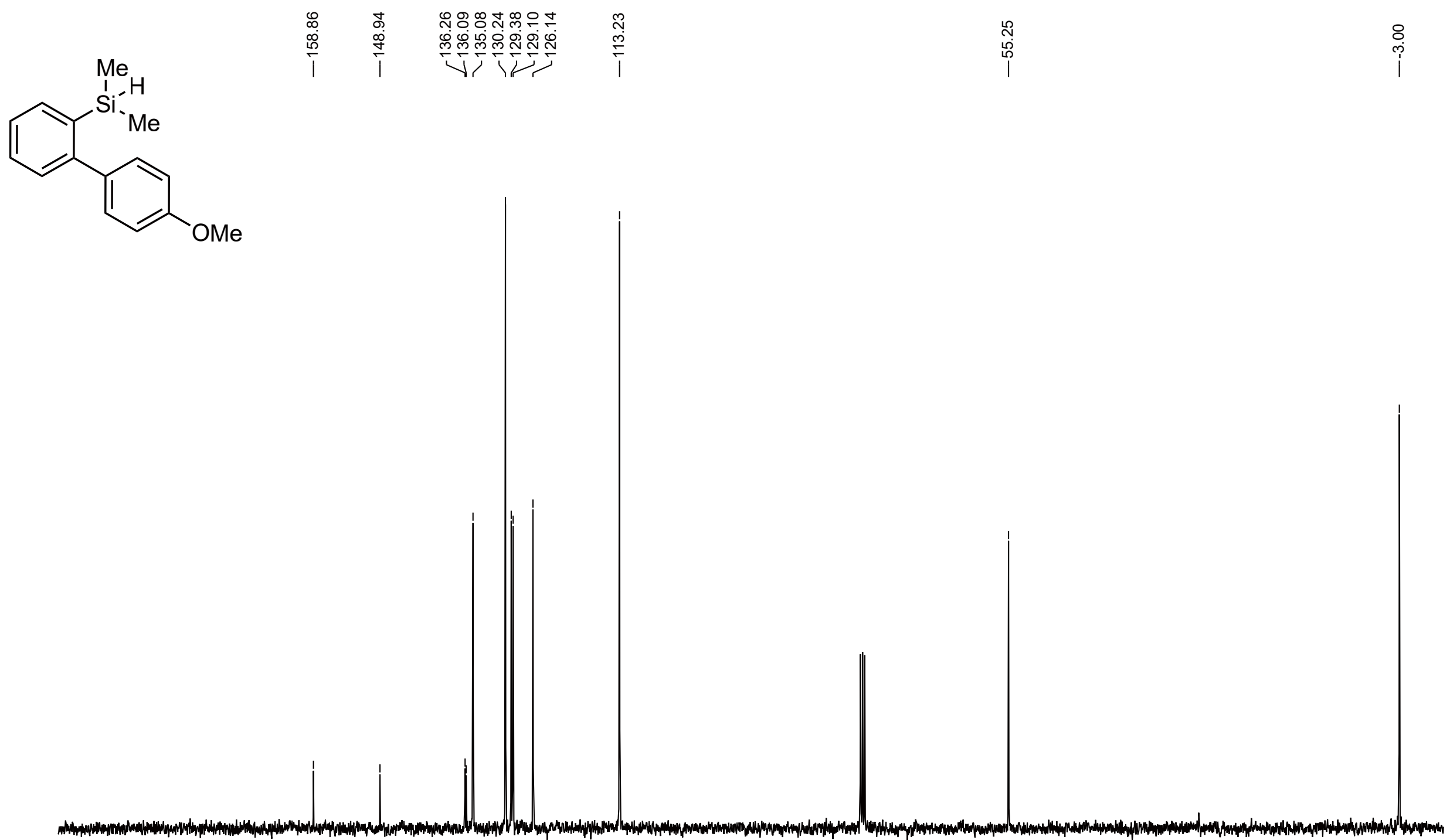




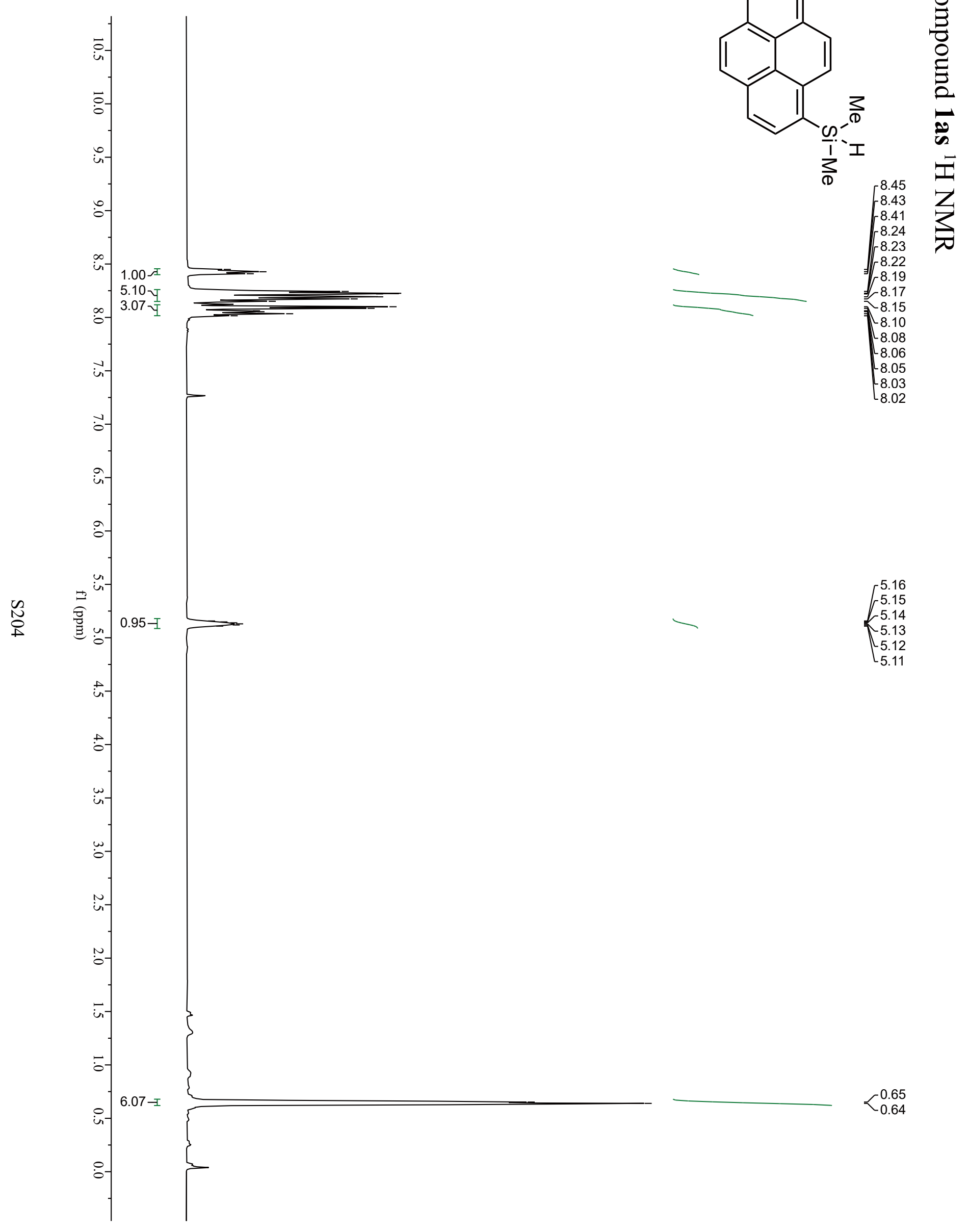




$$
E^{i}
$$




\section{Compound 1at ${ }^{1} \mathrm{H}$ NMR}

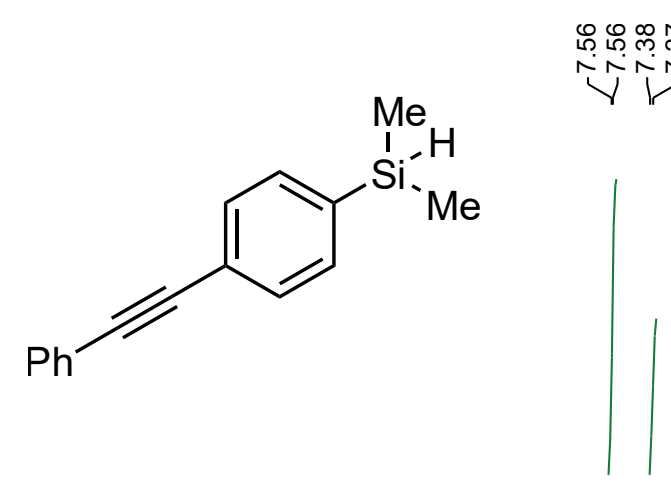

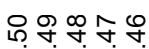

证有

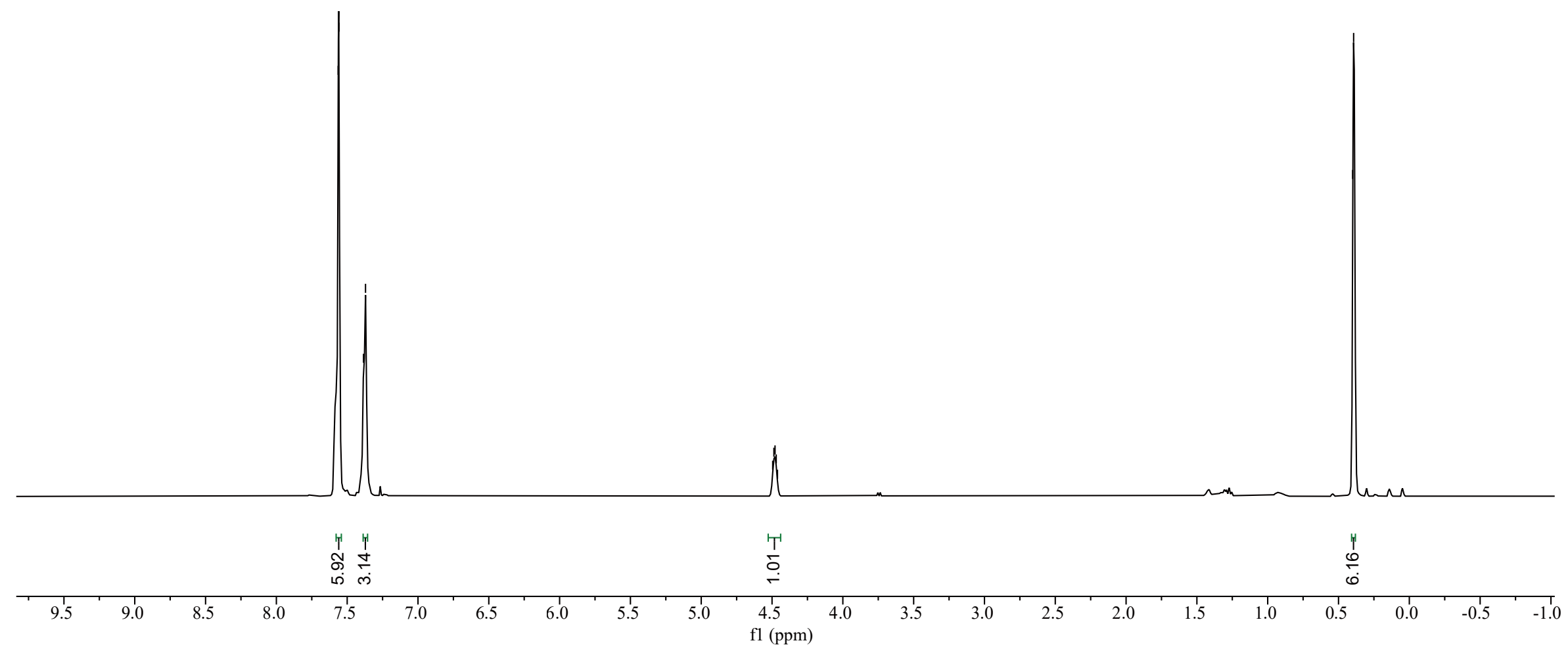


Compound 1at ${ }^{13} \mathrm{C}$ NMR

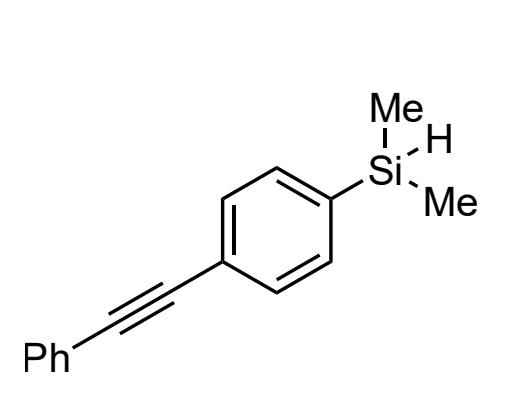

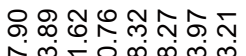

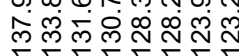

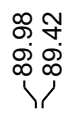

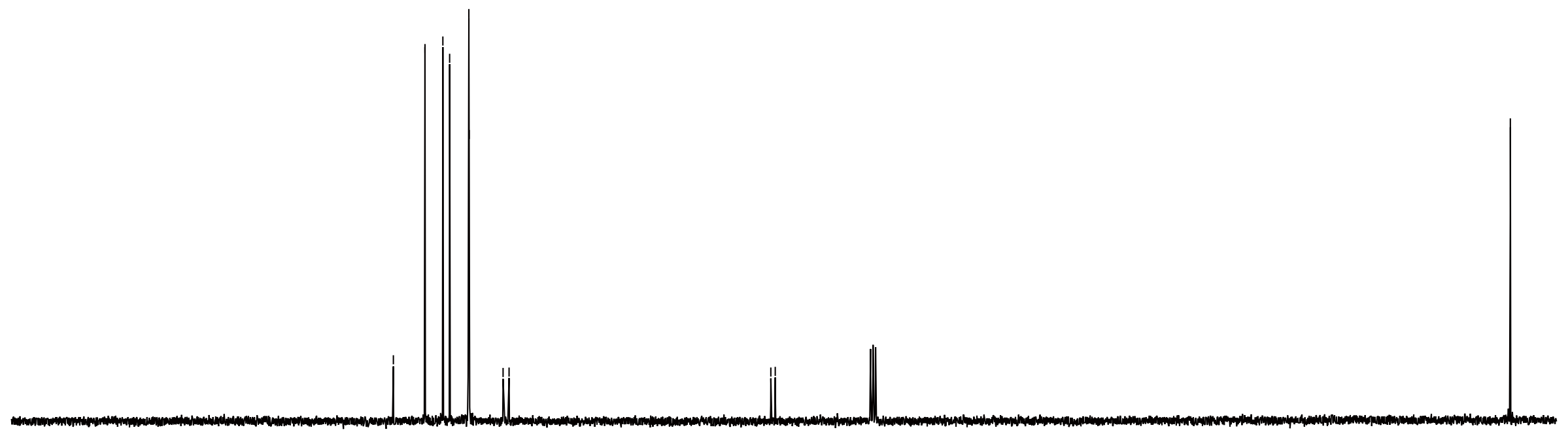


Compound 1au ${ }^{1} \mathrm{H}$ NMR

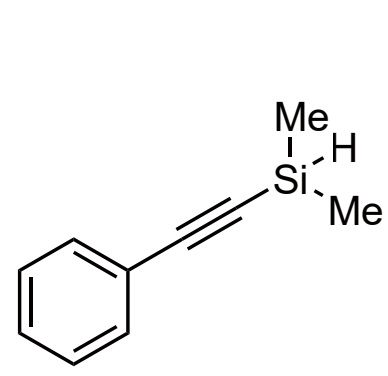

గํำ

해윰ำ

造证然

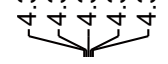

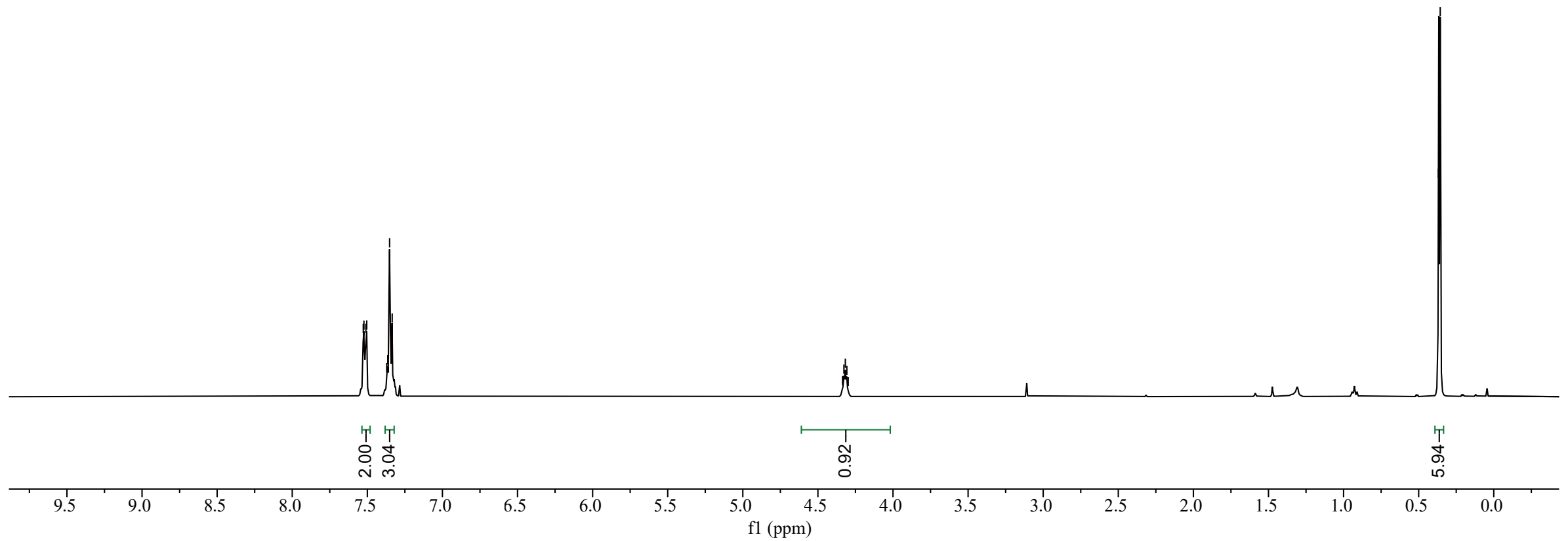


Compound 1au ${ }^{13} \mathrm{C}$ NMR

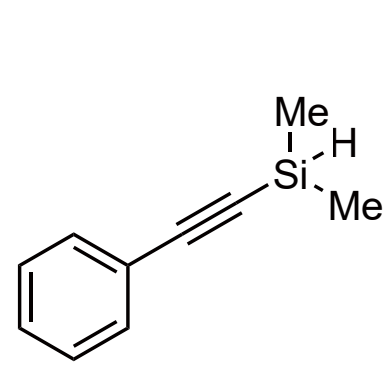

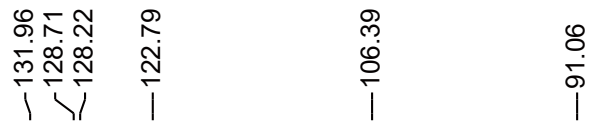

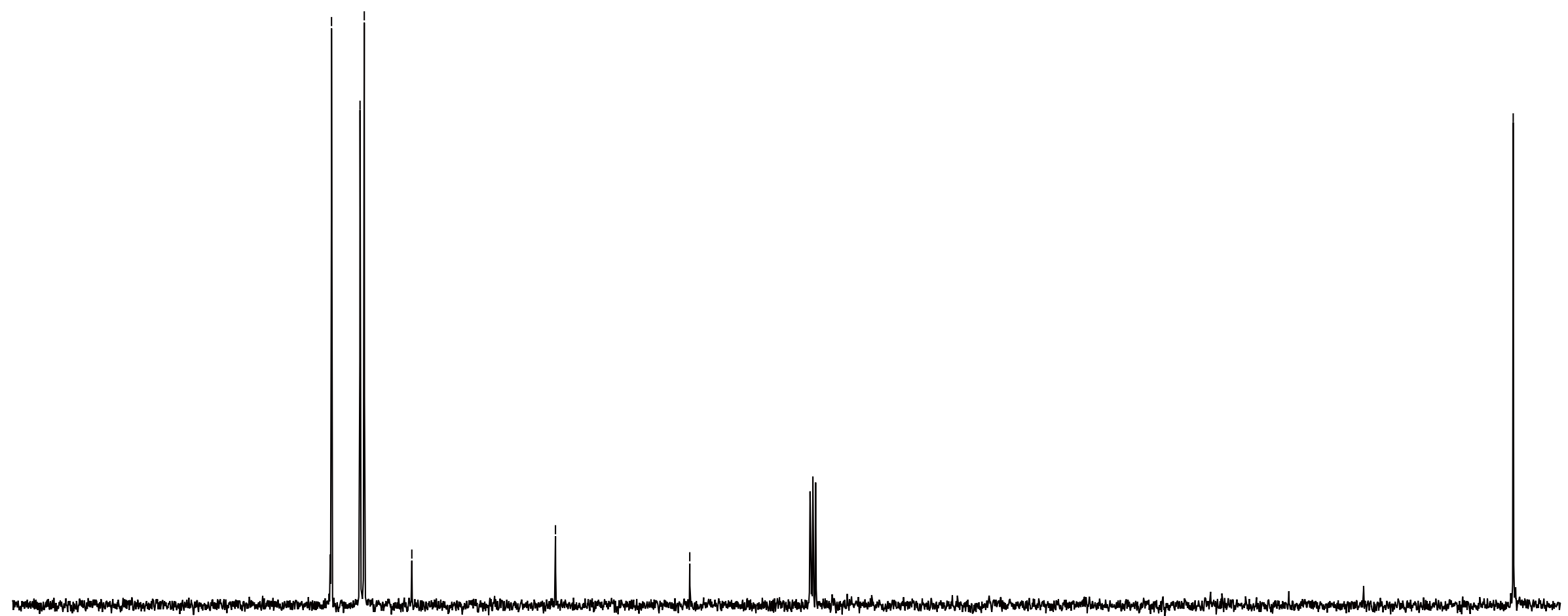




\section{Compound 1 av ${ }^{1} \mathrm{H}$ NMR}

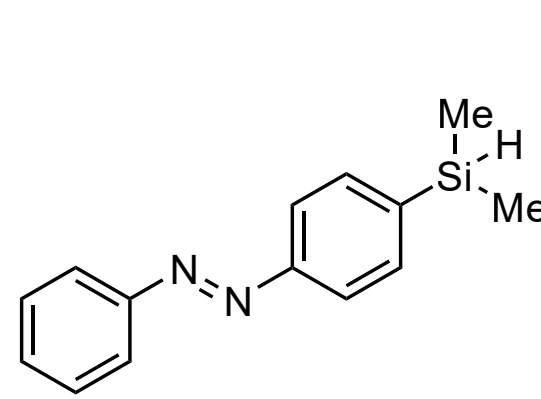

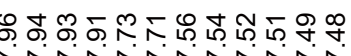

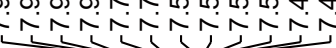

舟

$\sqrt[n]{.20}$

₹̛

$\mathrm{Me}$
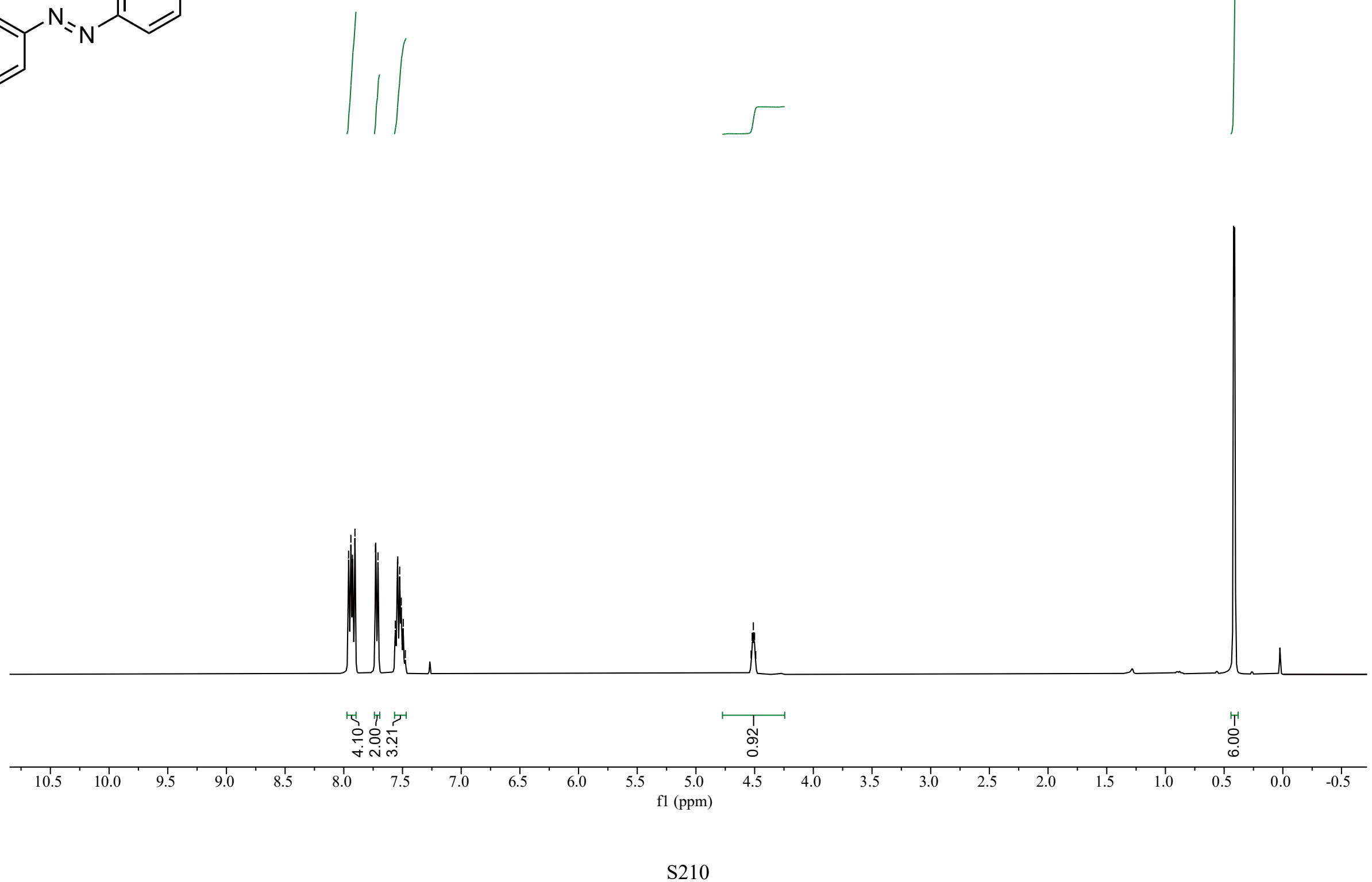
Compound 1 av ${ }^{13} \mathrm{C}$ NMR
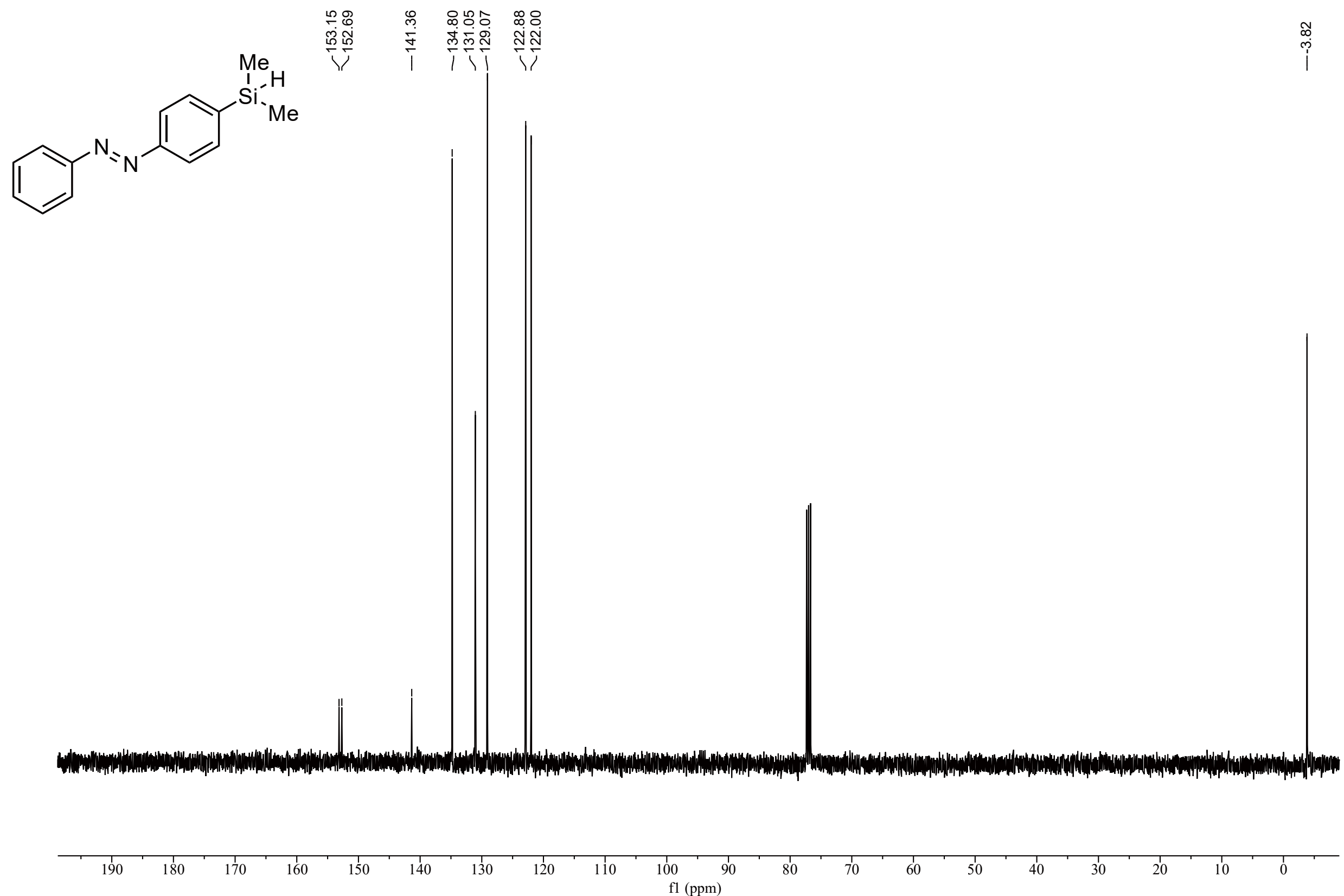
Compound 1aw ${ }^{1} \mathrm{H}$ NMR
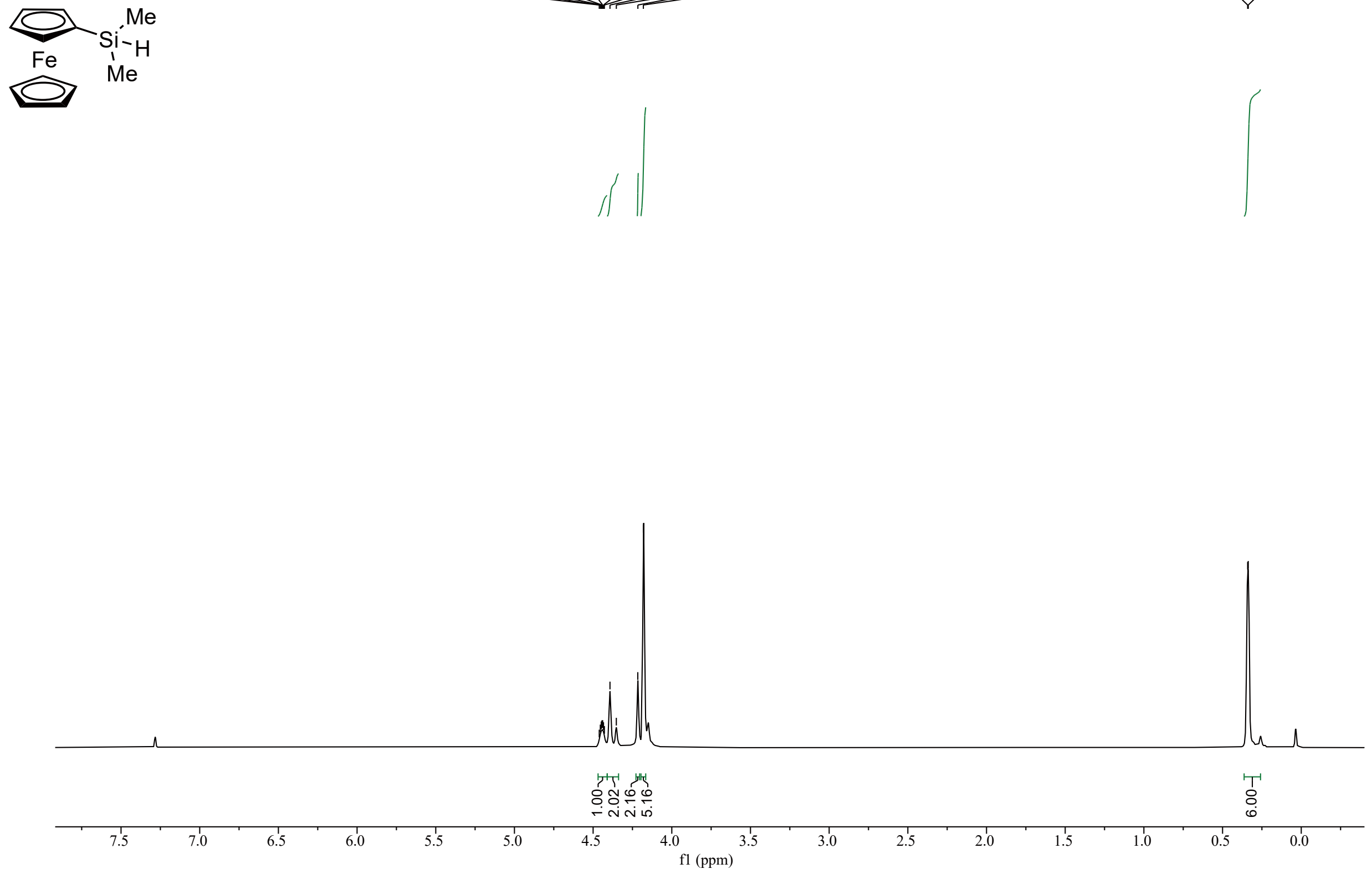
Compound 1aw ${ }^{13} \mathrm{C}$ NMR
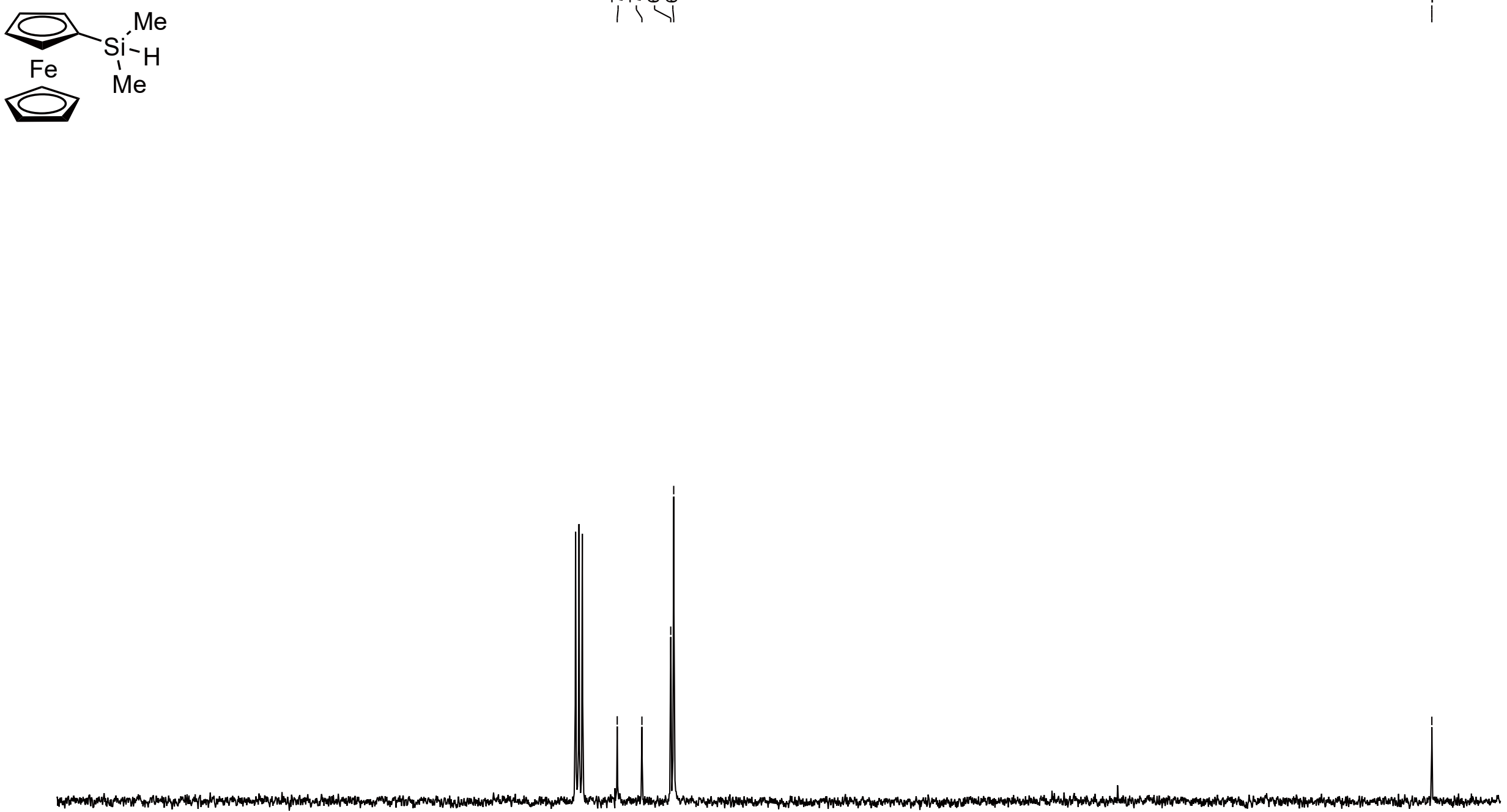


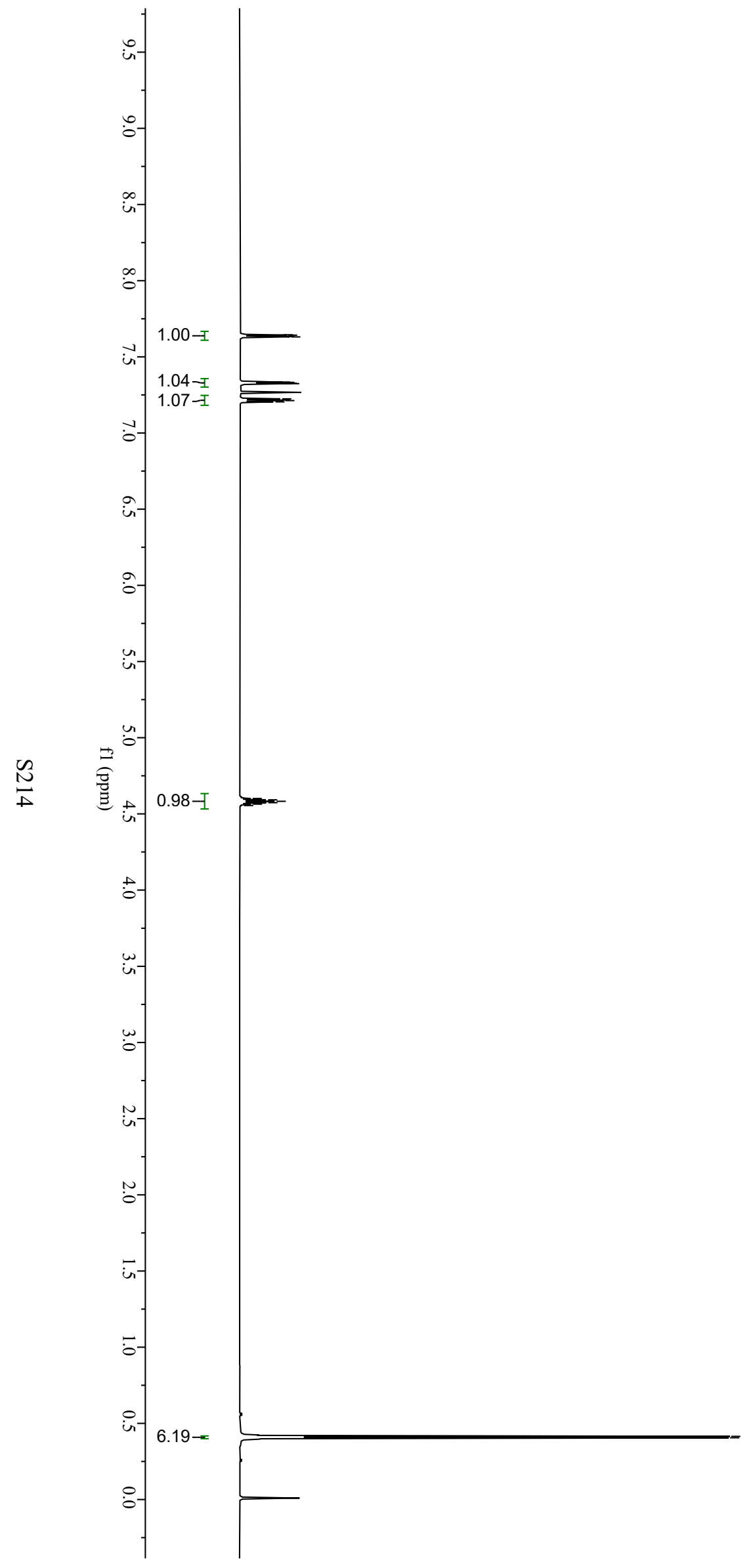


Compound 1ax ${ }^{1} \mathrm{H}$ NMR

수ㅇㅛㅛ

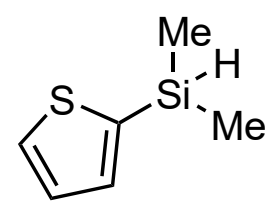

im
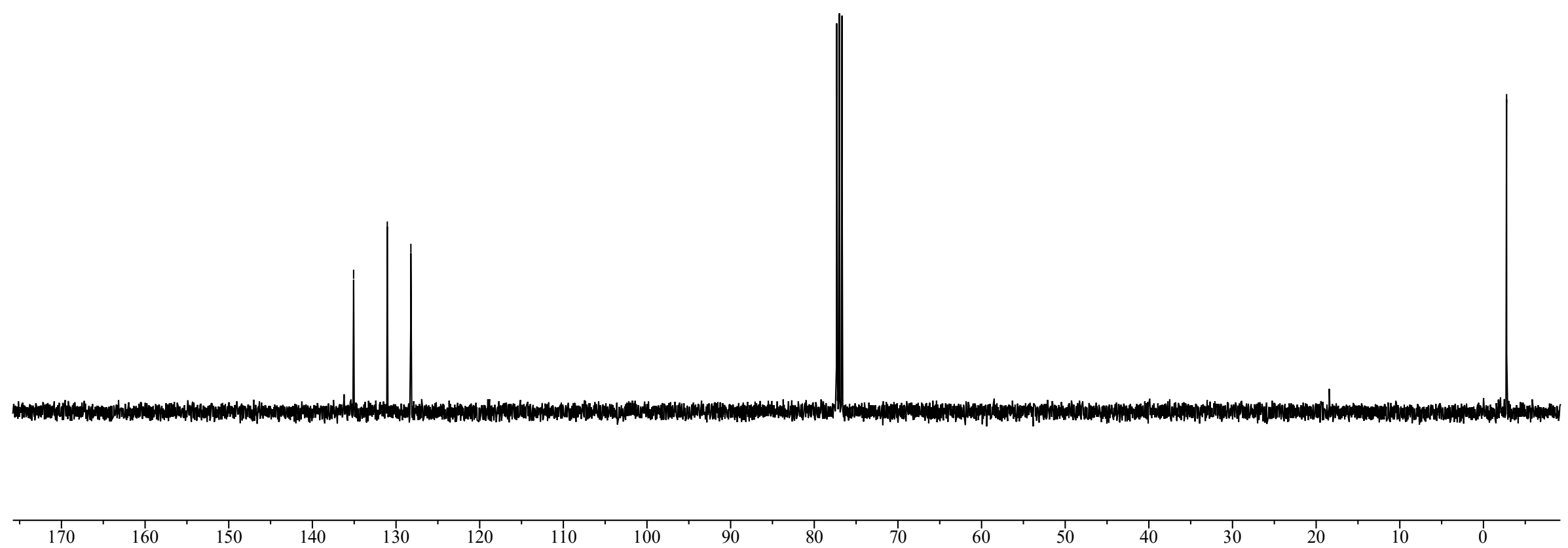
Compound 1ay ${ }^{1} \mathrm{H}$ NMR

$\overbrace{\mathrm{O}}^{\mathrm{Me}}$

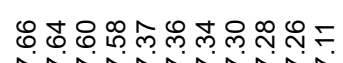

둥유융

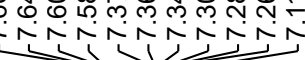

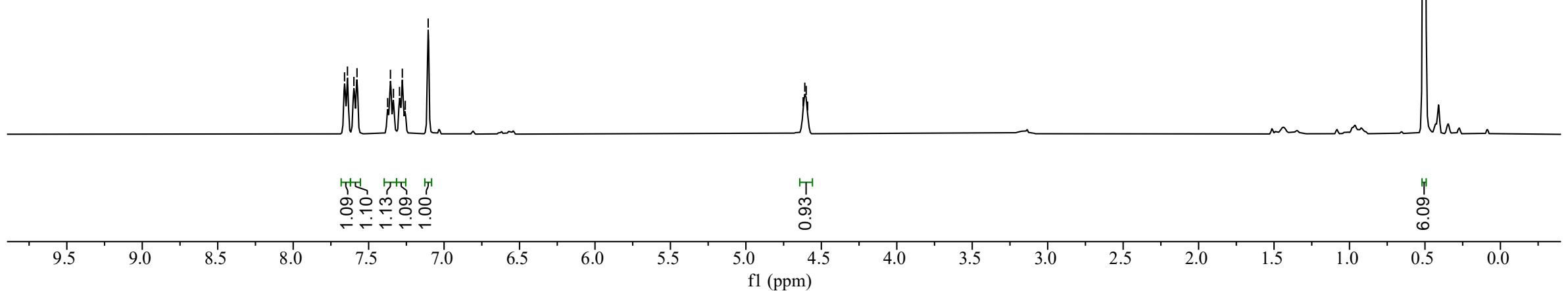


Compound 1ay ${ }^{13} \mathrm{C}$ NMR
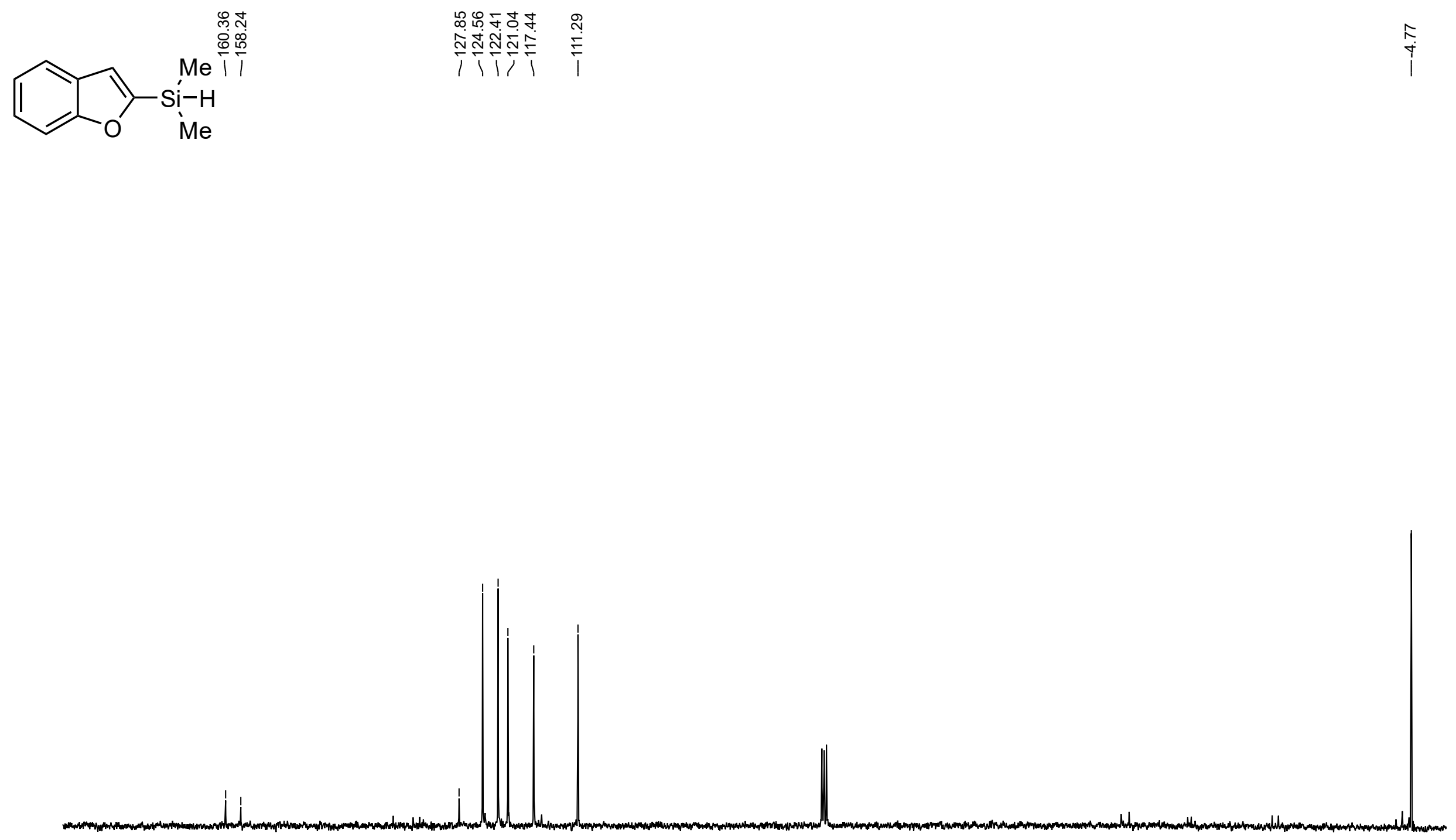


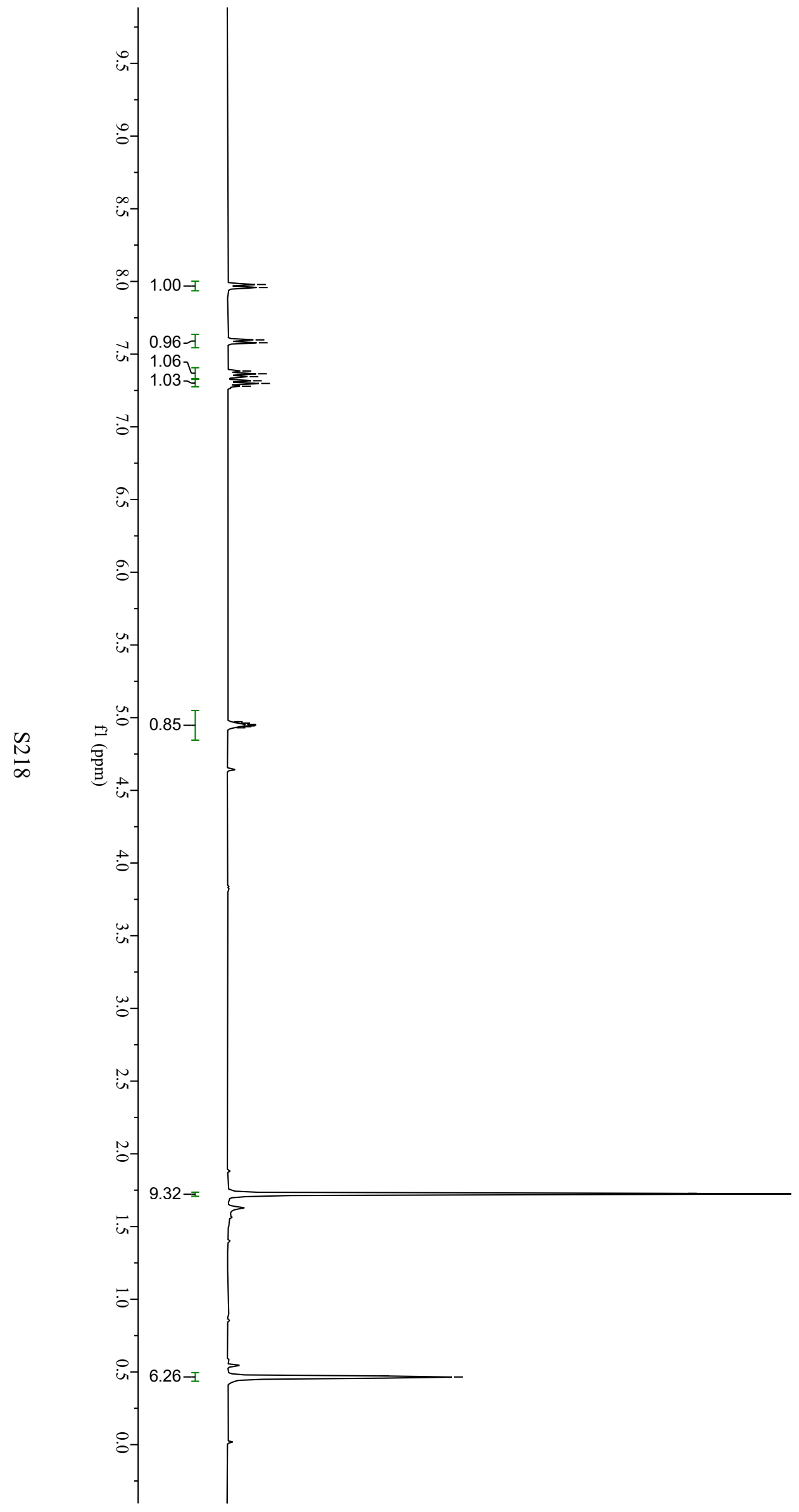


Compound $\mathbf{1 a z}{ }^{1} \mathrm{H}$ NMR<smiles>CC(=O)OC(=O)n1c([Si](C)(C)C)c(Br)c2ccccc21</smiles>

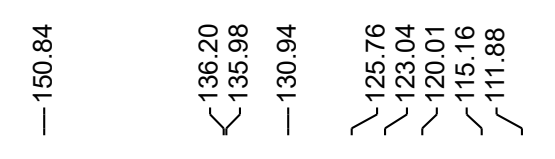

$\underset{\substack{1 \\ 0 \\ 0 \\ 0}}{9}$

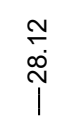

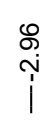
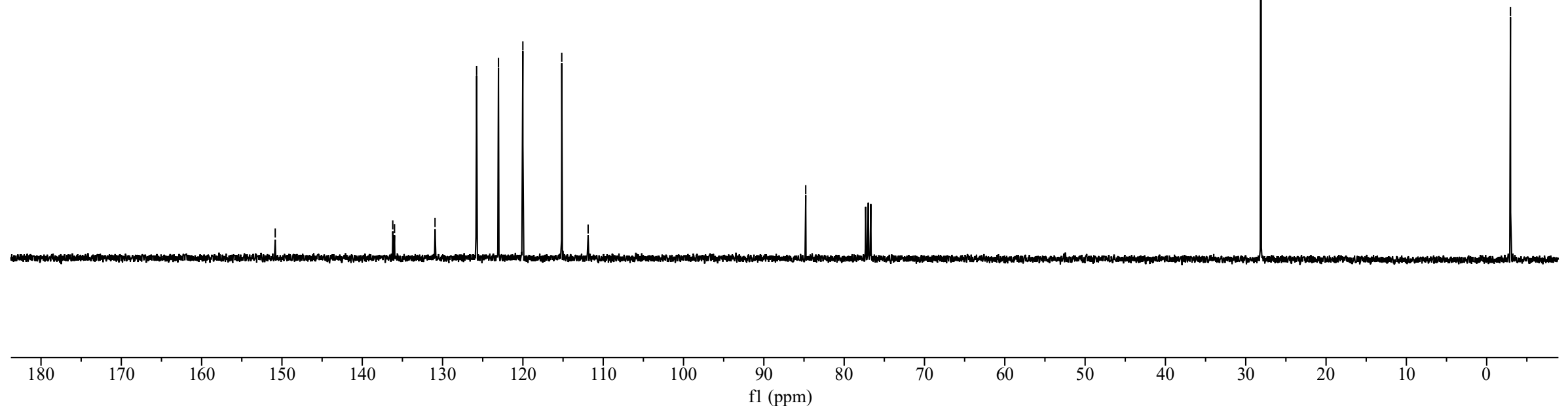

S219 

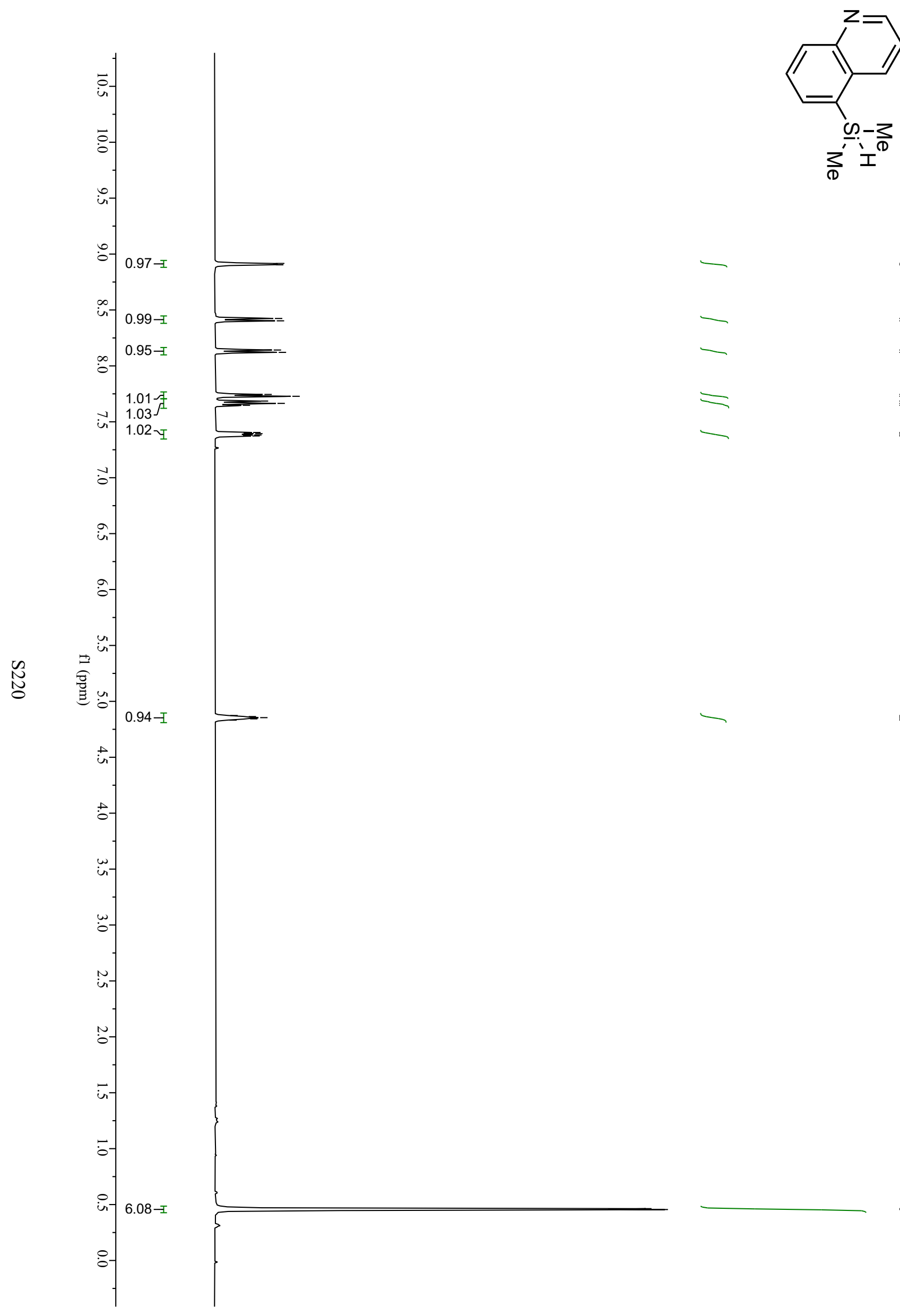

高

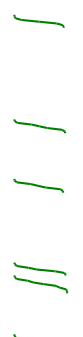

$\prec \begin{array}{r}8.92 \\ 8.90\end{array}$

吉

$-8.42$

8.40

$<_{8.12}^{8.14}$

7.74

$-7.73$

7.69

7.66

$\mathfrak{7 . 6 5}$

7.40

$-7.39$

7.38
7.37

4.87

$r^{4.86}$

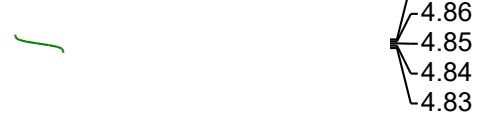

4.84
4.83 
Compound 1 ba ${ }^{13} \mathrm{C}$ NMR

20

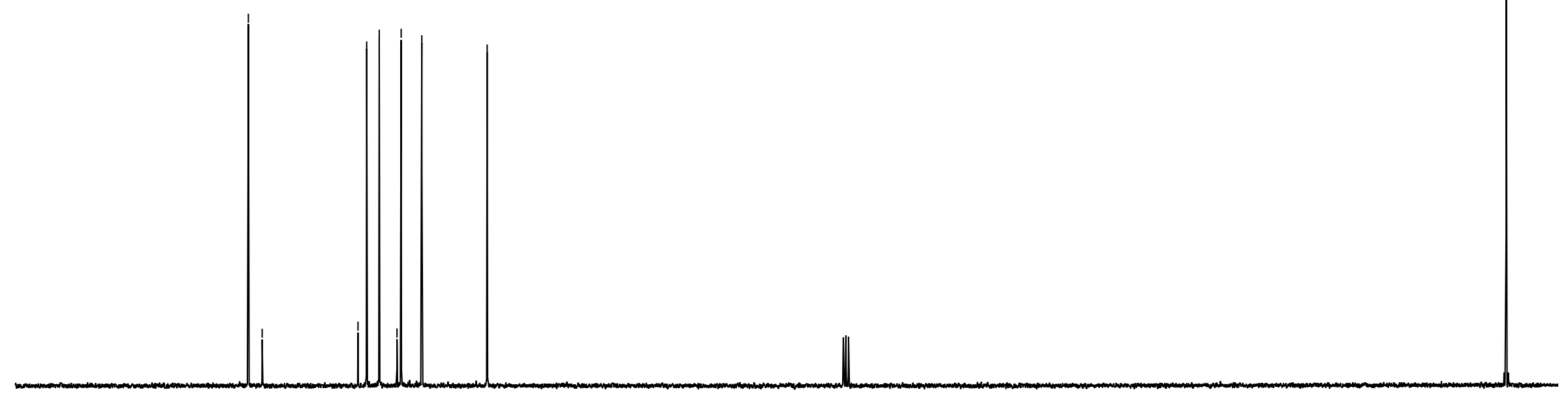




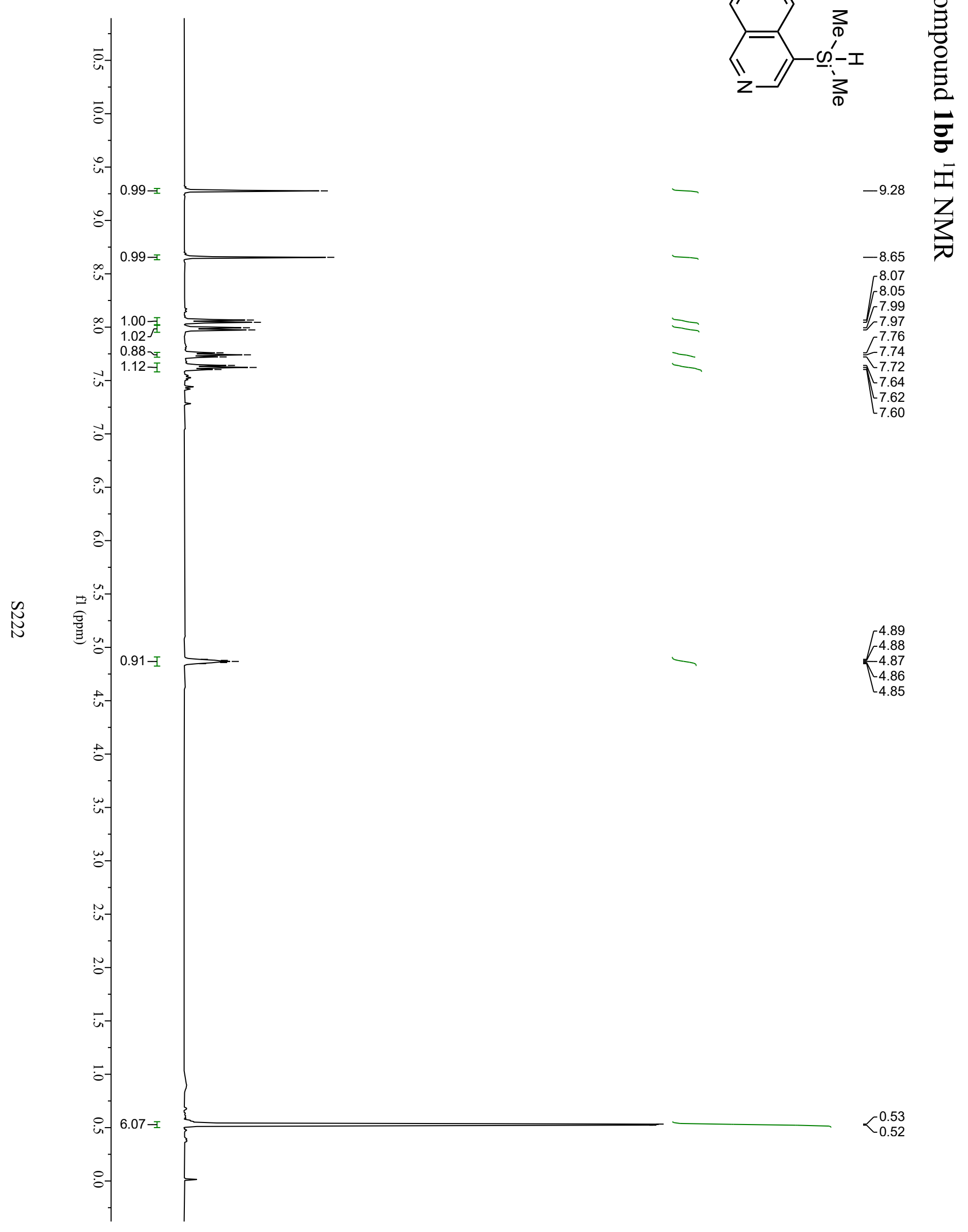


Compound $\mathbf{1 b b}{ }^{13} \mathrm{C}$ NMR
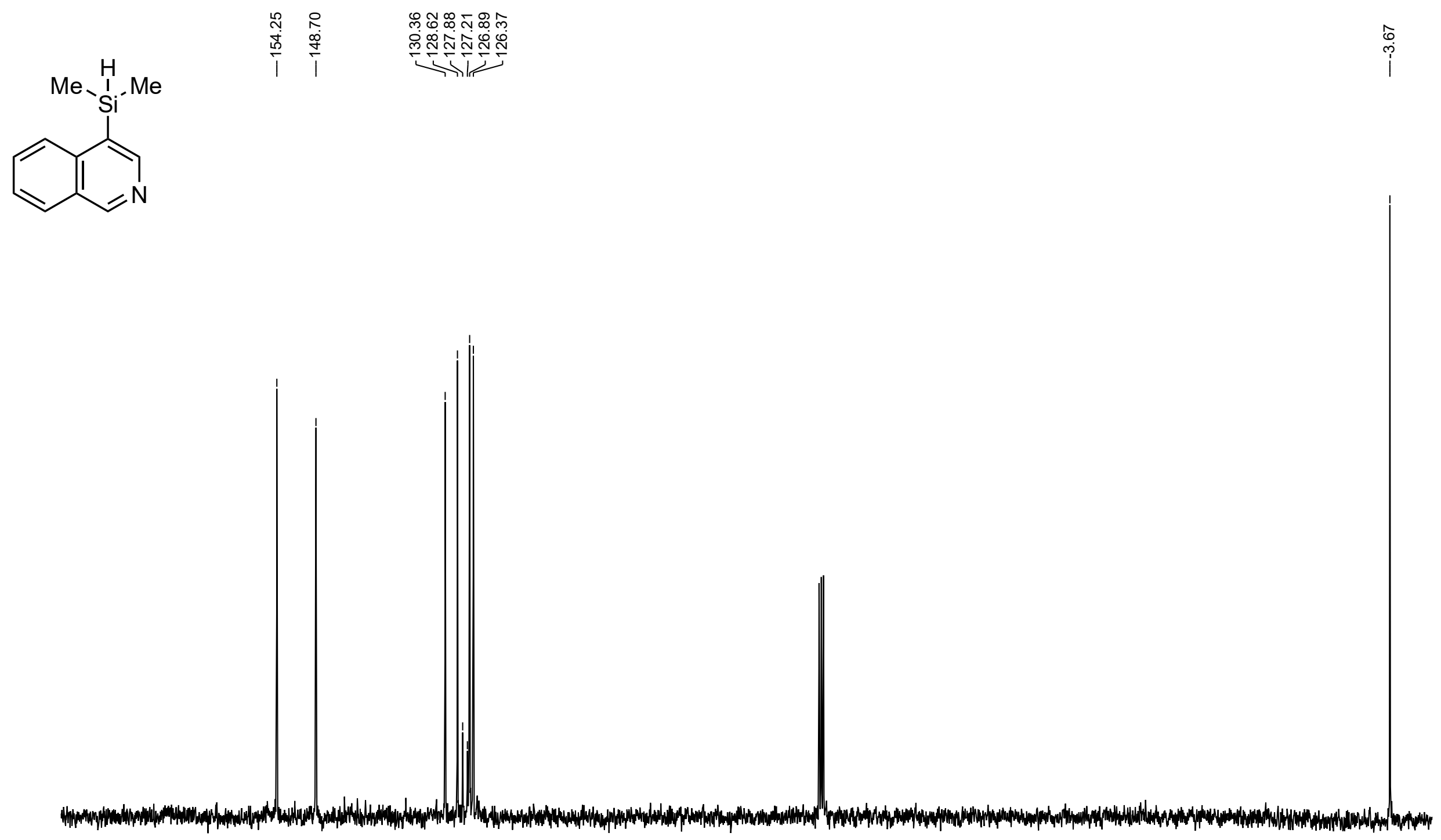

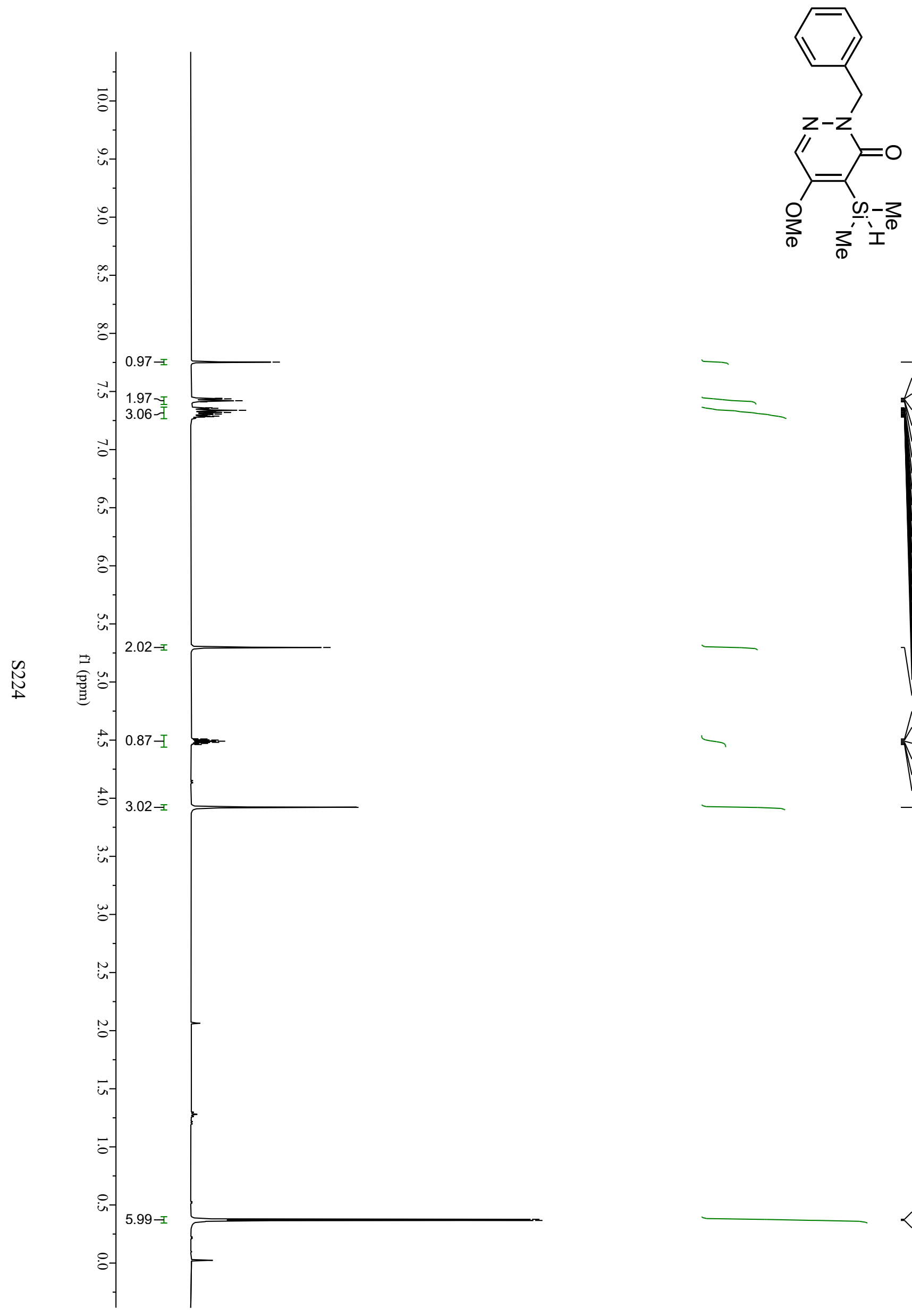

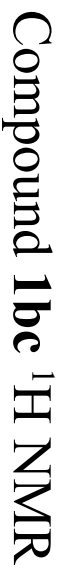

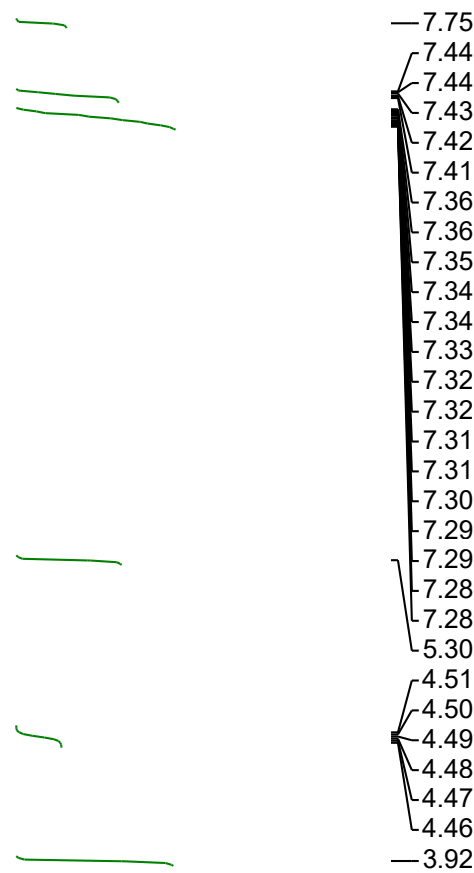

$<_{0.37}^{0.38}$ 
Compound 1 be ${ }^{13} \mathrm{C}$ NMR
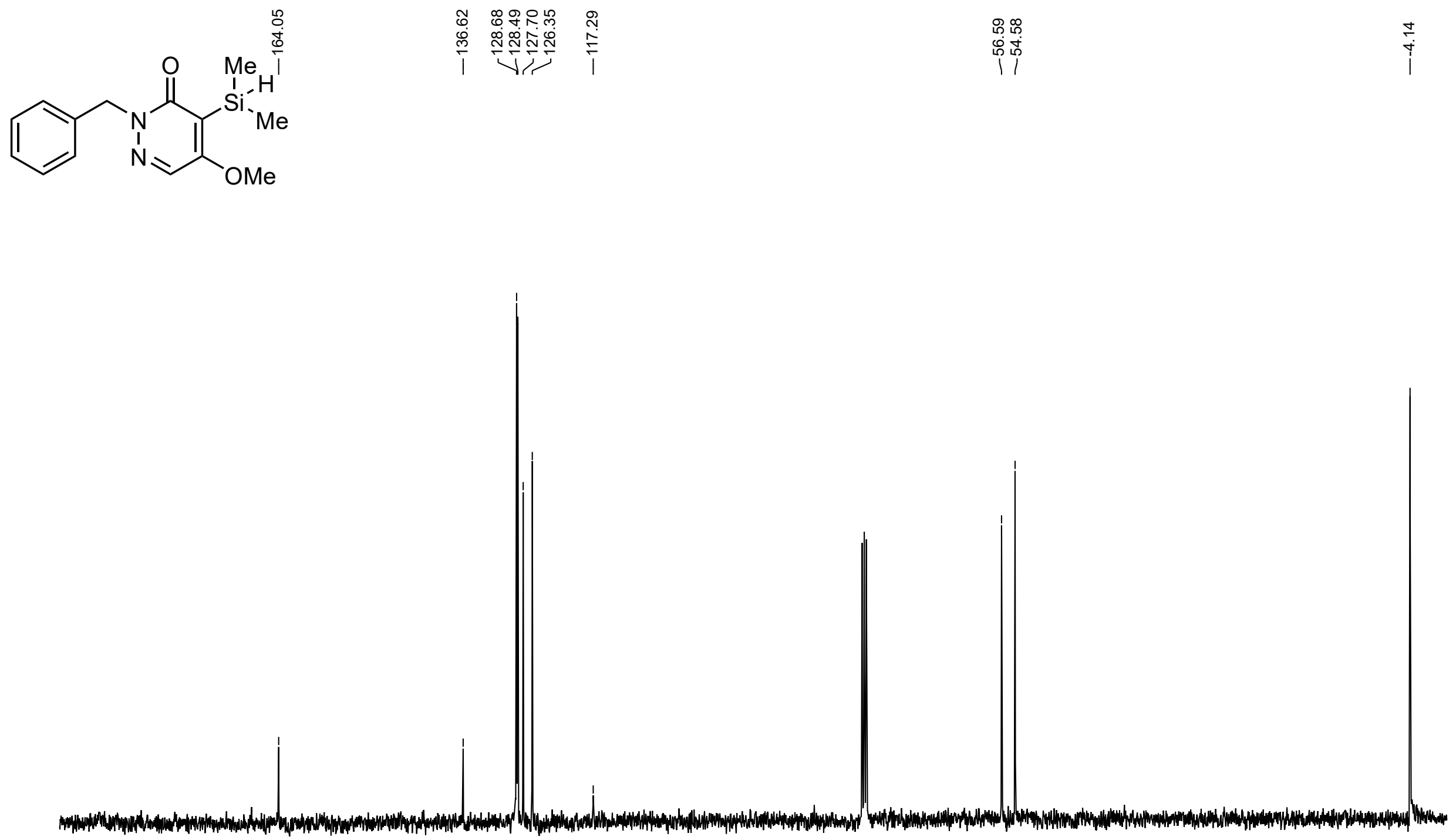


\section{Compound 1 bd ${ }^{1} \mathrm{H}$ NMR}
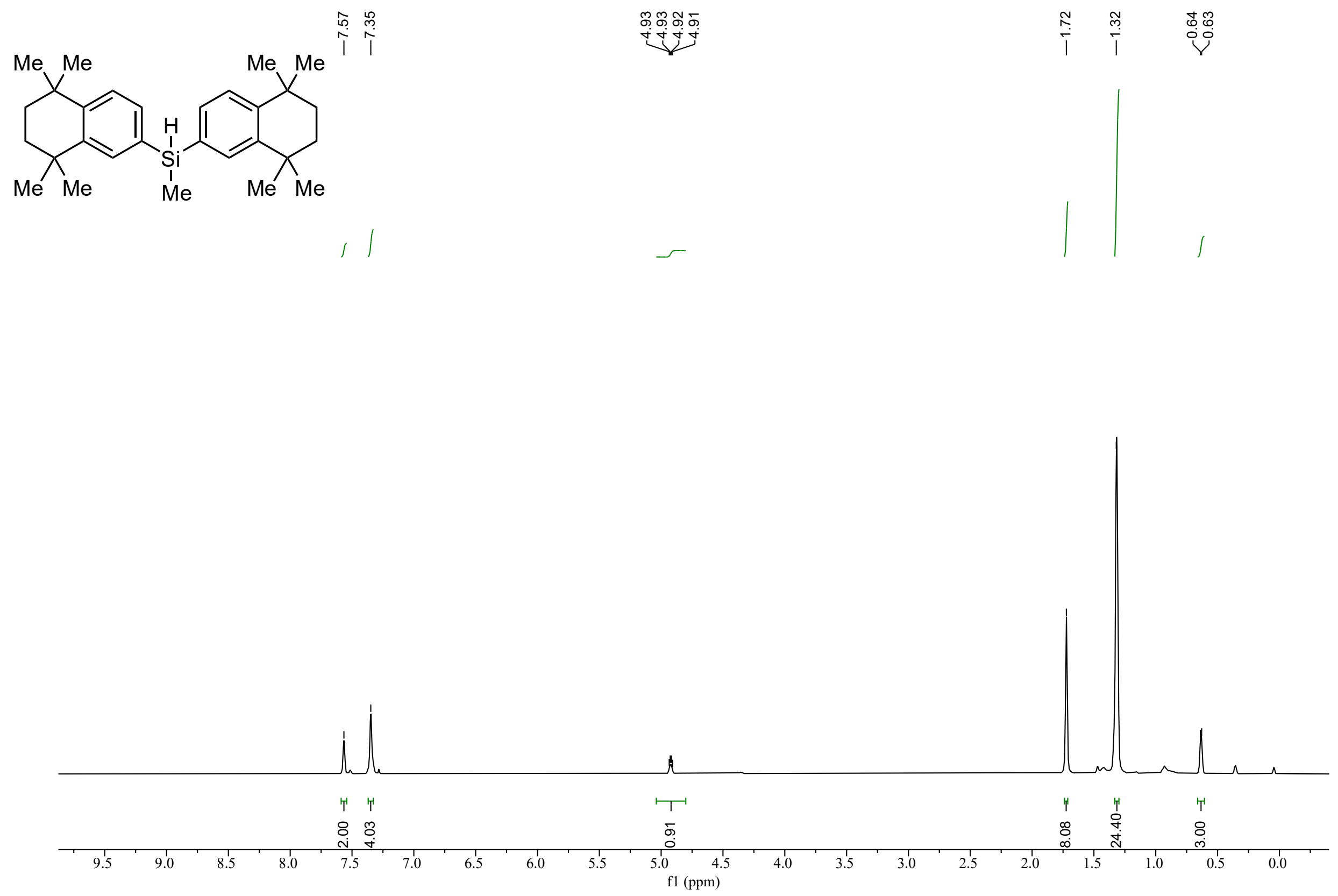
Compound $\mathbf{1 b d}{ }^{13} \mathrm{C}$ NMR
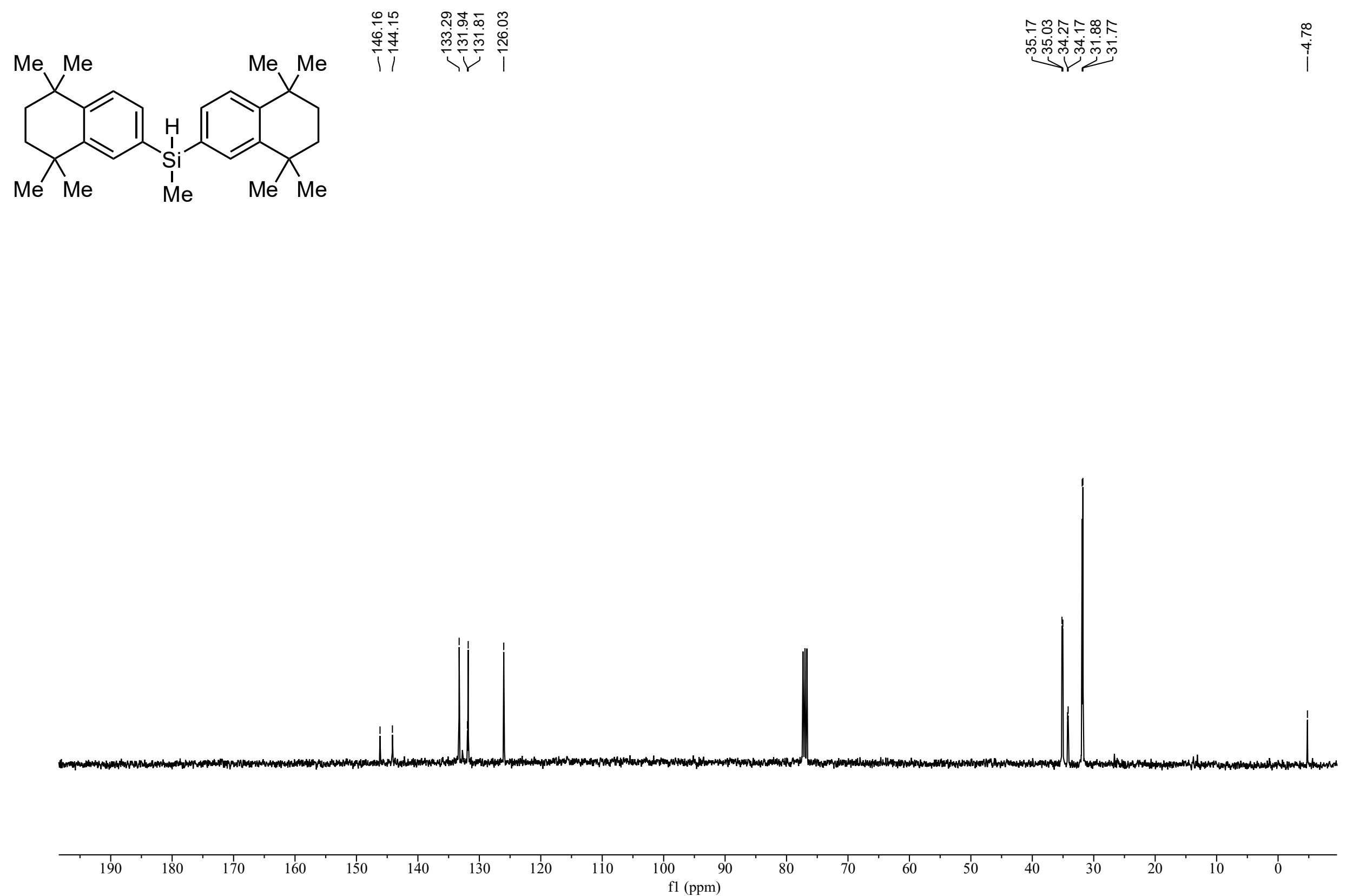

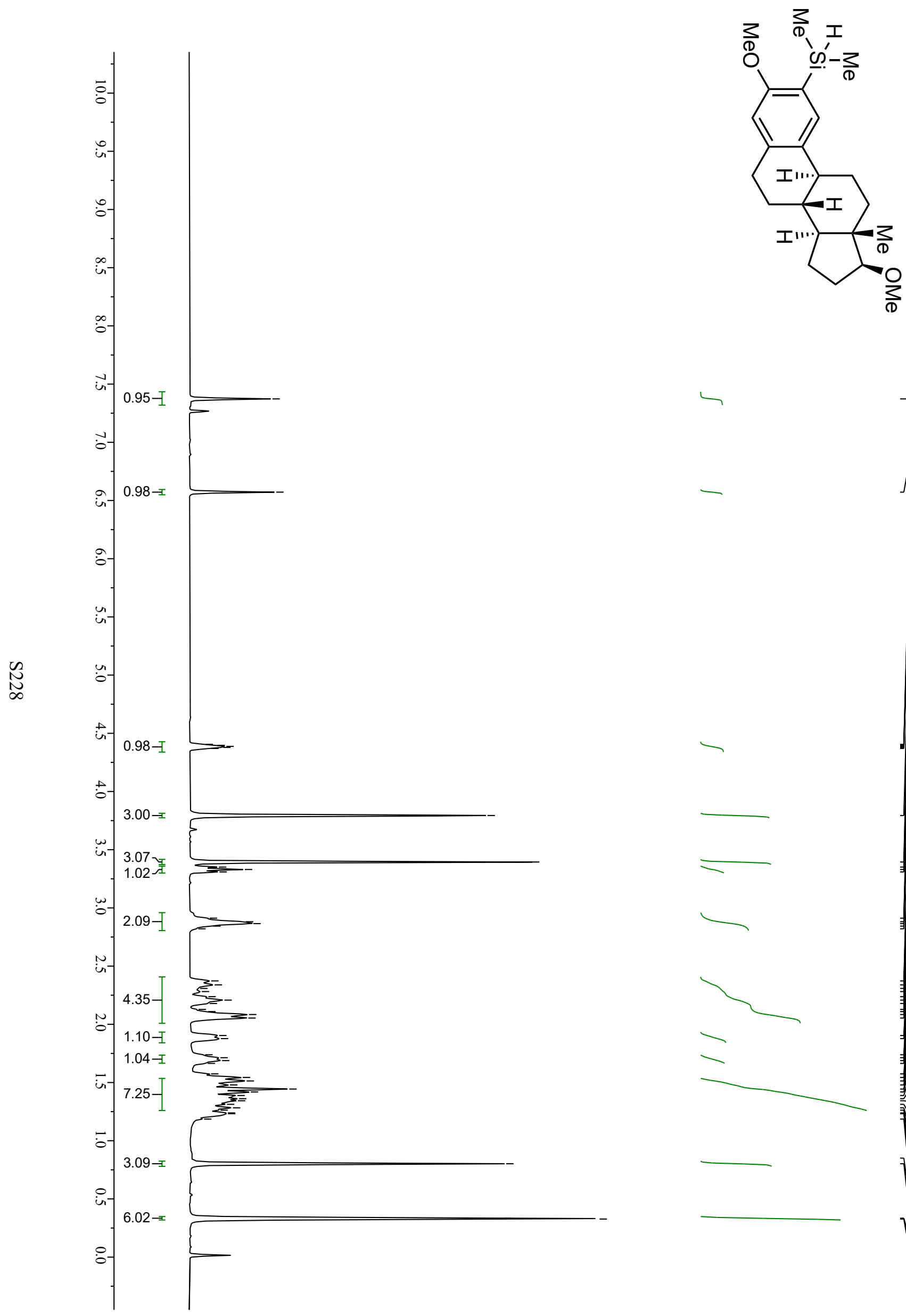

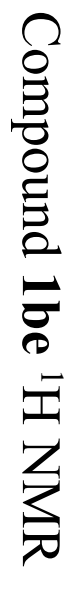

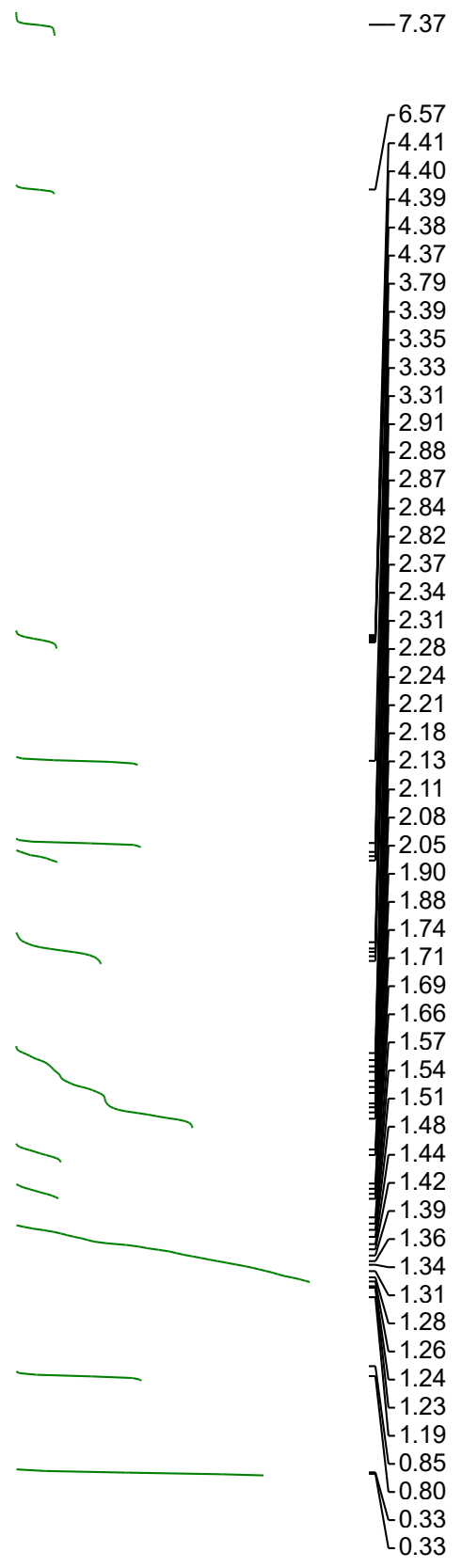



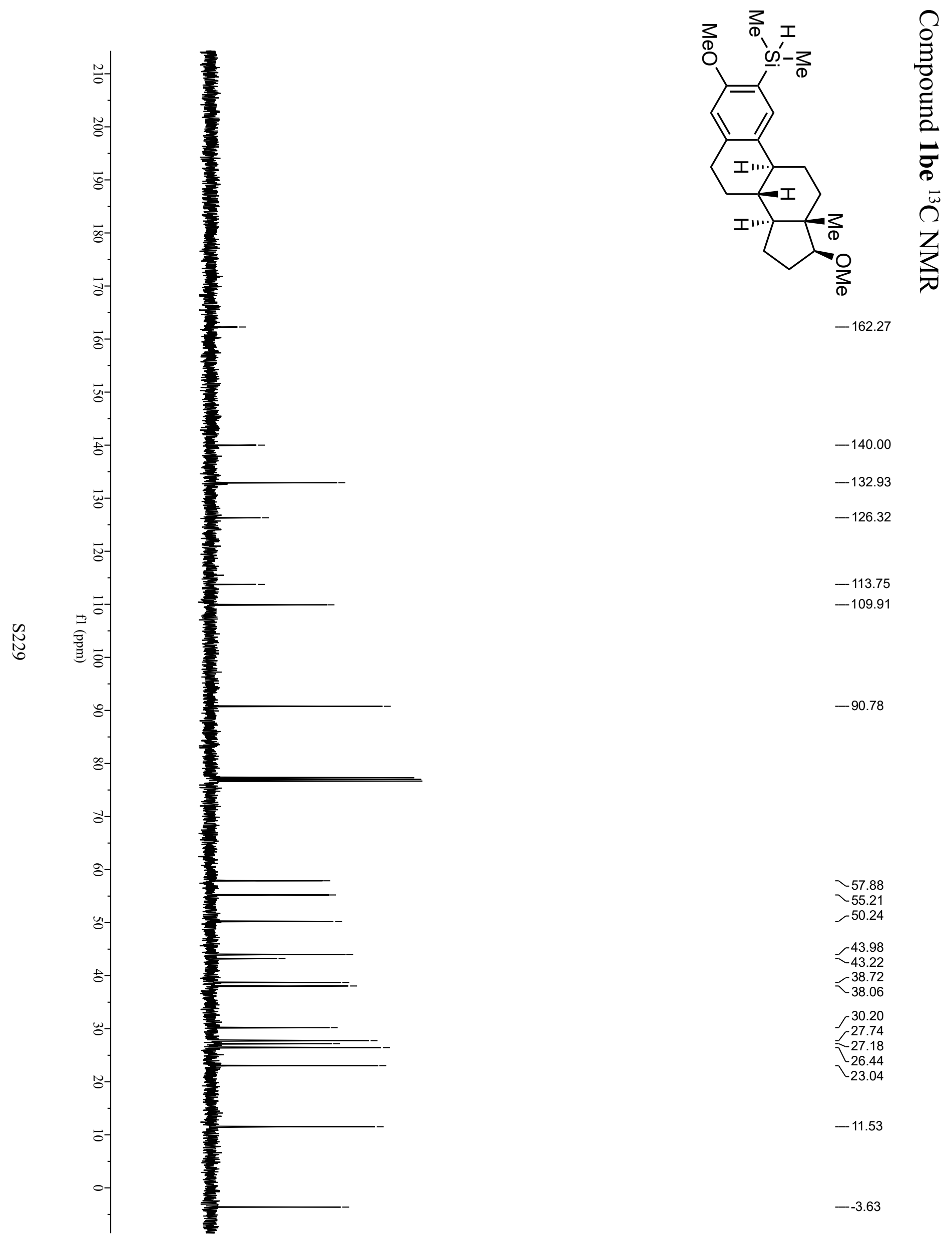

$-162.27$

$-140.00$

$-132.93$

$-126.32$

$-113.75$

$-109.91$

$-90.78$

$\sim 57.88$

$\backslash 55.21$

$-50.24$

43.98

$-43.22$

38.72

$\checkmark 38.06$

30.20

27.74

27.18

26.44

\23.04

$-11.53$

$--3.63$ 

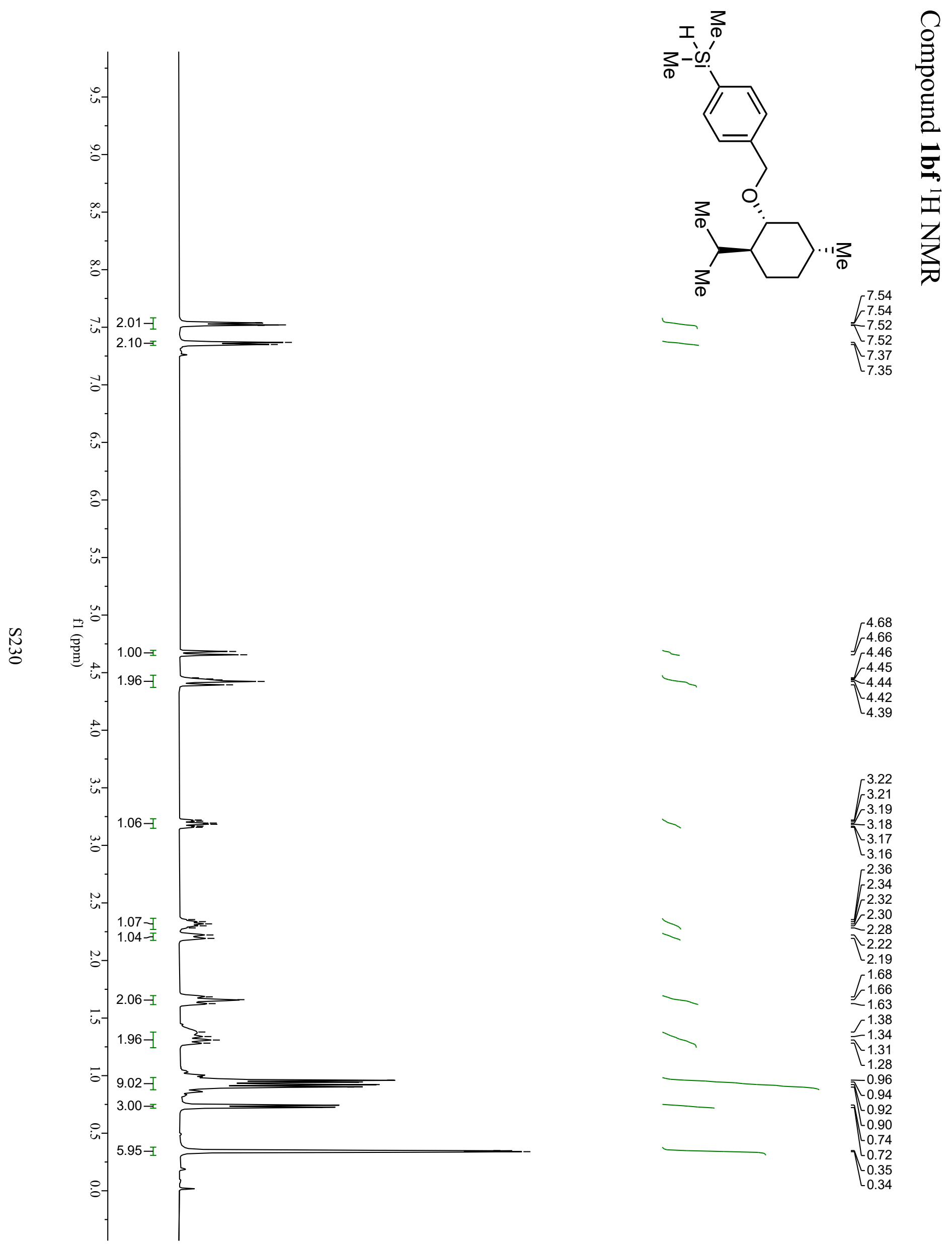
Compound $\mathbf{1 b f}{ }^{13} \mathrm{C}$ NMR

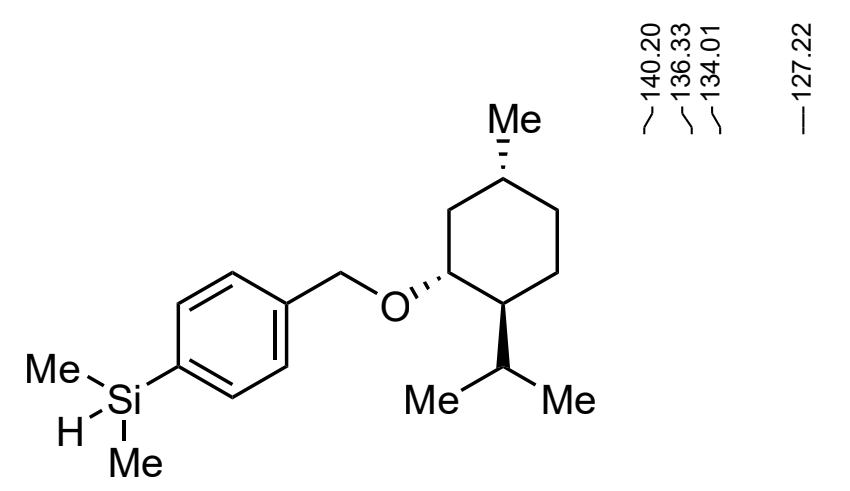

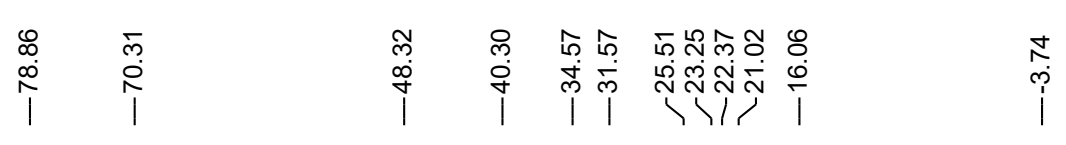

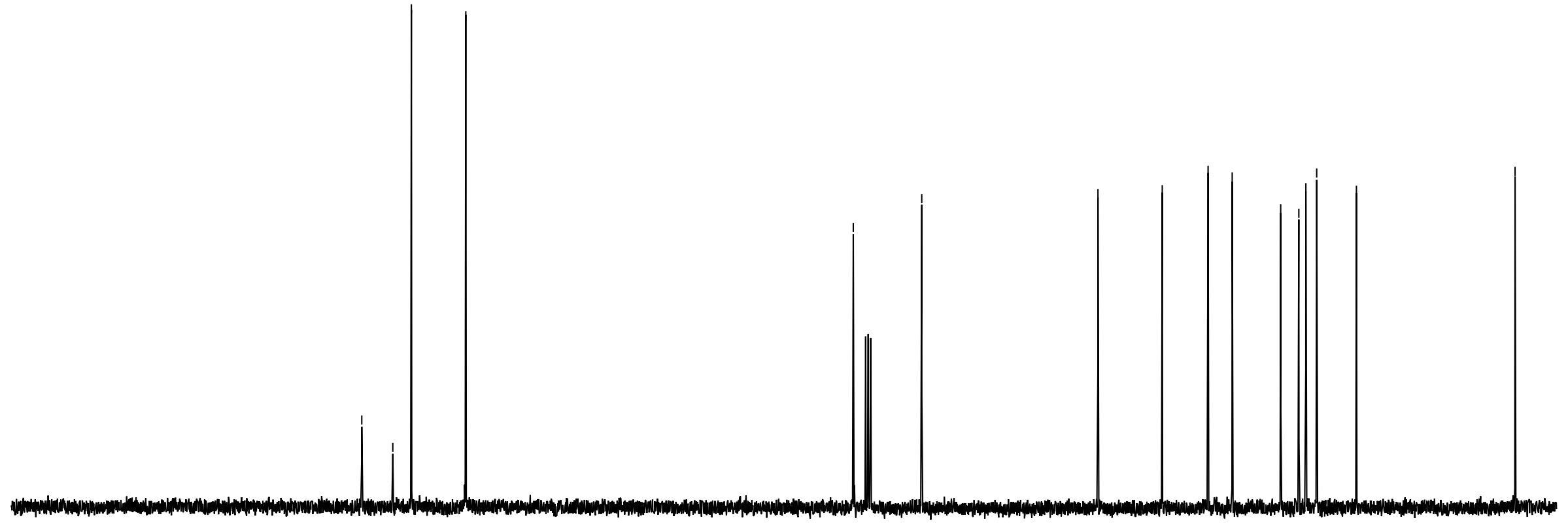




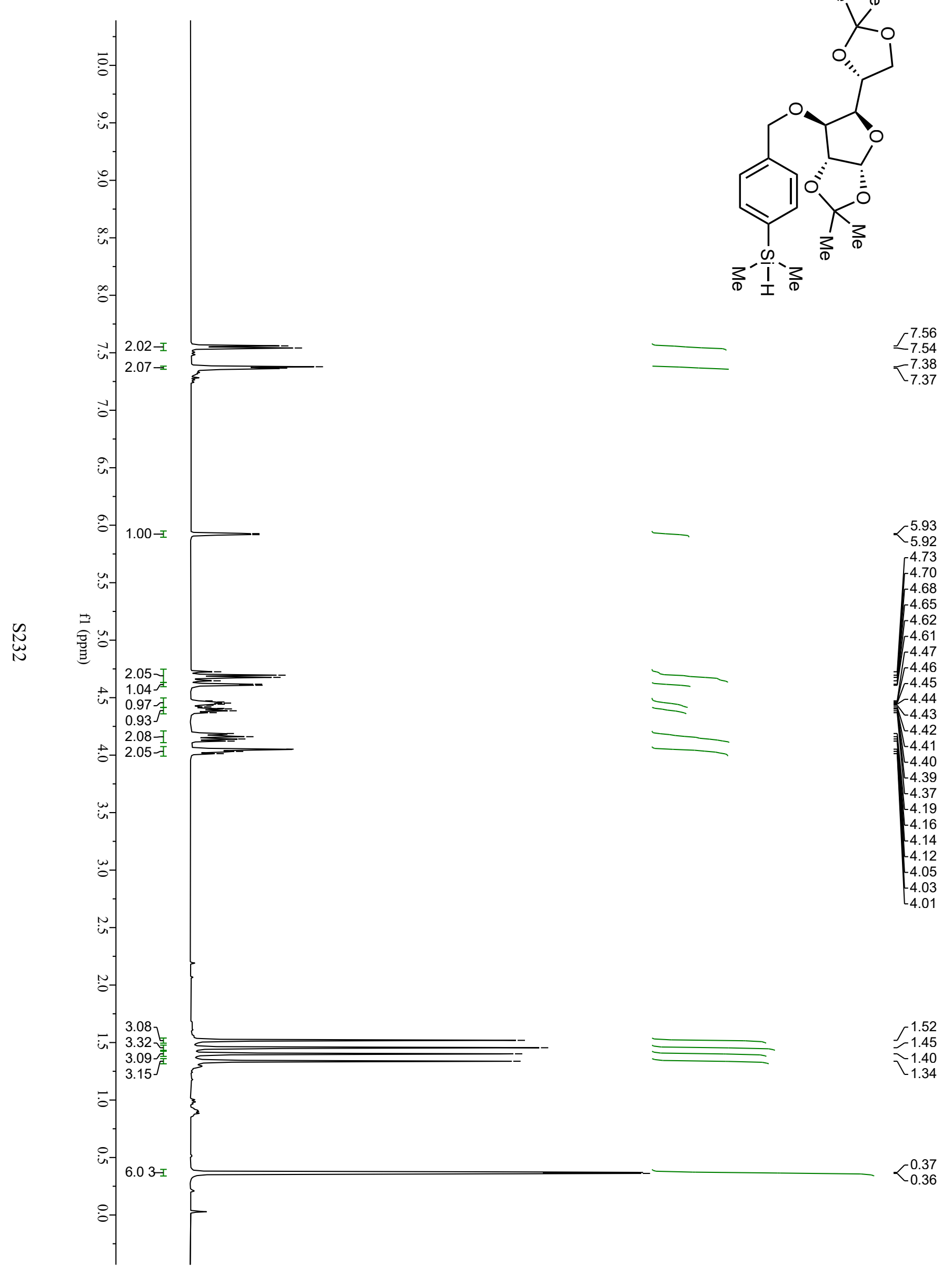


Compound 1 bg ${ }^{13} \mathrm{C}$ NMR

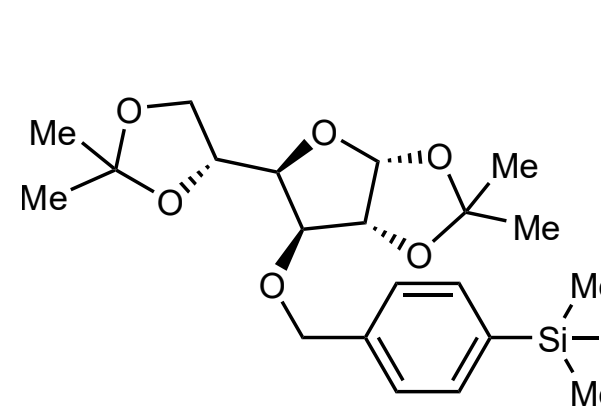

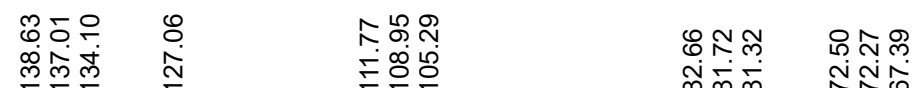

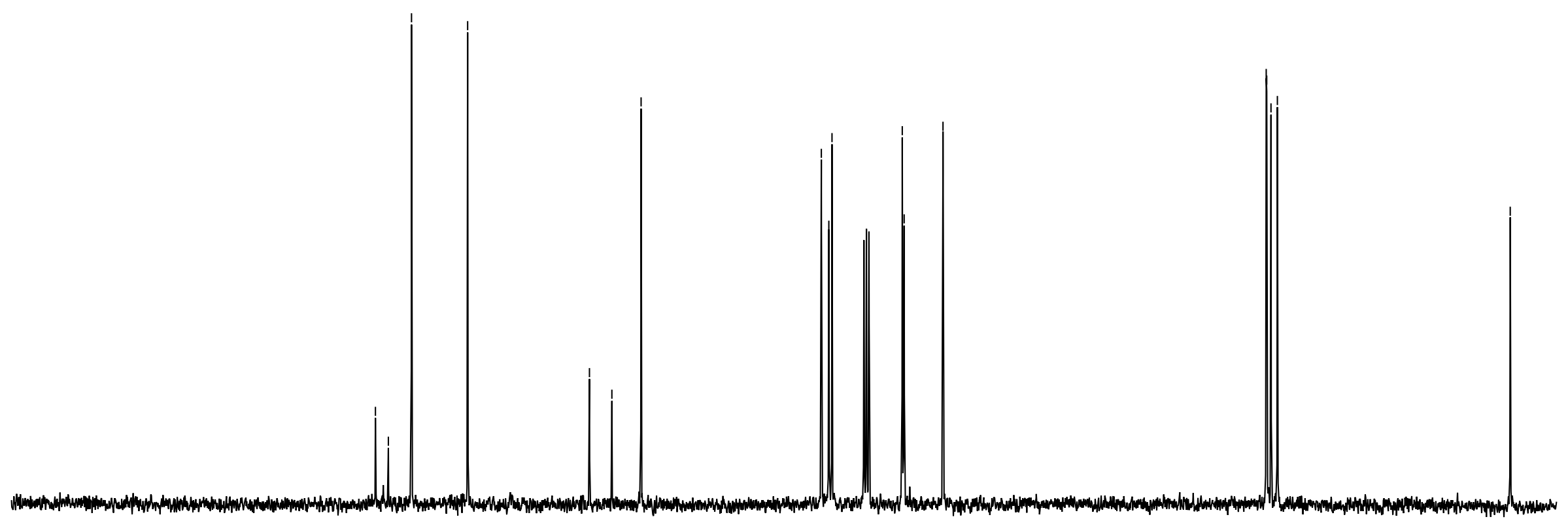



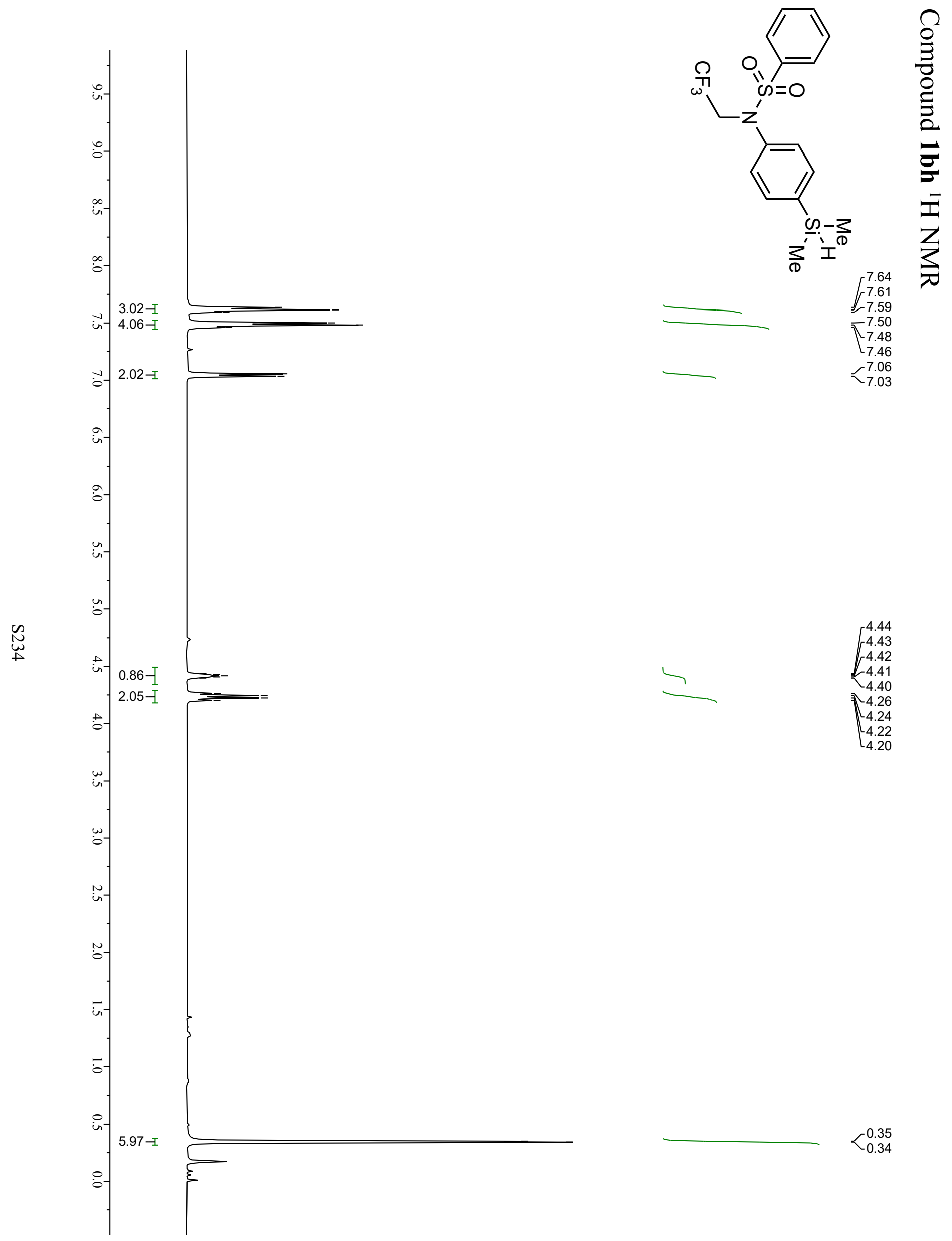

$<_{0.34}^{0.35}$ 

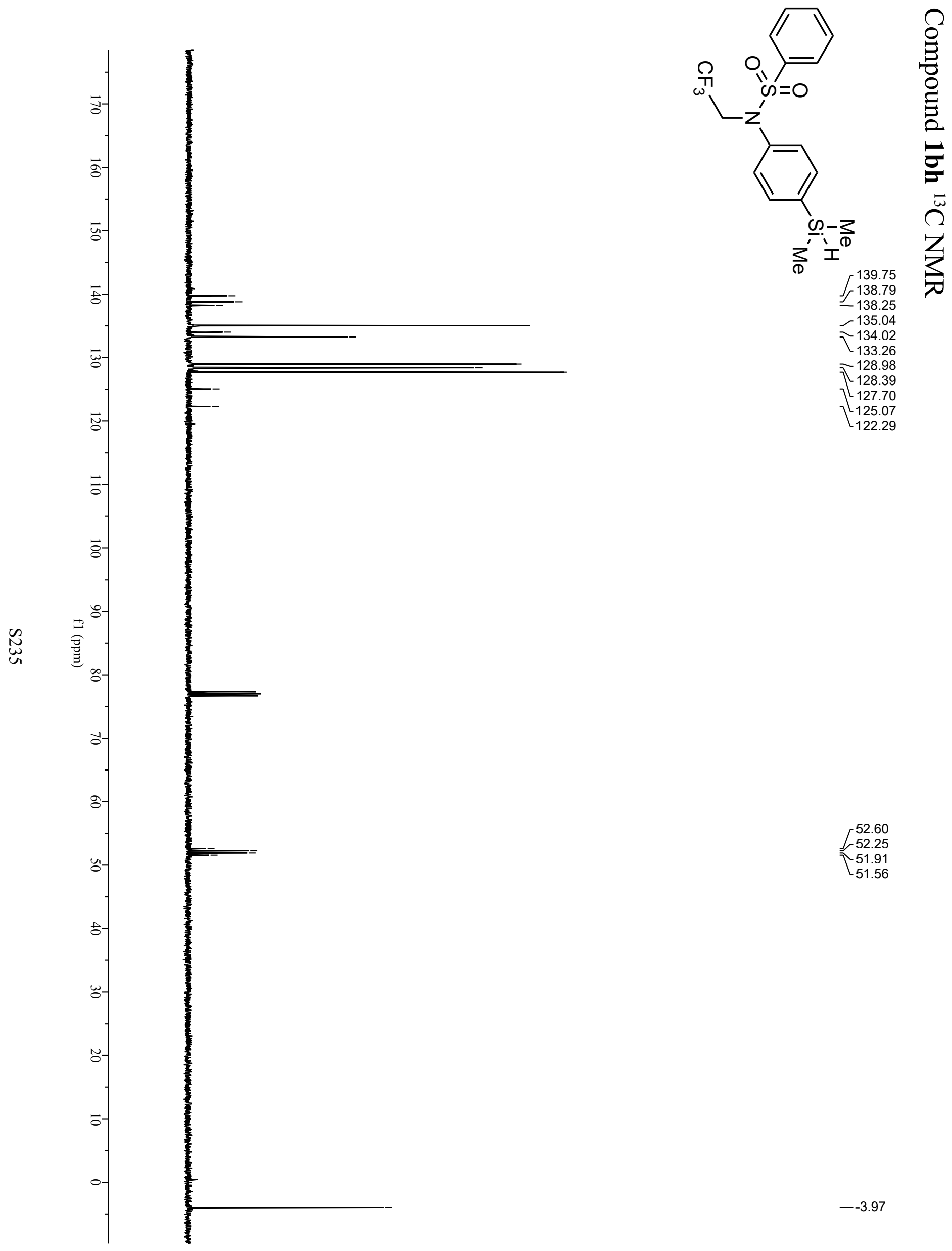

52.60
-52.25
51.91
51.56

$--3.97$ 
Compound 1bh ${ }^{19} \mathrm{~F}$ NMR<smiles>[Y][Si](C)([14CH3])c1ccc(N(CC(F)(F)F)S(=O)(=O)c2ccccc2)cc1</smiles> 


\section{Compound 1bi ${ }^{1} \mathrm{H}$ NMR}

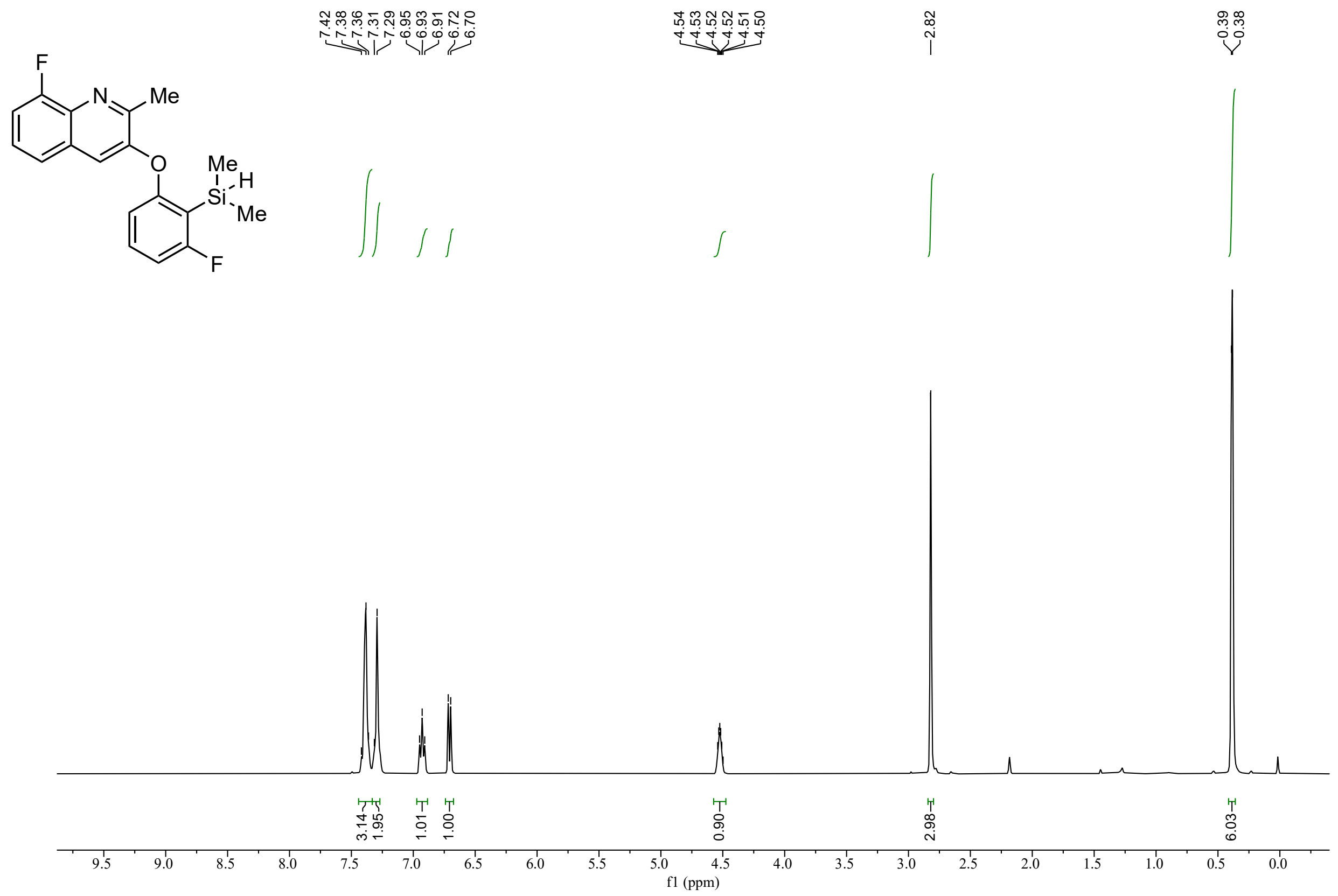




$$
\left.\right|^{\prime \prime 1}
$$




\section{Compound 1bi ${ }^{13} \mathrm{C}$ NMR}
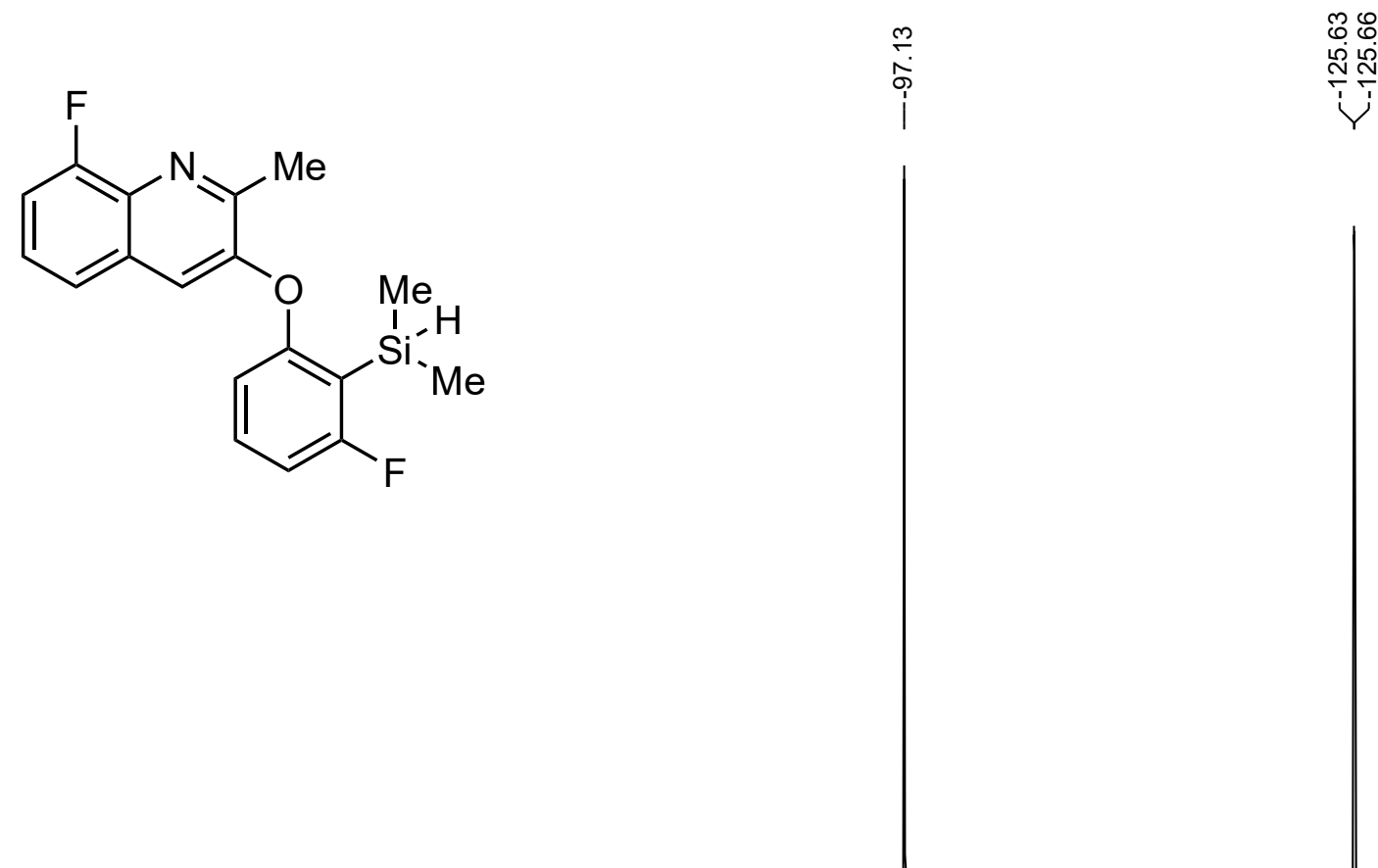
Compound 1bj ${ }^{1} \mathrm{H}$ NMR

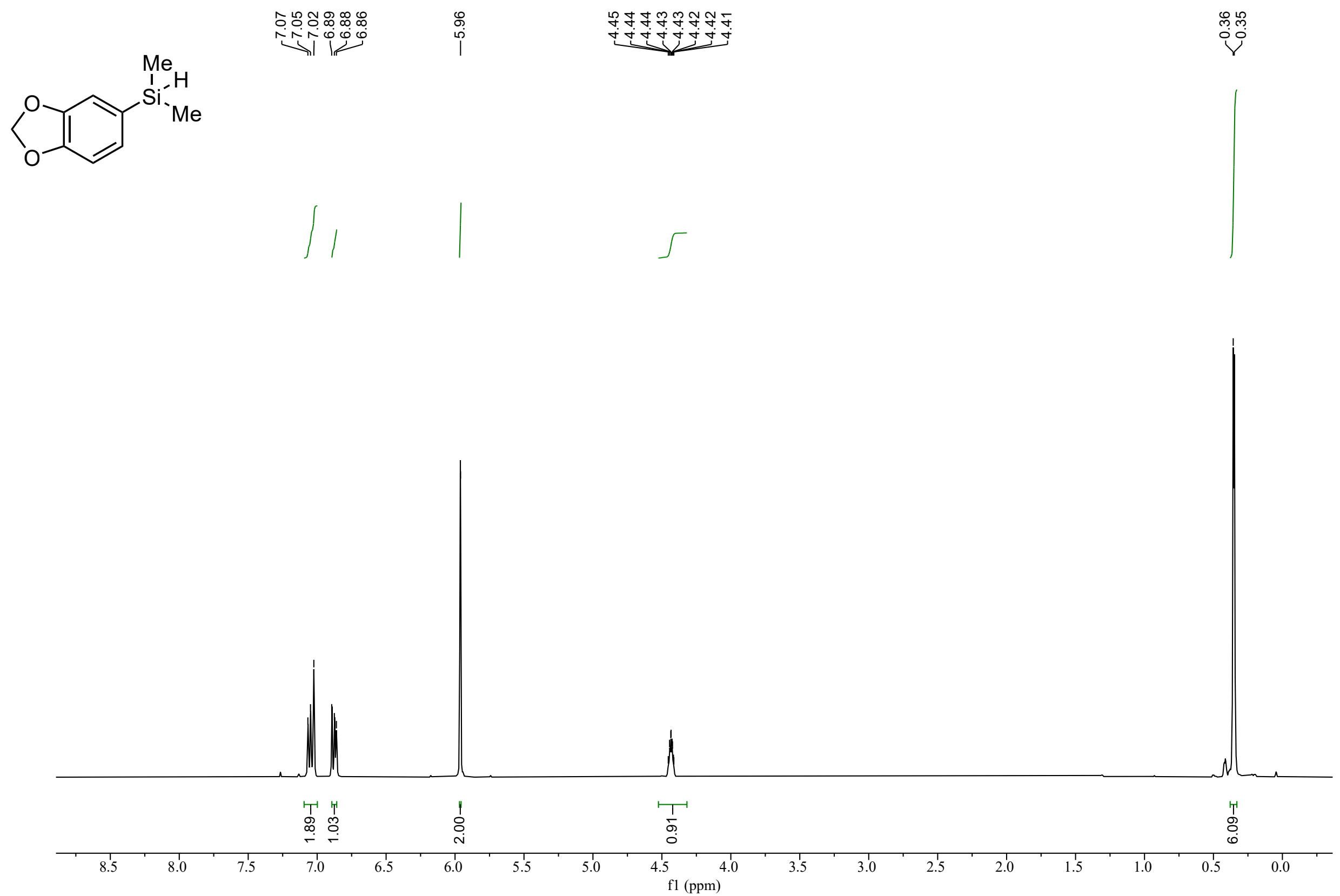


Compound 1bj ${ }^{13} \mathrm{C}$ NMR

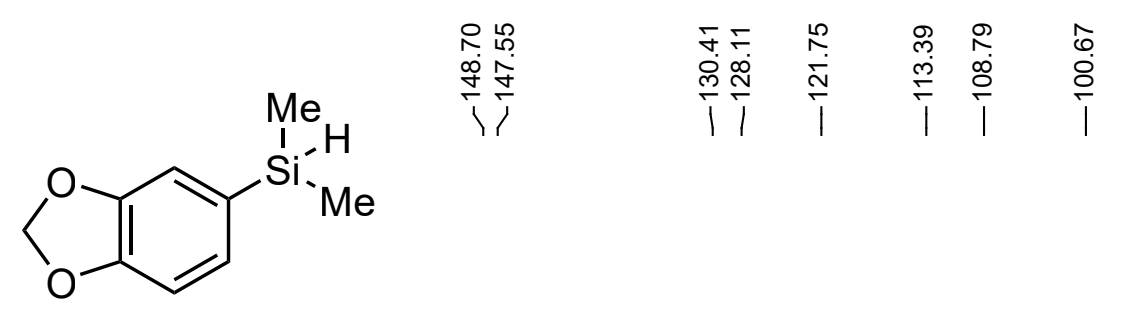

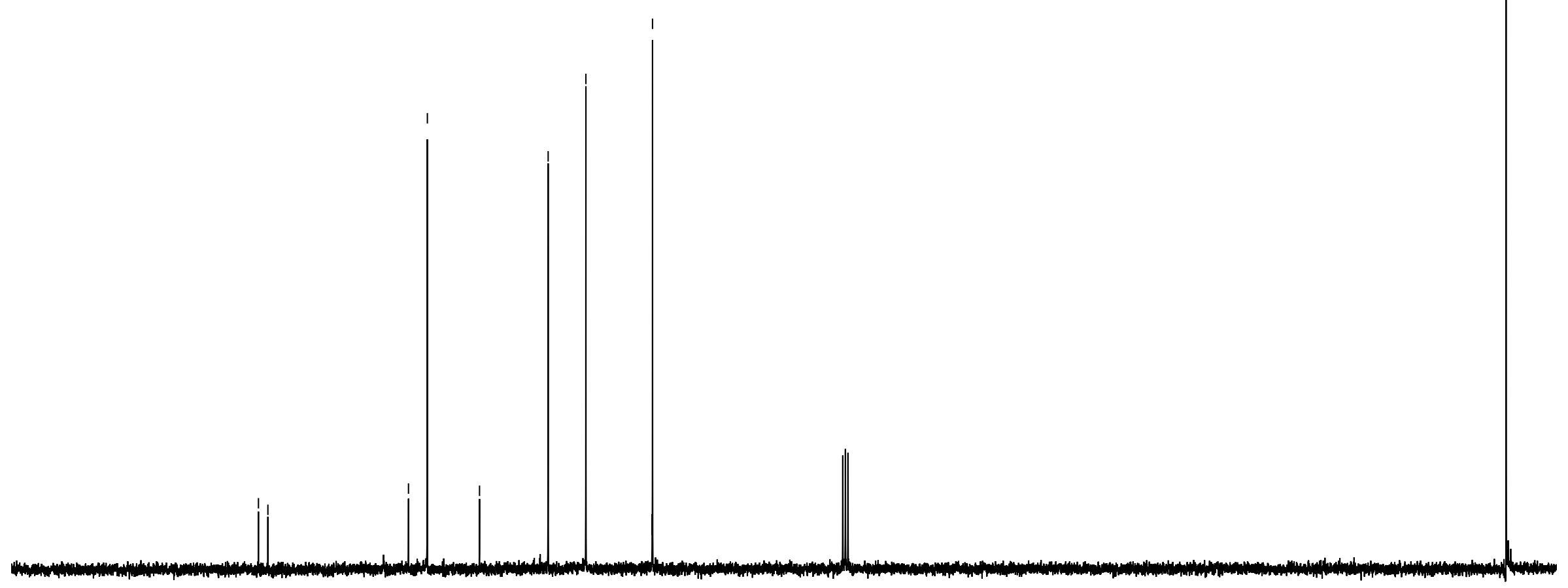

$170 \quad 160$

150

140

130 
Compound 1bk ${ }^{1} \mathrm{H}$ NMR
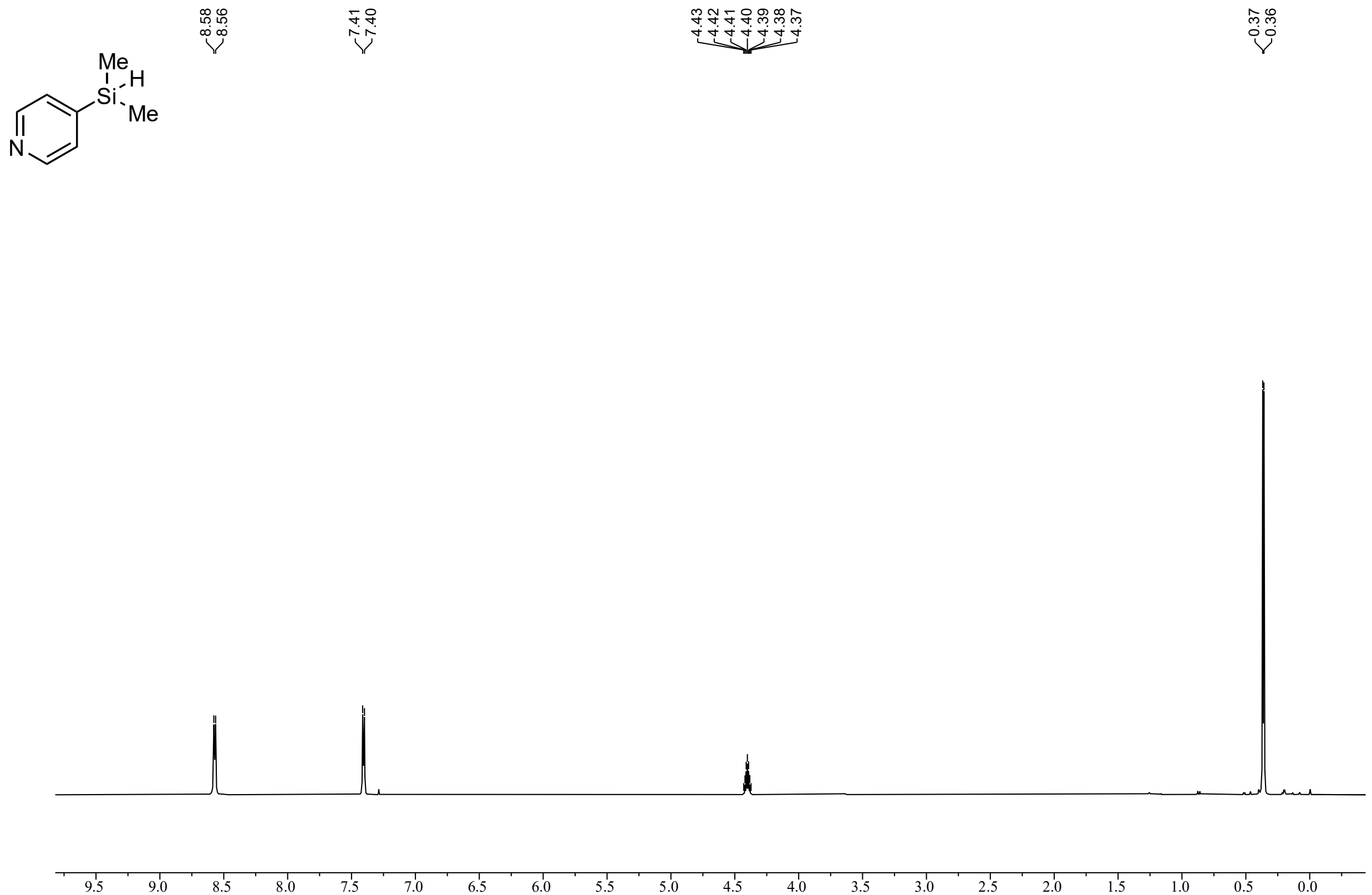
Compound 1bk ${ }^{13} \mathrm{C}$ NMR
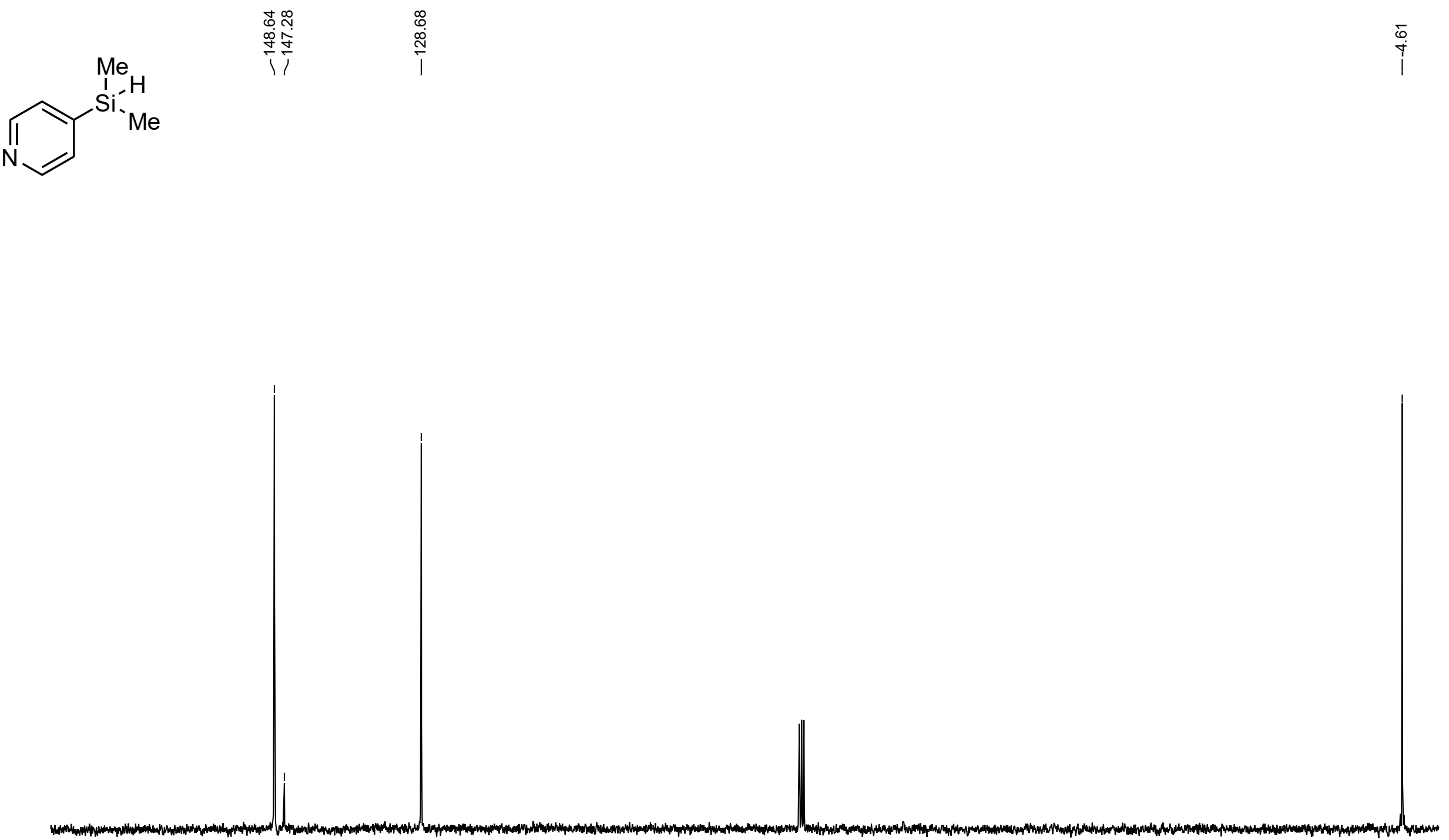

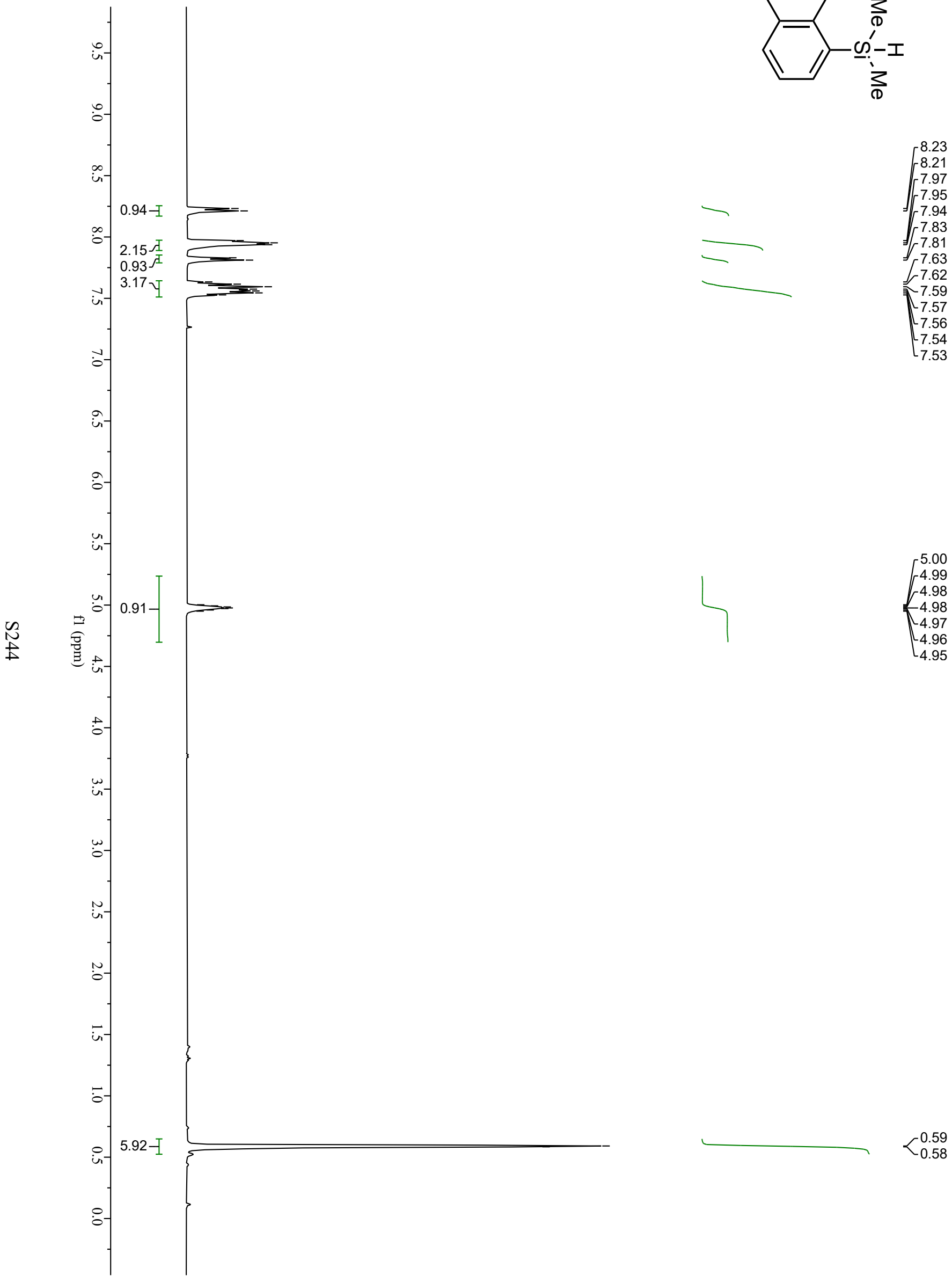

-4.99
-4.98 
Compound 1 bl ${ }^{13} \mathrm{C}$ NMR<smiles>C[Si](C)c1cccc2ccccc12</smiles>

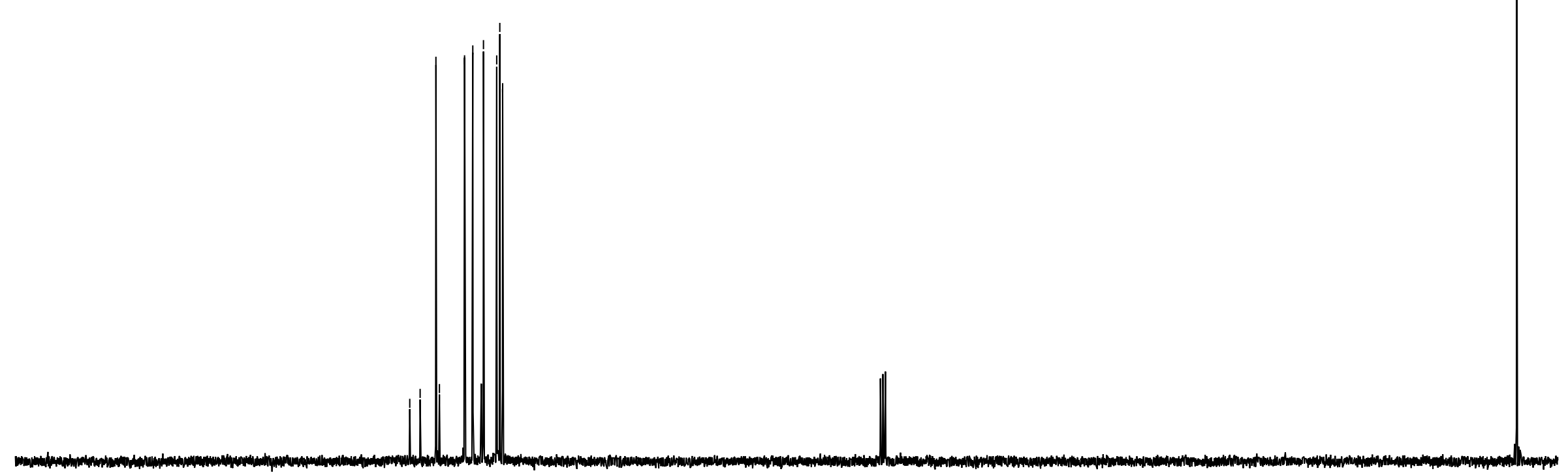




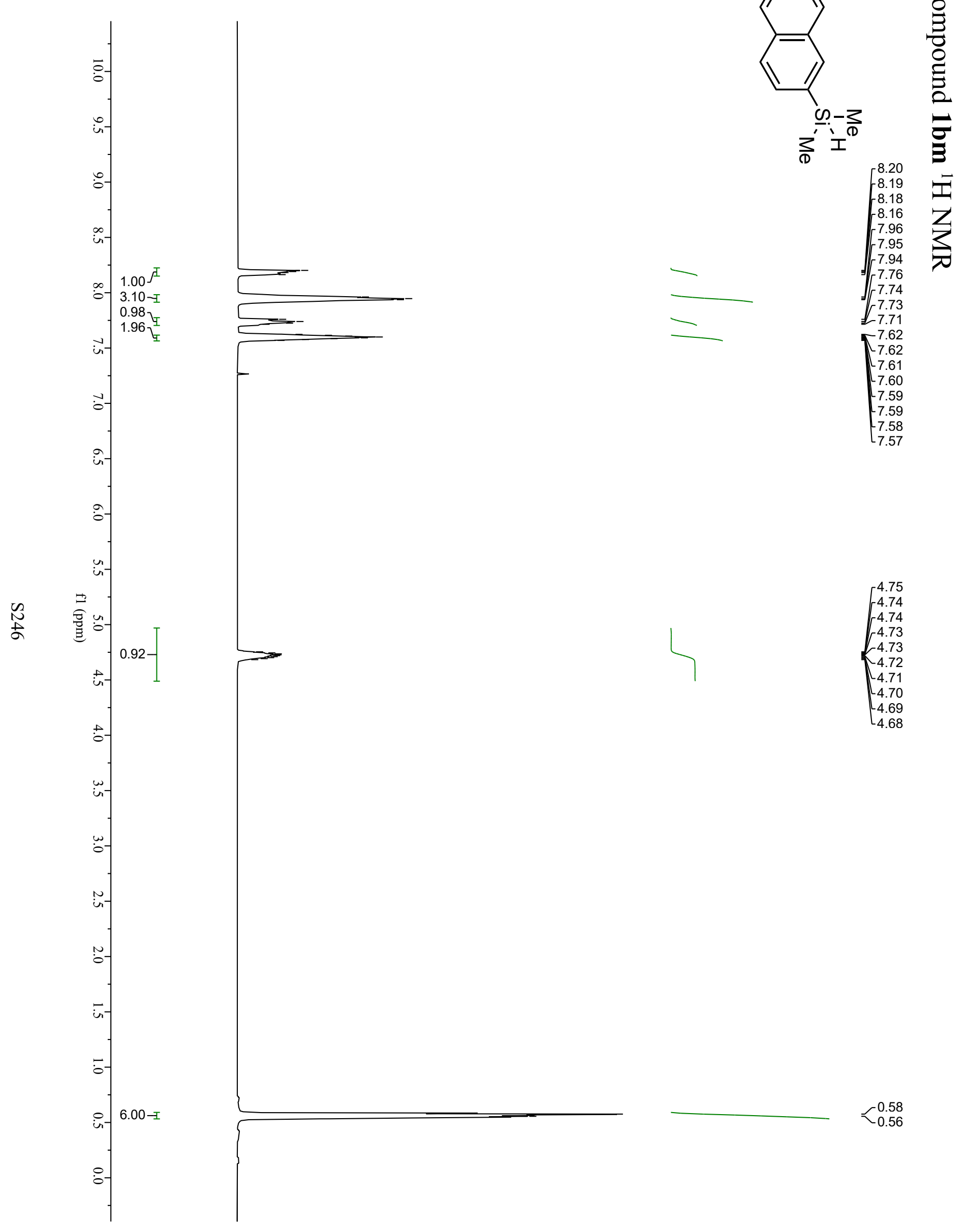


Compound 1 bm ${ }^{13} \mathrm{C}$ NMR
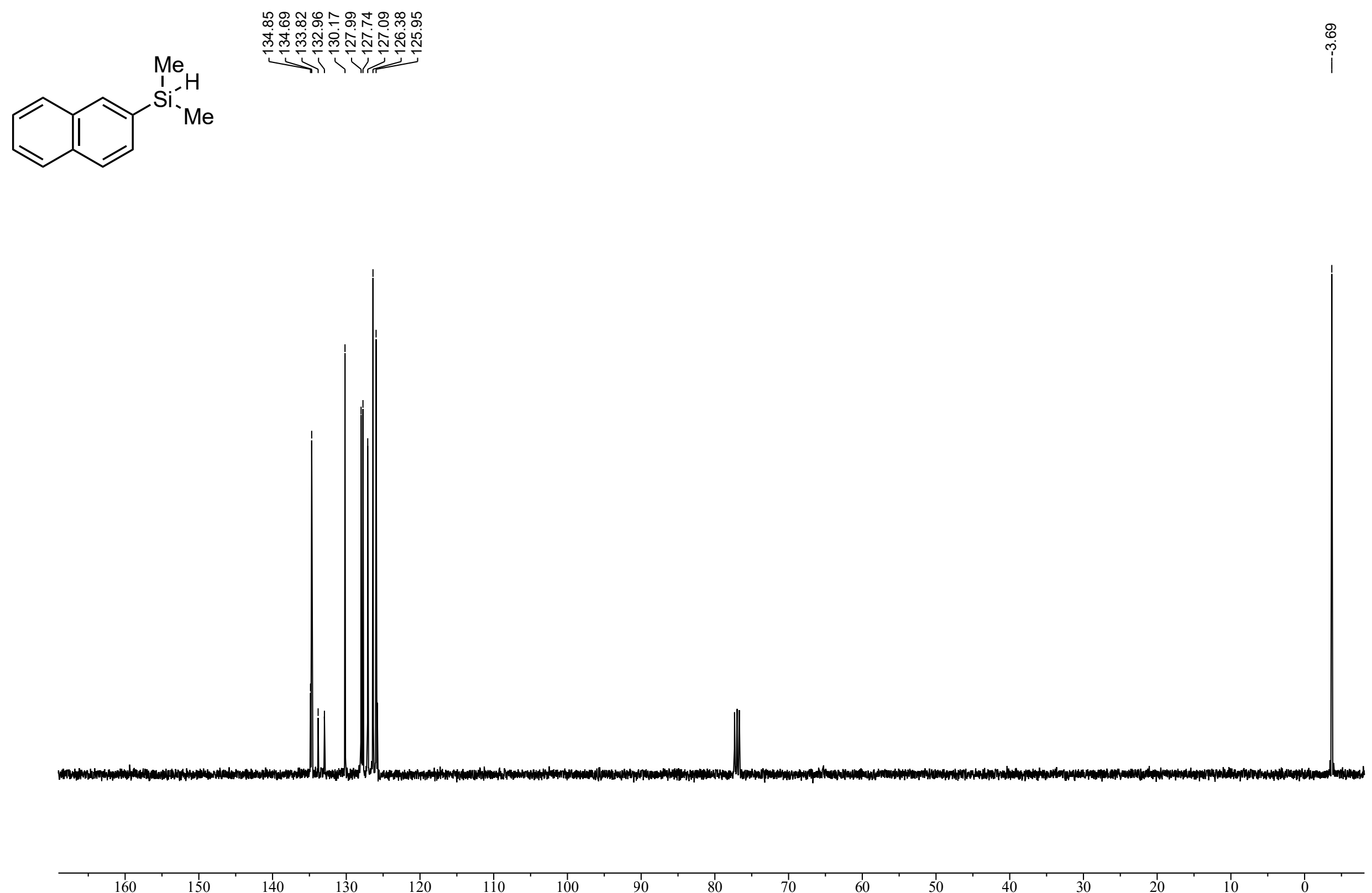


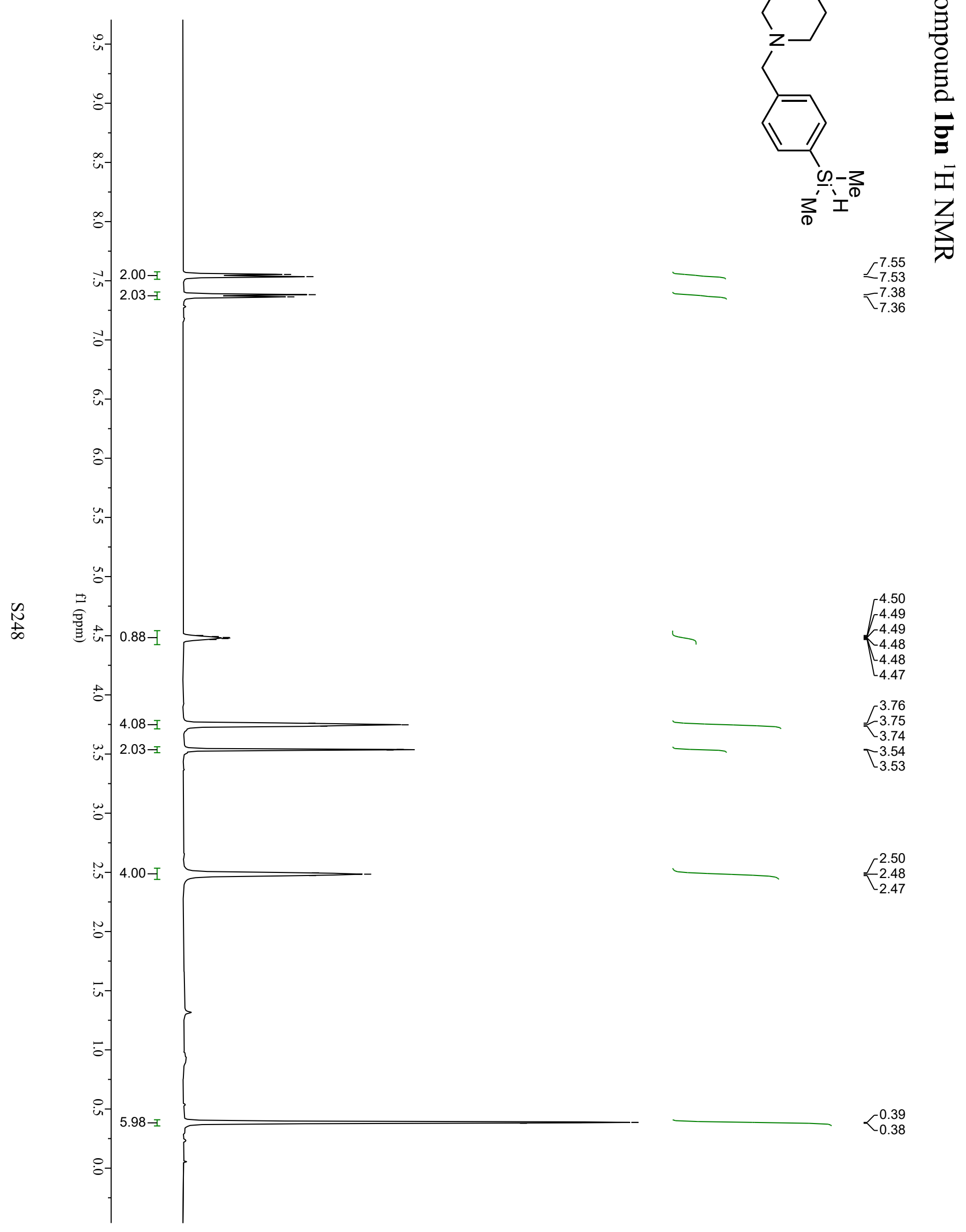


Compound 1bn ${ }^{13} \mathrm{C}$ NMR

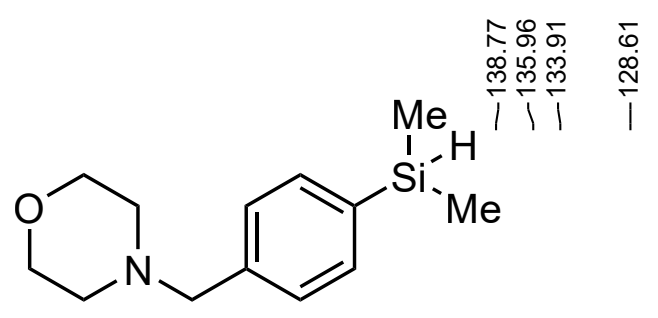

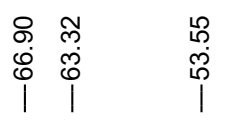

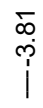
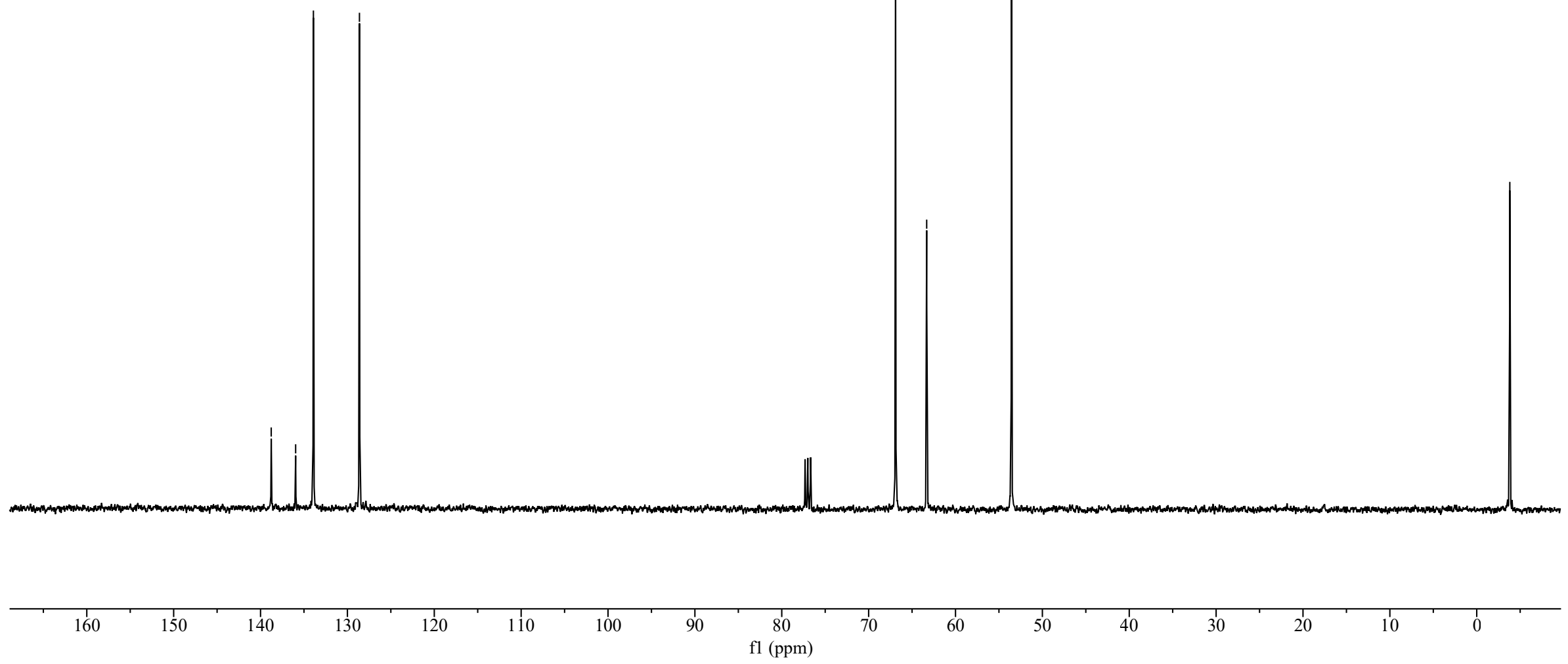

S249 
Compound 1 bo ${ }^{1} \mathrm{H}$ NMR

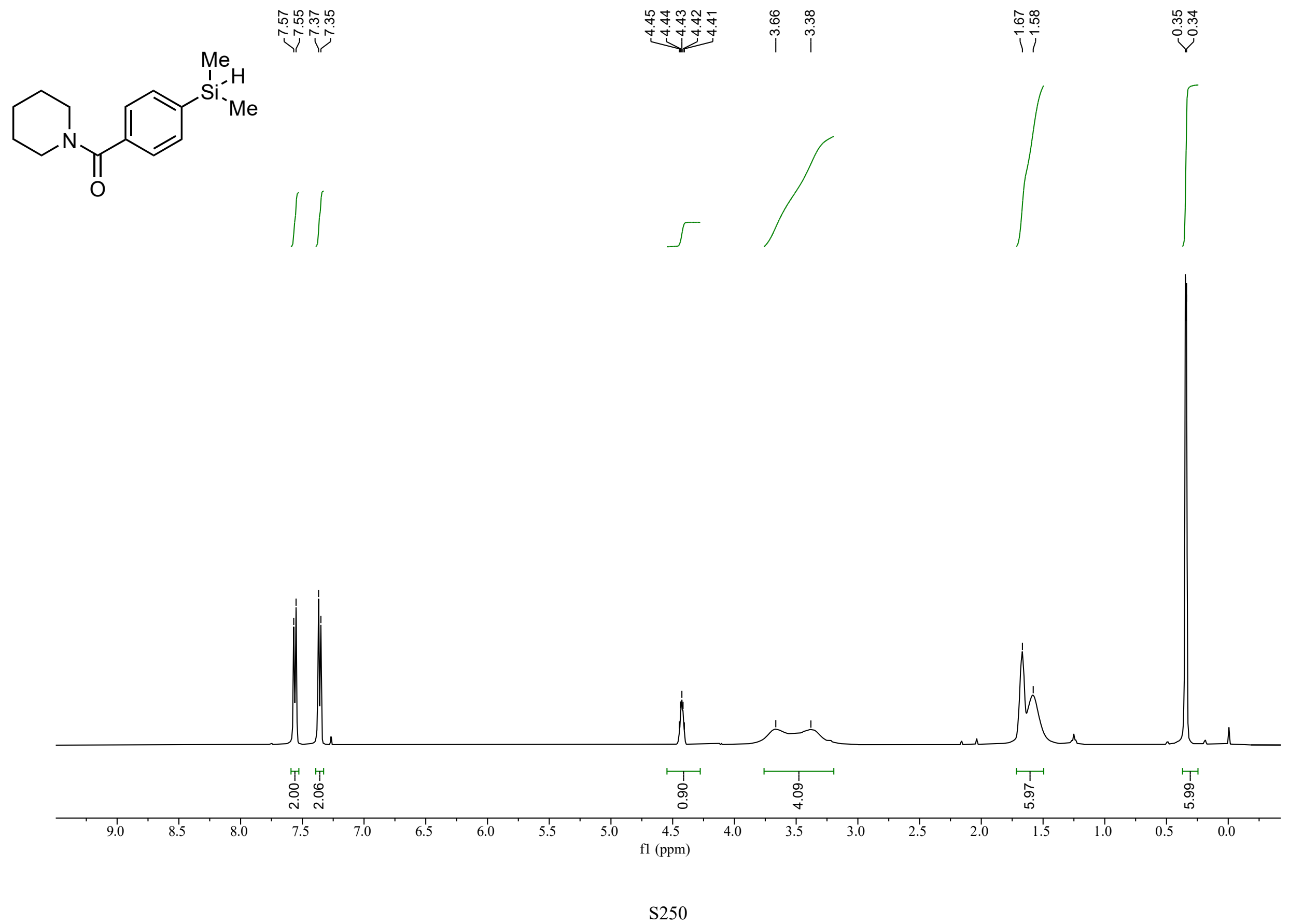


Compound 1 bo ${ }^{13} \mathrm{C}$ NMR
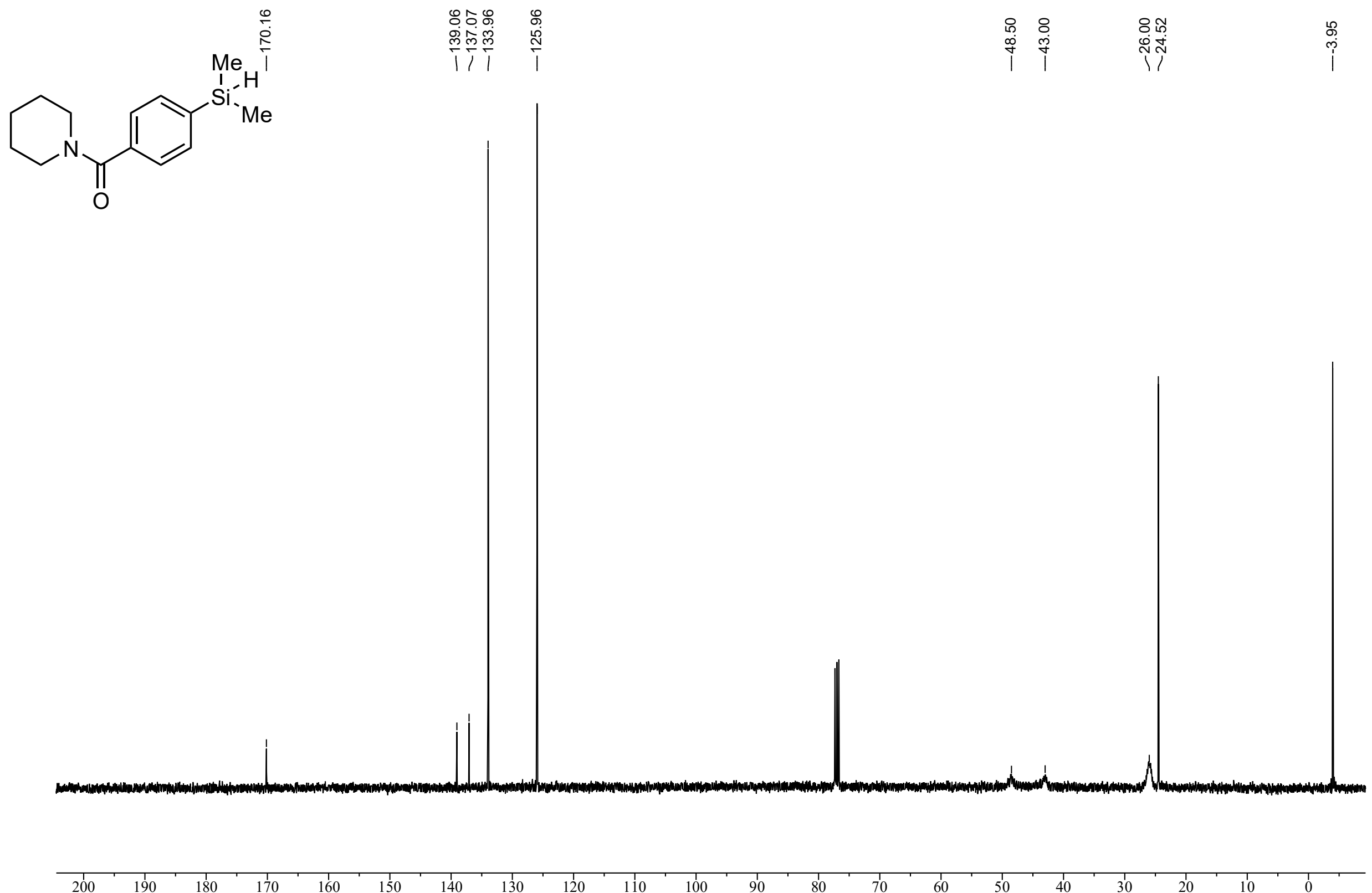

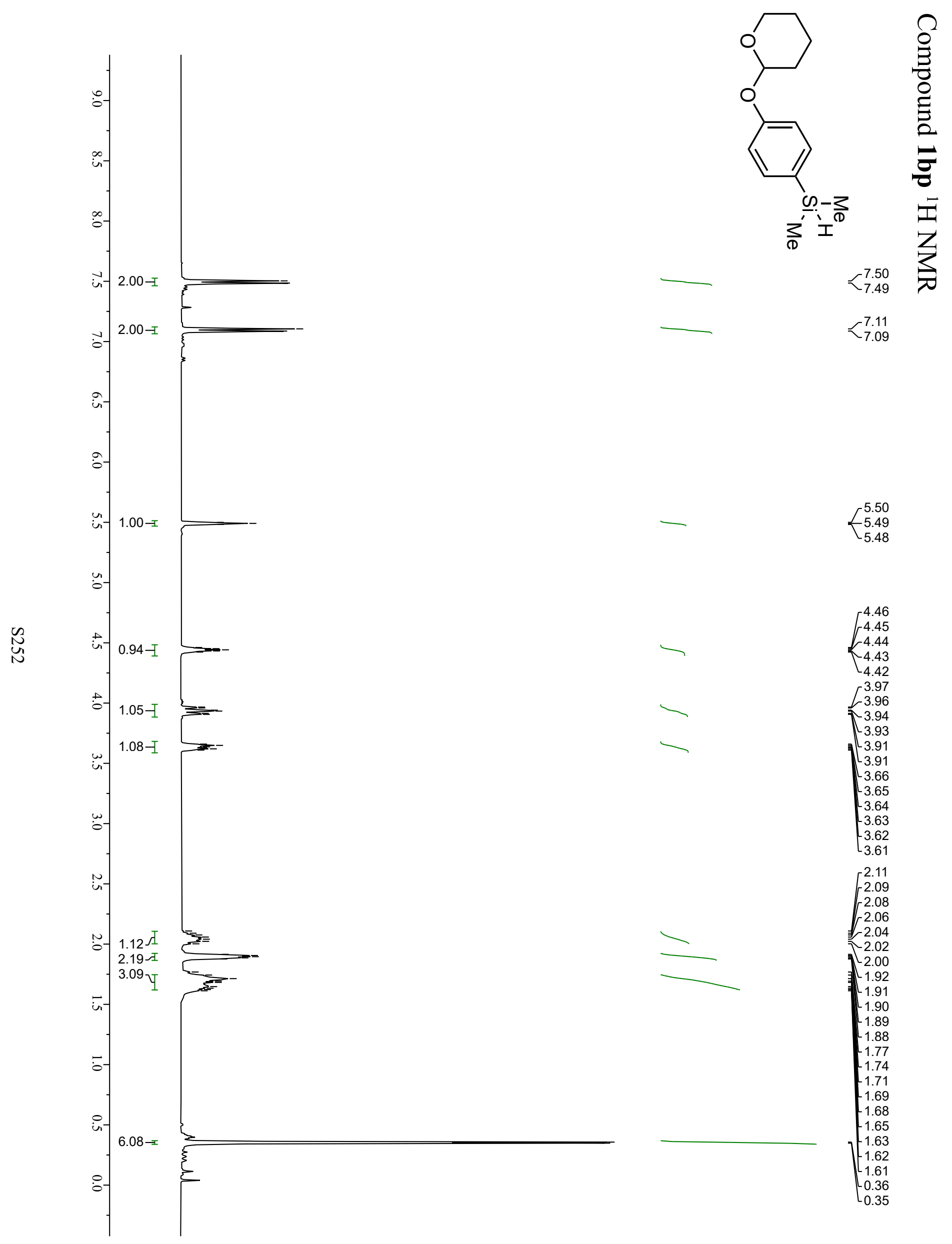
Compound 1bp ${ }^{13} \mathrm{C}$ NMR

r.
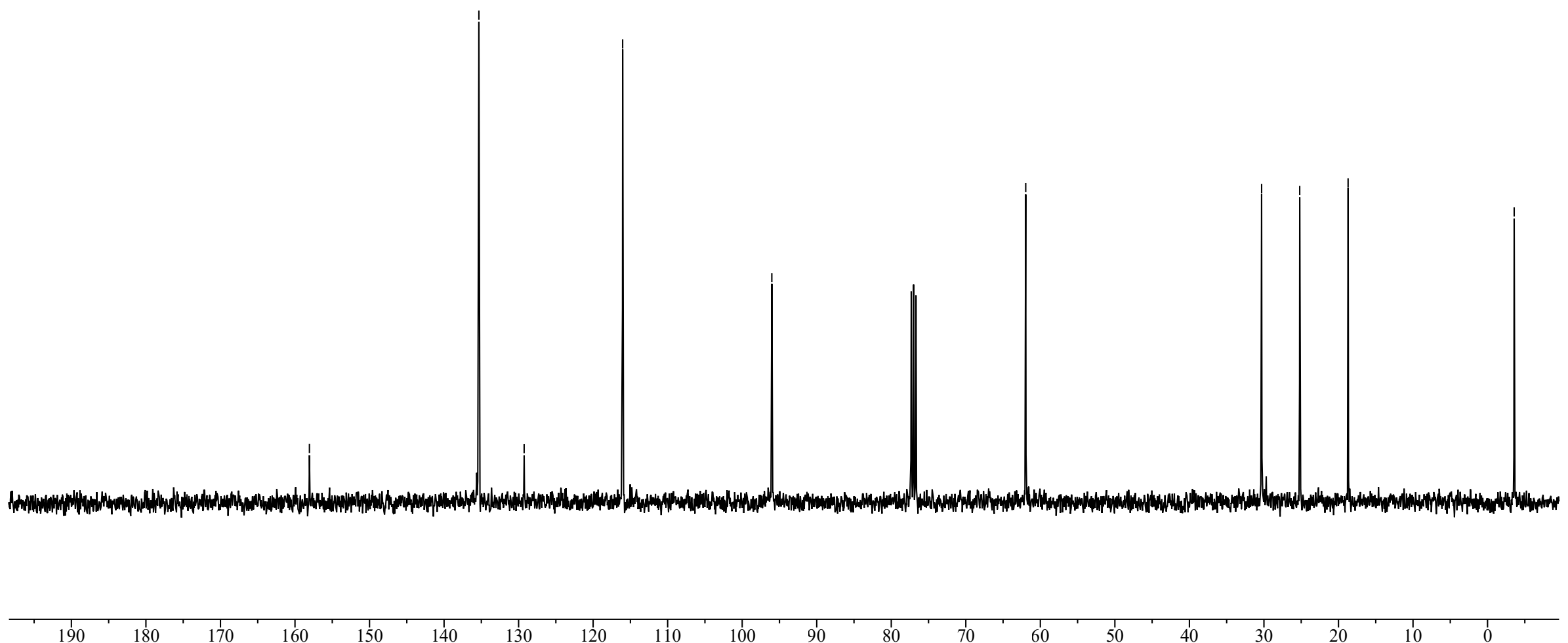

150 

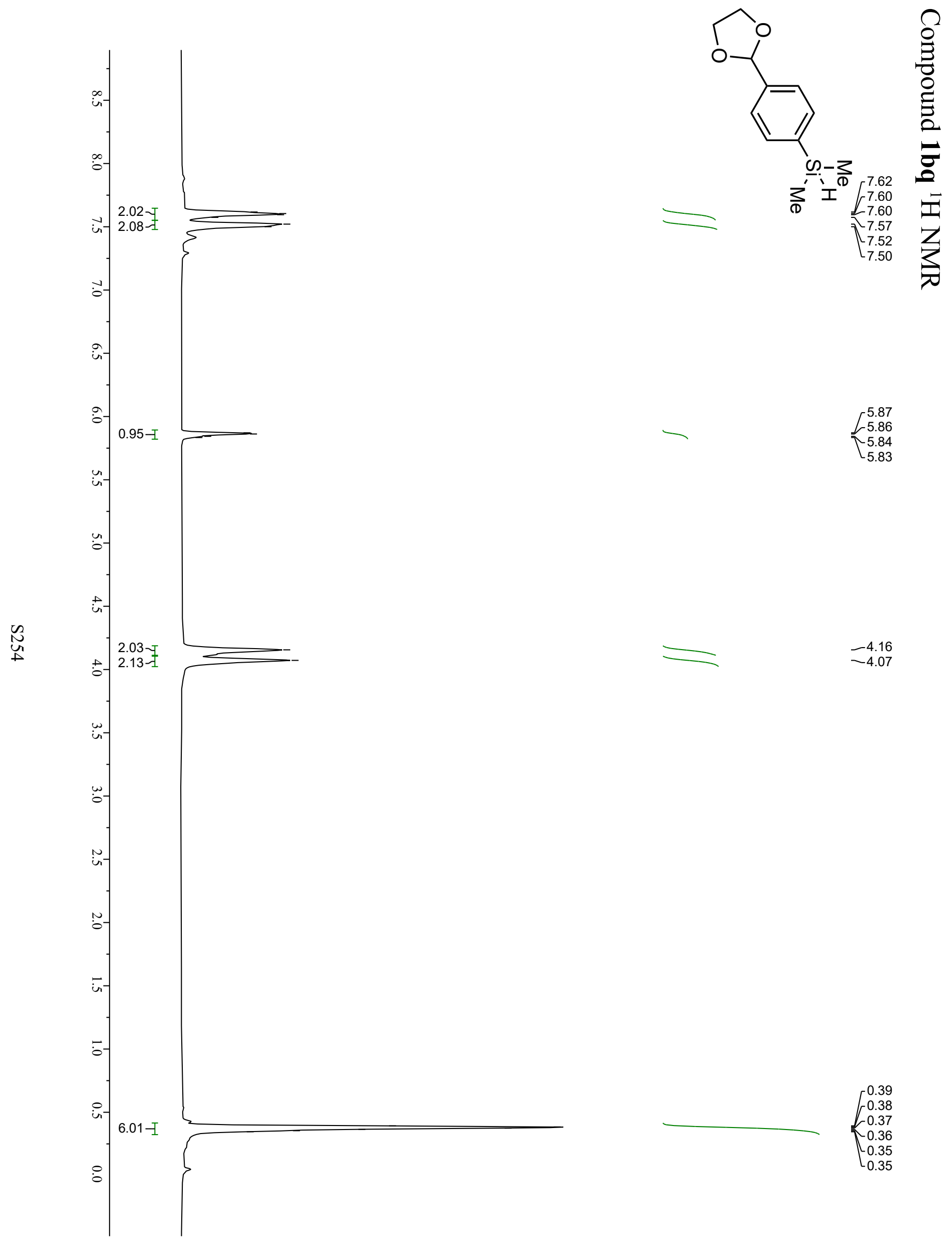
Compound $\mathbf{1 b q}{ }^{13} \mathrm{C}$ NMR
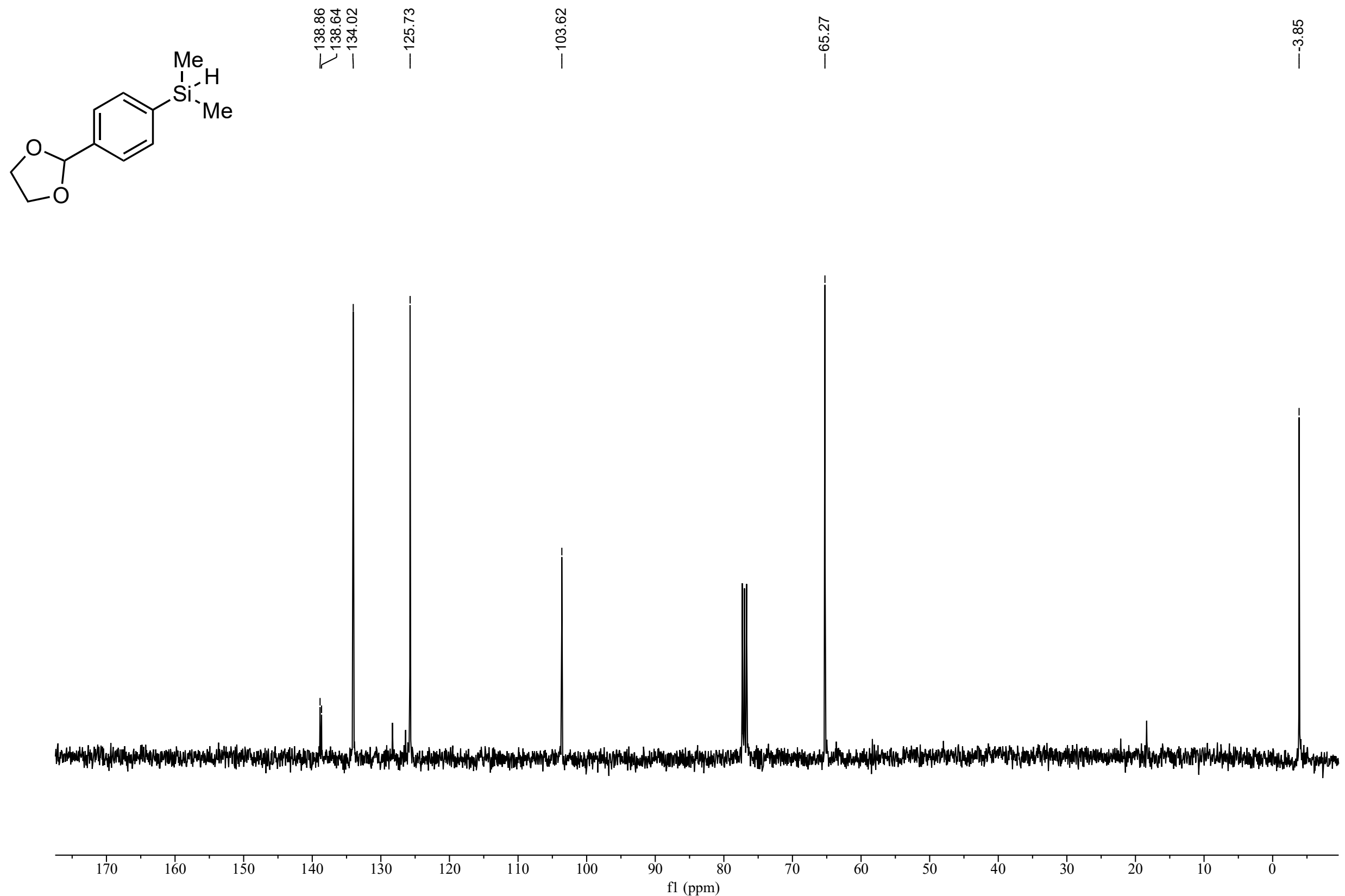


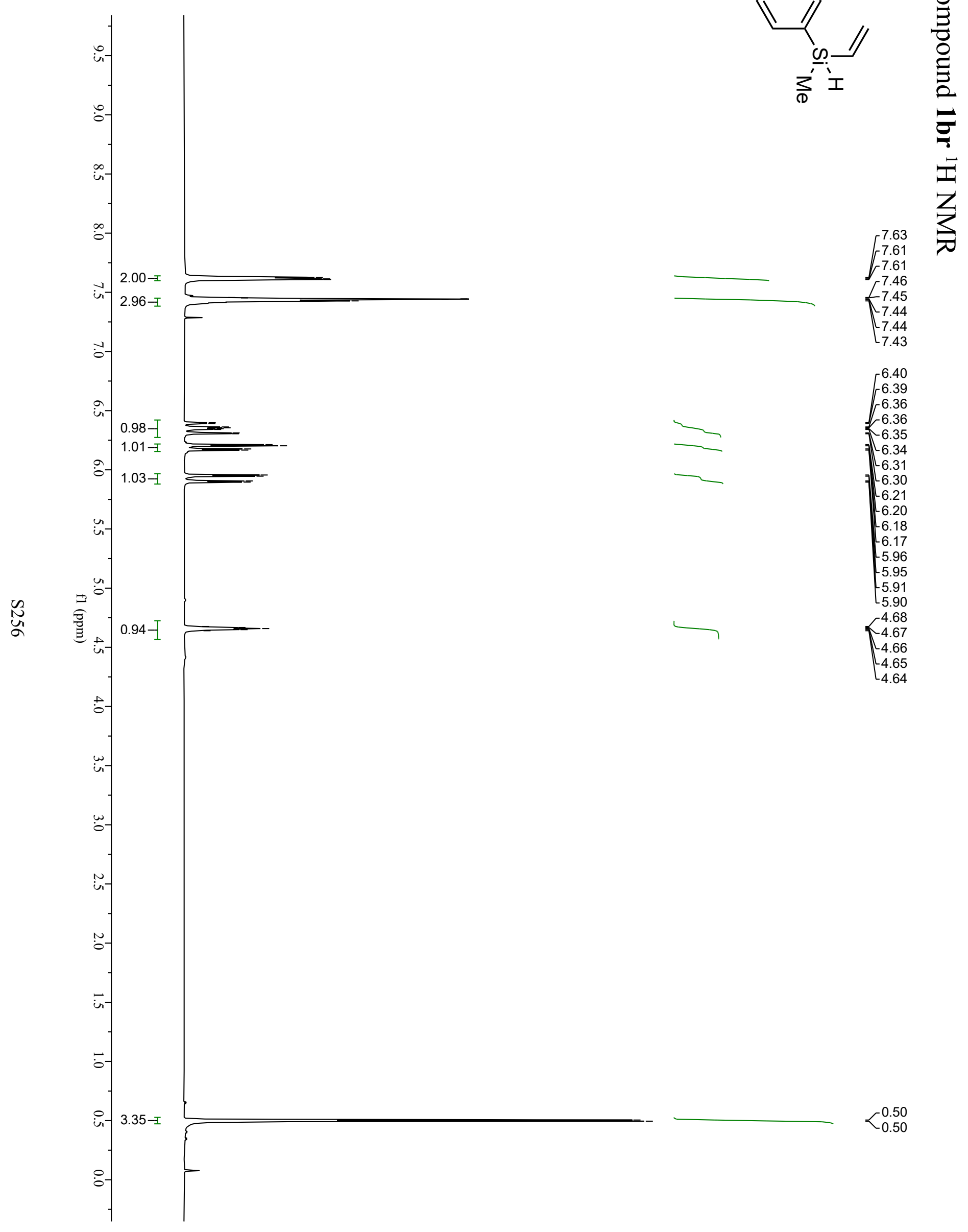


Compound $1 \mathrm{br}{ }^{13} \mathrm{C}$ NMR

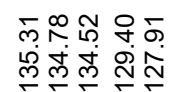

(1)

पा 11

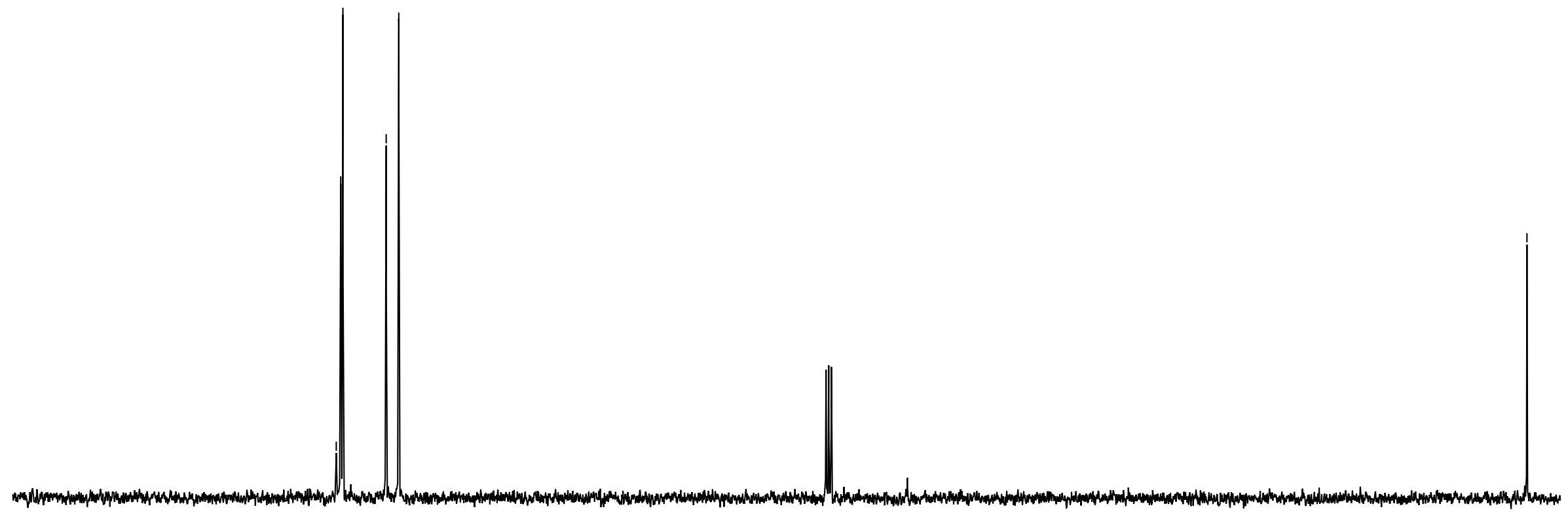



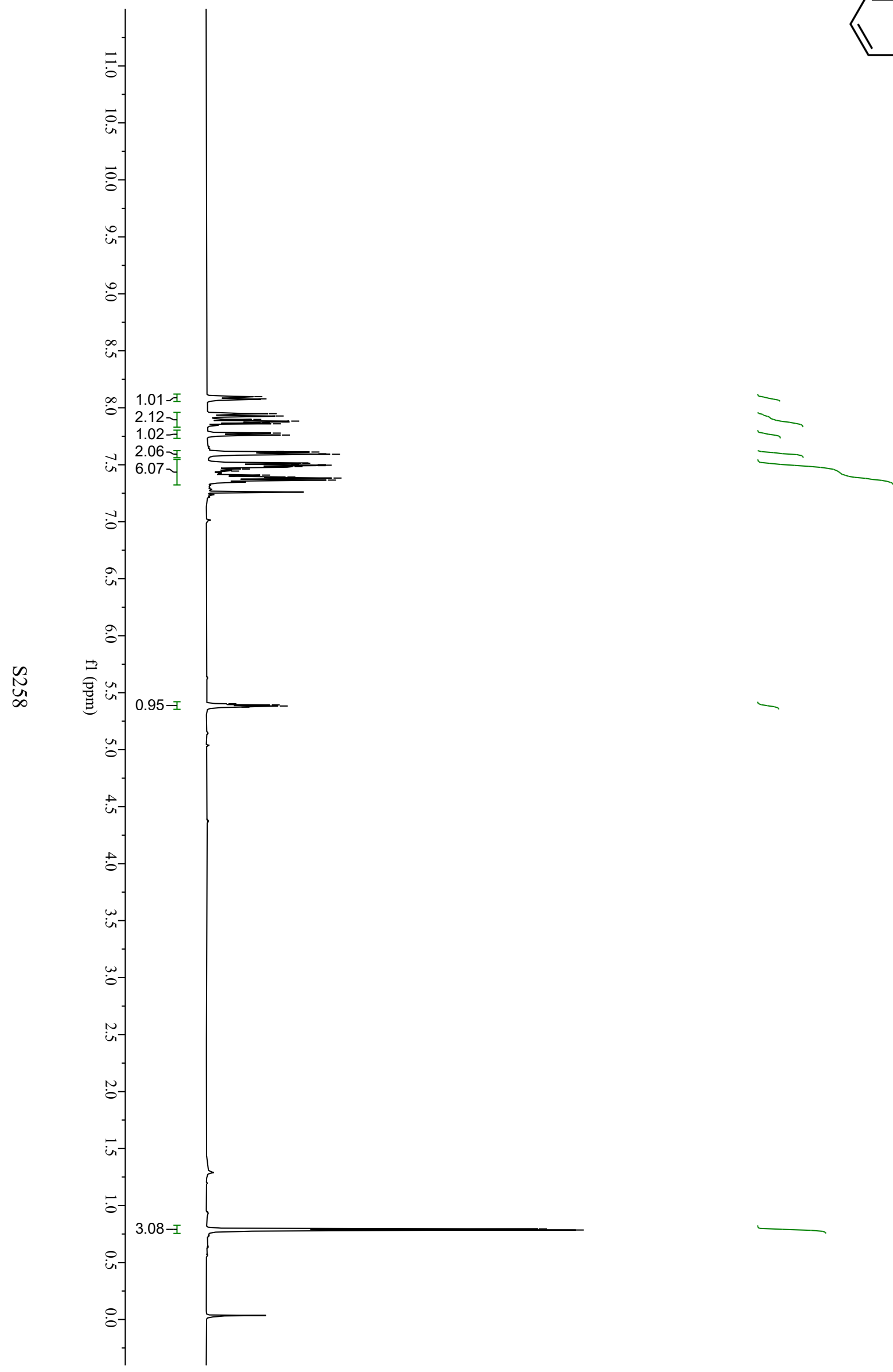

7.60

7.60

7.50

$-7.50$

7.49
7.48

-7.48
-7.46

$-7.44$

7.41
7.39

-7.38
7.37

5.40

$-5.39$

-5.38
5.37

플 
Compound 1b ${ }^{1} \mathrm{H}$ NMR
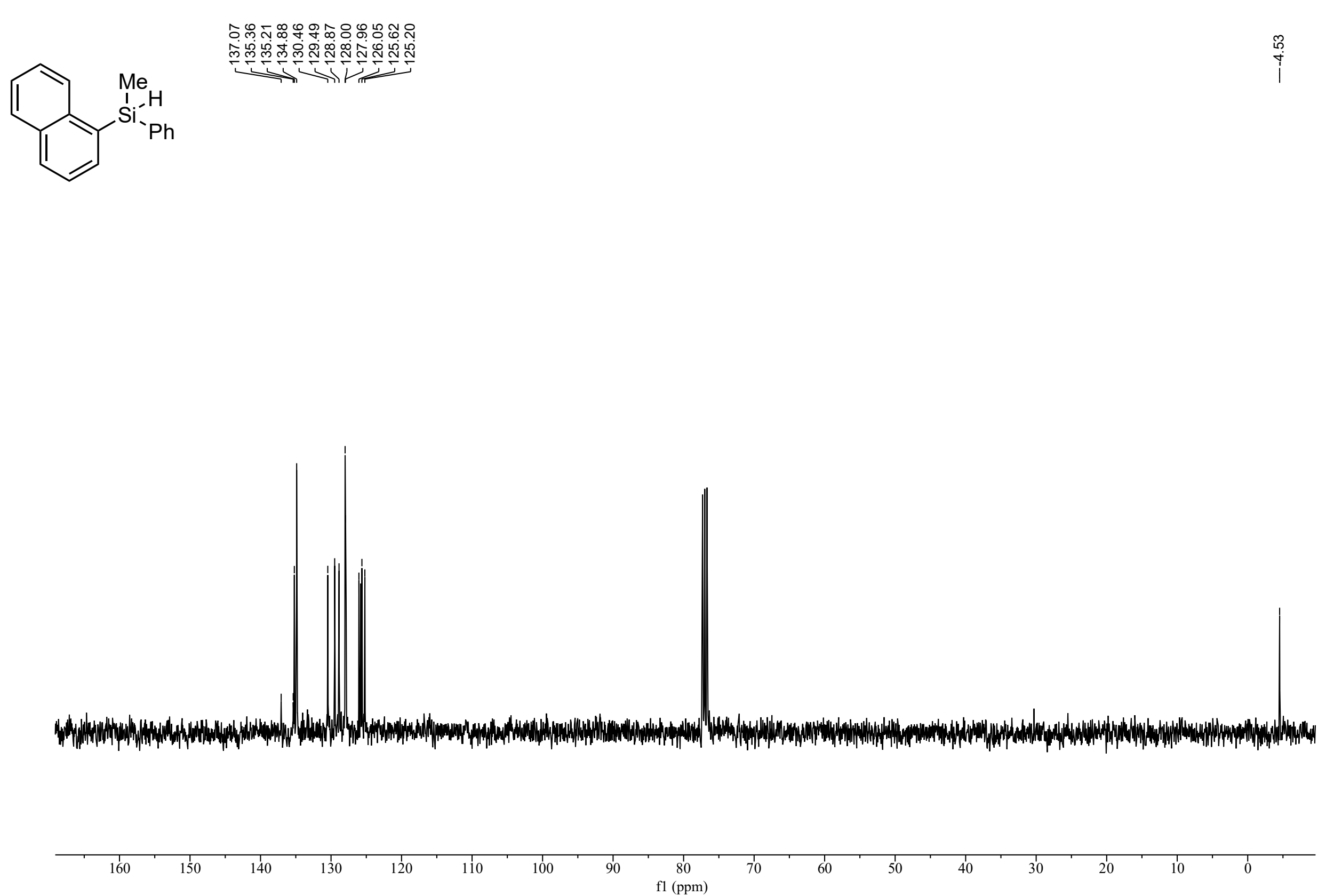

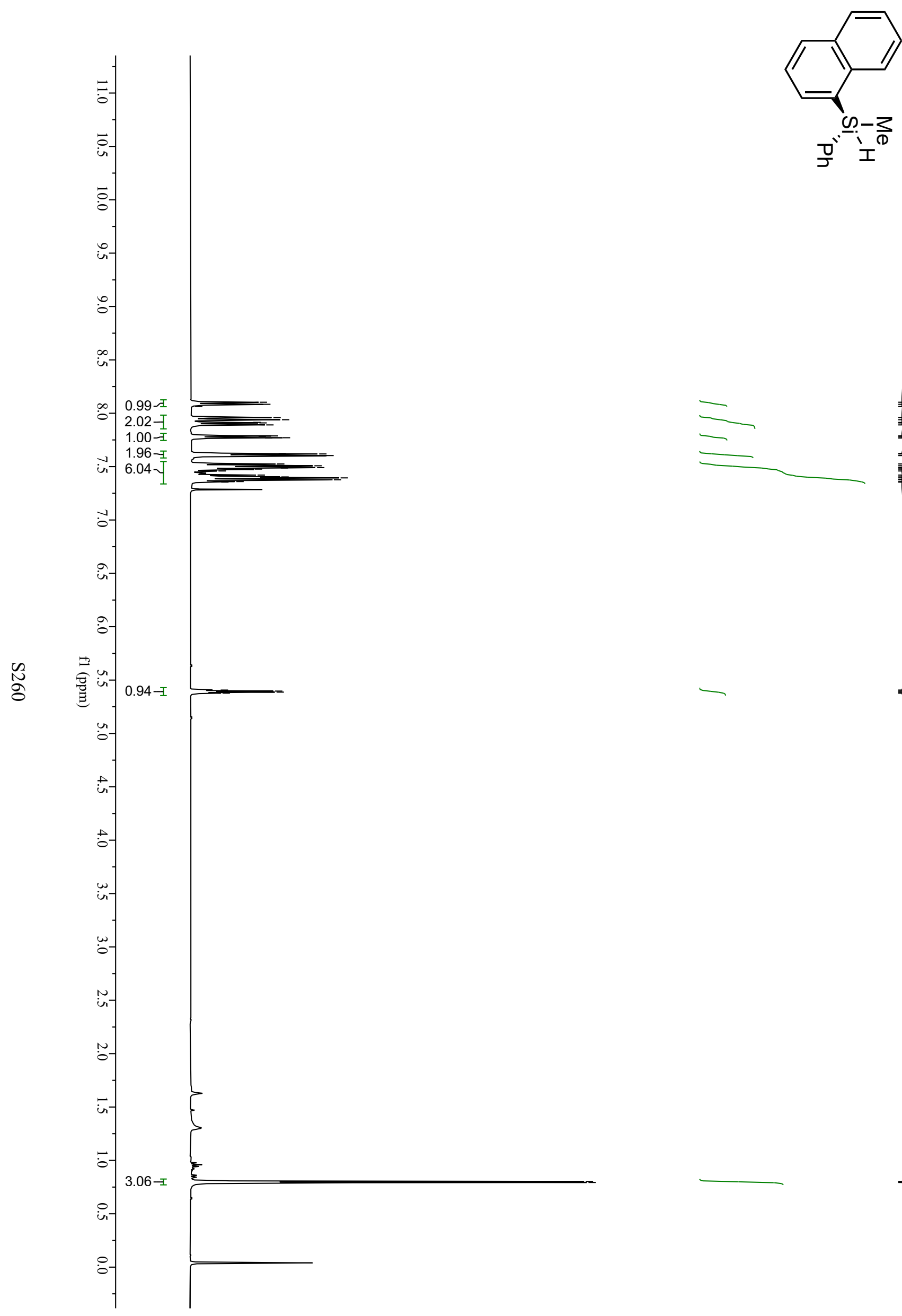

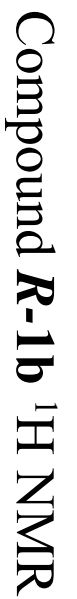




$$
=
$$


Compound 1a-d ${ }_{1}^{1} \mathrm{H}$ NMR

(l)

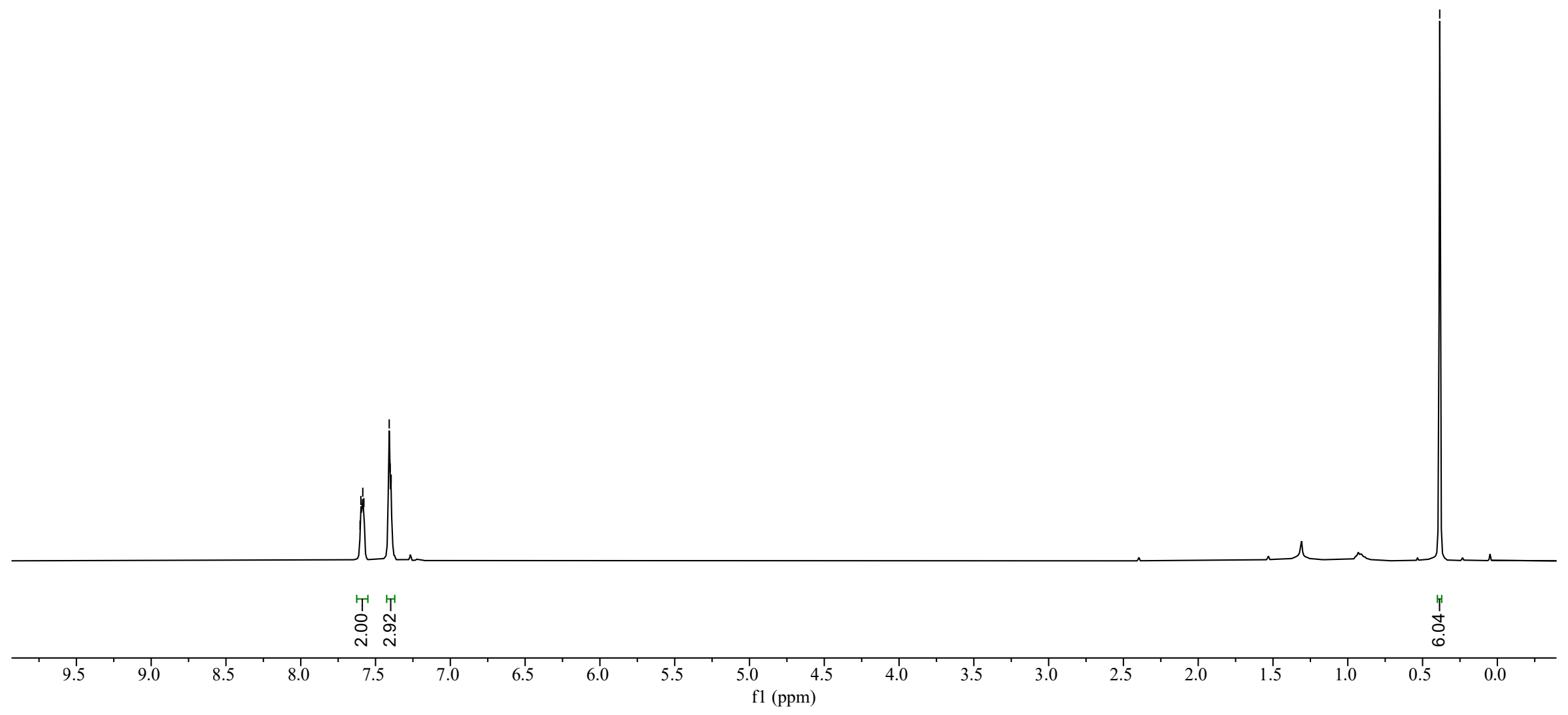


Compound 1a-d $1{ }^{13} \mathrm{C}$ NMR

(N)
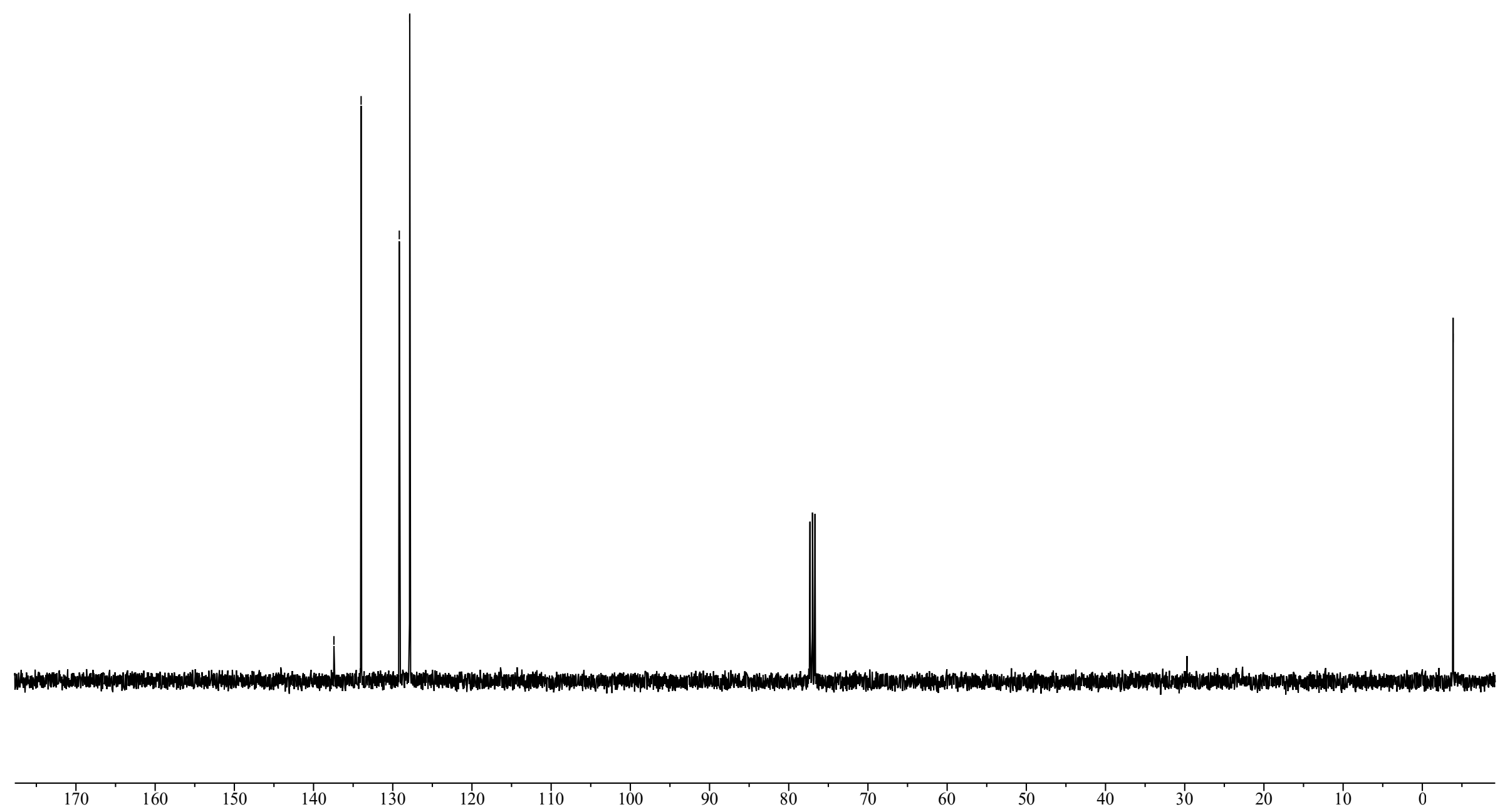
Compound 2a ${ }^{1} \mathrm{H}$ NMR

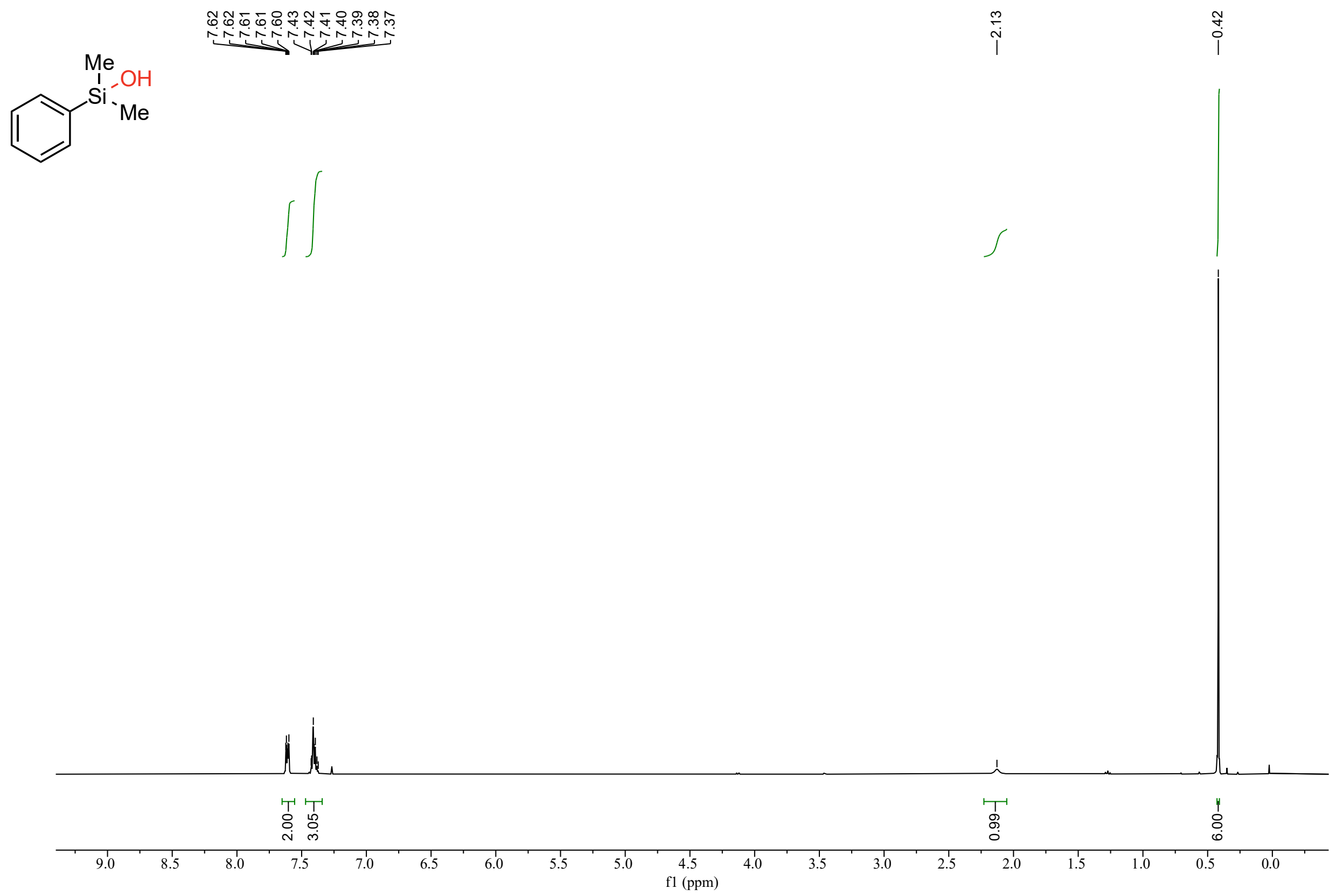


Compound 2a ${ }^{13} \mathrm{C}$ NMR

$\mathrm{SP}_{1 \mathrm{Me}}^{\mathrm{Me}}$

i

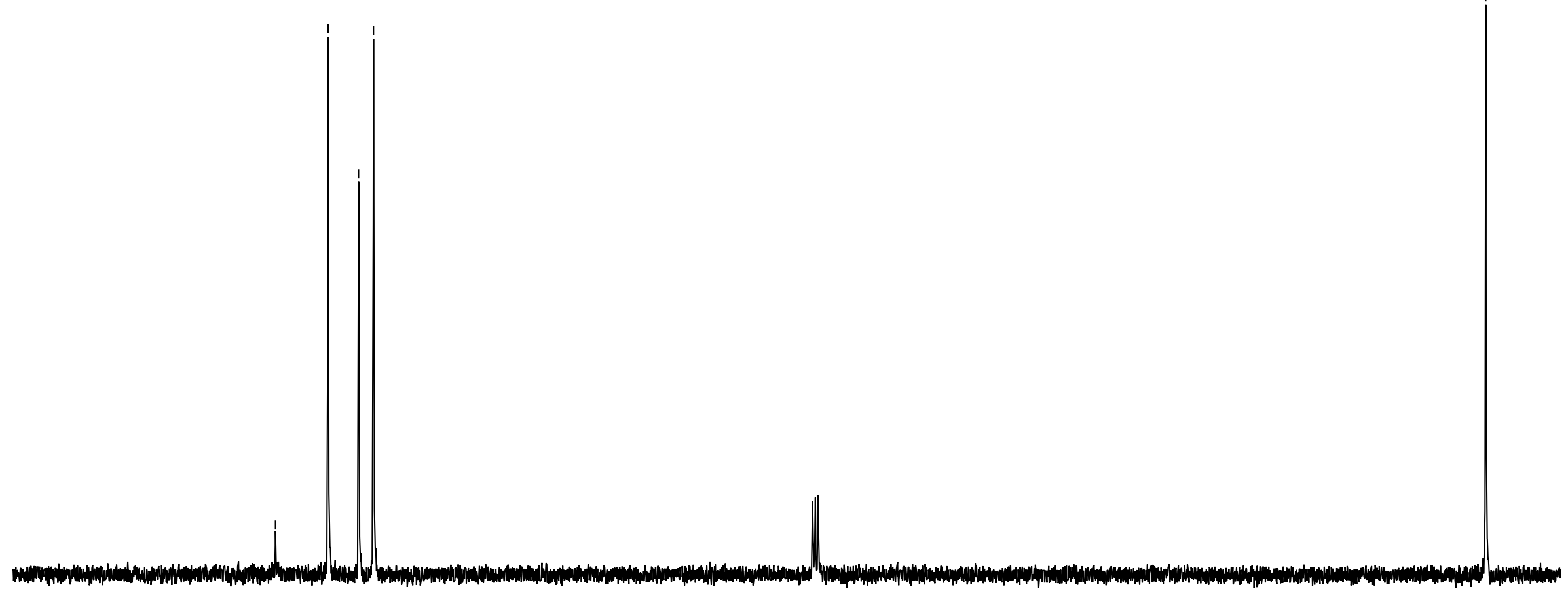



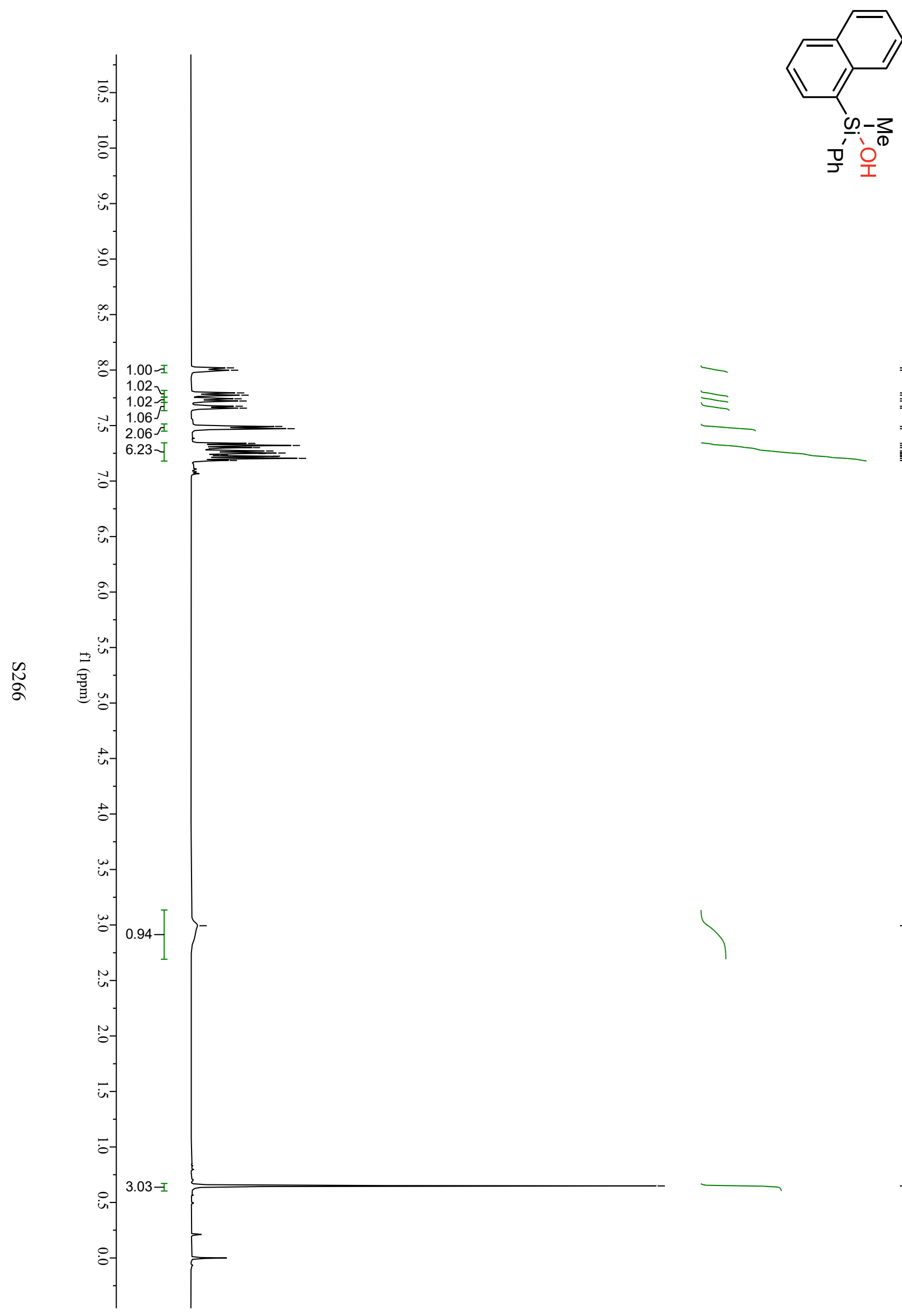

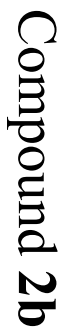
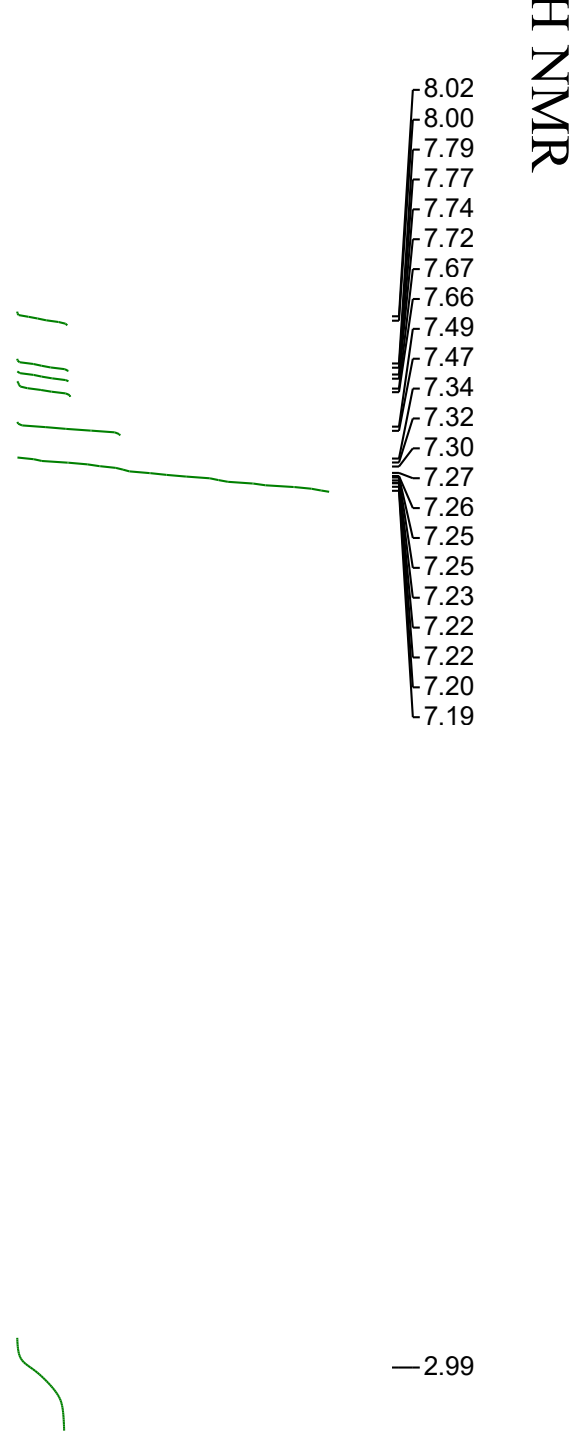

$-2.99$

$-0.65$ 


$$
[
$$



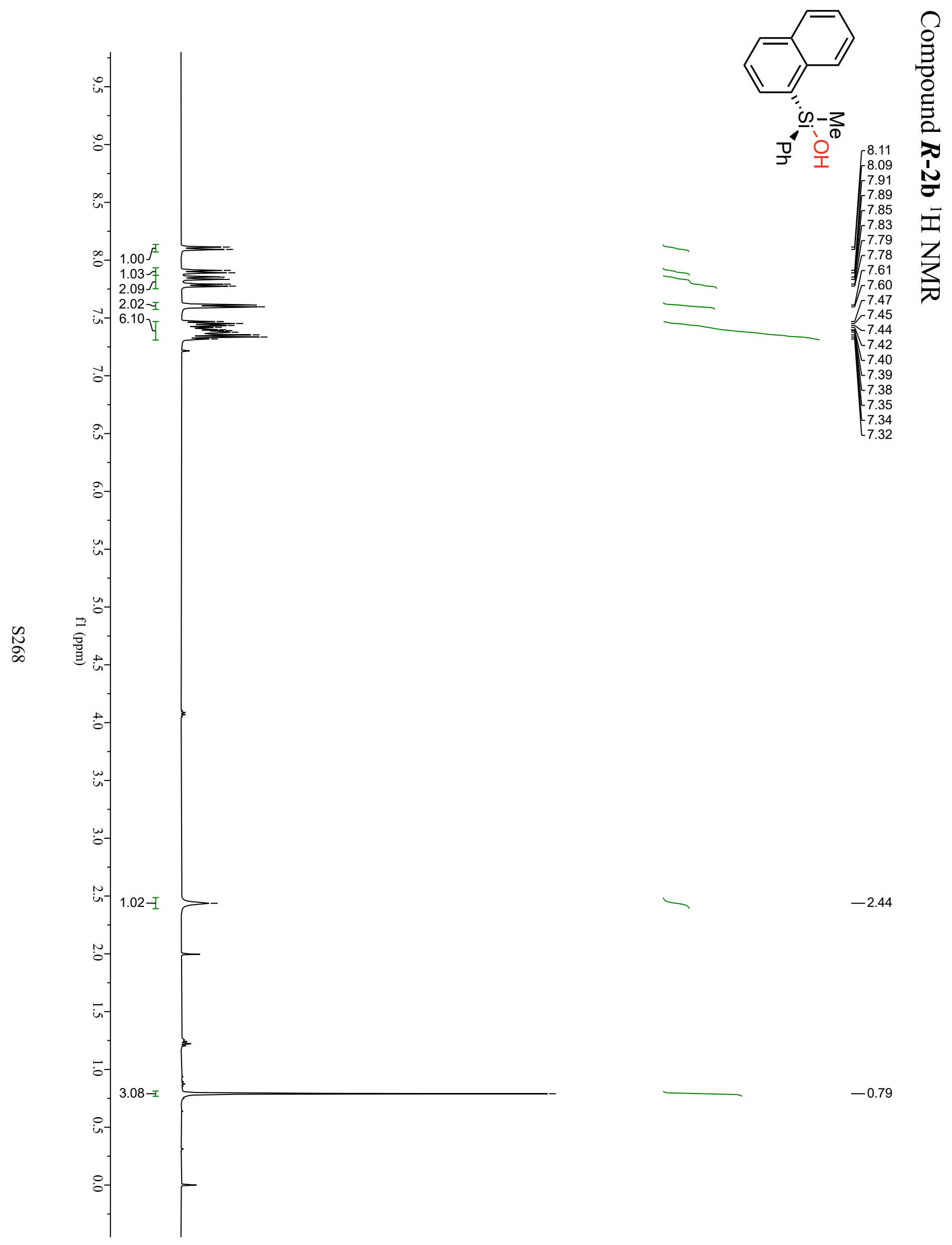

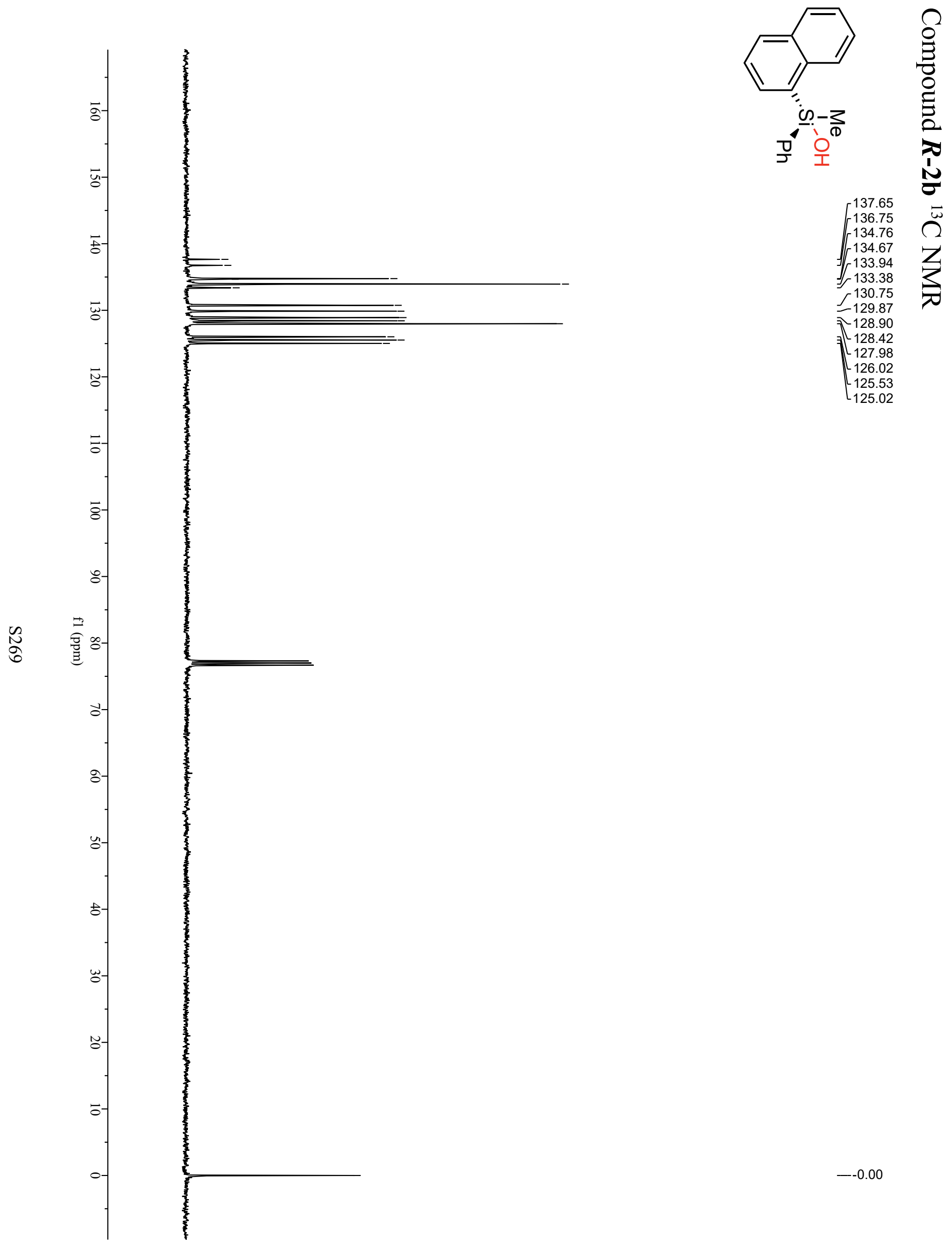
Compound 2c ${ }^{1} \mathrm{H}$ NMR
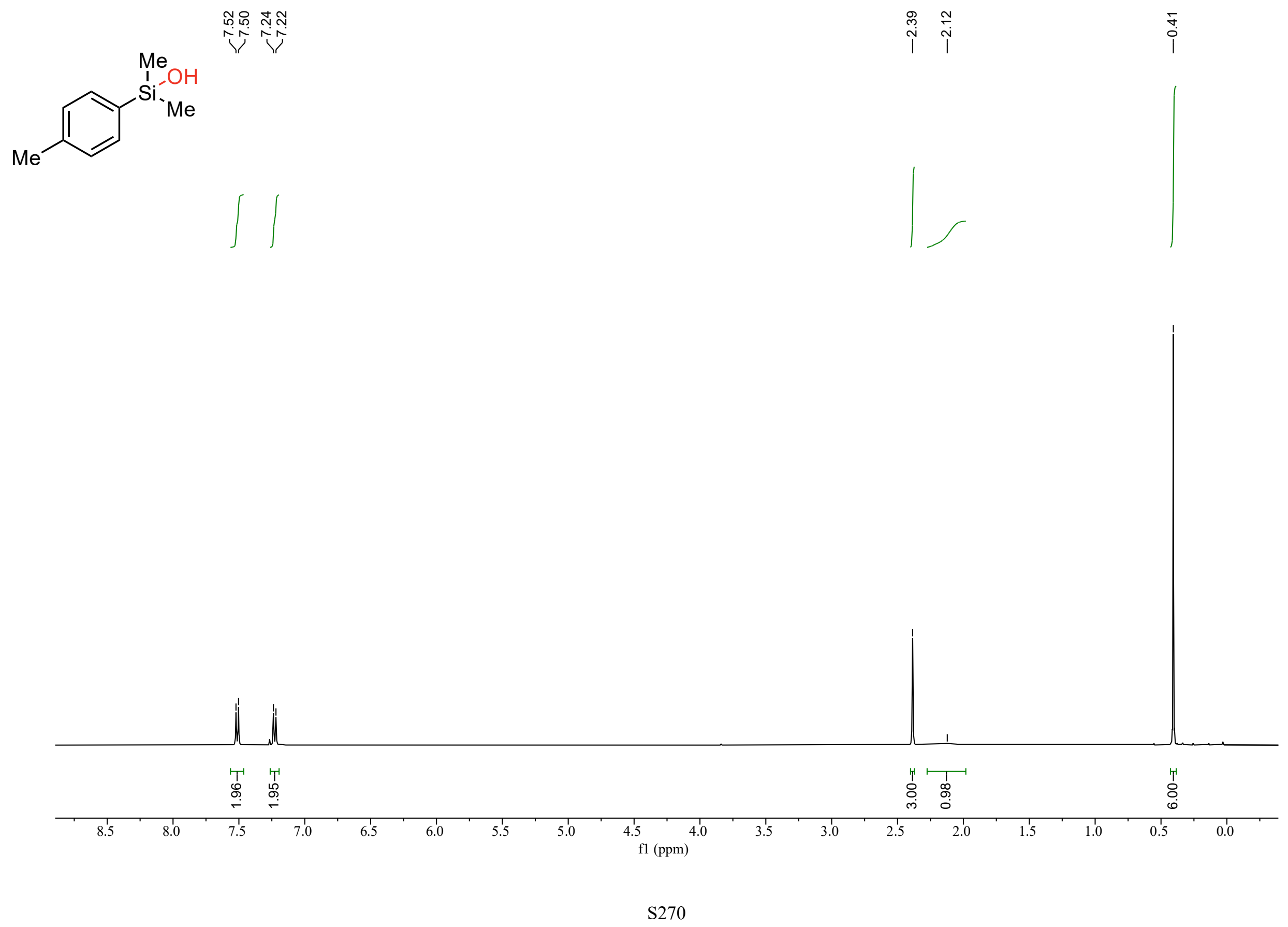
Compound 2c ${ }^{13} \mathrm{C}$ NMR

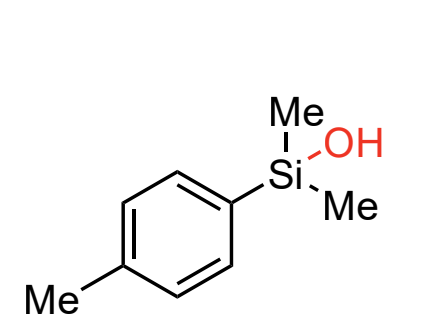

员战。

긴단

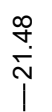

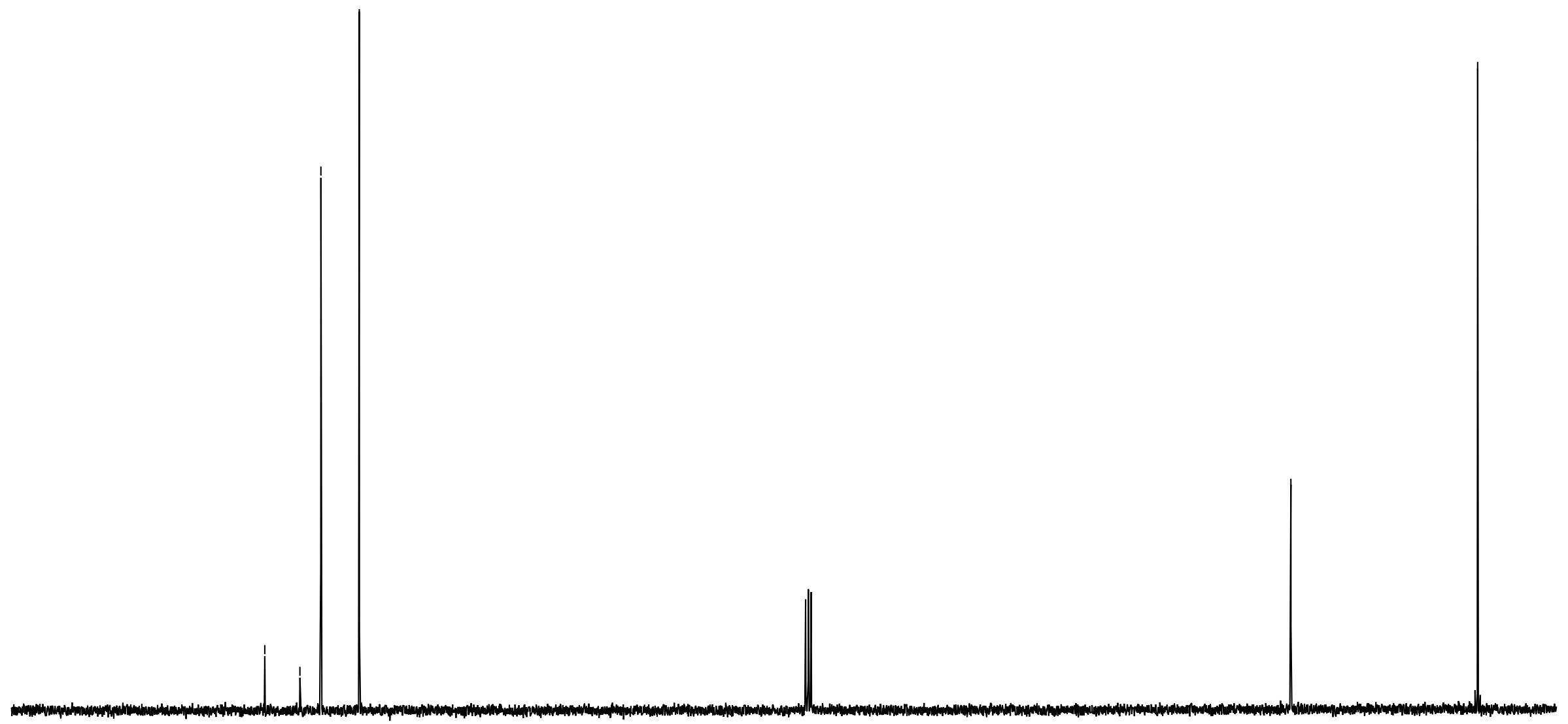


Compound 2d ${ }^{1} \mathrm{H}$ NMR

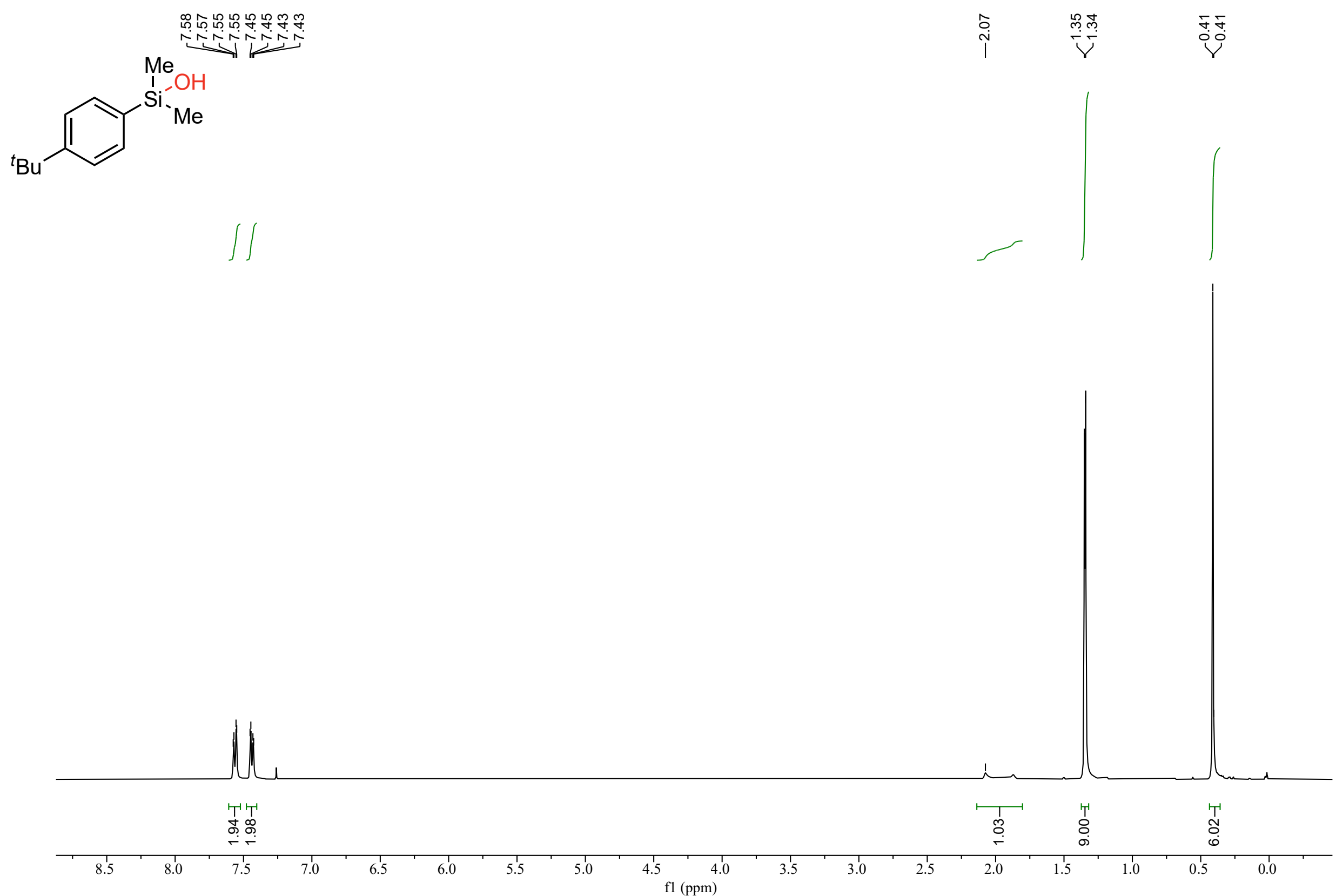


Compound 2d ${ }^{13} \mathrm{C}$ NMR

(1)

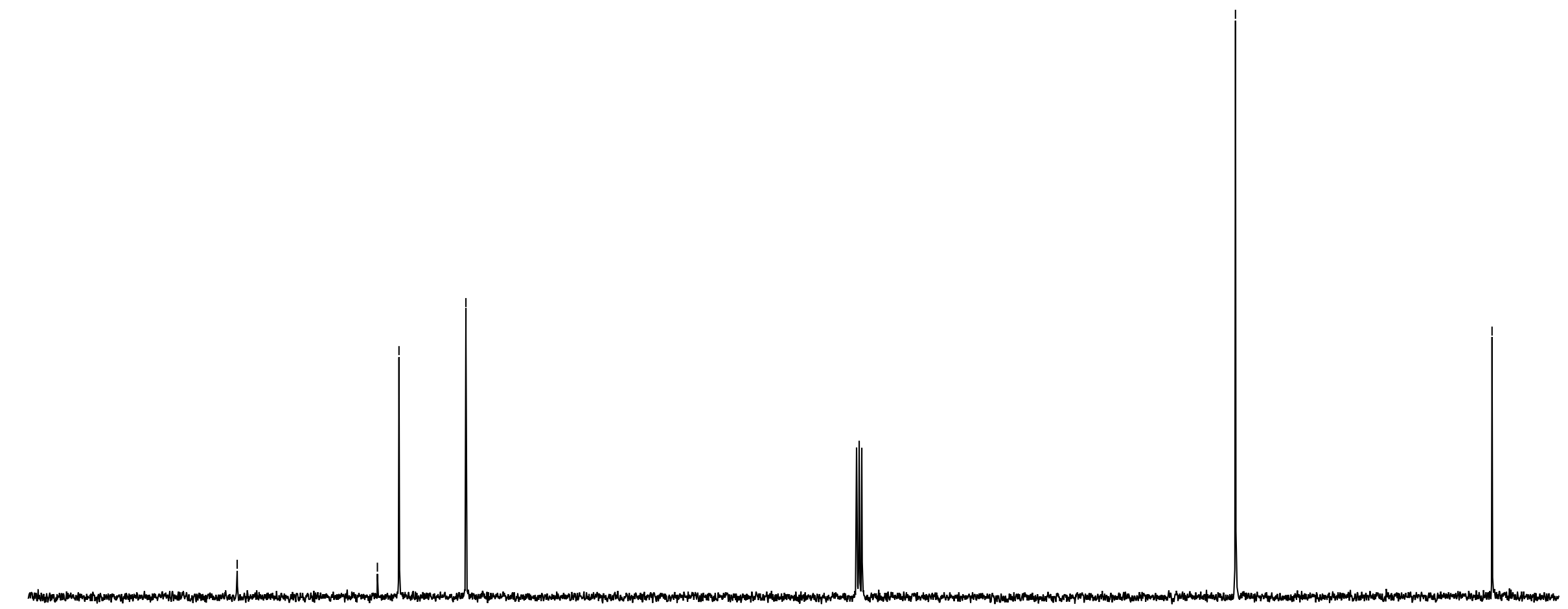




\section{Compound 2e ${ }^{1} \mathrm{H}$ NMR}
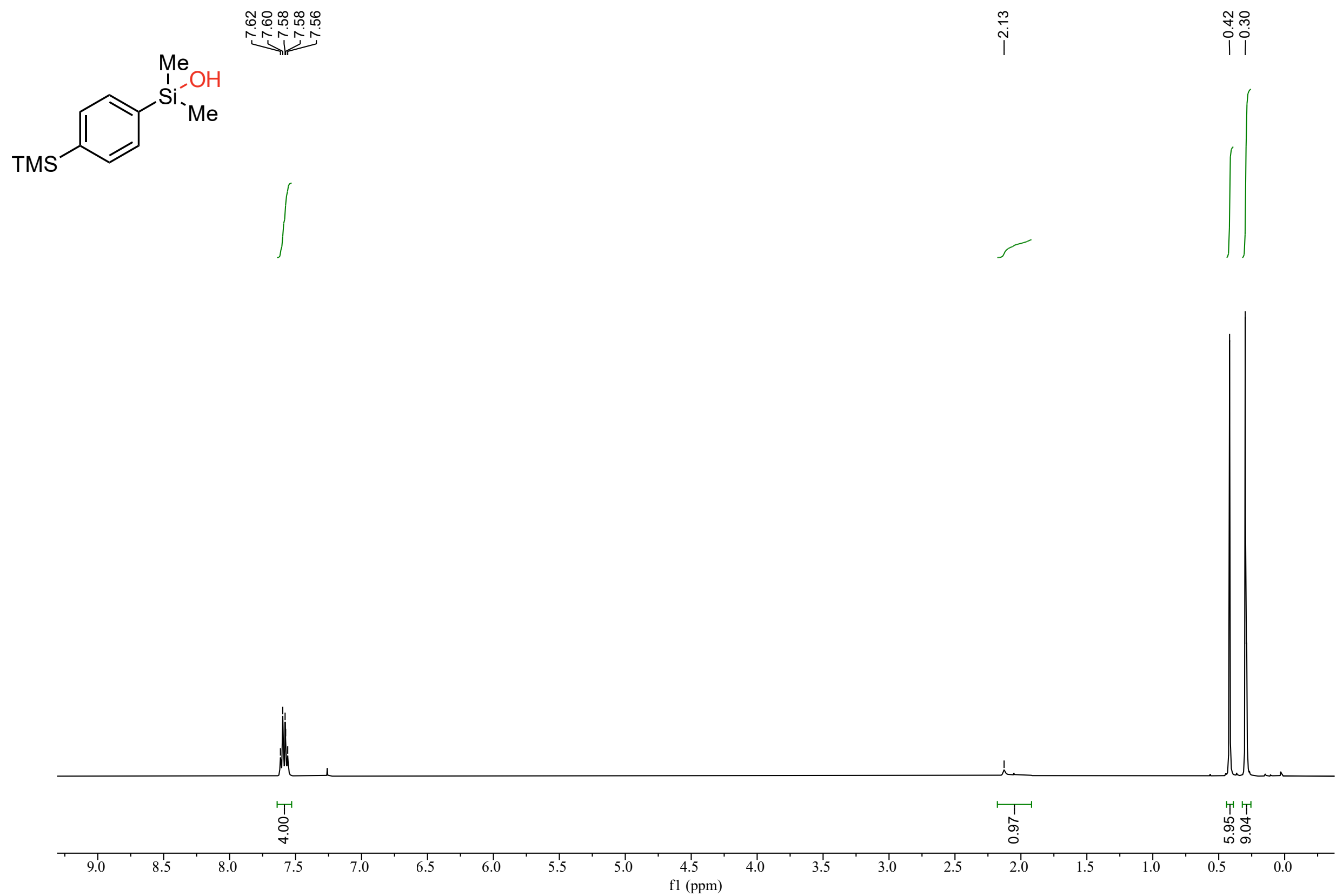
Compound 2e ${ }^{13} \mathrm{C}$ NMR

(1)
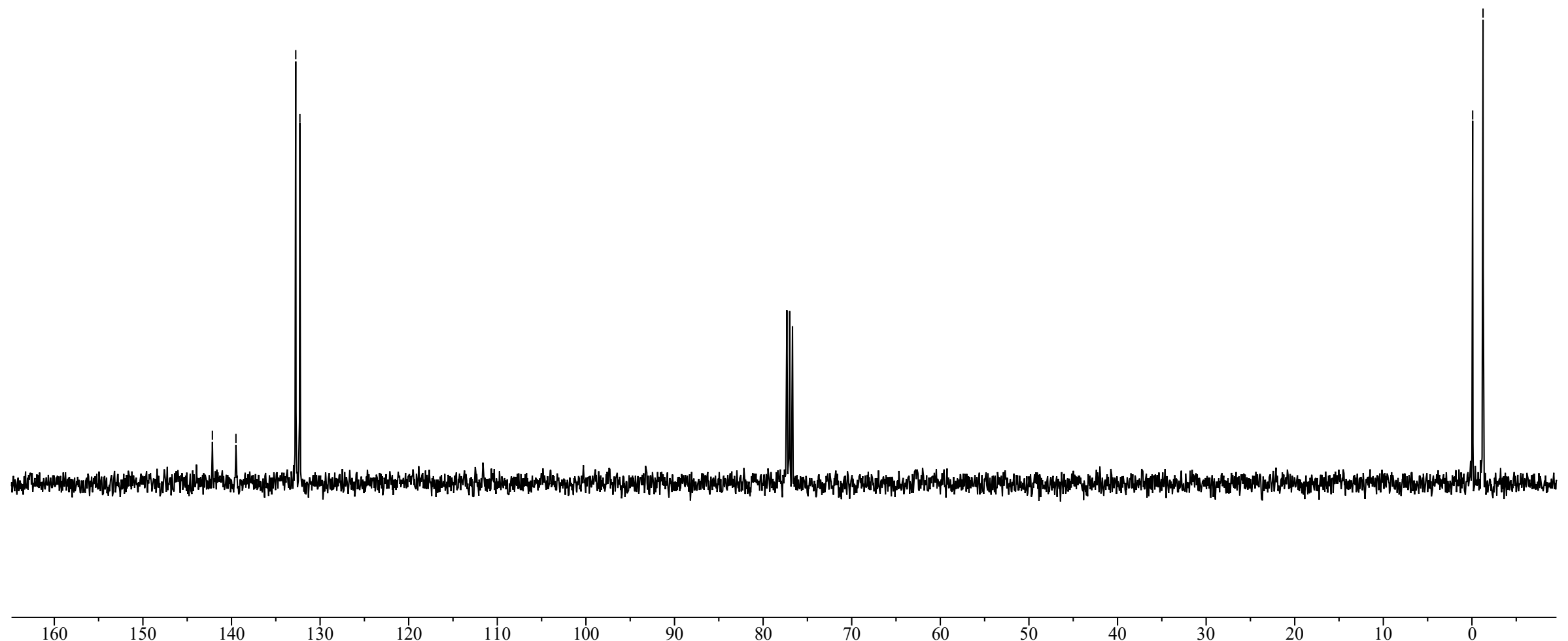


\section{Compound $2 \mathbf{f}^{1} \mathrm{H}$ NMR}
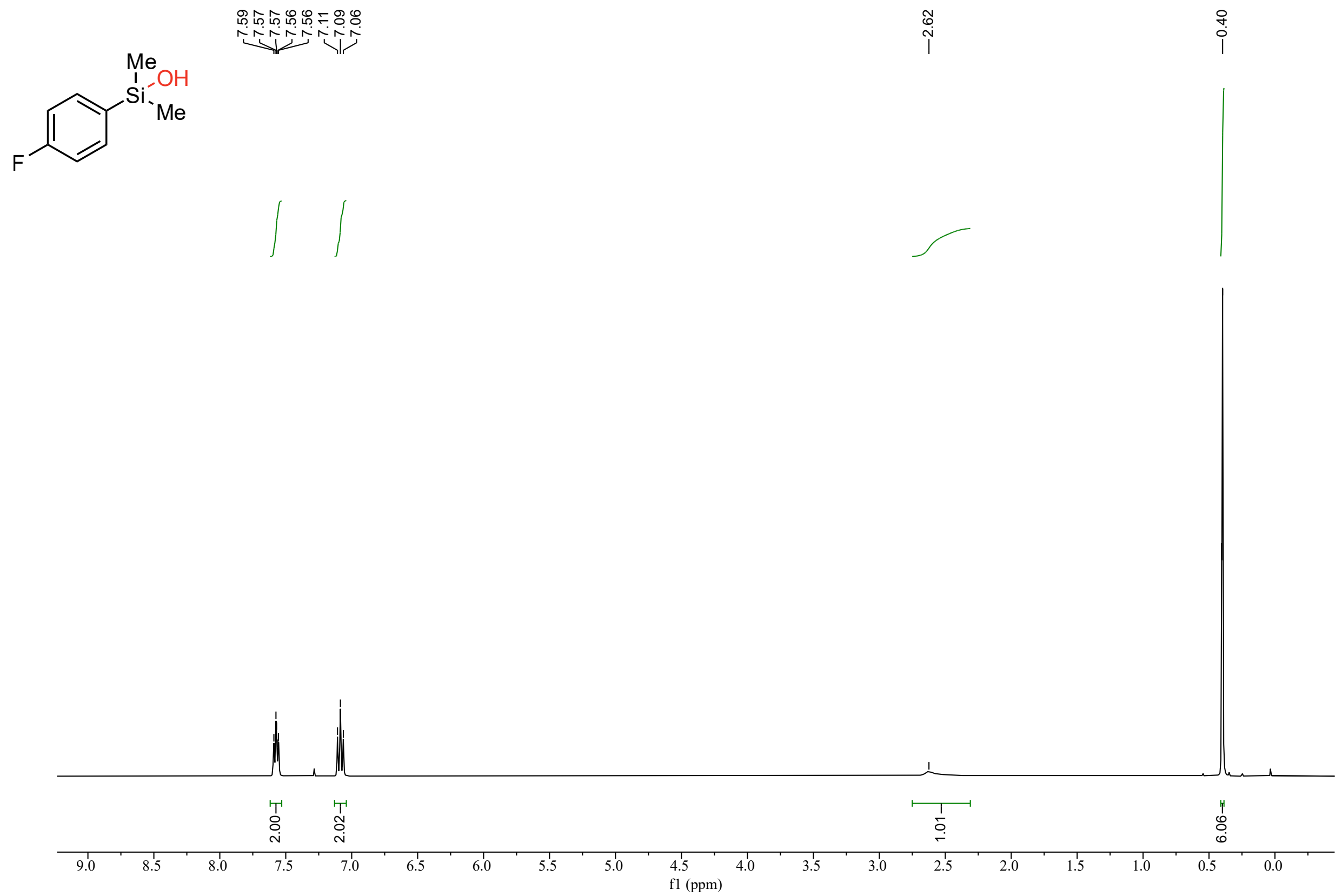
Compound $2 \mathrm{f}^{13} \mathrm{C}$ NMR
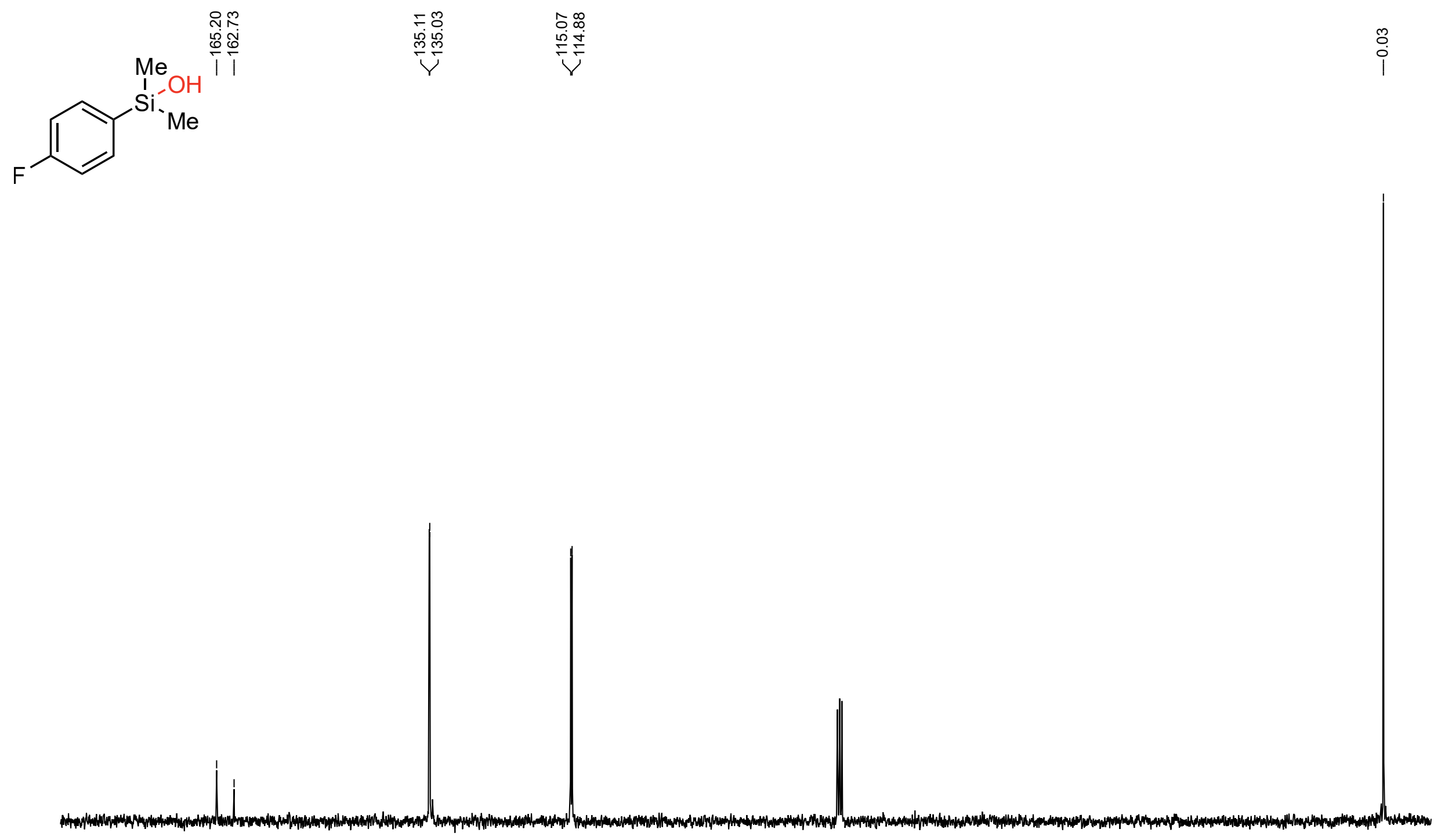
Compound $2 \mathbf{f f}^{19} \mathrm{~F}$ NMR<smiles>C[Si](O)(O)c1ccc(F)cc1</smiles>

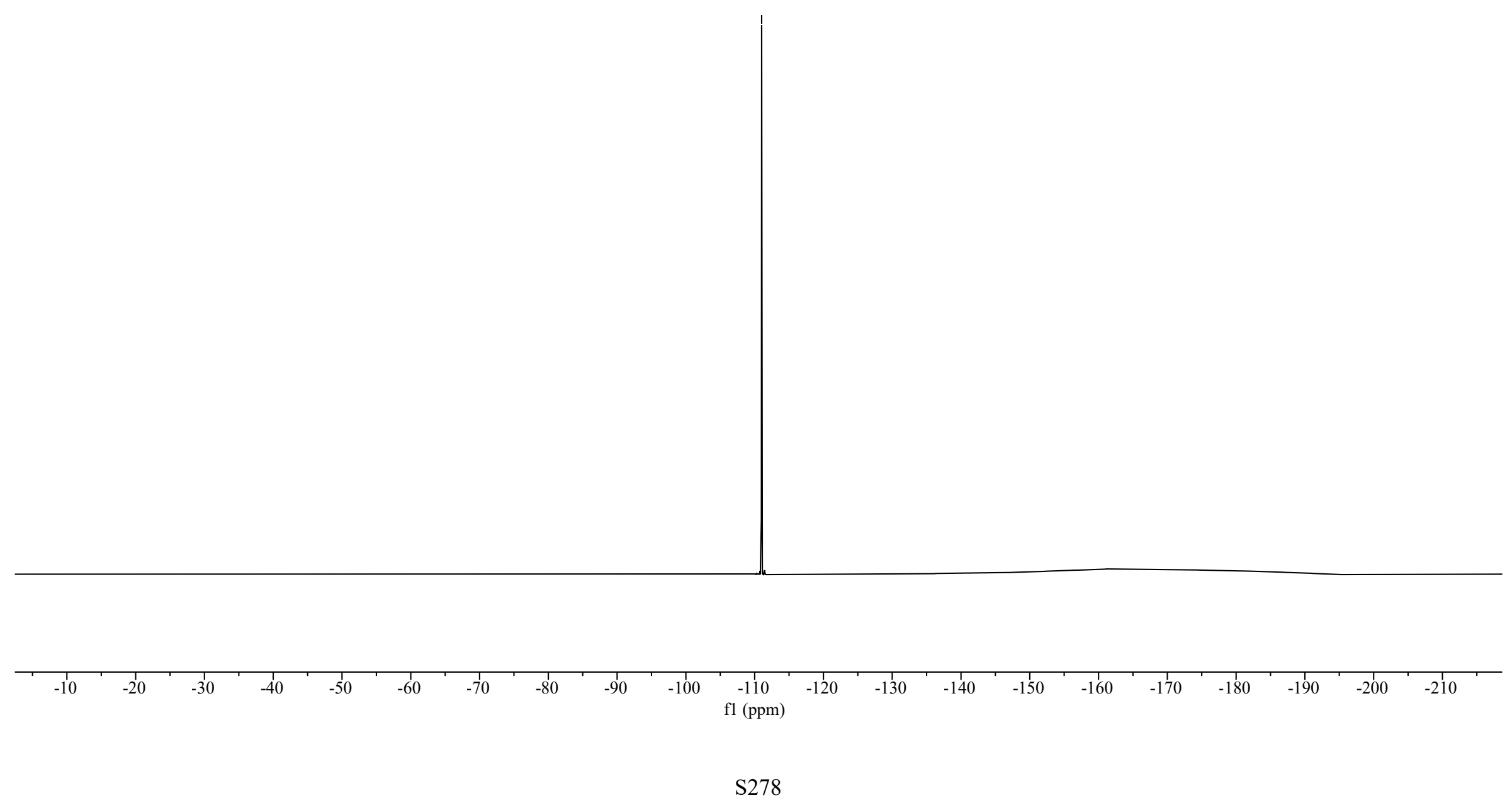


Compound 2g ${ }^{1} \mathrm{H}$ NMR
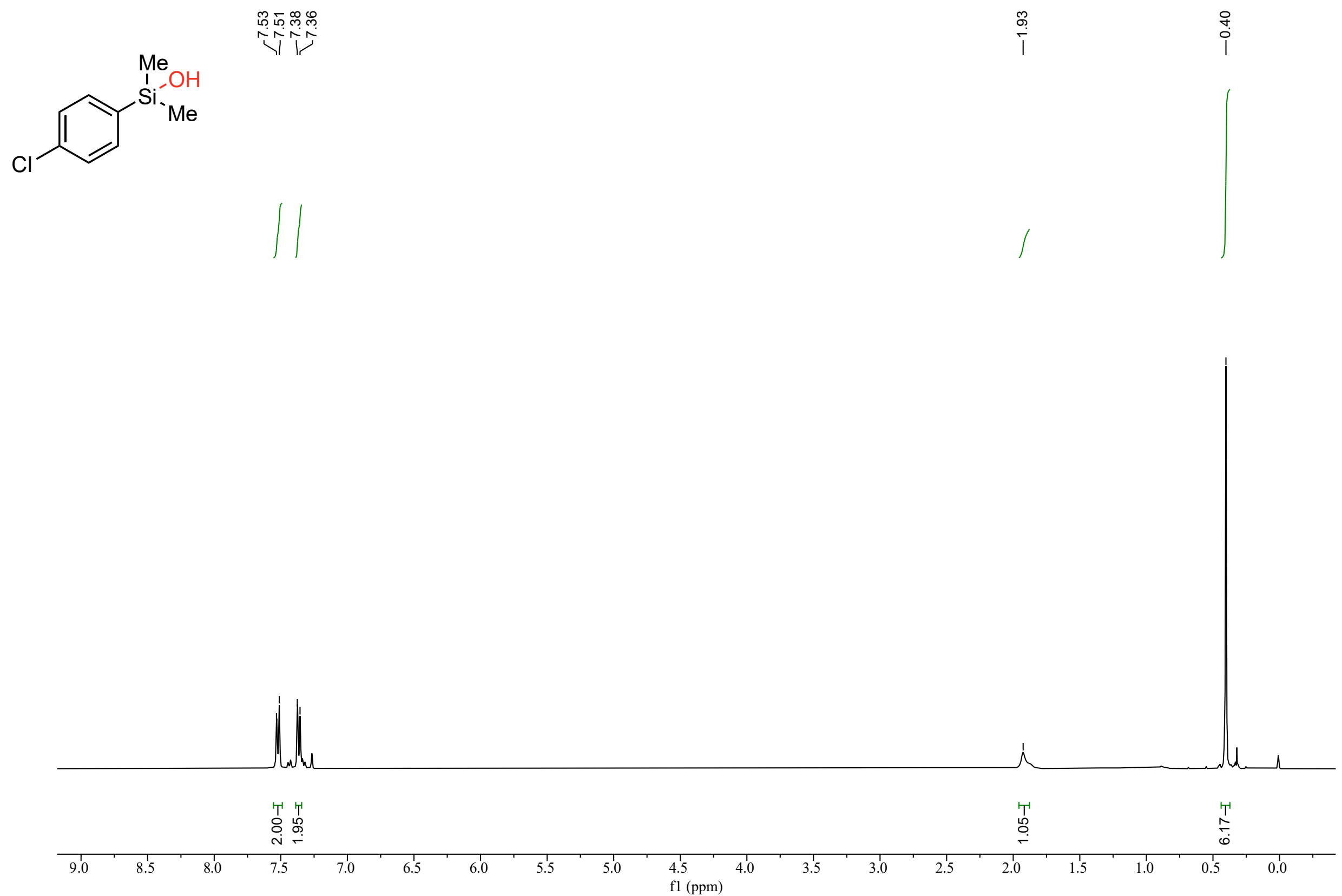
Compound 2g ${ }^{13} \mathrm{C}$ NMR

(1)

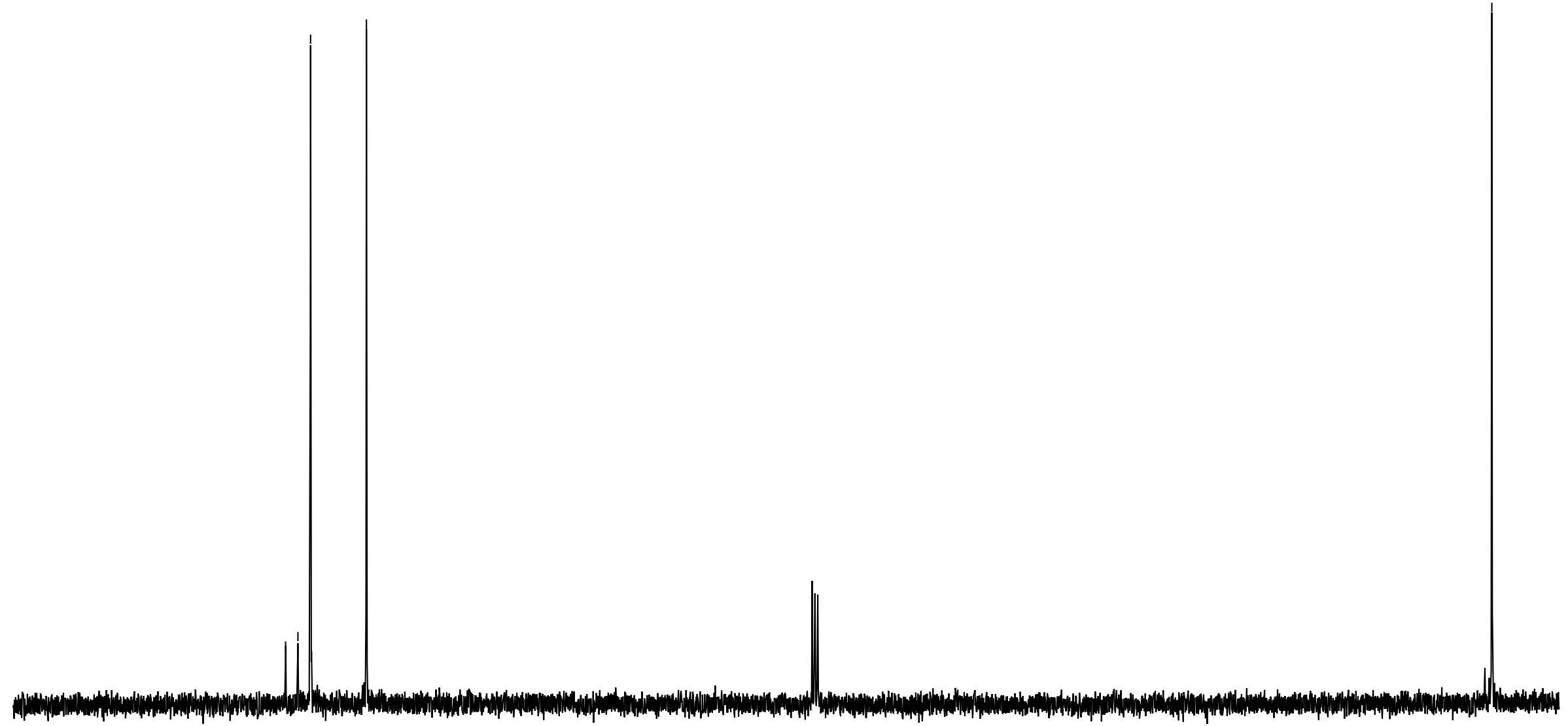


Compound $\mathbf{2 h}{ }^{1} \mathrm{H}$ NMR

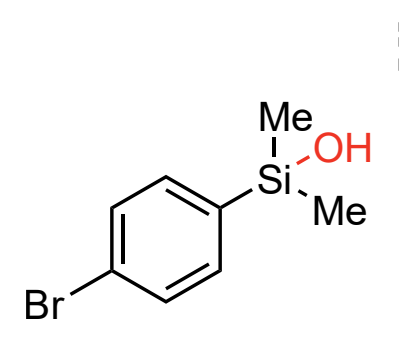

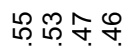

垈等

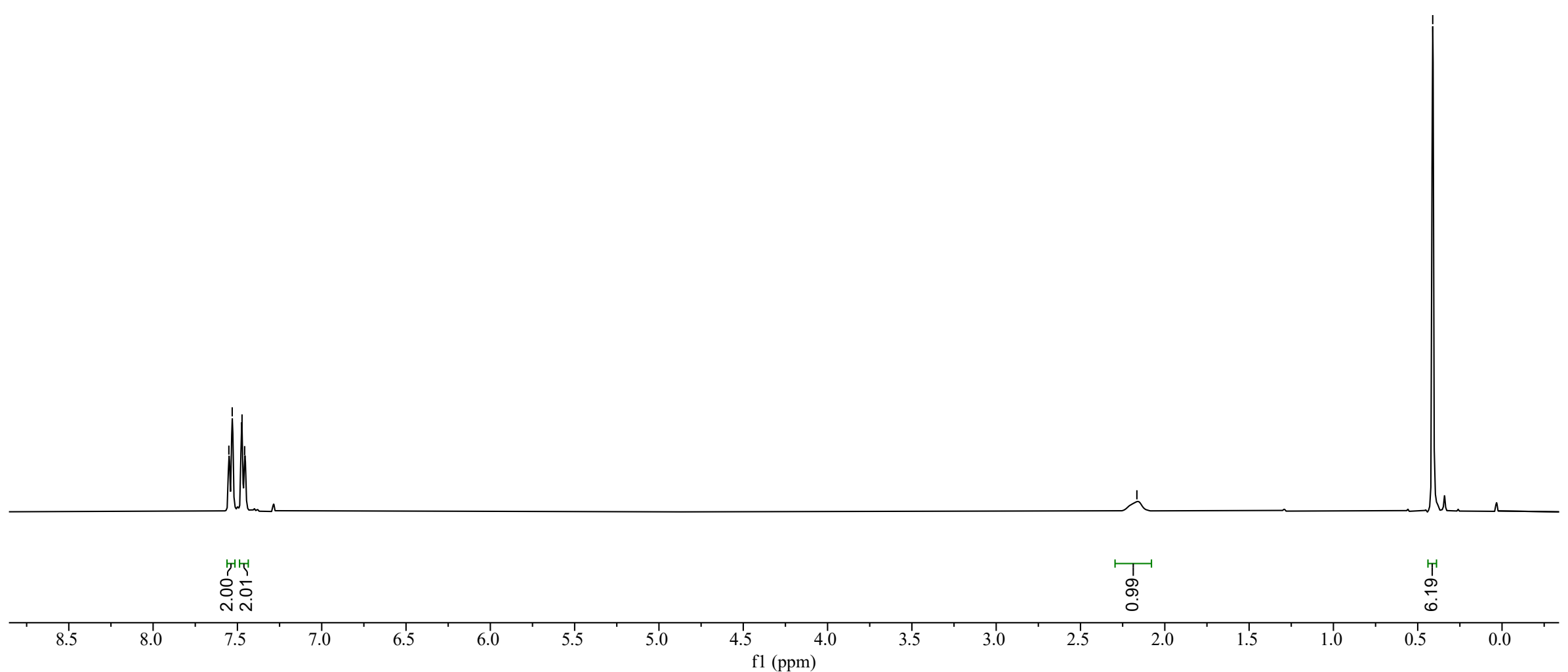


Compound $\mathbf{2 h}{ }^{13} \mathrm{C}$ NMR

(1)

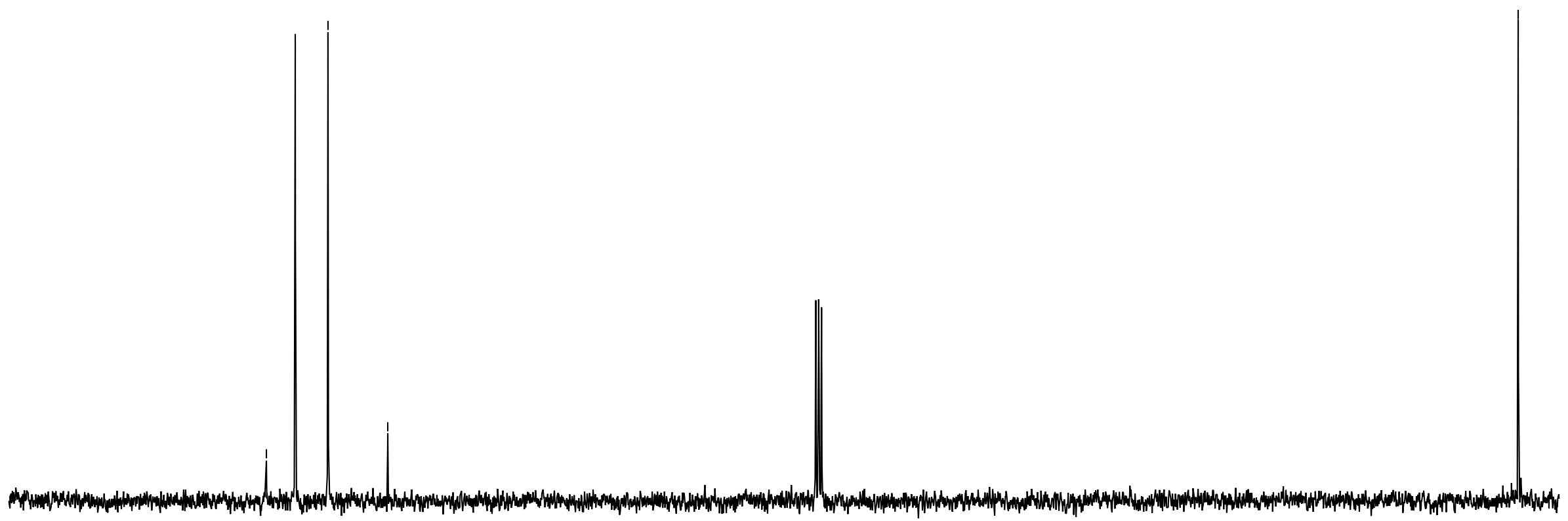


Compound $2 \mathbf{i}^{1} \mathrm{H}$ NMR

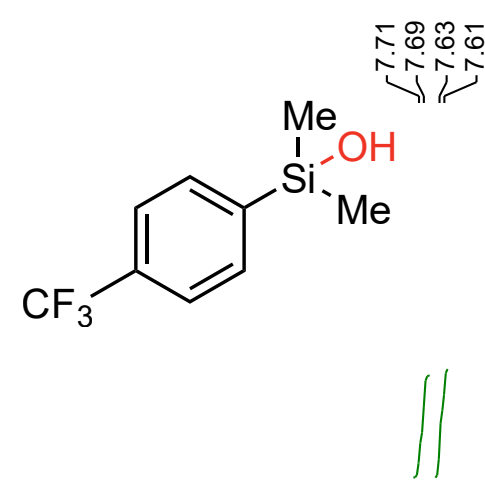

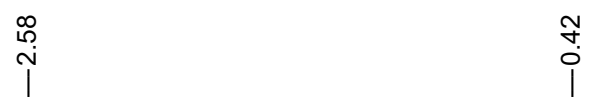
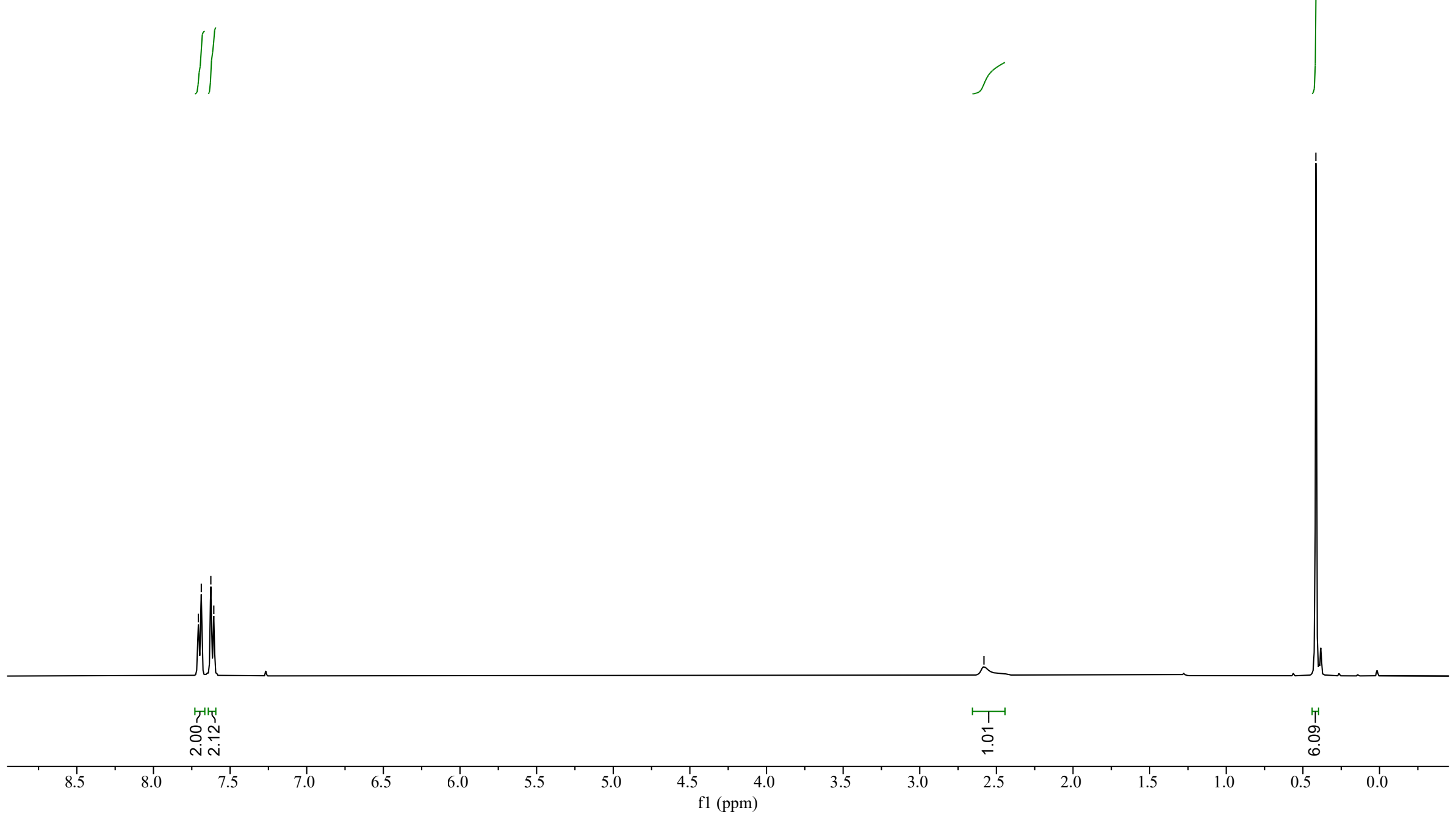


$$
E
$$


Compound $2 \mathbf{i}{ }^{19}$ F NMR<smiles>O[Si](O)([Mg])c1ccc(C(F)(F)F)cc1</smiles>

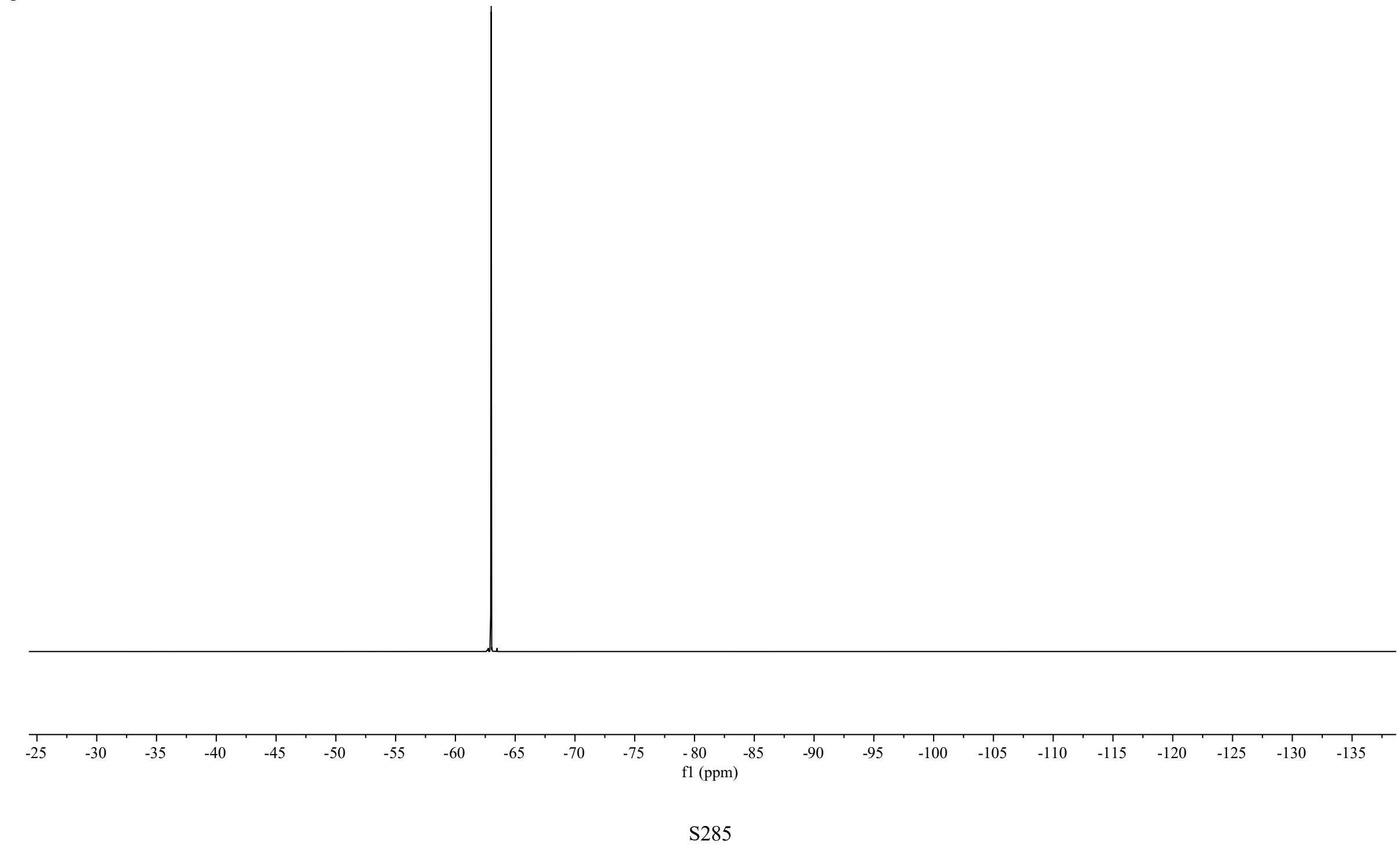




\section{Compound $\mathbf{2 j}{ }^{1} \mathrm{H}$ NMR}
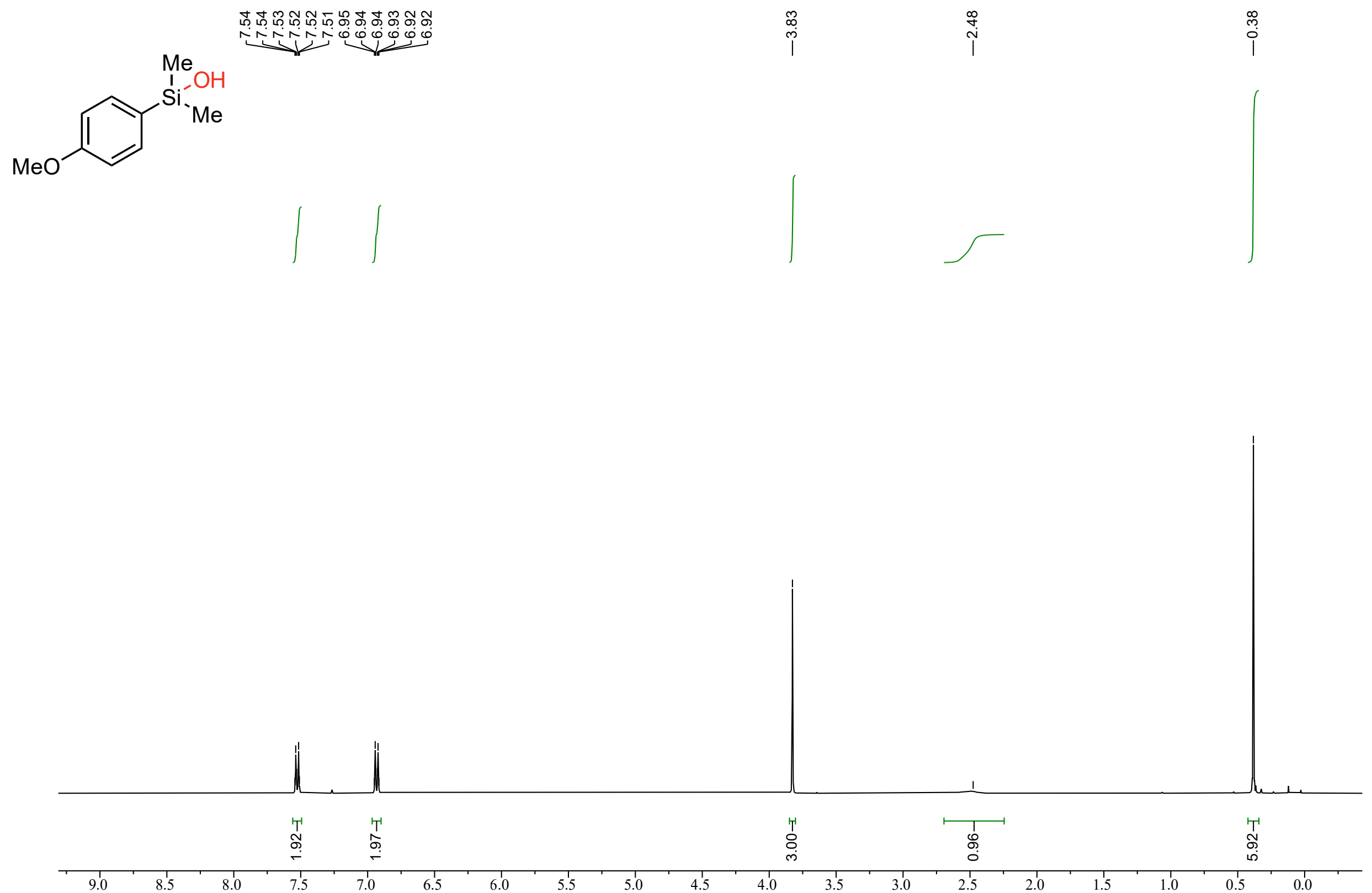
Compound $\mathbf{2 j}{ }^{13} \mathrm{C}$ NMR

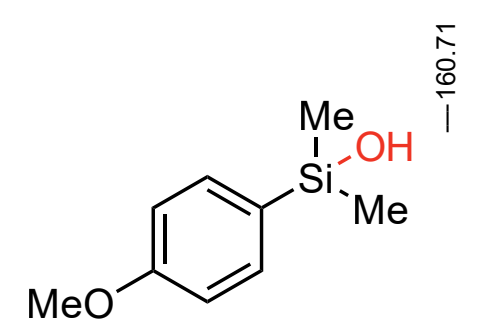

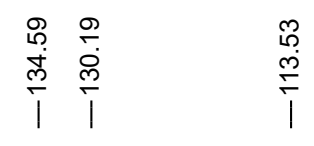

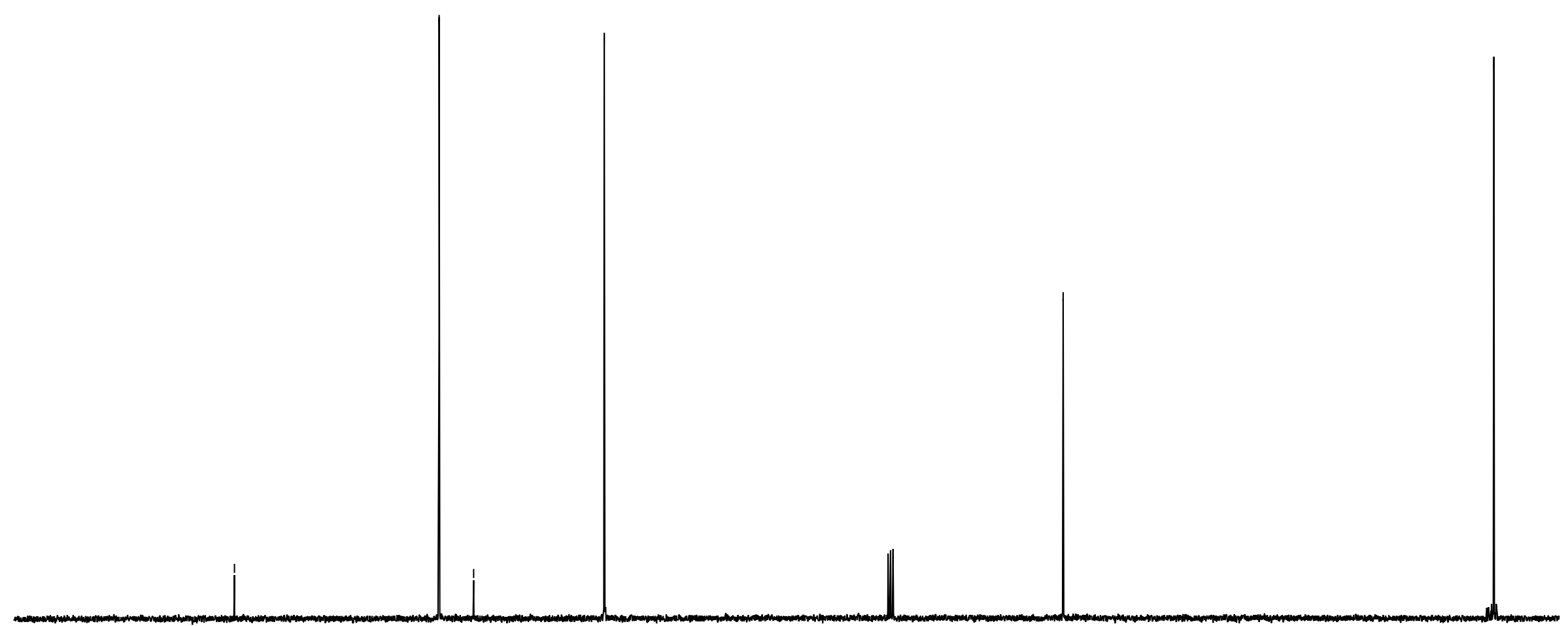




\section{Compound 2k ${ }^{1} \mathrm{H}$ NMR}
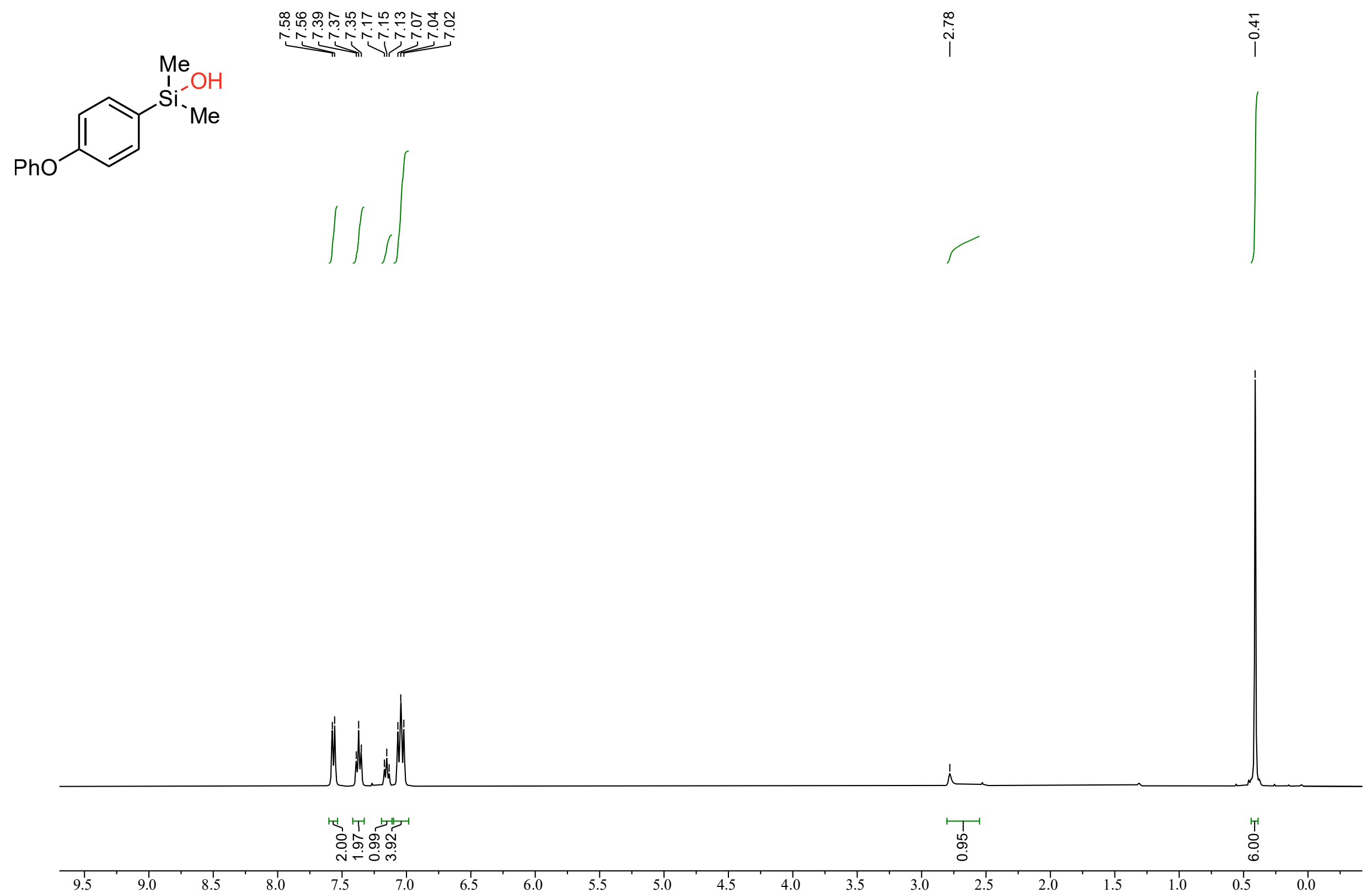
Compound $2 \mathbf{k}{ }^{13} \mathrm{C}$ NMR

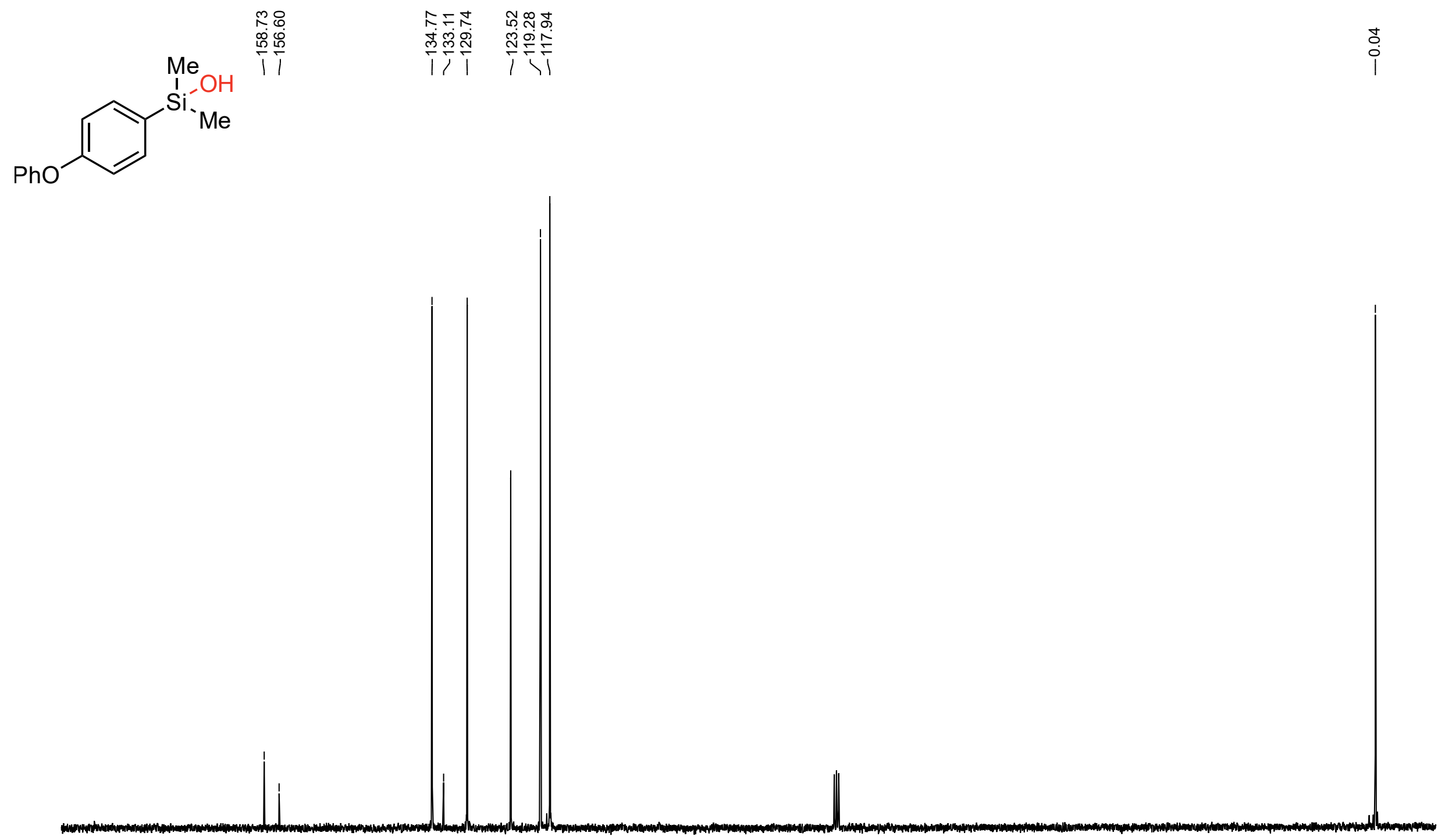




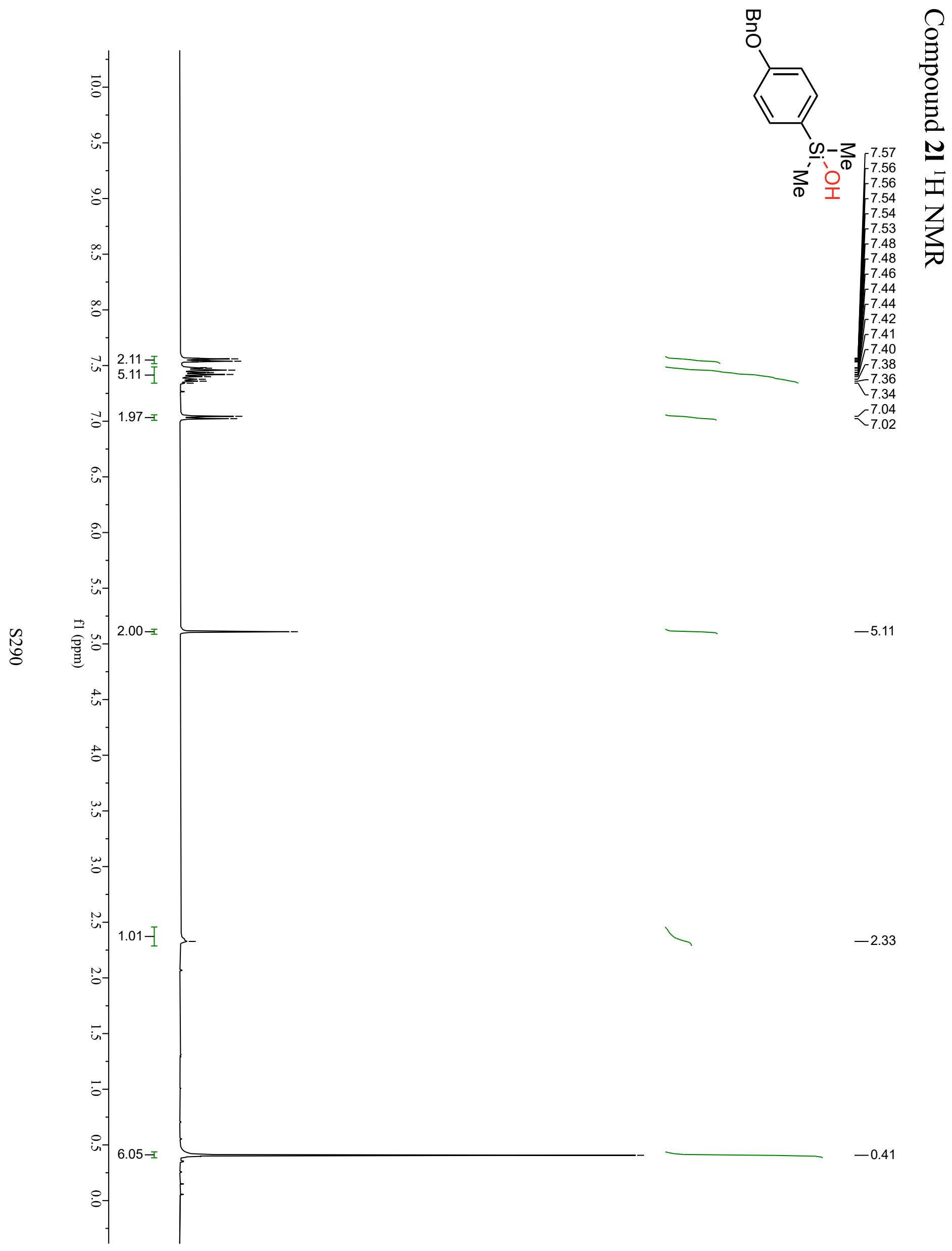


Compound 2I ${ }^{13} \mathrm{C}$ NMR

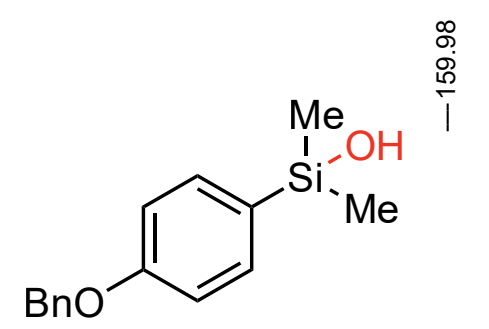

$\mathrm{BnO}$
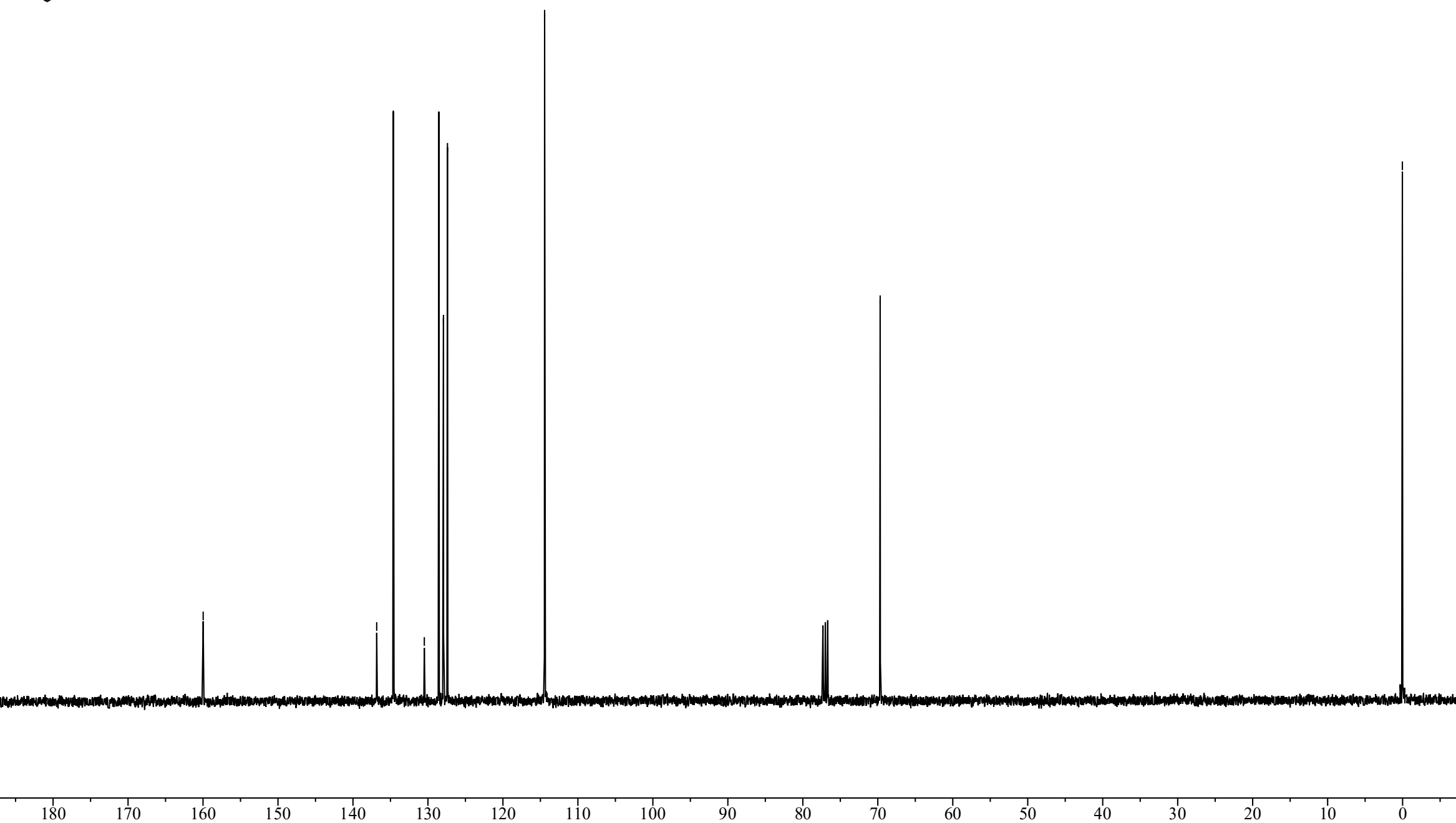


\section{Compound 2m ${ }^{1} \mathrm{H}$ NMR}

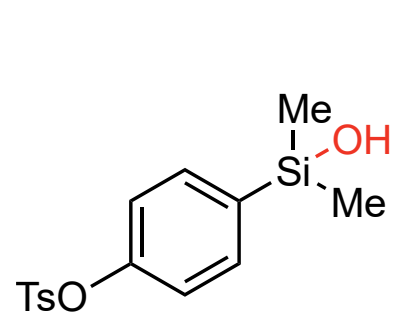

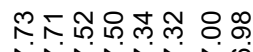

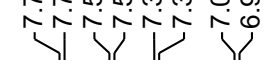

ช

i

) 111
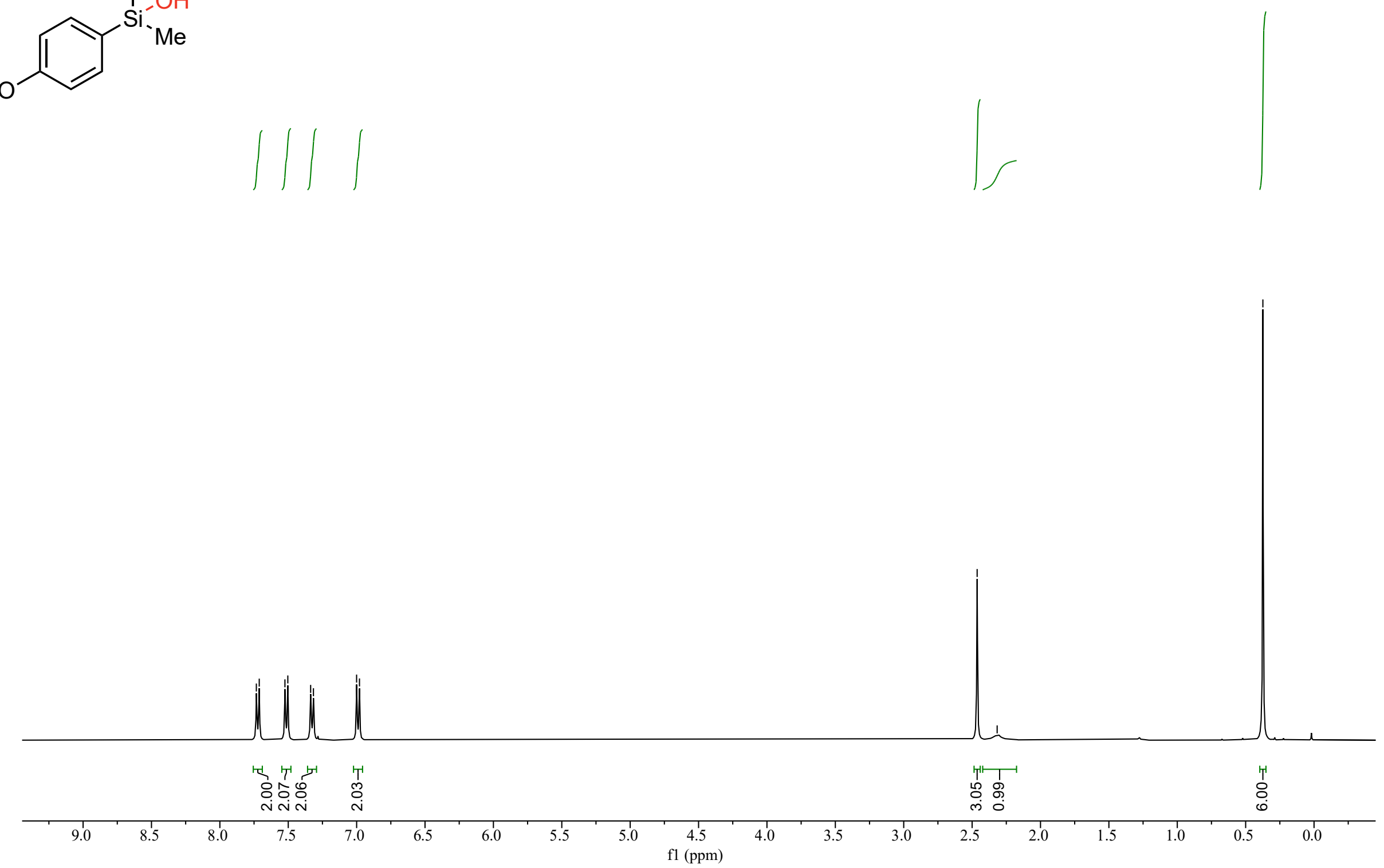


\section{Compound $2 \mathrm{~m}{ }^{13} \mathrm{C}$ NMR}
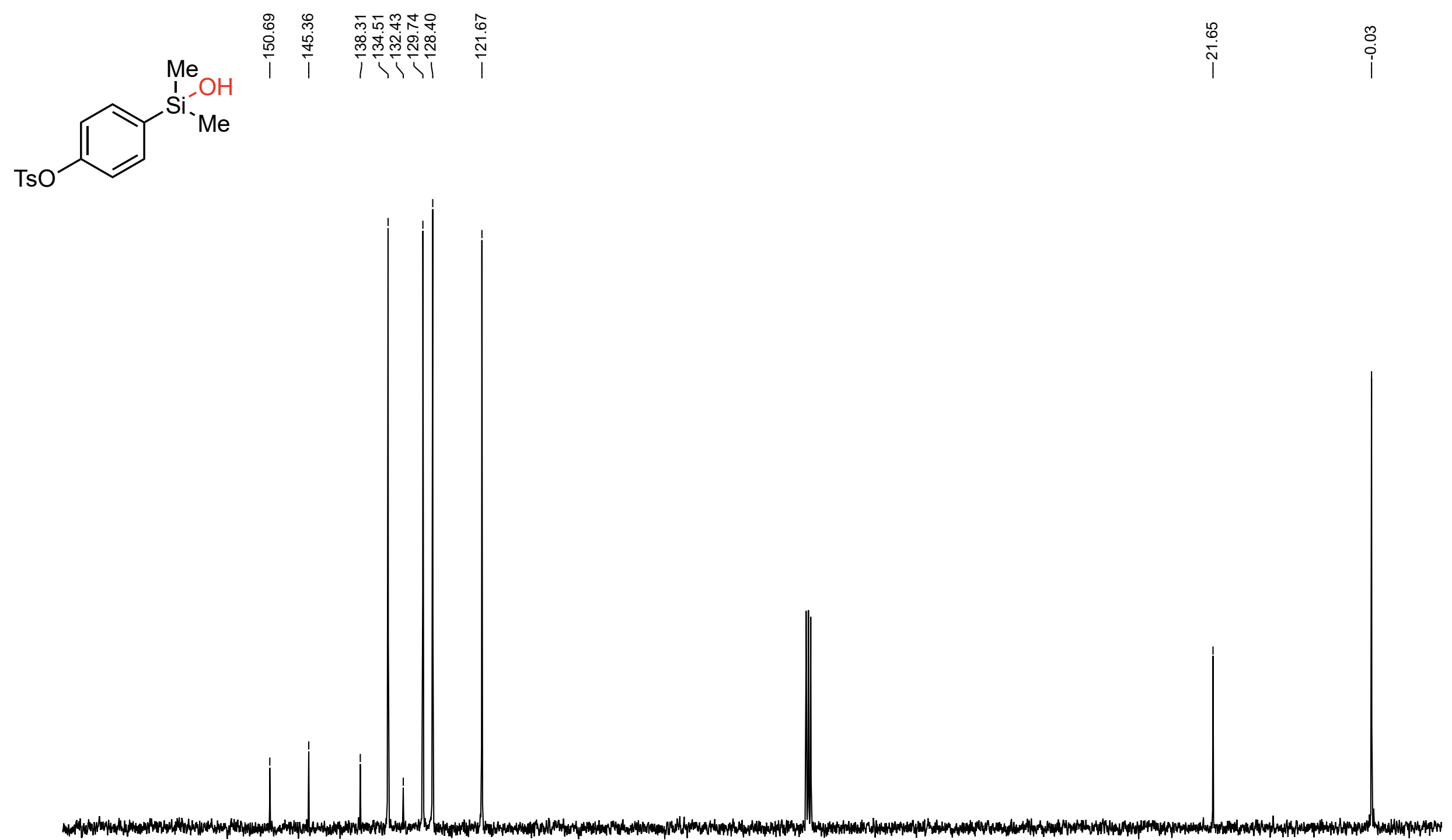
Compound 2n ${ }^{1} \mathrm{H}$ NMR
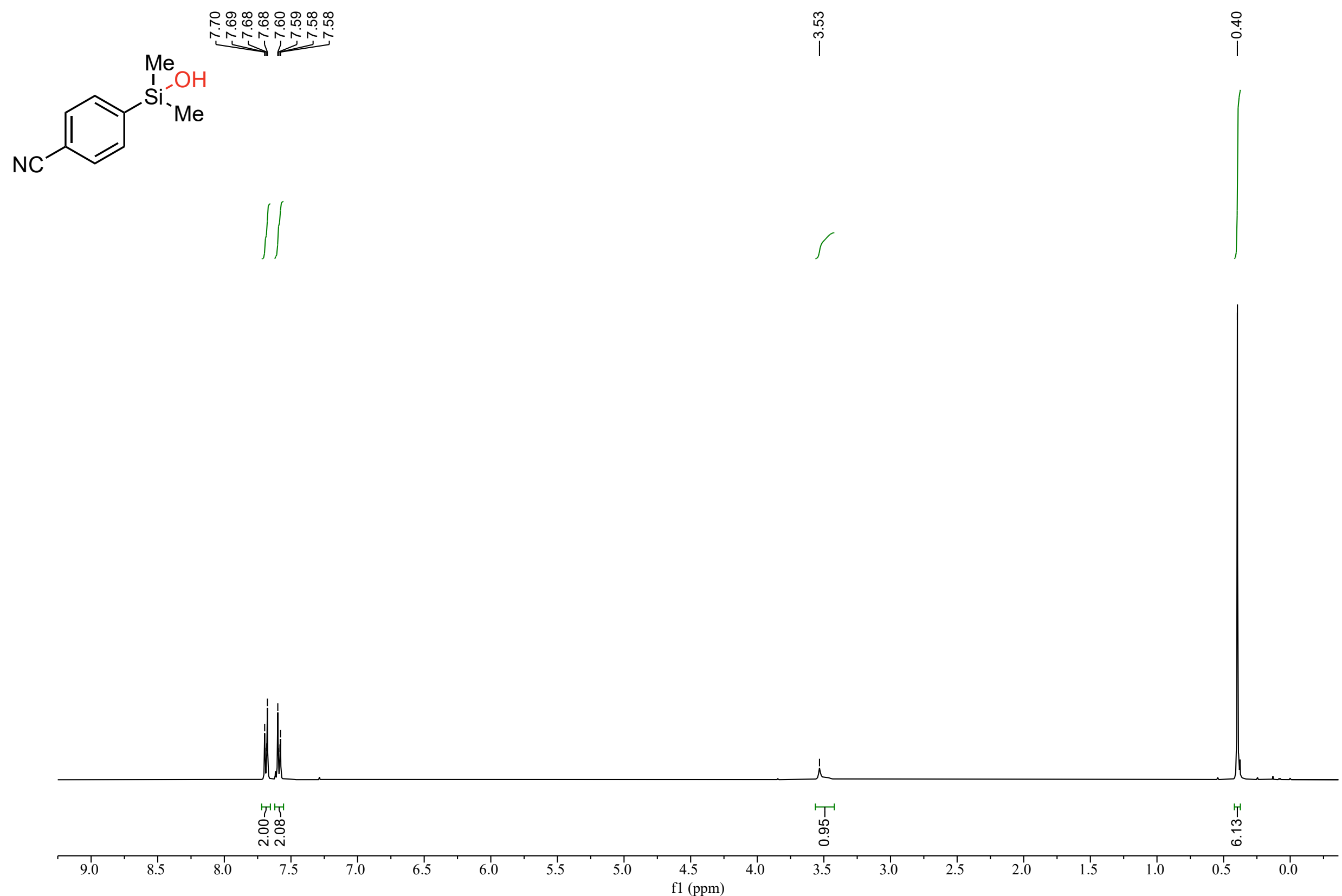
Compound 2n ${ }^{13} \mathrm{C}$ NMR
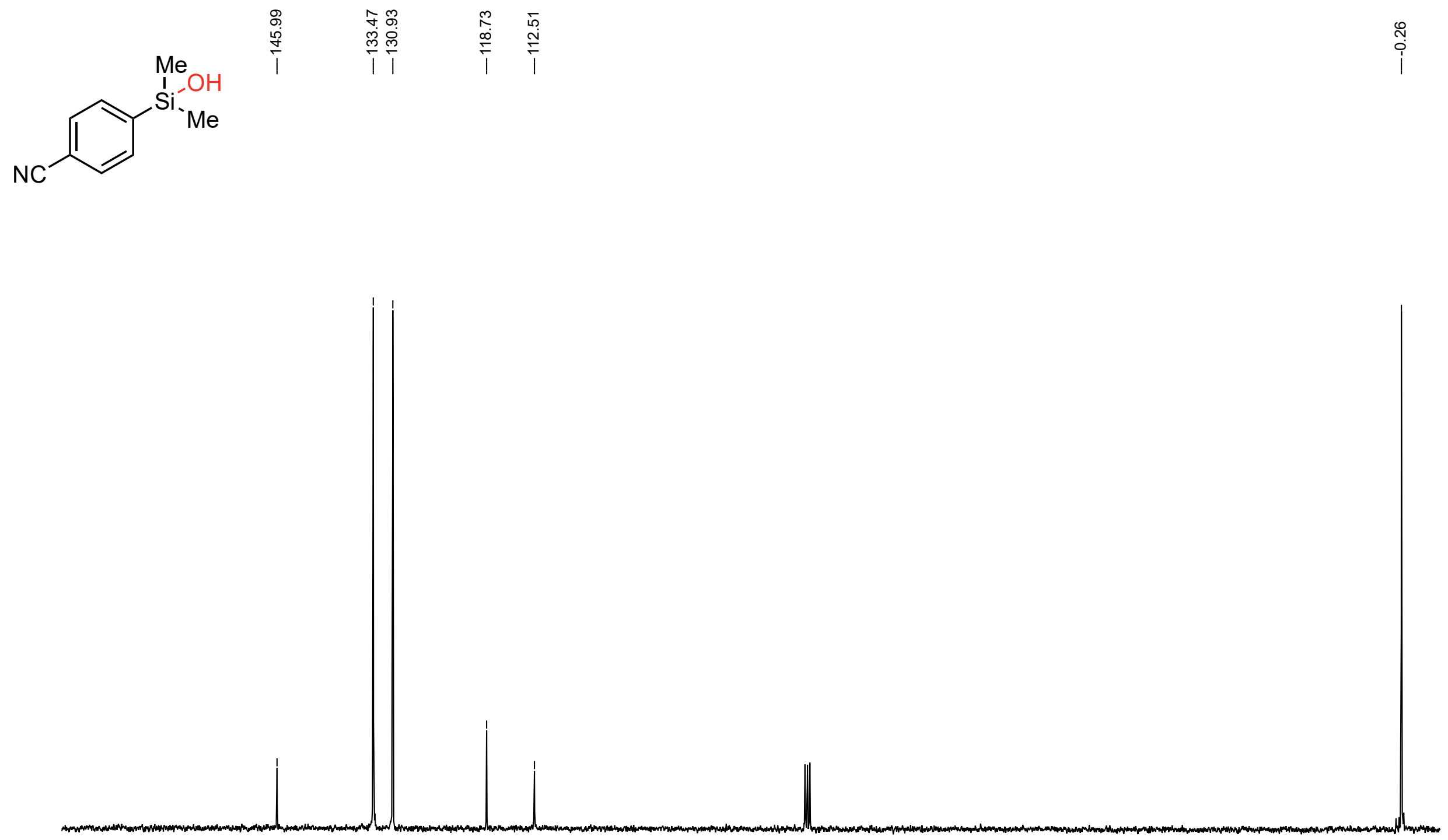
Compound 2o ${ }^{1} \mathrm{H}$ NMR

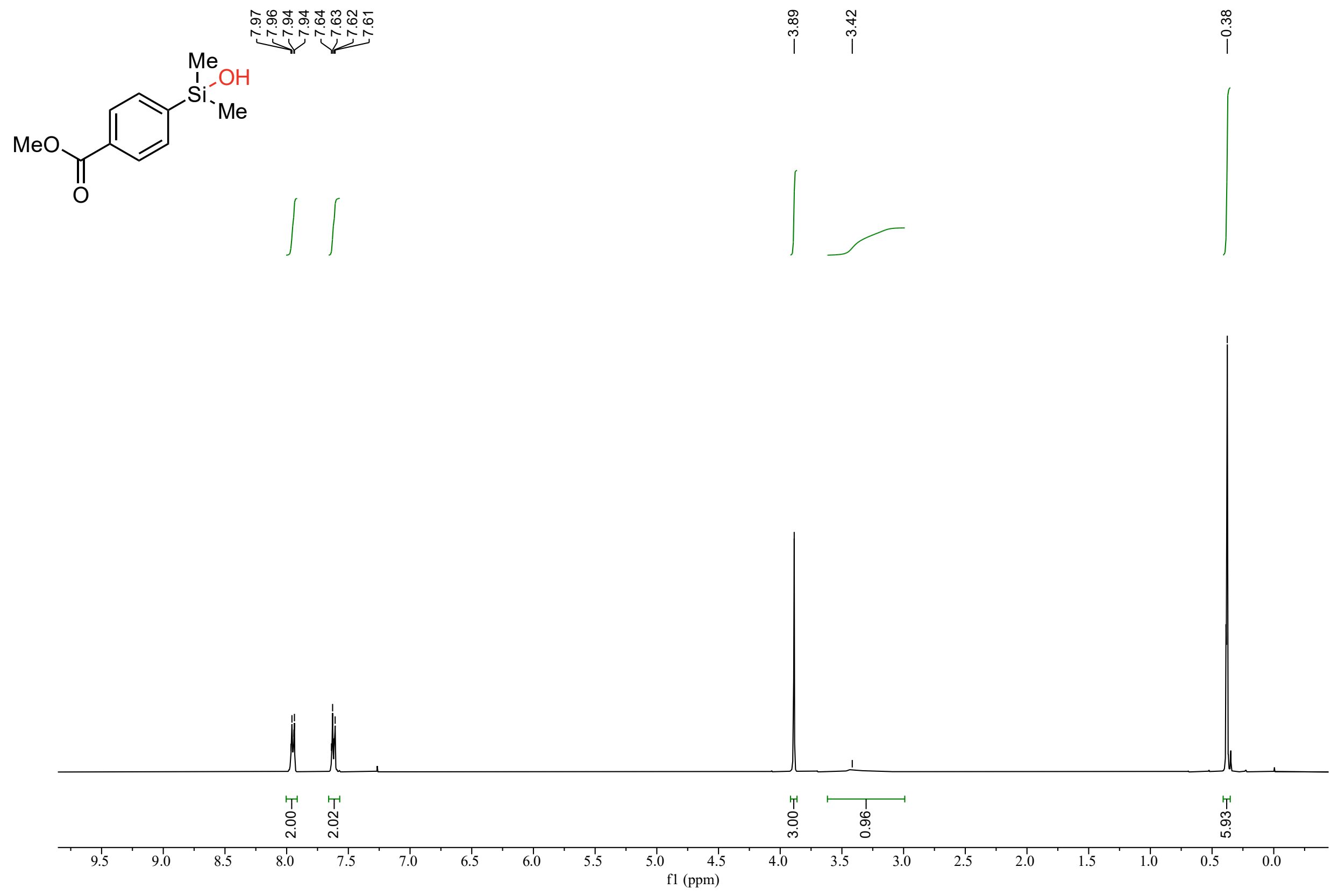


Compound 2o ${ }^{13} \mathrm{C}$ NMR

(1)

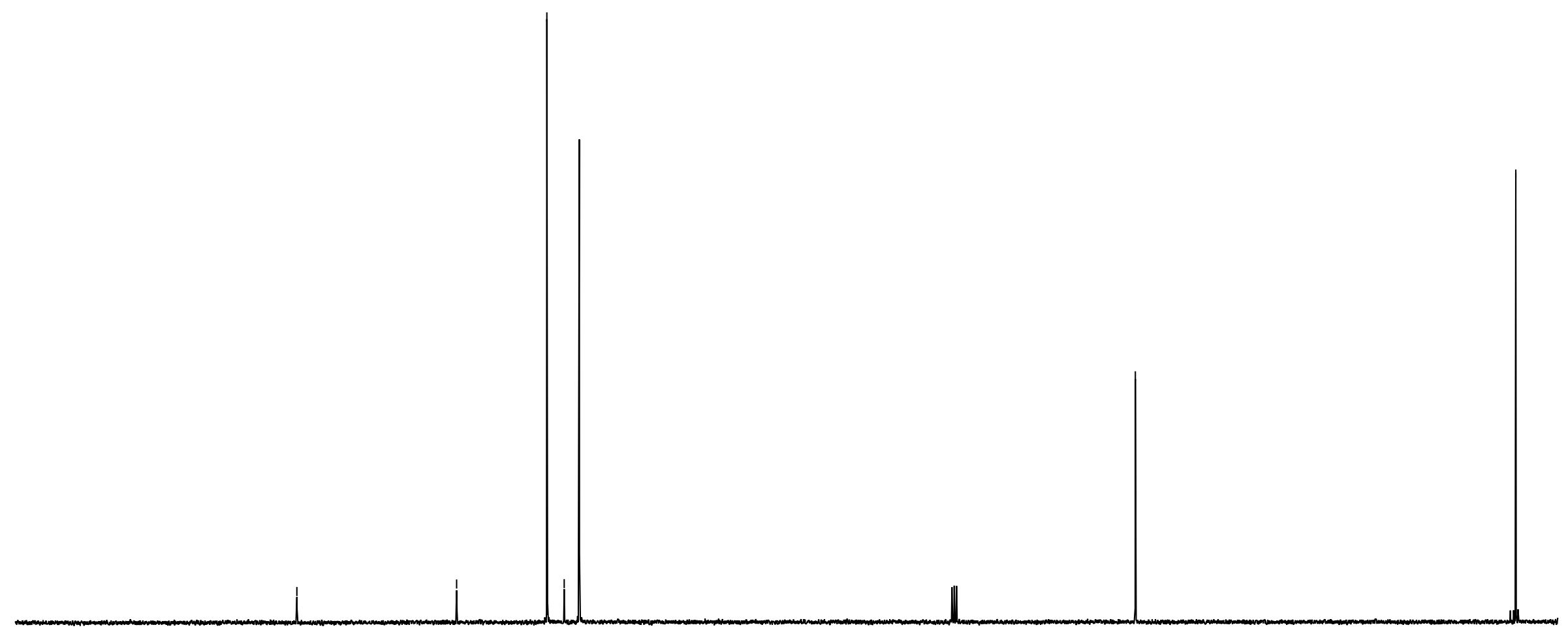




\section{Compound 2p ${ }^{1} \mathrm{H}$ NMR}

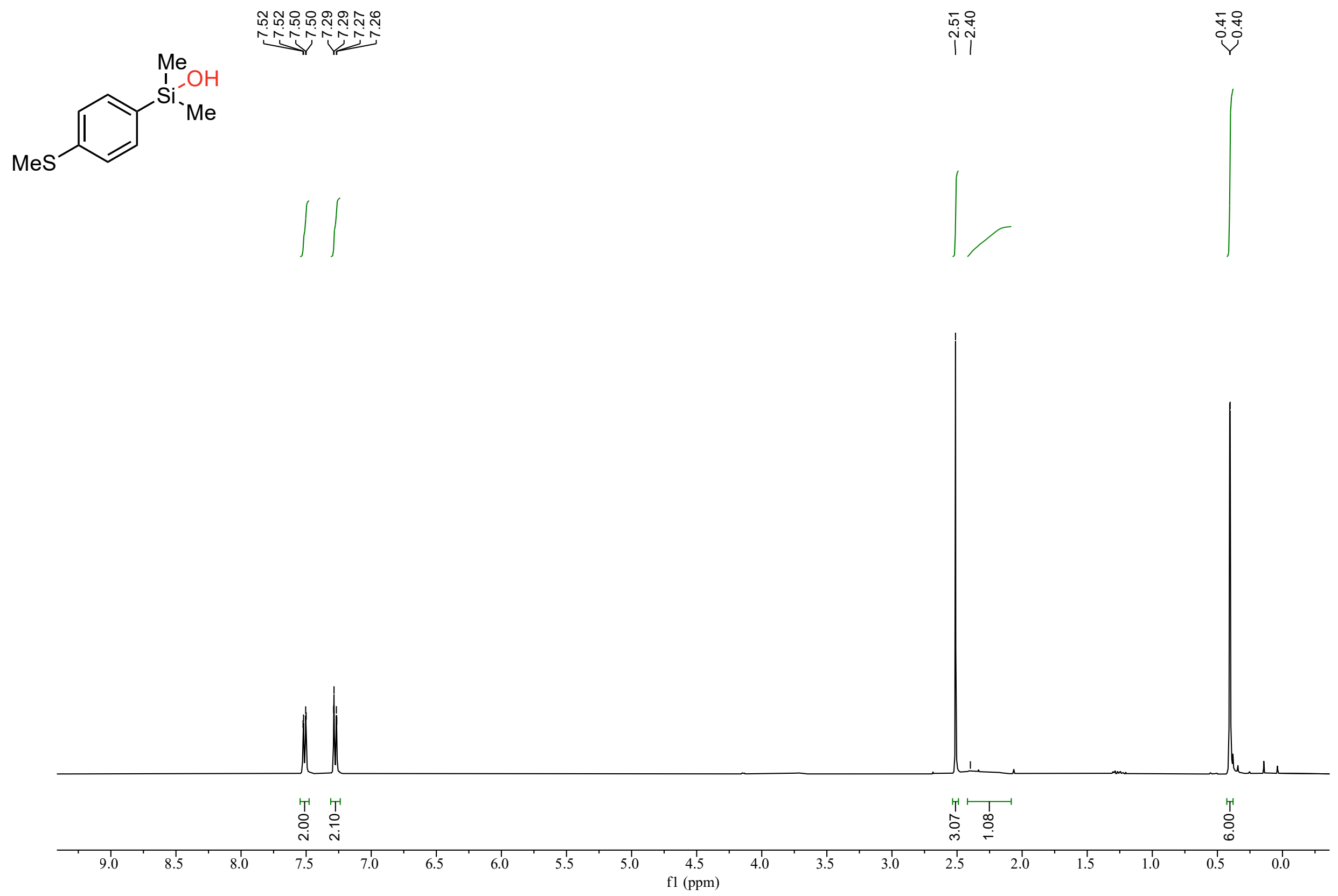


Compound 2p ${ }^{13} \mathrm{C}$ NMR

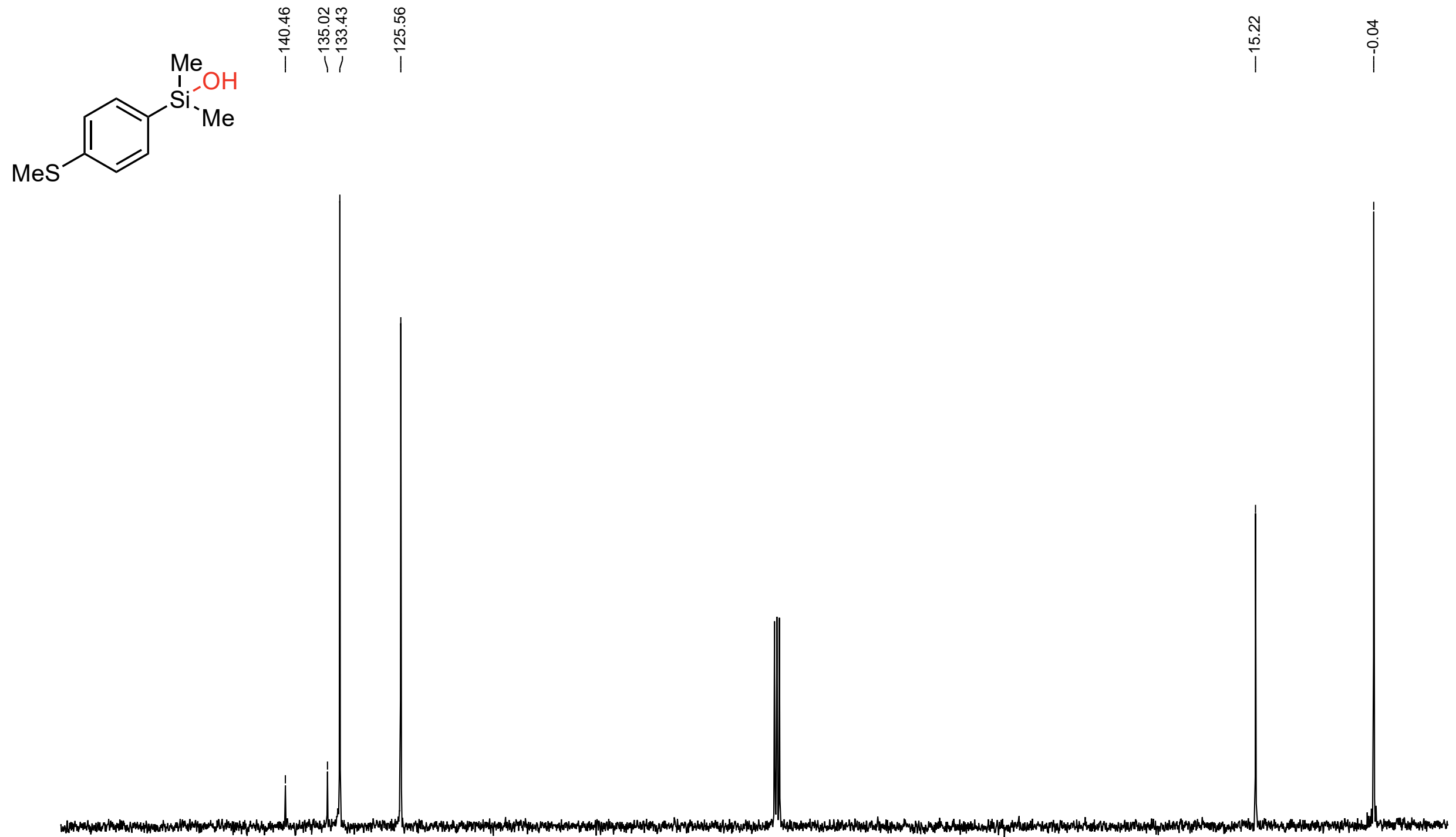


Compound aq ${ }^{1} \mathrm{H}$ NMR<smiles>CN(C)c1ccc([Si](C)(C)O)cc1</smiles>
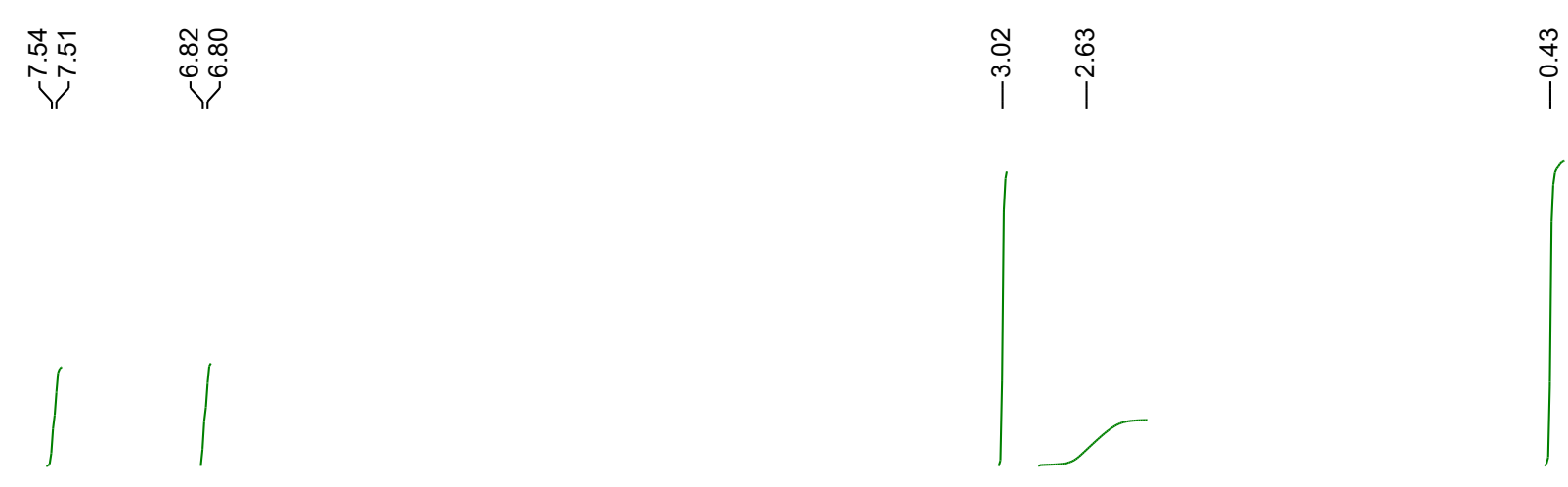

$\stackrel{N}{i}$

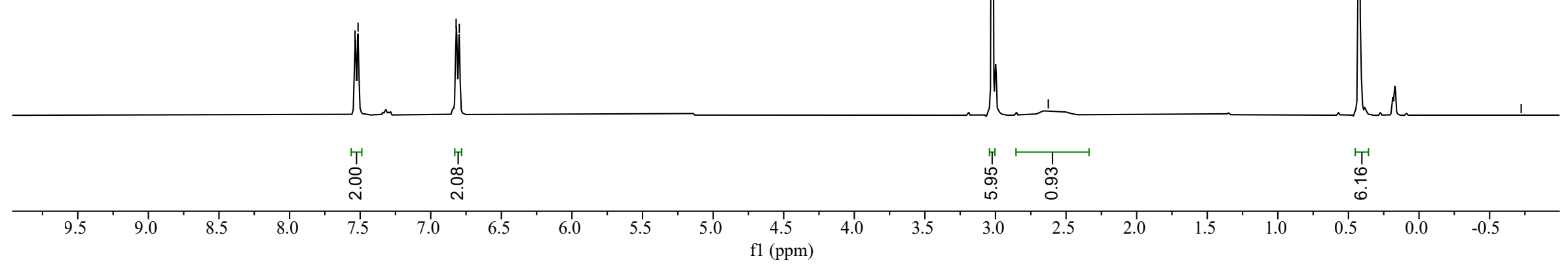

S300 
Compound $\mathbf{2 q}{ }^{13} \mathrm{C}$ NMR
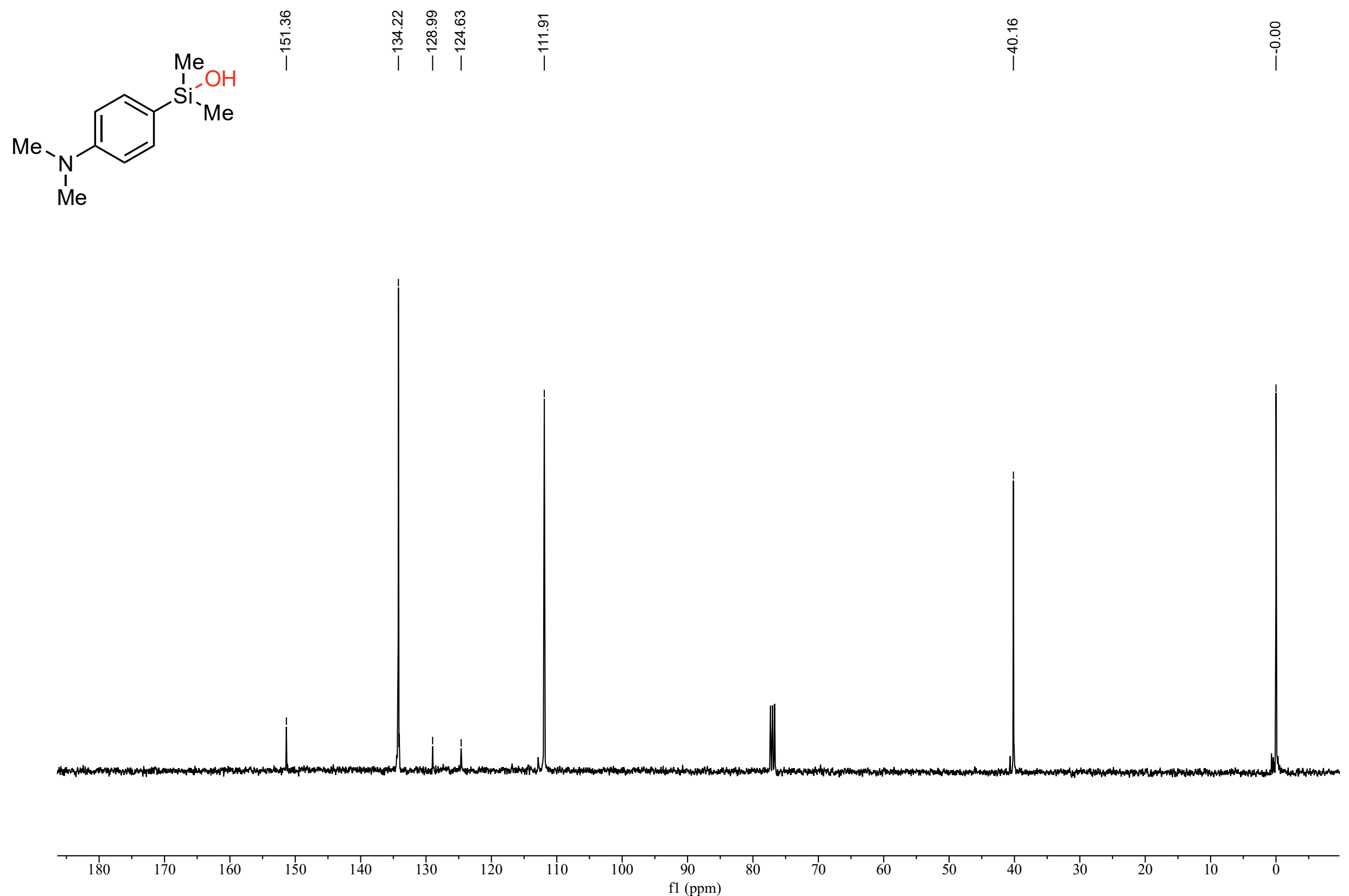


\section{Compound 2r ${ }^{1} \mathrm{H}$ NMR}
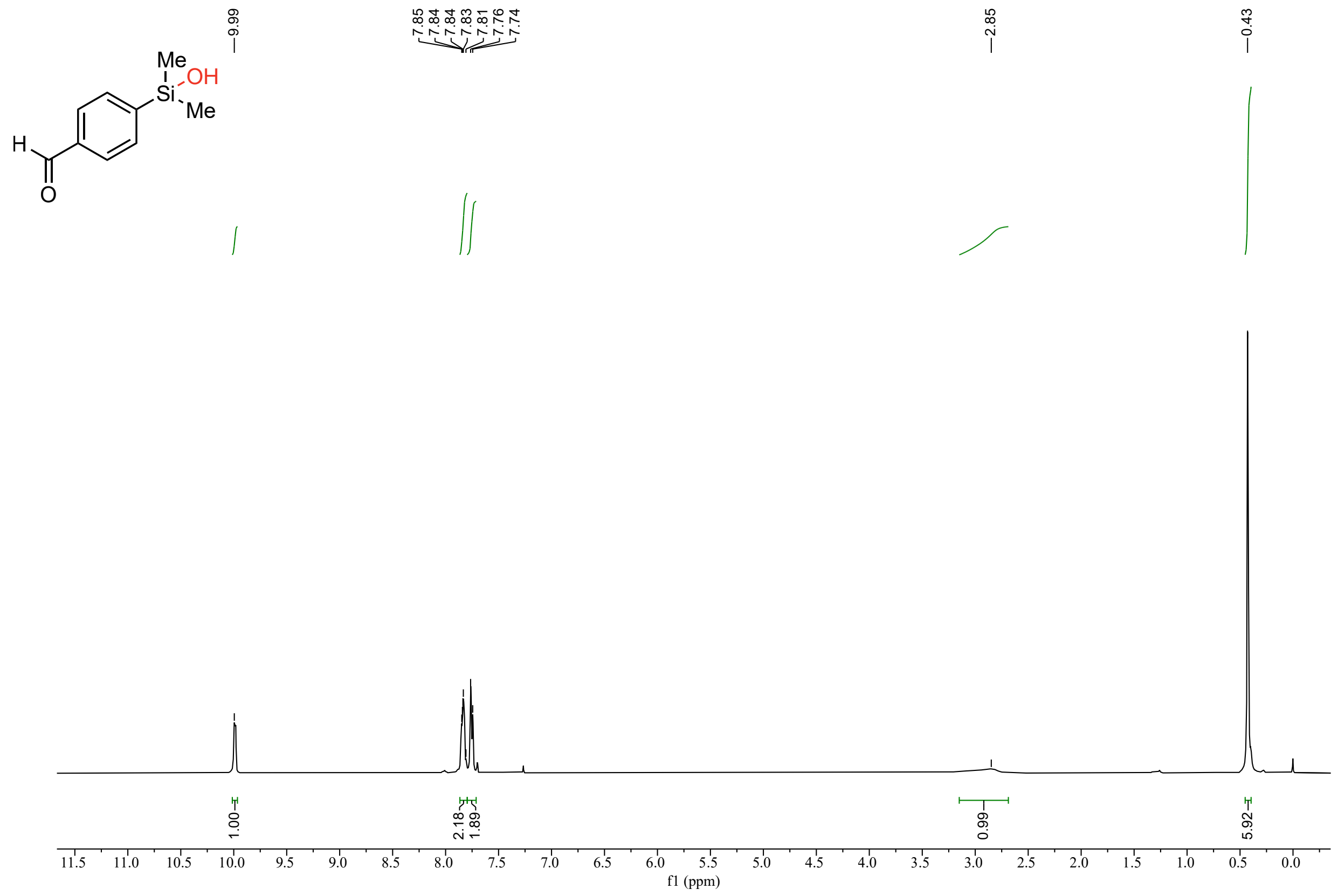
Compound $2 \mathbf{r}^{13} \mathrm{C}$ NMR
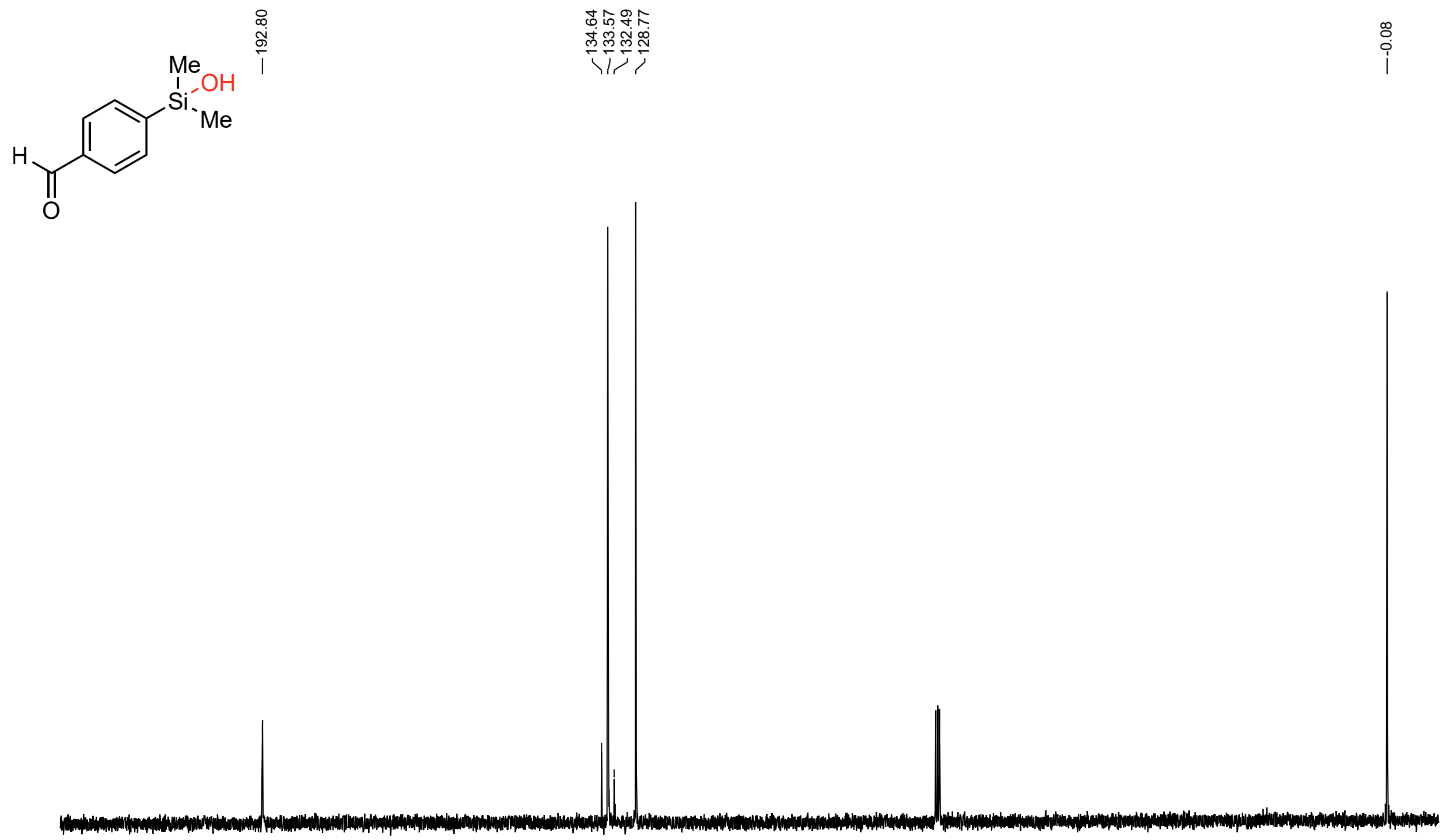


\section{Compound 2s ${ }^{1} \mathrm{H}$ NMR}

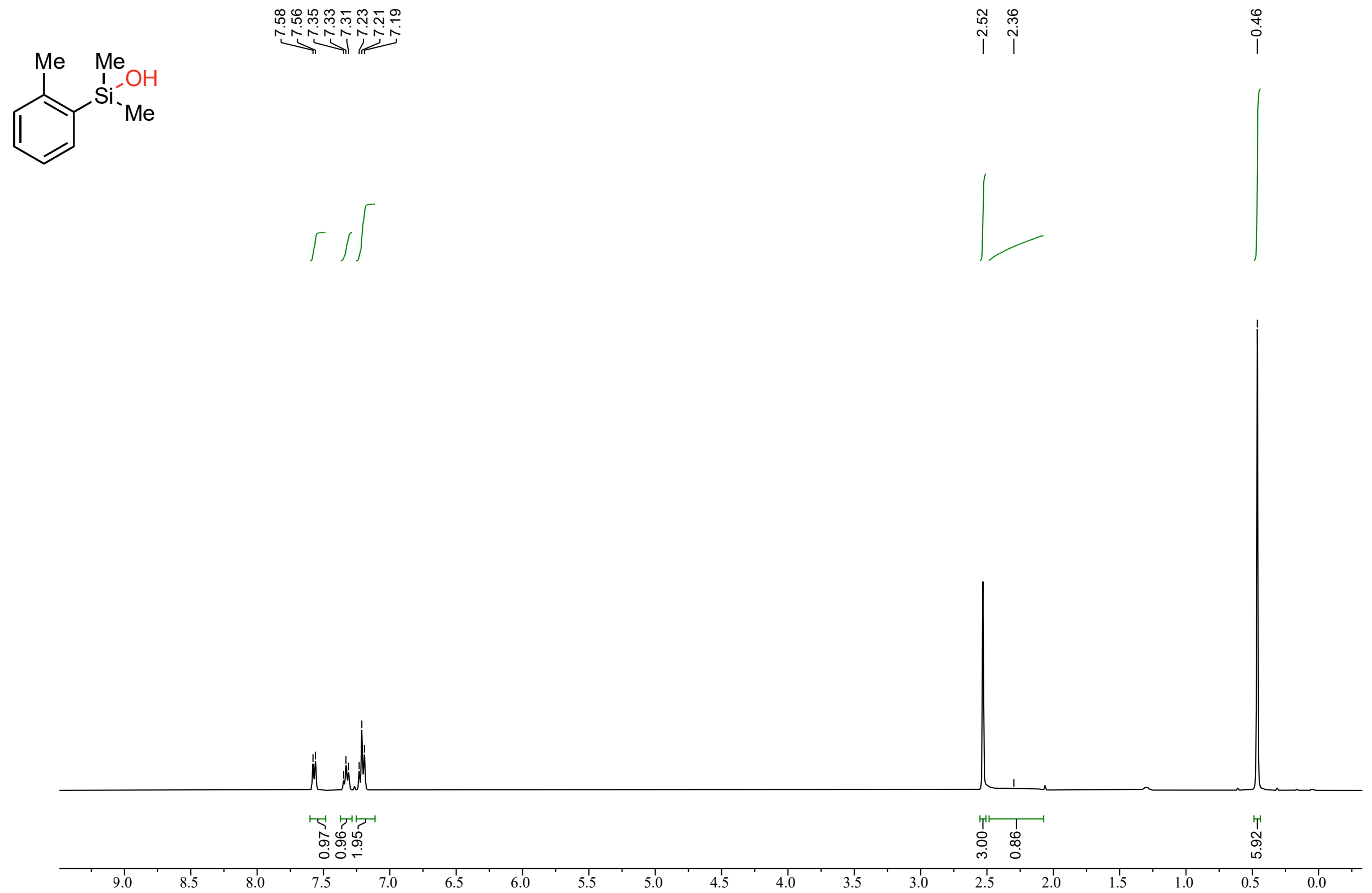


Compound 2s ${ }^{13} \mathrm{C}$ NMR
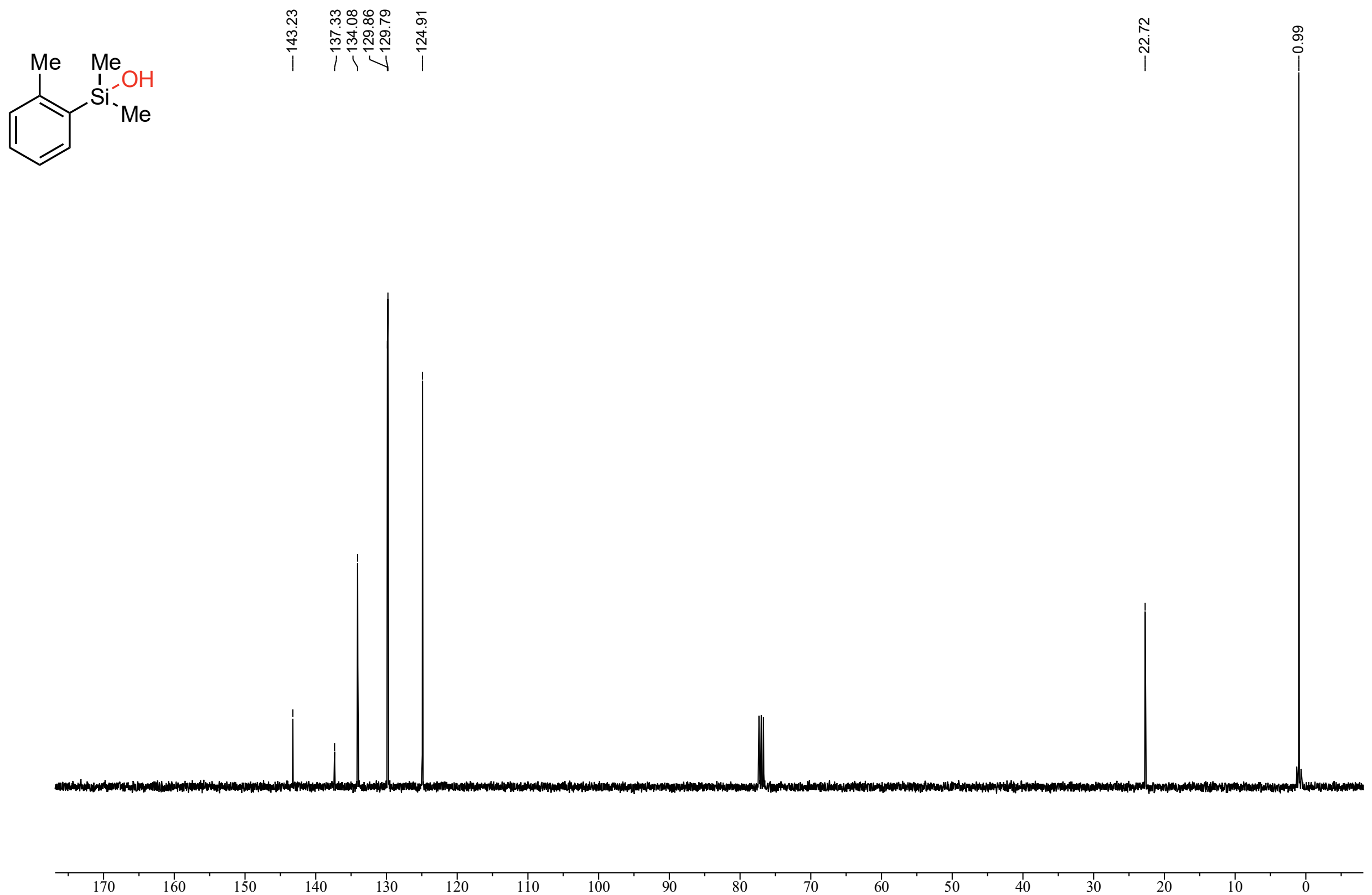


\section{Compound $2 \mathbf{t}^{1} \mathrm{H}$ NMR}

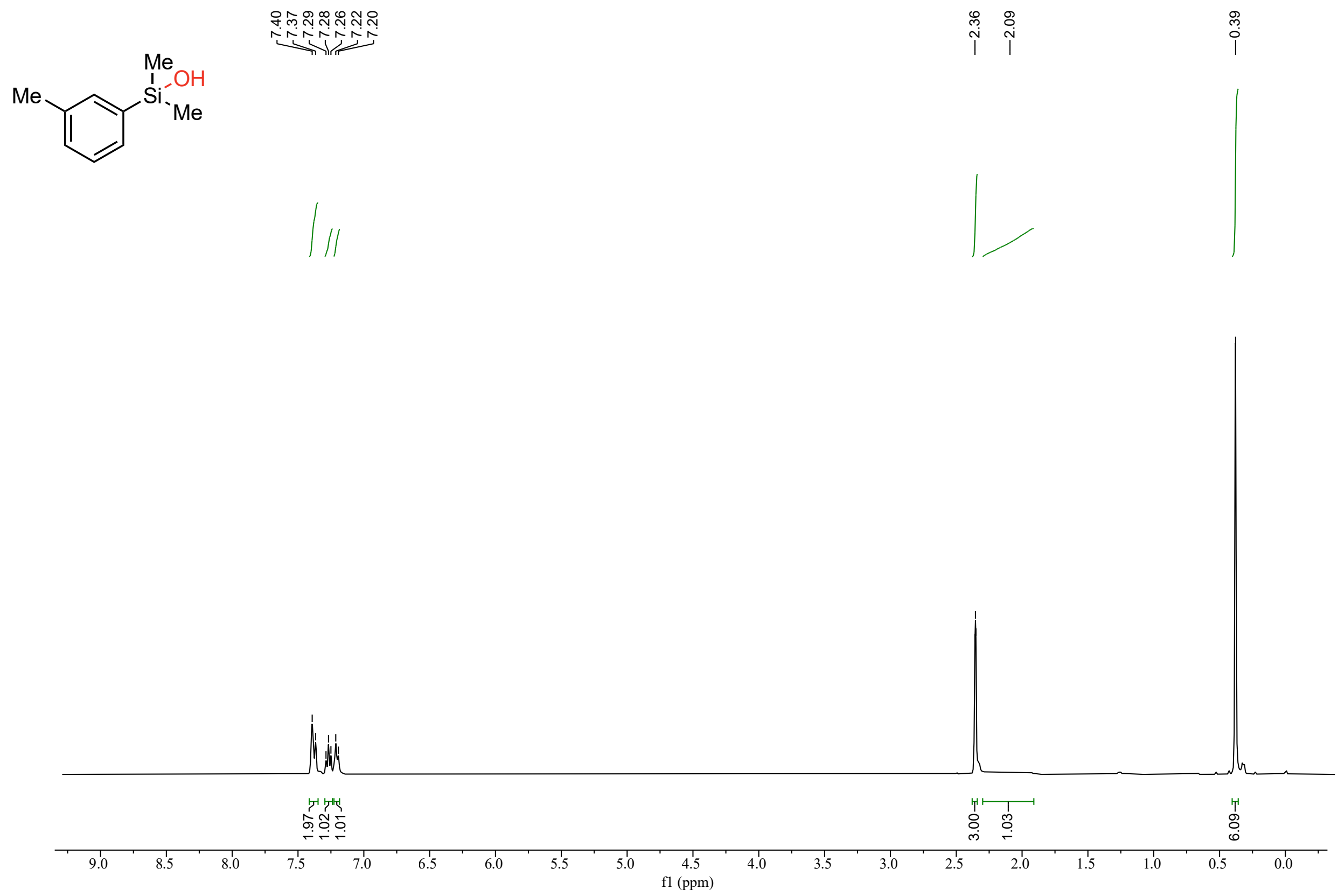


Compound $2 \mathrm{t}^{13} \mathrm{C}$ NMR
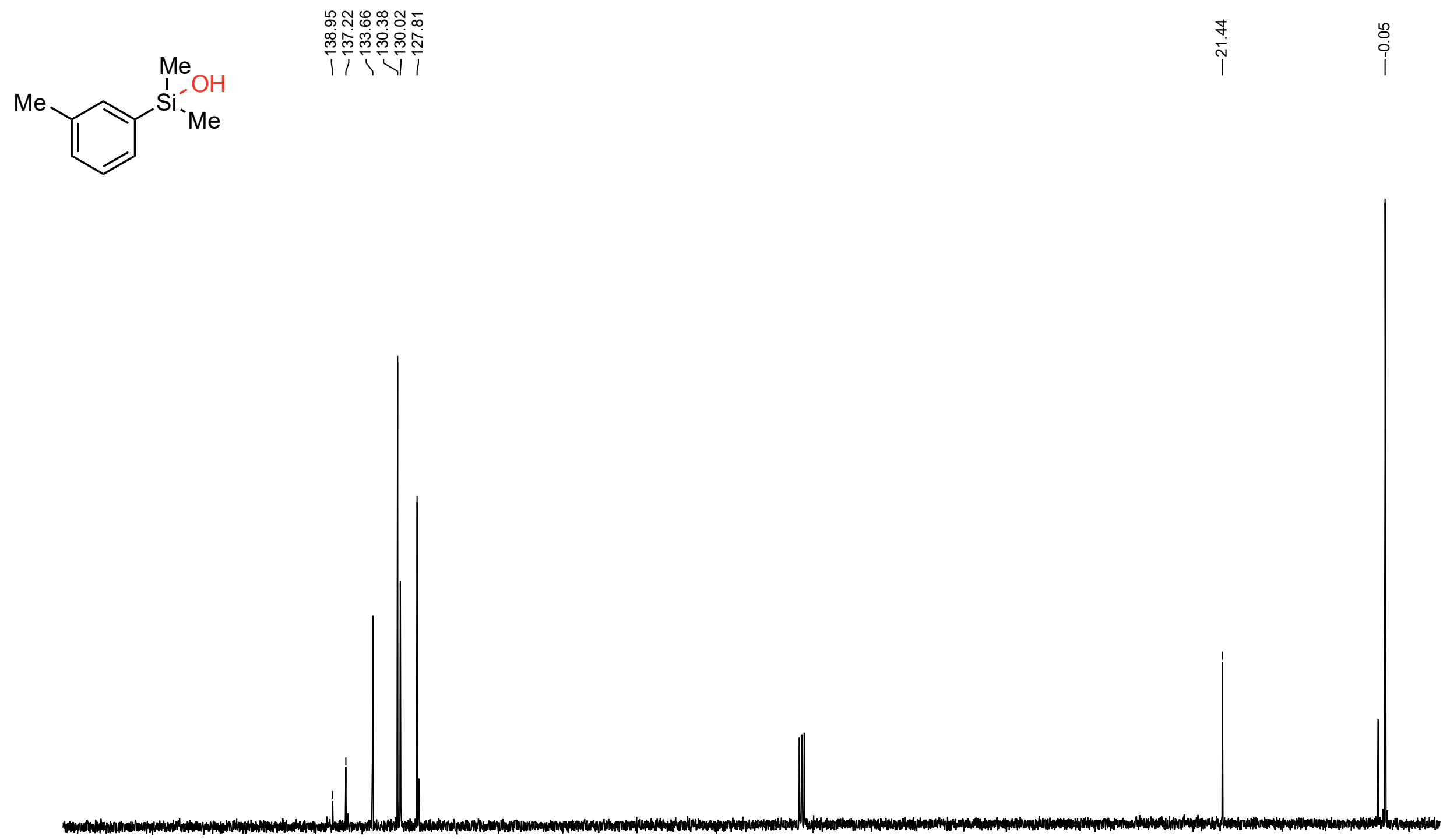


\section{Compound 2u ${ }^{1} \mathrm{H}$ NMR}

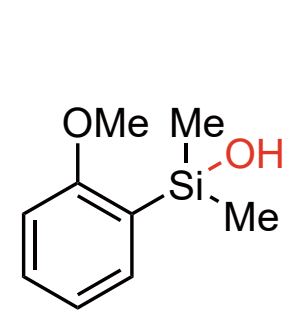

\section{t4.}

rivingog

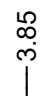

$\stackrel{\text { f̀ }}{\text { I }}$

ị

$$
1111
$$
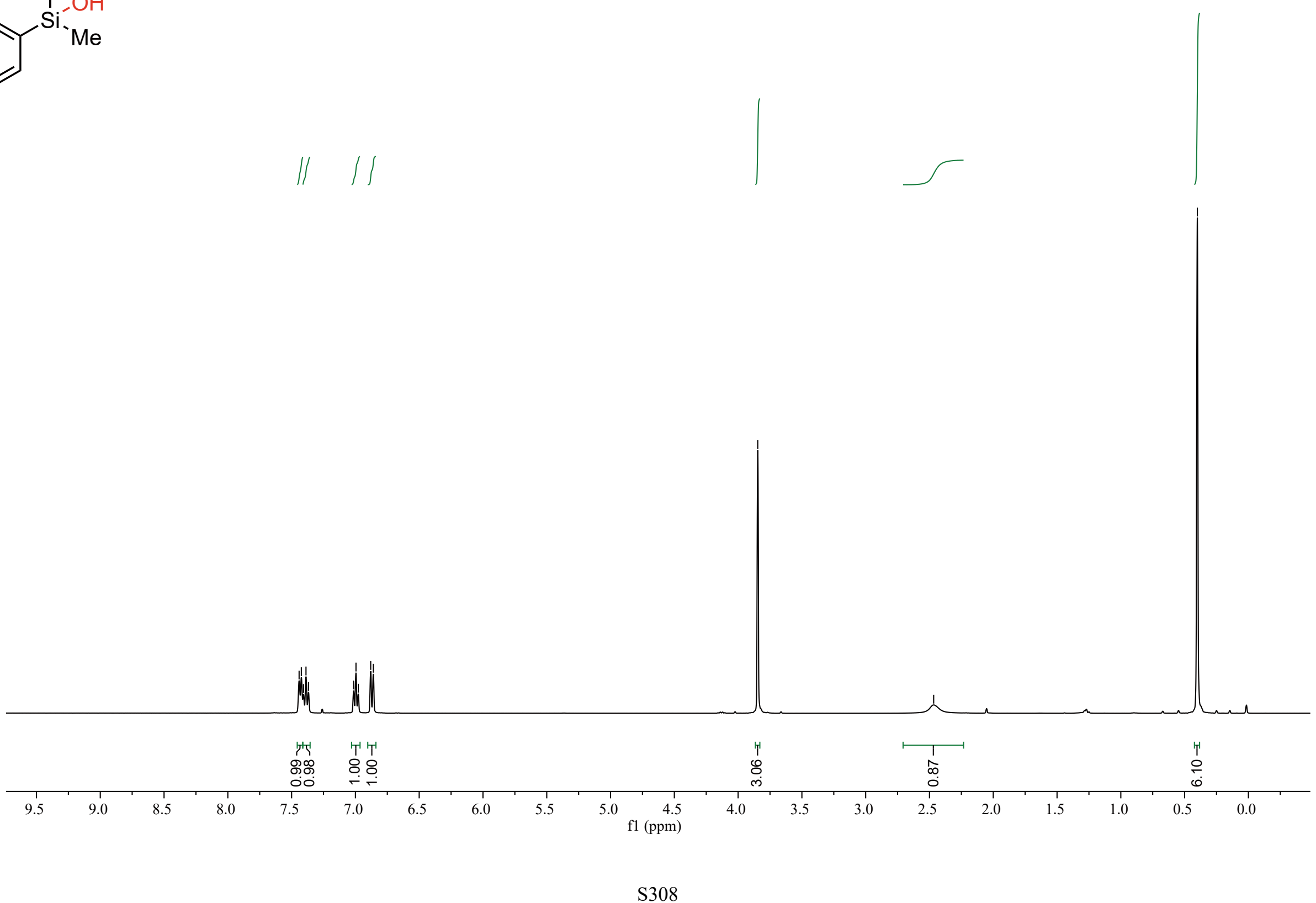
Compound $\mathbf{2 u}{ }^{13} \mathrm{C}$ NMR
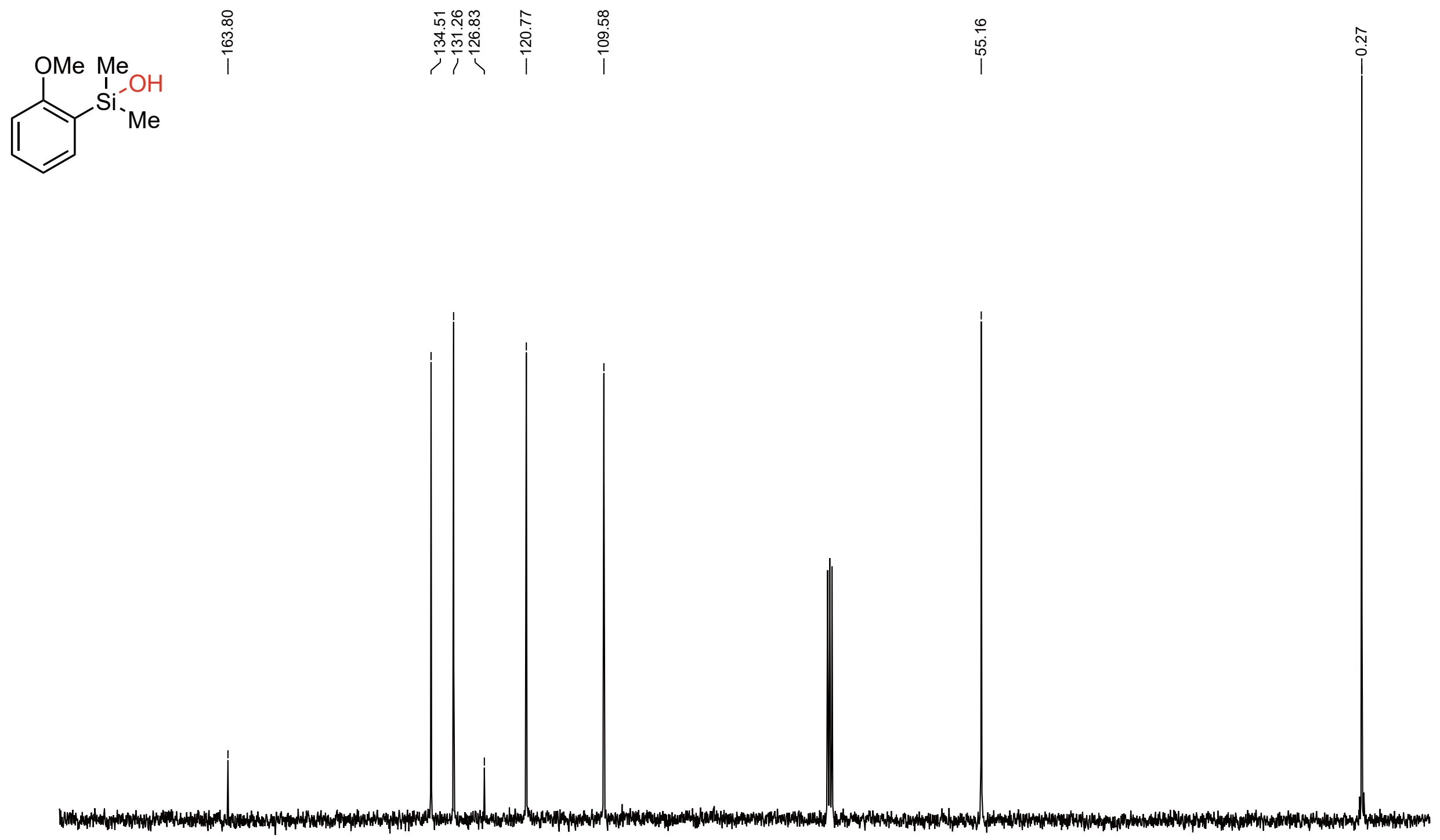


\section{Compound 2v ${ }^{1} \mathrm{H}$ NMR}
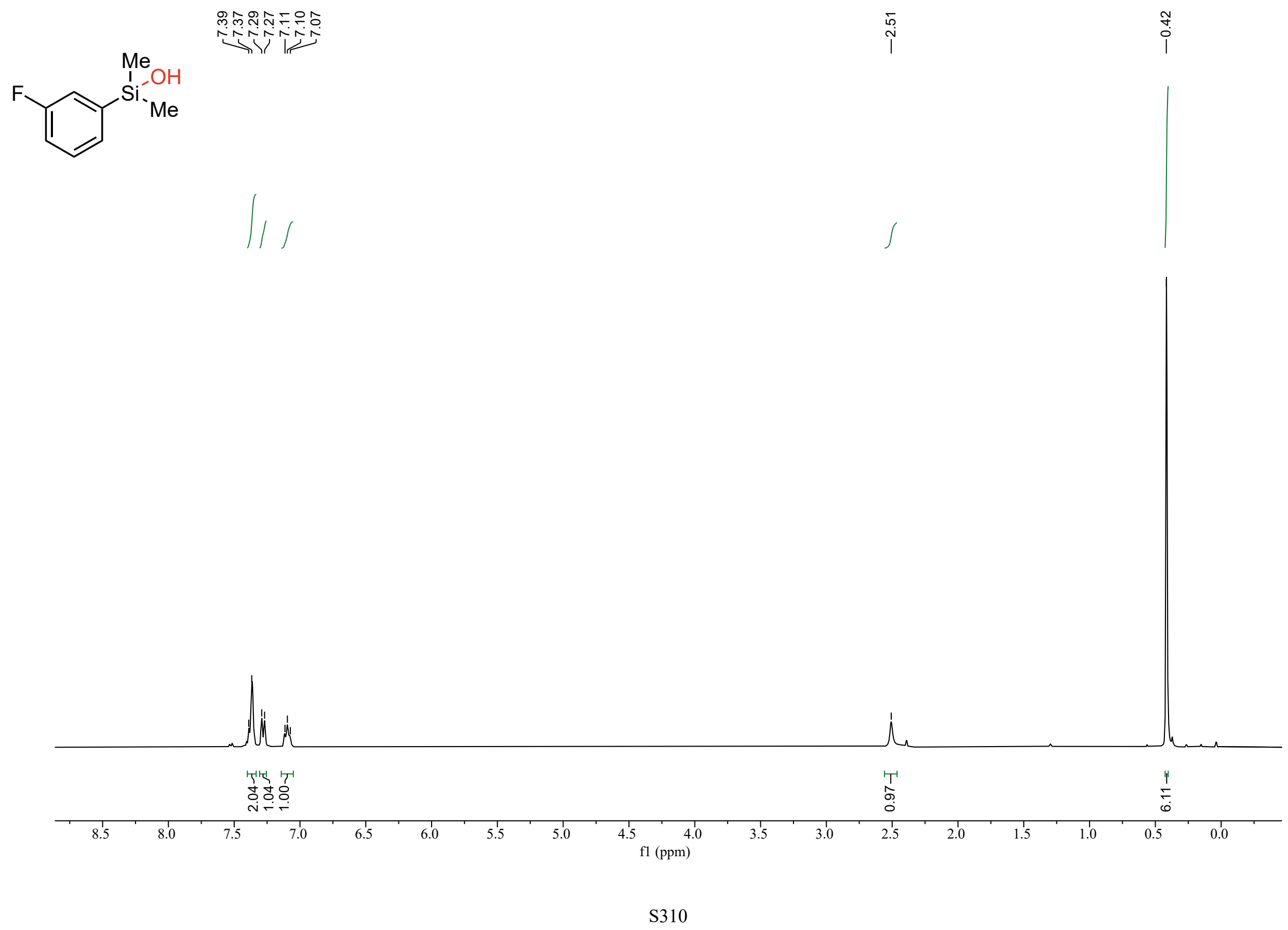
Compound $2 \mathbf{v}{ }^{13} \mathrm{C}$ NMR
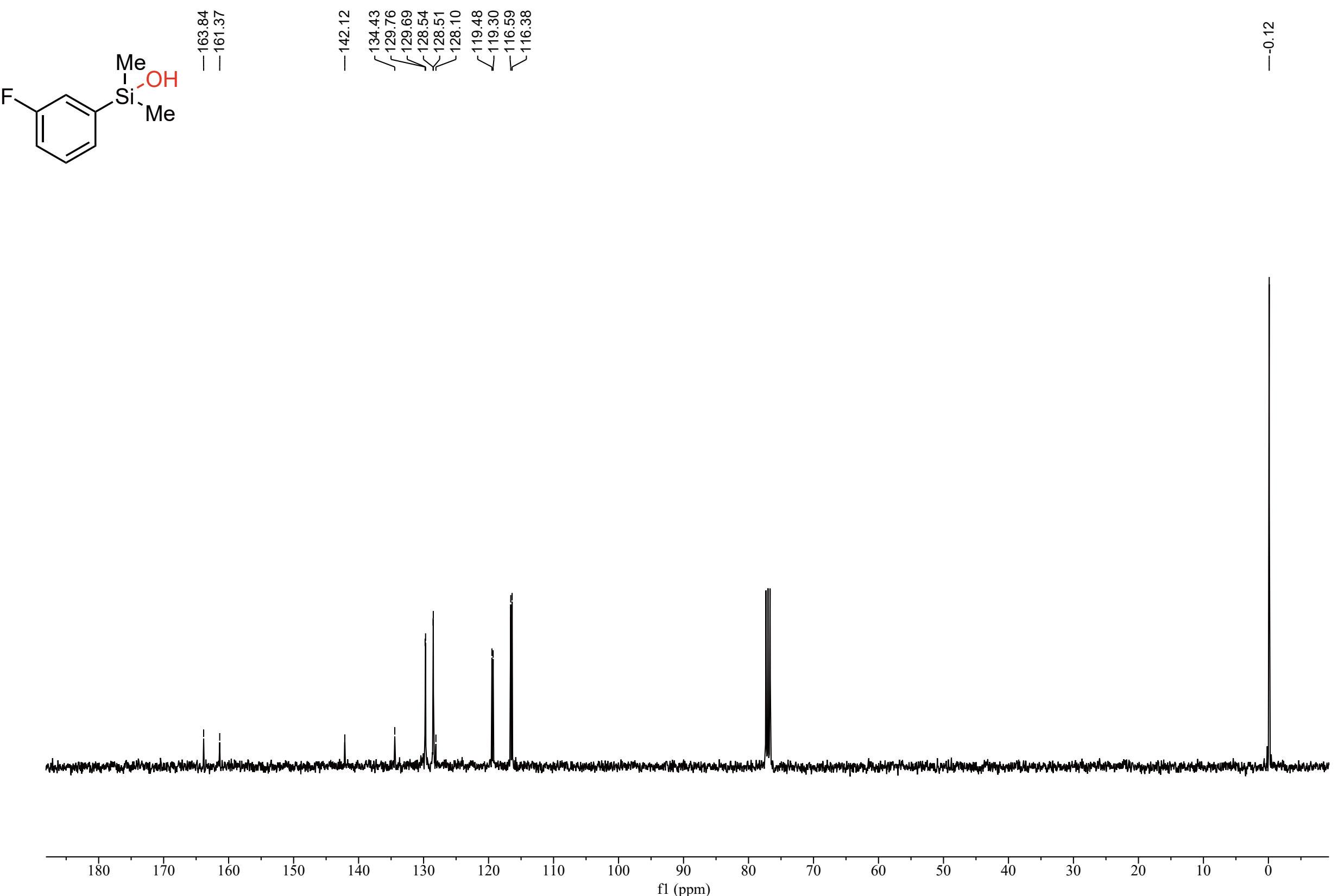
Compound 2v ${ }^{19} \mathrm{~F}$ NMR
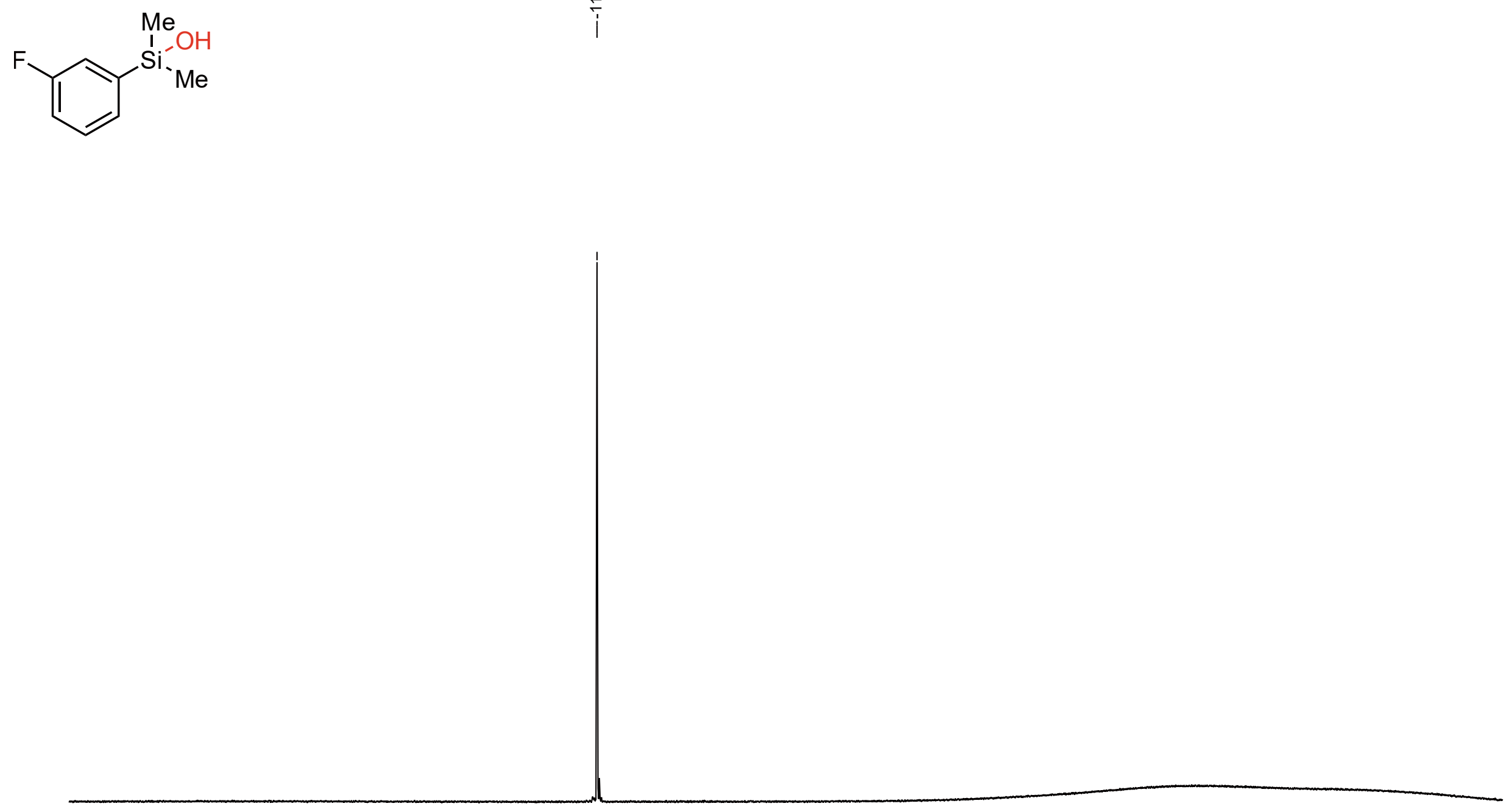

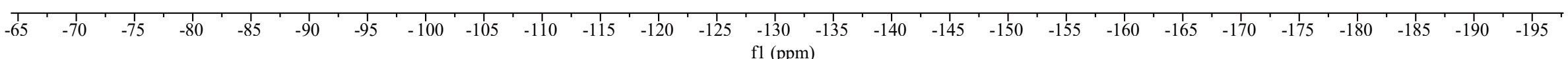


Compound 2w ${ }^{1} \mathrm{H}$ NMR

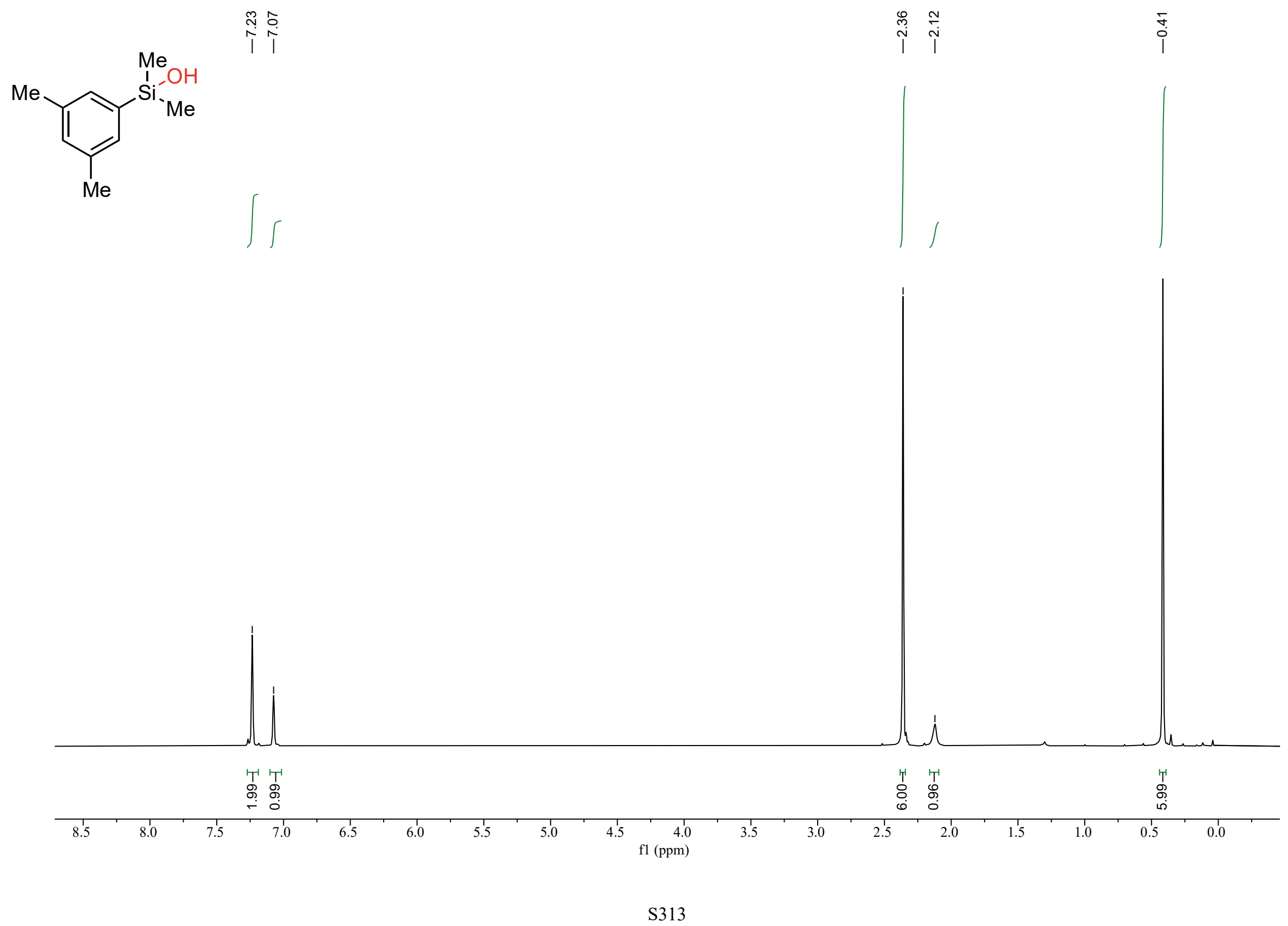


Compound $\mathbf{2 w}{ }^{13} \mathrm{C}$ NMR<smiles>Cc1cc(C)cc([Si](C)(O)O)c1</smiles>

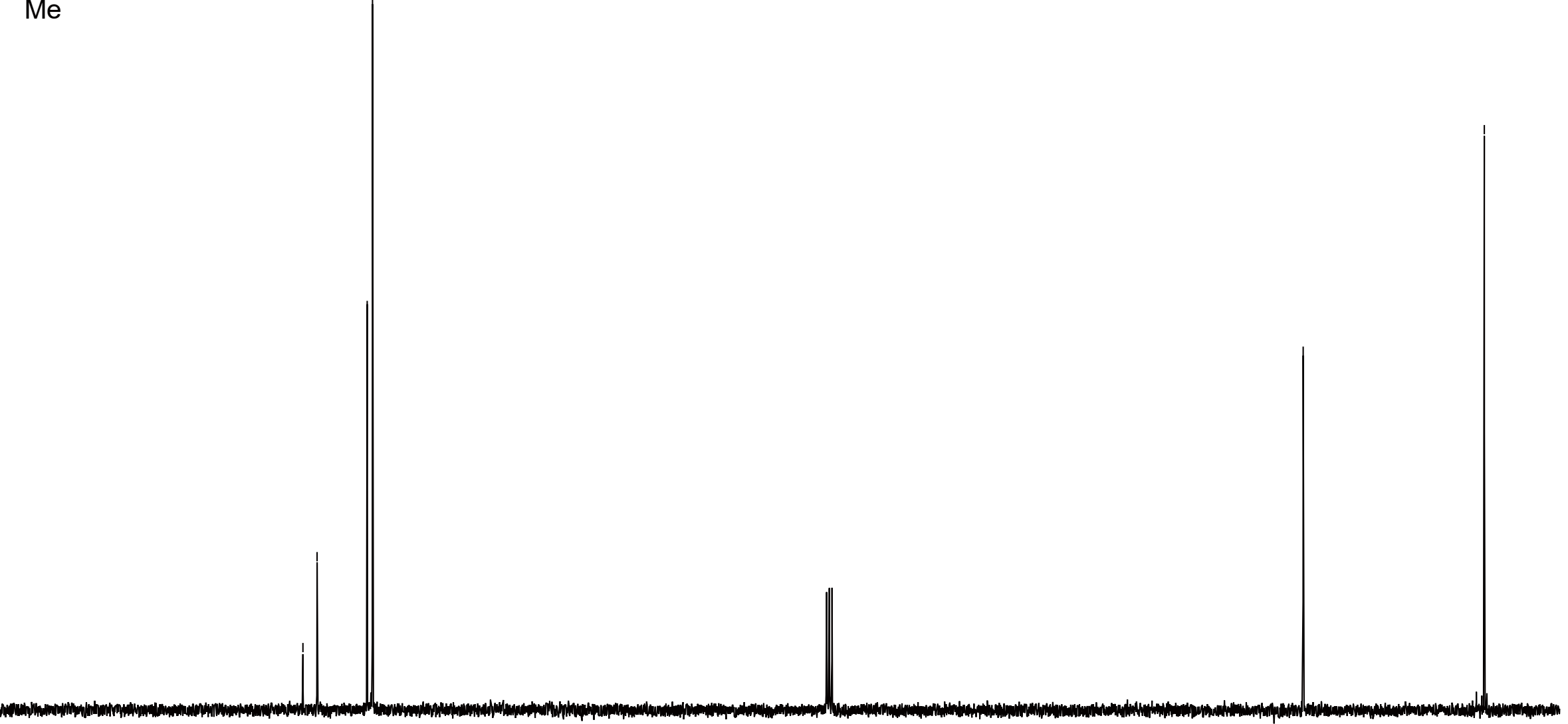




\section{Compound 2x ${ }^{1} \mathrm{H}$ NMR}
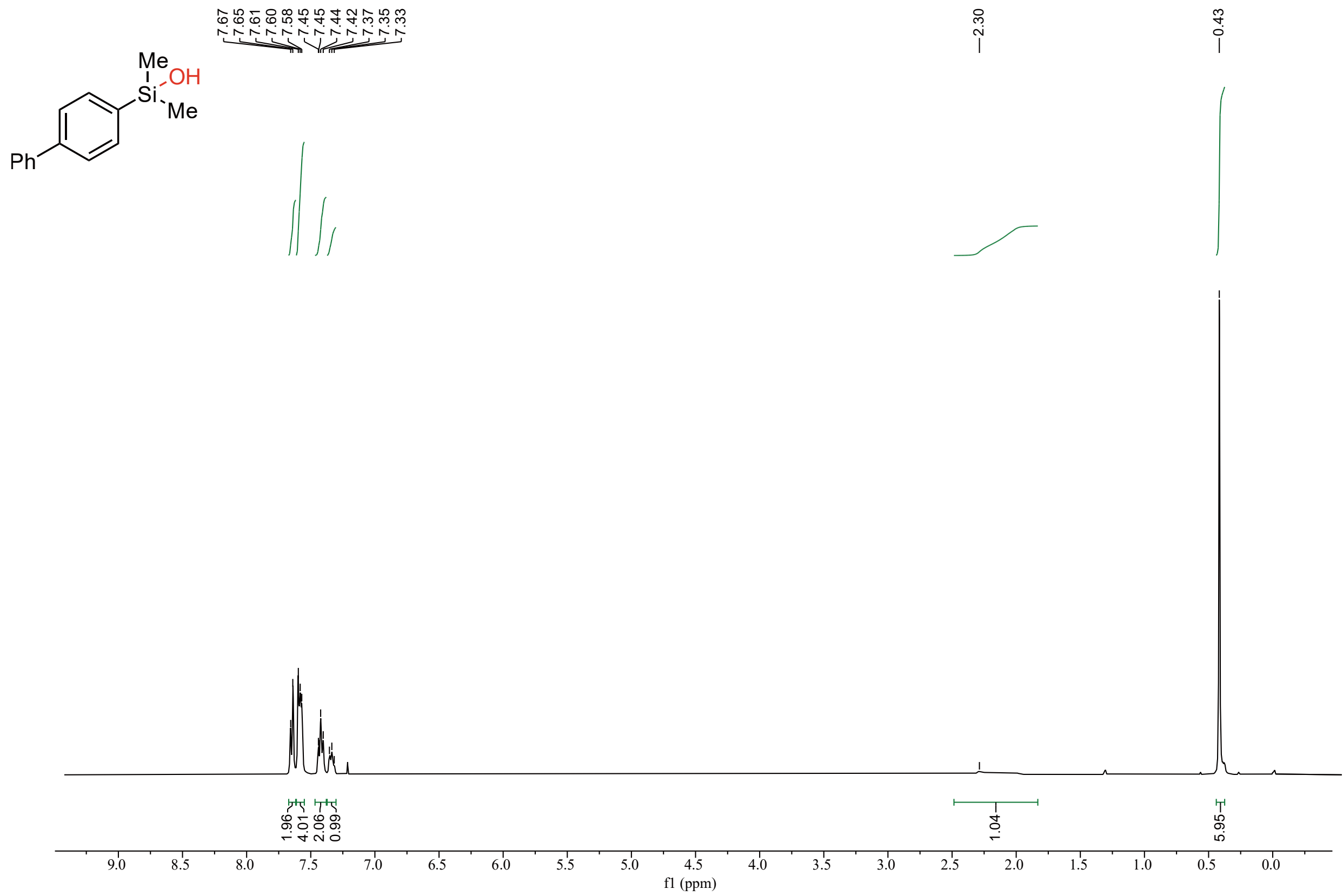
Compound 2x ${ }^{13} \mathrm{C}$ NMR

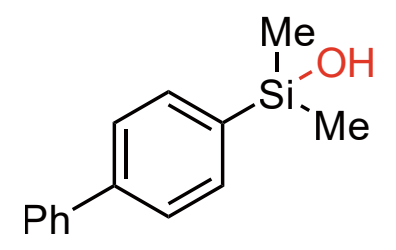

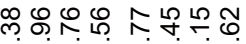

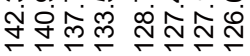

1), पर पर

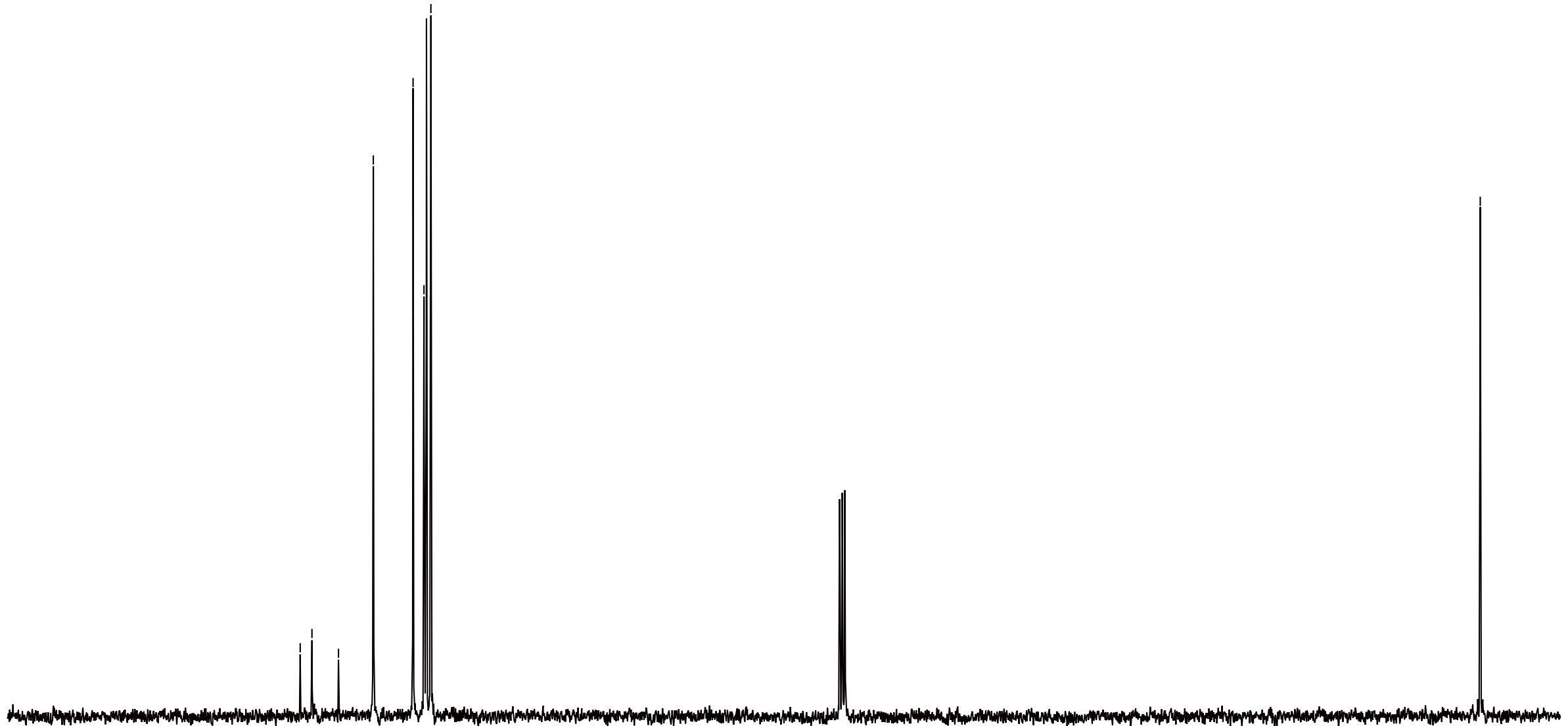


Compound $2 \mathbf{y}^{1} \mathrm{H}$ NMR

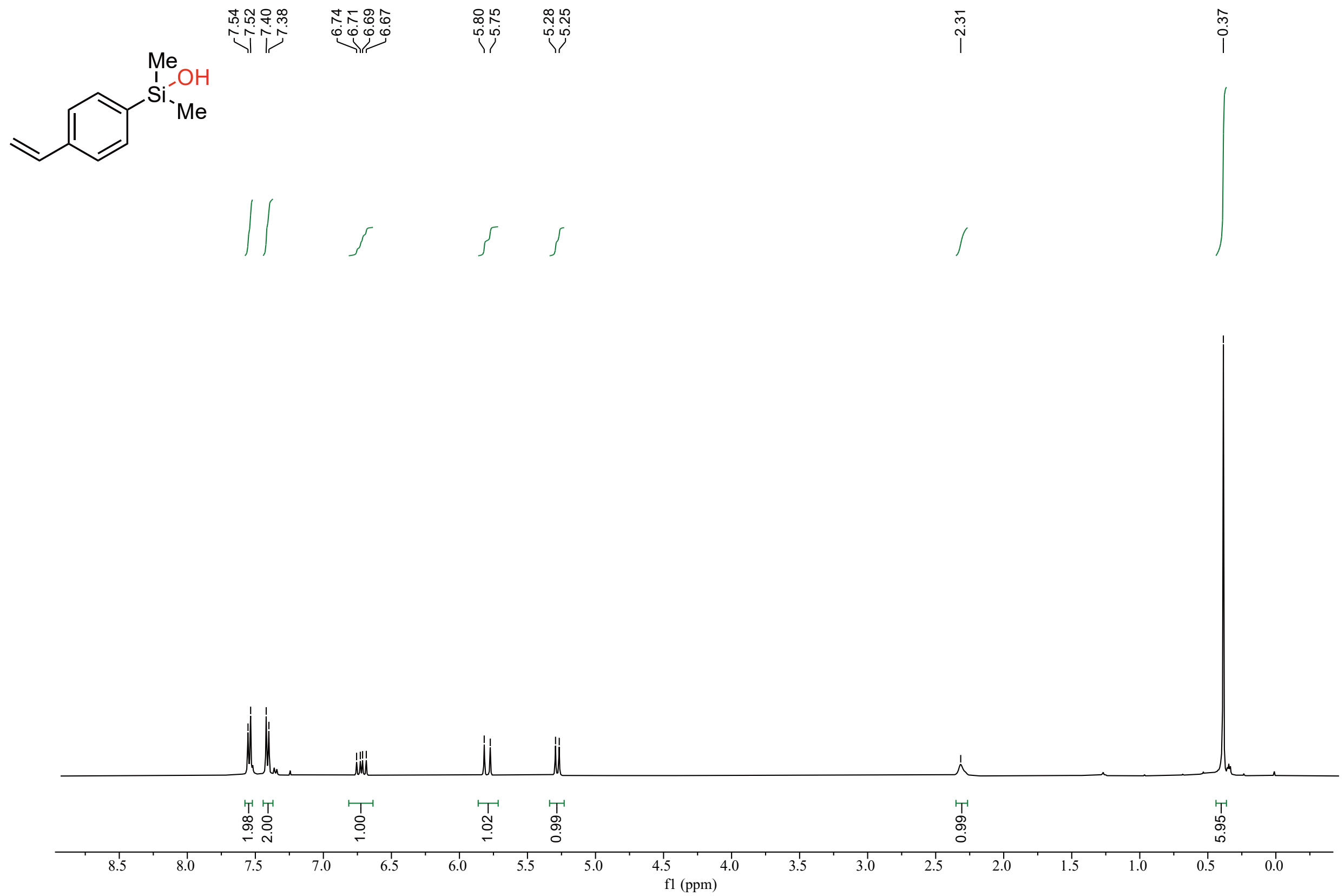


Compound 2y ${ }^{13} \mathrm{C}$ NMR
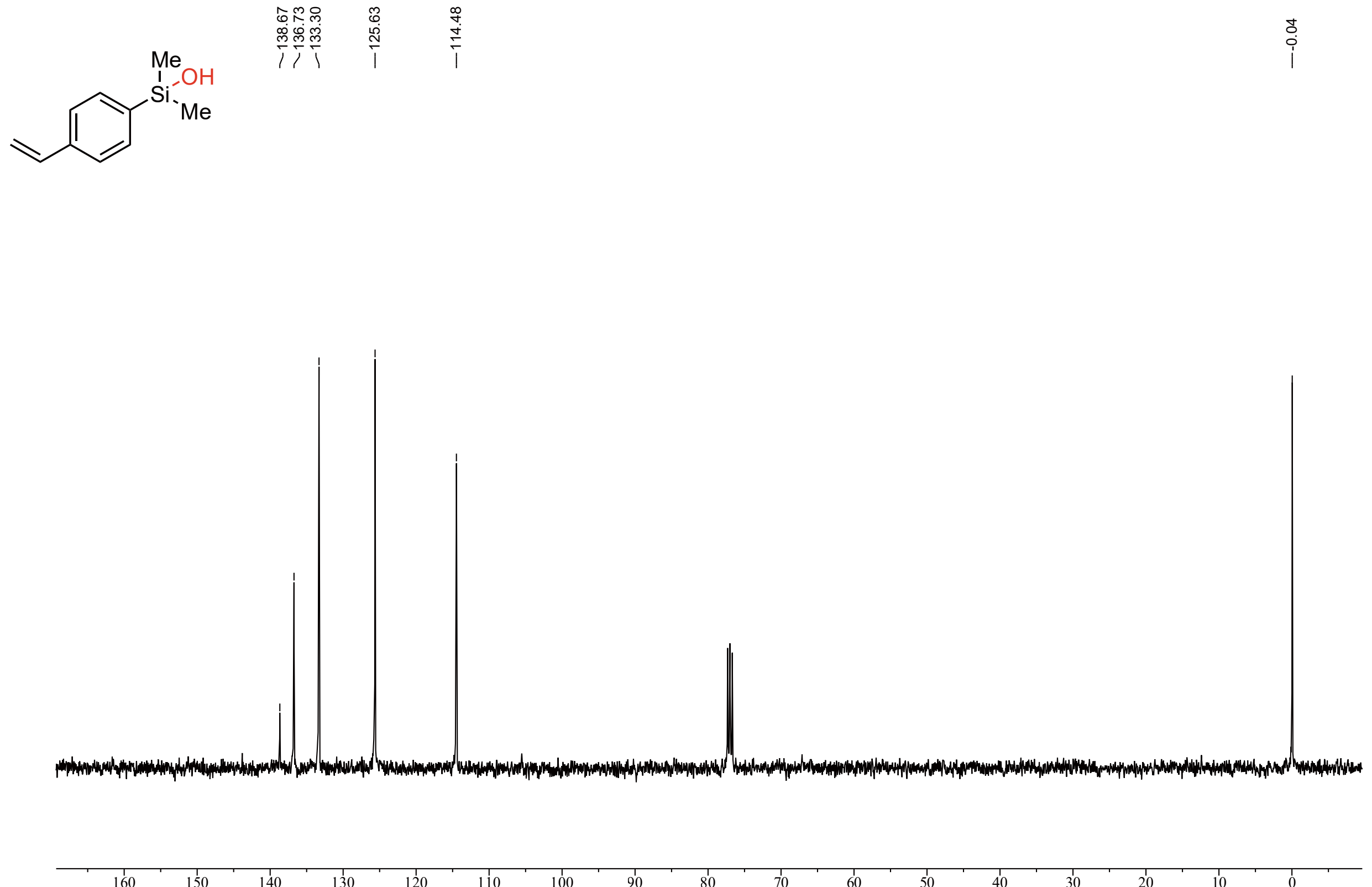
Compound $2 z^{1} \mathrm{H}$ NMR

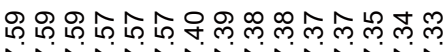
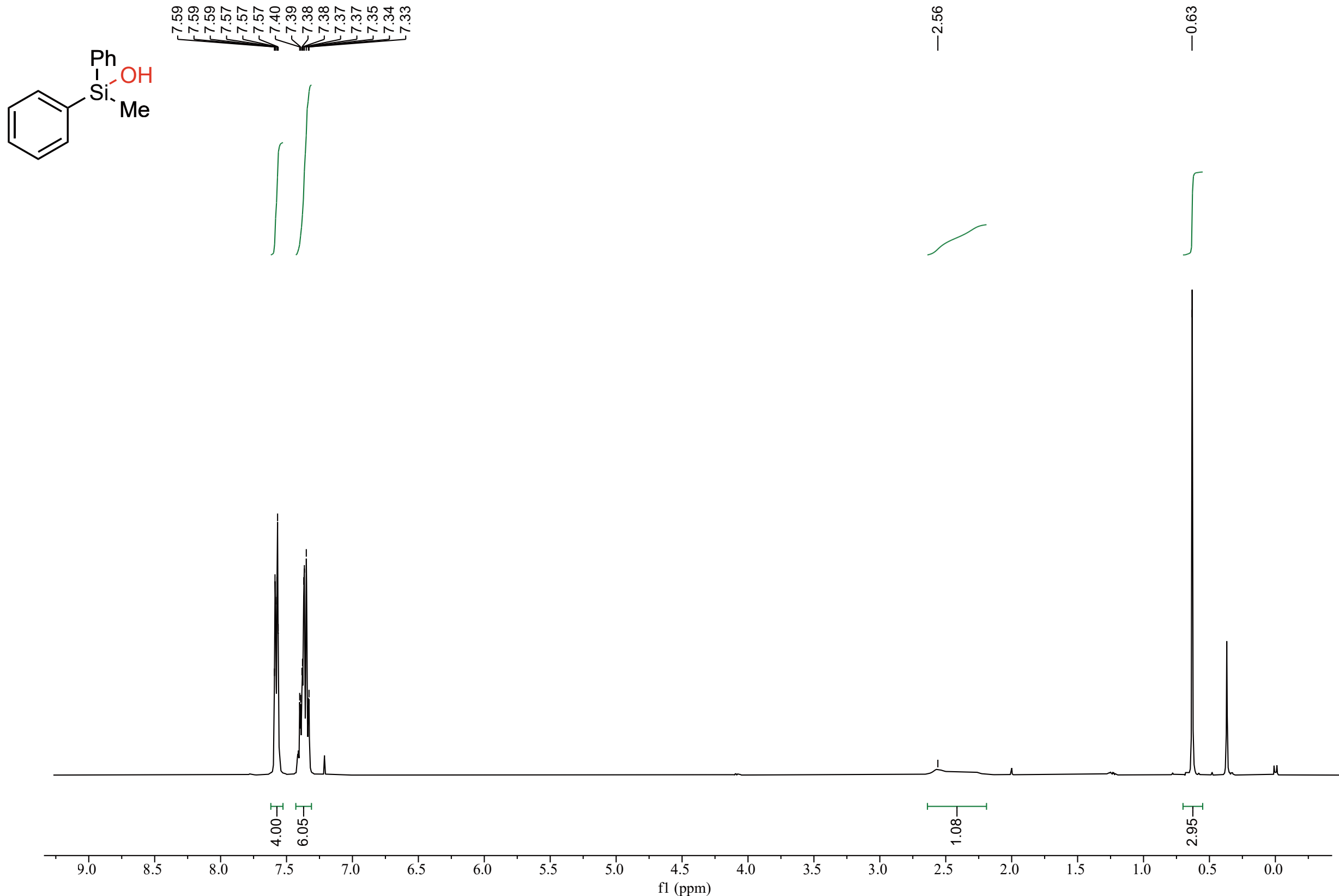
Compound $2 z^{13} \mathrm{C}$ NMR
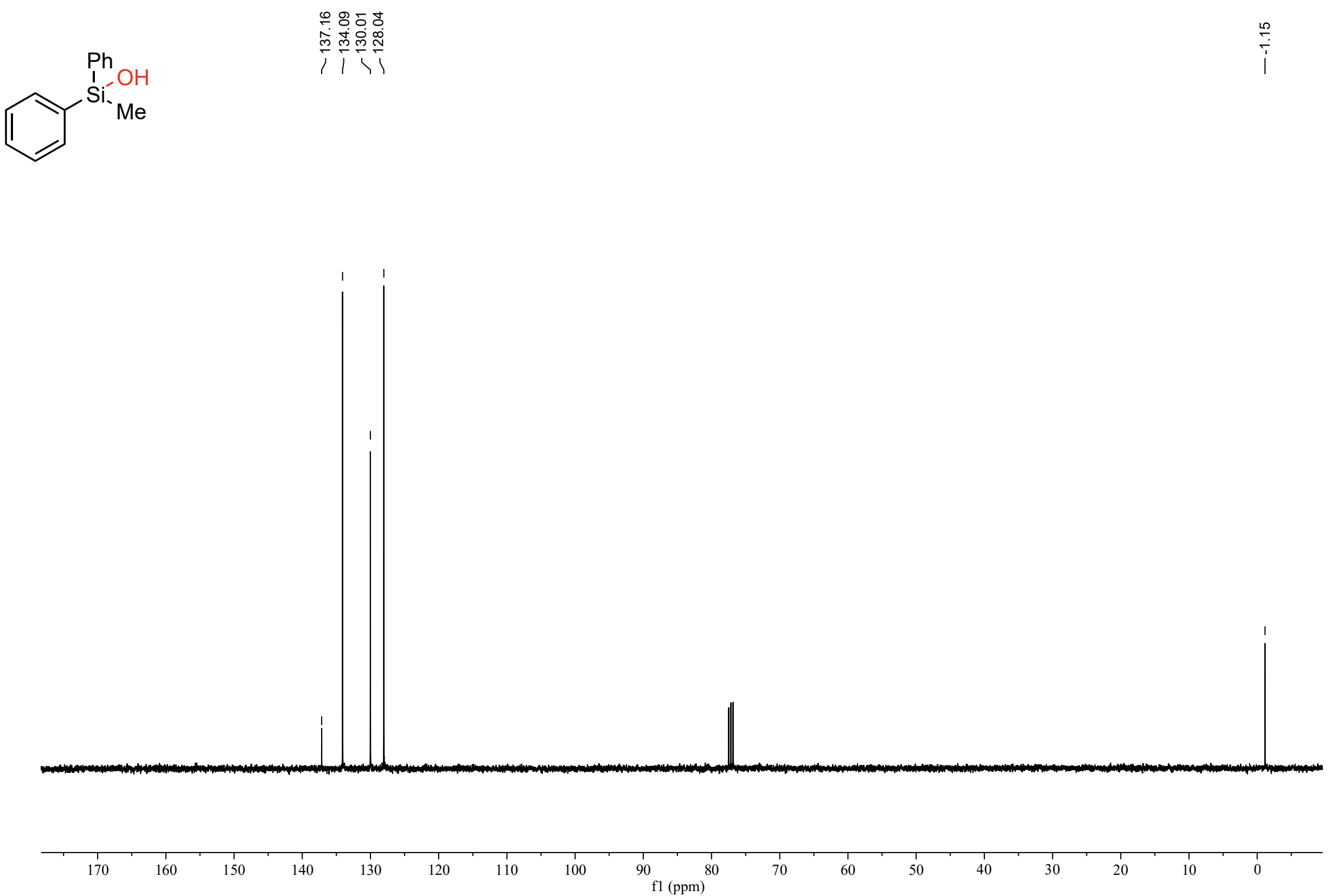
Compound 2aa ${ }^{1} \mathrm{H}$ NMR

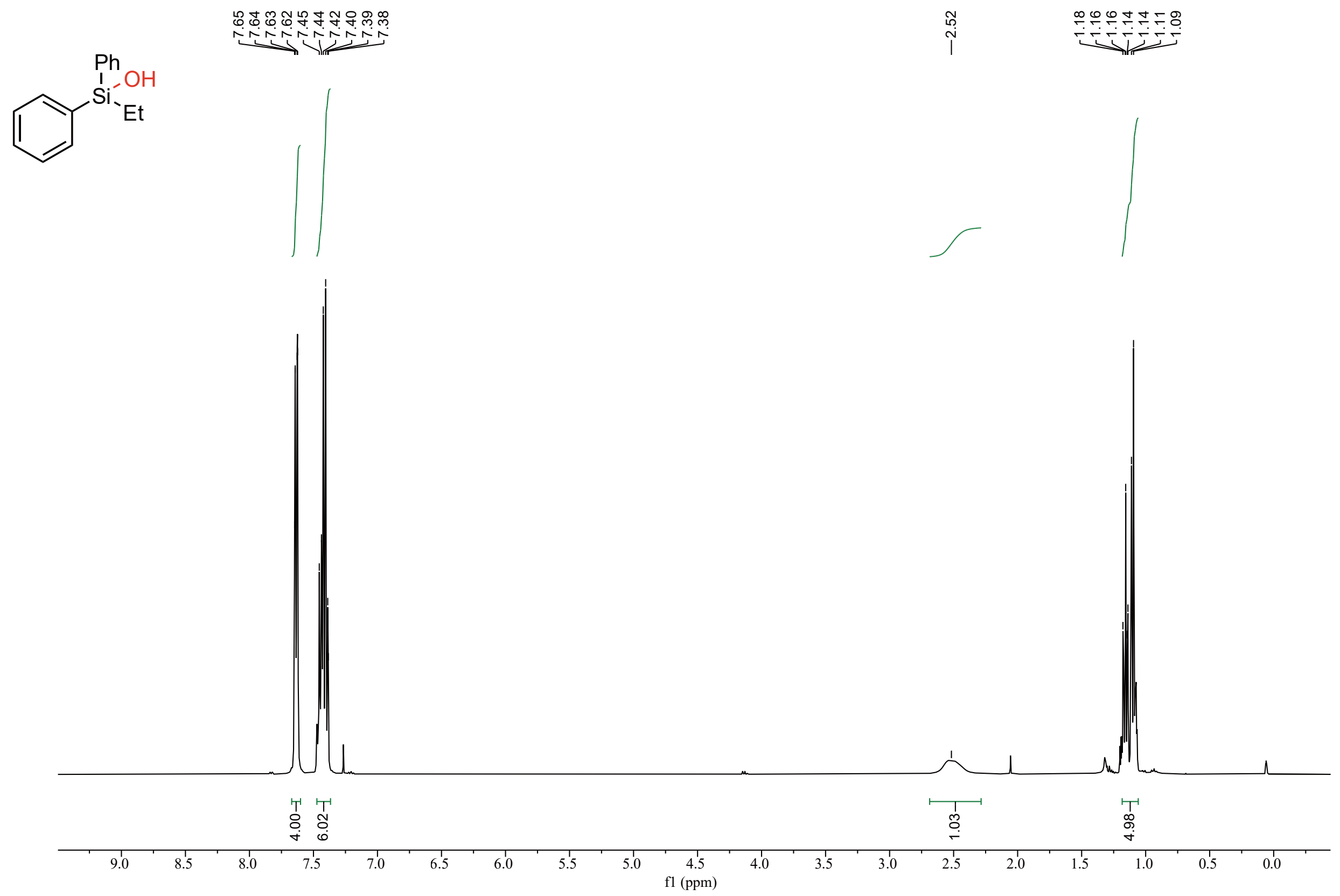




\section{Compound 2aa ${ }^{13} \mathrm{C}$ NMR}

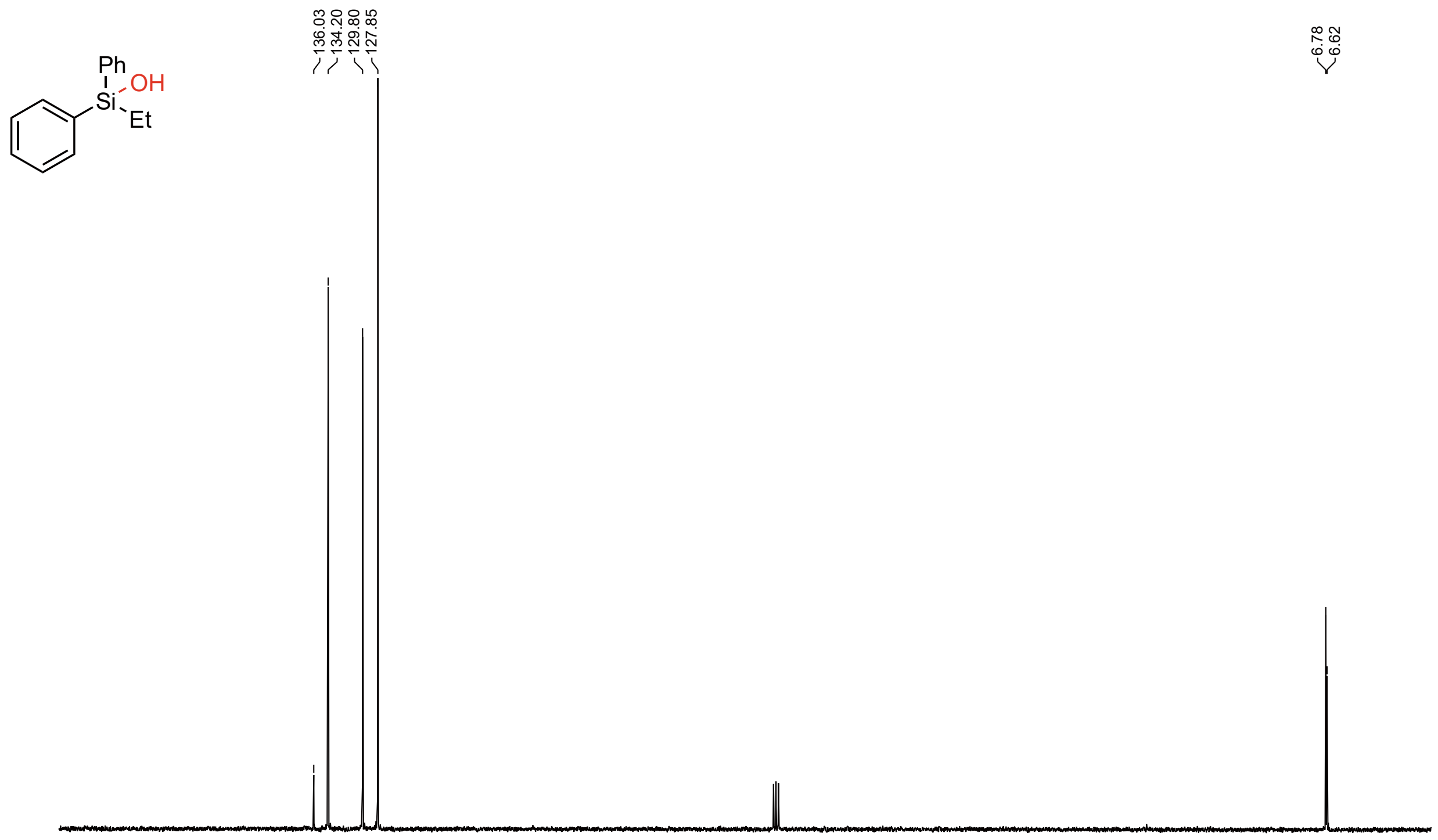


Compound 2ab ${ }^{1} \mathrm{H}$ NMR

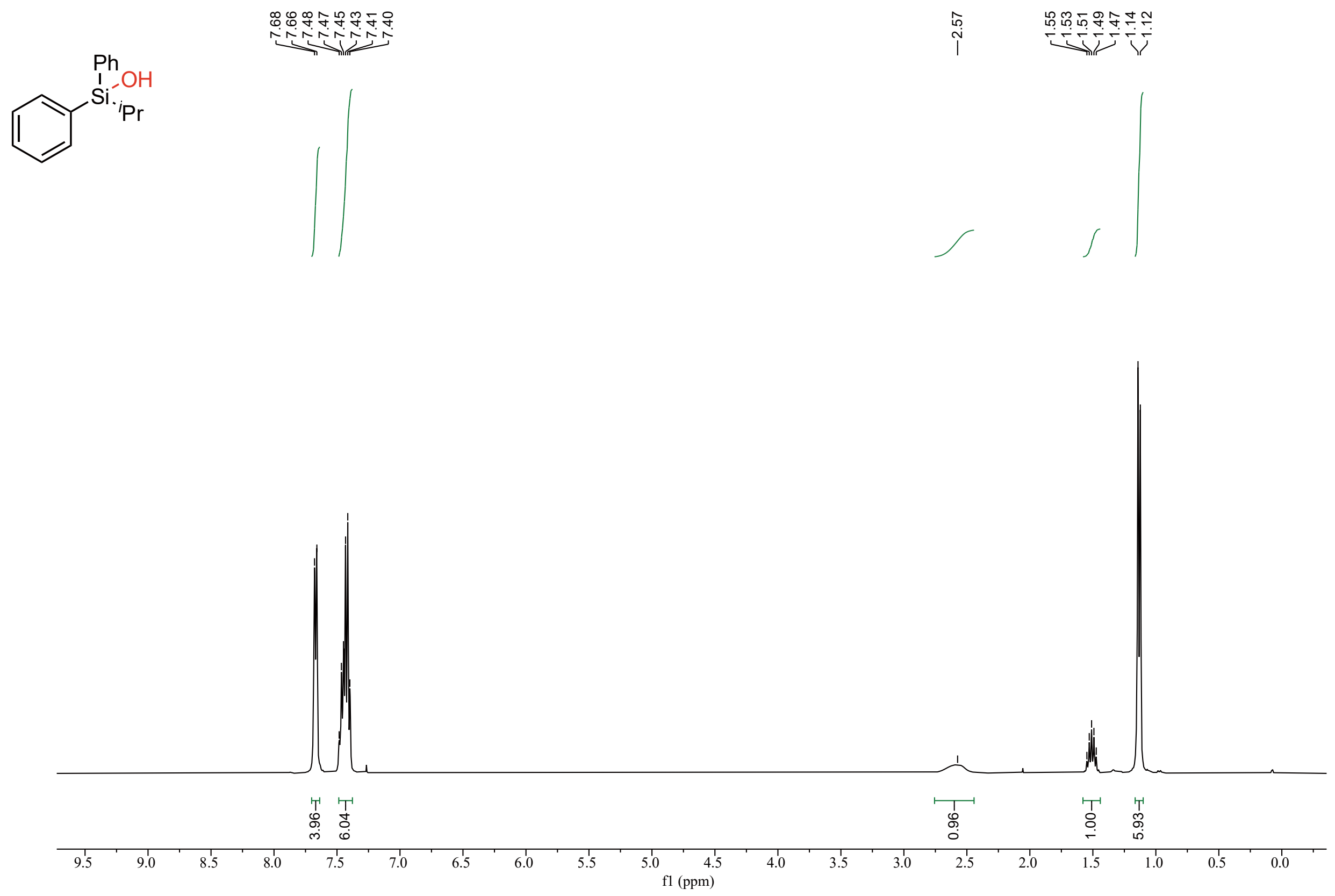




\section{Compound 2ab ${ }^{13} \mathrm{C}$ NMR}
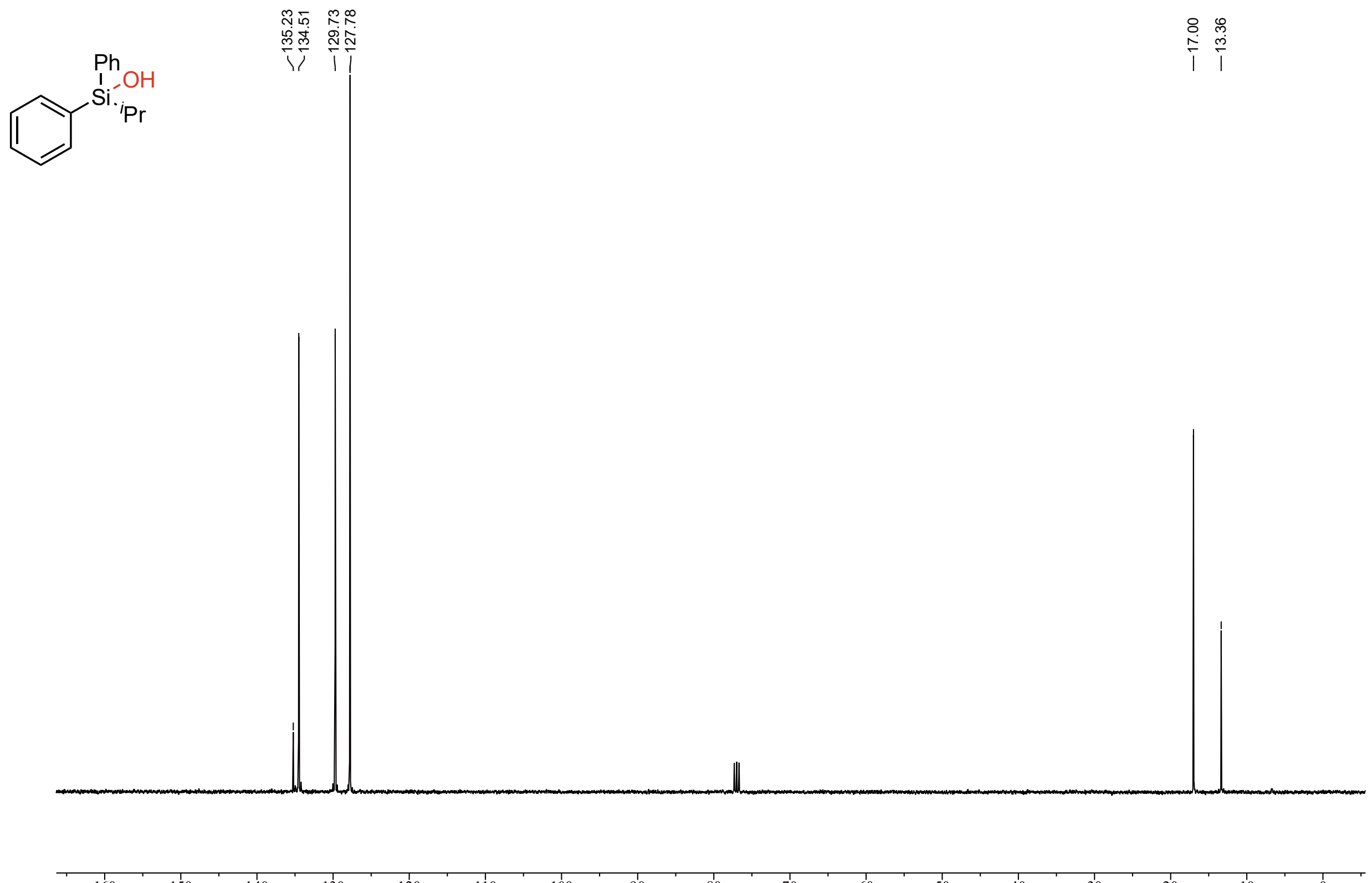
Compound 2ac ${ }^{1} \mathrm{H}$ NMR

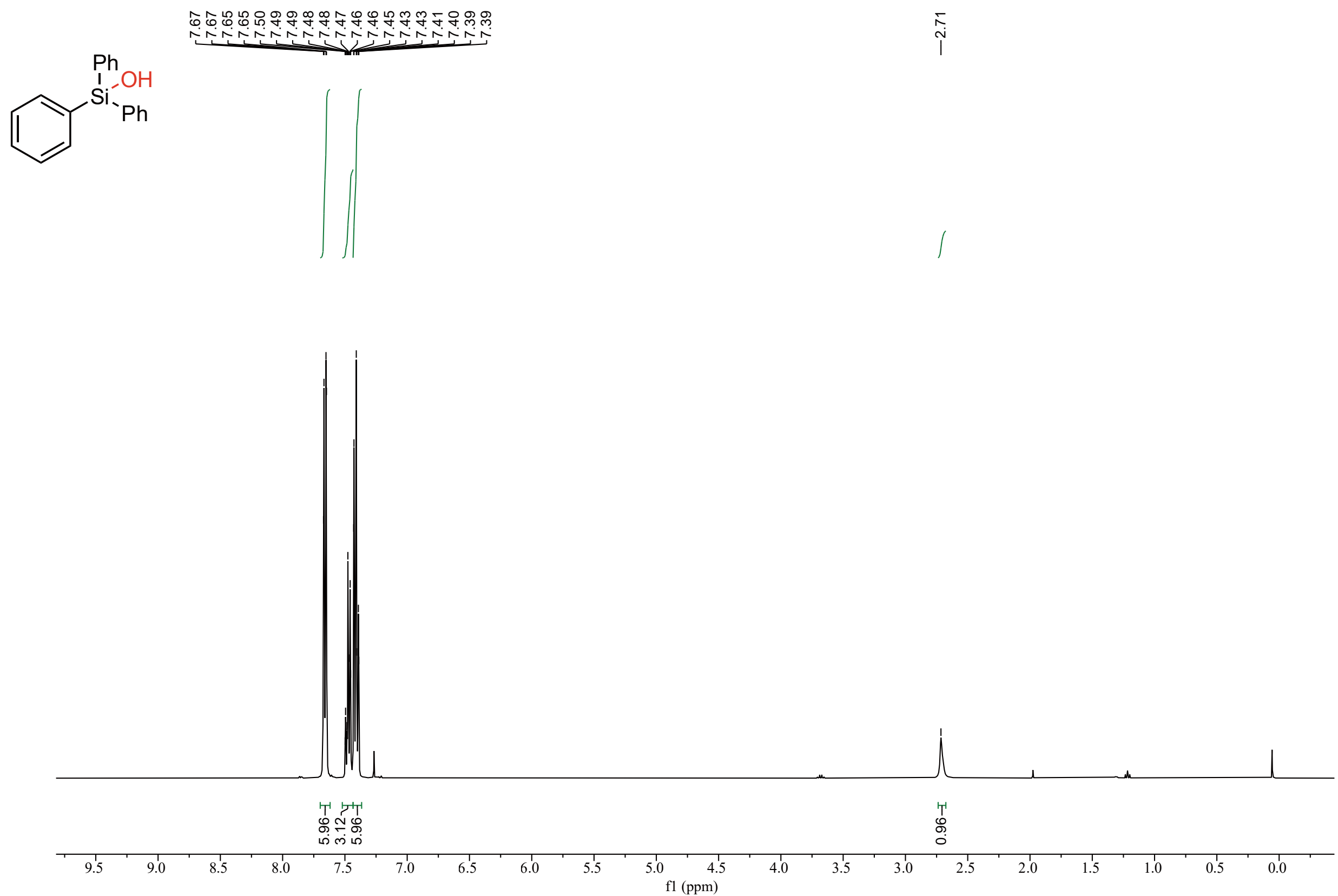


Compound 2ac ${ }^{13} \mathrm{C}$ NMR

(Ni=Ph

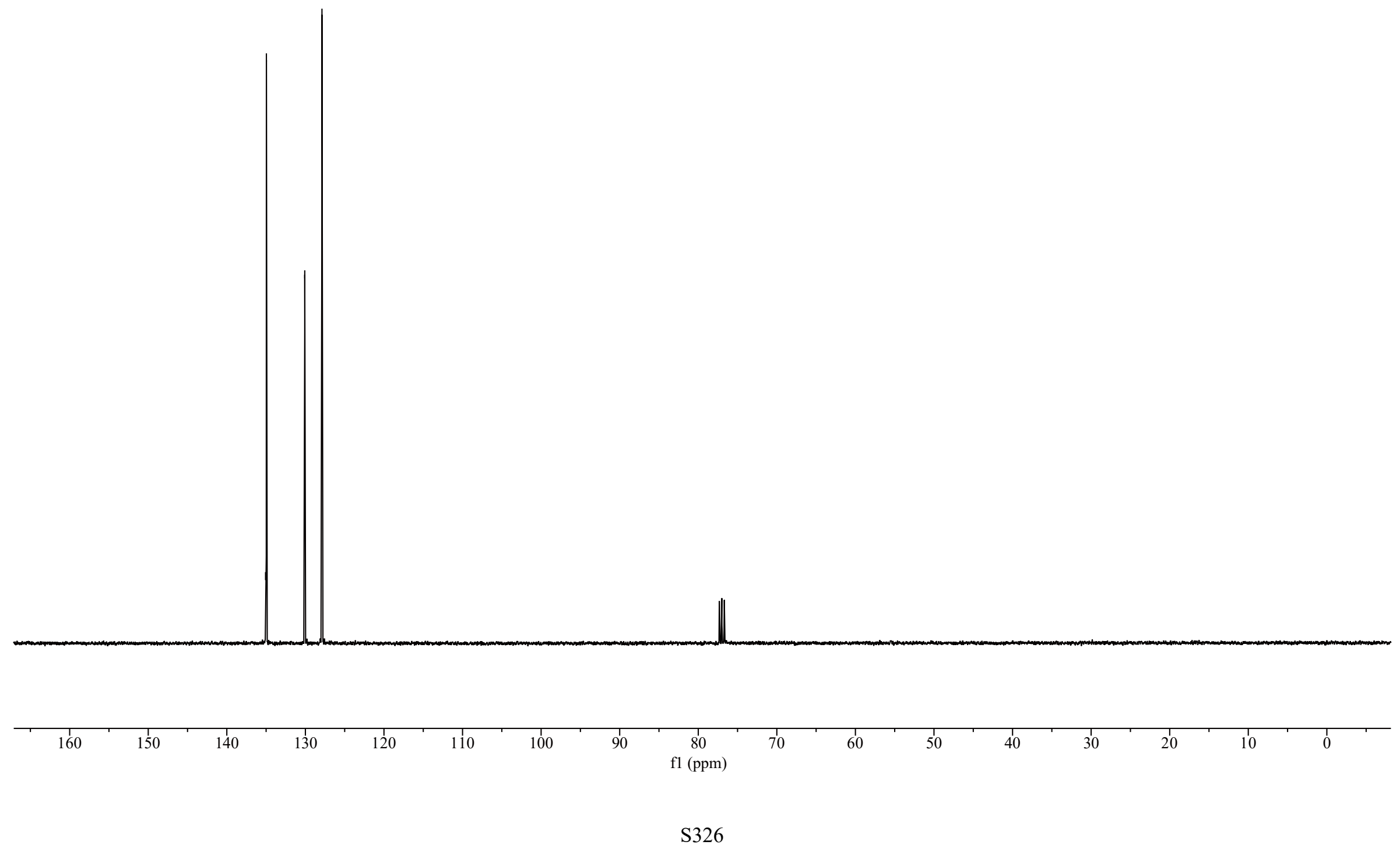




\section{Compound 2ad ${ }^{1} \mathrm{H}$ NMR}

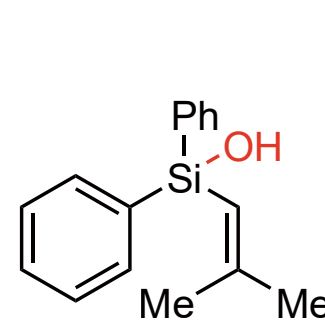

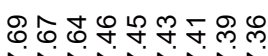

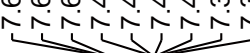

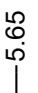

ֻ

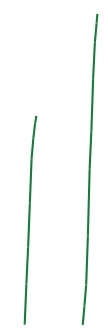

$\int \mid$

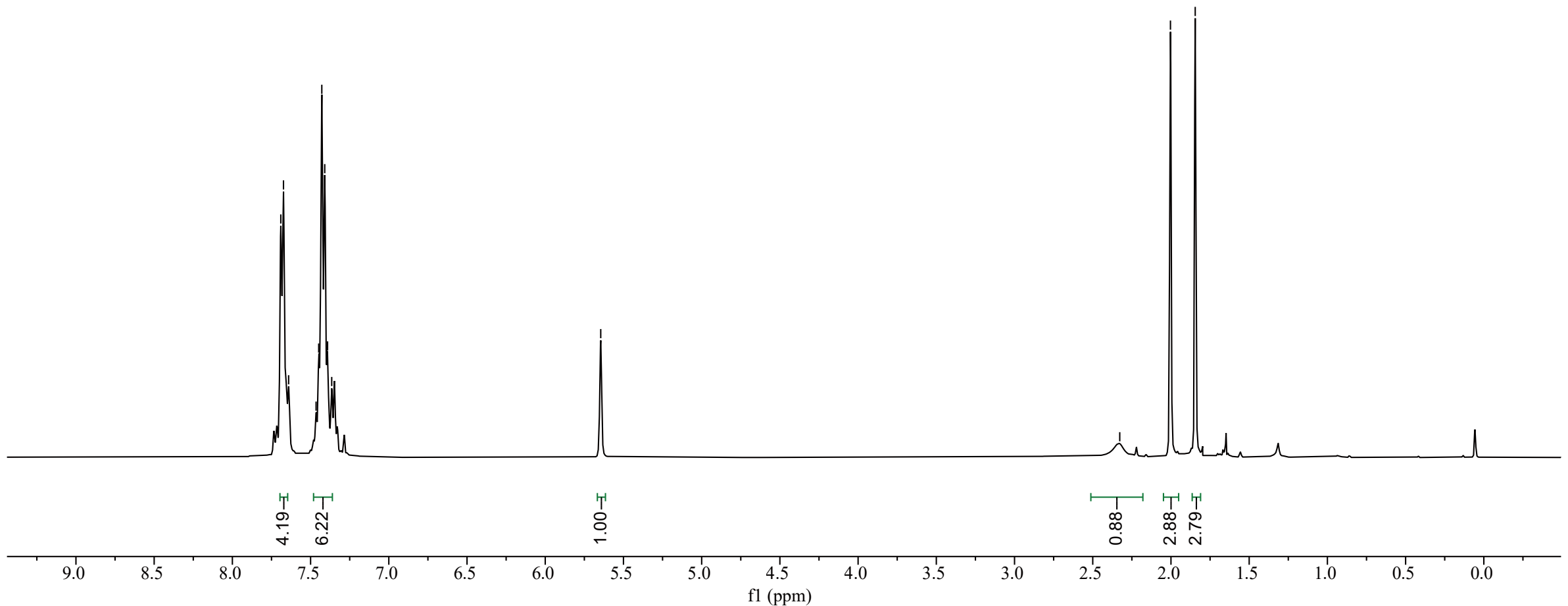


Compound 2ad ${ }^{13} \mathrm{C}$ NMR
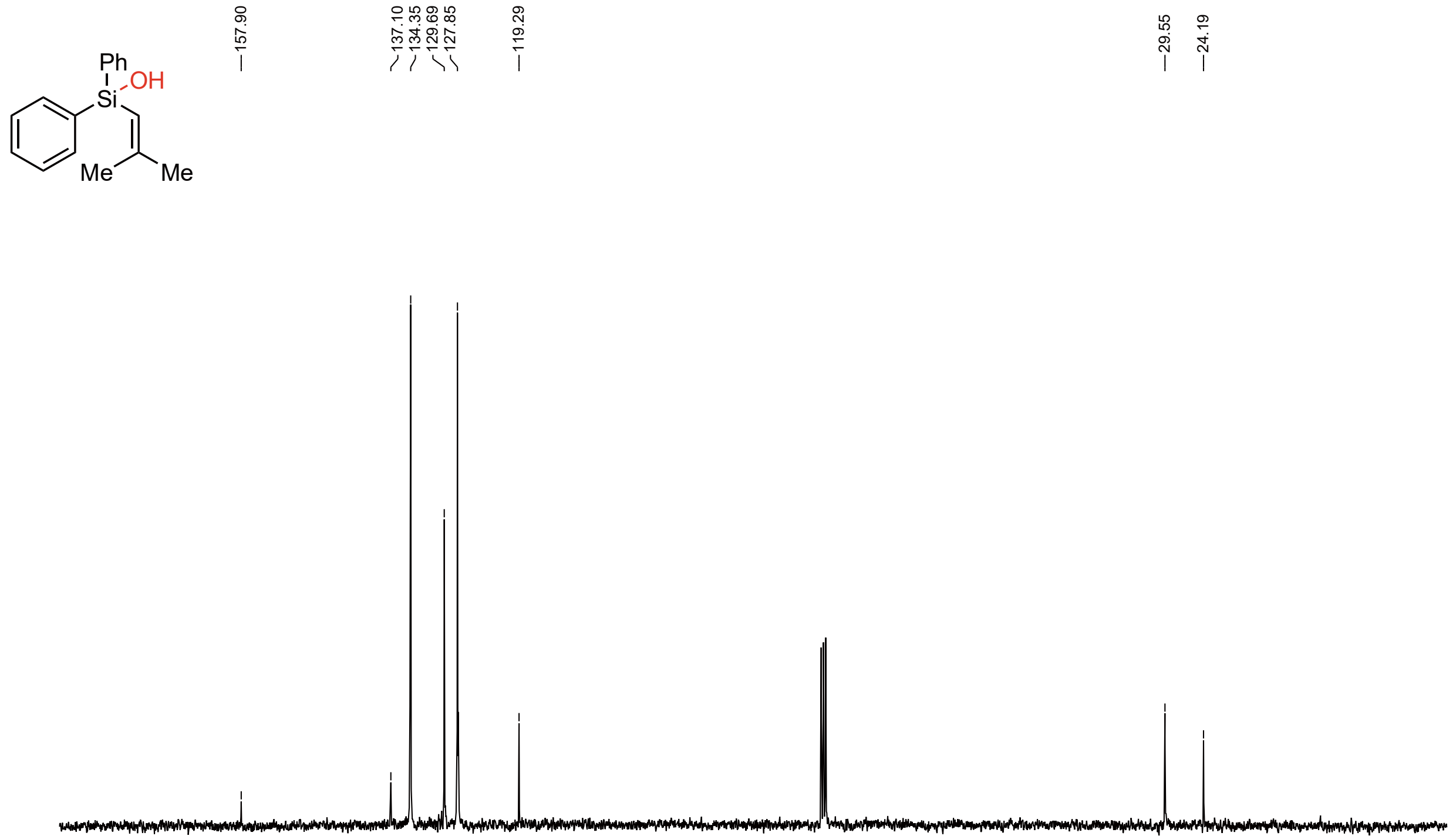
Compound 2ae ${ }^{1} \mathrm{H}$ NMR

\section{TMSO}

$\mathrm{Me}-\mathrm{Si}-\mathrm{OH}$

TMSO'

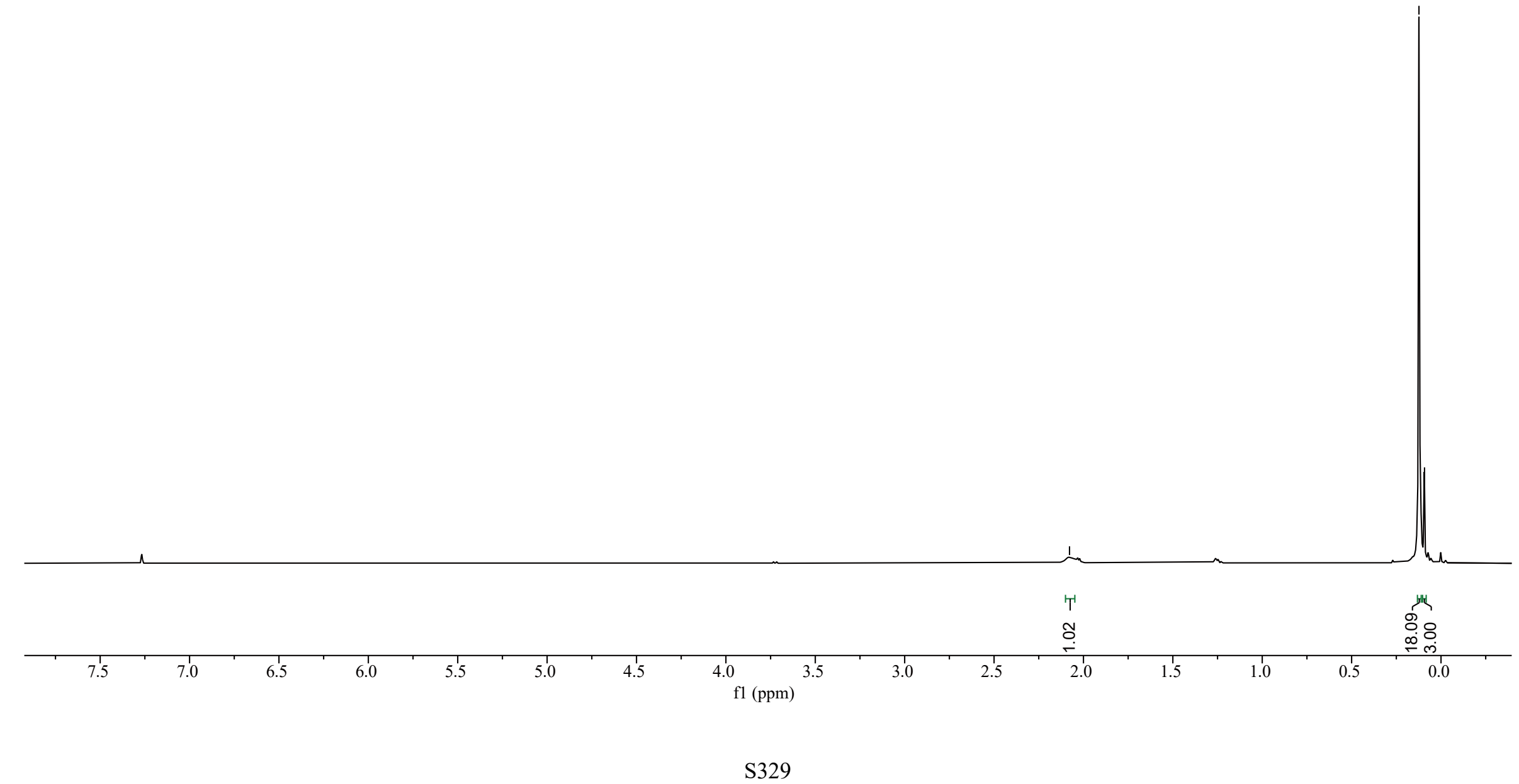


Compound 2ae ${ }^{13} \mathrm{C}$ NMR

TMSO

$\mathrm{Me}-\mathrm{Si}-\mathrm{OH}$

TMSO'

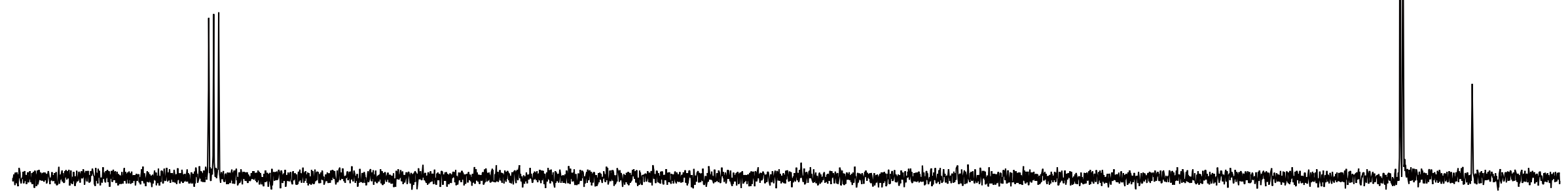


Compound 2af ${ }^{1} \mathrm{H}$ NMR
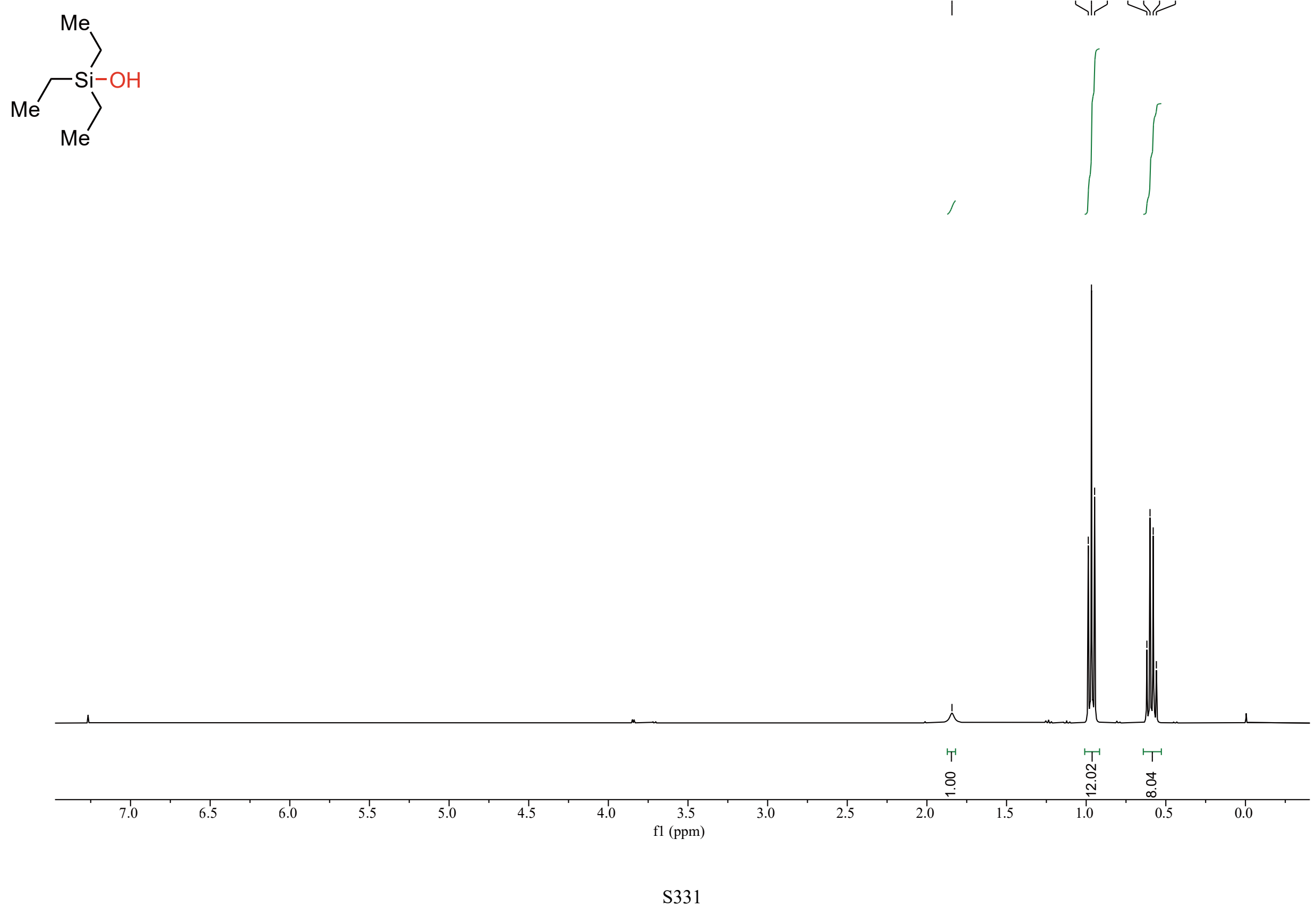
Compound 2af ${ }^{13} \mathrm{C}$ NMR

$\mathrm{Me}_{\mathrm{Me}}^{\mathrm{Me}} \sum^{\mathrm{Si}-\mathrm{OH}}$
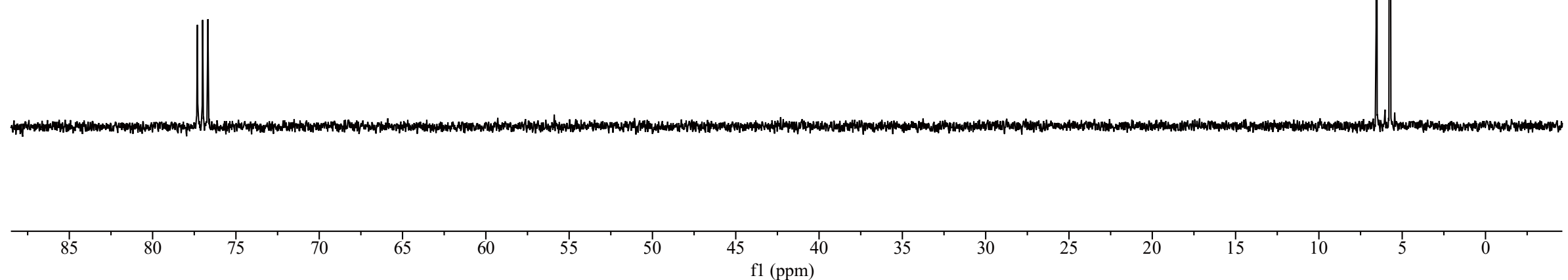
Compound 2ag ${ }^{1} \mathrm{H}$ NMR
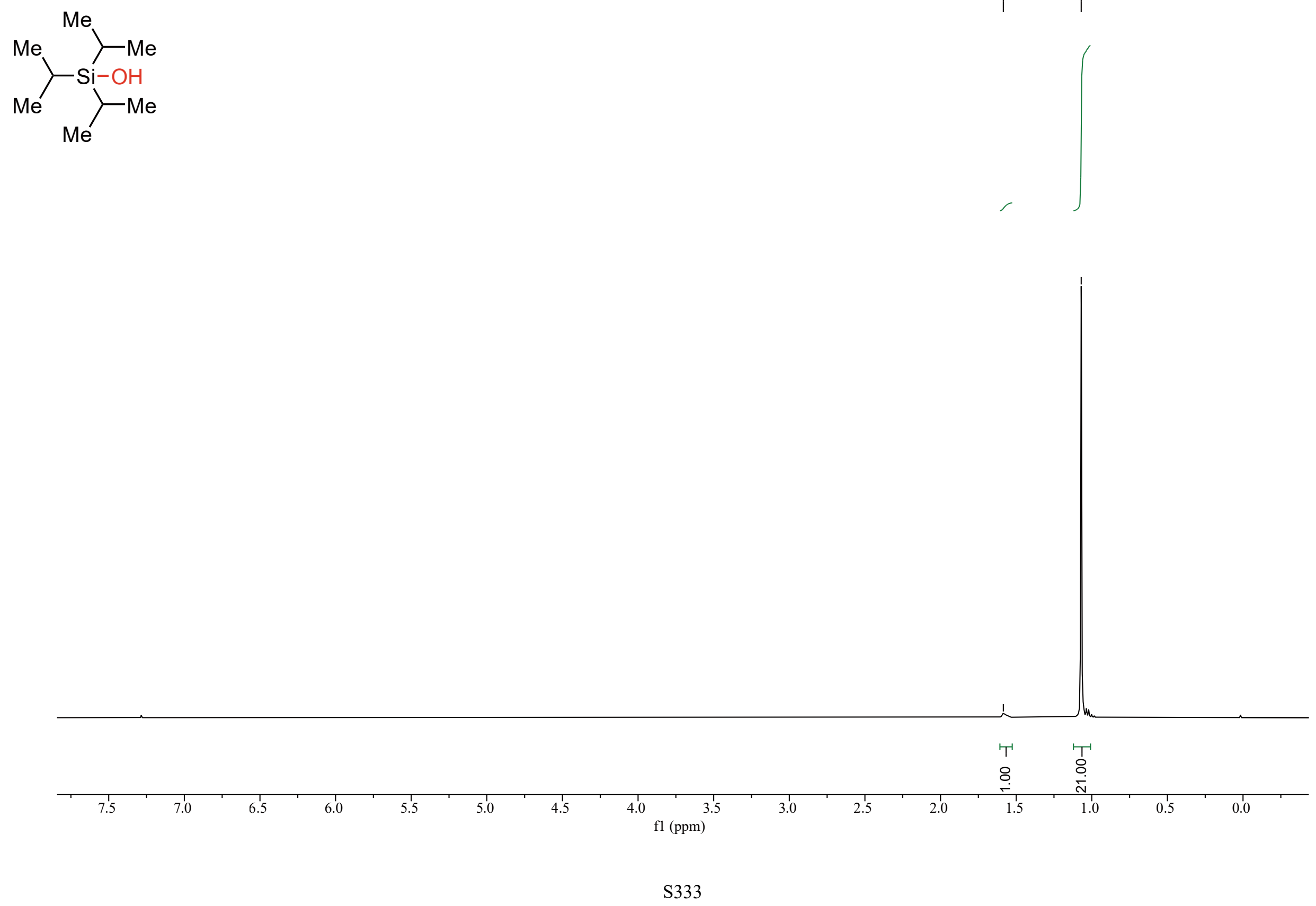
Compound 2ag ${ }^{13} \mathrm{C}$ NMR

$\mathrm{Me}_{\mathrm{Me}}^{\mathrm{Me}} \underset{\mathrm{Si}-\mathrm{OH}}{\succ-\mathrm{Me}}$

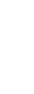
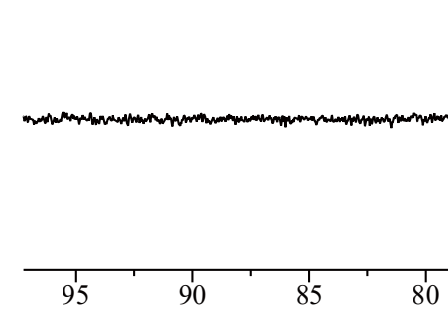

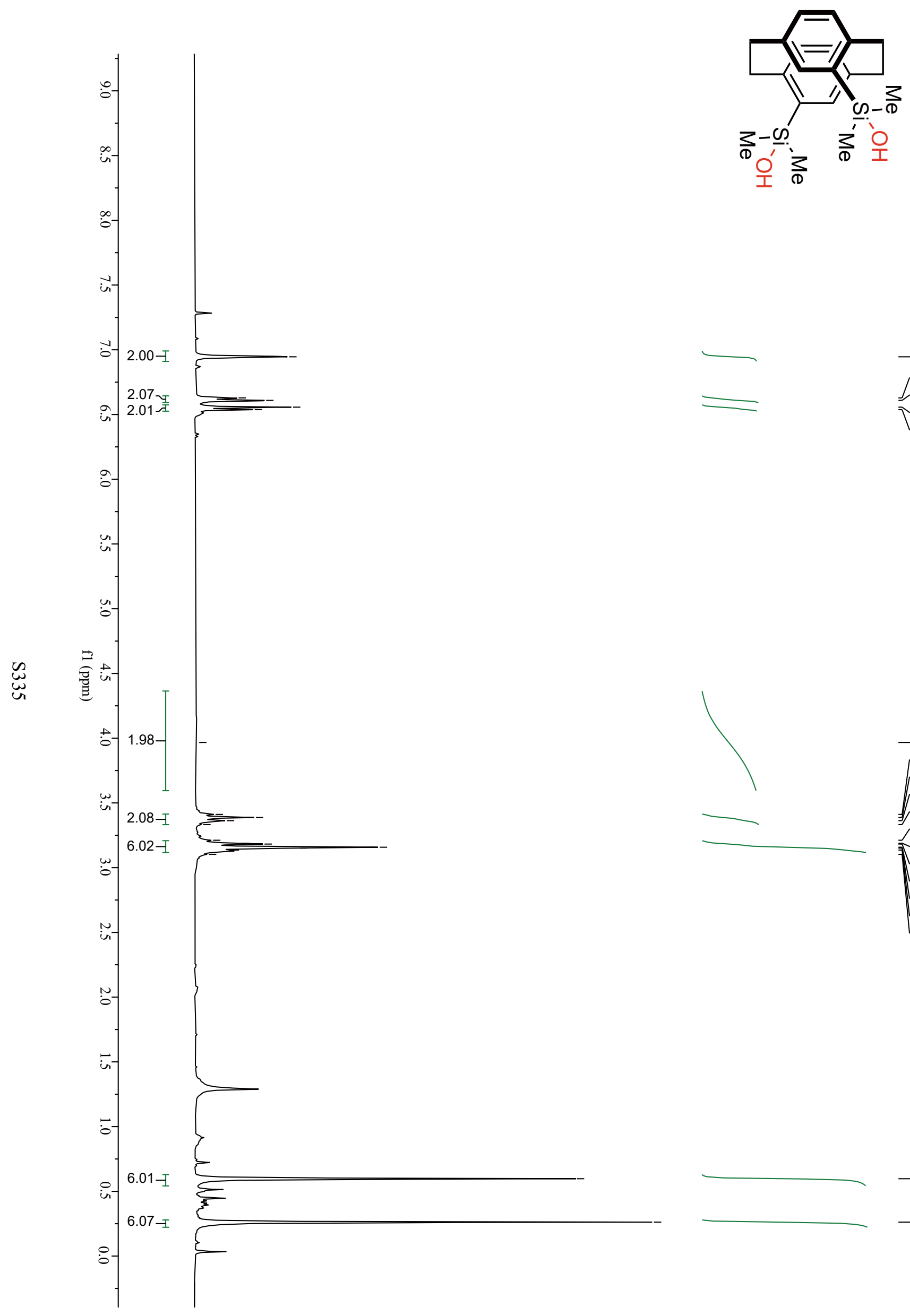

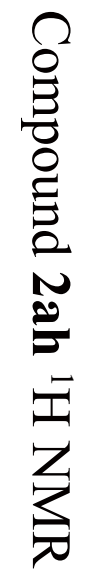
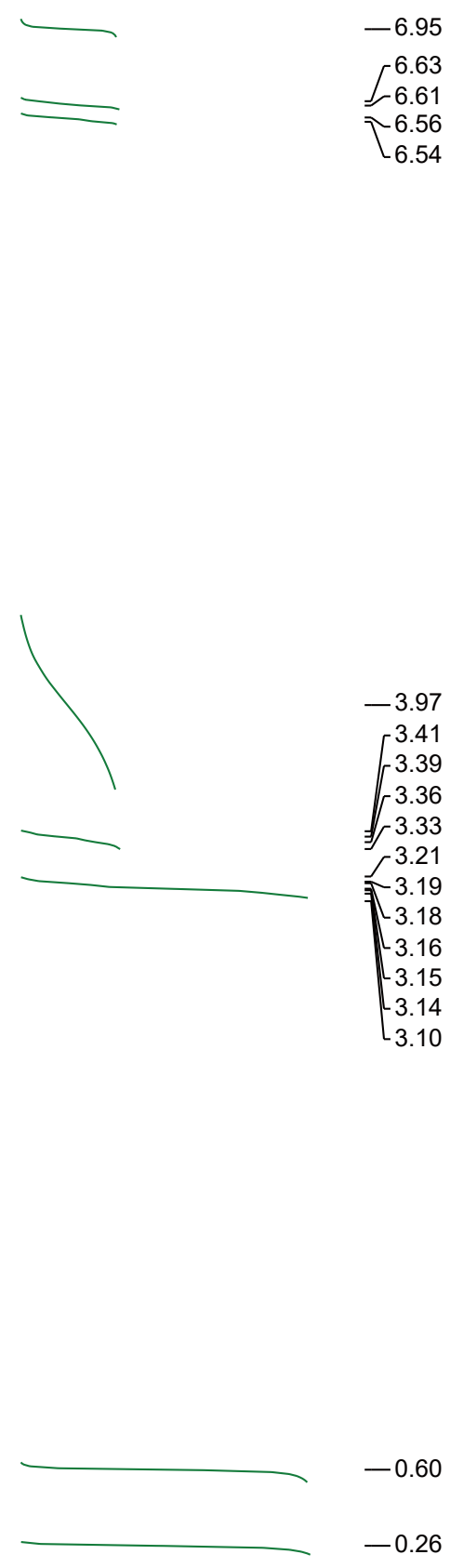
Compound 2ah ${ }^{13} \mathrm{C}$ NMR

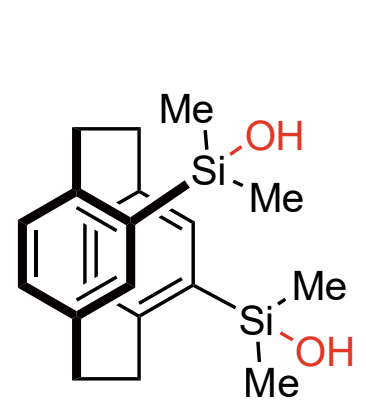

|

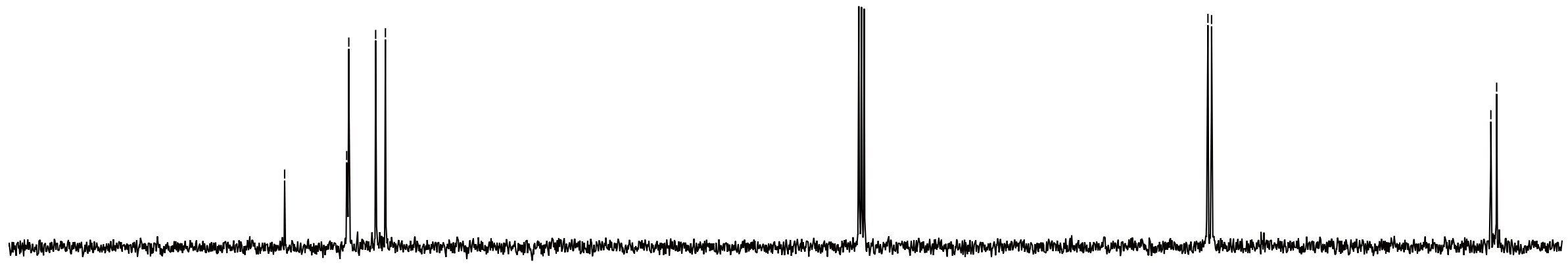


Compound 2ai ${ }^{1} \mathrm{H}$ NMR

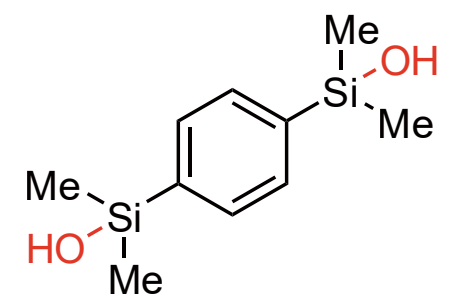

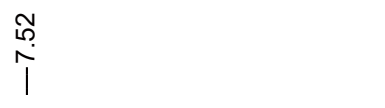

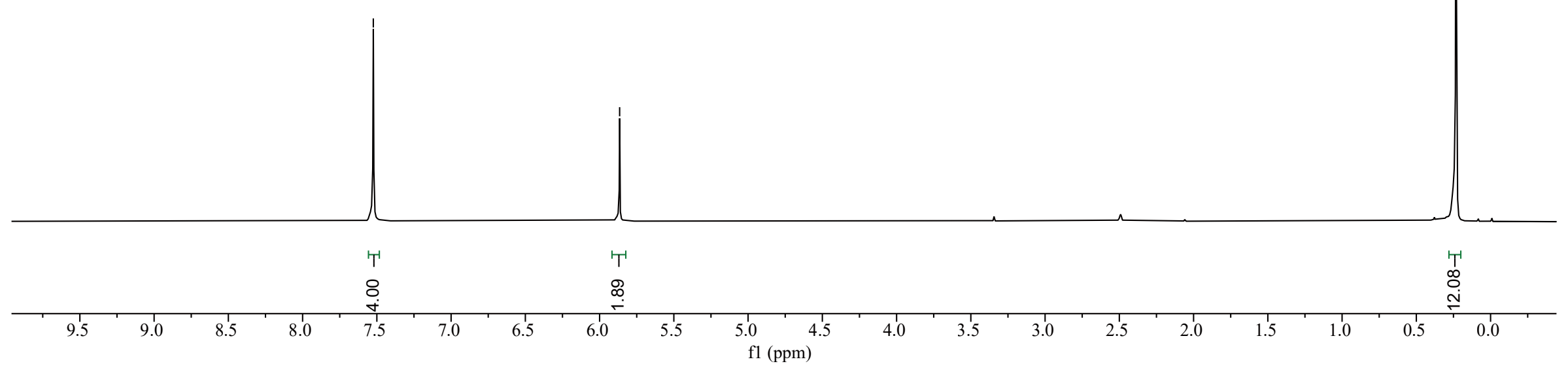


Compound 2ai ${ }^{13} \mathrm{C}$ NMR
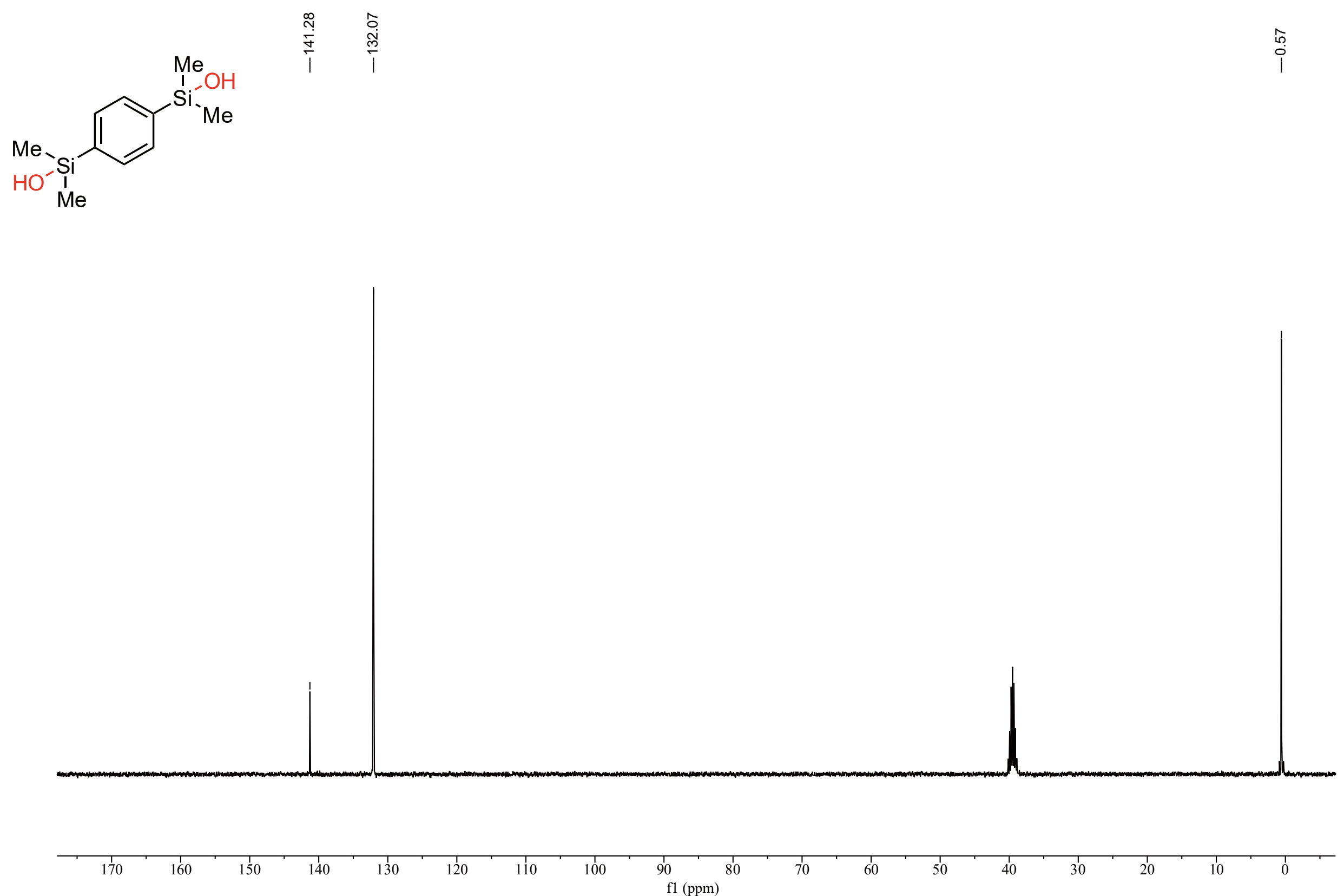


\section{Compound 2aj ${ }^{1} \mathrm{H}$ NMR}

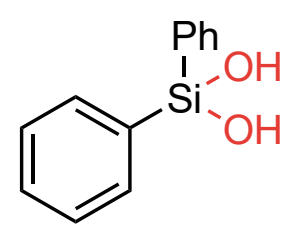

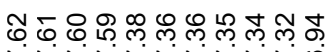

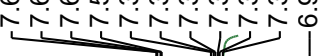

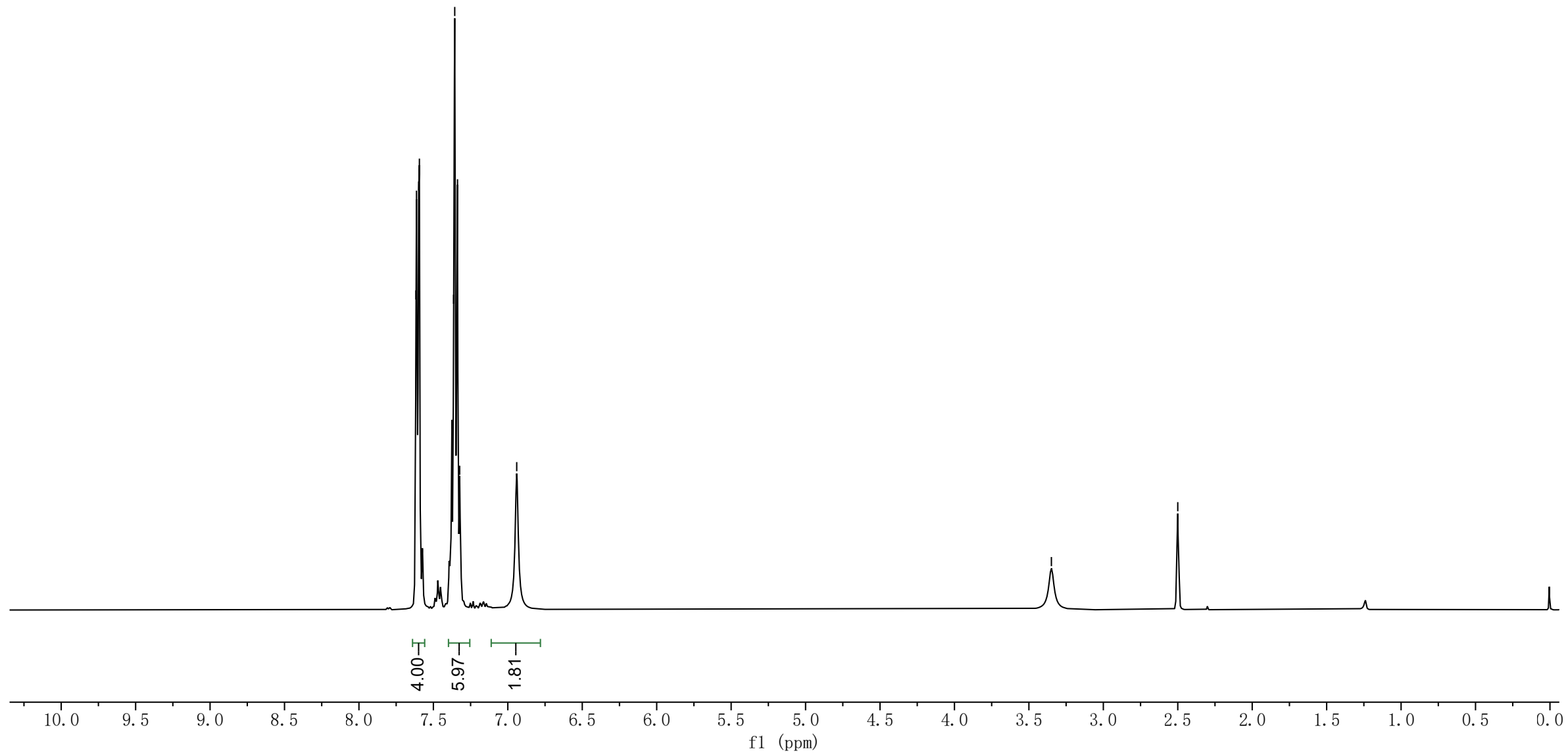


Compound 2aj ${ }^{13} \mathrm{C}$ NMR
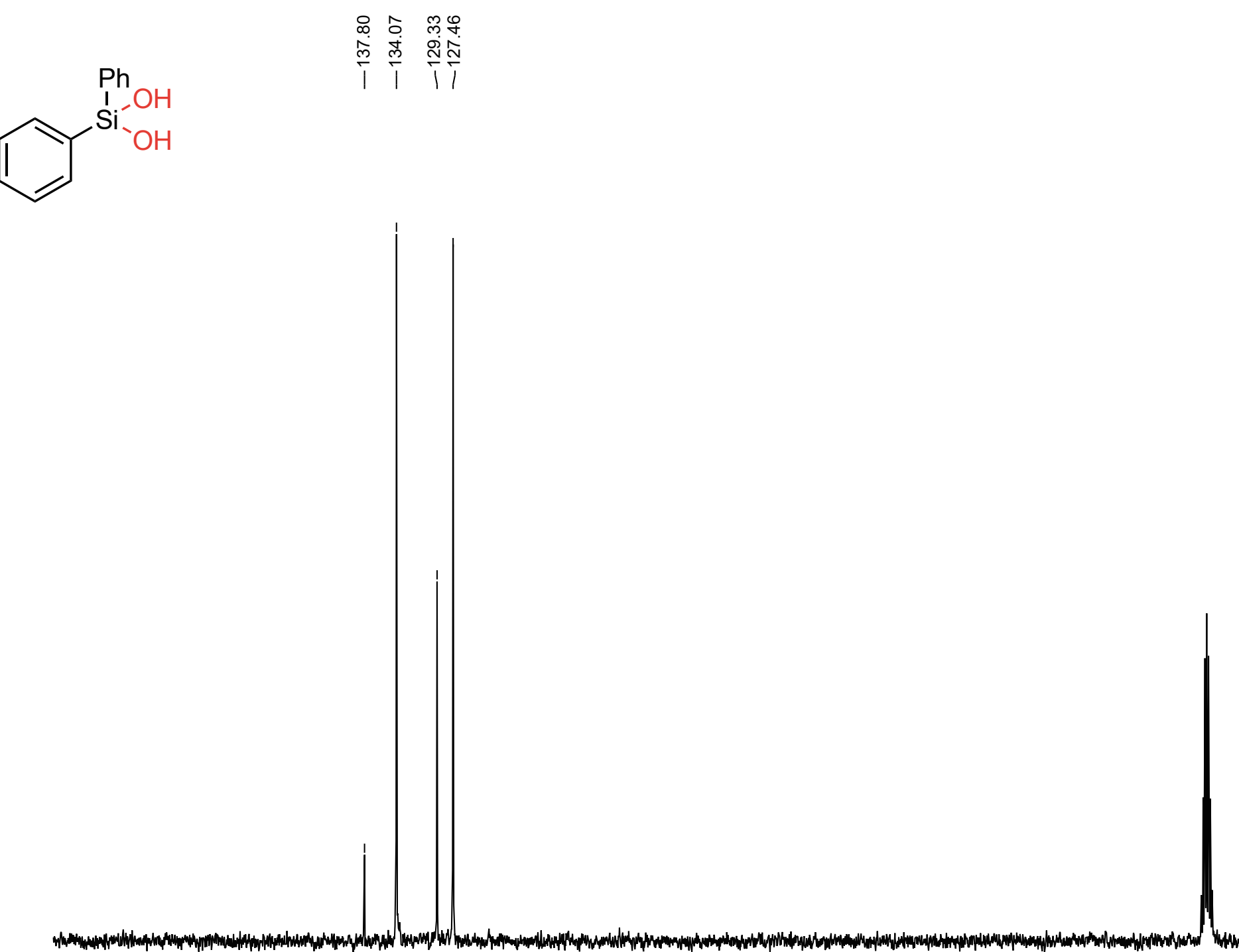

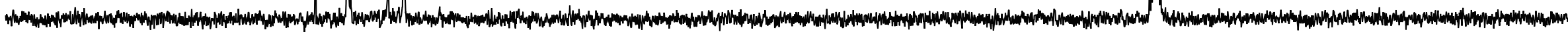

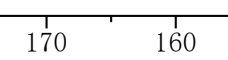



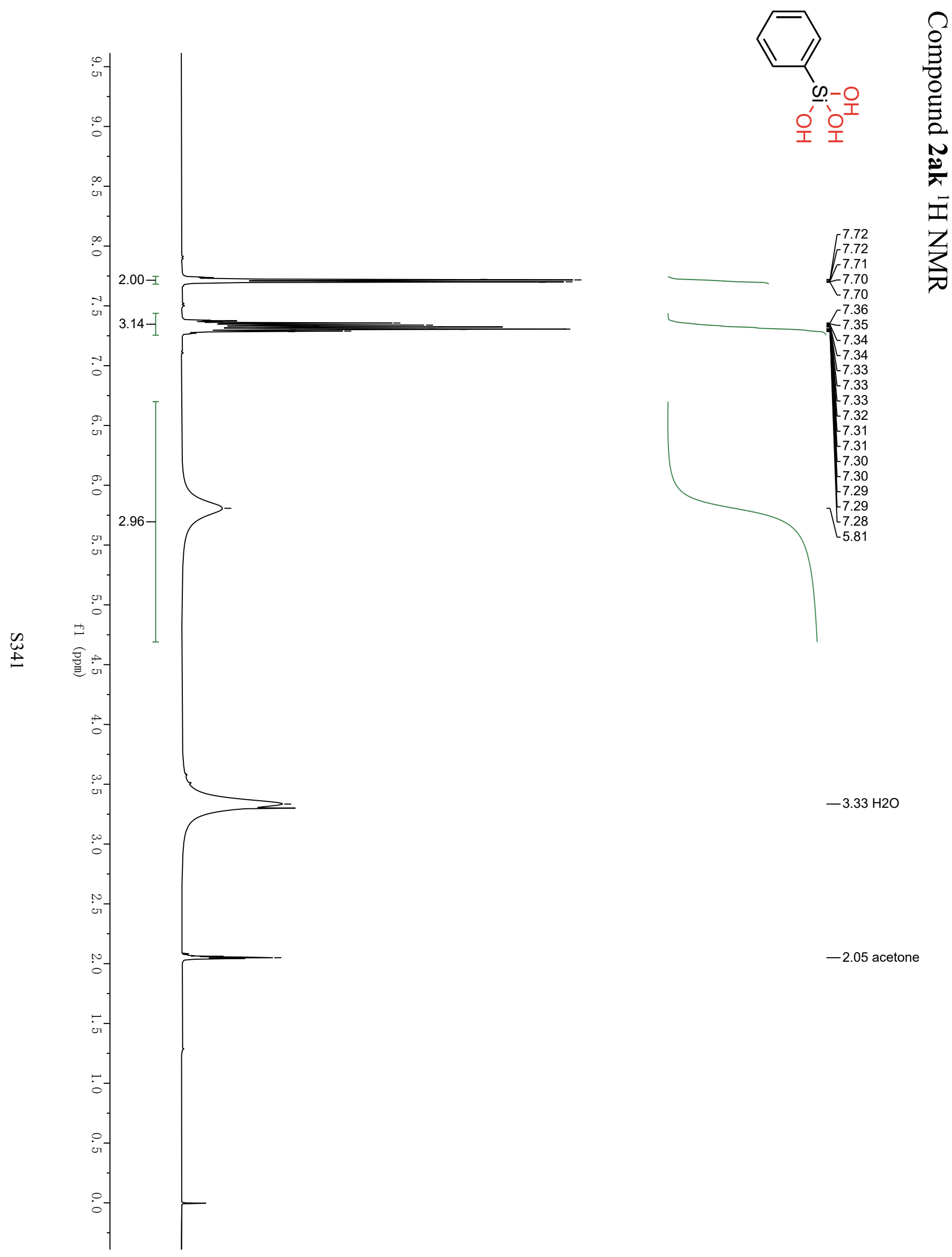
Compound 2ak ${ }^{13} \mathrm{C}$ NMR

중ํํำ

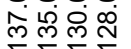

$\overbrace{}^{\mathrm{Si}_{i}^{\mathrm{OH}}}$

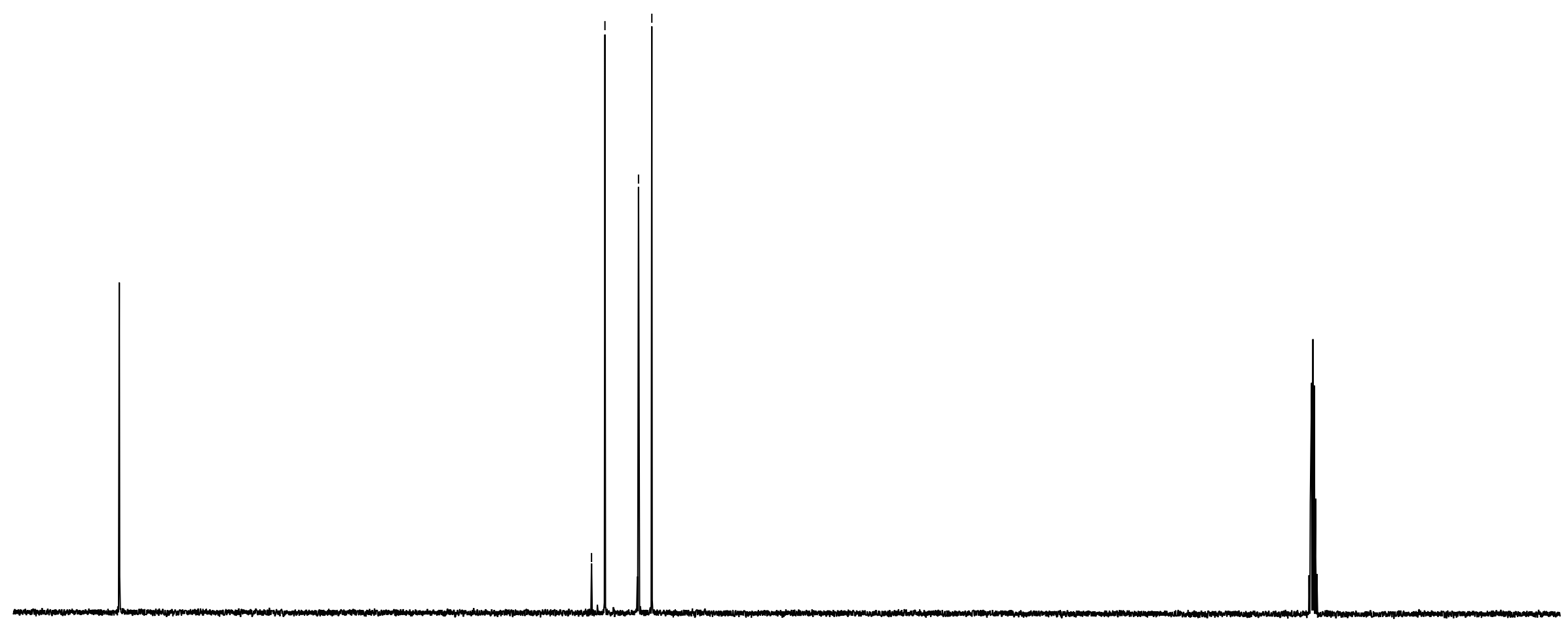

$\overline{220} 210$

190

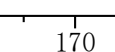

160
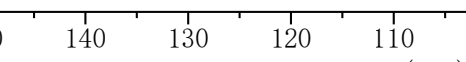

f1 (ppm) 


\section{Compound 2al ${ }^{1} \mathrm{H}$ NMR}

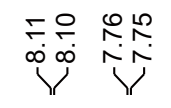
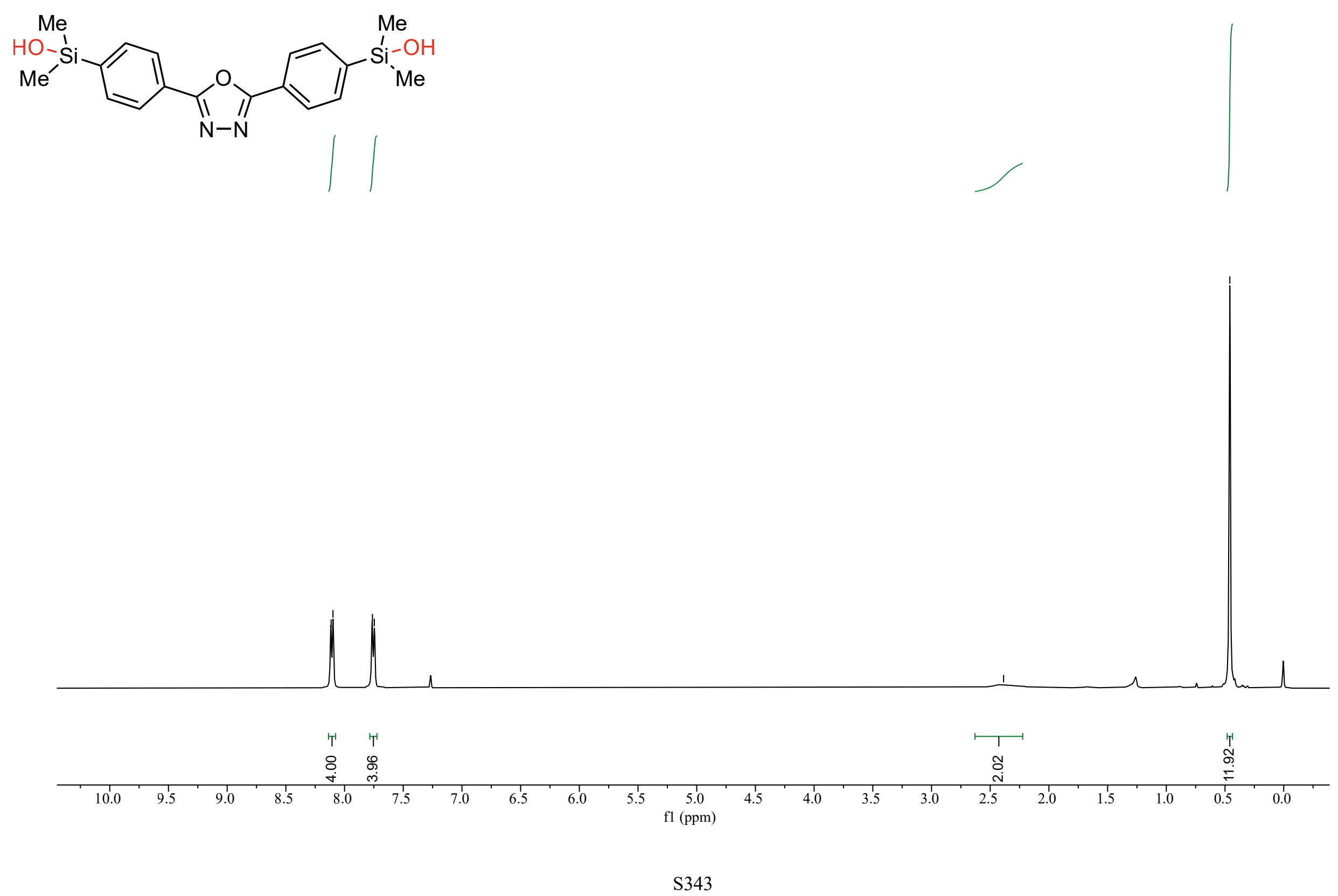
Compound 2al ${ }^{13} \mathrm{C}$ NMR

(1)
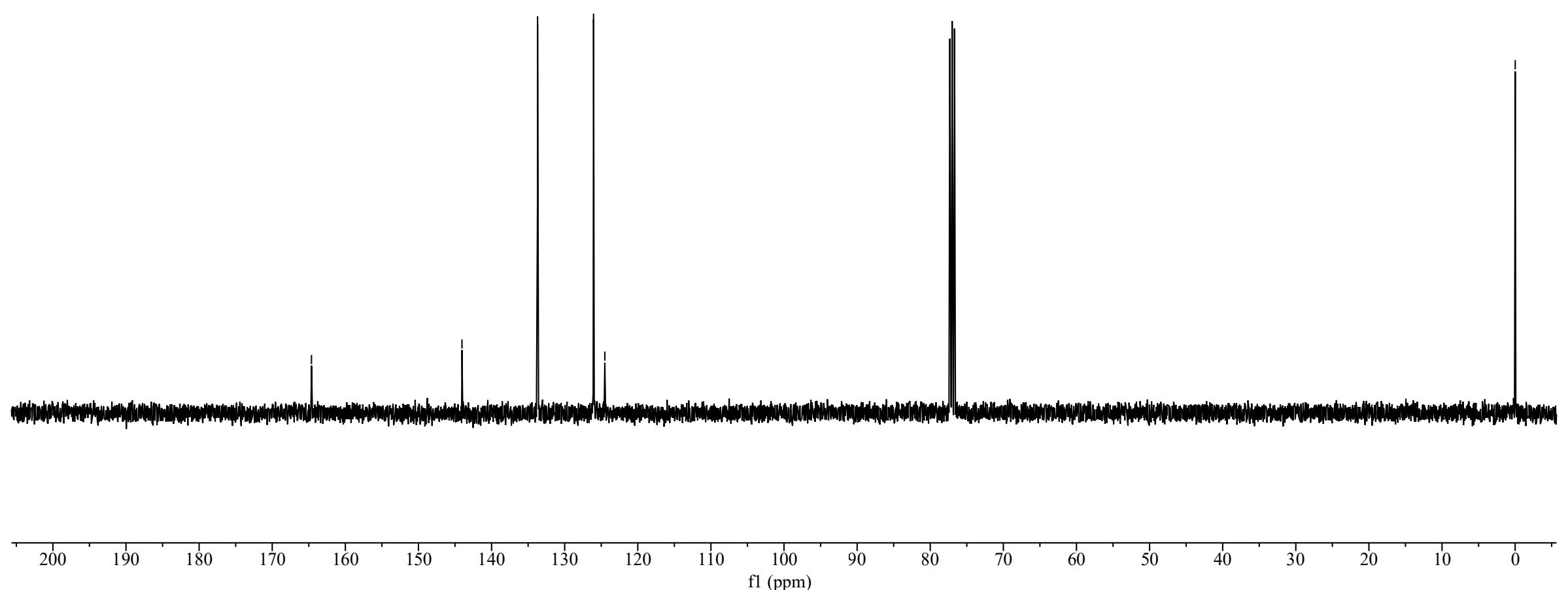
Compound 2am ${ }^{1} \mathrm{H}$ NMR



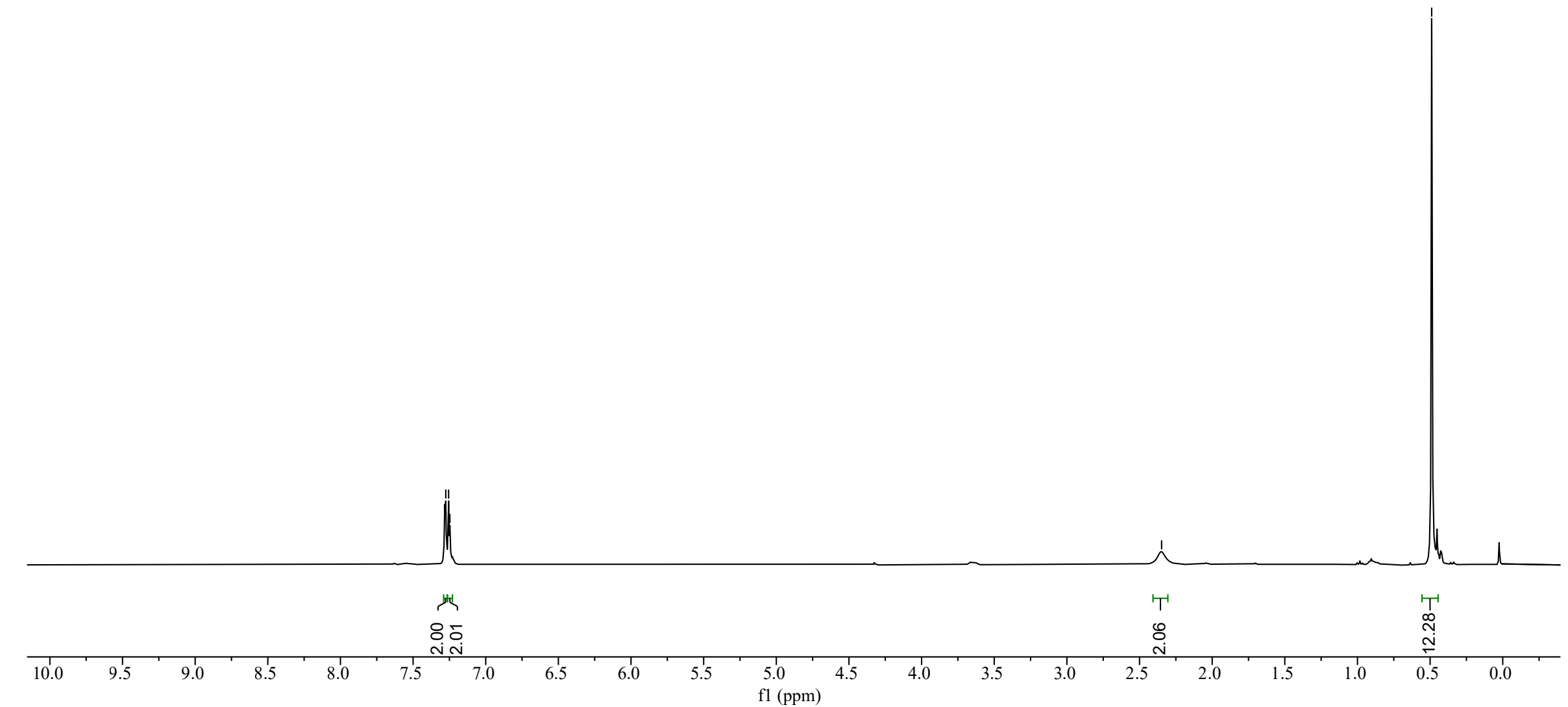


Compound 2am ${ }^{13} \mathrm{C}$ NMR
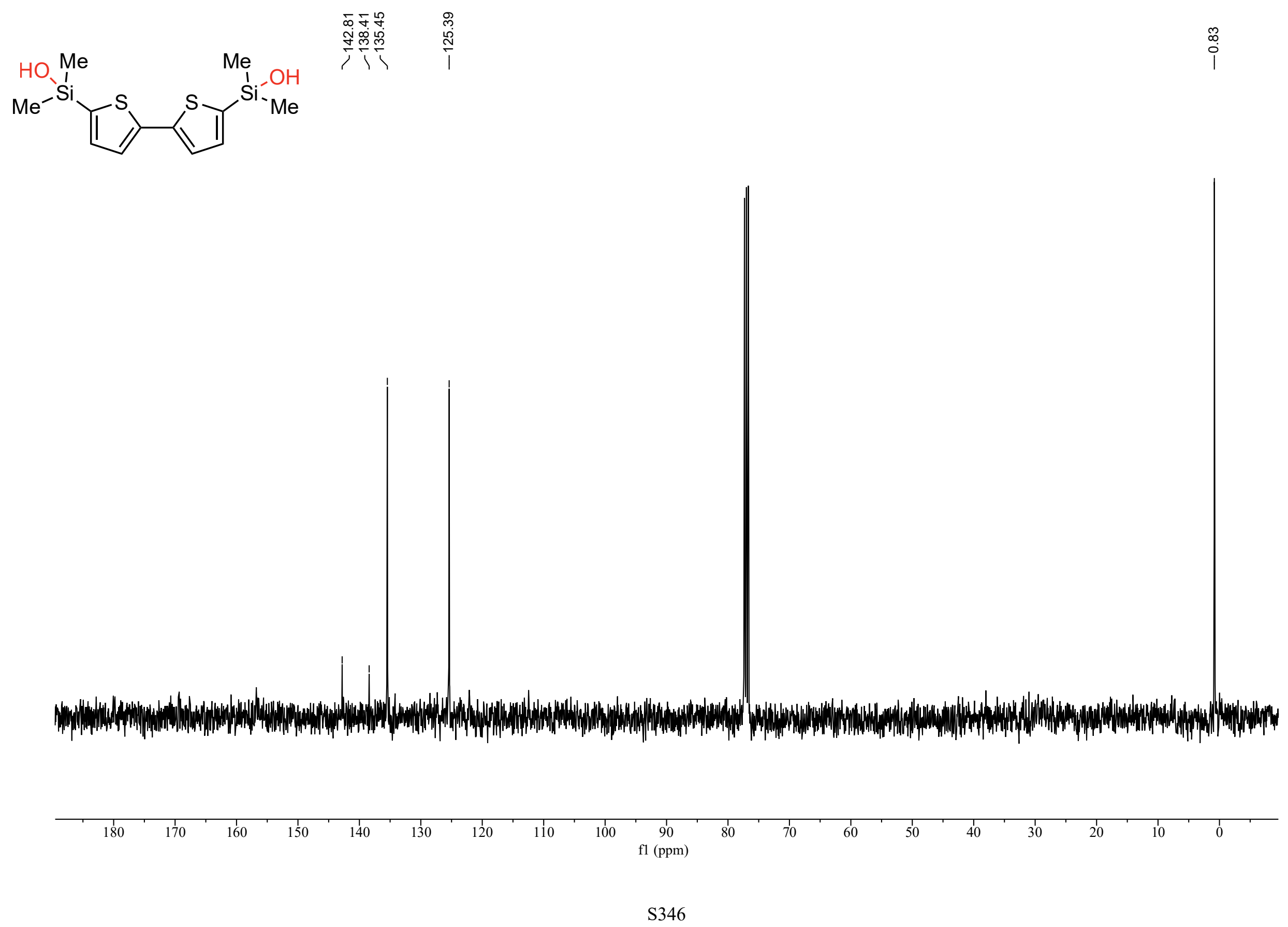


\section{Compound 2an ${ }^{1} \mathrm{H}$ NMR}

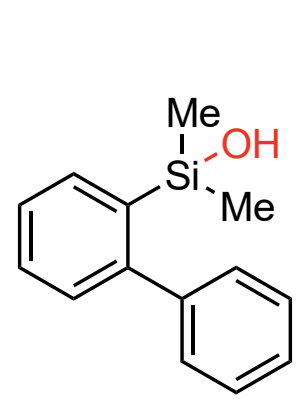

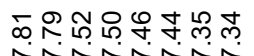

武造倍
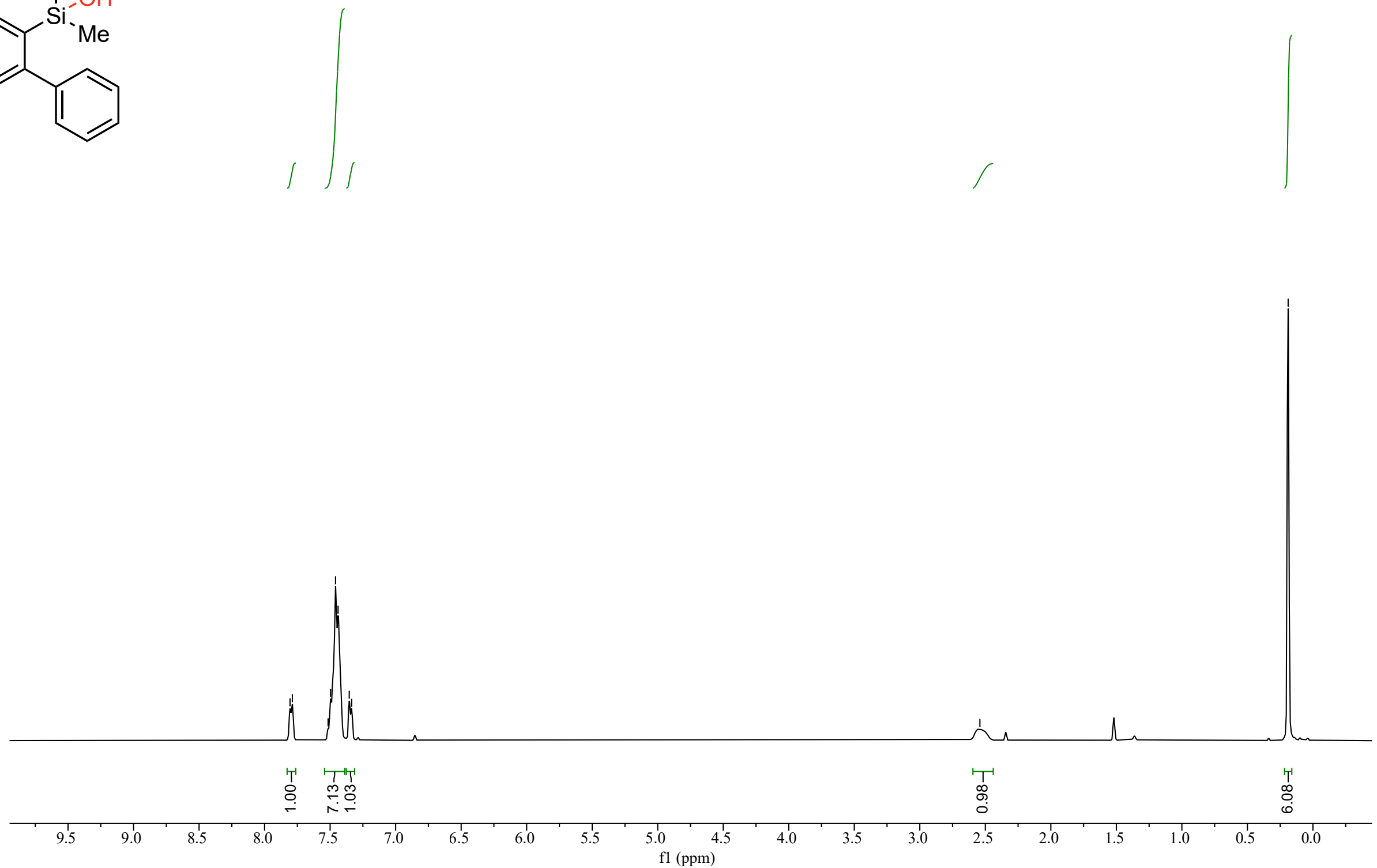
Compound 2an ${ }^{13} \mathrm{C}$ NMR

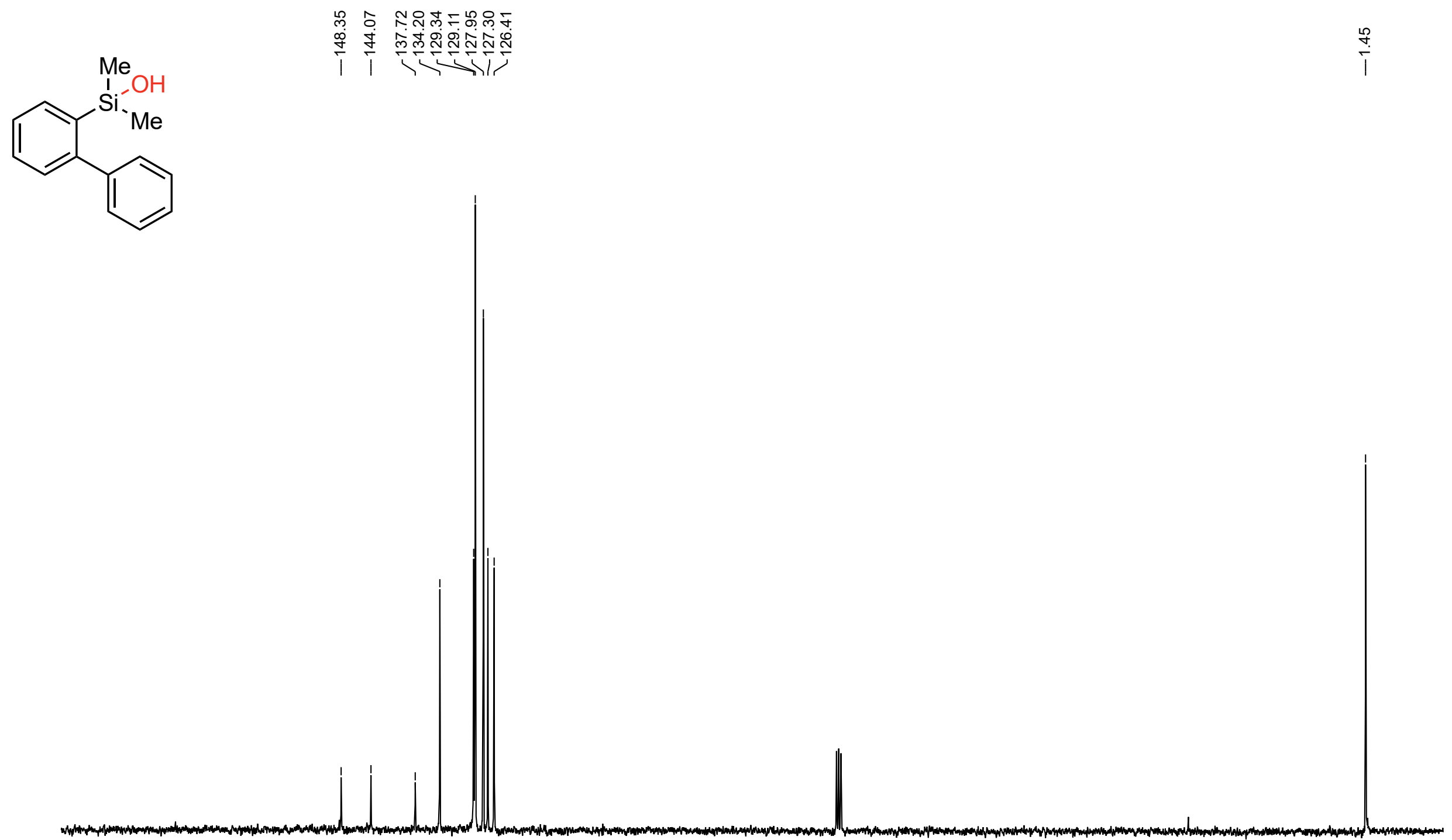



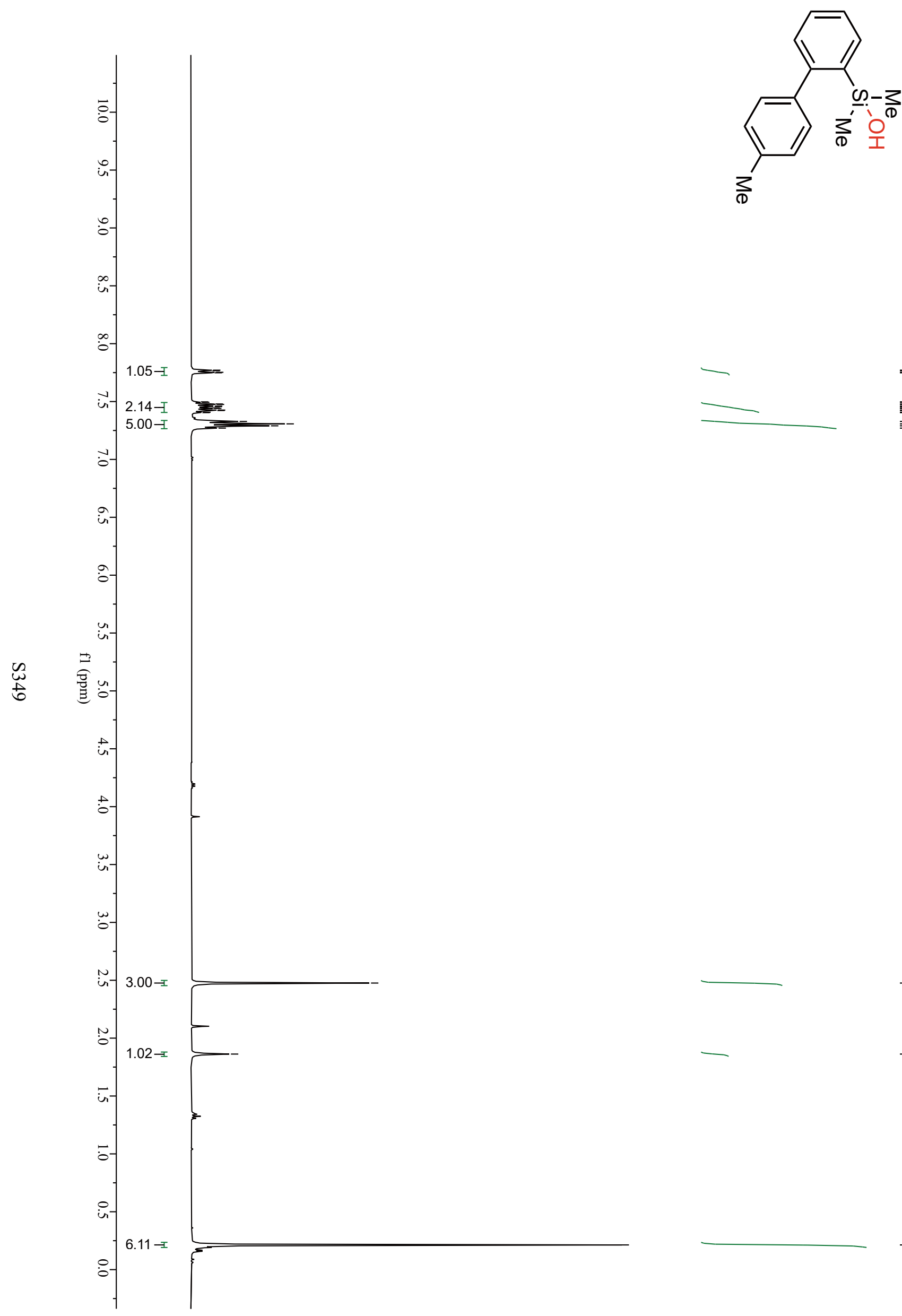

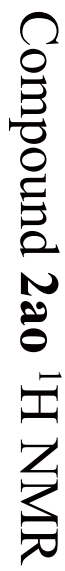
7.75 7.50 7.49 7.48 7.48

7.46

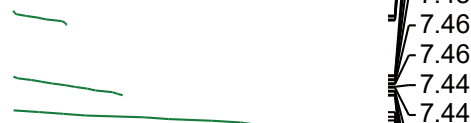

-7.44
-7.44

7.43
7.42

$-7.41$

$-7.40$

-7.33
-7.31

7.31

-7.29
7.27

$\underset{w}{w}$

$-2.48$

$-1.86$

$-0.21$ 
Compound 2ao ${ }^{13} \mathrm{C}$ NMR

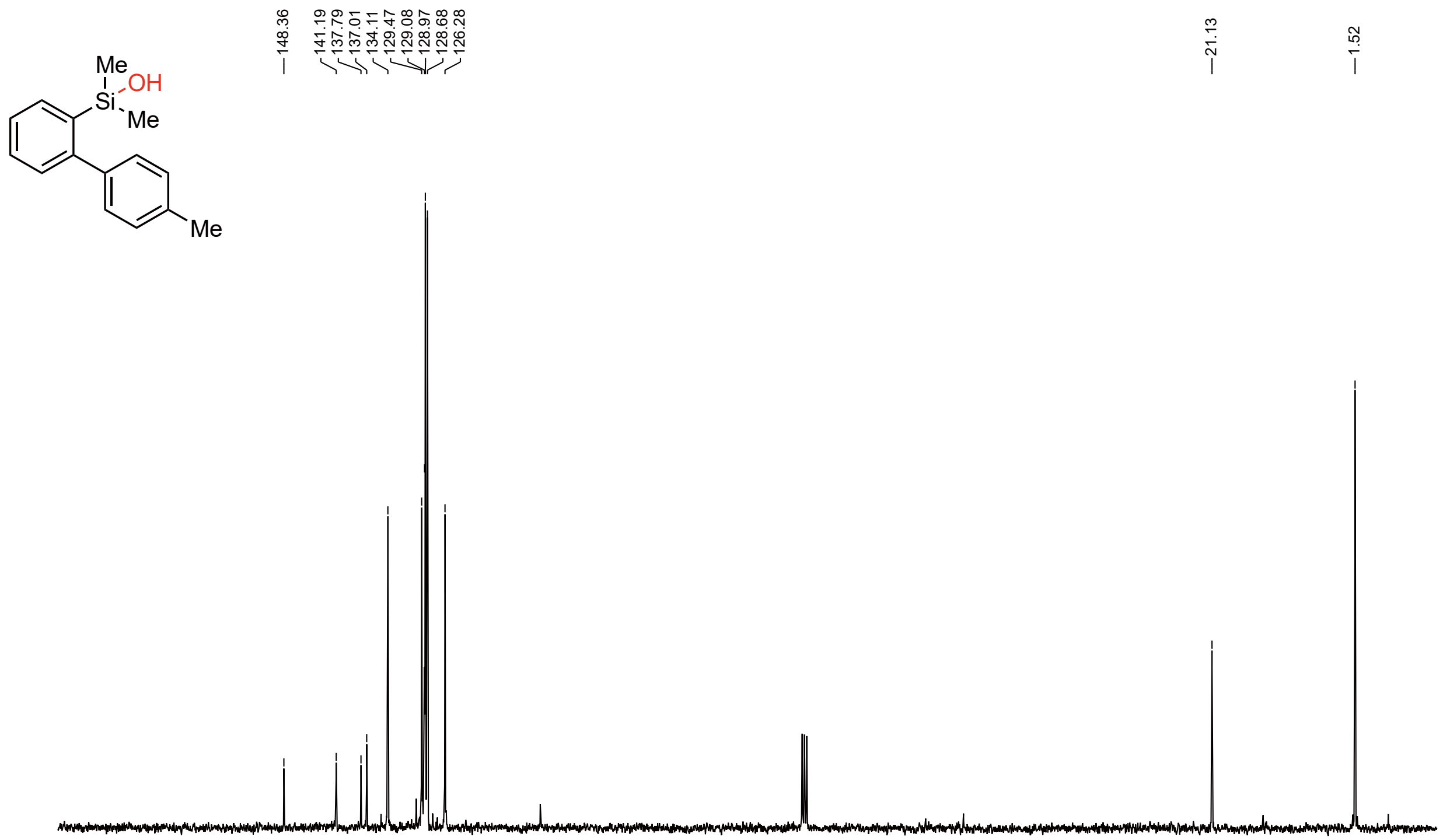




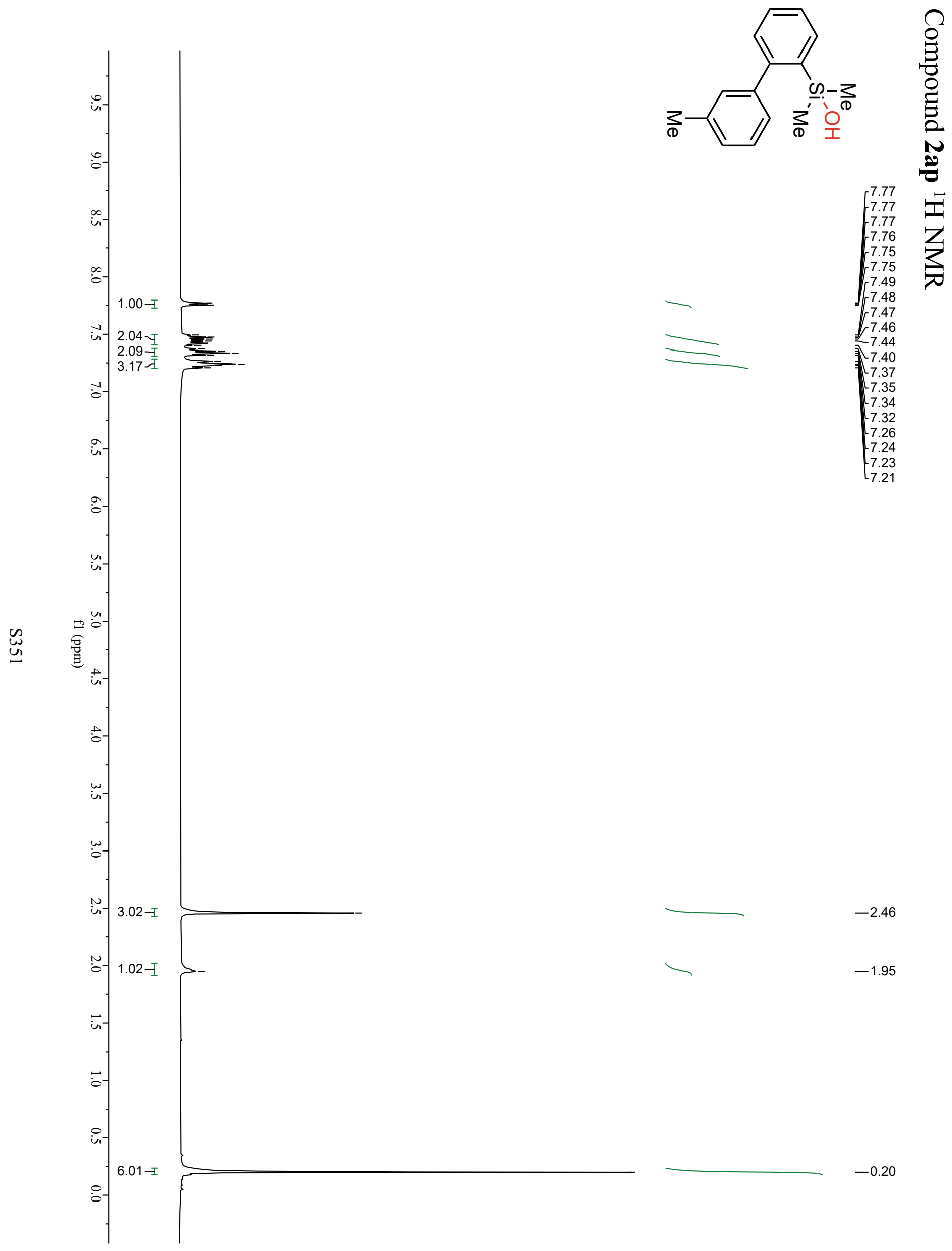


Compound 2ap ${ }^{13} \mathrm{C}$ NMR

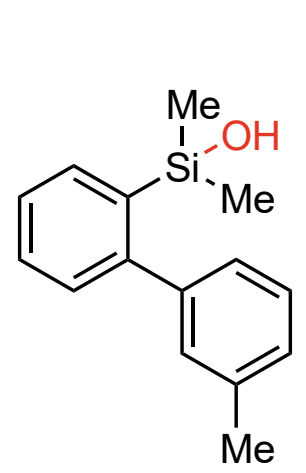

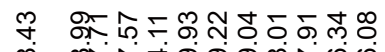

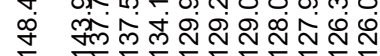

1

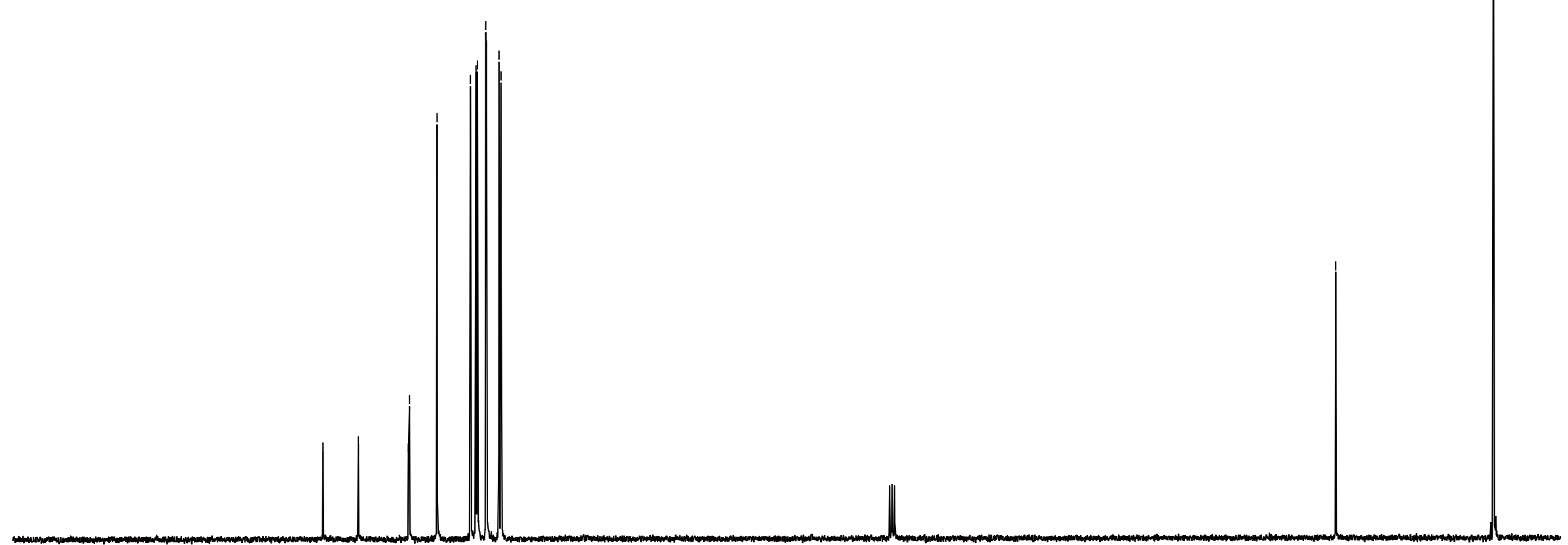




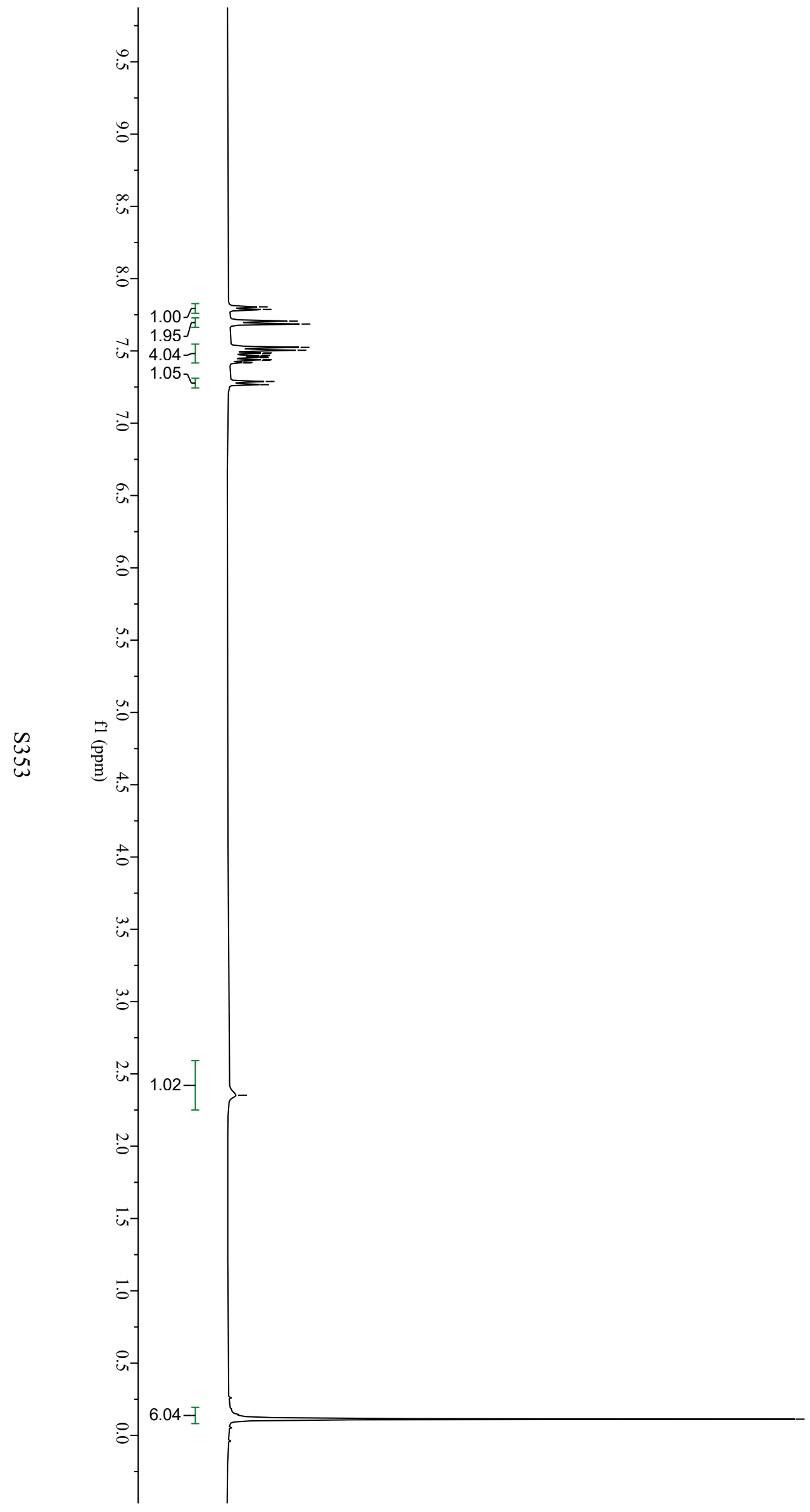

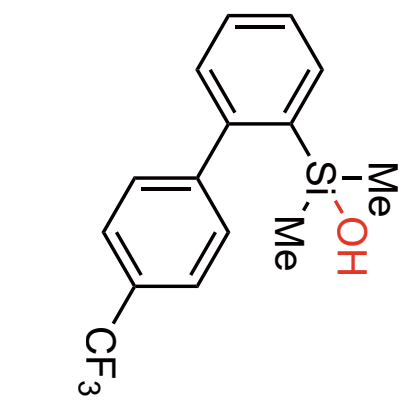

7.81 7.81
-7.79 7.71
7.69 7.69 7.52

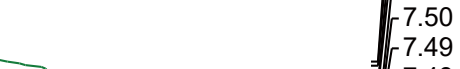

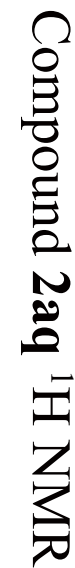

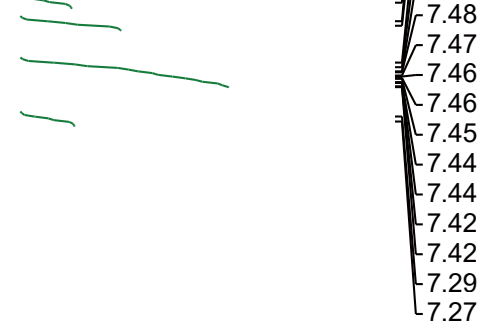

$-2.35$ 


\section{Compound 2aq ${ }^{13} \mathrm{C}$ NMR}
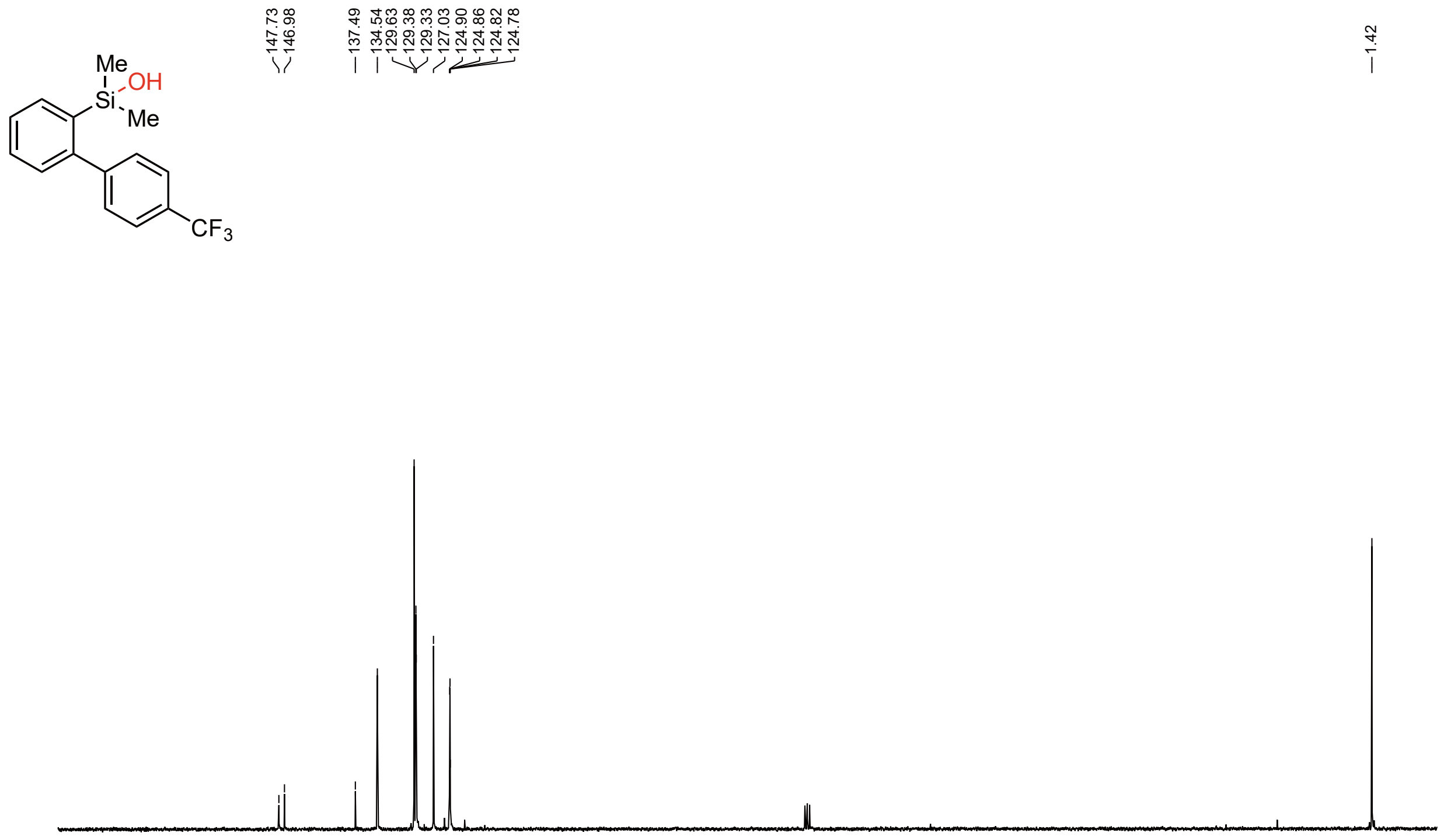
Compound 2aq ${ }^{19}$ F NMR

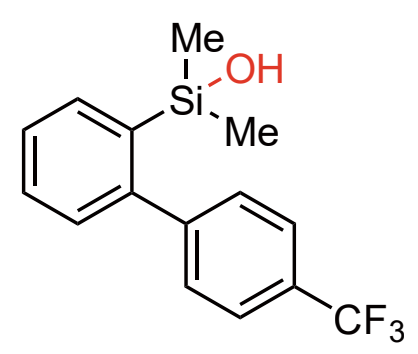


Compound 2ar ${ }^{1} \mathrm{H}$ NMR

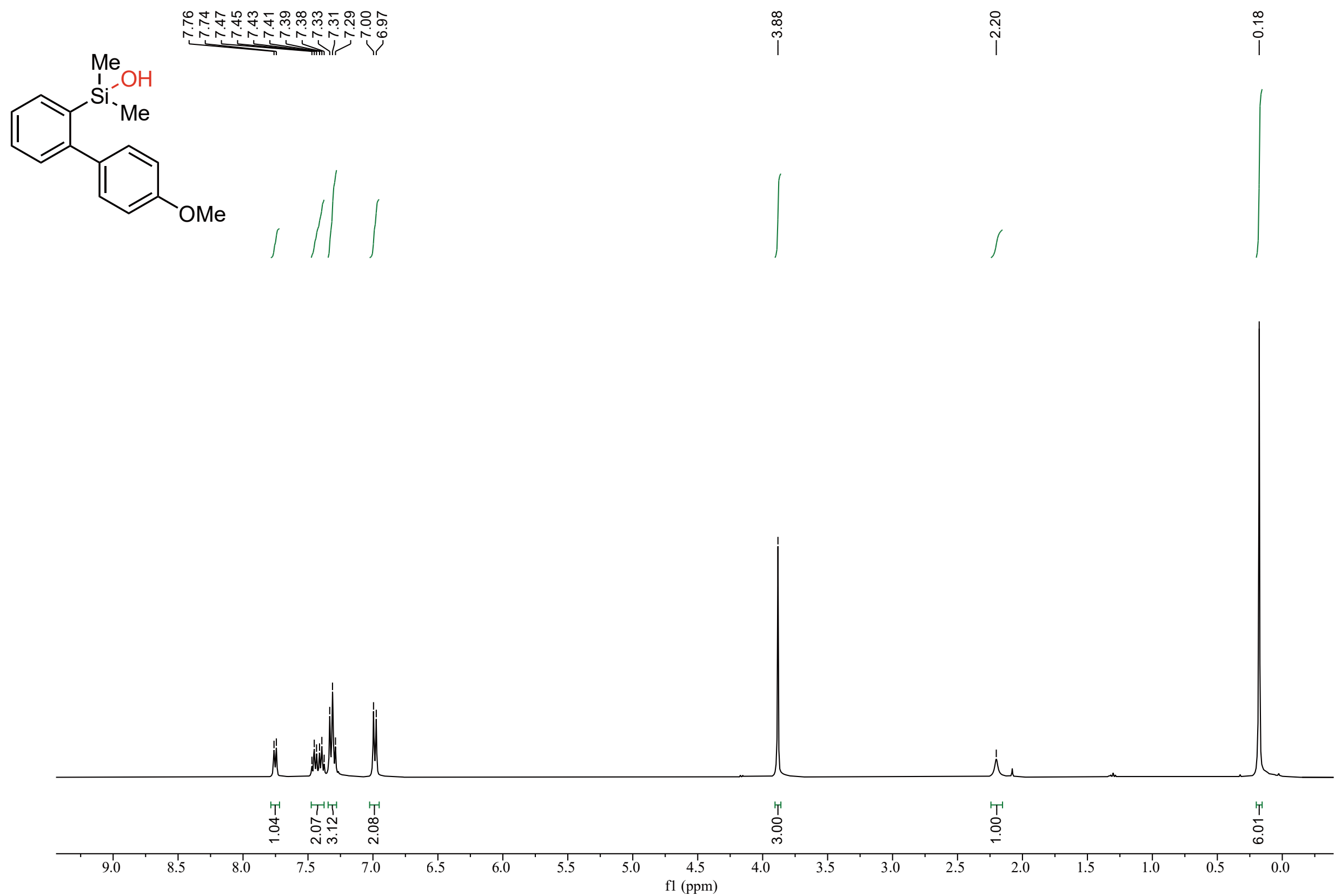


Compound 2ar ${ }^{13} \mathrm{C}$ NMR

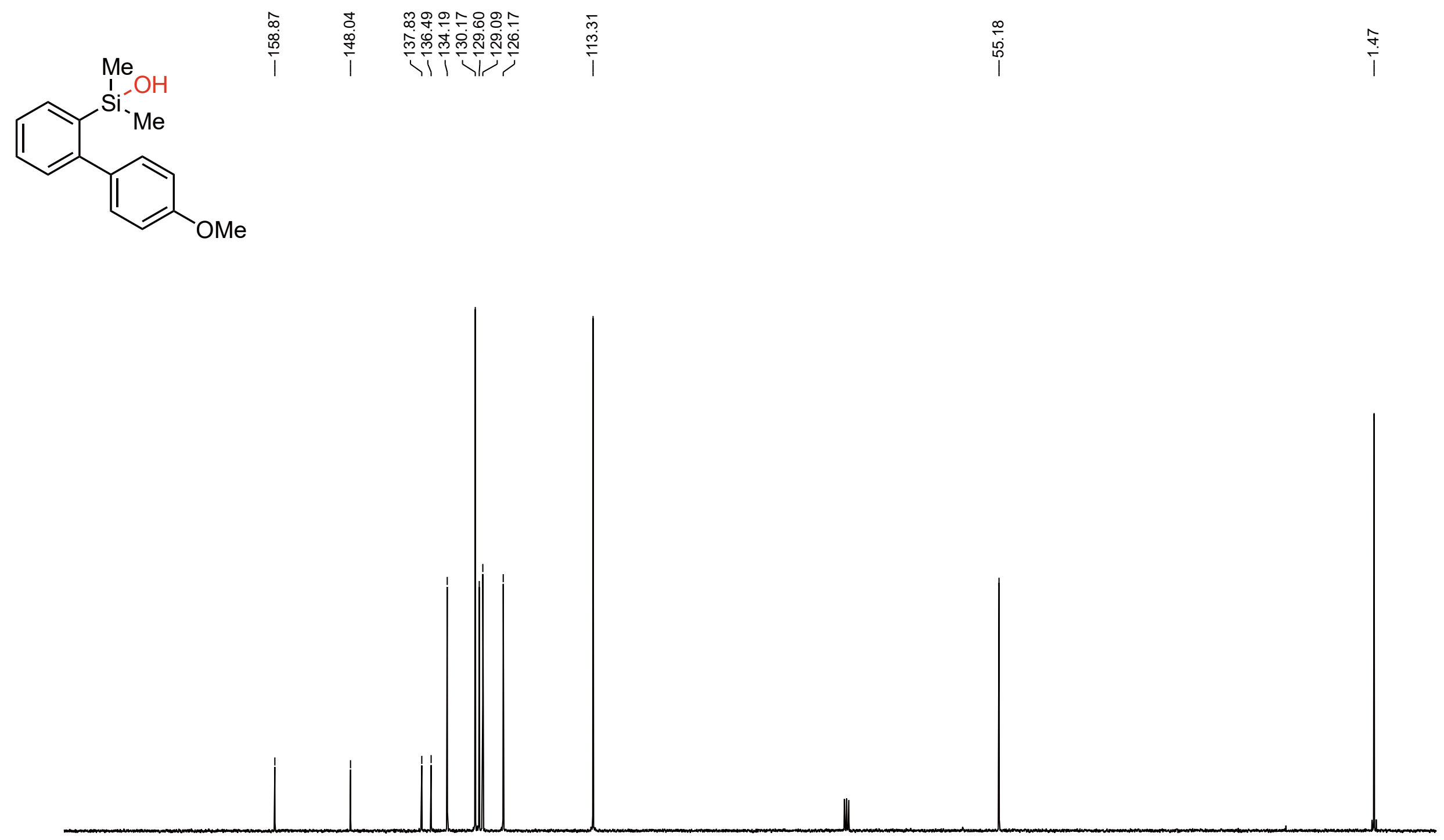


Compound 2as ${ }^{1} \mathrm{H}$ NMR

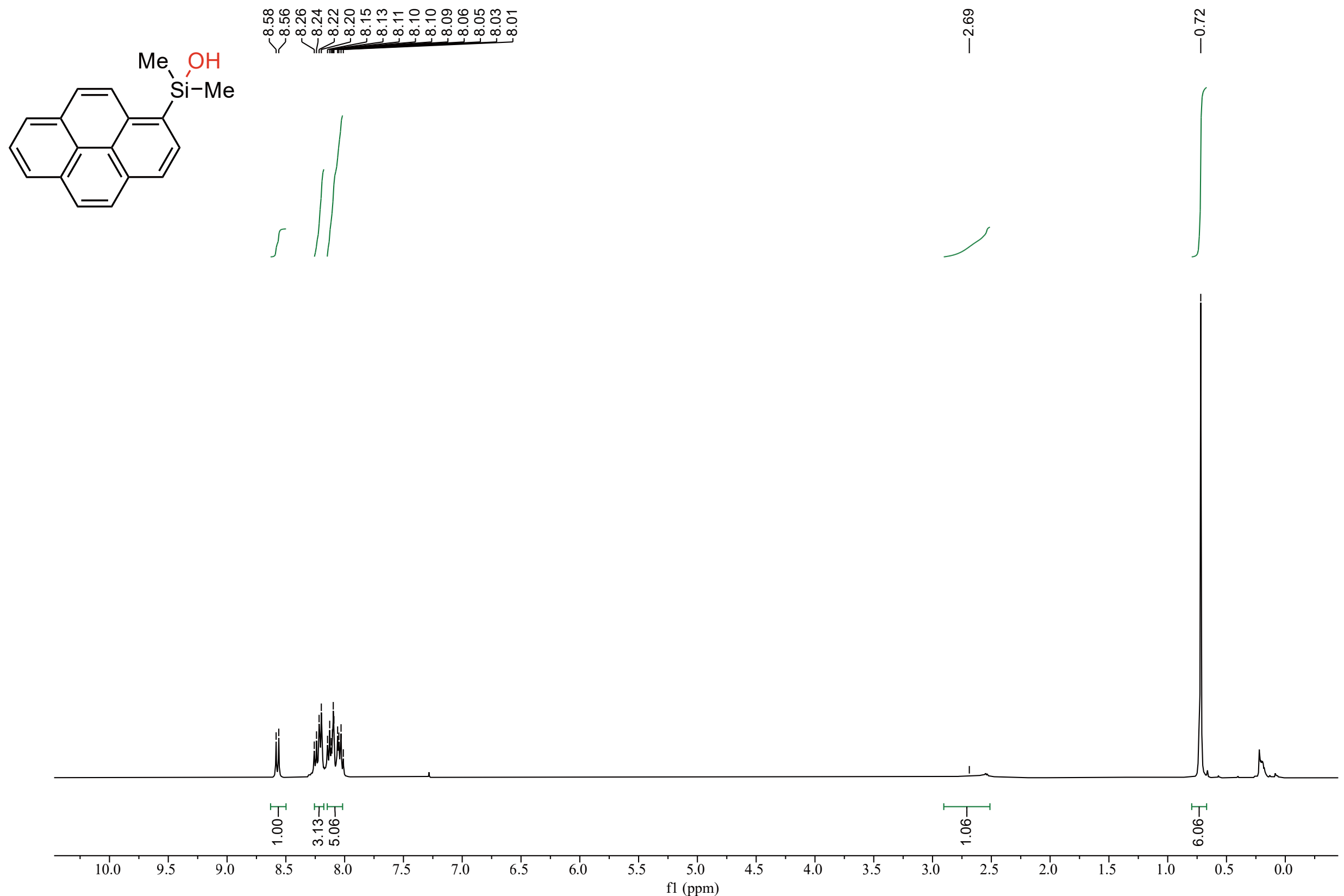




$$
E^{4}
$$




\section{Compound 2at ${ }^{1} \mathrm{H}$ NMR}
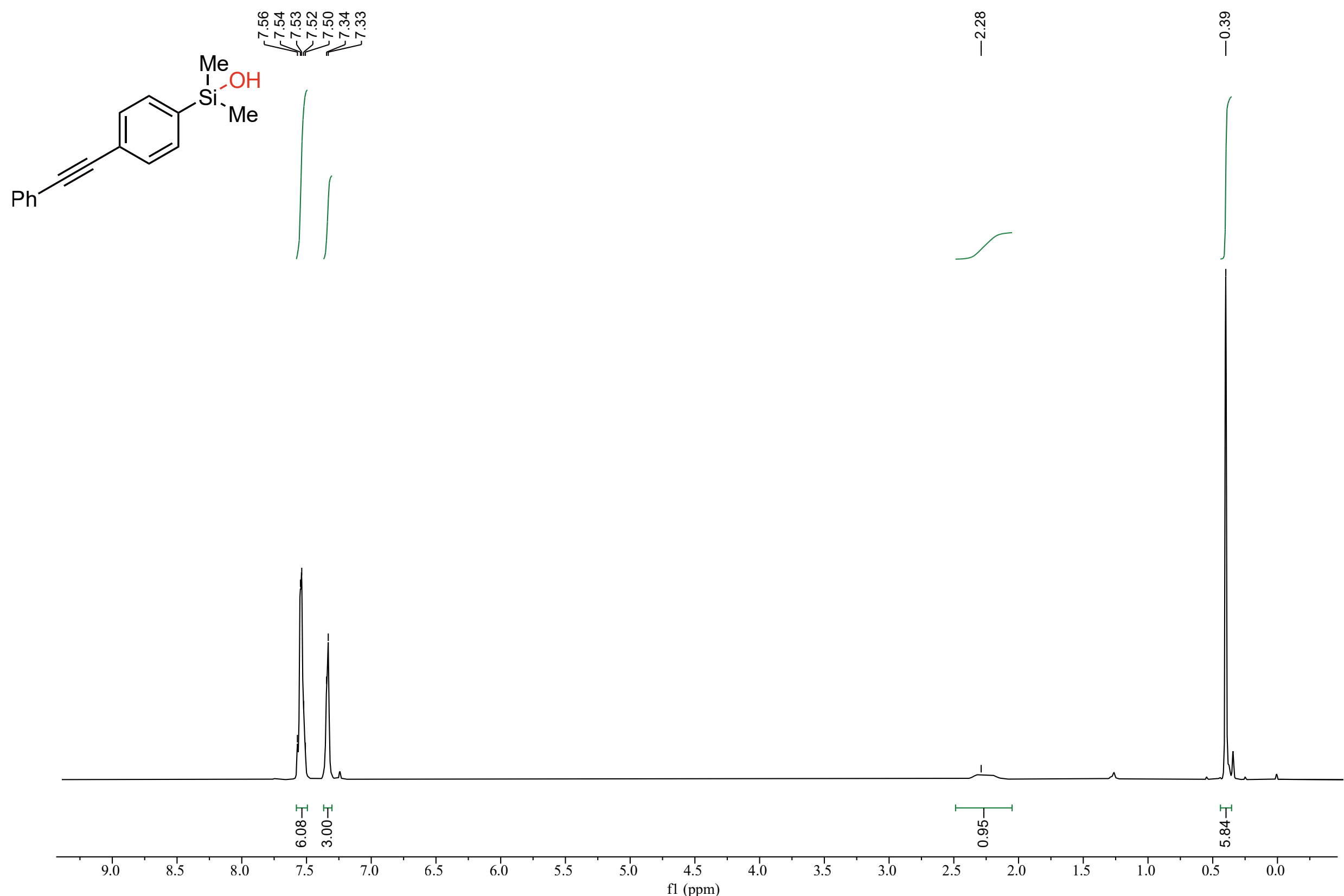
Compound 2at ${ }^{13} \mathrm{C}$ NMR

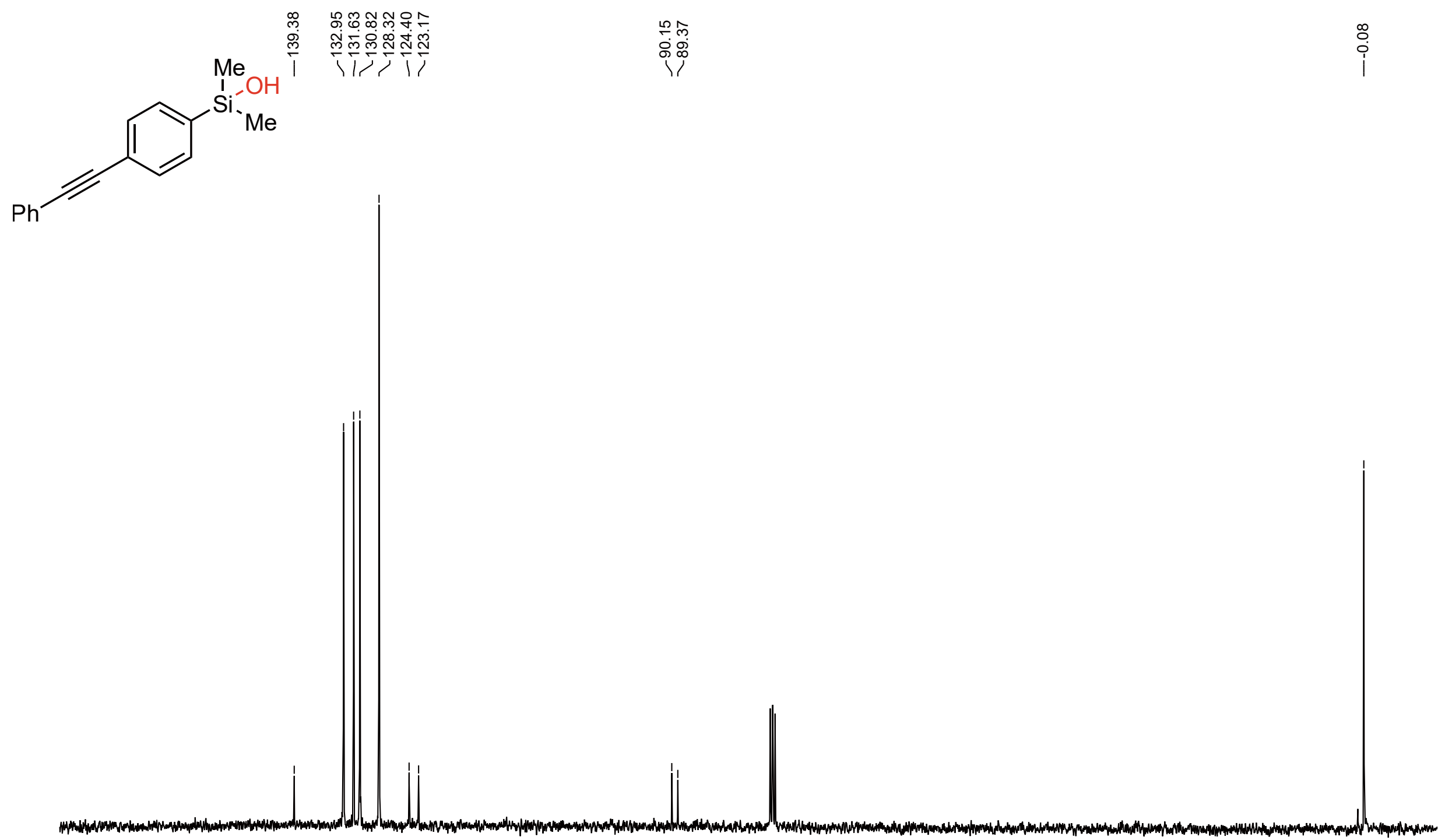


Compound 2au ${ }^{1} \mathrm{H}$ NMR

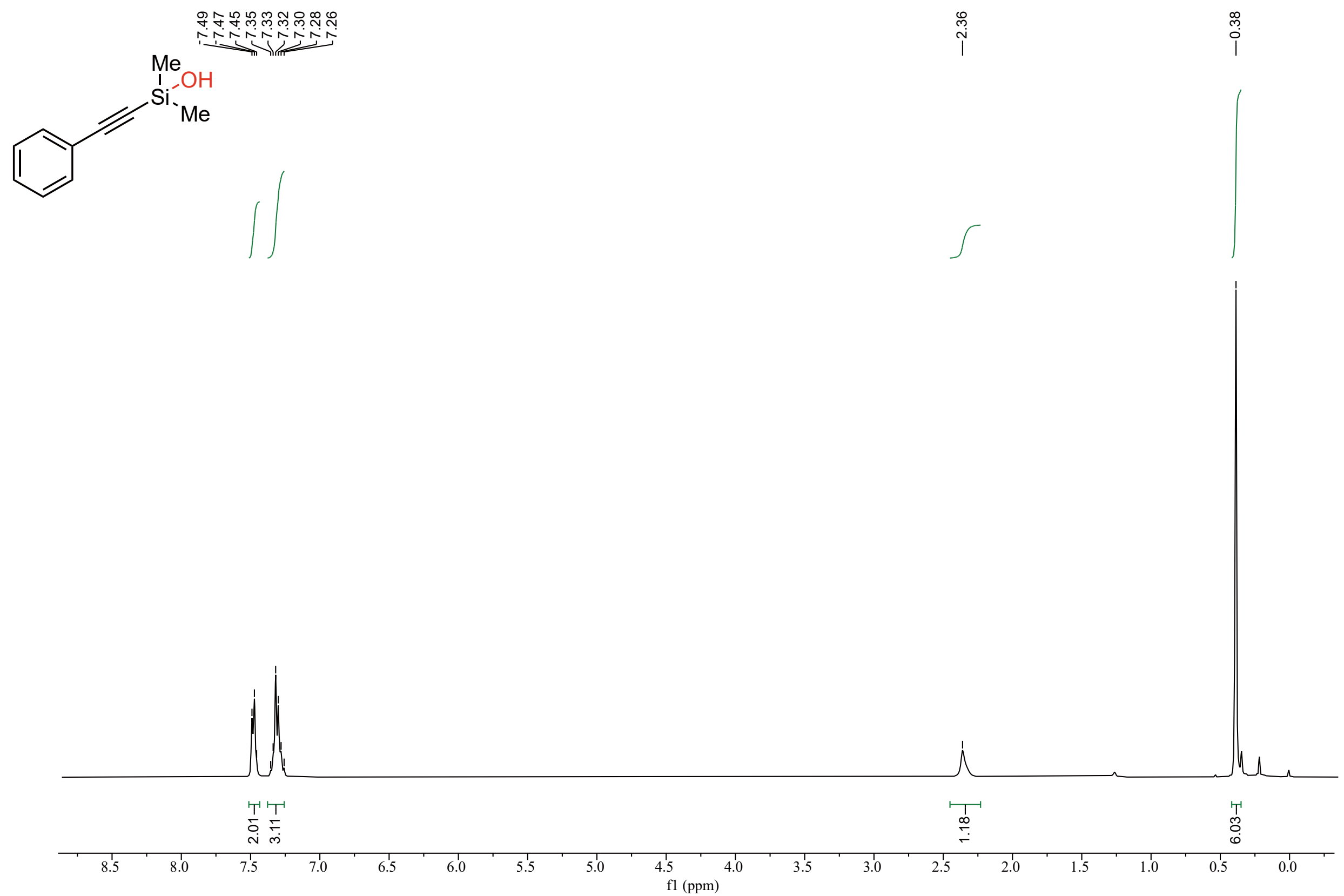


Compound 2au ${ }^{13} \mathrm{C}$ NMR

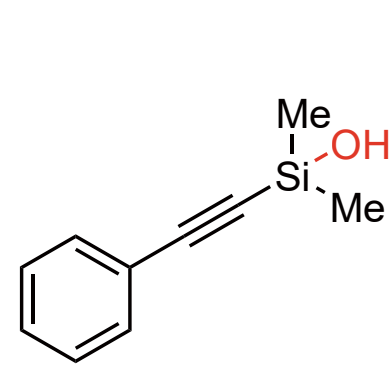

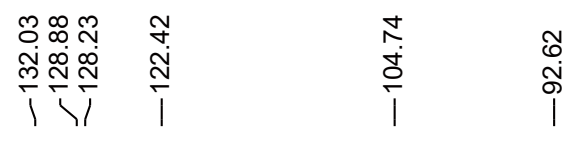

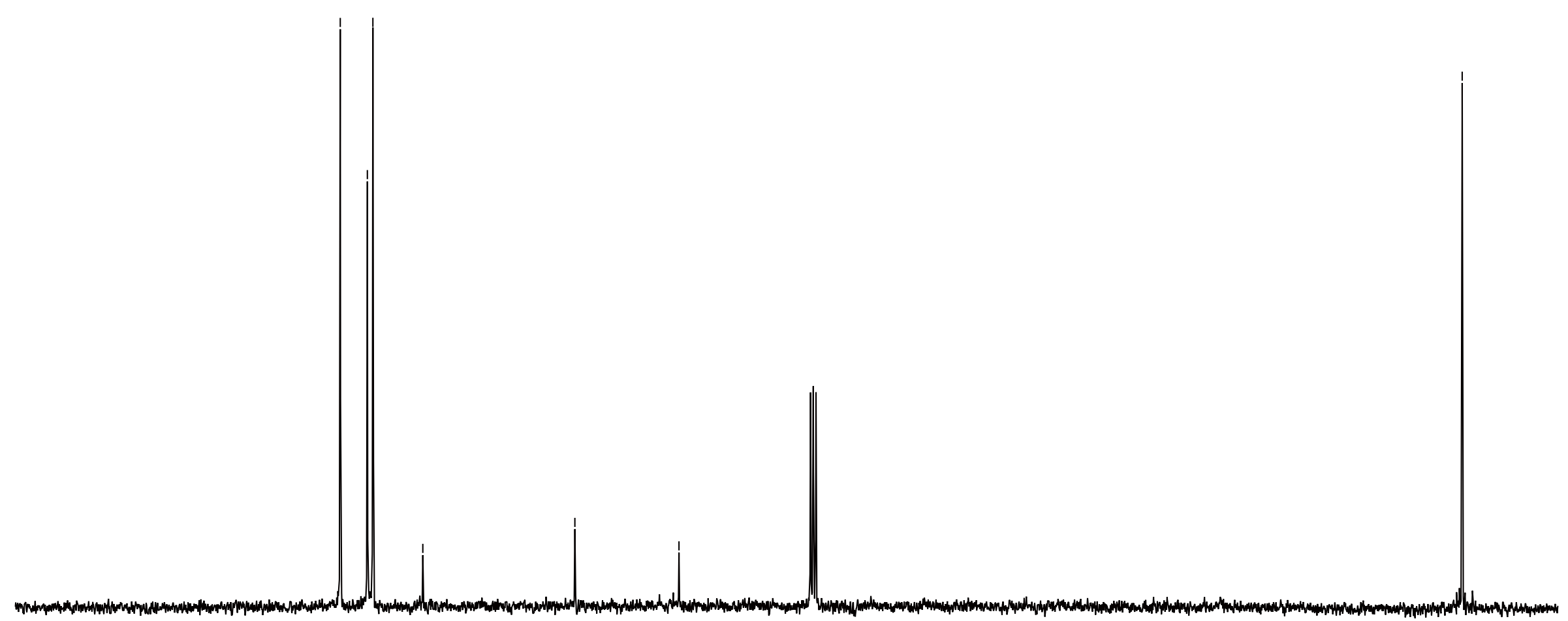


Compound 2av ${ }^{1} \mathrm{H}$ NMR

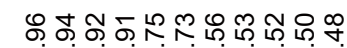

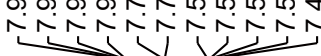
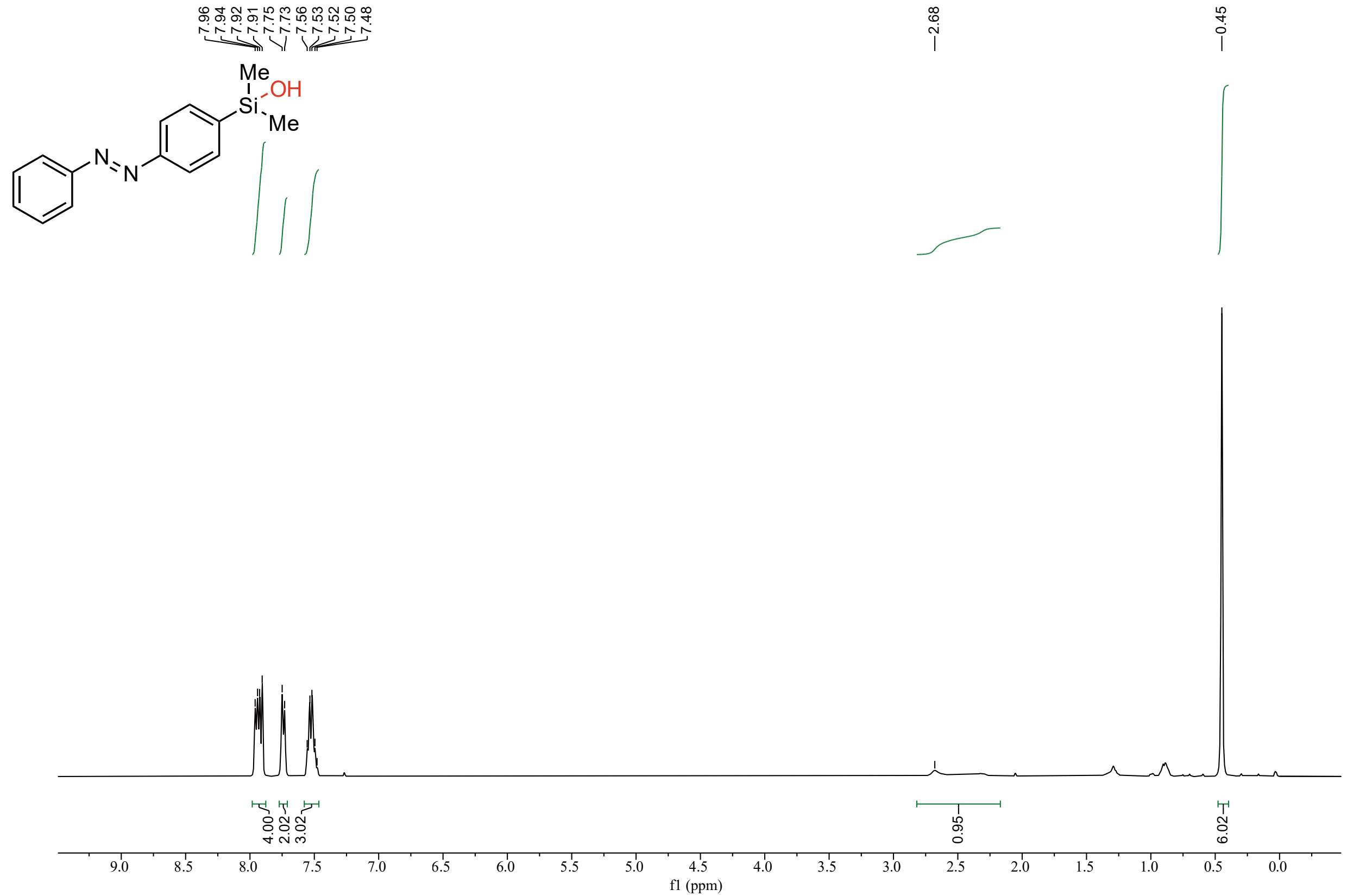
Compound 2av ${ }^{13} \mathrm{C}$ NMR
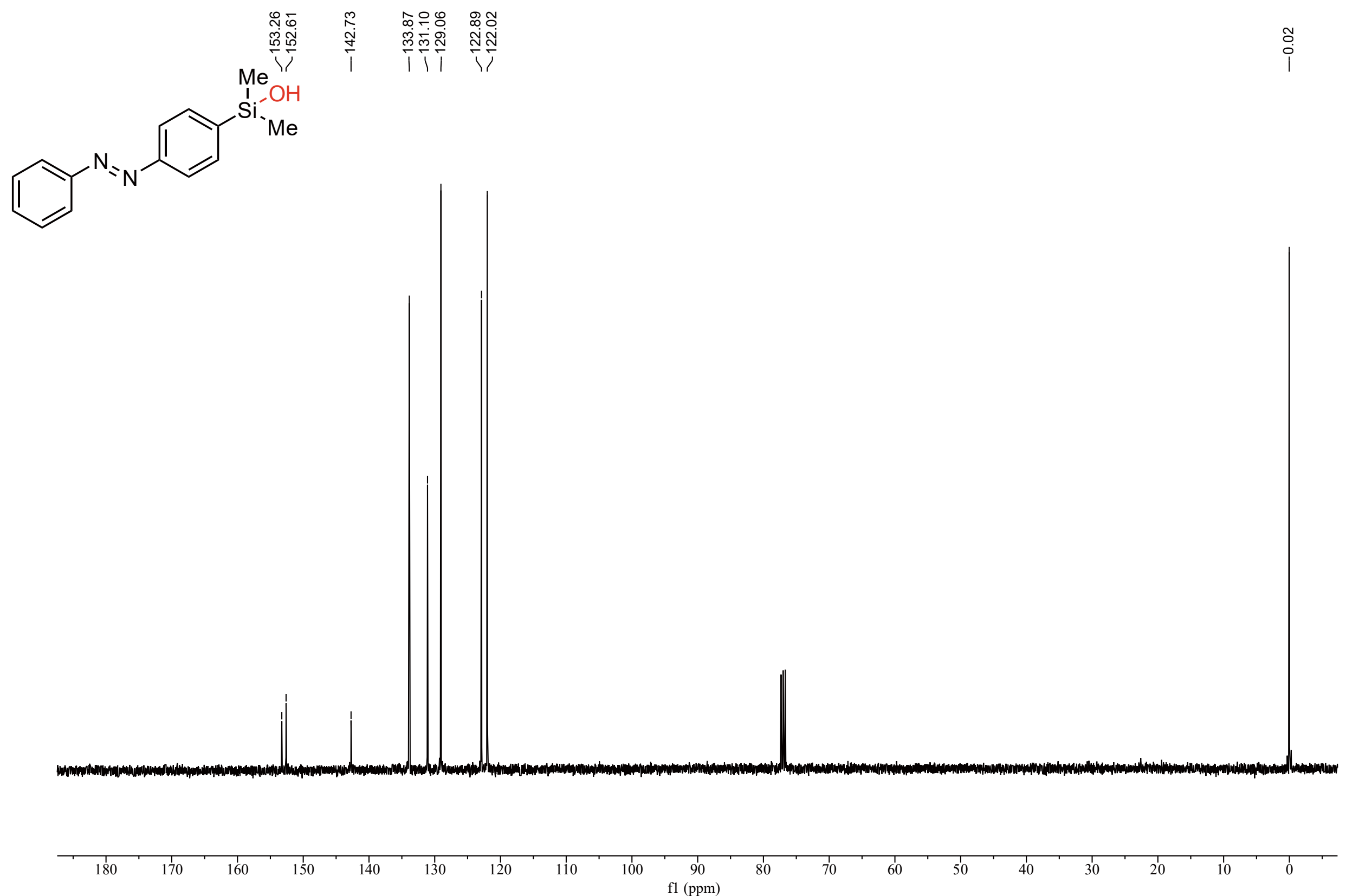
Compound 2aw ${ }^{1} \mathrm{H}$ NMR
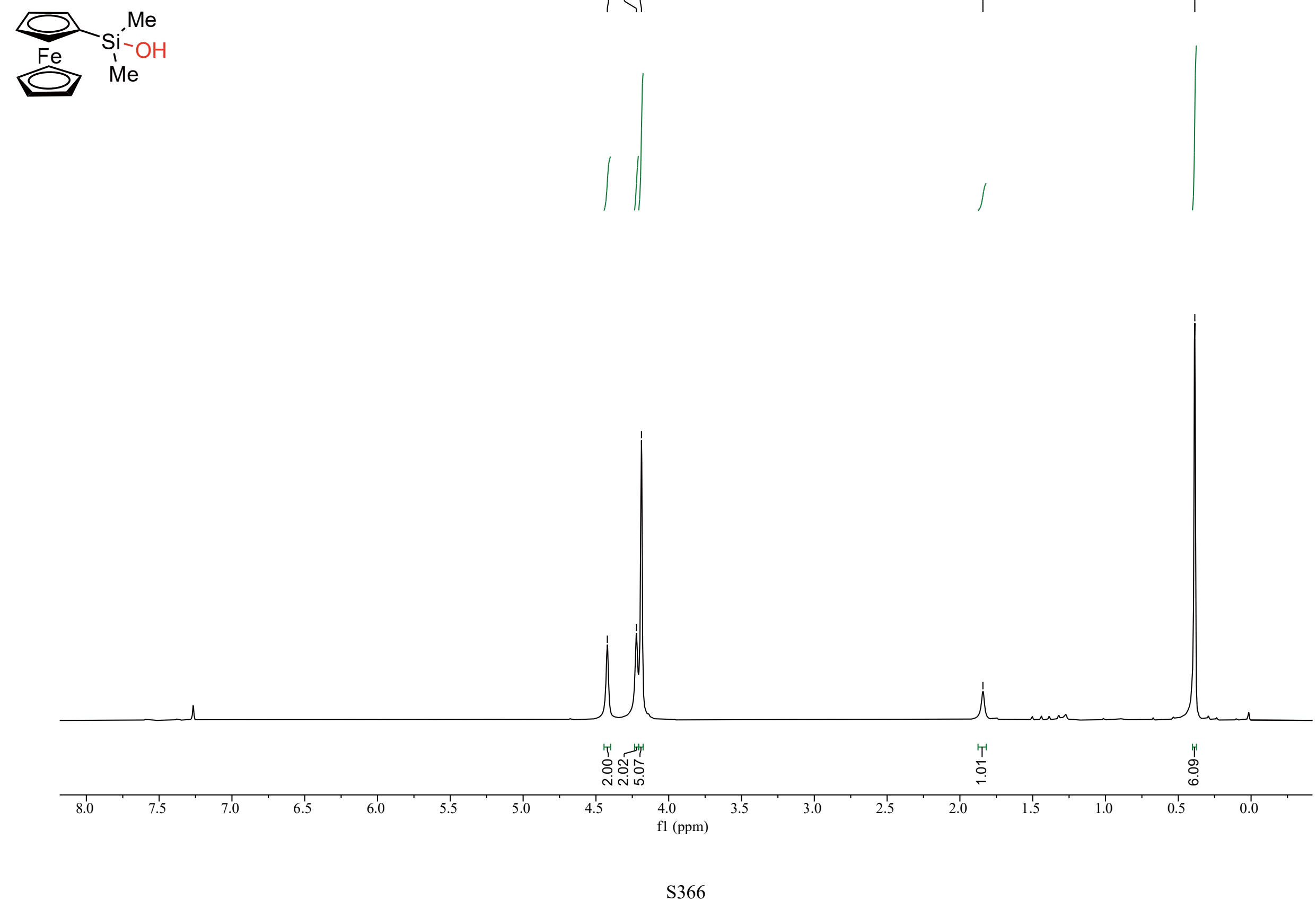
Compound 2aw ${ }^{13} \mathrm{C}$ NMR

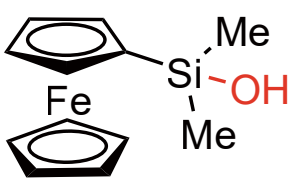
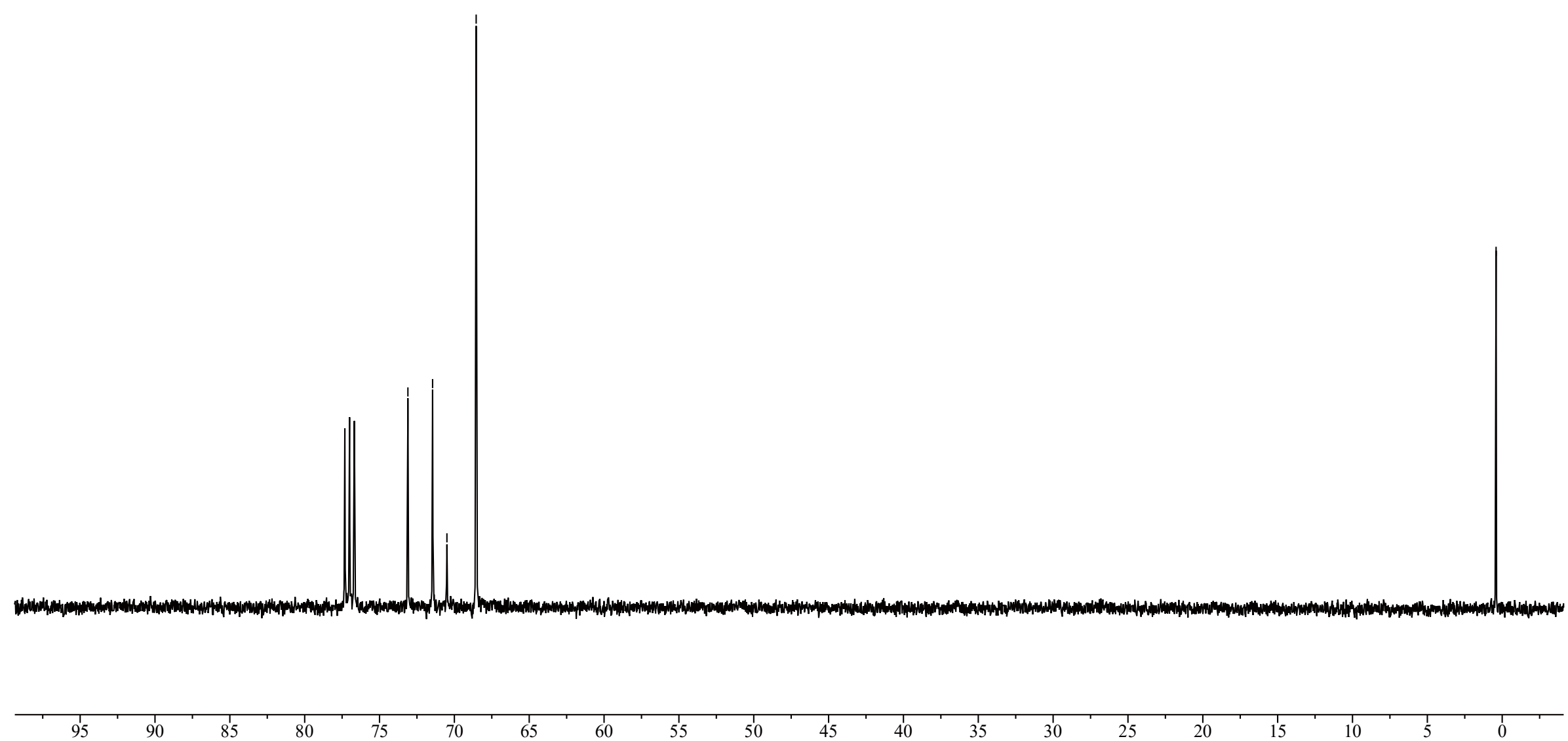


\section{Compound 2ax ${ }^{1} \mathrm{H}$ NMR}
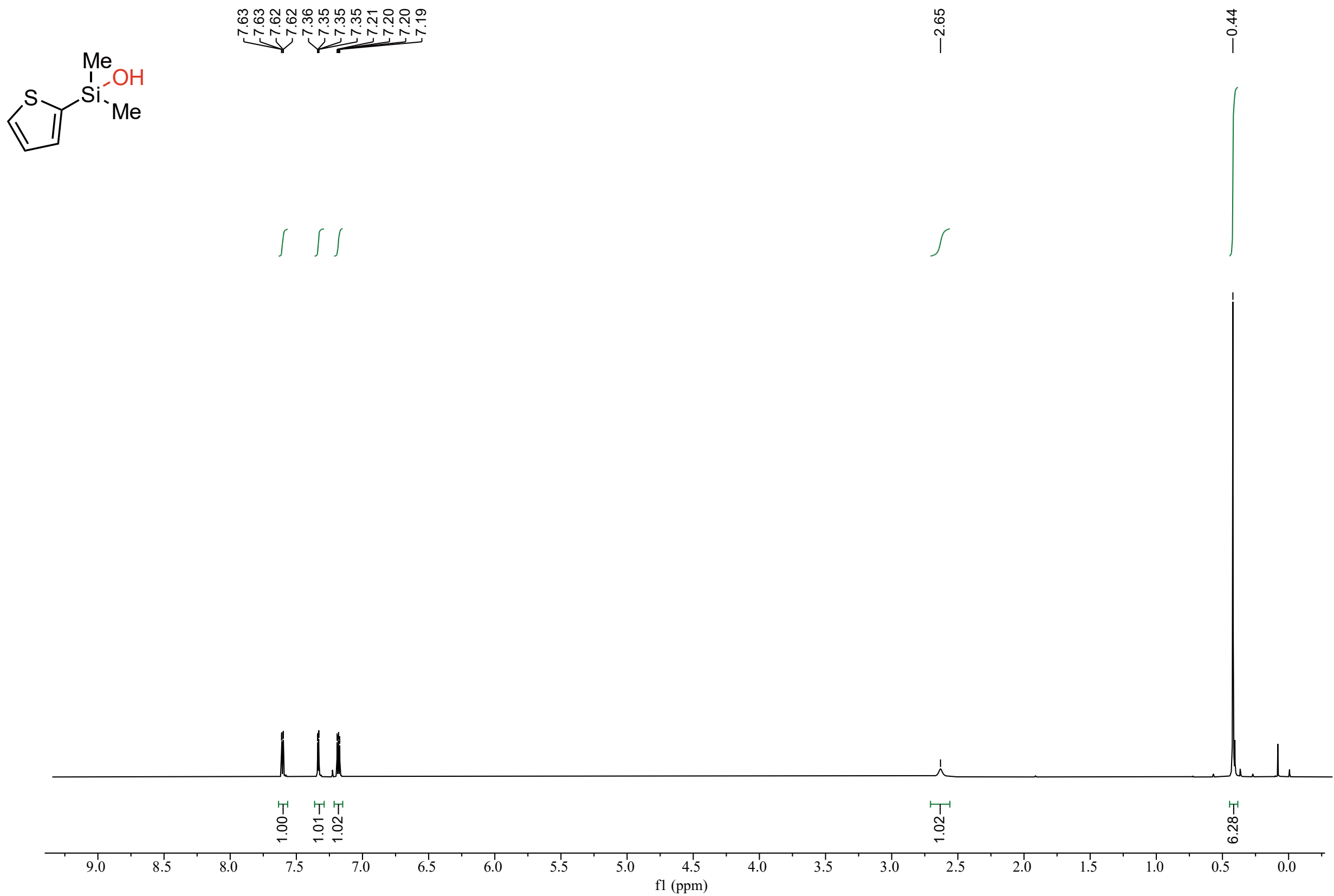
Compound 2ax ${ }^{13} \mathrm{C}$ NMR
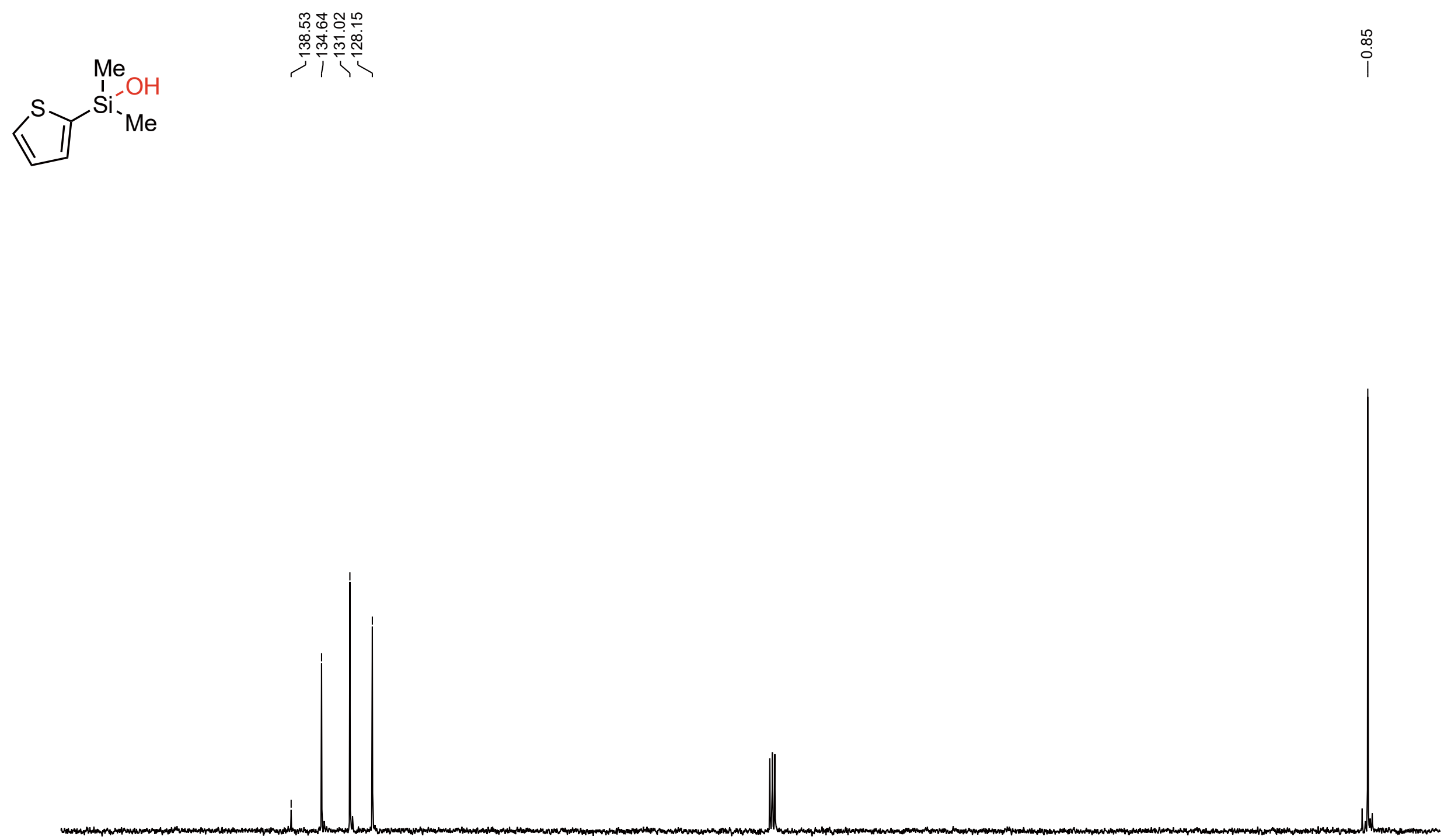


\section{Compound 2ay ${ }^{1} \mathrm{H}$ NMR}
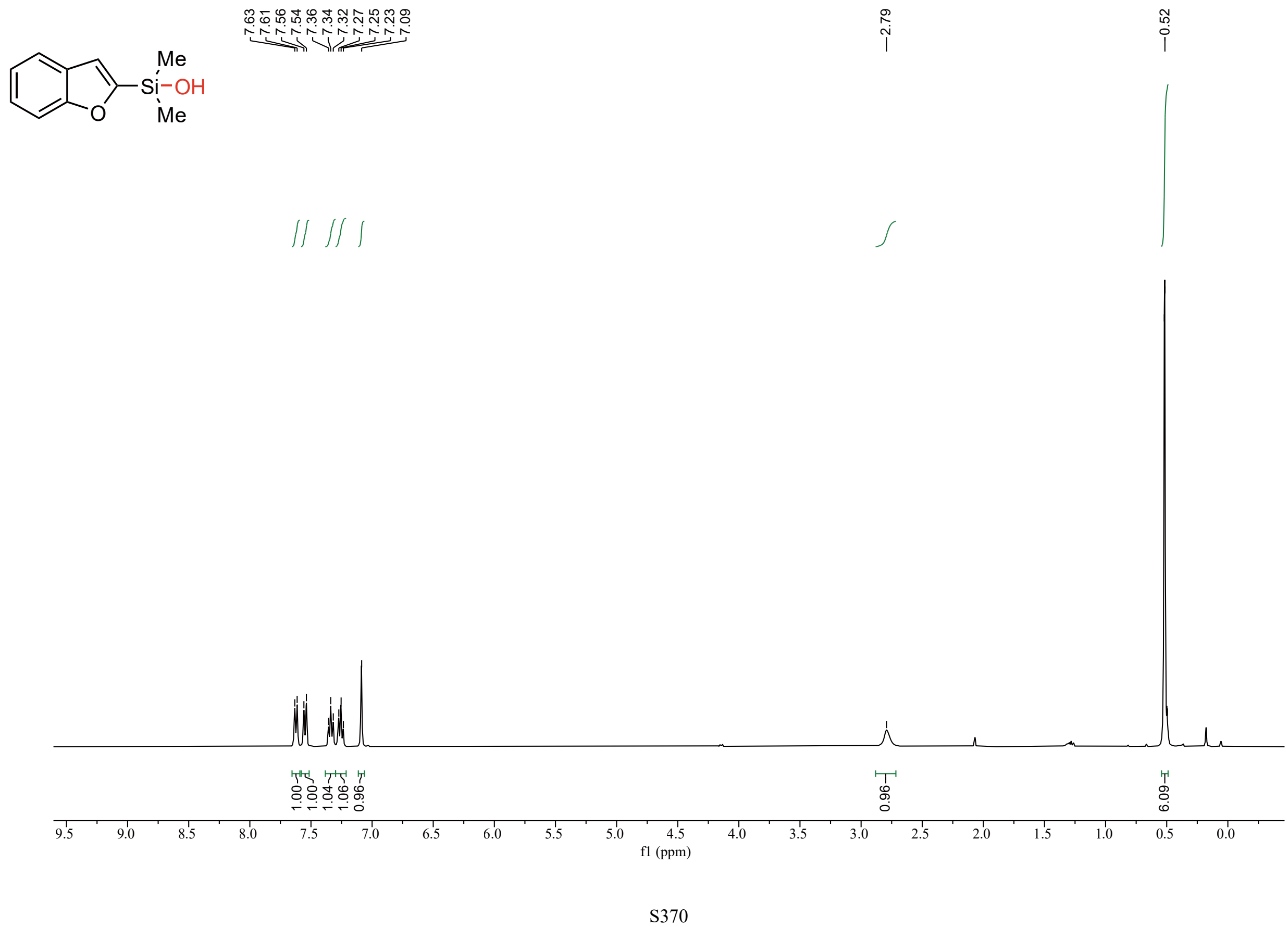
Compound 2ay ${ }^{13} \mathrm{C}$ NMR
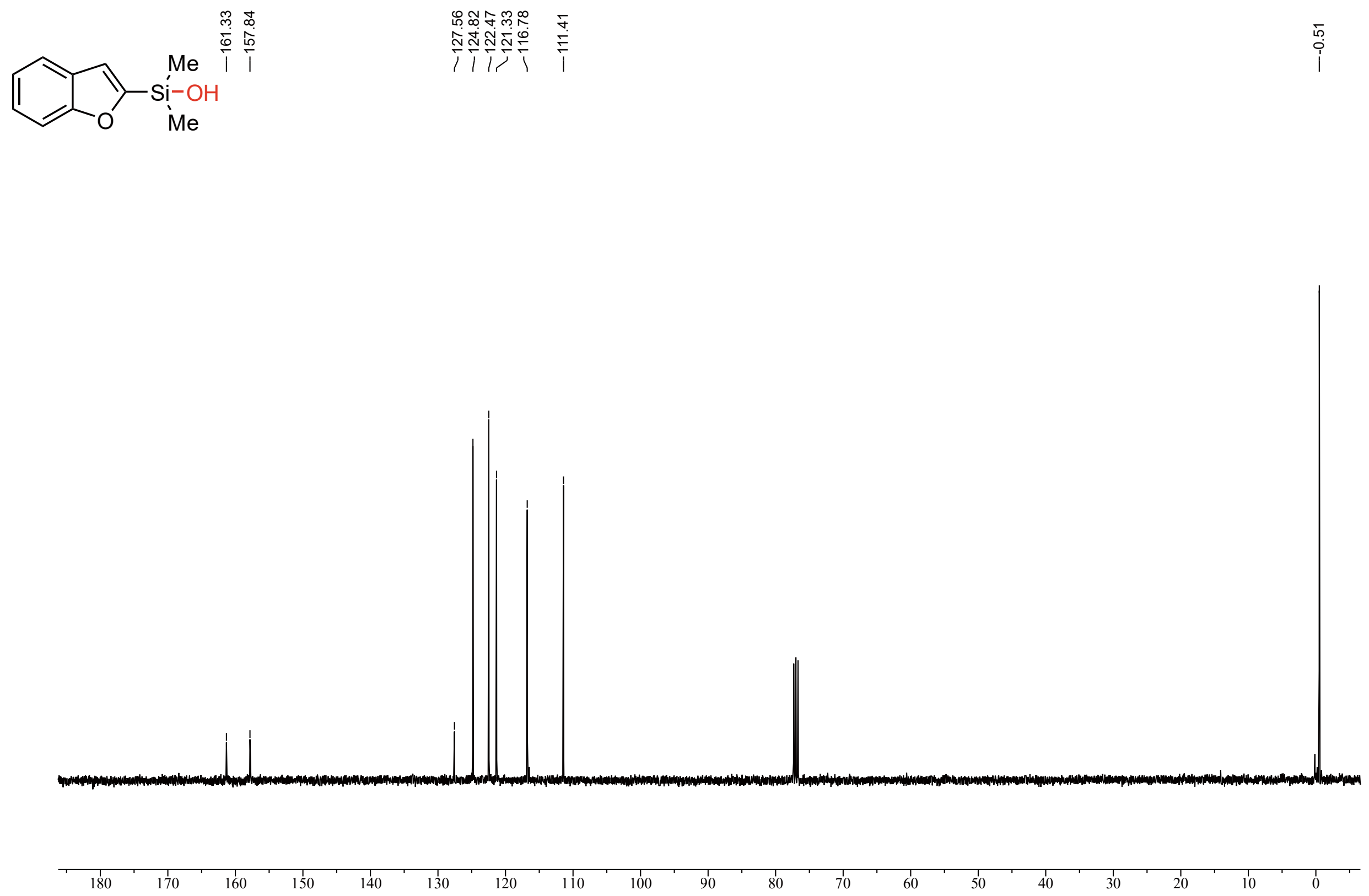


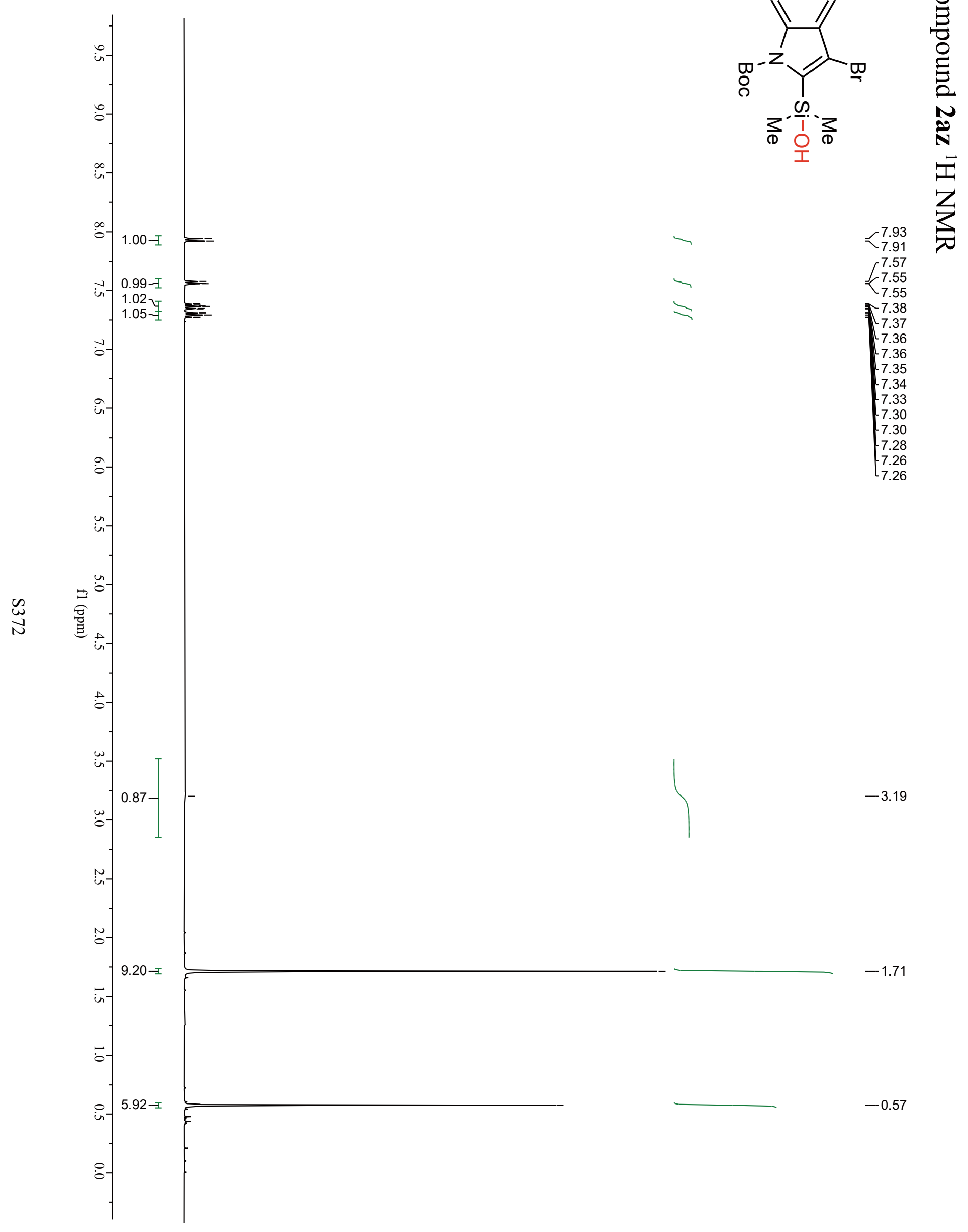




\section{Compound 2az ${ }^{13} \mathrm{C}$ NMR}

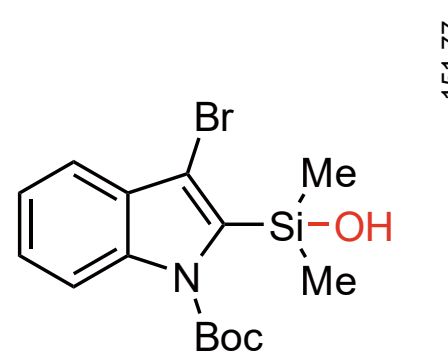

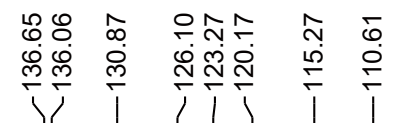

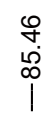

$\stackrel{\circ}{\stackrel{0}{i}}$

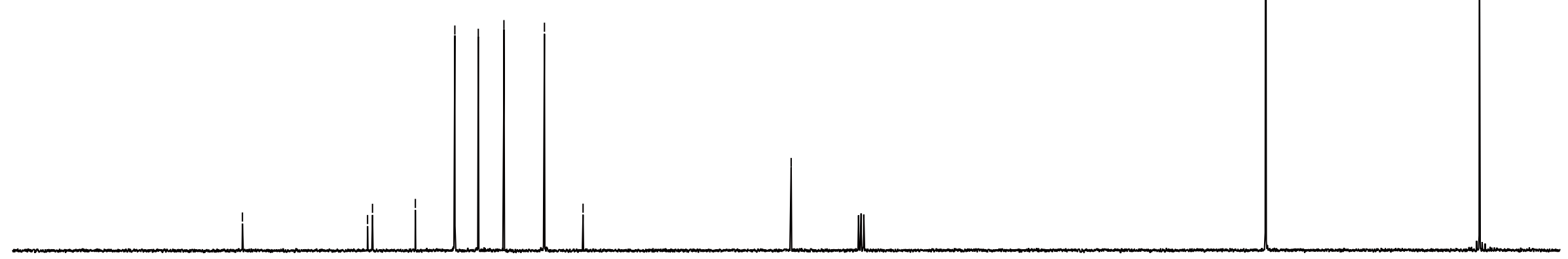




\section{Compound 2 ba ${ }^{1} \mathrm{H}$ NMR}<smiles>C[Si](O)(O)c1cccc2ncccc12</smiles>

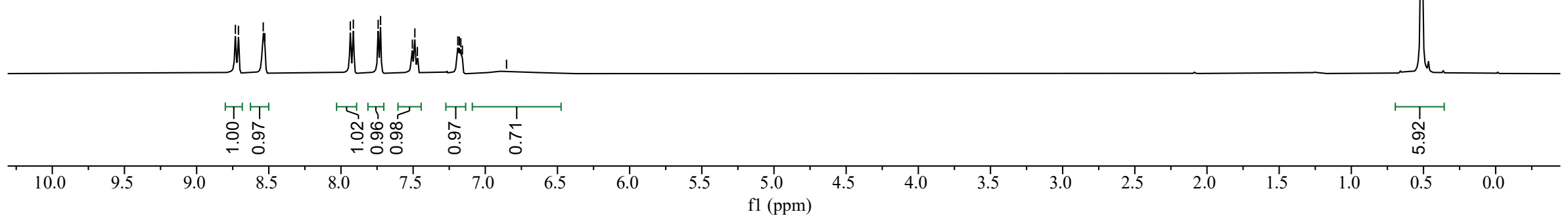




\section{Compound $\mathbf{2 b a}{ }^{13} \mathrm{C}$ NMR}
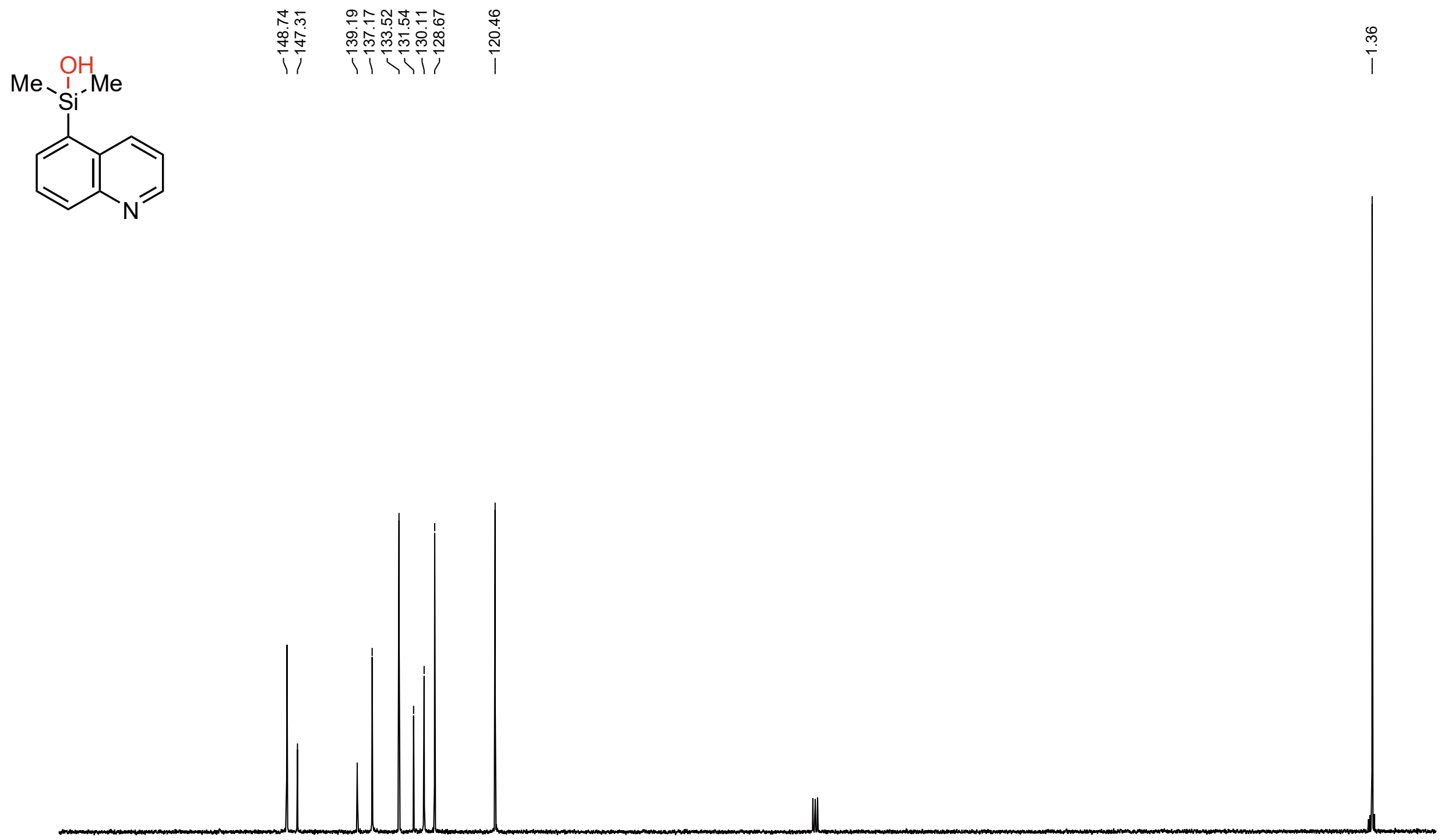

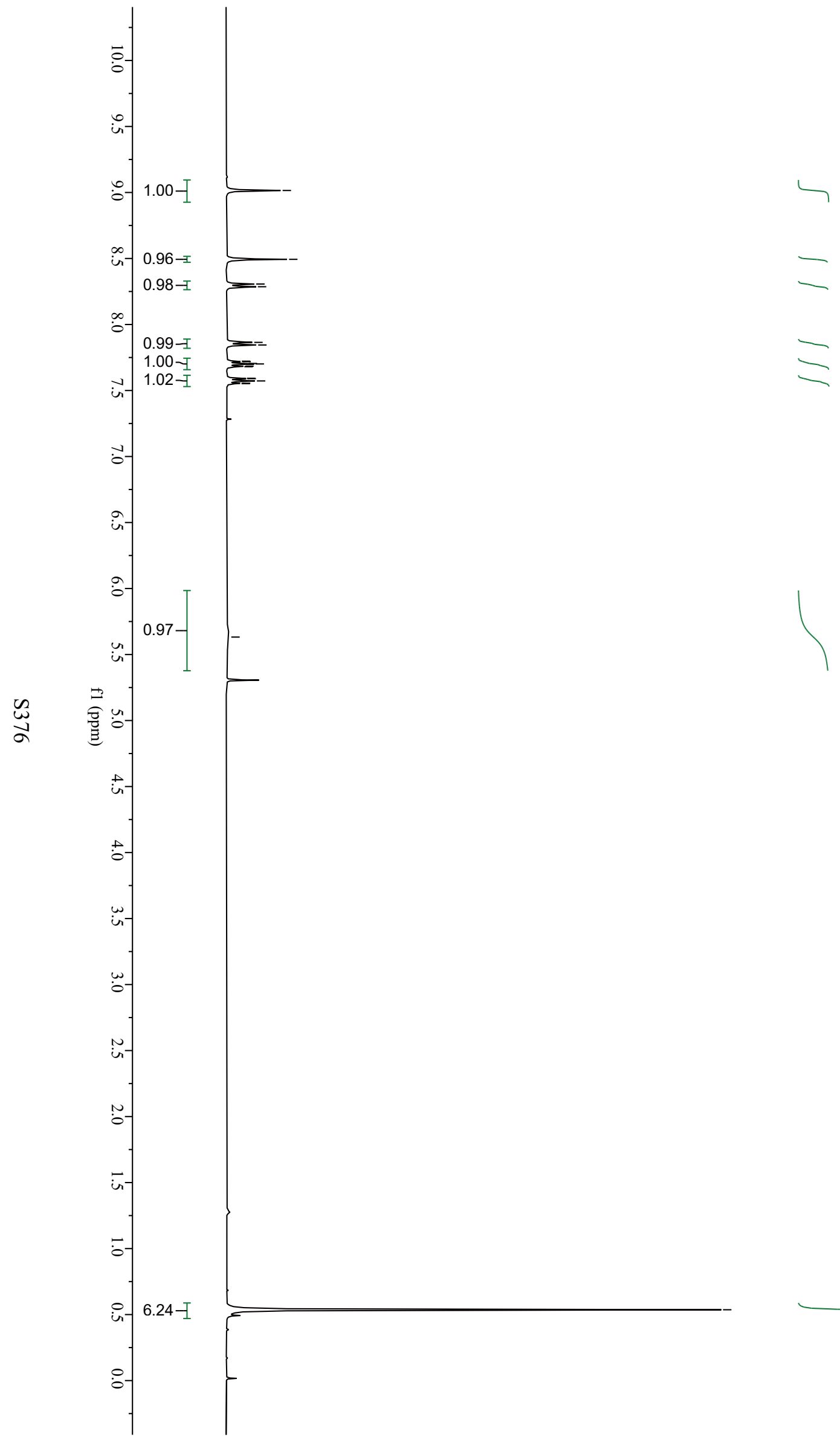

$-8.49$

$\mathcal{L}_{8.29}^{8.31} \equiv$

7.86

$-7.72$

7.70

7.70

7.70
7.68

$-7.68$

$-7.59$

-7.59
-7.57

$-7.57$

7.55

$-5.63$

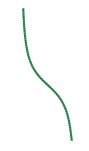

文 
Compound $\mathbf{2 b b}{ }^{13} \mathrm{C}$ NMR
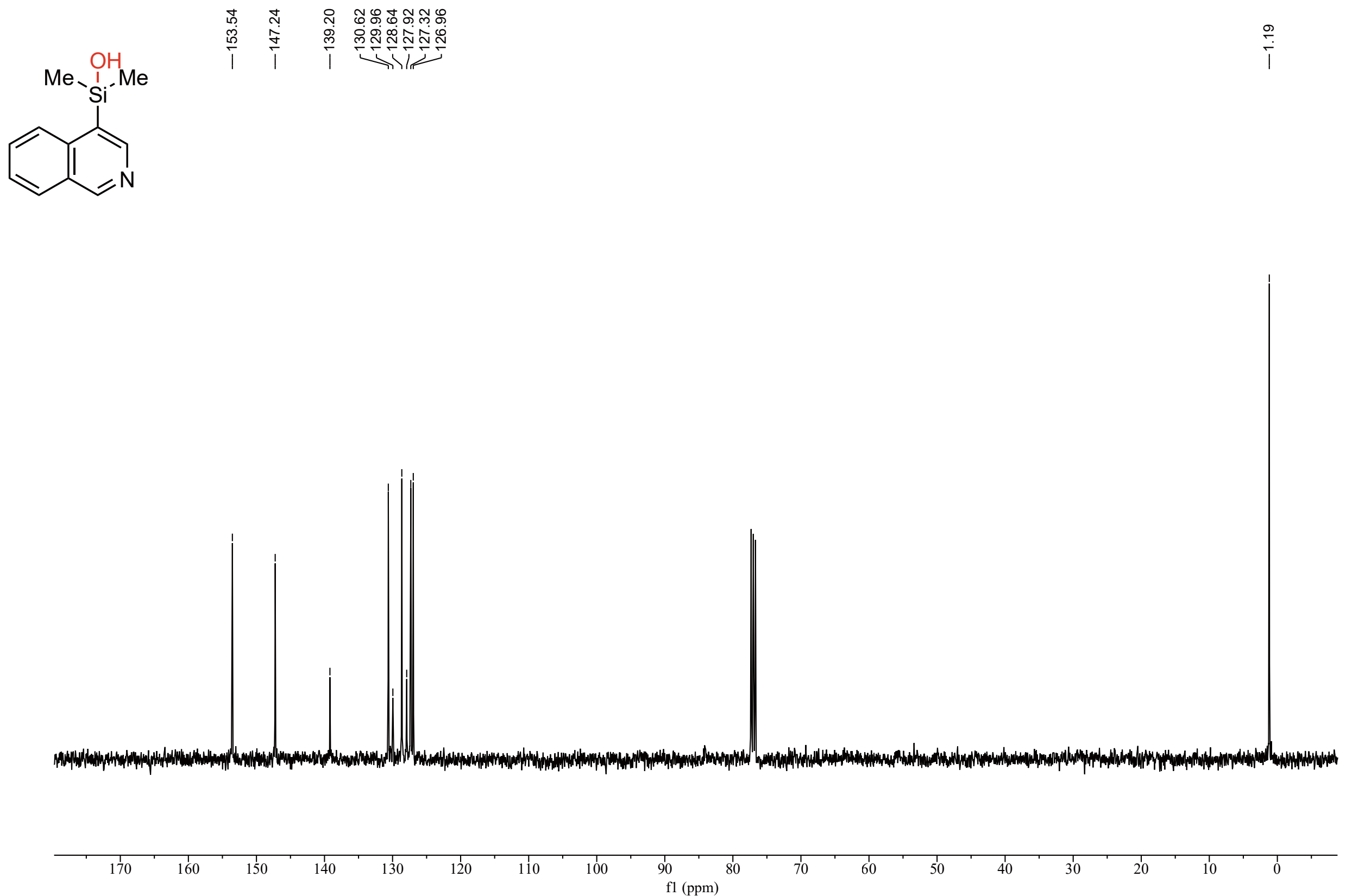


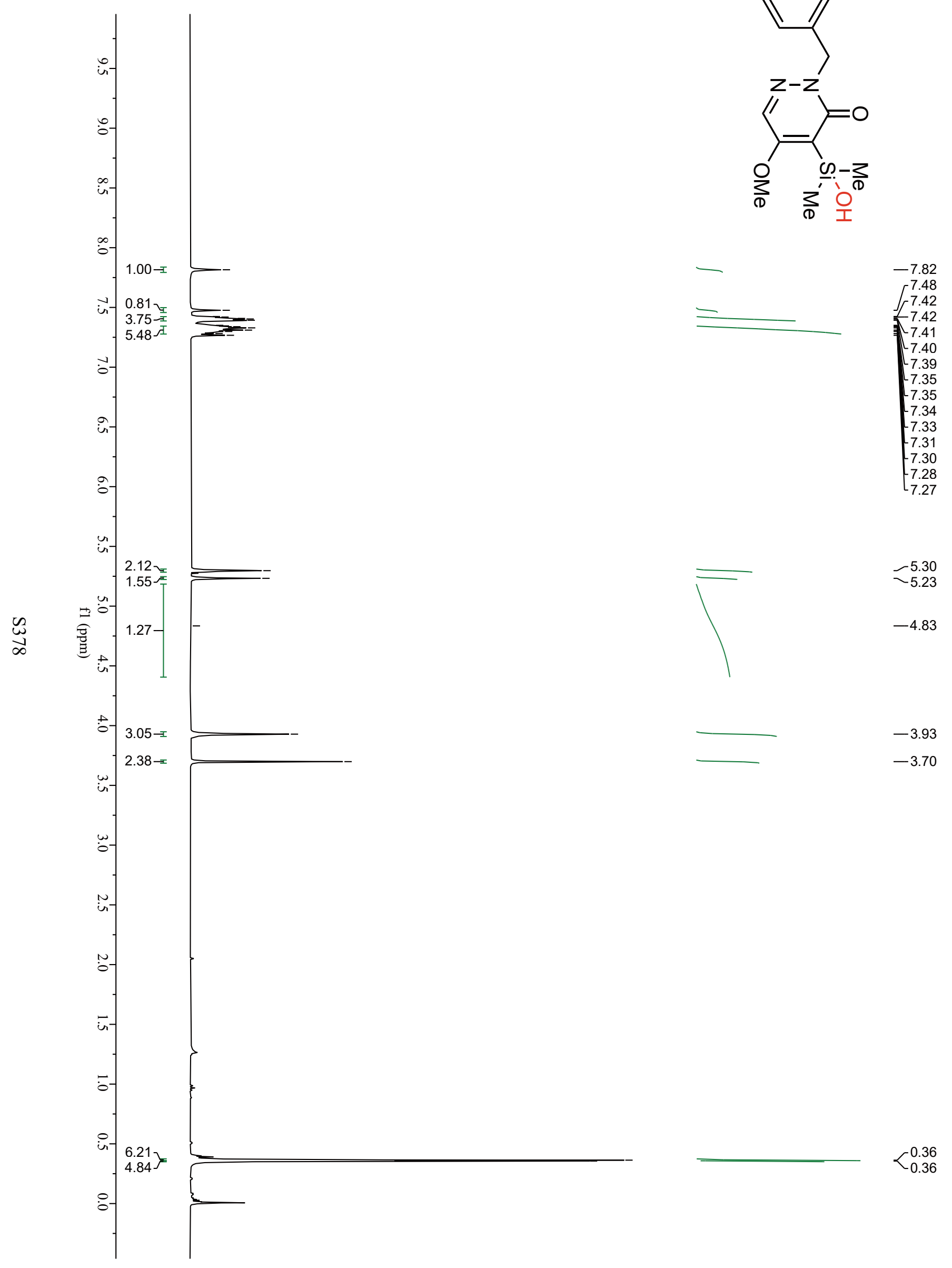



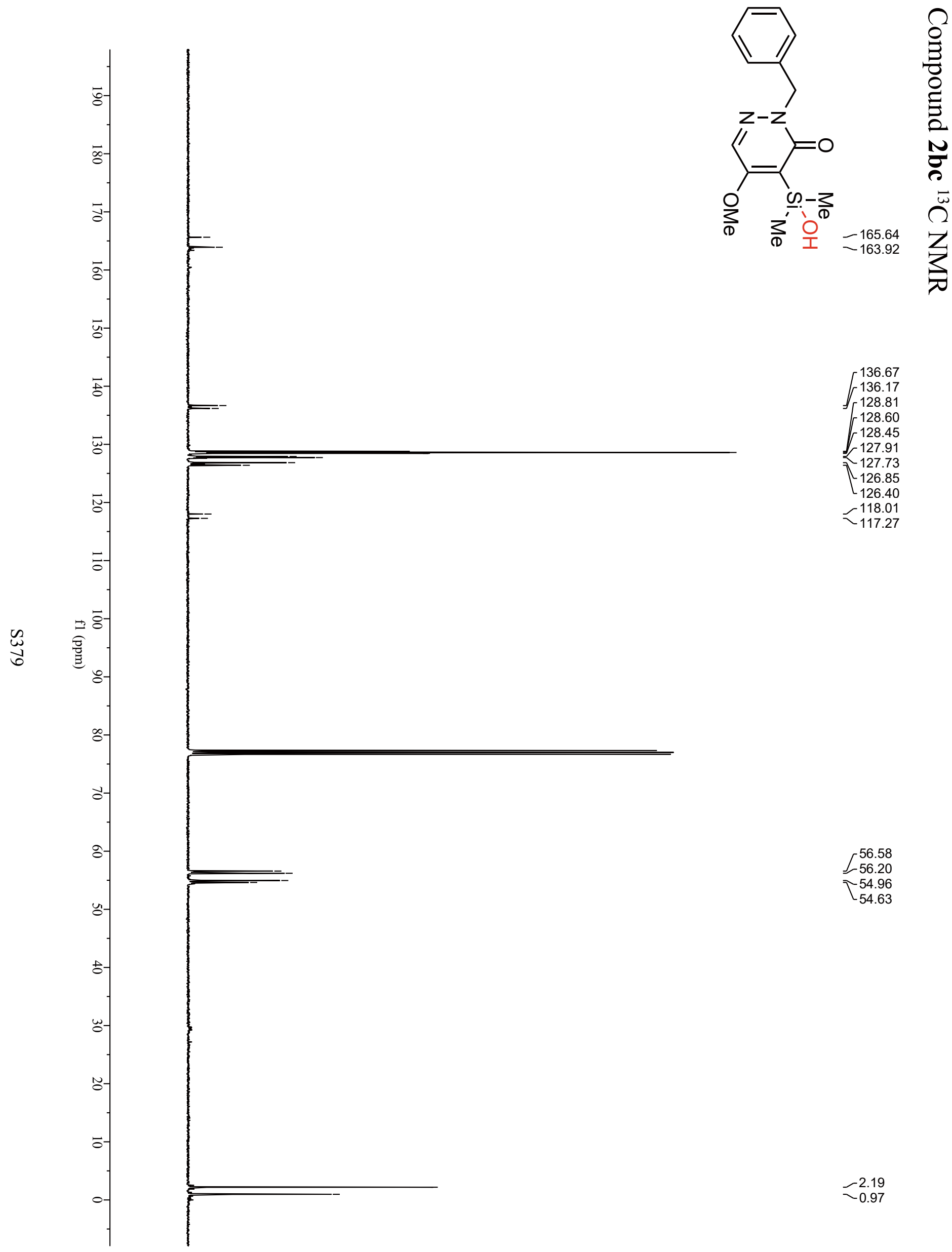

56.58

$-56.20$

$-54.96$

54.63 
Compound 2bd ${ }^{1} \mathrm{H}$ NMR

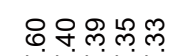

盗㲻

I
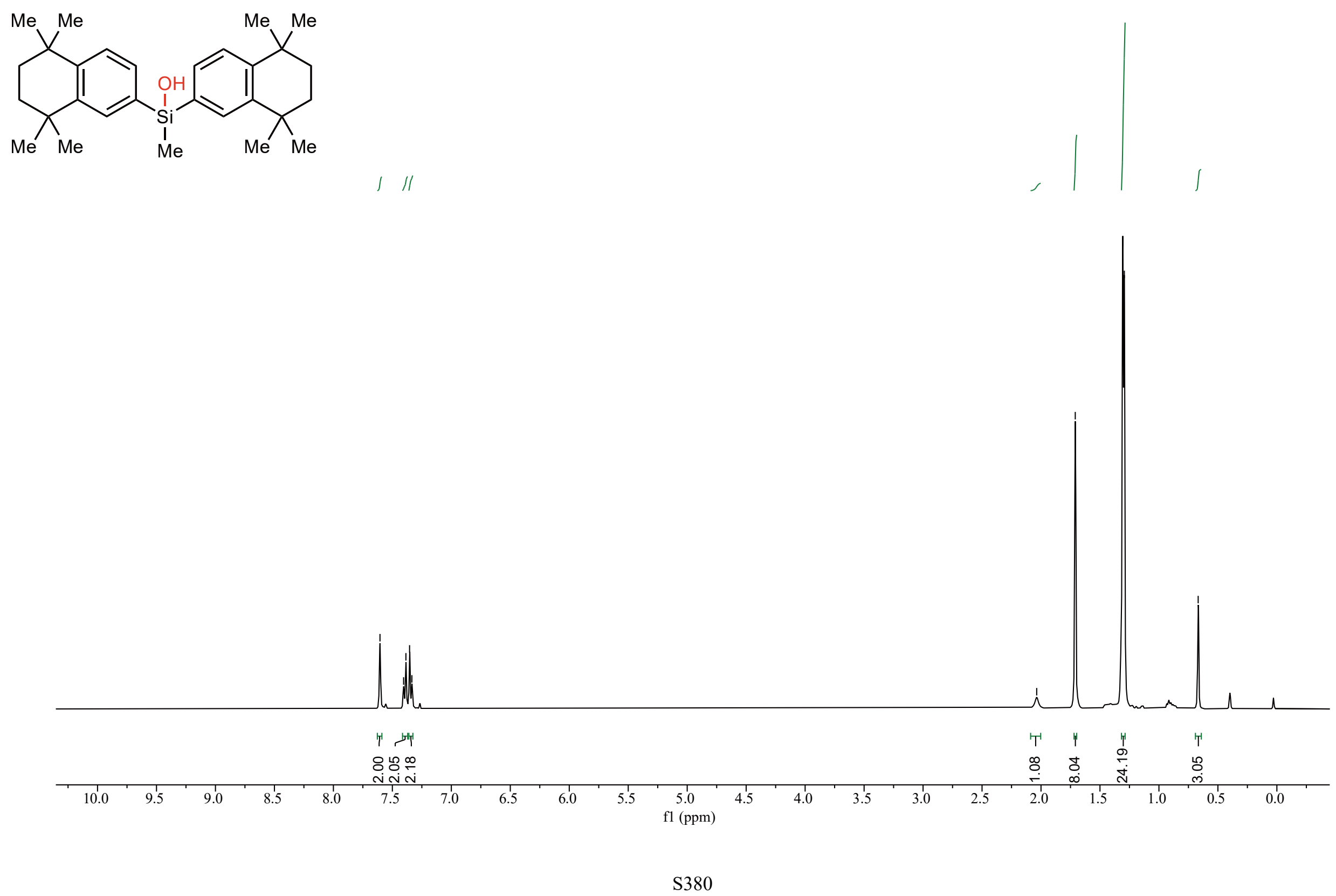
Compound 2bd ${ }^{13} \mathrm{C}$ NMR

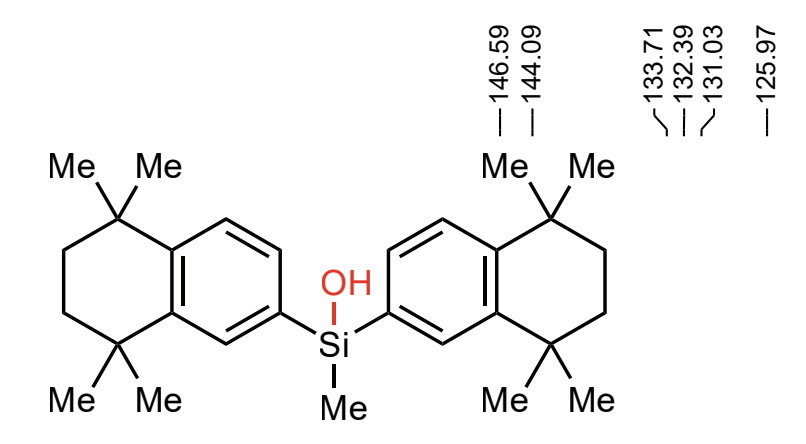

$$
\underbrace{3}
$$

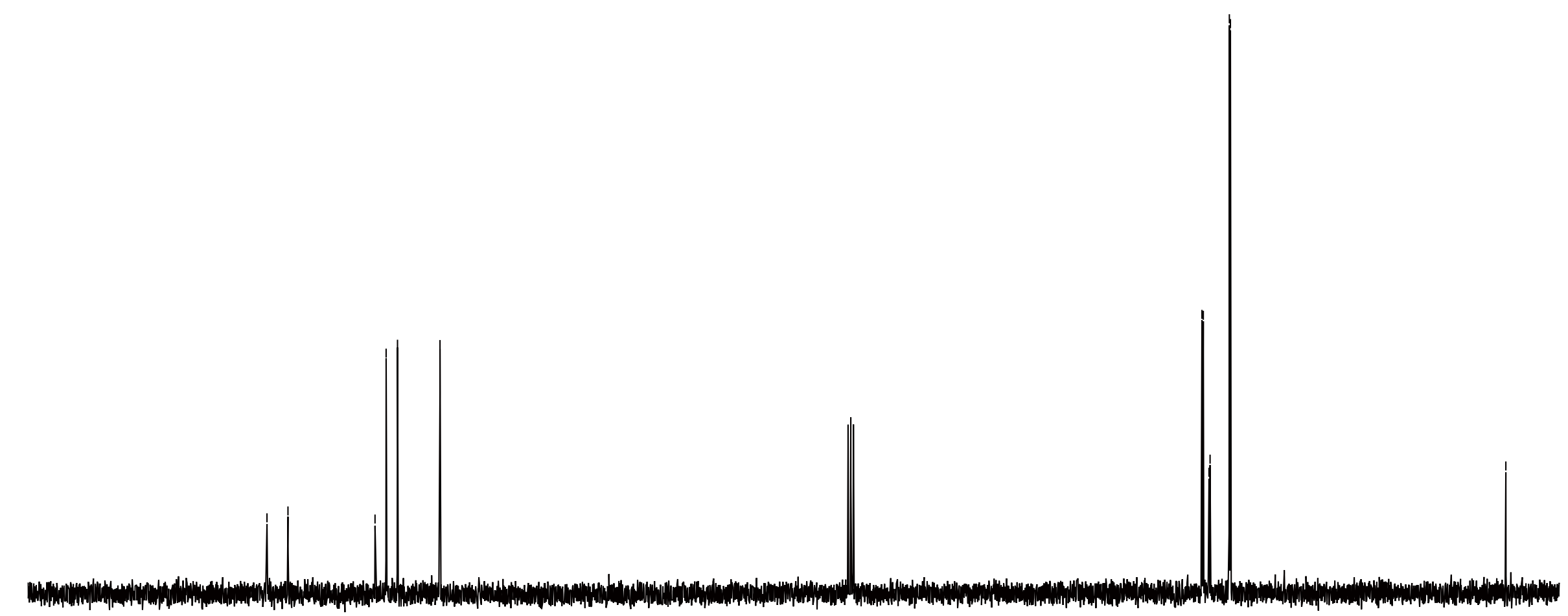



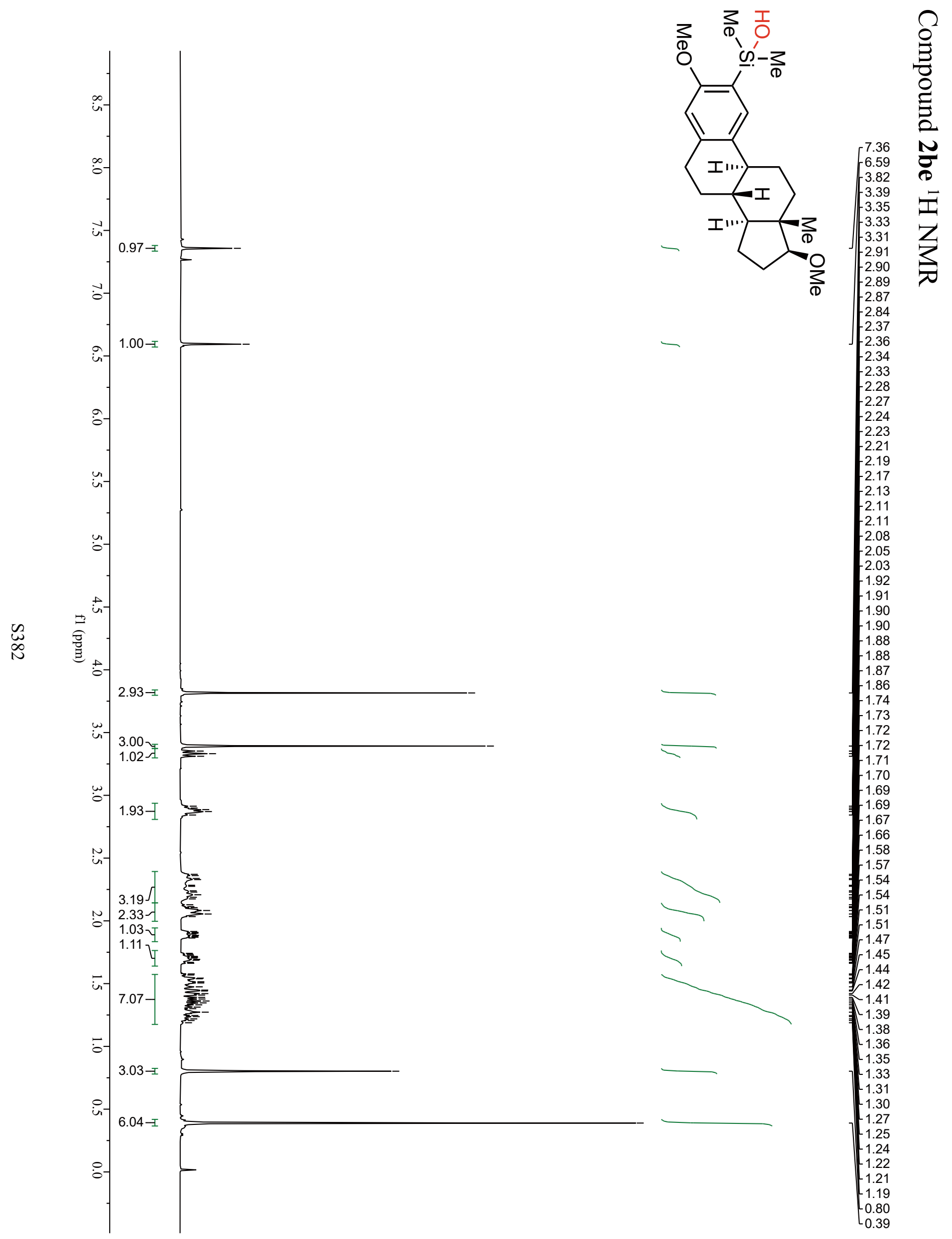


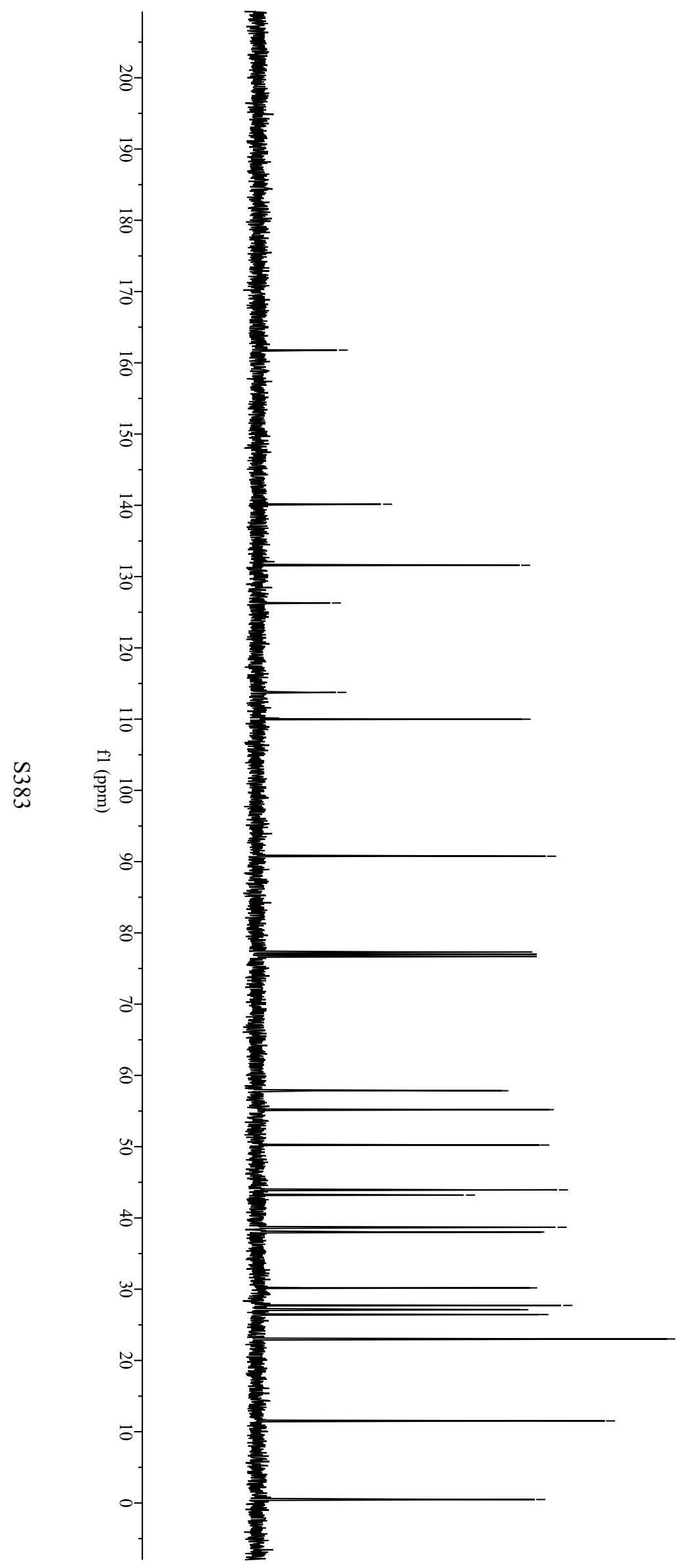

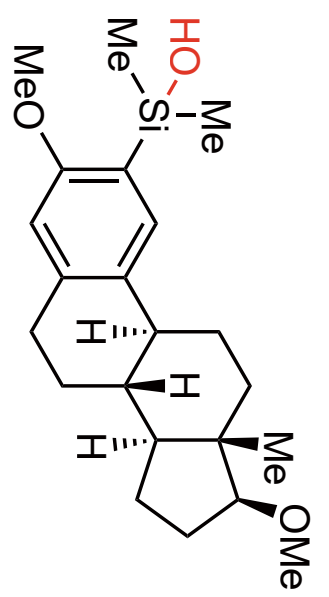

$-161.78$

$-140.16$

$-131.59$

$-126.29$

$-113.75$

$-109.99$

$-90.76$

$\backslash 57.85$

$\backslash 55.19$

$-50.23$

$-43.94$

43.21

$-38.69$

$\checkmark 38.02$

30.19

27.72

27.12

26.44

23.02

$-11.52$

$<_{0.44}^{0.49}$

.



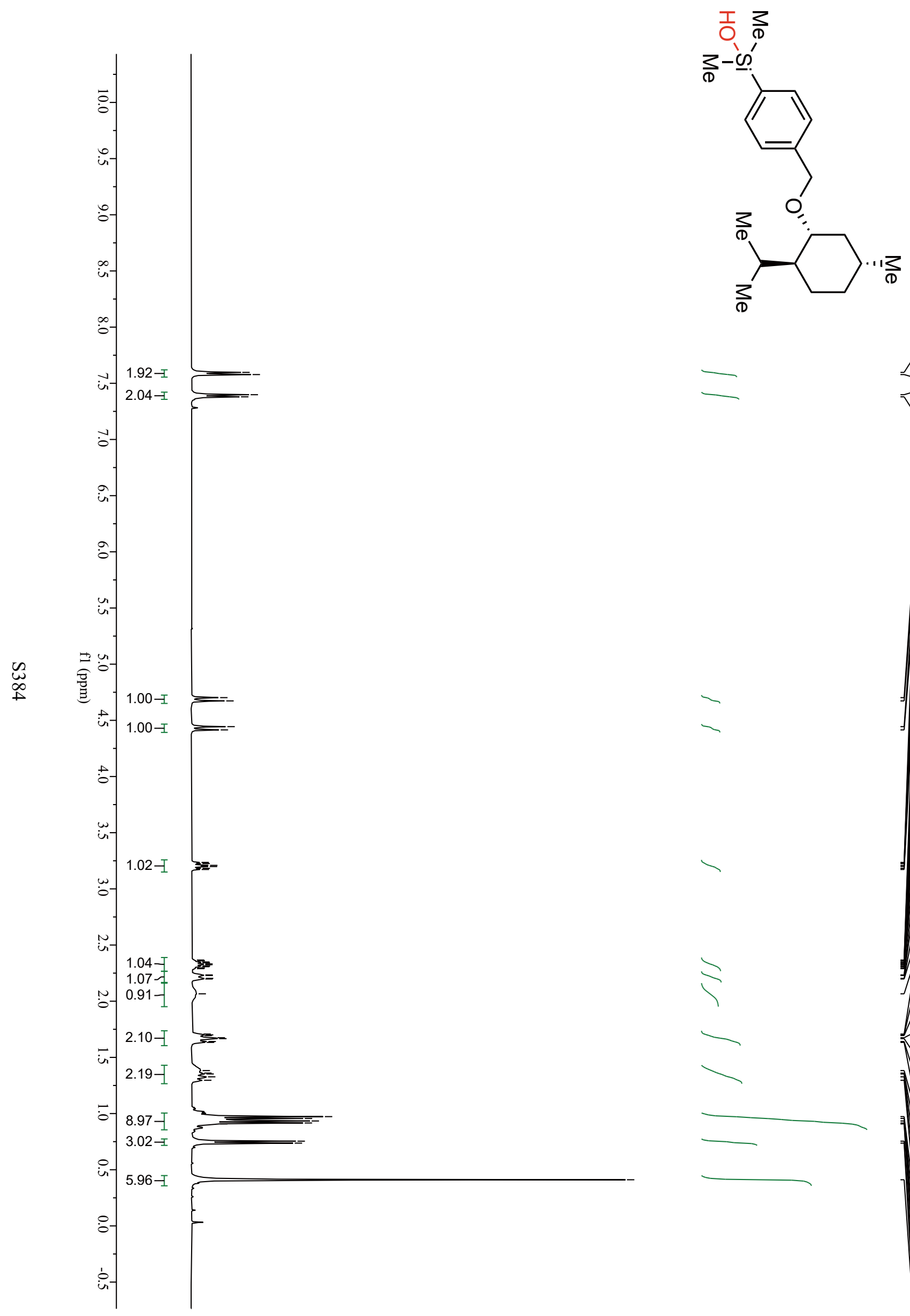

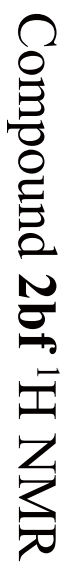
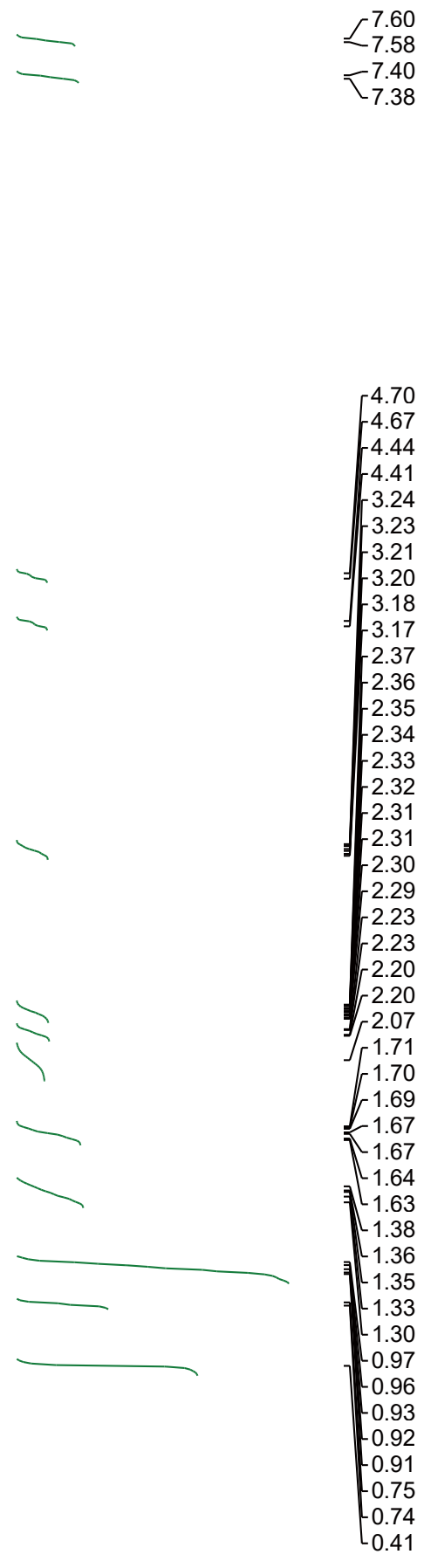
Compound $\mathbf{2 b f}{ }^{13} \mathrm{C}$ NMR
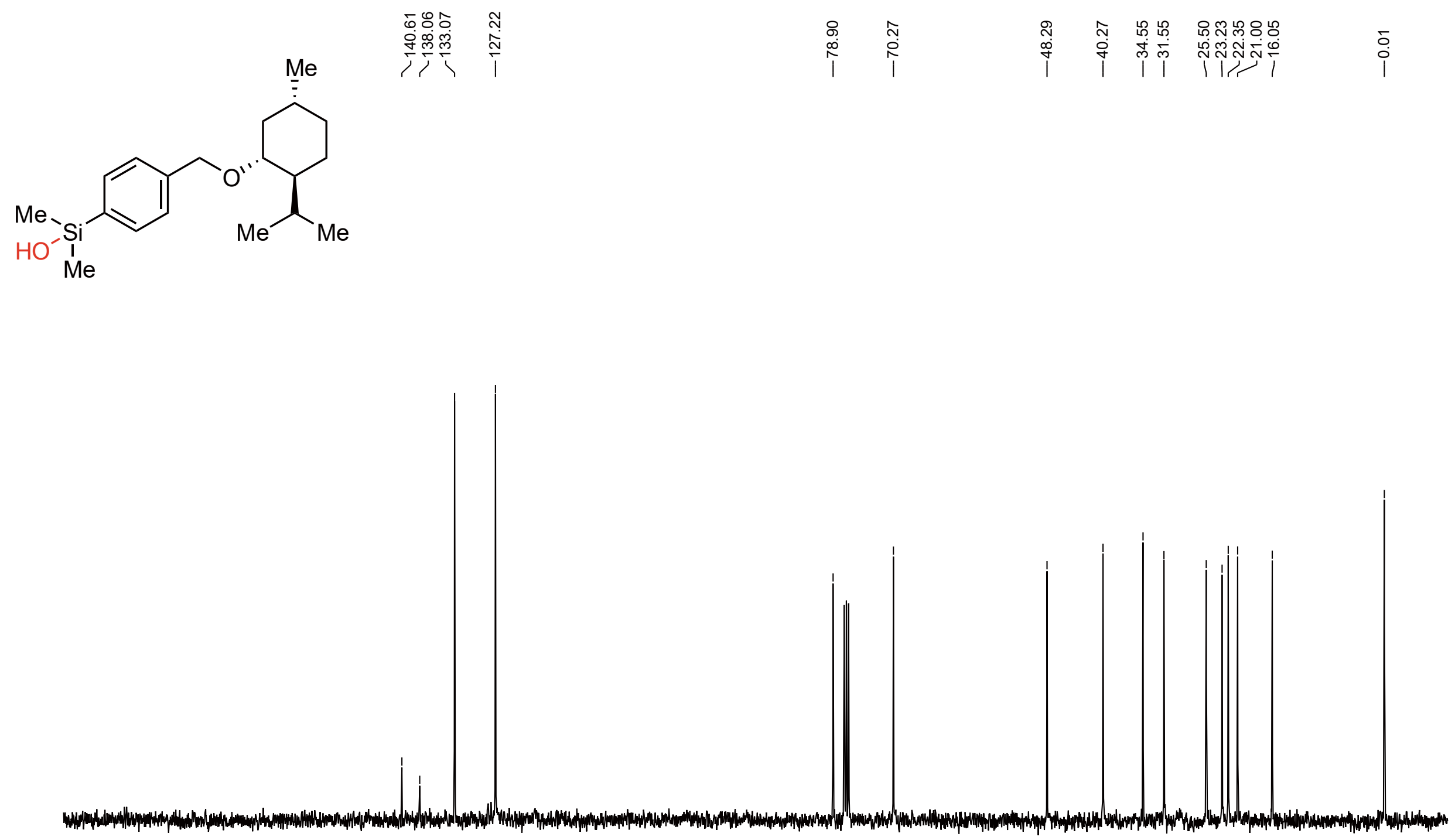

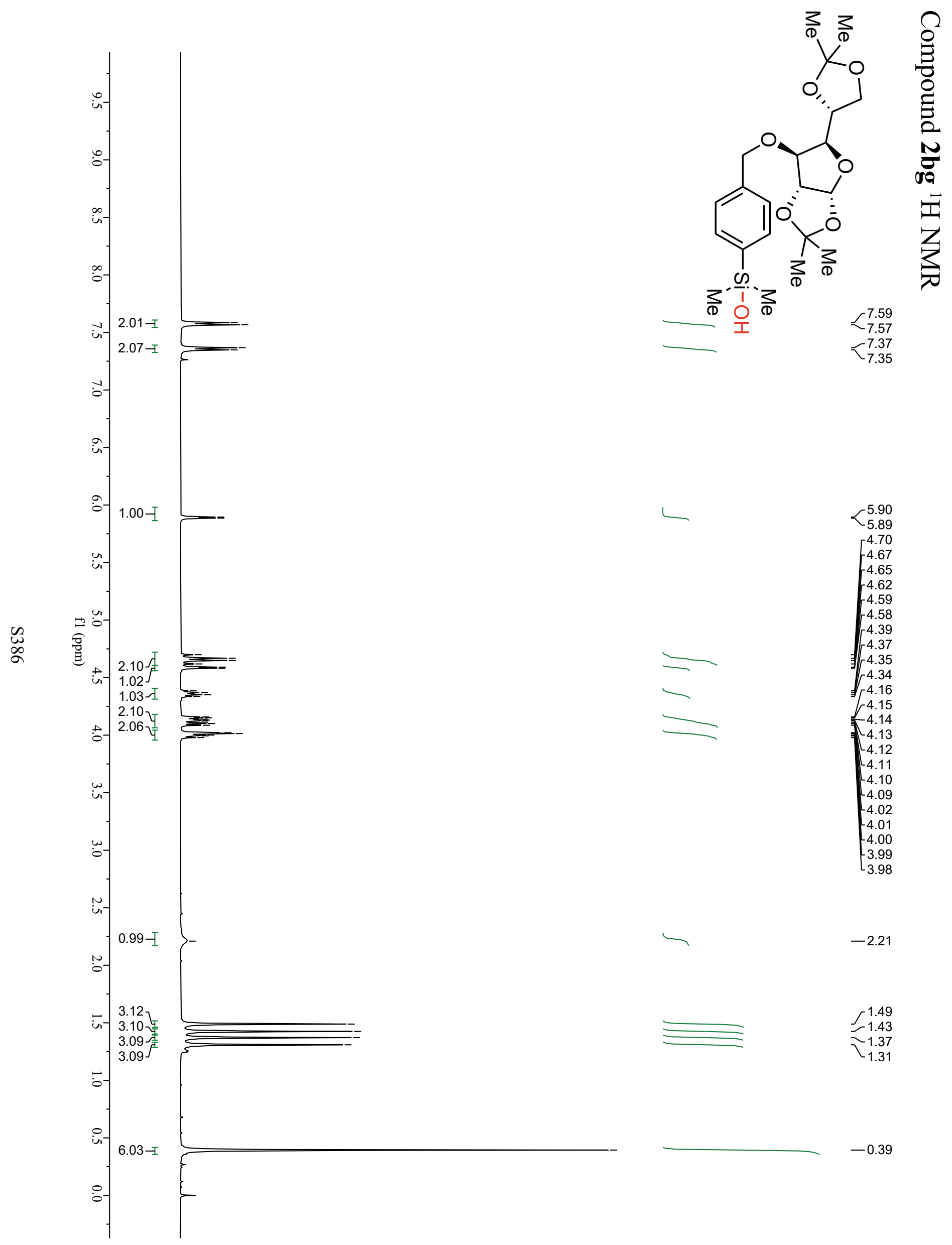

5.90
5.89

4.70
-4.67
-4.65

$-4.65$

$-4.62$

4.59

4.58

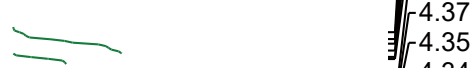

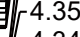

4.34
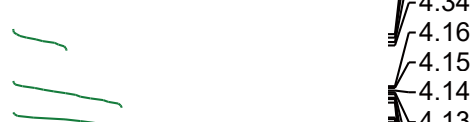

$-4.14$

4.13

$-4.13$

4.11

$-4.10$

$-4.09$

$-4.02$

$-4.01$

$-4.00$

-3.99
3.98

$-2.21$

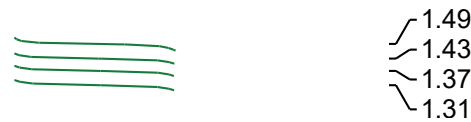

$-0.39$ 
Compound 2bg ${ }^{1} \mathrm{H}$ NMR
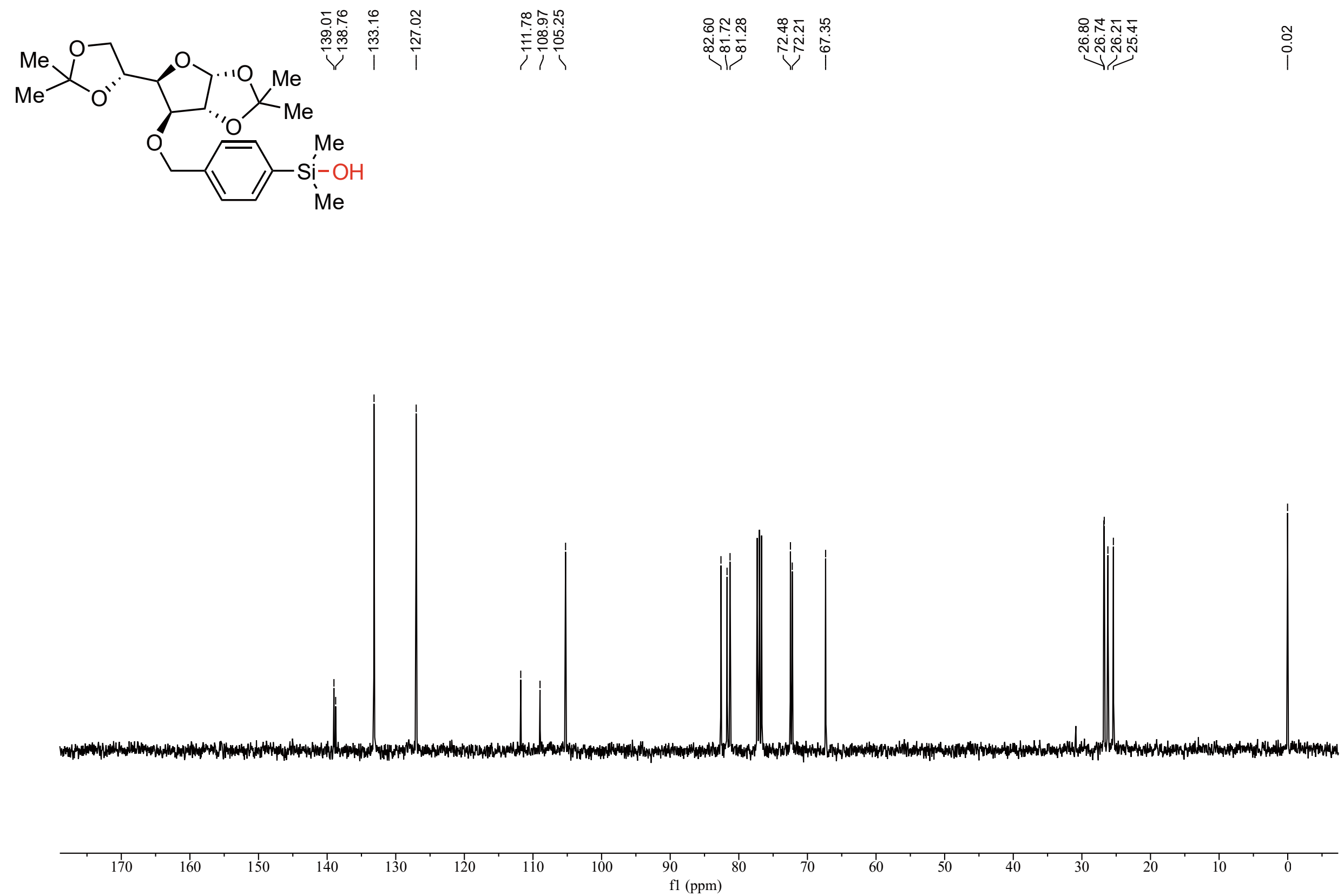


\section{Compound $\mathbf{2 b h}{ }^{1} \mathrm{H}$ NMR}
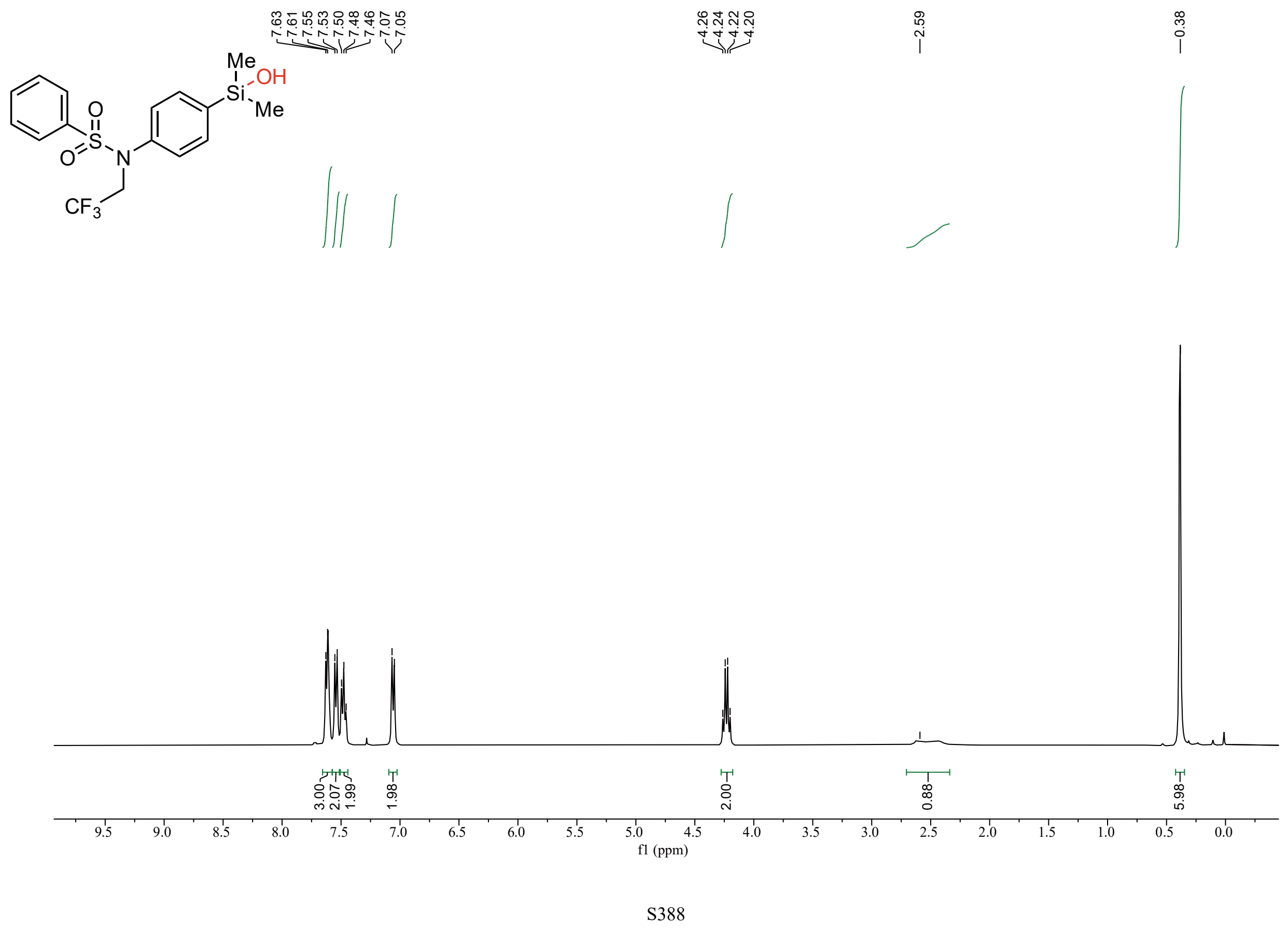
Compound 2bh ${ }^{13} \mathrm{C}$ NMR
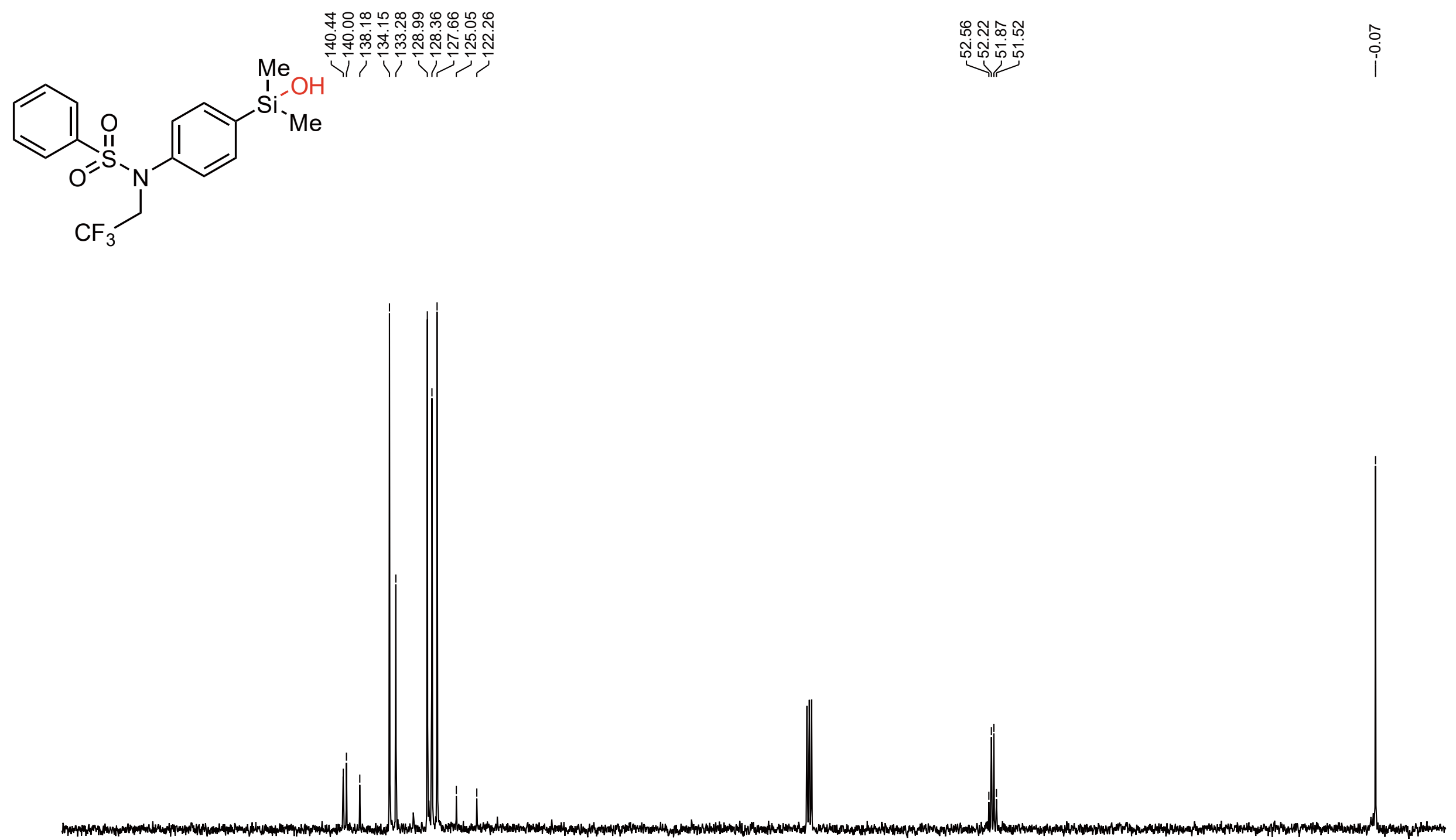
Compound $\mathbf{2 b h}{ }^{19} \mathrm{~F}$ NMR<smiles>C[Si](O)(O)c1ccc(N(CC(F)(F)F)S(=O)(=O)c2ccccc2)cc1</smiles>

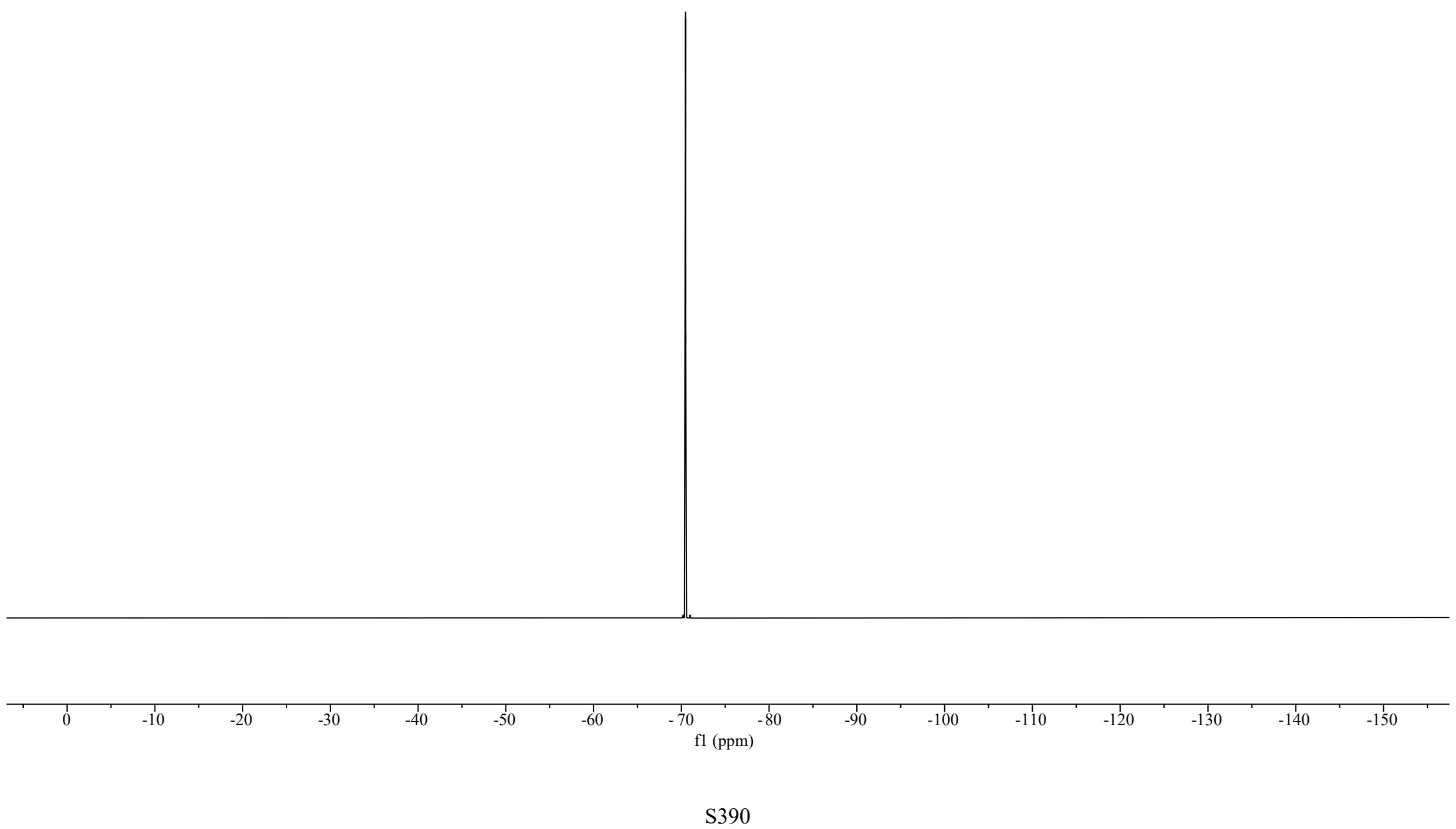



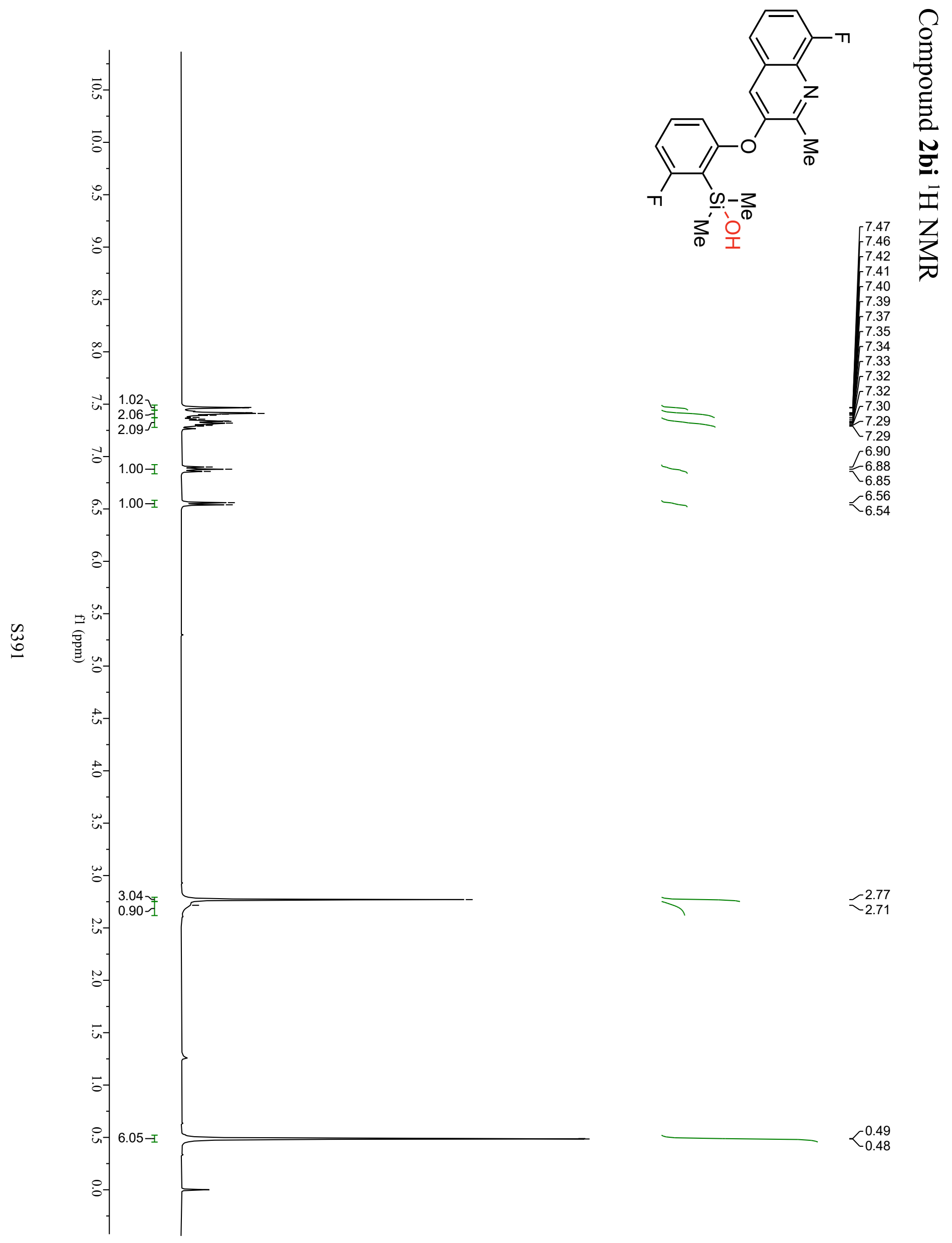

$<_{0.48}^{0.49}$ 


$$
z^{\prime \prime 4}
$$


Compound 2bi ${ }^{19} \mathrm{~F}$ NMR

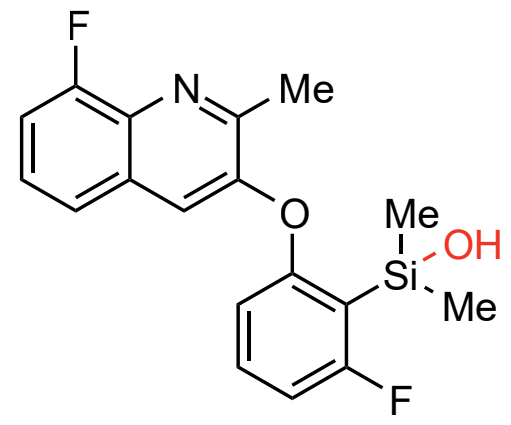

웅
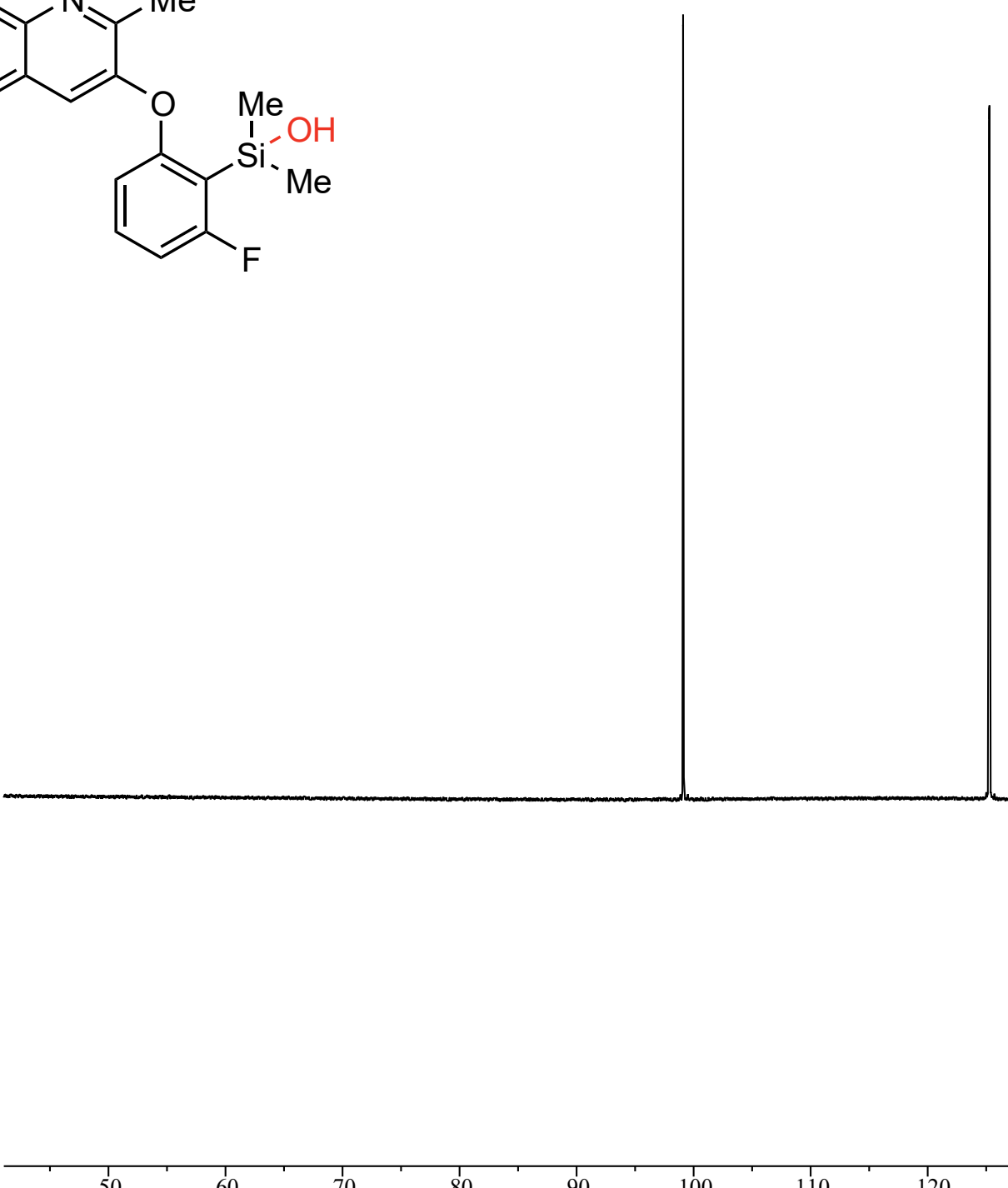


\section{Compound 2bj ${ }^{1} \mathrm{H}$ NMR}
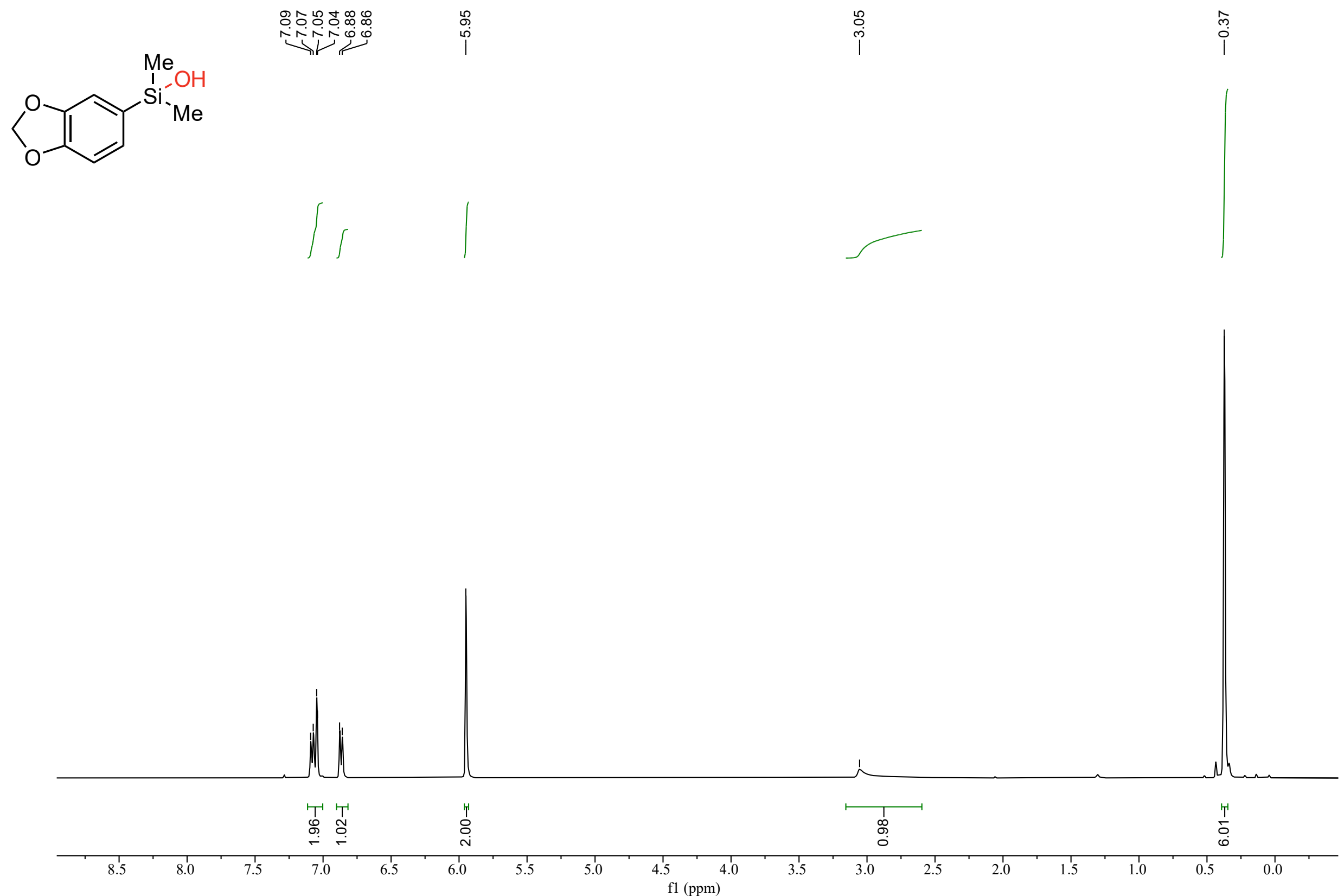
Compound 2bj ${ }^{13} \mathrm{C}$ NMR

(1)

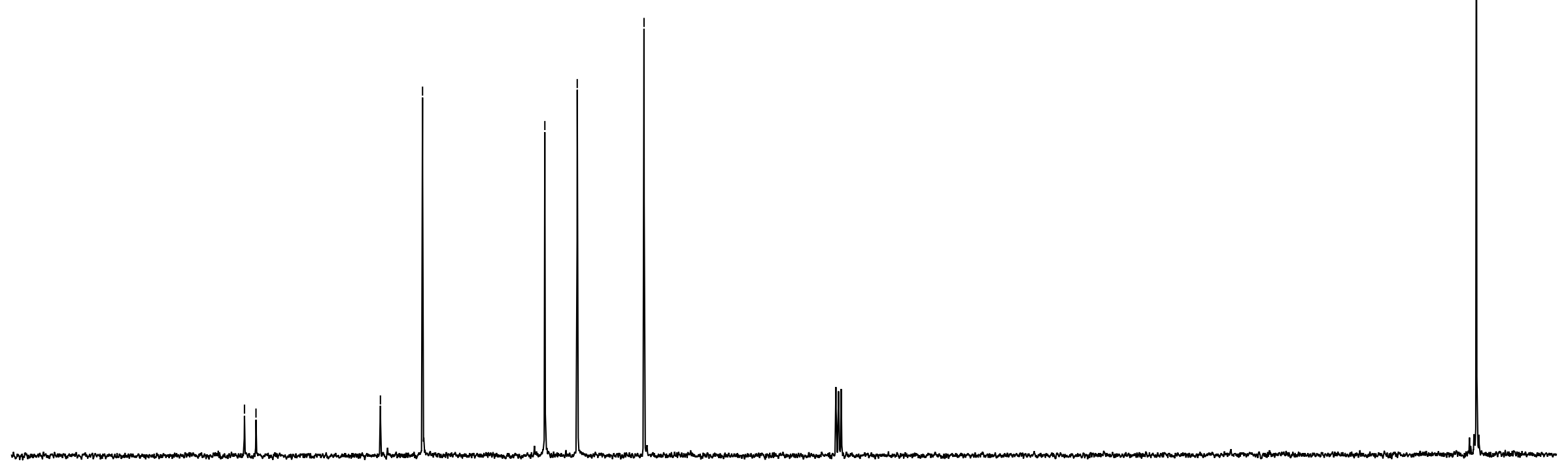


Compound 2bk ${ }^{1} \mathrm{H}$ NMR

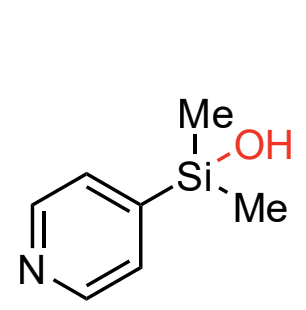

Me
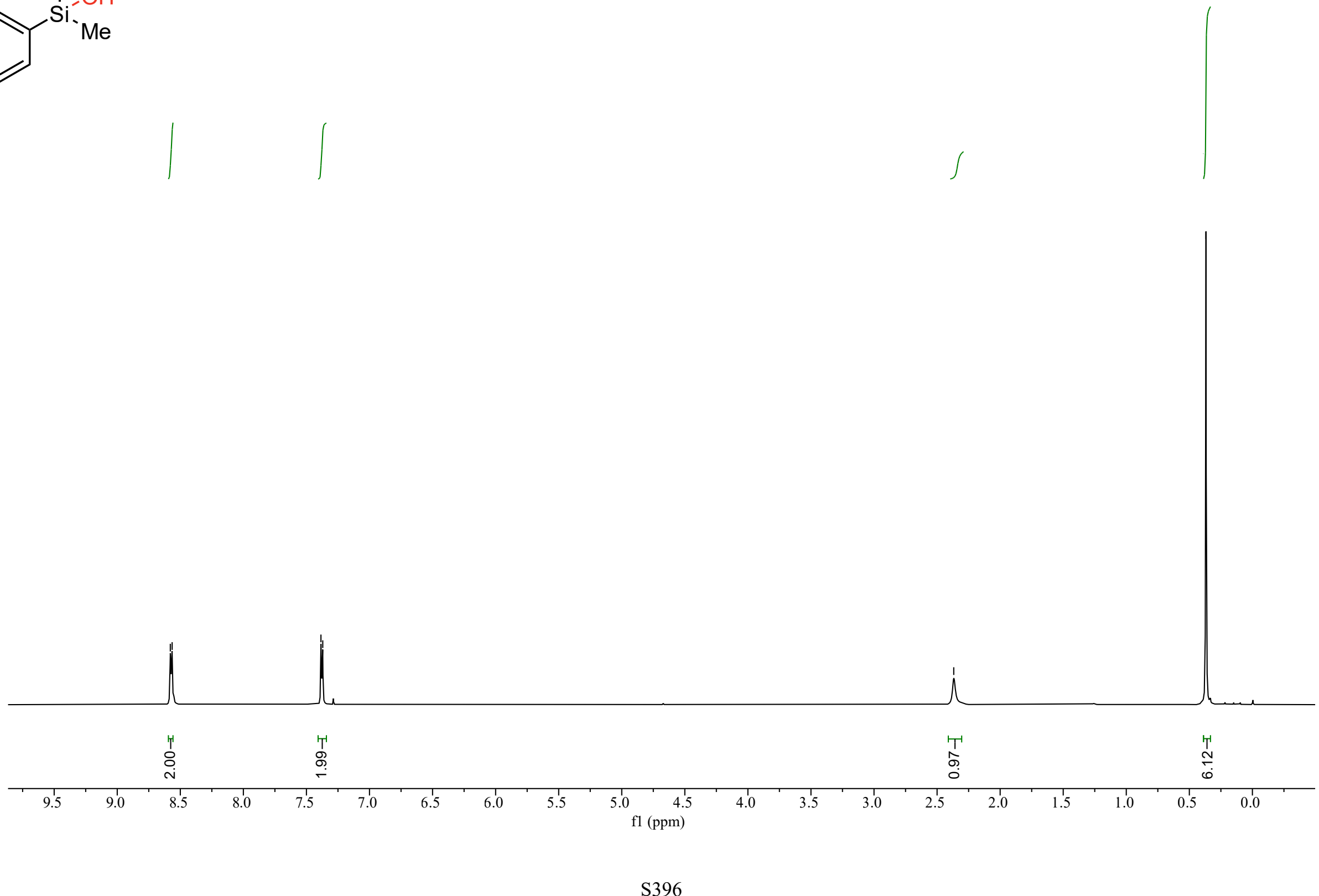
Compound 2bk ${ }^{13} \mathrm{C}$ NMR
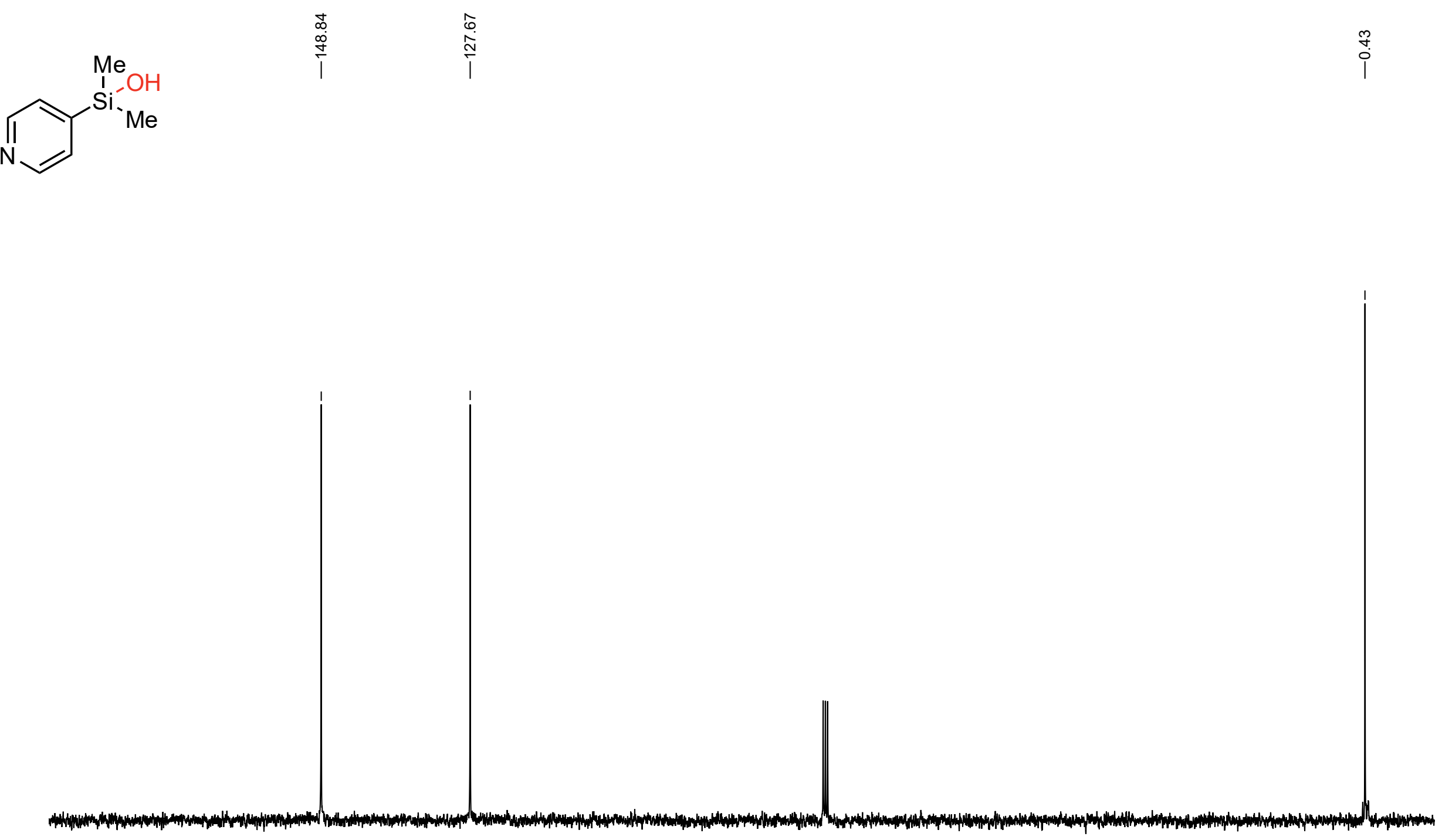


\section{Compound 2bl ${ }^{1} \mathrm{H}$ NMR}

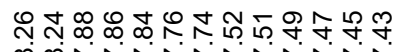

on

(1)
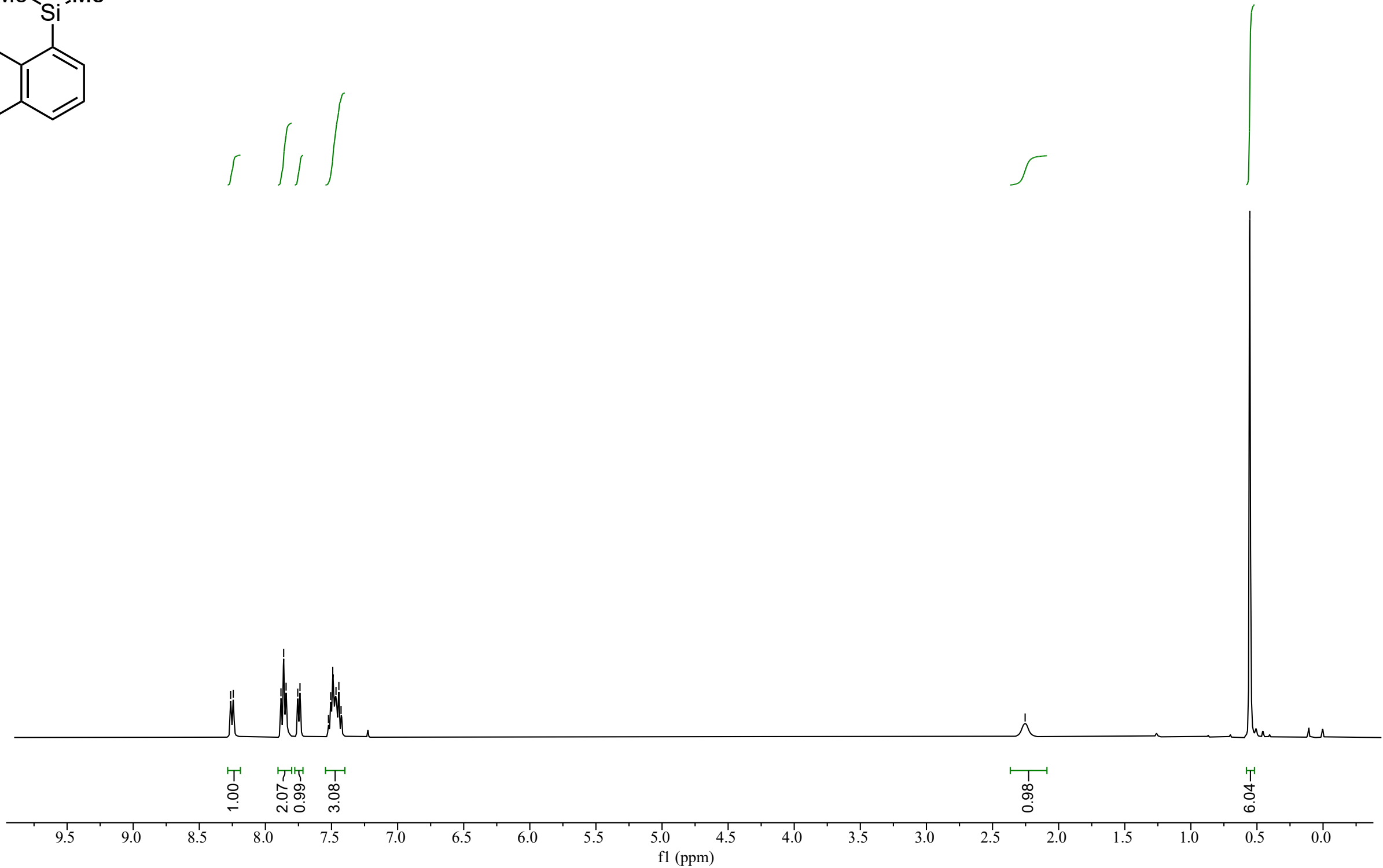
Compound 2bl ${ }^{13} \mathrm{C}$ NMR

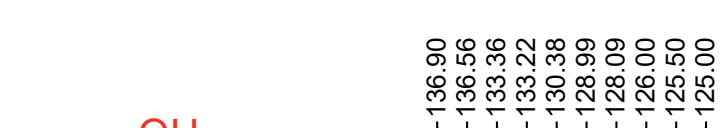

(1)

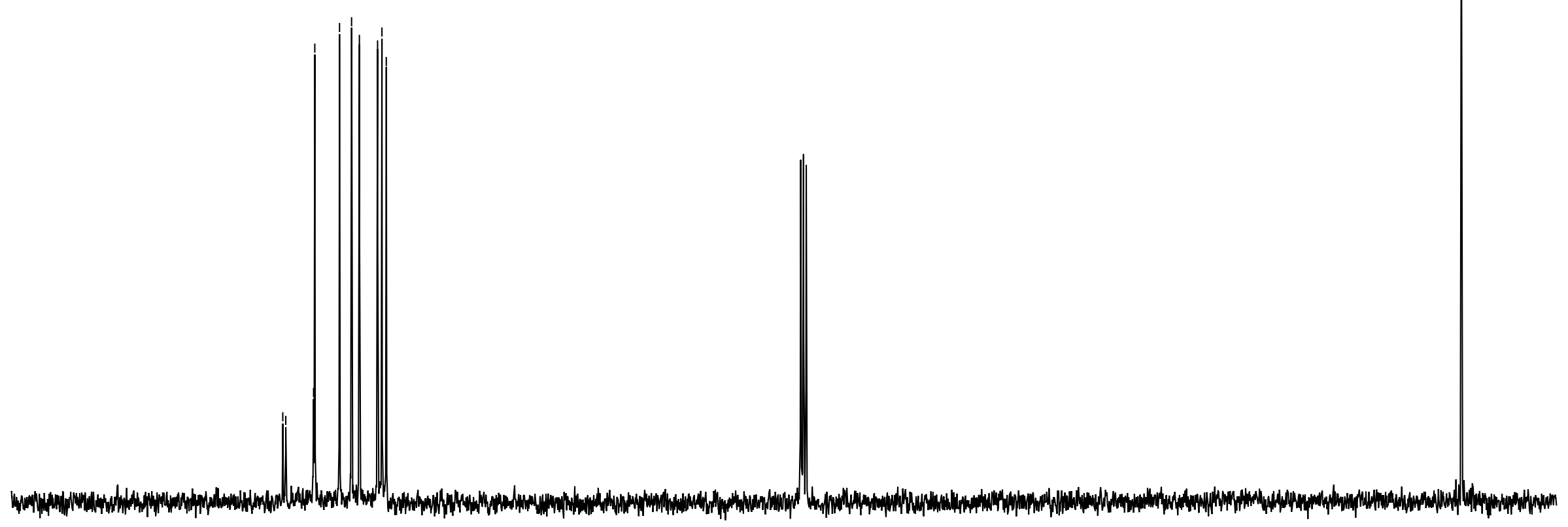




\section{Compound 2bm ${ }^{1} \mathrm{H}$ NMR}

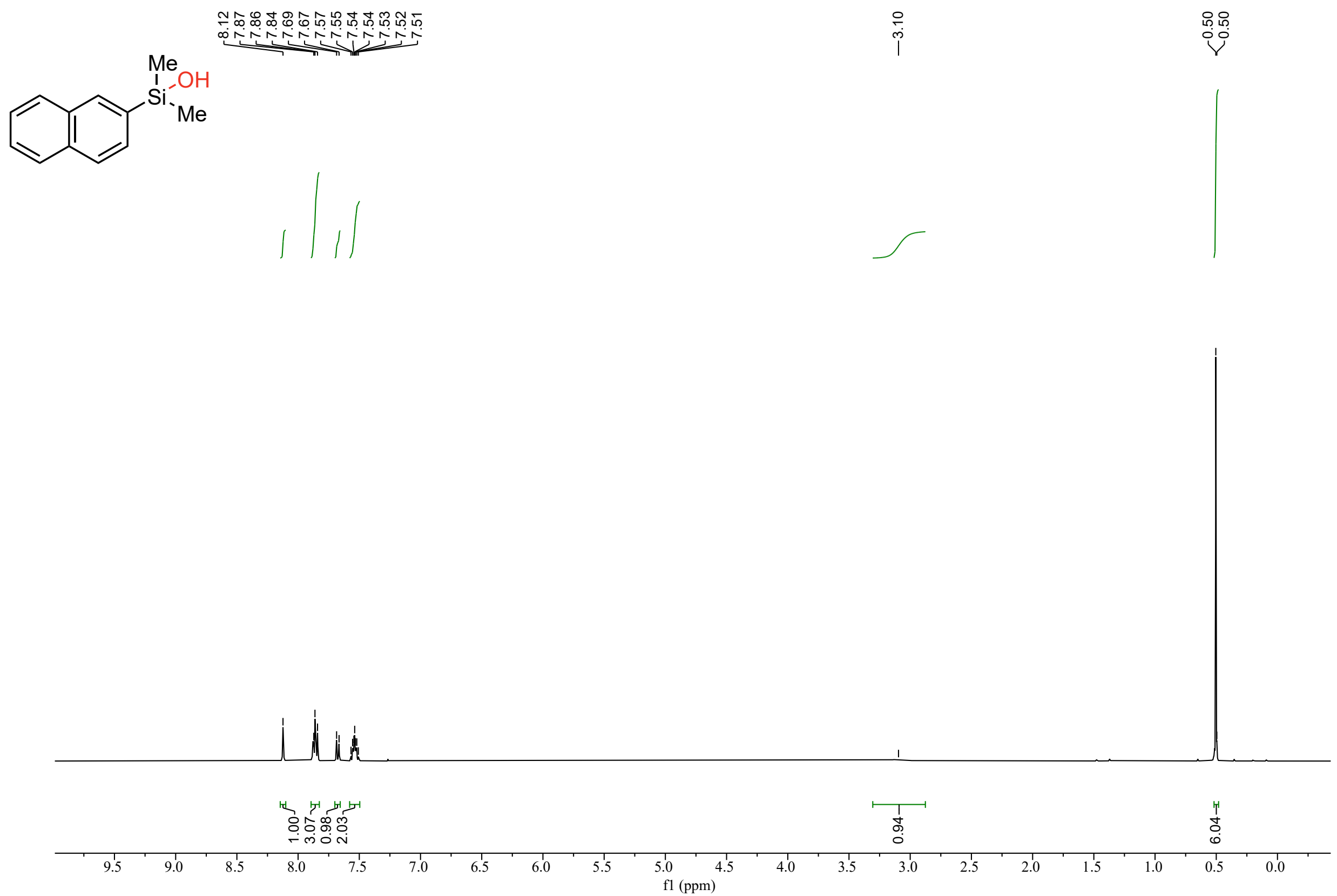




\section{Compound 2bm ${ }^{13} \mathrm{C}$ NMR}

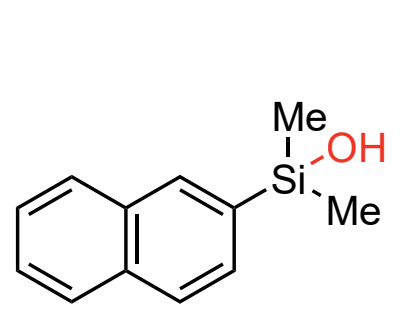

ช ชู

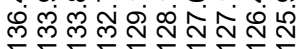

皮
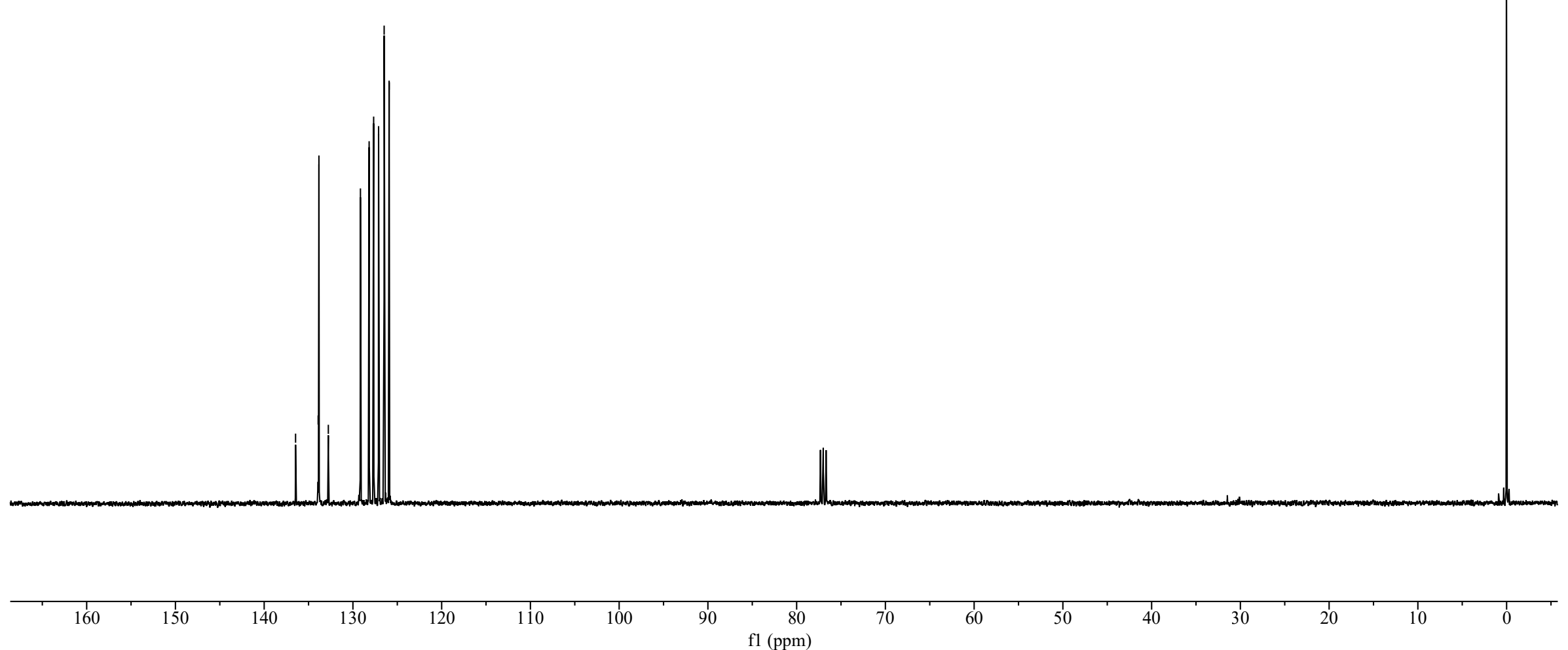


\section{Compound 2bn ${ }^{1} \mathrm{H}$ NMR}

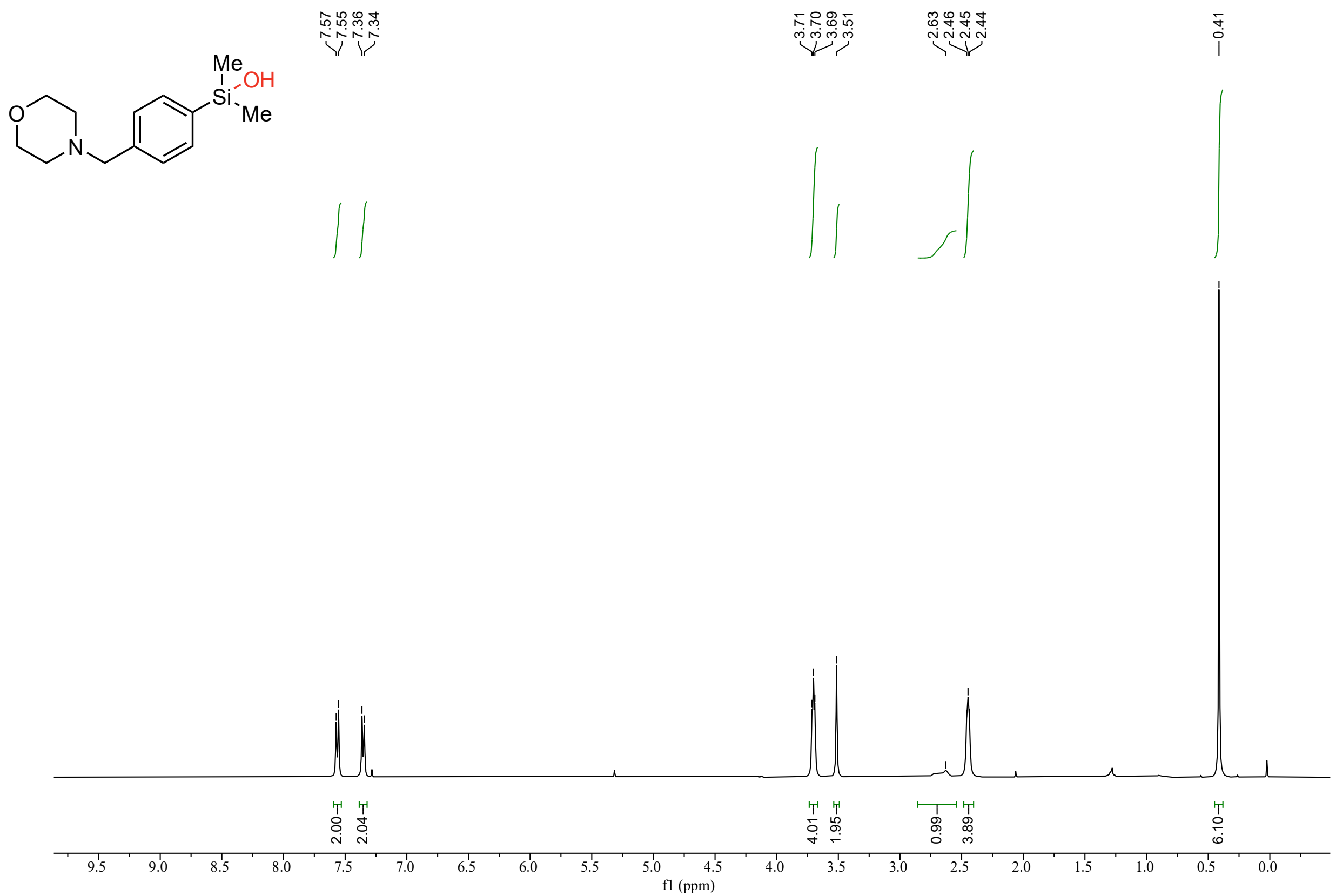


Compound 2bn ${ }^{13} \mathrm{C}$ NMR

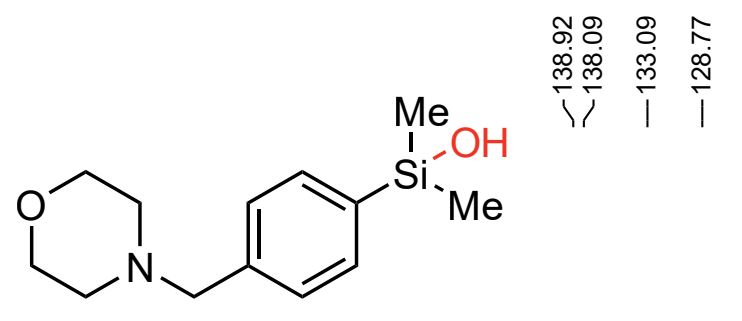

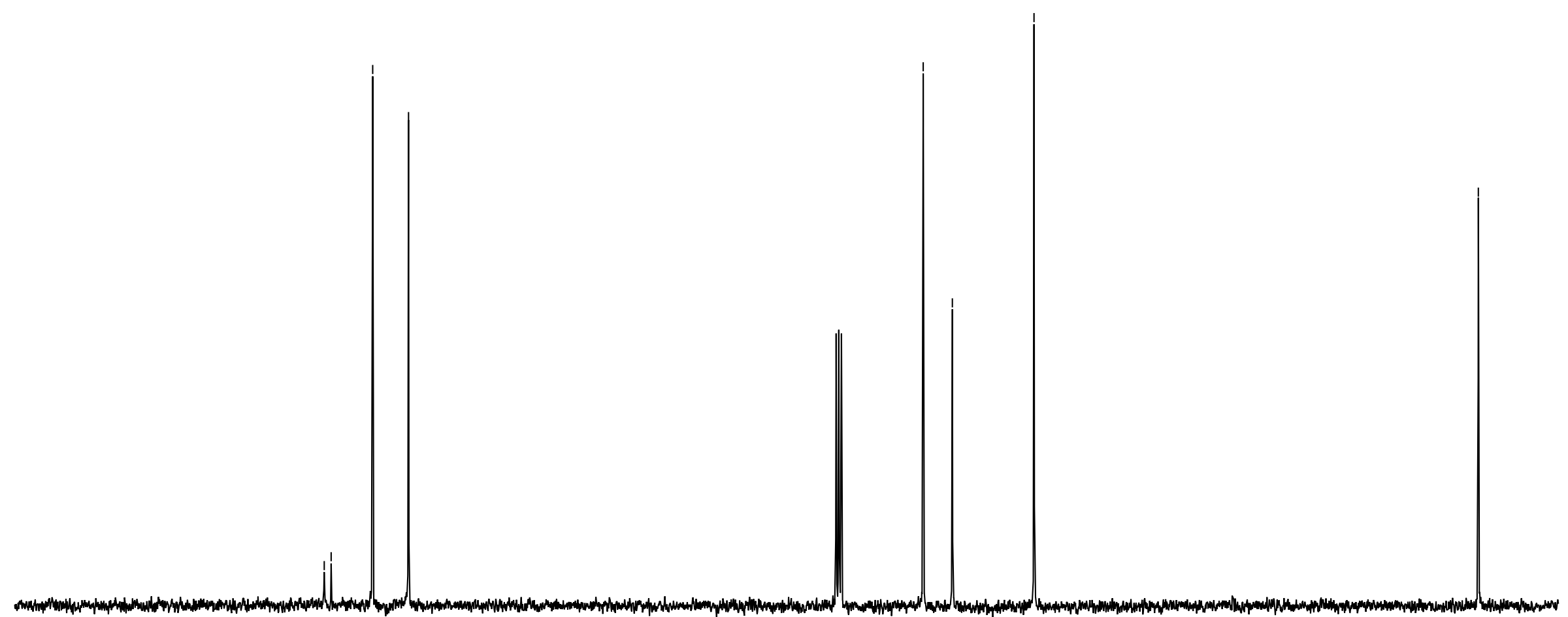


Compound 2 bo ${ }^{1} \mathrm{H}$ NMR
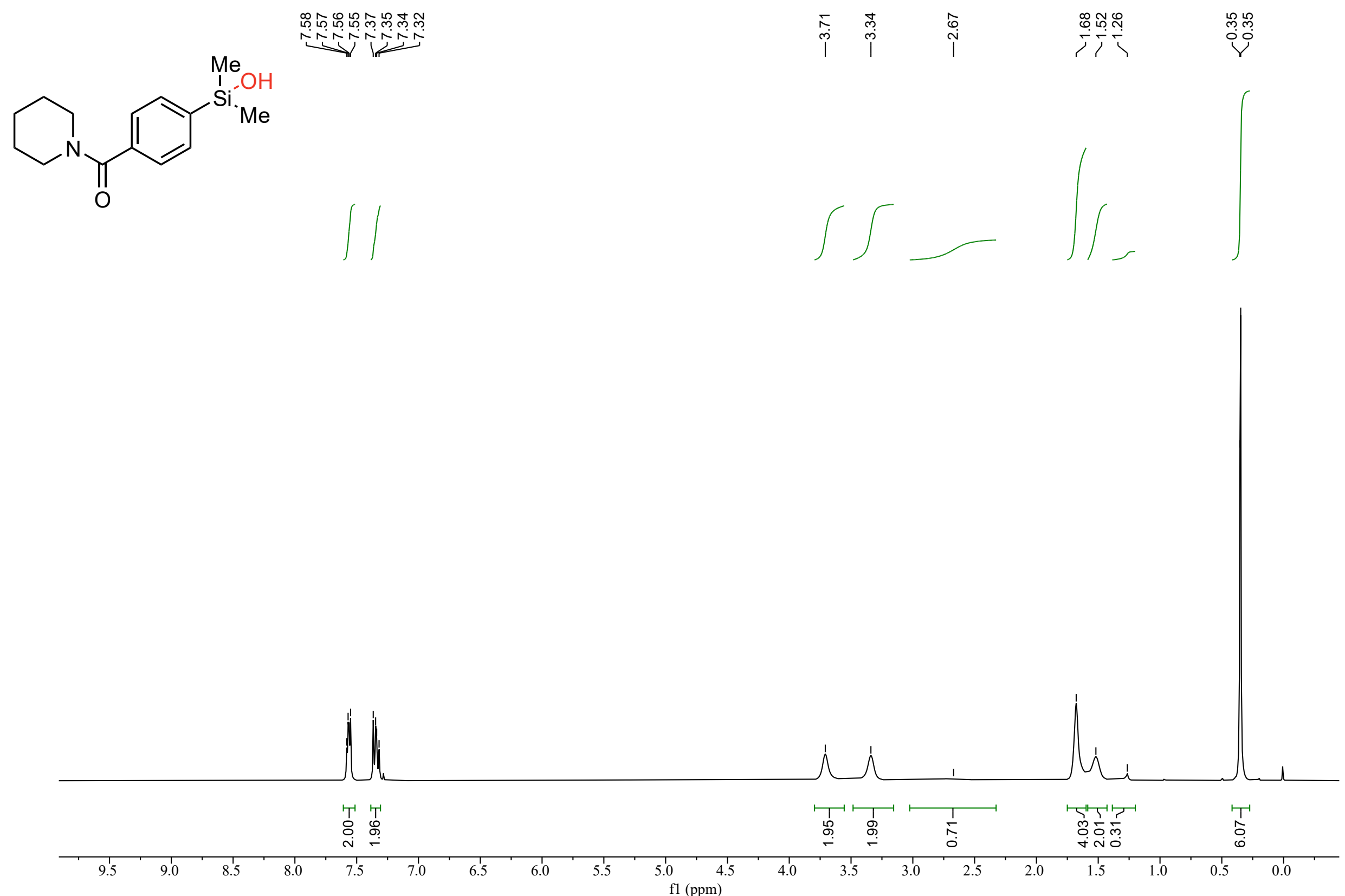
Compound 2 bo ${ }^{13} \mathrm{C}$ NMR
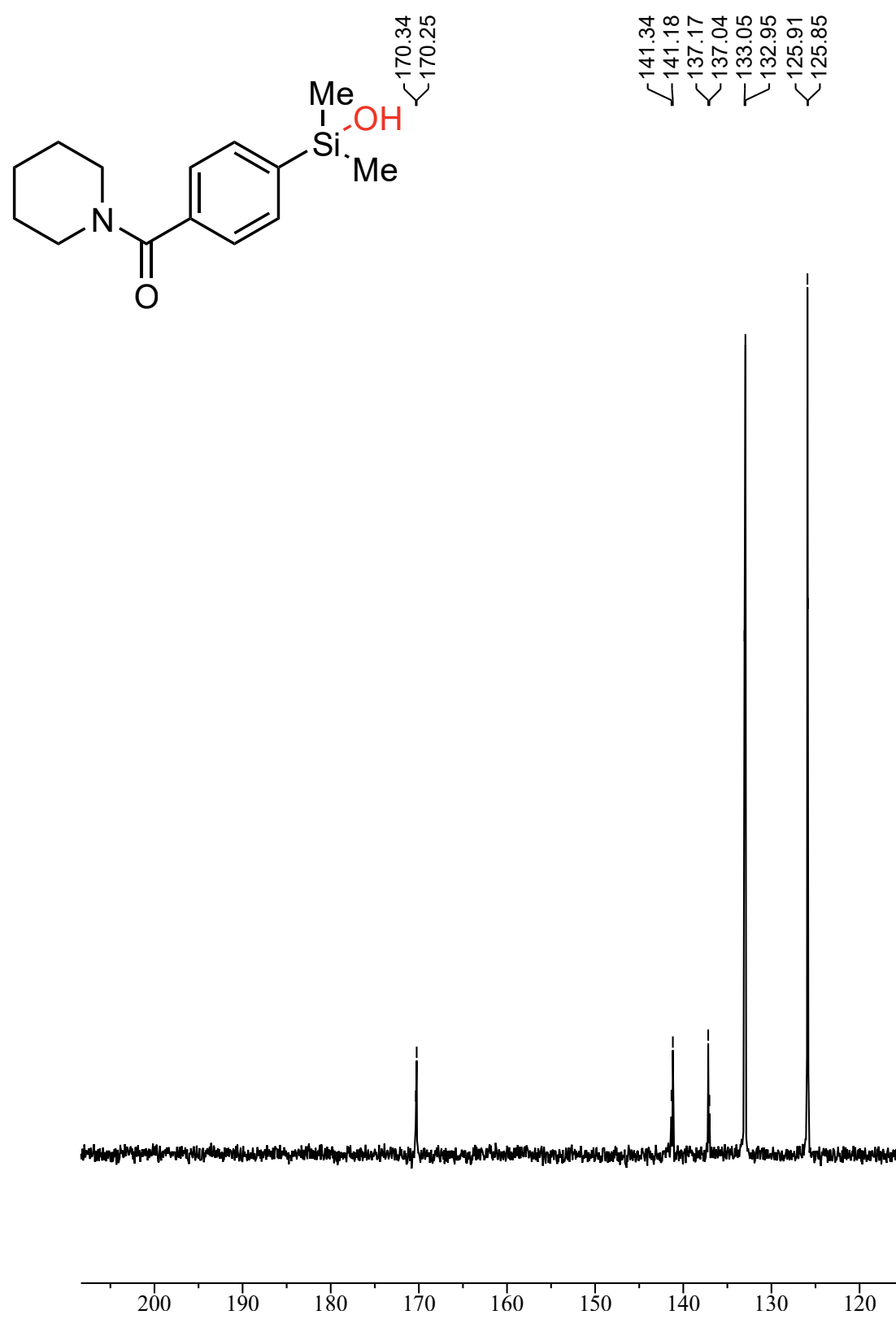

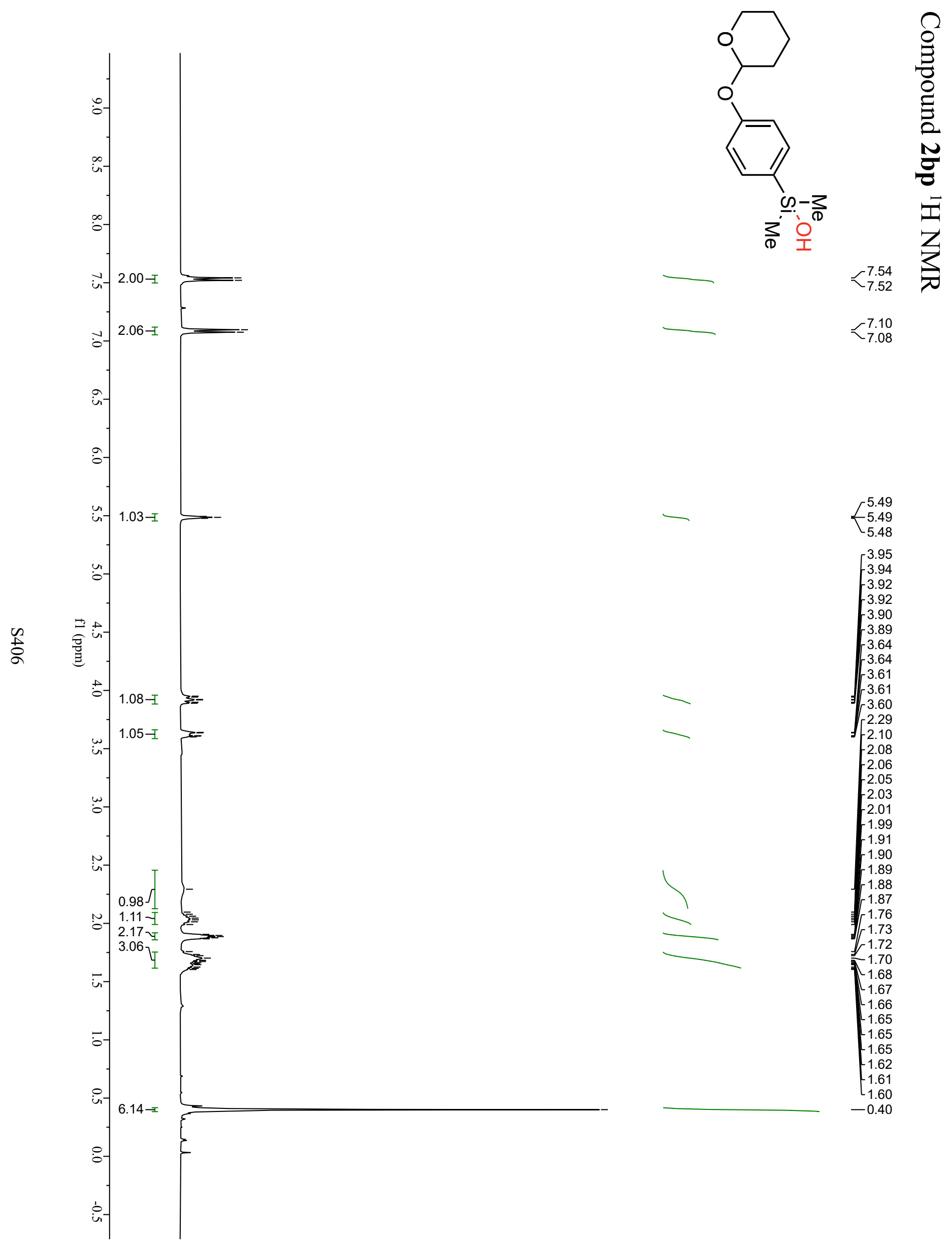
Compound 2 bp ${ }^{13} \mathrm{C}$ NMR

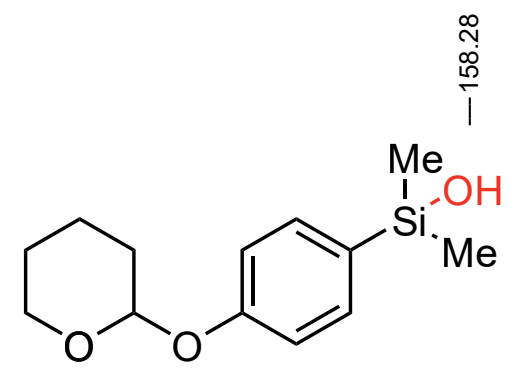

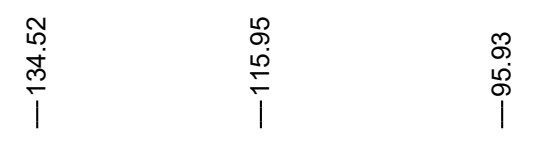

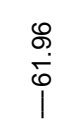

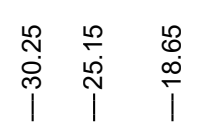

ta
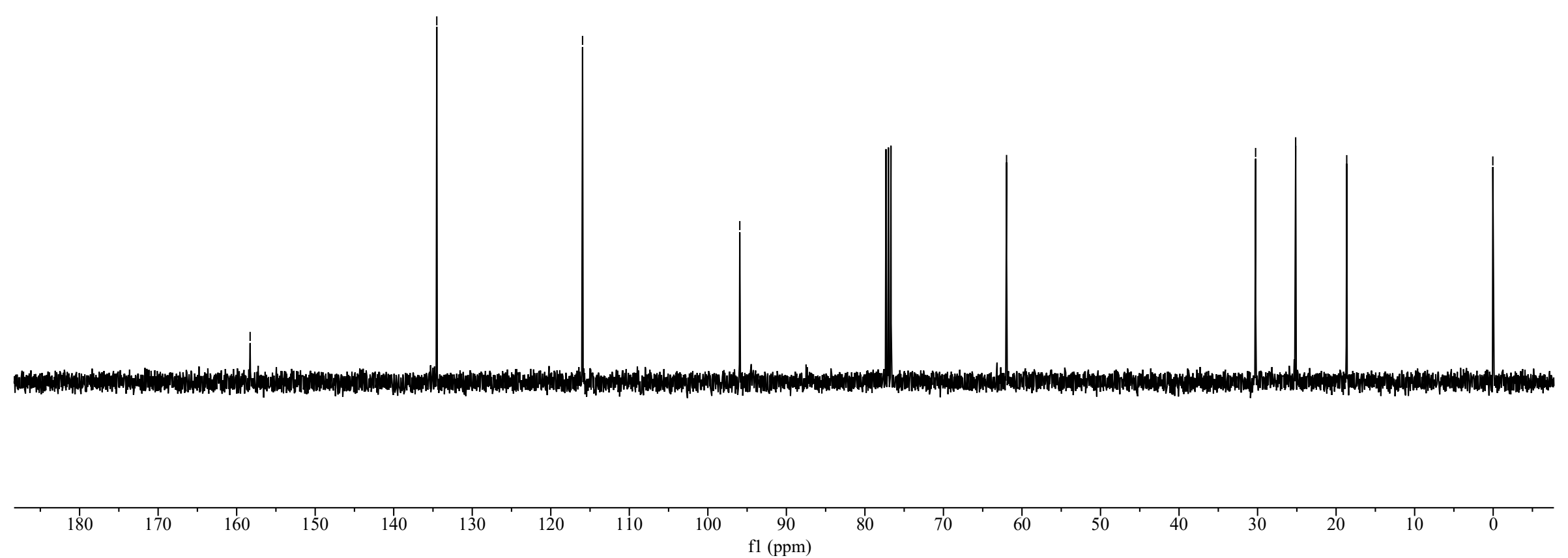

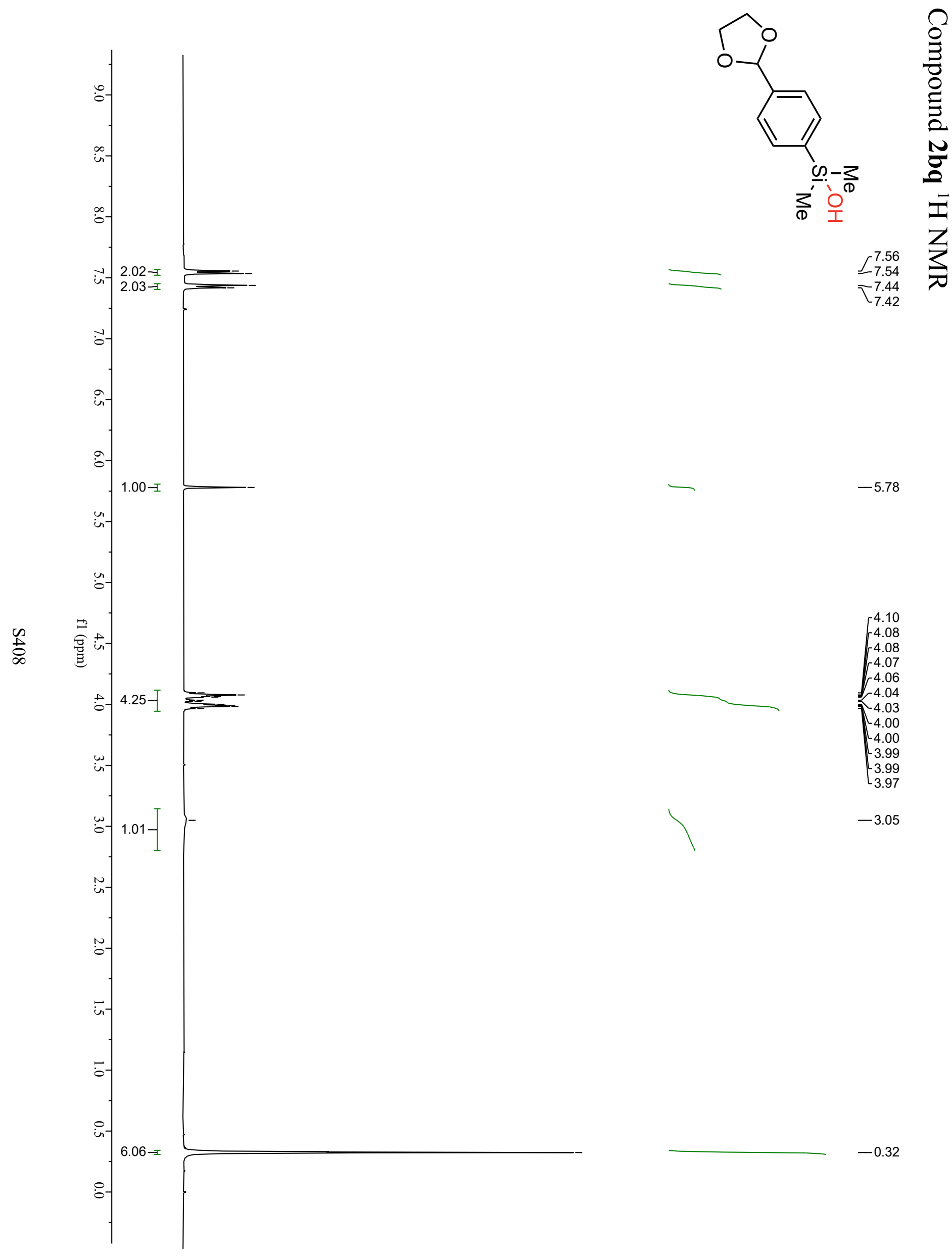
Compound 2bq ${ }^{13} \mathrm{C}$ NMR
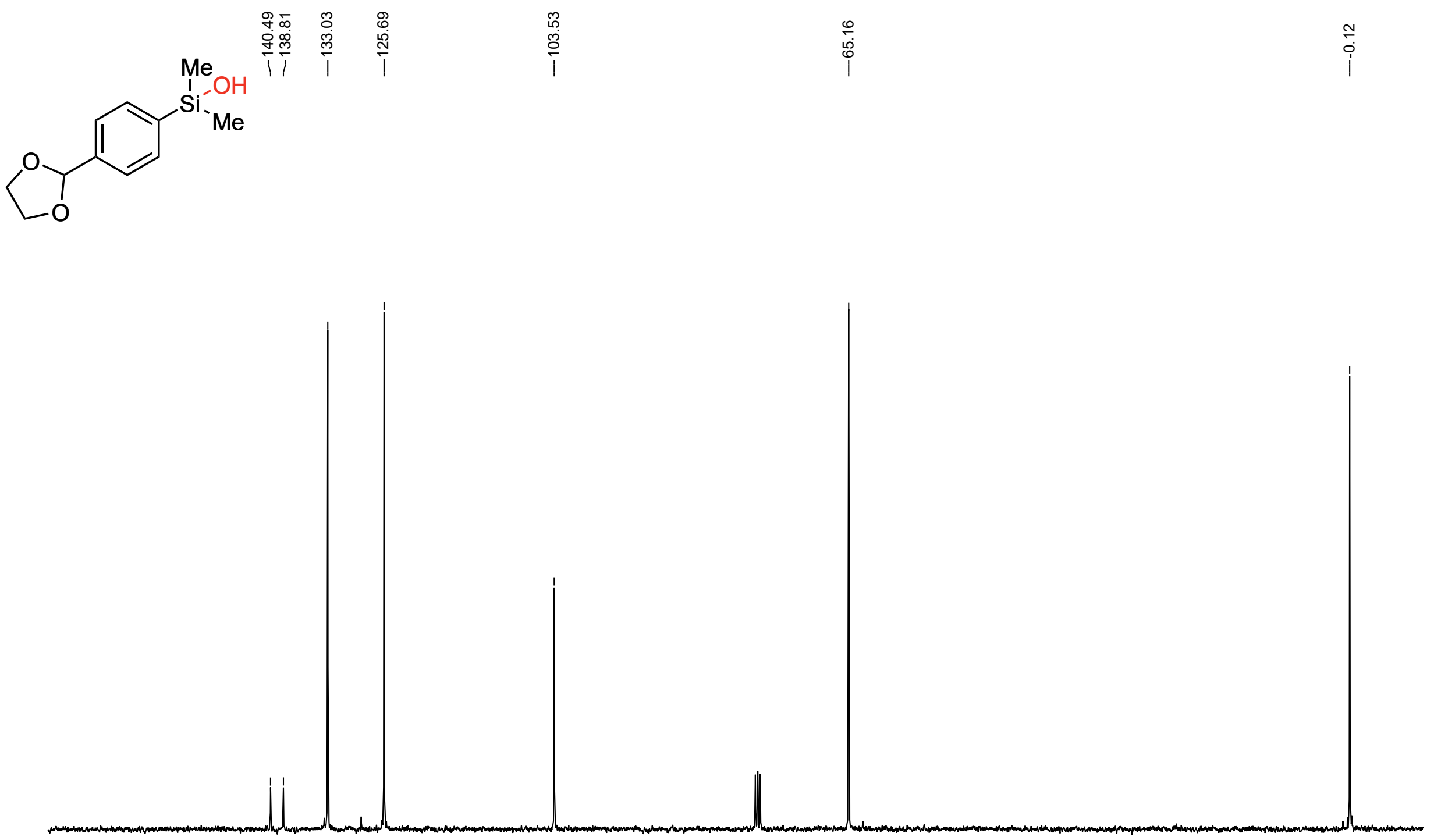


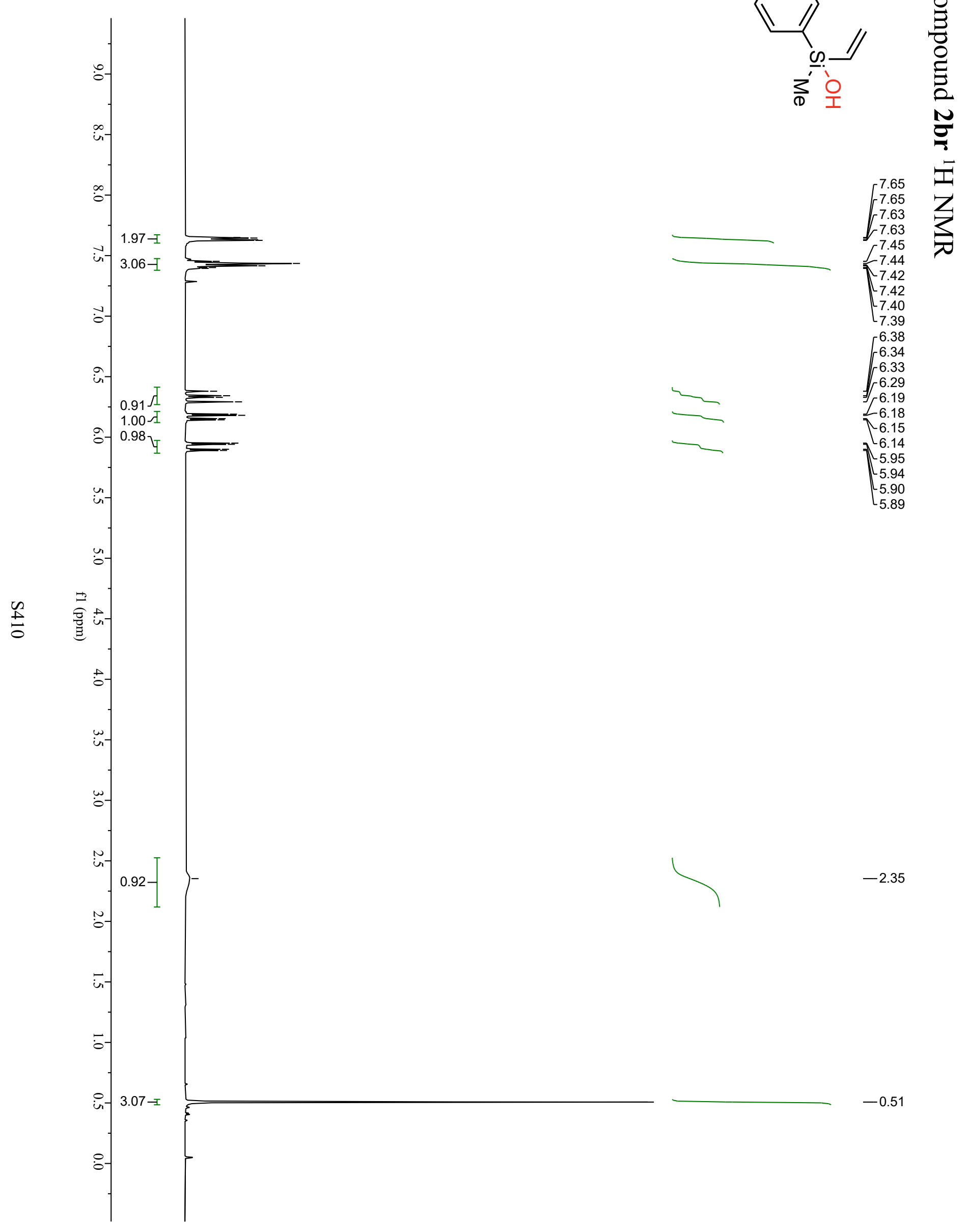


Compound 2br ${ }^{13} \mathrm{C}$ NMR

${ }_{\mathrm{Me}}^{\mathrm{OH}}$

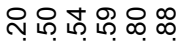

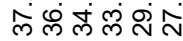

पIt II

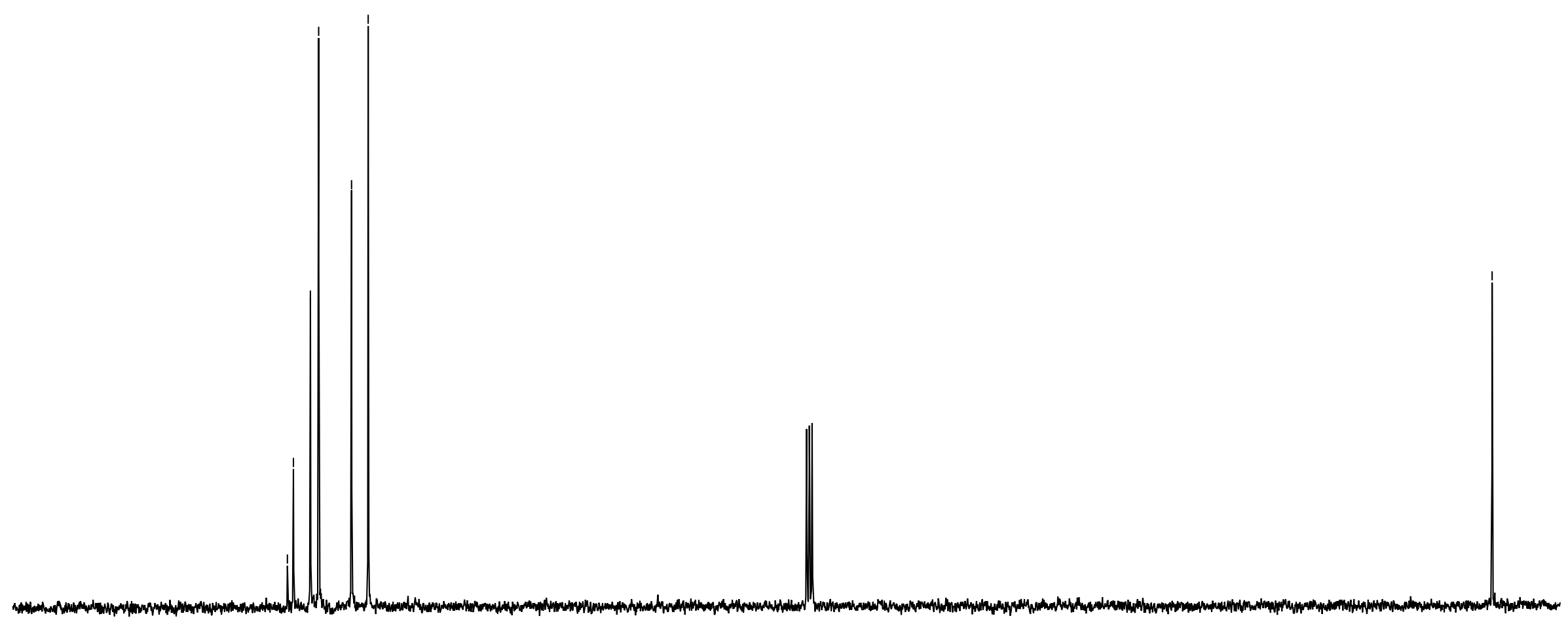


Compound 2bs ${ }^{1} \mathrm{H}$ NMR

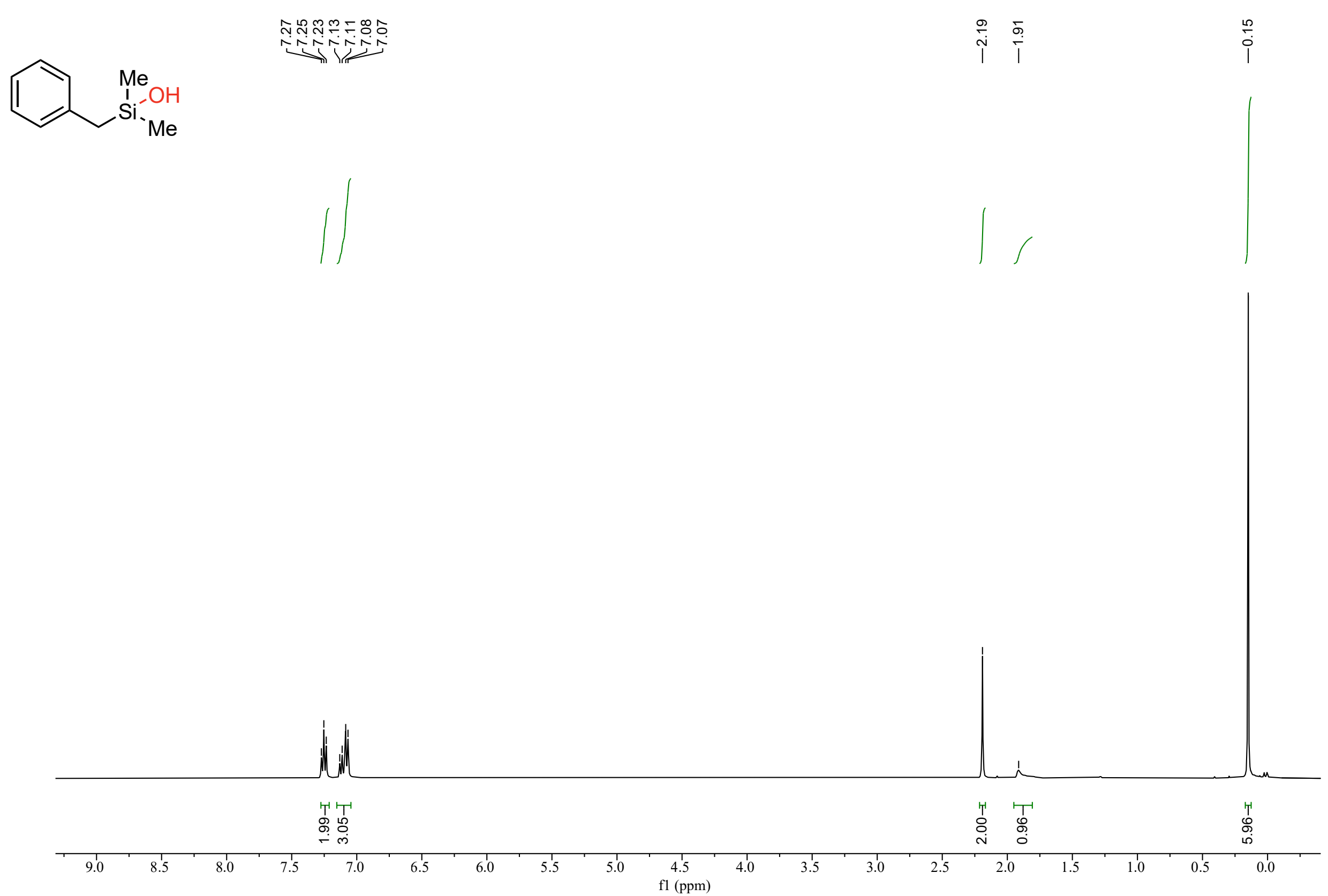


Compound 2bs ${ }^{13} \mathrm{C}$ NMR

$12 \mathrm{MiH}_{\mathrm{Me}}^{\mathrm{Me}} \mathrm{Si}^{\mathrm{OH}}$

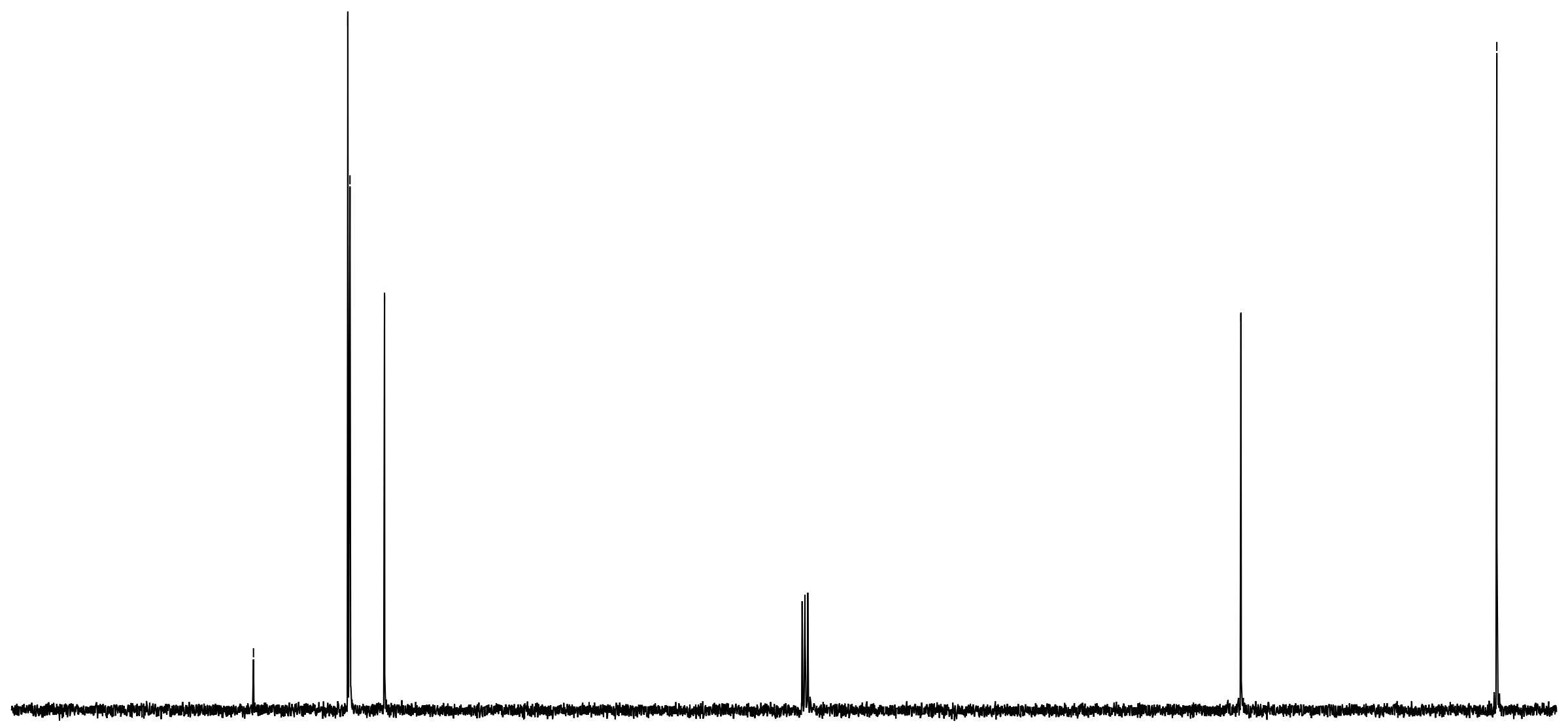


Compound 2 bt ${ }^{1} \mathrm{H}$ NMR
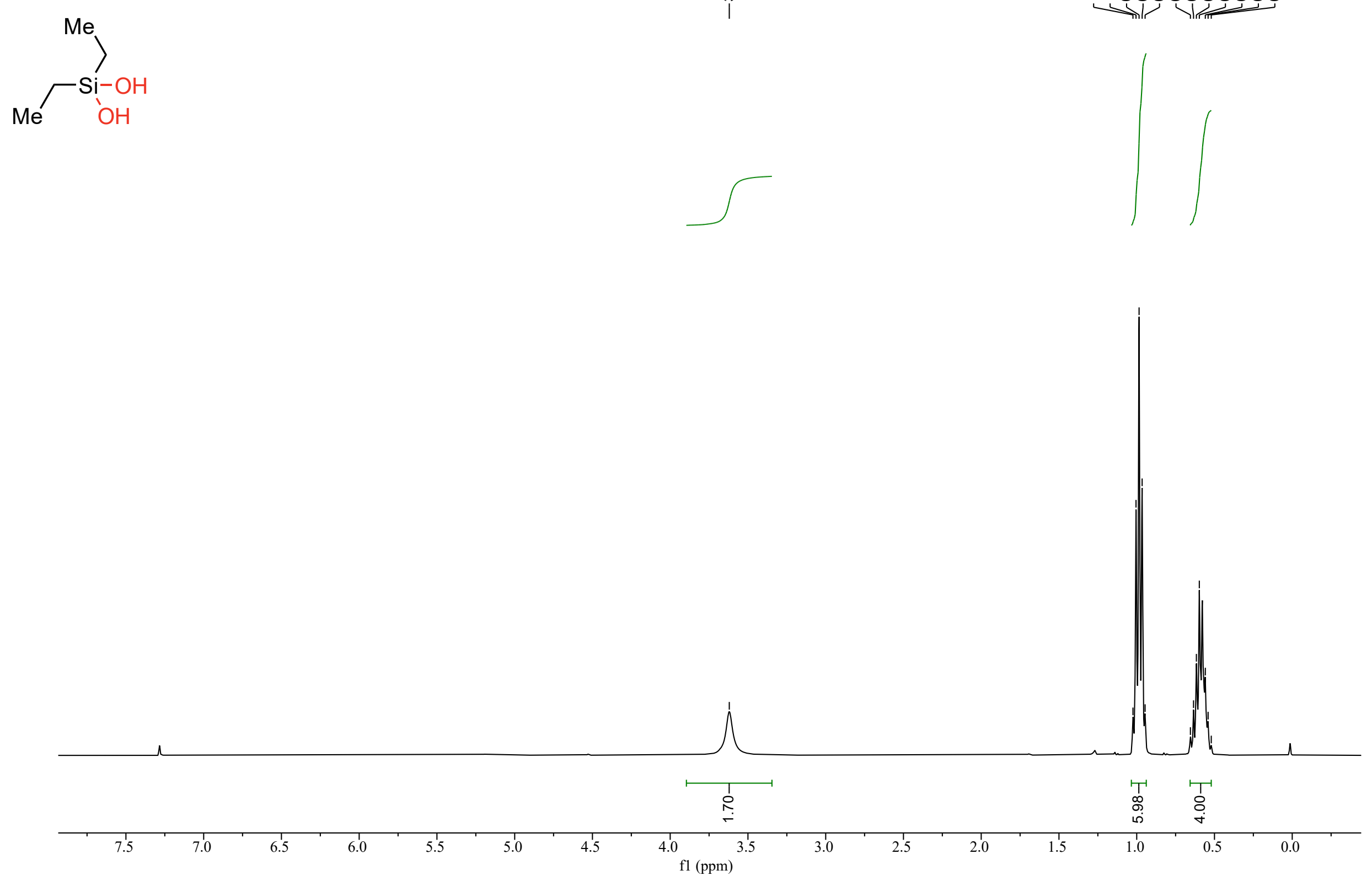
Compound $\mathbf{2 b t}{ }^{13} \mathrm{C}$ NMR
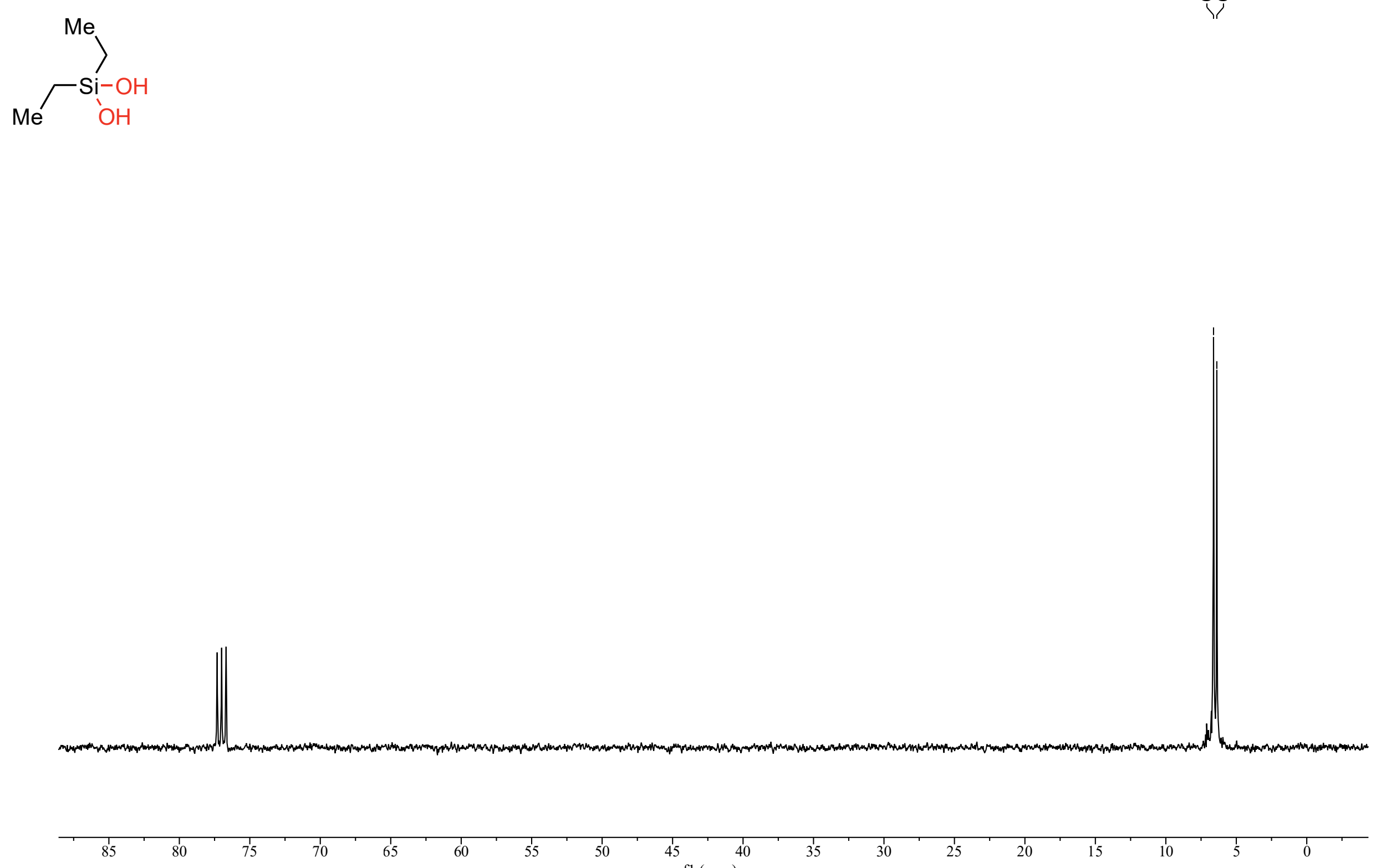
Compound 4a ${ }^{1} \mathrm{H}$ NMR

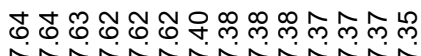

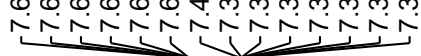

${ }^{\mathrm{Me}} \mathrm{Si}_{\mathrm{Me}}$

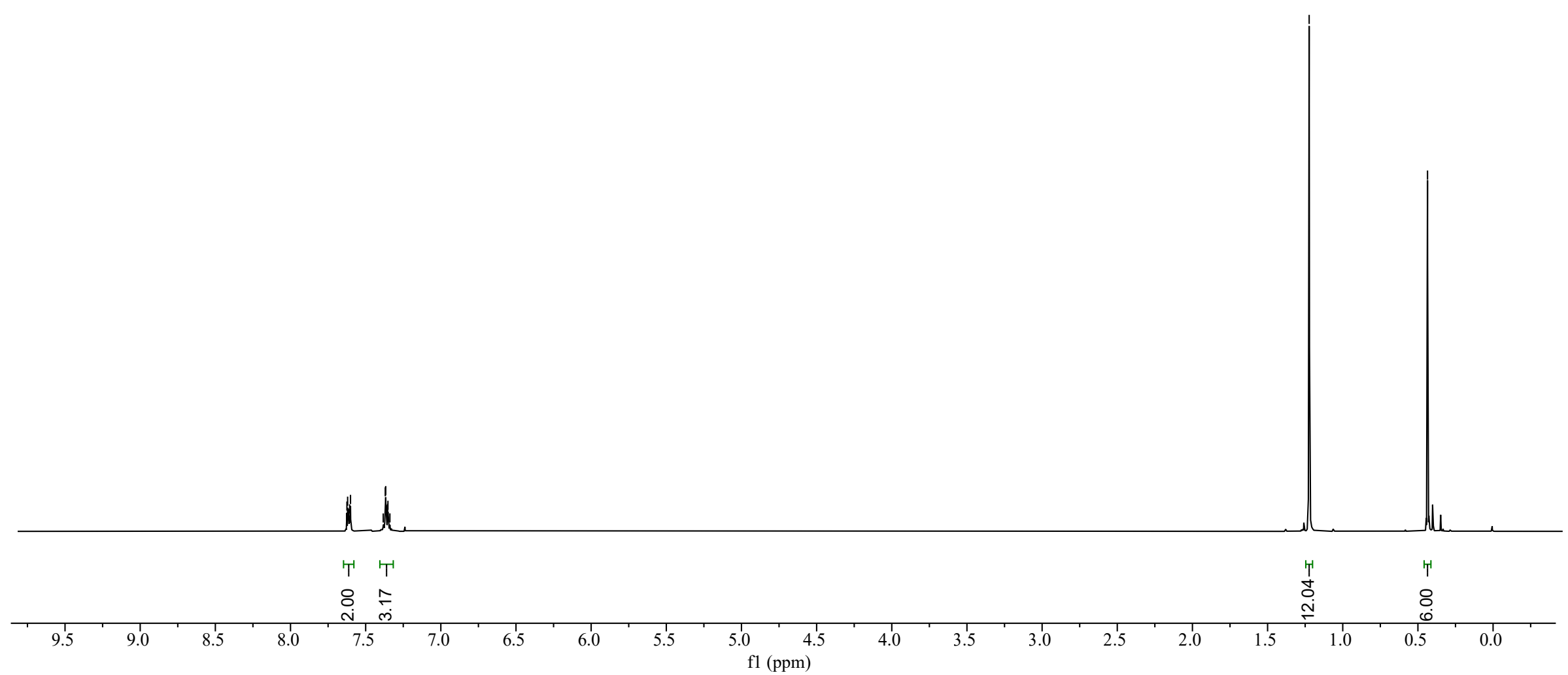




\section{Compound $4 a^{13} \mathrm{C}$ NMR}
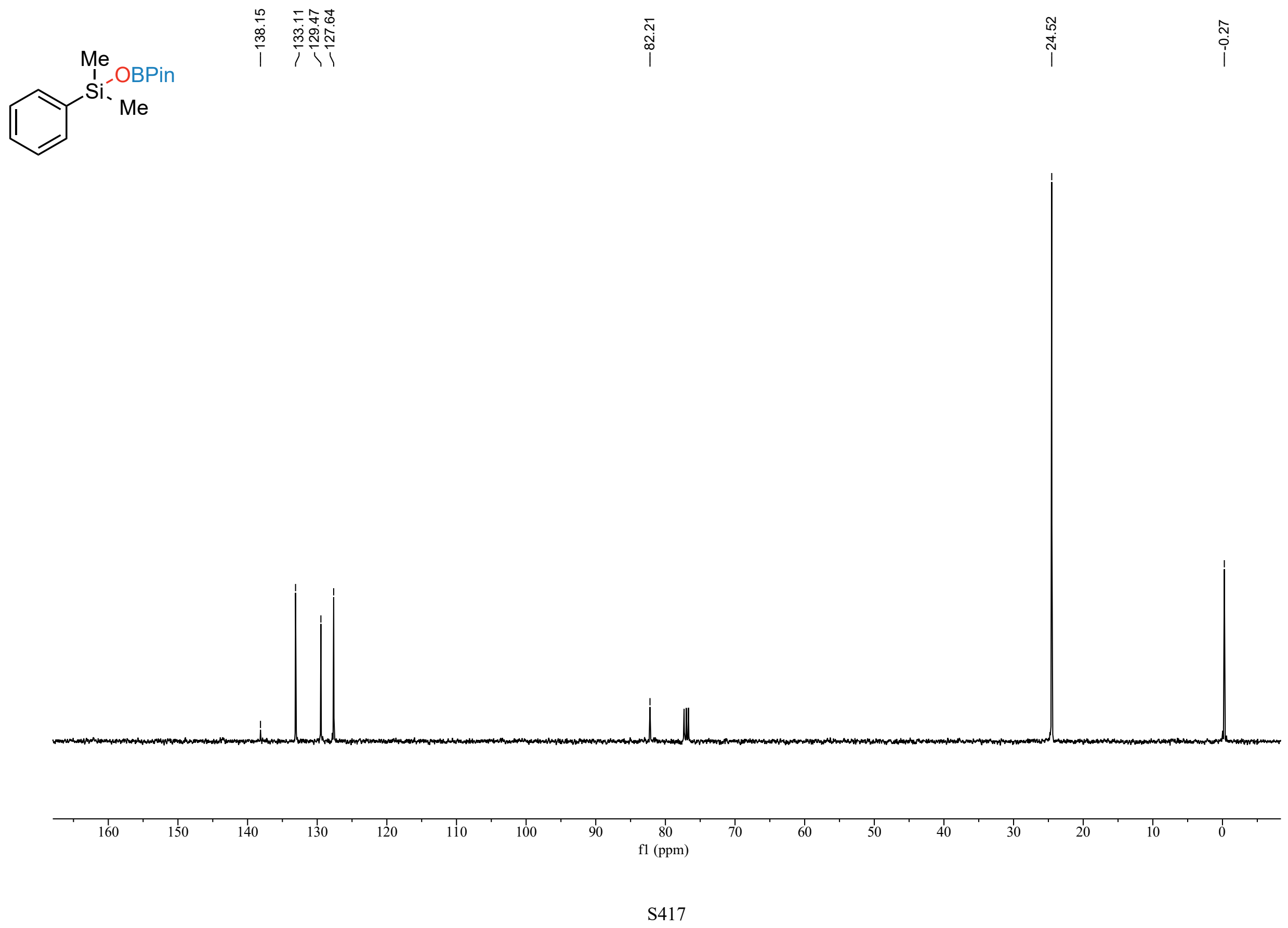


\section{Compound $\mathbf{4 b}{ }^{1} \mathrm{H}$ NMR}

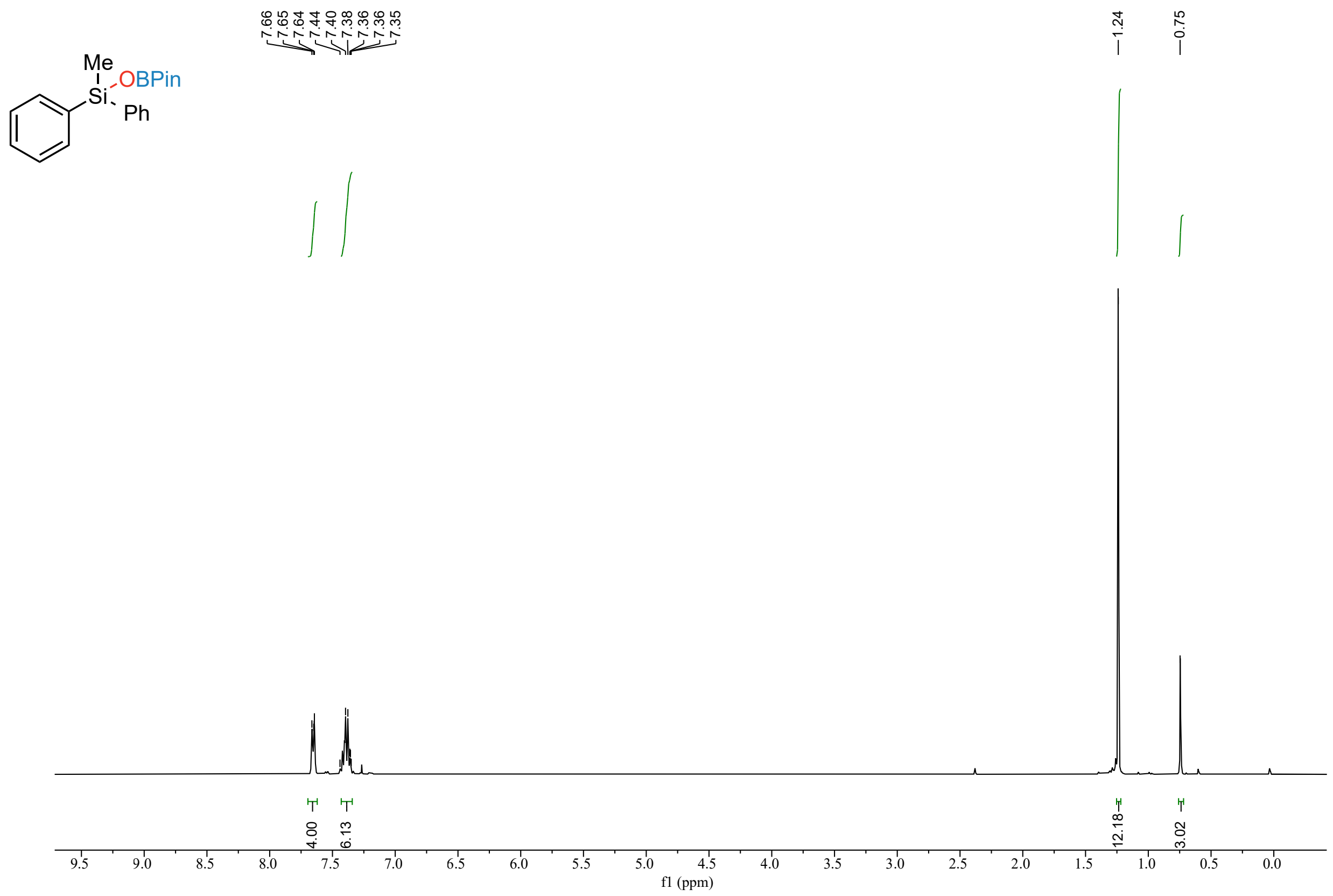


Compound $\mathbf{4 b}{ }^{13} \mathrm{C}$ NMR

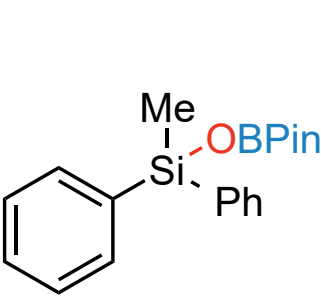

พิ่

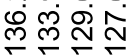

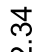

1111

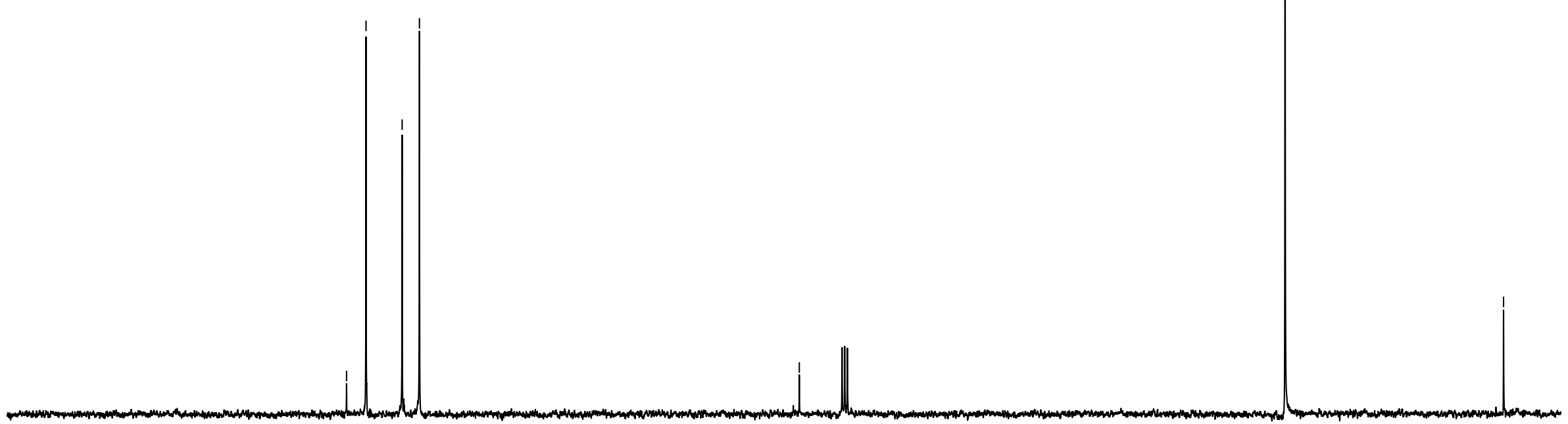




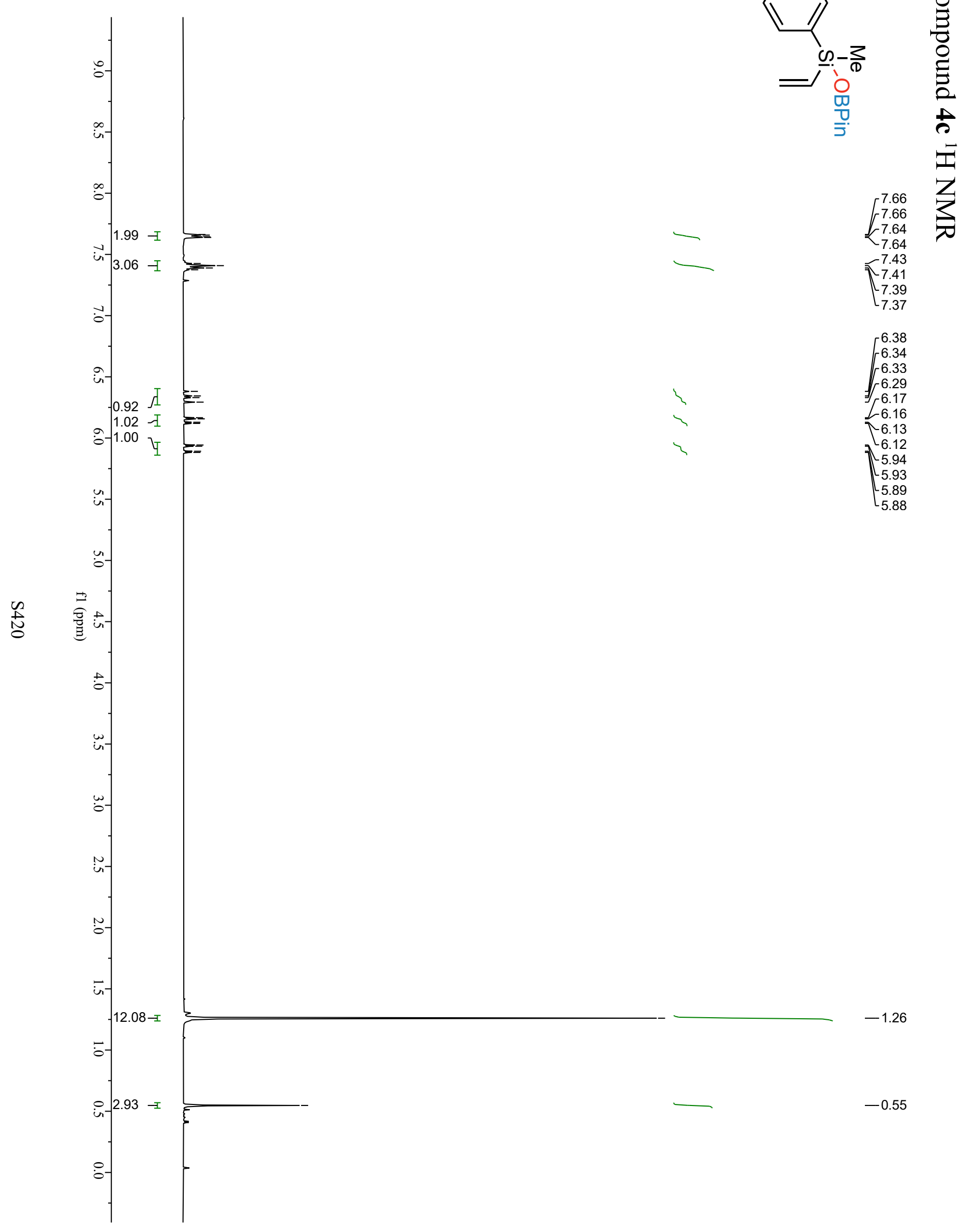


Compound $4 \mathbf{c}^{13} \mathrm{C}$ NMR

Siliobin

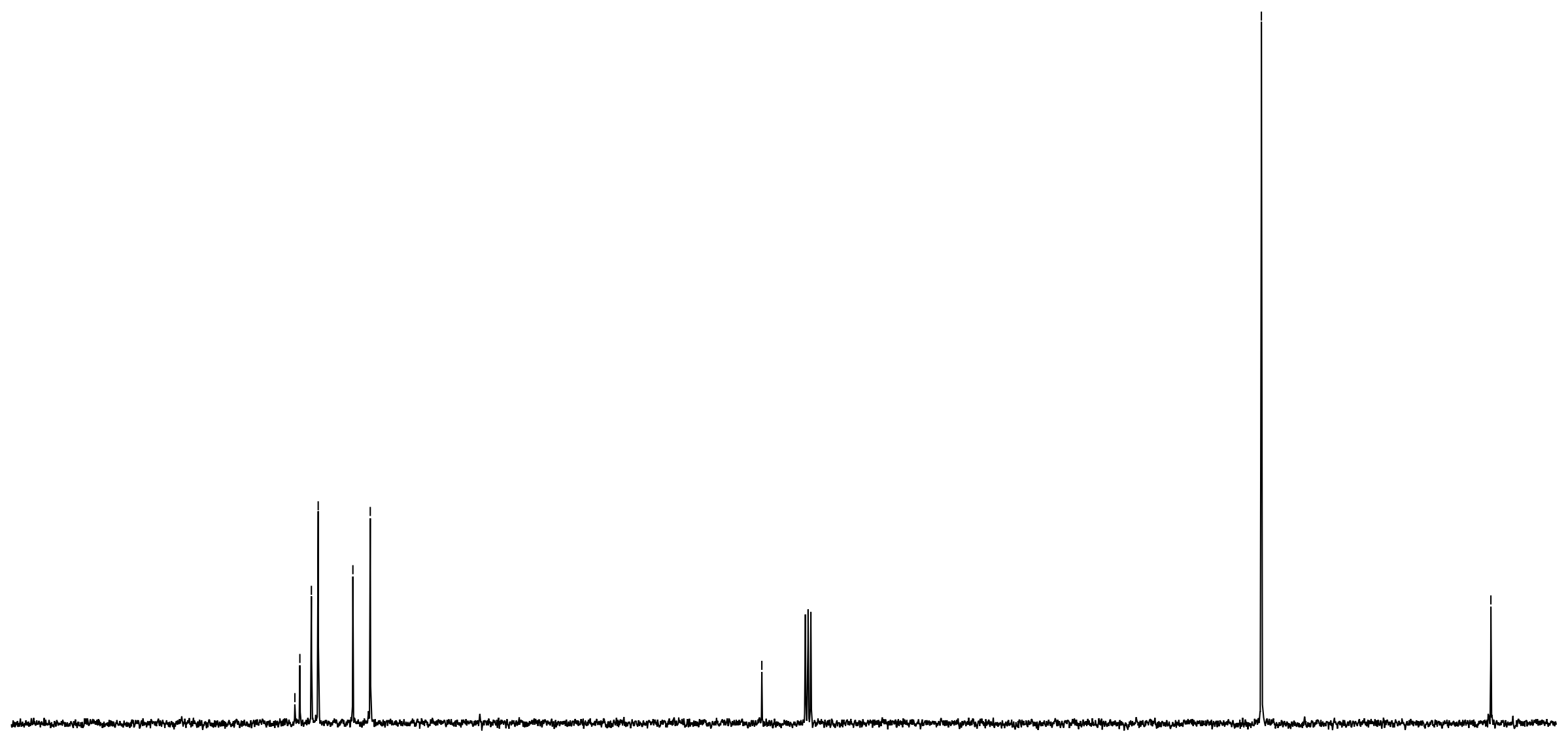


Compound 4d ${ }^{1} \mathrm{H}$ NMR

$\sum_{\mathrm{Me}}^{{ }_{\mathrm{Se}}^{\mathrm{Si}-\mathrm{OBPin}}}$

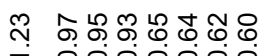

Y
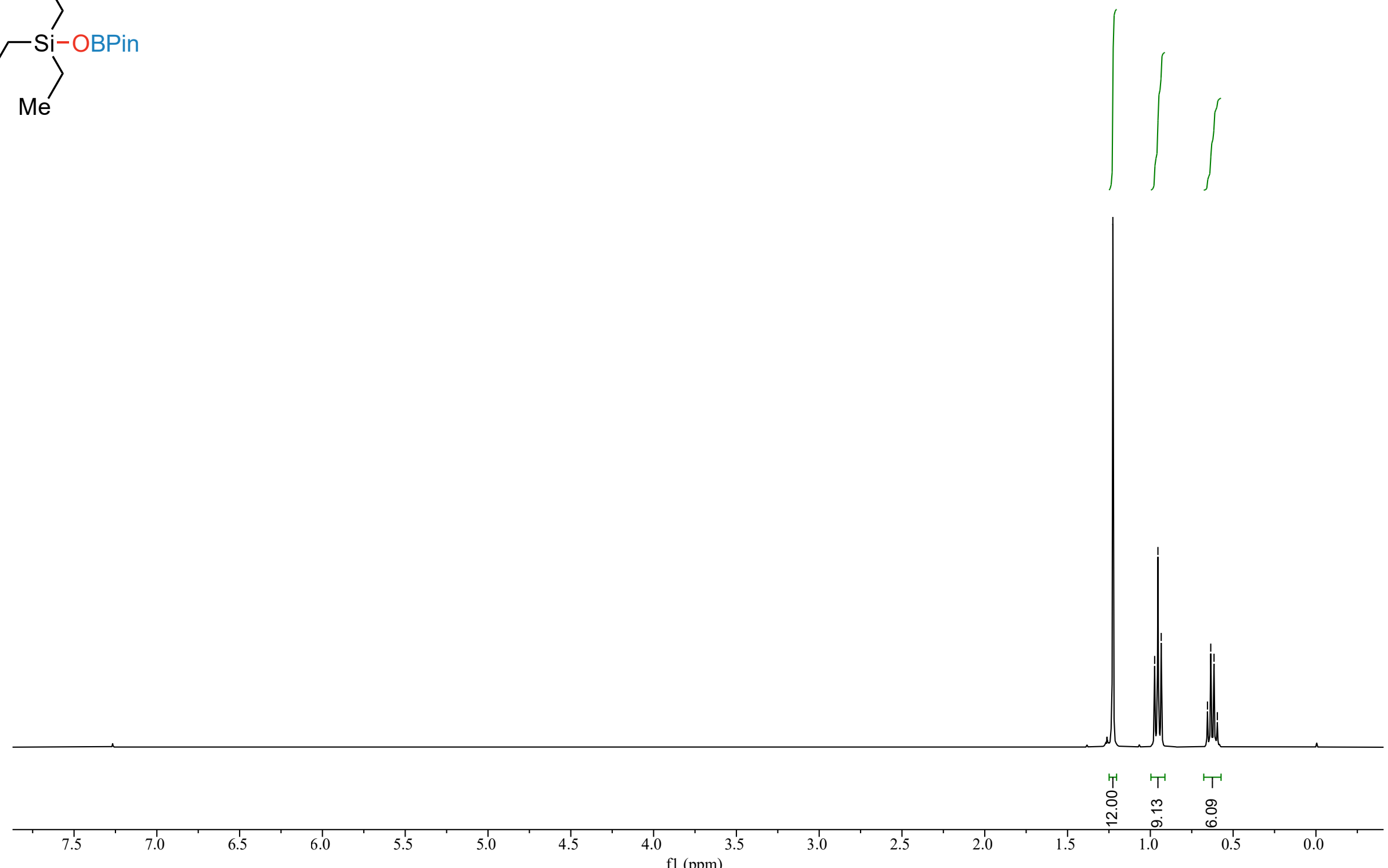
Compound 4d ${ }^{13} \mathrm{C}$ NMR

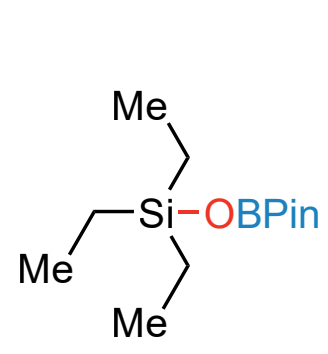




\section{Compound 4e ${ }^{1} \mathrm{H}$ NMR}
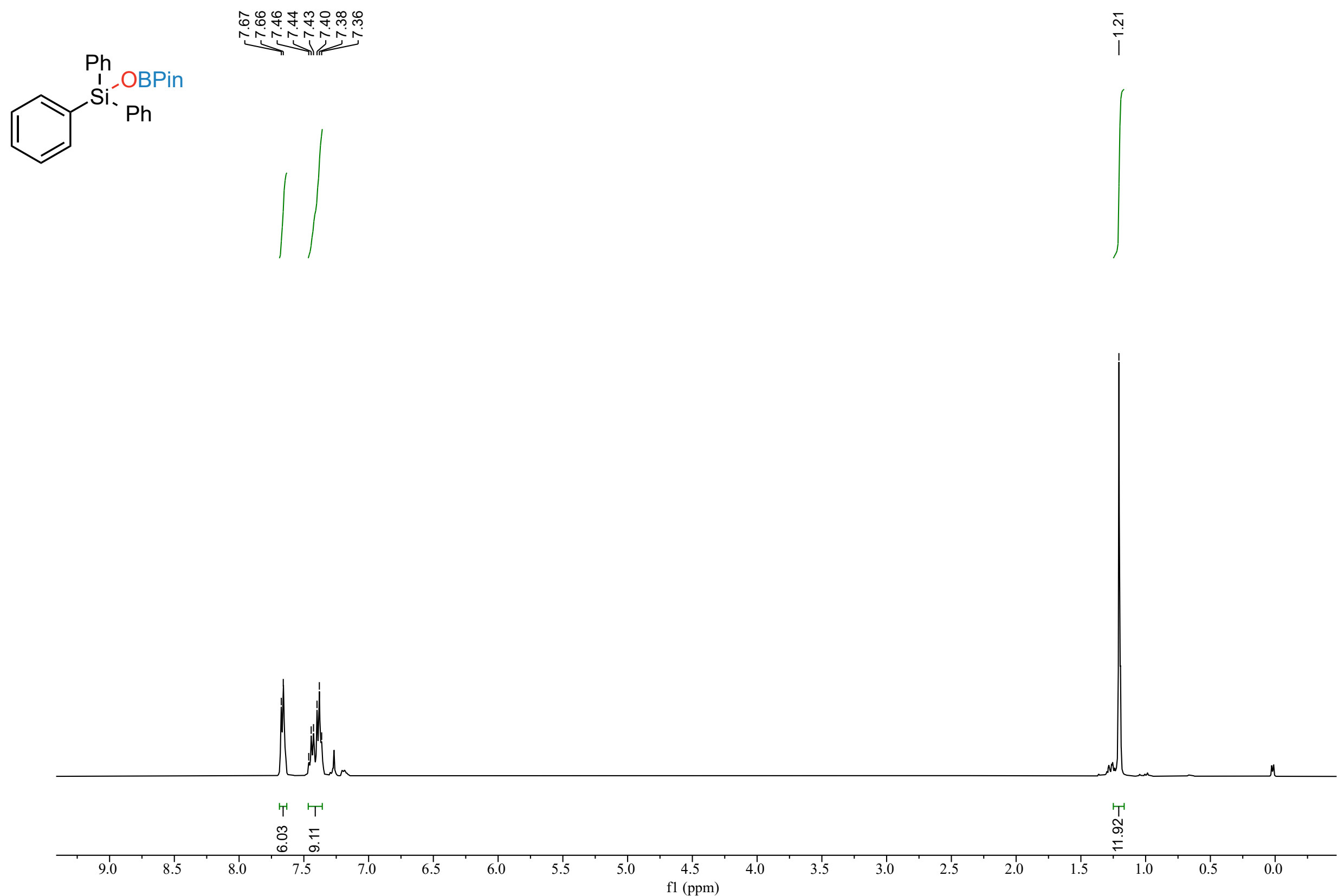
Compound $4 \mathrm{e}^{13} \mathrm{C}$ NMR
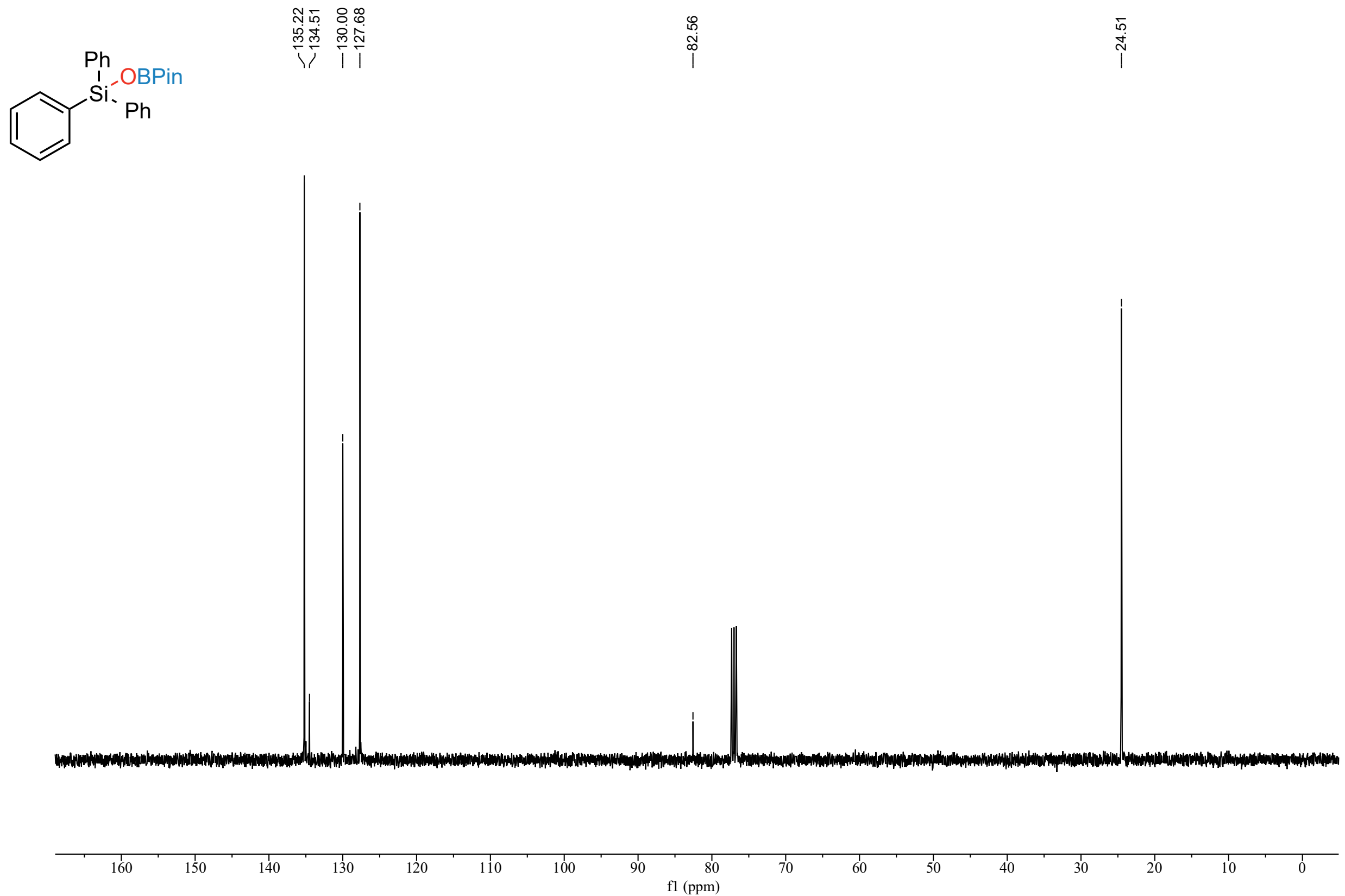


\section{Compound $\mathbf{4 f}{ }^{1} \mathrm{H}$ NMR}

伵

$\stackrel{m}{i} \quad \stackrel{?}{i}$
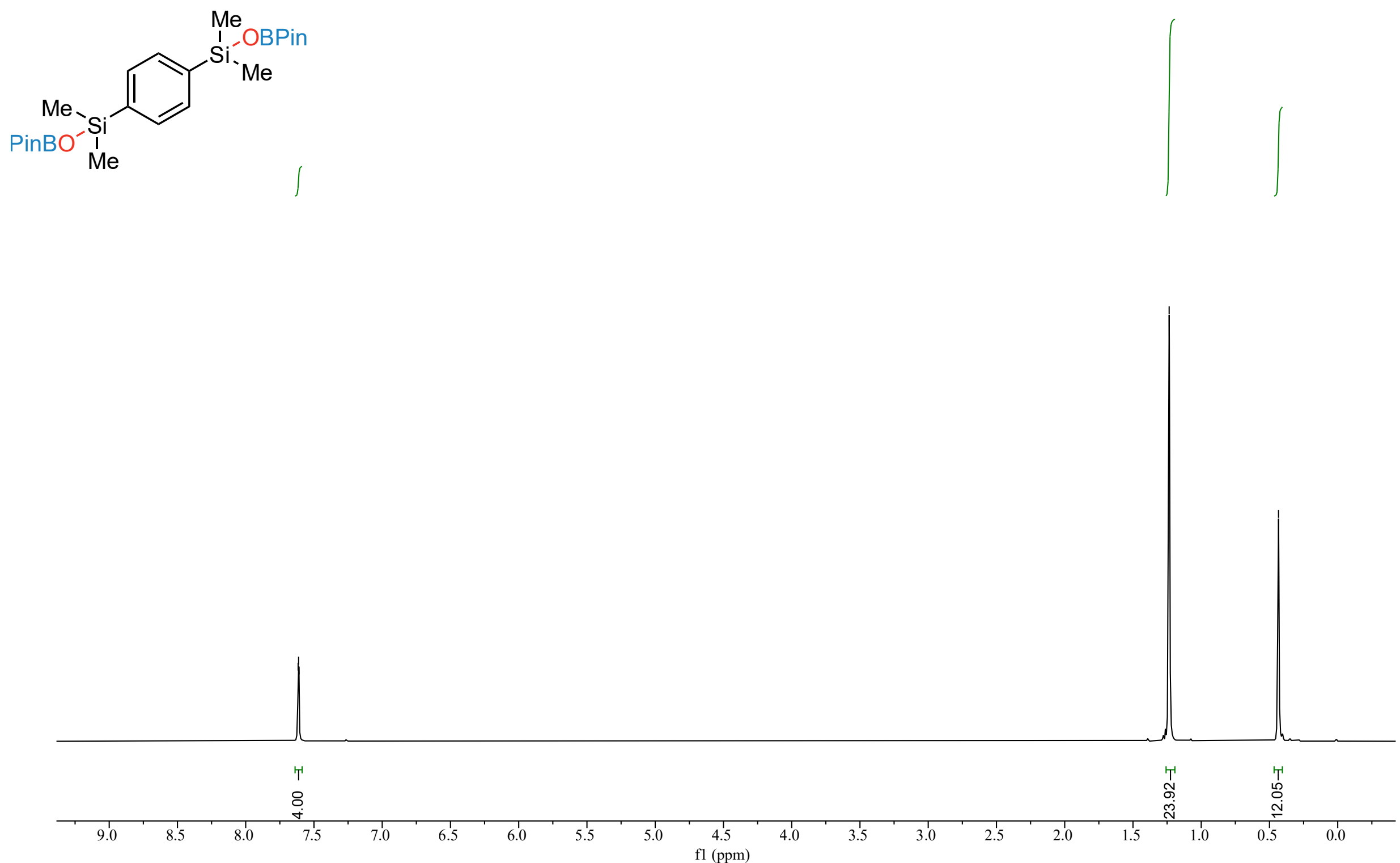


\section{Compound $4 f^{13} \mathrm{C}$ NMR}
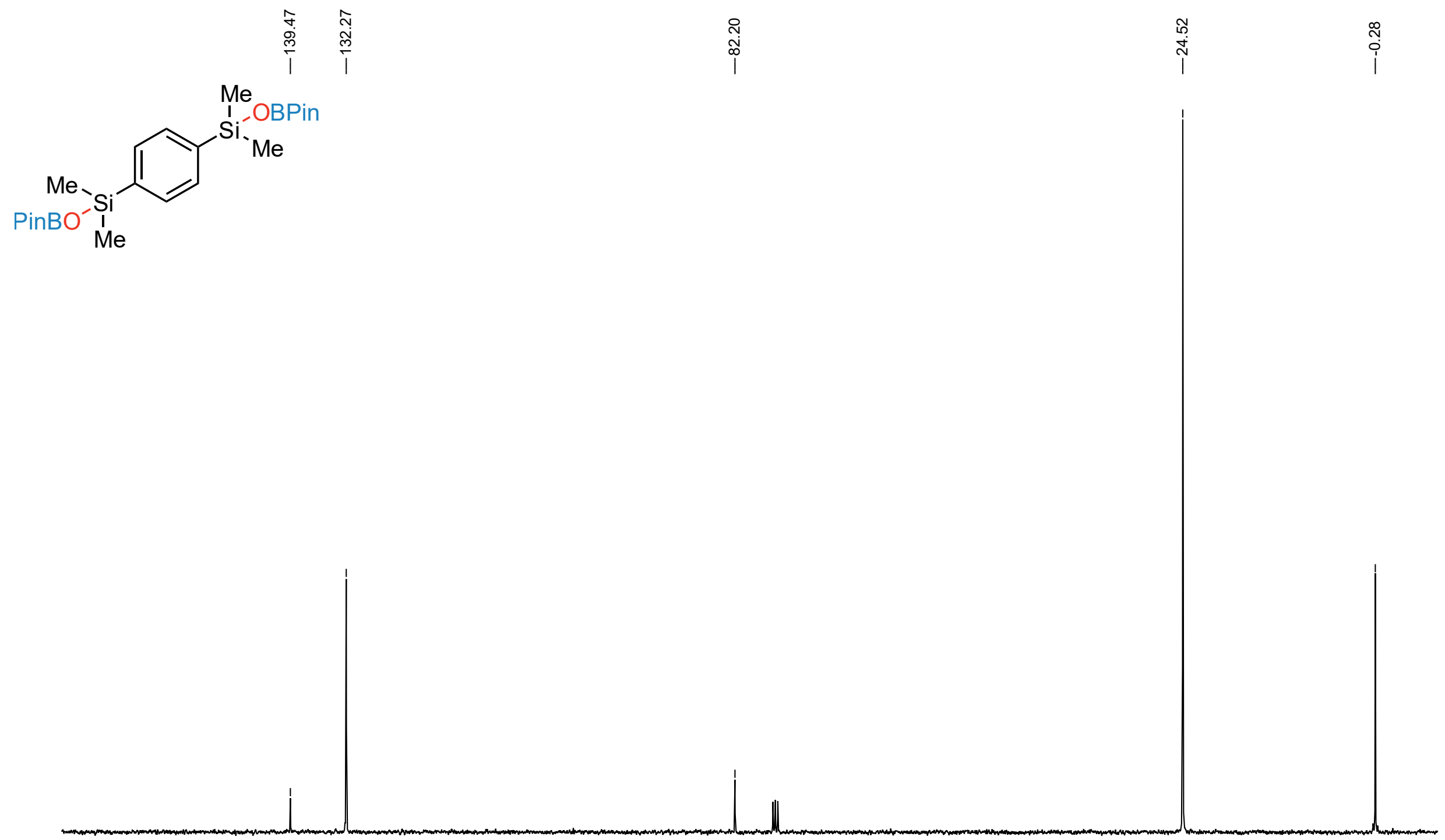
Compound 4g ${ }^{1} \mathrm{H}$ NMR

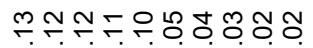

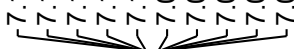

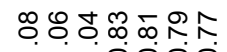

$\overbrace{O^{\prime}}^{\mathrm{B}-\mathrm{O}-\mathrm{SiEt}_{3}}$
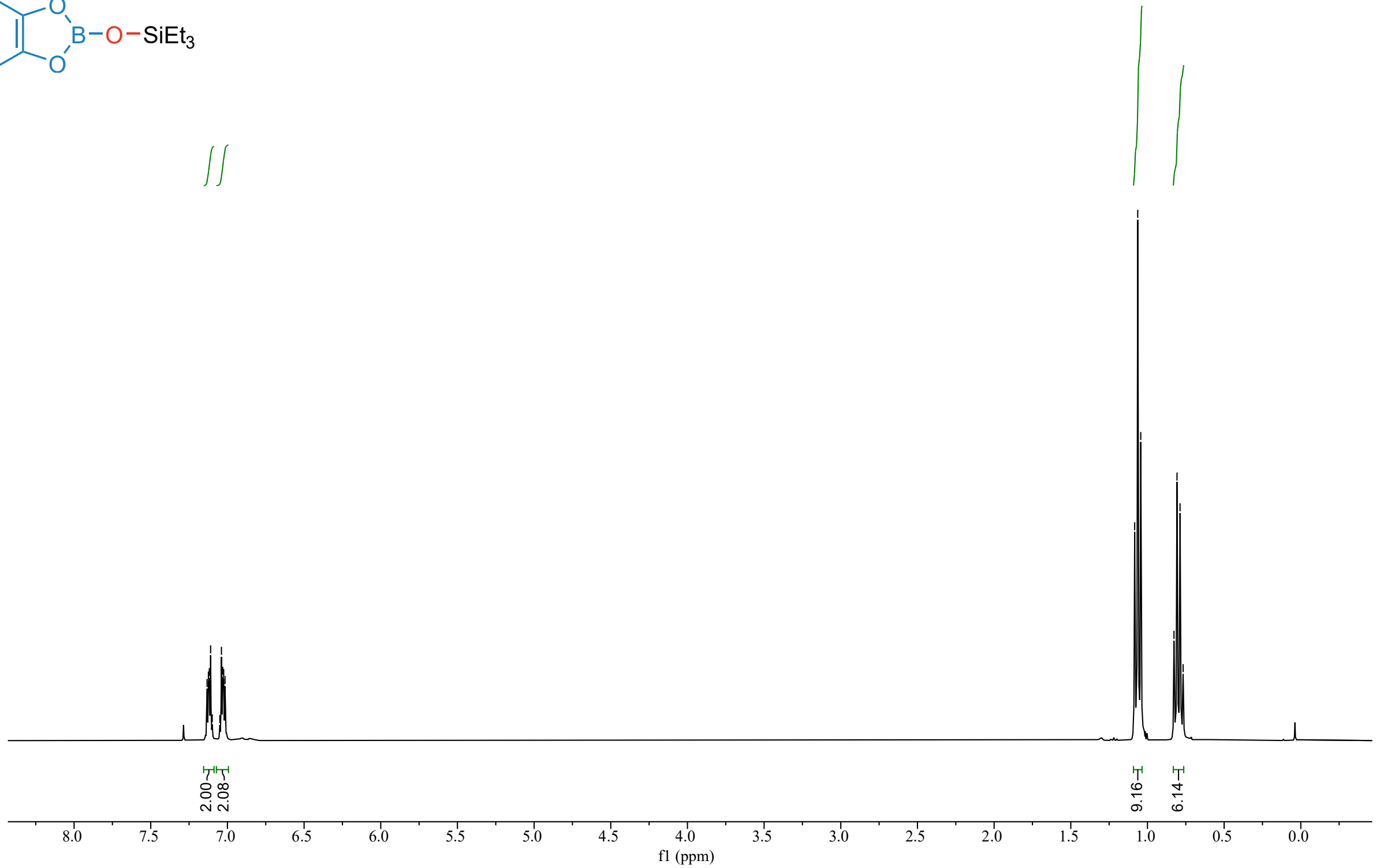
Compound $4 \mathrm{~g}{ }^{13} \mathrm{C}$ NMR

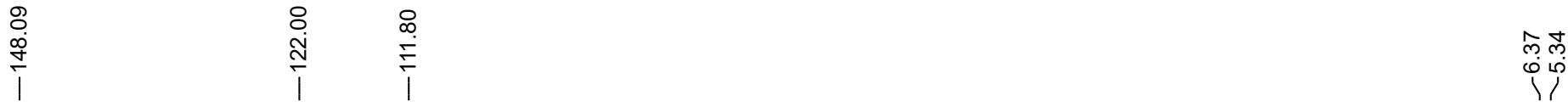

$\overbrace{\mathrm{O}^{\prime}}^{\mathrm{B}-\mathrm{O}-\mathrm{SiEt}_{3}}$

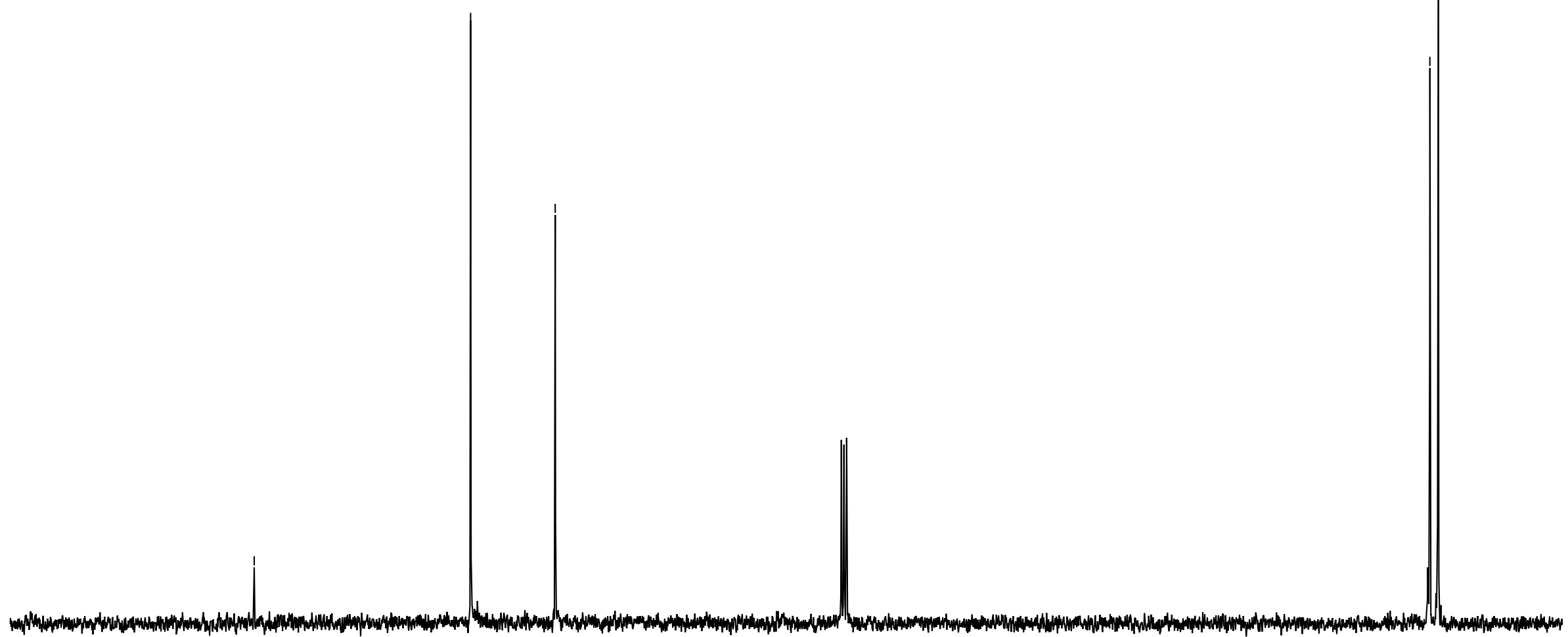




\section{Compound 4h ${ }^{1} \mathrm{H}$ NMR}

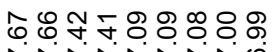

ivivitiog

$\mathrm{O}_{\mathrm{O}^{\prime} \mathrm{B}-\mathrm{O}-\mathrm{SiMe}_{2} \mathrm{Ph}}^{\mathrm{O}}$
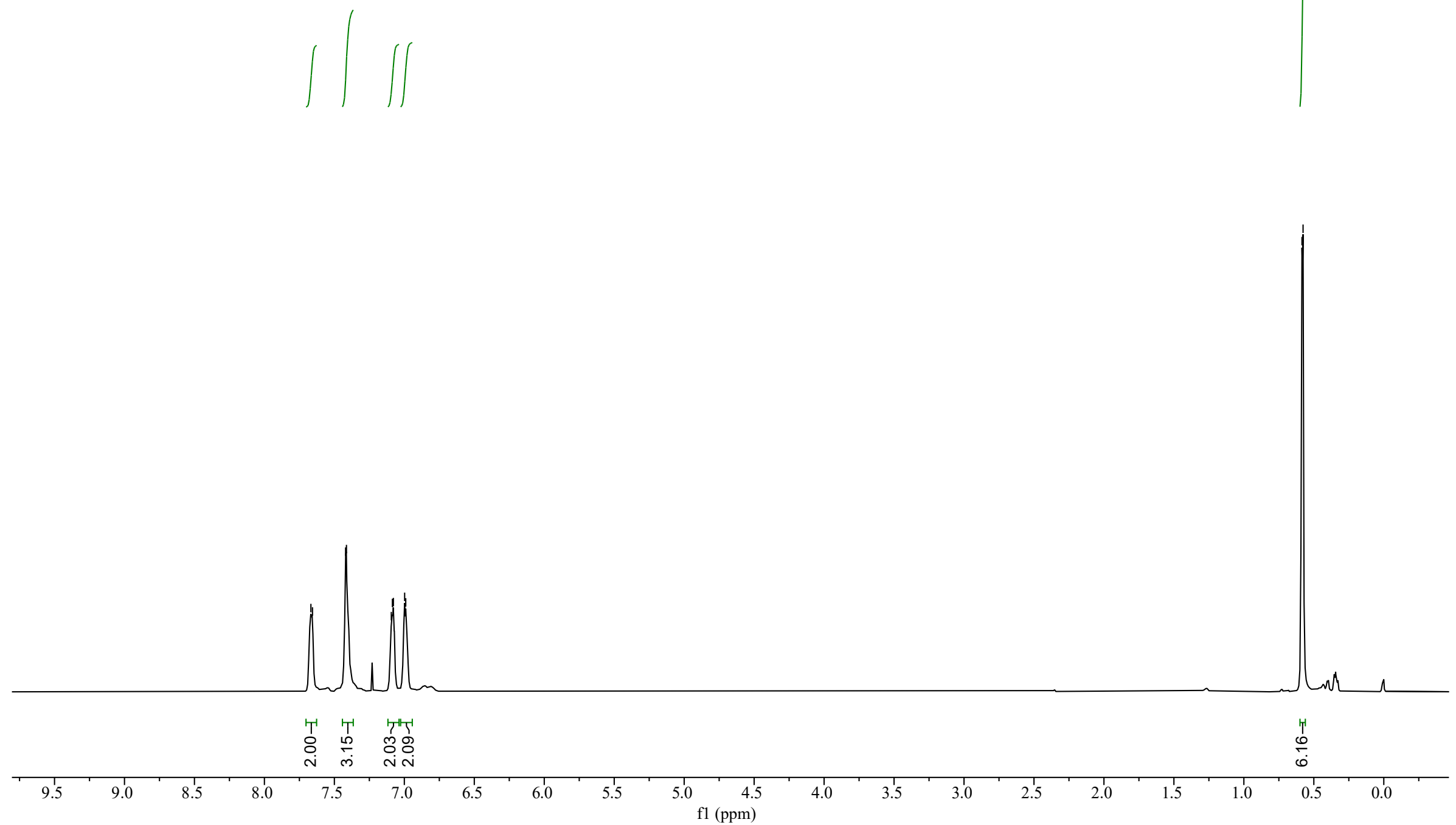
Compound $4 \mathbf{h}{ }^{13} \mathrm{C}$ NMR

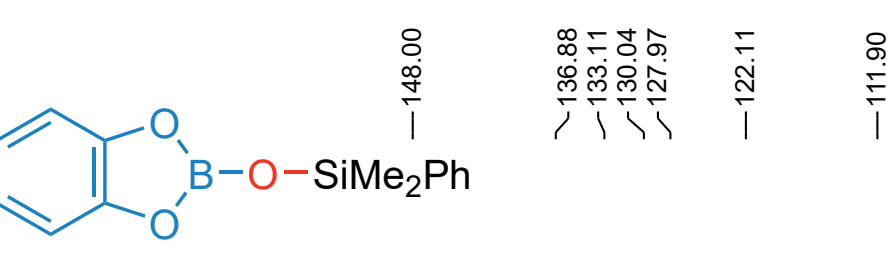

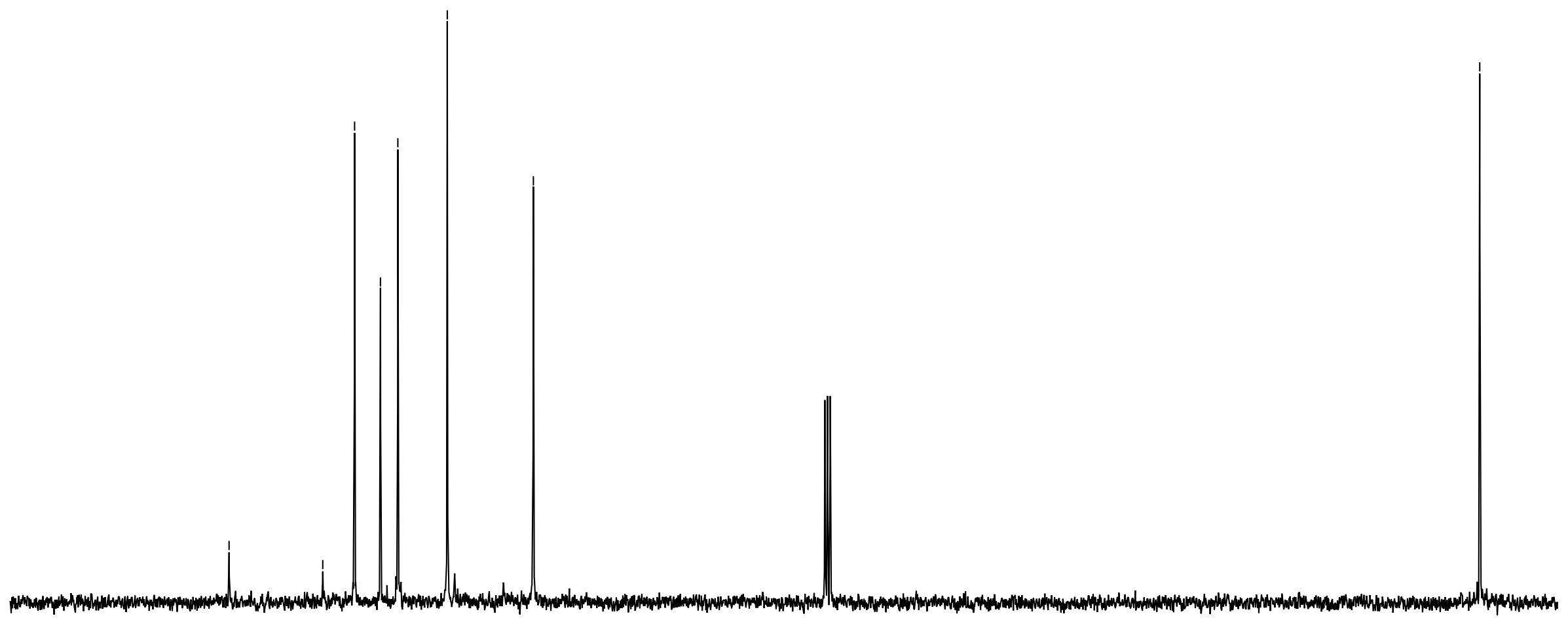




\section{Compound $4 \mathbf{i}{ }^{1} \mathrm{H}$ NMR}

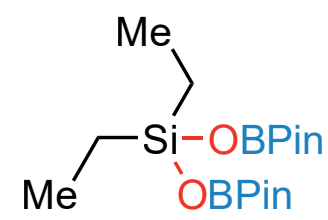

ำ

ivi

Me OBPin

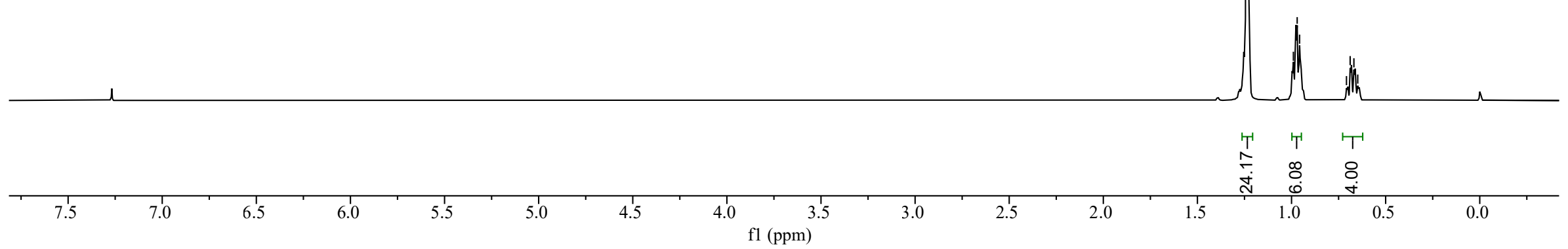


Compound $4 \mathbf{i}^{13} \mathrm{C}$ NMR
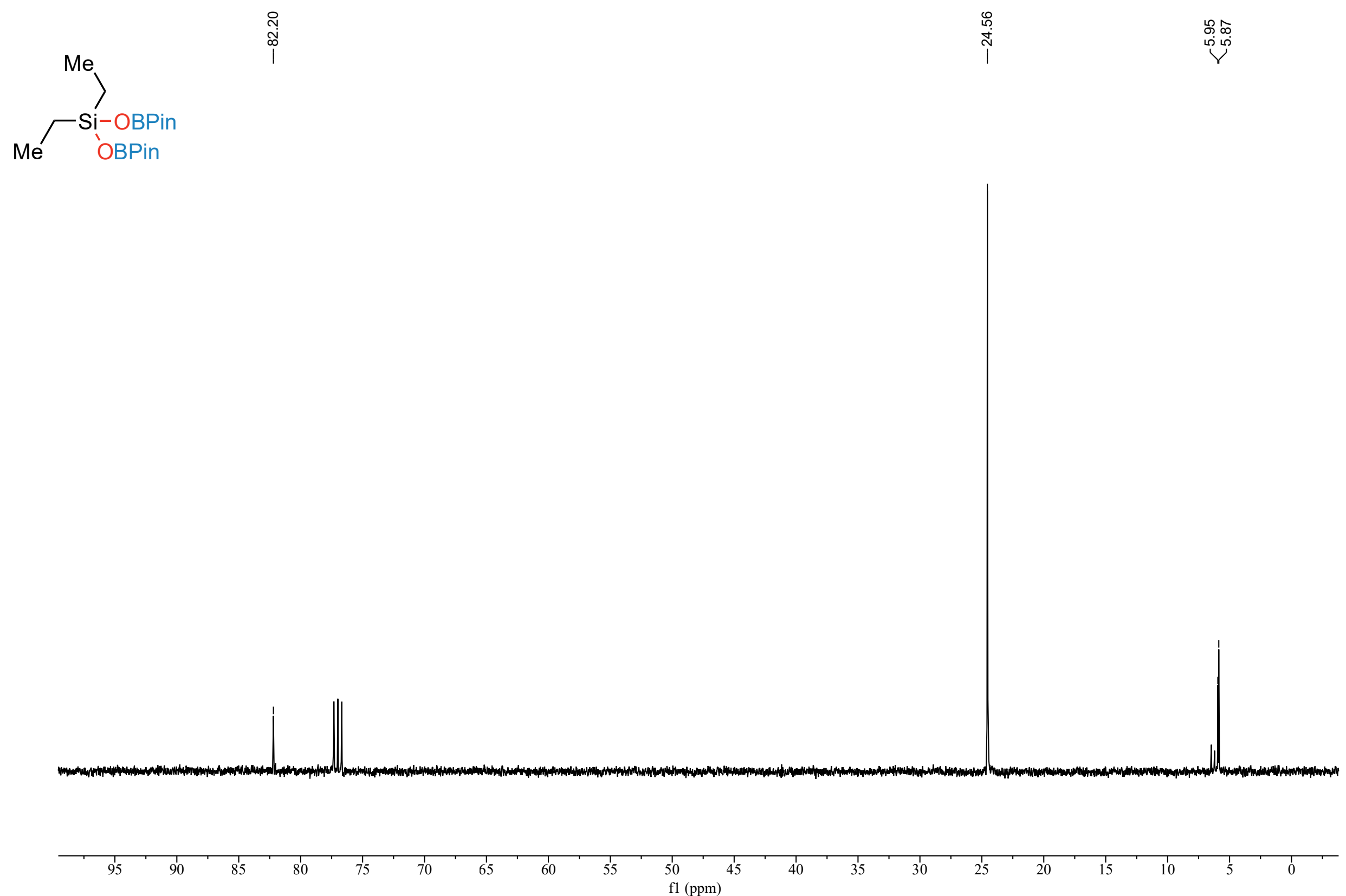


\section{Compound $\mathbf{4 j}{ }^{1} \mathrm{H}$ NMR}

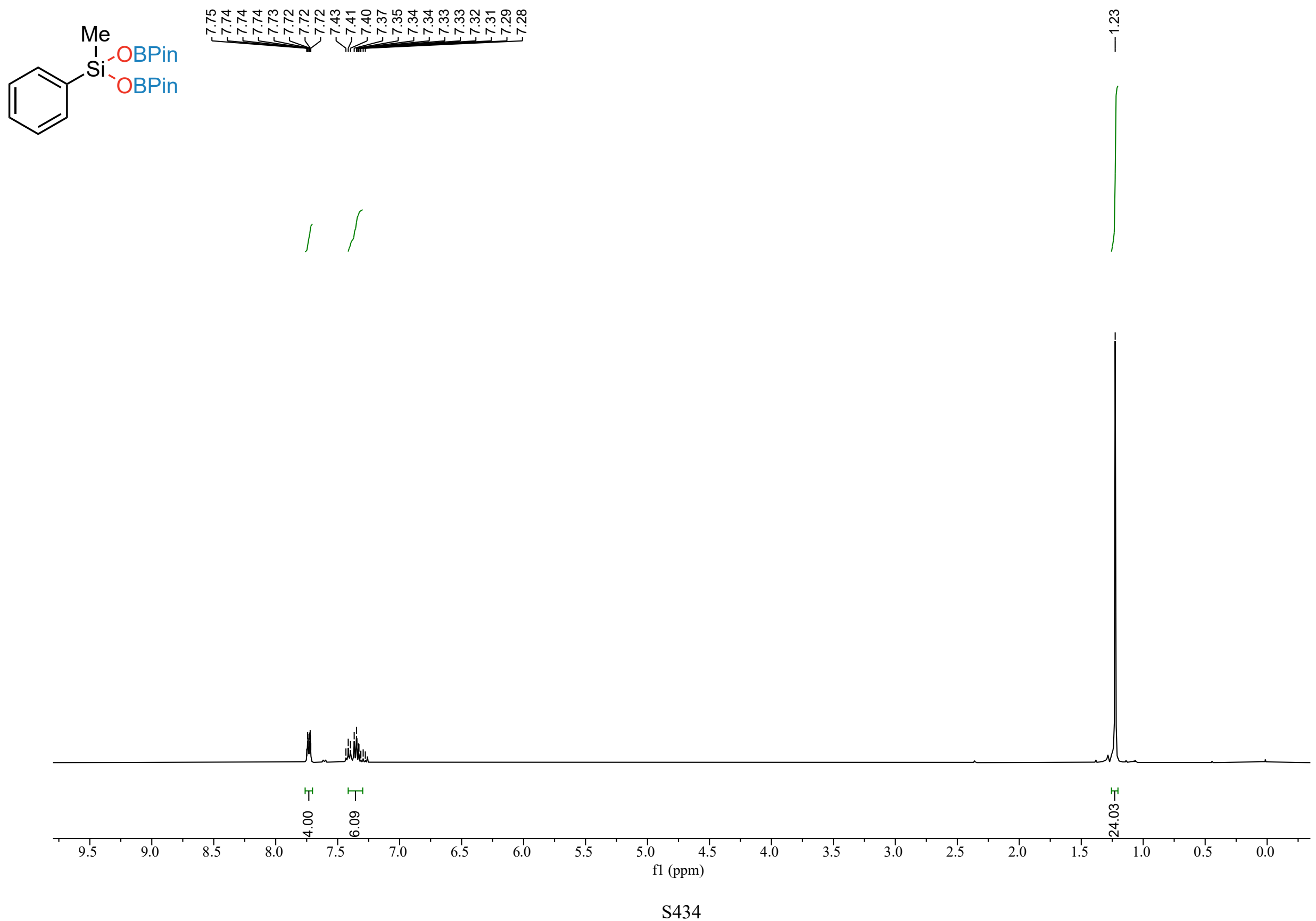




\section{Compound $\mathbf{4 j}{ }^{13} \mathrm{C}$ NMR}

Si-OBPin

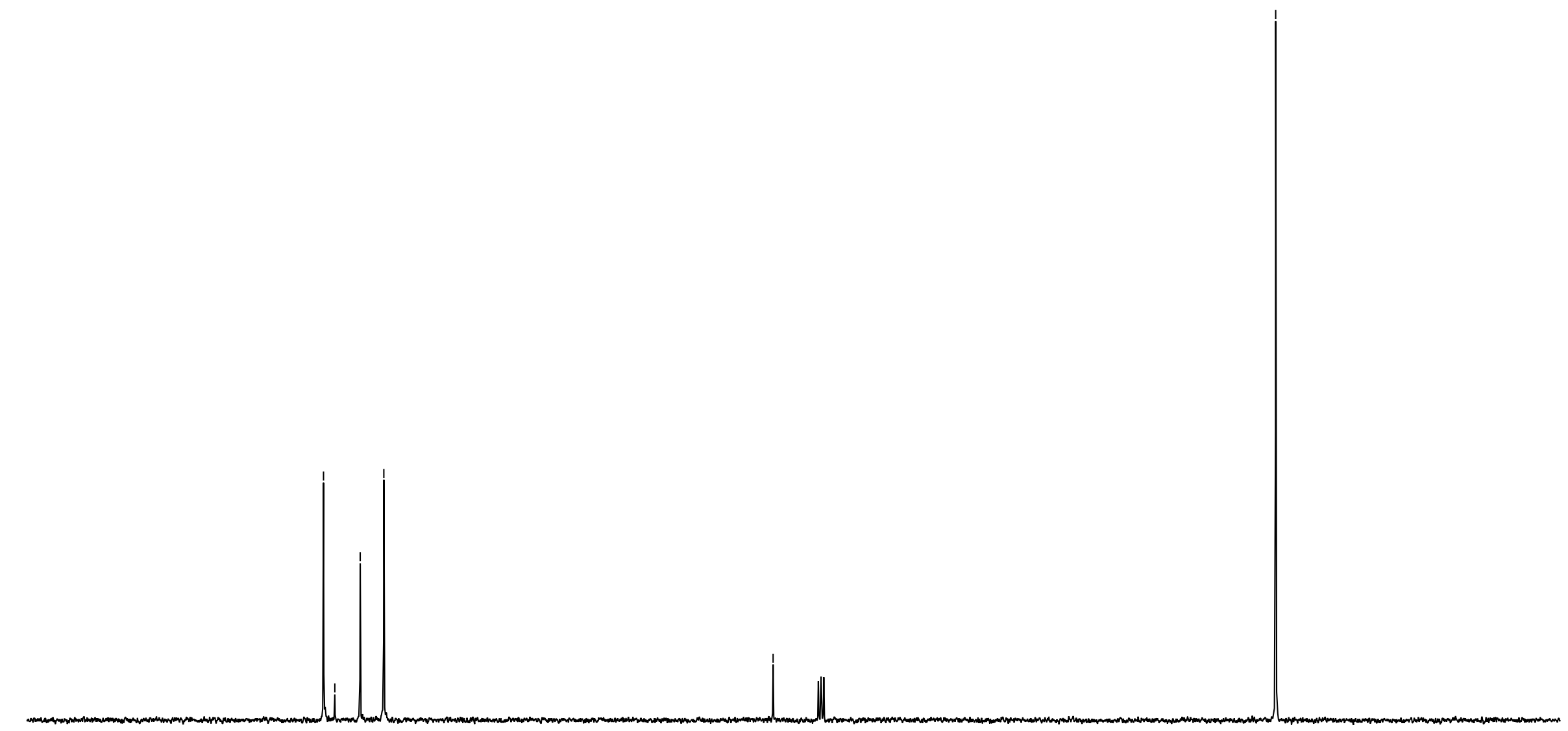




\section{Compound 6a ${ }^{1} \mathrm{H}$ NMR}

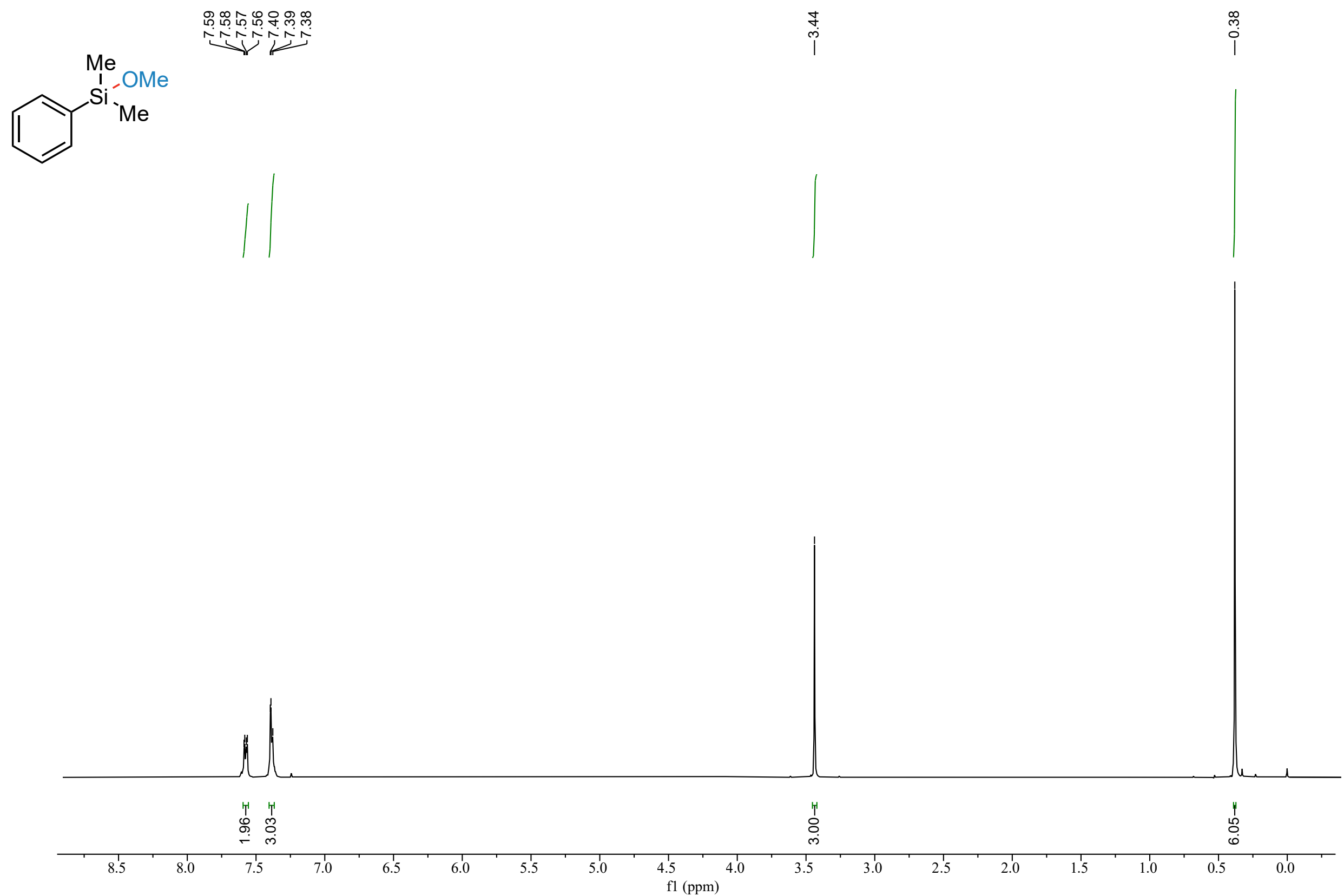


Compound 6a ${ }^{13} \mathrm{C}$ NMR

${ }_{\mathrm{Me}}^{\mathrm{Me}}$

$$
10
$$

잉ำ

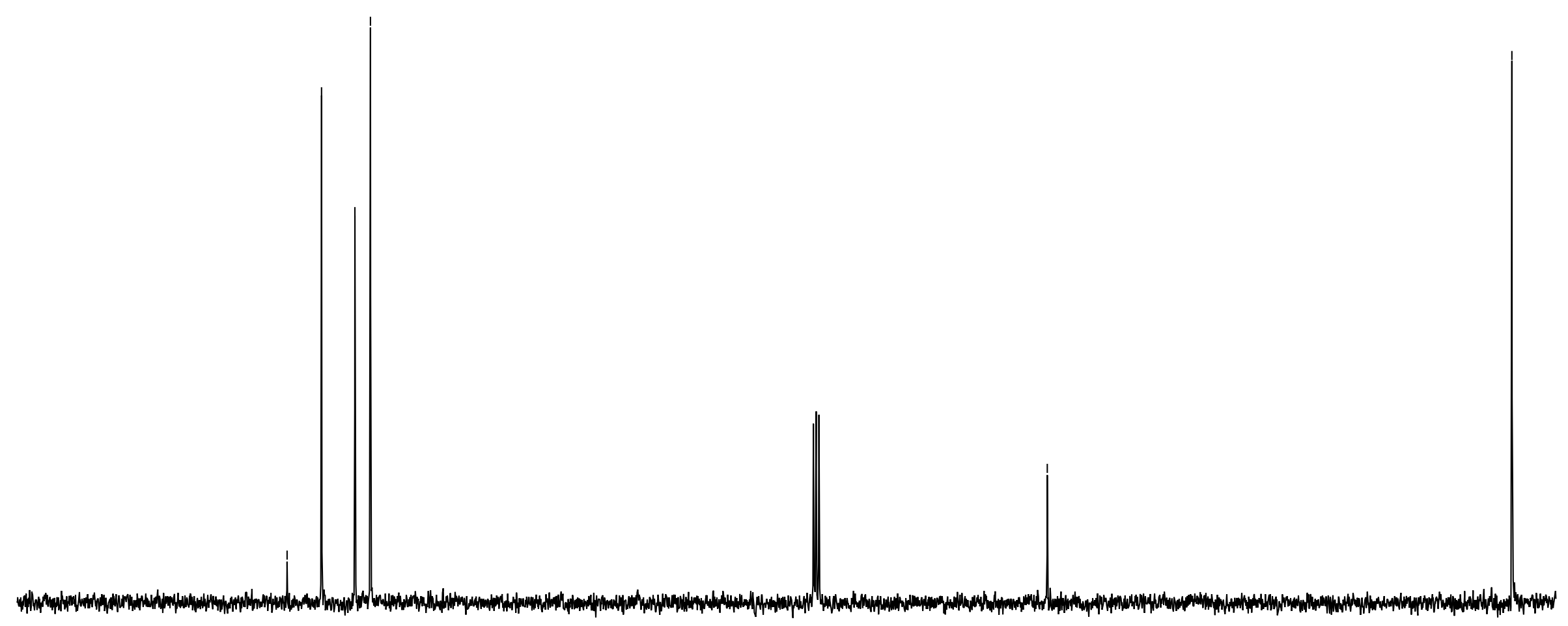




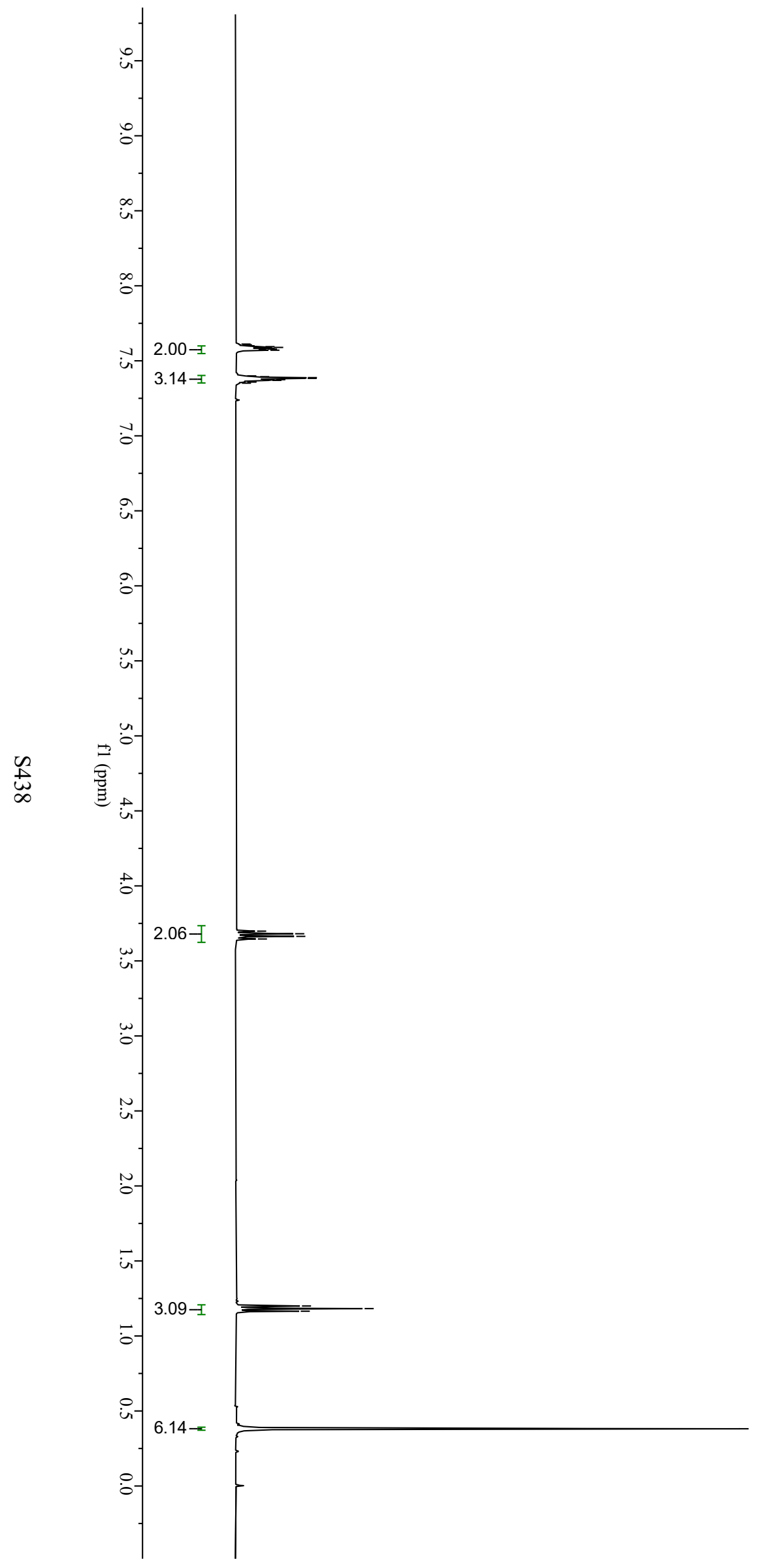

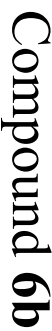

7.63

-7.62
-7.62

7.62
-7.61

7.60

7.60

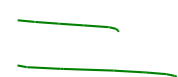

7.59
-7.59

7.41
7.41

7.40

7.40

$-7.39$

$-7.39$

-7.38
7.37

$-7.37$

3.71

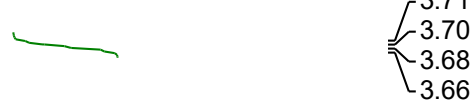

-3.68
3.66

1.20
-1.18

$\Upsilon_{1.16}$

$-0.38$ 
Compound $6 b^{13} \mathrm{C}$ NMR
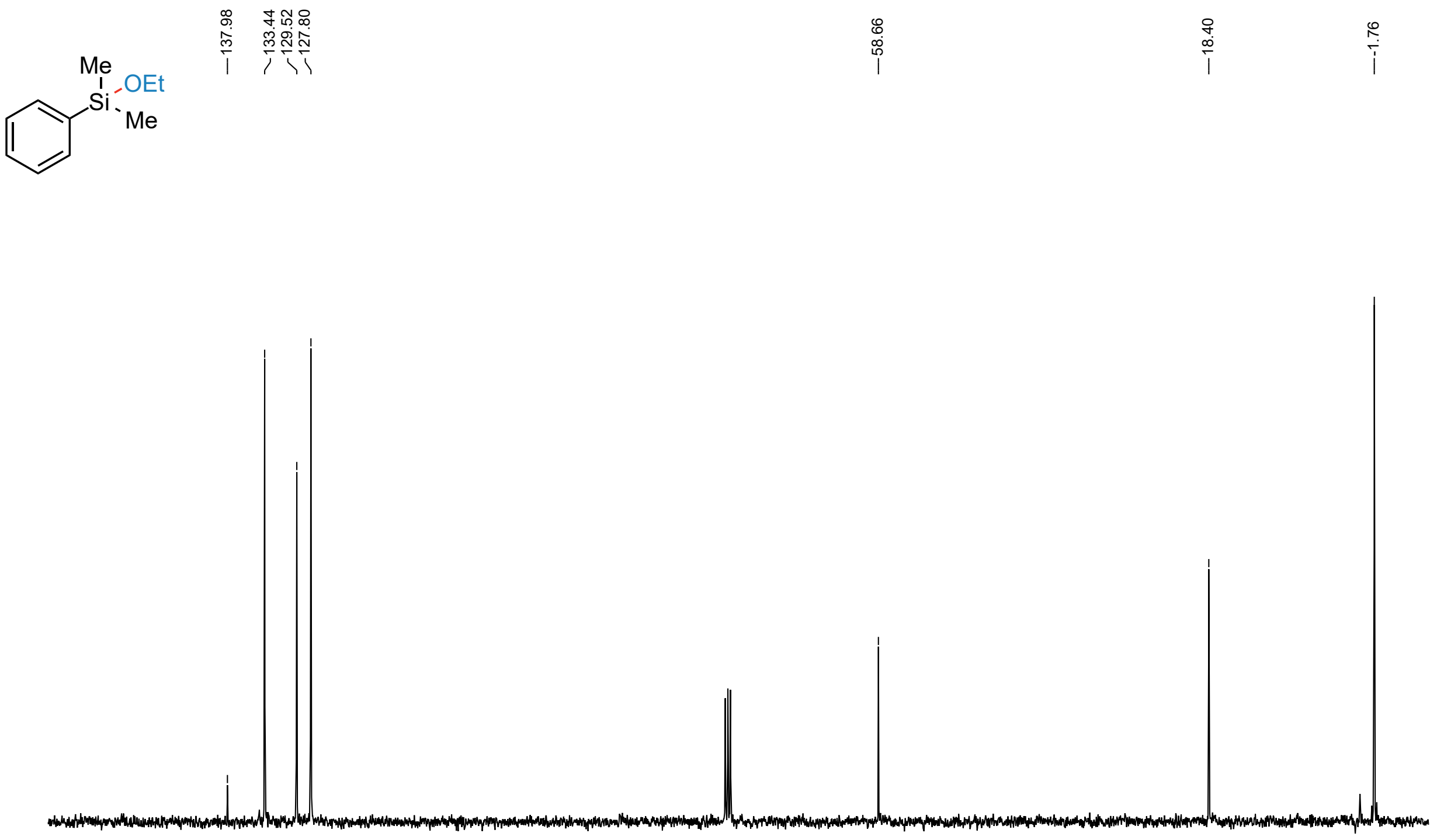


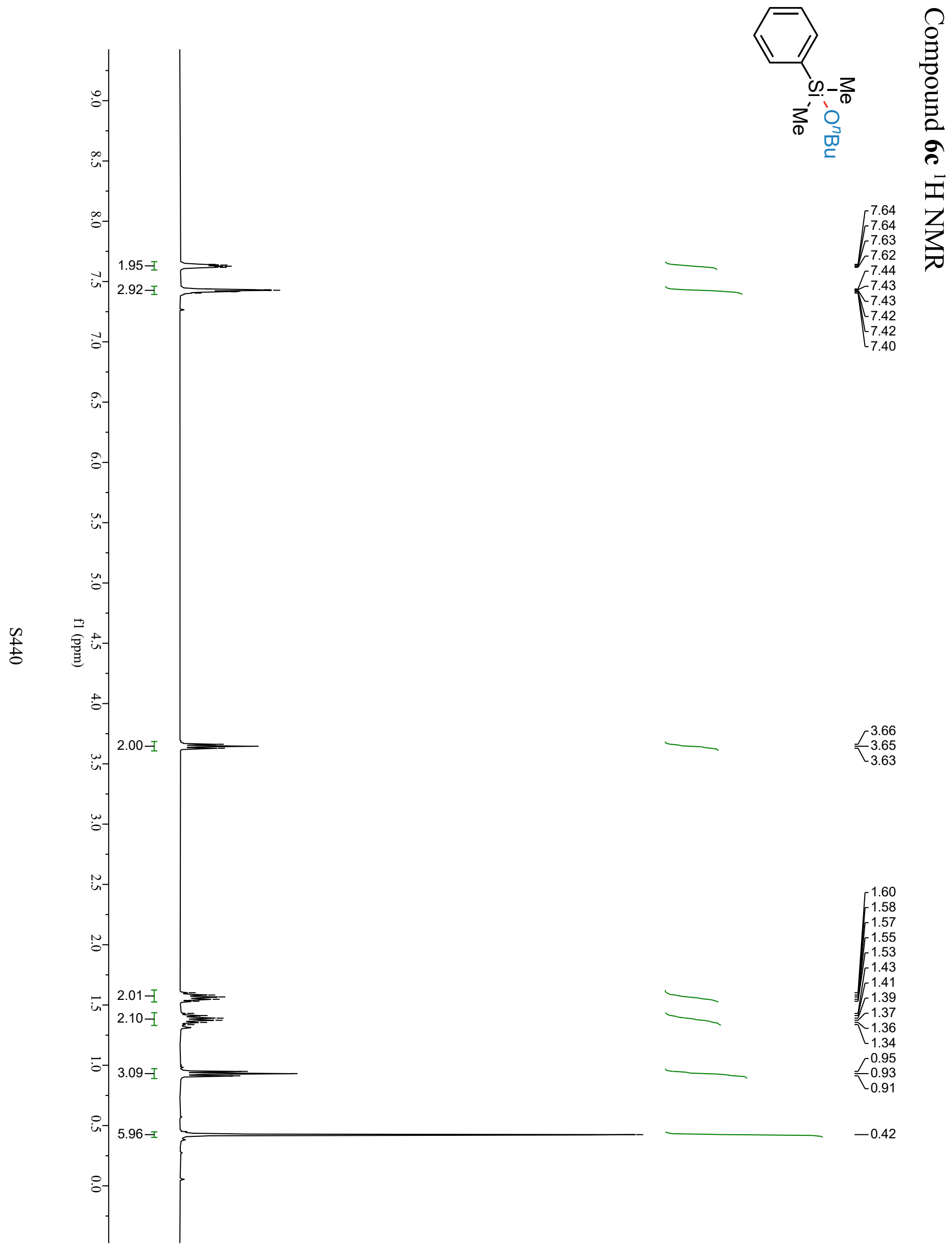


Compound $6 \mathbf{c}^{13} \mathrm{C}$ NMR

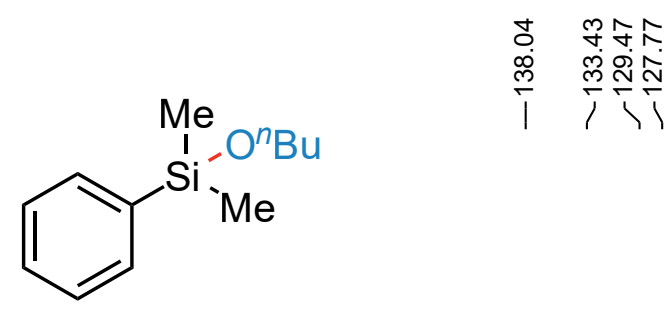

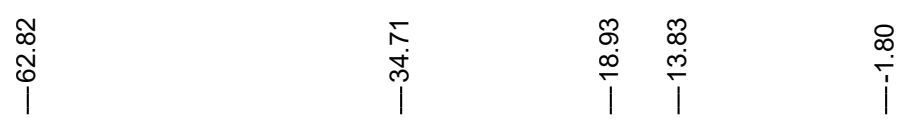

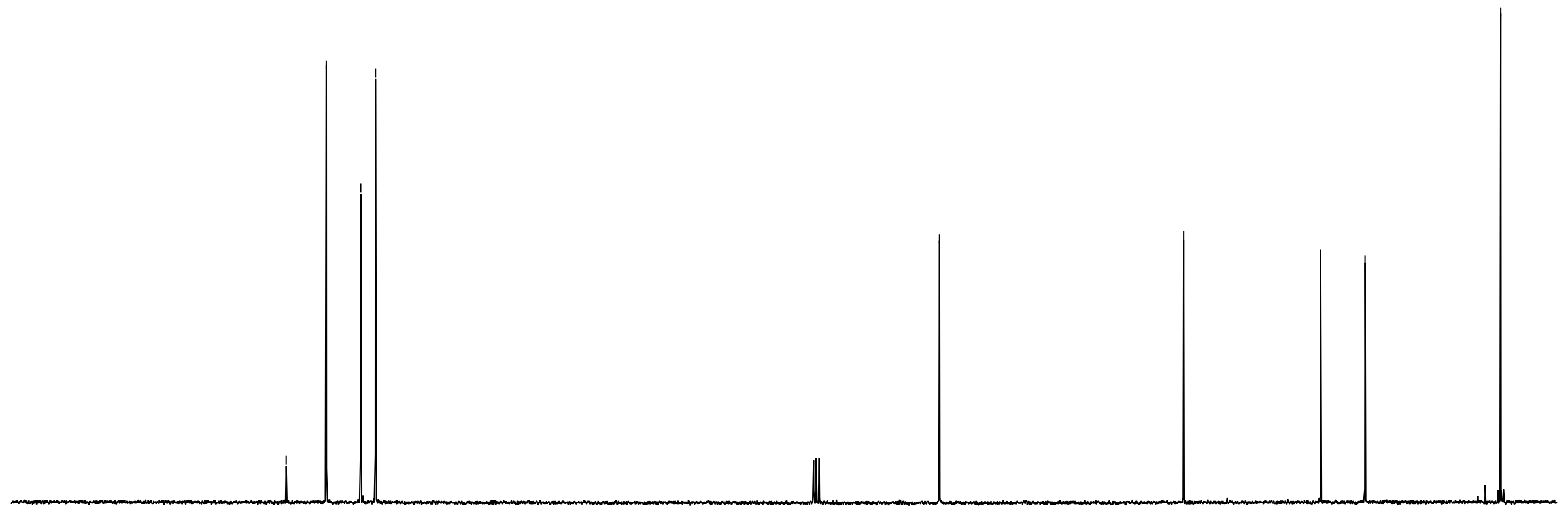



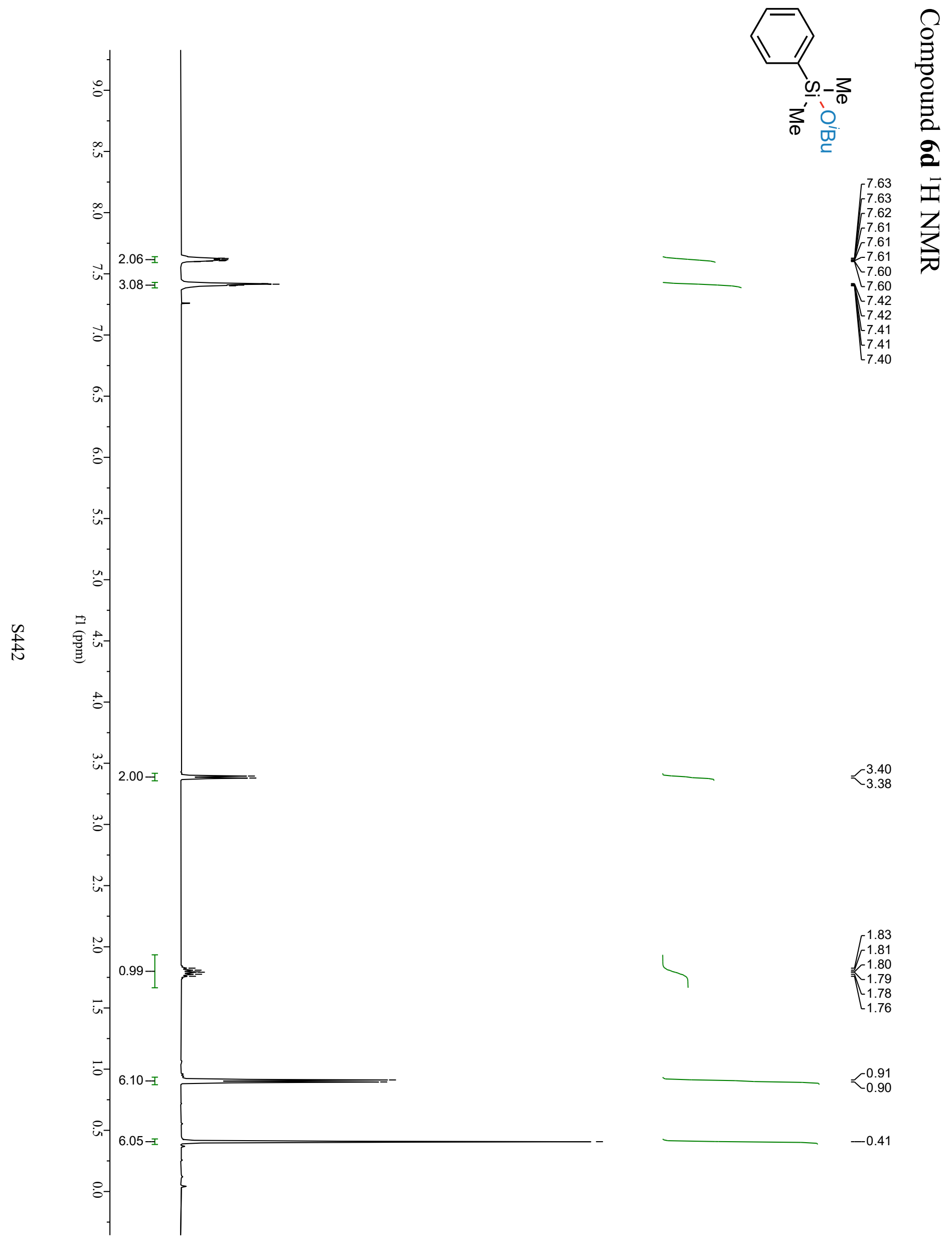

$\left[\begin{array}{r}1.83 \\ -1.81 \\ 1.80 \\ 1.79 \\ 1.78 \\ 1.76\end{array}\right.$

$\Upsilon_{0.90}^{0.91}$

$-0.41$ 


\section{Compound $\mathbf{6 d}{ }^{13} \mathrm{C}$ NMR}
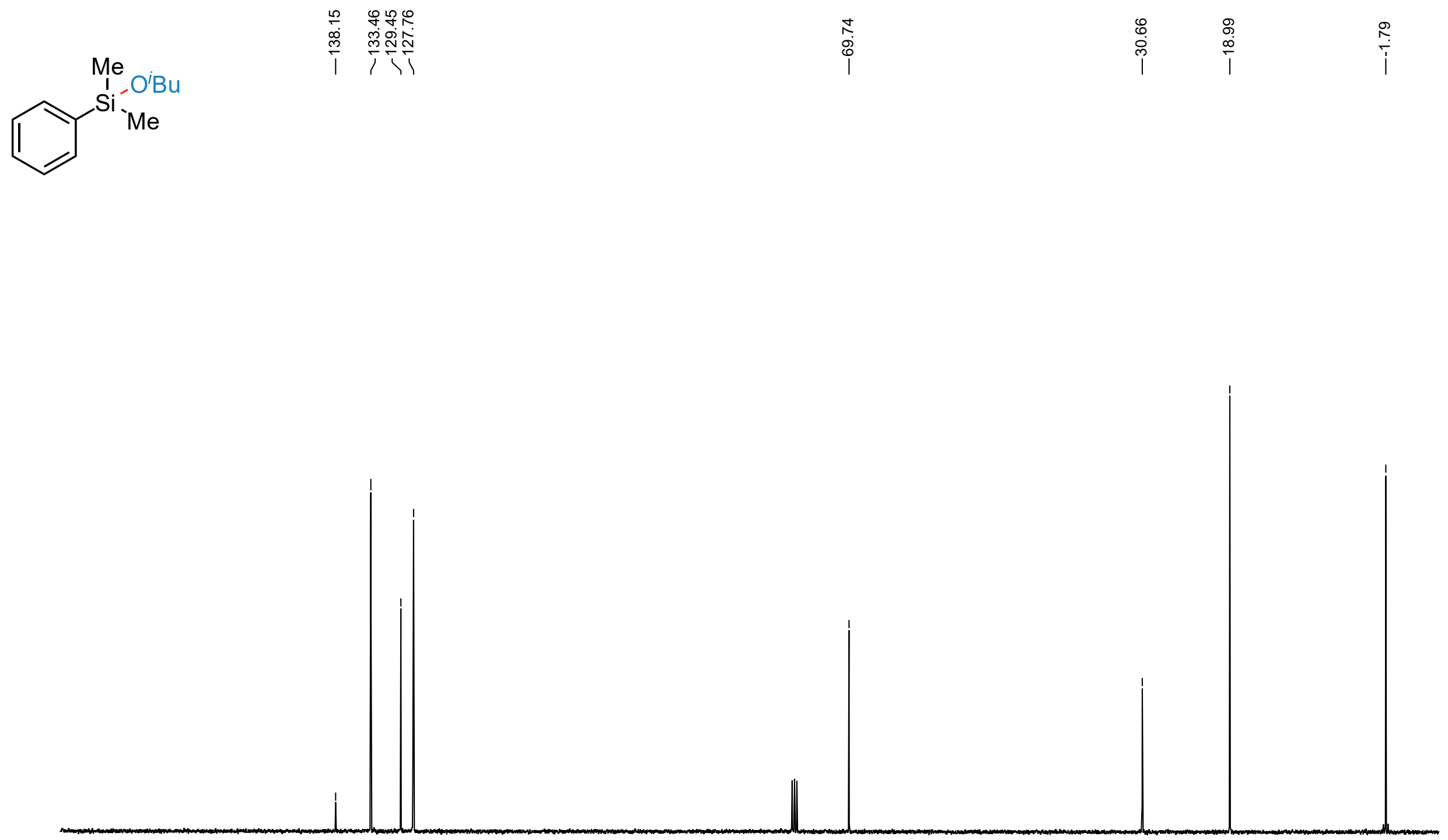


\section{Compound 6e ${ }^{1} \mathrm{H}$ NMR}

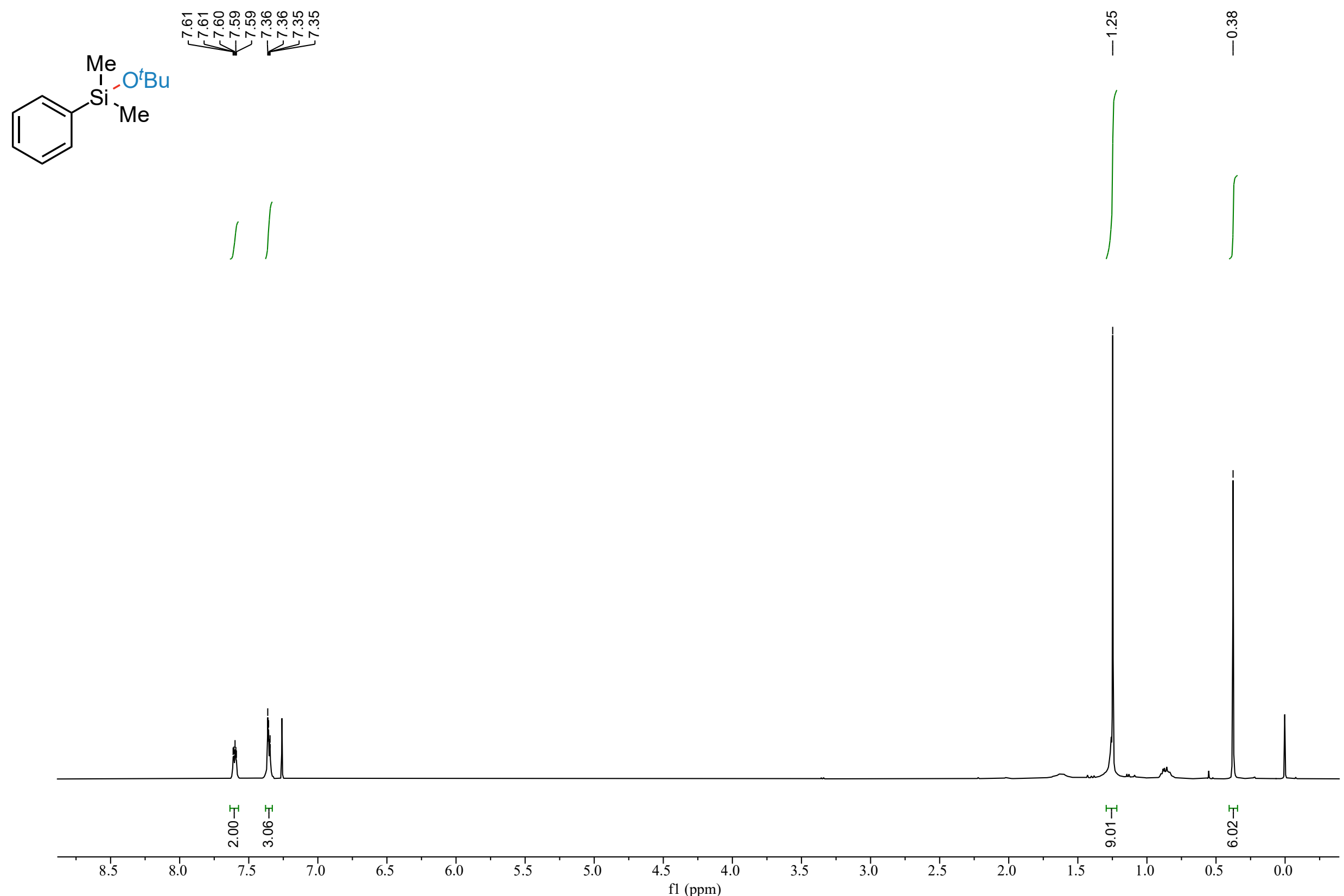


Compound $6 \mathrm{e}^{13} \mathrm{C}$ NMR
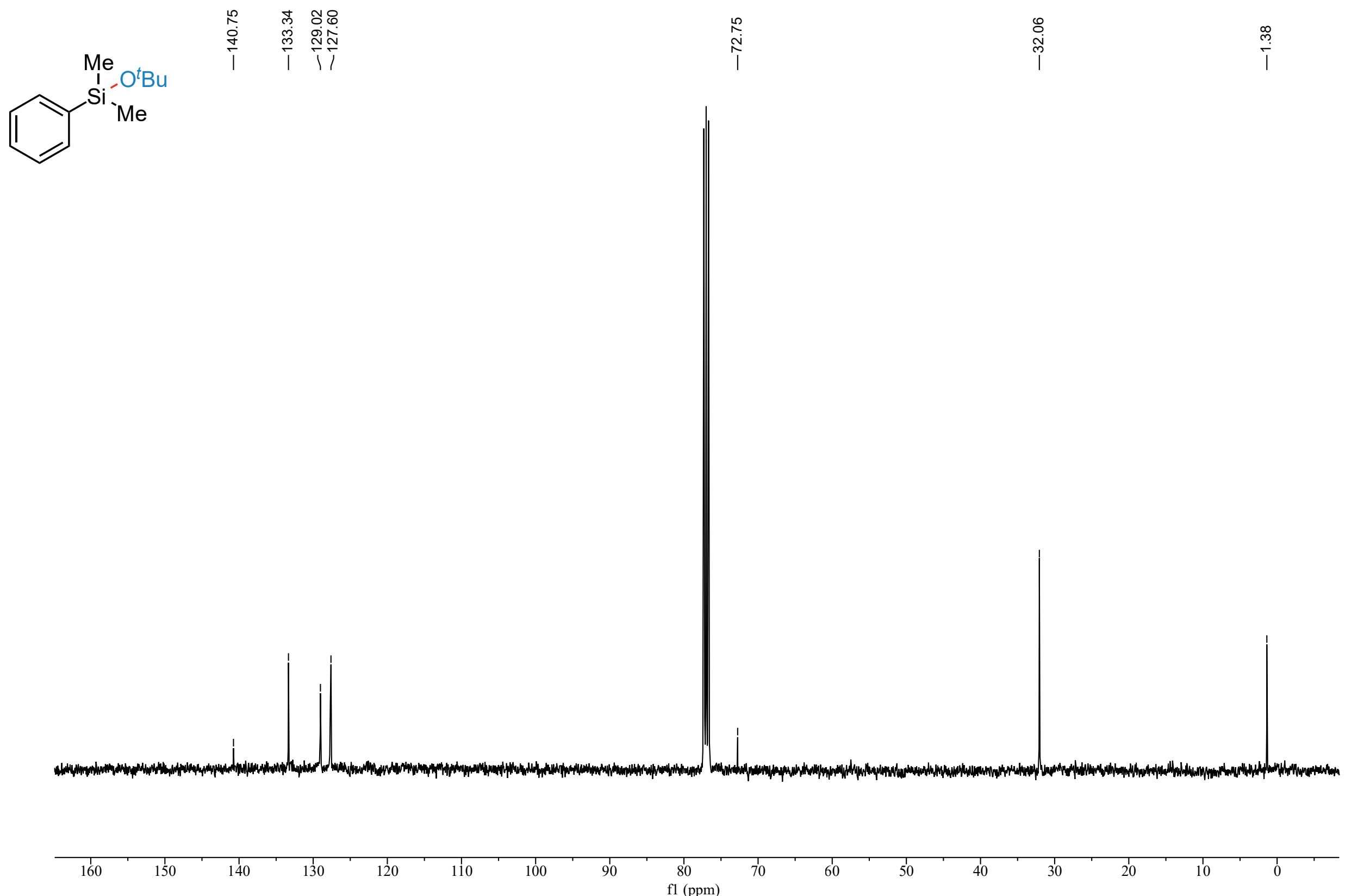


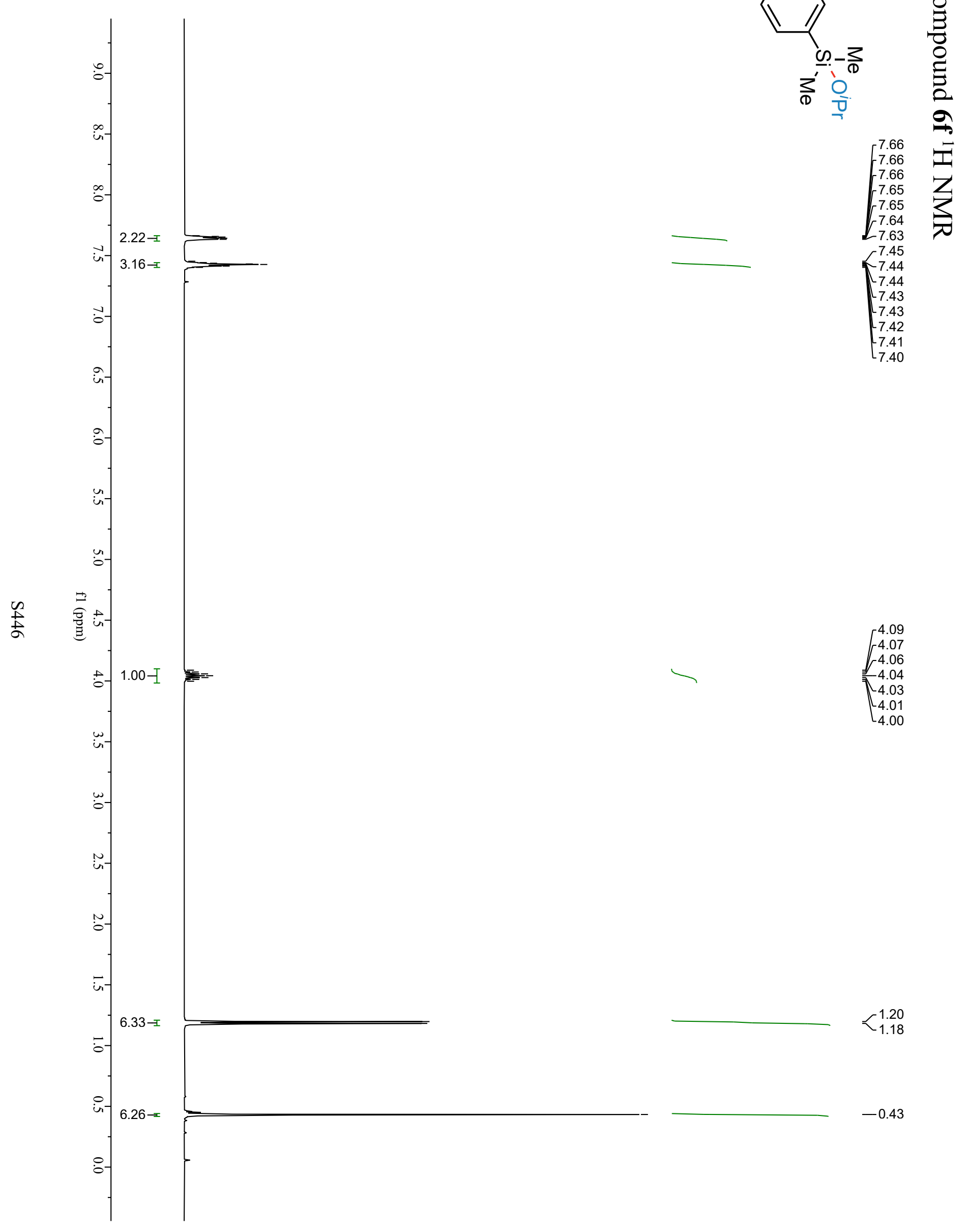


Compound $\mathbf{6 f}{ }^{13} \mathrm{C}$ NMR



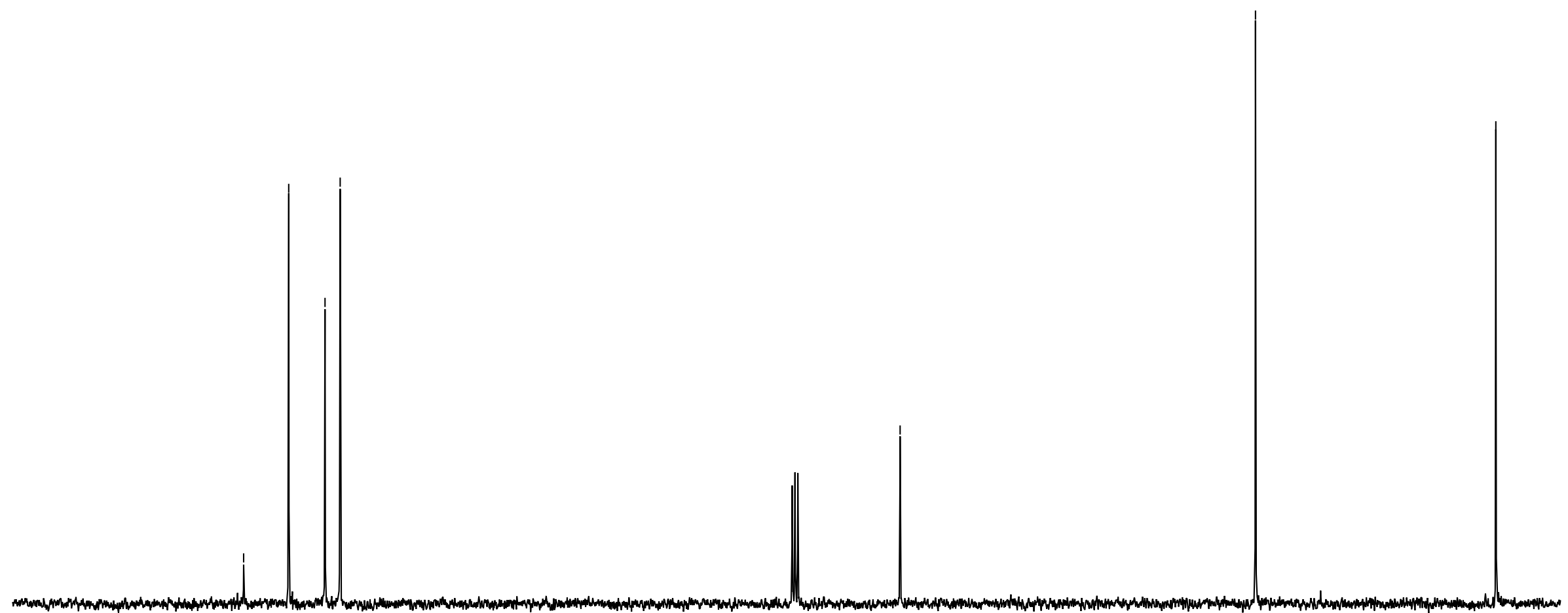



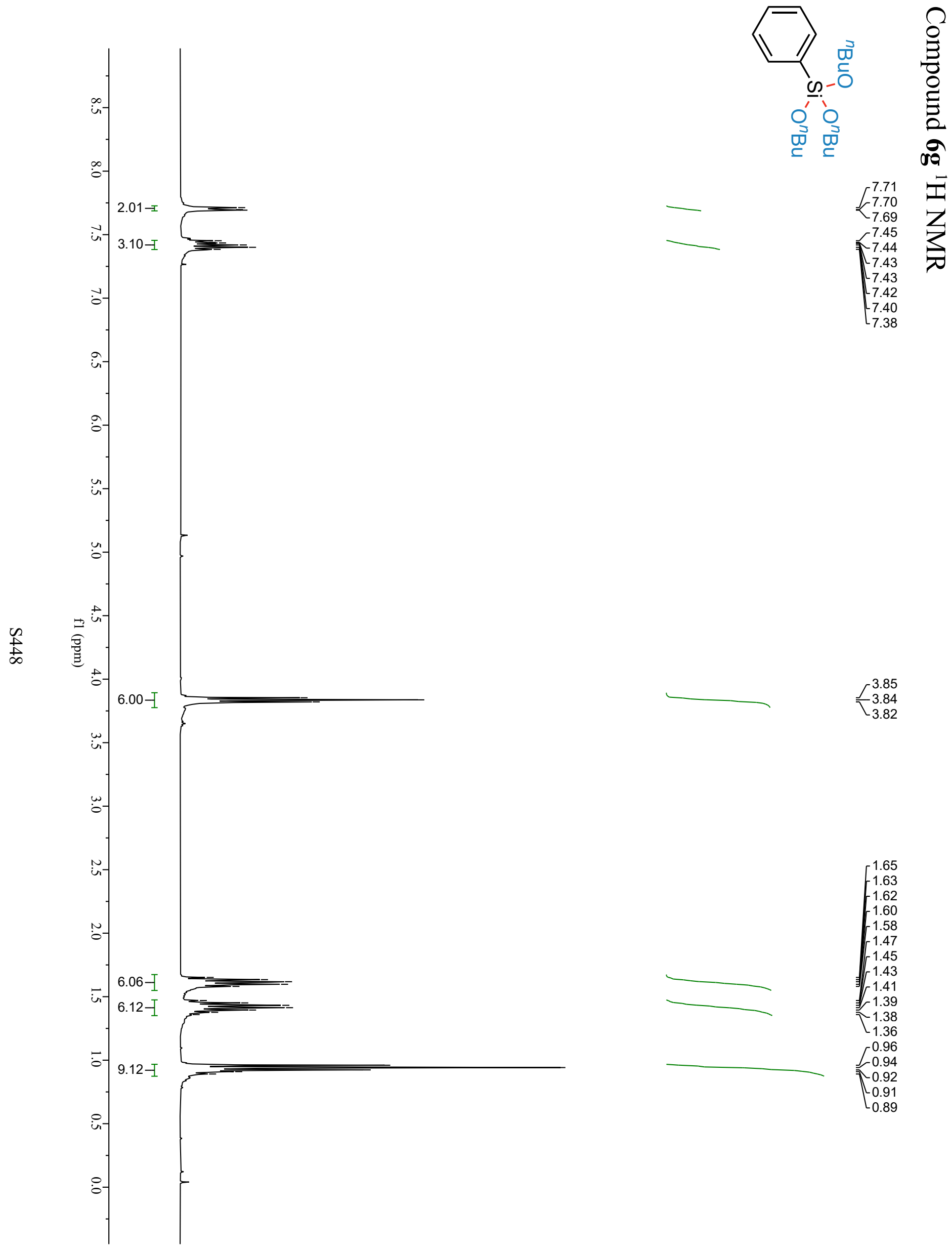

3.85
-3.84
3.82

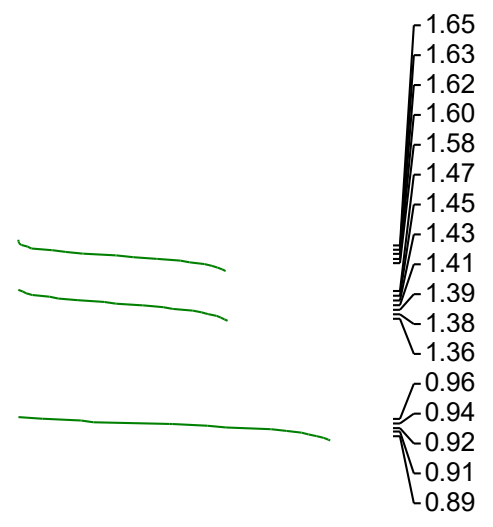


Compound 6g ${ }^{13} \mathrm{C}$ NMR
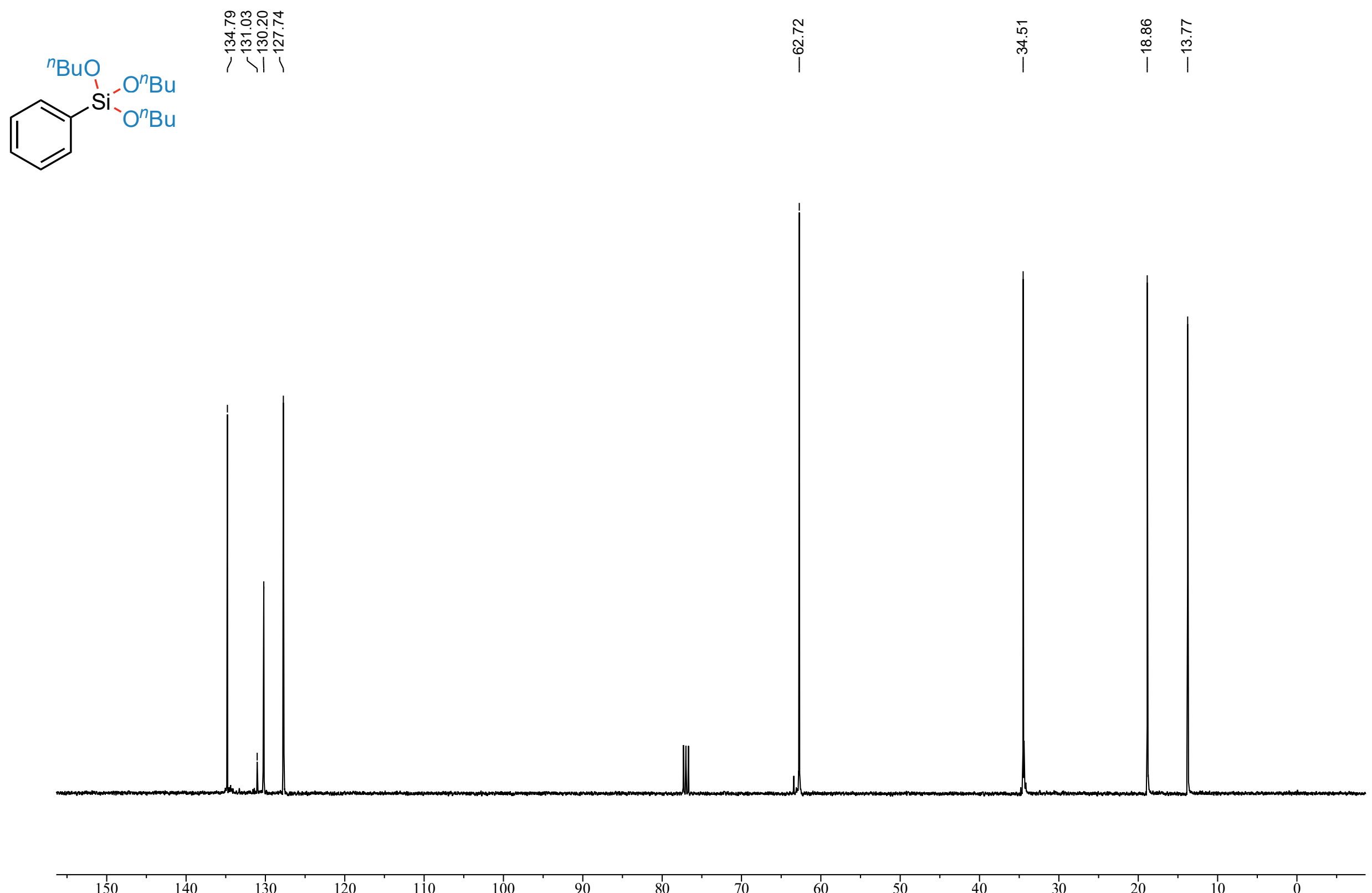


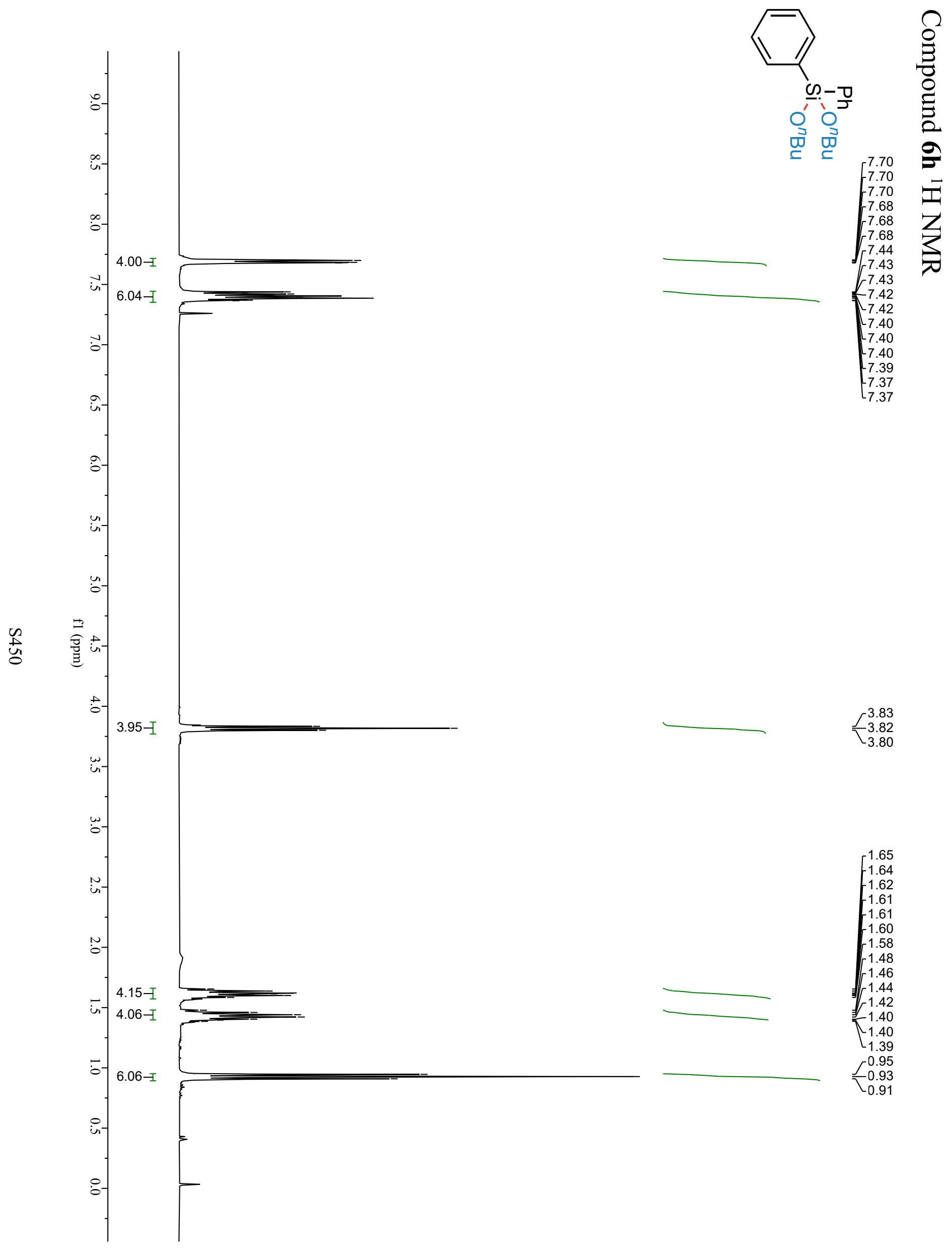


Compound $6 \mathbf{h}{ }^{13} \mathrm{C}$ NMR

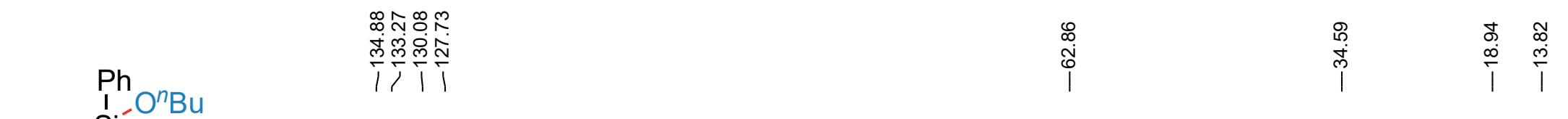

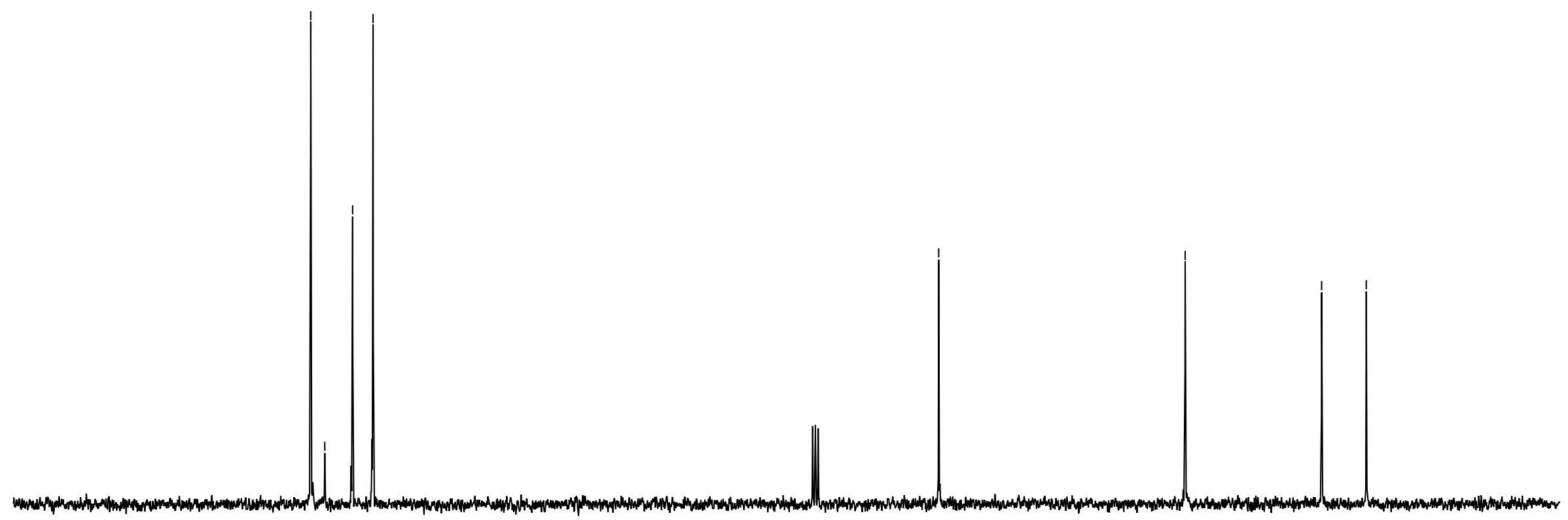




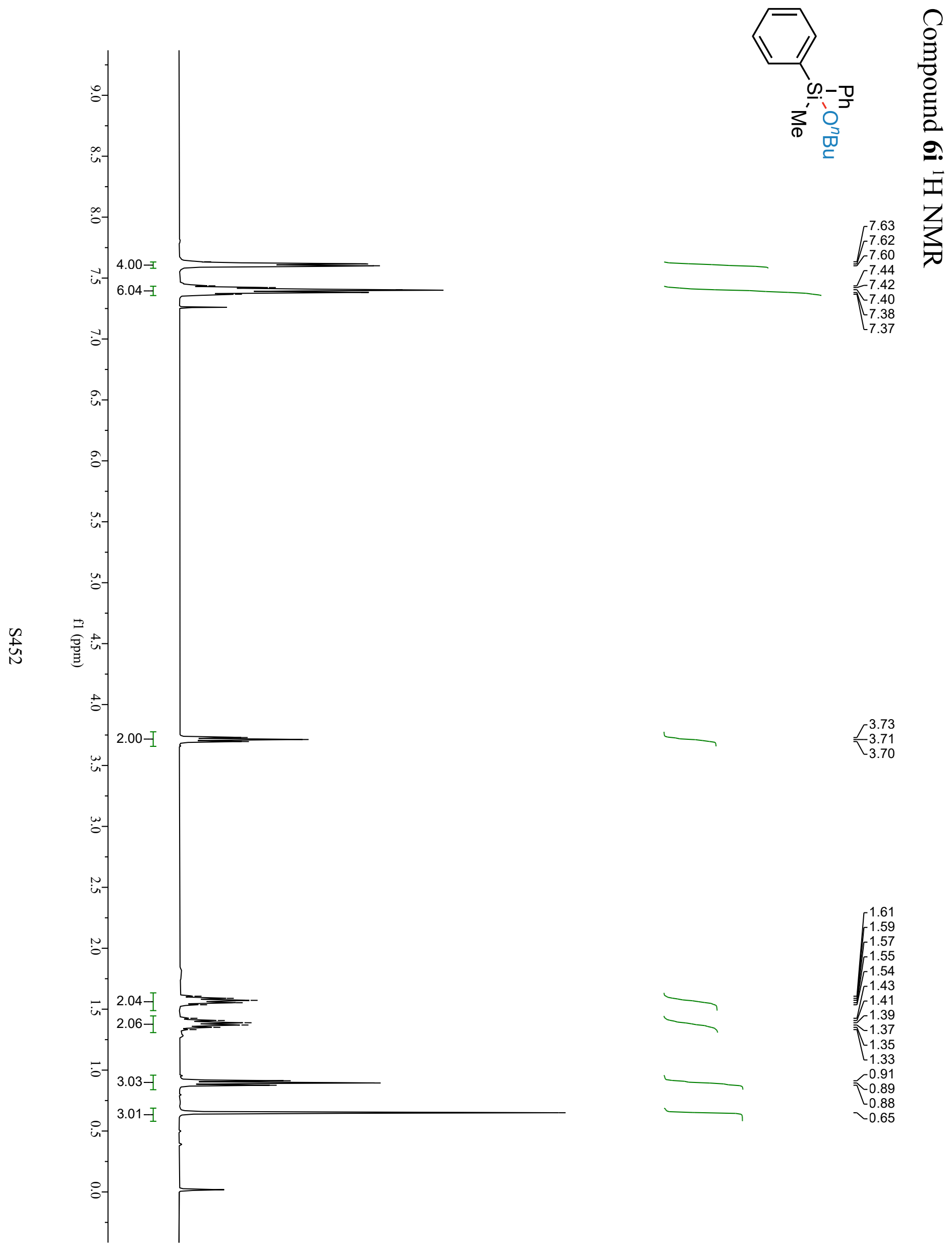


Compound $\mathbf{6} \mathbf{i}^{13} \mathrm{C}$ NMR
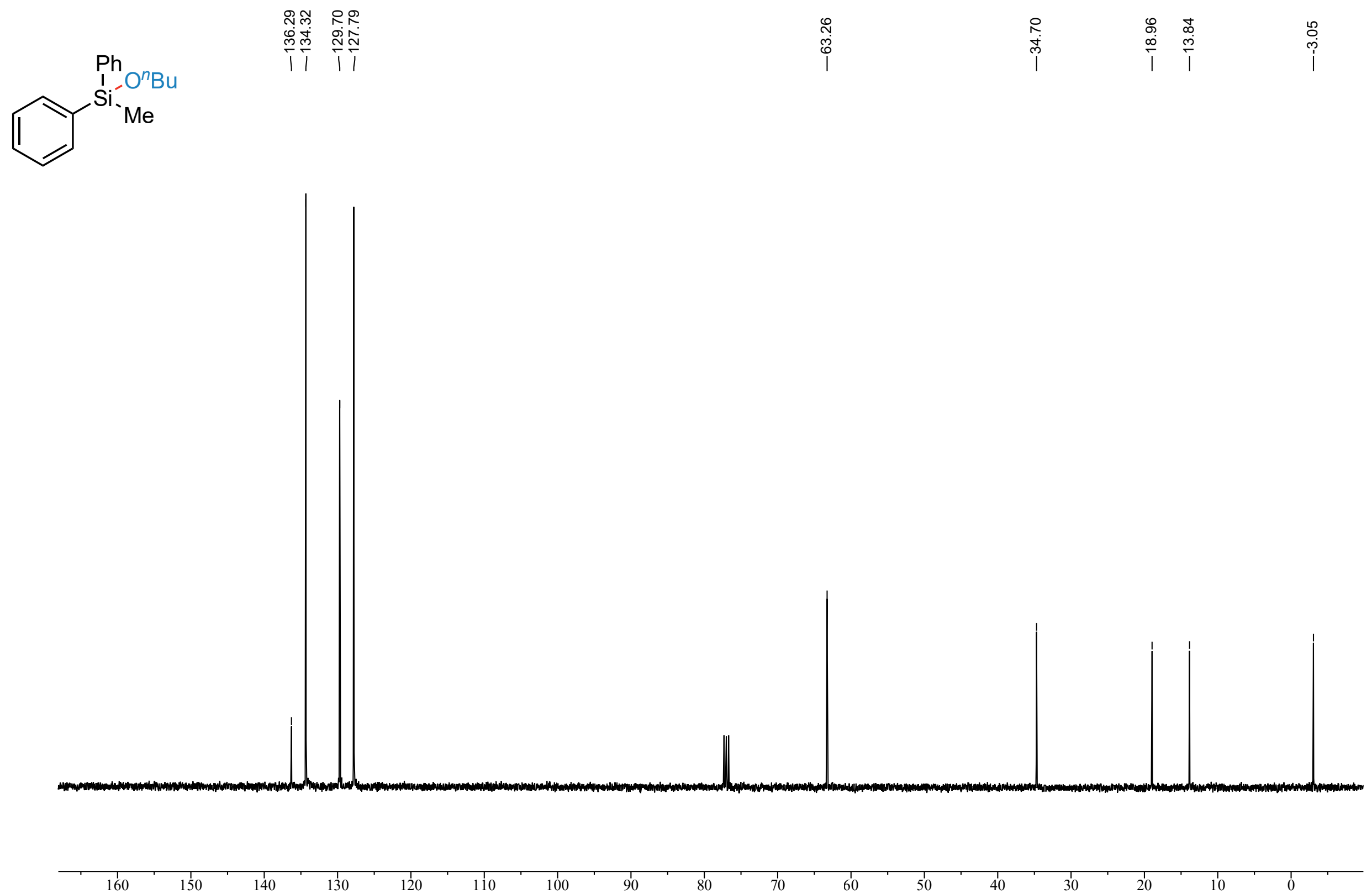


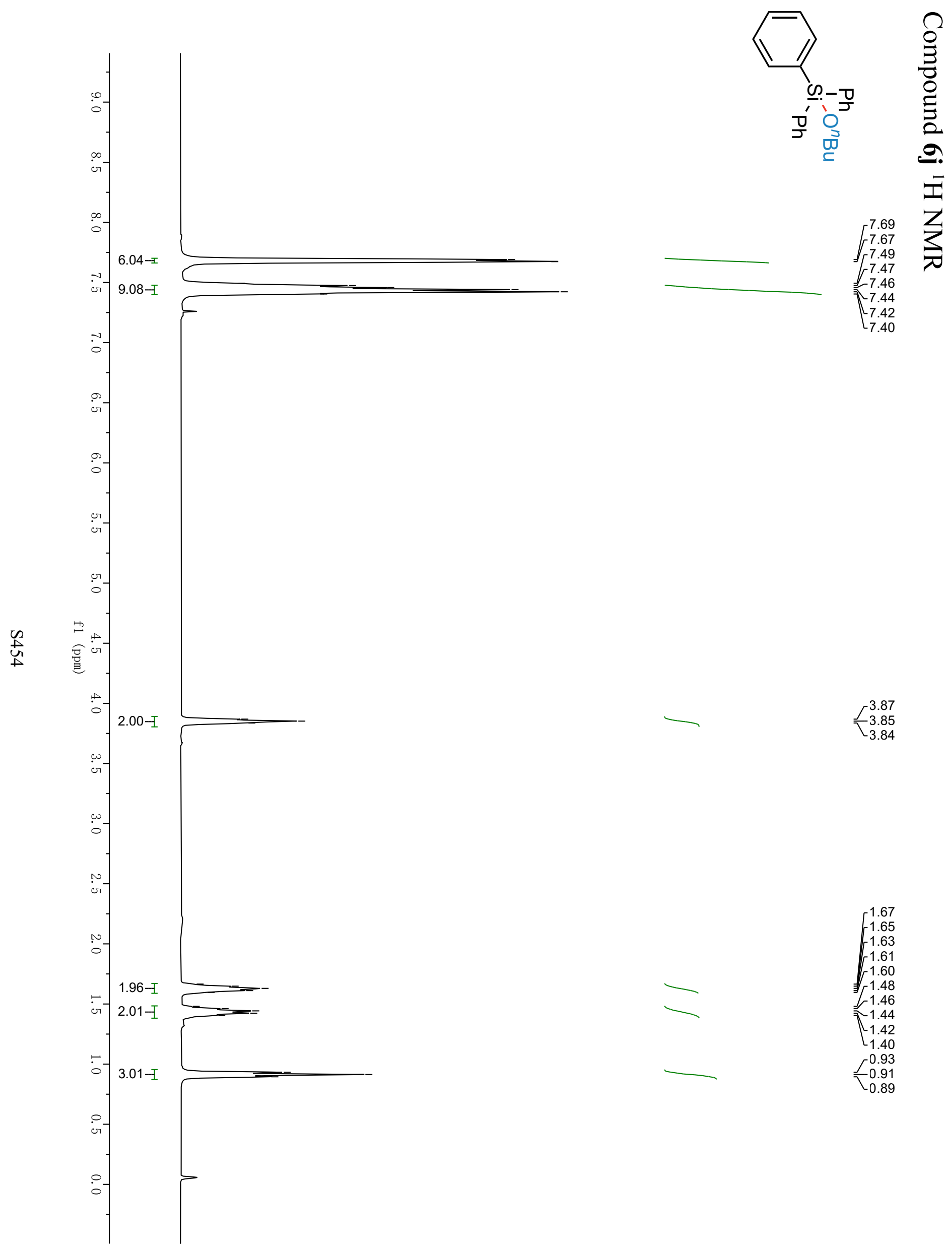


Compound $\mathbf{6 j}{ }^{13} \mathrm{C}$ NMR
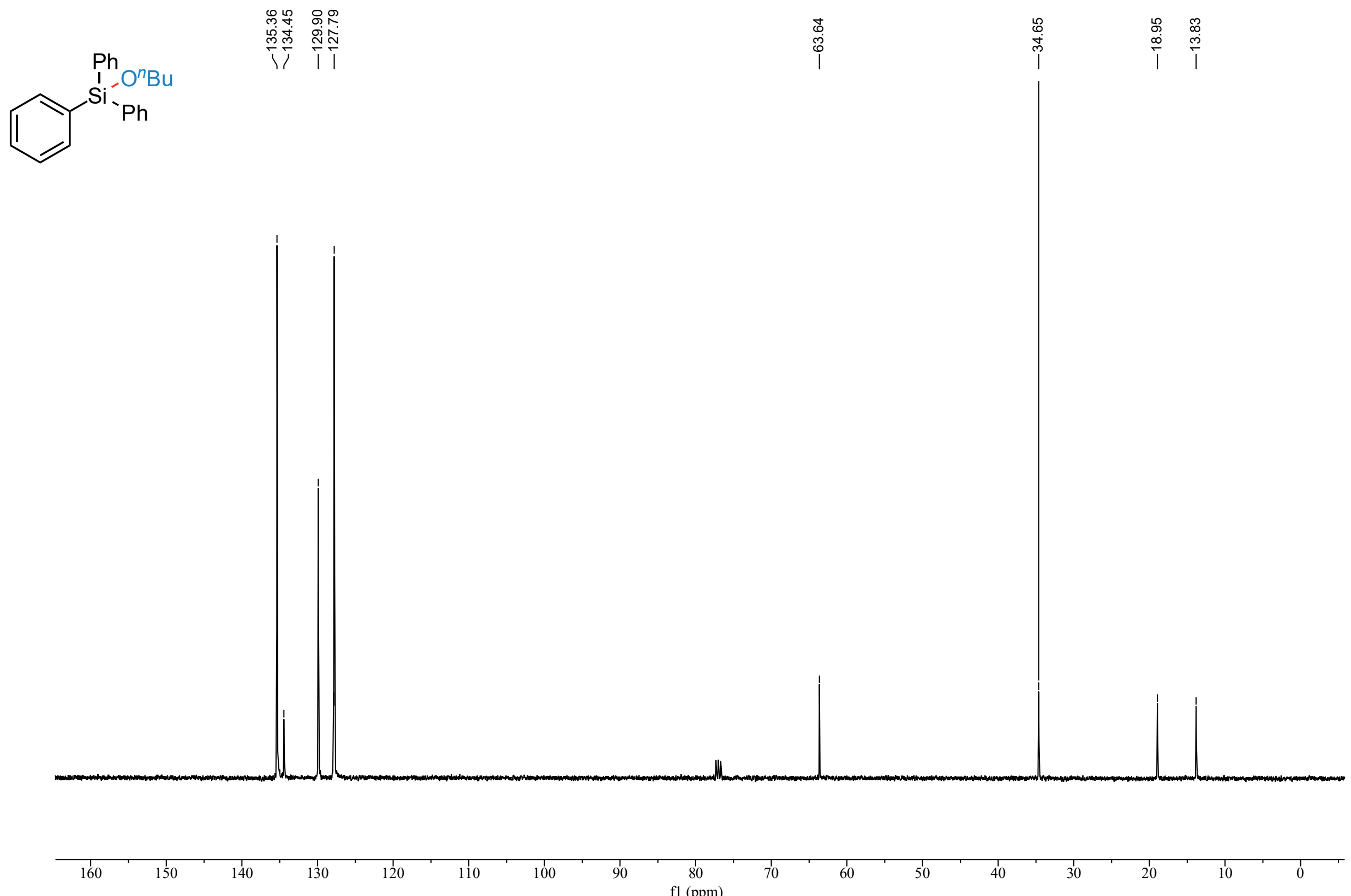


\section{Compound 6k ${ }^{1} \mathrm{H}$ NMR}

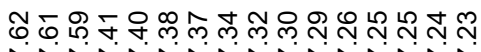
N

$\mathrm{O}^{-\mathrm{SiMe}_{2} \mathrm{Ph}}$
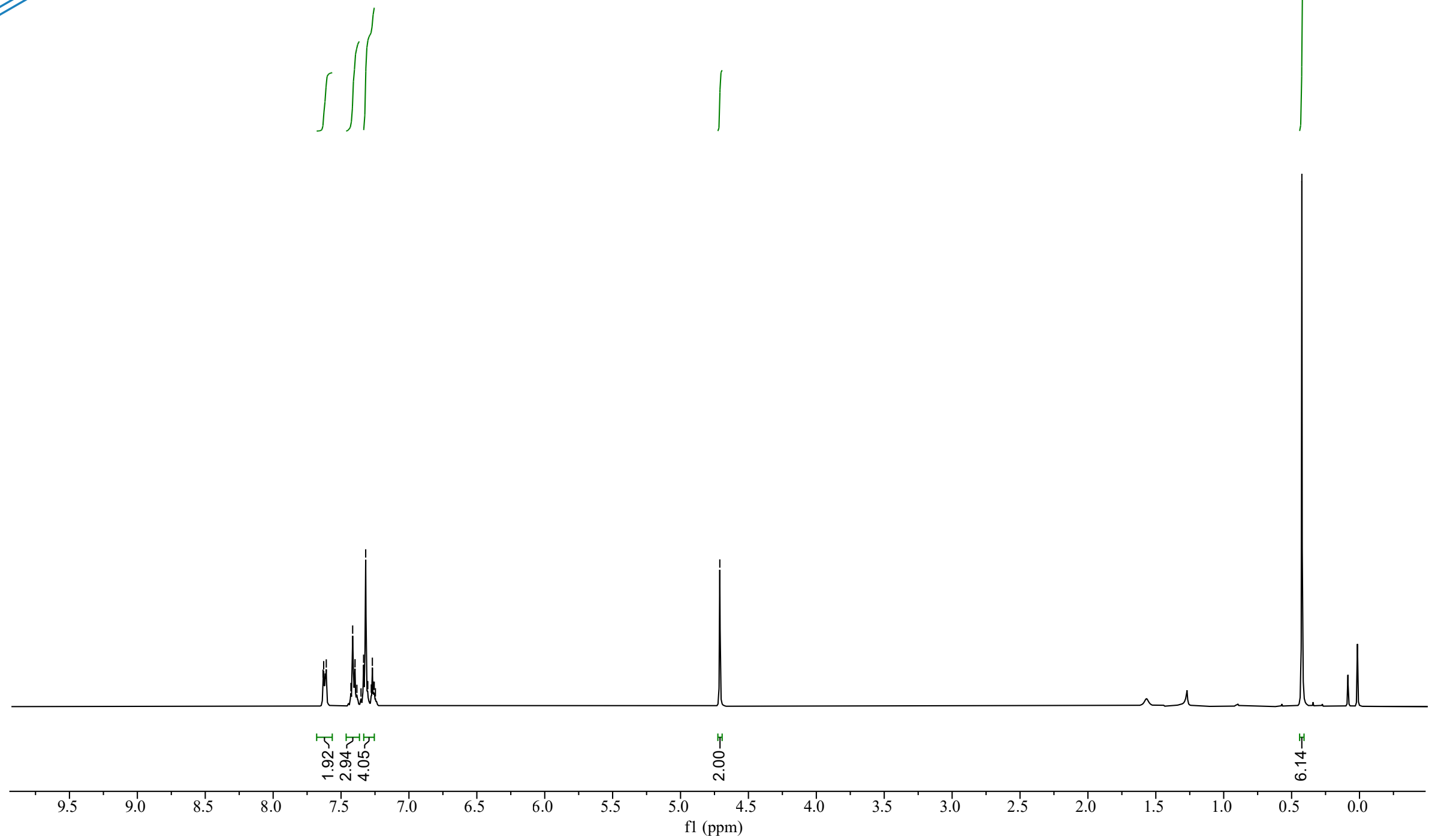
Compound $6 \mathbf{k}{ }^{13} \mathrm{C}$ NMR

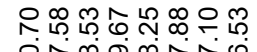

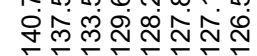

ڤิ

$\stackrel{i}{i}$

$\mathrm{O}^{-\mathrm{SiMe}_{2} \mathrm{Ph}}$

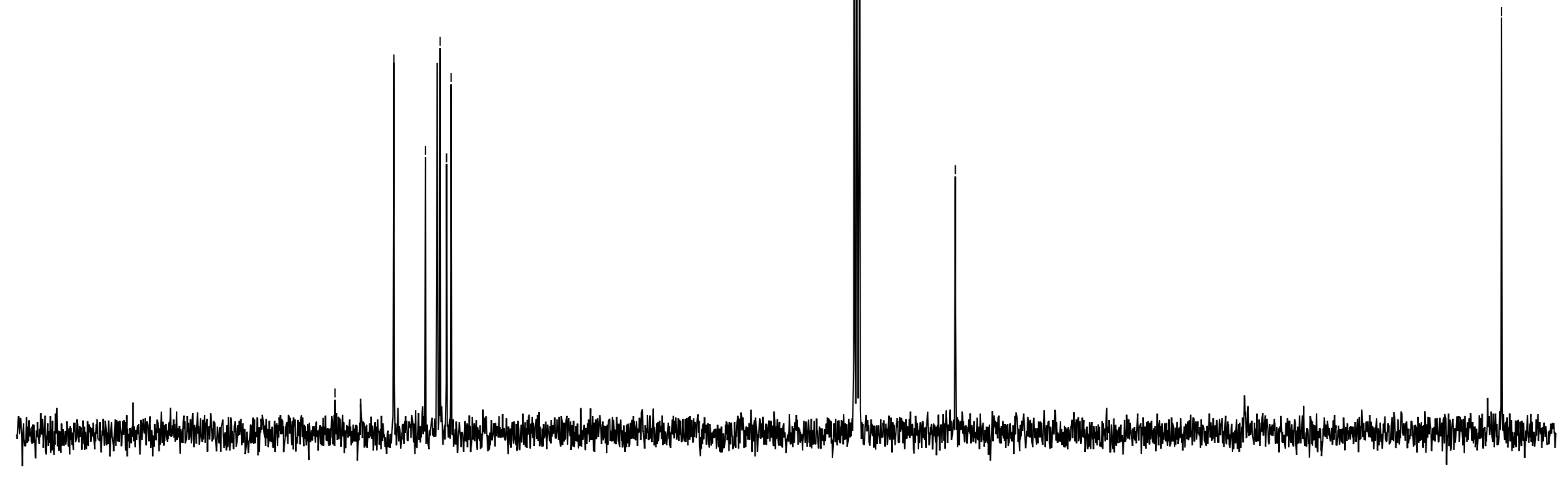




\section{Compound $6 \mathbf{I}^{1} \mathrm{H}$ NMR}

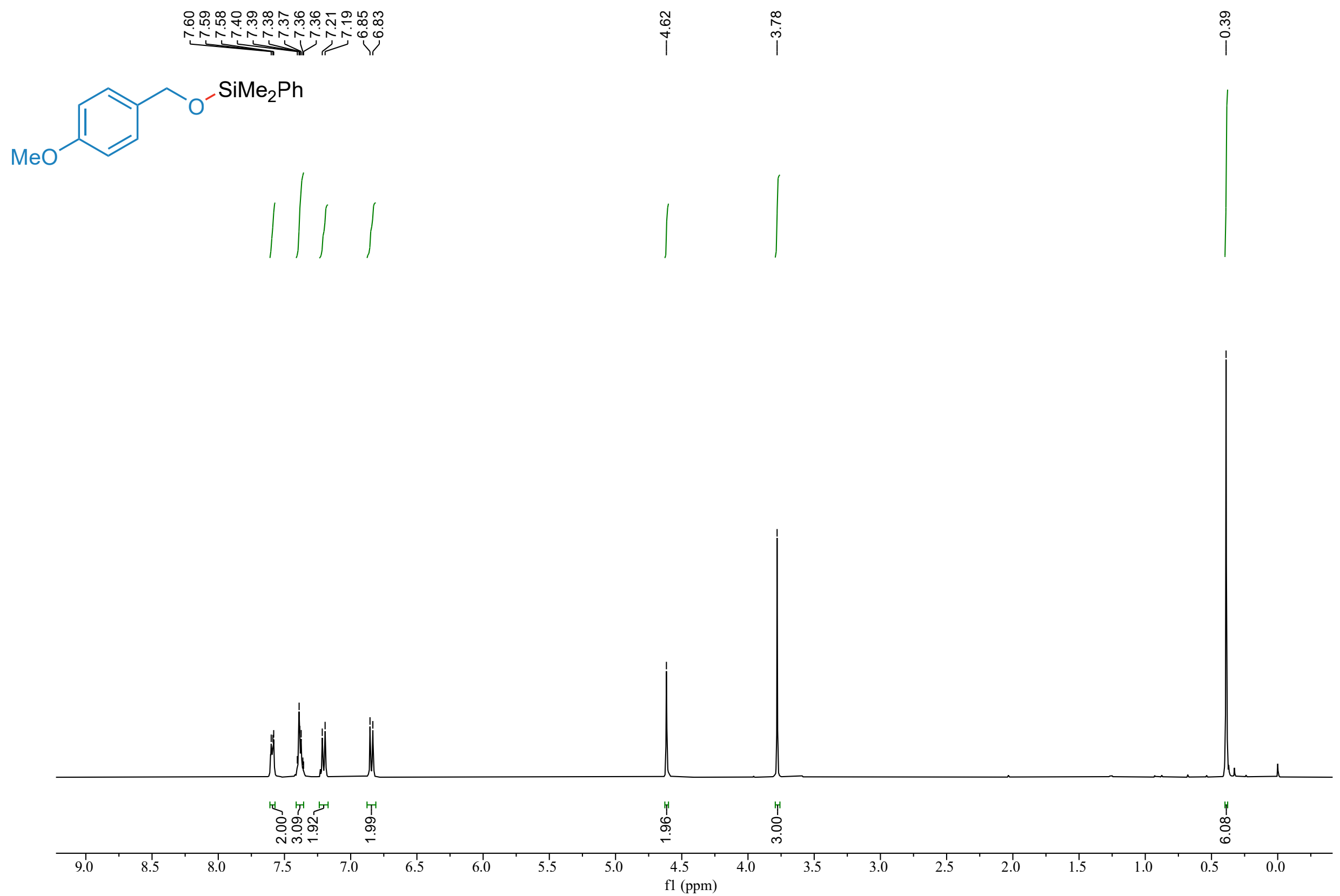


Compound 6I ${ }^{13} \mathrm{C}$ NMR
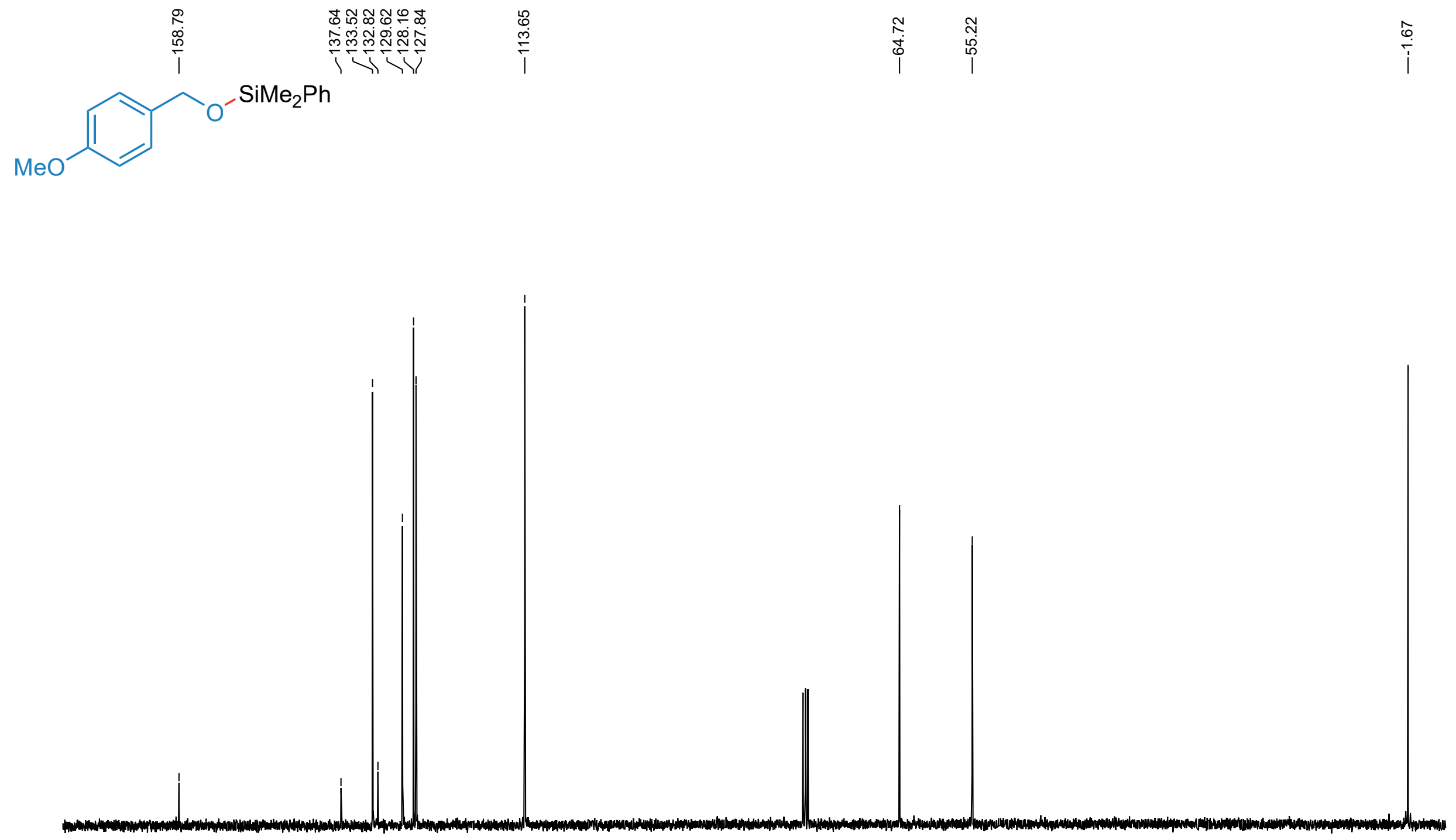


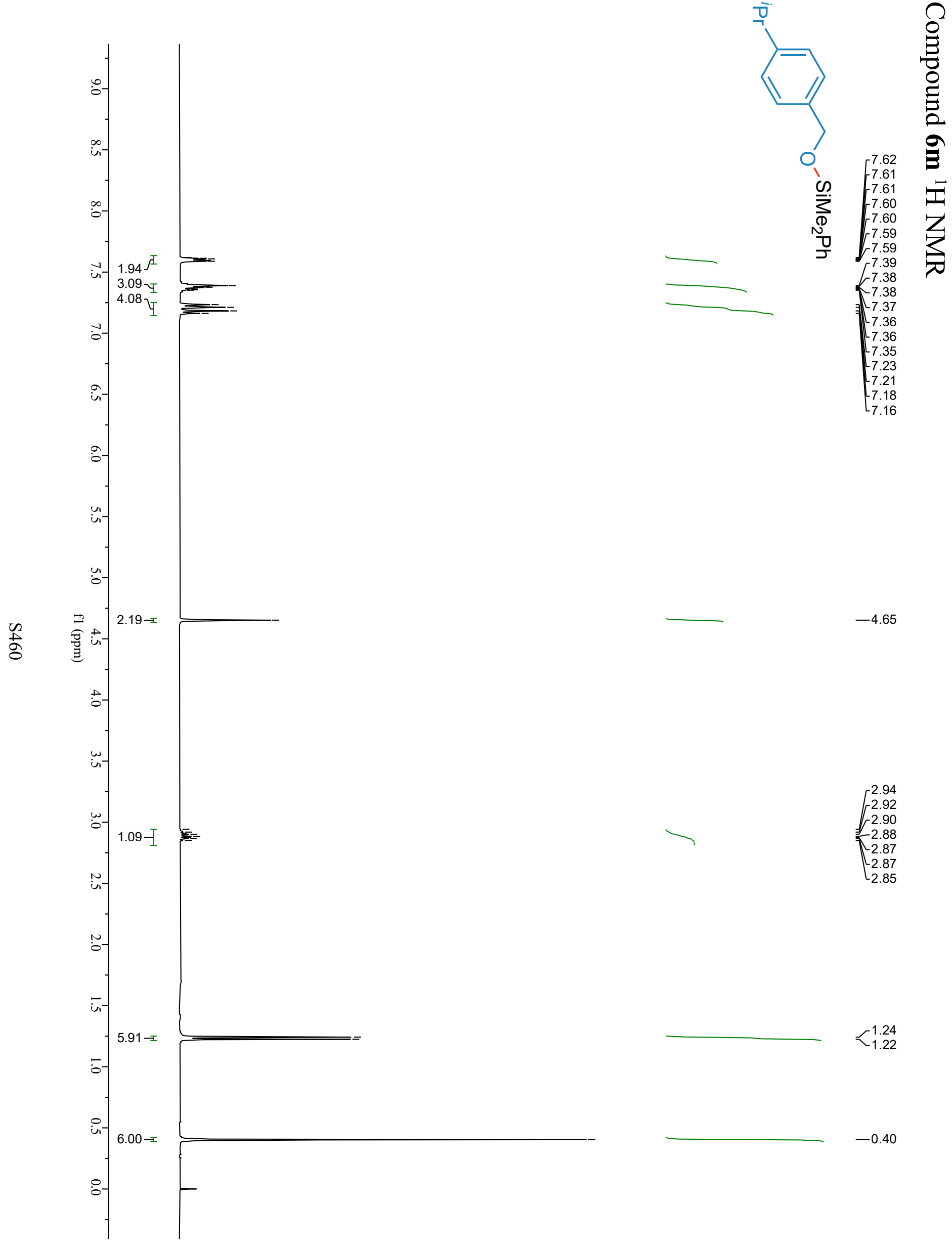


Compound $\mathbf{6 m}{ }^{13} \mathrm{C}$ NMR
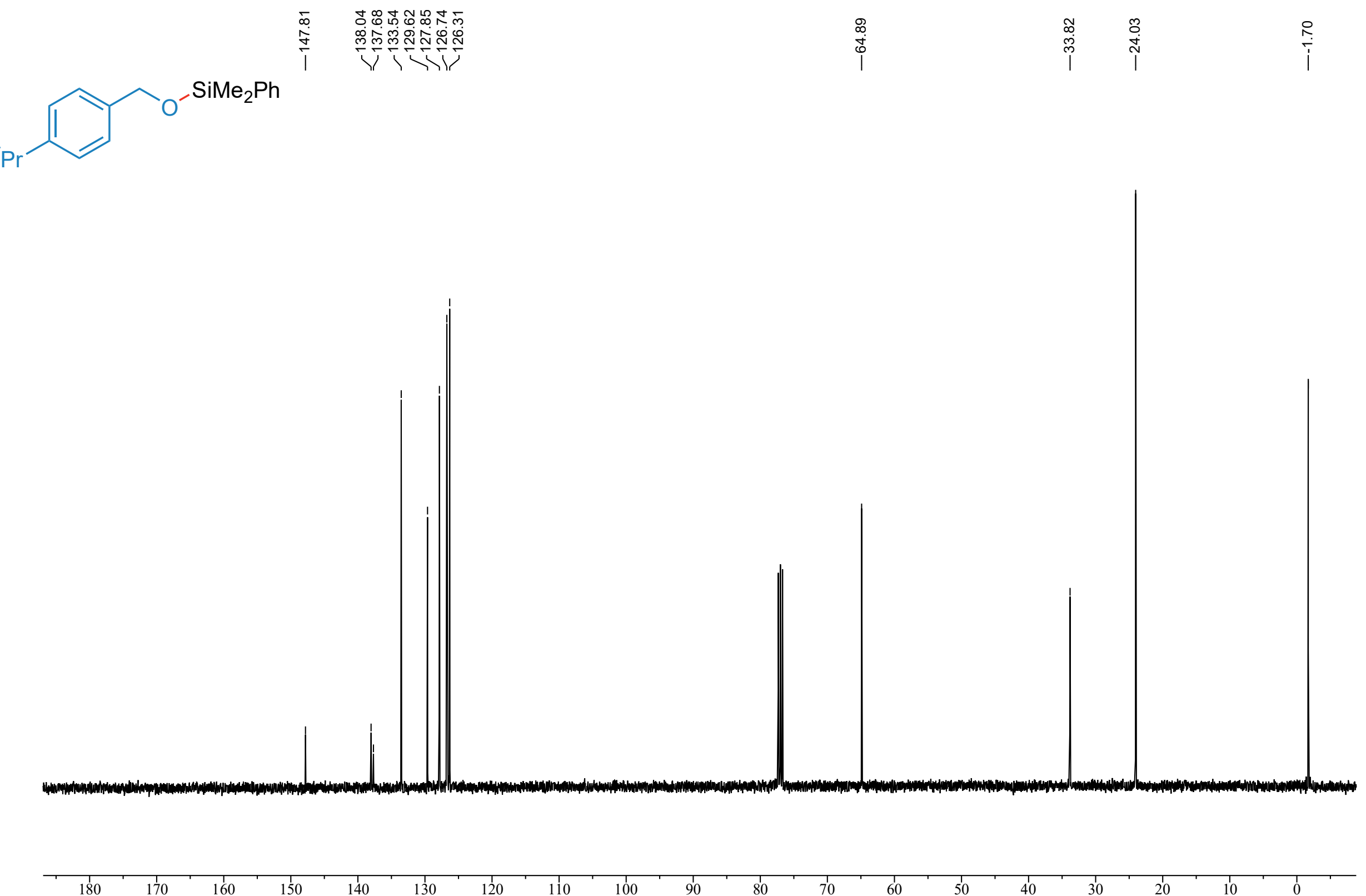
Compound 6n ${ }^{1} \mathrm{H}$ NMR

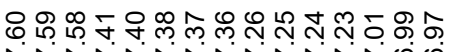

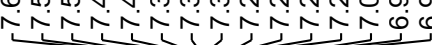

$>\mathrm{O}^{-\mathrm{SiMe}_{2} \mathrm{Ph}}$
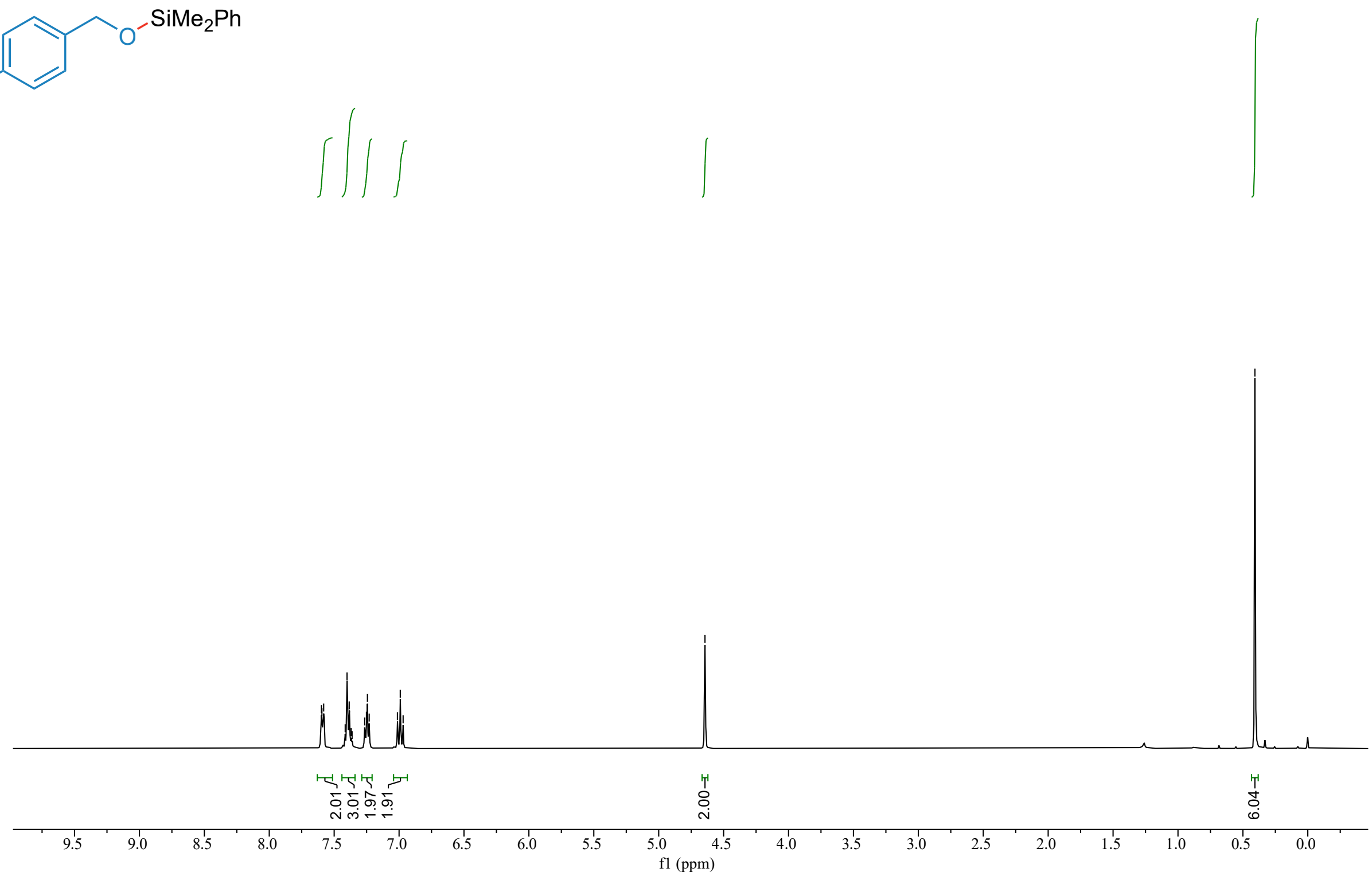
Compound 6n ${ }^{13} \mathrm{C}$ NMR
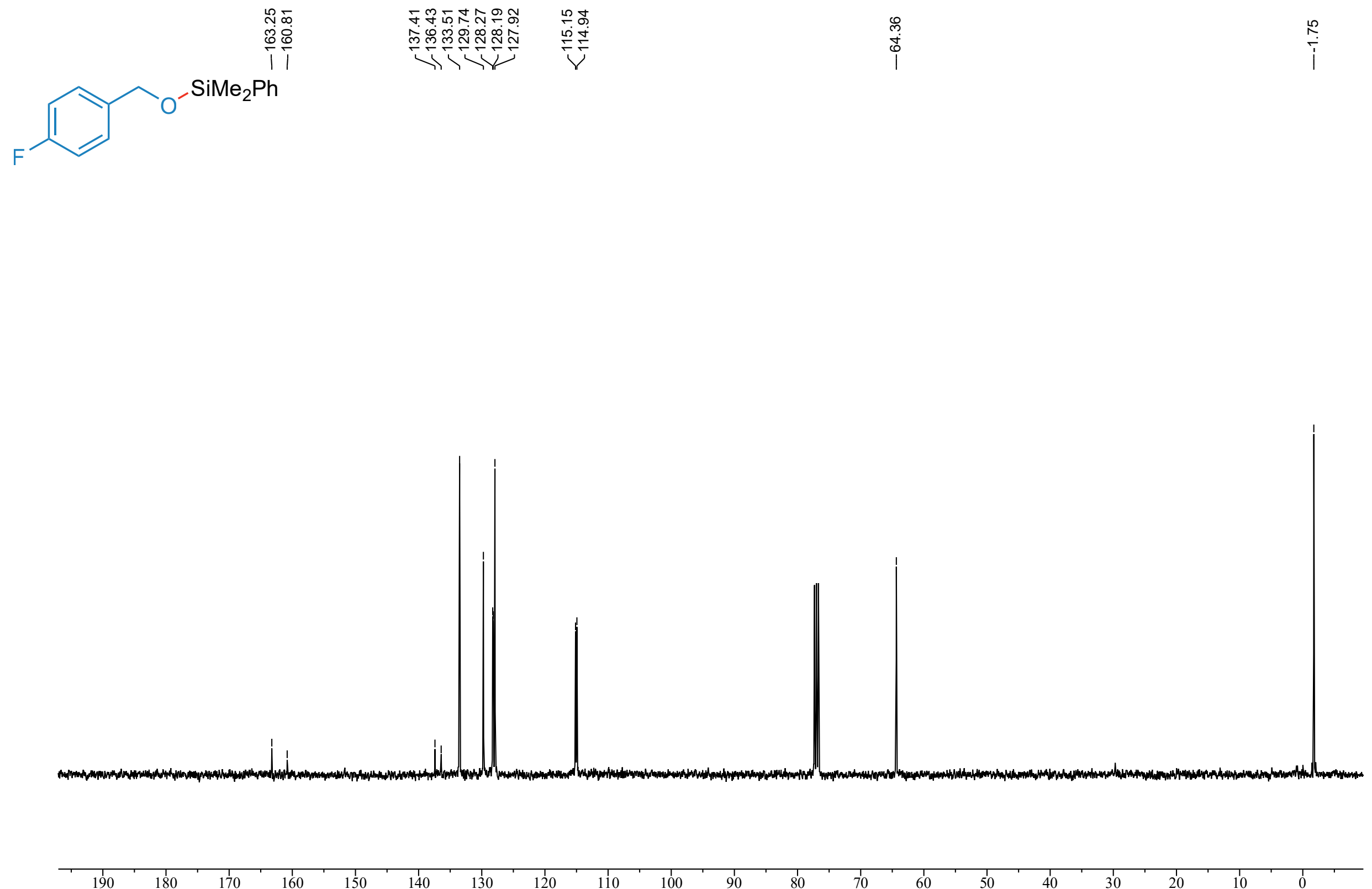
Compound 6n ${ }^{19} \mathrm{~F}$ NMR

$>\mathrm{O}^{-\mathrm{SiMe}_{2} \mathrm{Ph}}$

$\underset{\substack{\infty \\ \stackrel{i}{i}}}{\stackrel{i}{i}}$

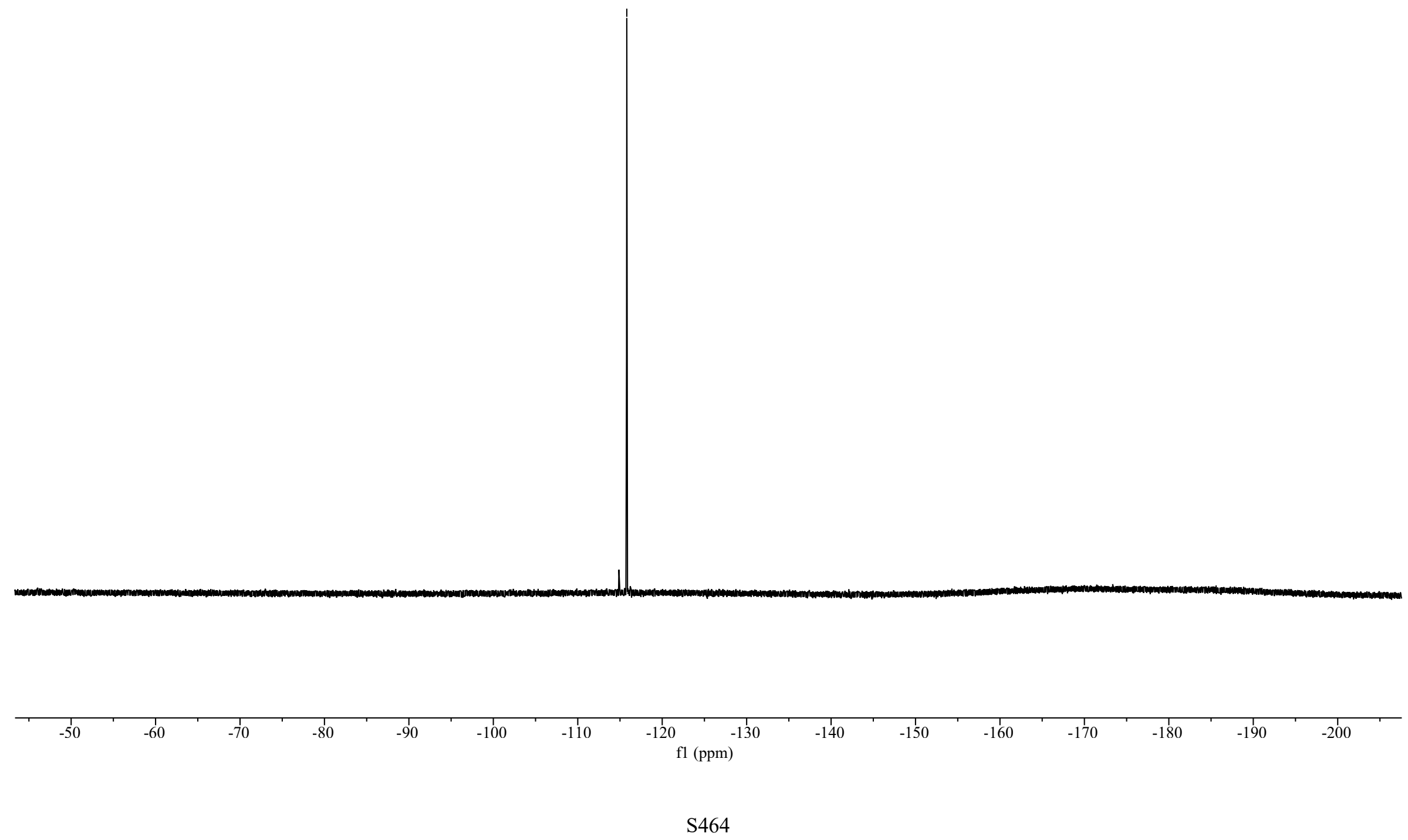


Compound $60^{1} \mathrm{H}$ NMR

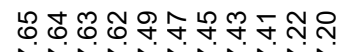

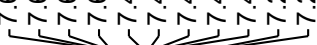
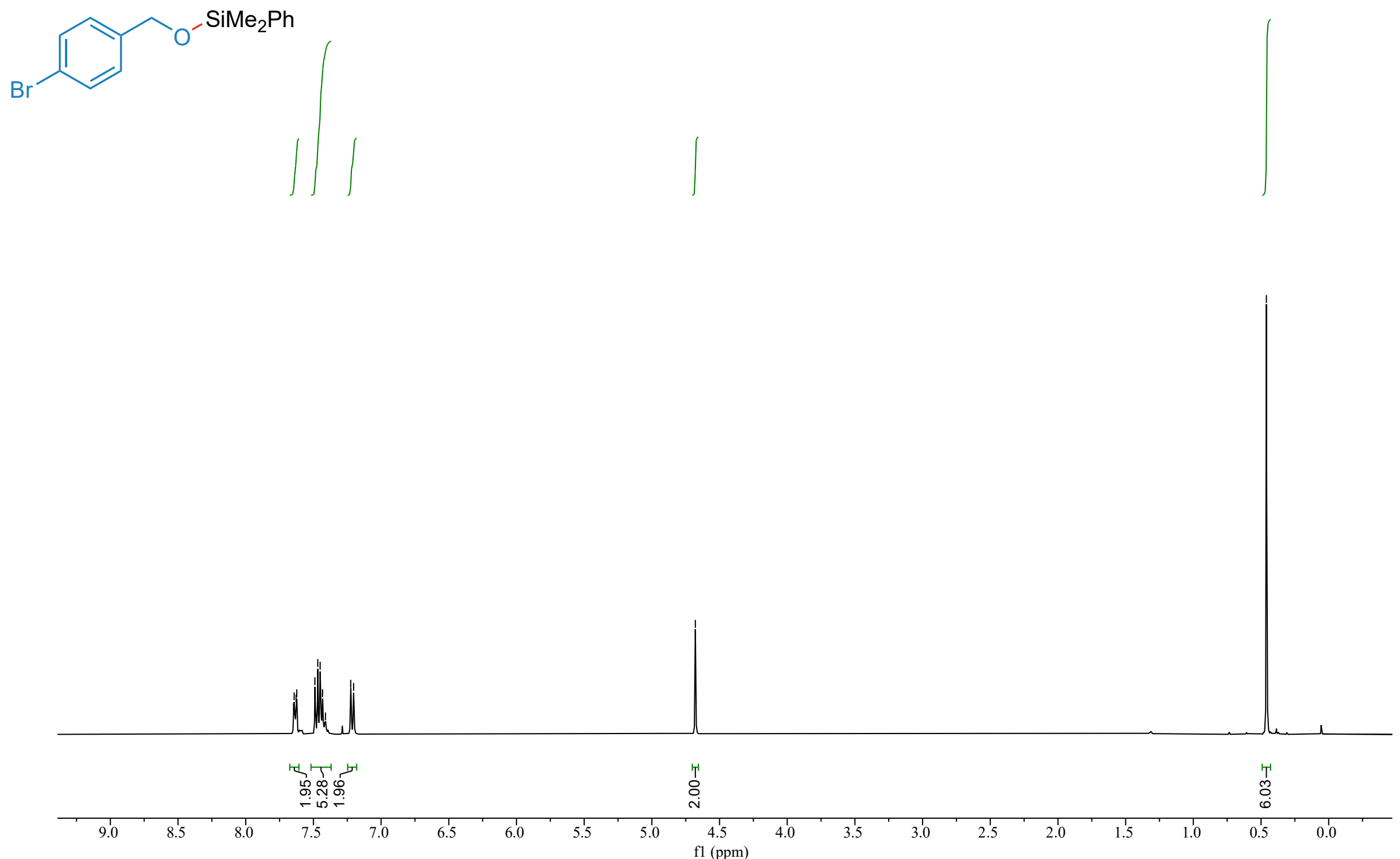
Compound $60{ }^{13} \mathrm{C}$ NMR

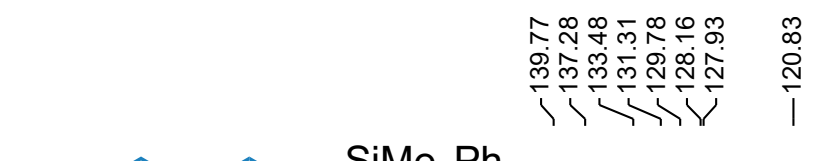

$\mathrm{Br}$

$-\mathrm{SiMe}_{2} \mathrm{Ph}$
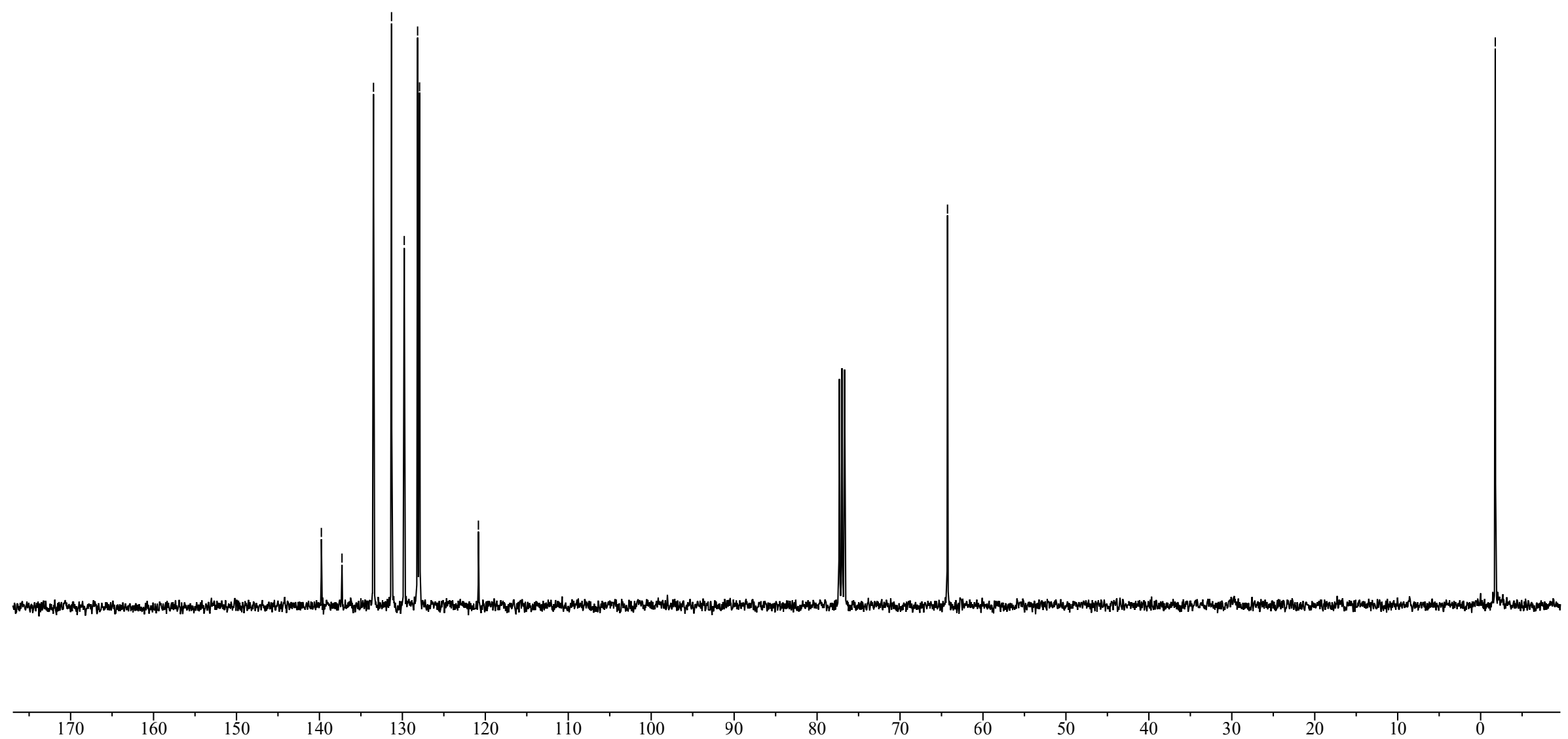


\section{Compound $6 \mathbf{p}{ }^{1} \mathrm{H}$ NMR}

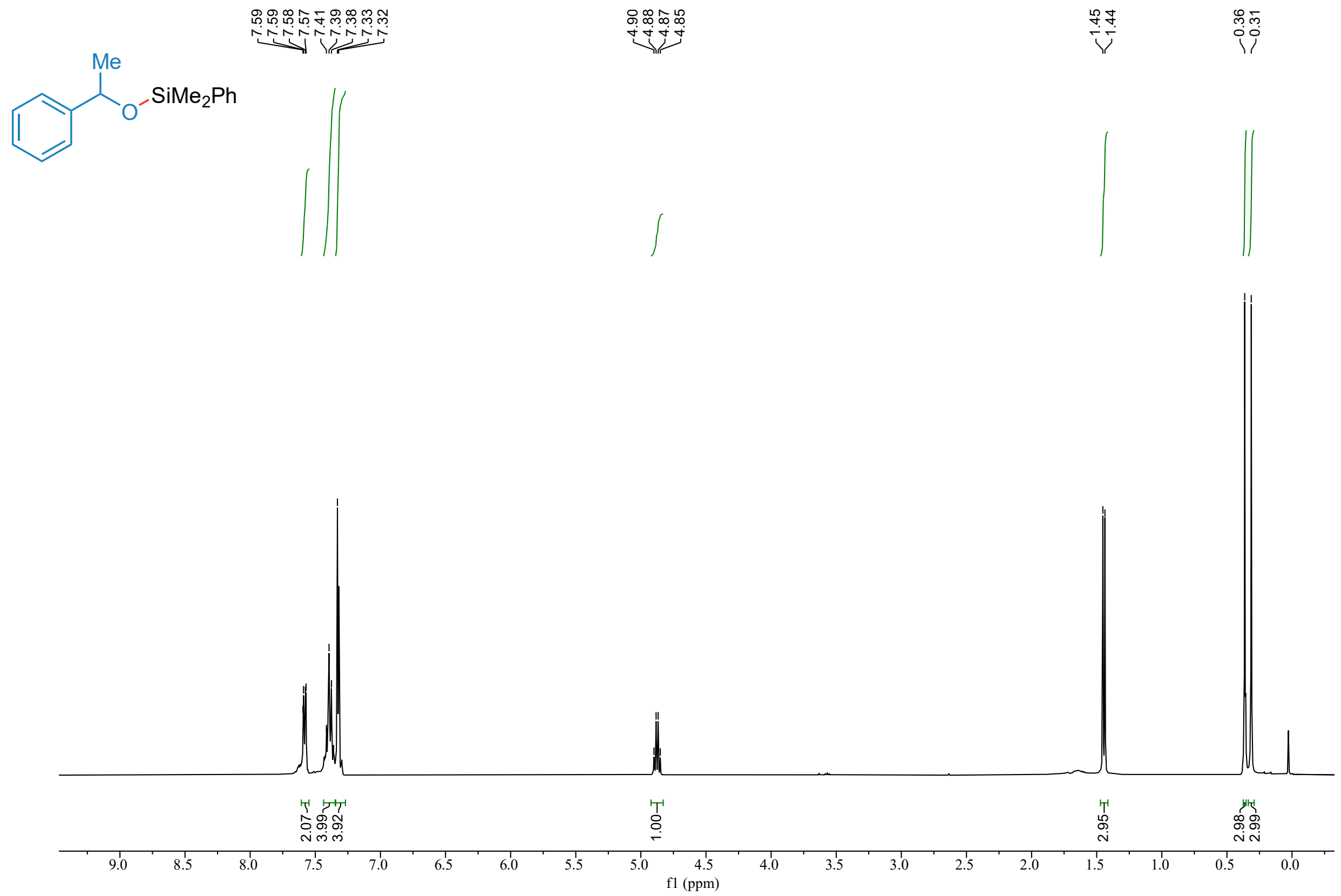


Compound $4 p{ }^{13} \mathrm{C}$ NMR

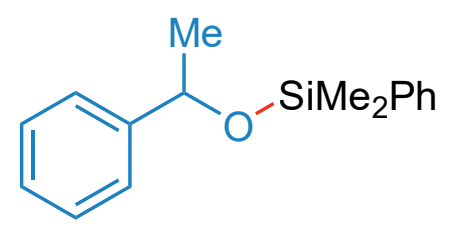

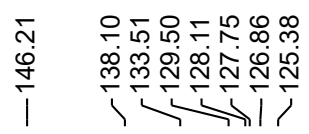

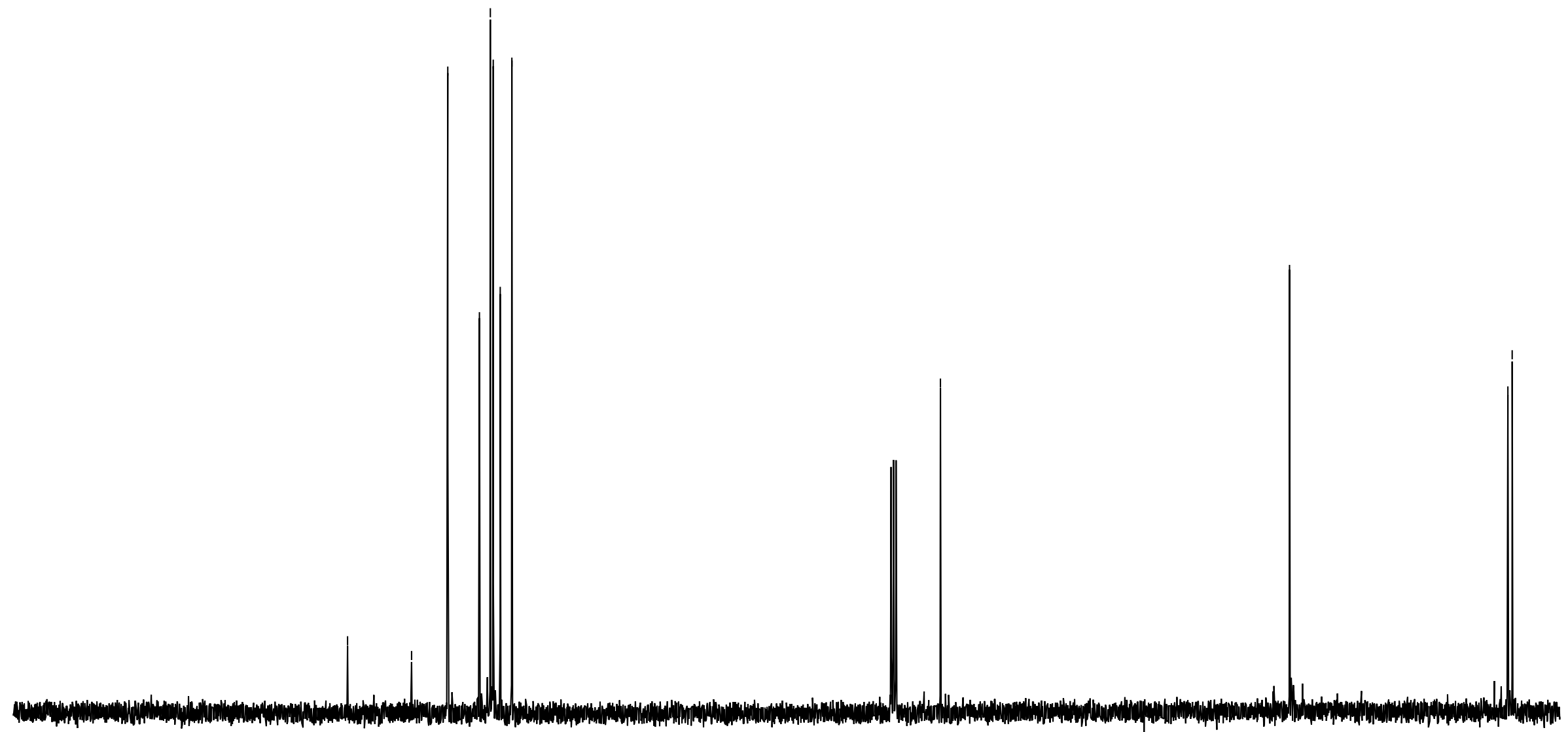




\section{Compound 6q ${ }^{1} \mathrm{H}$ NMR}

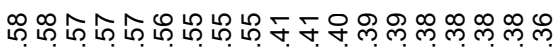

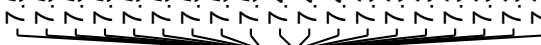

$\mathrm{PhMe}_{2} \mathrm{Si}-\mathrm{O} \mathrm{O}-\mathrm{SiMe}_{2} \mathrm{Ph}$
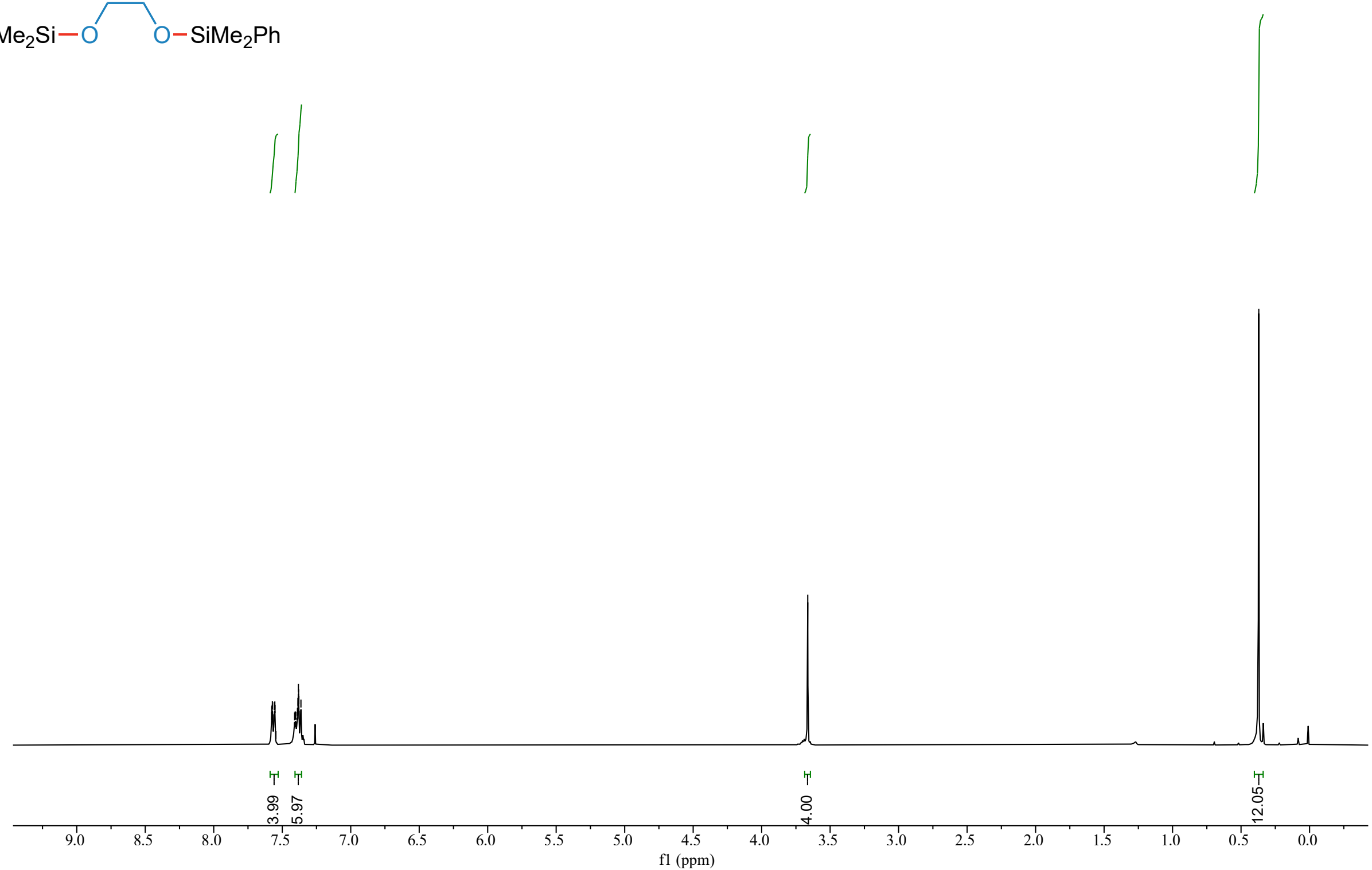
Compound $\mathbf{6 q}{ }^{13} \mathrm{C}$ NMR

$\mathrm{PhMe}_{2} \mathrm{Si}-\mathrm{O} \mathrm{O}-\mathrm{SiMe}_{2} \mathrm{Ph}$
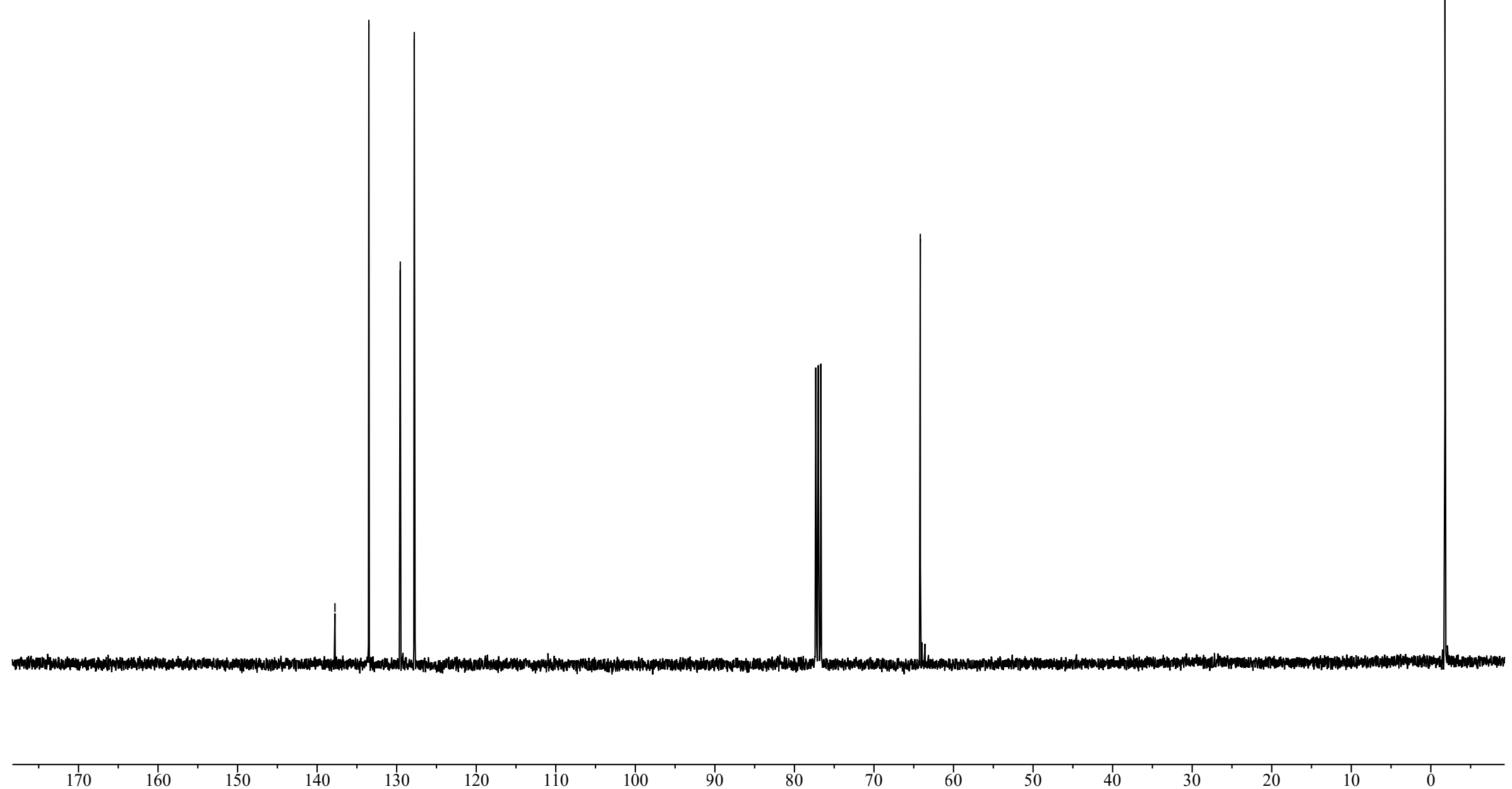

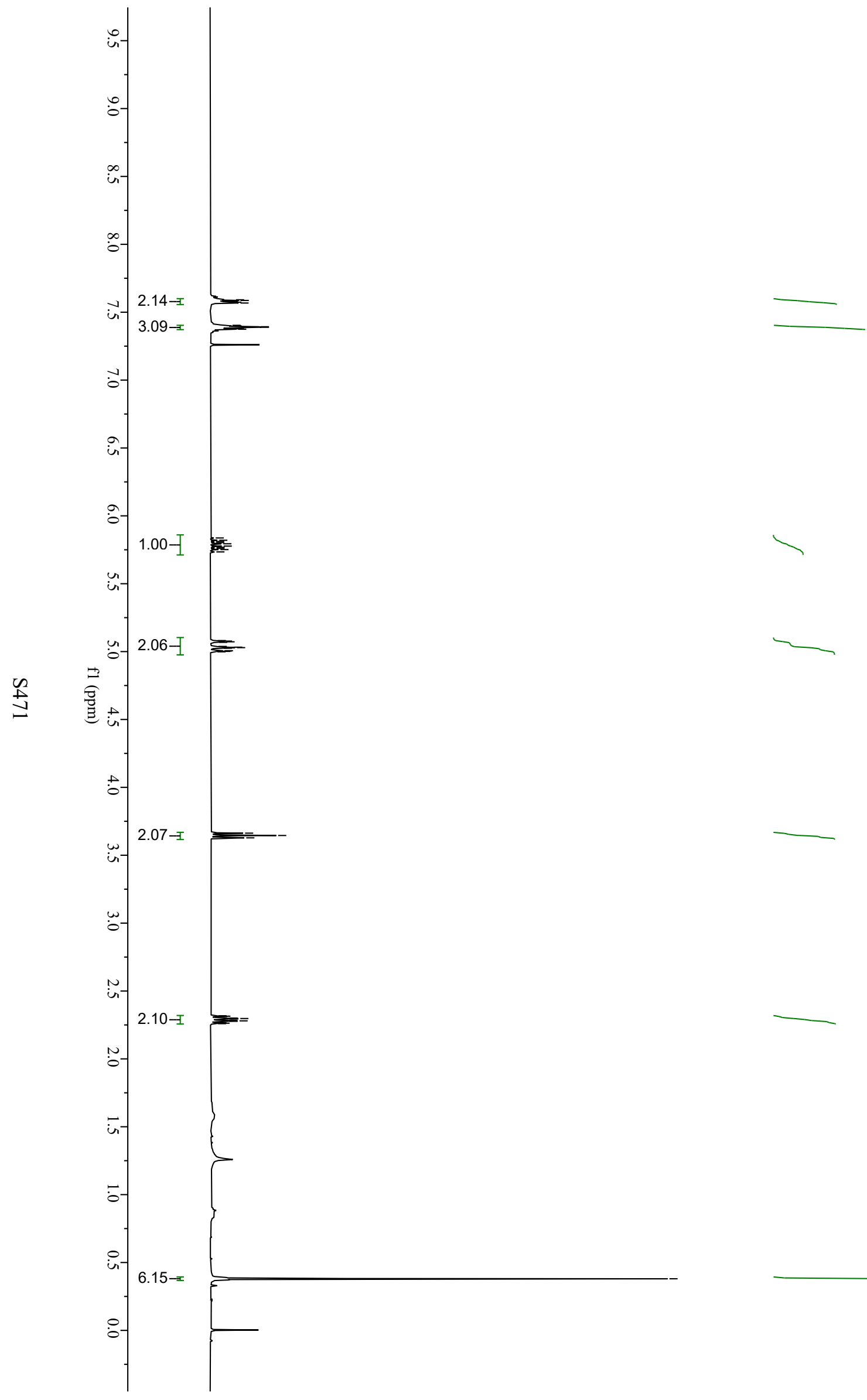

$-5.81$

5.80 5.79

5.78

5.77

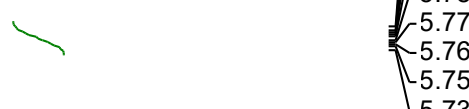

5.75
5.73

5.08
5.08

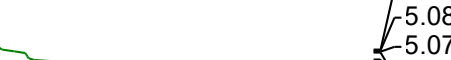

55.07

5.04

$-5.03$

5.03

$-5.02$

$-5.01$

$-5.00$

5.00

5.00

3.66

3.65

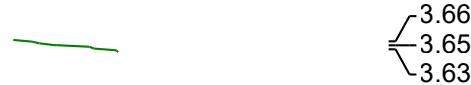

2.32
-2.31

2.31
-2.31
-2.30

2.30

2.30
2.30

2.29

2.28

2.28

2.28

2.27

$-2.26$

$-0.38$ 
Compound $6 \mathbf{r}^{13} \mathrm{C}$ NMR

$ح_{\mathrm{O}}^{-\mathrm{SiMe}_{2} \mathrm{Ph}}$

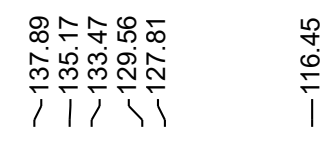

¿̊.

$\stackrel{m}{i m}$

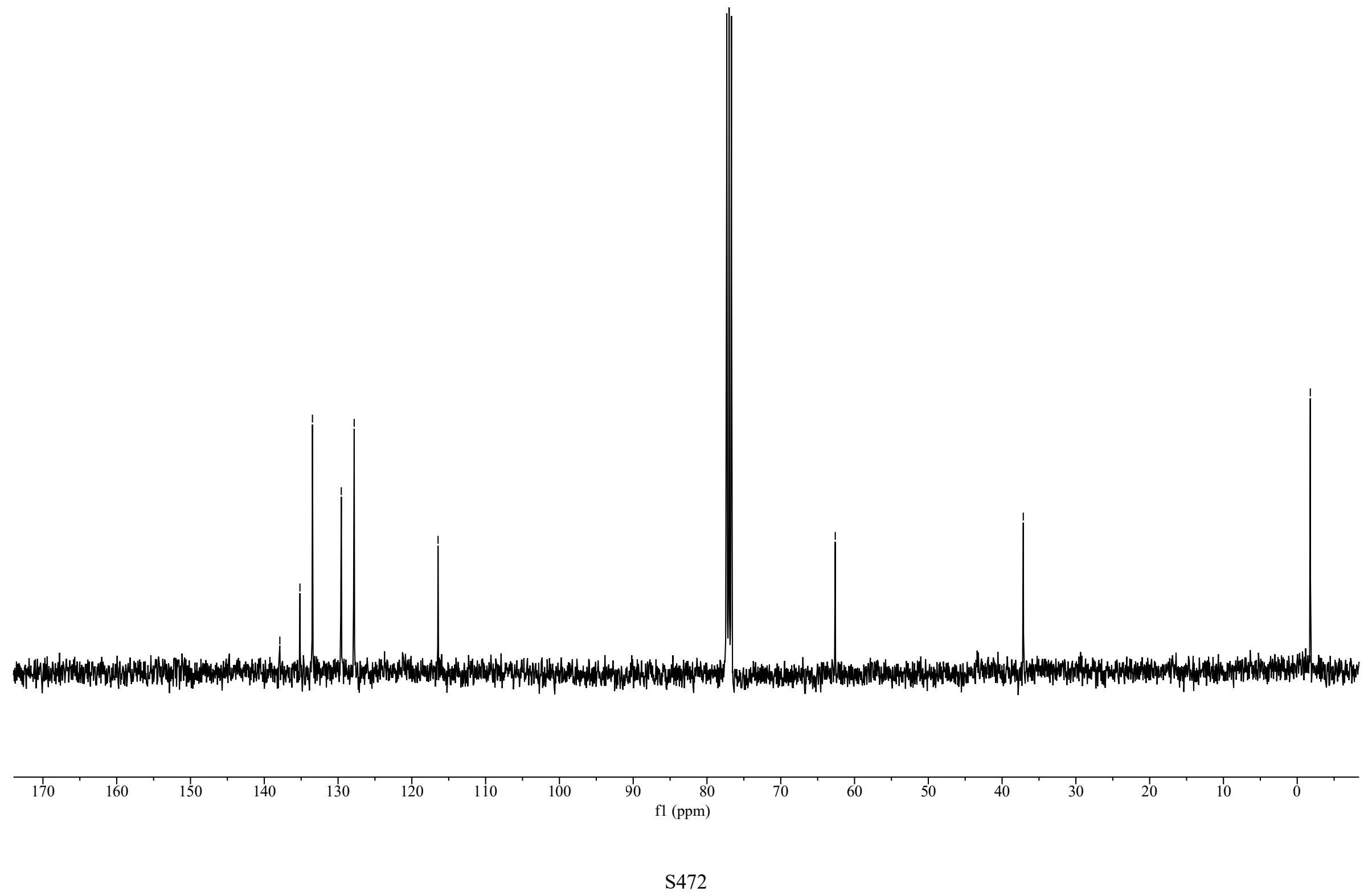




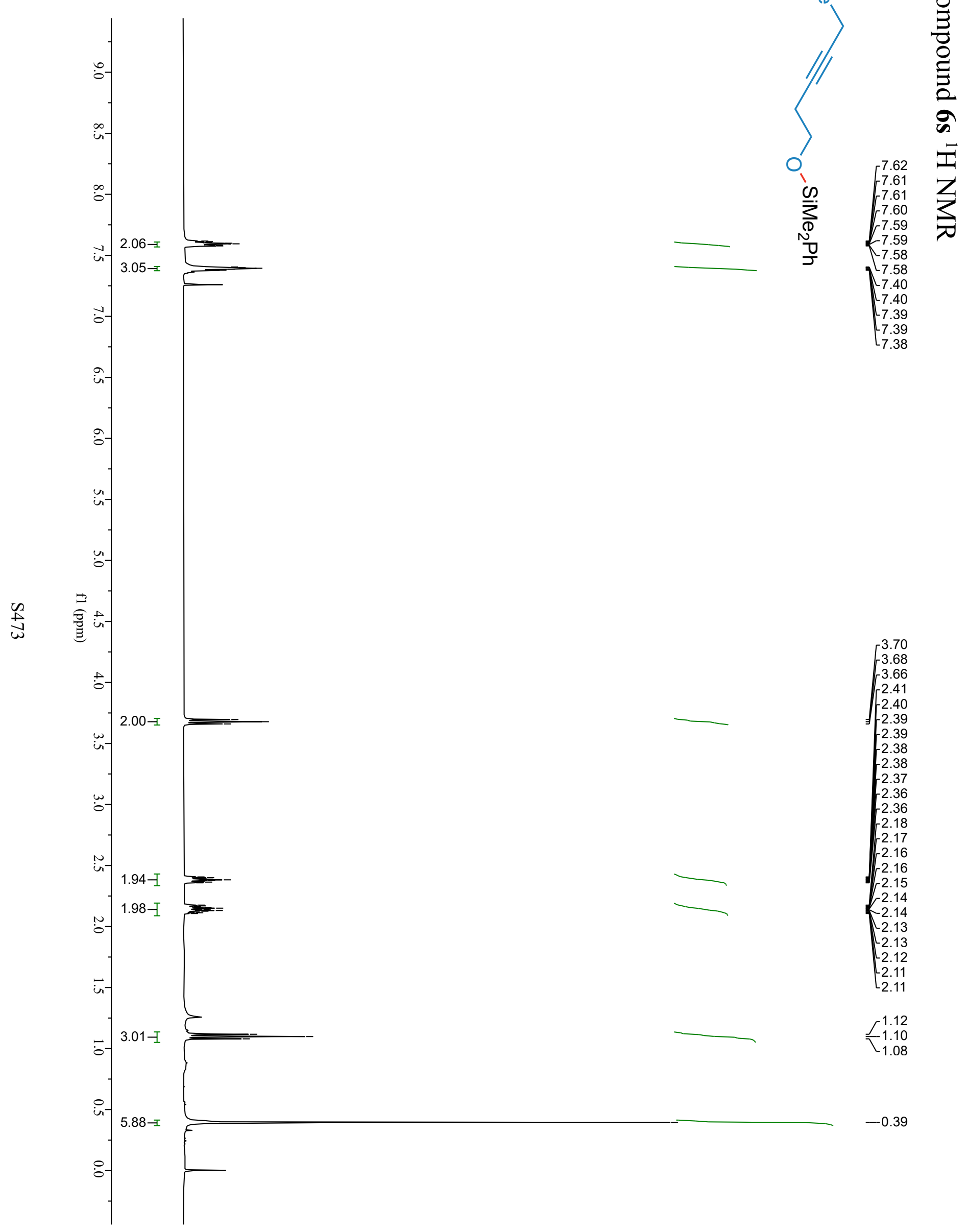


Compound $6 \mathbf{s}{ }^{13} \mathrm{C}$ NMR

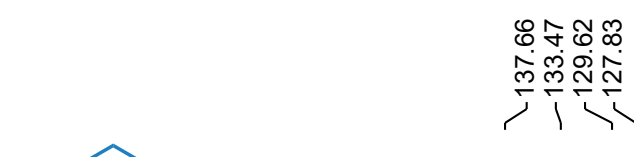

$\mathrm{Me}_{\mathrm{O}}-\mathrm{SiMe}_{2} \mathrm{Ph}$
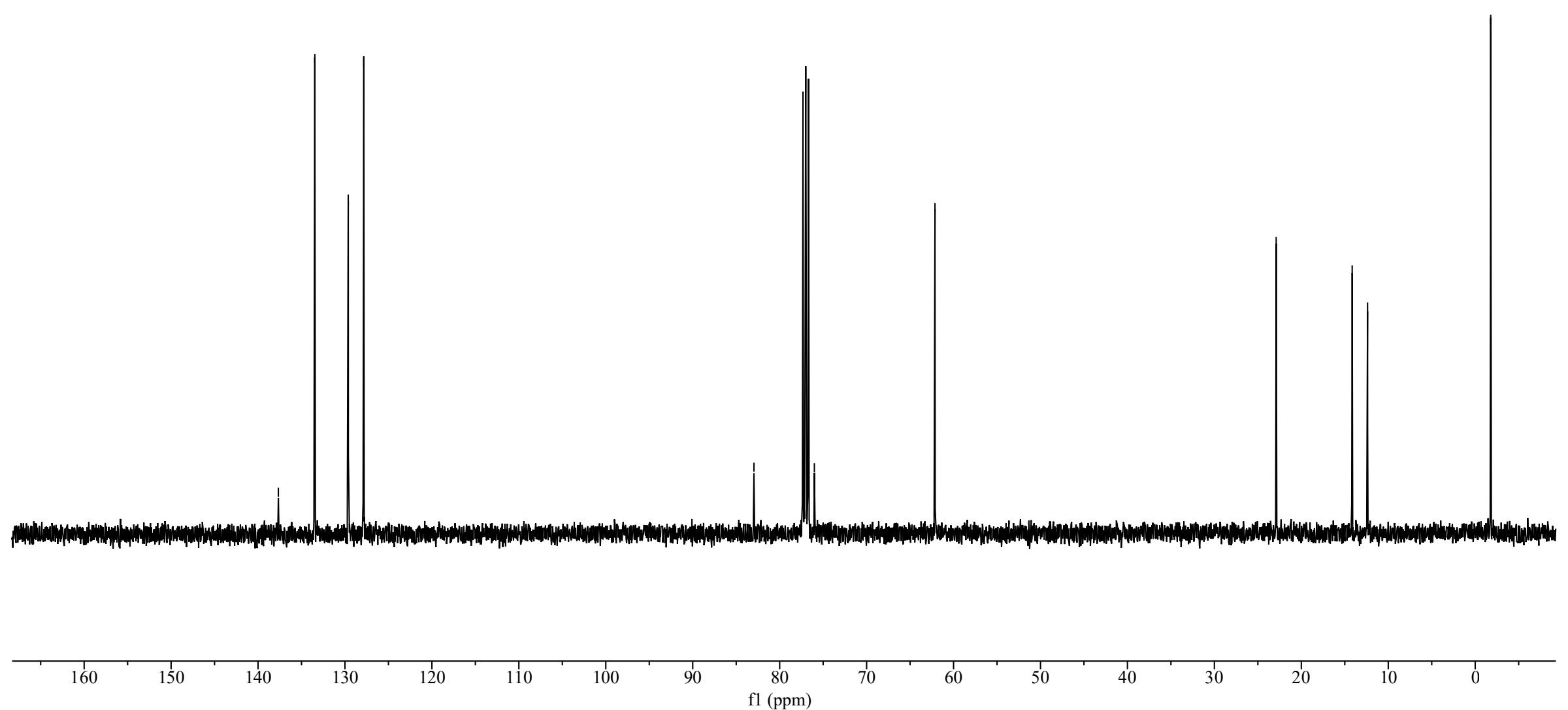

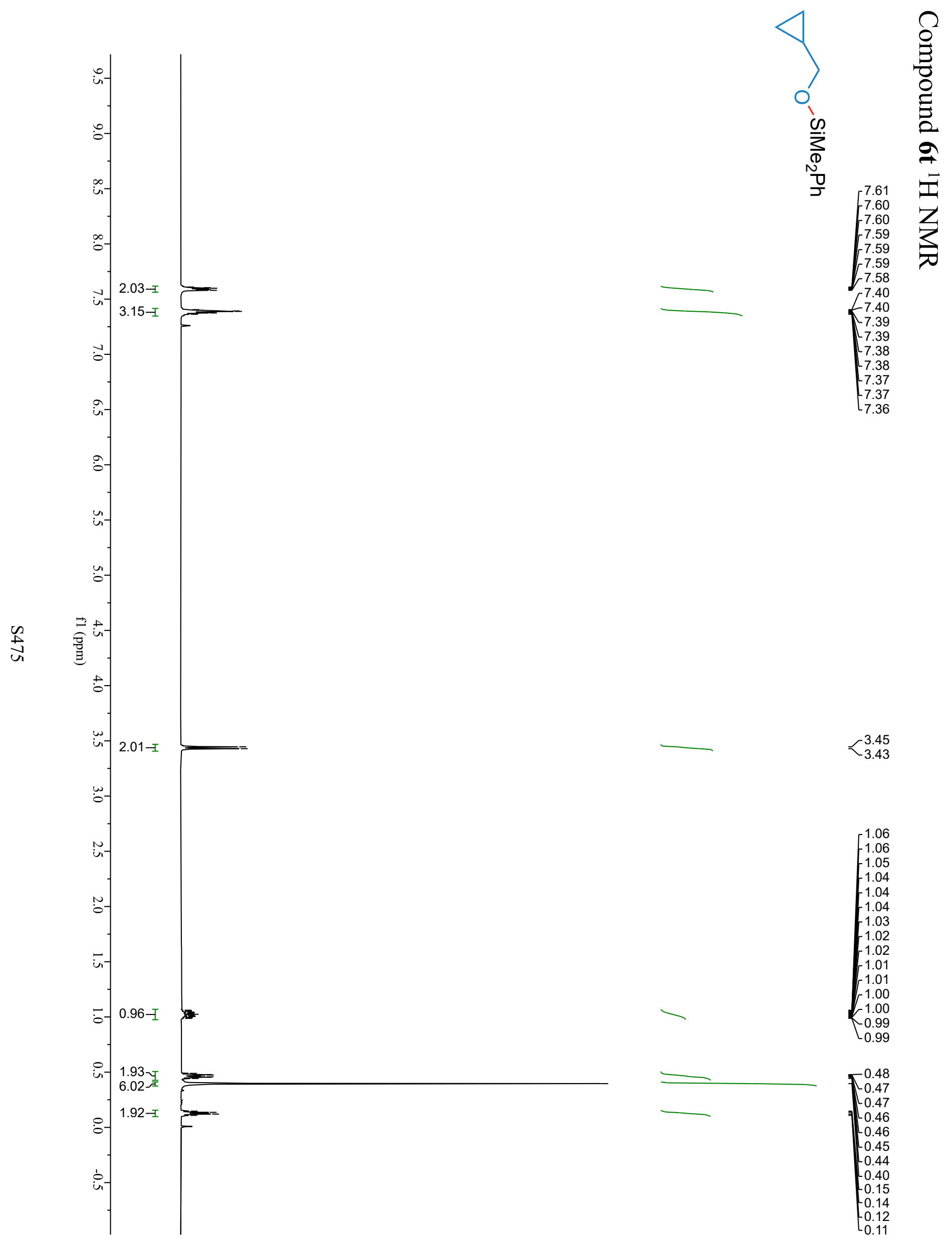
Compound $6 \mathrm{t}^{13} \mathrm{C}$ NMR

ㄷำ

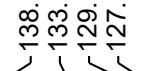

$-\mathrm{SiMe}_{2} \mathrm{Ph}$

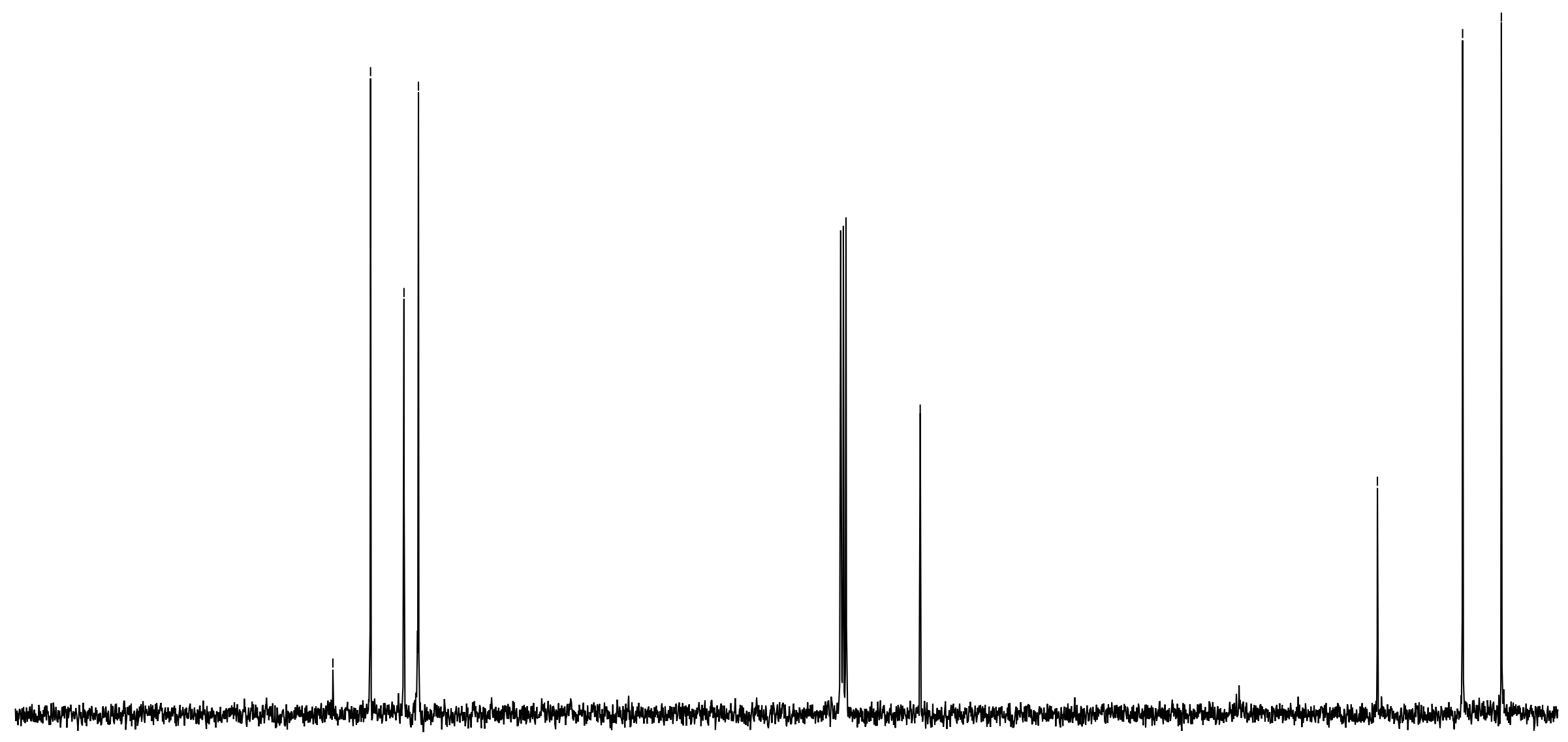



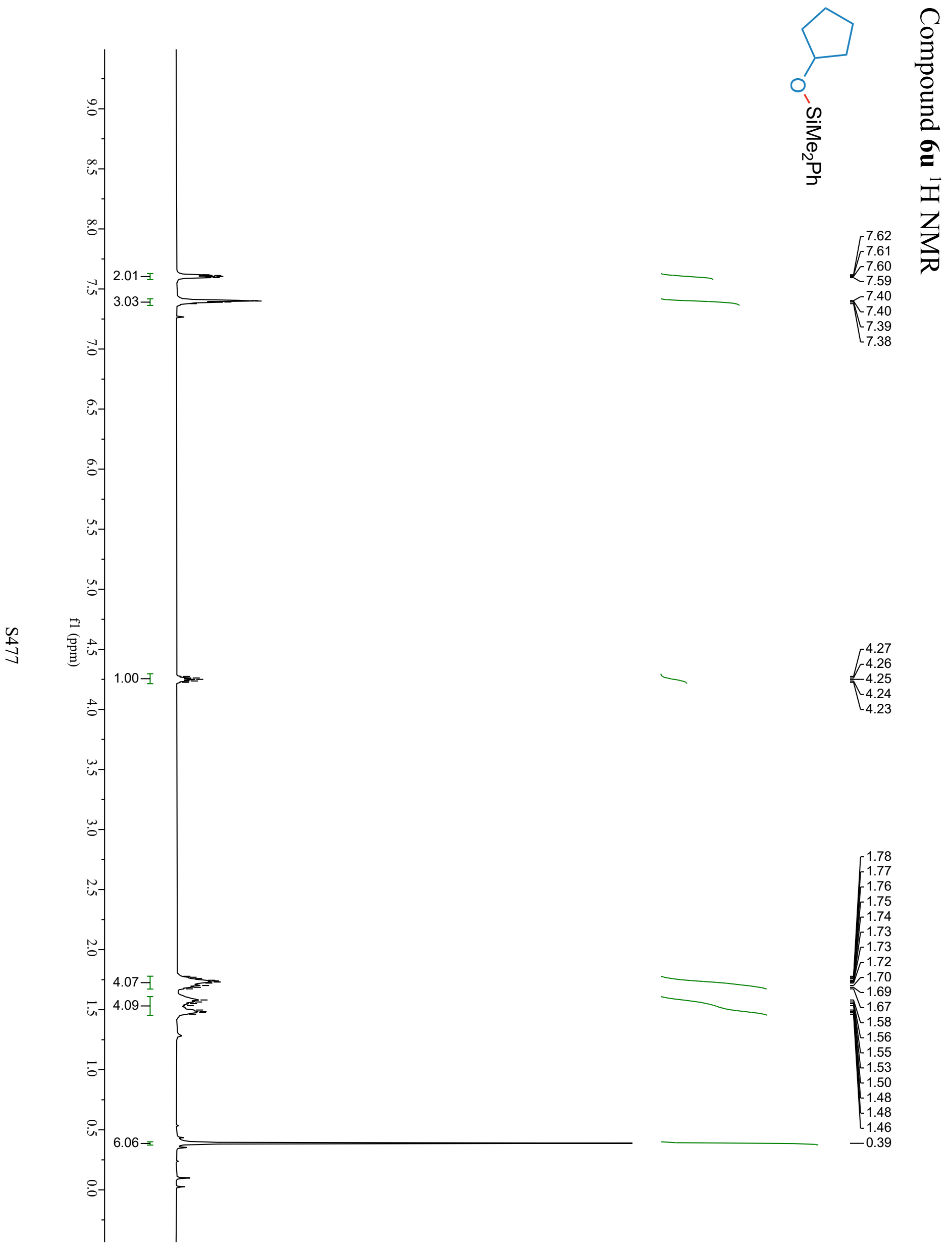
Compound $\mathbf{6 u}{ }^{13} \mathrm{C}$ NMR

$-\mathrm{SiMe}_{2} \mathrm{Ph}$
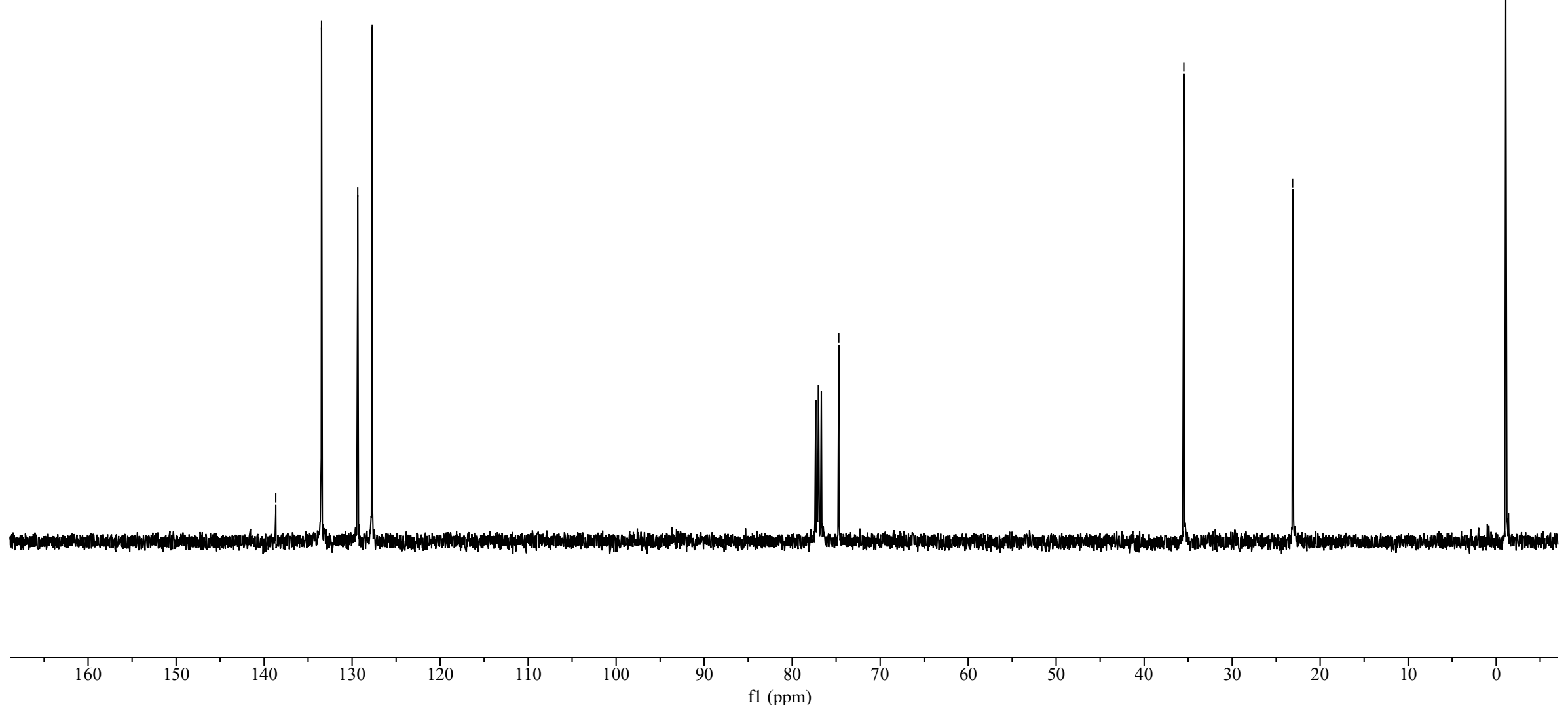


\section{Compound 6v ${ }^{1} \mathrm{H}$ NMR}

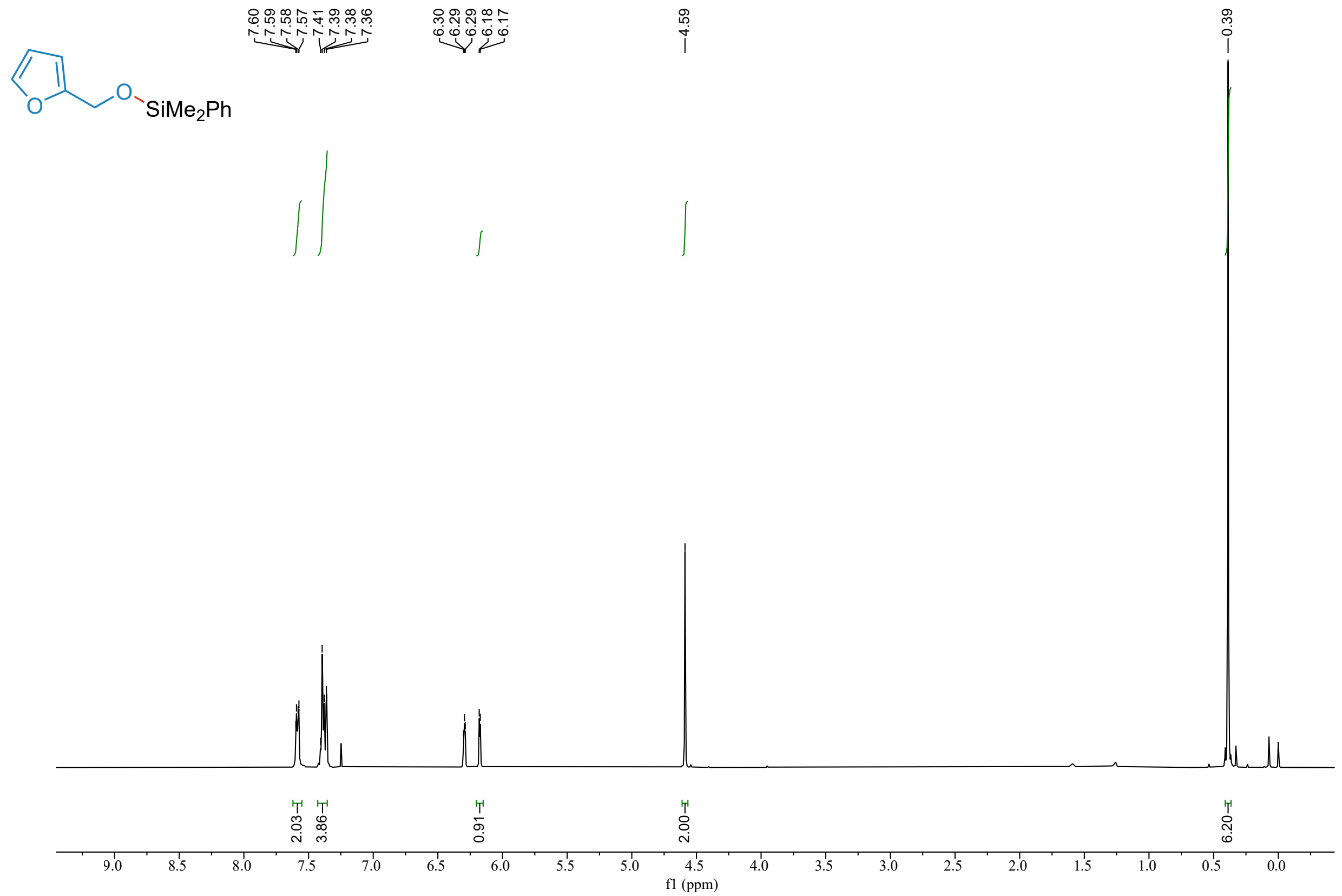


Compound $6 \mathbf{v}^{13} \mathrm{C}$ NMR

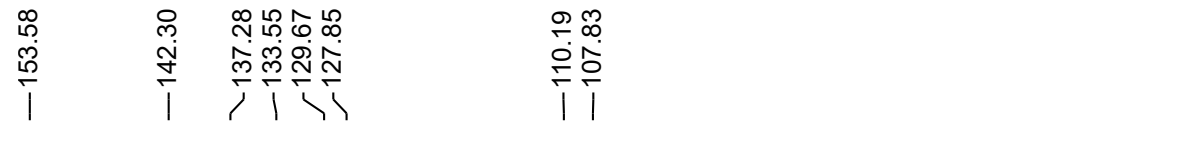

$\mathrm{SiMe}_{2} \mathrm{Ph}$
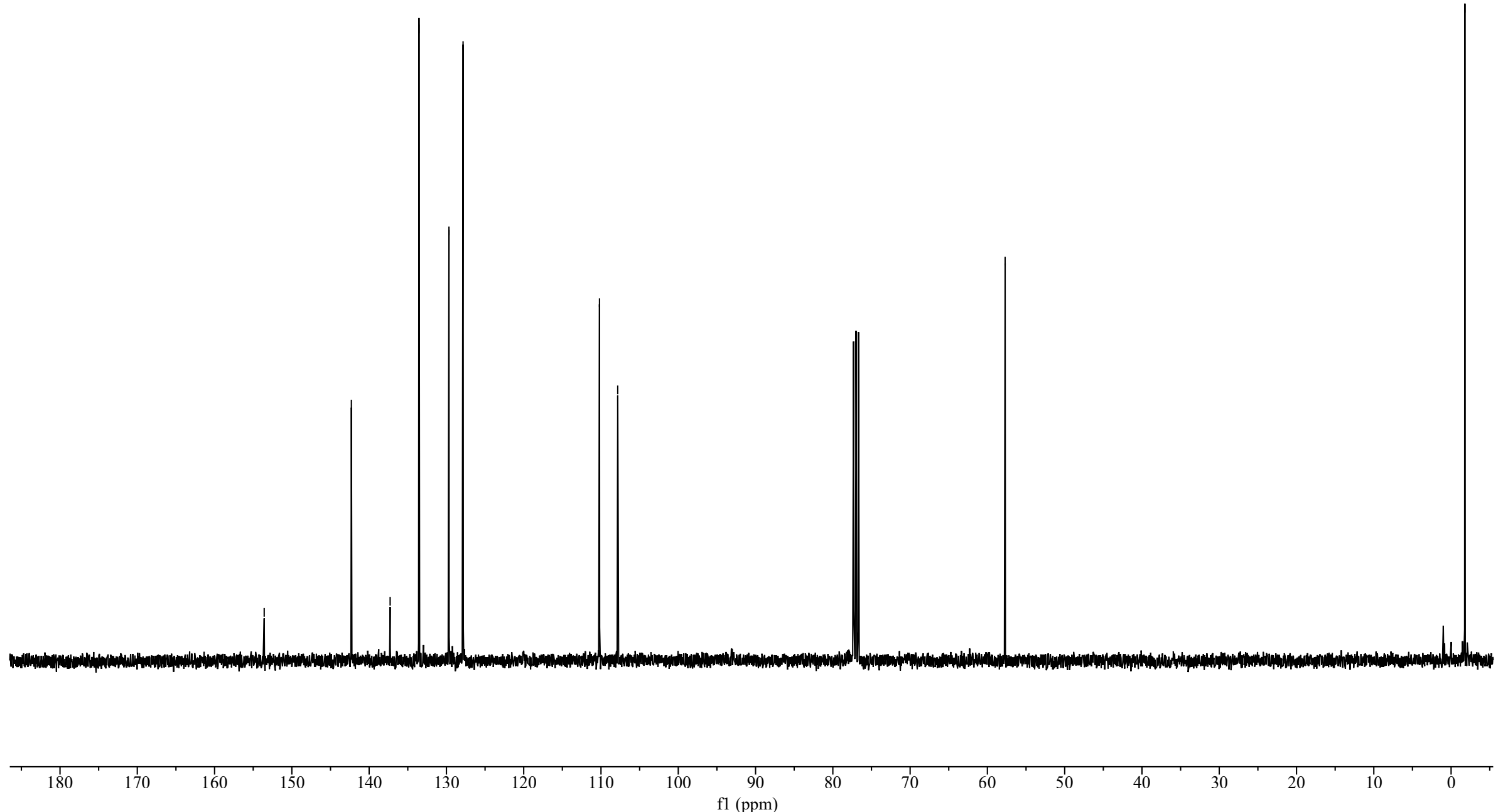


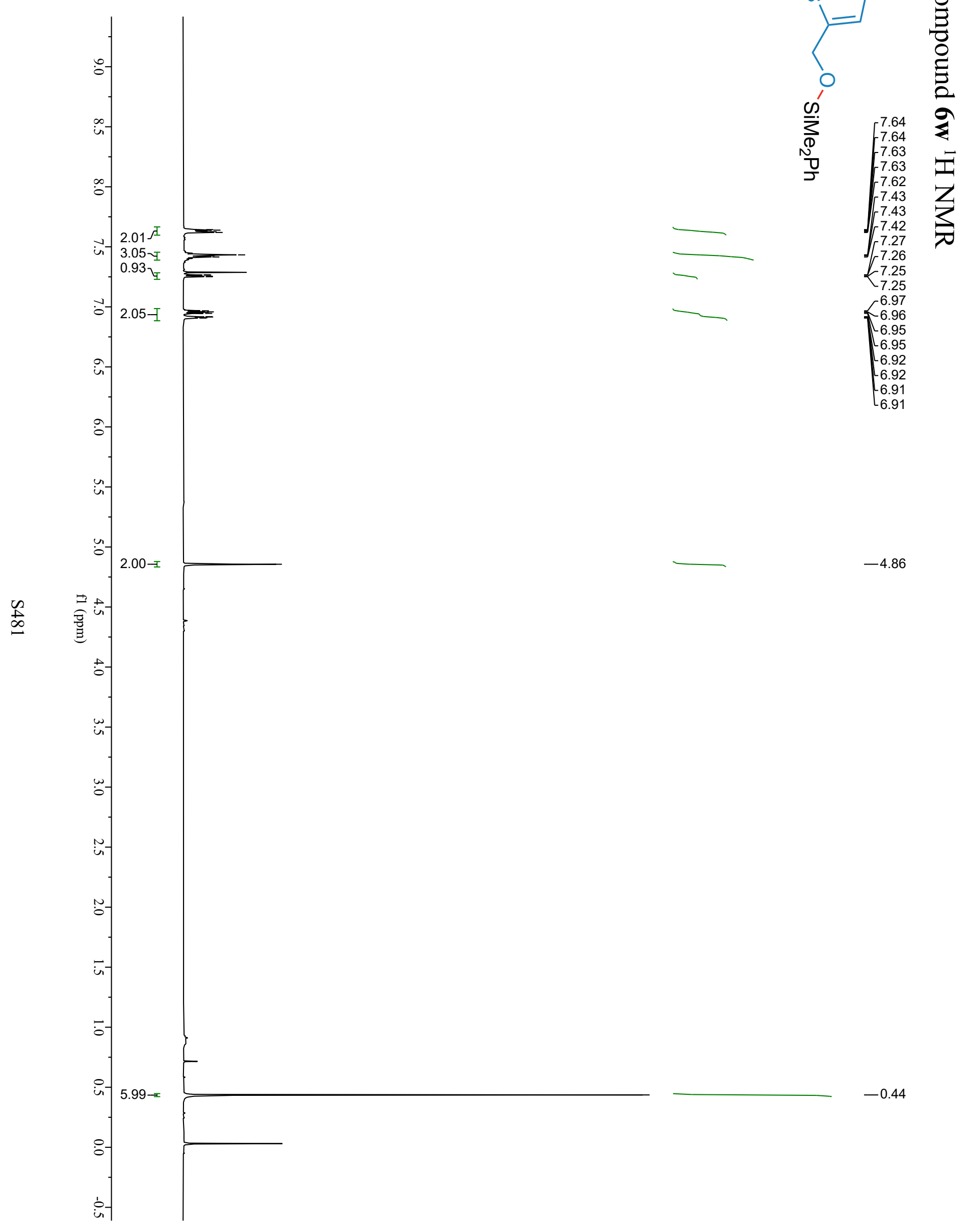


Compound $\mathbf{6 w}{ }^{13} \mathrm{C}$ NMR

둥요용요

$\left\langle\mathrm{S} \mathrm{O}_{\mathrm{SiMe}_{2} \mathrm{Ph}}\right.$

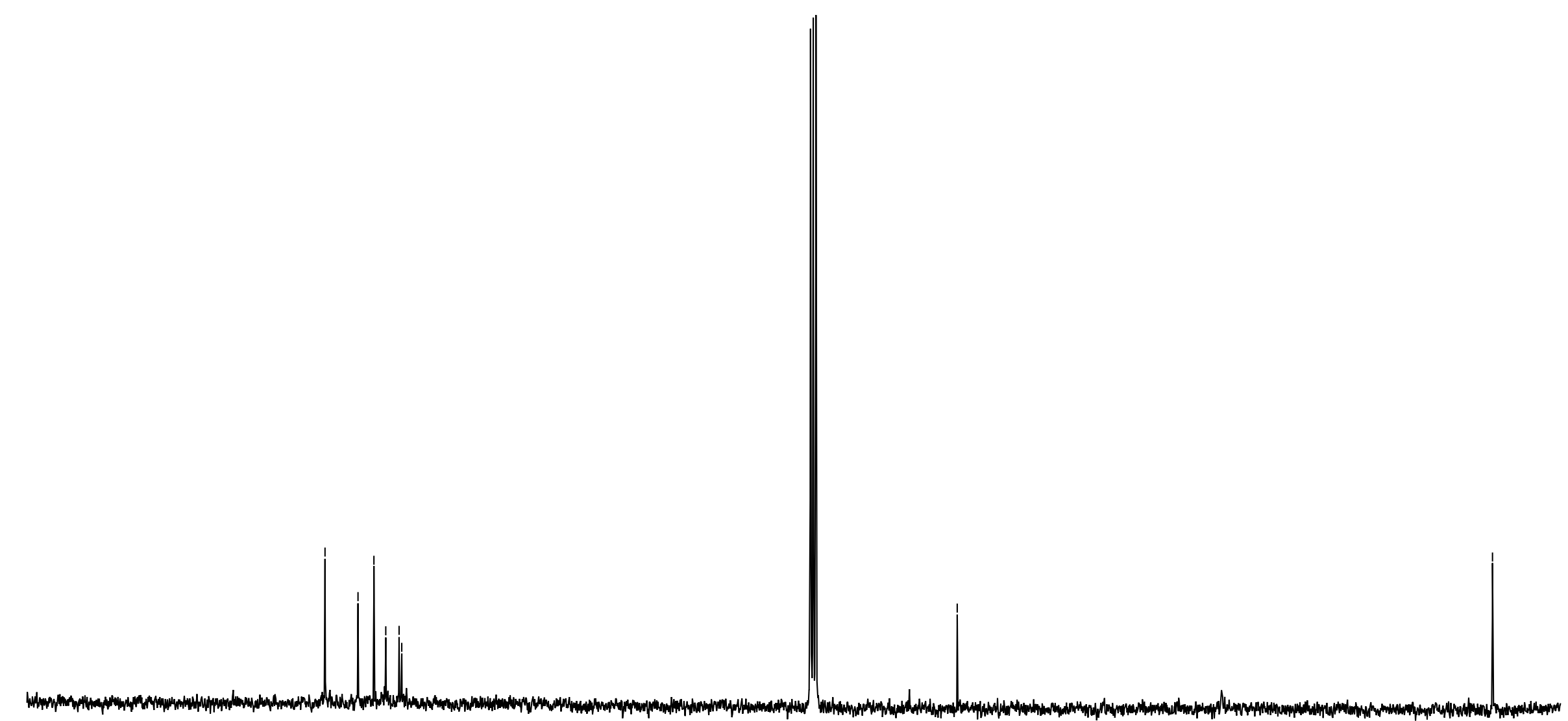




\section{Compound 6x ${ }^{1} \mathrm{H}$ NMR}

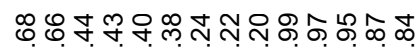
近

$\Longrightarrow \mathrm{O}^{\mathrm{SiMe}_{2} \mathrm{Ph}}$
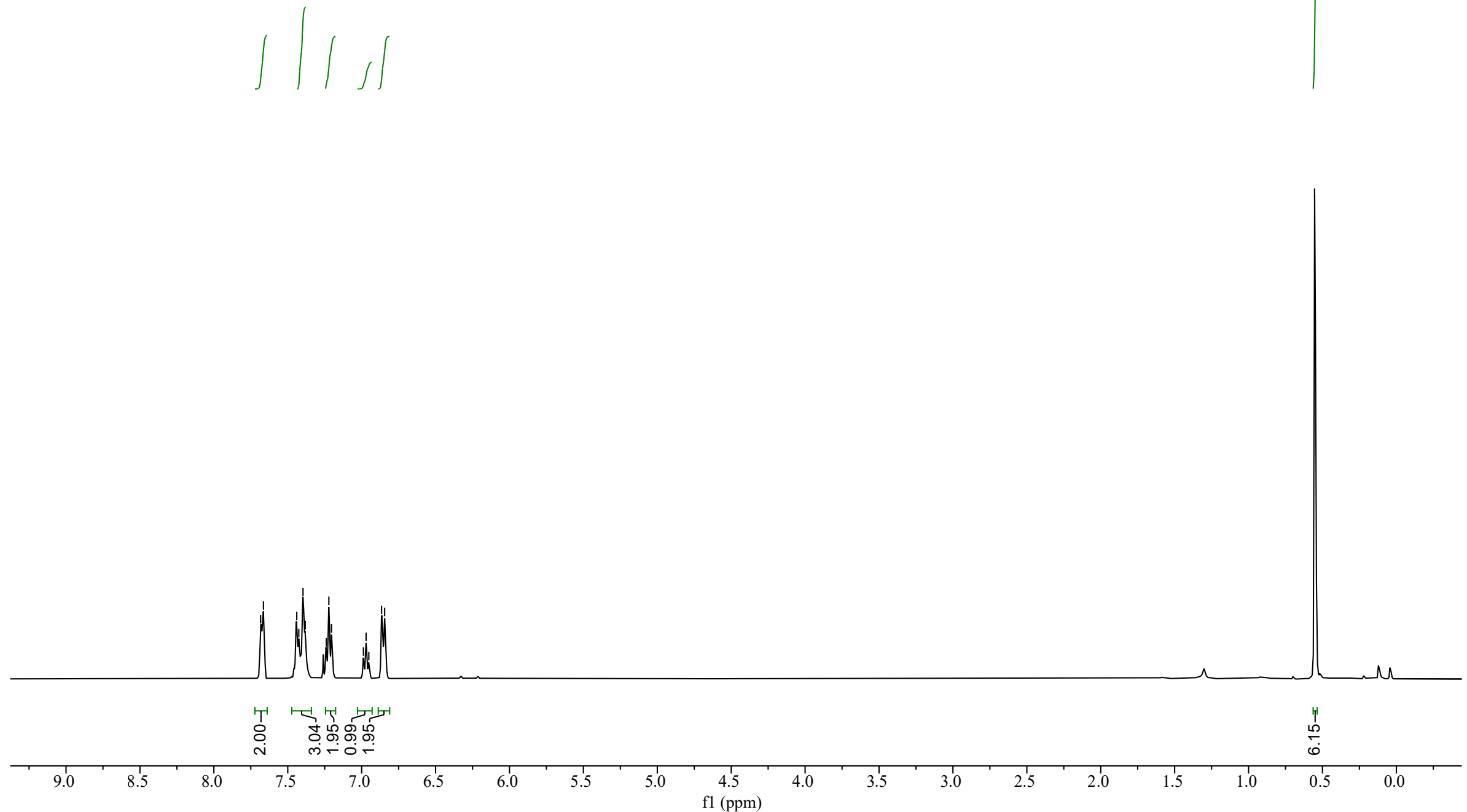
Compound $6 x{ }^{13} \mathrm{C}$ NMR
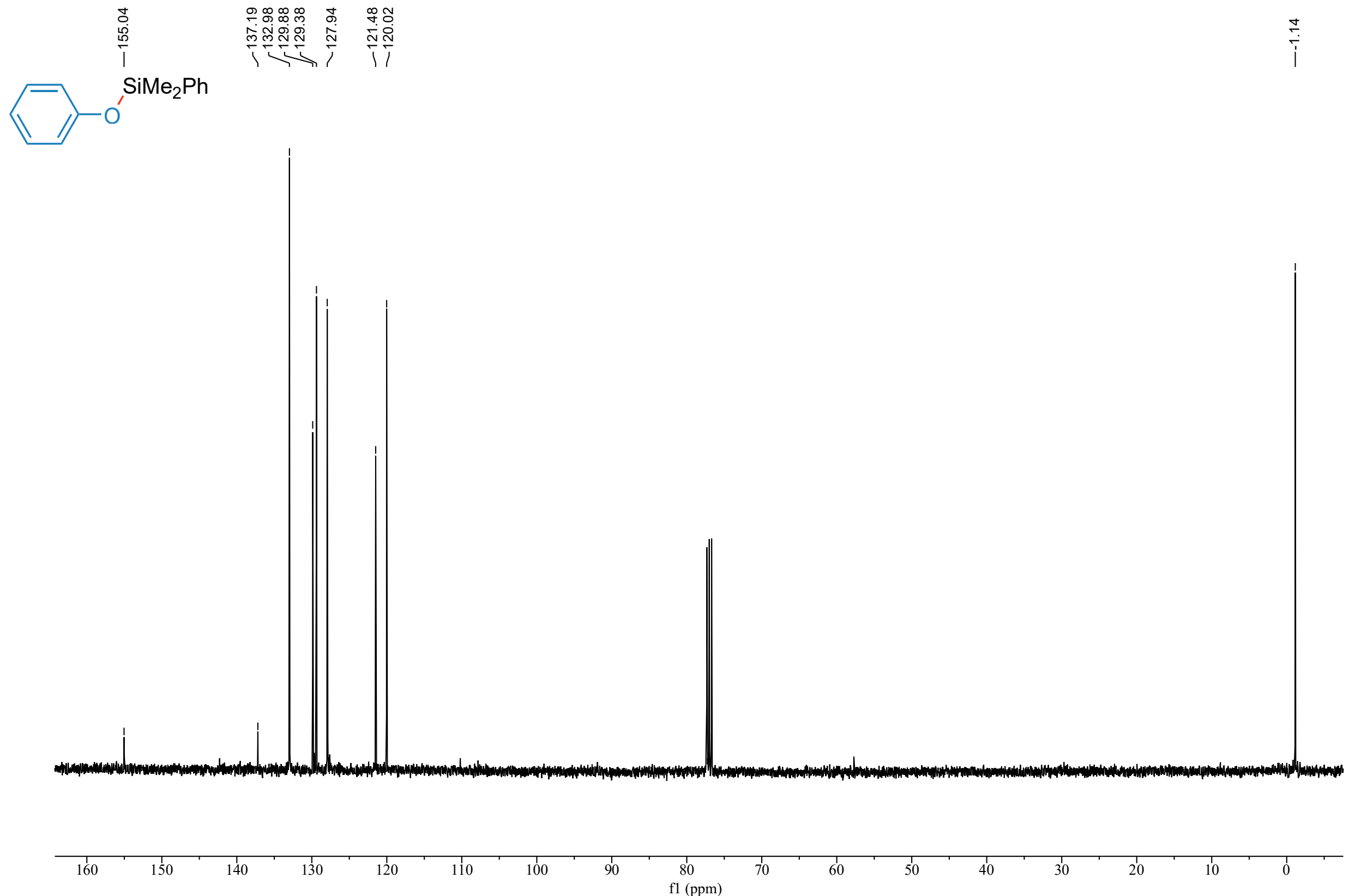

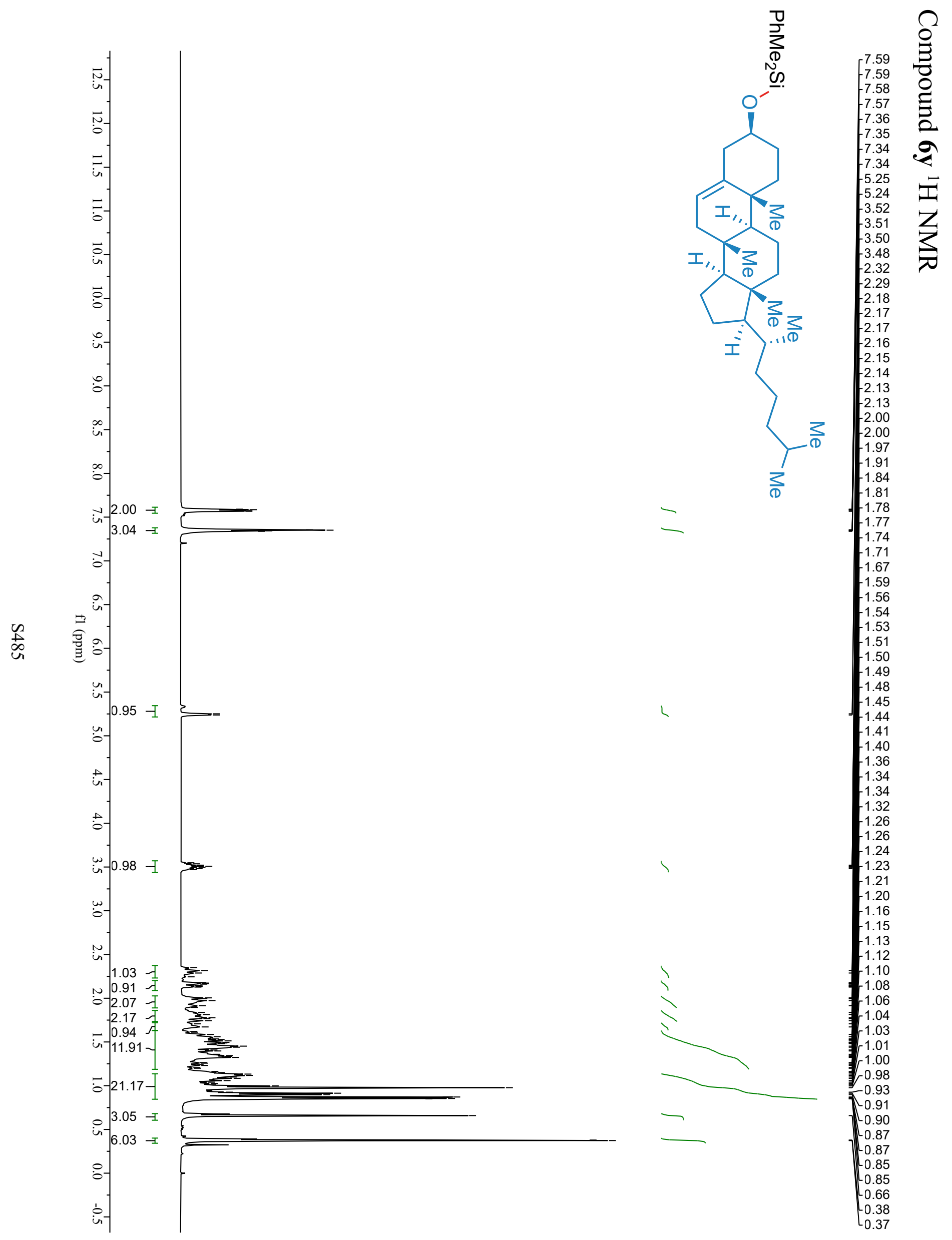


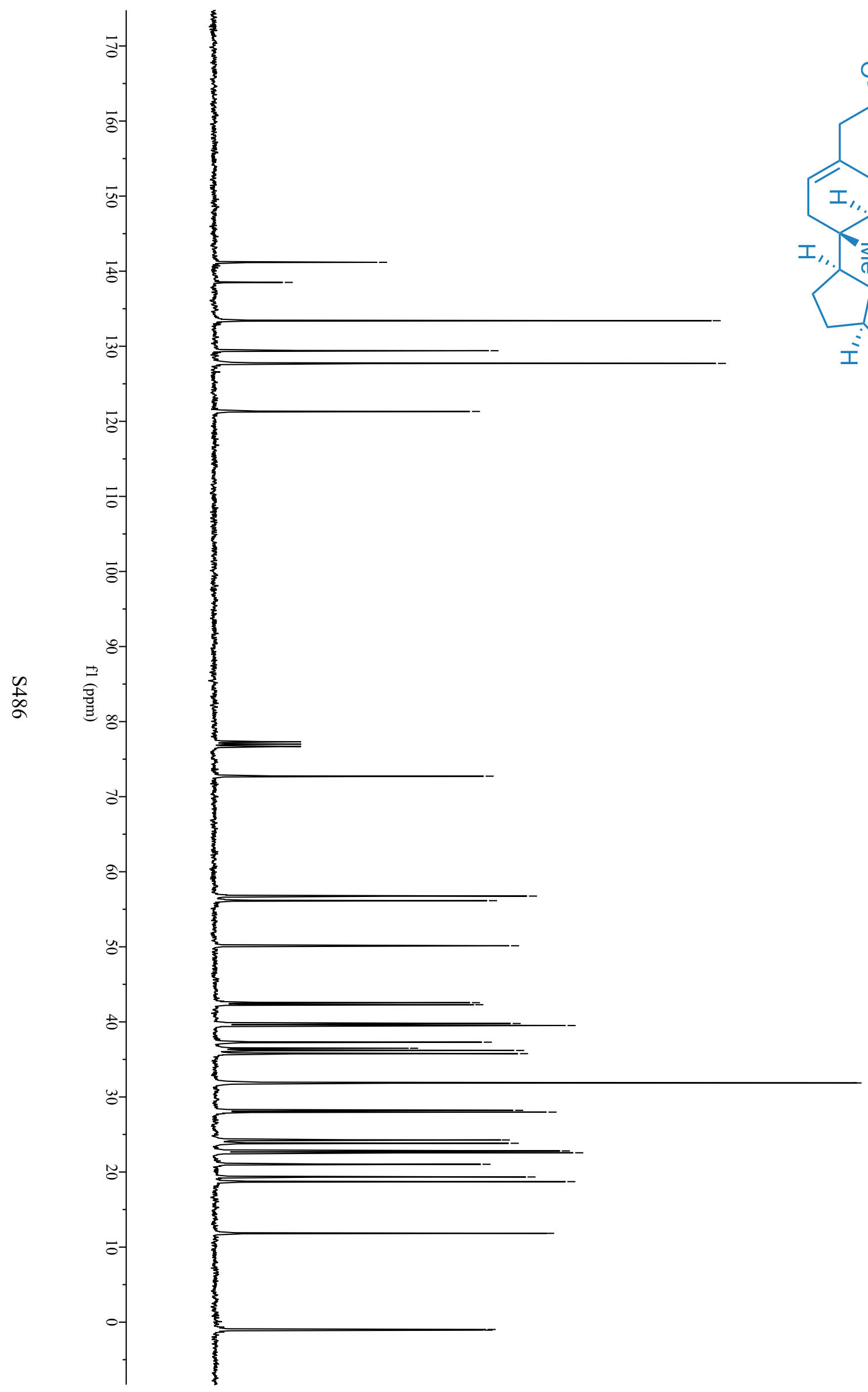

2
0
0
0
0
2
2
$\bar{\omega}$
2
3
0

$-50.14$ 42.56
-42.29 32.29 - 39.52 39.52
-37.30

36.19

35.77

31.86

$-28.22$

27.98

24.27

23.83

22.81

22.56

21.03

19.34

18.72
11.83 
Compound $8{ }^{1} \mathrm{H}$ NMR

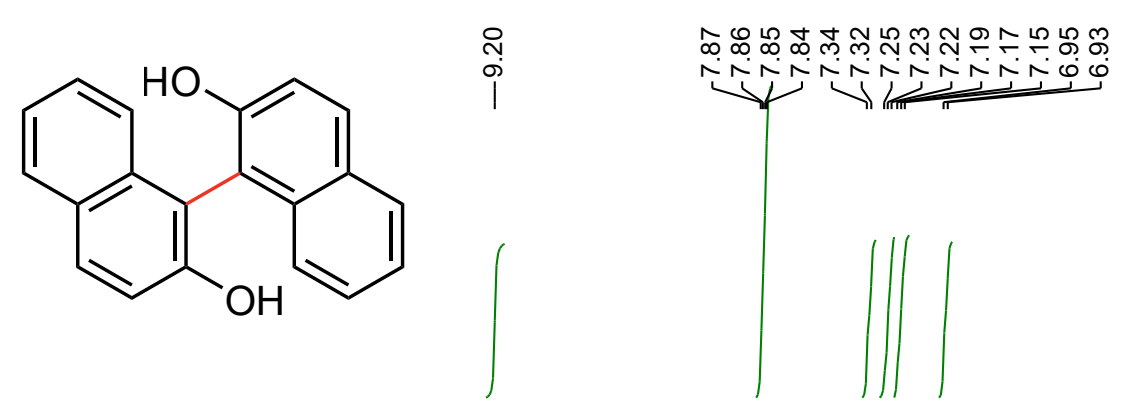

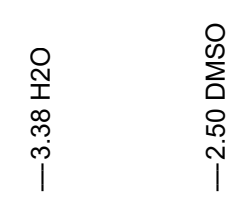

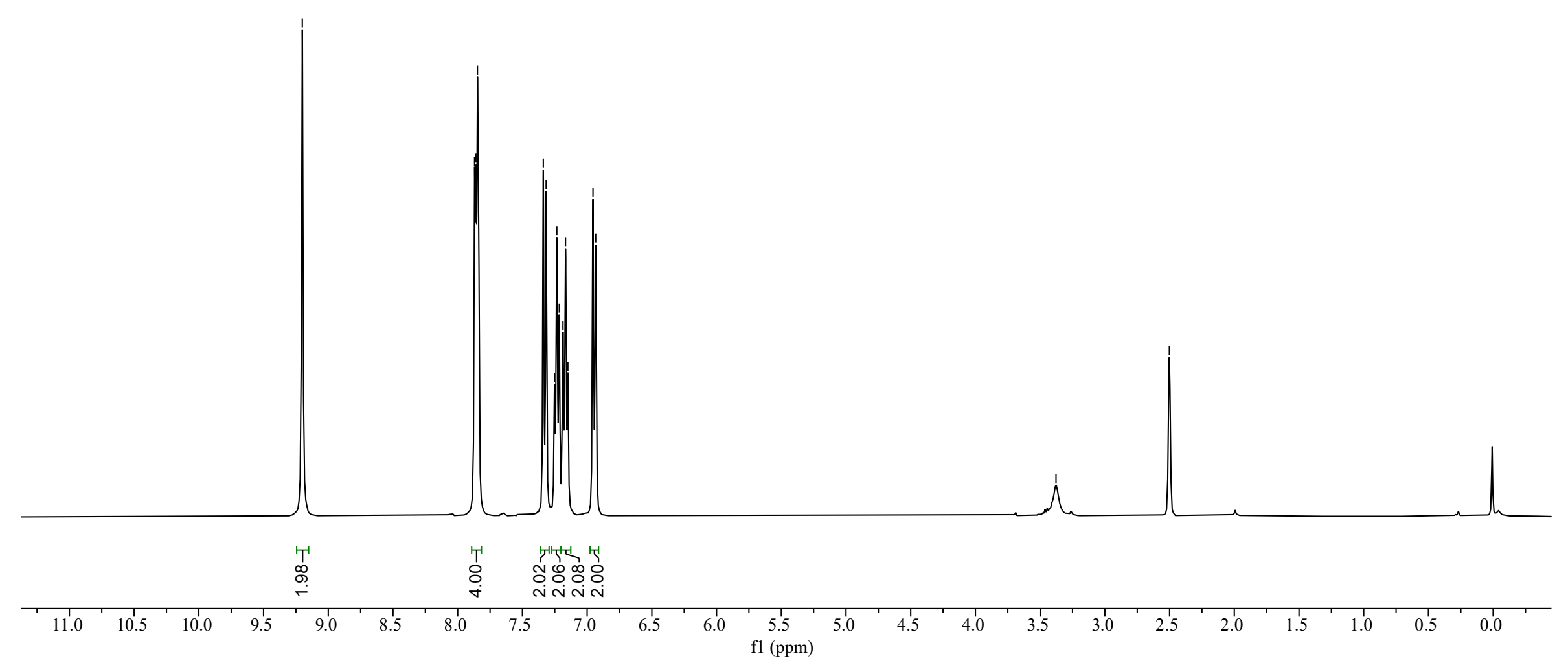


Compound $8{ }^{13} \mathrm{C}$ NMR<smiles>Oc1ccc2ccccc2c1-c1c(O)ccc2ccccc12</smiles>

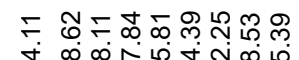

每

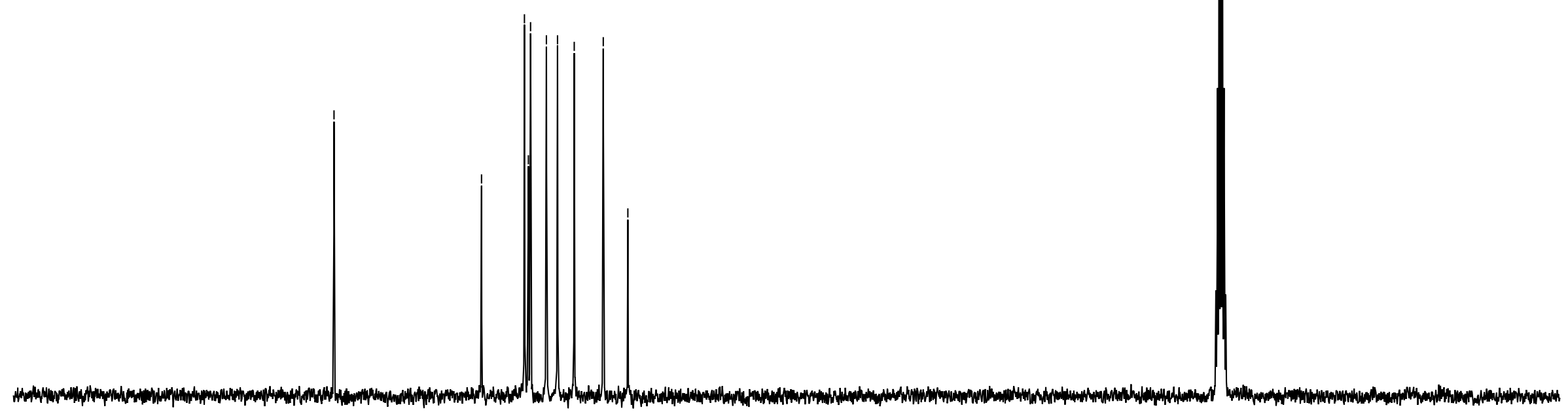


Compound $10{ }^{1} \mathrm{H}$ NMR

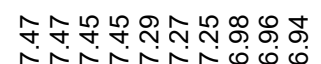

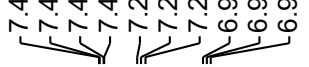

प)
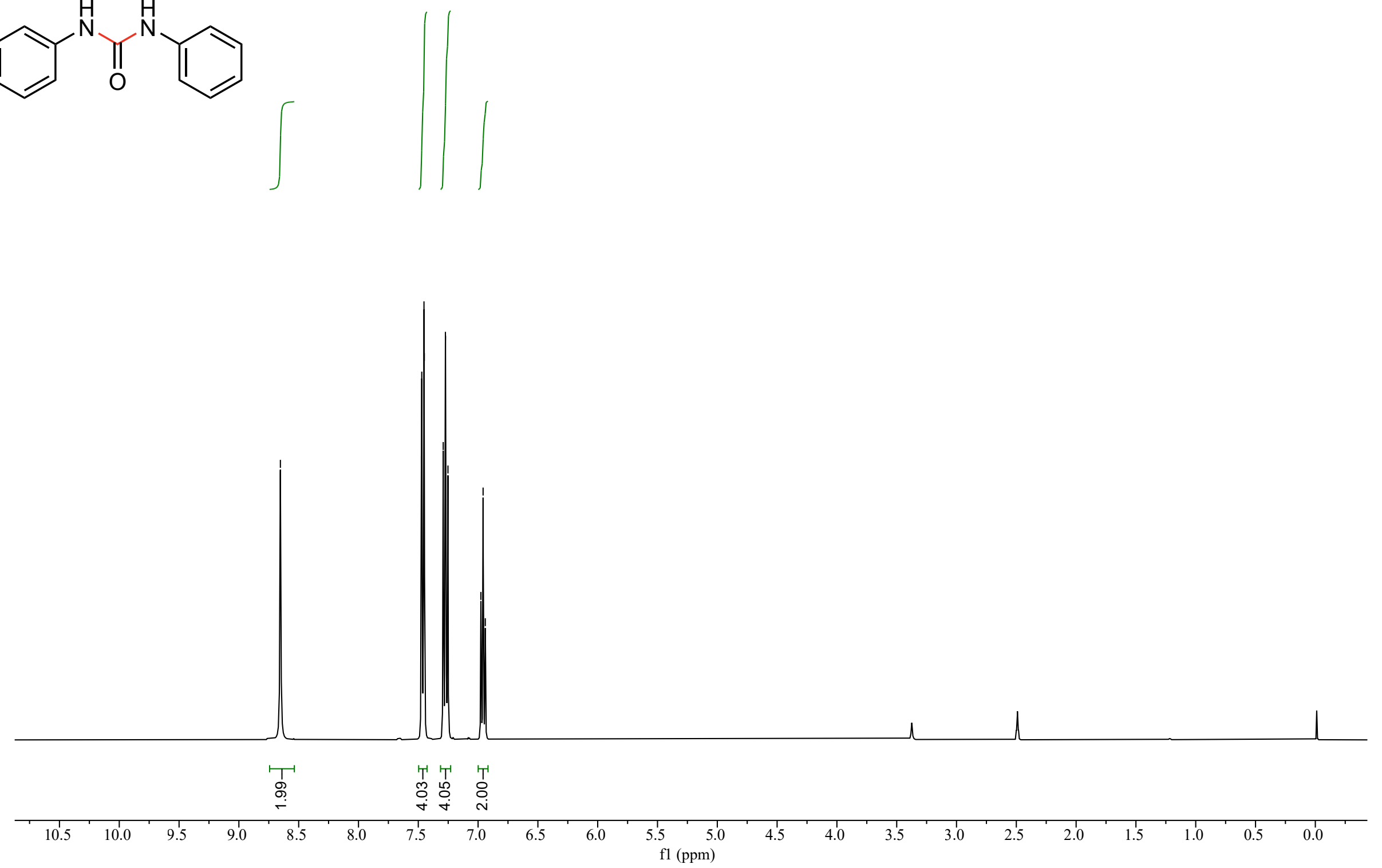


\section{Compound $10{ }^{13} \mathrm{C}$ NMR}

(N)

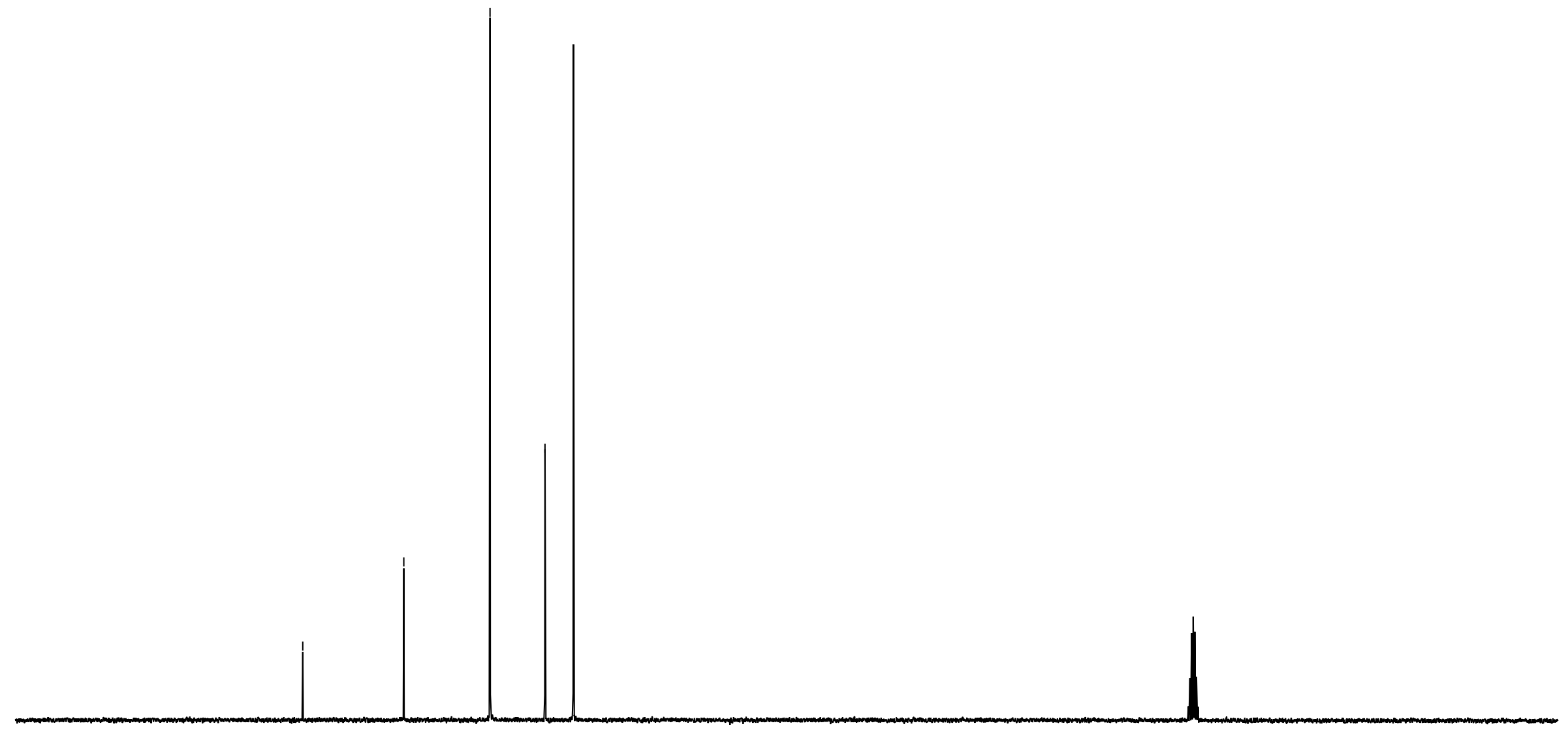



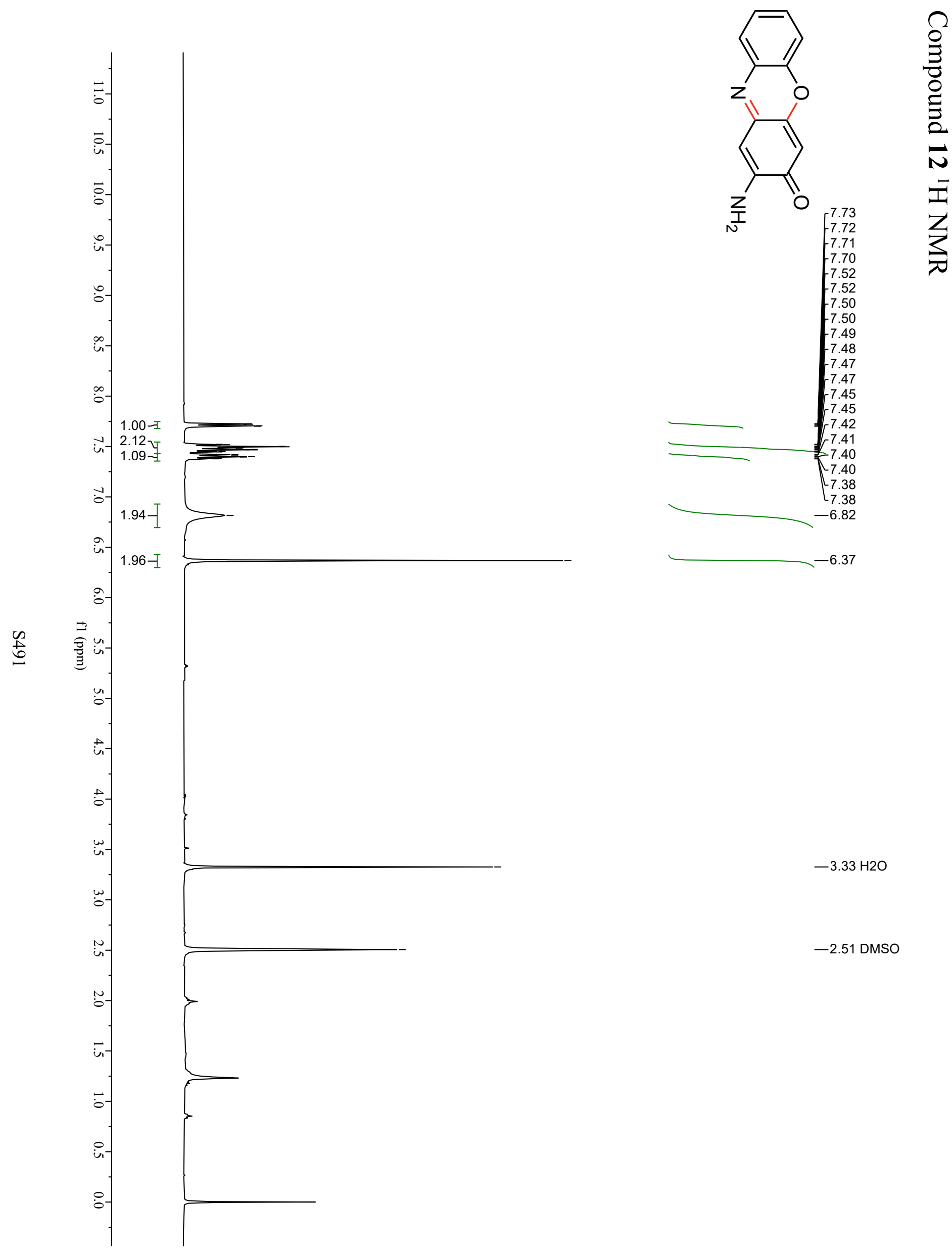

$-3.33 \mathrm{H} 2 \mathrm{O}$

$-2.51 \mathrm{DMSO}$ 
Compound $12{ }^{13} \mathrm{C}$ NMR

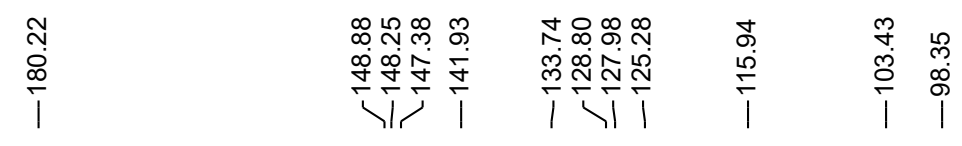<smiles>Nc1cc2nc3ccccc3oc-2cc1=O</smiles>
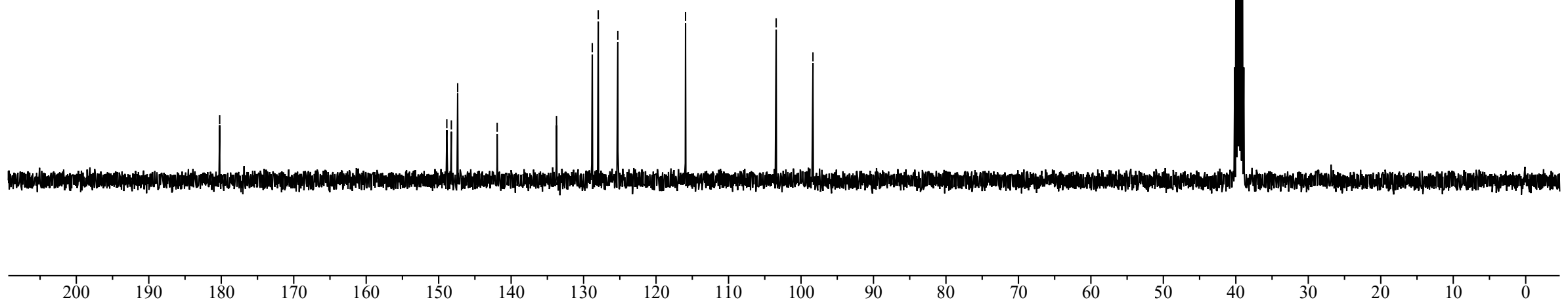


\section{Geometries and Cartesian coordinates for optimized structures}

$\mathrm{CoN}_{4} \mathrm{C}_{10}$

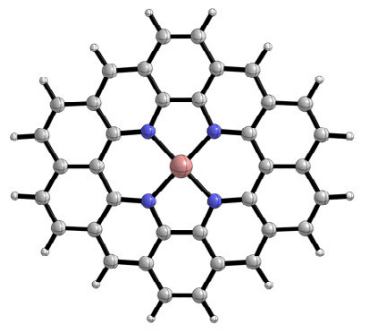

0.00127290

0.00227047

0.00441258

0.00436895

0.00249067

0.00514144

0.00347866

0.00030994

$-0.00158598$

$-0.00024603$

0.00257470

0.00218506

$-0.00120250$

0.00117415

0.00221287

0.00416795

0.00409310

0.00229091

0.00478248

0.00314973

0.00021304

$-0.00179276$

$-0.00065218$

0.00210779

0.00195476

$-0.00170897$

0.00589697

0.00612443

0.00627911
$\mathrm{H} \quad 5.43178459$

C $\quad 5.50037729$

$\mathrm{H} \quad 6.58483248$

$-4.87536605$

$-5.43179617$

$-4.75035945$

$-4.87498171$

$-5.43126391$

$-5.50019502$

$-6.58466101$

$-5.50038871$

$-6.58484374$

5.50018361

6.58464977

$-0.67914746$

0.67833242

1.24294520

$-0.67834483$

0.67913505

1.24389506

$-1.24295780$

$-1.24390748$

$-0.00000591$

1.31694761

1.31716784

$-1.31695956$

$-1.31717964$
2.42477077

3.35839551

1.22132114

1.15189256

$-2.42478168$

$-3.35840655$

0.00037152

2.42554361

3.35925657

1.22219295

1.15293616

$-1.22133063$

$-1.15190084$

$-1.22220242$

$-1.15294460$

$-5.06512286$

$-5.06523050$

$-5.99359225$

5.06521384

5.06510618

5.99337727

5.99357454

$-5.99339509$

$-0.00000608$

$-1.38944726$

1.38922582

1.38943466

$-1.38923849$
0.00393757

0.00340777

0.00625461

0.00769951

0.00629852

0.00653469

0.00679074

0.00458750

0.00416952

0.00692244

0.00849859

0.00776969

0.00927639

0.00725163

0.00865491

$-0.00042204$

$-0.00046531$

$-0.00150151$

$-0.00425322$

$-0.00435606$

$-0.00612382$

$-0.00593582$

$-0.00142186$

0.00362087

0.00344482

0.00236502

0.00252705

0.00356650

\section{CoPc}

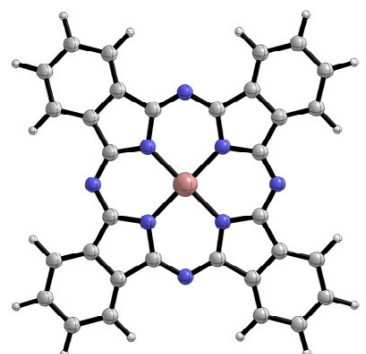

Co

0.00000000

0.00000000

1.79560300

$-0.71509100$

$-1.79560300$

0.71509200

$-0.00740900$
$-0.71509500$

$-1.79560400$

0.71509500

1.79560400

2.96589300
$-0.00013400$

$-0.00321500$

0.00296900

$-0.00322200$

0.00296700

0.02147500
C $\quad 1.77074400$

C $\quad-3.60391600$

C $\quad-4.12269100$

C $\quad-4.42371500$

C $\quad-5.48475200$
5.81664200 6.33382500

2.18916000

0.88670400

3.30415500

0.64157700
0.09235800

0.10216300

$-0.04100900$

$-0.05012700$

$-0.06082100$

$-0.08014600$ 


$\begin{array}{lrr}\text { C } & -2.14912100 & 2.04292400 \\ \text { C } & -2.96589300 & -0.00740700 \\ \text { C } & -2.04292000 & -2.14911900 \\ \text { C } & 0.00741000 & -2.96589400 \\ \text { C } & 2.14912100 & -2.04292400 \\ \text { C } & 2.04292100 & 2.14911900 \\ \text { C } & 2.96589300 & 0.00740700 \\ \text { C } & 3.60391600 & -2.18916100 \\ \text { C } & 4.12269100 & -0.88670400 \\ \text { C } & 4.42371500 & -3.30415500 \\ \text { C } & 5.48475200 & -0.64157700 \\ \text { C } & 5.81666100 & -3.06892700 \\ \text { C } & 6.33384100 & -1.77073200 \\ \text { C } & -0.88670400 & -4.12268700 \\ \text { C } & -2.18916000 & -3.60390900 \\ \text { C } & -0.64158500 & -5.48474500 \\ \text { C } & -3.30415800 & -4.42369900 \\ \text { C } & -1.77074500 & -6.33382500 \\ \text { C } & -3.06893700 & -5.81664100 \\ \text { C } & 2.18916000 & 3.60391000 \\ \text { C } & 0.88670500 & 4.12268700 \\ \text { C } & 3.30415900 & 4.42370000 \\ \text { C } & 0.64158400 & 5.48474500 \\ & & \end{array}$

\section{CoPc-16CI}

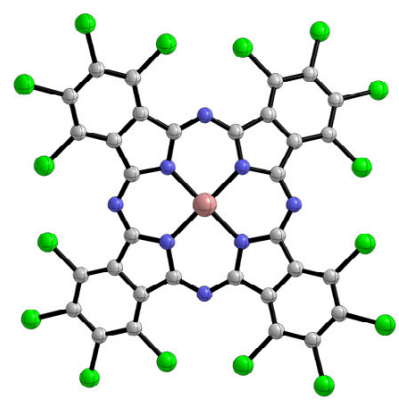

$\begin{array}{lrrr}\text { Co } & -0.00000264 & -0.00000335 & 0.14373554 \\ \text { N } & -0.00845213 & 1.98236276 & 0.07754870 \\ \text { N } & 1.96860141 & 0.00836896 & -0.03143203 \\ \text { N } & 0.00844950 & -1.98236856 & 0.07753338 \\ \text { N } & -1.96860858 & -0.00837388 & -0.03140425 \\ \text { C } & -2.73275442 & -1.12054916 & 0.20826450 \\ \text { C } & -1.09387839 & -2.74667343 & -0.18432320 \\ \text { C } & 1.11718967 & -2.73725106 & -0.18461795 \\ \text { C } & 2.74222469 & -1.09729180 & 0.20792466 \\ \text { C } & 2.73275072 & 1.12054295 & 0.20823662 \\ \text { C } & 1.09387034 & 2.74667200 & -0.18431089 \\ \text { C } & -2.74222737 & 1.09728439 & 0.20797351 \\ \text { C } & -1.11719770 & 2.73724625 & -0.18458236 \\ \text { C } & 0.68617254 & 4.07384270 & -0.67208248 \\ \text { C } & -0.72070531 & 4.06782437 & -0.67231489 \\ \text { C } & 1.39776443 & 5.18715975 & -1.07423581 \\ \text { C } & -1.44166574 & 5.17499500 & -1.07475030 \\ \text { C } & 0.66988798 & 6.32467344 & -1.48045783 \\ \text { C } & -0.72341812 & 6.31869190 & -1.48074721 \\ \text { C } & 4.07007400 & 0.72096844 & 0.66924556 \\ \text { C } & 4.07611687 & -0.68643774 & 0.66896014 \\ \text { C } & 5.18378037 & 1.44198651 & 1.05774424 \\ \text { C } & 5.19600436 & -1.39801755 & 1.05709247 \\ \text { C } & 6.33027985 & 0.72416059 & 1.45050619 \\ \text { C } & 6.33629679 & -0.67052701 & 1.45014445 \\ \text { C } & -4.07611342 & 0.68642718 & 0.66902497\end{array}$

$-0.01016200$

$-0.02163100$

0.00995700

0.02147700

$-0.01015100$

0.00995300

$-0.02163000$

$-0.04099900$

$-0.05012600$

$-0.06081000$

$-0.08015500$

$-0.09205400$

$-0.10177500$

0.05012400

0.04093700

0.08038000

0.06088900

0.10215500

0.09235500

0.04093500

0.05012500

0.06088700

0.08038700

$\begin{array}{rrrr}\mathrm{C} & -5.81666100 & 3.06892700 & -0.09205500 \\ \mathrm{C} & -6.33384100 & 1.77073300 & -0.10176600 \\ \mathrm{H} & -5.88402800 & -0.36685700 & -0.08718900 \\ \mathrm{H} & -7.40804300 & 1.62529600 & -0.12670300 \\ \mathrm{H} & -6.49670400 & 3.91343800 & -0.10892000 \\ \mathrm{H} & -4.01983500 & 4.31072800 & -0.05291700 \\ \mathrm{H} & -0.36684600 & 5.88402800 & 0.08752800 \\ \mathrm{H} & 1.62531100 & 7.40802200 & 0.12730900 \\ \mathrm{H} & 3.91345000 & 6.49667800 & 0.10934200 \\ \mathrm{H} & 4.31072900 & 4.01981500 & 0.05291700 \\ \mathrm{H} & 5.88402800 & 0.36685700 & -0.08720600 \\ \mathrm{H} & 7.40804200 & -1.62529500 & -0.12671900 \\ \mathrm{H} & 6.49670400 & -3.91343800 & -0.10891900 \\ \mathrm{H} & 4.01983500 & -4.31072800 & -0.05290000 \\ \mathrm{H} & 0.36684600 & -5.88402900 & 0.08751700 \\ \mathrm{H} & -1.62531200 & -7.40802200 & 0.12729600 \\ \mathrm{H} & -3.91345100 & -6.49667700 & 0.10933800 \\ \mathrm{H} & -4.31072900 & -4.01981400 & 0.05292300 \\ \mathrm{~N} & 3.08919200 & 1.32985300 & -0.00989800 \\ \mathrm{~N} & -1.32985500 & 3.08919400 & 0.00972000 \\ \mathrm{~N} & -3.08919100 & -1.32985300 & -0.00989200 \\ \mathrm{~N} & 1.32985500 & -3.08919400 & 0.00972800\end{array}$




$\begin{array}{lrrr}\mathrm{C} & -4.07007194 & -0.72097890 & 0.66929571 \\ \mathrm{C} & -5.19599385 & 1.39800441 & 1.05718133 \\ \mathrm{C} & -5.18377353 & -1.44199950 & 1.05780226\end{array}$

Pyridinic N

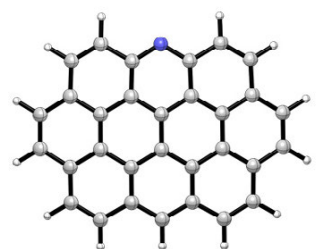

$\begin{array}{lrr}\mathrm{C} & 0.00000000 & -3.60324703 \\ \mathrm{C} & 0.00000000 & -3.66613767 \\ \mathrm{C} & 0.00000000 & -2.45223136 \\ \mathrm{C} & 0.00000000 & -1.21059742 \\ \mathrm{C} & 0.00000000 & -1.16151668 \\ \mathrm{C} & 0.00000000 & -2.41311963 \\ \mathrm{C} & 0.00000000 & -2.45979895 \\ \mathrm{C} & 0.00000000 & 0.00000000 \\ \mathrm{C} & 0.00000000 & 0.00000000 \\ \mathrm{C} & 0.00000000 & -1.22764742 \\ \mathrm{C} & 0.00000000 & 1.22764742 \\ \mathrm{C} & 0.00000000 & 2.45979895 \\ \mathrm{C} & 0.00000000 & 2.45223136 \\ \mathrm{C} & 0.00000000 & 1.21059742 \\ \mathrm{C} & 0.00000000 & 1.16151668 \\ \mathrm{H} & 0.00000000 & -4.54097389 \\ \mathrm{H} & 0.00000000 & -2.38016495 \\ \mathrm{C} & 0.00000000 & 2.41311963 \\ \mathrm{C} & 0.00000000 & 3.60324703 \\ \mathrm{H} & 0.00000000 & 2.38016495 \\ \mathrm{H} & 0.00000000 & 4.54097389 \\ \mathrm{C} & 0.00000000 & 3.66613767 \\ \mathrm{C} & 0.00000000 & 4.88587446\end{array}$

\section{Pyrrolic N}

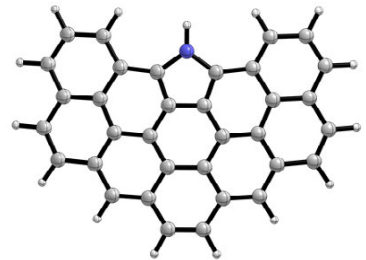

$\begin{array}{lrr}\mathrm{C} & -2.65058216 & -3.71418386 \\ \mathrm{C} & -1.21158151 & -3.75892462 \\ \mathrm{C} & -0.52150653 & -2.54802091 \\ \mathrm{C} & -1.29005112 & -1.38278100 \\ \mathrm{C} & -2.66041411 & -1.24564137 \\ \mathrm{C} & -3.42178534 & -2.47914042 \\ \mathrm{C} & 0.88288899 & -2.43047320 \\ \mathrm{C} & -0.70311923 & -0.12965379 \\ \mathrm{C} & 0.68405282 & 0.02590506 \\ \mathrm{C} & 1.49282454 & -1.12823208 \\ \mathrm{C} & 1.17251700 & 1.33123797 \\ \mathrm{C} & 0.21691830 & 2.40806466 \\ \mathrm{C} & -1.22558020 & 2.20986097 \\ \mathrm{C} & -1.68575973 & 0.83529164 \\ \mathrm{C} & -2.05232639 & 3.34271243 \\ \mathrm{C} & -1.50829219 & 4.62848143\end{array}$
$-2.87676500$

$-1.44049382$

$-0.71573297$

$-1.41358923$

$-2.83816706$

$-3.54737178$

0.70899464

$-0.69646834$

0.73418633

1.43267436

1.43267436

0.70899464

$-0.71573297$

$-1.41358923$

$-2.83816706$

$-3.42918138$

$-4.63462603$

$-3.54737178$

$-2.87676500$

$-4.63462603$

$-3.42918138$

$-1.44049382$

$-0.72019247$
0.00000850

0.00000850

0.00000850

0.00000850

0.00000850

0.00000850

0.00000850

0.00000850

0.00000850

0.00000850

0.00000850

0.00000850

0.00000850

0.00000850

0.00000850

0.00000850
$\begin{array}{lll}\mathrm{N} & 2.34405418 & 2.36609818\end{array}$

$\mathrm{N} \quad 2.36412516 \quad-2.34607959$
0.01016777

0.00965504
$\mathrm{H} \quad 0.00000000$

0.00000000

0.00000000

0.00000000

0.00000000

0.00000000

0.00000000

0.00000000

0.00000000

0.00000000

0.00000000

0.00000000

0.00000000

0.00000000

0.00000000

0.00000000

0.00000000

0.00000000

0.00000000

0.00000000

0.00000000

$\begin{array}{rr}4.89734525 & 0.66135731 \\ 5.82173509 & -1.27542221 \\ 5.84259015 & 1.20034703 \\ 3.69170212 & 1.40660761 \\ 3.66950525 & 2.84468229 \\ 4.62134205 & 3.37205198 \\ 2.49499753 & 3.53907199 \\ 2.49531321 & 4.62721916 \\ 1.22662671 & 2.85930234 \\ 0.00000000 & 3.54081675 \\ 0.00000000 & 4.63018958 \\ -1.22662671 & 2.85930234 \\ -2.49499753 & 3.53907199 \\ -2.49531321 & 4.62721916 \\ -3.66950525 & 2.84468229 \\ -3.69170212 & 1.40660761 \\ -4.62134205 & 3.37205198 \\ -4.89734525 & 0.66135731 \\ -5.84259015 & 1.20034703 \\ -4.88587446 & -0.72019247 \\ -5.82173509 & -1.27542221 \\ 0.00000000 & -3.52483035\end{array}$

4.89734525

3.69170212

2.84468229

37205198

07199

2.85930234

3.54081675

.53907199

.40660761

.20034703

$-1.27542221$

0.00000000

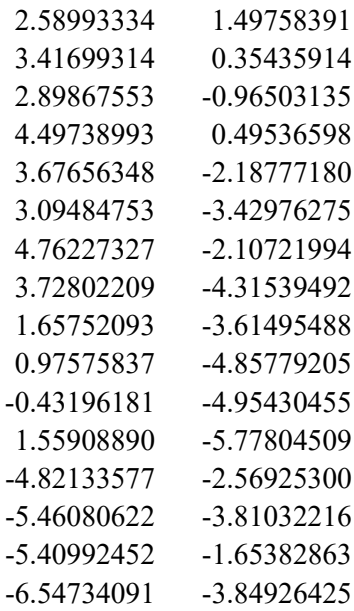

0.00000850 0.00000850 0.00000850 0.00000850 0.00000850 0.00000850 0.00000850 0.00000850 0.00000850 0.00000850 0.00000850 0.00000850 0.00000850 0.00000850 0.00000850 0.00000850 


$\begin{array}{lrl}\mathrm{H} & -3.13237994 & 3.20883631 \\ \mathrm{H} & -2.17396030 & 5.48810930 \\ \mathrm{C} & -0.12772509 & 4.82529686 \\ \mathrm{C} & 0.74815405 & 3.72790530 \\ \mathrm{H} & 0.28412738 & 5.83263680 \\ \mathrm{C} & 2.18867949 & 3.90203860 \\ \mathrm{C} & 3.06734914 & 2.86017995 \\ \mathrm{H} & 2.56143021 & 4.92503624 \\ \mathrm{H} & 4.14012991 & 3.04393739\end{array}$

\section{Graphitic N}

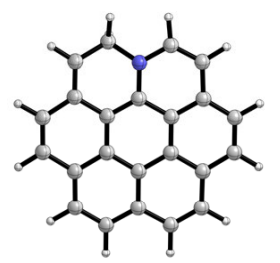

0.00000850 0.00000850 0.00000850 0.00000850 0.00000850 0.00000850 0.00000850 0.00000850 0.00000850
C $\quad-4.72818416$

C $\quad-3.32442051$

$\mathrm{H} \quad-5.23838071$

C $\quad-2.53598909$

C $\quad-1.17310049$

H $\quad-3.08324659$

$\mathrm{H} \quad-0.62748750$

$\mathrm{N} \quad-2.90586040$

$\mathrm{H} \quad-3.82551711$
$-4.99689991$

$-4.96723318$

$-5.95817807$

$-6.18535134$

$-6.19337972$

$-7.12661459$

$-7.13515031$

0.13803699

0.56878005
0.00000850 0.00000850 0.00000850 0.00000850 0.00000850 0.00000850 0.00000850 0.00000850 0.00000850
$-0.03732670$

$-0.00563065$

$-0.06696369$

0.04251560

0.01188774

0.37965565

0.04128456

$-0.02721422$

0.03930368

0.07727770

0.03968403

$-0.03792343$

$-0.12509494$

$-0.12339405$

$-0.21761732$

$-0.15871335$

$-0.19204103$

$-0.32033411$

$-0.11315890$
H 4.61743932

$\mathrm{H} \quad 4.62010262$

C $\quad-3.68020055$

C $\quad-3.67446225$

C $\quad-2.44895863$

$\mathrm{H} \quad-4.61373547$

$\mathrm{H} \quad-4.60745355$

C $\quad-2.43506636$

C $\quad-1.24725761$

C $\quad-0.00899422$

$\mathrm{H} \quad-3.38482785$

H $\quad-1.25606314$

C $\quad 2.43439679$

H $\quad 3.38105168$

C $\quad 1.25040408$

$\mathrm{H} \quad 1.23875861$

H -1.10315316
3.68858728

$-1.28677745$

1.18850302

0.67456643

$-0.67908108$

$-1.44278985$

1.22167400

$-1.23464393$

$-2.84939639$

$-3.56221910$

$-2.88664325$

$-3.37996896$

$-4.64993805$

$-2.89803609$

$-3.43523631$

$-3.57698349$

$-4.66480196$

4.50888289
1.47319207

$-0.03805117$

$-0.19569244$

$-0.23083980$

$-0.17967129$

$-0.03049346$

$-0.35126709$

$-0.26452128$

0.00226074

0.08488550

0.10703699

$-0.04672415$

0.11029988

0.10365251

0.11622426

0.13694551

0.17481456

$-0.09244792$

\section{1a_CoPc_O ${ }_{2}$}

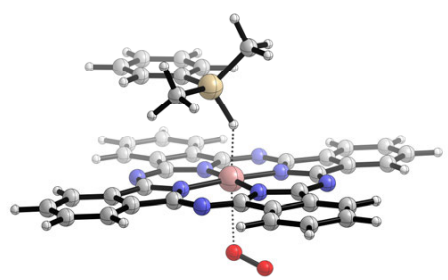

$\begin{array}{lrrr}\text { Co } & 0.44962190 & 0.04265487 & -0.44750559 \\ \mathrm{~N} & -1.39433378 & 0.46438842 & -0.83379503 \\ \mathrm{~N} & 0.87155994 & 1.93031779 & -0.39645475 \\ \mathrm{~N} & 2.32218919 & -0.37614471 & -0.19253280 \\ \mathrm{~N} & 0.05326949 & -1.83969232 & -0.62111272 \\ \mathrm{Si} & -0.45778456 & -0.47404903 & 2.66789078 \\ \mathrm{H} & 0.23525343 & 0.09906222 & 1.45848126 \\ \mathrm{C} & 0.94402256 & -2.87865768 & -0.50299015 \\ \mathrm{C} & 2.88011783 & -1.63253581 & -0.12540290 \\ \mathrm{C} & 3.34165479 & 0.52026794 & 0.01980489 \\ \mathrm{C} & 2.10261095 & 2.49098743 & -0.14671518 \\ \mathrm{C} & -0.01904071 & 2.96993603 & -0.51902714\end{array}$

$\begin{array}{lr}\text { H } & -3.07665696 \\ \text { C } & -0.23094783 \\ \text { H } & 1.77115343 \\ \text { H } & -2.28472188 \\ \text { H } & 0.08579165 \\ \text { C } & 4.31665612 \\ \text { C } & 4.61010052 \\ \text { C } & 5.29401782 \\ \text { C } & 5.89371026 \\ \text { C } & 6.60891477 \\ \text { H } & 5.06506605 \\ \text { C } & 6.90121897\end{array}$

$-4.61262804$ $-6.48685235$ $-5.69930870$ $-6.99208093$ $-7.52357209$ $-1.54574632$ $-0.17759375$ $-2.51548685$ 0.28148520 $-2.06114997$ $-3.57326686$ $-0.69758828$
-1.27353141
-0.82587835
-0.44735252
-1.20614241
-0.80263431
0.13087312
0.22290164
0.27553508
0.46394361
0.52258921
0.20345666
0.61448539 


$\begin{array}{lrrr}\mathrm{C} & -1.95041838 & 1.71928026 & -0.90498551 \\ \mathrm{C} & -1.18045382 & -2.40182934 & -0.86620166 \\ \mathrm{C} & -2.41724114 & -0.43225707 & -1.03264455 \\ \mathrm{C} & -3.38915120 & 1.63297541 & -1.15166001 \\ \mathrm{C} & -3.68495265 & 0.26482365 & -1.22637907 \\ \mathrm{C} & -4.37246774 & 2.60148141 & -1.25545657 \\ \mathrm{C} & -4.97760099 & -0.19709455 & -1.40743750 \\ \mathrm{C} & -5.69706219 & 2.14454507 & -1.43897247 \\ \mathrm{H} & -4.14278721 & 3.65961185 & -1.19072463 \\ \mathrm{C} & -5.99246247 & 0.78023041 & -1.51297872 \\ \mathrm{H} & -5.20477927 & -1.25684927 & -1.45412776 \\ \mathrm{H} & -6.50053784 & 2.86813890 & -1.52172066 \\ \mathrm{H} & -7.02068985 & 0.46478877 & -1.65121022 \\ \mathrm{C} & 0.66025986 & 4.25383682 & -0.35380535 \\ \mathrm{C} & 2.00846458 & 3.94951566 & -0.11647476 \\ \mathrm{C} & 0.20021419 & 5.55897408 & -0.39326779 \\ \mathrm{C} & 2.95613965 & 4.93686366 & 0.09212431 \\ \mathrm{C} & 1.15667300 & 6.57709439 & -0.18113790 \\ \mathrm{H} & -0.84308174 & 5.79212040 & -0.57676679 \\ \mathrm{C} & 2.50021131 & 6.27380155 & 0.05555063 \\ \mathrm{H} & 3.99852092 & 4.69944190 & 0.27543182 \\ \mathrm{H} & 0.84055957 & 7.61404492 & -0.20253810 \\ \mathrm{H} & 3.20821802 & 7.07958377 & 0.21427696 \\ \mathrm{C} & -1.08495099 & -3.85994594 & -0.89416522 \\ \mathrm{C} & 0.26496944 & -4.16283590 & -0.66367226 \\ \mathrm{C} & -2.03321442 & -4.84880264 & -1.09452717 \\ \mathrm{C} & 0.72636835 & -5.46755105 & -0.62397708 \\ \mathrm{C} & -1.57627415 & -6.18521354 & -1.05504932\end{array}$

$\begin{array}{rrrr}\mathrm{H} & 6.11874681 & 1.34018309 & 0.53462082 \\ \mathrm{H} & 7.40636067 & -2.78601116 & 0.64285670 \\ \mathrm{H} & 7.92153120 & -0.38347110 & 0.80447091 \\ \mathrm{C} & -0.04884522 & -2.28548704 & 2.78843890 \\ \mathrm{H} & 1.03573933 & -2.44466559 & 2.71639237 \\ \mathrm{H} & -0.37982629 & -2.67397922 & 3.76204611 \\ \mathrm{H} & -0.53774940 & -2.88452021 & 2.00917242 \\ \mathrm{C} & 0.19568669 & 0.50941443 & 4.10639554 \\ \mathrm{H} & 1.28426094 & 0.40154586 & 4.19875995 \\ \mathrm{H} & -0.03445850 & 1.57706366 & 3.99424293 \\ \mathrm{H} & -0.26410624 & 0.15766984 & 5.04027906 \\ \mathrm{C} & -2.28247743 & -0.18141029 & 2.41559003 \\ \mathrm{C} & -2.78249316 & 1.13900705 & 2.35719641 \\ \mathrm{C} & -3.20160714 & -1.24585436 & 2.30116721 \\ \mathrm{C} & -4.15036653 & 1.38692523 & 2.19439756 \\ \mathrm{H} & -2.09599704 & 1.98198283 & 2.44138577 \\ \mathrm{C} & -4.57169655 & -1.00098478 & 2.14128419 \\ \mathrm{H} & -2.84839503 & -2.27472602 & 2.34221635 \\ \mathrm{C} & -5.04677671 & 0.31484548 & 2.08867019 \\ \mathrm{H} & -4.51574610 & 2.40886815 & 2.14852064 \\ \mathrm{H} & -5.26466392 & -1.83326160 & 2.05954544 \\ \mathrm{H} & -6.10860850 & 0.50560087 & 1.96308755 \\ \mathrm{O} & 0.87513466 & 0.10043375 & -2.99874159 \\ \mathrm{O} & 1.87846606 & 0.67659112 & -3.33990935 \\ \mathrm{~N} & 2.24949480 & -2.79304533 & -0.26176949 \\ \mathrm{~N} & 3.24839065 & 1.84670821 & 0.04653725 \\ \mathrm{~N} & -1.32509243 & 2.88158258 & -0.75048477 \\ \mathrm{~N} & -2.32777275 & -1.76008839 & -1.04746146\end{array}$

\section{1a_CoPc-16Cl_O}

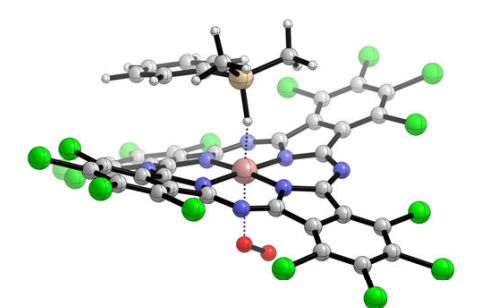

$\begin{array}{lrrr}\text { Co } & -0.26186541 & 0.02549205 & -0.29973551 \\ \mathrm{~N} & 1.66237294 & 0.08374446 & -0.51350209 \\ \mathrm{~N} & -0.20471052 & -1.90886150 & -0.32788492 \\ \mathrm{~N} & -2.19735771 & -0.03417979 & -0.29423273 \\ \mathrm{~N} & -0.32447315 & 1.95992886 & -0.32004324 \\ \mathrm{Si} & 0.27692350 & -0.17482512 & 2.93127713 \\ \mathrm{H} & -0.34638611 & 0.02962144 & 1.56543567 \\ \mathrm{C} & -1.43908788 & 2.70951354 & -0.08868871 \\ \mathrm{C} & -3.02990650 & 1.04680961 & -0.45696430 \\ \mathrm{C} & -2.95801865 & -1.16219391 & -0.38780351 \\ \mathrm{C} & -1.26725357 & -2.72358532 & -0.02397955 \\ \mathrm{C} & 0.93342426 & -2.65835861 & -0.24024086 \\ \mathrm{C} & 2.46477308 & -0.99548336 & -0.79687705 \\ \mathrm{C} & 0.77120530 & 2.77002407 & -0.13520739 \\ \mathrm{C} & 2.40761257 & 1.21165730 & -0.69915879 \\ \mathrm{C} & 3.77673614 & -0.53783715 & -1.27223181 \\ \mathrm{C} & 3.74489256 & 0.86545933 & -1.20137636 \\ \mathrm{C} & 4.91879550 & -1.21209968 & -1.65681729 \\ \mathrm{C} & 4.85735874 & 1.62251372 & -1.51346532 \\ \mathrm{C} & 6.05719763 & -0.44762924 & -1.98400084 \\ \mathrm{C} & 6.02693924 & 0.94335454 & -1.91344470 \\ \mathrm{C} & 0.61797733 & -4.00259556 & 0.26179943 \\ \mathrm{C} & -0.78223423 & -4.04637075 & 0.39343148\end{array}$

$\begin{array}{rrrr}\mathrm{H} & -1.45929413 & 1.16607256 & 4.06426481 \\ \mathrm{H} & 0.07687706 & 1.21792669 & 4.95902431 \\ \mathrm{H} & -0.13316302 & 2.20226055 & 3.49398672 \\ \mathrm{C} & -0.26659681 & -1.86811318 & 3.46675306 \\ \mathrm{H} & -1.36168034 & -1.94846520 & 3.44841288 \\ \mathrm{H} & 0.14653270 & -2.64758664 & 2.81263968 \\ \mathrm{H} & 0.07306964 & -2.07538964 & 4.49070886 \\ \mathrm{C} & 2.11474613 & -0.07254421 & 2.65252879 \\ \mathrm{C} & 2.88159114 & -1.23031217 & 2.40262142 \\ \mathrm{C} & 2.75520805 & 1.18245209 & 2.56243361 \\ \mathrm{C} & 4.23650456 & -1.13832900 & 2.05529859 \\ \mathrm{H} & 2.41334818 & -2.21315301 & 2.45498698 \\ \mathrm{C} & 4.10893071 & 1.27973164 & 2.21442997 \\ \mathrm{H} & 2.19092132 & 2.09571084 & 2.74926778 \\ \mathrm{C} & 4.84907933 & 0.11785650 & 1.95660629 \\ \mathrm{H} & 4.80619773 & -2.04173517 & 1.85225934 \\ \mathrm{H} & 4.57969527 & 2.25629827 & 2.13638127 \\ \mathrm{H} & 5.89593962 & 0.19128391 & 1.67496010 \\ \mathrm{Cl} & 4.98808233 & -2.97292608 & -1.73409878 \\ \mathrm{Cl} & 7.53820603 & -1.27403646 & -2.49112720 \\ \mathrm{Cl} & 7.47181822 & 1.87687288 & -2.33179457 \\ \mathrm{Cl} & 4.85640660 & 3.38274534 & -1.40683463 \\ \mathrm{Cl} & 2.80679647 & 5.23573024 & 0.82757728\end{array}$




$\begin{array}{lrrr}\text { C } & 1.40605403 & -5.07977645 & 0.61906033 \\ \text { C } & -1.42191712 & -5.17669034 & 0.86403178 \\ \text { C } & 0.76055544 & -6.24059793 & 1.09354036 \\ \text { C } & -0.62683332 & -6.28828432 & 1.21230704 \\ \text { C } & 0.34356318 & 4.08739771 & 0.35122288 \\ \text { C } & -1.06274029 & 4.05966848 & 0.35674356 \\ \text { C } & 1.04312314 & 5.20577142 & 0.76156044 \\ \text { C } & -1.79685414 & 5.16429991 & 0.74609315 \\ \text { C } & 0.30225793 & 6.33749147 & 1.15829714 \\ \text { C } & -1.09102631 & 6.31861823 & 1.14494728 \\ \text { C } & -4.38400991 & 0.58922233 & -0.79874332 \\ \text { C } & -4.34042105 & -0.81572256 & -0.74789190 \\ \text { C } & -5.54880719 & 1.25863903 & -1.11983584 \\ \text { C } & -5.46083789 & -1.57752243 & -1.01871507 \\ \text { C } & -6.69742793 & 0.48930364 & -1.39882677 \\ \text { C } & -6.65452293 & -0.90215853 & -1.34766673 \\ \text { C } & -0.36922981 & 1.23338503 & 3.95540391\end{array}$

I

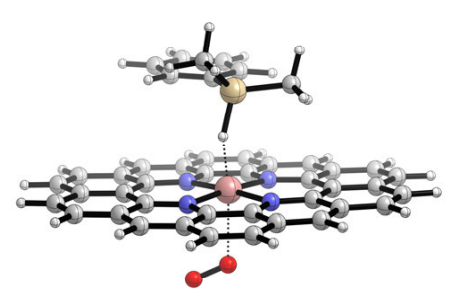

$\begin{array}{lll}\text { C } & -0.11841285 & -3.59075383\end{array}$

$\begin{array}{lll}\text { C } & 0.06296837 & -2.22449246\end{array}$

$\begin{array}{lll}\text { C } & -2.22152698 & -1.81022969\end{array}$

C $\quad-2.48298104 \quad-3.19719705$

C $\quad-1.42922240 \quad-4.06024408$

$\begin{array}{lll}\text { C } & -3.32651350 & -0.92005415\end{array}$

$\begin{array}{lll}\text { C } & -3.19948511 & 0.50346689\end{array}$

$\begin{array}{lll}\text { C } & -1.91623656 & 2.45972045\end{array}$

$\begin{array}{lll}\text { C } & -3.02635077 & 3.29306651\end{array}$

C $\quad-4.26924030 \quad 2.66218633$

$\begin{array}{lll}\text { C } & -4.37666417 & 1.28411878\end{array}$

$\mathrm{H} \quad-1.64658461$

H $\quad-5.17086130$

C $\quad 2.49095938$

C $\quad 1.38738348$

C $\quad 2.67978809$

C $\quad 3.85560542$

C $\quad 3.74009667$

C $\quad 2.80942599$

C $\quad 1.70048842$

C $\quad-0.59391028$

C $\quad-0.42017764$

C $\quad 0.89567752$

C $\quad 1.95878686$

H 4.64019256

$\mathrm{H} \quad 1.10841210$

C $\quad 5.14848458$

H $\quad 6.01573728$

C $\quad 4.11230427$

C $\quad 3.30126054$

H 3.45734852

C $\quad 4.33740911$

$\mathrm{H} \quad 5.36424303$

C $\quad-3.81915015$
$-1.32947617$

$-1.02746905$

$-0.68568298$

$-0.94786624$

$-1.26794317$

$-0.39373636$

$-0.15504013$

0.03417686

0.28477682

0.31818354

0.10493146

$-1.47139482$

0.51271642

$-1.34937700$

$-1.03993668$

$-0.74364491$

$-1.01906972$

$-1.31806988$

$-0.47446432$

$-0.23649525$

0.00647249

0.23073709

0.21308443

$-0.01166296$

$-1.53150286$

0.38051160

$-0.98929763$

$-1.19273628$

$-0.46674414$

$-0.00695315$

0.17380397

$-0.22203573$

$-0.22197159$

$-0.88856520$

$\begin{array}{lrrr}\mathrm{Cl} & 1.16096053 & 7.79315617 & 1.68402237 \\ \mathrm{Cl} & -1.99419978 & 7.75675861 & 1.64454022 \\ \mathrm{Cl} & -3.56100343 & 5.16404231 & 0.76420501 \\ \mathrm{Cl} & -5.62490571 & 3.01932589 & -1.20319171 \\ \mathrm{Cl} & -8.20546149 & 1.31118037 & -1.82777256 \\ \mathrm{Cl} & -8.11056154 & -1.84134874 & -1.71016029 \\ \mathrm{Cl} & -5.43094242 & -3.34103896 & -0.97316974 \\ \mathrm{Cl} & -3.17459536 & -5.25276616 & 1.0511124 \\ \mathrm{Cl} & -1.40851812 & -7.75470798 & 1.82187610 \\ \mathrm{Cl} & 1.73261102 & -7.64647837 & 1.55289005 \\ \mathrm{Cl} & 3.16743350 & -5.02471622 & 0.52704899 \\ \mathrm{O} & -0.41468135 & 0.08158120 & -2.99115704 \\ \mathrm{O} & -0.51917897 & -0.96987181 & -3.57230470 \\ \mathrm{~N} & 2.01760104 & 2.44551947 & -0.40704041 \\ \mathrm{~N} & -2.69056649 & 2.30653644 & -0.26936175 \\ \mathrm{~N} & -2.53954971 & -2.39575413 & -0.13982891 \\ \mathrm{~N} & 2.14686969 & -2.25829389 & -0.58807344\end{array}$




$\begin{array}{lrrr}\text { H } & -3.97497014 & -4.76209912 & -1.08525229 \\ \mathrm{C} & -4.62389124 & -1.47811936 & -0.35061867 \\ \mathrm{C} & -5.66306613 & 0.65925988 & 0.14804923 \\ \mathrm{H} & -6.52939125 & 1.28161739 & 0.35636974 \\ \mathrm{C} & -5.77717105 & -0.67439398 & -0.06975646 \\ \mathrm{H} & -6.74488548 & -1.16813736 & -0.04376529\end{array}$

\section{TS(I-II)}

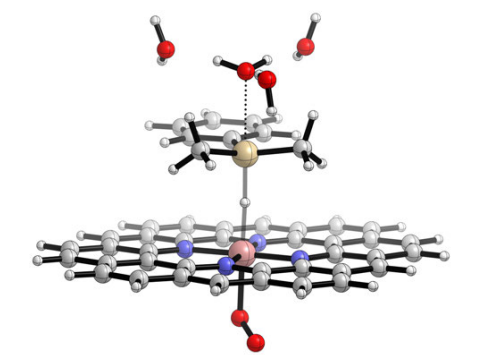

\begin{tabular}{|c|c|c|c|}
\hline $\mathrm{C}$ & -2.51039597 & -1.86136256 & -2.40030057 \\
\hline $\mathrm{C}$ & -1.34582500 & -1.22775346 & -1.91639211 \\
\hline $\mathrm{C}$ & -2.41494392 & 0.81687107 & -1.49528788 \\
\hline $\mathrm{C}$ & -3.64404302 & 0.23810876 & -1.95994408 \\
\hline $\mathrm{C}$ & -3.66862717 & -1.08731770 & -2.40744835 \\
\hline $\mathrm{C}$ & -2.40240554 & 2.19317312 & -1.04192894 \\
\hline $\mathrm{C}$ & -1.22676633 & 2.89633350 & -0.56917091 \\
\hline $\mathrm{C}$ & 1.05777205 & 2.97436243 & -0.03931521 \\
\hline $\mathrm{C}$ & 1.02125095 & 4.31645271 & 0.39669415 \\
\hline $\mathrm{C}$ & -0.21947730 & 4.94729413 & 0.32973121 \\
\hline $\mathrm{C}$ & -1.34451466 & 4.26208710 & -0.14147197 \\
\hline $\mathrm{H}$ & -4.60775781 & -1.51004541 & -2.75904615 \\
\hline $\mathrm{H}$ & -0.32513681 & 5.98208168 & 0.64940633 \\
\hline $\mathrm{C}$ & -0.07909042 & -3.31319086 & -2.26496458 \\
\hline $\mathrm{C}$ & -0.11171717 & -1.96457170 & -1.84791416 \\
\hline $\mathrm{C}$ & 2.15399779 & -1.91002513 & -1.24167700 \\
\hline $\mathrm{C}$ & 2.26214794 & -3.28831589 & -1.62847984 \\
\hline $\mathrm{C}$ & 1.14562329 & -3.96324638 & -2.13532366 \\
\hline $\mathrm{C}$ & 3.32357940 & -1.21766778 & -0.73967559 \\
\hline $\mathrm{C}$ & 3.34554299 & 0.17046230 & -0.32510573 \\
\hline $\mathrm{C}$ & 2.29172142 & 2.23712476 & 0.02935268 \\
\hline $\mathrm{C}$ & 3.45465849 & 2.86741621 & 0.52234266 \\
\hline $\mathrm{C}$ & 4.60223279 & 2.07899050 & 0.57353483 \\
\hline $\mathrm{C}$ & 4.56944361 & 0.74261042 & 0.16147173 \\
\hline $\mathrm{H}$ & 1.24605502 & -5.00617320 & -2.42929440 \\
\hline $\mathrm{H}$ & 5.53768713 & 2.49651976 & 0.94056048 \\
\hline $\mathrm{C}$ & 3.50936742 & -3.97761020 & -1.50658916 \\
\hline $\mathrm{H}$ & 3.55032775 & -5.02153460 & -1.80601362 \\
\hline $\mathrm{C}$ & 4.52851139 & -1.94985063 & -0.64388564 \\
\hline $\mathrm{C}$ & 5.75895790 & -0.04835065 & 0.23200959 \\
\hline $\mathrm{H}$ & 6.66562635 & 0.42139331 & 0.60419892 \\
\hline $\mathrm{C}$ & 5.73460777 & -1.34723045 & -0.15698169 \\
\hline $\mathrm{H}$ & 6.62739844 & -1.96500258 & -0.10987828 \\
\hline $\mathrm{C}$ & -4.84986156 & 1.00676277 & -1.96039757 \\
\hline $\mathrm{H}$ & -5.76048738 & 0.53168249 & -2.31597312 \\
\hline $\mathrm{C}$ & -3.62494826 & 2.90217587 & -1.06412955 \\
\hline $\mathrm{C}$ & -2.61007154 & 4.92719090 & -0.18854001 \\
\hline $\mathrm{H}$ & -2.65936535 & 5.96136671 & 0.14178297 \\
\hline $\mathrm{C}$ & -3.70788855 & 4.26691978 & -0.63339941 \\
\hline $\mathrm{H}$ & -4.67806611 & 4.75481375 & -0.67583826 \\
\hline $\mathrm{C}$ & -4.83719744 & 2.29074896 & -1.52318946 \\
\hline $\mathrm{H}$ & -5.74339132 & 2.89047720 & -1.51355929 \\
\hline $\mathrm{C}$ & 4.60035580 & -3.32824911 & -1.02944865 \\
\hline $\mathrm{H}$ & 5.55673471 & -3.83469754 & -0.92924815 \\
\hline
\end{tabular}

$\begin{array}{lrrr}\mathrm{O} & -0.41468135 & 0.08158120 & -2.99115704 \\ \mathrm{O} & -0.51917897 & -0.96987181 & -3.57230470 \\ \mathrm{~N} & 2.01760104 & 2.44551947 & -0.40704041 \\ \mathrm{~N} & -2.69056649 & 2.30653644 & -0.26936175 \\ \mathrm{~N} & -2.53954971 & -2.39575413 & -0.13982891 \\ \mathrm{~N} & 2.14686969 & -2.25829389 & -0.58807344\end{array}$


$\begin{array}{llll}\text { C } & -2.44603819 & -3.23697661 & -2.83483504\end{array}$

$\begin{array}{llll}\text { C } & -1.28173082 & -3.93175554 & -2.77149314\end{array}$
$\mathrm{H}$

$-0.65818605$

$-4.58930442$

4.97931778

$1.21231466 \quad 1.03929005$

4.82310503
II

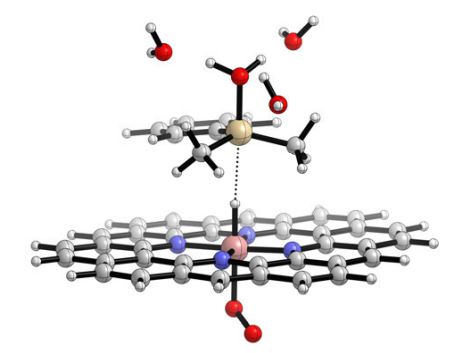

C $\quad-1.94725983 \quad-3.03797042$

C $\quad-1.03080859 \quad-2.00173037$

C $\quad-2.68502404 \quad-0.36152918$

C $\quad-3.67855583 \quad-1.36384263$

C $\quad-3.29161040 \quad-2.68317306$

$\begin{array}{lll}\text { C } & -3.10139114 & 1.00489369\end{array}$

C $\quad-2.19498166 \quad 2.10817005$

C $\quad-0.04130306 \quad 2.98431070$

C $\quad-0.49039452 \quad 4.30866197$

C $\quad-1.86639344 \quad 4.50127931$

C $\quad-2.72952314 \quad 3.42324380$

$\mathrm{H} \quad-4.05671691 \quad-3.42915128$

$\mathrm{H} \quad-2.28646755 \quad 5.49266206$

C $\quad 0.82557613 \quad-3.61317109$

C $\quad 0.37498782 \quad-2.29197726$

C $\quad 2.51908261 \quad-1.43713088$

C $\quad 3.05107493$

C 2.19446967

C $\quad 3.42413970$

C $\quad 3.01122532$

C $\quad 1.36505510$

C $\quad 2.28422504$

C $\quad 3.62340663$

C $\quad 4.00519464$

$\mathrm{H} \quad 2.61389177$

H 4.38872869

C $\quad 4.45701514$

$\mathrm{H} \quad 4.81910276$

C 4.80460523

C $\quad 5.38831164$

$\mathrm{H} \quad 6.10934444$

C $\quad 5.77045279$

$\mathrm{H} \quad 6.81701106$

C $\quad-5.06706219$

$\mathrm{H} \quad-5.78741164$

C $\quad-4.48760982$

C -4.14184002

$\mathrm{H} \quad-4.50641326$

C $\quad-4.98793757$

H $\quad-6.06460809$

C $\quad-5.45398827$

$\mathrm{H} \quad-6.50450977$

C $\quad 5.30018637$

$\mathrm{H} \quad 6.37239263$

C $\quad-1.45782979$

C $\quad-0.13090676$
$-1.89107826$

$-1.59943202$

$-1.37132979$

$-1.63543416$

$-1.89748611$

$-1.13453265$

$-0.89462953$

$-0.62899867$

$-0.42042796$

$-0.45322672$

$-0.68482686$

$-2.10296320$

$-0.29589500$

$-1.76341378$

$-1.53905826$

$-1.15214333$

$-1.33851665$

$-1.64320225$

$-0.87744720$

$-0.68839771$

$-0.56693370$

$-0.30770692$

$-0.24214074$

$-0.42962831$

$-1.78901199$

$-0.04415310$

$-1.23036355$

$-1.37290869$

$-0.79048372$

$-0.35790166$

$-0.16323971$

$-0.53005751$

$-0.47814908$

$-1.63840542$

$-1.83761121$

$-1.15111024$

$-0.70720721$

$-0.54291760$

$-0.93024757$

$-0.95220976$

$-1.39802332$

$-1.39502495$

$-0.96399681$

$-0.88192857$

$-2.13792685$

$-2.07480304$

\begin{tabular}{|c|c|c|c|}
\hline $\mathrm{H}$ & 0.24663306 & -5.65336897 & -2.25010425 \\
\hline $\mathrm{C}$ & 0.47246689 & 5.35802084 & -0.17622436 \\
\hline $\mathrm{C}$ & 1.79937590 & 5.08399970 & -0.12235571 \\
\hline $\mathrm{H}$ & 2.53055379 & 5.86570514 & 0.06552592 \\
\hline $\mathrm{H}$ & 0.09774184 & 6.36793515 & -0.03179319 \\
\hline $\mathrm{H}$ & -2.18558921 & -5.14814681 & -2.36596378 \\
\hline $\mathrm{C}$ & 1.74038870 & 0.21439803 & 2.50266672 \\
\hline $\mathrm{H}$ & 1.93688690 & 1.25390805 & 2.20827930 \\
\hline $\mathrm{H}$ & 2.28899854 & 0.01872385 & 3.43402466 \\
\hline $\mathrm{H}$ & 2.13782925 & -0.44780442 & 1.72227408 \\
\hline $\mathrm{C}$ & -1.27371536 & 1.35466551 & 2.46292260 \\
\hline $\mathrm{H}$ & -1.75791041 & 1.64935113 & 3.40306792 \\
\hline $\mathrm{H}$ & -0.74041036 & 2.23280957 & 2.07601924 \\
\hline $\mathrm{H}$ & -2.05316911 & 1.07406428 & 1.74253704 \\
\hline $\mathrm{C}$ & -0.70239248 & -1.69486325 & 2.14069524 \\
\hline $\mathrm{C}$ & 0.18519976 & -2.75771063 & 1.88528553 \\
\hline $\mathrm{C}$ & -2.08432332 & -1.92904400 & 1.99666213 \\
\hline $\mathrm{C}$ & -0.29200594 & -4.01725030 & 1.51056419 \\
\hline $\mathrm{H}$ & 1.26229613 & -2.60384768 & 1.97502804 \\
\hline $\mathrm{C}$ & -2.56398088 & -3.18629942 & 1.62067460 \\
\hline $\mathrm{H}$ & -2.79653593 & -1.12231141 & 2.17719934 \\
\hline $\mathrm{C}$ & -1.66676899 & -4.23168996 & 1.37972718 \\
\hline $\mathrm{H}$ & 0.40827832 & -4.82629948 & 1.31397228 \\
\hline $\mathrm{H}$ & -3.63399071 & -3.34954580 & 1.51236614 \\
\hline $\mathrm{H}$ & -2.03805671 & -5.20972433 & 1.08244602 \\
\hline $\mathrm{Co}$ & 0.16669815 & 0.35188790 & -1.08122335 \\
\hline $\mathrm{N}$ & 1.18079192 & -1.25612626 & -1.24637975 \\
\hline $\mathrm{N}$ & 1.71157412 & 1.40614652 & -0.73938916 \\
\hline $\mathrm{N}$ & -0.85374449 & 1.93797005 & -0.85104428 \\
\hline $\mathrm{N}$ & -1.38215404 & -0.72623134 & -1.35704844 \\
\hline $\mathrm{H}$ & 0.04626709 & 0.10958954 & 0.34506548 \\
\hline $\mathrm{O}$ & 0.58291858 & 1.85892554 & -3.39879121 \\
\hline $\mathrm{O}$ & 0.31453293 & 0.63725623 & -3.03655867 \\
\hline $\mathrm{Si}$ & -0.08317696 & -0.04296534 & 2.73922694 \\
\hline $\mathrm{O}$ & -0.19456117 & -0.27763889 & 4.52773931 \\
\hline $\mathrm{H}$ & -1.15213053 & -0.36004420 & 4.87757030 \\
\hline $\mathrm{H}$ & 0.37828026 & -1.05701877 & 4.85441823 \\
\hline $\mathrm{H}$ & 0.78078063 & 1.80496057 & 5.28981614 \\
\hline $\mathrm{O}$ & -2.65386713 & -0.46464247 & 5.18381662 \\
\hline $\mathrm{O}$ & 1.32277121 & -2.26309648 & 5.05453952 \\
\hline $\mathrm{O}$ & 0.77484513 & 2.50060198 & 4.61484615 \\
\hline $\mathrm{H}$ & -3.06740834 & -1.34498867 & 5.16591158 \\
\hline $\mathrm{H}$ & -3.02228909 & 0.01648020 & 5.94459720 \\
\hline $\mathrm{H}$ & 1.03100726 & -3.12836273 & 4.71936416 \\
\hline $\mathrm{H}$ & 1.77960713 & -2.41106237 & 5.90001154 \\
\hline $\mathrm{H}$ & 1.42460652 & 3.16024872 & 4.90137151 \\
\hline
\end{tabular}


III

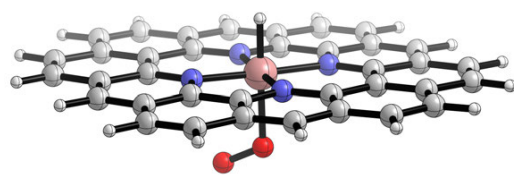

$\begin{array}{lrrr}\mathrm{C} & -1.39548453 & -3.81688216 & -0.10459466 \\ \mathrm{C} & -0.69472709 & -2.59000577 & -0.12829343 \\ \mathrm{C} & -2.63167658 & -1.27726317 & -0.16998673 \\ \mathrm{C} & -3.41493021 & -2.48103318 & -0.15996155 \\ \mathrm{C} & -2.78340578 & -3.72909554 & -0.12501290 \\ \mathrm{C} & -3.30358529 & 0.00577918 & -0.18545123 \\ \mathrm{C} & -2.62843604 & 1.28705970 & -0.16559126 \\ \mathrm{C} & -0.68818613 & 2.59476365 & -0.11938387 \\ \mathrm{C} & -1.38584678 & 3.82331402 & -0.09144007 \\ \mathrm{C} & -2.77398457 & 3.73909781 & -0.11216404 \\ \mathrm{C} & -3.40865221 & 2.49275969 & -0.15141709 \\ \mathrm{C} & 1.43927500 & -3.82025995 & -0.09054170 \\ \mathrm{C} & 0.74163672 & -2.59150692 & -0.11765525 \\ \mathrm{C} & 2.68294559 & -1.28400648 & -0.13735501 \\ \mathrm{C} & 3.46279444 & -2.48969332 & -0.12372503 \\ \mathrm{C} & 2.82735278 & -3.73620933 & -0.09729669 \\ \mathrm{C} & 3.35855140 & -0.00269200 & -0.14465893 \\ \mathrm{C} & 2.68618086 & 1.28029040 & -0.13292436 \\ \mathrm{C} & 0.74817683 & 2.59260807 & -0.10872728 \\ \mathrm{C} & 1.44891151 & 3.81949579 & -0.07734739 \\ \mathrm{C} & 2.83677339 & 3.73196768 & -0.08437240 \\ \mathrm{C} & 3.46906882 & 2.48395157 & -0.11511231 \\ \mathrm{C} & 4.89123112 & -2.42803383 & -0.13590958 \\ \mathrm{C} & 4.77168668 & -0.00446143 & -0.15232485 \\ \mathrm{C} & 4.89734577 & 2.41873019 & -0.12750078 \\ \mathrm{C} & 5.52242134 & 1.21556944 & -0.14955323 \\ \mathrm{C} & -4.84326531 & -2.41584331 & -0.18658532 \\ \mathrm{C} & -4.71591920 & 0.00759909 & -0.20749193 \\ \mathrm{C} & -4.83714776 & 2.43126763 & -0.17825848 \\ \mathrm{C} & & -3.81688216 & -0.10459466\end{array}$

$\begin{array}{lrrr}\mathrm{C} & -5.46423541 & 1.22961642 & -0.21008119 \\ \mathrm{C} & -5.46731674 & -1.21250975 & -0.21427318 \\ \mathrm{C} & 5.51934054 & -1.22638473 & -0.15379347 \\ \mathrm{C} & -0.65786262 & -5.05821591 & -0.06769496 \\ \mathrm{C} & 0.69858314 & -5.05992200 & -0.06181267 \\ \mathrm{C} & -0.64509810 & 5.06264877 & -0.05023596 \\ \mathrm{C} & 0.71134759 & 5.06091467 & -0.04433236 \\ \mathrm{H} & 3.44466868 & 4.63425382 & -0.06721593 \\ \mathrm{H} & -3.39149779 & -4.63136594 & -0.11481016 \\ \mathrm{H} & -3.37980047 & 4.64285749 & -0.09884450 \\ \mathrm{H} & 3.43297021 & -4.64008010 & -0.08328027 \\ \mathrm{H} & 5.44515559 & -3.36320891 & -0.12972907 \\ \mathrm{H} & 5.45362832 & 3.35247737 & -0.11807317 \\ \mathrm{H} & 6.60692988 & 1.14559034 & -0.15916737 \\ \mathrm{H} & -5.39978633 & -3.34951151 & -0.18329908 \\ \mathrm{H} & -5.39130956 & 3.36632127 & -0.17175763 \\ \mathrm{H} & -6.54877705 & 1.16183846 & -0.23069108 \\ \mathrm{H} & -6.55168381 & -1.14192399 & -0.23464466 \\ \mathrm{H} & 6.60402214 & -1.15910947 & -0.16317723 \\ \mathrm{H} & 1.26255808 & -5.98849309 & -0.03680458 \\ \mathrm{H} & 1.27766133 & 5.98796862 & -0.01609885 \\ \mathrm{H} & -1.20906178 & 5.99128147 & -0.02667230 \\ \mathrm{H} & -1.22416559 & -5.98549985 & -0.04733986 \\ \mathrm{Co} & 0.02092598 & 0.00148058 & -0.12167586 \\ \mathrm{~N} & 1.33387294 & -1.38421899 & -0.14041780 \\ \mathrm{~N} & 1.33736625 & 1.38391673 & -0.13565712 \\ \mathrm{~N} & -1.28067129 & 1.38855887 & -0.15884844 \\ \mathrm{O} & -1.28416962 & -1.38218061 & -0.16358600 \\ & 0.04467241 & -0.00199759 & 1.86068848 \\ & -1.13770327 & -0.00074806 & 2.41998154\end{array}$

\section{TS(III-IV)}

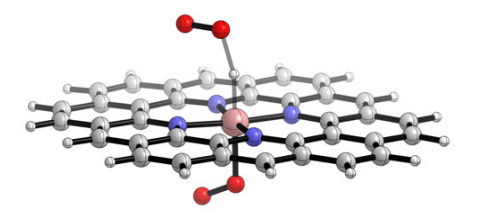

$\begin{array}{lrr}\mathrm{C} & -1.46993200 & -3.82436558 \\ \mathrm{C} & -0.77830060 & -2.59258472 \\ \mathrm{C} & -2.72626754 & -1.29364771 \\ \mathrm{C} & -3.50064443 & -2.50289703 \\ \mathrm{C} & -2.85915469 & -3.74622232 \\ \mathrm{C} & -3.40706805 & -0.01475371 \\ \mathrm{C} & -2.74119789 & 1.27155533 \\ \mathrm{C} & -0.80847998 & 2.59220127 \\ \mathrm{C} & -1.51440610 & 3.81550566 \\ \mathrm{C} & -2.90262732 & 3.72162830 \\ \mathrm{C} & -3.52960159 & 2.47160094 \\ \mathrm{H} & -3.46031734 & -4.65320762 \\ \mathrm{H} & -3.51430996 & 4.62143685 \\ \mathrm{C} & 1.36467636 & -3.80771320 \\ \mathrm{C} & 0.65898854 & -2.58423037 \\ \mathrm{C} & 2.59137185 & -1.26236740 \\ \mathrm{C} & 3.37983994 & -2.46268678\end{array}$

$-0.02091936$

$-0.02521690$

$-0.05557761$

$-0.06260854$

$-0.04163563$

$-0.06344630$

$-0.02952906$

0.02739447

0.05660097

0.03416546

$-0.01209310$

$-0.04513388$

0.04900479

$-0.01695450$

$-0.02125275$

$-0.05082774$

$-0.05776054$
$\mathrm{H}$

C

$\mathrm{H}$

C

C

$\mathrm{H}$

C

$\mathrm{H}$

C

$\mathrm{H}$

C

H

C

C

C

$\begin{array}{rr}5.41206114 & 1.25852455 \\ 6.49673432 & 1.19578462 \\ -4.92929672 & -2.44729052 \\ -5.47933987 & -3.38472559 \\ -4.82009692 & -0.02271396 \\ -4.95751385 & 2.40002500 \\ -5.51843299 & 3.33100792 \\ -5.57644209 & 1.19413844 \\ -6.66035013 & 1.11934347 \\ -5.56222537 & -1.24809513 \\ -6.64693347 & -1.18540994 \\ 5.42627724 & -1.18448135 \\ 6.51014344 & -1.10856047 \\ -0.72389872 & -5.06092245 \\ 0.63285834 & -5.05285037 \\ 1.20323781 & -5.97783344 \\ -0.78282108 & 5.06010295\end{array}$

$-0.08912094$ $-0.11755170$ $-0.09441589$ $-0.10463107$ $-0.08956029$ $-0.04517294$ $-0.03640857$ $-0.08672248$ $-0.11271876$ $-0.11154441$ $-0.13615667$ $-0.11389189$ $-0.14091434$ $-0.00565004$ $-0.00443662$ 0.00241374 0.09700779 


$\begin{array}{lrr}\text { C } & 2.75323520 & -3.71312675 \\ \mathrm{C} & 3.25749321 & 0.02405744 \\ \mathrm{C} & 2.57646065 & 1.30212080 \\ \mathrm{C} & 0.62881159 & 2.60050799 \\ \mathrm{C} & 1.32020597 & 3.83178898 \\ \mathrm{C} & 2.70977117 & 3.75378197 \\ \mathrm{C} & 3.35089658 & 2.51143020 \\ \mathrm{H} & 3.36486898 & -4.61310945 \\ \mathrm{H} & 3.31087745 & 4.66069556 \\ \mathrm{C} & 4.80817313 & -2.39088950 \\ \mathrm{H} & 5.36892898 & -3.32199667 \\ \mathrm{C} & 4.66908663 & 0.03256027 \\ \mathrm{C} & 4.77996426 & 2.45697641 \\ \mathrm{H} & 5.32984782 & 3.39453594\end{array}$

IV

$\begin{array}{lrrr}\mathrm{C} & 0.57393810 & 5.06780905 & 0.09819269 \\ \mathrm{H} & 1.13350721 & 5.99904356 & 0.12384606 \\ \mathrm{H} & -1.35319111 & 5.98477075 & 0.12159256 \\ \mathrm{H} & -1.28345871 & -5.99247830 & 0.00011027 \\ \mathrm{Co} & -0.07081068 & 0.00495472 & -0.09191043 \\ \mathrm{~N} & 1.24379302 & -1.37213317 & -0.03010224 \\ \mathrm{~N} & 1.22769128 & 1.39573317 & -0.00201145 \\ \mathrm{~N} & -1.39279854 & 1.38031415 & -0.01068901 \\ \mathrm{~N} & -1.37669197 & -1.38706113 & -0.03878353 \\ \mathrm{H} & -0.03191442 & -0.01049891 & 1.44255368 \\ \mathrm{O} & 0.43161974 & -0.02133729 & 2.84580514 \\ \mathrm{O} & 1.67363250 & -0.01414419 & 2.88670007 \\ \mathrm{O} & -0.11353130 & 0.02489662 & -2.08592646 \\ \mathrm{O} & 1.05777670 & 0.03745765 & -2.64117476\end{array}$

$-0.03568496$

$-0.06347936$

$-0.02481651$

0.03134444

0.06051359

0.04001905

$-0.00732846$

$-0.03818239$

0.05583093

$-0.09237402$

$-0.10137689$

$-0.09208140$

$-0.04322553$

$-0.03328528$
$-0.01358450$

$-0.00386824$

$-0.02890420$

$-0.04156203$

$-0.03255662$

$-0.03738420$

$-0.03420885$

$-0.01070303$

$-0.03294855$

$-0.05482210$

$-0.05664153$

$-0.03989257$

$-0.07017412$

0.03630185

0.02431510

0.08033265

0.09220872

0.06957730

0.10290591

0.07823146

0.01875594

0.02201696

0.05582955

0.08448266

0.08045774

0.06136856

0.13292673

0.14447347

0.13902936

0.12487033

0.13261249
$\begin{array}{lll}\mathrm{H} & 6.57067813 & 1.16466706\end{array}$

$\begin{array}{lll}\text { C } & -4.87582592 & -2.43597641\end{array}$

$\mathrm{H} \quad-5.43100483 \quad-3.37057253$

$\begin{array}{lll}\text { C } & -4.75548570 & -0.01039244\end{array}$

$\begin{array}{lll}\text { C } & -4.88640526 & 2.41525348\end{array}$

H $\quad-5.44564010 \quad 3.34732212$

$\begin{array}{lll}\text { C } & -5.50790879 & 1.20977679\end{array}$

$\mathrm{H} \quad-6.59237118 \quad 1.13755350$

$\begin{array}{lll}\text { C } & -5.50302324 & -1.23354469\end{array}$

H $\quad-6.58778098$

C $\quad 5.49131563$

$\mathrm{H} \quad 6.57542125$

C $\quad-0.67552034$

C $\quad 0.68109100$

H $\quad 1.24707049$

$\begin{array}{lr}\mathrm{C} & -0.69552082\end{array}$

C $\quad 0.66110307$

$\mathrm{H} \quad 1.22364715$

$\begin{array}{lr}\mathrm{H} & 1.22364715 \\ \mathrm{H} & -1.26091608\end{array}$

$\mathrm{H} \quad-1.23757202$

Co $\quad-0.01348713$

$\mathrm{N} \quad 1.30906265$

N 1.30320139

$\mathrm{N} \quad-1.32586633$

$\begin{array}{ll}\mathrm{N} & -1.32586633 \\ \mathrm{~N} & -1.31970118\end{array}$

O $\quad-0.00253032$

O $\quad-1.36396520$

$\mathrm{H} \quad-1.58915247$

O 0.58506007

O $\quad 1.72388411$

$-1.16612499$

$-1.21101750$

$-1.13917288$

$-5.06551701$

$-5.06287260$

$-5.99067350$

5.06155929

5.06387844

5.99379986

5.98958545

$-5.99572983$

$-0.00168532$

$-1.38496468$

1.38824130

1.38399208

$-1.39069557$

$-0.00815811$

$-0.05867954$

0.88251562

$-0.00501753$

$-0.00230928$

0.15340287

0.18452871

$-0.05993806$

$-0.06524928$

$-0.06142257$

$-0.07724562$

$-0.09041419$

$-0.07802961$

$-0.09290708$

$-0.06908081$

$-0.08341618$

0.15711230

0.18809234

$-0.00262257$

0.02052525

0.02979576

$-0.03002580$

$-0.00390822$

$-0.00092107$

$-0.04820099$

$-0.01209626$

0.13560172

0.04270813

0.04349036

$-0.01032393$

$-0.01201432$

2.15166170

2.64303130

2.73271734

$-3.35875456$

$-2.96150791$

\section{V}

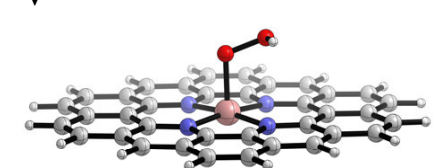

C $\quad 1.37699425 \quad 3.82541754$

$-0.16337556$

C $\quad 0.67962757 \quad 2.59639631$

$-0.17054074$

H $\quad-5.46620518$

$-3.36118235$

$-0.07506432$

C -5.53533695

$-1.22396914$

$-0.07176750$ 


$\begin{array}{lrrr}\mathrm{C} & 2.62257411 & 1.28982300 & -0.17975145 \\ \mathrm{C} & 3.40364494 & 2.49445543 & -0.17900806 \\ \mathrm{C} & 2.76639492 & 3.74092711 & -0.16991942 \\ \mathrm{C} & 3.29655382 & 0.00471752 & -0.18029686 \\ \mathrm{C} & 2.62634836 & -1.28200568 & -0.17957690 \\ \mathrm{C} & 0.68358942 & -2.59237316 & -0.16538757 \\ \mathrm{C} & 1.38307992 & -3.82012430 & -0.16538625 \\ \mathrm{C} & 2.77259330 & -3.73327025 & -0.17583038 \\ \mathrm{C} & 3.40874317 & -2.48619455 & -0.18355252 \\ \mathrm{H} & 3.37136042 & 4.64554588 & -0.16662230 \\ \mathrm{H} & 3.37865188 & -4.63715979 & -0.17659375 \\ \mathrm{C} & -1.46036836 & 3.82302981 & -0.14145768 \\ \mathrm{C} & -0.76093207 & 2.59532938 & -0.15751924 \\ \mathrm{C} & -2.70266894 & 1.28578807 & -0.12728389 \\ \mathrm{C} & -3.48546260 & 2.48946853 & -0.11589391 \\ \mathrm{C} & -2.84952673 & 3.73665361 & -0.12355688 \\ \mathrm{C} & -3.37380844 & -0.00061748 & -0.10990148 \\ \mathrm{C} & -2.70049758 & -1.28593191 & -0.12270183 \\ \mathrm{C} & -0.75685509 & -2.59263195 & -0.15052651 \\ \mathrm{C} & -1.45424046 & -3.82141572 & -0.13677132 \\ \mathrm{C} & -2.84364584 & -3.73716184 & -0.11806698 \\ \mathrm{C} & -3.48135749 & -2.49105985 & -0.11048885 \\ \mathrm{H} & -3.45554430 & 4.64048154 & -0.11218877 \\ \mathrm{H} & -3.44828459 & -4.64190478 & -0.10721003 \\ \mathrm{C} & -4.91375719 & 2.42338390 & -0.09012088 \\ \mathrm{H} & -5.47172147 & 3.35626593 & -0.08137357 \\ \mathrm{C} & -4.78677815 & -0.00180567 & -0.08510438 \\ \mathrm{C} & -4.90982847 & -2.42737354 & -0.08507688\end{array}$

$\begin{array}{rrrr}\mathrm{H} & -6.61989196 & -1.15525785 & -0.05144631 \\ \mathrm{C} & 4.83265367 & 2.43096108 & -0.18285026 \\ \mathrm{H} & 5.38906369 & 3.36484055 & -0.18068640 \\ \mathrm{C} & 4.70934511 & 0.00559421 & -0.18685691 \\ \mathrm{C} & 4.83749323 & -2.42018832 & -0.19013460 \\ \mathrm{H} & 5.39576978 & -3.35292325 & -0.19197030 \\ \mathrm{C} & 5.46041194 & -1.21546171 & -0.19106632 \\ \mathrm{H} & 6.54506379 & -1.14455662 & -0.19460280 \\ \mathrm{C} & 5.45842346 & 1.22777715 & -0.18708664 \\ \mathrm{H} & 6.54319427 & 1.15900733 & -0.18970203 \\ \mathrm{C} & -5.53742481 & 1.21896459 & -0.07475687 \\ \mathrm{H} & -6.62187612 & 1.14851993 & -0.05434753 \\ \mathrm{C} & 0.63560168 & 5.06579618 & -0.14934244 \\ \mathrm{C} & -0.72117666 & 5.06476474 & -0.13953568 \\ \mathrm{H} & -1.28605564 & 5.99321183 & -0.12783725 \\ \mathrm{C} & 0.64353923 & -5.06152849 & -0.15306504 \\ \mathrm{C} & -0.71327780 & -5.06204012 & -0.13937242 \\ \mathrm{H} & -1.27692927 & -5.99124288 & -0.12908542 \\ \mathrm{H} & 1.20804763 & -5.99026722 & -0.15398053 \\ \mathrm{H} & 1.19895509 & 5.99526125 & -0.14570028 \\ \mathrm{Co} & -0.03499933 & 0.00313444 & -0.05507391 \\ \mathrm{~N} & -1.35433966 & 1.38756212 & -0.15408993 \\ \mathrm{~N} & -1.35188129 & -1.38534130 & -0.14643463 \\ \mathrm{~N} & 1.27767894 & -1.38458922 & -0.17227871 \\ \mathrm{~N} & 1.27492059 & 1.39022499 & -0.18030009 \\ \mathrm{O} & -0.06660345 & 0.01495888 & 1.96429358 \\ \mathrm{O} & 1.28890957 & 0.06476792 & 2.47258901 \\ \mathrm{H} & 1.51192810 & -0.87654183 & 2.56612340\end{array}$

VI

$\begin{array}{lrrr} & & \\ \mathrm{C} & & & \\ \mathrm{C} & 0.67083147 & 2.58657326 & -0.15774484 \\ \mathrm{C} & 2.61618889 & 1.27571575 & -0.17909810 \\ \mathrm{C} & 3.39576307 & 2.48175358 & -0.18086085 \\ \mathrm{C} & 2.75817257 & 3.72660969 & -0.17230025 \\ \mathrm{C} & 3.28962499 & -0.00812119 & -0.18833322 \\ \mathrm{C} & 2.61922093 & -1.29357120 & -0.19258392 \\ \mathrm{C} & 0.67569423 & -2.60825916 & -0.18911783 \\ \mathrm{C} & 1.37422154 & -3.83404817 & -0.19793959 \\ \mathrm{C} & 2.76559739 & -3.74414482 & -0.20457957 \\ \mathrm{C} & 3.40116022 & -2.49811727 & -0.20213889 \\ \mathrm{H} & 3.36296327 & 4.63123546 & -0.17407442 \\ \mathrm{H} & 3.37200820 & -4.64768131 & -0.21114540 \\ \mathrm{C} & -1.46836014 & 3.81105141 & -0.13438454 \\ \mathrm{C} & -0.76951534 & 2.58576081 & -0.14115494 \\ \mathrm{C} & -2.71365124 & 1.27029105 & -0.12043158 \\ \mathrm{C} & -3.49539213 & 2.47485478 & -0.11044298 \\ \mathrm{C} & -2.85967833 & 3.72075251 & -0.11800404 \\ \mathrm{C} & -3.38399544 & -0.01524330 & -0.11815674 \\ \mathrm{C} & -2.71073491 & -1.29945089 & -0.14070828 \\ \mathrm{C} & -0.76478017 & -2.60987125 & -0.17654121 \\ \mathrm{C} & -1.46090295 & -3.83690108 & -0.17577466 \\ \mathrm{C} & -2.85233455 & -3.74996040 & -0.15881859 \\ \mathrm{C} & -3.49039583 & -2.50541039 & -0.14041834 \\ \mathrm{H} & -3.46619199 & 4.62418779 & -0.11052089 \\ \mathrm{H} & -3.45694158 & -4.65470016 & -0.15778419 \\ & & & \\ \mathrm{C} & & & \\ \mathrm{C} & & & \end{array}$

$\begin{array}{lrrr}\text { C } & -5.54557256 & -1.23924154 & -0.09763885 \\ \text { H } & -6.63000640 & -1.17096458 & -0.08111605 \\ \mathrm{C} & 4.82522426 & 2.41870081 & -0.19056826 \\ \mathrm{H} & 5.38072086 & 3.35292568 & -0.19093346 \\ \mathrm{C} & 4.70236013 & -0.00667392 & -0.19752146 \\ \mathrm{C} & 4.83047560 & -2.43193052 & -0.20842954 \\ \mathrm{H} & 5.38807086 & -3.36488550 & -0.21469258 \\ \mathrm{C} & 5.45387206 & -1.22769170 & -0.20612530 \\ \mathrm{H} & 6.53828935 & -1.15689269 & -0.21086719 \\ \mathrm{C} & 5.45132875 & 1.21593479 & -0.19749258 \\ \mathrm{H} & 6.53588355 & 1.14752243 & -0.20390066 \\ \mathrm{C} & -5.54795892 & 1.20434200 & -0.08300612 \\ \mathrm{H} & -6.63226307 & 1.13371540 & -0.06755145 \\ \mathrm{C} & 0.62640908 & 5.05408445 & -0.15354114 \\ \mathrm{C} & -0.73090691 & 5.05295485 & -0.14161511 \\ \mathrm{H} & -1.29635179 & 5.98083081 & -0.13635421 \\ \mathrm{C} & 0.63642035 & -5.07588591 & -0.19892427 \\ \mathrm{C} & -0.72087571 & -5.07727747 & -0.18866132 \\ \mathrm{H} & -1.28451392 & -6.00628422 & -0.18856659 \\ \mathrm{H} & 1.20184343 & -6.00377983 & -0.20708194 \\ \mathrm{H} & 1.19002008 & 5.98310567 & -0.15780559 \\ \mathrm{Co} & -0.04489960 & -0.01274742 & -0.13026946 \\ \mathrm{~N} & -1.36513316 & 1.37610123 & -0.13148024 \\ \mathrm{~N} & -1.36279128 & -1.40226879 & -0.16125396 \\ \mathrm{~N} & 1.27146469 & -1.39942589 & -0.18578311 \\ \mathrm{~N} & 1.26842347 & 1.37861634 & -0.16581216\end{array}$




$\begin{array}{lrrrrrrr}\mathrm{C} & -4.92445543 & 2.40861572 & -0.09093148 & \mathrm{O} & 0.00029367 & -0.11755008 & 2.13708921 \\ \mathrm{H} & -5.48194802 & 3.34158816 & -0.08239952 & \mathrm{O} & 1.34155690 & 0.07441721 & 2.57675740 \\ \mathrm{C} & -4.79687519 & -0.01666950 & -0.09866265 & \mathrm{H} & 1.47523850 & -0.69978120 & 3.15871261 \\ \mathrm{C} & -4.91958706 & -2.44206653 & -0.11838265 & \mathrm{H} & -0.48745126 & 0.59504437 & 2.59888465\end{array}$




\section{Supplementary References}

1. Achar, B. N., Fohlen, G. M., Parker, J. A. \& Keshavayya, J. Synthesis and structural studies of metal(II) 4,9,16,23-phthalocyanine tetraamines. Polyhedron 6, 1463-1467; 10.1016/S02775387(00)80910-9 (1987).

2. Alves, M. C. M., Dodelet, J. P., Guay, D., Ladouceur, M. \& Tourillon, G. Origin of the electrocatalytic properties for oxygen reduction of some heat-treated polyacrylonitrile and phthalocyanine cobalt compounds adsorbed on carbon black as probed by electrochemistry and x-ray absorption spectroscopy. J. Phys. Chem. 96, 10898-10905; 10.1021/j100205a054 (1992).

3. Chang, Q. et al. Determination of hydrogen peroxide with the aid of peroxidase-like $\mathrm{Fe}_{3} \mathrm{O}_{4}$ magnetic nanoparticles as the catalyst. Microchim. Acta. 165, 299-305; 10.1007/s00604-008-0133-z (2009).

4. Kattel, S., Atanassov, P. \& Kiefer, B. Catalytic activity of Co- $\mathrm{N}_{\mathrm{x}} / \mathrm{C}$ electrocatalysts for oxygen reduction reaction: A density functional theory study. Phys. Chem. Chem. Phys. 15, 148-153; 10.1039/C2CP42609A (2013).

5. Wu, G. et al. Synthesis-structure-performance correlation for polyaniline-Me-C non-precious metal cathode catalysts for oxygen reduction in fuel cells. J. Mater. Chem. 21, 11392; 10.1039/C0JM03613G (2011).

6. He, L., Weniger, F., Neumann, H. \& Beller, M. Synthesis, characterization, and application of metal nanoparticles supported on nitrogen-doped carbon: Catalysis beyond electrochemistry. Angew. Chem. Int. Ed. 55, 12582-12594; 10.1002/anie.201603198 (2016).

7. Chong, L. et al. Ultralow-loading platinum-cobalt fuel cell catalysts derived from imidazolate frameworks. Science 362, 1276-1281; 10.1126/science.aau0630 (2018).

8. Zitolo, A. et al. Identification of catalytic sites in cobalt-nitrogen-carbon materials for the oxygen reduction reaction. Nat. Commun. 8, 957; 10.1038/s41467-017-01100-7 (2017).

9. Li, J. et al. Atomically dispersed manganese catalysts for oxygen reduction in proton-exchange membrane fuel cells. Nat. Catal. 1, 935-945; 10.1038/s41929-018-0164-8 (2018).

10. Weisz, P. B. \& Prater, C. D. Interpretation of measurements in experimental catalysis. Adv. Catal. 6, 143-196; 10.1016/S0360-0564(08)60390-9 (1954).

11. Vannice, M. A. Kinetics of catalytic reactions (Springer US, Boston, MA, 2005).

12. Ternan, M. The diffusion of liquids in pores. Can. J. Chem. Eng. 65, 244-249; 10.1002/cjce.5450650208 (1987).

13. Chantong, A. \& Massoth, F. E. Restrictive diffusion in aluminas. AIChE J. 29, 725-731; 10.1002/aic.690290505 (1983).

14. Wilke, C. R. \& Chang, P. Correlation of diffusion coefficients in dilute solutions. AIChE J. 1, 264-270; 10.1002/aic.690010222 (1955).

15. Yaws, C. L. Chemical properties handbook. Physical, thermodynamic, environmental, transport, safety, and health related properties for organic and inorganic chemicals (McGraw-Hill, New York, London, 1999).

16. Yaws, C. L. Thermophysical properties of chemicals and hydrocarbons (William Andrew, Norwich, N.Y., 2008).

17. Fujita, M. \& Hiyama, T. Fluoride ion-catalyzed reduction of aldehydes and ketones with hydrosilanes. Synthetic and mechanistic aspects and an application to the threo-directed reduction of .alpha.substituted alkanones. J. Org. Chem. 53, 5405-5415; 10.1021/jo00258a003 (1988).

18. Rayment, E. J., Summerhill, N. \& Anderson, E. A. Synthesis of phenols via fluoride-free oxidation of 
arylsilanes and arylmethoxysilanes. J. Org. Chem. 77, 7052-7060; 10.1021/jo301363h (2012).

19. Ma, Y., Zhang, L., Luo, Y., Nishiura, M. \& Hou, Z. B $\left(\mathrm{C}_{6} \mathrm{~F}_{5}\right)_{3}$-catalyzed C-Si/Si-H cross-metathesis of hydrosilanes. J. Am. Chem. Soc. 139, 12434-12437; 10.1021/jacs.7b08053 (2017).

20. Seyferth, D. \& White, D. L. Acid-catalyzed isomerization of 1,2-bis(trimethylsilyl)benzene and related compounds. J. Am. Chem. Soc. 94, 3132-3138; 10.1021/ja00764a040 (1972).

21. Wang, K. et al. Selective manganese-catalyzed oxidation of hydrosilanes to silanols under neutral reaction conditions. Angew. Chem. Int. Ed. 58, 6380-6384; 10.1002/anie.201900342 (2019).

22. B. N. Kang, C. E. Ahn, J. C. Lee, H. B. Joo, S. S. Song, J. Y. Han. Novel synthesis method of silane compound for packaging optical devices (2015).

23. Cho, S. H. \& Hartwig, J. F. Iridium-catalyzed diborylation of benzylic C-H bonds directed by a hydrosilyl group: Synthesis of 1,1-benzyldiboronate esters. Chem. Sci. 5, 694-698; 10.1039/C3SC52824C (2014).

24. Nagai, Y., Ohtsuki, M., Nakano, T. \& Watanabe, H. Correlaiton of Hammett $\sigma$ constants with NMR parameters for substituted phenylsilanes, phenylmethylsilanes and phenyldimethylsilanes. $J$.

Organomet. Chem. 35, 81-90; 10.1016/S0022-328X(00)86885-0 (1972).

25. Lipowitz, J. Fluorine-19 substituent chemical shifts of fluorophenylsilanes. Substituent effects on silicon. J. Am. Chem. Soc. 94, 1582-1589; 10.1021/ja00760a025 (1972).

26. Ohshita, J. et al. Polymeric organosilicon systems. XXIX. Thermal properties of poly[(disilanylene)oligophenylenes]. J. Organomet. Chem. 564, 47-56; 10.1016/S0022328X(98)00702-5 (1998).

27. Omann, L. \& Oestreich, M. A catalytic $\mathrm{S}_{\mathrm{E}} \mathrm{Ar}$ approach to dibenzosiloles functionalized at both benzene cores. Angew. Chem. Int. Ed. 54, 10276-10279; 10.1002/anie.201504066 (2015).

28. Lee, M., Ko, S. \& Chang, S. Highly selective and practical hydrolytic oxidation of organosilanes to silanols catalyzed by a ruthenium complex. J. Am. Chem. Soc. 122, 12011-12012; 10.1021/ja003079g (2000).

29. Manson, B. W., Morrison, J. J., Coupar, P. I., Jaffrès, P.-A. \& Morris, R. E. Synthesis of aldehyde functionalised polyhedral oligomeric silsesquioxanes. J. Chem. Soc., Dalton Trans. 2001, 1123-1127; 10.1039/B100568P (2001).

30. Kan, S. B. J., Lewis, R. D., Chen, K. \& Arnold, F. H. Directed evolution of cytochrome c for carbonsilicon bond formation: Bringing silicon to life. Science 354, 1048-1051; 10.1126/science.aah6219 (2016).

31. Hevesi, L., Dehon, M., Crutzen, R. \& Lazarescu-Grigore, A. Kinetic control in the cleavage of unsymmetrical disilanes. J. Org. Chem. 62, 2011-2017; 10.1021/jo961131e (1997).

32. Beemelmanns, C., Husmann, R., Whelligan, D. K., Özçubukçu, S. \& Bolm, C. Planar-chiral bissilanols and diols as H-bonding asymmetric organocatalysts. Eur. J. Org. Chem. 2012, 3373-3376; 10.1002/ejoc.201200548 (2012).

33. Chen, C.-H. et al. Controlling conformations in alternating dialkylsilylene-spaced donor-acceptor copolymers by a cooperative Thorpe-Ingold effect and polymer folding. Chem. Eur. J. 18, 334-346; 10.1002/chem.201102032 (2012).

34. Naka, A. et al. Preparation of branched molecules by regioselective hydrosilation of tetrakis(ethynyldimethylsilyl)silanes and some of their properties. J. Organomet. Chem. 846, 360-366; 10.1016/j.jorganchem.2017.07.015 (2017).

35. Moitra, N. et al. Surface functionalization of silica by Si-H activation of hydrosilanes. J. Am. Chem. Soc. 136, 11570-11573; 10.1021/ja504115d (2014). 
36. Itami, K., Mitsudo, K., Nishino, A. \& Yoshida, J. Metal-catalyzed hydrosilylation of alkenes and alkynes using dimethyl(pyridyl)silane. J. Org. Chem. 67, 2645-2652; 10.1021/jo0163389 (2002).

37. Sumida, Y., Harada, R., Sumida, T., Hashizume, D. \& Hosoya, T. Hydrosilyl group-directed iridiumcatalyzed peri-selective C-H borylation of ring-fused (hetero)arenes. Chem. Lett. 47, 1251-1254; 10.1246/cl.180594 (2018).

38. Wiltse, H. R., Johnson, A. N., Durand, R. J., Brennessel, W. \& Chin, R. M. Syntheses, characterization, and reactivity of diruthenium hydrido complexes. Organometallics 35, 1079-1085; 10.1021/acs.organomet.6b00067 (2016).

39. Nielsen, L. \& Skrydstrup, T. Sequential C-Si bond formations from diphenylsilane: Application to silanediol peptide isostere precursors. J. Am. Chem. Soc. 130, 13145-13151; 10.1021/ja804720p (2008).

40. N. Kazuki, M. Nirmalya, K. Kazuyoshi, H. Toyoshi. Method for producing surface-modified base material, method for producing joined body, new hydrosilane compound, surface treatment agent, surface treatment agent kit, and surface-modified base material (2017).

41. Nakamura, M., Matsumoto, Y., Toyama, M., Baba, M. \& Hashimoto, Y. Organosilicon compounds as adult T-cell leukemia cell proliferation inhibitors. Chem. Pharm. Bull. 61, 237-241; 10.1248/cpb.c1200839 (2013).

42. Rakita, P. E. \& Worsham, L. S. ${ }^{13} \mathrm{C}$ NMR studies of organosilanes: IV. vinyl- and allyl-silanes. $J$. Organomet. Chem. 139, 135-142; 10.1016/S0022-328X(00)85465-0 (1977).

43. Kim, Y. \& Chang, S. Borane-catalyzed reductive $\alpha$-silylation of conjugated esters and amides leaving carbonyl groups intact. Angew. Chem. Int. Ed. 55, 218-222; 10.1002/anie.201508669 (2016).

44. Takale, B. S. et al. Exclusive chemoselective reduction of imines in the coexistence of aldehydes using AuNPore catalyst. Org. Lett. 16, 2558-2561; 10.1021/o1500958p (2014).

45. Denmark, S. E. \& Kallemeyn, J. M. Palladium-catalyzed silylation of aryl bromides leading to functionalized aryldimethylsilanols. Org. Lett. 5, 3483-3486; 10.1021/o1035288m (2003).

46. Yoshida, H., Yamaryo, Y., Ohshita, J. \& Kunai, A. Activator-free oxidative homocoupling of organosilanes catalysed by a palladium-DPPP complex. Chem. Commun. 2003, 1510-1511; 10.1039/B303852A (2003).

47. Rayment, E. J., Mekareeya, A., Summerhill, N. \& Anderson, E. A. Mechanistic study of arylsilane oxidation through ${ }^{19}$ F NMR spectroscopy. J. Am. Chem. Soc. 139, 6138-6145; 10.1021/jacs.7b00357 (2017).

48. Everson, D. A., Jones, B. A. \& Weix, D. J. Replacing conventional carbon nucleophiles with electrophiles: Nickel-catalyzed reductive alkylation of aryl bromides and chlorides. J. Am. Chem. Soc. 134, 6146-6159; 10.1021/ja301769r (2012).

49. Takeda, D., Oyama, R. \& Yamada, S. A novel method for preparing silanols from silylmethanols. Chem. Lett. 38, 532-533; 10.1246/cl.2009.532 (2009).

50. Denmark, S. E., Smith, R. C., Chang, W.-T. T. \& Muhuhi, J. M. Cross-coupling reactions of aromatic and heteroaromatic silanolates with aromatic and heteroaromatic halides. J. Am. Chem. Soc. 131, 3104-3118; 10.1021/ja8091449 (2009).

51. Koo, J., Kim, S. H. \& Hong, S. H. Hydrogenation of silyl formates: Sustainable production of silanol and methanol from hydrosilane and carbon dioxide. Chem. Commun. 54, 4995-4998;

10.1039/C8CC02276C (2018).

52. Igarashi, M., Matsumoto, T., Sato, K., Ando, W. \& Shimada, S. Nonhydrolytic synthesis of silanols by the hydrogenolysis of benzyloxysilanes. Chem. Lett. 43, 429-431; 10.1246/cl.131079 (2014). 
53. Lyčka, A., Šnobl, D., Handlír, K., Holeček, J. \& Nádvorník, M. ${ }^{29} \mathrm{Si}$ and ${ }^{13} \mathrm{C}$ NMR spectra of some alkyldiphenylchlorosilanes, alkyldiphenylsilanoles and bis(alkyldiphenylsilyl)chromates. Collect. Czech. Chem. Commun. 47, 603-612; 10.1135/cccc19820603 (1982).

54. Goncharova, I. K. et al. Aerobic Co-/N-hydroxysuccinimide-catalyzed oxidation of $p$-tolylsiloxanes to $p$-carboxyphenylsiloxanes: Synthesis of functionalized siloxanes as promising building blocks for siloxane-based materials. J. Am. Chem. Soc. 141, 2143-2151; 10.1021/jacs.8b12600 (2019).

55. Korkin, S. et al. Phenylsilanetriol—synthesis, stability, and reactivity. J. Organomet. Chem. 686, 313320; 10.1016/S0022-328X(03)00721-6 (2003).

56. Missaghi, M. N., Galloway, J. M. \& Kung, H. H. Bis(pyridyl)siloxane-Pd(II) complex catalyzed oxidation of alcohol to aldehyde: Effect of ligand tethering on catalytic activity and deactivation behavior. Appl. Catal. A-Gen. 391, 297-304; 10.1016/j.apcata.2010.09.008 (2011).

57. Ito, M., Itazaki, M. \& Nakazawa, H. Selective boryl silyl ether formation in the photoreaction of bisboryloxide/boroxine with hydrosilane catalyzed by a transition-metal carbonyl complex. J. Am. Chem. Soc. 136, 6183-6186; 10.1021/ja500465x (2014).

58. Yoshimura, A. et al. A convenient and clean synthetic method for borasiloxanes by Pd-catalysed reaction of silanols with diborons. Chem. Commun. 53, 5822-5825; 10.1039/C7CC02420G (2017).

59. Blandez, J. F., Primo, A., Asiri, A. M., Álvaro, M. \& García, H. Copper nanoparticles supported on doped graphenes as catalyst for the dehydrogenative coupling of silanes and alcohols. Angew. Chem. Int. Ed. 53, 12581-12586; 10.1002/anie.201405669 (2014).

60. Yuan, Y., Kumar, P. \& Guo, S. Metal-Free ammonium iodide catalyzed oxidative dehydrocoupling of silanes with alcohols. Synlett 28, 1620-1623; 10.1055/s-0036-1588816 (2017).

61. Mitsudome, T. et al. Highly efficient etherification of silanes by using a gold nanoparticle catalyst: Remarkable effect of $\mathrm{O}_{2}$. Chem. Eur. J. 19, 14398-14402; 10.1002/chem.201302807 (2013).

62. Toutov, A. A., Betz, K. N., Haibach, M. C., Romine, A. M. \& Grubbs, R. H. Sodium hydroxide catalyzed dehydrocoupling of alcohols with hydrosilanes. Org. Lett. 18, 5776-5779; 10.1021/acs.orglett.6b01687 (2016).

63. Dhiman, M., Chalke, B. \& Polshettiwar, V. Organosilane oxidation with a half million turnover number using fibrous nanosilica supported ultrasmall nanoparticles and pseudo-single atoms of gold. $J$. Mater. Chem. A 5, 1935-1940; 10.1039/C6TA09434A (2017).

64. Brown, C. A., Abrahamse, M. \& Ison, E. A. Re-silane complexes as frustrated lewis pairs for catalytic hydrosilylation. Dalton. Trans. 49, 11403-11411; 10.1039/D0DT02084B (2020).

65. Lorenz, C. \& Schubert, U. An efficient catalyst for the conversion of hydrosilanes to alkoxysilanes. Chem. Ber. 128, 1267-1269; 10.1002/cber.19951281220 (1995).

66. Gao, D. \& Cui, C. N-heterocyclic carbene organocatalysts for dehydrogenative coupling of silanes and hydroxyl compounds. Chem. Eur. J. 19, 11143-11147; 10.1002/chem.201301893 (2013).

67. Ojima, Y., Yamaguchi, K. \& Mizuno, N. An efficient solvent-free route to silyl esters and silyl ethers. Adv. Synth. Catal. 351, 1405-1411; 10.1002/adsc.200900230 (2009).

68. Wang, B. et al. Active cobalt catalyst for the cleavage of benzyl ether. J. Org. Chem. 76, 9531-9535; 10.1021/jo2018284 (2011).

69. Ageshina, A. A. et al. Mixed er-NHC/phosphine Pd(II) complexes and their catalytic activity in the Buchwald-Hartwig reaction under solvent-free conditions. Dalton. Trans. 48, 3447-3452; 10.1039/C9DT00216B (2019).

70. Xu, M. et al. Synthesis of urea derivatives from $\mathrm{CO}_{2}$ and silylamines. Angew. Chem. Int. Ed. 58, 57075711; 10.1002/anie.201900058 (2019). 
71. Ferlin, F., Luque Navarro, P. M., Gu, Y., Lanari, D. \& Vaccaro, L. Waste minimized synthesis of pharmaceutically active compounds via heterogeneous manganese catalysed $\mathrm{C}-\mathrm{H}$ oxidation in flow. Green Chem. 22, 397-403; 10.1039/C9GC02961C (2020). 